\title{
CORRECTIVE ACTION DECISION DOCUMENT/CLOSURE REPORT FOR CORRECTIVE ACTION UNIT 383: AREA 12 E-TUNNEL SITES, NEVADA TEST SITE
}

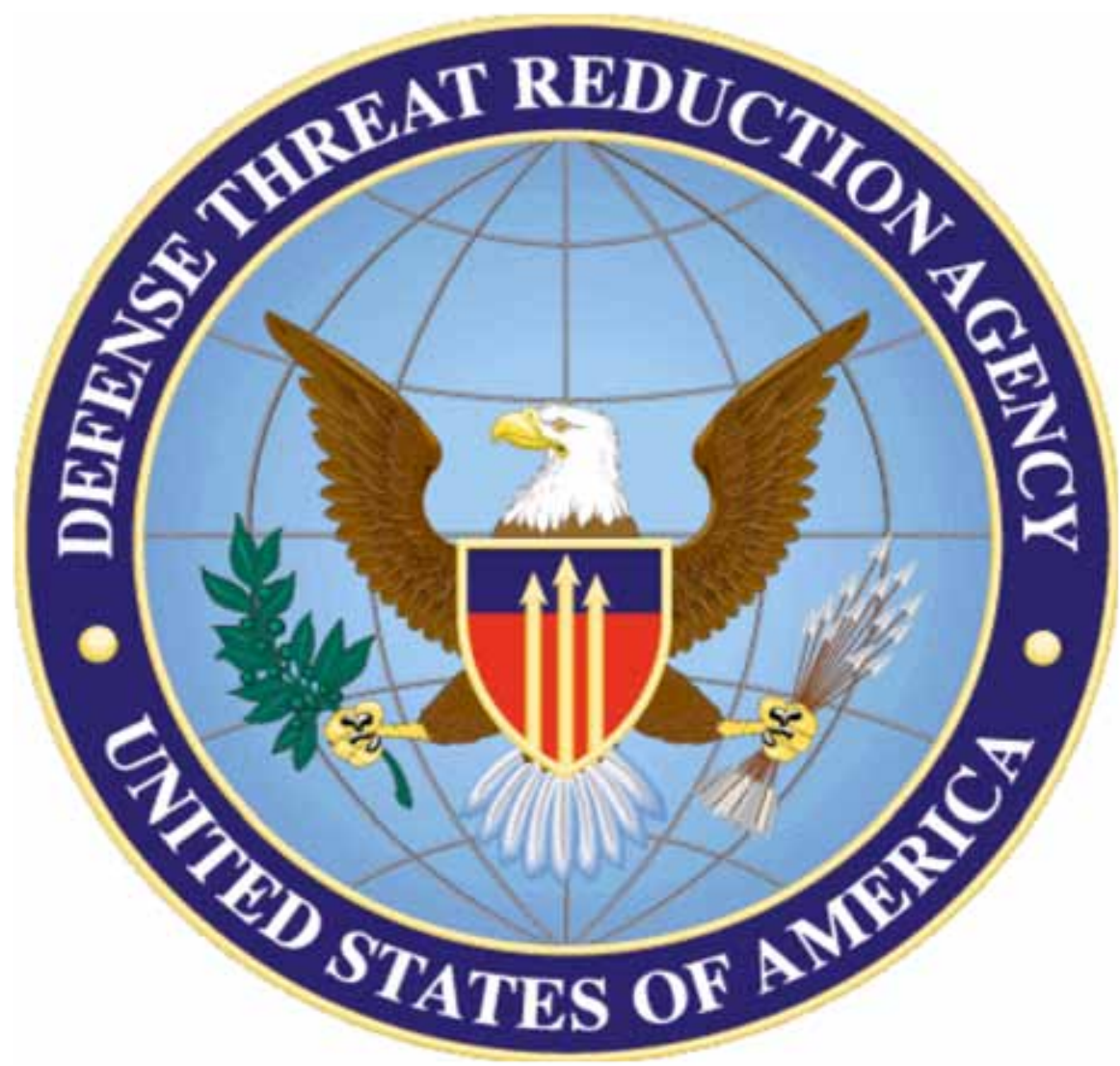

Controlled Copy No:

Revision No.: 0

February 2007

(Republished March 2010) 


\title{
DISCLAIMER
}

Reference herein to any specific commercial product, process, or service by trade name, trademark, manufacturer, or otherwise, does not necessarily constitute or imply its endorsement, recommendation, or favoring by the United States Government or any agency thereof.

This report has been reproduced directly from the best available copy.

Available for sale to the public from:

\author{
U.S. Department of Commerce \\ National Technical Information Service \\ 5301 Shawnee Road \\ Alexandria, VA 22312 \\ Telephone: (800) 553-6847 \\ Fax: (703) 605-6900 \\ E-mail: orders@ntis.gov \\ Online ordering: http://www.ntis.gov/help/ordermethods.aspx
}

Available electronically at http://www.osti.gov/bridge.

Available for a processing fee to the U.S. Department of Energy and its contractors, in paper, from:
U.S. Department of Energy
Office of Scientific and Technical Information
P.O. Box 62
Oak Ridge, TN 37831-0062
Telephone: (865) 576-8401
Fax: (865) 576-5728
E-mail: reports@adonis.osti.gov

(This document was republished. A front cover and back cover were added to reflect document numbers, disclaimer, availability, and library distribution. No other changes were made.) 


\title{
CORRECTIVE ACTION DECISION DOCUMENT/CLOSURE REPORT FOR CORRECTIVE ACTION UNIT 383: AREA 12 E-TUNNEL SITES, NEVADA TEST SITE
}

\author{
Prepared by \\ Defense Threat Reduction Agency \\ Mercury, Nevada
}

Controlled Copy No.

Revision No.: 0

February 2007

(Republished March 2010) 


\section{CORRECTIVE ACTION DECISION DOCUMENT/CLOSURE REPORT \\ FOR CORRECTIVE ACTION UNIT 383: \\ AREA 12 E-TUNNEL SITES, NEVADA TEST SITE}

\section{Approved by: APPROVED SIGNATURE}

Tiffany A. Lantow

Environmental Program Manager

Nevada Operations Office

Defense Threat Reduction Agency

Date: $2 / 21 / 2007$

Approved by: APPROVED SIGNATURE

Dat e: $2 / 21 / 2007$

Kevin J. Cabble

Acting Federal Project Director

Environmental Restoration Project

U.S. Department of Energy, National Nuclear Security Administration

Nevada Site Office 
CAU 383 CADD/CR

\section{Table of Contents}

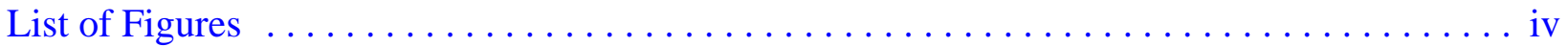

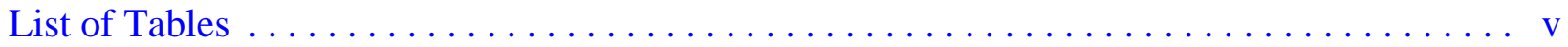

List of Acronyms and Abbreviations $\ldots \ldots \ldots \ldots \ldots \ldots \ldots \ldots \ldots \ldots \ldots \ldots \ldots \ldots$

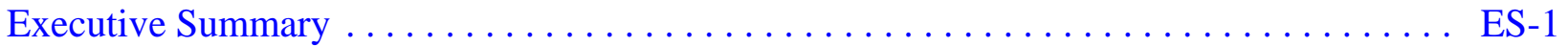

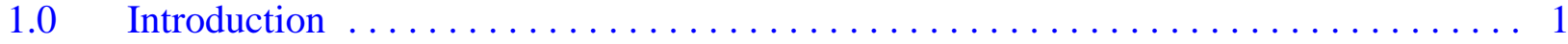

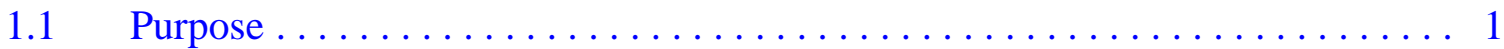

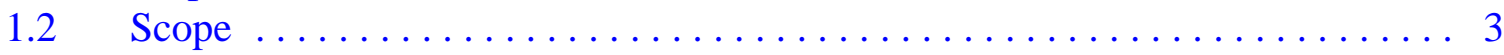

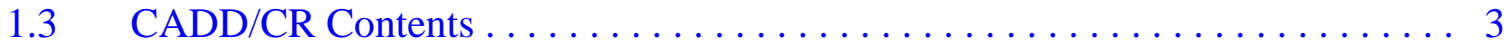

$2.0 \quad$ Corrective Action Investigation Summary $\ldots \ldots \ldots \ldots \ldots \ldots \ldots \ldots \ldots$

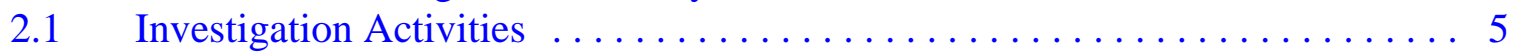

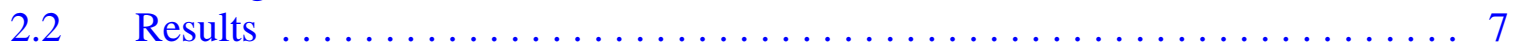

2.2.1 Summary of Analytical Data . . . . . . . . . . . . . . 7

2.2.1.1 Muckpile (CAS 12-06-06); Drainage; and Ponds 1, 2, and 3 . . 7

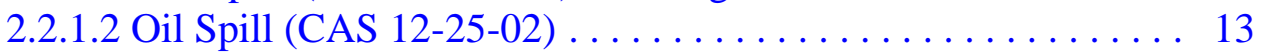

2.2.1.3 Radioactive Material (CAS 12-28-02) . . . . . . . . . . . 15

2.2.2 Data Assessment Summary ....................... 18

2.3 Justification for No Further Action $\ldots \ldots \ldots \ldots \ldots \ldots \ldots \ldots \ldots \ldots$

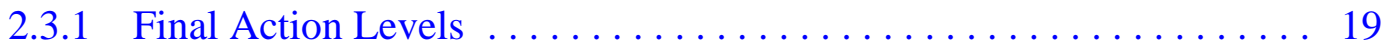

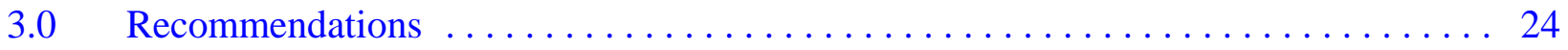

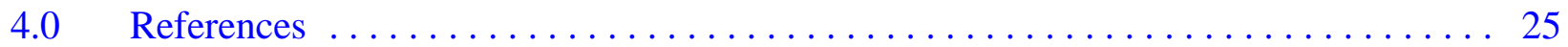

Appendix A - Corrective Action Investigation Results

Attachment A - Soil Boring Logs

Attachment B - Cross Sections

Attachment C - Analytical Results Table

Appendix B - Data Quality Objective Process for CAU 383, Area 12 E-Tunnel Sites

B.1.0 Seven-Step DQO Process Characterization of CAU 383 (Area 12 E-Tunnel Sites) .. B-1

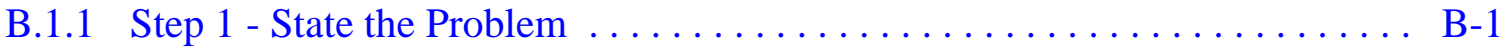

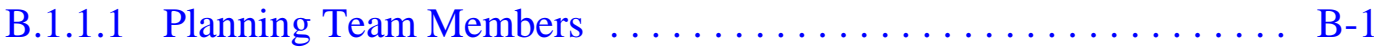


CAU 383 CADD/CR

\section{Table of Contents (Continued)}

B.1.1.2 State the Problem $\ldots \ldots \ldots \ldots \ldots \ldots \ldots \ldots \ldots$ B-2

B.1.1.3 Conceptual Site Model $\ldots \ldots \ldots \ldots \ldots \ldots \ldots \ldots \ldots \ldots$ B-2

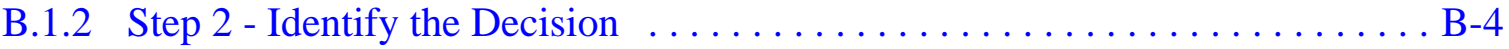

B.1.2.1 Develop the Decision Statement . . . . . . . . . . . . . . . B-4

B.1.3 Step 3 - Identify Inputs to the Decision $\ldots \ldots \ldots \ldots \ldots \ldots \ldots \ldots \ldots \ldots$

B.1.3.1 Information Needs and Information Sources . . . . . . . . . . . B-6

B.1.3.1.1 Quantitative Data ......................

B.1.3.1.2 Semiquantitative Data ................. B-6

B.1.3.1.3 Qualitative Data .................... B-8

B.1.3.1.4 Determine the Basis for the Preliminary Action Levels . . B-8

B.1.3.1.5 Potential Sampling Techniques and Appropriate

Analytical Methods ................... B-9

B.1.3.1.5.1 Radiological Surveys .............. B-9

B.1.3.1.5.2 Soil Sampling ............... B-9

B.1.4 Step 4 - Define the Boundaries of the Study $\ldots \ldots \ldots \ldots \ldots \ldots \ldots \ldots$ B-10

B.1.4.1 Define the Target Population . . . . . . . . . . . . . . B-12

B.1.4.3 Identify Practical Constraints on the Characterization . . . . . . . . . B-12

B.1.4.4 Define the Scale of the Decision Making . . . . . . . . . . . . B-12

B.1.5 Step 5 - Develop a Decision Rule . . . . . . . . . . . . . . . . B-12

B.1.5.1 Specify the Population Parameter . . . . . . . . . . . . . . B-13

B.1.5.2 Choose an Action Level . . . . . . . . . . . . . . . . . . . . B-13

B.1.5.3 Measurement and Analysis Methods . . . . . . . . . . . . B-13

B.1.5.4 Decision Rule . . . . . . . . . . . . . . . . . . . . B-13

B.1.6 Step 6 - Specify the Tolerable Limits on the Decision Errors . . . . . . . . . B B-13

B.1.6.1 False Positive Decision Error . . . . . . . . . . . . . . . B-14

B.1.6.2 False Negative Decision Error . . . . . . . . . . . . . . . B-14

B.1.6.3 Statistical Model . . . . . . . . . . . . . . . . . . . B-14

B.1.6.4 Quality Assurance/Quality Control .................. B-18

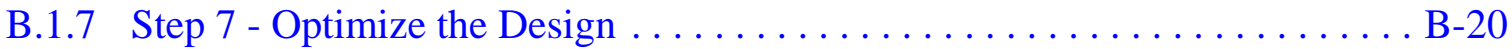

B.1.7.1 Radiological Survey Methodologies and Instruments . . . . . . . . . B-20

B.1.7.2 Intrusive Investigation $\ldots \ldots \ldots \ldots \ldots \ldots \ldots \ldots \ldots \ldots \ldots \ldots \ldots \ldots \ldots \ldots$

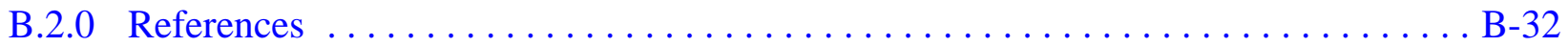


CAU 383 CADD/CR

\section{Table of Contents (Continued)}

\section{Appendix C - Data Assessment}

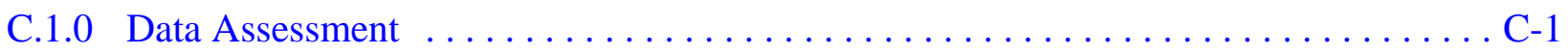

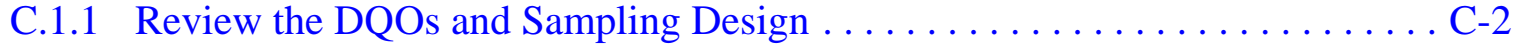

C.1.1.1 Review DQOs ............................

C.1.1.2 Sampling Design . . . . . . . . . . . . . . . . . . . C-8

C.1.2 Conduct a Preliminary Data Review .................... C-9

C.1.3 Select a Test . . . . . . . . . . . . . . . . . . . . . . . . C-9

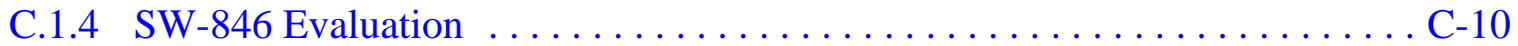

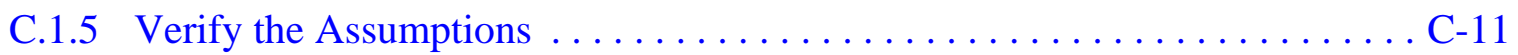

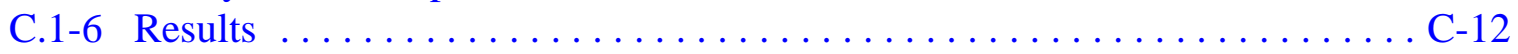

C.1.6.1 Decision Rules for CAU $383 \ldots \ldots \ldots \ldots \ldots \ldots \ldots$. . . . . . . . . .

C.2.0 References . ................................ C-14

\section{Appendix D - Risk Assessment for CAU 383}

D.1.0 Risk-Based Corrective Action Process $\ldots \ldots \ldots \ldots \ldots \ldots \ldots \ldots \ldots \ldots \ldots$ D-1

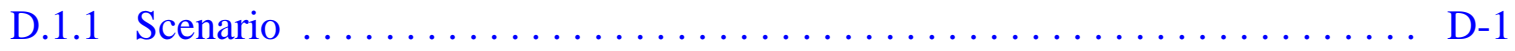

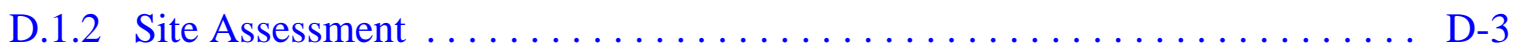

D.1.3 Site Classification and Initial Response Action . . . . . . . . . . . . D D-12

D.1.4 Development of Tier 1 Look-up Table of Risk-Based Screening

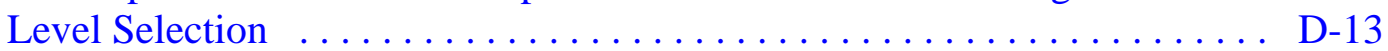

D.1.5 Exposure Pathway Evaluation $\ldots \ldots \ldots \ldots \ldots \ldots \ldots \ldots \ldots \ldots \ldots \ldots \ldots \ldots \ldots$

D.1.6 Comparison of Site Conditions with Tier 1 Risk-Based Screening Levels . . D-15

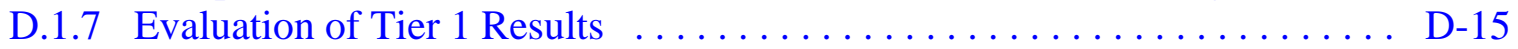

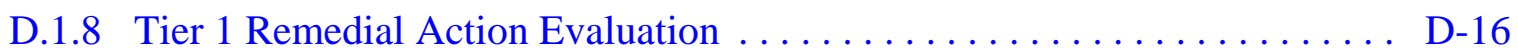

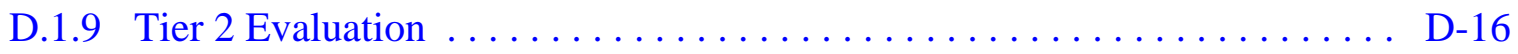

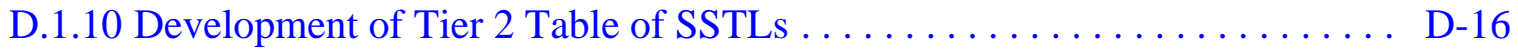

D.1.11 Comparison of Site Conditions with Tier 2 FALs . . . . . . . . . . . D-20

D.1.12 Tier 2 Remedial Action Evaluation $\ldots \ldots \ldots \ldots \ldots \ldots \ldots \ldots \ldots \ldots \ldots \ldots \ldots \ldots \ldots$

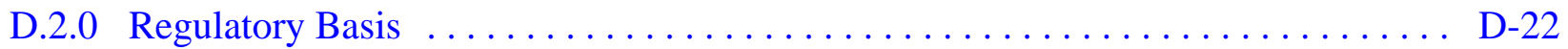

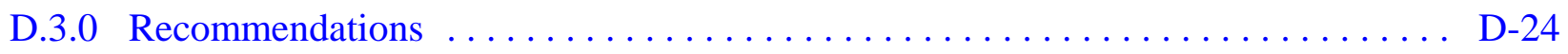


CAU $383 \mathrm{CADD} / \mathrm{CR}$

Date: February 2007

Page iv of ix

\section{Table of Contents (Continued)}

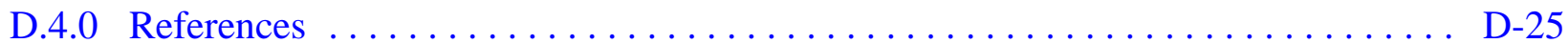

Attachment A - RESRAD Report

Exhibit 1 - RESRAD Summary Report: CAU 383 CAS 12-06-06

Exhibit 2 - RESRAD Summary Report: CAU 383 CAS 12-25-02

Exhibit 3 - RESRAD Summary Report: CAU 383 CAS 12-28-02

Appendix E - Closure Summary

E.1.0 Closure Summary $\ldots \ldots \ldots \ldots \ldots \ldots \ldots \ldots \ldots \ldots \ldots \ldots \ldots \ldots \ldots \ldots \ldots \ldots \ldots$ 
CAU 383 CADD/CR

\section{List of Figures}

Number

Title

Page

1-1 E-Tunnel Sites Location Map, Area 12, Nevada Test Site $\ldots . . . . . . . . . .2$

B.1-1 Generalized Conceptual Site Model for CAU $383 \ldots \ldots \ldots \ldots \ldots$. . . .

B.1-2 Decision Performance Curve for Cesium-137 in Muckpiles from Previous

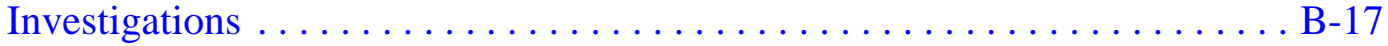

B.1-3 Decision Performance Curve for Cesium-137 in Ponds from

Previous Investigation $\ldots \ldots \ldots \ldots \ldots \ldots \ldots \ldots \ldots \ldots \ldots \ldots \ldots \ldots \ldots \ldots$

B.1-4 Decision Performance Curve for Cesium-137 in Drainages from

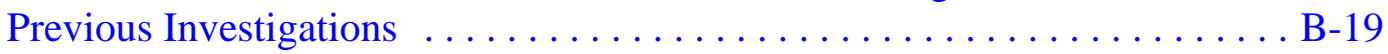

B.1-5 Borehole and Sampling Locations on the Muckpile $\ldots \ldots \ldots \ldots \ldots \ldots$ B-22

B.1-6 Borehole and Sampling Locations in the Ponds $\ldots \ldots \ldots \ldots \ldots \ldots . . \ldots \ldots$

B.1-7 Borehole and Sampling Locations in the Drainage ................ B-24

B.1-8 Sampling Strategy for the Muckpile $\ldots \ldots \ldots \ldots \ldots \ldots \ldots \ldots \ldots$ B-27

B.1-9 Sampling Strategy for the Ponds $\ldots \ldots \ldots \ldots \ldots \ldots \ldots \ldots \ldots \ldots \ldots \ldots \ldots$

B.1-10 Sampling Strategy for the Drainage $\ldots \ldots \ldots \ldots \ldots \ldots \ldots \ldots \ldots \ldots . .29$

B.1.11 Sampling Strategy for the Radioactive Material Area $\ldots . \ldots \ldots \ldots \ldots . . .6-30$

B.1-12 Sampling Strategy for the Oil Spill $\ldots \ldots \ldots \ldots \ldots \ldots \ldots \ldots \ldots \ldots$ B-31

D.1-1 ASTM Method E 1739-95 Risk-Based Corrective Action

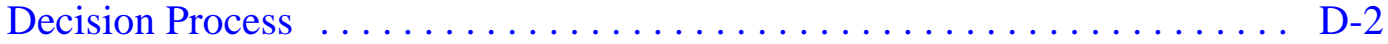

E.1-1 CAU 383 Area 12 E-Tunnel Sites 
CAU 383 CADD/CR

\section{List of Tables}

Number

Title

Page

2-1 Maximum Reported Chemical Values for CAS 12-06-06;

Ponds 1, 2, and 3; and Drainage $\ldots \ldots \ldots \ldots \ldots \ldots \ldots \ldots \ldots \ldots \ldots \ldots \ldots \ldots \ldots \ldots$

2-2 Maximum Reported Radiological Values for CAS 12-06-06;

Ponds 1 , 2, and 3; and Drainage $\ldots \ldots \ldots \ldots \ldots \ldots \ldots \ldots \ldots \ldots \ldots \ldots \ldots \ldots \ldots$

2-3 Maximum Reported Chemical Values for CAS $12-25-02 \ldots \ldots \ldots \ldots \ldots . . \ldots 14$

2-4 Maximum Reported Radiological Values for CAS 12-25-02 . . . . . . . . 16

2-5 Maximum Reported Chemical Values for CAS $12-28-02 \ldots \ldots \ldots \ldots \ldots$

2-6 Maximum Reported Radiological Values for CAS 12-28-02 . . . . . . . . 18

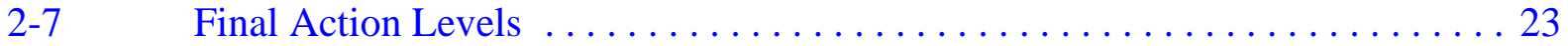

B.1-1 DQO Meeting Participants $\ldots \ldots \ldots \ldots \ldots \ldots \ldots \ldots \ldots \ldots \ldots \ldots \ldots \ldots \ldots \ldots$

B.1-2 Information Needs to Resolve the Decision $\ldots \ldots \ldots \ldots \ldots \ldots \ldots \ldots$. $\ldots \ldots$

B.1-3 Analyses to Be Performed $\ldots \ldots \ldots \ldots \ldots \ldots \ldots \ldots \ldots \ldots \ldots \ldots \ldots \ldots \ldots \ldots \ldots \ldots$

B.1-4 Proposed VOCs for Analysis $\ldots \ldots \ldots \ldots \ldots \ldots \ldots \ldots \ldots \ldots \ldots \ldots \ldots \ldots \ldots$

B.1-5 Proposed SVOCs for Analysis $\ldots \ldots \ldots \ldots \ldots \ldots \ldots \ldots \ldots \ldots \ldots \ldots \ldots$

B.1-6 Coordinates for Intrusive Investigation Locations $\ldots \ldots \ldots \ldots \ldots \ldots \ldots$. . . . .

C.1-1 CAU 383 Number of Samples Submitted per Analyte . . . . . . . . . . C-4

C.1-2 Chemical Analytes Failing Sensitivity Criteria for CAU $383 \ldots \ldots \ldots \ldots$. . . C-4

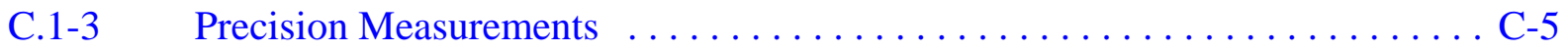


CAU 383 CADD/CR

\section{List of Tables (Continued)}

Number

C.1-4 Accuracy Measurements

C.1-5 Rejected Measurements for Completeness $\ldots \ldots \ldots \ldots \ldots \ldots \ldots \ldots$ C-8

C.1-6 SW-846 Evaluation of the Number of Samples and Comparison of $90 \%$ Confidence Level with the PAL . . . . . . . . . . . . . . C-11

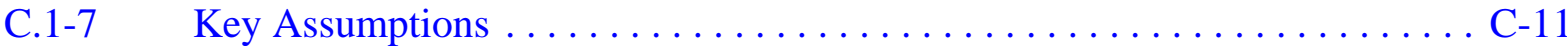

D.1-1 Maximum Reported Chemical Values for Tier 1 Comparison $\ldots . . \ldots \ldots$. . D-4

D.1-2 Maximum Reported Radiological Values for Tier 1 Comparison .......... D-9

D.1-3 COPCs Detected Above Preliminary Action Levels . . . . . . . . . . . . . D-15

D.1-4 Tier 2 SSTLs and CAU 383 Results for Hazardous Constituents of Diesel . . . . . . . . . . . . . . . . . . . . . . . . D-17

D.1-5 Tier 2 SSTLs and CAU 383 Results for Chemical Constituents . . . . . . . D D-18

D.1-6 Specific Input Parameters with Proposed NTS Standard Values . . . . . . . D-19

D.1-7 Tier 2 SSTLs and CAU 383 Results for Radiological Constituents ....... D-20 


\section{List of Acronyms and Abbreviations}

\begin{tabular}{|c|c|}
\hline Am & Americium \\
\hline ASTM & American Society for Testing and Materials \\
\hline bgs & Below ground surface \\
\hline CADD/CR & Corrective Action Decision Document/Closure Report \\
\hline CAI & Corrective Action Investigation \\
\hline CAIP & Corrective Action Investigation Plan \\
\hline CAS & Corrective Action Site \\
\hline CAU & Corrective Action Unit \\
\hline $\mathrm{cm}^{3} / \mathrm{cm}^{3}$ & Cubic centimeters per cubic centimeter \\
\hline Co & Cobalt \\
\hline COC & Contaminant of concern \\
\hline COPC & Contaminant of potential concern \\
\hline Cs & Cesium \\
\hline CSM & Conceptual site model \\
\hline day/yr & Days per year \\
\hline $\mathrm{DOE}$ & U.S. Department of Energy \\
\hline DQA & Data quality assessment \\
\hline DQI & Data quality indicator \\
\hline DQO & Data quality objective \\
\hline DRO & Diesel-range organics \\
\hline DTRA & Defense Threat Reduction Agency \\
\hline EPA & U.S. Environmental Protection Agency \\
\hline FAL & Final action level \\
\hline FFACO & Federal Facility Agreement and Consent Order \\
\hline $\mathrm{ft}$ & Foot \\
\hline $\mathrm{g} / \mathrm{g}$ & Grams per gram \\
\hline HASL & Health and Safety Laboratory \\
\hline $\mathrm{kg} /$ day & Kilograms per day \\
\hline MDC & Minimum detectable concentration \\
\hline $\mathrm{mg} / \mathrm{kg}$ & Milligrams per kilogram \\
\hline N/A & Not applicable \\
\hline NAC & Nevada Administrative Code \\
\hline NCRP & National Council of Radiation Protection and Measur \\
\hline
\end{tabular}




\section{List of Acronyms and Abbreviations (Continued)}

$\begin{array}{ll}\text { NDEP } & \text { Nevada Division of Environmental Protection } \\ \text { NTS } & \text { Nevada Test Site } \\ \text { PAL } & \text { Preliminary action level } \\ \text { pCi/g } & \text { Picocuries per gram } \\ \text { POC } & \text { Performance Objective for the Certification of Nonradioactive Hazardous Waste } \\ \text { PRG } & \text { Preliminary remediation goal } \\ \text { QA } & \text { Quality assurance } \\ \text { QC } & \text { Quality control } \\ \text { ORNL } & \text { Oak Ridge National Laboratory } \\ \text { Pu } & \text { Plutonium } \\ \text { QAPP } & \text { Quality Assurance Project Plan } \\ \text { RBSL } & \text { Risk-based screening level } \\ \text { RCRA } & \text { Resource Conservation and Recovery Act } \\ \text { RESRAD } & \text { Residual Radioactive } \\ \text { RPD } & \text { Relative percent difference } \\ \text { RT } & \text { Regulatory threshold } \\ \text { Sr } & \text { Strontium } \\ \text { SSTL } & \text { Site-specific target level } \\ \text { SVOC } & \text { Semivolatile organic compound } \\ \text { Th } & \text { Thorium } \\ \text { TPH } & \text { Total petroleum hydrocarbons } \\ \text { VOC } & \text { Volatile organic compound } \\ \text { yd } & \text { Cubic yard } \\ \text { yr } & \text { Year } \\ \text { Fg/kg } & \text { Micrograms per kilogram } \\ \end{array}$




\section{Executive Summary}

This Corrective Action Decision Document/Closure Report (CADD/CR) was prepared by the Defense Threat Reduction Agency (DTRA) for Corrective Action Unit (CAU) 383, Area 12 E-Tunnel Sites, which is the joint responsibility of DTRA and the U.S. Department of Energy (DOE), National Nuclear Security Administration Nevada Site Office (NNSA/NSO). This $\mathrm{CADD} / \mathrm{CR}$ is consistent with the requirements of the Federal Facility Agreement and Consent Order (FFACO) agreed to by the State of Nevada, the DOE, and the U.S. Department of Defense. Corrective Action Unit 383 is comprised of three Corrective Action Sites (CASs) and two adjacent areas:

- CAS 12-06-06, Muckpile

- CAS 12-25-02, Oil Spill

- CAS 12-28-02, Radioactive Material

- Drainage below the Muckpile

- $\quad$ Ponds 1, 2, and 3

The purpose of this CADD/CR is to provide justification and documentation to support the recommendation for closure with no further corrective action, by placing use restrictions at the three CASs and two adjacent areas of CAU 383. To support this recommendation, a corrective action investigation (CAI) was performed in May and June 2004. The purpose of the CAI was to fulfill the following data needs as defined during the data quality objective (DQO) process:

- Determine whether contaminants of concern (COCs) are present.

- If COCs are present, determine their nature and extent.

- Provide sufficient information and data to determine appropriate corrective actions.

The CAU 383 dataset from the CAI was evaluated based on the data quality indicator parameters. This evaluation demonstrated the quality and acceptability of the dataset for use in fulfilling the DQO data needs.

Analytes detected during the CAI were evaluated against final action levels (FALs) established in this document. Tier 2 FALS were calculated for the semivolatile organic compound (SVOC) benzo(a)pyrene and the radionuclides americium (Am)-241, cesium (Cs)-137, plutonium (Pu)-238, and Pu-239. The Tier 2 FALs were calculated for the detected SVOC (benzo(a)pyrene) and 
radionuclides using site-specific information (see Appendix D of this document). The SVOC FAL was calculated using the Oak Ridge National Laboratory (ORNL) risk calculations provided on the website http://risk.lsd.ornl.gov/cgi-bin/prg/PRG_search. The radionuclide FALs were calculated using the Residual Radioactive (RESRAD) code (version 6.21). Although all detected radionuclides at a CAS are used in the sum-of-fractions calculation and a unique Tier 2 action level is developed for all radionuclides, only the five radionuclides that initially exceeded Tier 1 levels have a Tier 2-based FAL. The CAS-specific FALS established for these radionuclides are the site-specific target levels listed in Table D.1-7.

Based on the field investigation, no chemical or radiological constituents exceeded their FALs at CAS 12-28-02, the drainage, and the ponds. However, the following contaminants were determined to be present at concentrations exceeding their corresponding FALs:

- Benzo(a)pyrene at one location in CAS 12-25-02

- Am-241, Cs-137, Pu-238, and Pu-239 at CAS 12-06-06

Based on the data and risk evaluations (Tier 2 evaluations), the DQO data needs presented in the Corrective Action Investigation Plan (CAIP) were met, and the data accurately represent the radiological and chemical risks present at CAU 383. Based on the results of the CAI data evaluation and results showing there was no lateral or vertical migration, it was determined that the closure in place with use restrictions is the appropriate corrective action for CAU 383 and that use restrictions will effectively control exposure to future land users. Therefore, DTRA and NNSA/NSO provide the following recommendations:

- Close COCs in place at CAS 12-06-06 and 12-25-02 with use restrictions.

- $\quad$ No further action for CAU 383.

- A Notice of Completion be issued to DTRA and NNSA/NSO by the Nevada Division of Environmental Protection for closure of CAU 383.

- $\quad$ Move CAU 383 from Appendix III to Appendix IV of the FFACO. 


\subsection{Introduction}

This Corrective Action Decision Document/Closure Report (CADD/CR) has been prepared for Corrective Action Unit (CAU) 383, Area 12 E-Tunnel Sites, Nevada Test Site (NTS), Nevada. The corrective action proposed in this document is in accordance with the Federal Facility Agreement and Consent Order (FFACO) that was agreed to by the State of Nevada, the U.S. Department of Energy (DOE), and the U.S. Department of Defense (FFACO, 1996).

The E-Tunnel Muckpile is identified under FFACO classification as CAU 383, Area 12 E-Tunnel Sites. The CAU consists of three Corrective Action Sites (CASs): 12-06-06, Muckpile; 12-25-02, Oil Spill; and 12-28-02 Radioactive Material. In addition, the drainage between the Muckpile and the ponds and Ponds 1, 2, and 3 were included in the investigation. The E-Tunnel Sites are located approximately 42 miles north of Mercury in Area 12 of the NTS (Figure 1-1).

This CADD/CR describes the corrective action that is selected as a result of the investigation activities and the rationale for its selection. The rationale consists of a justification for closure in place with use restrictions in accordance with Sections IV.8 and IV.11 of the FFACO (1996).

\subsection{Purpose}

The purpose of this CADD/CR is to provide justification for the closure of CAU 383 with no further action based on the results of the corrective action investigation (CAI). The CAI was conducted in accordance with the Corrective Action Investigation Plan (CAIP) for Corrective Action Unit 383: Area 12 E-Tunnel Sites, Nevada Test Site (DTRA, 2003), which provides additional information relating to the history, planning, and scope of the investigation.

The E-Tunnel was used for nine nuclear weapons tests between 1958 and 1977. The Muckpile contains an estimated 355,000 cubic yards $\left(\mathrm{yd}^{3}\right)$ of material consisting primarily of mining debris (rock) generated during excavation of the underground facilities; construction/re-entry debris comprise the remainder of the Muckpile. The drainage and Ponds 1, 2, and 3, which were included in this investigation, trend easterly and extend approximately 350 feet (ft) down canyon from the Muckpile. Corrective Action Site 12-28-02 (Radioactive Material) is located at the E-Tunnel main portal and is part of the Muckpile. Corrective Action Site 12-25-02 (Oil Spill) is located on the blower pad, which is on a bench approximately $100 \mathrm{ft}$ above the E-Tunnel south portal. 


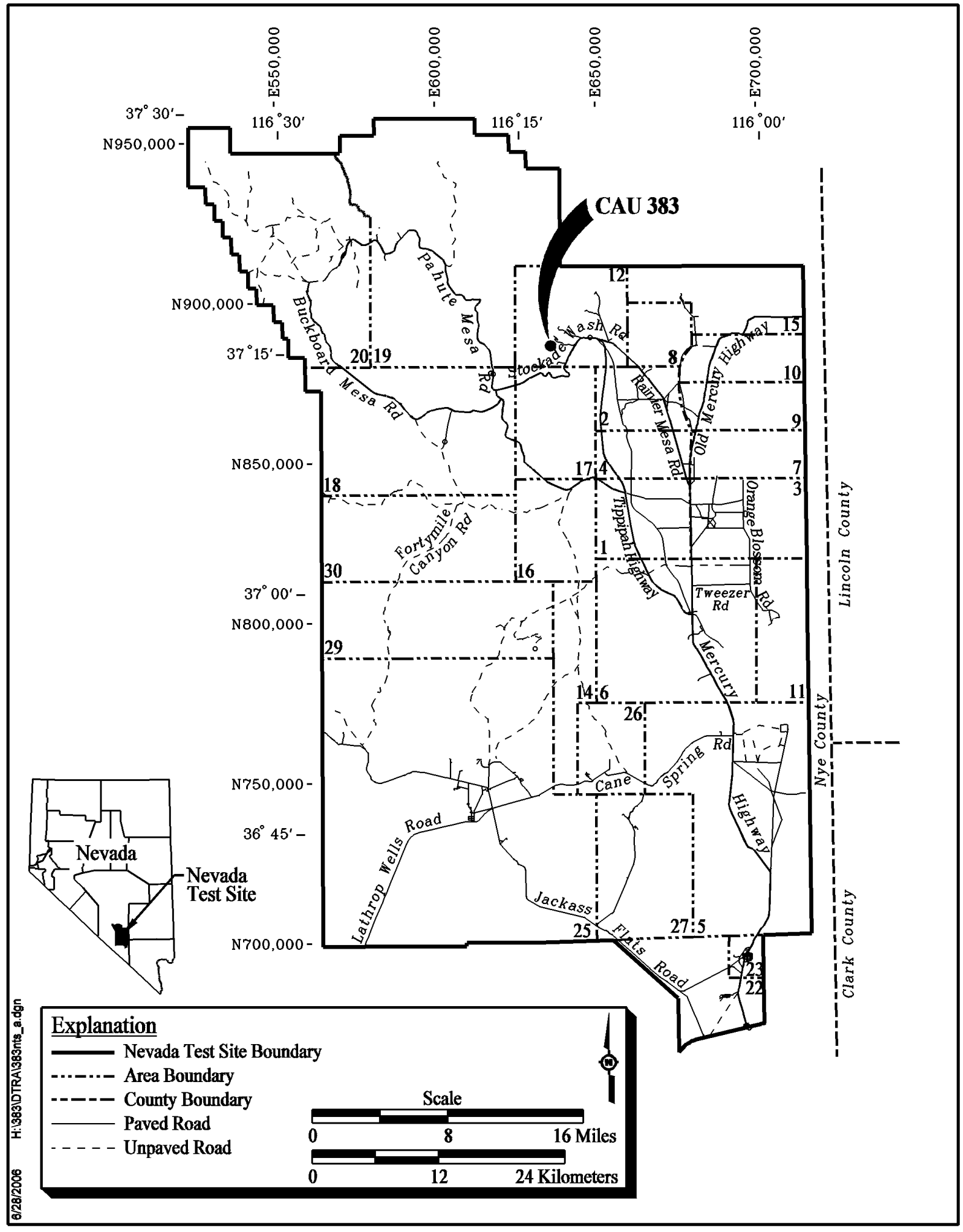

Figure 1-1

E-Tunnel Sites Location Map, Area 12, Nevada Test Site 


\subsection{Scope}

The scope of this CADD/CR is to justify and recommend that no further corrective action is required at CAU 383, Area 12 E-Tunnel Sites. To achieve this scope, the following actions were implemented:

- Evaluation of current site conditions, including the nature and extent of contaminants of concern (COCs).

- Closure in place with use restrictions to prevent exposure of industrial and construction workers to unacceptable risks.

\subsection{CADD/CR Contents}

This CADD/CR is divided into the following sections:

- Section 1.0 - Introduction: Summarizes of the purpose, scope, and contents of this $\mathrm{CADD} / \mathrm{CR}$.

- Section 2.0 - CAI Summary: Summarizes the investigation field activities, the results of the investigation, and the data quality objective (DQO) assessment.

- Section 3.0 - Recommendation: States why no further action is required.

- Section 4.0 - References: Lists all documents referenced in the CADD/CR.

- Appendix A - Corrective Action Investigation Results

- Appendix B - Data Quality Objective Process for CAU 383, Area 12 E-Tunnel Sites

- Appendix C - Data Assessment

- $\quad$ Appendix D - Risk Assessment for CAU 383

All work was performed in accordance with the following documents:

- Corrective Action Investigation Plan for Corrective Action Unit 383: E-Tunnel Sites, Nevada Test Site, Nevada, Rev. 0 (DTRA, 2003)

- Industrial Sites Quality Assurance Project Plan (QAPP), Nevada Test Site, Nevada, Rev. 3 (NNSA/NV, 2002)

- Federal Facility Agreement and Consent Order (FFACO, 1996) 
- SNJV Site-Specific Health and Safety Plan, DTRA CAU 383 E-Tunnel Muckpile, Nevada Test Site, Rev 0. (SNJV, 2003)

The DQOs identified in the CAIP are as follows:

- Determine whether COCs are present.

- If COCs are present, determine their nature and extent. Obtain sufficient information to evaluate potential corrective action alternatives.

The data quality indicators (DQIs) as defined in the Industrial Sites QAPP (NNSA/NV, 2002) were achieved, and the DQOs established in the CAIP were met. 


\subsection{Corrective Action Investigation Summary}

The following sections describe and summarize the results of the CAI activities conducted at CAU 383. For detailed CAI results, refer to Appendix A.

\subsection{Investigation Activities}

Corrective action investigation activities were performed at the E-Tunnel Sites as set forth in the CAIP (DTRA, 2003) between May 19 and June 24, 2004. The purpose of the CAI was to determine whether or not the E-Tunnel Muckpile and ponds; the underlying native soils; and/or the drainage below the Muckpile, the Oil Spill, and Radioactive Material have been impacted by COCs at concentrations that exceed final action levels (FALs), and to provide sufficient information and data to justify closure in place with administrative controls. In accordance with the CAIP (DTRA, 2003), the following tasks were performed:

- Radiological surveys - Before commencing drilling and sampling, walk-over and drive-over radiological surveys were conducted to identify surface and near-surface areas with elevated readings.

- Sampling of the Muckpile (muck) and underlying native material (CAS 12-06-06) Thirty-four locations were drilled or hand sampled on the Muckpile to characterize the muck and the native material under the Muckpile. A total of 1,039.5 ft was drilled in 32 boreholes ranging from 1.5 to $91.5 \mathrm{ft}$ deep. Two locations were sampled by hand. Up to three samples were collected from each location. One location was drilled but not sampled. During the characterization, 66 environmental samples were collected. These included 41 muck samples and 25 native material samples. All samples were sent to Paragon Analytics, Inc., for analysis. Analyses performed included volatile organic compounds (VOCs), semivolatile organic compounds (SVOCs), total petroleum hydrocarbons (TPH)-diesel-range organics (DRO), total Resource Conservation and Recovery Act (RCRA) metals, beryllium, and radionuclides (including strontium [Sr]-90 and isotopic plutonium [Pu]).

- Sampling of the drainage (included with CAS 12-06-06) - Thirty-five locations were drilled or hand sampled in the drainage to characterize the site. A total of $10 \mathrm{ft}$ was drilled in two boreholes $5 \mathrm{ft}$ deep. Thirty-three locations were sampled by hand. One sample was collected from each location. During the characterization, 35 environmental samples were collected. All samples were sent to Paragon Analytics, Inc., to be analyzed for the same analyses as the Muckpile samples. 
- Sampling of the pond sediments and underlying native material (included with CAS 12-06-06) - Nine locations were drilled or hand sampled in the ponds to characterize the sediment and the native material under the sediments. In Pond 1, a total of $80 \mathrm{ft}$ was drilled in five boreholes ranging from depths of 18 to $21 \mathrm{ft}$. Due to soil wetness in Ponds 2 and 3, only two locations were sampled in each pond, and each of the four locations was hand sampled. During the characterization, 14 environmental samples were collected. These included nine sediment samples and five native material samples. All samples were sent to Paragon Analytics, Inc., to be analyzed for the same analyses as the Muckpile samples.

- Sampling of the Oil Spill (CAS 12-25-02) - One sample was collected from each of eight locations in the Oil Spill to characterize the site. All samples were sent to Paragon Analytics, Inc., to be analyzed for the same analyses as the Muckpile samples.

- Sampling of the Radioactive Material (CAS 12-28-02) - One sample was collected from each of four locations in the Radioactive Material area to characterize the site. All samples were sent to Paragon Analytics, Inc., to be analyzed for the same analyses as the Muckpile samples.

- Additional Sampling - Background and preliminary soil samples were collected at eight locations on and around the Muckpile. Three background samples were hand collected at three undisturbed locations around the Muckpile. Five preliminary samples were collected by hand at five biased locations on the Muckpile. Seven of the samples were analyzed for beryllium, RCRA metals, and radionuclides; and one sample, 383GB0901, was analyzed only for radionuclides (Sr-90, isotopic Pu, and gamma spec). Analysis was conducted by Paragon Analytics, Inc.

The conceptual site model (CSM) postulated the following:

- The majority of the Muckpile does not contain contaminants of potential concern (COPCs), and if any are present, they are probably isolated.

- The location of buried COPCs cannot be predicted, but surface radiological surveys can be used to increase the probability of finding impacted areas.

- It is possible, but unlikely based on experience, that the native soil beneath the Muckpile and ponds has been impacted by downward migration of COPCs.

- Releases to the Muckpile from surface activities of concern (equipment maintenance and storage of equipment and petroleum products) may be locally significant. These releases, if present, are expected to have limited lateral and vertical extent. 
- The most likely pathway for migration of contaminants away from the Muckpile is in stormwater runoff. Migration might also result when precipitation infiltrates into the thin, peripheral portions of the Muckpile and enters the native material and/or drains, downgrading along the contact between the fill and the underlying native alluvium or bedrock.

Although the first CSM postulate was shown to be incorrect, the other postulates and the assumptions used to develop the CSM (DTRA, 2003) were proven to be correct by the CAI. The fact that the contamination is more widespread than anticipated does not invalidate the rest of the model. The more important concepts of the model, that there was no leaching into the native material and that migration is taking place from the Muckpile in storm runoff, were shown to be correct.

\subsection{Results}

The following is a summary of the data obtained during the CAI.

\subsubsection{Summary of Analytical Data}

Chemical and radiological results for investigation samples collected at each of the CASs are summarized in Sections 2.2.1.1 through 2.2.1.3. Preliminary action levels (PALs) for the CAU 383 investigation were determined during the DQO process and are discussed in Section 3.3 of the CAIP (DTRA, 2003). Final action levels are presented in Section 2.3.1, and details about the methods used during this investigation and a comparison of the environmental sample results to the FALs are presented in Appendix D.

\subsubsection{Muckpile (CAS 12-06-06); Drainage; and Ponds 1, 2, and 3}

During the CAI, TPH-DRO was found above the PAL of 100 milligrams per kilogram (mg/kg) in three soil samples and one pond sediment sample, so it was moved into a Tier 2 evaluation. The Tier 2 evaluation involved evaluating the individual hazardous constituents of TPH-DRO as prescribed in the American Society for Testing and Materials (ASTM) Method E 1739-95, Section 6.4.3, “Use of Total Petroleum Hydrocarbon Measurements” (ASTM, 1995). The Tier 2 evaluation determined that none of the hazardous constituents of TPH-DRO exceeded their respective PALs. Therefore, for those chemical constituents, the PALs are identified as the FALs.

For the chemical constituents, only benzo(a)pyrene was detected in one sample (383MB1000.5) that exceeded the PAL of $0.21 \mathrm{mg} / \mathrm{kg}$, so it was moved to a Tier 2 analysis, and the Oak Ridge 
National Laboratory (ORNL) website was used to calculate the site-specific FAL (ORNL, 2004). For all other constituents, the PALs are identified as the FALs. The maximum concentration of each detected chemical contaminant at this CAS is listed in Table 2-1.

Table 2-1

Maximum Reported Chemical Values for CAS 12-06-06; Ponds 1, 2, and 3; and Drainage

(Page 1 of 4)

\begin{tabular}{|c|c|c|c|c|c|}
\hline Contaminant of Concern & Results & Sample Number & $\begin{array}{c}\text { Depth } \\
\text { (ft bgs) }\end{array}$ & FAL & Units \\
\hline \multicolumn{6}{|c|}{ Muckpile } \\
\hline Arsenic & 6 & 383МН0353.5 & $53-54$ & 23 & $\mathrm{mg} / \mathrm{kg}$ \\
\hline Barium & 7,000 & 383МB0600.5 & $0-1$ & 67,000 & $\mathrm{mg} / \mathrm{kg}$ \\
\hline Beryllium & 2.4 & 383MB1200.5 & $0-1$ & 1,900 & $\mathrm{mg} / \mathrm{kg}$ \\
\hline Cadmium & 15 & 383МB0400.5 & $0-1$ & 450 & $\mathrm{mg} / \mathrm{kg}$ \\
\hline Chromium & 33 & 383МВ0200.5 & $0-1$ & 450 & $\mathrm{mg} / \mathrm{kg}$ \\
\hline Lead & 160 & 383МH1734.5 & $34-35$ & 800 & $\mathrm{mg} / \mathrm{kg}$ \\
\hline Selenium & 0.99 & 383МB0559.5 & $59-60$ & 310 & $\mathrm{mg} / \mathrm{kg}$ \\
\hline Silver & 2.8 & 383МH1734.5 & $34-35$ & 5,100 & $\mathrm{mg} / \mathrm{kg}$ \\
\hline Mercury & 0.51 & 383МН0353.5 & $53-54$ & 5,100 & $\mathrm{mg} / \mathrm{kg}$ \\
\hline Diesel-Range Organics ${ }^{1}$ & 700 & 383МH0600.5 & $0-1$ & 100 & $\mathrm{mg} / \mathrm{kg}$ \\
\hline 1,2,4-Trimethylbenzene & 0.0072 & 383МВ0800.5 & $0-1$ & 170 & $\mathrm{mg} / \mathrm{kg}$ \\
\hline 1,3,5-Trimethybenzene & 0.0072 & 383MB0800.5 & $0-1$ & 70 & $\mathrm{mg} / \mathrm{kg}$ \\
\hline Acetone & 0.018 & 383MH1422.0 & $21.5-22.5$ & 54,000 & $\mathrm{mg} / \mathrm{kg}$ \\
\hline Hexachlorobutadiene & 0.0015 & 383MH1503.5 & $3-4$ & 22 & $\mathrm{mg} / \mathrm{kg}$ \\
\hline Ethylbenzene & 0.12 & 383МВ0800.5 & $0-1$ & 400 & $\mathrm{mg} / \mathrm{kg}$ \\
\hline M+P-Xylene & 1.5 & 383MB0800.5 & $0-1$ & 420 & $\mathrm{mg} / \mathrm{kg}$ \\
\hline Methylene Chloride & 0.0013 & 383МВ0586.0 & $0-1$ & 21 & $\mathrm{mg} / \mathrm{kg}$ \\
\hline O-Xylene & 1 & 383МВ0800.5 & $0-1$ & 420 & $\mathrm{mg} / \mathrm{kg}$ \\
\hline Acenaphthene & 0.022 & 383MB1000.5 & $0-1$ & 29,000 & $\mathrm{mg} / \mathrm{kg}$ \\
\hline Anthracene & 0.17 & $383 \mathrm{MB} 1000.5$ & $0-1$ & 100,000 & $\mathrm{mg} / \mathrm{kg}$ \\
\hline Benzo(A)Anthracene & 1.2 & 383MB1000.5 & $0-1$ & 2.1 & $\mathrm{mg} / \mathrm{kg}$ \\
\hline Benzo(A)Pyrene & 1.3 & 383MB1000.5 & $0-1$ & 152 & $\mathrm{mg} / \mathrm{kg}$ \\
\hline Benzo(B)Fluoranthene & 1.7 & 383MB1000.5 & $0-1$ & 2.1 & $\mathrm{mg} / \mathrm{kg}$ \\
\hline Benzo(K)Fluoranthene & 0.65 & 383MB1000.5 & $0-1$ & 21 & $\mathrm{mg} / \mathrm{kg}$ \\
\hline
\end{tabular}


Table 2-1

Maximum Reported Chemical Values for CAS 12-06-06;

Ponds 1, 2, and 3; and Drainage

(Page 2 of 4 )

\begin{tabular}{|c|c|c|c|c|c|}
\hline Contaminant of Concern & Results & Sample Number & $\begin{array}{c}\text { Depth } \\
\text { (ft bgs) } \\
\end{array}$ & FAL & Units \\
\hline Bis(2-Ethylhexyl)Phthalate & 0.22 & 383МB1425.5 & $25-26$ & 120 & $\mathrm{mg} / \mathrm{kg}$ \\
\hline Butyl Benzyl Phthalate & 0.035 & $383 \mathrm{MH} 1100.5$ & $0-1$ & 100,000 & $\mathrm{mg} / \mathrm{kg}$ \\
\hline Carbazole & 0.057 & 383MB1000.5 & $0-1$ & 86 & $\mathrm{mg} / \mathrm{kg}$ \\
\hline Chrysene & 1 & 383MB1000.5 & $0-1$ & 210 & $\mathrm{mg} / \mathrm{kg}$ \\
\hline Dibenzofuran & 0.065 & 383MB1000.5 & $0-1$ & 1,600 & $\mathrm{mg} / \mathrm{kg}$ \\
\hline Fluoranthene & 1.5 & 383MB1000.5 & $0-1$ & 22,000 & $\mathrm{mg} / \mathrm{kg}$ \\
\hline Fluorene & 0.016 & 383MB1000.5 & $0-1$ & 26,000 & $\mathrm{mg} / \mathrm{kg}$ \\
\hline Ideno(1,2,3-Cd)Pyrene & 0.63 & 383MB1000.5 & $0-1$ & 2.1 & $\mathrm{mg} / \mathrm{kg}$ \\
\hline Naphthalene & 0.019 & $383 \mathrm{MB} 1000.5$ & $0-1$ & 190 & $\mathrm{mg} / \mathrm{kg}$ \\
\hline Pyrene & 1.6 & $383 \mathrm{MB} 1000.5$ & $0-1$ & 29,000 & $\mathrm{mg} / \mathrm{kg}$ \\
\hline \multicolumn{6}{|c|}{ Ponds } \\
\hline Arsenic & 14 & 383РН0101.0 & $0.5-1.5$ & 23 & $\mathrm{mg} / \mathrm{kg}$ \\
\hline Barium & 6,300 & 383РН0101.0 & $0.5-1.5$ & 67,000 & $\mathrm{mg} / \mathrm{kg}$ \\
\hline Beryllium & 2 & $383 \mathrm{PH} 0306.5$ & $6-7$ & 1,900 & $\mathrm{mg} / \mathrm{kg}$ \\
\hline Cadmium & 0.34 & 383РН0300.5 & $0-1$ & 450 & $\mathrm{mg} / \mathrm{kg}$ \\
\hline Chromium & 12 & $383 \mathrm{PH} 0101.0$ & $0.5-1.5$ & 450 & $\mathrm{mg} / \mathrm{kg}$ \\
\hline Lead & 38 & 383РВ0300.5 & $0-1$ & 800 & $\mathrm{mg} / \mathrm{kg}$ \\
\hline Silver & 0.48 & $383 \mathrm{PH} 0217.5$ & $17-18$ & 5,100 & $\mathrm{mg} / \mathrm{kg}$ \\
\hline Mercury & 0.12 & $383 \mathrm{PH} 0320.5$ & $20-21$ & 5,100 & $\mathrm{mg} / \mathrm{kg}$ \\
\hline Diesel-Range Organics ${ }^{1}$ & 330 & $383 \mathrm{PH} 0216.0$ & $15.5-16.5$ & 100 & $\mathrm{mg} / \mathrm{kg}$ \\
\hline Acetone & 0.025 & $383 \mathrm{PH} 0217.5$ & $17-18$ & 54,000 & $\mathrm{mg} / \mathrm{kg}$ \\
\hline Hexachlorobutadiene & 0.0017 & 383РН0418.5 & $18-19$ & 22 & $\mathrm{mg} / \mathrm{kg}$ \\
\hline Ethylbenzene & 0.0016 & 383РВ0400.5 & $0-1$ & 400 & $\mathrm{mg} / \mathrm{kg}$ \\
\hline M+P-Xylene & 0.0083 & 383РВ0400.5 & $0-1$ & 420 & $\mathrm{mg} / \mathrm{kg}$ \\
\hline O-Xylene & 0.0033 & 383РВ0400.5 & $0-1$ & 420 & $\mathrm{mg} / \mathrm{kg}$ \\
\hline Benzo(A)Anthracene & 0.026 & $383 \mathrm{PH} 0216.0$ & $15.5-16.5$ & 2.1 & $\mathrm{mg} / \mathrm{kg}$ \\
\hline Benzo(A)Pyrene & 0.028 & $383 \mathrm{PH} 0216.0$ & $15.5-16.5$ & 0.21 & $\mathrm{mg} / \mathrm{kg}$ \\
\hline Benzo(B)Fluoranthene & 0.059 & 383РН0216.0 & $15.5-16.5$ & 2.1 & $\mathrm{mg} / \mathrm{kg}$ \\
\hline
\end{tabular}




\section{Table 2-1 \\ Maximum Reported Chemical Values for CAS 12-06-06; \\ Ponds 1, 2, and 3; and Drainage}

(Page 3 of 4 )

\begin{tabular}{|c|c|c|c|c|c|}
\hline Contaminant of Concern & Results & Sample Number & $\begin{array}{l}\text { Depth } \\
\text { (ft bgs) }\end{array}$ & FAL & Units \\
\hline Benzo(K)Fluoranthene & 0.031 & 383РH0216.0 & $15.5-16.5$ & 21 & $\mathrm{mg} / \mathrm{kg}$ \\
\hline Bis(2-Ethylhexyl)Phthalate & 0.33 & 383РH0216.0 & $15.5-16.5$ & 120 & $\mathrm{mg} / \mathrm{kg}$ \\
\hline Chrysene & 0.066 & 383РH0216.0 & $15.5-16.5$ & 210 & $\mathrm{mg} / \mathrm{kg}$ \\
\hline Fluoranthene & 0.031 & 383PH0216.0 & $15.5-16.5$ & 22,000 & $\mathrm{mg} / \mathrm{kg}$ \\
\hline Pyrene & 0.063 & $383 \mathrm{PH} 0216.0$ & $15.5-16.5$ & 29,000 & $\mathrm{mg} / \mathrm{kg}$ \\
\hline \multicolumn{6}{|c|}{ Drainage } \\
\hline Arsenic & 5.3 & 383DH3100.5 & $0-1$ & 23 & $\mathrm{mg} / \mathrm{kg}$ \\
\hline Barium & 6,600 & 383DH3200.5 & $0-1$ & 67,000 & $\mathrm{mg} / \mathrm{kg}$ \\
\hline Beryllium & 2.1 & $383 \mathrm{DH} 1400.5$ & $0-1$ & 1,900 & $\mathrm{mg} / \mathrm{kg}$ \\
\hline Chromium & 9.2 & 383DH3200.5 & $0-1$ & 450 & $\mathrm{mg} / \mathrm{kg}$ \\
\hline Lead & 52 & 383DH0900.5 & $0-1$ & 800 & $\mathrm{mg} / \mathrm{kg}$ \\
\hline Mercury & 0.083 & $383 \mathrm{DH} 1400.5$ & $0-1$ & 5,100 & $\mathrm{mg} / \mathrm{kg}$ \\
\hline Diesel-Range Organics $^{1}$ & 68 & $383 \mathrm{DH} 3500.5$ & $0-1$ & 100 & $\mathrm{mg} / \mathrm{kg}$ \\
\hline Acetone & 0.039 & $383 \mathrm{DH} 0200.5$ & $0-1$ & 54,000 & $\mathrm{mg} / \mathrm{kg}$ \\
\hline 2-Butanone & 0.01 & 383DH0200.5 & $0-1$ & 110,000 & $\mathrm{mg} / \mathrm{kg}$ \\
\hline Ethylbenzene & 0.011 & 383DH0300.5 & $0-1$ & 400 & $\mathrm{mg} / \mathrm{kg}$ \\
\hline Hexachlorobutadiene & 0.0016 & $383 \mathrm{DH} 2700.5$ & $0-1$ & 22 & $\mathrm{mg} / \mathrm{kg}$ \\
\hline M+P-Xylene & 0.057 & 383DH0300.5 & $0-1$ & 420 & $\mathrm{mg} / \mathrm{kg}$ \\
\hline Methylene Chloride & 0.004 & 383DH0200.5 & $0-1$ & 21 & $\mathrm{mg} / \mathrm{kg}$ \\
\hline O-Xylene & 0.023 & 383DH0300.5 & $0-1$ & 420 & $\mathrm{mg} / \mathrm{kg}$ \\
\hline Styrene & 0.0036 & 383DH0300.5 & $0-1$ & 1,700 & $\mathrm{mg} / \mathrm{kg}$ \\
\hline Toluene & 0.0016 & 383DH0500.5 & $0-1$ & 520 & $\mathrm{mg} / \mathrm{kg}$ \\
\hline Benzo(A)Anthracene & 0.066 & $383 \mathrm{DH} 2600.5$ & $0-1$ & 2.1 & $\mathrm{mg} / \mathrm{kg}$ \\
\hline Benzo(A)Pyrene & 0.064 & $383 \mathrm{DH} 2600.5$ & $0-1$ & 0.21 & $\mathrm{mg} / \mathrm{kg}$ \\
\hline Benzo(B)Fluoranthene & 0.062 & 383DH2600.5 & $0-1$ & 2.1 & $\mathrm{mg} / \mathrm{kg}$ \\
\hline Benzo(K)Fluoranthene & 0.057 & $383 \mathrm{DH} 2600.5$ & $0-1$ & 21 & $\mathrm{mg} / \mathrm{kg}$ \\
\hline Bis(2-Ethylhexyl)Phthalate & 2.4 & $383 \mathrm{DH} 2200.5$ & $0-1$ & 120 & $\mathrm{mg} / \mathrm{kg}$ \\
\hline Butyl Benzyl Phthalate & 0.04 & 383DH0500.5 & $0-1$ & 100,000 & $\mathrm{mg} / \mathrm{kg}$ \\
\hline
\end{tabular}




\section{Table 2-1 \\ Maximum Reported Chemical Values for CAS 12-06-06; \\ Ponds 1, 2, and 3; and Drainage}

(Page 4 of 4 )

\begin{tabular}{||c|c|c|c|c|c||}
\hline \hline Contaminant of Concern & Results & Sample Number & $\begin{array}{c}\text { Depth } \\
\text { (ft bgs) }\end{array}$ & FAL & Units \\
\hline \hline Chrysene & 0.1 & $383 \mathrm{DH} 2200.5$ & $0-1$ & 210 & $\mathrm{mg} / \mathrm{kg}$ \\
\hline Dimethyl Phthalate & 0.014 & $383 \mathrm{DH} 2200.5$ & $0-1$ & 100,000 & $\mathrm{mg} / \mathrm{kg}$ \\
\hline Di-N-Octyl Phthalate & 0.059 & $383 \mathrm{DH} 2200.5$ & $0-1$ & 25,000 & $\mathrm{mg} / \mathrm{kg}$ \\
\hline Fluoranthene & 0.12 & $383 \mathrm{DH} 2600.5$ & $0-1$ & 22,000 & $\mathrm{mg} / \mathrm{kg}$ \\
\hline Pyrene & 0.19 & $383 \mathrm{DH} 2600.5$ & $0-1$ & 29,000 & $\mathrm{mg} / \mathrm{kg}$ \\
\hline \hline
\end{tabular}

RR1 - Indicates the sample was re-analyzed at a higher dilution due to higher-than-expected results.

$\mathrm{ft}$ bgs - Feet below ground surface

${ }^{1}$ Diesel-range organics (i.e., petroleum hydrocarbons) level is defined by Nevada Administrative Code 445.2272 (NAC, 2005b).

${ }^{2}$ Tier 2 FAL

The radionuclide americium (Am)-241 was detected at concentrations above the PAL (7.62 picocuries per gram [pCi/g]) in three of the 115 environmental samples analyzed. Cesium (Cs)-137 was detected at concentrations above the PAL ( $7.3 \mathrm{pCi} / \mathrm{g})$ in 54 of the 115 environmental samples analyzed. Plutonium-238 was detected at concentrations above the PAL (7.78 pCi/g) in one of the 115 environmental samples analyzed. Plutonium-239 was detected at concentrations above the PAL (7.62 pCi/g) in four of the 115 environmental samples analyzed. Americium-241, Cs-137, Pu-238, and Pu-239 were moved to a Tier 2 evaluation. The Tier 2 evaluation was completed using the Residual Radioactive (RESRAD) model and computer code to determine the site-specific FALs for Am-241, Cs-137, Pu-238, and Pu-239. Sites contaminated with two or more radionuclides are evaluated using a sum-of-fractions method to determine the total exposure from the radionuclides present. The RESRAD methods and results are explained in detail in Attachment A of Appendix D in this report. The Am-241, Cs-137, and Pu-238 all exceeded FALs in three samples from the Muckpile. The Pu-239 exceeded its FAL in four samples from the Muckpile. No other radionuclide exceeded PALs, so the PALs for those radionuclides are identified as the FALs. The maximum concentration of each detected radiological contaminant at this CAS is listed in Table 2-2. 
Table 2-2

Maximum Reported Radiological Values for CAS 12-06-06;

Ponds 1, 2, and 3; and Drainage

(Page 1 of 2)

\begin{tabular}{|c|c|c|c|c|c|}
\hline Contaminant of Concern & Results & Sample Number & $\begin{array}{c}\text { Depth } \\
\text { (ft bgs) }\end{array}$ & FAL & Units \\
\hline \multicolumn{6}{|c|}{ Muckpile } \\
\hline Actinium-228 & 2.89 & 383MB0400.5 & $0-1$ & 5 & $\mathrm{pCi} / \mathrm{g}$ \\
\hline Americium-241 & 28.8 & 383MB0543.5 & $43-44$ & $7.32^{1}$ & $\mathrm{pCi} / \mathrm{g}$ \\
\hline Bismuth-214 & 2.08 & 383MB0900.5 & $0-1$ & 5 & $\mathrm{pCi} / \mathrm{g}$ \\
\hline Cesium-137 & 1,060 & 383MB0543.5 & $43-44$ & $269.56^{1}$ & $\mathrm{pCi} / \mathrm{g}$ \\
\hline Europium-152 & 2.64 & 383МB0543.5 & $43-44$ & 5.7 & $\mathrm{pCi} / \mathrm{g}$ \\
\hline Lead-212 & 3.37 & 383MB0900.5 & $0-1$ & 5 & $\mathrm{pCi} / \mathrm{g}$ \\
\hline Lead-214 & 2.08 & 383МB0900.5 & $0-1$ & 5 & $\mathrm{pCi} / \mathrm{g}$ \\
\hline Thallium-208 & 1.02 & 383МВ0900.5 & $0-1$ & 5 & $\mathrm{pCi} / \mathrm{g}$ \\
\hline Strontium-90 & 54 & 383МH0559.5 & $59-60$ & 503 & $\mathrm{pCi} / \mathrm{g}$ \\
\hline Plutonium-238 & 15.6 & 383МB0543.5 & $43-44$ & $3.97^{1}$ & $\mathrm{pCi} / \mathrm{g}$ \\
\hline Plutonium-239 & 84 & 383MB0543.5 & $43-44$ & $21.36^{1}$ & $\mathrm{pCi} / \mathrm{g}$ \\
\hline \multicolumn{6}{|c|}{ Ponds } \\
\hline Actinium-228 & 2.4 & 383РН0116.5 & $16-17$ & 5 & $\mathrm{pCi} / \mathrm{g}$ \\
\hline Bismuth-214 & 1.72 & 383РН0306.5 & $6-7$ & 5 & $\mathrm{pCi} / \mathrm{g}$ \\
\hline Cesium-137 & 142 & 383РН0216.0 & $16-17$ & $269.56^{1}$ & $\mathrm{pCi} / \mathrm{g}$ \\
\hline Lead-212 & 2.76 & 383РН0320.5 & $20-21$ & 5 & $\mathrm{pCi} / \mathrm{g}$ \\
\hline Lead-214 & 1.81 & 383РН0408.0X & $7.5-8.5$ & 5 & $\mathrm{pCi} / \mathrm{g}$ \\
\hline Thallium-208 & 1.05 & 383РН0116.5 & $16-17$ & 5 & $\mathrm{pCi} / \mathrm{g}$ \\
\hline Strontium-90 & 3.62 & 383РН0300.5X & $0-1$ & 503 & $\mathrm{pCi} / \mathrm{g}$ \\
\hline Plutonium-238 & 0.74 & 383РН0216.0 & $15.5-16.5$ & 7.78 & $\mathrm{pCi} / \mathrm{g}$ \\
\hline Plutonium-239 & 5.37 & 383РН0216.0 & $15.5-16.5$ & 7.62 & $\mathrm{pCi} / \mathrm{g}$ \\
\hline
\end{tabular}


Table 2-2

Maximum Reported Radiological Values for CAS 12-06-06;

Ponds 1, 2, and 3; and Drainage

(Page 2 of 2 )

\begin{tabular}{|c|c|c|c|c|c|}
\hline Contaminant of Concern & Results & Sample Number & $\begin{array}{c}\text { Depth } \\
\text { (ft bgs) } \\
\end{array}$ & FAL & Units \\
\hline \multicolumn{6}{|c|}{ Drainage } \\
\hline Actinium-228 & 2.26 & 383DH0200.5 & $0-1$ & 5 & $\mathrm{pCi} / \mathrm{g}$ \\
\hline Aluminum-26 & 0.105 & 383DH1600.5 & $0-1$ & 2.32 & $\mathrm{pCi} / \mathrm{g}$ \\
\hline Americium-241 & 1.88 & 383DH0200.5 & $0-1$ & 7.62 & $\mathrm{pCi} / \mathrm{g}$ \\
\hline Bismuth-214 & 1.48 & 383DH1400.5 & $0-1$ & 5 & $\mathrm{pCi} / \mathrm{g}$ \\
\hline Cesium-137 & 78.1 & $383 \mathrm{DH} 2100.5$ & $0-1$ & $269.56^{1}$ & $\mathrm{pCi} / \mathrm{g}$ \\
\hline Lead-212 & 2.54 & 383DH0600.5 & $0-1$ & 5 & $\mathrm{pCi} / \mathrm{g}$ \\
\hline Lead-214 & 1.68 & $383 \mathrm{DH} 1400.5$ & $0-1$ & 5 & $\mathrm{pCi} / \mathrm{g}$ \\
\hline Thorium-234 & 2.4 & 383DH0500.5 & $0-1$ & 5 & $\mathrm{pCi} / \mathrm{g}$ \\
\hline Thallium-208 & 0.87 & $383 \mathrm{DH} 1400.5$ & $0-1$ & 5 & $\mathrm{pCi} / \mathrm{g}$ \\
\hline Strontium-90 & 1.88 & 383DH0100.5 & $0-1$ & 503 & $\mathrm{pCi} / \mathrm{g}$ \\
\hline Plutonium-238 & 0.73 & 383DH0100.5 & $0-1$ & 7.78 & $\mathrm{pCi} / \mathrm{g}$ \\
\hline Plutonium-239 & 4.21 & 383DH1400.5 & $0-1$ & 7.62 & $\mathrm{pCi} / \mathrm{g}$ \\
\hline
\end{tabular}

\footnotetext{
${ }^{1}$ Tier 2 FAL
}

These results show that there is no vertical migration into the native material and only limited migration in storm runoff. The runoff is constrained by the drainage and ponds.

\subsubsection{Oil Spill (CAS 12-25-02)}

During the CAI, TPH-DRO was found above the PAL of $100 \mathrm{mg} / \mathrm{kg}$ in five soil samples, so it was moved into a Tier 2 evaluation as described in the previous section. Of the hazardous constituents of TPH-DRO, benzo(a)anthracene exceeded the PAL $(2.1 \mathrm{mg} / \mathrm{kg})$ in two of the samples, and benzo(a)pyrene exceeded the PAL $(0.21 \mathrm{mg} / \mathrm{kg})$ in three of the samples. None of the other hazardous constituents of TPH-DRO exceeded the PALs; therefore, for those constituents, the PALs are identified as the FALs.

For the chemical constituents, other than the hazardous constituents of TPH-DRO reported above, benzo(a)pyrene (PAL $0.21 \mathrm{mg} / \mathrm{kg}$ ), benzo(b)fluoranthene (PAL $2.1 \mathrm{mg} / \mathrm{kg}$ ), dibenzo(a,h)anthracene (PAL $0.21 \mathrm{mg} / \mathrm{kg}$ ), and ideno(1,2,3-cd)pyrene (PAL $2.1 \mathrm{mg} / \mathrm{kg}$ ) exceeded their PALs, so they were moved to a Tier 2 analysis and the ORNL website was used to calculate the site-specific 
FALs (ORNL, 2004). For all other constituents, the PALs as identified in the CAIP (DTRA, 2003) are identified as the FALs. The Tier 2 methodology results are presented and explained in Appendix D of this report. Benzo(a)pyrene exceeded its FAL in one sample; no other COCs exceeded their respective FALs. The maximum concentration of each detected chemical contaminant at this CAS is listed in Table 2-3.

Table 2-3

Maximum Reported Chemical Values for CAS 12-25-02

(Page 1 of 2)

\begin{tabular}{|c|c|c|c|c|c|}
\hline Contaminant of Concern & Results & Sample Number & $\begin{array}{c}\text { Depth } \\
\text { (ft bgs) }\end{array}$ & FAL & Units \\
\hline \multicolumn{6}{|c|}{ Oil Spill } \\
\hline Arsenic & 7.1 & 3830 O०0800.5 & $0-1$ & 23 & $\mathrm{mg} / \mathrm{kg}$ \\
\hline Barium & 300 & $3830 B 0400.5$ & $0-11$ & 67,000 & $\mathrm{mg} / \mathrm{kg}$ \\
\hline Beryllium & 1.2 & $3830 B 0500.5$ & $0-1$ & 1,900 & $\mathrm{mg} / \mathrm{kg}$ \\
\hline Cadmium & 1.4 & 3830 OB0200.5 & $0-1$ & 450 & $\mathrm{mg} / \mathrm{kg}$ \\
\hline Chromium & 10 & $3830 B 0700.5$ & $0-1$ & 450 & $\mathrm{mg} / \mathrm{kg}$ \\
\hline Lead & 31 & $3830 B 0700.5$ & $0-1$ & 800 & $\mathrm{mg} / \mathrm{kg}$ \\
\hline Selenium & 1.2 & $3830 B 0800.5$ & $0-1$ & 310 & $\mathrm{mg} / \mathrm{kg}$ \\
\hline Diesel-Range Organics $^{1}$ & 3,400 & $3830 B 0100.5$ & $0-1$ & 100 & $\mathrm{mg} / \mathrm{kg}$ \\
\hline Acetone & 0.072 & $3830 B 0100.5$ & $0-1$ & 54,000 & $\mathrm{mg} / \mathrm{kg}$ \\
\hline Acenaphthene & 0.093 & $3830 B 0800.5$ & $0-1$ & 29,000 & $\mathrm{mg} / \mathrm{kg}$ \\
\hline Anthracene & 0.47 & $3830 B 0800.5$ & $0-1$ & 100,000 & $\mathrm{mg} / \mathrm{kg}$ \\
\hline Benzo(A)Anthracene & 17 & $3830 B 0800.5$ & $0-1$ & $147^{2}$ & $\mathrm{mg} / \mathrm{kg}$ \\
\hline Benzo(A)Pyrene & 20 & 3830B0800.5RR1 & $0-1$ & $15^{2}$ & $\mathrm{mg} / \mathrm{kg}$ \\
\hline Benzo(B)Fluoranthene & 37 & $3830 B 0800.5$ & $0-1$ & $150^{2}$ & $\mathrm{mg} / \mathrm{kg}$ \\
\hline Benzo(K)Fluoranthene & 10 & $3830 B 0800.5$ & $0-1$ & 21 & $\mathrm{mg} / \mathrm{kg}$ \\
\hline Bis(2-Ethylhexyl)Phthalate & 4 & $3830 B 0800.5$ & $0-1$ & 120 & $\mathrm{mg} / \mathrm{kg}$ \\
\hline 2 Butanone & 0.017 & 3830 OB0100.5 & $0-1$ & 110,000 & $\mathrm{mg} / \mathrm{kg}$ \\
\hline Carbazole & 0.79 & $3830 B 0800.5$ & $0-1$ & 86 & $\mathrm{mg} / \mathrm{kg}$ \\
\hline Chrysene & 21 & 3830 OB0800.5 & $0-1$ & 210 & $\mathrm{mg} / \mathrm{kg}$ \\
\hline
\end{tabular}




\section{Table 2-3 \\ Maximum Reported Chemical Values for CAS 12-25-02}

(Page 2 of 2)

\begin{tabular}{|c|c|c|c|c|c|}
\hline Contaminant of Concern & Results & Sample Number & $\begin{array}{c}\text { Depth } \\
\text { (ft bgs) }\end{array}$ & FAL & Units \\
\hline Dibenzo $(\mathrm{A}, \mathrm{H})$ Anthracene & 10 & 383 OB0800.5 & $0-1$ & $15^{2}$ & $\mathrm{mg} / \mathrm{kg}$ \\
\hline Fluoranthene & 10 & 383ОВ0800.5 & $0-1$ & 22,000 & $\mathrm{mg} / \mathrm{kg}$ \\
\hline Fluorene & 0.068 & 3830 O०0800.5 & $0-1$ & 26,000 & $\mathrm{mg} / \mathrm{kg}$ \\
\hline Ideno(1,2,3-Cd)Pyrene & 22 & 383OB0800.5 & $0-1$ & $150^{2}$ & $\mathrm{mg} / \mathrm{kg}$ \\
\hline Naphthalene & 0.056 & $3830 B 0800.5$ & $0-1$ & 190 & $\mathrm{mg} / \mathrm{kg}$ \\
\hline Pyrene & 89 & 383ОВ0800.5 & $0-1$ & 29,000 & $\mathrm{mg} / \mathrm{kg}$ \\
\hline
\end{tabular}

RR1 - Indicates the sample was re-analyzed at a higher dilution due to higher-than-expected results. $\mathrm{ft}$ bgs - Feet below ground surface

\footnotetext{
${ }^{1}$ Diesel-range organics (i.e., petroleum hydrocarbons) level is defined by Nevada Administrative Code 445.2272 (NAC, 2005b).

2 Tier 2 FAL
}

For the radionuclides, only Cs-137 was detected at concentrations above the PAL (7.3 pCi/g) in two of the eight environmental samples analyzed, so it was moved to a Tier 2 evaluation. No other radionuclides exceeded their PALs as defined in the CAIP (DTRA, 2003), so the PALs for those radionuclides are identified as the FALs. The Tier 2 evaluation was completed by using the RESRAD model and computer code to determine the site-specific FALs for Cs-137 (Appendix D). None of the radionuclides exceeded their respective FALs in this CAS. The maximum concentration of each detected radiological contaminant at this CAS is listed in Table 2-4.

\subsubsection{Radioactive Material (CAS 12-28-02)}

During the CAI, TPH-DRO was not found above the PAL of $100 \mathrm{mg} / \mathrm{kg}$; therefore, the PAL is identified as the FAL for TPH-DRO.

For the chemical constituents, only benzo(a)pyrene was detected in one sample that exceeded the PAL (0.21 mg/kg), so it was moved to a Tier 2 analysis as described above. Benzo(a)pyrene did not exceed the calculated FAL (Appendix D). The maximum concentration of each detected chemical contaminant at this CAS and its FAL are listed in Table 2-5. 
Table 2-4

Maximum Reported Radiological Values for CAS 12-25-02

\begin{tabular}{|c|c|c|c|c|c|}
\hline Contaminant of Concern & Results & Sample Number & $\begin{array}{c}\text { Depth } \\
\text { (ft bgs) }\end{array}$ & FAL & Units \\
\hline \multicolumn{6}{|c|}{ Oil Spill } \\
\hline Actinium-228 & 2.21 & $3830 B 0100.5$ & $0-1$ & 5 & $\mathrm{pCi} / \mathrm{g}$ \\
\hline Bismuth-214 & 1.1 & $3830 B 0800.5$ & $0-1$ & 5 & $\mathrm{pCi} / \mathrm{g}$ \\
\hline Cesium-137 & 20.5 & 3830 О 0700.5 & $0-1$ & $275^{1}$ & $\mathrm{pCi} / \mathrm{g}$ \\
\hline Lead-212 & 3.1 & $3830 B 0500.5$ & $0-1$ & 5 & $\mathrm{pCi} / \mathrm{g}$ \\
\hline Lead-214 & 1.06 & 3830 О 0600.5 & $0-1$ & 5 & $\mathrm{pCi} / \mathrm{g}$ \\
\hline Thallium-208 & 0.97 & $3830 B 0500.5$ & $0-1$ & 5 & $\mathrm{pCi} / \mathrm{g}$ \\
\hline Plutonium-239 & 1.04 & 3830 OB0800.5 & $0-1$ & 7.62 & $\mathrm{pCi} / \mathrm{g}$ \\
\hline
\end{tabular}

${ }^{1}$ Tier 2 FAL

Table 2-5

Maximum Reported Chemical Values for CAS 12-28-02

(Page 1 of 2)

\begin{tabular}{||c|c|c|c|c|c||}
\hline Contaminant of Concern & Results & Sample Number & $\begin{array}{c}\text { Depth } \\
\text { (ft bgs) }\end{array}$ & FAL & Units \\
\hline \hline \multicolumn{5}{|c|}{ Radioactive Material } \\
\hline \hline Arsenic & 7.1 & $383 R B 0200.5$ & $0-1$ & 23 & $\mathrm{mg} / \mathrm{kg}$ \\
\hline Barium & 260 & $383 R B 0300.5$ & $0-1$ & 67,000 & $\mathrm{mg} / \mathrm{kg}$ \\
\hline Beryllium & 1.7 & $383 R B 0100.5$ & $0-1$ & 1,900 & $\mathrm{mg} / \mathrm{kg}$ \\
\hline Cadmium & 0.57 & $383 R B 0300.5$ & $0-1$ & 450 & $\mathrm{mg} / \mathrm{kg}$ \\
\hline Lead & 61 & $383 R B 0300.5$ & $0-1$ & 800 & $\mathrm{mg} / \mathrm{kg}$ \\
\hline Mercury & 0.16 & $383 R B 0100.5$ & $0-1$ & 5,100 & $\mathrm{mg} / \mathrm{kg}$ \\
\hline Diesel-Range Organics ${ }^{1}$ & 15 & $383 R B 0400.5$ & $0-1$ & 100 & $\mathrm{mg} / \mathrm{kg}$ \\
\hline Benzo(A)Anthracene & 0.17 & $383 R B 0300.5$ & $0-1$ & 2.1 & $\mathrm{mg} / \mathrm{kg}$ \\
\hline Benzo(A)Pyrene & 0.23 & $383 R B 0400.5$ & $0-1$ & $15^{2}$ & $\mathrm{mg} / \mathrm{kg}$ \\
\hline Benzo(B)Fluoranthene & 0.62 & $383 R B 0400.5$ & $0-1$ & 2.1 & $\mathrm{mg} / \mathrm{kg}$ \\
\hline Benzo(K)Fluoranthene & 0.23 & $383 R B 0300.5$ & $0-1$ & 21 & $\mathrm{mg} / \mathrm{kg}$ \\
\hline Bis(2-Ethylhexyl)Phthalate & 1.2 & $383 R B 0300.5$ & $0-1$ & 120 & $\mathrm{mg} / \mathrm{kg}$ \\
\hline
\end{tabular}




\section{Table 2-5 \\ Maximum Reported Chemical Values for CAS 12-28-02}

(Page 2 of 2 )

\begin{tabular}{||c|c|c|c|c|c||}
\hline Contaminant of Concern & Results & Sample Number & $\begin{array}{c}\text { Depth } \\
\text { (ft bgs) }\end{array}$ & FAL & Units \\
\hline \hline Butyl Benzyl Phthalate & 0.78 & $383 R B 0100.5$ & $0-1$ & 100,000 & $\mathrm{mg} / \mathrm{kg}$ \\
\hline Carbazole & 0.023 & $383 R B 0400.5$ & $0-1$ & 86 & $\mathrm{mg} / \mathrm{kg}$ \\
\hline Chrysene & 0.48 & $383 R B 0400.5$ & $0-1$ & 210 & $\mathrm{mg} / \mathrm{kg}$ \\
\hline Dibenzo(A,H)Anthracene & 0.027 & $383 R B 0400.5$ & $0-1$ & 21 & $\mathrm{mg} / \mathrm{kg}$ \\
\hline Di-N-Butyl Phthalate & 4.5 & $383 R B 0100.5$ & $0-1$ & 62,000 & $\mathrm{mg} / \mathrm{kg}$ \\
\hline Fluoranthene & 0.51 & $383 R B 0400.5$ & $0-1$ & 22,000 & $\mathrm{mg} / \mathrm{kg}$ \\
\hline Ideno(1,2,3-Cd)Pyrene & 0.08 & $383 R B 0400.5$ & $0-1$ & 2.1 & $\mathrm{mg} / \mathrm{kg}$ \\
\hline Naphthalene & 0.018 & $383 R B 0400.5$ & $0-1$ & 190 & $\mathrm{mg} / \mathrm{kg}$ \\
\hline Pyrene & 0.43 & $383 R B 0400.5$ & $0-1$ & 29,000 & $\mathrm{mg} / \mathrm{kg}$ \\
\hline
\end{tabular}

$\mathrm{ft}$ bgs - Feet below ground surface

${ }^{1}$ Diesel-range organics (i.e., petroleum hydrocarbons) level is defined by Nevada Administrative Code 445.2272 (NAC, 2005b).

2 Tier 2 FAL

For the radionuclides, Cs-137 and thorium (Th)-234 were detected at concentrations above their PALs (7.3 and $5 \mathrm{pCi} / \mathrm{g}$, respectively) in one of the four environmental samples analyzed.

Plutonium-238 was detected at concentrations above the PAL (7.78 pCi/g) in one of the 115 environmental samples analyzed. Plutonium-239 was detected at concentrations above the PAL (7.62 pCi/g) in four of the 115 environmental samples analyzed. Only Cs-137, Pu-238, and Pu-239 were moved to a Tier 2 evaluation. The half life of Th-234 is too short (24 days) to be considered in the Tier 2 evaluation. No other radionuclides exceeded their PALs, so the PALs for those radionuclides are identified as the FALs (Appendix D). Based on the PAL and FAL comparisons, none of the radionuclides exceed the FALs. The maximum concentration of each detected radiological contaminant at this CAS is listed in Table 2-6. 
Table 2-6

Maximum Reported Radiological Values for CAS 12-28-02

\begin{tabular}{|c|c|c|c|c|c|}
\hline Contaminant of Concern & Results & Sample Number & $\begin{array}{c}\text { Depth } \\
\text { (ft bgs) }\end{array}$ & FAL & Units \\
\hline \multicolumn{6}{|c|}{ Radioactive Material } \\
\hline Actinium-228 & 2.9 & 383RB0100.5 & $0-1$ & 5 & $\mathrm{pCi} / \mathrm{g}$ \\
\hline Bismuth-212 & 3.2 & 383RB0100.5 & $0-1$ & 5 & $\mathrm{pCi} / \mathrm{g}$ \\
\hline Bismuth-214 & 1.12 & 383RB0200.5 & $0-1$ & 5 & $\mathrm{pCi} / \mathrm{g}$ \\
\hline Cesium-137 & 114 & 383RB0100.5 & $0-1$ & $275.2^{1}$ & $\mathrm{pCi} / \mathrm{g}$ \\
\hline Lead-212 & 2.96 & 383RB0100.5 & $0-1$ & 5 & $\mathrm{pCi} / \mathrm{g}$ \\
\hline Lead-214 & 1.04 & 383RB0400.5 & $0-1$ & 5 & $\mathrm{pCi} / \mathrm{g}$ \\
\hline Thorium-227 & 4.1 & 383RB0100.5 & $0-1$ & 5 & $\mathrm{pCi} / \mathrm{g}$ \\
\hline Thorium-234 & 7.2 & 383RB0100.5 & $0-1$ & 5 & $\mathrm{pCi} / \mathrm{g}$ \\
\hline Thallium-208 & 0.85 & 383RB0100.5 & $0-1$ & 5 & $\mathrm{pCi} / \mathrm{g}$ \\
\hline Plutonium-238 & 0.232 & 383RB0400.5 & $0-1$ & 7.78 & $\mathrm{pCi} / \mathrm{g}$ \\
\hline Plutonium-239 & 2.09 & 383RB0200.5 & $0-1$ & 7.62 & $\mathrm{pCi} / \mathrm{g}$ \\
\hline
\end{tabular}

${ }^{1}$ Tier 2 FAL

\subsubsection{Data Assessment Summary}

The Data Quality Assessment (DQA) is presented in Appendix C and includes an evaluation of the DQIs to determine the degree of acceptability and usability of the reported data in the decision-making process. The DQO process ensures that the right type, quality, and quantity of data will be available to support the resolution of those decisions at an appropriate level of confidence. Using both the DQO and DQA processes ensures that DQO decisions are sound and defensible.

The DQA process as presented in Appendix C is comprised of the following steps:

- Step 1 - Review DQOs and Sampling Design.

- Step 2 - Conduct a Preliminary Data Review.

- Step 3 - Select the Test.

- Step 4 - Verify the Assumptions.

- Step 5 - Draw Conclusions from the Data. 
Sample locations that support the presence and/or extent of contamination at each CAS are shown in Appendix A. Based on the results of the DQA presented in Appendix C, the DQO requirements have been met, and the close in place corrective action alternative was selected as the closure alternative at CAU 383, Area 12 E-Tunnel Sites. The DQA also determined that information generated during the investigation supports the CSM assumptions, and the data collected support the intended use in the decision-making process.

\subsection{Justification for No Further Action}

No further action is justified based on an evaluation of risk (see Appendix D) to ensure protection of the public and the environment in accordance with Nevada Administrative Code (NAC) 445A (NAC, 2005a), feasibility, and cost effectiveness. The corrective action was determined from DQO decision statements based on a comparison of the analyte concentrations detected in CAI soil samples to the FALs defined in Section 2.3.1. Because the extent of the COCs is limited and the CAI demonstrated that there has been no vertical migration and only limited lateral migration of COCs in storm runoff, the corrective action to close in place with use restrictions is justified at all three CASs; the drainage; and Ponds 1, 2, and 3. Appendix D presents an evaluation of risk associated with the recommended closure alternative.

\subsubsection{Final Action Levels}

The CAU 383 FALs are risk-based cleanup goals that, if met, will ensure that each release site will not pose an unacceptable risk to human health and the environment under the identified use scenario, and that conditions at each site are in compliance with all applicable laws and regulations. The process described in this section to define and determine the FALs conforms with NAC Section 445A.227 (NAC, 2005b), which lists the requirements for sites with soil contamination. For the evaluation of corrective actions, NAC Section 445A.22705 (NAC, 2005c) requires the use of ASTM Method E 1739-95 to "conduct an evaluation of the site, based on the risk it poses to public health and the environment, to determine the necessary remediation standards (i.e., FALs) or to establish that corrective action is not necessary.” 
The ASTM procedure (ASTM, 1995) defines three tiers (or levels) of evaluation involving increasingly sophisticated analyses as follows.

- Tier 1 Evaluation - Sample results from source areas (highest concentrations) are compared to action levels based on generic (non-site-specific) conditions (i.e., the PALs established in the CAIP). The FALs may then be established as the Tier 1 action levels, or the FALs may be calculated using a Tier 2 evaluation.

- Tier 2 Evaluation - Conducted by calculating Tier 2 site-specific target levels (SSTLs) using site-specific information as inputs to the same or similar methodology used to calculate Tier 1 action levels. The Tier 2 SSTLs are then compared to individual sample results from reasonable points of exposure (as opposed to the source areas as is done in Tier 1) on a point-by-point basis. Total TPH concentrations are not used for risk-based decisions under Tier 2 or Tier 3. Rather, the individual hazardous constituents in TPH will be compared to their SSTLs.

Alternatively, the Tier 2 risk-based corrective action process SSTLs may be compared to the predicted concentration or activity of the contaminant at the point of exposure based on attenuation from the source using relatively simplistic mathematical models. Points of exposure are defined as those locations at which an individual or population may come in contact with a COC originating from a CAS. If a Tier 2 evaluation is conducted, the calculations used to derive the SSTLs and the contaminant attenuation calculations will be provided as an appendix to the investigation report. If remediation to Tier 2 SSTLs is not practical, a Tier 3 evaluation may be conducted.

- Tier 3 Evaluation - Conducted by calculating Tier 3 SSTLs on the basis of more sophisticated risk analyses using methodologies described in ASTM Method E 1739-95 that consider site-, pathway-, and receptor-specific parameters. Tier 3 evaluation is much more complex than Tiers 1 and 2, because it may include additional site characterization, probabilistic evaluations, and sophisticated chemical fate/transport models. The Tier 3 SSTLs are then compared to the upper 95 percent confidence limit of the mean of sample results from reasonable points of exposure (as opposed to individual sample results as is done in Tier 2). Contaminant concentrations exceeding 
Tier 3 SSTLs require corrective action. If a Tier 3 evaluation is conducted, the calculations used to derive the SSTLs and the upper confidence limit of the means will be provided as an appendix to the investigation report.

A Tier 1 evaluation was conducted for all COPCs to determine whether contaminant levels satisfy the criteria for a quick regulatory closure or warrant a more site-specific assessment. This was accomplished by comparing individual source area contaminant concentration results to the Tier 1 actions levels (the PALs established in the CAIP).

The constituents detected at CAU 383 that exceeded Tier 1 action levels were:

- TPH-DRO at CAS 12-06-06, the drainage, the ponds, and CAS 12-25-02

- Benzo(a)pyrene at CAS 12-06-06, CAS 12-25-02, and CAS 12-28-02

- Benzo(a)anthracene at CAS 12-25-02

- Benzo(b)fluoranthene at CAS 12-25-02

- Dibenzo(a,h)anthracene at CAS 12-25-02

- Ideno(1,2,3-cd)pyrene at CAS 12-25-02

- Am-241 at CAS 12-06-06

- $\quad$ Cs-137 at CAS 12-06-06, the drainage, the ponds, CAS 12-25-02, and CAS 12-28-02

- $\quad \mathrm{Pu}-238$ at CAS 12-06-06

- $\quad \mathrm{Pu}-239$ at CAS 12-06-06

The concentration of all constituents not listed above were below Tier 1 action levels, and the corresponding PALs were established as the Tier 1 FALs. All of the constituents at CAU 383 that exceeded Tier 1 action levels were moved to a Tier 2 evaluation.

The Tier 2 evaluation of TPH-DRO compared the concentrations of the individual hazardous constituents of TPH-DRO to the Tier 1 action levels in each sample that exceeded for TPH-DRO. For the constituent concentrations that exceeded the Tier 1 action levels, a site-specific action level was calculated using site-specific inputs to the risk equations in the ORNL website (ORNL, 2004). These calculated concentrations were then established as the FALs for the hazardous constituents in TPH-DRO at CAU 383.

For the non-TPH chemicals that exceeded the PALs, a FAL was calculated using the Tier 2 evaluation process. The calculated concentrations were then established as the FALs for those 
constituents at CAU 383 (Appendix D). The Tier 2 calculated FALs are presented in Table 2-7. Additional details of the Tier 2 evaluation is provided in Appendix D.

The Tier 2 evaluation for the radionuclides was conducted by entering site-specific radionuclide information and physical characteristics of the site into the RESRAD program to calculate the site-specific action levels. This calculated the site-specific activities needed ensure the collective exposures from individual radionuclides do not exceed the 25-millirem-per-year annual dose constraint.

Although all detected radionuclides as a CAS are used in the sum-of-fractions calculation, and a unique Tier 2 action level is developed for all radionuclides, only the five radionuclides that initially exceeded Tier 1 levels have a Tier 2-based FAL. The CAS-specific FALs established for these radionuclides are the SSTLs listed in Table D.1-7. These calculated activities were established as the FALs for each radionuclide at each CAS that exceeded a Tier 1 action level. The Tier 2 calculated FALs are presented in Table 2-7. Additional details of the Tier 2 evaluation are provided in Appendix D. 
Table 2-7

Final Action Levels

\begin{tabular}{|c|c|c|c|}
\hline cOPCs & Tier 1 FALs & Tier 2 FALs & $\begin{array}{l}\text { Tier } 3 \\
\text { FALs }\end{array}$ \\
\hline VOCs & PALS & N/A & N/A \\
\hline SVOCs & $\begin{array}{c}\text { PALs except as listed } \\
\text { under Tier } 2\end{array}$ & $\begin{array}{ll}\text { Benzo(a)anthracene } & 147 \mathrm{mg} / \mathrm{kg} \\
\text { Benzo(a)pyrene } & 15 \mathrm{mg} / \mathrm{kg} \\
\text { Benzo(b)fluoranthene } & 150 \mathrm{mg} / \mathrm{kg} \\
\text { Benzo(k)fluoranthene } & 1,500 \mathrm{mg} / \mathrm{kg} \\
\text { Dibenzo(a,h)anthracene } & 15 \mathrm{mg} / \mathrm{kg} \\
\text { Ideno(1,2,3-cd)pyrene } & 150 \mathrm{mg} / \mathrm{kg}\end{array}$ & N/A \\
\hline $\begin{array}{l}\text { RCRA Metals + } \\
\text { Beryllium }\end{array}$ & PALS & $\mathrm{N} / \mathrm{A}$ & N/A \\
\hline TPH-DRO & PALS & $\begin{array}{l}\text { PALs except for } \\
\text { benzo(a)pyrene }\end{array}$ & N/A \\
\hline Radionuclides & $\begin{array}{c}\text { PALs except as listed } \\
\text { under Tier } 2\end{array}$ & 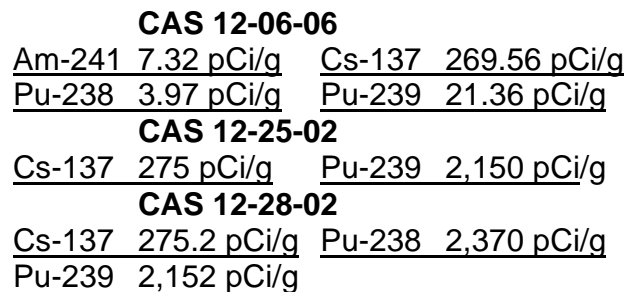 & $N / A$ \\
\hline
\end{tabular}

N/A = Not applicable 


\subsection{Recommendations}

The data generated by the CAI show that the FALs were exceeded for radionuclides in one surface sample and three subsurface samples in CAS 12-06-06 (Muckpile), and for benzo(a)pyrene in one surface sample at CAS 12-25-02 (Oil Spill). Because FALs were exceeded at CAU 383, a use restriction is recommended to be put in place that will prevent exposure of NTS workers and the public to the COCs. On a conservative basis, CASs 12-06-06, CAS 12-25-02, and CAS 12-28-02 will be use restricted from the surface to a depth of $150 \mathrm{ft}$ bgs, and the drainage and Ponds 1, 2, and 3 will be use restricted from the surface to a depth of $50 \mathrm{ft}$ bgs. The future use of CAU 383 will be restricted from any activity that will alter or modify the containment controls unless concurrence is obtained from the Nevada Division of Environmental Protection (NDEP). Because removal of the contaminants within the Muckpile is not feasible, the close in place with administrative controls corrective action alternative is appropriate. It will prevent inadvertent contact with the COCs, and meets all applicable state and federal regulations for closure of the site.

An annual post-closure inspection is associated with the use restriction to certify that markers and postings are in place, intact, and readable. Results of these inspections will be provided in the annual Post Closure Inspection and Monitoring Report.

In conclusion, DTRA and NNSA/NSO request that NDEP issue a Notice of Completion for this CAU and approval to move the CAU from Appendix III to Appendix IV of the FFACO. 


\subsection{References}

ASTM, see American Society for Testing and Materials.

American Society for Testing and Materials. 1995. Standard Guide for Risk-Based Corrective Action Applied at Petroleum Release Sites/American Society for Testing and Materials, ASTM E 1739-95 (Reapproved 2002). Philadelphia, PA.

DTRA, see Defense Threat Reduction Agency.

Defense Threat Reduction Agency. 2003. Corrective Action Investigation Plan for Corrective Action Unit 383: Area 12 E-Tunnel Sites, Nevada Test Site, Rev. 0. Prepared by SNJV. Las Vegas, NV.

FFACO, see Federal Facility Agreement and Consent Order.

Federal Facility Agreement and Consent Order. 1996 (as amended). Agreed to by the State of Nevada, the U.S. Department of Energy, and the U.S. Department of Defense.

NAC, see Nevada Administrative Code.

NNSA/NV, see U.S. Department of Energy, National Nuclear Security Administration Nevada Operations Office.

Nevada Administrative Code. 2005a. NAC 445A, "Water Controls.” Carson City, NV.

Nevada Administrative Code. 2005b. NAC 445A.2272, “Contamination of Soil: Establishment of Action Levels.” Carson City, NV.

Nevada Administrative Code. 2005c. NAC 445A.22705, “Contamination of Soil: Evaluation of Site by Owner or Operator; Review of Evaluation by Division.” Carson City, NV.

ORNL, see Oak Ridge National Laboratory

Oak Ridge National Laboratory. 2004. Risk Assessment Information System. As accessed at http://risk.lsd.ornl.gov/cgi-bin/prg/PRG_search on 25 April 2006.

SNJV, see Stoller Navarro Joint Venture.

Stoller-Navarro Joint Venture. 2003. SNJV Site-Specific Health and Safety Plan, DTRA CAU 383 E-Tunnel Muckpile, Nevada Test Site, Rev. 0. Las Vegas, NV. 
Date: February 2007

Page 26 of 26

U.S. Department of Energy, National Nuclear Security Administration Nevada Operations Office. 2002. Industrial Sites Quality Assurance Project Plan, Nevada Test Site, Nevada, DOE/NV--372, Rev. 3. Las Vegas, NV. 


\section{Appendix A}

\section{Corrective Action Investigation Results}




\section{Table of Contents}

List of Figures $\ldots \ldots \ldots \ldots \ldots \ldots \ldots \ldots \ldots \ldots \ldots \ldots \ldots \ldots \ldots \ldots \ldots \ldots \ldots \ldots$ A-iii

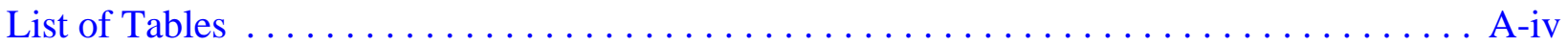

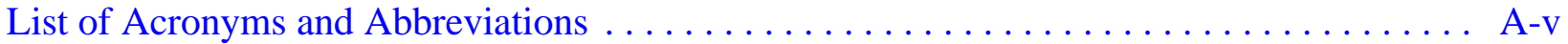

Executive Summary . . . . . . . . . . . . . . . . . . . . . . ES-1

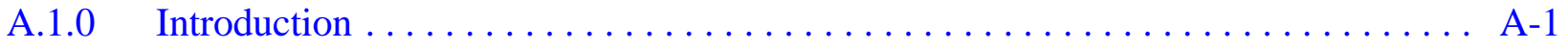

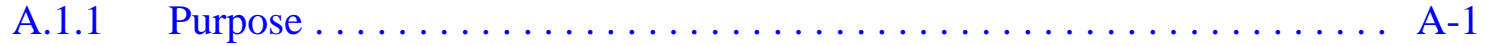

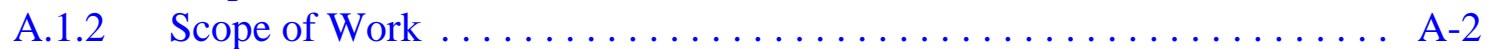

A.2.0 Project Description $\ldots \ldots \ldots \ldots \ldots \ldots \ldots \ldots \ldots \ldots \ldots \ldots \ldots \ldots \ldots \ldots \ldots \ldots$

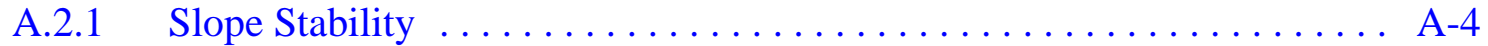

A.2.2 Surface Radiological Surveys $\ldots \ldots \ldots \ldots \ldots \ldots \ldots \ldots \ldots \ldots$ A-4

A.2.3 Muckpile Sample Locations . . . . . . . . . . . . . . . . . A-7

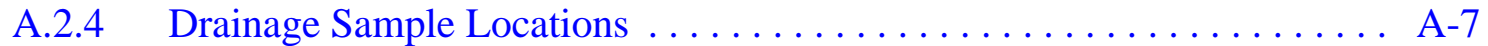

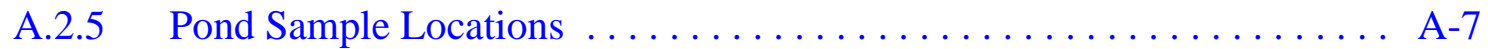

A.2.6 Radioactive Material (CAS 12-28-02) Sample Locations . . . . . . . . . . . A-8

A.2.7 Oil Spill (CAS 12-25-02) Sample Locations . . . . . . . . . . . . . . . A-8

A.2.8 Background and Preliminary Sample Locations $\ldots \ldots \ldots \ldots \ldots \ldots \ldots$ A-8

A.2.9 Checklists . . . . . . . . . . . . . . . . . . . . . . . . A-8

A.3.0 Muckpile Investigation $\ldots \ldots \ldots \ldots \ldots \ldots \ldots \ldots \ldots \ldots \ldots \ldots \ldots \ldots \ldots$ A-10

A.3.1 Work Packages . . . . . . . . . . . . . . . . . . . . . A A-10

A.3.2 Surface Radiological Surveys $\ldots \ldots \ldots \ldots \ldots \ldots \ldots \ldots \ldots \ldots \ldots$. . . . . . . . . . .

A.3.3 Muckpile Samples, CAS 12-06-06 . . . . . . . . . . . . . . A A-12

A.3.4 Drainage Soil Samples . . . . . . . . . . . . . . . . . . A-16

A.3.5 Pond Soil Samples ........................... A-19

A.3.6 Oil Spill Soil Samples, CAU 12-25-02 . . . . . . . . . . . . . . . . A-21

A.3.7 Radioactive Material Soil Samples, CAU 12-28-02 . . . . . . . . . . . A-21

A.3.8 Background and Preliminary Soil Samples . . . . . . . . . . . . . A-22

A.3.9 Other Sampling ......................... A-26

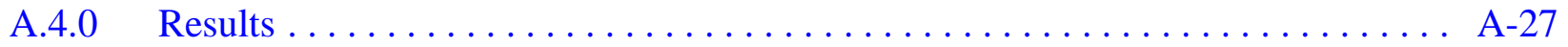

A.5.0 Waste Management . . . . . . . . . . . . . . . . . . . . . . . . . A-37

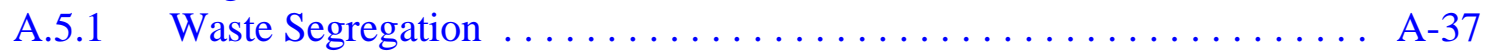

A.5.2 Waste Generation and Accumulation $\ldots \ldots \ldots \ldots \ldots \ldots \ldots \ldots \ldots$ A-37

A.5.2.1 Rinsates ....................... A-38

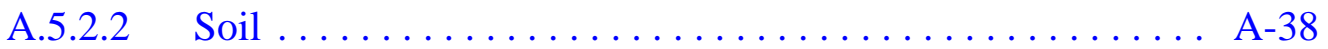




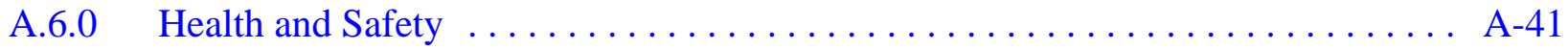

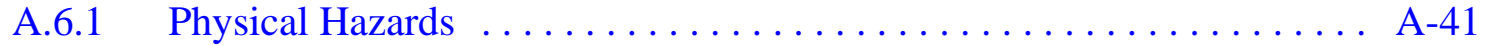

A.6.2 Chemical and Radiological Hazards $\ldots \ldots \ldots \ldots \ldots \ldots \ldots \ldots \ldots$. A-41

A.6.3 Drilling .................................. A-42

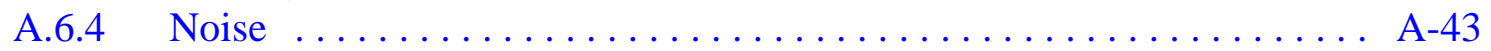

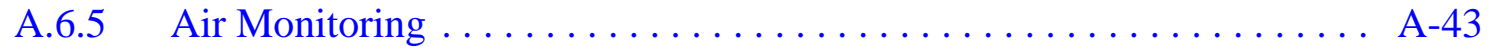

A.6.6 Personnel Monitoring .......................... A-43

A.6.7 Training $\ldots \ldots \ldots \ldots \ldots \ldots \ldots \ldots \ldots \ldots \ldots \ldots \ldots \ldots \ldots \ldots \ldots \ldots \ldots \ldots \ldots \ldots$

A.7.0 Lessons Learned $\ldots \ldots \ldots \ldots \ldots \ldots \ldots \ldots \ldots \ldots \ldots \ldots \ldots \ldots \ldots \ldots \ldots \ldots$

A.8.0 References $\ldots \ldots \ldots \ldots \ldots \ldots \ldots \ldots \ldots \ldots \ldots \ldots \ldots \ldots \ldots \ldots \ldots \ldots \ldots$ A-46

Attachment A - Soil Boring Logs

Attachment B - Cross Sections

Attachment C - Analytical Results Table 


\section{List of Figures}

Number

Title

Page

A.2-1 Area 12 E-Tunnel Location . . . . . . . . . . . . . . . . . . . . . . . A-5

A.2-2 CAU 383 (E-Tunnel Sites) CASs . . . . . . . . . . . . . . . . . . . . A-6

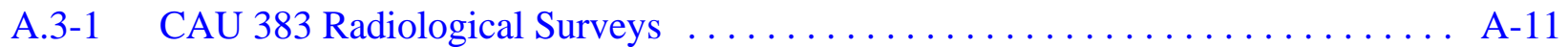

A.3-2 CAU 383 Muckpile Sampling Locations $\ldots . \ldots \ldots \ldots \ldots \ldots \ldots \ldots$. . . . . . . . .

A.3-3 CAU 383 Drainage Sampling Locations ...................... A-17

A.3-4 CAU 383 Ponds Sampling Locations $\ldots . \ldots \ldots \ldots \ldots \ldots \ldots \ldots \ldots \ldots$ A-20

A.3-5 CAU 383 Oil Spill and Radioactive Material Sampling Locations . ......... A A-23

A.3-6 CAU 383 Background and Preliminary Sampling Locations $\ldots . \ldots \ldots \ldots \ldots$ A-25 


\section{List of Tables}

Number

Title

Page

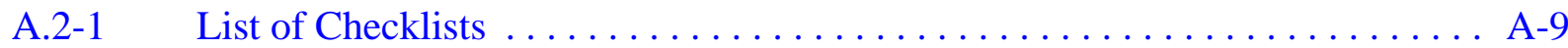

A.3-1 As-Built Muckpile Sampling Locations and Total Depth . . . . . . . . . . . . A-15

A.3-2 As-Built Drainage Sampling Locations and Total Depths . . . . . . . . . . . A A-18

A.3-3 As-Built Ponds Sampling Locations and Total Depths ............. A-21

A.3-4 As-Built Oil Spill and Radioactive Material

Sampling Locations and Total Depths ..................... A-24

A.3-5 As-Built Background and Preliminary Sampling Locations Total Depths . . . A-26

A.4-1 Analytical Result Detects for Muckpile and Pond Samples ........... A-28

A-4-2 Analytical Result Detects for the Radioactive Material,

Oil Spill, Drainage, and QC Water Samples ................. A-32

A.4-3 Analytical Results for Background / Preliminary Samples . . . . . . . . . A A-36

A.5-1 Drum Count $\ldots \ldots \ldots \ldots \ldots \ldots \ldots \ldots \ldots \ldots \ldots \ldots \ldots \ldots \ldots \ldots \ldots \ldots \ldots$ 


\section{List of Acronyms and Abbreviations}

Ac

$\mathrm{Al}$

Am

$\mathrm{Bi}$

$\mathrm{BN}$

CADD

CAI

CAIP

CAS

CAU

COPC

cps

Cs

DLAPS

DOE

DRO

DTRA

$\mathrm{Eu}$

EZ

FADL

FFACO

FID

$\mathrm{ft}$

GPS

$\mathrm{H} \& \mathrm{~S}$

HWAA

IDW

$\mathrm{K}$

$\mathrm{mg} / \mathrm{kg}$

$\mathrm{mg} / \mathrm{L}$
Actinium

Aluminum

Americium

Bismuth

Bechtel Nevada

Corrective Action Decision Document

Corrective Action Investigation

Corrective Action Investigation Plan

Corrective Action Site

Corrective Action Unit

Contaminant of potential concern

Counts per second

Cesium

Dual Large-Area Plastic Scintillation Detector

U.S. Department of Energy

Diesel-range organics

Defense Threat Reduction Agency

Europium

Exclusion Zone

Field Activity Daily Log

Federal Facility Agreement and Consent Order

Flame-ionization detector

Foot

Global Positioning System

Health and Safety

Hazardous waste accumulation area

Investigation-derived waste

Potassium

Milligrams per kilogram

Milligrams per liter 


\section{List of Acronyms and Abbreviations (Continued)}

\begin{tabular}{|c|c|}
\hline ND & Not detected \\
\hline \multirow[t]{2}{*}{ NNSA/NSO } & U.S. Department of Energy, National Nuclear Security Administration Nevada Site \\
\hline & Office \\
\hline NTS & Nevada Test Site \\
\hline $\mathrm{Pb}$ & Lead \\
\hline $\mathrm{pCi} / \mathrm{g}$ & Picocuries per gram \\
\hline $\mathrm{pCi} / \mathrm{L}$ & Picocuries per liter \\
\hline PPE & Personal protective equipment \\
\hline $\mathrm{Pu}$ & Plutonium \\
\hline QA & Quality assurance \\
\hline QC & Quality control \\
\hline RCRA & Resource Conservation and Recovery Act \\
\hline REOP & Real Estate/Operations Permit \\
\hline SAPS & Small Area Plastic Scintillation Detector \\
\hline SCL & Sample Collection Log \\
\hline SNJV & Stoller-Navarro Joint Venture \\
\hline $\mathrm{Sr}$ & Strontium \\
\hline SS & Site Supervisor \\
\hline SSHASP & Site-Specific Health and Safety Plan \\
\hline SSO & Site Safety Officer \\
\hline SVOC & Semivolatile organic compound \\
\hline TD & Total depth \\
\hline Th & Thorium \\
\hline $\mathrm{Tl}$ & Thallium \\
\hline TID & Tamper-indicating device \\
\hline TPH & Total petroleum hydrocarbons \\
\hline VOC & Volatile organic compound \\
\hline $\mathrm{Fg} / \mathrm{kg}$ & Micrograms per kilogram \\
\hline $\mathrm{Fg} / \mathrm{L}$ & Micrograms per liter \\
\hline
\end{tabular}




\section{Executive Summary}

The Corrective Action Investigation (CAI) of the E-Tunnel Sites and drainage, identified under the Federal Facility Agreement and Consent Order (FFACO) (1996) classification as Corrective Action Unit (CAU) 383, Area 12 E-Tunnel Sites, consists of three Corrective Action Sites (CASs): 12-06-06, Muckpile; 12-25-02, Oil Spill; and 12-28-02, Radioactive Material. The following activities were completed during the CAI: a drive-over radiological survey, a walk-over radiological survey, rotosonic drilling and soil sampling, and hand soil sampling.

In September 2003, drive-over and walk-over radiological surveys of the E-Tunnel Sites and the drainage below the Muckpile, including the ponds were completed. These surveys identified surface and near-surface areas with elevated readings on the Muckpile, in the drainage and ponds, and near the blower pad.

The next stage of the investigation consisted of rotosonic drilling and hand sampling to collect soil samples from within and beneath the Muckpile and ponds, the drainage, the radioactive material area, and the oil spill (blower pad). This was conducted between May 19 and June 24, 2004. Ninety locations were drilled or hand sampled to characterize CAU 383. A total of 1,129.5 feet (ft) was drilled in 39 boreholes ranging from 1.5 to $91.5 \mathrm{ft}$ deep. Fifty-one locations were sampled by hand. One to three samples were collected from 89 locations, and one borehole was not sampled. During the characterization, 129 environmental samples were collected. These included 40 muck samples, eight oil spill samples, four contamination area samples, 37 samples from the drainage, 10 pond sediment samples, and 30 native material samples. Seven duplicate and six lab soil samples were collected for quality control (QC) purposes. Thirty-seven QC water samples were collected (seven equipment rinsate samples, seven field blank samples, one source blank, and 22 trip blanks). All soil and water samples were sent to Paragon Analytics, Inc., for analysis. Analyses performed included volatile organic compounds (VOCs), semivolatile organic compounds (SVOCs), total petroleum hydrocarbons (TPH)-diesel-range organics (DRO), total Resource Conservation and Recovery Act (RCRA) metals, beryllium, and radionuclides (gamma spec, isotopic plutonium and strontium-90). In addition, three background, five preliminary, one duplicate, and one lab QC soil samples were collected in February and March 2004. These soil samples were also sent to Paragon Analytics, Inc., for analysis. 
Varying amounts of VOCs, SVOCs, TPH-DRO, beryllium, total RCRA metals, and radionuclides were detected in the muck and/or native soil samples from all three CASs included in this investigation.

During the CAI, seven drums of investigation-derived waste were accumulated: four drums of rinsate from decontamination activities, two drums of hydrocarbon-contaminated plastic, and one drum of personal protective equipment. The environmental sample analytical results were used to make the waste determination that none of the drums were considered to be hazardous or radioactive. All of the drums were disposed of as sanitary waste except for the two drums of hydrocarbon-contaminated plastic, which were sent to the hydrocarbon landfill. 


\section{A.1.0 Introduction}

The Corrective Action Investigation (CAI) of the Area 12 E-Tunnel Sites and associated drainage was conducted in accordance with the Federal Facility Agreement and Consent Order (FFACO) (1996), that was agreed to by the U.S. Department of Defense, Defense Special Weapons Agency (predecessor to the Defense Threat Reduction Agency [DTRA]); the U.S. Department of Energy (DOE); and the Nevada Division of Environmental Protection. The investigation was controlled and guided by the Corrective Action Unit (CAU) 383 Corrective Action Investigation Plan (CAIP) (DTRA, 2003); Field Instructions (DTRA, 2004), which referenced the operational checklists; and the site-specific health and safety plan (SSHASP) (SNJV, 2003). The E-Tunnel Muckpile and associated sites are identified under FFACO classification as CAU 383, Area 12 E-Tunnel Sites. Corrective Action Unit 383 is a joint DTRA and DOE, National Nuclear Security Administration Nevada Site Office (NNSA/NSO) site. The CAU consists of three Corrective Action Sites (CASs): 12-06-06, Muckpile; 12-25-02, Oil Spill; and 12-28-02, Radioactive Material. In addition to these sites, the drainage between the Muckpile and the first pond, and Ponds 1, 2, and 3 were sampled. This report presents a summary of the field activities and the data collected during the field effort.

Section 1.0 is the introduction, which includes a description of the purpose and scope of the project. Section 2.0 is the project description. Section 3.0 details the Area 12 E-Tunnel Sites investigation and provides a description of the sample collection activities and locations.

Section 4.0 is a summary of the sample analytical results. Section 5.0 provides a description of the waste management activities. Section 6.0 covers the health and safety aspects of the project. Section 7.0 discusses the lessons learned during the project, and Section 8.0 lists the references. Attachments A through C provide copies of the field and analytical data.

\section{A.1.1 Purpose}

The purpose of the Area 12 E-Tunnel Sites CAI was to determine whether the Area 12 E-Tunnel Sites and/or the underlying soils have been impacted by the contaminants of concern (COCs). The data collected during the field effort, which are presented in this report, enabled DTRA and NNSA/NSO to make informed decisions about the future use or closure of the site. The 
Muckpile primarily consists of mining debris (rock) generated during tunnel excavation and construction in support of nuclear weapons effects testing. The investigation was conducted to achieve the following goals:

- Determine whether identified COPCs (both chemical and radiological) are present at the surface, within, or beneath the Muckpile, the Oil Spill, the Radioactive Material, the drainage, and/or the first three ponds at concentrations exceeding FALs.

- Provide sufficient information and data to develop appropriate corrective action strategies for the Muckpile and associated sites, and support the selected corrective action alternative.

\section{A.1.2 Scope of Work}

The scope of the Area 12 E-Tunnel Muckpile investigation included the following:

- Conducted walk-over and drive-over radiological surveys using field-screening instruments to locate areas with elevated radiological levels.

- Collected random and biased Muckpile and native soil samples using the dry rotosonic drilling method or hand tools.

- Collected random and biased soil samples from the drainage below the Muckpile and the ponds using the dry rotosonic drilling method or hand tools.

- Conducted volatile organic compound (VOC) and radiological field screening for health and safety monitoring and to identify the presence or absence of COPCs.

- Logged the drill cores to describe soil characteristics and identify the muck/native material contact.

- Conducted laboratory analysis of the environmental and quality control (QC) samples to determine the presence or absence of COPCs.

- Collected three background samples from undisturbed areas around the Muckpile.

The sampling was a combination of simple random and judgmental (biased) sampling. The random sampling locations on the Muckpile and in the drainage were selected based on a simple random sampling method as described in Chapter 4 of Statistical Methods for Environmental Pollution Monitoring (Gilbert, 1987). The random sampling locations in the first pond were 
selected based on a stratified random sampling method as described in Chapter 5 of the same reference (Gilbert, 1987). The biased samples were collected from locations where elevated radiological levels were identified by the drive-over and/or walk-over surveys, process knowledge indicates surface operations could have contributed COPCs to the environment, or visual evidence indicates impacted soils. 


\section{A.2.0 Project Description}

The Area 12 E-Tunnel Sites, CAU 383, is located approximately 42 miles north of Mercury in Area 12 of the Nevada Test Site (NTS) (Figure A.2-1). Corrective Action Unit 383

(Figure A.2-2) consists of three CASs: 12-06-06, Muckpile; 12-25-02, Oil Spill; and 12-28-02, Radioactive Material. The E-Tunnel was used for nine nuclear weapons effects tests between 1958 and 1977. The E-Tunnel Muckpile (CAS 12-06-06) consists of an estimated 355,000 cubic yards of material mined out of the tunnel. The surface elevation at the top of the Muckpile is about 6,120 feet (ft) above mean sea level. The Muckpile was deposited in an easterly trend in front of the tunnel portals. The muckpile is approximately $200 \mathrm{ft}$ across and 1,200 ft long in an east-west direction. The thickness of the Muckpile ranges from less than $5 \mathrm{ft}$ at the west end to approximately $100 \mathrm{ft}$ at the northeast side. The drainage that was included in this investigation trends easterly along the base of the Muckpile.

Corrective Action Site 12-28-02 (Radioactive Material) is located at the E-Tunnel Main portal and is part of the Muckpile.

Corrective Action Site 12-25-02 (Oil Spill) is located on the blower pad, which is on a bench approximately $100 \mathrm{ft}$ above the E-Tunnel south portal. The pad was constructed in the hillside and is about $100 \mathrm{ft}$ long north to south and $50 \mathrm{ft}$ wide. Fill on the pad varies in thickness from less than $1 \mathrm{ft}$ next to the slope to about $10 \mathrm{ft}$ on the north edge of the pad.

\section{A.2.1 Slope Stability}

Given the site conditions and proposed operating parameters, a slope stability analysis was prepared for the E-Tunnel Muckpile. The work restriction, based on the analysis, was that drilling would not be conducted within $20 \mathrm{ft}$ of the edge of the Muckpile.

\section{A.2.2 Surface Radiological Surveys}

Before beginning drilling and sampling, walk-over and drive-over radiological surveys were conducted to identify surface and near surface areas with elevated readings (see Figure A.3-1). 


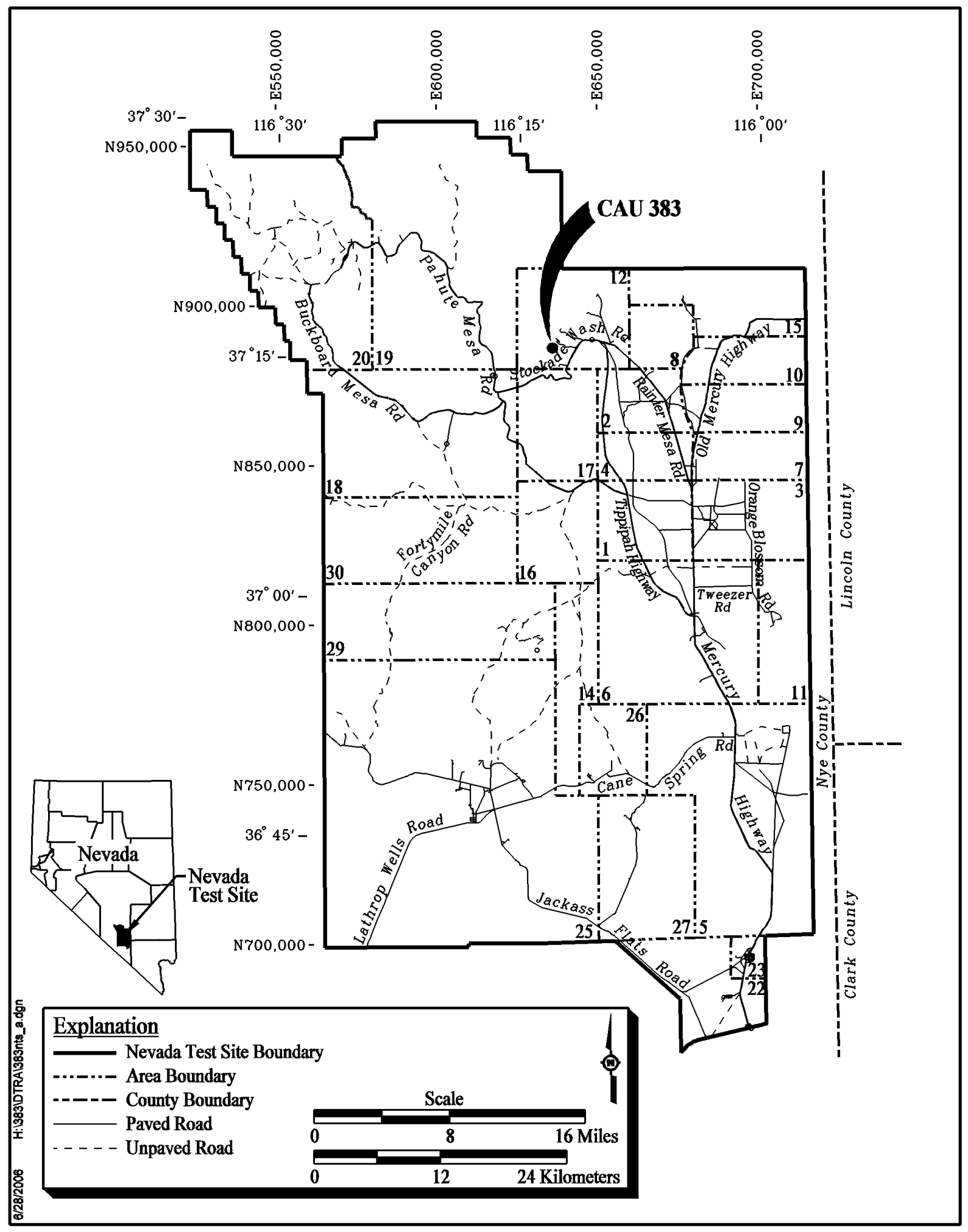

Figure A.2-1

Area 12 E-Tunnel Location 


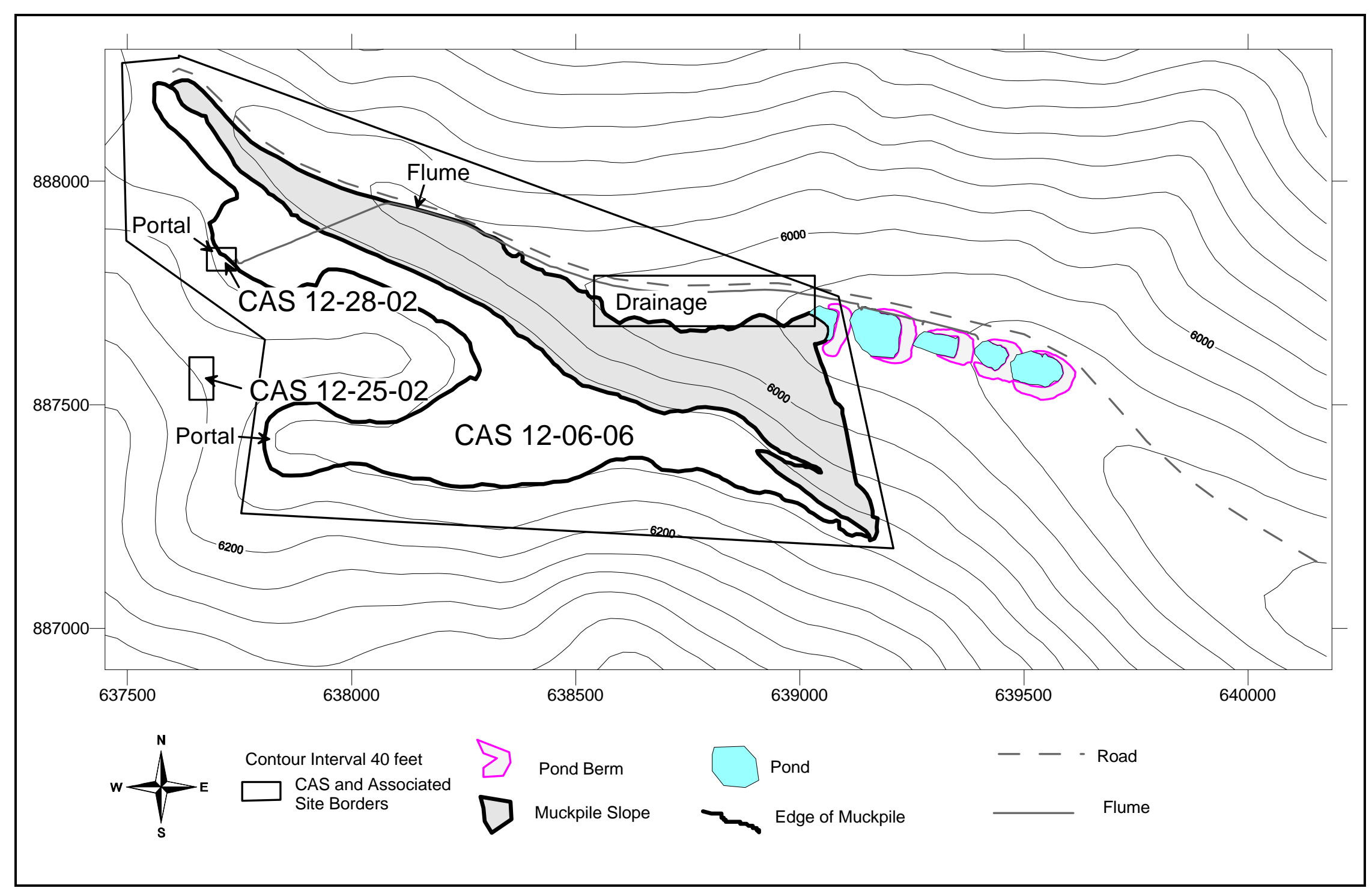

Figure A.2-2

CAU 383 (E-Tunnel Sites) CASs 


\section{A.2.3 Muckpile Sample Locations}

A total of 33 Muckpile locations were sampled: 15 biased locations (two locations were hand sampled) and 18 random locations. The Muckpile random sample locations at CAU 383 were identified using a simple random sampling strategy as defined in Gilbert (1987). Before commencing the drilling operations, Stoller-Navarro Joint Venture (SNJV) scientists used a Trimble Global Positioning System (GPS) total station surveying instrument to locate and mark each proposed sample location. The CAIP listed six biased sampling locations; the additional nine biased locations were identified and marked during a site visit by the Site Supervisor (SS) and DTRA to provide additional information on the outer edge of the Muckpile. This information was collected to support a potential corrective action that would have required this area to be excavated and cut away. After drilling operations were completed, all sampling locations were surveyed using a GPS to record the as-built locations.

\section{A.2.4 Drainage Sample Locations}

A total of 35 random locations were sampled in the drainage; 33 were hand sampled and two were sampled using the drill rig. The drainage random sample locations were identified using a simple random sampling strategy as defined in Gilbert (1987). Before beginning sampling operations, SNJV scientists used the Trimble GPS total station surveying instrument to identify and mark the proposed sample locations. After sampling operations were completed, all sampling locations were surveyed using the GPS to record the as-built locations.

\section{A.2.5 Pond Sample Locations}

Nine locations were sampled in three of the ponds as specified in the CAIP (DTRA, 2003).

Ponds 4, 5, and 6 were not sampled because they are still receiving water from E-Tunnel. Four random locations and one biased location were sampled in Pond 1, two biased location in Pond 2, and two biased locations in Pond 3 (Ponds 2 and 3 were hand sampled). Before beginning drilling operations, SNJV scientists used the Trimble GPS total station surveying instrument to identify and mark the proposed random sample locations. The biased locations were chosen and marked by the client and SS during drilling operations. After sampling operations were completed, all sampling locations were surveyed using the GPS to record the as-built locations. 


\section{A.2.6 Radioactive Material (CAS 12-28-02) Sample Locations}

Four biased locations in the Radioactive Material CAS were sampled, all by hand. Before beginning sampling operations, SNJV scientists marked the proposed sample locations. After sampling operations were completed, all sampling locations were surveyed using the GPS to record the as-built locations.

\section{A.2.7 Oil Spill (CAS 12-25-02) Sample Locations}

Eight biased locations in the Oil Spill CAS were sampled, all by hand. Before beginning sampling operations, SNJV scientists marked the proposed sample locations. After sampling operations were completed, all sampling locations were surveyed using a GPS to record the as-built locations.

\section{A.2.8 Background and Preliminary Sample Locations}

Three biased locations were hand sampled in undisturbed areas near the Muckpile for background information. In addition, five biased locations were hand sampled on the Muckpile for preliminary safety and health information. These samples were collected in February and March 2004, before beginning drilling activities. The SNJV scientists used the Trimble GPS total station surveying instrument to record the sample locations.

\section{A.2.9 Checklists}

To increase the efficiency and completeness of the field effort, checklists were used during the E-Tunnel Sites characterization to track individual field tasks. Table A.2-1 contains a list of the checklists, as well as detailing when and how often each checklist was used. 
Table A.2-1

List of Checklists

\begin{tabular}{|c|c|c|c|c|}
\hline Checklist & Purpose & $\begin{array}{l}\text { Responsible } \\
\text { Persons }\end{array}$ & Frequency & Timing \\
\hline $\begin{array}{l}\text { Waste } \\
\text { Management } \\
\text { Pre-Field }\end{array}$ & $\begin{array}{l}\text { Ensure that all appropriate } \\
\text { documentation has been prepared } \\
\text { and issued, and everything is ready to } \\
\text { properly handle all waste streams } \\
\text { generated at the site. }\end{array}$ & $\begin{array}{l}\text { Waste } \\
\text { Management } \\
\text { Coordinator }\end{array}$ & Once & Before mobilization \\
\hline $\begin{array}{l}\text { Health \& Safety } \\
\text { Pre-Field and } \\
\text { Mobilization }\end{array}$ & $\begin{array}{l}\text { Ensure all documents and required } \\
\text { forms have been prepared and are } \\
\text { available, all facilities have been } \\
\text { notified, all personnel have the } \\
\text { required training and certifications, all } \\
\text { health and safety (H\&S) monitoring } \\
\text { equipment is available and } \\
\text { operational, and all H\&S supplies are } \\
\text { available at the site. This will mitigate } \\
\text { potential exposures to personnel and } \\
\text { reduce the risk of accidents. }\end{array}$ & $\begin{array}{l}\text { Site Safety } \\
\text { Officer }\end{array}$ & Once & $\begin{array}{l}\text { Before and during } \\
\text { mobilization }\end{array}$ \\
\hline Mobilization & $\begin{array}{l}\text { Ensure that the site is ready for the } \\
\text { field activities, all documentation is in } \\
\text { place, and equipment and supplies } \\
\text { are available on site. }\end{array}$ & Site Supervisor & Once & $\begin{array}{l}\text { Before field work } \\
\text { begins }\end{array}$ \\
\hline Geologist & $\begin{array}{l}\text { Ensure the hole is properly field } \\
\text { screened and logged, all required } \\
\text { information is entered on the borehole } \\
\text { log, and the hole is properly } \\
\text { abandoned and marked. }\end{array}$ & Rig Geologist & $\begin{array}{l}\text { One for each } \\
\text { borehole }\end{array}$ & $\begin{array}{l}\text { During and after } \\
\text { drilling }\end{array}$ \\
\hline $\begin{array}{l}\text { Drill Rig Safety } \\
\text { Inspection }\end{array}$ & $\begin{array}{l}\text { Ensure the drill rig meets all required } \\
\text { safety standards to mitigate possible } \\
\text { personnel injury resulting from faulty } \\
\text { or worn equipment, and/or inadequate } \\
\text { safety devices. }\end{array}$ & $\begin{array}{l}\text { Site Supervisor } \\
\text { and/or Rig } \\
\text { Geologist and } \\
\text { Site Safety } \\
\text { Officer }\end{array}$ & Once & $\begin{array}{l}\text { Before the start of } \\
\text { field work }\end{array}$ \\
\hline Sampling & $\begin{array}{l}\text { Ensure all supplies are ready for the } \\
\text { day's sampling, the samples are } \\
\text { collected and handled properly, that } \\
\text { required quality assurance (QA)/QC } \\
\text { samples are collected, and all } \\
\text { paperwork is completed correctly. }\end{array}$ & Sampler & Daily & $\begin{array}{l}\text { Before, during, and } \\
\text { after sampling }\end{array}$ \\
\hline Decontamination & $\begin{array}{l}\text { Ensure adequate equipment and } \\
\text { supplies are available for the day, } \\
\text { decontaminated equipment is properly } \\
\text { handled and screened if } \\
\text { contamination is encountered, and } \\
\text { rinsate and any sediment generated is } \\
\text { properly handled. }\end{array}$ & $\begin{array}{l}\text { Rig Geologist } \\
\text { and/or Site } \\
\text { Safety Officer }\end{array}$ & $\begin{array}{l}\text { Daily on days } \\
\text { when decon } \\
\text { is done }\end{array}$ & $\begin{array}{l}\text { Before, during, and } \\
\text { after } \\
\text { decontamination }\end{array}$ \\
\hline Pressure Washer & $\begin{array}{l}\text { Ensure the pressure washer is safe } \\
\text { and operational. }\end{array}$ & $\begin{array}{l}\text { Equipment } \\
\text { Operator and/or } \\
\text { Rig Geologist }\end{array}$ & $\begin{array}{l}\text { Initial entry to } \\
\text { site, daily } \\
\text { before use }\end{array}$ & $\begin{array}{l}\text { Before use of the } \\
\text { equipment }\end{array}$ \\
\hline
\end{tabular}




\section{A.3.0 Muckpile Investigation}

The drive-over and walk-over radiological surveys at CAU 383 were completed in September 2003. Mobilization of equipment and supplies and site setup for the characterizations at E-Tunnel Sites took place May 17 and 18, 2004. The CAI of the E-Tunnel Sites was conducted over 27 workdays between May 19 and June 24, 2004. The drillers mobilized to the site on May 18. Drilling commenced on May 19 and continued to June 14. From June 14 through June 24, hand samples were collected, as-built GPS surveys were conducted, and demobilization of equipment was completed.

All visitors coming on site had to check in with the Facility Manager Representative, the SS, or the Site Safety Officer (SSO) to receive a site-specific health and safety briefing and the tailgate safety briefing before being allowed on site.

\section{A.3.1 Work Packages}

At CAU 383, DTRA is the primary Real Estate/Operations Permit (REOP) holder (REOP Number DTRA-12) and is responsible for safety at the site. Three Work Packages were prepared by Shaw/SNJV and approved by DTRA so that the work could be conducted under the DTRA REOP. Work package Shaw-2003-DTRA-01 covered the drive-over and walk-over surveys. Work package SNJV 04 E 01 covered mobilization and demobilization from the site and all activities involved in the characterization. Work package SNJV 04 E 02 covered the waste management activities, which continued after the field work was completed. All of the waste has been removed from the site.

Before beginning field work, an NTS Operations Schedule B form was filled out and sent to the Site Operations Center and Bechtel Nevada $(\mathrm{BN})$ to advise them of the planned work activities, locations, dates, and times.

\section{A.3.2 Surface Radiological Surveys}

The walk-over and drive-over radiological surveys were completed in September 2003. Results from both the walk-over and drive-over surveys are shown in Figure A.3-1. 
Date: February 2007

Page A-11 of A-47

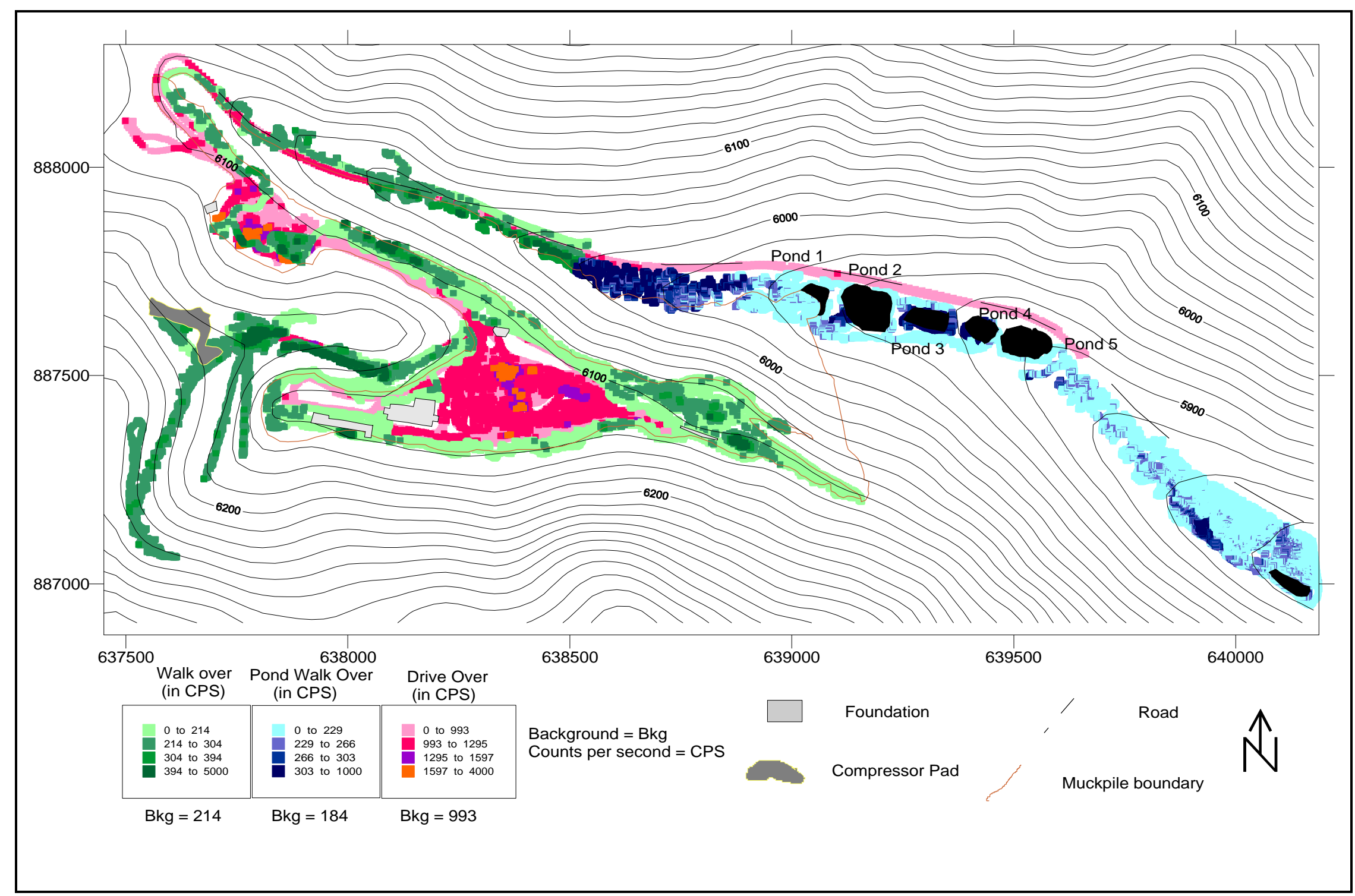

Figure A.3-1

CAU 383 Radiological Surveys 
The drive-over radiological survey of the Muckpile was conducted by towing a Dual Large-Area Plastic Scintillation (DLAPS) Detector Model VRM-3, with a TSA Model SC-755 Controller, and a Trimble Pathfinder Pro XRS ${ }^{\mathrm{TM}}$ GPS receiver with a TSC1 ${ }^{\mathrm{TM}}$ data logger behind a fourwheel-drive vehicle. Each radiological measurement was taken with the DLAPS detector and recorded on the TSC1 data logger. Each point was then stored with its related GPS location measurement in a combined file. Approximately 6 acres were surveyed and a total of 6,782 beta/gamma measurements were recorded. The highest beta/gamma measurement detected was 3,959 counts per second (cps) at Nevada State Plane coordinates E 637,788.0, N 887,586.0. This high level is four times greater than the background radiation emission rate of $993 \mathrm{cps}$ (Figure A.3-1).

The walk-over radiological survey was conducted by walking over the drainage carrying a Small Area Plastic Scintillation (SAPS) Detector Model 8204, with a TSA Model SC-755 Controller, and a Trimble Pathfinder Pro XRS ${ }^{\mathrm{TM}}$ GPS receiver with a TSC1 ${ }^{\mathrm{TM}}$ data logger. Each radiological measurement was taken with the SAPS detector and recorded on the TSC1 data logger and stored with its related GPS measurement in a combined file. Approximately 2 acres were surveyed and more than 25,000 beta/gamma measurements were recorded. The highest beta/gamma measurement detected was 1,160 cps at Nevada State Plane coordinates E 638,076.6, $\mathrm{N} 887,817.5$. This high level is 5.2 times greater than the background radiation emission rate of 214 cps (Figure A.3-1).

\section{A.3.3 Muckpile Samples, CAS 12-06-06}

Soil sampling was accomplished by either drilling or hand sampling. Drilling consisted of drilling boreholes through the Muckpile into the native material underneath using the rotosonic drilling method. If the native material was alluvial in nature, the borehole was advanced $5 \mathrm{ft}$ into the native material or until refusal. If the native material was bedrock, the borehole was only advanced $2 \mathrm{ft}$ into the native material or until refusal. Copies of the soil boring logs for all boreholes are in Attachment A. Hand sampling consisted of using hand tools to collect a sample from a depth of 0 to $0.5 \mathrm{ft}$ or until refusal. A total of $1,039.5 \mathrm{ft}$ of drilling was completed in 32 boreholes. Samples were collected to characterize the Muckpile and/or the native material under the Muckpile in 31 of the boreholes (borehole EMH02 was not sampled; borehole EMH2A was drilled and sampled as the alternate). Two locations were not accessible to the drill rig and were sampled by hand. Cross sections of the Muckpile are in Attachment B. The random locations were designated as EMH01 through EMH18 and the biased locations were designated 
EMB01 through EMB15 (Figure A.3-2). The boreholes were drilled to depths ranging from 1.5 to $91.5 \mathrm{ft}$.

Up to three soil samples were collected from each random borehole (Table A.3-1). In each borehole one sample was collected at a randomly selected depth (the z-depth) and one from the bottom of the borehole. In addition, a sample could be collected from sections of core where field screening indicated elevated alpha, beta, gamma, or flame-ionization detector (FID) levels. Three soil samples could have been collected from each biased borehole: one from the top of the borehole and additional samples from sections of core where field screening indicated elevated readings. One sample was collected from each of the hand-sample locations. If the field screening identified elevated readings for any hand sample, additional samples could be taken. No additional samples were taken from the hand-sample locations due to refusal. A total of 41 environmental soil samples were collected to characterize the muckpile, and 25 environmental soil samples were collected to characterize the native material underneath the muckpile. All of the soil samples were sent to an off-site laboratory to be analyzed for VOCs, semivolatile organic compounds (SVOCs), total petroleum hydrocarbons (TPH)-diesel-range organics (DRO), beryllium, total Resource Conservation and Recovery Act (RCRA) metals, and radionuclides. 


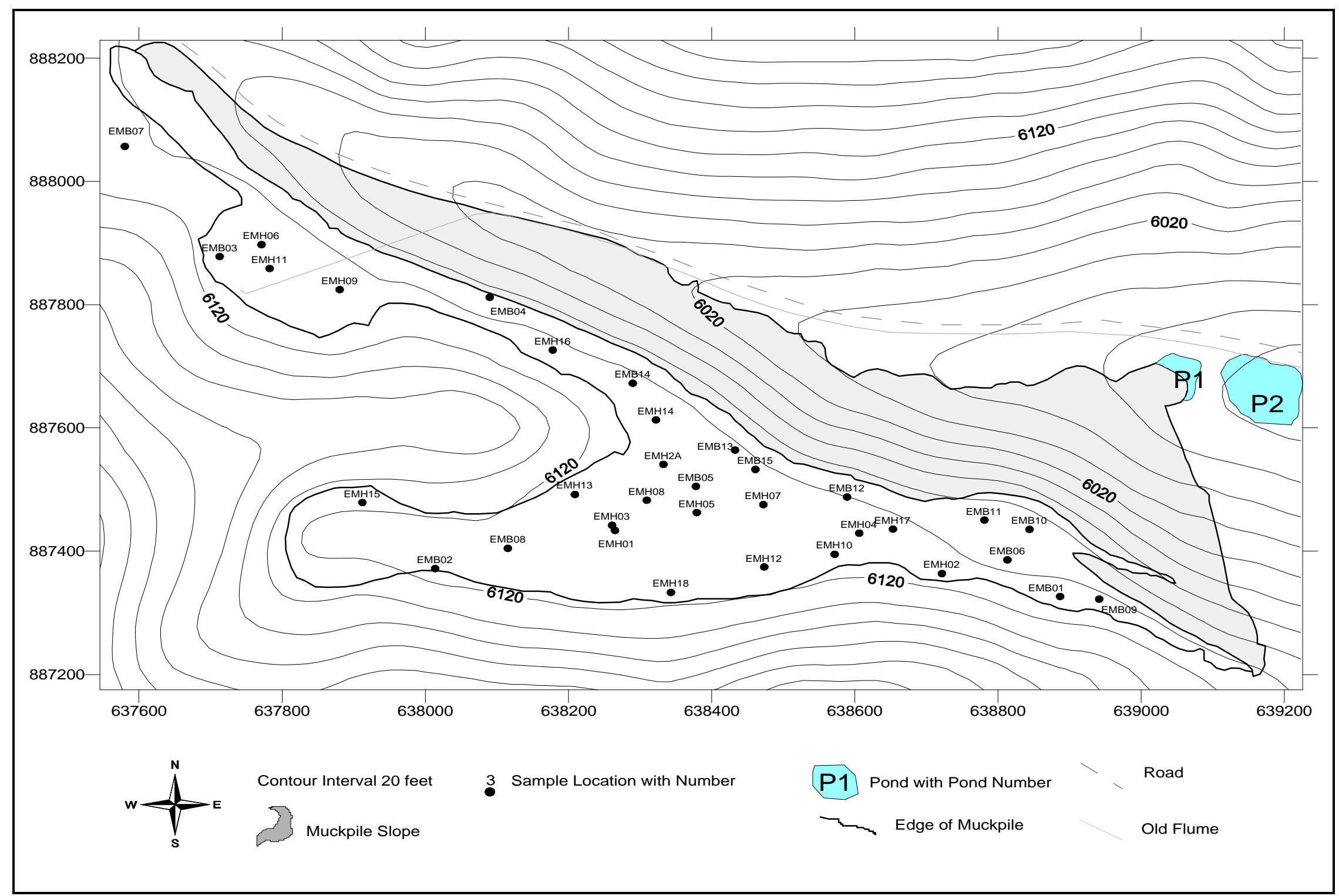

Figure A.3-2

CAU 383 Muckpile Sampling Locations 
Table A.3-1

As-Built Muckpile Sampling Locations and Total Depth

(Page 1 of 2)

\begin{tabular}{|c|c|c|c|c|c|c|c|}
\hline Hole \# & North & East & Elevation & $\begin{array}{c}\text { Sampling } \\
\text { Method }\end{array}$ & $\begin{array}{c}\text { Drill TD in } \\
\mathbf{f t}\end{array}$ & $\begin{array}{l}\text { Hand TD } \\
\text { in } \mathrm{ft}\end{array}$ & $\begin{array}{c}\text { \# of } \\
\text { Samples }\end{array}$ \\
\hline \multicolumn{8}{|c|}{ Biased Muckpile Locations } \\
\hline EMB01 & 887326.5 & 638886.7 & 6123.5 & drill & 12 & & 1 \\
\hline EMB02 & 887371.8 & 638013.7 & 6136.0 & drill & 3.5 & & 1 \\
\hline EMB03 & 887878.1 & 637712.6 & 6121.5 & drill & 9.5 & & 2 \\
\hline EMB04 & 887812.1 & 638089.9 & 6119.3 & hand & & 0.5 & 1 \\
\hline EMB05 & 887505.2 & 638378.0 & 6117.8 & drill & 86.5 & & 3 \\
\hline EMB06 & 887386.0 & 638813.1 & 6105.0 & drill & 7.5 & & 1 \\
\hline EMB07 & 888056.7 & 637580.3 & 6123.1 & drill & 5 & & 1 \\
\hline EMB08 & 887404.7 & 638115.3 & 6132.5 & drill & 31.5 & & 2 \\
\hline EMB09 & 887322.3 & 638941.3 & 6122.3 & drill & 14 & & 3 \\
\hline EMB10 & 887435.4 & 638844.0 & 6102.2 & drill & 37 & & 1 \\
\hline EMB11 & 887450.5 & 638781.0 & 6107.2 & drill & 25.5 & & 3 \\
\hline EMB12 & 887487.9 & 638589.1 & 6123.1 & drill & 75 & & 1 \\
\hline EMB13 & 887564.0 & 638432.6 & 6127.8 & drill & 91.5 & & 2 \\
\hline EMB14 & 887672.4 & 638289.8 & 6121.6 & drill & 29 & & 3 \\
\hline \multirow[t]{2}{*}{ EMB15 } & 887532.7 & 638461.1 & 6119.0 & hand & & 0.5 & 1 \\
\hline & & & & Total & 427.5 & 1.0 & 26 \\
\hline \multicolumn{8}{|c|}{ Random Muckpile Locations } \\
\hline EMH01 & 887433.7 & 638265.0 & 6128.6 & drill & 69 & & 2 \\
\hline $\mathrm{EMHO2}$ & 887363.7 & 638721.6 & 6118.8 & drill & 5 & & 0 \\
\hline $\mathrm{EMH} 2 \mathrm{~A}$ & 887540.8 & 638332.6 & 6123.4 & drill & 45.5 & & 3 \\
\hline EMH03 & 887442.1 & 638260.8 & 6131.4 & drill & 61.5 & & 3 \\
\hline EMH04 & 887429.3 & 638606.1 & 6115.4 & drill & 42 & & 2 \\
\hline EMH05 & 887462.7 & 638379.3 & 6123.6 & drill & 70 & & 3 \\
\hline EMH06 & 887897.5 & 637771.1 & 6119.0 & drill & 1.5 & & 1 \\
\hline EMH07 & 887475.6 & 638472.2 & 6123.5 & drill & 68 & & 3 \\
\hline EMH08 & 887482.7 & 638309.3 & 6125.9 & drill & 59 & & 2 \\
\hline EMH09 & 887824.4 & 637880.4 & 6121.9 & drill & 25 & & 2 \\
\hline EMH10 & 887395.0 & 638571.8 & 6120.8 & drill & 20 & & 2 \\
\hline EMH11 & 887858.6 & 637782.5 & 6116.5 & drill & 4.5 & & 2 \\
\hline
\end{tabular}


Table A.3-1

As-Built Muckpile Sampling Locations and Total Depth

(Page 2 of 2)

\begin{tabular}{||c|c|c|c|c|c|c|c||}
\hline Hole \# & North & East & Elevation & Sampling & Drill TD in & Hand TD in & \# of \\
ft & & & Samples \\
\hline \hline EMH12 & 887374.4 & 638473.4 & 6124.1 & drill & 25.5 & & 2 \\
\hline EMH13 & 887492.0 & 638209.0 & 6136.5 & drill & 21 & & 2 \\
\hline EMH14 & 887613.0 & 638322.2 & 6129.7 & drill & 22.5 & & 2 \\
\hline EMH15 & 887479.0 & 637912.1 & 6133.6 & drill & 4 & & 2 \\
\hline EMH16 & 887726.3 & 638178.0 & 6127.8 & drill & 19.5 & & 3 \\
\hline EMH17 & 887435.9 & 638653.1 & 6122.8 & drill & 36.5 & & 2 \\
\hline EMH18 & 887333.0 & 638343.0 & 6129.9 & drill & 12 & & 2 \\
\hline
\end{tabular}

$\mathrm{TD}=$ Total depth

\section{A.3.4 Drainage Soil Samples}

There were 35 locations sampled in the drainage, and all were random locations collected to determine whether contamination is migrating in storm runoff. Due to the inaccessibility of most of the drainage to the drill rig, only two locations were drilled (Figure A.3-3), and 33 locations were sampled using hand tools. At the locations sampled with the rotosonic drill rig, a hole was drilled to a depth of $5 \mathrm{ft}$, and one sample was collected. At the hand-sampled locations, one sample was collected between 0 and $0.5 \mathrm{ft}$ bgs. A total of $10 \mathrm{ft}$ of rotosonic drilling and $16.5 \mathrm{ft}$ of hand digging was completed in two boreholes and 33 hand-sample locations to characterize the drainage. Once the drill core was recovered, it was field screened and sampled. If the field screening did not identify elevated readings for any sections of core, a sample was collected between 0 and $1 \mathrm{ft}$, and no other samples were collected. No sections of drill core were found that had elevated radiological readings. The hand samples were collected between 0 and $0.5 \mathrm{ft}$. As stated in the CAIP (DTRA, 2003), if the field screening identified elevated readings for any hand sample, additional samples would be taken. No additional samples were taken from the hand-sample locations due to refusal. A total of 35 environmental soil samples were collected from 35 locations to characterize the soil in the drainage. Sample locations in the drainage were designated EDH01 through EDH35. All of the soil samples were sent to an off-site laboratory to be analyzed for VOCs, SVOCs, TPH-DRO, beryllium, total RCRA metals, and radionuclides. The drainage sampling locations and total depths are shown in Table A.3-2. 


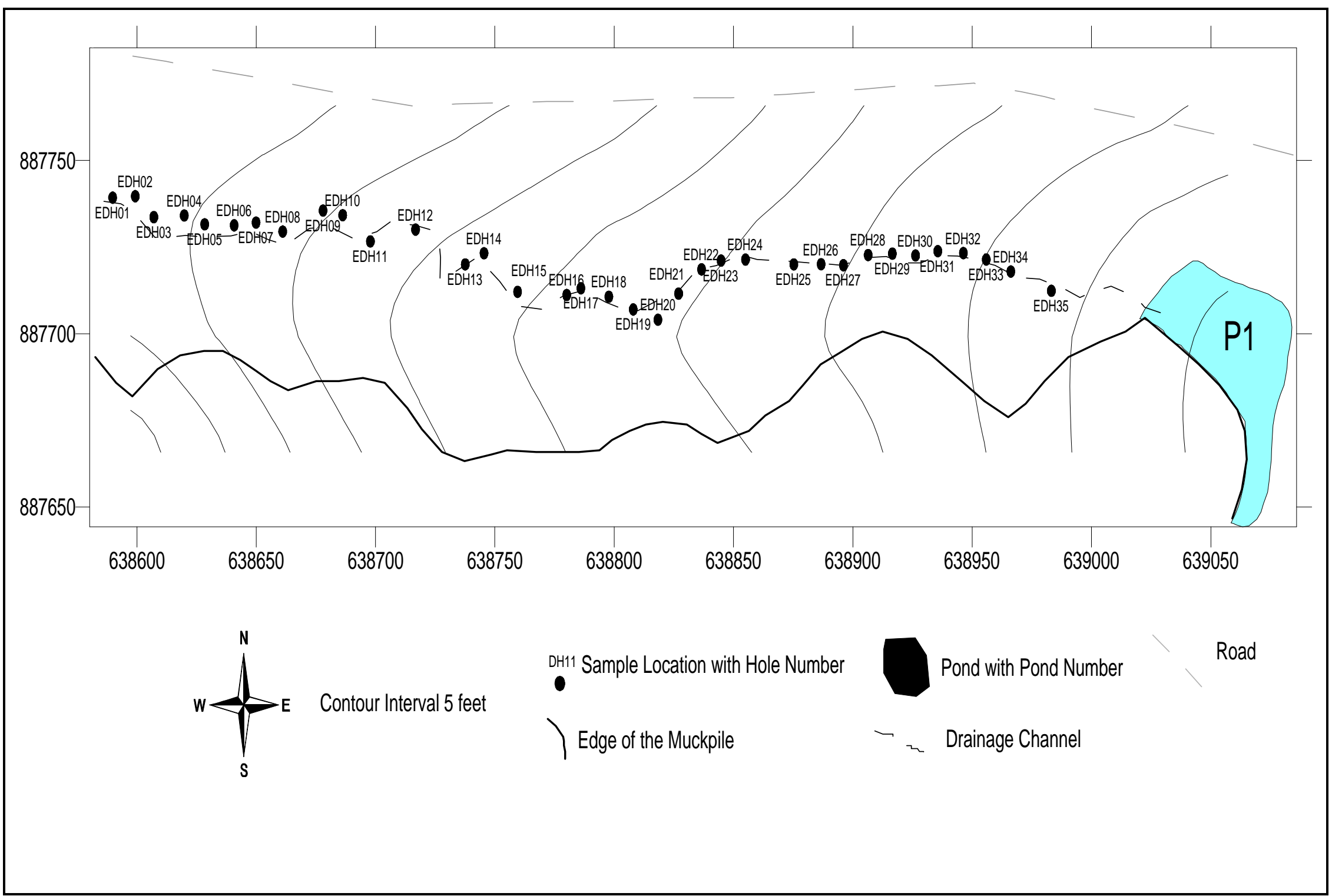

Figure A.3-3

CAU 383 Drainage Sampling Locations 
Table A.3-2

As-Built Drainage Sampling Locations and Total Depths

(Page 1 of 2)

\begin{tabular}{|c|c|c|c|c|c|c|c|}
\hline Hole \# & North & East & Elevation & $\begin{array}{l}\text { Sampling } \\
\text { Method }\end{array}$ & $\begin{array}{l}\text { Drill TD } \\
\text { in } \mathrm{ft}\end{array}$ & $\begin{array}{c}\text { Hand TD } \\
\text { in ft }\end{array}$ & $\begin{array}{c}\text { \# of } \\
\text { Samples }\end{array}$ \\
\hline \multicolumn{8}{|c|}{ Random Drainage Locations } \\
\hline EDH01 & 887739.2 & 638589.8 & 6007.8 & hand & & 0.5 & 1 \\
\hline EDH02 & 887739.6 & 638599.3 & 6006.8 & hand & & 0.5 & 1 \\
\hline EDH03 & 887733.6 & 638607.1 & 6004.1 & hand & & 0.5 & 1 \\
\hline EDH04 & 887734.1 & 638619.8 & 6003 & hand & & 0.5 & 1 \\
\hline EDH05 & 887731.5 & 638628.4 & 6003.1 & hand & & 0.5 & 1 \\
\hline EDH06 & 887731.2 & 638640.7 & 5998.9 & hand & & 0.5 & 1 \\
\hline EDH07 & 887732 & 638649.9 & 5997.1 & hand & & 0.5 & 1 \\
\hline EDH08 & 887729.5 & 638661.1 & 5999.9 & hand & & 0.5 & 1 \\
\hline EDHO9 & 887735.5 & 638678 & 5997.2 & hand & & 0.5 & 1 \\
\hline EDH10 & 887734.2 & 638686.2 & 5995.1 & hand & & 0.5 & 1 \\
\hline EDH11 & 887726.6 & 638697.8 & 5984.3 & hand & & 0.5 & 1 \\
\hline EDH12 & 887730 & 638716.7 & 5983.9 & hand & & 0.5 & 1 \\
\hline EDH13 & 887720 & 638737.5 & 5970.7 & hand & & 0.5 & 1 \\
\hline EDH14 & 887723.2 & 638745.4 & 5982.0 & hand & & 0.5 & 1 \\
\hline EDH15 & 887712.1 & 638759.5 & 5976.8 & hand & & 0.5 & 1 \\
\hline EDH16 & 887711.1 & 638780.1 & 5983.1 & hand & & 0.5 & 1 \\
\hline EDH17 & 887713.1 & 638786.0 & 5978.8 & hand & & 0.5 & 1 \\
\hline EDH18 & 887710.6 & 638797.7 & 5977.6 & hand & & 0.5 & 1 \\
\hline EDH19 & 887707.0 & 638808.0 & 5975.1 & hand & & 0.5 & 1 \\
\hline EDH20 & 887704.0 & 638818.3 & 5972.7 & hand & & 0.5 & 1 \\
\hline EDH21 & 887711.5 & 638827 & 5976.5 & hand & & 0.5 & 1 \\
\hline EDH22 & 887718.5 & 638836.6 & 5972 & hand & & 0.5 & 1 \\
\hline EDH23 & 887721.1 & 638844.8 & 5976.1 & hand & & 0.5 & 1 \\
\hline $\mathrm{EDH} 24$ & 887721.4 & 638855 & 5972.1 & hand & & 0.5 & 1 \\
\hline EDH25 & 887720 & 638875.3 & 5968 & hand & & 0.5 & 1 \\
\hline EDH26 & 887720 & 638886.7 & 5971.4 & hand & & 0.5 & 1 \\
\hline EDH27 & 887719.7 & 638896 & 5970.9 & hand & & 0.5 & 1 \\
\hline EDH28 & 887722.7 & 638906.4 & 5972.1 & hand & & 0.5 & 1 \\
\hline EDH29 & 887723.1 & 638916.6 & 5969.8 & hand & & 0.5 & 1 \\
\hline
\end{tabular}


Table A.3-2

As-Built Drainage Sampling Locations and Total Depths

(Page 2 of 2)

\begin{tabular}{||c|c|c|c|c|c|c|c||}
\hline Hole \# & North & East & Elevation & $\begin{array}{c}\text { Sampling } \\
\text { Method }\end{array}$ & $\begin{array}{c}\text { Drill TD } \\
\text { in ft }\end{array}$ & $\begin{array}{c}\text { Hand TD } \\
\text { in ft }\end{array}$ & $\begin{array}{c}\text { \# of } \\
\text { Samples }\end{array}$ \\
\hline \hline EDH30 & 887722.5 & 638926.3 & 5971.0 & hand & & 0.5 & 1 \\
\hline EDH31 & 887723.8 & 638935.6 & 5969.6 & hand & & 0.5 & 1 \\
\hline EDH32 & 887723.2 & 638946.3 & 5968.2 & hand & & 0.5 & 1 \\
\hline EDH33 & 887721.4 & 638955.9 & 5963.7 & hand & & 0.5 & 1 \\
\hline EDH34 & 887717.9 & 638966.2 & 5964.4 & drill & 5.0 & & 1 \\
\hline EDH35 & 887712.3 & 638983.2 & 5961.7 & drill & 5.0 & & 1 \\
\hline & & & & Total & 10.0 & 16.5 & 35 \\
\hline
\end{tabular}

\section{A.3.5 Pond Soil Samples}

Fourteen pond soil samples were collected from nine pond locations. Four random and one biased sampling locations were drilled in Pond 1. Ponds 2 and 3 had two biased hand-sampling locations each (Figure A.3-4). At the random locations, a hole was drilled to depths of 18 to 21 $\mathrm{ft}$, and two samples were collected. One sample was collected at a randomly selected depth (the z-depth) and one from the bottom of the borehole. Two soil samples were collected from the biased borehole as well, one from above the native material contact and one from the bottom of the borehole (this borehole was $5 \mathrm{ft}$ deep). At the hand-sampled locations (Ponds 2 and 3), a hole was dug to $0.5 \mathrm{ft}$ deep, and one sample was collected. Pond 1 had five sample locations drilled. Due to the wetness of the soil in Ponds 2 and 3, only two locations were hand sampled in each pond. As stated in the CAIP (DTRA, 2003) if the field screening identified elevated readings for any hand sample, additional samples could be taken. No additional samples were taken from the hand-sample locations due to refusal. A total of $80 \mathrm{ft}$ of rotosonic drilling and $2 \mathrm{ft}$ of hand digging was completed in five boreholes and four hand-sample locations. Drilling consisted of drilling boreholes through the pond sediments (muck) into the native material underneath using the rotosonic drill rig. Once the core was recovered, it was field screened and sampled. A total of 14 environmental soil samples, nine muckpile material and five native material, were collected from nine locations to characterize the ponds. Ponds 4, 5, and 6 were not sampled because they are still active and receiving discharge water from E-Tunnel.

In the ponds, random sample locations were designated EPH01 through EPH04, and the biased samples location were designated as EPB01 through EPB05. All of the soil samples were sent to 


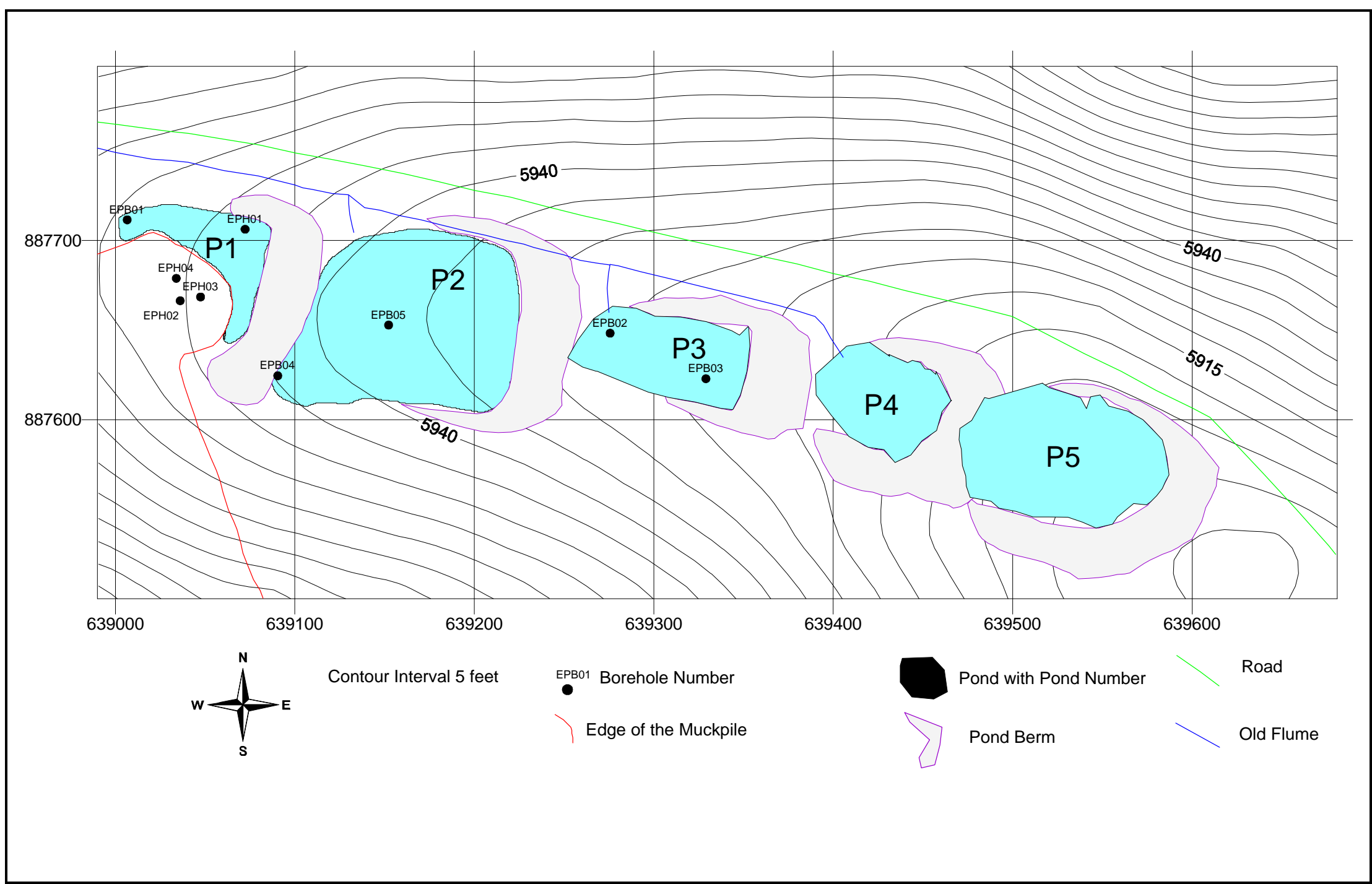

Figure A.3-4

CAU 383 Ponds Sampling Locations 
Table A.3-3

As-Built Ponds Sampling Locations and Total Depths

\begin{tabular}{|c|c|c|c|c|c|c|c||}
\hline Hole \# & North & East & Elevation & $\begin{array}{c}\text { Sampling } \\
\text { Method }\end{array}$ & $\begin{array}{c}\text { Drill TD } \\
\text { in ft }\end{array}$ & $\begin{array}{c}\text { Hand TD } \\
\text { in ft }\end{array}$ & $\begin{array}{c}\text { \# of } \\
\text { Samples }\end{array}$ \\
\hline \hline \multicolumn{7}{|c||}{ Biased Pond Locations } \\
\hline \hline EPB01 & 887711.6 & 639006.6 & 5959.0 & drill & 5.0 & & 2 \\
\hline EPB02 & 887648.3 & 639275.7 & 5917.5 & hand & & 0.5 & 1 \\
\hline EPB03 & 887622.8 & 639329.0 & 5927.1 & hand & & 0.5 & 1 \\
\hline EPB04 & 887624.5 & 639090.5 & 5942.3 & hand & & 0.5 & 1 \\
\hline EPB05 & 887652.8 & 639152.3 & 5935.2 & hand & & 0.5 & 1 \\
\hline & & & & Total & 5.0 & 2.0 & 6 \\
\hline \hline & & \multicolumn{7}{|c|}{ Random Pond Location } & & 2 \\
\hline \hline EPH01 & 887706.3 & 639072.3 & 5954.9 & drill & 17.0 & & 2 \\
\hline EPH02 & 887666.4 & 639036.1 & 5954.3 & drill & 18.0 & & 2 \\
\hline EPH03 & 887668.5 & 639047.4 & 5964.2 & drill & 21.0 & & 2 \\
\hline EPH04 & 887678.9 & 639033.9 & 5943.2 & drill & 19.0 & & 2 \\
\hline & & & & Total & 75.0 & & 2 \\
\hline
\end{tabular}

an off-site laboratory to be analyzed for VOCs, SVOCs, TPH-DRO, beryllium, total RCRA metals, and radionuclides. The ponds sampling locations and total depths are shown in Table A.3-3.

\section{A.3.6 Oil Spill Soil Samples, CAU 12-25-02}

Eight soil samples were collected from eight Oil Spill locations (Figure A.3-5). All of the locations were biased locations, and all were hand sampled. At each location, one sample was collected from 0 to $0.5 \mathrm{ft}$ bgs. Four feet of hand digging was completed at eight hand-sample locations. In accordance with the CAIP (DTRA, 2003), if the field screening identified elevated readings for any hand-sample, additional samples could be taken. No additional samples were taken from the hand-sample locations due to refusal. In this CAS, the biased sample locations were designated as EOB01 through EOB08. All of the soil samples were sent to an off-site laboratory to be analyzed for VOCs, SVOCs, TPH-DRO, beryllium, total RCRA metals, and radionuclides. The Oil Spill sampling locations and total depths are shown in Table A.3-4.

\section{A.3.7 Radioactive Material Soil Samples, CAU 12-28-02}

Four soil samples were collected from four Radioactive Material area locations (Figure A.3-5). All of the locations were biased locations, and all were hand sampled. At each location, one sample was collected from 0 to $0.5 \mathrm{ft}$ bgs. In accordance with the CAIP (DTRA, 2003), $2 \mathrm{ft}$ 
of hand digging was completed at four hand-sample locations. If the field screening identified elevated readings for any hand-sample additional samples could be taken; however, no additional samples were taken due to refusal. In this CAS, the biased sample locations were designated as ERB01 through ERB04. All of the soil samples were sent to an off-site laboratory to be analyzed for VOCs, SVOCs, TPH-DRO, beryllium, total RCRA metals, and radionuclides. The Radioactive Material area sampling locations and total depths are shown in Table A.3-4.

\section{A.3.8 Background and Preliminary Soil Samples}

The client and SS selected three undisturbed locations adjacent to the E-Tunnel Sites for collection of background native soil samples, and four locations from the surface of the Muckpile for the collection of preliminary samples. All the sampling locations were hand-sampled biased locations, and all the samples were collected at $0.5 \mathrm{ft}$ below ground surface in February 2004 (Figure A.3-6). Both sets of samples were sent to an off-site laboratory and analyzed for beryllium, lead, arsenic, and radionuclides. In March 2004, a biased hand sample was collected from a contaminated layer, approximately 6 inches below the surface in front of the E-Tunnel main portal (location EGB09). The sample was sent to an off-site laboratory, and analyzed for strontium-90, isotopic plutonium, and gamma spec. These sample locations were designated as EGB01 through EGB09. Locations EGB05, EGB06, and EGB07 are the background sampling locations, and EGB01, EGB02, EGB03, EGB04, and EGB09 are the preliminary sampling locations (there is no location EGB08). The sampling location coordinates and total depths are shown in Table A.3-5. 


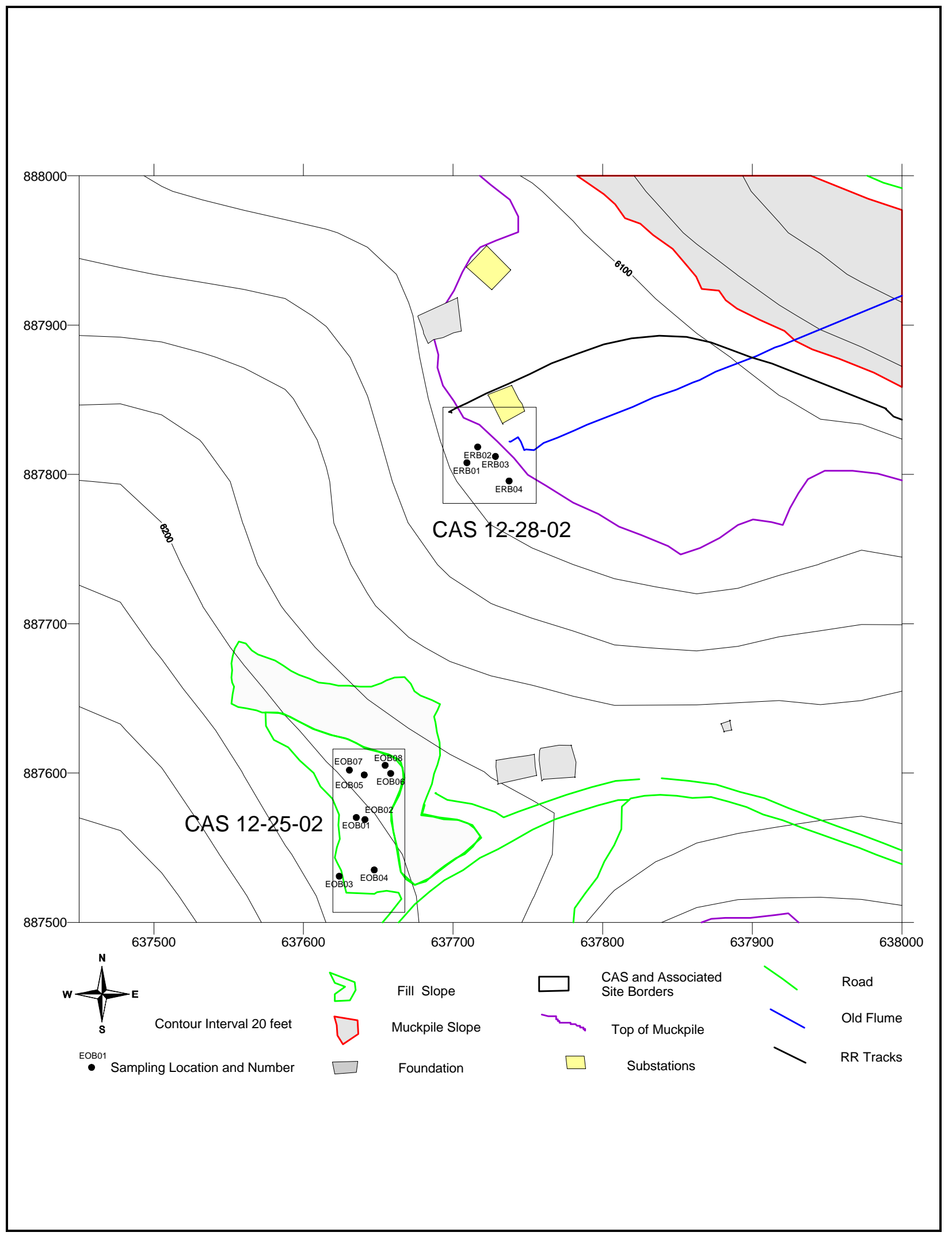

Figure A.3-5

CAU 383 Oil Spill and Radioactive Material Sampling Locations 
Table A.3-4

As-Built Oil Spill and Radioactive Material Sampling Locations and Total Depths

\begin{tabular}{|c|c|c|c|c|c|c|c|}
\hline Hole \# & North & East & Elevation & $\begin{array}{l}\text { Sampling } \\
\text { Method }\end{array}$ & $\begin{array}{l}\text { Drill TD } \\
\text { in } \mathbf{f t}\end{array}$ & $\begin{array}{c}\text { Hand TD } \\
\text { in } \mathrm{ft}\end{array}$ & $\begin{array}{c}\text { \# of } \\
\text { Samples }\end{array}$ \\
\hline \multicolumn{8}{|c|}{ Biased Oil Spill Locations } \\
\hline EOB01 & 637635.3 & 887570.3 & 6217.6 & hand & & 0.5 & 1 \\
\hline EOB02 & 637641.1 & 887568.9 & 6218.0 & hand & & 0.5 & 1 \\
\hline EOB03 & 637623.9 & 887531.0 & 6213.9 & hand & & 0.5 & 1 \\
\hline EOB04 & 637647.3 & 887535.2 & 6220.8 & hand & & 0.5 & 1 \\
\hline EOB05 & 637630.7 & 887602.0 & 6218.3 & hand & & 0.5 & 1 \\
\hline EOB06 & 637658.3 & 887599.8 & 6213.0 & hand & & 0.5 & 1 \\
\hline ЕOB07 & 637640.6 & 887598.8 & 6207.4 & hand & & 0.5 & 1 \\
\hline \multirow[t]{2}{*}{ EOB08 } & 637654.6 & 887605.2 & 6220.9 & hand & & 0.5 & 1 \\
\hline & & & & Total & & 4.0 & 8 \\
\hline \multicolumn{8}{|c|}{ Biased Radiation Material Locations } \\
\hline ERB01 & 637709.2 & 887807.9 & 6122.6 & hand & & 0.5 & 1 \\
\hline ERB02 & 637716.4 & 887818.4 & 6119.5 & hand & & 0.5 & 1 \\
\hline ERB03 & 637728.3 & 887812.1 & 6121.3 & hand & & 0.5 & 1 \\
\hline \multirow[t]{2}{*}{ ERB04 } & 637737.4 & 887795.6 & 6118.7 & hand & & 0.5 & 1 \\
\hline & & & & Total & & 2.0 & 4 \\
\hline
\end{tabular}




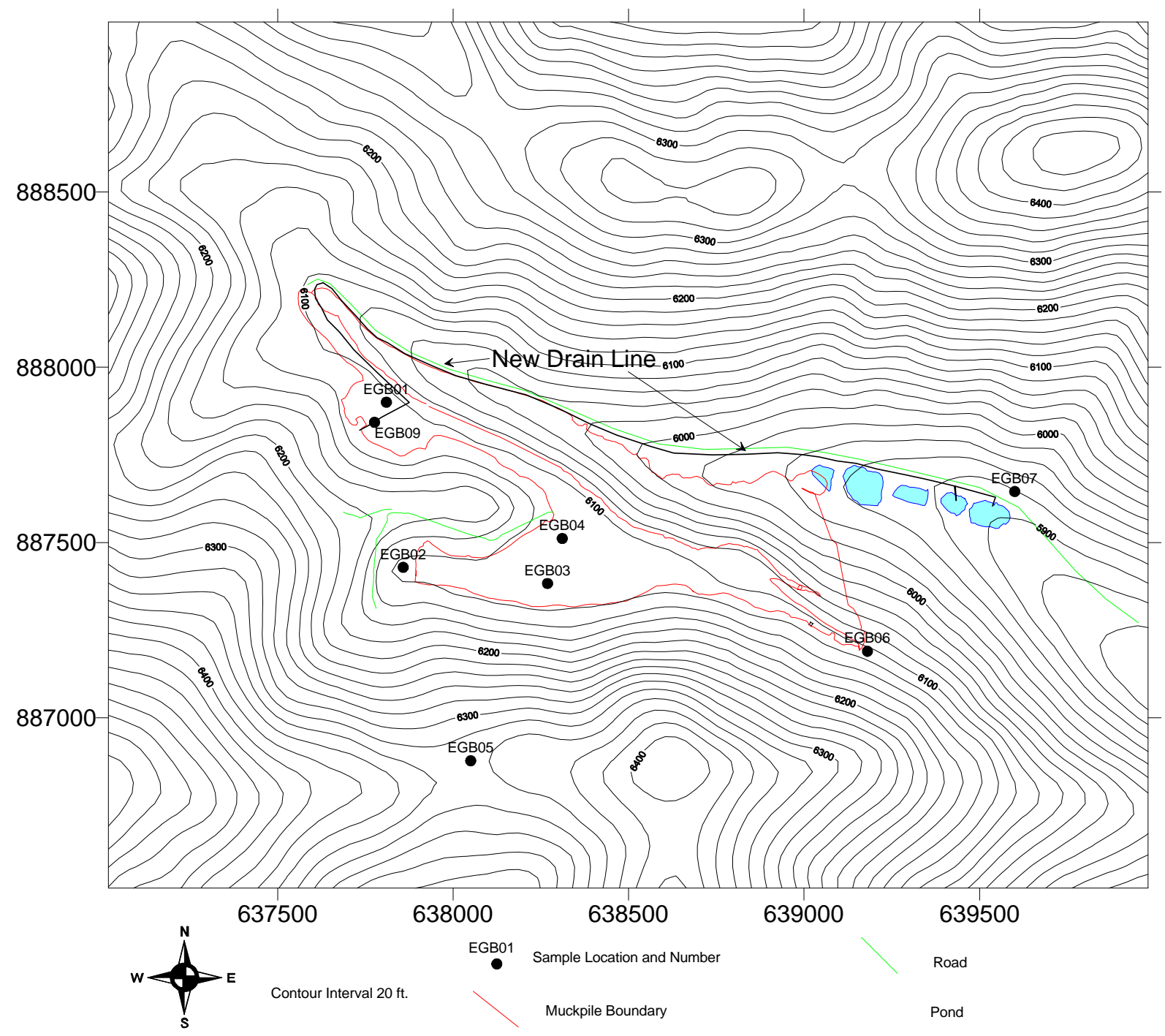

Figure A.3-6

CAU 383 Background and Preliminary Sampling Locations 
Table A.3-5

As-Built Background and Preliminary Sampling Locations Total Depths

\begin{tabular}{||c|c|c|c|c|c|c|c|c||}
\hline Hole \# & North & East & Elevation & $\begin{array}{c}\text { Sampling } \\
\text { Method }\end{array}$ & $\begin{array}{c}\text { Drill TD } \\
\text { in ft }\end{array}$ & $\begin{array}{c}\text { Hand TD in } \\
\text { ft }\end{array}$ & $\begin{array}{c}\text { \# of } \\
\text { Samples }\end{array}$ \\
\hline \hline \multicolumn{7}{|c||}{ Biased Background / Preliminary Locations } \\
\hline \hline EGB01 & 887900.2 & 637809.9 & 6122.7 & hand & & 0.5 & 1 \\
\hline EGB02 & 887429.3 & 637857.8 & 6130.6 & hand & & 0.5 & 1 \\
\hline EGB03 & 887383.3 & 638269.0 & 6129.3 & hand & & 0.5 & 1 \\
\hline EGB04 & 887511.6 & 638310.9 & 6123.5 & hand & & 0.5 & 1 \\
\hline EGB05 & 886877.4 & 638050.2 & 6356.9 & hand & & 0.5 & 1 \\
\hline EGB06 & 887189.7 & 639180.4 & 6132.5 & hand & & 0.5 & 1 \\
\hline EGB07 & 887645.5 & 639600.3 & 5913.3 & hand & & 0.5 & 1 \\
\hline EGB09 & 887856.9 & 637771.0 & 6122.7 & hand & & 0.5 & 1 \\
\hline
\end{tabular}

\section{A.3.9 Other Sampling}

In addition to the environmental samples, $51 \mathrm{QA} / \mathrm{QC}$ and other samples were collected during the site characterization. The QA/QC samples included eight blind duplicate samples, collected and analyzed to check on the laboratory's precision; seven laboratory QC samples, collected to check for matrix interference; seven rinsate samples, collected to check on the effectiveness of the decontamination procedures; seven field blanks, collected to check on possible environmental interferences; and 22 trip blanks, sent with the VOC samples.

Other sampling consisted of collecting a water sample from the driller's water truck (a source blank). All of the QC samples were sent to the off-site laboratory for analysis. 


\section{A.4.0 Results}

A spreadsheet of the analytical results from the CAU 383 E-Tunnel Sites investigation is provided in Attachment C. The spreadsheet includes only those analytical results where COPCs were reported above the measured detectable concentration, even if the detect was qualified as estimated ( $\mathrm{J}$ qualifier) or the radionuclide identification was tentative (TI qualifier). A detect indicates that the parameter was identified in the sample, and does not have any reference to action levels or regulatory limits. The analytical results from the investigation are summarized in the following tables. Results from duplicate samples are not included. If a sample was reanalyzed by the laboratory, the re-analysis results are included in the table, not the original results. Table A.4-1 is a summary of the detects from the muckpile and Ponds, muck and native soil samples, which were analyzed for VOCs, SVOCs, TPH-DRO, beryllium, total metals, and radionuclides. Table A.4-2 is a summary of the detects from the Radioactive Material area, Oil Spill, drainage, and QC water samples, which were analyzed for VOCs, SVOCs, TPH-DRO, beryllium, total metals, and radionuclides. All the QC water sample results were included. Table A.4-3 is a summary of the detects from the background/preliminary soil samples, collected before beginning characterization, which were analyzed for beryllium, total metals, and radionuclides. An analysis of the data, including comparisons to action levels and regulatory limits, is discussed in Section 2.0 of the main document and in Appendices C and D. 
Table A.4-1

Analytical Result Detects for Muckpile and Pond Samples

Analytical Result Detects for Muckpile
(Page 1 of 4 )

\begin{tabular}{|c|c|c|c|c|c|c|c|c|}
\hline & \multicolumn{4}{|c|}{ Muckpile } & \multicolumn{4}{|c|}{ Ponds } \\
\hline & \multicolumn{2}{|c|}{ Native } & \multicolumn{2}{|c|}{ Muck } & \multicolumn{2}{|c|}{ Native } & \multicolumn{2}{|c|}{ Muck } \\
\hline & Range & $\begin{array}{l}\text { No. of } \\
\text { detects }\end{array}$ & Range & $\begin{array}{c}\text { No. of } \\
\text { detects }\end{array}$ & Range & $\begin{array}{l}\text { No. of } \\
\text { detects }\end{array}$ & Range & $\begin{array}{c}\text { No. of } \\
\text { detects }\end{array}$ \\
\hline Volatile Compounds & $\mu \mathrm{g} / \mathrm{kg}$ & & $\mu \mathrm{g} / \mathrm{kg}$ & & $\mu \mathrm{g} / \mathrm{kg}$ & & $\mu \mathrm{g} / \mathrm{kg}$ & \\
\hline 1,2,4-Trimethylbenzene & ND & 0 & 7.2 & 1 & ND & 0 & ND & 0 \\
\hline 1,3,5-Trimethylbenzene & ND & 0 & 7.2 & 1 & ND & 0 & ND & 0 \\
\hline Acetone & $5.7-18$ & 11 & $7.2-10.0$ & 9 & $6.7-25$ & 5 & $7.2-14.0$ & 3 \\
\hline Ethylbenzene & ND & 0 & 80 & 1 & ND & 0 & 1.6 & 1 \\
\hline Hexachlorobutadiene & 1.5 & 1 & ND & 0 & 1.7 & 1 & ND & 0 \\
\hline M+P-Xylene & ND & 0 & 1200 & 1 & ND & 0 & 8.3 & 1 \\
\hline Methylene Chloride & $0.78-1.6$ & 13 & $0.83-9.5$ & 23 & ND & 0 & $0.95-1.2$ & 3 \\
\hline N-Propylbenzene & ND & 0 & 5.9 & 1 & ND & 0 & ND & 0 \\
\hline O-Xylene & ND & 0 & $1.2-780.0$ & 2 & ND & 0 & 3.3 & 1 \\
\hline P-Isopropyltoluene & ND & 0 & $1.3-13.0$ & 2 & ND & 0 & ND & 0 \\
\hline Semivolatile Compounds & $\mu \mathrm{g} / \mathbf{k g}$ & & $\mu \mathrm{g} / \mathbf{k g}$ & & $\mu \mathrm{g} / \mathbf{k g}$ & & $\mu \mathrm{g} / \mathbf{k g}$ & \\
\hline 2-Methylnaphthalene & ND & 0 & 68 & 1 & ND & 0 & ND & 0 \\
\hline Acenaphthene & ND & 0 & 22 & 1 & ND & 0 & ND & 0 \\
\hline Acenaphthylene & ND & 0 & 14 & 1 & ND & 0 & ND & 0 \\
\hline Anthracene & ND & 0 & 170 & 1 & ND & 0 & ND & 0 \\
\hline Benzo(A)Anthracene & 22 & 1 & $18-1200$ & 10 & ND & 0 & $21-26$ & 2 \\
\hline Benzo(A)Pyrene & 16 & 1 & $15-1300$ & 7 & ND & 0 & $18-28$ & 2 \\
\hline Benzo(B)Fluoranthene & 28 & 1 & $18-1700$ & 8 & ND & 0 & $31-59$ & 2 \\
\hline
\end{tabular}

\section{Uncontrolled When Printed}


Table A.4-1

Analytical Result Detects for Muckpile and Pond Samples

(Page 2 of 4)

\begin{tabular}{|c|c|c|c|c|c|c|c|c|}
\hline & \multicolumn{4}{|c|}{ Muckpile } & \multicolumn{4}{|c|}{ Ponds } \\
\hline & \multicolumn{2}{|c|}{ Native } & \multicolumn{2}{|c|}{ Muck } & \multicolumn{2}{|c|}{ Native } & \multicolumn{2}{|c|}{ Muck } \\
\hline & Range & $\begin{array}{c}\text { No. of } \\
\text { detects }\end{array}$ & Range & $\begin{array}{c}\text { No. of } \\
\text { detects }\end{array}$ & Range & $\begin{array}{c}\text { No. of } \\
\text { detects }\end{array}$ & Range & $\begin{array}{c}\text { No. of } \\
\text { detects }\end{array}$ \\
\hline Benzo(G,H,I)Perylene & ND & 0 & $68-760$ & 4 & ND & 0 & ND & 0 \\
\hline Benzo(K)Fluoranthene & ND & 0 & $34-650$ & 6 & ND & 0 & 31 & 1 \\
\hline Bis(2-Ethylhexyl)Phthalate & $84-220$ & 2 & $72-170$ & 6 & ND & 0 & $85-330$ & 2 \\
\hline Butyl Benzyl Phthalate & ND & 0 & 35 & 1 & ND & 0 & ND & 0 \\
\hline Butyl Benzyl Phthalate & ND & 0 & 35 & 1 & ND & 0 & ND & 0 \\
\hline Carbazole & ND & 0 & 57 & 1 & ND & 0 & ND & 0 \\
\hline Chrysene & 23 & 1 & $21-1000$ & 8 & ND & 0 & $30-66$ & 2 \\
\hline Dibenzofuran & ND & 0 & 65 & 1 & ND & 0 & ND & 0 \\
\hline Di-N-Butyl Phthalate & ND & 0 & 26 & 2 & ND & 0 & ND & 0 \\
\hline Fluoranthene & 23 & 1 & $21-1500$ & 8 & ND & 0 & $20-31$ & 3 \\
\hline Fluorene & ND & 0 & 16 & 1 & ND & 0 & ND & 0 \\
\hline Indeno(1,2,3-Cd)Pyrene & ND & 0 & $27-630$ & 5 & ND & 0 & ND & 0 \\
\hline Naphthalene & ND & 0 & 19 & 1 & ND & 0 & ND & 0 \\
\hline Phenanthrene & ND & 0 & $30-350$ & 6 & ND & 0 & ND & 0 \\
\hline Pyrene & ND & 0 & $91-1600$ & 6 & ND & 0 & $50-63$ & 2 \\
\hline Petroleum Hydrocarbons & $\mathrm{mg} / \mathrm{kg}$ & & $\mathrm{mg} / \mathrm{kg}$ & & $\mathrm{mg} / \mathrm{kg}$ & & $\mathrm{mg} / \mathrm{kg}$ & \\
\hline Diesel-Range Organics & 700 & 1 & $3.2-300$ & 16 & ND & 0 & $1.9-330$ & 7 \\
\hline Metals & $\mathrm{mg} / \mathrm{kg}$ & & $\mathrm{mg} / \mathrm{kg}$ & & $\mathrm{mg} / \mathrm{kg}$ & & $\mathrm{mg} / \mathrm{kg}$ & \\
\hline Arsenic & $0.31-3.8$ & 23 & $0.7-6.0$ & 41 & $1.7-2.9$ & 5 & $1.0-14.0$ & 9 \\
\hline
\end{tabular}

\section{Uncontrolled When Printed}


Table A.4-1

Analytical Result Detects for Muckpile and Pond Samples

(Page 3 of 4)

\begin{tabular}{|c|c|c|c|c|c|c|c|c|}
\hline & \multicolumn{4}{|c|}{ Muckpile } & \multicolumn{4}{|c|}{ Ponds } \\
\hline & \multicolumn{2}{|c|}{ Native } & \multicolumn{2}{|c|}{ Muck } & \multicolumn{2}{|c|}{ Native } & \multicolumn{2}{|c|}{ Muck } \\
\hline & Range & $\begin{array}{c}\text { No. of } \\
\text { detects }\end{array}$ & Range & $\begin{array}{c}\text { No. of } \\
\text { detects }\end{array}$ & Range & $\begin{array}{c}\text { No. of } \\
\text { detects }\end{array}$ & Range & $\begin{array}{c}\text { No. of } \\
\text { detects }\end{array}$ \\
\hline Barium & $2.6-370$ & 25 & $22.0-7000$ & 41 & $71.0-3200$ & 5 & $320-6300$ & 9 \\
\hline Beryllium & $0.15-1.0$ & 25 & $0.42-2.4$ & 41 & $0.65-1.0$ & 5 & $0.19-2.0$ & 9 \\
\hline Cadmium & $0.019-1.0$ & 20 & $0.03-15.0$ & 28 & $0.092-0.17$ & 5 & $0.052-0.39$ & 9 \\
\hline Chromium & $0.91-5.8$ & 25 & $0.49-33.0$ & 41 & $1.7-3.4$ & 5 & $1.4-12.0$ & 9 \\
\hline Lead & $1.1-160$ & 16 & $3.4-120$ & 41 & $5.7-12.0$ & 5 & $5.3-38$ & 9 \\
\hline Mercury & $0.008-0.33$ & 25 & $0.0012-0.51$ & 40 & $0.011-0.12$ & 5 & $0.0016-0.048$ & 9 \\
\hline Selenium & $0.26-0.29$ & 2 & $0.24-0.99$ & 12 & ND & 0 & $0.31-0.65$ & 4 \\
\hline Silver & $0.071-2.8$ & 20 & $0.1-1.1$ & 5 & 0.48 & 1 & 0.077 & 1 \\
\hline Radionuclides & $\mathrm{pCi} / \mathrm{g}$ & & $\mathrm{pCi} / \mathrm{g}$ & & $\mathrm{pCi} / \mathrm{g}$ & & $\mathrm{pCi} / \mathrm{g}$ & \\
\hline Actinium-228 & $0.42-2.14$ & 5 & $0.5-2.89$ & 39 & $1.67-2.4$ & 5 & $1.48-2.31$ & 7 \\
\hline Aluminum-26 & ND & 0 & 0.108 & 1 & ND & 0 & ND & 0 \\
\hline Americium-241 & 1.1 & 1 & $0.52-28.8$ & 9 & ND & 0 & 0.72 & 1 \\
\hline Bismuth-212 & ND & 0 & $1.77-3.7$ & 6 & ND & 0 & ND & 0 \\
\hline Bismuth-214 & $0.26-0.92$ & 13 & $0.39-2.08$ & 36 & $0.96-1.49$ & 5 & $0.95-1.72$ & 8 \\
\hline Cesium-137 & $0.121-9.8$ & 9 & $0.15-1060$ & 39 & $0.33-4.34$ & 2 & $0.78-142$ & 8 \\
\hline Europium-152 & ND & 0 & 2.64 & 1 & ND & 0 & ND & 0 \\
\hline Europium-155 & 0.32 & 1 & ND & 0 & ND & 0 & ND & 0 \\
\hline Potassium-40 & $2.05-31.7$ & 11 & $3.7-43.8$ & 41 & $26.2-33.2$ & 5 & 8.1-33.7 & 9 \\
\hline Lead-212 & $0.212-2.27$ & 10 & $0.35-3.37$ & 39 & $1.87-2.76$ & 5 & $0.27-2.73$ & 9 \\
\hline
\end{tabular}

\section{Uncontrolled When Printed}


Table A.4-1

Analytical Result Detects for Muckpile and Pond Samples

(Page 4 of 4 )

\begin{tabular}{|c|c|c|c|c|c|c|c|c|}
\hline & \multicolumn{4}{|c|}{ Muckpile } & \multicolumn{4}{|c|}{ Ponds } \\
\hline & \multicolumn{2}{|c|}{ Native } & \multicolumn{2}{|c|}{ Muck } & \multicolumn{2}{|c|}{ Native } & \multicolumn{2}{|c|}{ Muck } \\
\hline & Range & $\begin{array}{c}\text { No. of } \\
\text { detects }\end{array}$ & Range & $\begin{array}{l}\text { No. of } \\
\text { detects }\end{array}$ & Range & $\begin{array}{c}\text { No. of } \\
\text { detects }\end{array}$ & Range & $\begin{array}{c}\text { No. of } \\
\text { detects }\end{array}$ \\
\hline Plutonium-239 & $0.03-4.55$ & 7 & $0.016-84.0$ & 28 & 0.154 & 1 & $0.305-5.37$ & 6 \\
\hline Strontium-90 & $0.048-0.33$ & 2 & $0.21-54.0$ & 16 & ND & 0 & $0.76-3.58$ & 5 \\
\hline Thorium-234 & 1.8 & 1 & 2.7 & 1 & ND & 0 & ND & 0 \\
\hline Thallium-208 & $0.093-0.76$ & 7 & $0.131-1.02$ & 34 & $0.59-1.05$ & 5 & $0.51-0.8$ & 8 \\
\hline Tot & & 25 & & 41 & & 5 & & 9 \\
\hline
\end{tabular}

$\mathrm{mg} / \mathrm{kg}=$ Milligrams per kilogram

$\mathrm{ND}=$ Not detected

$\mathrm{pCi} / \mathrm{g}=$ Picocuries per gram

$\mu \mathrm{g} / \mathrm{kg}=$ Micrograms per kilogram 
Table A.4-2

Analytical Result Detects for the Radioactive Material, Oil Spill, Drainage, and QC Water Samples

(Page 1 of 4)

\begin{tabular}{|c|c|c|c|c|c|c|c|c|}
\hline & \multicolumn{2}{|c|}{ "Radioactive Material } & \multicolumn{2}{|c|}{ Oil Spill } & \multicolumn{2}{|c|}{ Drainage } & \multicolumn{2}{|c|}{ QC Water } \\
\hline & Range & $\begin{array}{c}\text { No. of } \\
\text { detects }\end{array}$ & Range & $\begin{array}{c}\text { No. of } \\
\text { detects }\end{array}$ & Range & $\begin{array}{l}\text { No. of } \\
\text { detects }\end{array}$ & Range & $\begin{array}{l}\text { No. of } \\
\text { detects }\end{array}$ \\
\hline Volatile Compounds & $\mu \mathrm{g} / \mathrm{kg}$ & & $\mu \mathrm{g} / \mathrm{kg}$ & & $\mu \mathrm{g} / \mathrm{kg}$ & & $\mu \mathrm{g} / \mathrm{L}$ & \\
\hline 2-Butanone & ND & 0 & $11.0-17.0$ & 2 & 10 & 1 & ND & 0 \\
\hline Acetone & ND & 0 & $7.2-72.0$ & 3 & $6.2-39.0$ & 12 & $6.9-25.0$ & 9 \\
\hline Chloromethane & ND & 0 & ND & 0 & ND & 0 & $0.58-0.71$ & 2 \\
\hline Ethylbenzene & ND & 0 & ND & 0 & $1.2-11.0$ & 6 & ND & 0 \\
\hline Hexachlorobutadiene & ND & 0 & ND & 0 & 1.6 & 1 & ND & 0 \\
\hline M+P-Xylene & ND & 0 & ND & 0 & $2.1-57.0$ & 11 & ND & 0 \\
\hline Methylene Chloride & ND & 0 & ND & 0 & $0.82-4.0$ & 28 & $0.75-15.0$ & 18 \\
\hline O-Xylene & ND & 0 & ND & 0 & $1.0-23.0$ & 7 & ND & 0 \\
\hline Styrene & ND & 0 & ND & 0 & $0.99-3.6$ & 4 & ND & 0 \\
\hline Toluene & ND & 0 & ND & 0 & $1.0-1.6$ & 4 & $0.61-1.3$ & 4 \\
\hline Semivolatile Compounds & $\mu \mathrm{g} / \mathrm{kg}$ & & $\mu \mathrm{g} / \mathrm{kg}$ & & $\mu \mathrm{g} / \mathrm{kg}$ & & $\mu \mathrm{g} / \mathrm{L}$ & \\
\hline Acenaphthene & ND & 0 & $39-93$ & 2 & ND & 0 & ND & 0 \\
\hline Anthracene & ND & 0 & $86-470$ & 3 & ND & 0 & ND & 0 \\
\hline Benzo(A)Anthracene & $56-170$ & 4 & $14-18000$ & 8 & $26-66$ & 3 & ND & 0 \\
\hline Benzo(A)Pyrene & $39-230$ & 4 & $64-23000$ & 6 & $37-64$ & 3 & ND & 0 \\
\hline Benzo(B)Fluoranthene & $84-620$ & 4 & $140-43000$ & 6 & $51-62$ & 3 & ND & 0 \\
\hline Benzo(G,H,I)Perylene & 90 & 1 & $59-16000$ & 4 & 46 & 1 & ND & 0 \\
\hline
\end{tabular}

\section{Uncontrolled When Printed}


Table A.4-2

Analytical Result Detects for the Radioactive Material, Oil Spill, Drainage, and QC Water Samples

(Page 2 of 4)

\begin{tabular}{|c|c|c|c|c|c|c|c|c|}
\hline & \multicolumn{2}{|c|}{ Radioactive Material } & \multicolumn{2}{|c|}{ Oil Spill } & \multicolumn{2}{|c|}{ Drainage } & \multicolumn{2}{|c|}{ QC Water } \\
\hline & Range & $\begin{array}{l}\text { No. of } \\
\text { detects }\end{array}$ & Range & $\begin{array}{c}\text { No. of } \\
\text { detects }\end{array}$ & Range & $\begin{array}{c}\text { No. of } \\
\text { detects }\end{array}$ & Range & $\begin{array}{l}\text { No. of } \\
\text { detects }\end{array}$ \\
\hline Benzo(K)Fluoranthene & $41-230$ & 4 & $45-19000$ & 6 & $25-57$ & 3 & ND & 0 \\
\hline Bis(2-Ethylhexyl)Phthalate & $76-1200$ & 4 & $710-3900$ & 2 & $82-2400$ & 8 & $5.9-42.0$ & 14 \\
\hline Butyl Benzyl Phthalate & $23-630$ & 2 & ND & 0 & $33-40$ & 2 & ND & 0 \\
\hline Carbazole & 23 & 1 & $160-790$ & 3 & ND & 0 & ND & 0 \\
\hline Chrysene & $63-480$ & 4 & $110-23000$ & 8 & $55-100$ & 3 & ND & 0 \\
\hline Dibenzo $(\mathrm{A}, \mathrm{H})$ Anthracene & 27 & 1 & $2100-10000$ & 2 & ND & 0 & ND & 0 \\
\hline Dimethyl Phthalate & ND & 0 & ND & 0 & 14 & 1 & ND & 0 \\
\hline Di-N-Butyl Phthalate & $45-5100$ & 4 & ND & 0 & 23 & 1 & ND & 0 \\
\hline Di-N-Octyl Phthalate & ND & 0 & ND & 0 & 59 & 1 & ND & 0 \\
\hline Fluoranthene & $86-510$ & 4 & $93-13000$ & 8 & $13-120$ & 4 & ND & 0 \\
\hline Fluorene & ND & 0 & $31-68$ & 2 & ND & 0 & ND & 0 \\
\hline Indeno(1,2,3-Cd)Pyrene & 80 & 1 & $54-12000$ & 3 & ND & 0 & ND & 0 \\
\hline Naphthalene & 18 & 1 & $15-56$ & 4 & ND & 0 & ND & 0 \\
\hline Phenanthrene & $38-210$ & 4 & $32-2500$ & 8 & 49 & 1 & ND & 0 \\
\hline Pyrene & $73-430$ & 4 & $75-64000$ & 8 & $41-190$ & 3 & ND & 0 \\
\hline Petroleum Hydrocarbons & $\mathrm{mg} / \mathrm{kg}$ & & $\mathrm{mg} / \mathrm{kg}$ & & $\mathrm{mg} / \mathrm{kg}$ & & $\mathrm{mg} / \mathrm{L}$ & \\
\hline Diesel-Range Organics & $3.0-15$ & 3 & $63-3400$ & 6 & $1.8-6.8$ & 8 & ND & 0 \\
\hline
\end{tabular}

\section{Uncontrolled When Printed}


Table A.4-2

Analytical Result Detects for the Radioactive Material, Oil Spill, Drainage, and QC Water Samples

(Page 3 of 4)

\begin{tabular}{|c|c|c|c|c|c|c|c|c|}
\hline & \multicolumn{2}{|c|}{ Radioactive Material } & \multicolumn{2}{|c|}{ Oil Spill } & \multicolumn{2}{|c|}{ Drainage } & \multicolumn{2}{|c|}{ QC Water } \\
\hline & Range & $\begin{array}{l}\text { No. of } \\
\text { detects }\end{array}$ & Range & $\begin{array}{c}\text { No. of } \\
\text { detects }\end{array}$ & Range & $\begin{array}{l}\text { No. of } \\
\text { detects }\end{array}$ & Range & $\begin{array}{c}\text { No. of } \\
\text { detects }\end{array}$ \\
\hline Metals & $\mathrm{mg} / \mathrm{kg}$ & & $\mathrm{mg} / \mathrm{kg}$ & & $\mathrm{mg} / \mathrm{kg}$ & & $\mu g / L$ & \\
\hline Arsenic & $4.5-7.1$ & 4 & $2.9-7.1$ & 8 & $2.2-5.3$ & 35 & $3.7-19.0$ & 2 \\
\hline Barium & $140-260$ & 4 & $140-300$ & 8 & $750-6600$ & 35 & $0.49-13.0$ & 6 \\
\hline Beryllium & $0.84-1.7$ & 4 & $0.64-1.2$ & 8 & $0.77-2.1$ & 35 & $0.06-0.09$ & 2 \\
\hline Cadmium & $0.56-0.57$ & 2 & $0.58-1.4$ & 4 & $0.11-0.37$ & 35 & 0.18 & 1 \\
\hline Chromium & $3.2-9.1$ & 4 & $2.5-10.0$ & 8 & $1.1-9.2$ & 35 & 1.7 & 1 \\
\hline Lead & $18.0-61.0$ & 4 & $12.0-31.0$ & 8 & $14.0-52.0$ & 35 & ND & 0 \\
\hline Mercury & 0.16 & 1 & ND & 0 & $0.0088-0.083$ & 35 & $0.0066-0.03$ & 10 \\
\hline Selenium & ND & 0 & 1.2 & 1 & $0.29-1.1$ & 9 & 2.4 & 1 \\
\hline Silver & ND & 0 & ND & 0 & ND & 0 & $0.66-1.7$ & 7 \\
\hline Actinium-228 & $1.26-2.9$ & 4 & $1.27-2.21$ & 8 & $0.7-2.26$ & 35 & ND & 0 \\
\hline Aluminum-26 & ND & 0 & ND & 0 & 0.105 & 1 & ND & 0 \\
\hline Radionuclides & $\mathrm{pCi} / \mathrm{g}$ & & $\mathrm{pCi} / \mathrm{g}$ & & $\mathrm{pCi} / \mathrm{g}$ & & $\mathrm{pCi} / \mathrm{L}$ & \\
\hline Americium-241 & ND & 0 & ND & 0 & 1.88 & 1 & ND & 0 \\
\hline Bismuth-212 & 3.2 & 1 & ND & 0 & ND & 0 & ND & 0 \\
\hline Bismuth-214 & $0.7-1.12$ & 3 & $0.58-1.1$ & 8 & $0.76-1.48$ & 35 & ND & 0 \\
\hline Cesium-137 & $7.06-114$ & 4 & $0.53-20.5$ & 8 & $2.72-78.1$ & 35 & ND & 0 \\
\hline Potassium-40 & $21.4-27.8$ & 4 & $19.8-25.0$ & 8 & $17.0-34.5$ & 35 & ND & 0 \\
\hline
\end{tabular}

\section{Uncontrolled When Printed}


Table A.4-2

Analytical Result Detects for the Radioactive Material, Oil Spill, Drainage, and QC Water Samples

(Page 4 of 4 )

\begin{tabular}{|c|c|c|c|c|c|c|c|c|}
\hline & \multicolumn{2}{|c|}{ "Radioactive Material } & \multicolumn{2}{|c|}{ Oil Spill } & \multicolumn{2}{|c|}{ Drainage } & \multicolumn{2}{|c|}{ QC Water } \\
\hline & Range & $\begin{array}{l}\text { No. of } \\
\text { detects }\end{array}$ & Range & $\begin{array}{c}\text { No. of } \\
\text { detects }\end{array}$ & Range & $\begin{array}{c}\text { No. of } \\
\text { detects }\end{array}$ & Range & $\begin{array}{l}\text { No. of } \\
\text { detects }\end{array}$ \\
\hline Lead-212 & $1.52-2.96$ & 4 & $1.83-3.1$ & 8 & $0.74-2.54$ & 35 & ND & 0 \\
\hline Lead-214 & $0.93-1.04$ & 3 & 0.86-1.06 & 7 & $0.8-1.68$ & 34 & ND & 0 \\
\hline Plutonium-238 & $0.076-0.232$ & 3 & ND & 0 & $0.04-0.73$ & 32 & ND & 0 \\
\hline Plutonium-239 & $0.166-2.09$ & 4 & $0.051-1.04$ & 6 & $0.071-4.21$ & 35 & ND & 0 \\
\hline Strontium-90 & ND & 0 & ND & 0 & $0.45-1.88$ & 25 & ND & 0 \\
\hline Thorium-227 & 4.1 & 1 & ND & 0 & ND & 0 & ND & 0 \\
\hline Thorium-234 & 7.2 & 1 & ND & 0 & 2.4 & 1 & ND & 0 \\
\hline Thallium-208 & $0.47-0.85$ & 4 & 0.54-0.97 & 8 & $0.3-0.87$ & 34 & ND & 0 \\
\hline \multicolumn{2}{|c|}{ Total No. of Samples Collected } & 4 & & 8 & & 35 & & 37 \\
\hline
\end{tabular}

$\mathrm{mg} / \mathrm{kg}=$ Milligrams per kilogram

$\mathrm{mg} / \mathrm{L}=$ Milligrams per liter

ND $=$ Not detected

$\mathrm{pCi} / \mathrm{L}=$ Picocuries per liter

$\mathrm{pCi} / \mathrm{g}=$ Picocuries per gram

$\mu \mathrm{g} / \mathrm{kg}=$ Micrograms per kilogram

$\mu \mathrm{g} / \mathrm{L}=$ Micrograms per liter

\section{Uncontrolled When Printed}


Table A.4-3

Analytical Results for Background / Preliminary Samples

\begin{tabular}{|c|c|c|}
\hline & \multicolumn{2}{|c|}{ Background/Preliminary } \\
\hline & Range & No. of Detects \\
\hline Radionuclides & $\mathrm{pCi} / \mathrm{g}$ & \\
\hline Actinium-228 & $1.44-2.27$ & 6 \\
\hline Americium-241 & 2.83 & 1 \\
\hline Bismuth-214 & $0.45-1.41$ & 4 \\
\hline Cesium-137 & $0.46-180$ & 7 \\
\hline Potassium-40 & 8.8-30.7 & 8 \\
\hline Lead-212 & $0.72-2.47$ & 8 \\
\hline Lead-214 & $0.6-1.07$ & 4 \\
\hline Plutonium-238 & $0.032-11.3$ & 5 \\
\hline Plutonium-239 & $0.093-61.0$ & 8 \\
\hline Strontium-90 & 18.8 & 1 \\
\hline Thallium-208 & $0.247-0.83$ & 5 \\
\hline \multicolumn{2}{|c|}{ Total No. of Samples Collected } & 8 \\
\hline Metals & $\mathrm{mg} / \mathrm{kg}$ & \\
\hline Arsenic & $2.6-5.3$ & 7 \\
\hline Barium & $130-290$ & 3 \\
\hline Beryllium & $0.72-1.3$ & 6 \\
\hline Cadmium & $0.11-0.32$ & 2 \\
\hline Chromium & $5.0-8.4$ & 3 \\
\hline Lead & $12.0-75.0$ & 7 \\
\hline Mercury & $0.032-0.065$ & 3 \\
\hline Selenium & $0.52-0.71$ & 2 \\
\hline \multicolumn{2}{|c|}{ Total No. of Samples Collected } & $7^{*}$ \\
\hline
\end{tabular}

$\mathrm{mg} / \mathrm{kg}=$ Milligrams per kilogram

$\mathrm{pCi} / \mathrm{g}=$ Picocuries per gram

* Sample 383GB901 was not analyzed for metals 


\section{A.5.0 Waste Management}

Management of the investigation-derived waste (IDW) was guided by the CAU 383 CAIP (DTRA, 2003) requirements, field observations, and the results of the laboratory analysis of the E-Tunnel Sites characterization samples. Administrative controls were used to minimize the amount of waste generated during the investigation.

\section{A.5.1 Waste Segregation}

All wastes generated on the site were divided into either sanitary waste or IDW. The sanitary waste was disposed of in the dumpster located by Building 153. The IDW was segregated into solid (personal protective equipment [PPE] and plastic) and liquid (rinsate) waste streams to limit the amount of potentially contaminated waste.

\section{A.5.2 Waste Generation and Accumulation}

Waste generated during the field work was either sanitary waste or IDW. The sanitary waste consisted mostly of paper and kitchen debris generated in the field office and, in accordance with the waste minimization strategy, all PPE that was not visibly contaminated and not used when elevated field-screening results were found. The IDW consisted of potentially contaminated PPE, plastic, and all rinsates. This waste was dealt with as described in the rest of this section.

All IDW was put in U.S. Department of Transportation compliant, 55-gallon, steel drums. For drums receiving solid IDW, an absorbent $\left(\right.$ Stergo $^{\circledR}$ ) pad was placed in the bottom, and a plastic liner was inserted. After receiving waste, the drums were stored on plastic spill pallets in the fenced Hazardous Waste Accumulation Area (HWAA). After a drum received waste, it was marked with a unique identification number, labeled with a "Hazardous Waste Pending Analysis" sticker and a "Radioactive Material Pending Analysis" sticker, and secured with a tamperindicating device (TID). A waste management logbook was used to track the drums used, the contents of each drum, where the material was used, and an estimate of how much material was in each drum. The volume of material recorded for each drum is an estimate of what was put in the drum. 


\section{A.5.2.1 Rinsates}

The drill rig, tools, core barrels, drill bits, and sampling equipment (stainless-steel bowls) were decontaminated at the E-Tunnel decontamination pad before and after the work at E-Tunnel. After being decontaminated in the decontamination tub, the stainless-steel bowls, core barrels, and drill bits were cased in plastic sleeves to prevent them from getting contaminated before use. Each hole was drilled using the same core barrel and drill bit for the entire hole. When the hole was completed, they were decontaminated and sealed in plastic again. A clean stainless-steel bowl was used for each sample, after which it was decontaminated and sealed in plastic again. All liquid from the decontamination tub was containerized as rinsate.

Rinsate from the decontamination tub was transferred into an unlined 55-gallon drum staged next to the decontamination pad. Once used, each drum was marked and controlled (as stated above) and stored on a plastic spill pallet in the fenced HWAA. Waste was added to the drum until it was full (leaving some ullage). It was then secured (as stated before) and moved back to the HWAA. The waste management logbook was used to track the drums used, where the equipment decontaminated with the rinsate was used, and an estimate of how much rinsate was in each drum.

\section{A.5.2.2 Soil}

No significant quantity of soil accumulated in the bottom of the decontamination tub, nor was any soil contaminated by spills, so no soil required disposal other than the drill core, which was disposed of in the borehole from which it came.

\section{A.5.2.3 Personal Protective Equipment, Sampling Equipment, and Plastic Sleeves}

The PPE used in the exclusion zone consisted of Tyvek ${ }^{\circledR}$ anti-contamination clothing, boot covers, cotton gloves, and nitrile or latex gloves. At the end of each shift, the PPE was evaluated with respect to the radiation emissions and the presence of visible contamination. If there were no elevated radiation emissions and there was no visible contamination, the PPE was determined to be sanitary waste and was disposed of in the roll off bin at Building 153. During the E-Tunnel Sites and drainage characterization no PPE was determined to be IDW (it was all disposed of as sanitary waste). 
The disposable sampling equipment was collected in a plastic bag inside the exclusion zone as the sampling was conducted. The wastes were accumulated until the bag was filled, and then it was taped closed. If there were no elevated radiation emissions and there was no visible contamination, the waste was disposed of as sanitary waste at Building 153. None of the sampling wastes were determined to be IDW.

Plastic sleeves were used to keep the decontaminated core barrels clean before use and to containerize slough and core as it was removed from the boreholes. After sampling, the core and slough material was returned to the borehole. The empty sleeves were containerized in a clear plastic bag and handled in the same manner as the PPE.

\section{A.5.2.4 Plastic Liners}

Plastic liners were used under the drill rig and the support truck to prevent hydrocarbon leaks from getting on the ground. To minimize waste generation, each liner was used at several locations unless it was damaged or leaked on, or field screening of the liner indicated the presence of contamination, in which case the liner was replaced at the completion of the hole. When the plastic liners became damaged, leaked on or contaminated, the liner was containerized in a clear plastic bag, a waste tracking tag was attached, and the bag was put into a lined drum and treated as solid IDW (as described above). The samples collected while the plastic liner was in use were entered into the waste management logbook. The waste management logbook was updated each time additional liners were added to the drum. No liners were containerized due to the presence of contamination other than minor hydrocarbon leaks.

\section{A.5.3 Waste Characterization}

The IDW was considered potentially contaminated waste by virtue of contact with potentially contaminated media or elevated radiological emissions. No IDW was containerized due to elevated radiological emissions. Therefore, additional sampling and analysis of the IDW, separate from the borehole soil sampling and analysis, was not required unless the sample results showed the potential for the IDW to be contaminated. The data generated as a result of the site characterization were used to make waste determinations of the IDW.

The IDW was characterized by identifying the borehole samples associated with each waste container and then comparing the analytical results from those samples to various standards. For the hazardous waste determination, the borehole sample results were compared to the RCRA 
waste characterization levels for hazardous waste. For total petroleum hydrocarbon wastes, the sample results were compared to the Nevada Administrative Code for TPH-DRO determination (NAC, 2005). For the low-level radioactive waste determination, the sample results were compared to the BN 2000 Application for Permit to Operate a Class II Solid Waste Disposal Site at the NTS (BN, 2000), and to the National Council on Radiation Protection and Measurements report entitled Recommended Screening Limits for Contaminated Surface Soil and Review of Factors Relevant to Site-Specific Studies (NCRP, 1999) and DOE Order 5400.5 Change 2, "Radiation Protection of the Public and Environment” (DOE, 1993).

\section{A.5.4 Waste Disposal}

The hazardous waste determination did not identify any drums that were associated with samples that contained a high-enough concentration of hazardous constituents to be considered hazardous. However, the two drums that contained hydrocarbon-stained plastic were shipped to the hydrocarbon landfill for disposal as hydrocarbon waste.

The preliminary radiological waste determination identified several samples that had radionuclide levels above the preliminary action level. However, when comparing the volume of soil in the drums required to be present to be regulated to the most conservative theoretical maximum amount of soil actually present in the drums, the action level was not exceeded. Therefore, the drums were nonradioactive

Table A.5-1 provides a tally of the number of drums used, what they contained, and their final disposition. Five of the drums of IDW were disposed of as sanitary waste, and two drums (\#383M04 and \#383M05) were disposed of as hydrocarbon waste.

Table A.5-1

Drum Count

\begin{tabular}{|c|c|c|c||}
\hline Number of Drums & $\begin{array}{c}\text { Volume } \\
\text { (Gallons) }\end{array}$ & Waste Stream & Disposition \\
\hline \hline 4 & 182 & Rinsate & Sanitary \\
\hline 1 & 10 & PPE & Sanitary \\
\hline 2 & 60 & Plastic & Hydrocarbon Landfill \\
\hline \hline Total Number 7 & & & \\
\hline
\end{tabular}




\section{A.6.0 Health and Safety}

At the start of the project, a preliminary hazard assessment was prepared and used to guide the preparation of the SSHASP (SNJV, 2003), the Radiation Work Permit (SN2004-CAU383-01), and the CAU 383 CAIP (DTRA, 2003). The work was then performed in accordance with these documents.

Prefield H\&S preparation was accomplished using a project-specific H\&S checklist. The checklist was developed to ensure that all required items were verified and/or available before mobilization to the field.

\section{A.6.1 Physical Hazards}

The primary physical hazards fell into three general categories: fatigue; heat stress; and slip, trip, and fall. The sources of these hazards were the long drive to and from the work site, the general hazards of working around moving machinery, the high ambient air temperatures at NTS during that time of year, and working on uneven ground and around the slope of the Muckpile.

The hazards associated with fatigue; heat stress; and slip, trip, and fall were dealt with by discussing the long drive; the hazards involved with the machinery; heat stress symptoms; and site-specific slip, trip, and fall hazards during the morning tailgate safety meetings. The length of the workday was limited, and workers were observed by the SSO and SS for fatigue and heat stress and were encouraged to drink water. This ensured that workers did not become overly fatigued and could safely drive back to Mercury at the end of the day.

The hazard posed by the slope of the Muckpile was addressed by applying a restriction to drill no closer than $20 \mathrm{ft}$ from the edge of the Muckpile. This conservative restriction ensured personnel did not work too close to the edge of the Muckpile and ensured that the vibrations and weight of the rotosonic drill rig would not cause the slope to fail.

\section{A.6.2 Chemical and Radiological Hazards}

Chemical hazards believed to be present included mostly hydrocarbon products, epoxies, and chemical grouts that may have been disposed of in the Muckpile. These products were used for fuels, ground support, and various other applications during mining operations. Radiological 
hazards could possibly have come from the rock and construction waste deposited in the Muckpile during re-entry mining operations following nuclear testing.

Drive-over and walk-over radiological surveys were conducted at the E-Tunnel Muckpile and the drainage associated with the Muckpile. These surveys identified surface and near surface areas with elevated readings on the Muckpile, in the drainage and ponds, and near the compressor pad. The highest level was approximately 5.2 times greater than the background radiation emission rate. To ensure that radiological safety, additional precautions were taken during drilling and sampling activities, high-volume air samples were taken. No airborne radioactive particles were detected in the high-volume air sample filters during field activities. All personnel wore PPE in the exclusion zone. All the PPE was determined to be sanitary waste. After each shift, all personnel in the exclusion zone (EZ) were monitored for elevated radiation emission as they left the EZ. No elevated emissions were detected.

Products brought on site to assist with the characterization investigation were required to have a material safety data sheet before receiving approval for use. These items generally consisted of fuels, lubricants, and cooling and cleaning agents.

Action levels for the chemical constituents and radiological hazards were posted on the tailgate safety briefing as noted in the SSHASP. All intrusive work required real-time monitoring be conducted and the results documented on the Sample Collection Logs (SCLs) and the Soil Characterization Log. Field-screening results ensured action limits for occupational threats to personnel during the drilling and sampling operations were not exceeded.

As a precaution, all personnel wore gloves, boot covers, and Tyvek ${ }^{\circledR}$ anti-contamination clothing in the exclusion zone.

\section{A.6.3 Drilling}

An equipment inspection of each Boart-Longyear drill rig was performed by SNJV personnel upon their arrival on site and before any drilling activity. These inspections were documented on the Drill Rig Inspection form as described in the Field Instructions (DTRA, 2004). All safety devices were present and operational. In addition, the drill rig was inspected daily by the driller. This was recorded in the geologist's Field Activity Daily Log (FADL). The rotosonic drill rigs were operated safely throughout the project. 


\section{A.6.4 Noise}

During rotosonic drilling operations personnel exposure to noise is always a concern.

Noise-level monitoring was conducted before startup and during actual drilling, with a sound-level meter set on the "A" scale with a slow response. Measurements indicated that average noise levels of 85 to 110 decibels were common within $35 \mathrm{ft}$ of the drill rig. With this knowledge, all personnel working or observing operations within this distance were required to wear hearing protection. Personnel were also diligent in watching out for one another to make certain that hearing protection was available and in use before equipment startup.

\section{A.6.5 Air Monitoring}

A Photovac Micro FID was used for real-time air monitoring for VOCs during all intrusive work. The FID measures concentrations of photoionizable gases and records the values. The FID cannot distinguish between individual pollutants, it merely lets the operator know that something is present. During drilling activities at the Area 12 E-Tunnel Sites, the FID was used to measure concentrations in a worker's breathing zone. All concentrations logged during field activities were below the established action level of one part per million sustained in the breathing zone.

In addition to the VOC monitoring, radiation monitoring was conducted daily through high-volume air sampling. One air sample was collected in the exclusion zone side of the sample van and another sample was collected in the clean side of the sample van. Two more air samplers were used to collect air samples (one at the exclusion zone hot line and one on the drill rig). These samples were collected by continuously drawing air through the two filters during work hours. Once work ceased for the day, the filters were removed and counted on a Tennelec series 5XLB, low-background alpha/beta counter. No airborne radioactive particles were detected in the high-volume air sample filters during field activities.

\section{A.6.6 Personnel Monitoring}

All personnel were required to wear a thermal luminescent dosimeter while working at the E-Tunnel site.

\section{A.6.7 Training}

Before assignment, all necessary training documentation for specific tasks was reviewed and verified as to current status. By verifying all documentation before personnel arrived on site, delays to track down missing or incomplete training documentation were minimized. 
Training requirements for this project were outlined in the SSHASP and included 40-hour Hazardous Waste Operations and Emergency Response, annual 8-hour refresher, medical surveillance, and Radiological Worker II. Additional training for specific personnel included 8-hour supervisory training, first aid and cardiopulmonary resuscitation, bloodborne pathogens training, and classroom and field waste management training. 


\section{A.7.0 Lessons Learned}

A lessons learned meeting was not held following the field work. The work went well, and because this was the sixth Mudpit/Muckpile field effort and no issues came out in the lessons learned meeting at the end of the field efforts, it was decided that little could be gained by holding an additional lessons learned meeting. 


\section{A.8.0 References}

BN, see Bechtel Nevada.

Bechtel Nevada. 2000. Application for Permit to Operate a Class II Solid Waste Disposal Site at the Nevada Test Site. Las Vegas, NV

DOE, see U.S. Department of Energy

DTRA, see Defense Threat Reduction Agency.

Defense Threat Reduction Agency. 2003. Corrective Action Investigation Plan for Corrective Action Unit 383: Area 12 E-Tunnel Sites, Nevada Test Site, Rev. 0. Prepared by SNJV. Las Vegas, NV.

Defense Threat Reduction Agency. 2004. Field Instructions for CAU 383: Area 12 E-Tunnel Sites, Rev. 0. Prepared by SNJV. Las Vegas, NV.

FFACO, see Federal Facility Agreement and Consent Order.

Federal Facility Agreement and Consent Order. 1996 (as amended). Agreed to by the State of Nevada, the U.S. Department of Energy, and the U.S. Department of Defense.

Las Vegas, NV.

Gilbert, R.O. 1987. Statistical Methods for Environmental Pollution Monitoring, Chapter 4. New York, NY: Van Nostrand Reinhold.

NAC, see Nevada Administrative Code.

NCRP, See National Council on Radiation Protection and Measurements.

National Council on Radiation Protection and Measurements. 1999. Recommended Screening Limits for Contaminated Surface Soil and Review of Factors Relevant to Site-Specific Studies. NCRP Report No. 129. Bethesda MD.

Nevada Administrative Code. 2005. NAC 445A.2272, "Contamination of Soils: Establishment of Action Levels.” Carson City, NV.

Stoller-Navarro Joint Venture. 2003. SNJV Site-Specific Health and Safety Plan, CAU 383: Area 12 E-Tunnel Sites, Nevada Test Site, Rev. 0. Las Vegas, NV. 
CAU $383 \mathrm{CADD} / \mathrm{CR}$

Appendix A

Revision: 0

Date: February 2007

Page $A-47$ of $A-47$

U.S. Department of Energy. 1993. "Radiation Protection of the Public and Environment.” DOE Order 5400.5 Change 2, 7 January. Washington, DC. 


\section{Attachment A}

\section{Soil Boring Logs}

(96 Pages) 
Page 1 of 1

\begin{tabular}{|c|c|c|c|c|c|c|c|c|c|}
\hline \multicolumn{6}{|c|}{$\begin{array}{l}\text { Project Hame: CAU } 383 \text { E-Tunnel Sites } \\
\text { Project Humber: DTO4-320 } \\
\text { Borehole Humber: EDHO } 1 \\
\text { Logged By: M. McLane } \\
\text { Drilled By: M. McLane } \\
\text { Drilling Method: Hand Sampled }\end{array}$} & \multicolumn{4}{|c|}{$\begin{array}{l}\text { Date Started: } 6 / 07 / 04 \\
\text { Date Completed: } 6 / 07 / 04 \\
\text { Elevation: } 6007.8 \\
\text { Horthing: } 887739.2 \\
\text { Easting: } 638589.8 \\
\text { Total Depth: } 0.5 \mathrm{ft} .\end{array}$} \\
\hline \multirow[b]{2}{*}{ 点 } & \multirow[b]{2}{*}{ 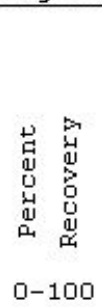 } & \multirow{2}{*}{\multicolumn{2}{|c|}{ 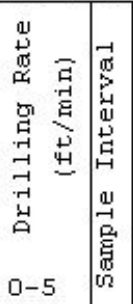 }} & \multirow[b]{2}{*}{ 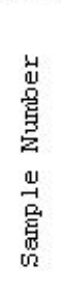 } & \multirow[b]{2}{*}{ 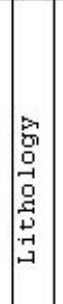 } & \multirow[b]{2}{*}{$\begin{array}{c}\text { Lithologic Description } \\
\text { Comments }\end{array}$} & Field & Screening & Results \\
\hline & & & & & & & $\begin{array}{l}\text { VOCs } \\
\text { (ppm) }\end{array}$ & $\begin{array}{l}\text { Alpha } \\
\text { (dpm) }\end{array}$ & $\begin{array}{l}\text { Beta } \\
\text { (dpm) }\end{array}$ \\
\hline
\end{tabular}

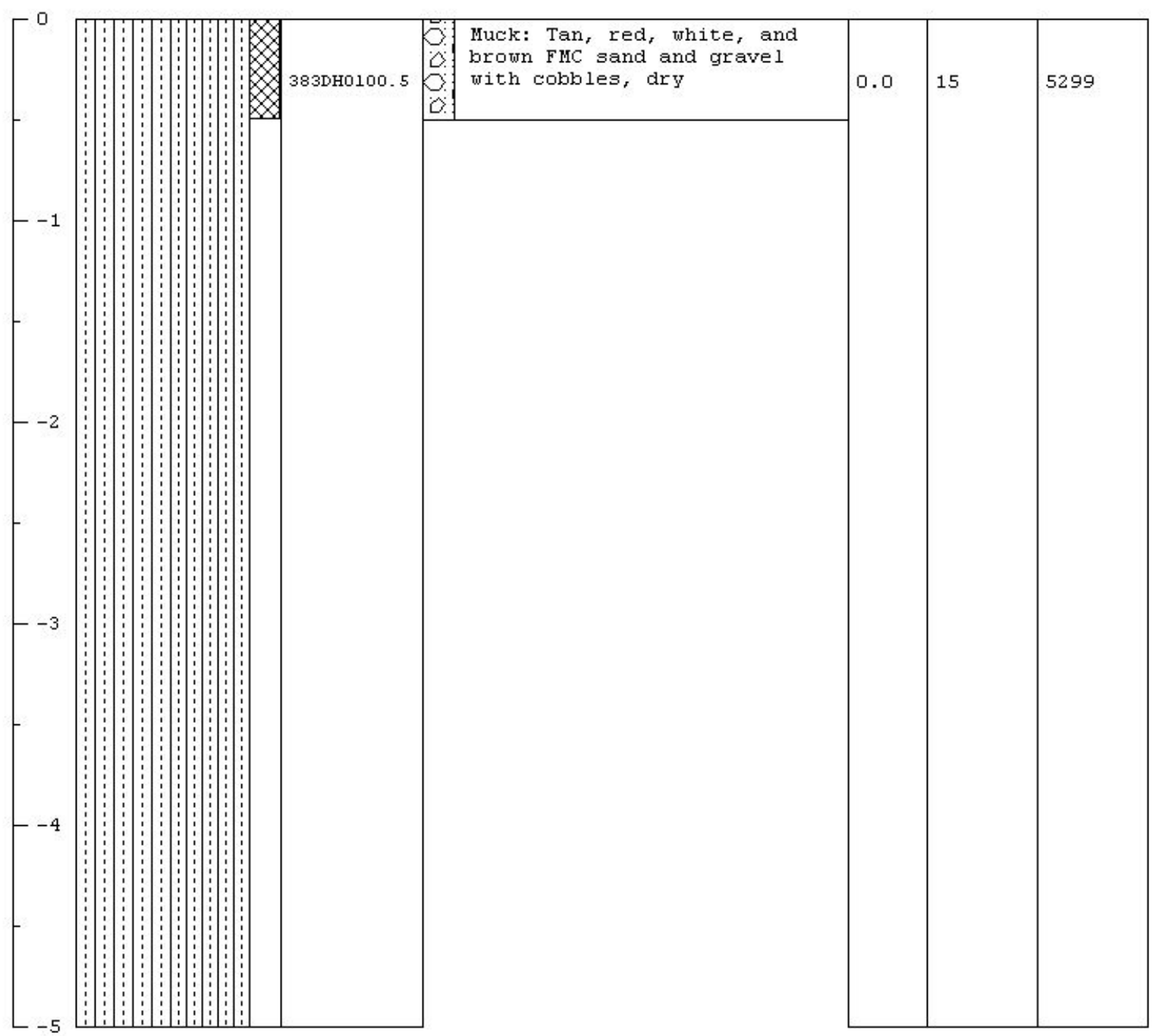


Page 1 of 1

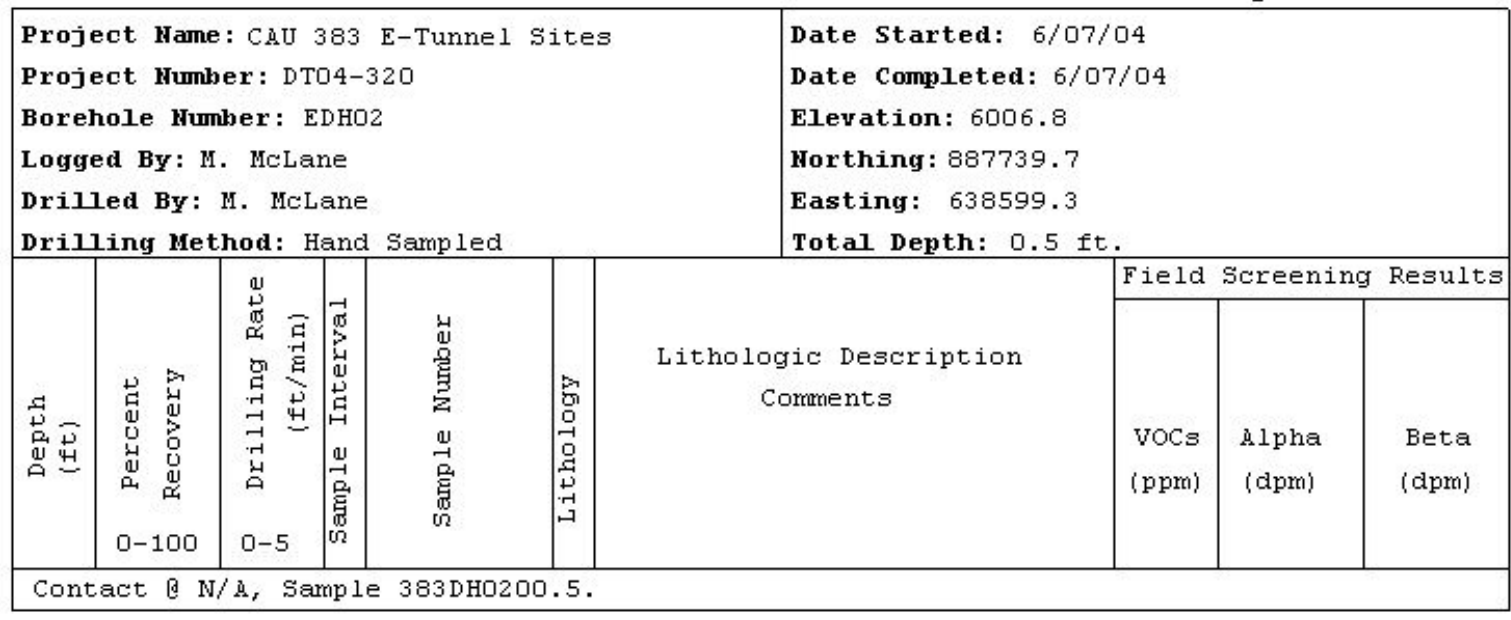

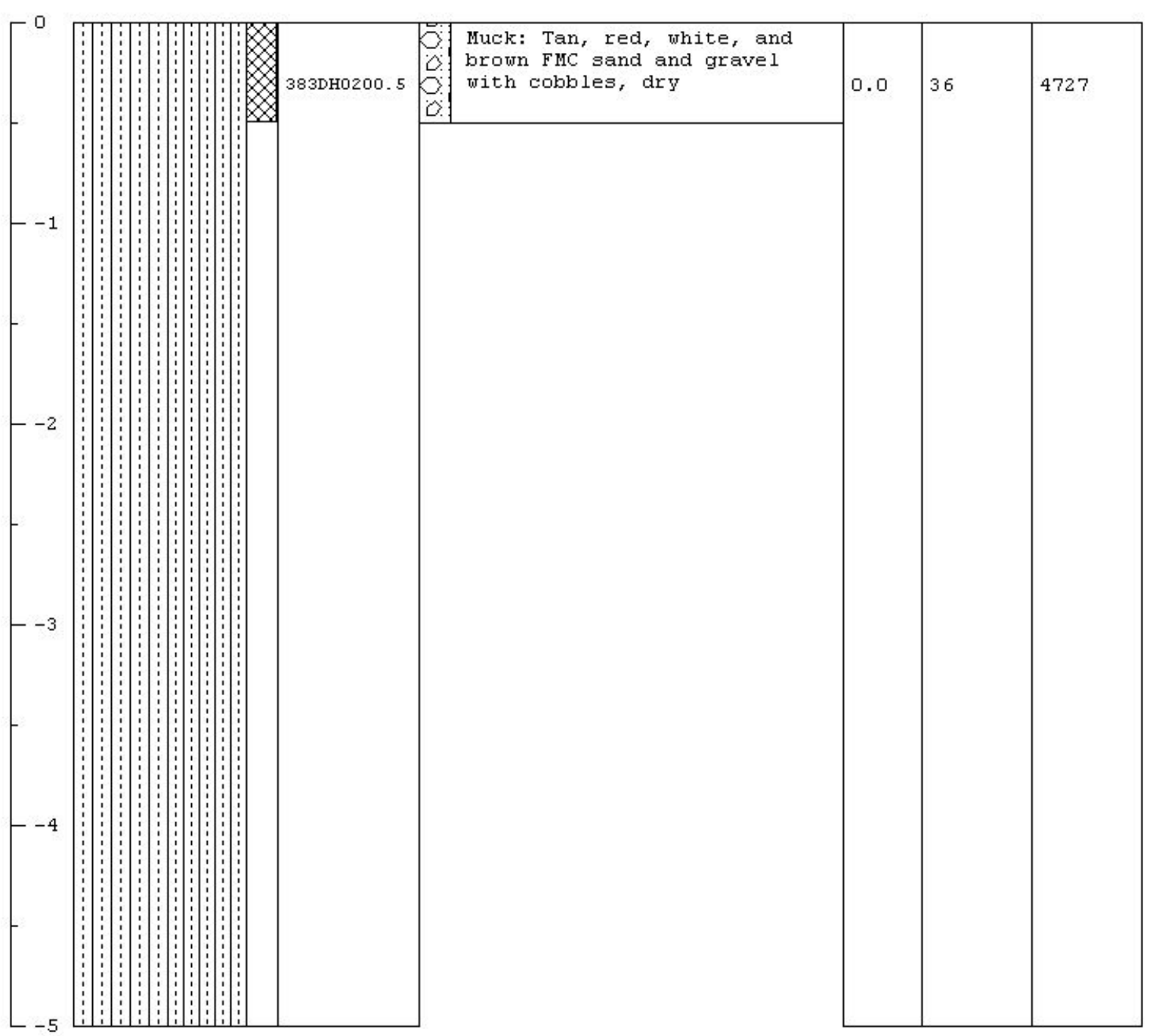


Page 1 of 1

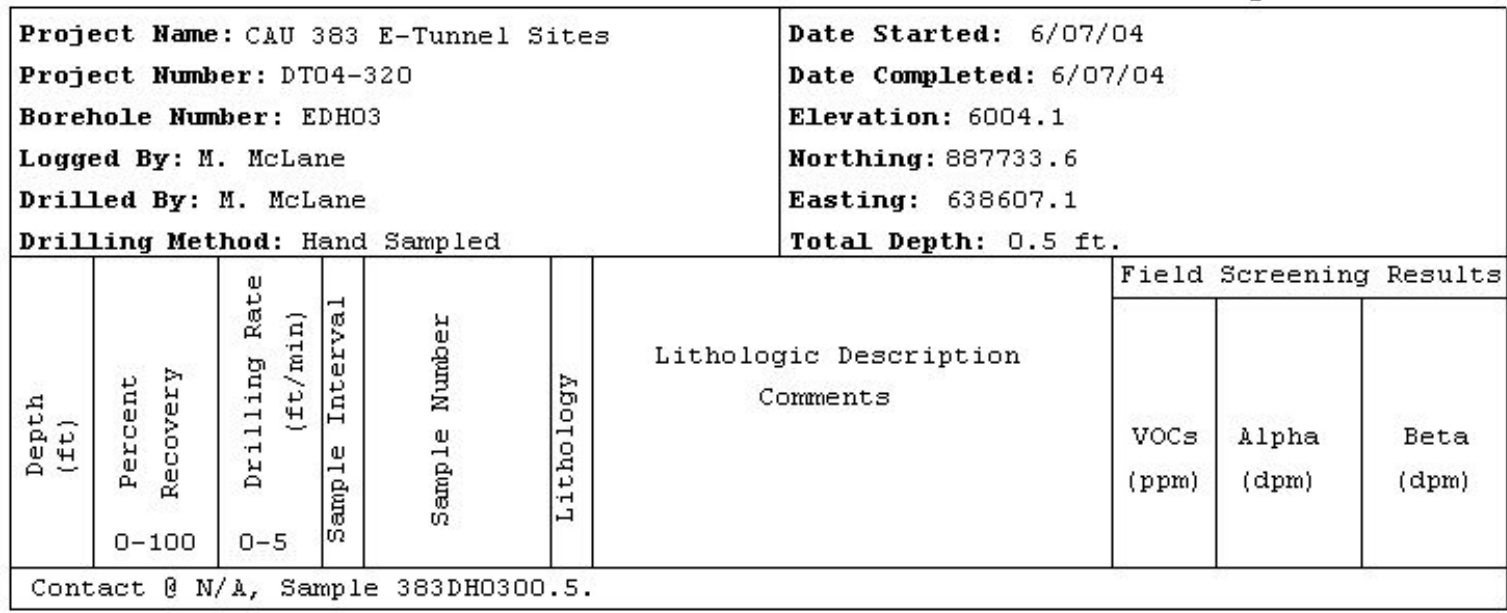

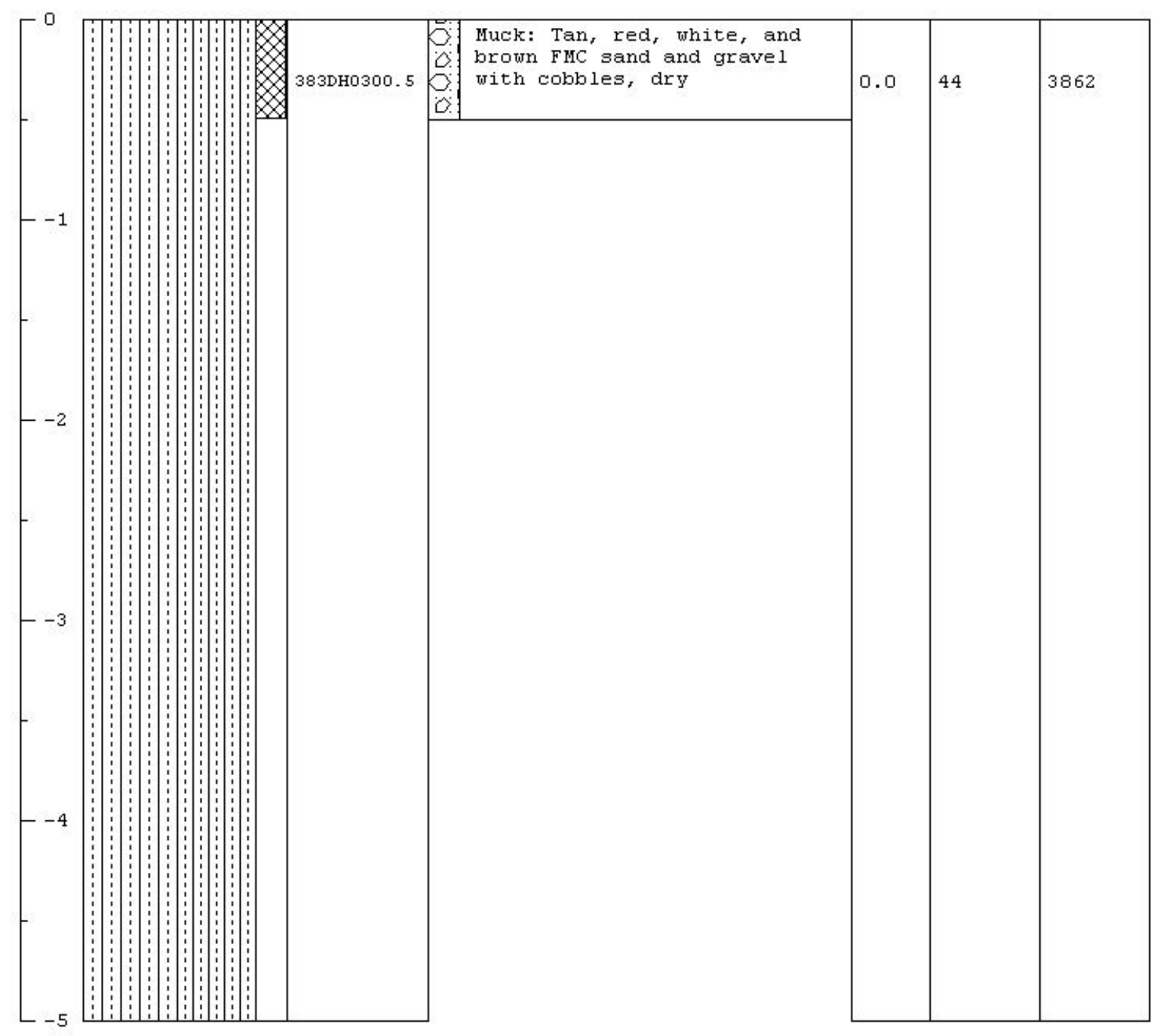


Page 1 of 1

\begin{tabular}{|c|c|c|c|c|c|c|c|c|c|}
\hline \multicolumn{6}{|c|}{$\begin{array}{l}\text { Project Hame: CAU } 383 \text { E-Tunnel sites } \\
\text { Project Humber: DTO } 4-320 \\
\text { Borehole Humber: EDHO4 } \\
\text { Logged By: M. McLane } \\
\text { Drilled By: M. McLane } \\
\text { Drilling Method: Hand Sampled }\end{array}$} & \multicolumn{4}{|c|}{$\begin{array}{l}\text { Date Started: } 6 / 07 / 04 \\
\text { Date Completed: } 6 / 07 / 04 \\
\text { Elevation: } 6003.0 \\
\text { Horthing: } 887734.1 \\
\text { Easting: } 638619.8 \\
\text { Total Depth: } 0.5 \mathrm{ft} . \\
\end{array}$} \\
\hline \multirow[b]{2}{*}{ 总出 } & \multirow[b]{2}{*}{ 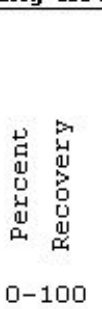 } & \multirow{2}{*}{\multicolumn{2}{|c|}{ 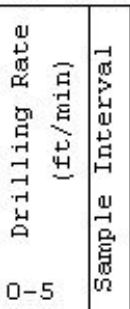 }} & \multirow[b]{2}{*}{ 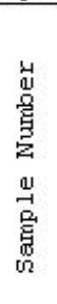 } & \multirow[b]{2}{*}{ 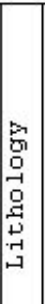 } & \multirow[b]{2}{*}{$\begin{array}{c}\text { Lithologic Description } \\
\text { Comments }\end{array}$} & \multicolumn{3}{|c|}{ Field Screening Results } \\
\hline & & & & & & & $\begin{array}{l}\text { voCs } \\
\text { (ppm) }\end{array}$ & $\begin{array}{l}\text { Alpha } \\
\text { (dpm) }\end{array}$ & $\begin{array}{l}\text { Beta } \\
\text { (dpm) }\end{array}$ \\
\hline
\end{tabular}

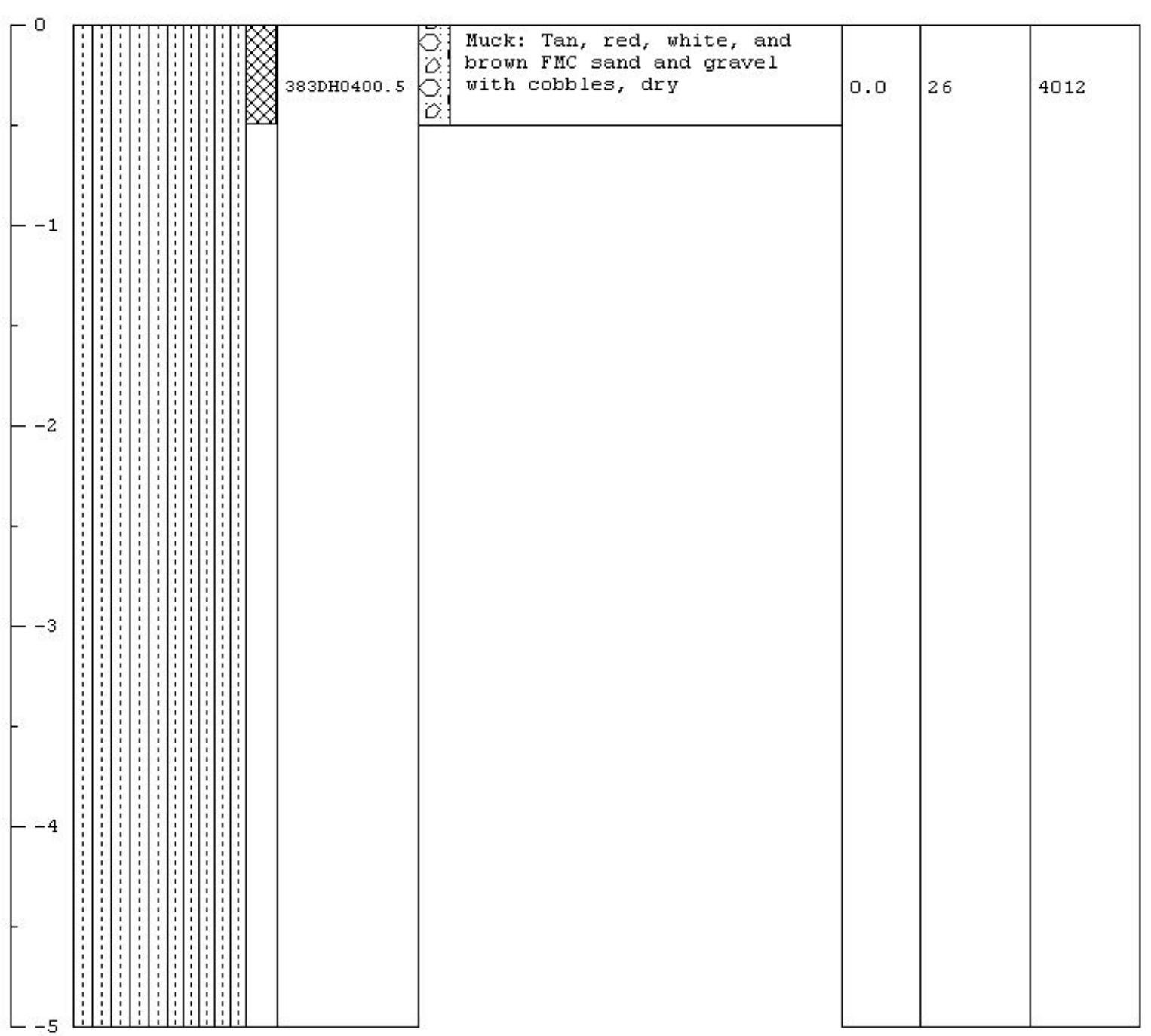


Page 1 of 1

\begin{tabular}{|c|c|c|c|c|c|c|c|c|}
\hline \multicolumn{5}{|c|}{$\begin{array}{l}\text { Project Hame: CAU } 383 \text { E-Tunnel Sites } \\
\text { Project Humber: DTO } 4-320 \\
\text { Borehole Humber: EDHO } 5 \\
\text { Logged By: M. McLane } \\
\text { Drilled By: M. McLane } \\
\text { Drilling Method: Hand Sampled }\end{array}$} & \multicolumn{4}{|c|}{$\begin{array}{l}\text { Date Started: } 6 / 07 / 04 \\
\text { Date Completed: } 6 / 07 / 04 \\
\text { Elevation: } 6003.1 \\
\text { Horthing: } 887731.5 \\
\text { Easting: } 638628.4 \\
\text { Total Depth: } 0.5 \mathrm{ft} .\end{array}$} \\
\hline \multirow[b]{2}{*}{ 点 } & \multirow[b]{2}{*}{ 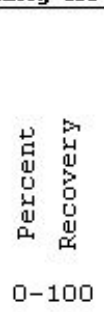 } & \multirow[b]{2}{*}{ 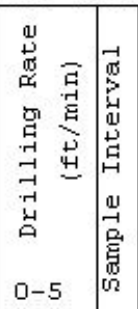 } & \multirow[b]{2}{*}{ 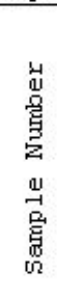 } & \multirow[b]{2}{*}{ 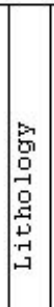 } & \multirow[b]{2}{*}{$\begin{array}{c}\text { Lithologic Description } \\
\text { Comments }\end{array}$} & Field & Screening & Results \\
\hline & & & & & & $\begin{array}{l}\text { VOCs } \\
\text { (ppm) }\end{array}$ & $\begin{array}{l}\text { Alpha } \\
\text { (dpm) }\end{array}$ & $\begin{array}{r}\text { Beta } \\
\text { (dpm) }\end{array}$ \\
\hline
\end{tabular}

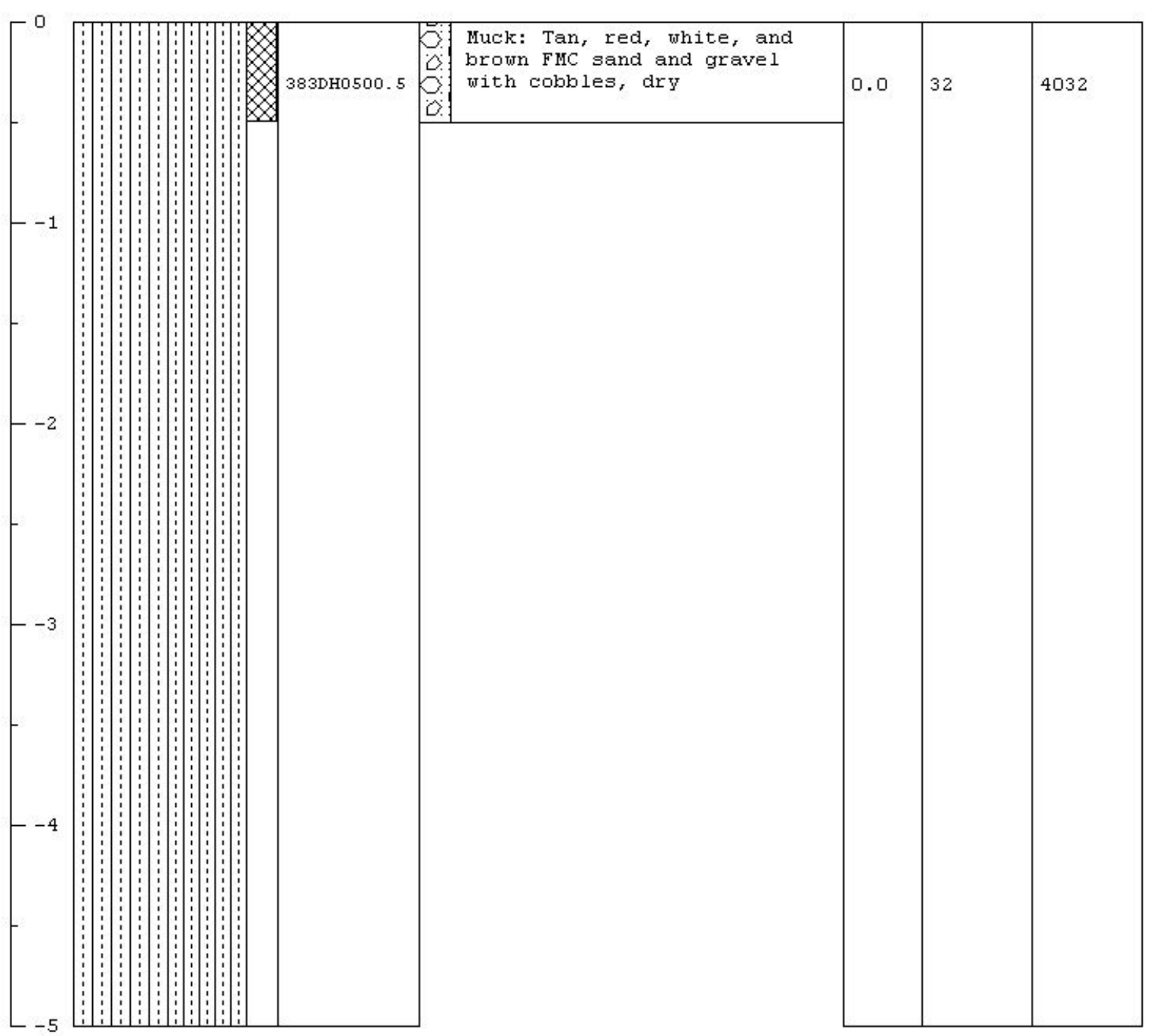


Page 1 of 1

\begin{tabular}{|c|c|c|c|c|c|c|c|c|c|}
\hline \multicolumn{6}{|c|}{$\begin{array}{l}\text { Project Hame: CAU } 383 \text { E-Tunnel Sites } \\
\text { Project Humber: DTO } 4-320 \\
\text { Borehole Humber: EDHO } 6 \\
\text { Logged By: M. McLane } \\
\text { Drilled By: M. McLane } \\
\text { Drilling Method: Hand Sampled }\end{array}$} & \multicolumn{4}{|c|}{$\begin{array}{l}\text { Date Started: } 6 / 07 / 04 \\
\text { Date Completed: } 6 / 07 / 04 \\
\text { Elevation: } 5998.9 \\
\text { Northing: } 887731.2 \\
\text { Easting: } 638640.7 \\
\text { Total Depth: } 0.5 \mathrm{ft} .\end{array}$} \\
\hline \multirow[b]{2}{*}{ 吉 } & \multirow[b]{2}{*}{ 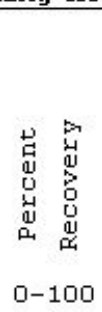 } & \multirow{2}{*}{\multicolumn{2}{|c|}{ 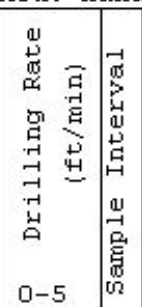 }} & \multirow[b]{2}{*}{ 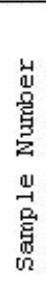 } & \multirow[b]{2}{*}{ 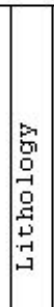 } & \multirow[b]{2}{*}{$\begin{array}{c}\text { Lithologic Description } \\
\text { Comments }\end{array}$} & Field & Screening & Results \\
\hline & & & & & & & $\begin{array}{l}\text { VOCs } \\
\text { (ppm) }\end{array}$ & $\begin{array}{l}\text { Alpha } \\
\text { (dpm) }\end{array}$ & $\begin{array}{l}\text { Beta } \\
\text { (dpm) }\end{array}$ \\
\hline
\end{tabular}

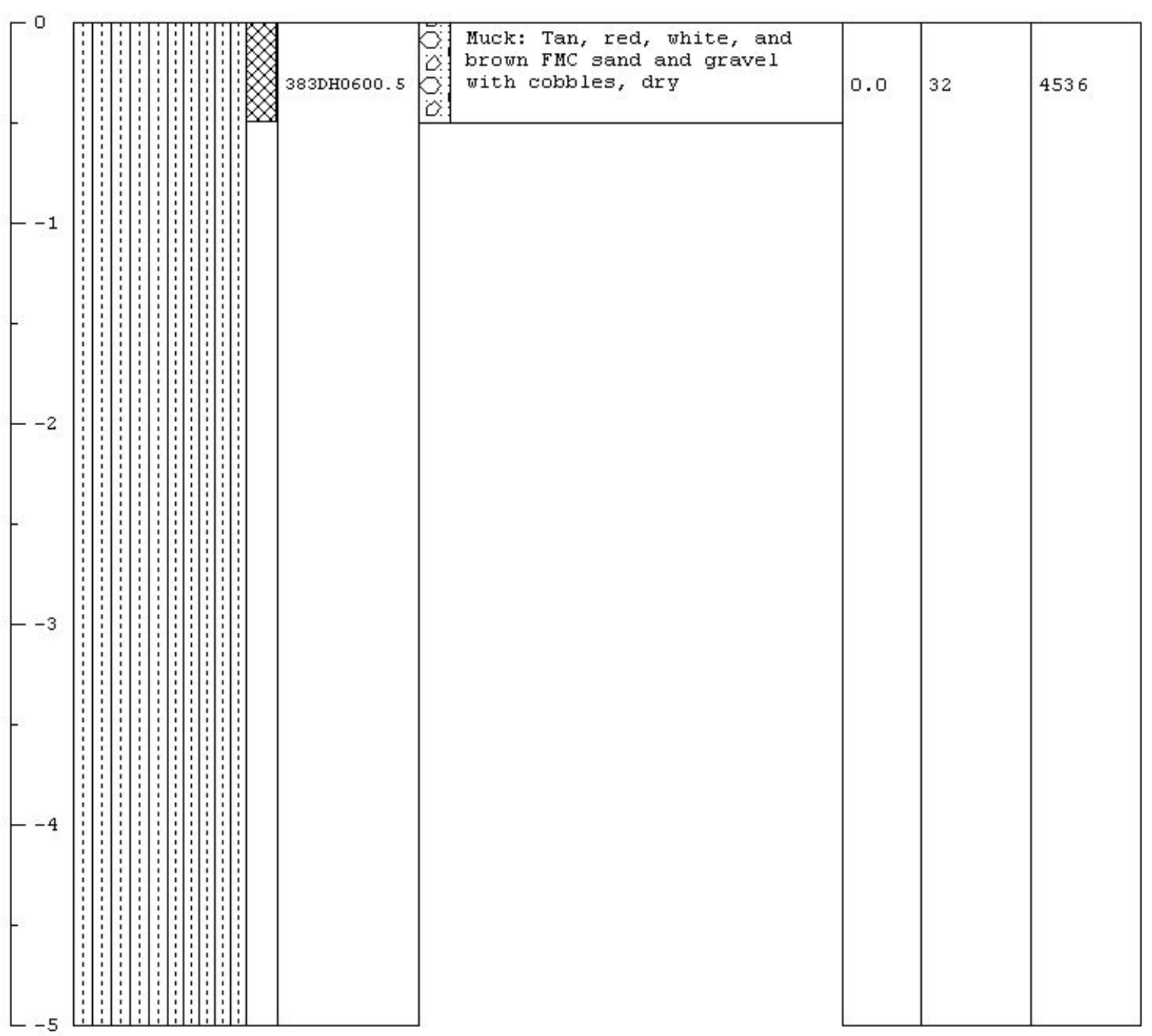


Page 1 of 1

Project Name: CAU 383 E-Tunnel sites

Project Humber: DTO4-320

Borehole Number: EDHO7

Logged By: M. McLane

Drilled By: M. McLane

Drilling Method: Hand Sampled
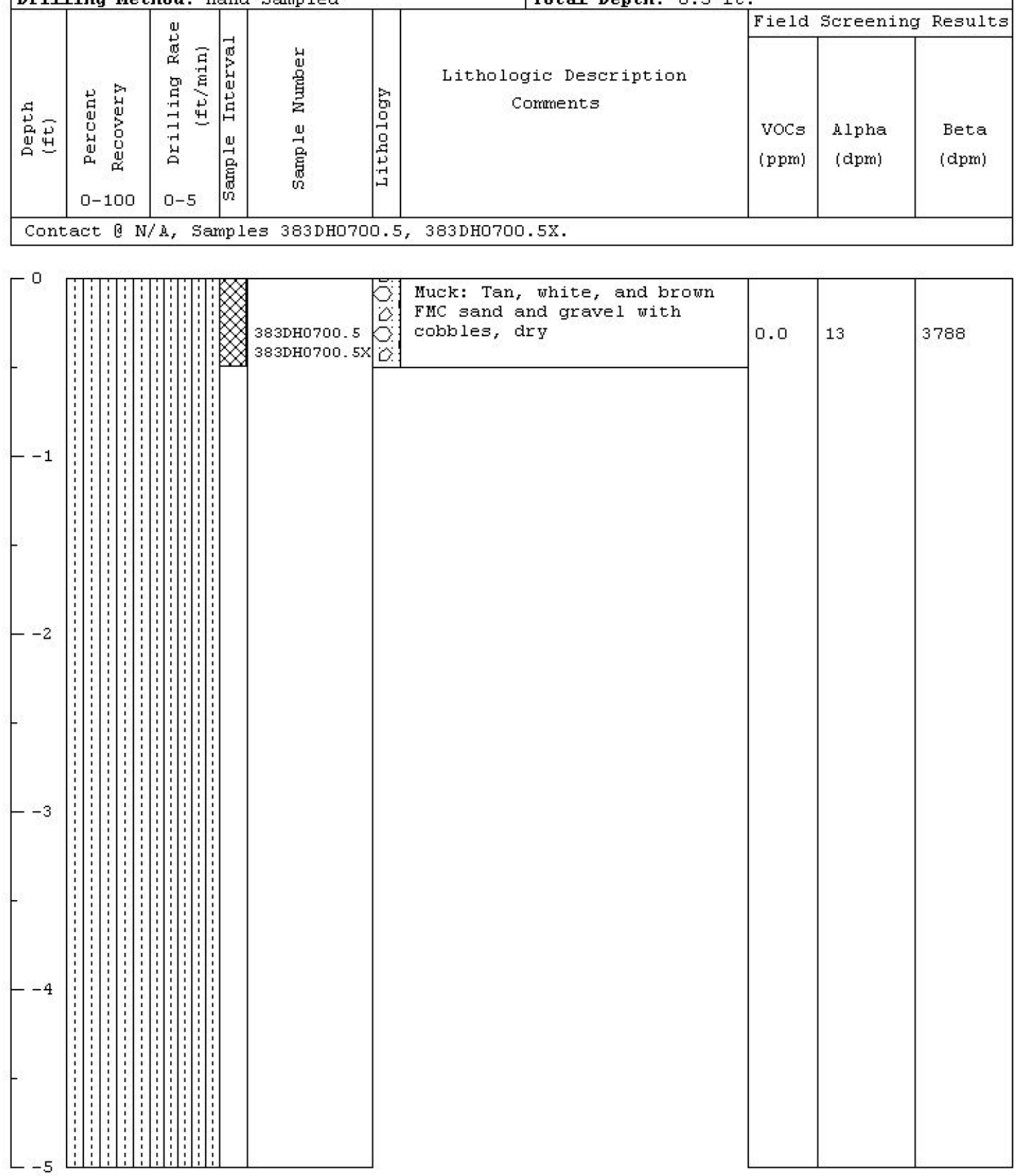
Page 1 of 1

\begin{tabular}{|c|c|c|c|c|c|c|c|c|}
\hline \multicolumn{5}{|c|}{$\begin{array}{l}\text { Project Hame: CAU } 383 \text { E-Tunnel Sites } \\
\text { Project Humber: DTO } 4-320 \\
\text { Borehole Humber: EDHOs } \\
\text { Logged By: M. MeLane } \\
\text { Drilled By: M. McLane } \\
\text { Drilling Method: Hand Sampled }\end{array}$} & \multicolumn{4}{|c|}{$\begin{array}{l}\text { Date Started: } 6 / 07 / 04 \\
\text { Date Completed: } 6 / 07 / 04 \\
\text { Elevation: } 5999.9 \\
\text { Horthing: } 887729.5 \\
\text { Easting: } 638661.1 \\
\text { Total Depth: } 0.5 \mathrm{ft} .\end{array}$} \\
\hline \multirow[b]{2}{*}{ 点 } & \multirow[b]{2}{*}{ 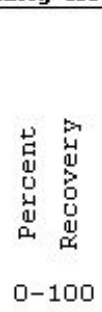 } & \multirow[b]{2}{*}{ 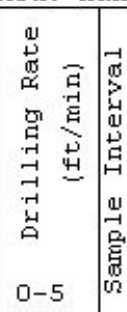 } & \multirow[b]{2}{*}{ 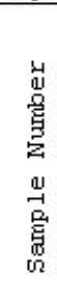 } & \multirow[b]{2}{*}{ 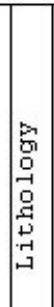 } & \multirow[b]{2}{*}{$\begin{array}{c}\text { Lithologic Description } \\
\text { Comments }\end{array}$} & Field & Screening & Results \\
\hline & & & & & & $\begin{array}{l}\text { VOCs } \\
\text { (ppm) }\end{array}$ & $\begin{array}{l}\text { Alpha } \\
\text { (dpm) }\end{array}$ & $\begin{array}{l}\text { Beta } \\
\text { (dpm) }\end{array}$ \\
\hline
\end{tabular}

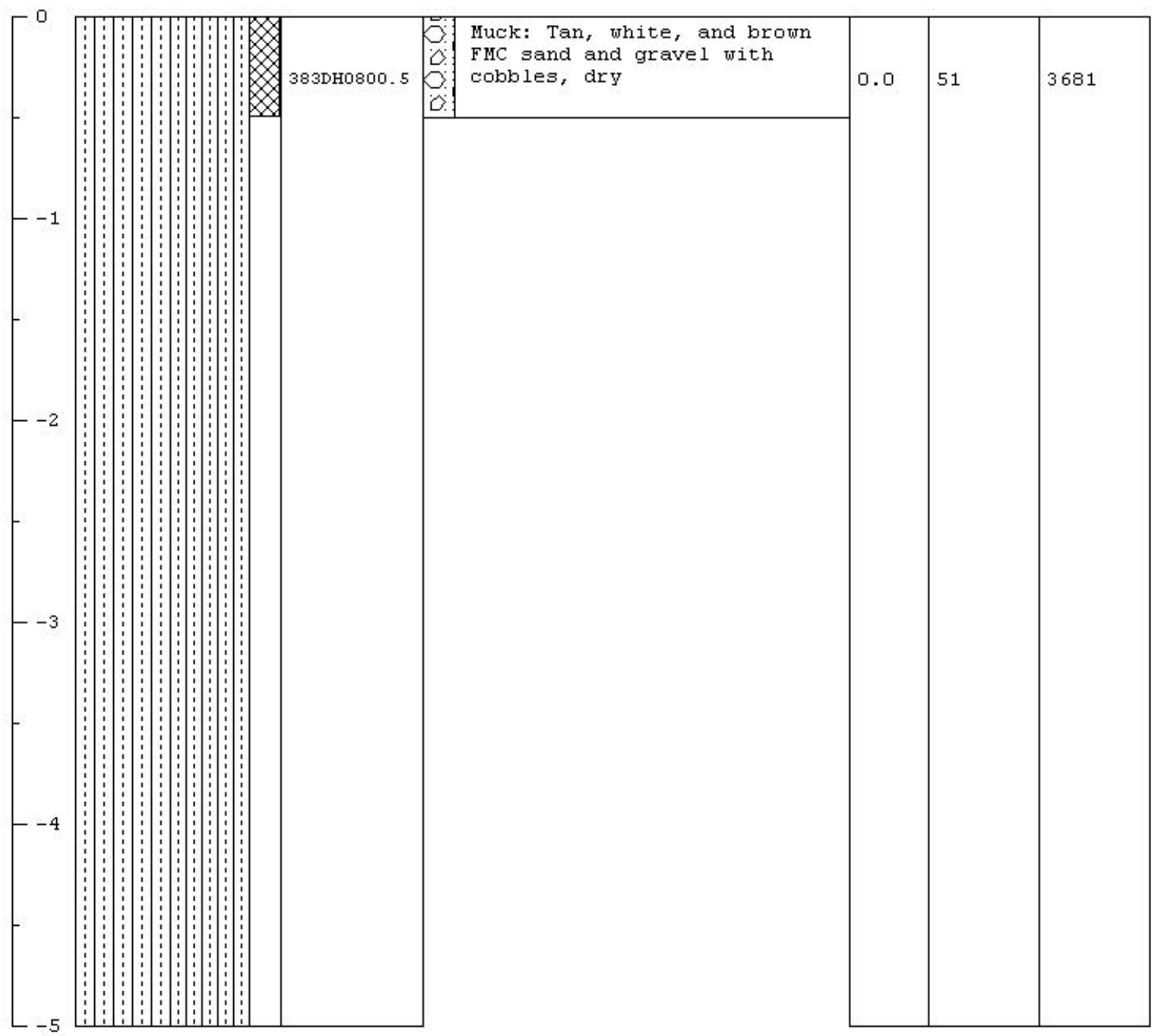


Page 1 of 1

\begin{tabular}{|c|c|c|c|c|c|c|c|c|c|}
\hline $\begin{array}{l}\text { Proj } \\
\text { Proj } \\
\text { Bore } \\
\text { Logg } \\
\text { Dril } \\
\text { Dril }\end{array}$ & $\begin{array}{l}\text { ct Nam } \\
\text { ct Huml } \\
\text { ole Hu } \\
\text { d By: } \\
\text { ed By: } \\
\text { ing Me }\end{array}$ & $\begin{array}{l}\text { CAU } 3 \\
\text { er: DTO } \\
\text { er: ED } \\
\text { MeLan } \\
\text { 1. MCLa } \\
\text { Lod: Ha }\end{array}$ & $\begin{array}{l}383 \\
\text { DH-3 } \\
\text { DHe } \\
\text { ane } \\
\text { and }\end{array}$ & Tun & site & $\begin{array}{l}\text { Date Started: } \\
\text { Date Completed: } \\
\text { Elevation: } 5997 \\
\text { Horthing: } 887735 \\
\text { Easting: } 638678 \\
\text { Total Depth: } 0 .\end{array}$ & $\begin{array}{l}7 / 04 \\
07 / 04 \\
\\
\text { Et. }\end{array}$ & & \\
\hline & & & & & & & Field & Screening & Results \\
\hline 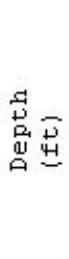 & 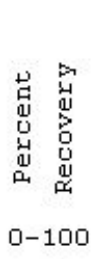 & 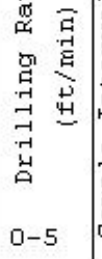 & 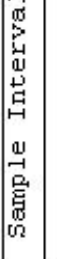 & 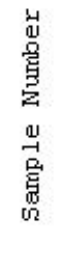 & 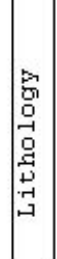 & $\begin{array}{c}\text { Lithologic Description } \\
\text { Comments }\end{array}$ & $\begin{array}{l}\text { VOCs } \\
\text { (ppm) }\end{array}$ & $\begin{array}{l}\text { Alpha } \\
\text { (dpm) }\end{array}$ & $\begin{array}{l}\text { Beta } \\
\text { (dpm) }\end{array}$ \\
\hline
\end{tabular}

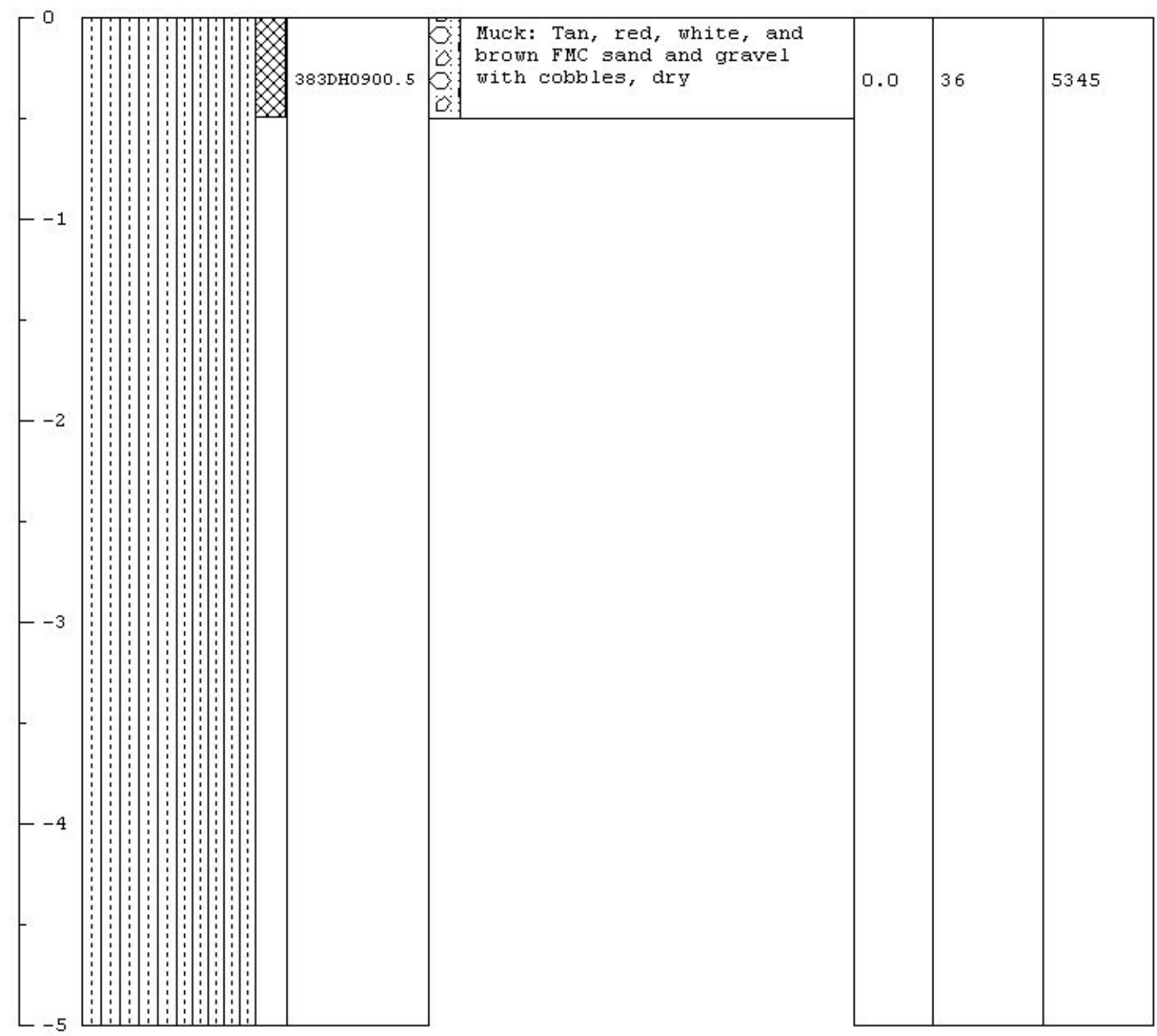


Page 1 of 1

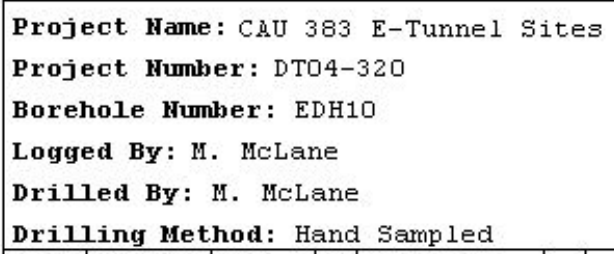

\begin{tabular}{|c|c|c|c|c|c|c|c|c|c|}
\hline \multirow[b]{2}{*}{ 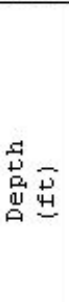 } & \multirow[b]{2}{*}{ 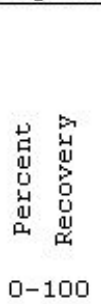 } & \multirow[b]{2}{*}{ 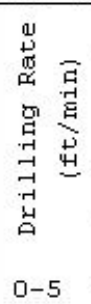 } & \multirow[b]{2}{*}{ 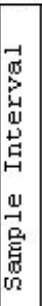 } & \multirow[b]{2}{*}{ 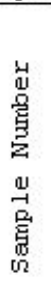 } & \multirow[b]{2}{*}{ 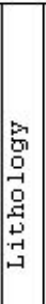 } & \multirow[b]{2}{*}{$\begin{array}{c}\text { Lithologic Description } \\
\text { Comments }\end{array}$} & \multicolumn{3}{|c|}{ Field Screening Results } \\
\hline & & & & & & & $\begin{array}{l}\text { VOCs } \\
\text { (ppm) }\end{array}$ & $\begin{array}{l}\text { Àlpha } \\
\text { (dpm) }\end{array}$ & $\begin{array}{l}\text { Beta } \\
\text { (dpm) }\end{array}$ \\
\hline
\end{tabular}

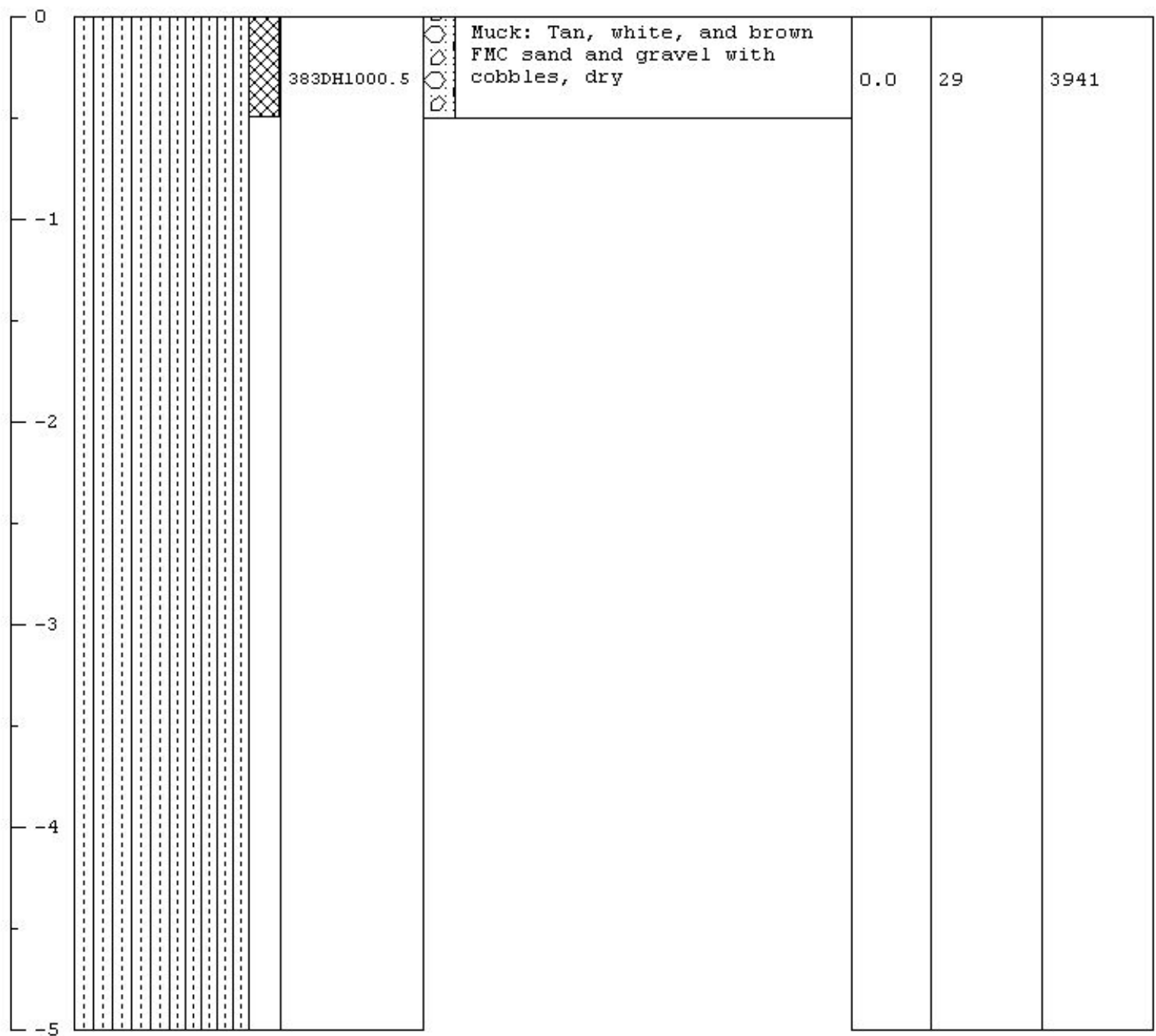


Page 1 of 1

\begin{tabular}{|c|c|c|c|c|c|c|c|c|c|}
\hline \multicolumn{6}{|c|}{$\begin{array}{l}\text { Project Hame: CAU } 383 \text { E-Tunnel Sites } \\
\text { Project Humber: DTO } 4-320 \\
\text { Borehole Humber: EDH } 11 \\
\text { Logged By: M. McLane } \\
\text { Drilled By: M. McLane } \\
\text { Drilling Method: Hand Sampled } \\
\end{array}$} & \multicolumn{4}{|c|}{$\begin{array}{l}\text { Date Started: } 6 / 07 / 04 \\
\text { Date Completed: } 6 / 07 / 04 \\
\text { Elevation: } 5984.3 \\
\text { Horthing: } 887726.6 \\
\text { Easting: } 638697.8 \\
\text { Total Depth: } 0.5 \mathrm{ft} \text {. }\end{array}$} \\
\hline \multirow[b]{2}{*}{ 袁 } & \multirow[b]{2}{*}{ 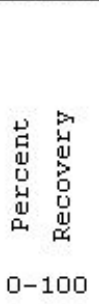 } & \multirow[b]{2}{*}{ 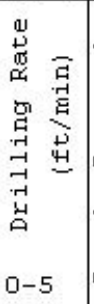 } & \multirow[b]{2}{*}{ 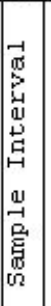 } & \multirow[b]{2}{*}{ 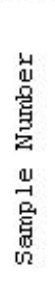 } & \multirow[b]{2}{*}{ 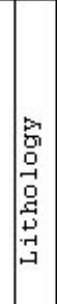 } & \multirow[b]{2}{*}{$\begin{array}{c}\text { Lithologic Description } \\
\text { Comments }\end{array}$} & \multirow[b]{2}{*}{$\begin{array}{l}\text { Field } \\
\text { vocs } \\
\text { (ppm) }\end{array}$} & Screening & Results \\
\hline & & & & & & & & $\begin{array}{l}\text { Alpha } \\
\text { (dpm) }\end{array}$ & $\begin{array}{l}\text { Beta } \\
\text { (dpm) }\end{array}$ \\
\hline
\end{tabular}

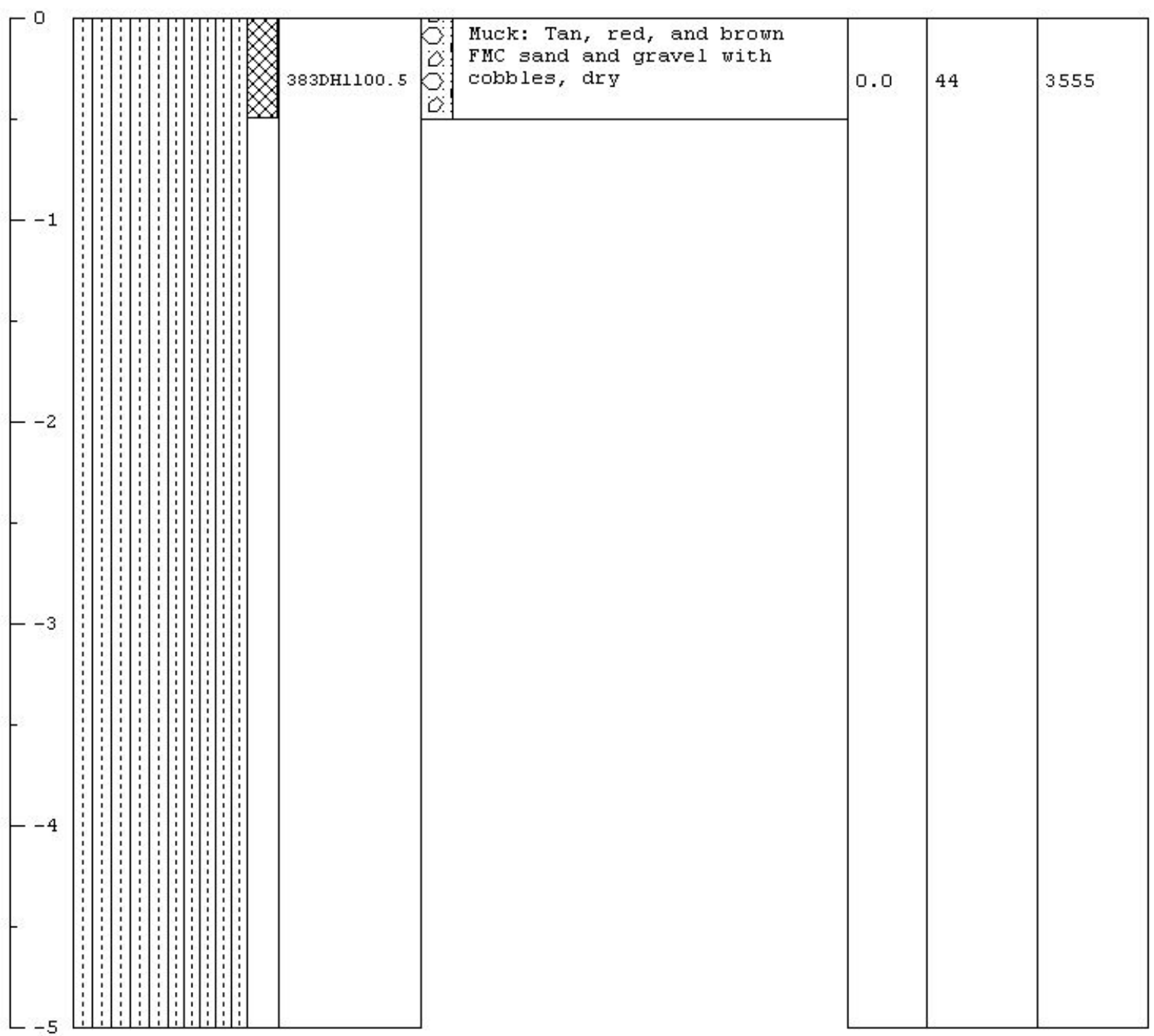


Page 1 of 1

Project Hame: CAU 383 E-Tunnel Sites

Project Humber: DTO4-320

Date Started: $6 / 07 / 04$

Date Completed: $6 / 07 / 04$

Borehole Number: EDH12

Elevation: 5983.9

Logged BY: $M$. McLane

Horthing : 887730.0

Drilled By: $M$. McLane

Easting: 638716.7

Drilling Method: Hand Sampled

Total Depth: $0.5 \mathrm{ft}$.

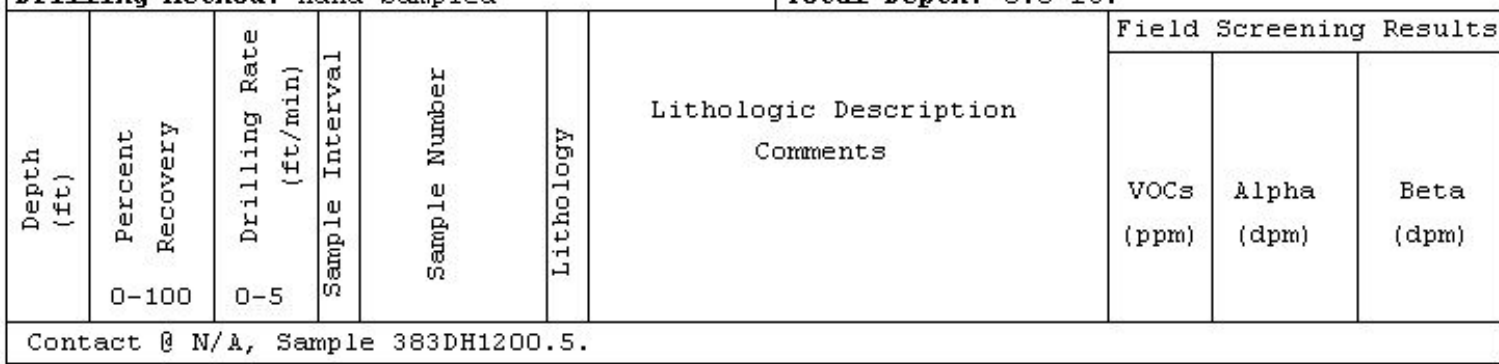

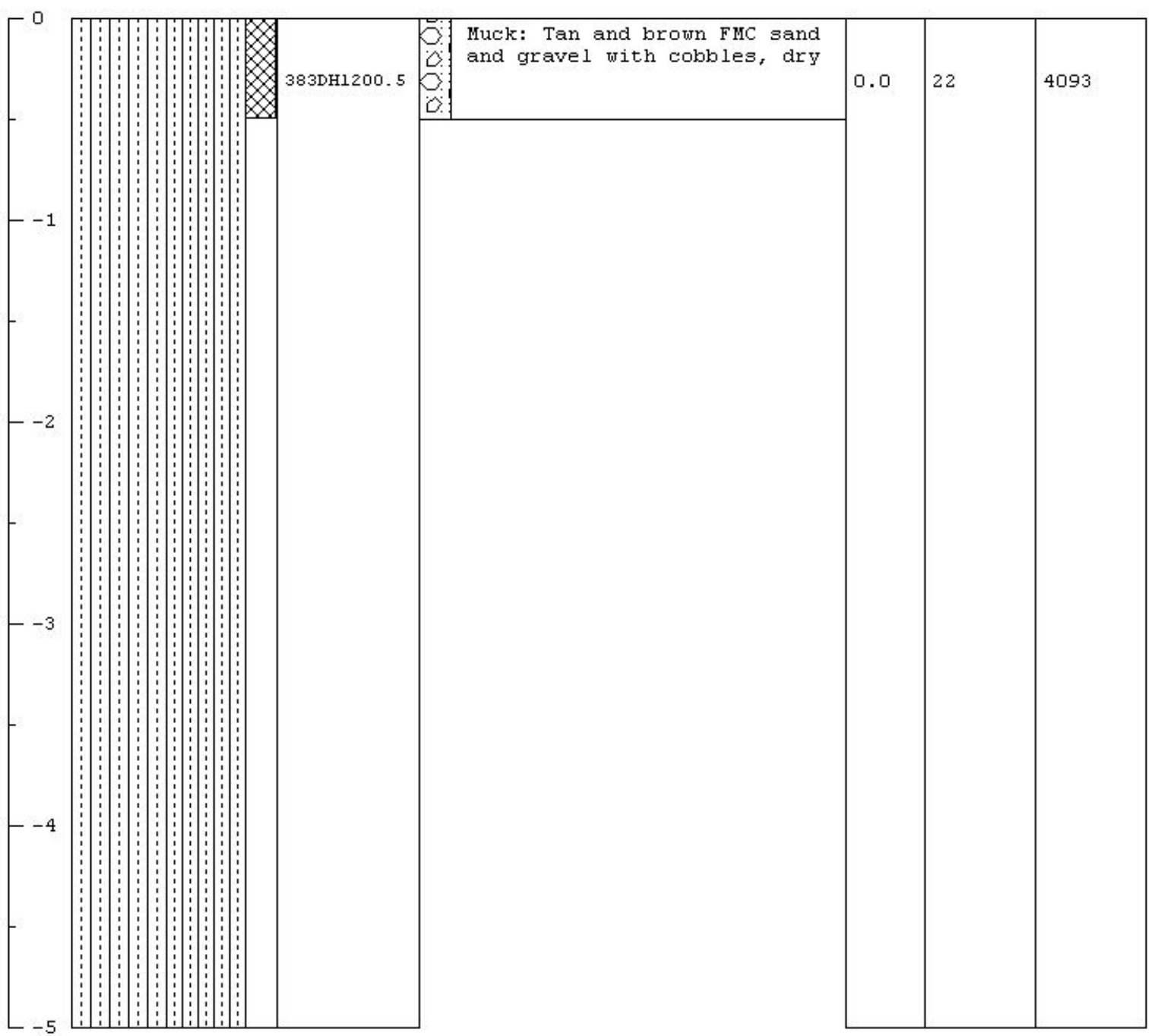


Page 1 of 1

\begin{tabular}{|c|c|c|c|c|c|c|c|c|c|}
\hline \multicolumn{6}{|c|}{$\begin{array}{l}\text { Project Hame: CAU } 383 \text { E-Tunnel Sites } \\
\text { Project Humber: DTO } 4-320 \\
\text { Borehole Humber: EDH } 13 \\
\text { Logged By: M. MeLane } \\
\text { Drilled By: M. McLane } \\
\text { Drilling Method: Hand Sampled }\end{array}$} & \multicolumn{4}{|c|}{$\begin{array}{l}\text { Date Started: } 6 / 07 / 04 \\
\text { Date Completed: } 6 / 07 / 04 \\
\text { Elevation: } 5970.7 \\
\text { Northing: } 887720.0 \\
\text { Easting: } 638737.5 \\
\text { Total Depth: } 0.5 \mathrm{ft} .\end{array}$} \\
\hline \multirow[b]{2}{*}{ 袁 } & \multirow[b]{2}{*}{ 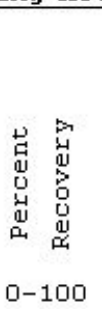 } & \multirow{2}{*}{\multicolumn{2}{|c|}{ 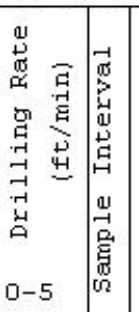 }} & \multirow[b]{2}{*}{ 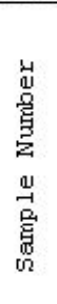 } & \multirow[b]{2}{*}{ 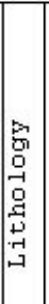 } & \multirow[b]{2}{*}{$\begin{array}{c}\text { Lithologic Description } \\
\text { Corments }\end{array}$} & \multirow[b]{2}{*}{\begin{tabular}{|c|} 
\\
Field \\
vocs \\
(ppm)
\end{tabular}} & Screening & Results \\
\hline & & & & & & & & $\begin{array}{l}\text { Alpha } \\
\text { (dpm) }\end{array}$ & $\begin{array}{l}\text { Beta } \\
\text { (dpm) }\end{array}$ \\
\hline
\end{tabular}

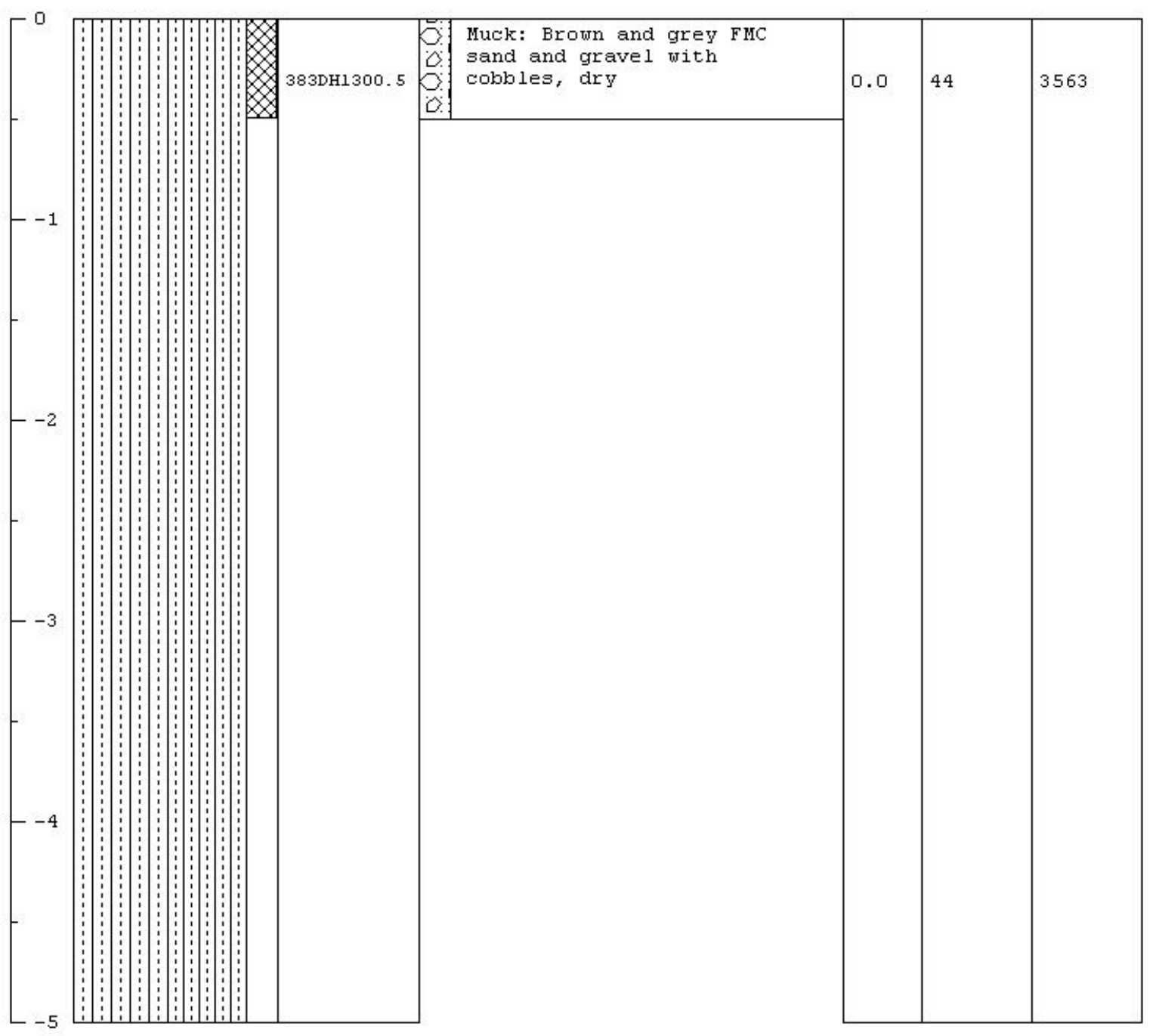


Page 1 of 1

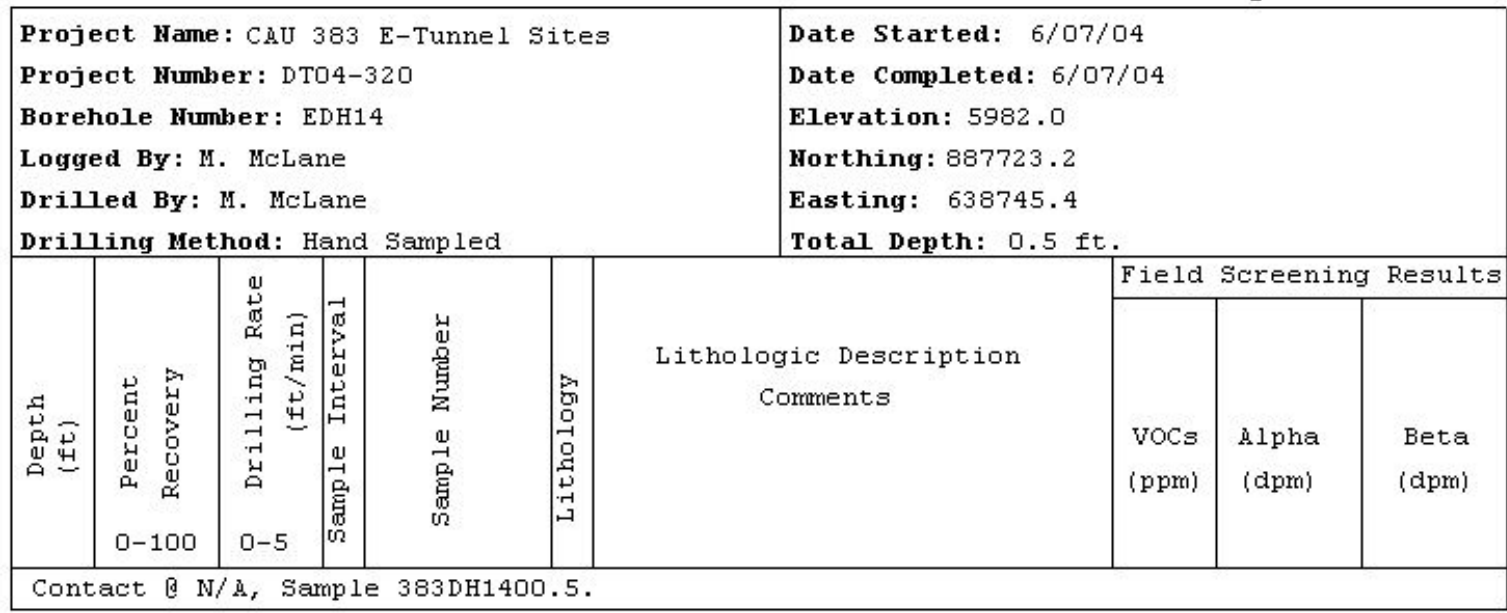

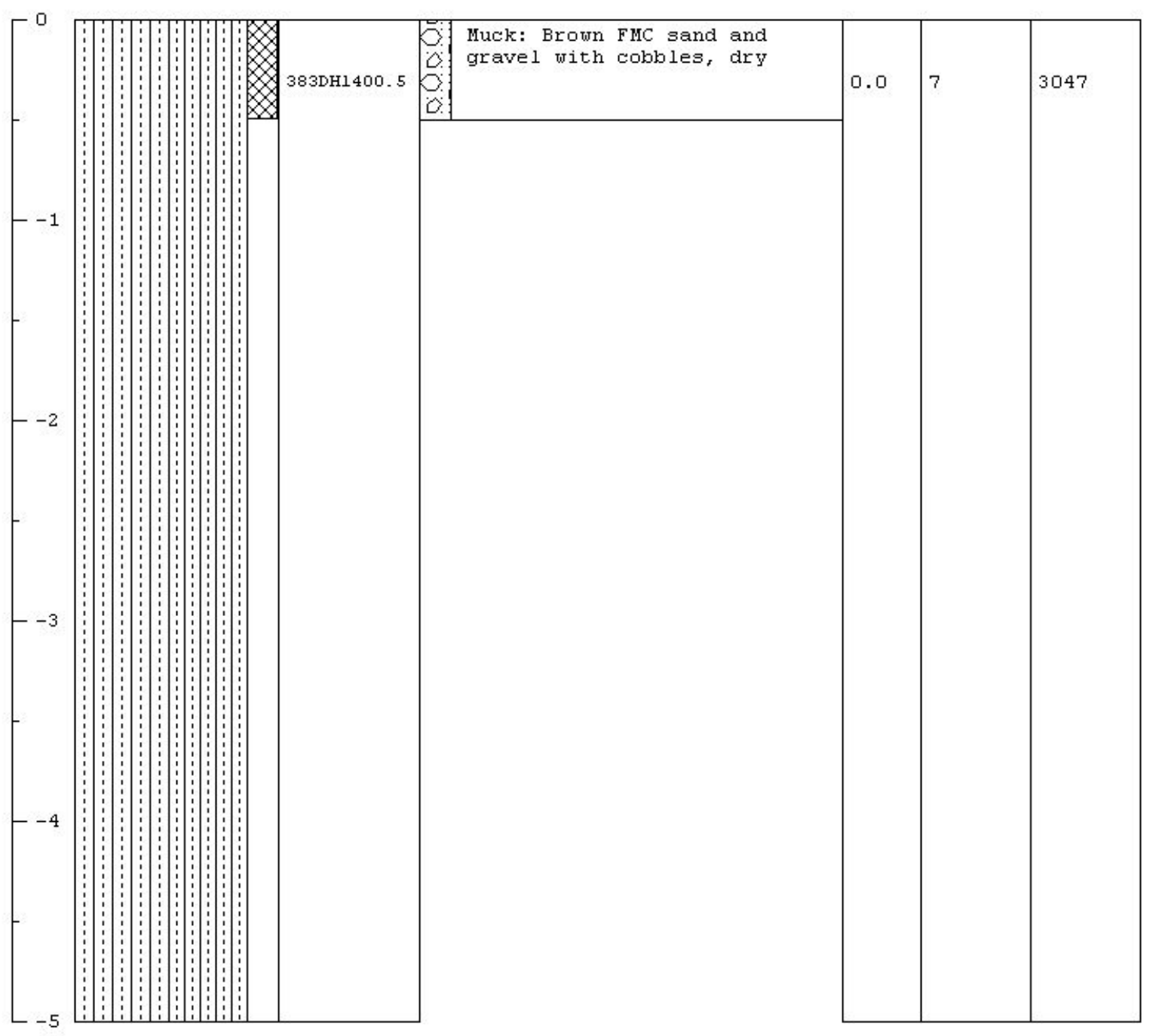


Page 1 of 1

\begin{tabular}{|c|c|c|c|c|c|c|c|c|}
\hline \multicolumn{5}{|c|}{$\begin{array}{l}\text { Project Hame: CAU } 383 \text { E-Tunnel Sites } \\
\text { Project Humber: DTO } 4-320 \\
\text { Borehole Humber: EDH } 15 \\
\text { Logged By: M. McLane } \\
\text { Drilled By: M. McLane } \\
\text { Drilling Method: Hand Sampled }\end{array}$} & \multicolumn{4}{|c|}{$\begin{array}{l}\text { Date Started: } 6 / 07 / 04 \\
\text { Date Completed: } 6 / 07 / 04 \\
\text { Elevation: } 5976.8 \\
\text { Horthing: } 887712.1 \\
\text { Easting: } 638759.5 \\
\text { Total Depth: } 0.5 \mathrm{ft} .\end{array}$} \\
\hline \multirow[b]{2}{*}{ 点 } & \multirow[b]{2}{*}{ 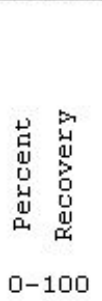 } & \multirow[b]{2}{*}{ 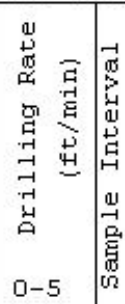 } & \multirow[b]{2}{*}{ 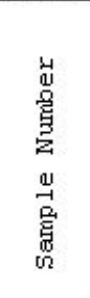 } & \multirow[b]{2}{*}{ 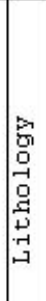 } & \multirow[b]{2}{*}{$\begin{array}{c}\text { Lithologic Description } \\
\text { Comments }\end{array}$} & Field & Screening & Results \\
\hline & & & & & & $\begin{array}{l}\text { VOCs } \\
\text { (ppm) }\end{array}$ & $\begin{array}{l}\text { Alpha } \\
\text { (dpm) }\end{array}$ & $\begin{array}{l}\text { Beta } \\
\text { (dpm) }\end{array}$ \\
\hline
\end{tabular}

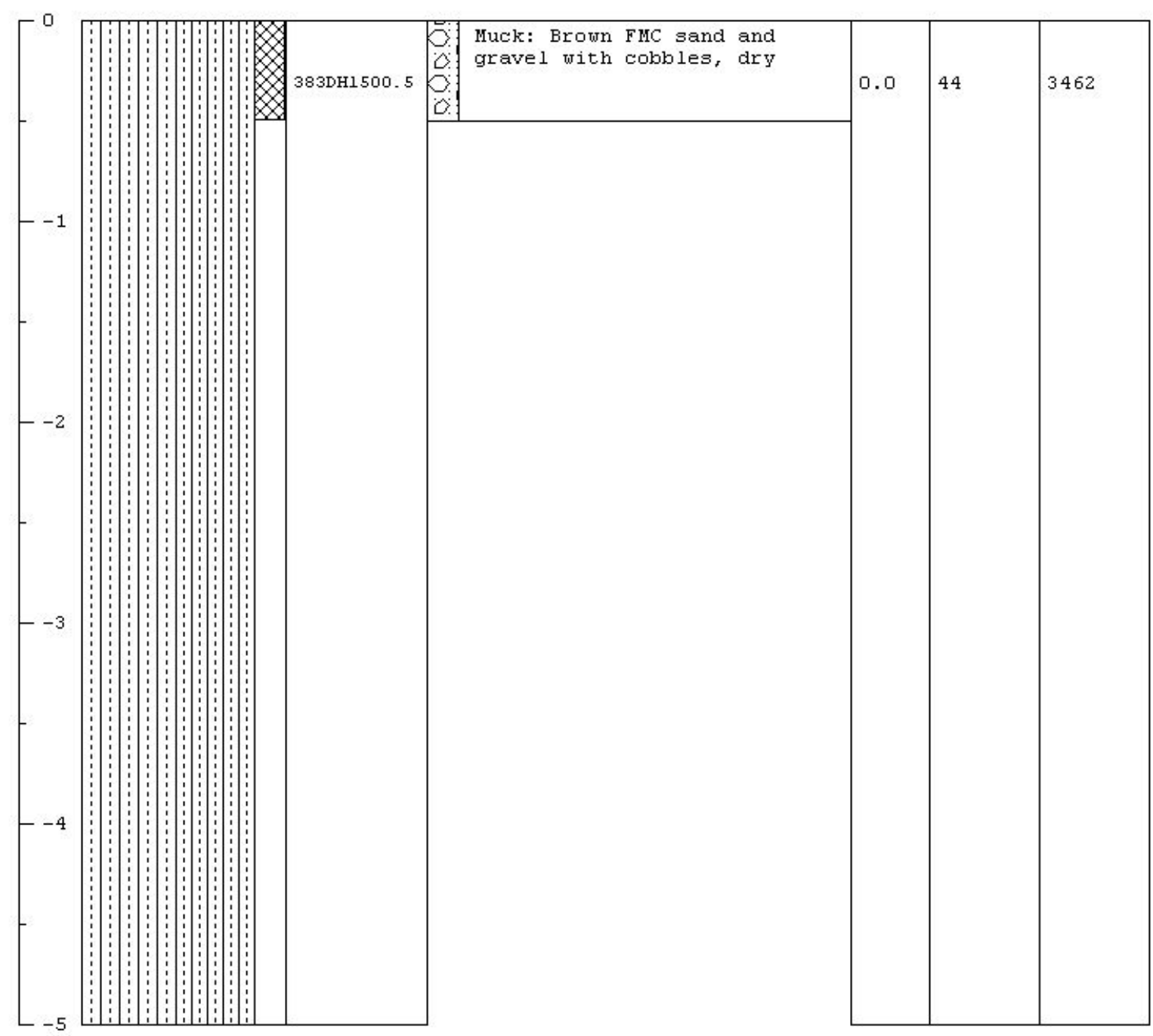




\begin{tabular}{|c|c|c|c|c|c|c|c|c|c|}
\hline \multicolumn{6}{|c|}{$\begin{array}{l}\text { Project Hame: CAU } 383 \text { E-Tunnel Sites } \\
\text { Project Humber: DTO4-320 } \\
\text { Borehole Number: EDH } 16 \\
\text { Logged By: M. McLane } \\
\text { Drilled By: M. McLane } \\
\text { Drilling Method: Hand Sampled }\end{array}$} & \multicolumn{4}{|c|}{$\begin{array}{l}\text { Date Started: } 6 / 07 / 04 \\
\text { Date Completed: } 6 / 07 / 04 \\
\text { Elevation: } 5983.1 \\
\text { Horthing: } 887711.1 \\
\text { Easting: } 638780.1 \\
\text { Total Depth: } 0.5 \mathrm{ft} . \\
\end{array}$} \\
\hline \multirow[b]{2}{*}{ 点 } & \multirow[b]{2}{*}{ 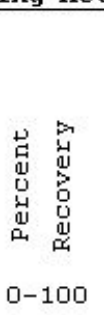 } & \multirow[b]{2}{*}{ 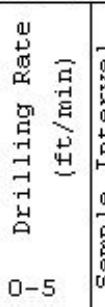 } & & \multirow[b]{2}{*}{ 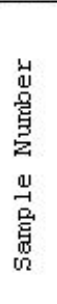 } & \multirow[b]{2}{*}{ 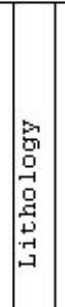 } & \multirow[b]{2}{*}{$\begin{array}{c}\text { Lithologic Description } \\
\text { Comments }\end{array}$} & Field & Screening & Results \\
\hline & & & 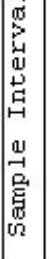 & & & & $\begin{array}{l}\text { VOCs } \\
\text { (ppm) }\end{array}$ & $\begin{array}{l}\text { Alpha } \\
\text { (dpm) }\end{array}$ & $\begin{array}{r}\text { Beta } \\
\text { (dpm) }\end{array}$ \\
\hline
\end{tabular}

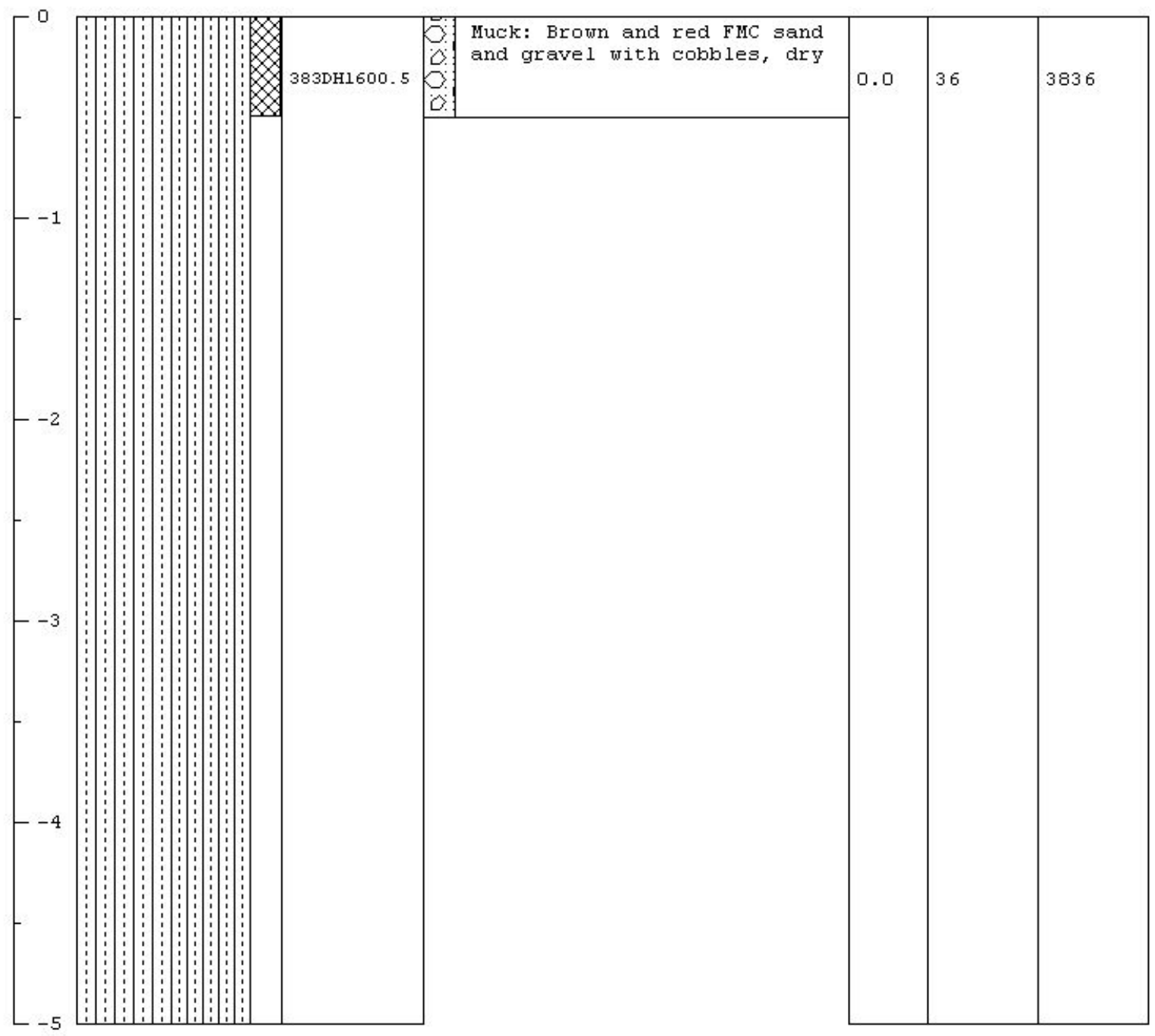




\begin{tabular}{|c|c|c|c|c|c|c|c|c|c|}
\hline \multicolumn{6}{|c|}{$\begin{array}{l}\text { Project Hame: CAU } 383 \text { E-Tunnel Sites } \\
\text { Project Humber: DTO } 4-320 \\
\text { Borehole Humber: EDH } 17 \\
\text { Logged By: M. McLane } \\
\text { Drilled By: M. McLane } \\
\text { Drilling Method: Hand Sampled }\end{array}$} & \multicolumn{4}{|c|}{$\begin{array}{l}\text { Date Started: } 6 / 07 / 04 \\
\text { Date Completed: } 6 / 07 / 04 \\
\text { Elevation: } 5978.8 \\
\text { Horthing: } 887713.1 \\
\text { Easting: } 638786.0 \\
\text { Total Depth: } 0.5 \mathrm{ft} .\end{array}$} \\
\hline \multirow[b]{2}{*}{ 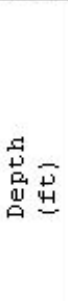 } & \multirow[b]{2}{*}{ 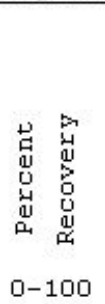 } & \multirow{2}{*}{\multicolumn{2}{|c|}{ 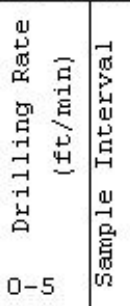 }} & \multirow[b]{2}{*}{ 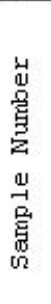 } & \multirow[b]{2}{*}{ 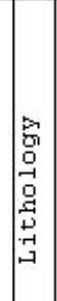 } & \multirow[b]{2}{*}{$\begin{array}{c}\text { Lithologic Description } \\
\text { Comments }\end{array}$} & \multirow{2}{*}{\begin{tabular}{|l} 
Field \\
vocs \\
(ppm)
\end{tabular}} & Screening & Results \\
\hline & & & & & & & & $\begin{array}{l}\text { Alpha } \\
\text { (dpm) }\end{array}$ & $\begin{array}{l}\text { Beta } \\
\text { (dpm) }\end{array}$ \\
\hline
\end{tabular}

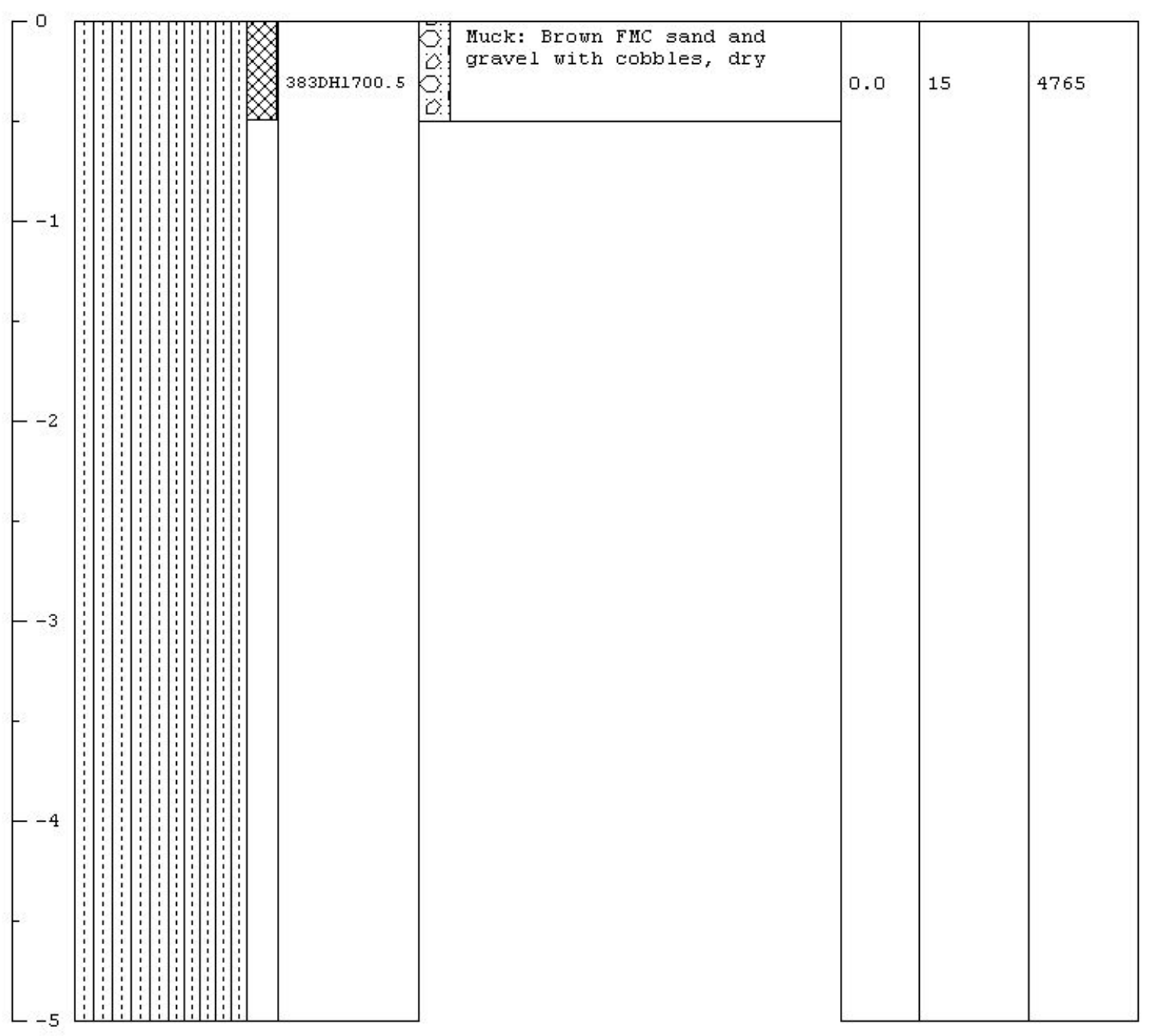


Page 1 of 1

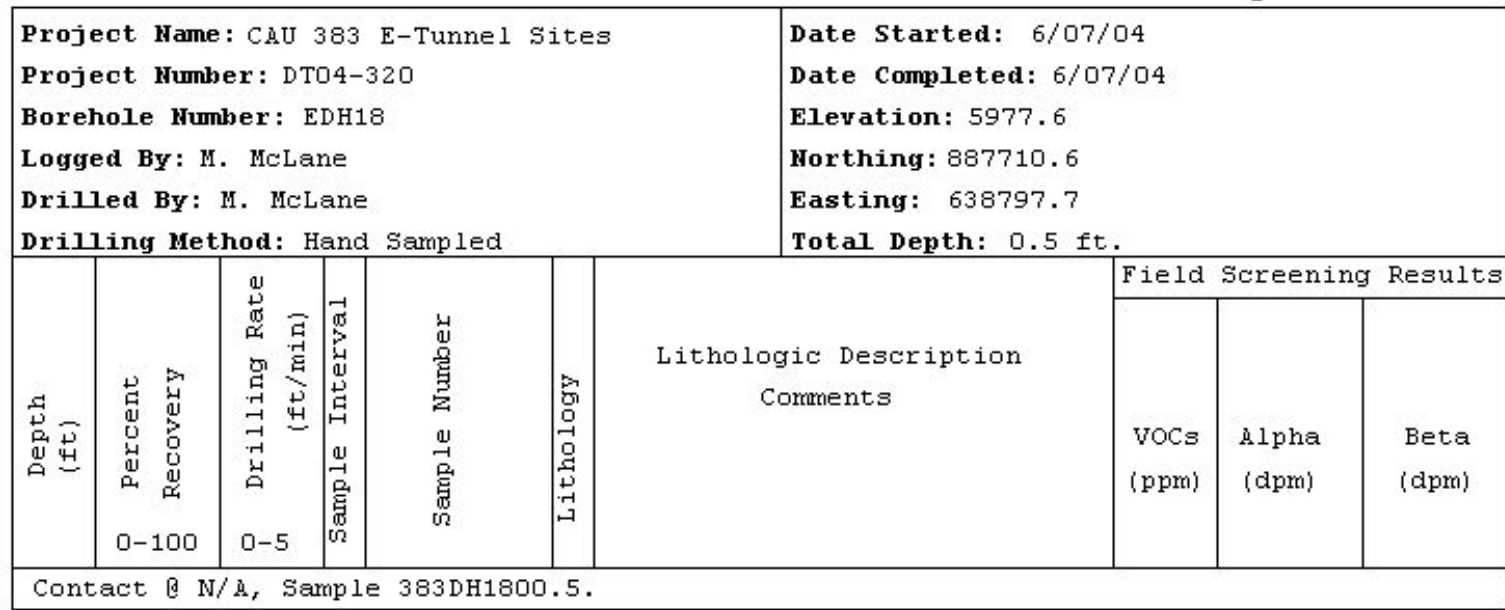

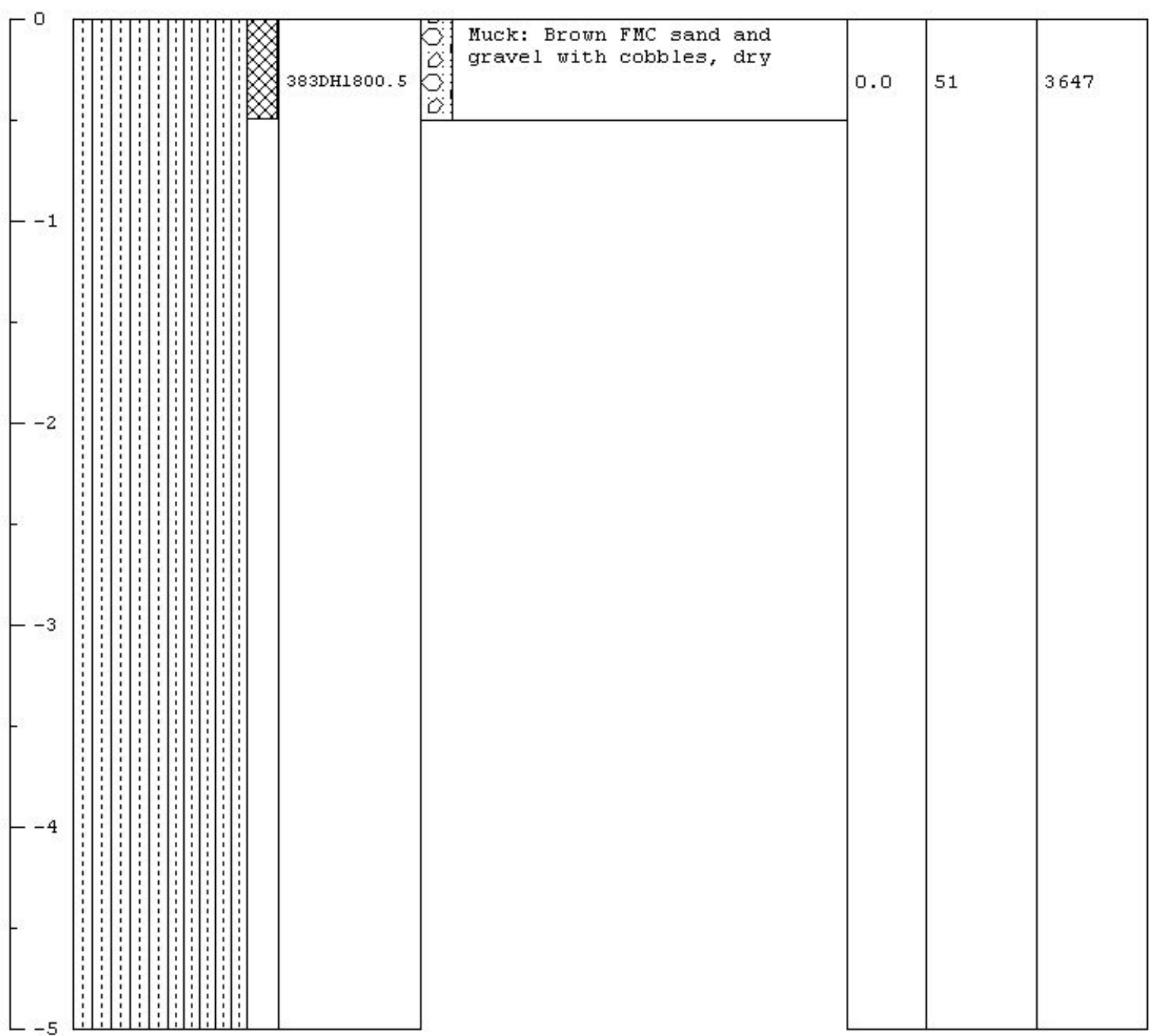


Page 1 of 1

Project Hame: CAU 383 E-Tunnel Sites

Project Humber: DTO4-320

Borehole Number: EDH19

Logged BY: M. McLane

Drilled By: $M$. McLane

Drilling Method: Hand Sampled
Date Started: $6 / 07 / 04$

Date Completed: $6 / 07 / 04$

Elevation: 5975.1

Horthing: 887707.0

Easting : 638808.0

Total Depth: $0.5 \mathrm{ft}$.
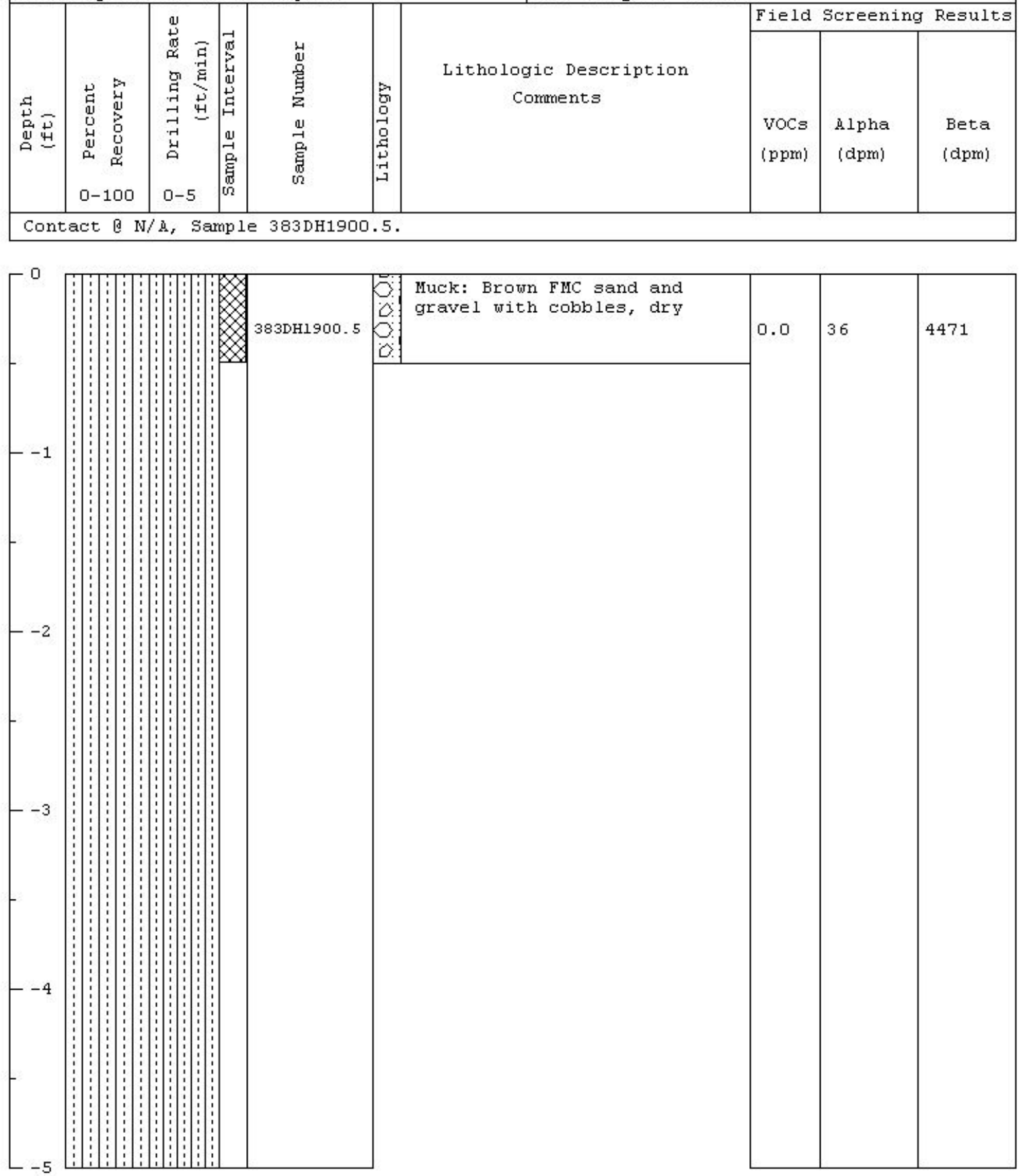
Page 1 of 1

\begin{tabular}{|c|c|c|c|c|c|c|c|c|c|}
\hline \multicolumn{6}{|c|}{$\begin{array}{l}\text { Project Hame: CAU } 383 \text { E-Tunnel Sites } \\
\text { Project Humber: DTO } 4-320 \\
\text { Borehole Humber: EDH } 20 \\
\text { Logged By: M. MeLane } \\
\text { Drilled By: M. MeLane } \\
\text { Drilling Method: Hand Sampled }\end{array}$} & \multicolumn{4}{|c|}{$\begin{array}{l}\text { Date Started: } 6 / 07 / 04 \\
\text { Date Completed: } 6 / 07 / 04 \\
\text { Elevation: } 5972.7 \\
\text { Horthing: } 887704.0 \\
\text { Easting: } 638818.3 \\
\text { Total Depth: } 0.5 \mathrm{ft} . \\
\end{array}$} \\
\hline \multirow[b]{2}{*}{ 点 } & \multirow[b]{2}{*}{ 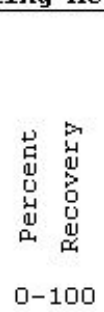 } & \multirow{2}{*}{\multicolumn{2}{|c|}{ 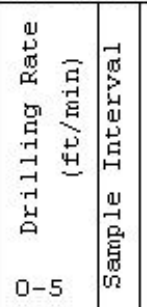 }} & \multirow[b]{2}{*}{ 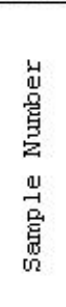 } & \multirow[b]{2}{*}{ 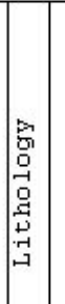 } & \multirow[b]{2}{*}{$\begin{array}{c}\text { Lithologic Description } \\
\text { Comments }\end{array}$} & Field & Screening & Results \\
\hline & & & & & & & $\begin{array}{l}\text { VoCs } \\
\text { (ppm) }\end{array}$ & $\begin{array}{l}\text { Alpha } \\
\text { (dpm) }\end{array}$ & $\begin{array}{l}\text { Beta } \\
\text { (dpm) }\end{array}$ \\
\hline
\end{tabular}

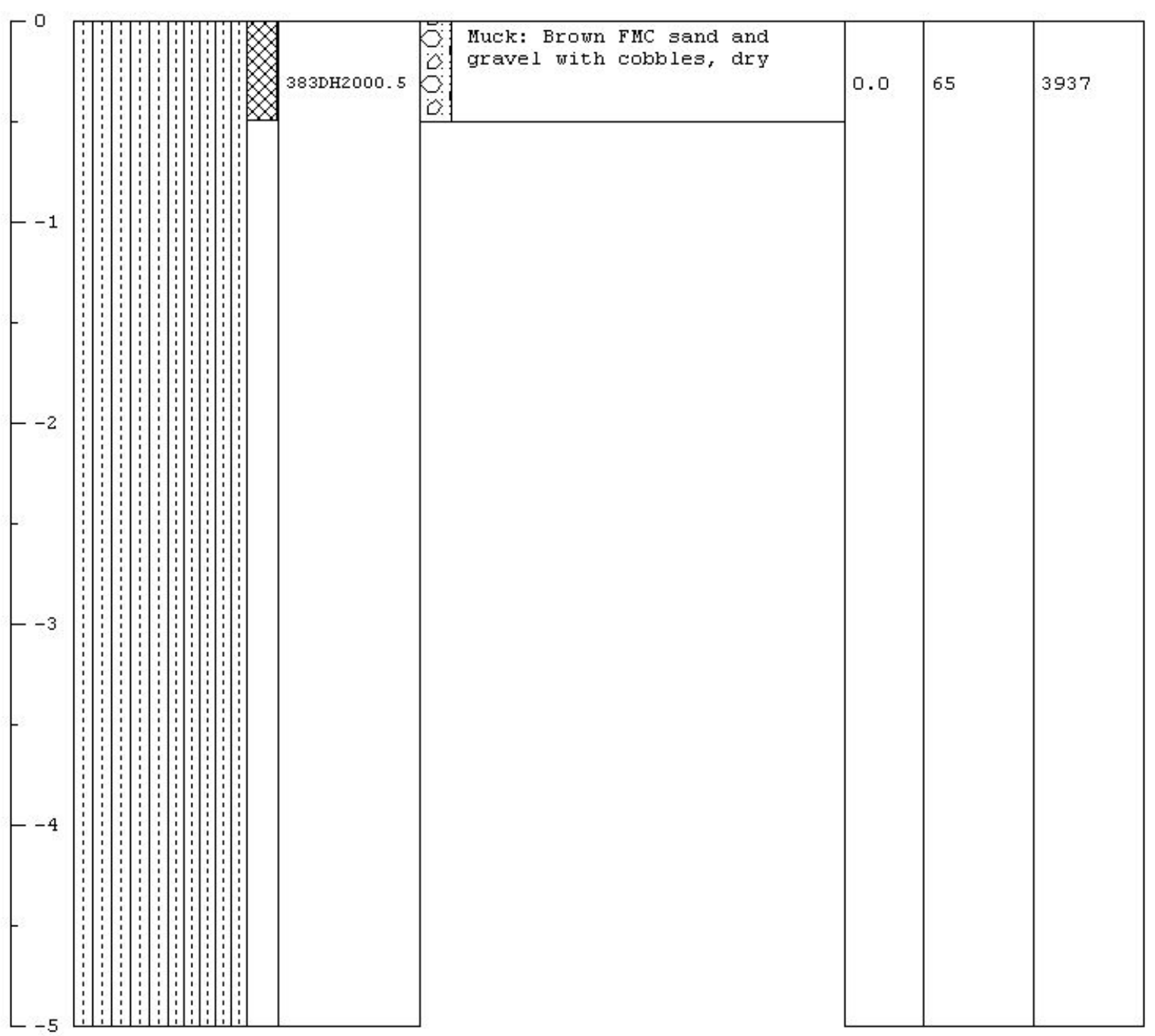


Page 1 of 1

\begin{tabular}{|c|c|c|c|c|c|c|c|c|}
\hline $\begin{array}{l}\text { Proj } \\
\text { Proj } \\
\text { Borel } \\
\text { Logg } \\
\text { Dril } \\
\text { Dril }\end{array}$ & $\begin{array}{l}\text { et Nam } \\
\text { ct Hum } \\
\text { ole Hu } \\
\text { d By: } \\
\text { ed By: } \\
\text { ing Me }\end{array}$ & $\begin{array}{l}\text { : CAU } 383 \\
\text { er: DTO4- } \\
\text { ber: EDH } \\
\text {. McLane } \\
\text { M. MeLan } \\
\text { hod: Han }\end{array}$ & Tun & ite & $\begin{array}{l}\text { Date Started: } \\
\text { Date Completed: } \\
\text { Elevation: } 5976 \\
\text { Horthing: } 88771 \\
\text { Easting: } 63882 \\
\text { Total Depth: } 0 .\end{array}$ & $\begin{array}{l}04 \\
5 / 04 \\
\\
\end{array}$ & . & 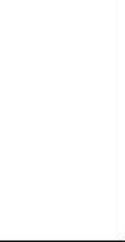 \\
\hline & & & & & & Field & Screening & Results \\
\hline 点 & 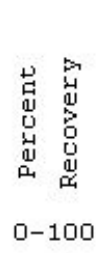 & 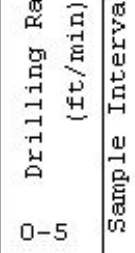 & 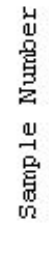 & 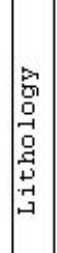 & $\begin{array}{c}\text { Lithologic Description } \\
\text { Comments }\end{array}$ & $\begin{array}{l}\text { VOCs } \\
\text { (ppm) }\end{array}$ & $\begin{array}{l}\text { Alpha } \\
\text { (dpm) }\end{array}$ & $\begin{array}{l}\text { Beta } \\
\text { (dpm) }\end{array}$ \\
\hline
\end{tabular}

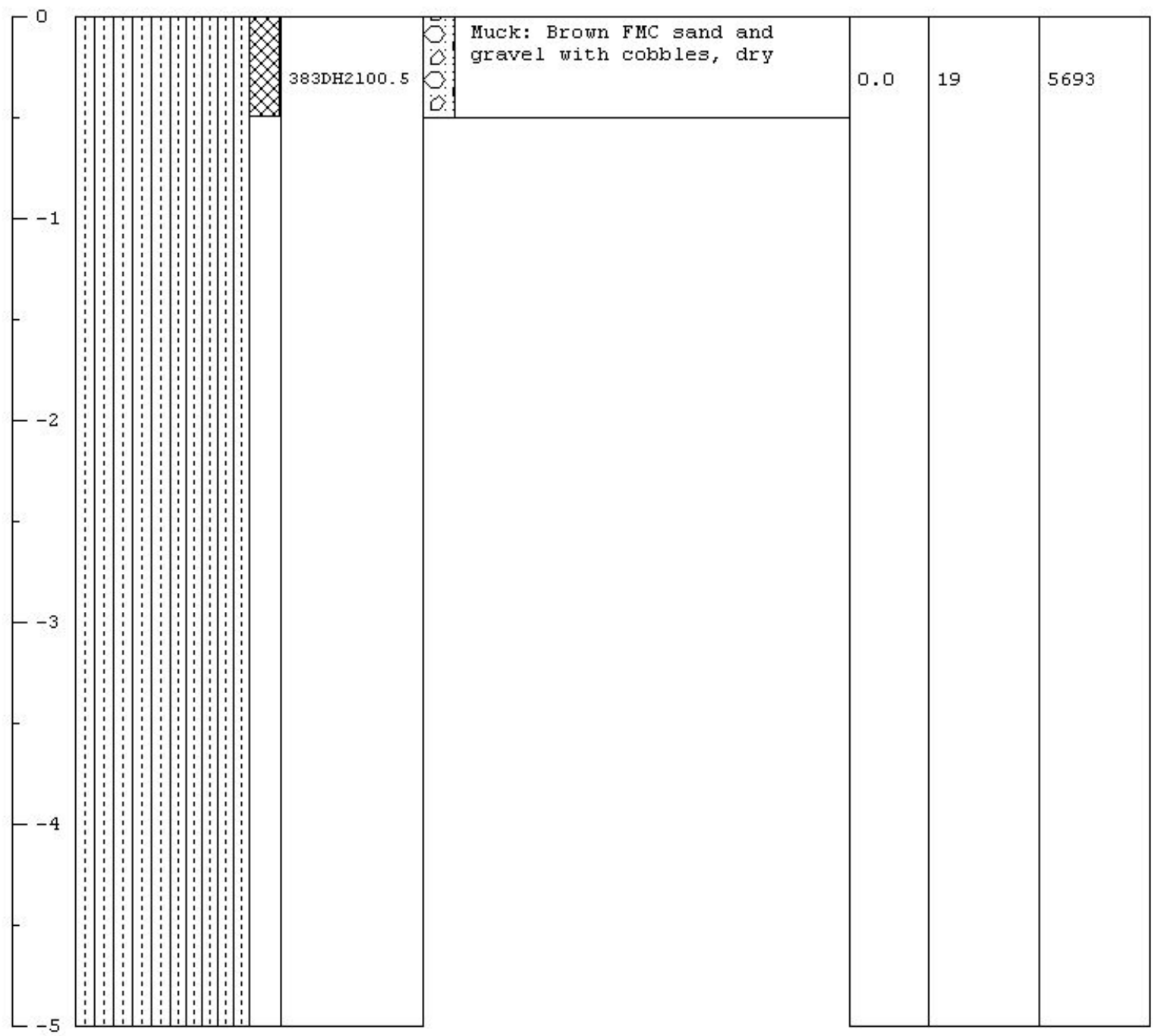


Page 1 of 1

Project Name: CAU 383 E-Tunnel sites

Project Humber: DTO4-320

Borehole Number: EDH 22

Logged By: M. McLane

Drilled By: M. McLane

Drilling Method: Hand Sampled
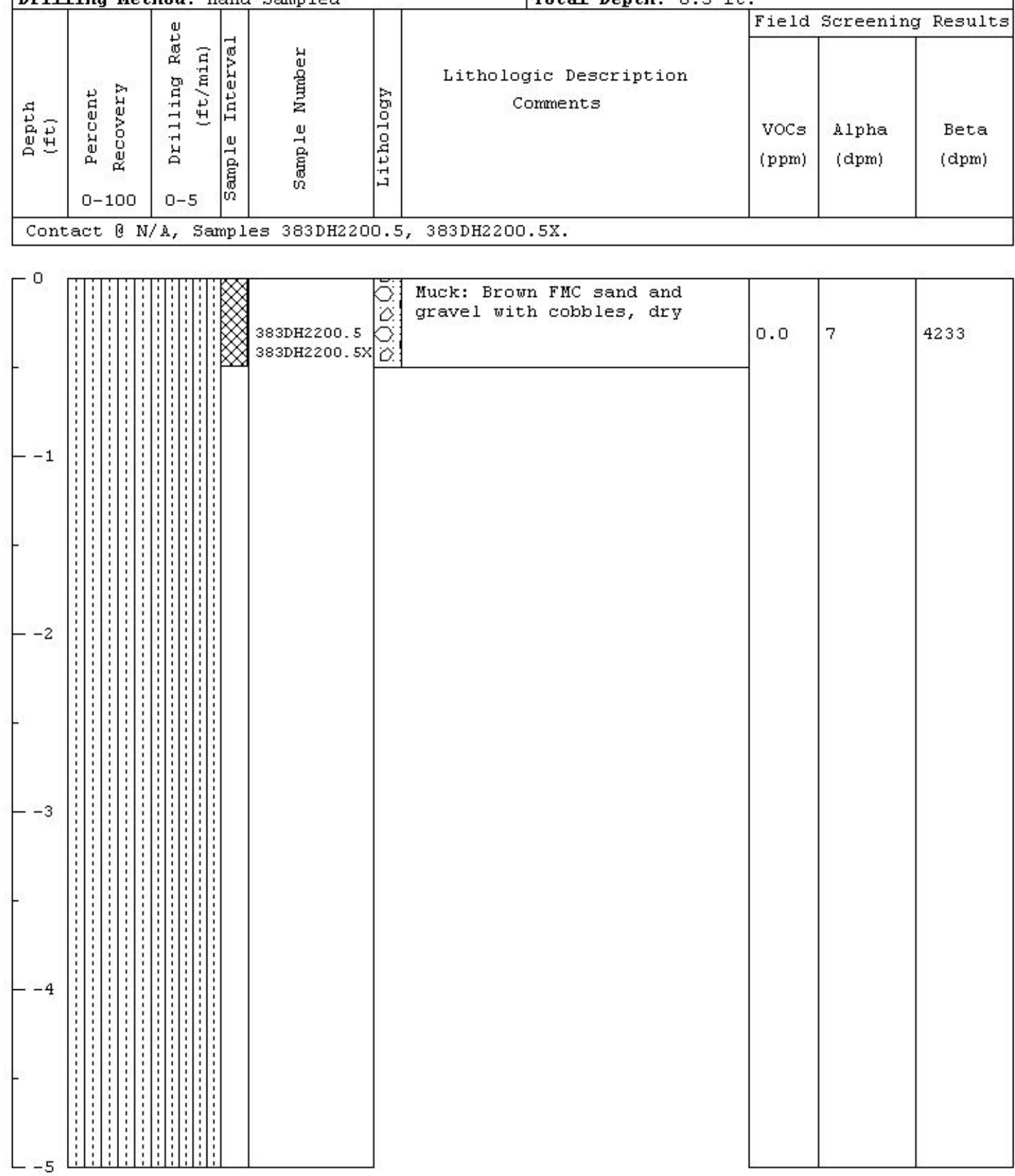
Page 1 of 1

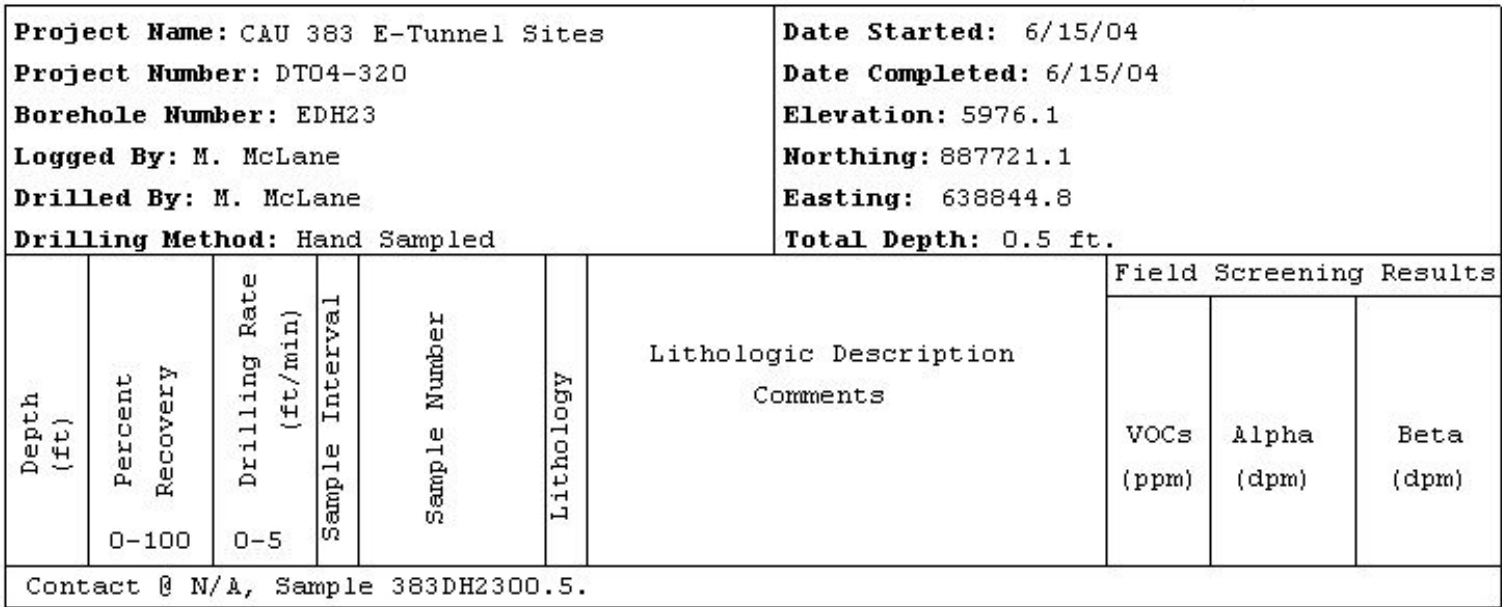

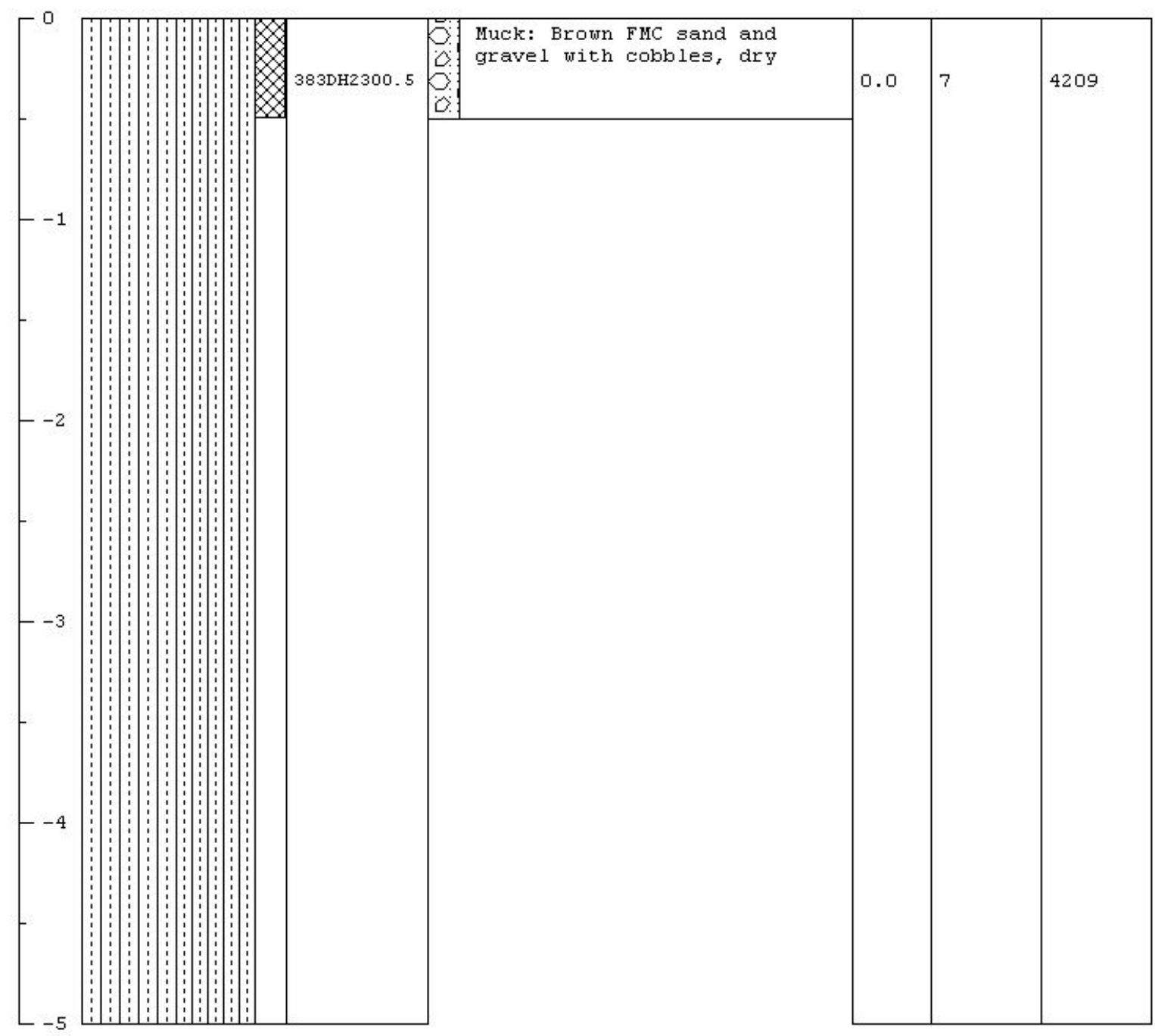


Page 1 of 1

\begin{tabular}{|c|c|c|c|c|c|c|c|c|c|}
\hline \multicolumn{6}{|c|}{$\begin{array}{l}\text { Project Hame: CAU } 383 \text { E-Tunnel Sites } \\
\text { Project Humber: DTO } 4-320 \\
\text { Borehole Humber: EDH } 4 \\
\text { Logged By: M. McLane } \\
\text { Drilled By: M. MeLane } \\
\text { Drilling Method: Hand Sampled }\end{array}$} & \multicolumn{4}{|c|}{$\begin{array}{l}\text { Date Started: } 6 / 15 / 04 \\
\text { Date Completed: } 6 / 15 / 04 \\
\text { Elevation: } 5972.1 \\
\text { Horthing: } 887721.4 \\
\text { Easting: } 638855.0 \\
\text { Total Depth: } 0.5 \mathrm{ft} .\end{array}$} \\
\hline \multirow[b]{2}{*}{ 点点 } & \multirow[b]{2}{*}{ 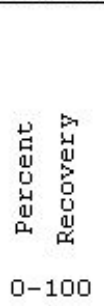 } & \multirow{2}{*}{\multicolumn{2}{|c|}{ 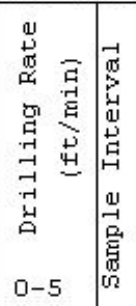 }} & \multirow[b]{2}{*}{ 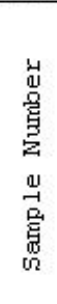 } & \multirow[b]{2}{*}{ 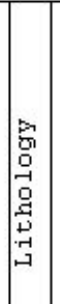 } & \multirow[b]{2}{*}{$\begin{array}{c}\text { Lithologic Description } \\
\text { Comments }\end{array}$} & \multirow{2}{*}{\begin{tabular}{|l} 
Field \\
vocs \\
(ppm)
\end{tabular}} & Screening & Results \\
\hline & & & & & & & & $\begin{array}{l}\text { Alpha } \\
\text { (dpm) }\end{array}$ & $\begin{array}{l}\text { Beta } \\
\text { (dpm) }\end{array}$ \\
\hline
\end{tabular}

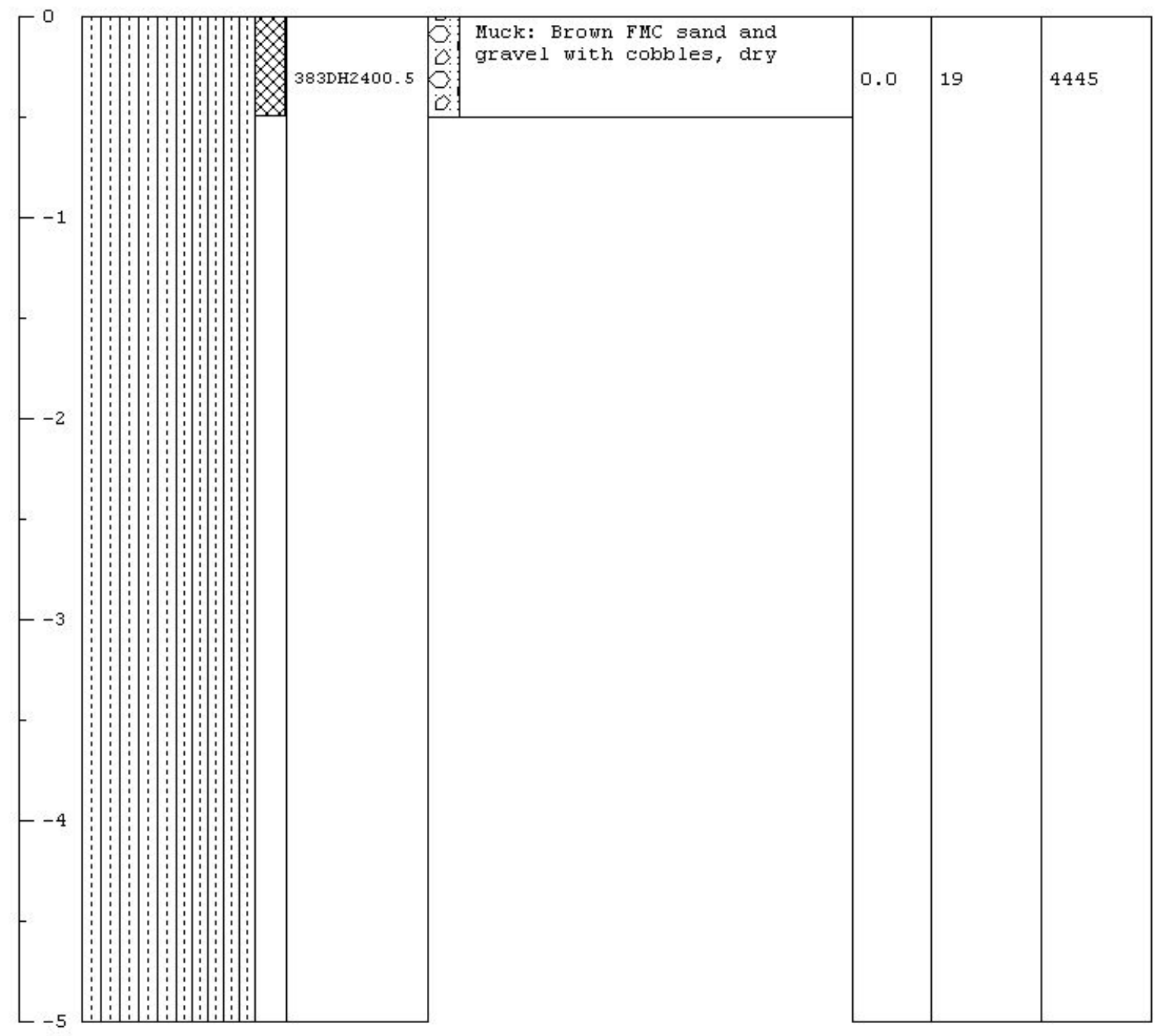


Page 1 of 1

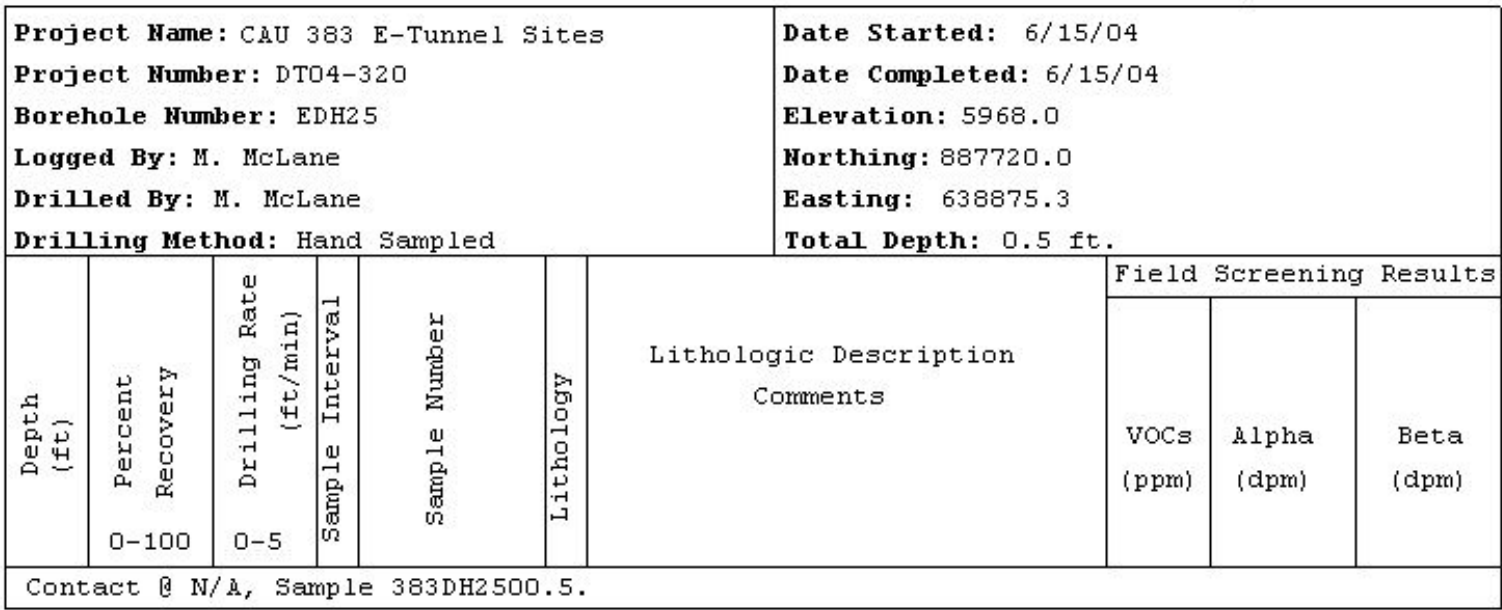

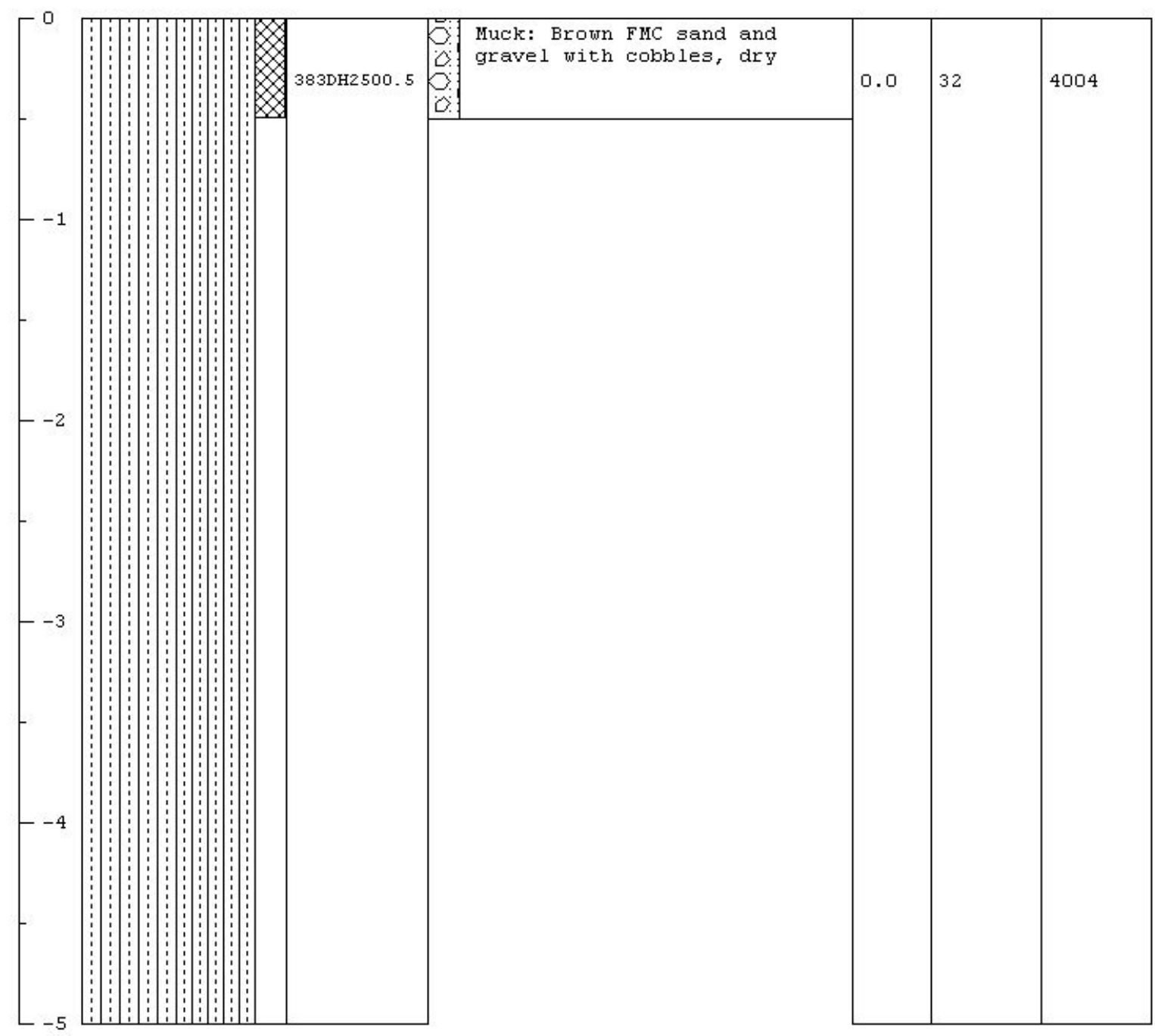


Page 1 of 1

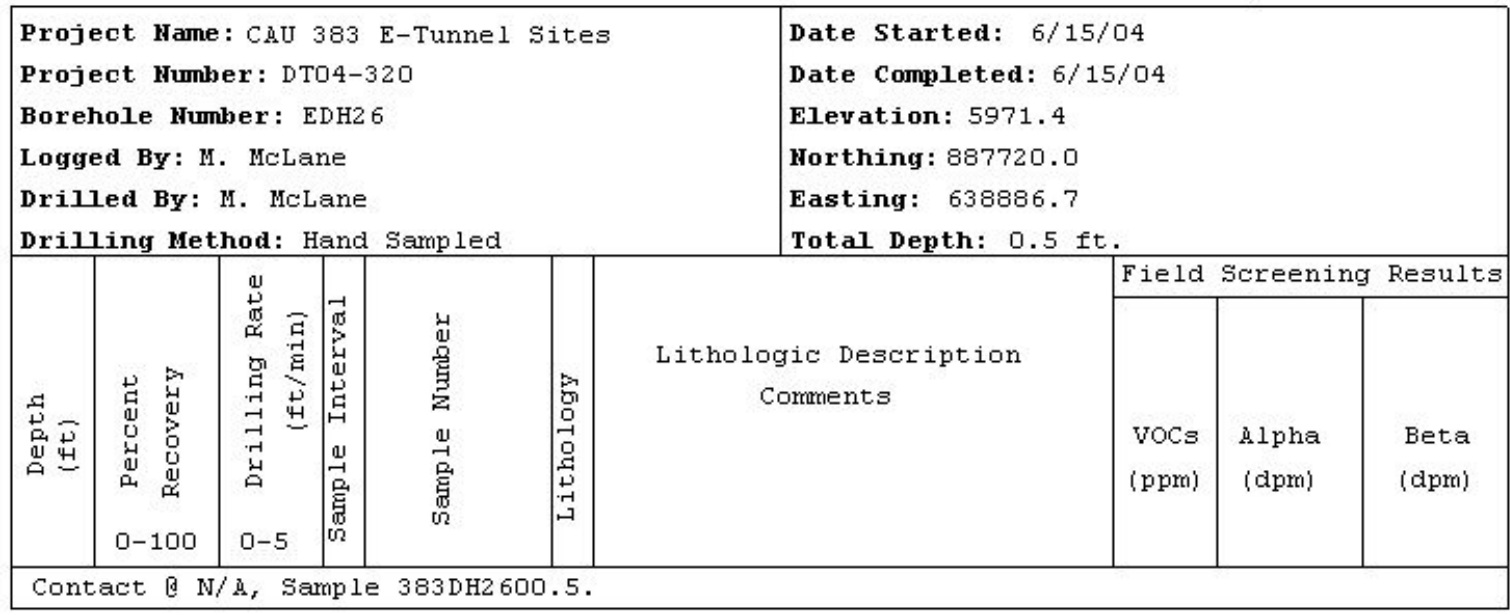

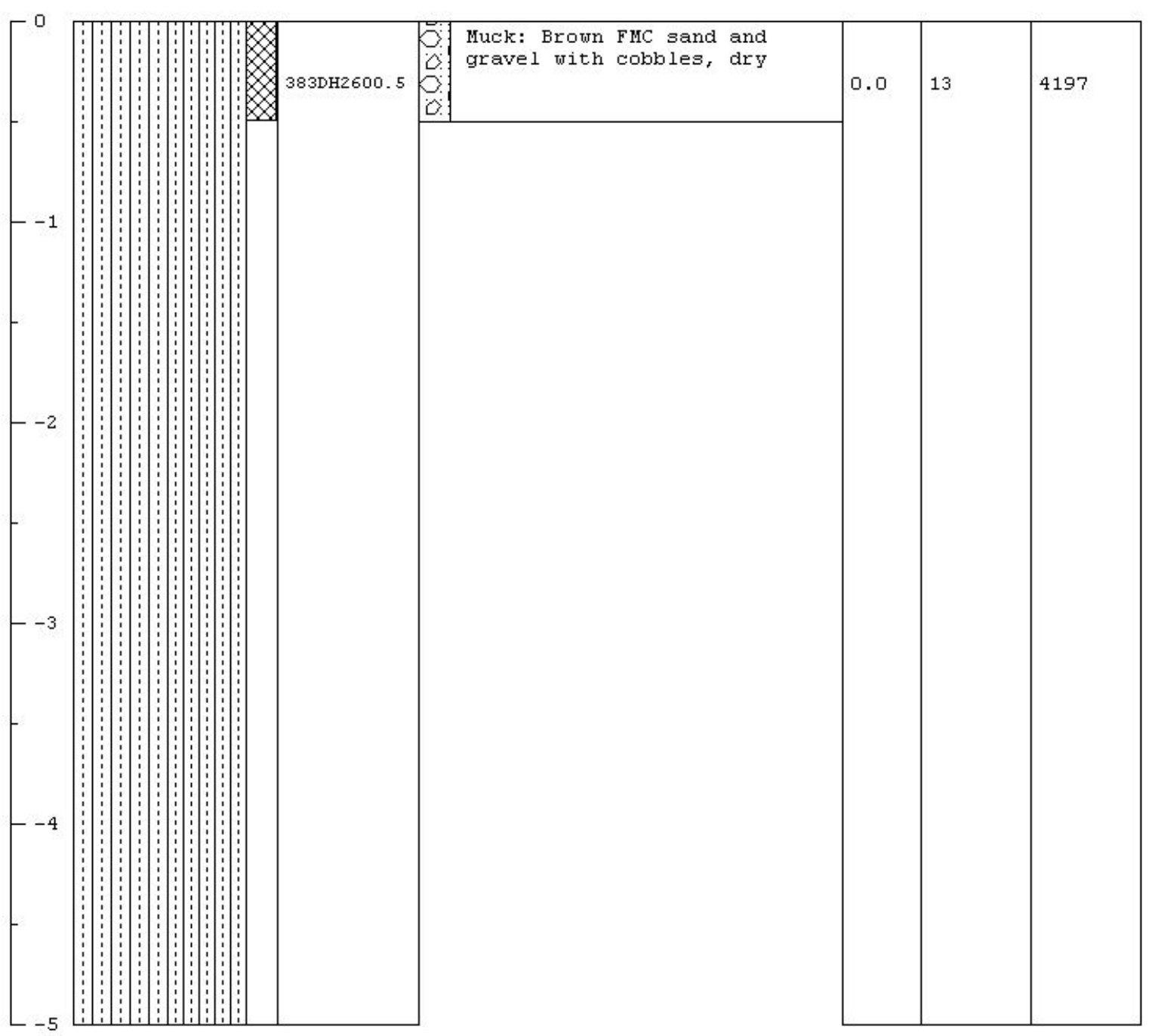


Page 1 of 1

\begin{tabular}{|c|c|c|c|c|c|c|c|c|}
\hline \multicolumn{5}{|c|}{$\begin{array}{l}\text { Project Hame: CAU } 383 \text { E-Tunnel Sites } \\
\text { Project Humber: DTO4-320 } \\
\text { Borehole Humber: EDH } 27 \\
\text { Logged By: M. McLane } \\
\text { Drilled By: M. McLane } \\
\text { Drilling Method: Hand Sampled }\end{array}$} & \multicolumn{4}{|c|}{$\begin{array}{l}\text { Date Started: } 6 / 16 / 04 \\
\text { Date Completed: } 6 / 16 / 04 \\
\text { Elevation: } 5970.9 \\
\text { Northing: } 887719.7 \\
\text { Easting: } 638896.0 \\
\text { Total Depth: } 0.5 \mathrm{ft} .\end{array}$} \\
\hline \multirow[b]{2}{*}{ 穿开 } & \multirow[b]{2}{*}{ 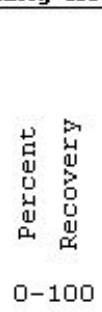 } & \multirow[b]{2}{*}{ 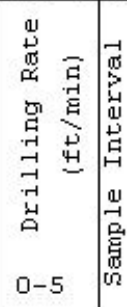 } & \multirow[b]{2}{*}{ 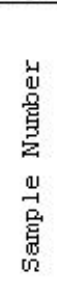 } & \multirow[b]{2}{*}{ 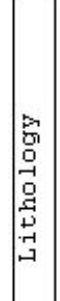 } & \multirow[b]{2}{*}{$\begin{array}{c}\text { Lithologic Description } \\
\text { Comments }\end{array}$} & Field & Screening & Results \\
\hline & & & & & & $\begin{array}{l}\text { VOCs } \\
\text { (ppm) }\end{array}$ & $\begin{array}{l}\text { Alpha } \\
\text { (dpm) }\end{array}$ & $\begin{array}{l}\text { Beta } \\
\text { (dpm) }\end{array}$ \\
\hline
\end{tabular}

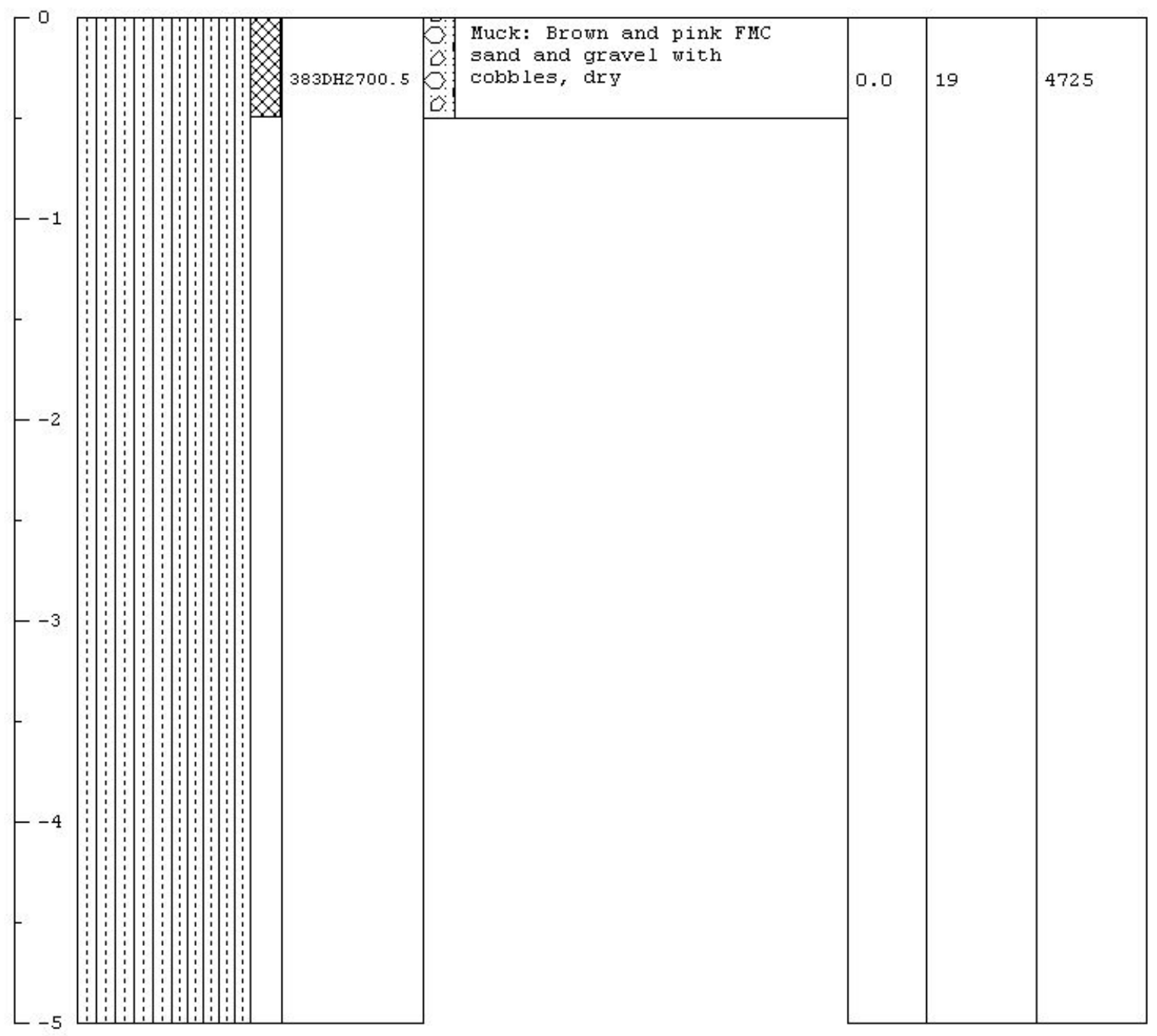


Page 1 of 1

\begin{tabular}{|c|c|c|c|c|c|c|c|c|c|}
\hline $\begin{array}{l}\text { Proj } \\
\text { Proj } \\
\text { Bore } \\
\text { Logg } \\
\text { Dril } \\
\text { Dril }\end{array}$ & $\begin{array}{l}\text { ct Nam } \\
\text { ct Hum } \\
\text { ole Hu } \\
\text { d By: } \\
\text { ed By: } \\
\text { ing Me }\end{array}$ & $\begin{array}{l}\text { CAU } \\
\text { r: DTO } \\
\text { er: E } \\
\text { MeLar } \\
\text { I. MeL } \\
\text { Lod: H }\end{array}$ & $\begin{array}{l}883 \\
\text { DH2 } \\
\text { ne } \\
\text { ane } \\
\text { and }\end{array}$ & Tun & ites & $\begin{array}{l}\text { Date Started: } \\
\text { Date Completed: } \\
\text { Elevation: } 5972 \\
\text { Horthing: } 887722 \\
\text { Easting: } 638906 \\
\text { Total Depth: } 0 .\end{array}$ & $\begin{array}{l}5 / 04 \\
16 / 04 \\
t .\end{array}$ & & - \\
\hline & & & & & & & Field & Screeni & Results \\
\hline 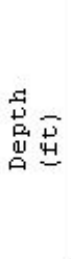 & 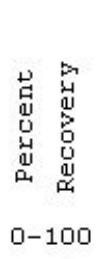 & 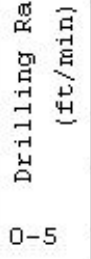 & 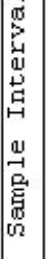 & 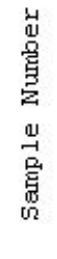 & 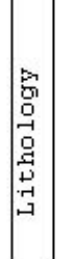 & $\begin{array}{c}\text { Lithologic Description } \\
\text { Comments }\end{array}$ & $\begin{array}{l}\text { VOCs } \\
\text { (ppm) }\end{array}$ & $\begin{array}{l}\text { Alpha } \\
\text { (dpm) }\end{array}$ & $\begin{array}{l}\text { Beta } \\
\text { (dpm) }\end{array}$ \\
\hline
\end{tabular}

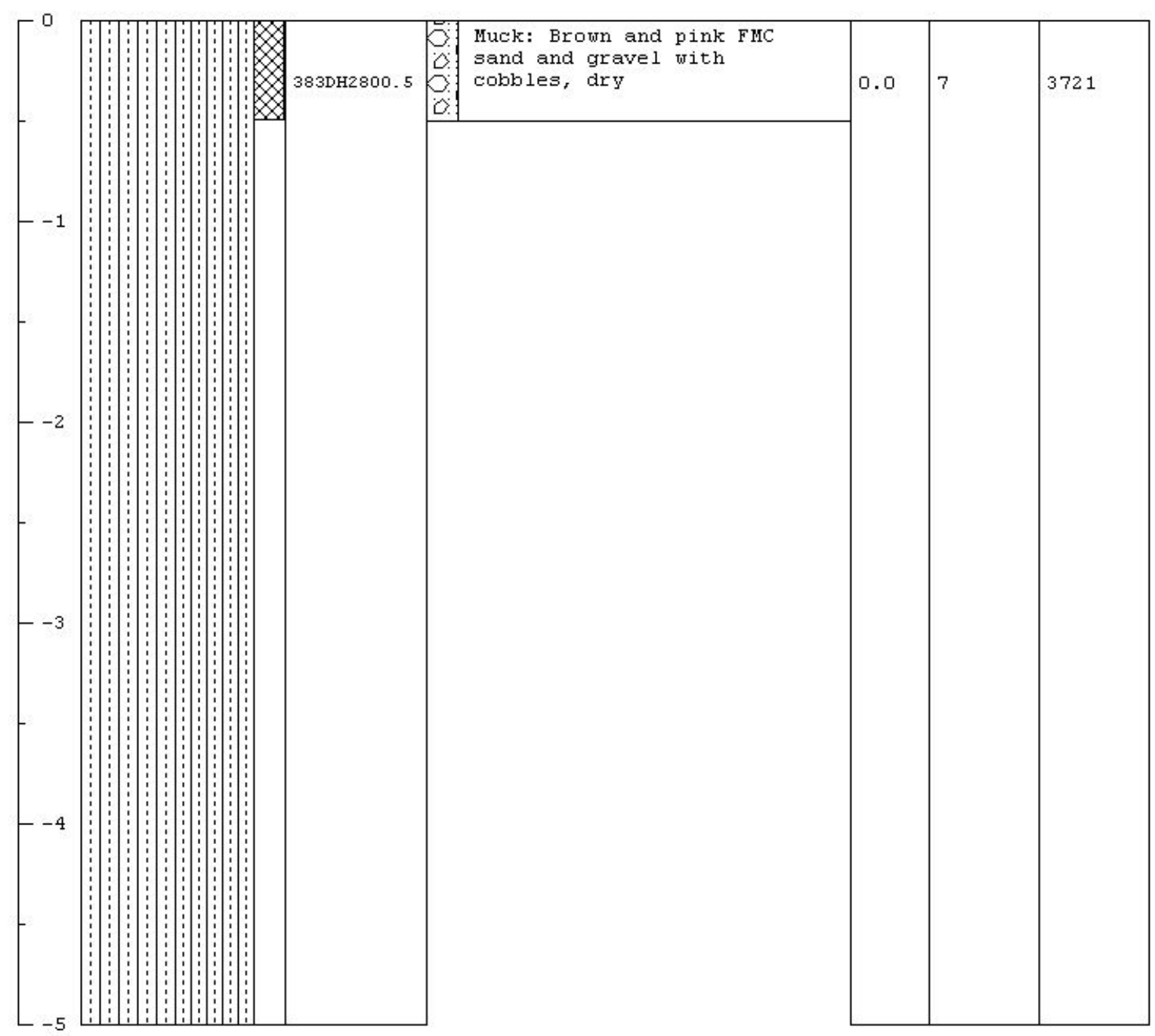


Page 1 of 1

\begin{tabular}{|c|c|c|c|c|c|c|c|c|c|}
\hline \multicolumn{6}{|c|}{$\begin{array}{l}\text { Project Hame: CAU } 383 \text { E-Tunnel Sites } \\
\text { Project Humber: DTO } 4-320 \\
\text { Borehole Humber: EDH } 29 \\
\text { Logged By: M. McLane } \\
\text { Drilled By: M. McLane } \\
\text { Drilling Method: Hand Sampled }\end{array}$} & \multicolumn{4}{|c|}{$\begin{array}{l}\text { Date Started: } 6 / 16 / 04 \\
\text { Date Completed: } 6 / 16 / 04 \\
\text { Elevation: } 5969.8 \\
\text { Horthing: } 887723.1 \\
\text { Easting: } 638916.6 \\
\text { Total Depth: } 0.5 \mathrm{ft} .\end{array}$} \\
\hline \multirow[b]{2}{*}{ 窝 } & \multirow[b]{2}{*}{ 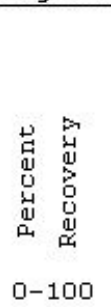 } & \multirow{2}{*}{\multicolumn{2}{|c|}{ 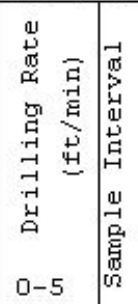 }} & \multirow[b]{2}{*}{ 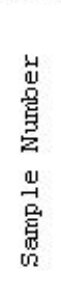 } & \multirow[b]{2}{*}{ 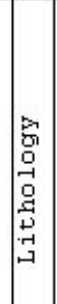 } & \multirow[b]{2}{*}{$\begin{array}{c}\text { Lithologic Description } \\
\text { Comments }\end{array}$} & Field & Screening & Results \\
\hline & & & & & & & $\begin{array}{l}\text { VOCs } \\
\text { (ppm) }\end{array}$ & $\begin{array}{l}\text { Alpha } \\
\text { (dpm) }\end{array}$ & $\begin{array}{l}\text { Beta } \\
\text { (dpm) }\end{array}$ \\
\hline
\end{tabular}

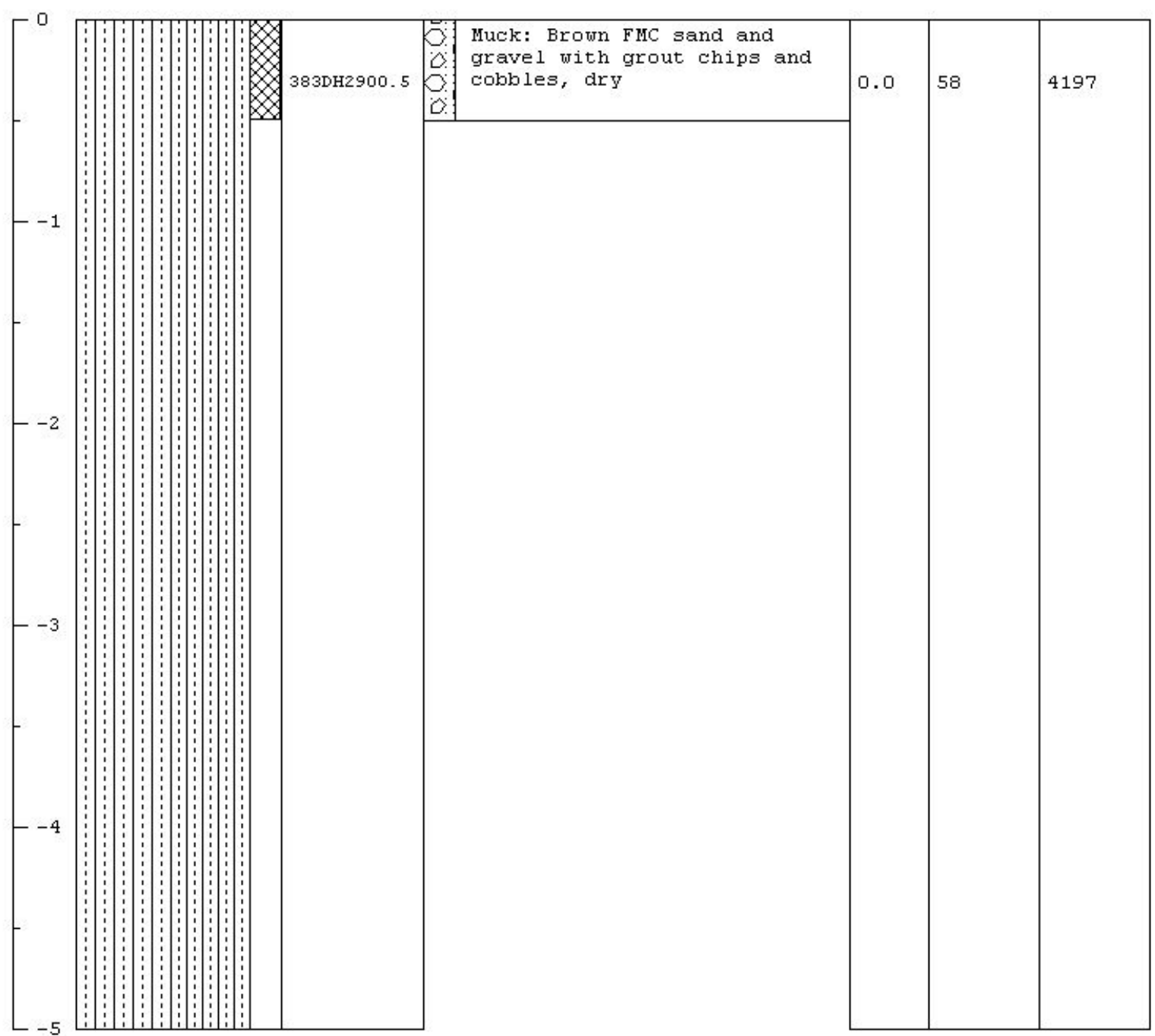


Page 1 of 1

Project Hame: CAU 383 E-Tunnel sites

Project Humber: DTO4-320

Borehole Number: EDH30

Logged By: M. MeLane

Drilled By: M. McLane

Drilling Method: Hand Sampled
Date Started: $6 / 16 / 04$

Date Completed: $6 / 16 / 04$

Elevation: 5971.0

Horthing: 887722.5

Easting: 638926.3

Total Depth: $0.5 \mathrm{ft}$.
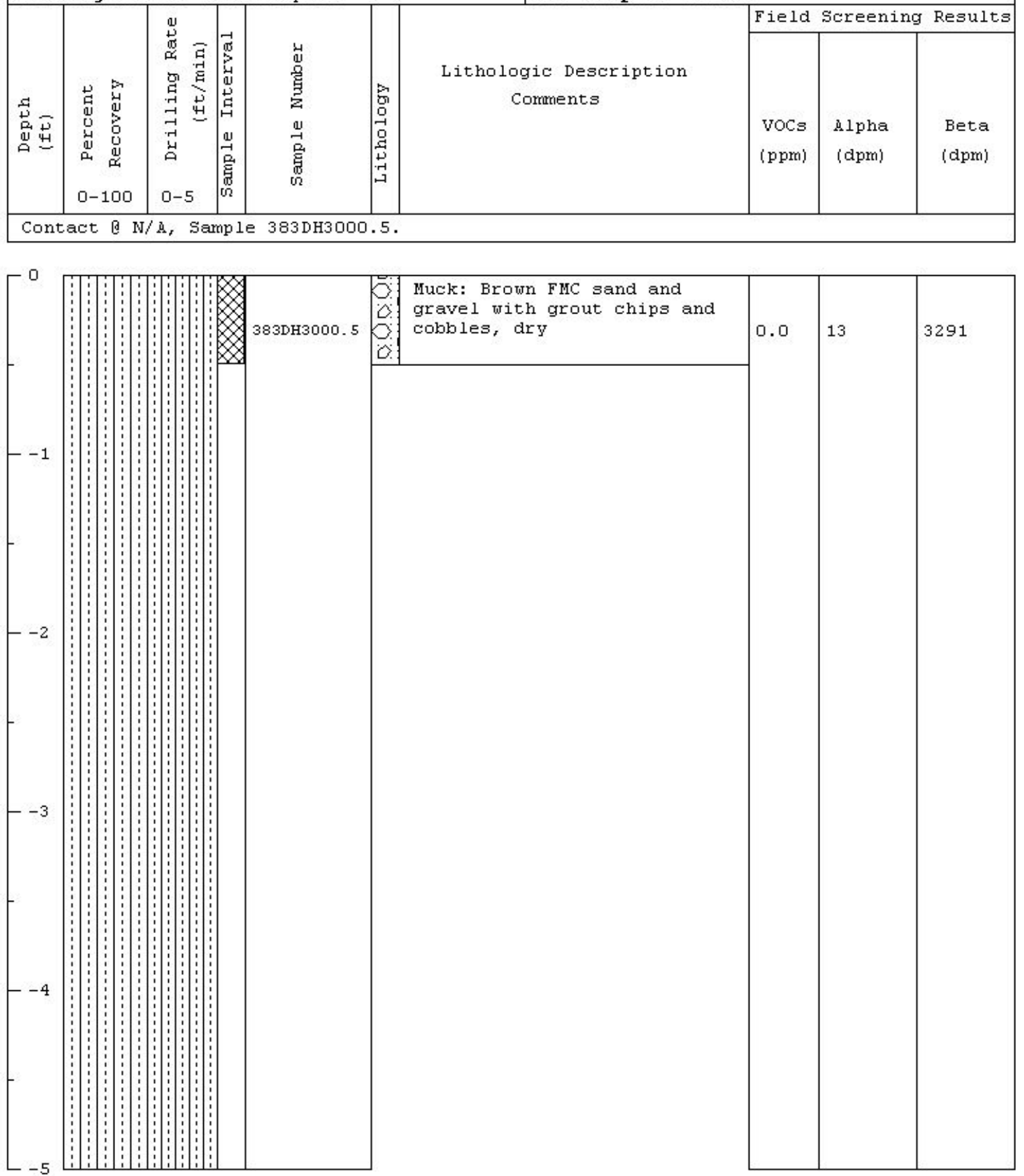
Page 1 of 1

\begin{tabular}{|c|c|c|c|c|c|c|c|c|c|}
\hline \multicolumn{6}{|c|}{$\begin{array}{l}\text { Project Hame: CAU } 383 \text { E-Tunnel Sites } \\
\text { Project Humber: DTO4-320 } \\
\text { Borehole Humber: EDH } 1 \\
\text { Logged By: M. MeLane } \\
\text { Drilled By: M. MeLane } \\
\text { Drilling Method: Hand Sampled }\end{array}$} & \multicolumn{4}{|c|}{$\begin{array}{l}\text { Date Started: } 6 / 16 / 04 \\
\text { Date Completed: } 6 / 16 / 04 \\
\text { Elevation: } 5969.6 \\
\text { Horthing: } 887723.8 \\
\text { Easting: } 638935.6 \\
\text { Total Depth: } 0.5 \mathrm{ft} \text {. }\end{array}$} \\
\hline \multirow[b]{2}{*}{ 点 } & \multirow[b]{2}{*}{ 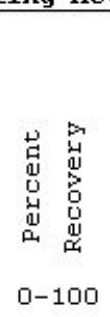 } & \multirow{2}{*}{\multicolumn{2}{|c|}{ 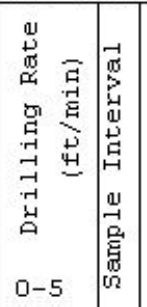 }} & \multirow[b]{2}{*}{ 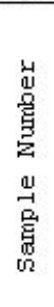 } & \multirow[b]{2}{*}{ 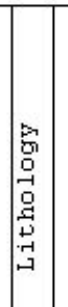 } & \multirow[b]{2}{*}{$\begin{array}{c}\text { Lithologic Description } \\
\text { Comments }\end{array}$} & Field & Screening & Results \\
\hline & & & & & & & $\begin{array}{l}\text { VOCs } \\
\text { (ppm) }\end{array}$ & $\begin{array}{l}\text { Alpha } \\
\text { (dpm) }\end{array}$ & $\begin{array}{l}\text { Beta } \\
\text { (dpm) }\end{array}$ \\
\hline
\end{tabular}

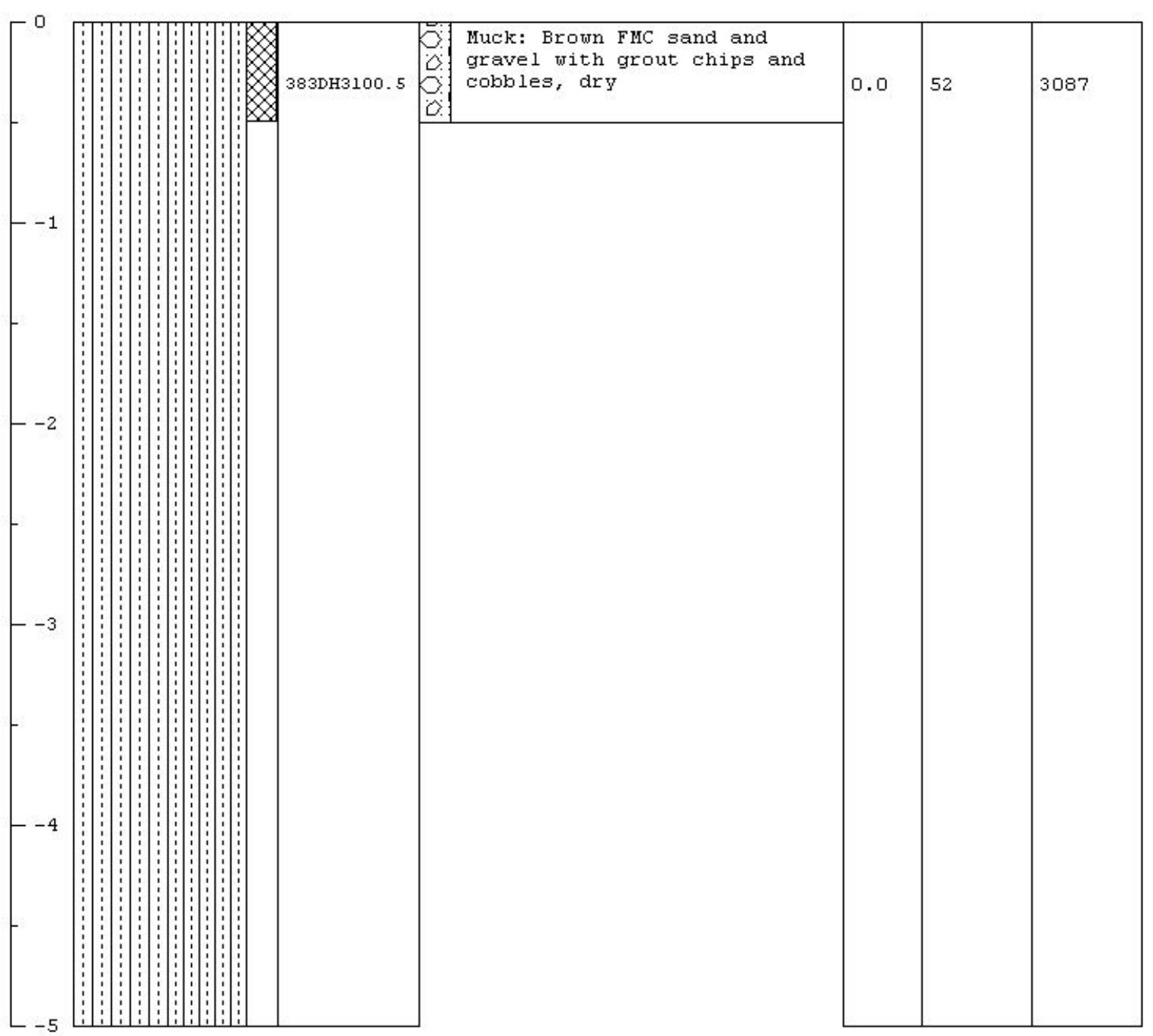


Page 1 of 1

Project Hame: CAU 383 E-Tunnel Sites

Project Number: DTO4-320

Borehole Humber: EDH32

Logged By: M. McLane

Drilled By: M. MeLane

Drilling Method: Hand Sampled
Date Started: $6 / 16 / 04$

Date Completed: $6 / 16 / 04$

Elevation: 5968.2

Horthing: 887723.2

Easting : 638946.3

Total Depth: $0.5 \mathrm{ft}$.
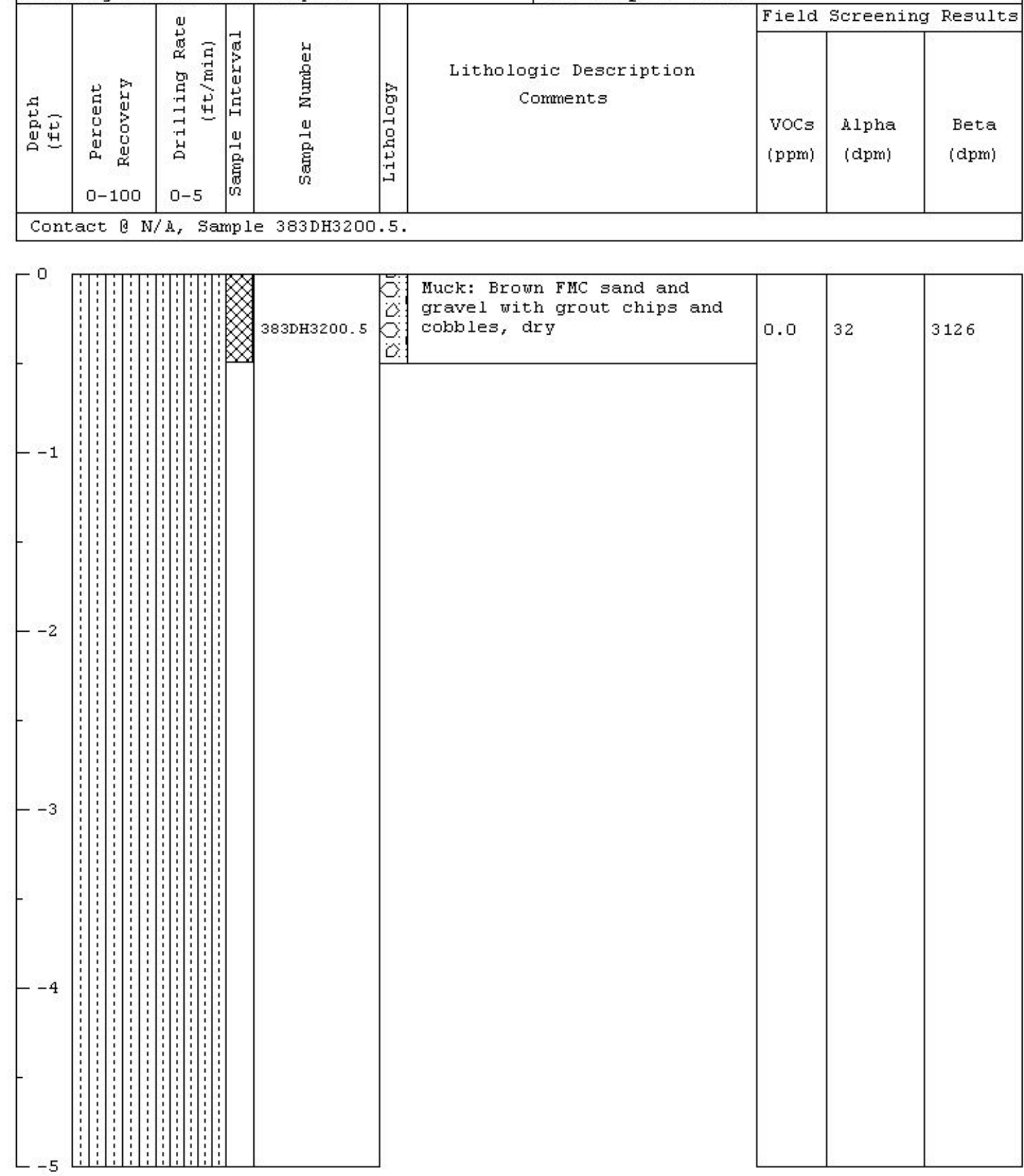
Page 1 of 1

\begin{tabular}{|c|c|c|c|c|c|c|c|c|}
\hline \multicolumn{5}{|c|}{$\begin{array}{l}\text { Project Hame: CAU } 383 \text { E-Tunnel Sites } \\
\text { Project Humber: DTO } 4-320 \\
\text { Borehole Humber: EDH } 33 \\
\text { Logged By: M. McLane } \\
\text { Drilled By: M. McLane } \\
\text { Drilling Method: Hand Sampled }\end{array}$} & \multicolumn{4}{|c|}{$\begin{array}{l}\text { Date Started: } 6 / 16 / 04 \\
\text { Date Completed: } 6 / 16 / 04 \\
\text { Elevation: } 5963.7 \\
\text { Horthing: } 887721.4 \\
\text { Easting: } 638955.9 \\
\text { Total Depth: } 0.5 \mathrm{ft} .\end{array}$} \\
\hline \multirow[b]{2}{*}{ 豆 } & \multirow[b]{2}{*}{ 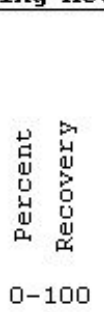 } & \multirow[b]{2}{*}{ 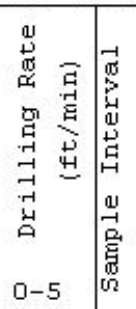 } & \multirow[b]{2}{*}{ 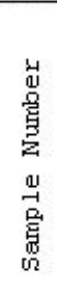 } & \multirow[b]{2}{*}{ 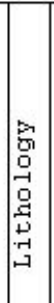 } & \multirow[b]{2}{*}{$\begin{array}{c}\text { Lithologic Description } \\
\text { Comments }\end{array}$} & Field & Screening & Results \\
\hline & & & & & & $\begin{array}{l}\text { VoCs } \\
\text { (ppm) }\end{array}$ & $\begin{array}{l}\text { Alpha } \\
\text { (dpm) }\end{array}$ & $\begin{array}{l}\text { Beta } \\
\text { (dpm) }\end{array}$ \\
\hline
\end{tabular}

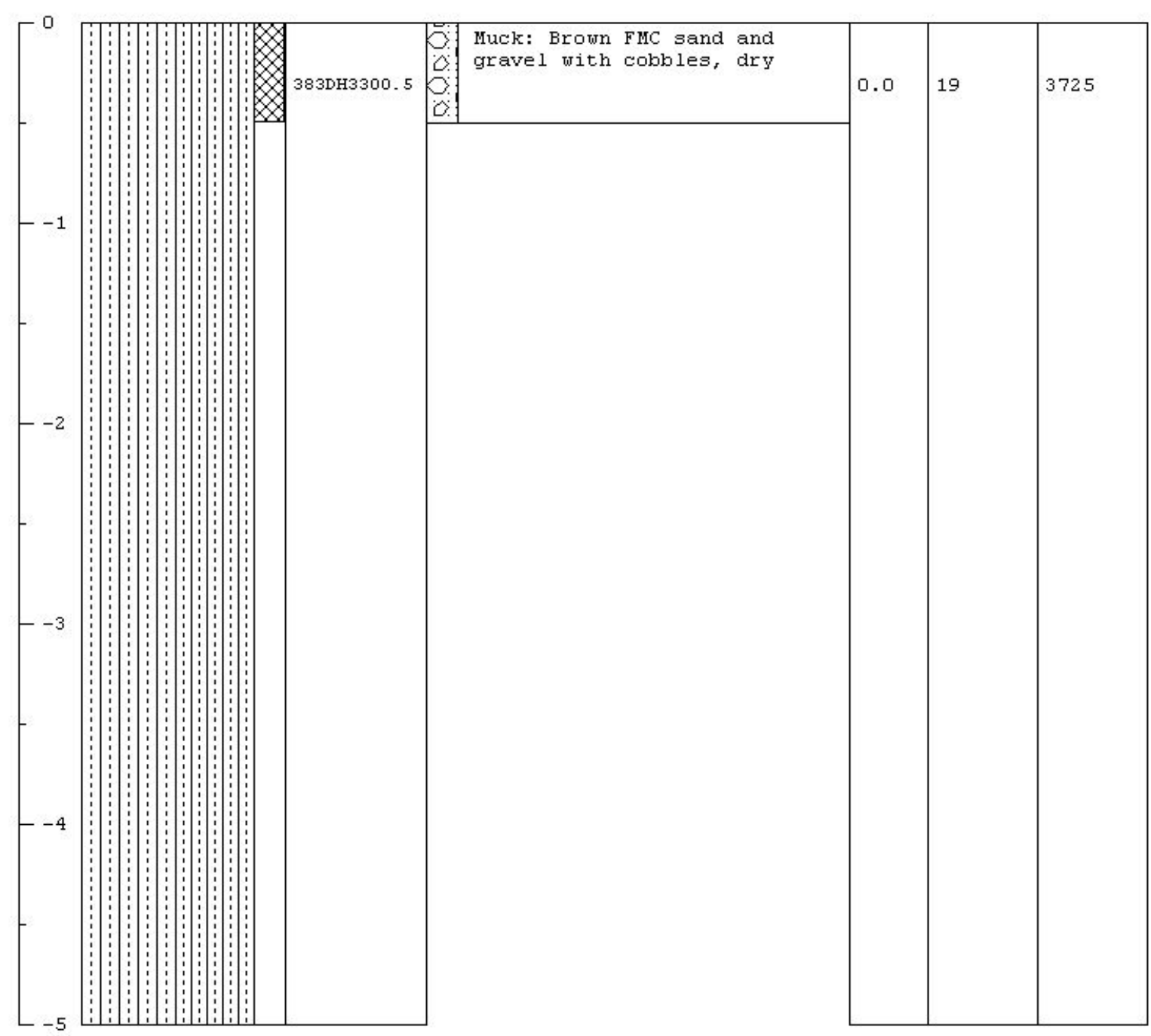




\begin{tabular}{|c|c|c|c|c|c|c|c|c|}
\hline \multicolumn{5}{|c|}{$\begin{array}{l}\text { Project Hame: CAU } 383 \text { E-Tunnel sites } \\
\text { Project Humber: DTO } 4-320 \\
\text { Borehole Humber: EDH } 34 \\
\text { Logged By: M. MeLane } \\
\text { Drilled By: Boart-Longyear } \\
\text { Drilling Method: Rotosonic }\end{array}$} & \multicolumn{4}{|c|}{$\begin{array}{l}\text { Date Started: } 6 / 10 / 04 \\
\text { Date Completed: } 6 / 10 / 04 \\
\text { Elevation: } 5964.4 \\
\text { Horthing: } 887717.9 \\
\text { Easting: } 638966.2 \\
\text { Total Depth: } 5.0 \mathrm{ft} .\end{array}$} \\
\hline \multirow[b]{2}{*}{ 点 } & \multirow[b]{2}{*}{ 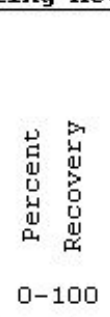 } & \multirow[b]{2}{*}{ 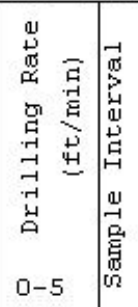 } & \multirow[b]{2}{*}{ 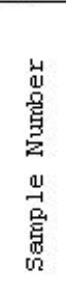 } & \multirow[b]{2}{*}{ 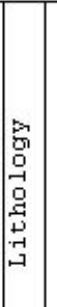 } & \multirow[b]{2}{*}{$\begin{array}{c}\text { Lithologic Description } \\
\text { Corments }\end{array}$} & Field & Screening & Results \\
\hline & & & & & & $\begin{array}{l}\text { VOCs } \\
\text { (ppm) }\end{array}$ & $\begin{array}{l}\text { Alpha } \\
\text { (dpm) }\end{array}$ & $\begin{array}{l}\text { Beta } \\
\text { (dpm) }\end{array}$ \\
\hline
\end{tabular}

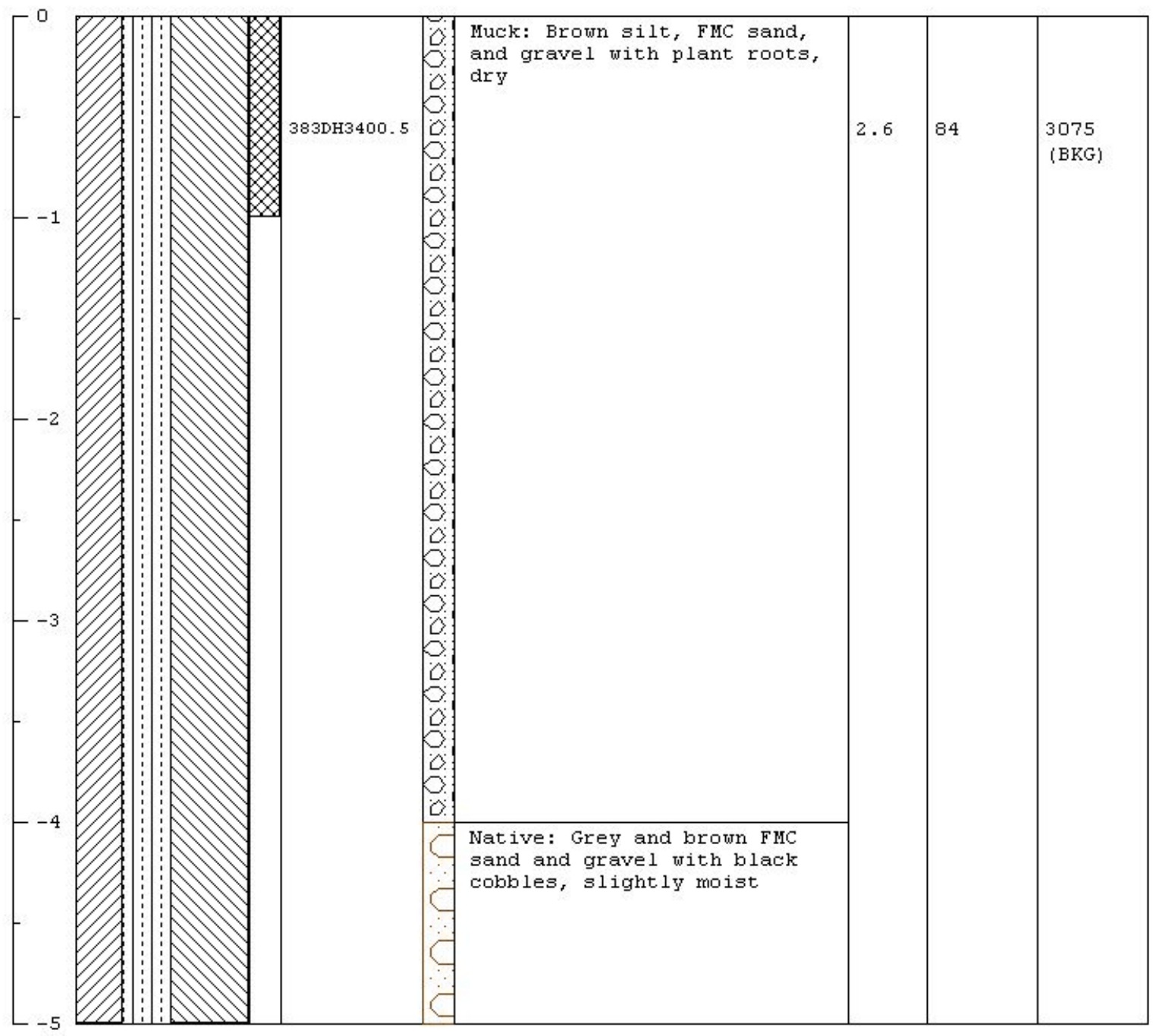


Page 1 of 1

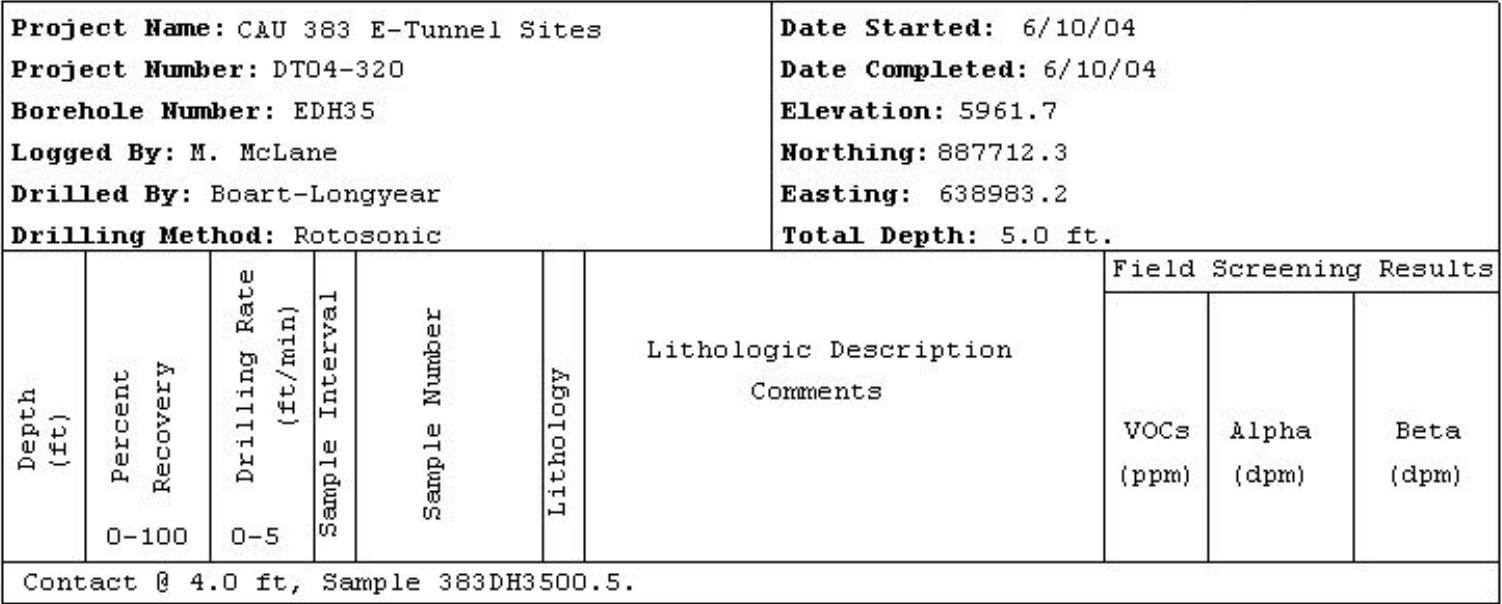

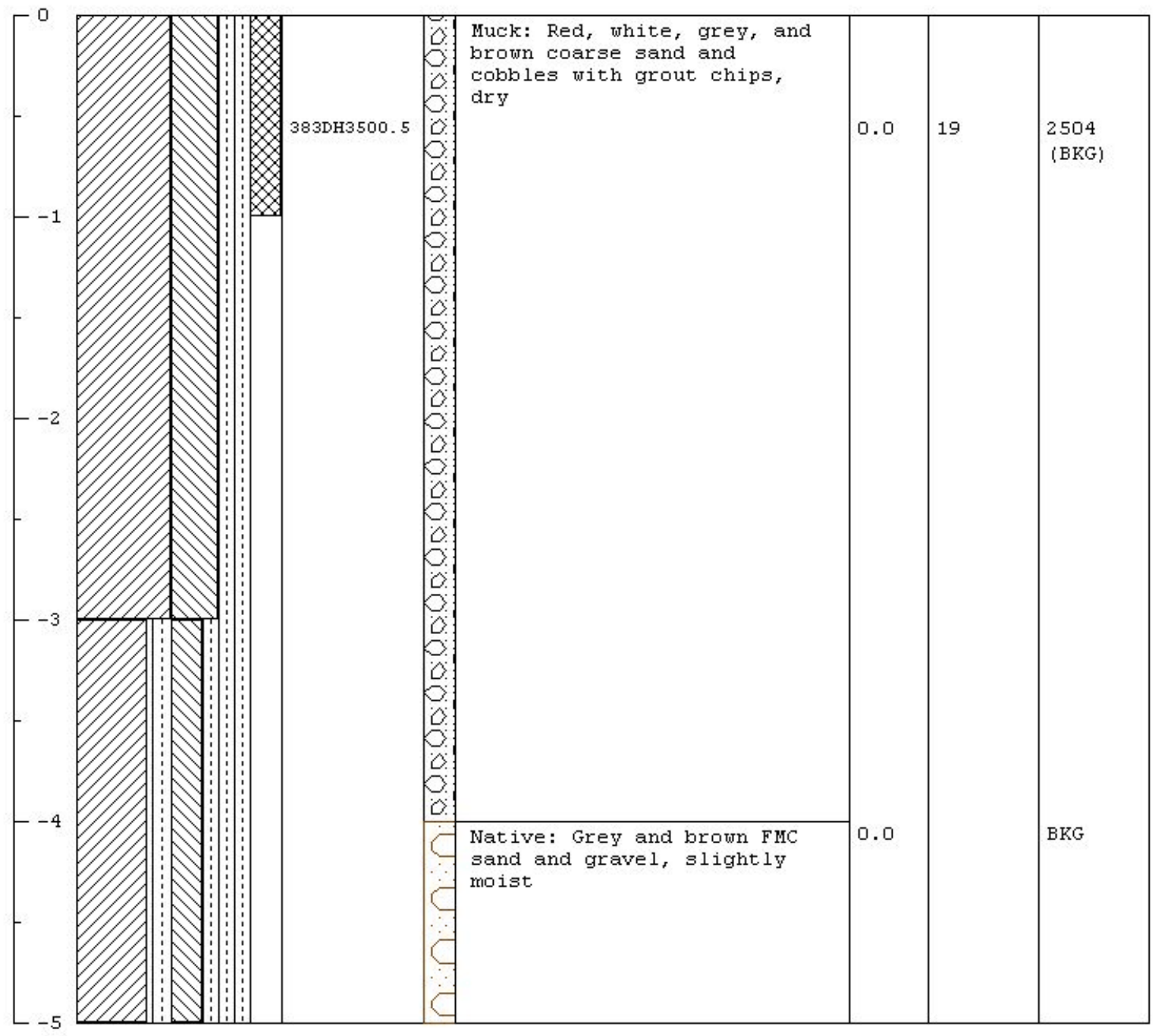


Page 1 of 1

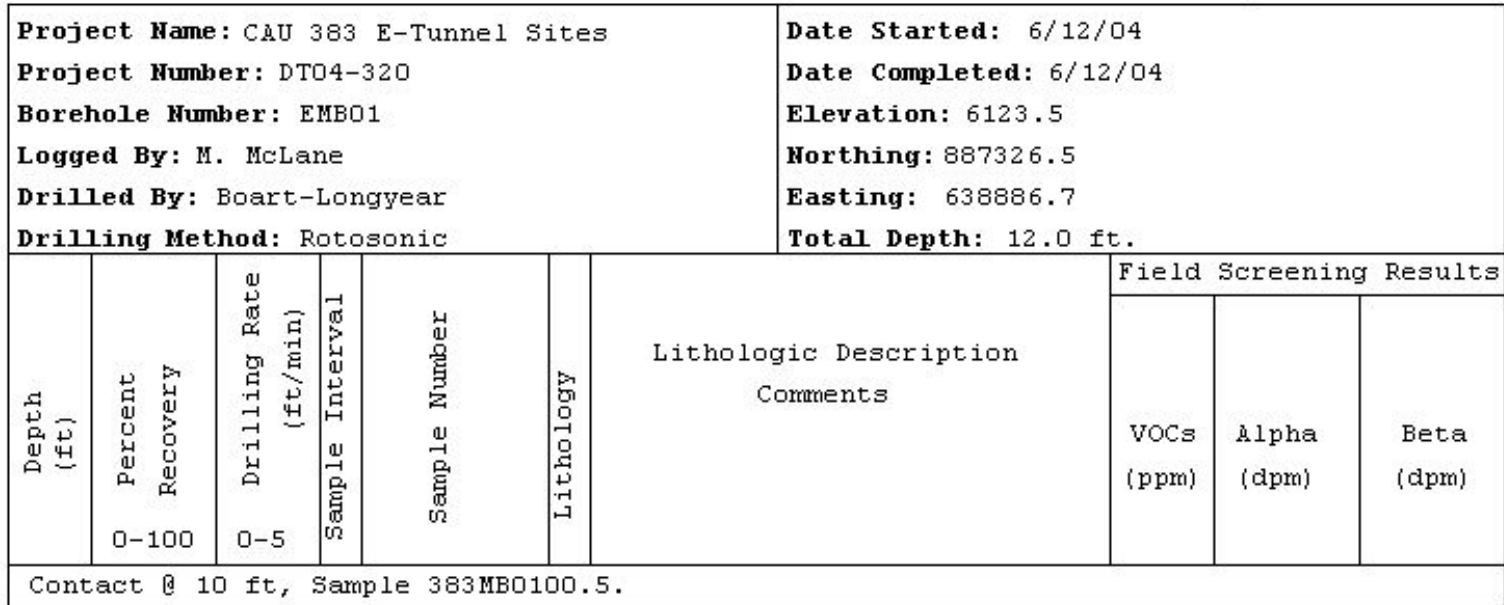

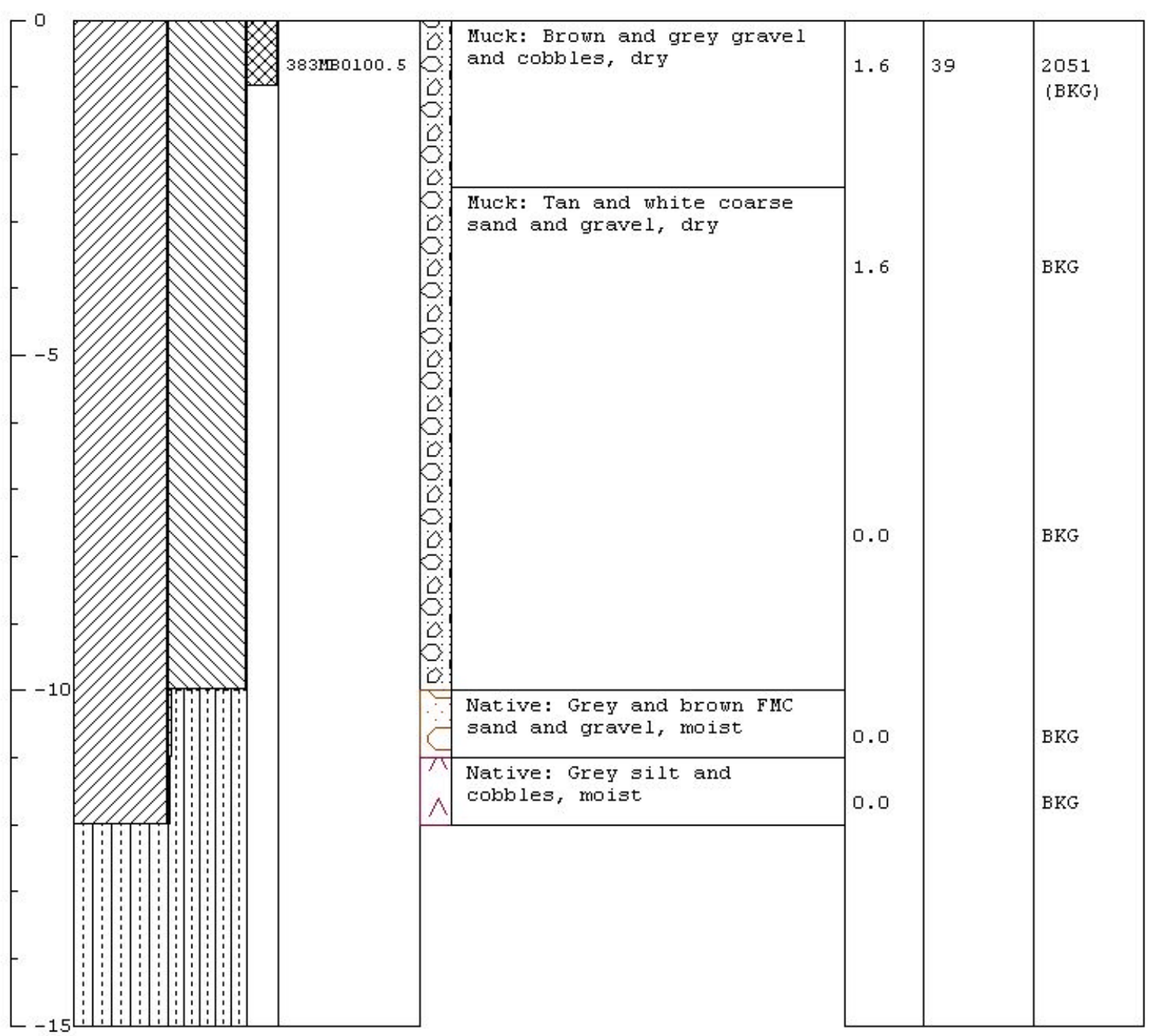


Page 1 of 1

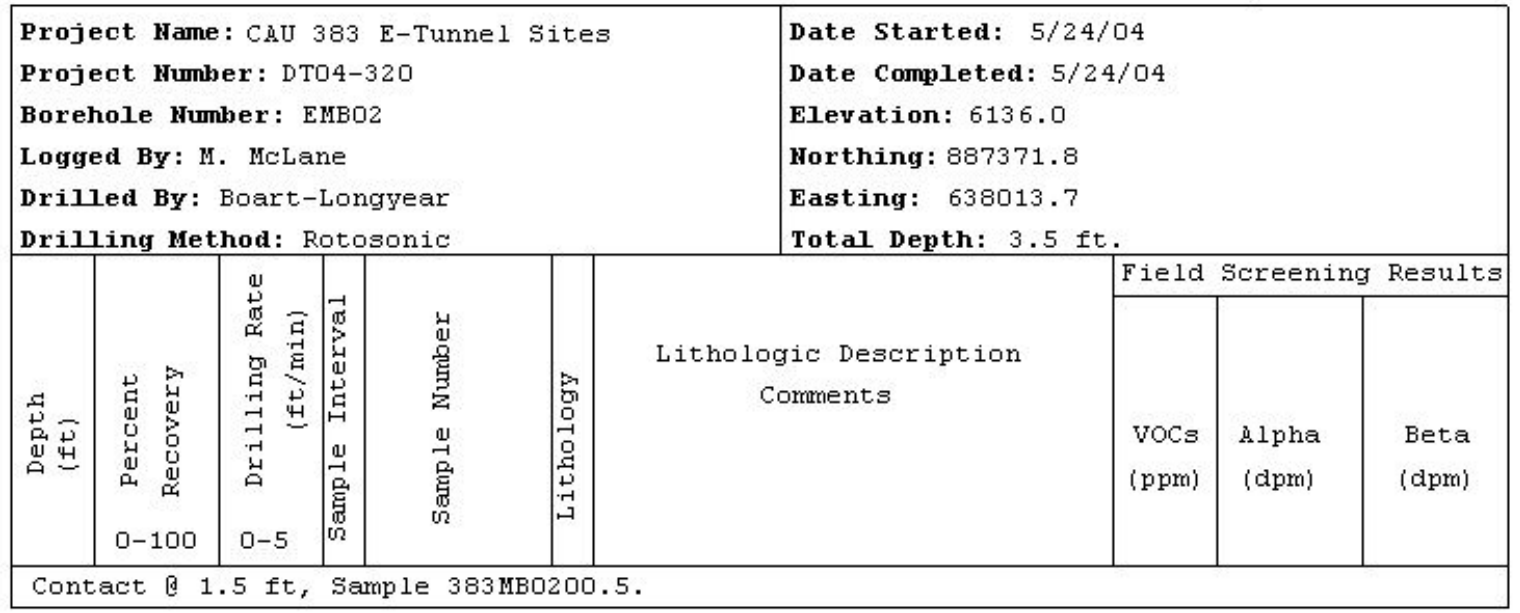

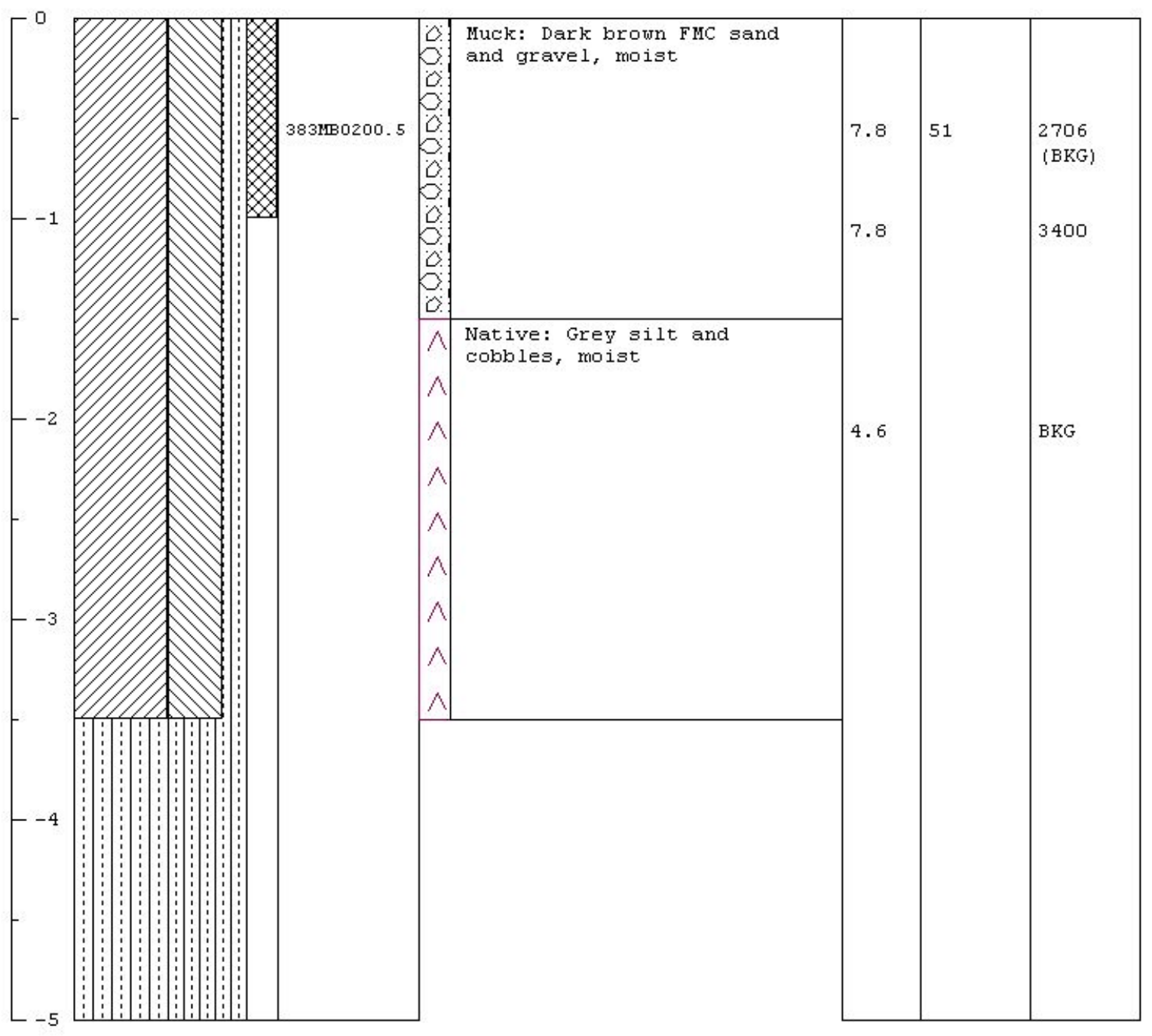




\begin{tabular}{|c|c|c|c|c|c|c|c|c|}
\hline \multicolumn{5}{|c|}{$\begin{array}{l}\text { Project Hame: CAU } 383 \text { E-Tunnel Sites } \\
\text { Project Humber: DT04-320 } \\
\text { Borehole Humber: EMBO3 } \\
\text { Logged By: M. McLane } \\
\text { Drilled By: Boart-Longyear } \\
\text { Drilling Method: Rotosonic }\end{array}$} & \multicolumn{4}{|c|}{$\begin{array}{l}\text { Date Started: } 6 / 11 / 04 \\
\text { Date Completed: } 6 / 11 / 04 \\
\text { Elevation: } 6121.5 \\
\text { Horthing: } 887878.1 \\
\text { Easting: } 637712.6 \\
\text { Total Depth: } 9.5 \mathrm{ft} .\end{array}$} \\
\hline \multirow[b]{2}{*}{ 总出 } & \multirow[b]{2}{*}{ 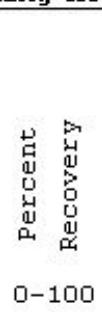 } & \multirow[b]{2}{*}{ 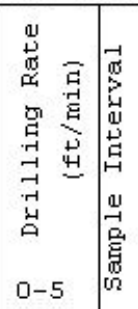 } & \multirow[b]{2}{*}{ 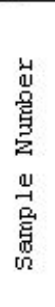 } & \multirow[b]{2}{*}{ 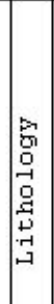 } & \multirow[b]{2}{*}{$\begin{array}{c}\text { Lithologic Description } \\
\text { Comments }\end{array}$} & \multirow[b]{2}{*}{\begin{tabular}{|l} 
Field \\
\\
voCs \\
(ppm)
\end{tabular}} & Screening & Results \\
\hline & & & & & & & $\begin{array}{l}\text { Alpha } \\
\text { (dpm) }\end{array}$ & $\begin{array}{r}\text { Beta } \\
\text { (dpm) }\end{array}$ \\
\hline
\end{tabular}

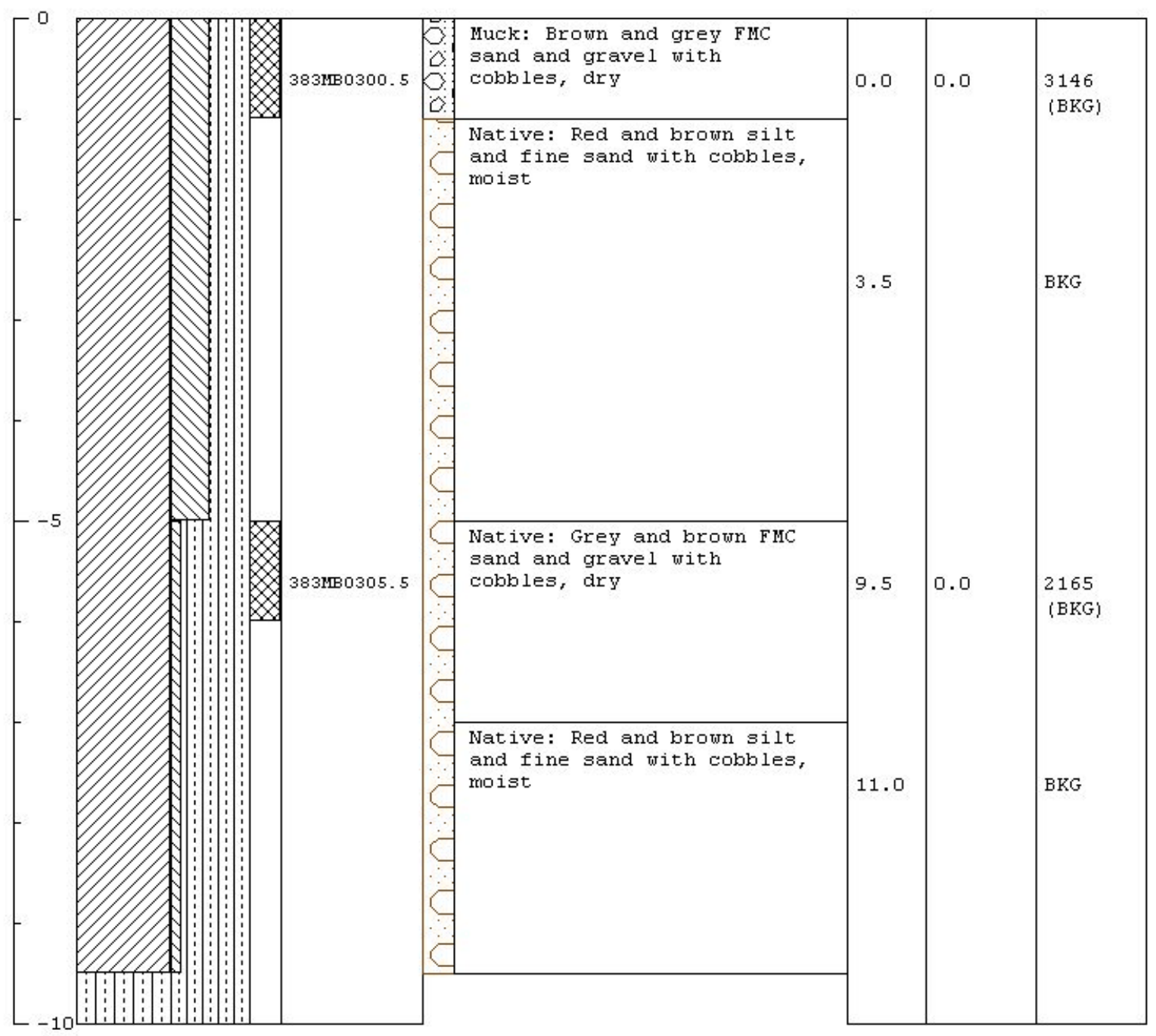


Page 1 of 1

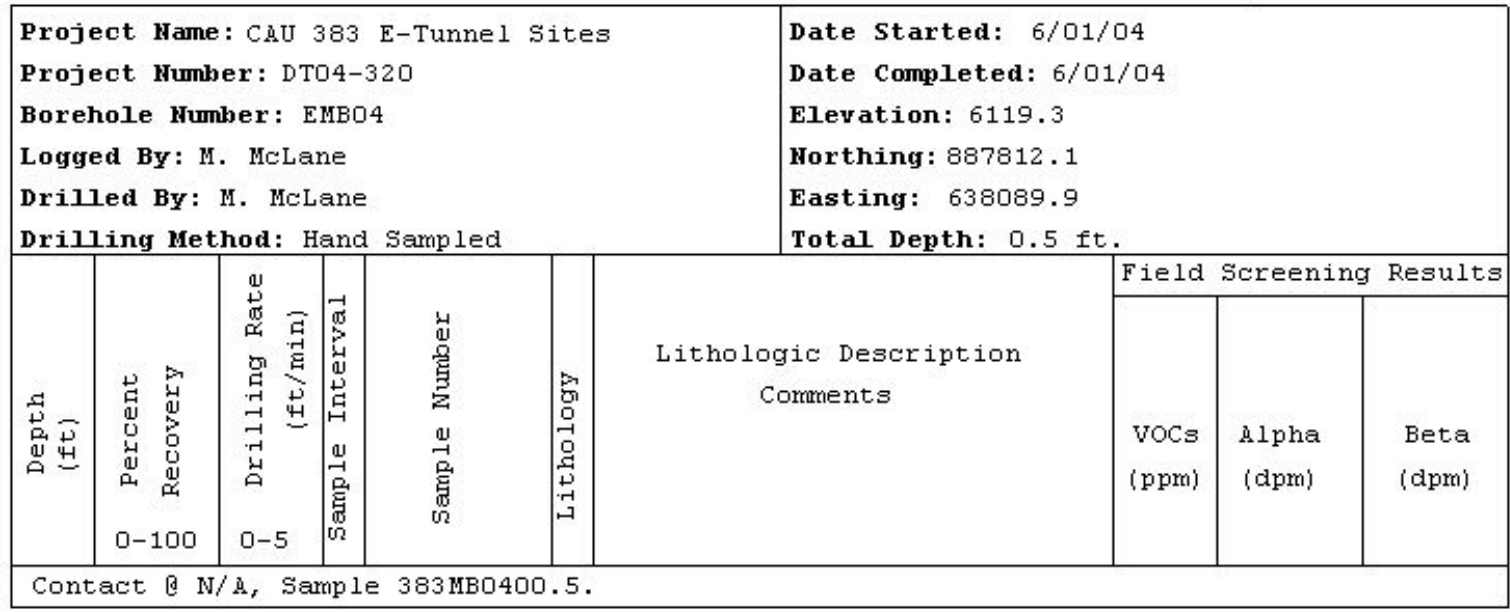

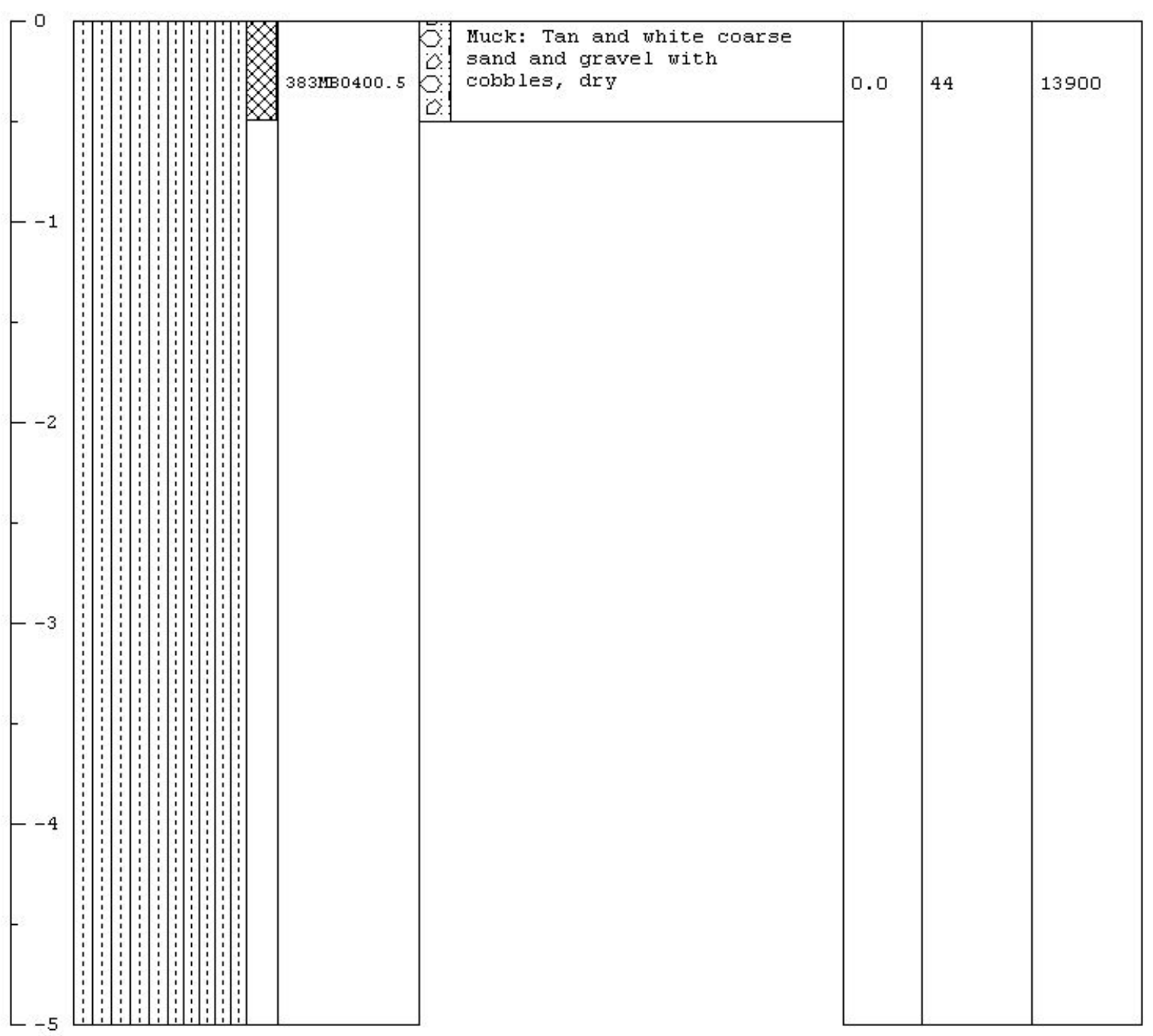


Page 1 of 2

\begin{tabular}{|c|c|c|c|c|c|c|c|c|}
\hline \multicolumn{5}{|c|}{$\begin{array}{l}\text { Project Hame: CAU } 383 \text { E-Tunnel Sites } \\
\text { Project Humber: DTO } 4-320 \\
\text { Borehole Humber: EMB } 05 \\
\text { Logged By: M. McLane } \\
\text { Drilled By: Boart-Longyear } \\
\text { Drilling Method: Rotosonic } \\
\end{array}$} & \multicolumn{4}{|c|}{$\begin{array}{l}\text { Date Started: } 6 / 02 / 04 \\
\text { Date Completed: } 6 / 03 / 04 \\
\text { Elevation: } 6117.8 \\
\text { Horthing: } 887505.2 \\
\text { Easting: } 638378.0 \\
\text { Total Depth: } 86.5 \mathrm{ft} .\end{array}$} \\
\hline \multirow[b]{2}{*}{ 总 } & \multirow[b]{2}{*}{ 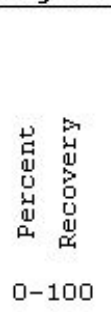 } & \multirow[b]{2}{*}{ 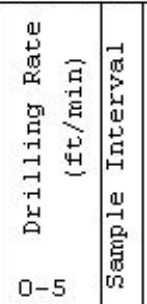 } & \multirow[b]{2}{*}{ 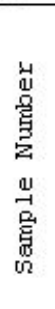 } & \multirow[b]{2}{*}{ 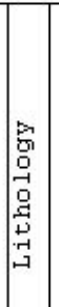 } & \multirow[b]{2}{*}{$\begin{array}{c}\text { Lithologic Description } \\
\text { Comments }\end{array}$} & Field & Screening & Results \\
\hline & & & & & & $\begin{array}{l}\text { voCs } \\
\text { (ppm) }\end{array}$ & $\begin{array}{l}\text { Alpha } \\
\text { (dpm) }\end{array}$ & $\begin{array}{l}\text { Beta } \\
\text { (dpm) }\end{array}$ \\
\hline
\end{tabular}

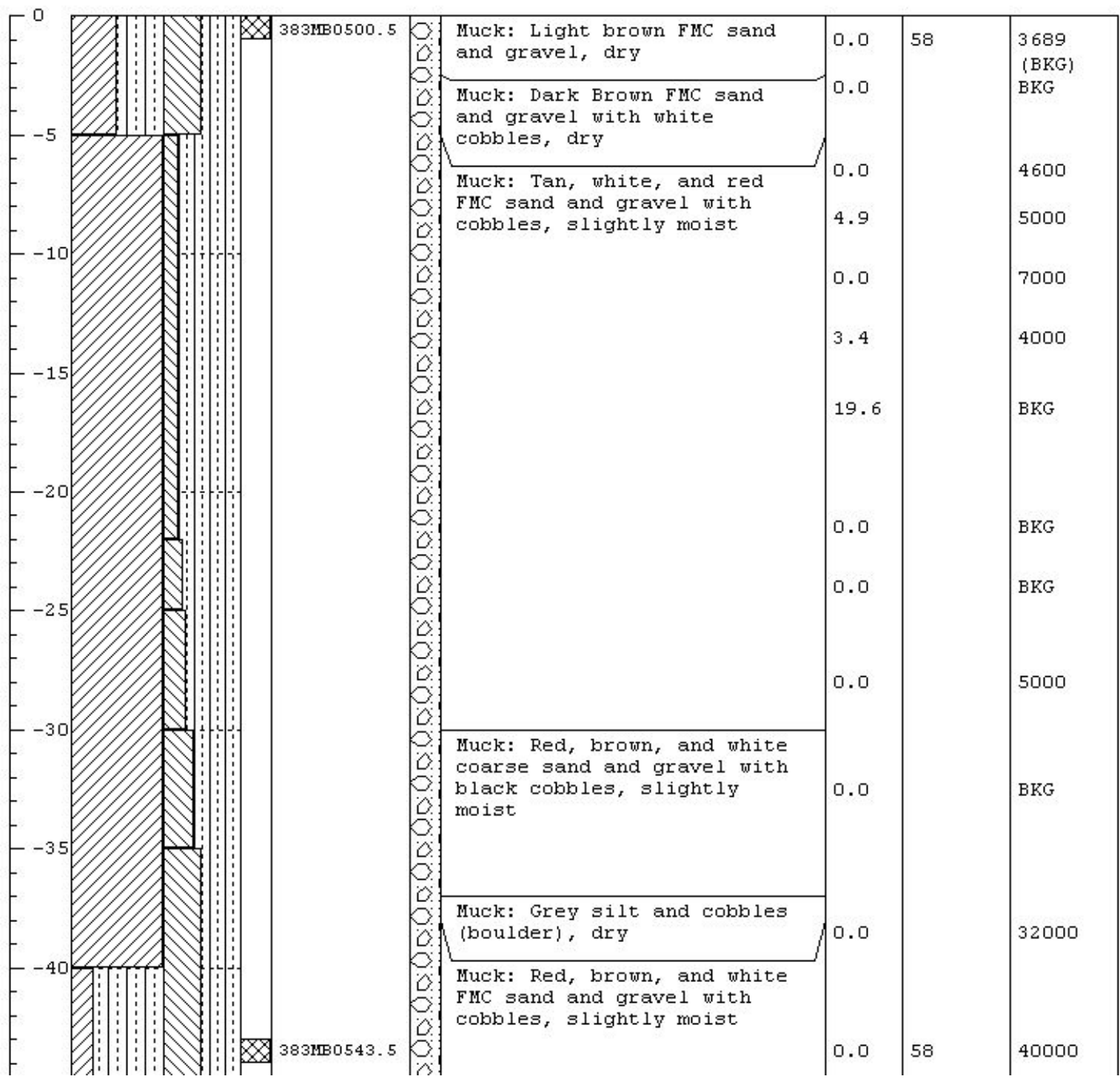




\begin{tabular}{|c|c|c|c|c|c|c|c|c|c|}
\hline \multicolumn{3}{|c|}{ Borehole Number: } & \multicolumn{7}{|c|}{ EMBO5 } \\
\hline \multirow[b]{2}{*}{ 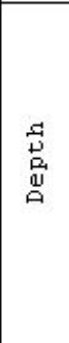 } & \multirow[b]{2}{*}{ 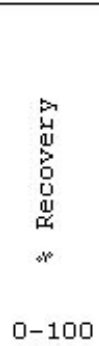 } & \multirow[b]{2}{*}{ 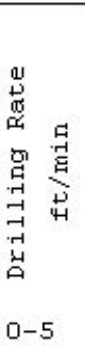 } & \multirow[b]{2}{*}{ 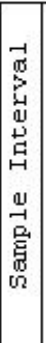 } & \multirow[b]{2}{*}{ 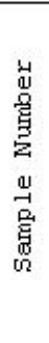 } & \multirow[b]{2}{*}{ 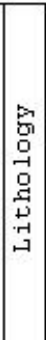 } & \multirow[b]{2}{*}{$\begin{array}{c}\text { Lithologic Description } \\
\text { Comments }\end{array}$} & \multirow{2}{*}{\begin{tabular}{|c} 
Field \\
\\
vocs \\
(ppm)
\end{tabular}} & Screening & Results \\
\hline & & & & & & & & $\begin{array}{l}\text { Alpha } \\
\text { (dpm) }\end{array}$ & $\begin{array}{l}\text { Beta } \\
\text { (dpm) }\end{array}$ \\
\hline
\end{tabular}

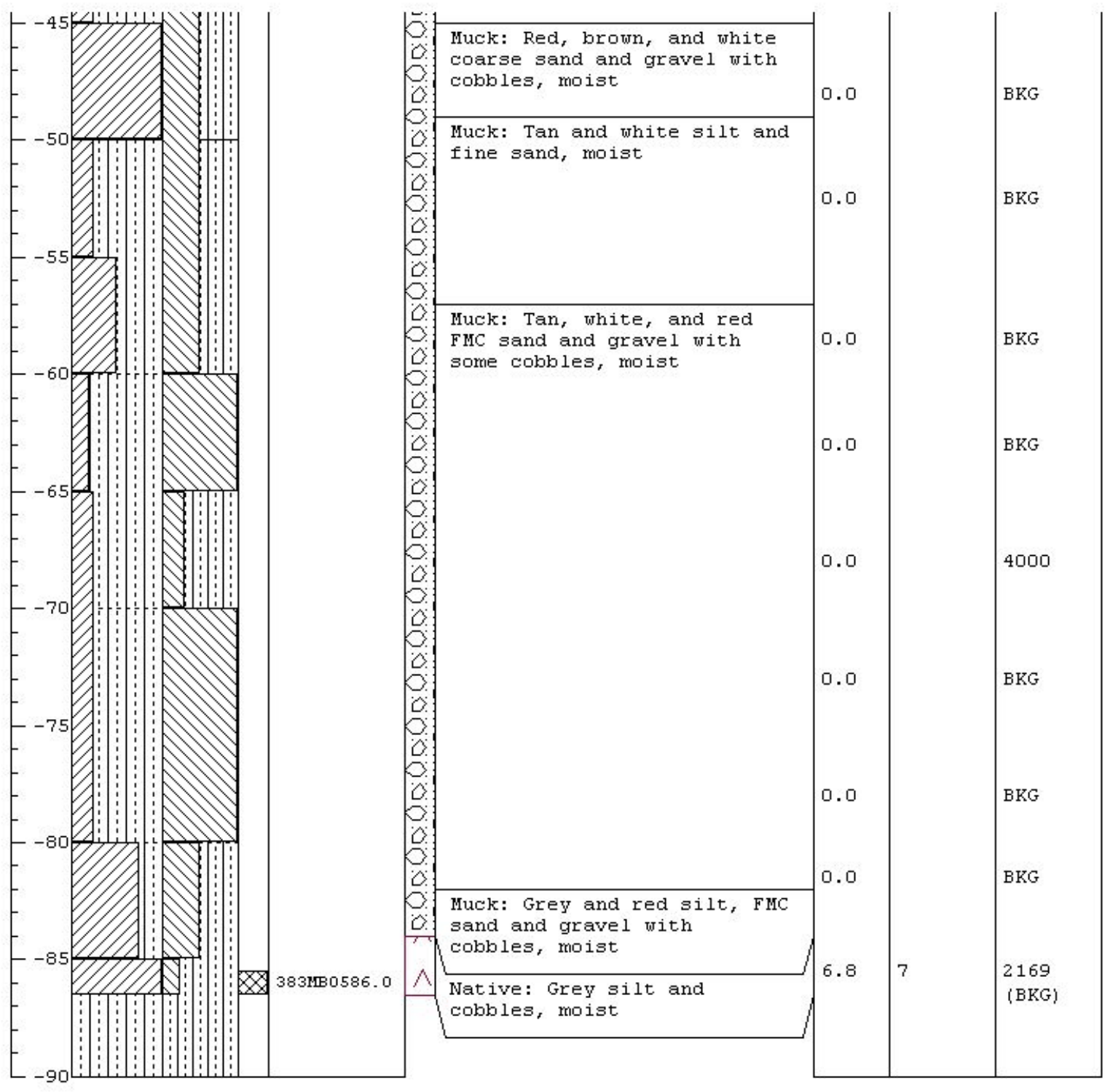


Page 1 of 1

\begin{tabular}{|c|c|c|c|c|c|c|c|c|}
\hline \multicolumn{5}{|c|}{$\begin{array}{l}\text { Project Hame: CAU } 383 \text { E-Tunnel Sites } \\
\text { Project Humber: DTO } 4-320 \\
\text { Borehole Humber: EMBO } 6 \\
\text { Logged By: M. McLane } \\
\text { Drilled By: Boart-Longyear } \\
\text { Drilling Method: Rotosonic }\end{array}$} & \multicolumn{4}{|c|}{$\begin{array}{l}\text { Date Started: } 6 / 12 / 04 \\
\text { Date Completed: } 6 / 12 / 04 \\
\text { Elevation: } 6105.0 \\
\text { Horthing: } 887386.0 \\
\text { Easting: } 638813.1 \\
\text { Total Depth: } 7.5 \mathrm{ft} \text {. }\end{array}$} \\
\hline \multirow[b]{2}{*}{ 总点 } & \multirow[b]{2}{*}{ 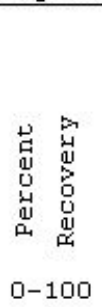 } & \multirow[b]{2}{*}{ 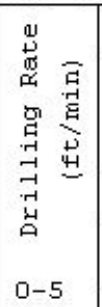 } & \multirow[b]{2}{*}{ 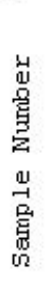 } & \multirow[b]{2}{*}{ 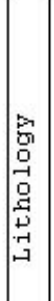 } & \multirow[b]{2}{*}{$\begin{array}{c}\text { Lithologic Description } \\
\text { Comments }\end{array}$} & Field & Screening & Results \\
\hline & & & & & & $\begin{array}{l}\text { VoCs } \\
\text { (ppm) }\end{array}$ & $\begin{array}{l}\text { Alpha } \\
\text { (dpm) }\end{array}$ & $\begin{array}{l}\text { Beta } \\
\text { (dpm) }\end{array}$ \\
\hline
\end{tabular}

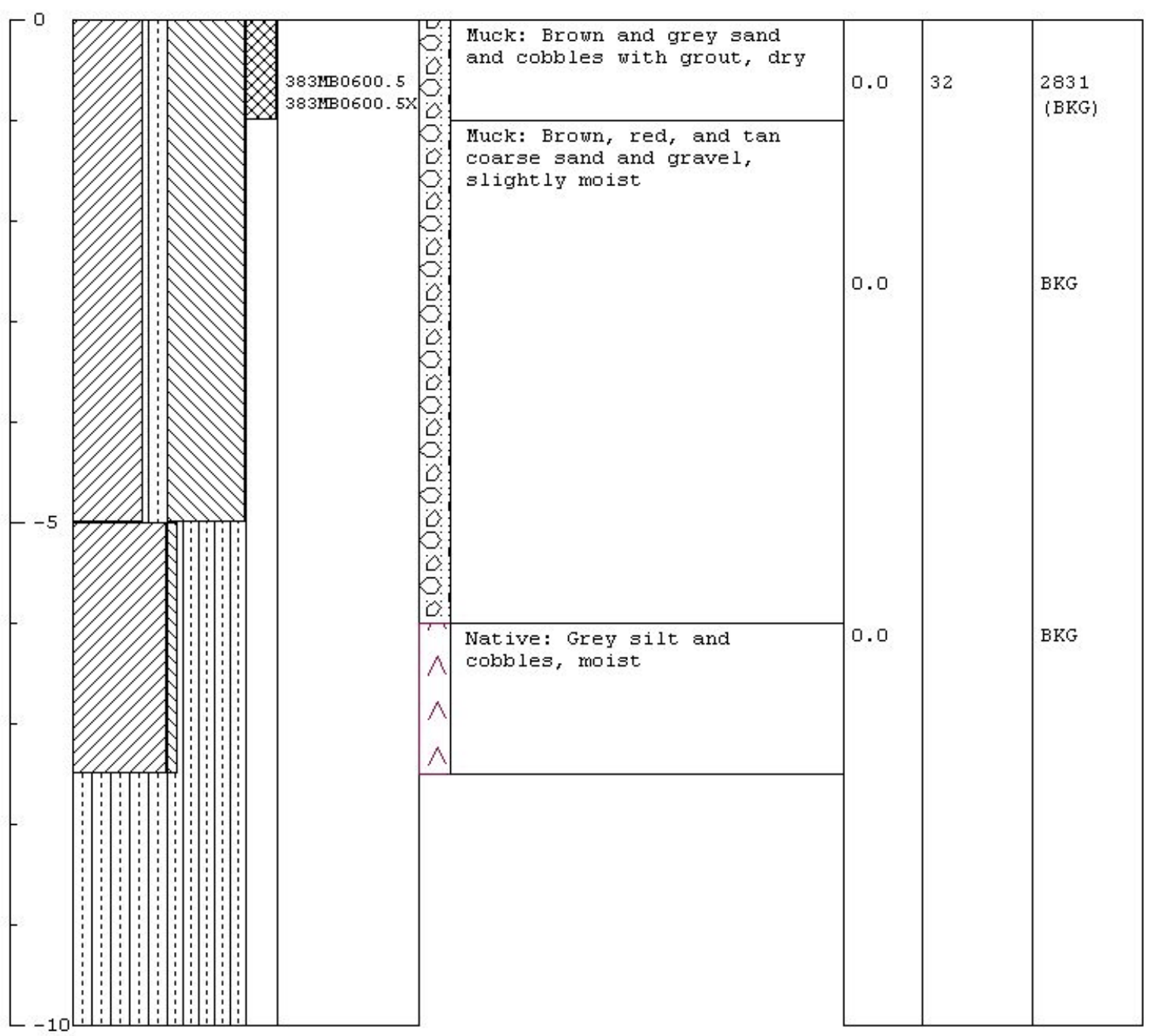


Page 1 of 1

Project Hame: CAU 383 E-Tunnel Sites

Project Number: DT04-320

Borehole Humber: EMBO7

Logged By: M. MeLane

Drilled By: Boart-Longyear Drilling Method: Rotosonic
Date Started: $5 / 19 / 04$

Date Completed : 5/19/04

Elevation: 6123.1

Horthing: 888056.7

Easting: 637580.3

Total Depth: 5.0 ft.
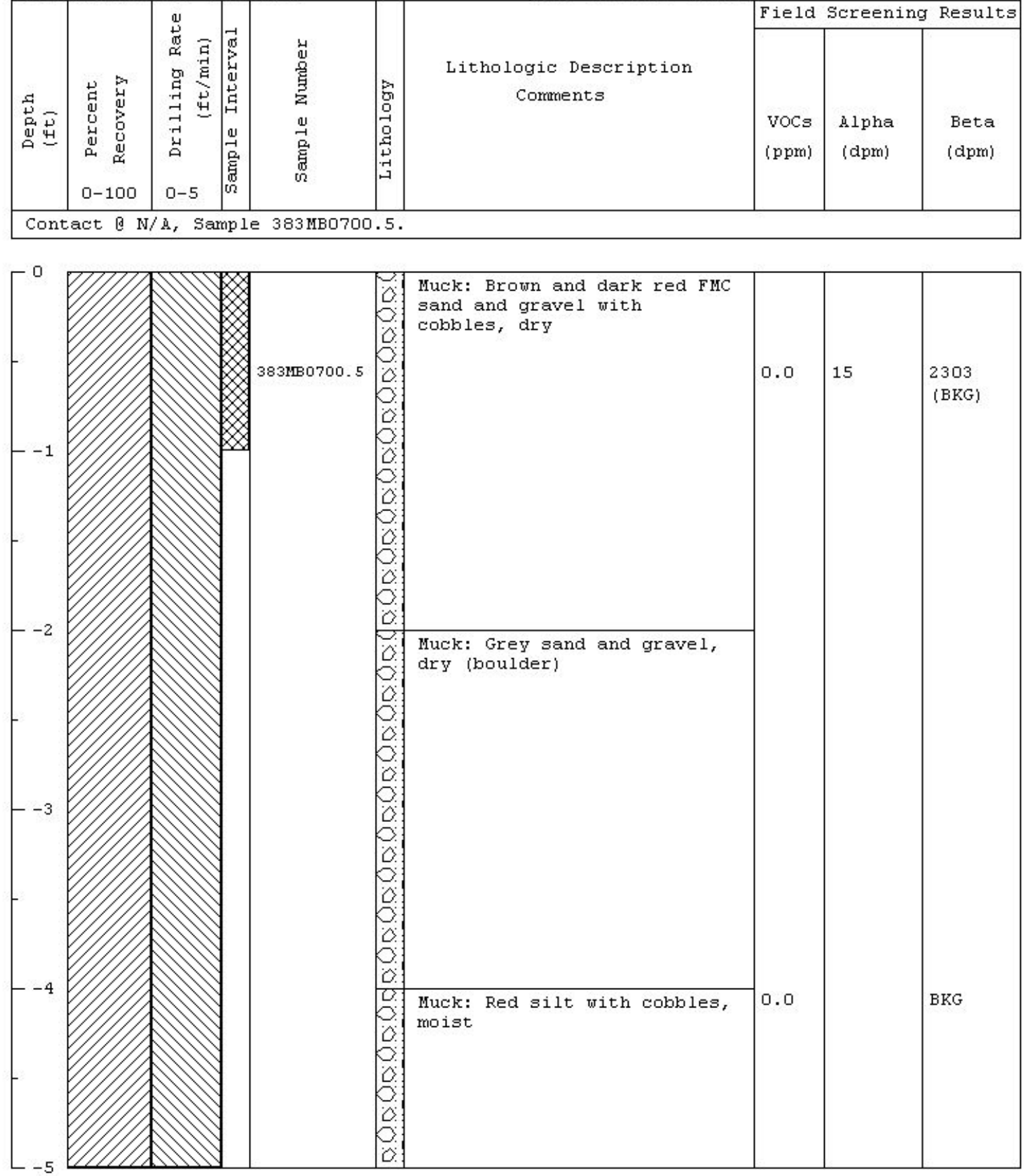
Project Hame: CAU 383 E-Tunnel sites

Project Humber: DTO4-320

Borehole Number: EMBO8

Logged By: M. McLane

Drilled By: Boart-Longyear

Drilling Method: Rotosonic
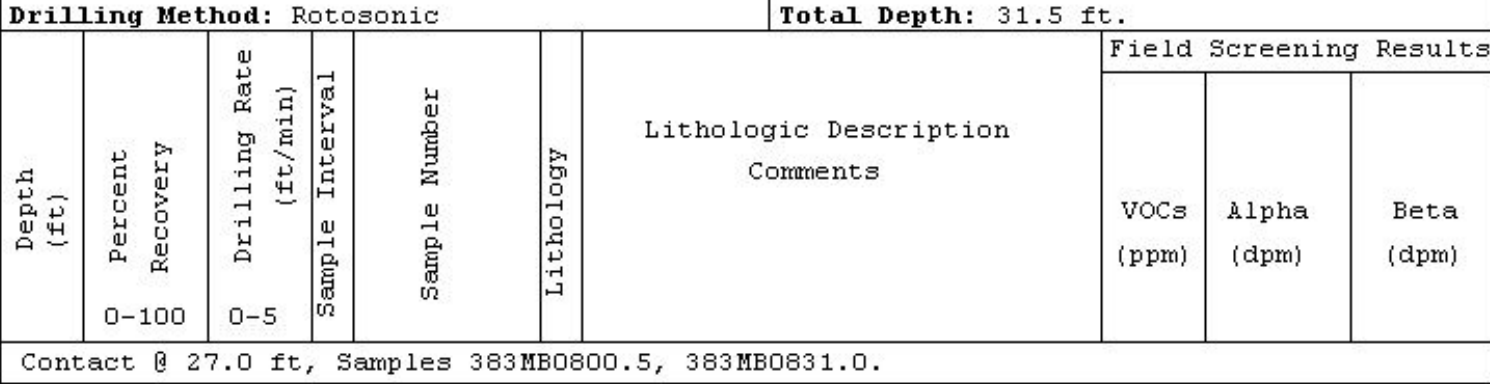

Date Started: $5 / 24 / 04$

Elevation: 6132.5

Horthing: 887404.7

Easting : 638115.3

t.

Field Screening Results
Date Completed: $5 / 24 / 04$

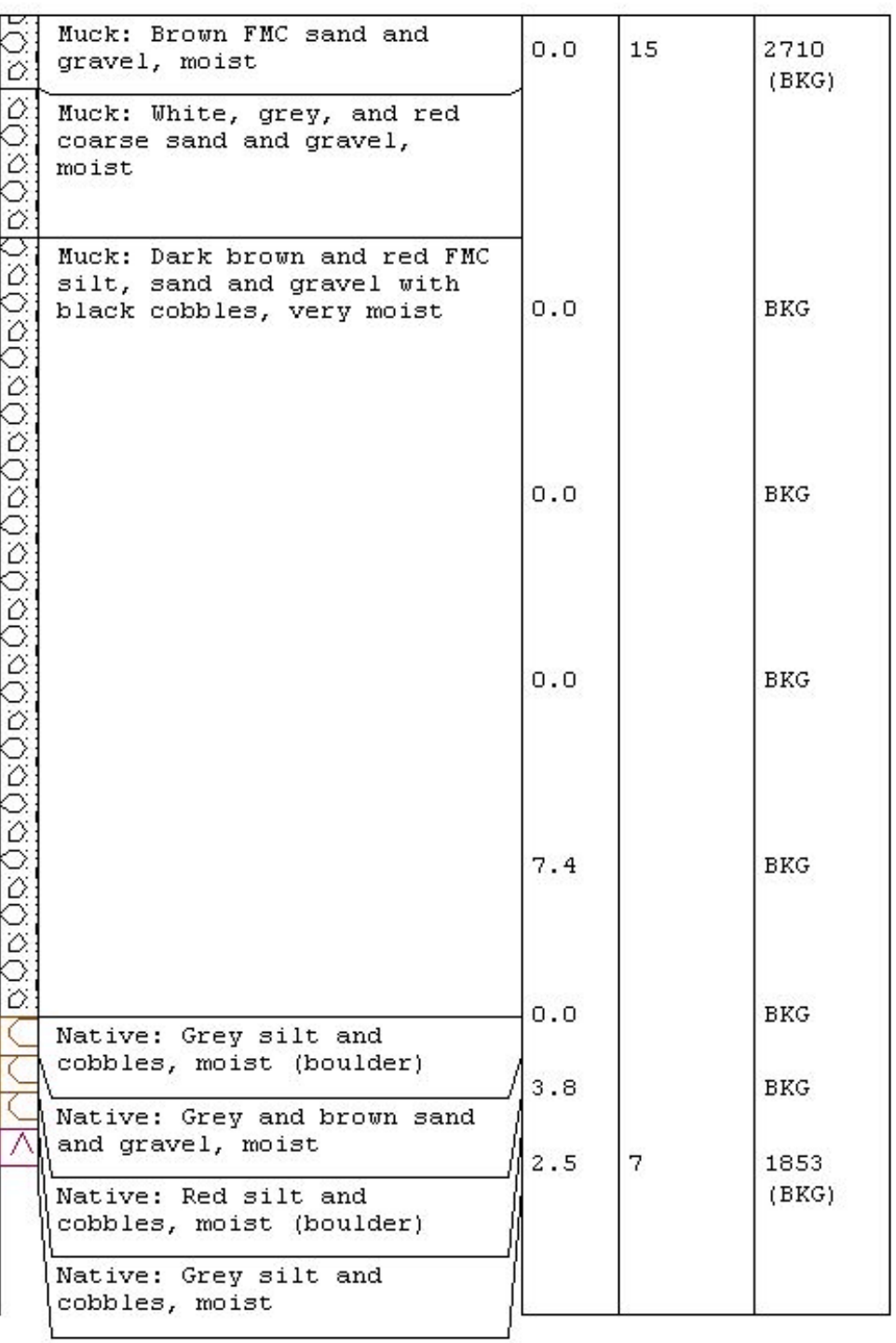


Page 1 of 1

\begin{tabular}{|c|c|c|c|c|c|c|c|c|}
\hline \multicolumn{5}{|c|}{$\begin{array}{l}\text { Project Hame: CAU } 383 \text { E-Tunnel Sites } \\
\text { Project Humber: DTO } 4-320 \\
\text { Borehole Number: EMBO } \\
\text { Logged By: M. McLane } \\
\text { Drilled By: Boart-Longyear } \\
\text { Drilling Method: Rotosonic }\end{array}$} & \multicolumn{4}{|c|}{$\begin{array}{l}\text { Date Started: } 6 / 12 / 04 \\
\text { Date Completed: } 6 / 12 / 04 \\
\text { Elevation: } 6122.3 \\
\text { Horthing: } 887322.3 \\
\text { Easting: } 638941.3 \\
\text { Total Depth: } 14.0 \mathrm{ft} .\end{array}$} \\
\hline \multirow[b]{2}{*}{ 窇 } & \multirow[b]{2}{*}{ 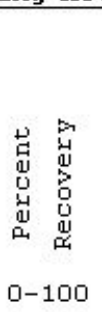 } & \multirow[b]{2}{*}{ 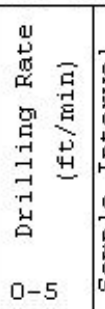 } & \multirow[b]{2}{*}{ 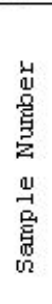 } & \multirow[b]{2}{*}{ 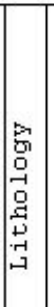 } & \multirow[b]{2}{*}{$\begin{array}{c}\text { Lithologic Description } \\
\text { Comments }\end{array}$} & Field & Screening & Results \\
\hline & & & & & & $\begin{array}{l}\text { VOCs } \\
\text { (ppm) }\end{array}$ & $\begin{array}{l}\text { Alpha } \\
\text { (dpm) }\end{array}$ & $\begin{array}{l}\text { Beta } \\
\text { (dpm) }\end{array}$ \\
\hline
\end{tabular}

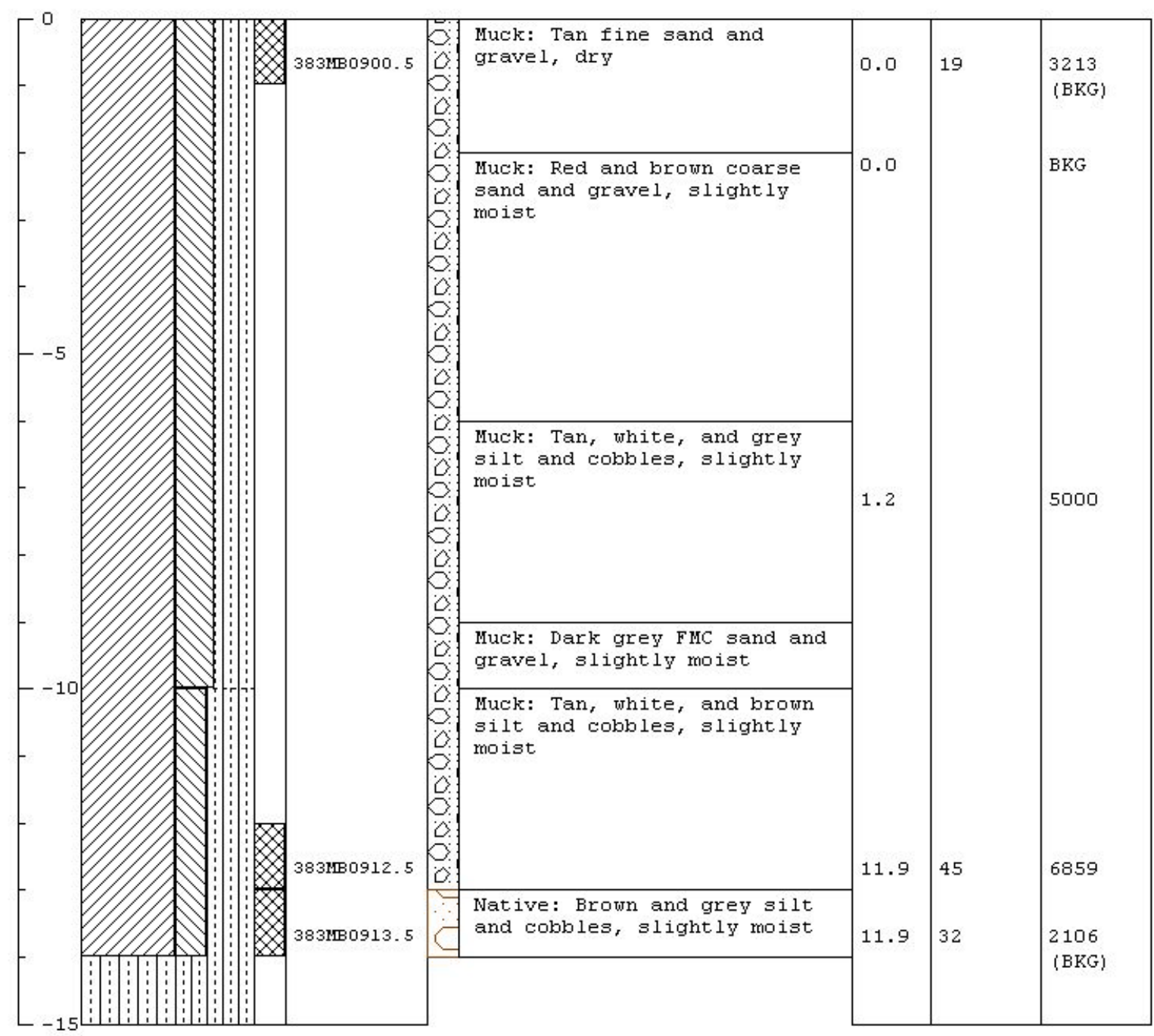


Page 1 of 1

Project Hame: CAU 383 E-Tunnel sites

Project Humber: DTO4-320

Borehole Number: EMB10

Logged By: M. McLane

Drilled By: Boart-Longyear

Drilling Method: Rotosonic
Date Started: $6 / 12 / 04$

Date Completed: $6 / 12 / 04$

Elevation: 6102.2

Horthing: 887435.4

Easting: 638844.0

Total Depth: $37.0 \mathrm{ft}$.

\begin{tabular}{|c|c|c|c|c|c|c|c|c|c|}
\hline \multirow[b]{2}{*}{ 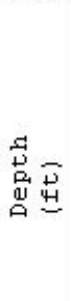 } & \multirow[b]{2}{*}{ 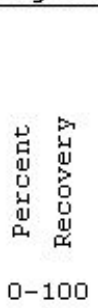 } & \multirow[b]{2}{*}{ 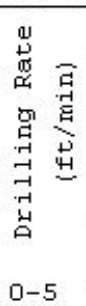 } & \multirow[b]{2}{*}{ 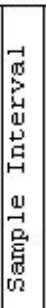 } & \multirow[b]{2}{*}{ 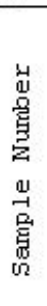 } & \multirow[b]{2}{*}{ 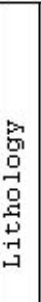 } & \multirow[b]{2}{*}{$\begin{array}{c}\text { Lithologic Description } \\
\text { Comments }\end{array}$} & \multicolumn{3}{|c|}{ Field Screening Results } \\
\hline & & & & & & & (poCs & $\begin{array}{l}\text { Al lpha } \\
\text { (dpm) }\end{array}$ & $\begin{array}{l}\text { Beta } \\
\text { (dpm) }\end{array}$ \\
\hline
\end{tabular}

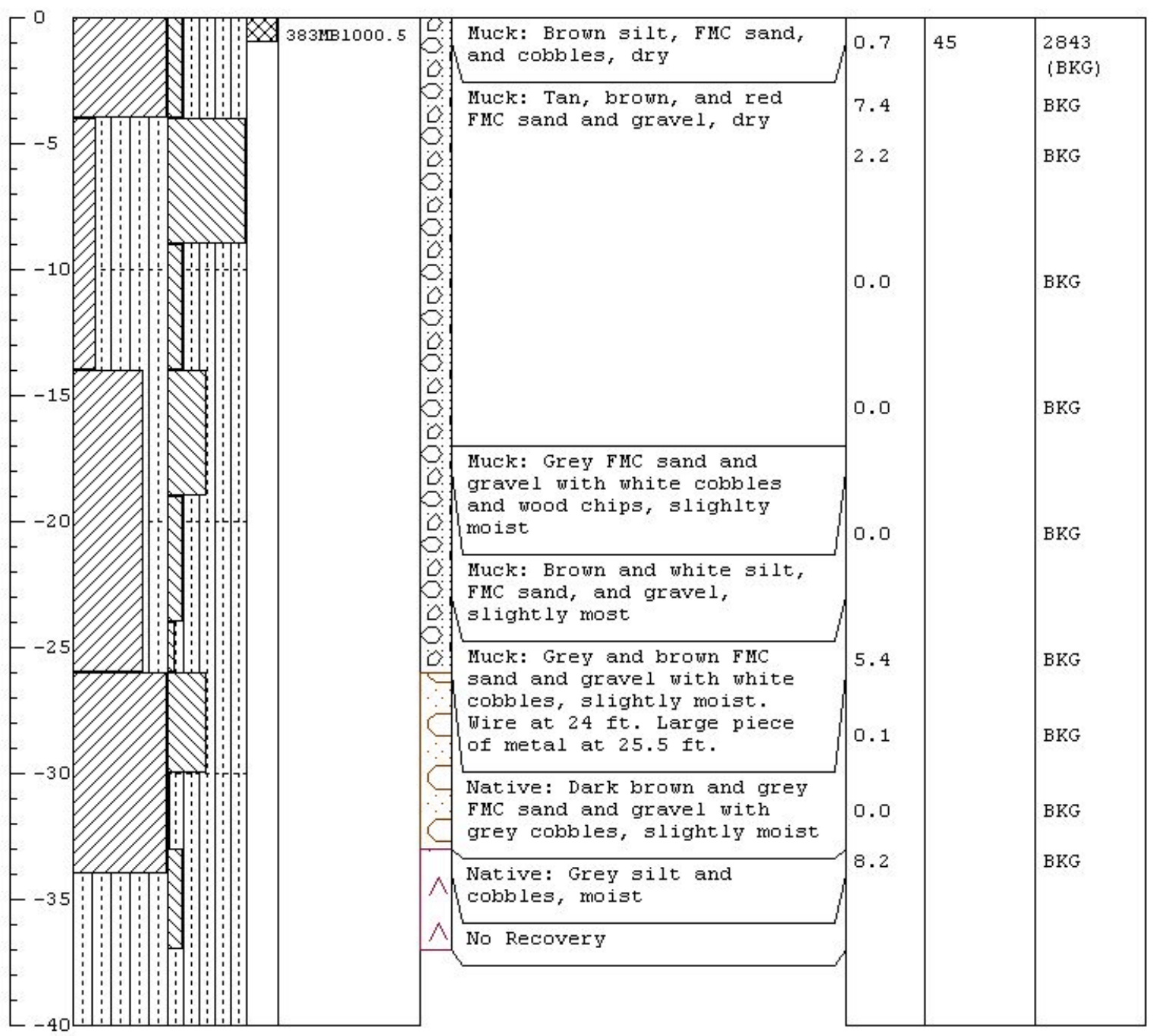


Project Hame: CAU 383 E-Tunnel sites

Project Humber: DTO4-320

Borehole Number: EMB11

Logged By: $M$. McLane

Drilled By: Boart-Longyear

Drilling Method: Rotosonic

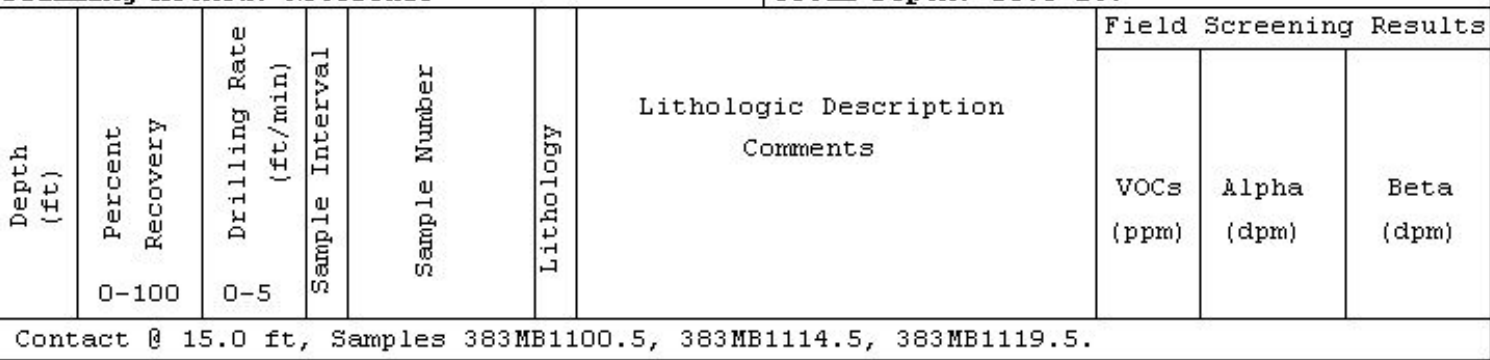

Date Started: $6 / 12 / 04$

Date Completed: $6 / 12 / 04$

Elevation: 6107.2

Horthing: 887450.5

Easting: 638781.0

Total Depth: 25.5 ft.

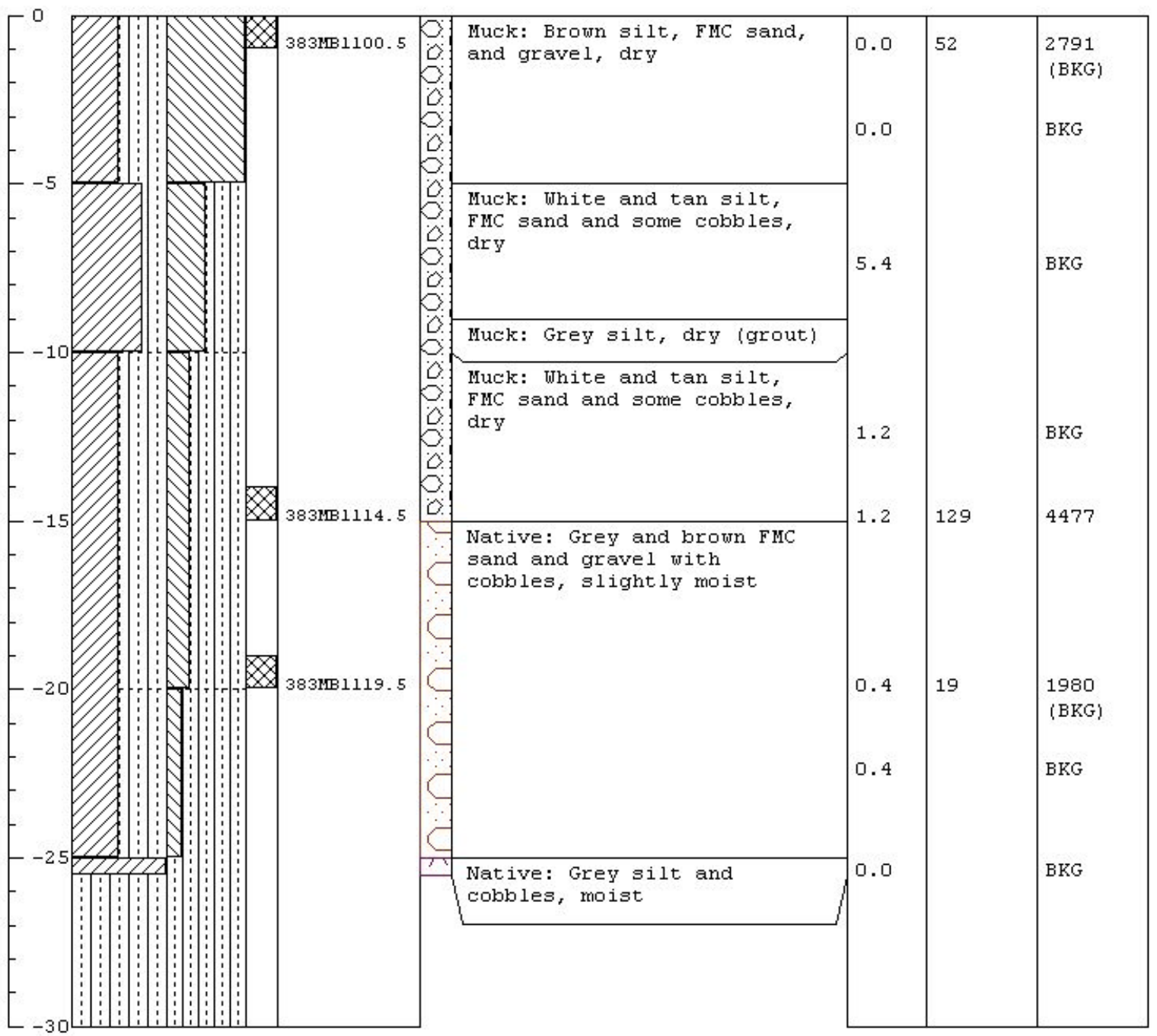




\begin{tabular}{|c|c|c|c|c|c|c|c|c|}
\hline \multicolumn{5}{|c|}{$\begin{array}{l}\text { Project Hame: CAU } 383 \text { E-Tunnel sites } \\
\text { Project Humber: DTO } 4-320 \\
\text { Borehole Humber: EMB } 12 \\
\text { Logged By: M. McLane } \\
\text { Drilled By: Boart-Longyear } \\
\text { Drilling Method: Rotosonic }\end{array}$} & \multicolumn{4}{|c|}{$\begin{array}{l}\text { Date Started: } 6 / 13 / 04 \\
\text { Date Completed: } 6 / 13 / 04 \\
\text { Elevation: } 6123.1 \\
\text { Horthing: } 887487.9 \\
\text { Easting: } 638589.1 \\
\text { Total Depth: } 75 \mathrm{ft} .\end{array}$} \\
\hline \multirow[b]{2}{*}{ 茧出 } & \multirow[b]{2}{*}{ 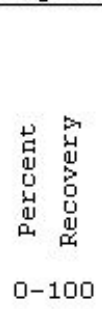 } & \multirow[b]{2}{*}{ 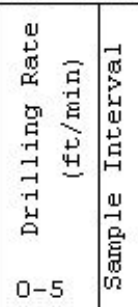 } & \multirow[b]{2}{*}{ 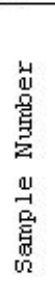 } & \multirow[b]{2}{*}{ 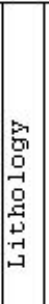 } & \multirow[b]{2}{*}{$\begin{array}{c}\text { Lithologic Description } \\
\text { Corments }\end{array}$} & Field & Screening & Results \\
\hline & & & & & & $\begin{array}{l}\text { VOCs } \\
\text { (ppm) }\end{array}$ & $\begin{array}{l}\text { Alpha } \\
\text { (dpm) }\end{array}$ & $\begin{array}{l}\text { Beta } \\
\text { (dpm) }\end{array}$ \\
\hline
\end{tabular}

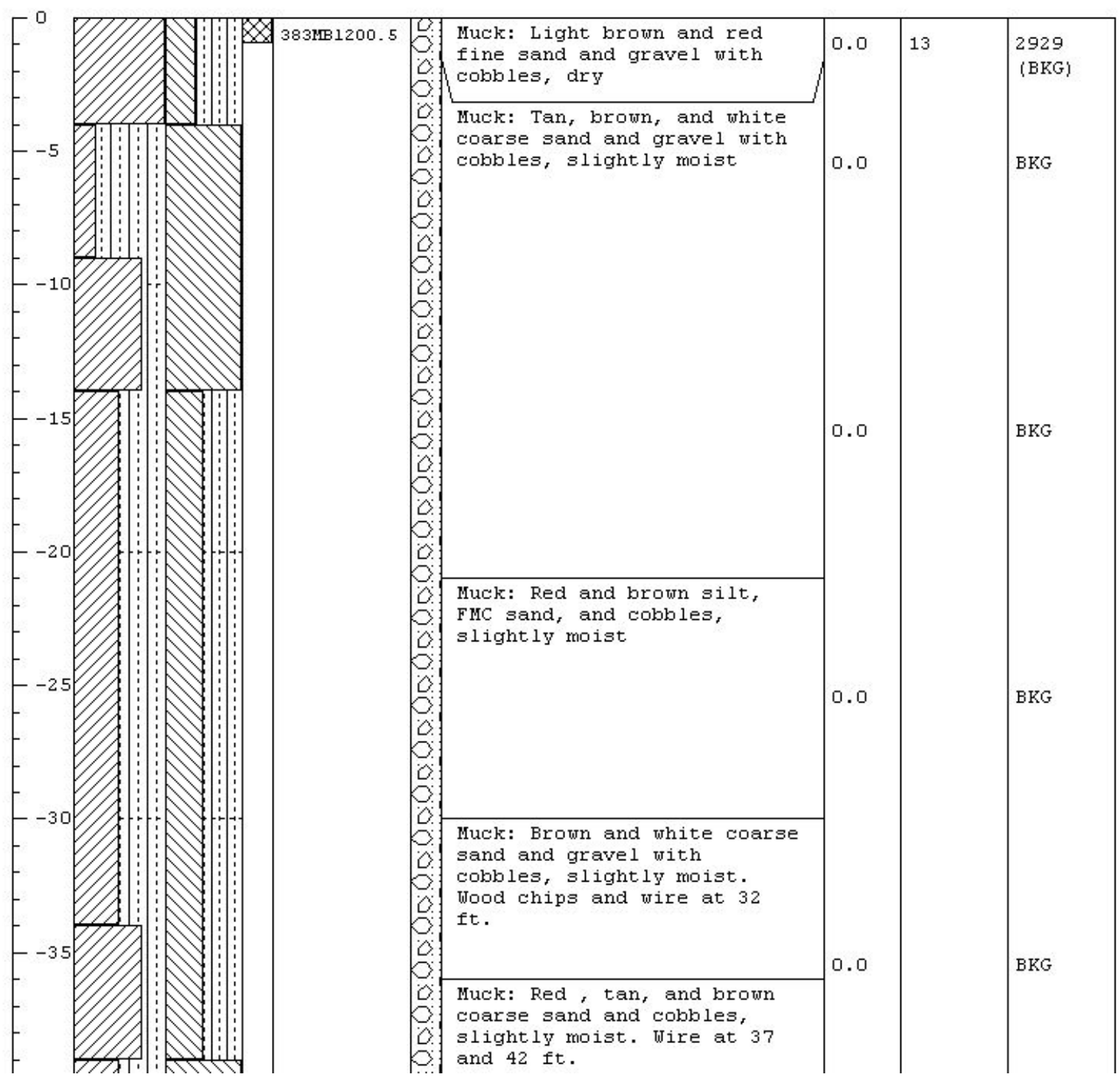




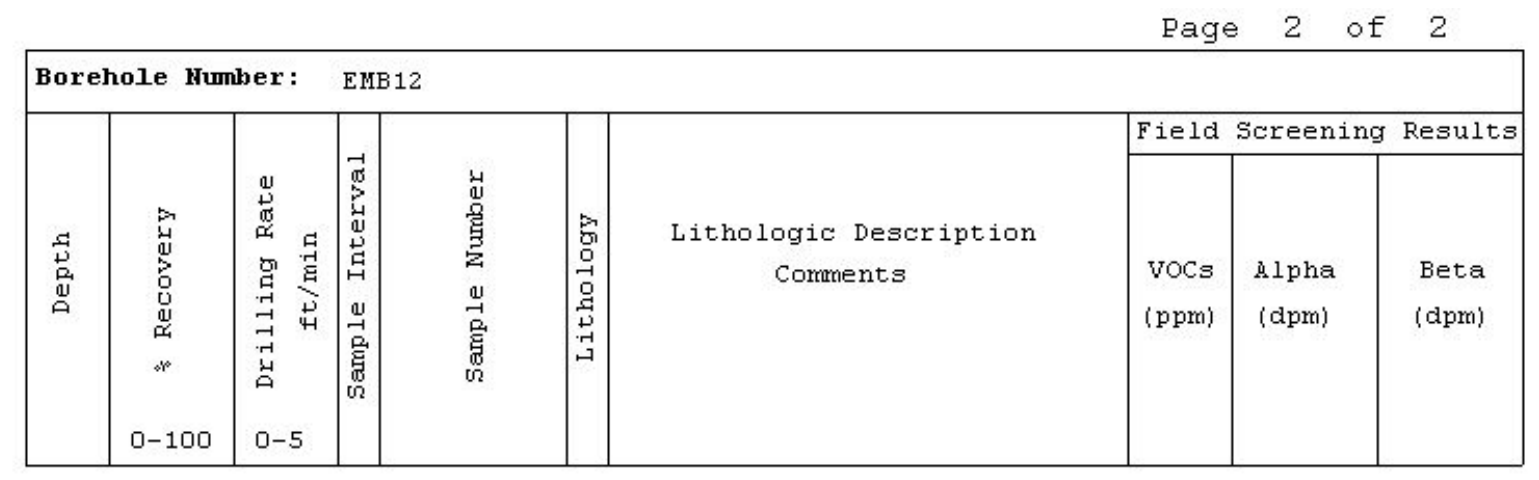

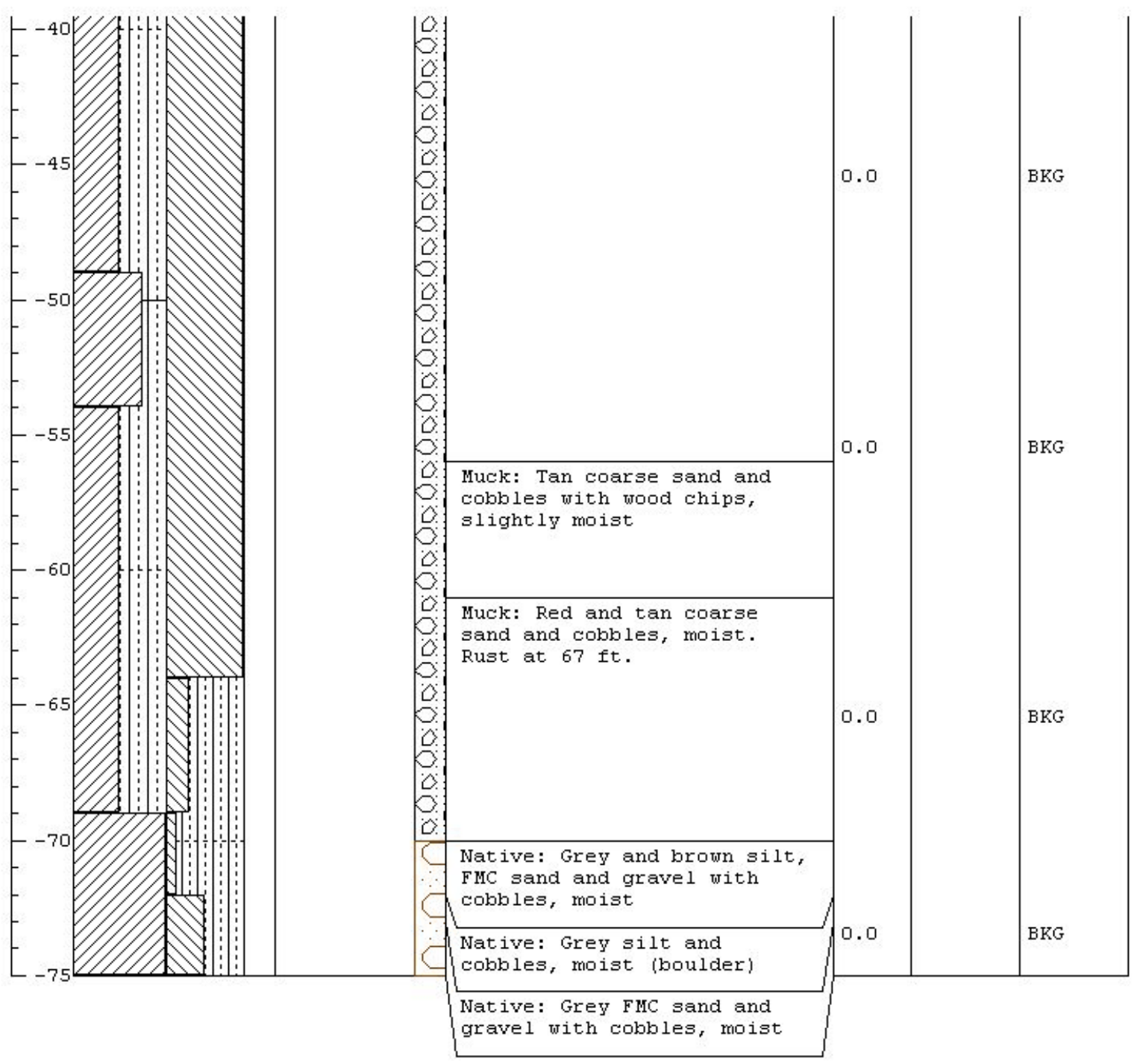


Page 1 of 2

Project Name: CAU 383 E-Tunnel Sites

Project Humber: DTO4-320

Borehole Humber: EMB13

Logged By: $M$. McLane

Drilled By: Boart-Longyear

Drilling Method: Rotosonic
Date Started: $6 / 13 / 04$

Date Completed: $6 / 13 / 04$

Elevation: 6127.8

Horthing: 887564.0

Easting: 638432.6

Total Depth: $91.5 \mathrm{ft}$.

\begin{tabular}{|c|c|c|c|c|c|c|c|c|c|}
\hline \multirow[b]{2}{*}{ 虫出 } & \multirow[b]{2}{*}{ 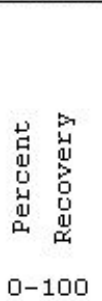 } & \multirow{2}{*}{\multicolumn{2}{|c|}{ 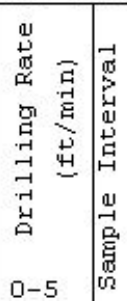 }} & \multirow[b]{2}{*}{ 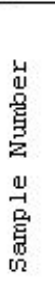 } & \multirow[b]{2}{*}{ 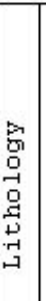 } & \multirow[b]{2}{*}{$\begin{array}{c}\text { Lithologic Description } \\
\text { Comments }\end{array}$} & \multicolumn{3}{|c|}{ Field Screening Results } \\
\hline & & & & & & & $\begin{array}{r}\text { VOCs } \\
\text { (ppm) }\end{array}$ & $\begin{array}{l}\text { Alpha } \\
\text { (dpm) }\end{array}$ & $\begin{array}{l}\text { Beta } \\
\text { (dpm) }\end{array}$ \\
\hline
\end{tabular}

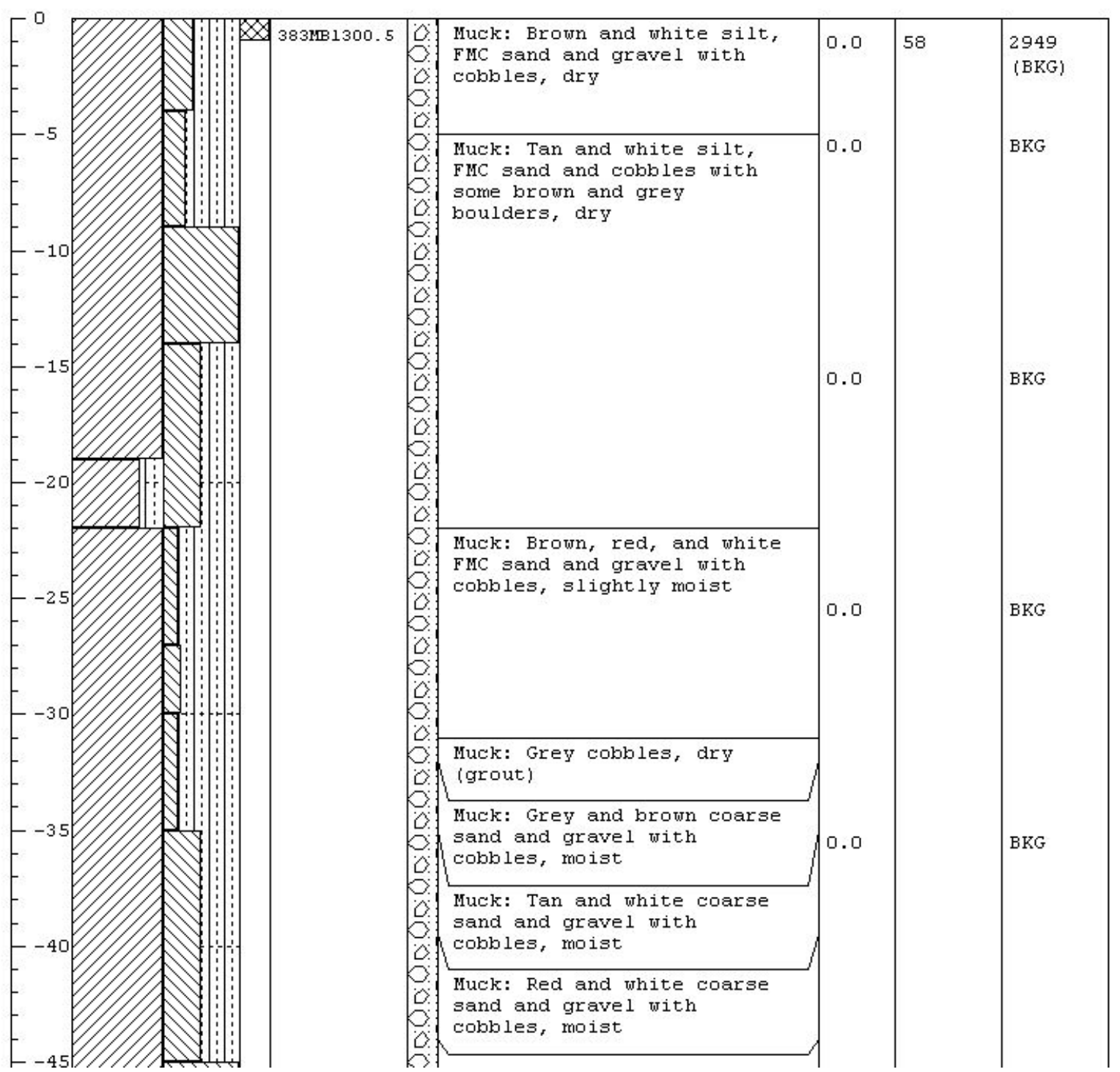


Page 2 of 2

\begin{tabular}{|c|c|c|c|c|c|c|c|c|c|}
\hline \multicolumn{3}{|c|}{ Borehole Number: } & \multicolumn{7}{|c|}{ EMB 13} \\
\hline \multirow[b]{2}{*}{$\begin{array}{l}\text { 吾 } \\
\text { 总 } \\
\text { 岁 }\end{array}$} & \multirow[b]{2}{*}{ 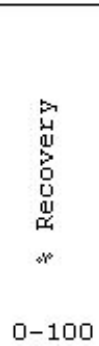 } & \multirow[b]{2}{*}{ 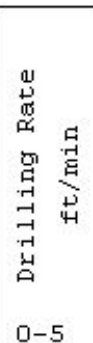 } & \multirow[b]{2}{*}{ 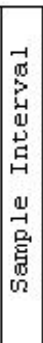 } & \multirow[b]{2}{*}{ 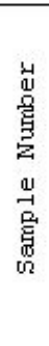 } & \multirow[b]{2}{*}{ 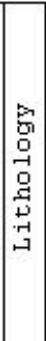 } & \multirow[b]{2}{*}{$\begin{array}{c}\text { Lithologic Description } \\
\text { Comments }\end{array}$} & \multicolumn{3}{|c|}{ Field Screening Results } \\
\hline & & & & & & & $\begin{array}{l}\text { VoCs } \\
\text { (ppm) }\end{array}$ & $\begin{array}{l}\text { Alpha } \\
\text { (dpm) }\end{array}$ & $\begin{array}{l}\text { Beta } \\
\text { (dpm) }\end{array}$ \\
\hline
\end{tabular}

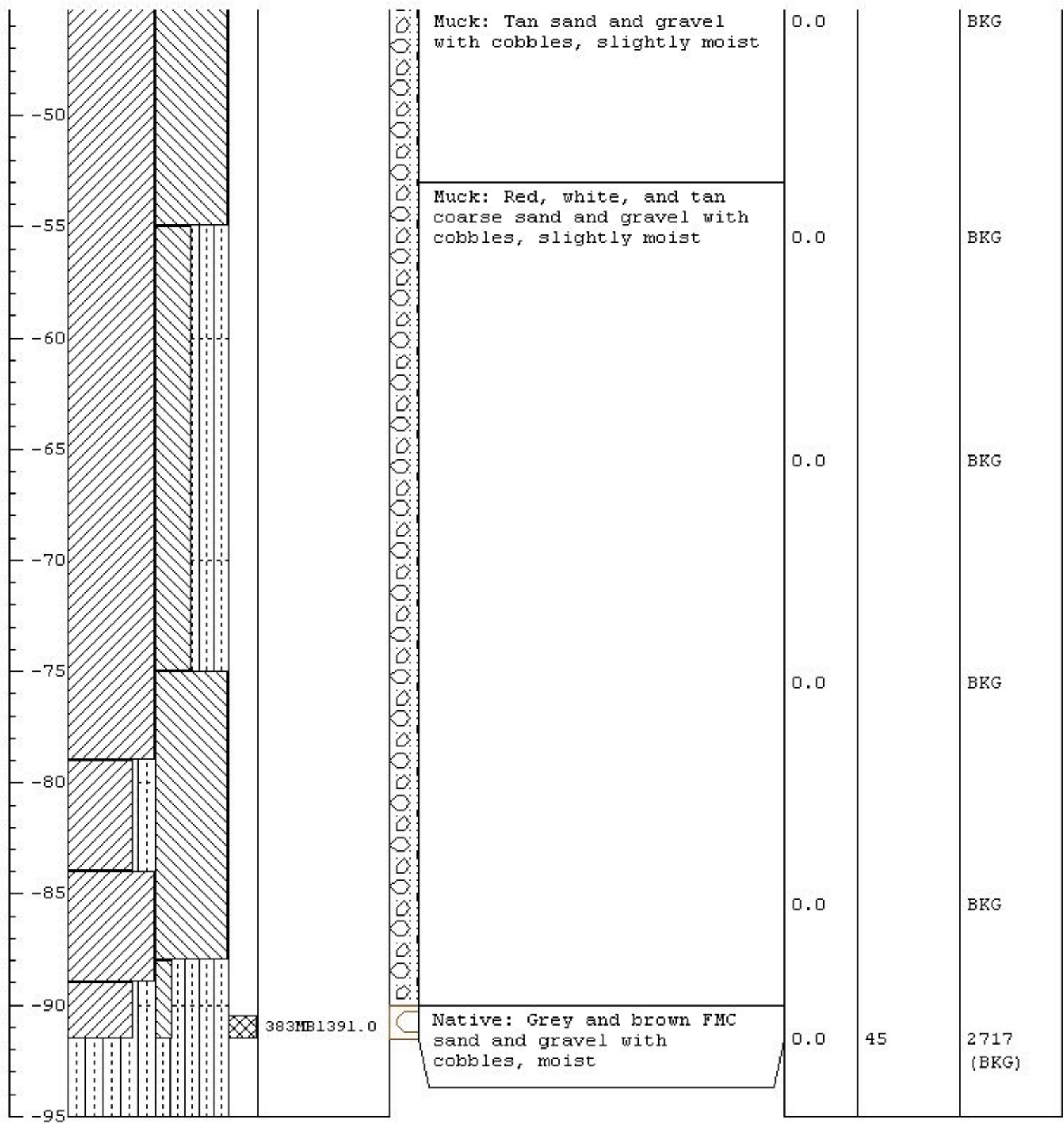


Page 1 of 1

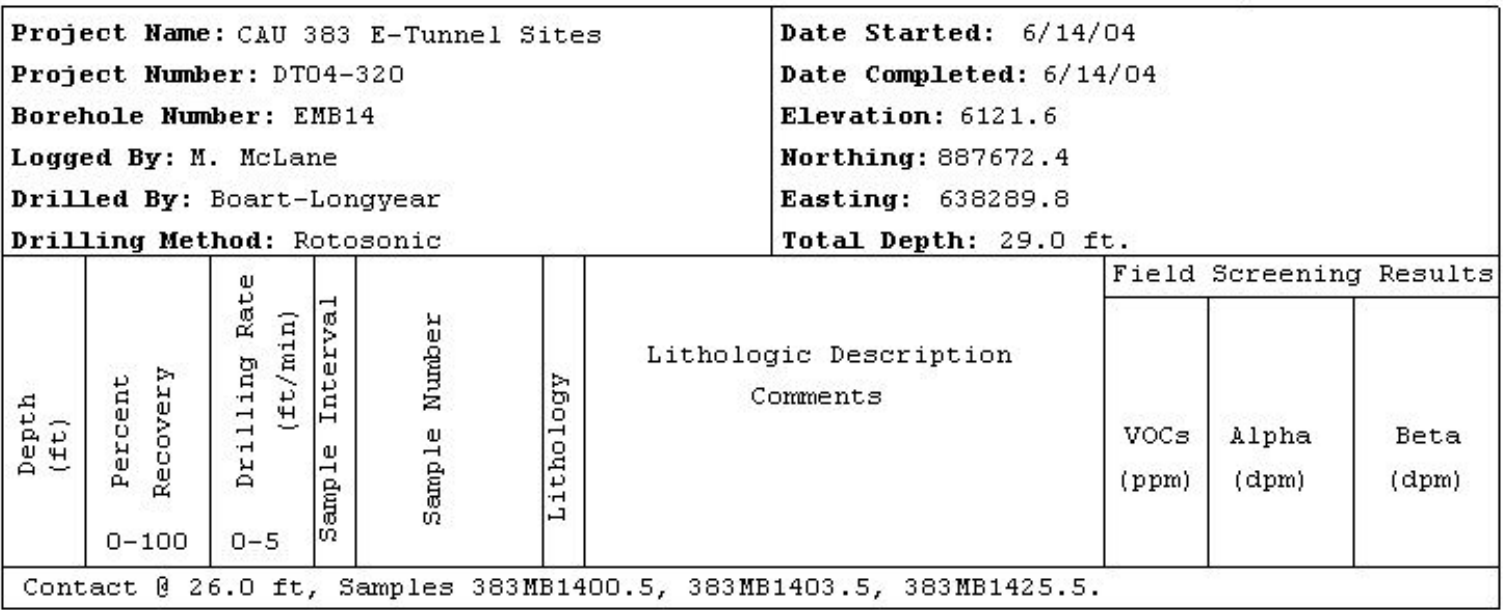

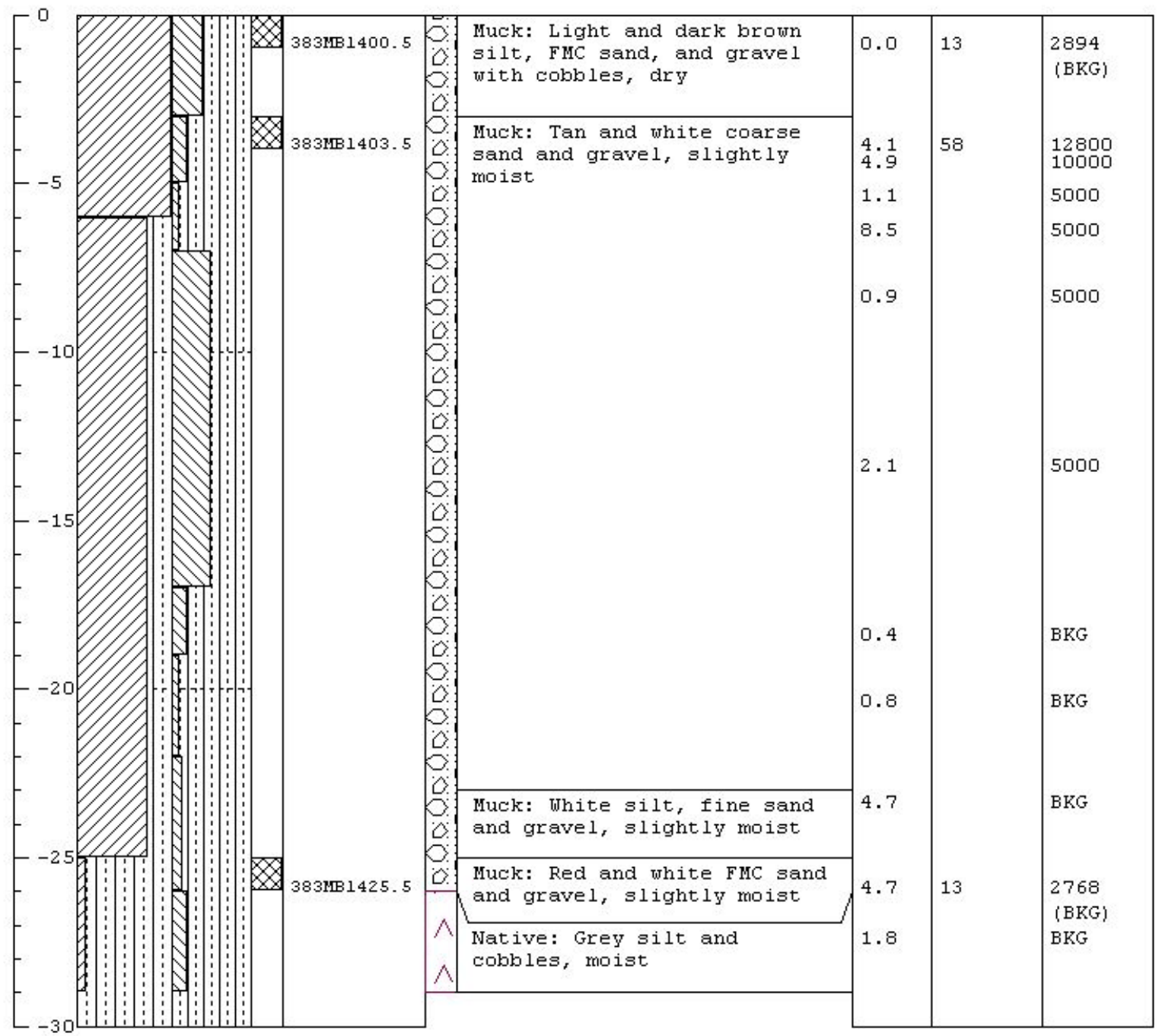


Page 1 of 1

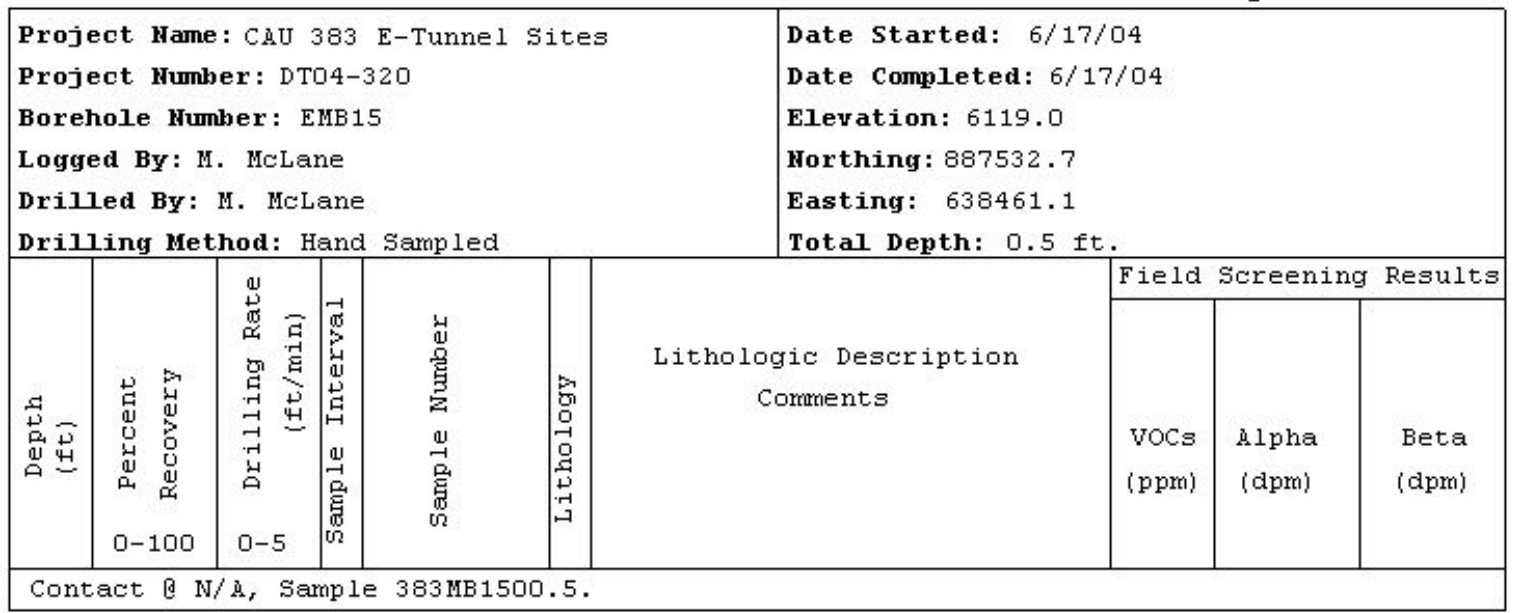

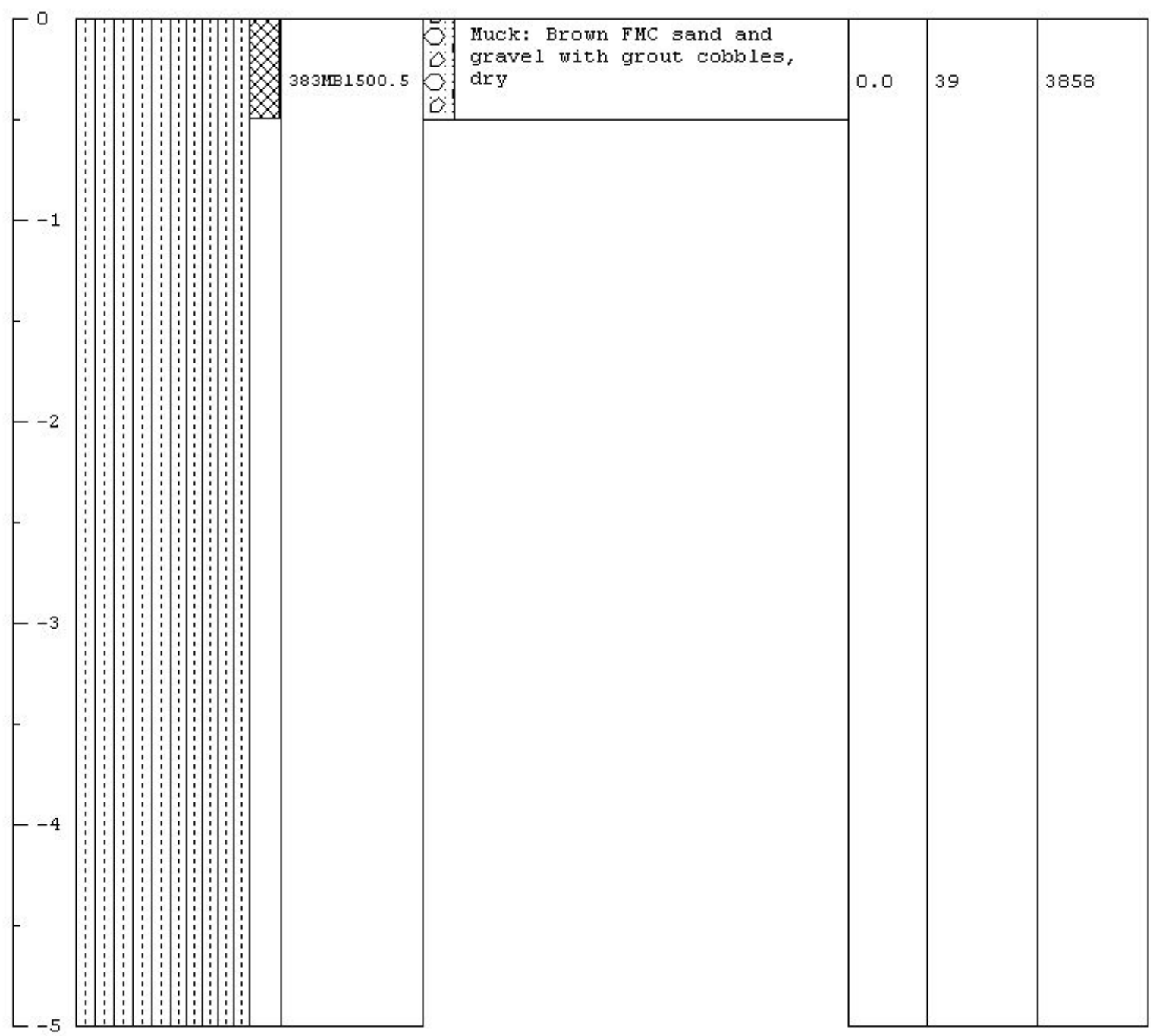


Page 1 of 2

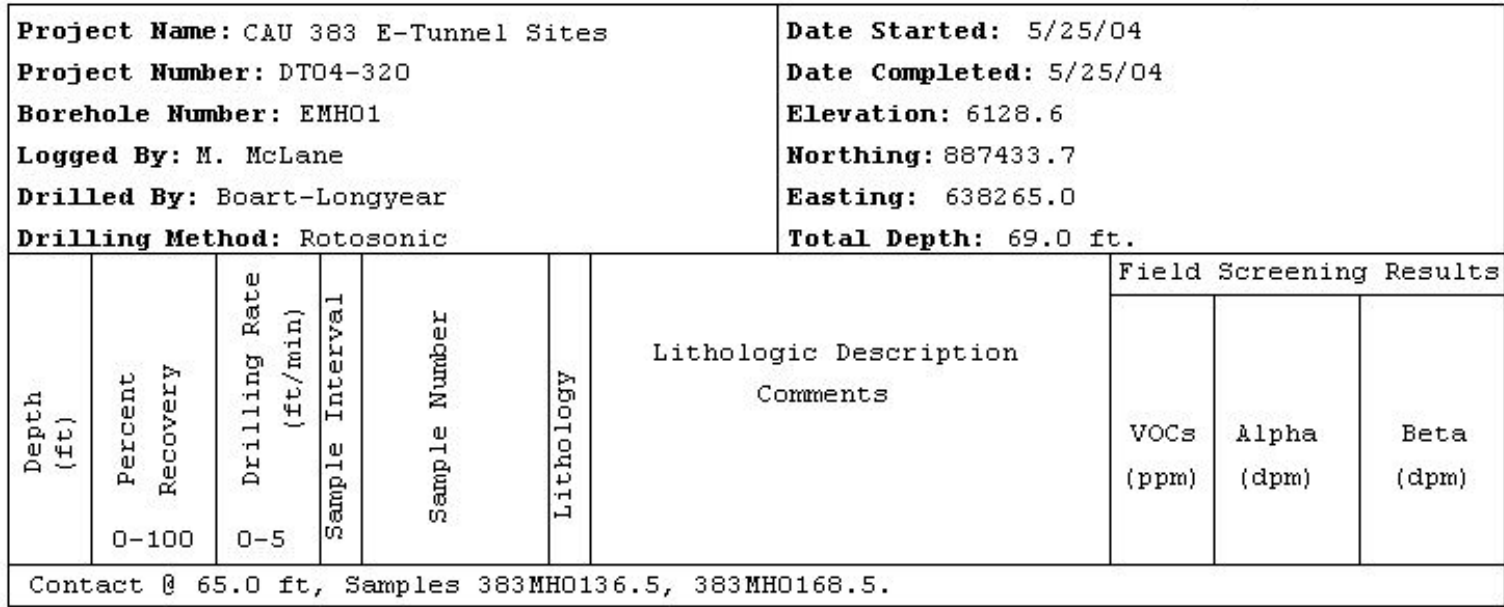

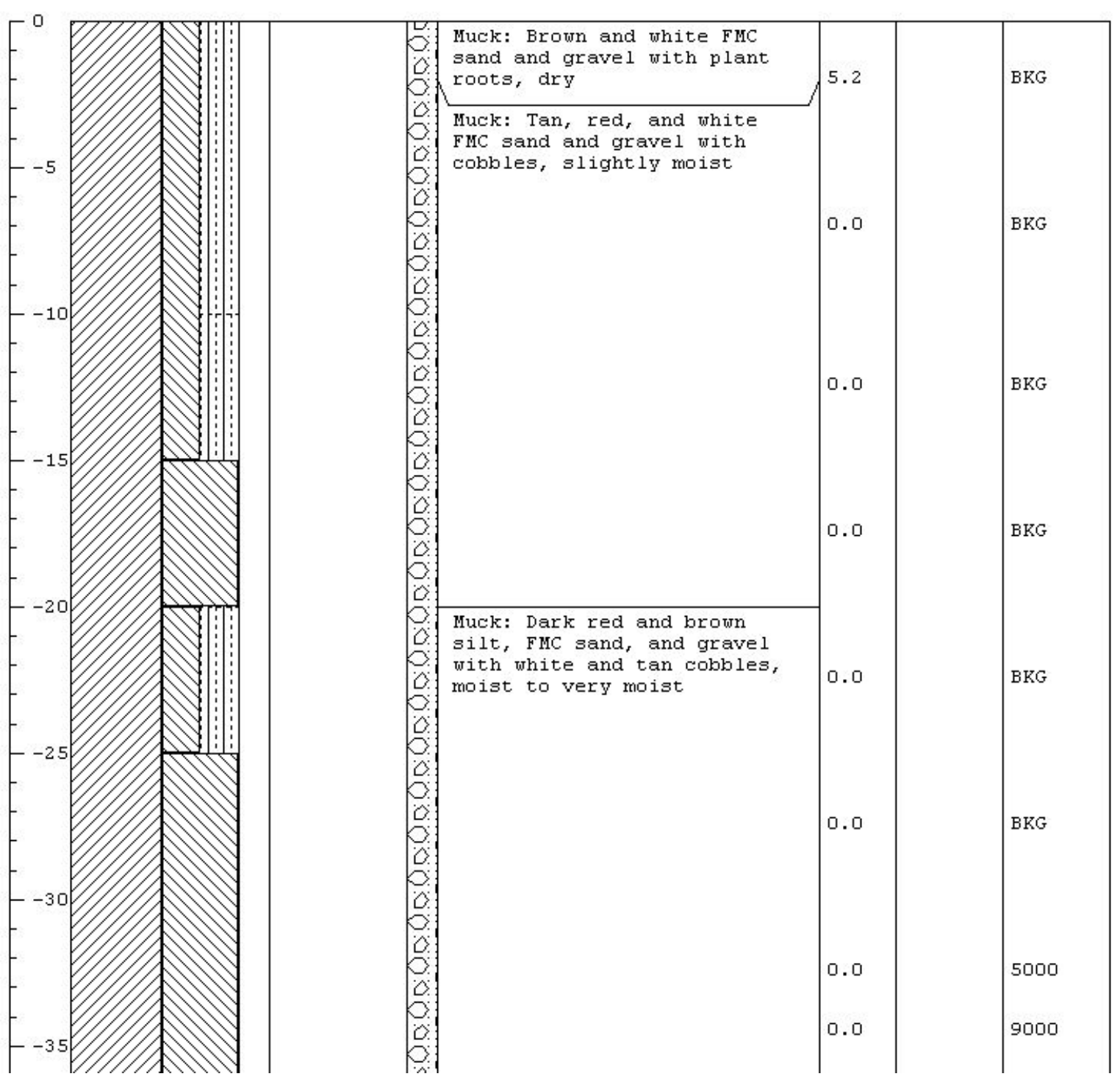


Page 2 of 2

\begin{tabular}{|c|c|c|c|c|c|c|c|c|c|}
\hline \multicolumn{10}{|c|}{ Borehole Humber: } \\
\hline \multirow[b]{2}{*}{ 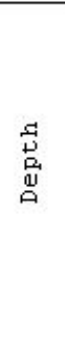 } & \multirow[b]{2}{*}{ 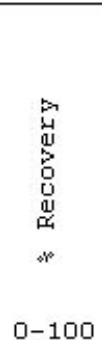 } & \multirow[b]{2}{*}{ 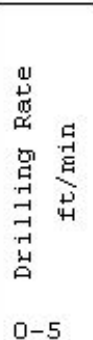 } & \multirow[b]{2}{*}{ 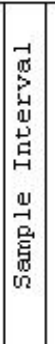 } & \multirow[b]{2}{*}{ 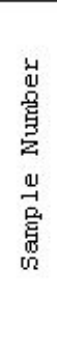 } & \multirow[b]{2}{*}{ 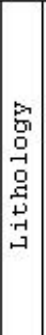 } & \multirow[b]{2}{*}{$\begin{array}{c}\text { Lithologic Description } \\
\text { Comments }\end{array}$} & \multicolumn{2}{|c|}{ Field screening } & Results \\
\hline & & & & & & & $\begin{array}{l}\text { VOCs } \\
\text { (ppm) }\end{array}$ & $\begin{array}{l}\text { Alpha } \\
\text { (dpm) }\end{array}$ & $\begin{array}{l}\text { Beta } \\
\text { (dpm) }\end{array}$ \\
\hline
\end{tabular}

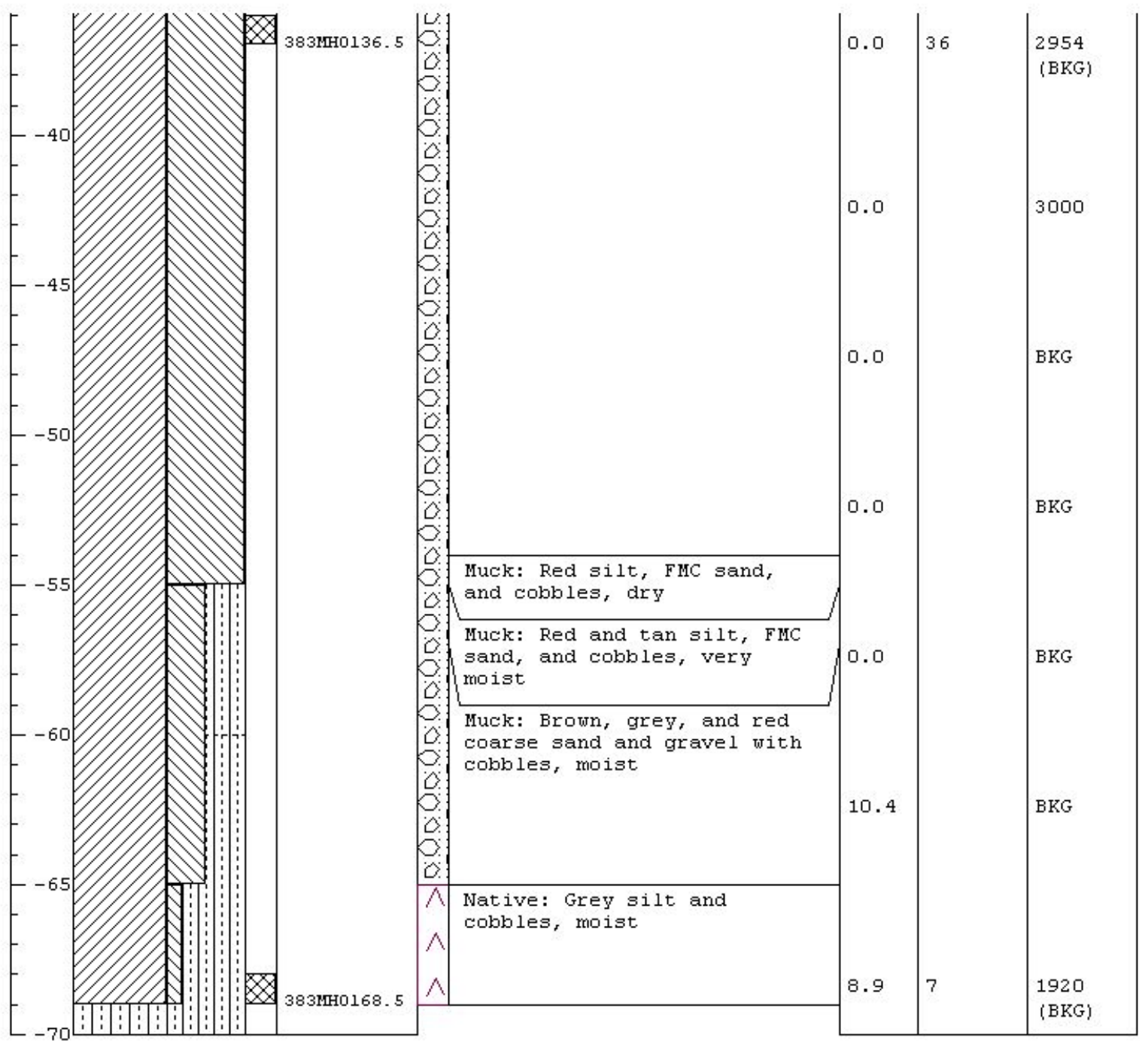




\begin{tabular}{|c|c|c|c|c|c|c|c|c|}
\hline \multicolumn{5}{|c|}{$\begin{array}{l}\text { Project Hame: CAU } 383 \text { E-Tunnel Sites } \\
\text { Project Humber: DTO } 4-320 \\
\text { Borehole Humber: EMHO2 } \\
\text { Logged By: M. McLane } \\
\text { Drilled By: Boart-Longyear } \\
\text { Drilling Method: Rotosonic }\end{array}$} & \multicolumn{4}{|c|}{$\begin{array}{l}\text { Date Started: } 5 / 22 / 04 \\
\text { Date Completed: } 5 / 22 / 04 \\
\text { Elevation: } 6118.8 \\
\text { Horthing: } 887363.7 \\
\text { Easting: } 638721.6 \\
\text { Total Depth: } 5.0 \mathrm{ft} .\end{array}$} \\
\hline \multirow[b]{2}{*}{ 点 } & \multirow[b]{2}{*}{ 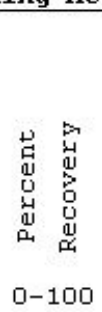 } & \multirow[b]{2}{*}{ 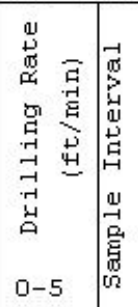 } & \multirow[b]{2}{*}{ 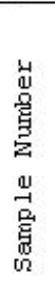 } & \multirow[b]{2}{*}{ 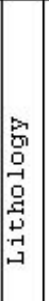 } & \multirow[b]{2}{*}{$\begin{array}{c}\text { Lithologic Description } \\
\text { Comments }\end{array}$} & Field & Screening & Results \\
\hline & & & & & & $\begin{array}{l}\text { VoCs } \\
\text { (ppm) }\end{array}$ & $\begin{array}{l}\text { Alpha } \\
\text { (dpm) }\end{array}$ & $\begin{array}{l}\text { Beta } \\
\text { (dpm) }\end{array}$ \\
\hline
\end{tabular}

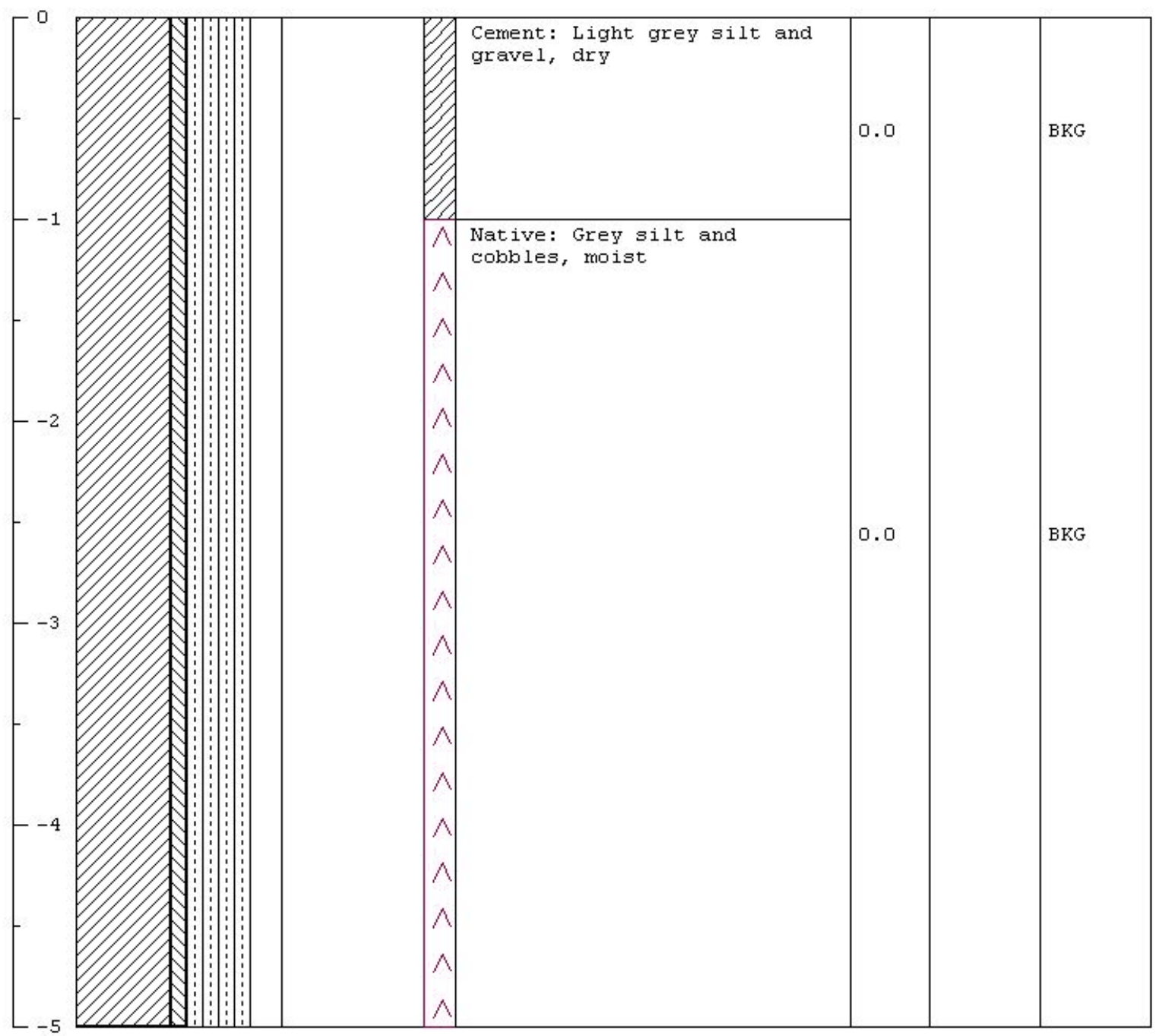


Page 1 of 1

\begin{tabular}{|c|c|c|c|c|c|c|c|c|}
\hline \multicolumn{5}{|c|}{$\begin{array}{l}\text { Project Hame: CAU } 383 \text { E-Tunnel Sites } \\
\text { Project Humber: DTO } 4-320 \\
\text { Borehole Humber: EMH } 2 \text { A } \\
\text { Logged By: M. MeLane } \\
\text { Drilled By: Boart-Longyear } \\
\text { Drilling Method: Rotosonic }\end{array}$} & \multicolumn{4}{|c|}{$\begin{array}{l}\text { Date Started: } 6 / 03 / 04 \\
\text { Date Completed: } 6 / 08 / 04 \\
\text { Elevation: } 6123.4 \\
\text { Horthing: } 887540.8 \\
\text { Easting: } 638332.6 \\
\text { Total Depth: } 45.5 \mathrm{ft} .\end{array}$} \\
\hline \multirow[b]{2}{*}{ 点出 } & \multirow[b]{2}{*}{ 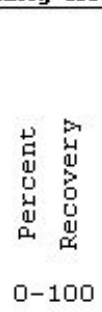 } & \multirow[b]{2}{*}{ 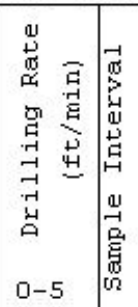 } & \multirow[b]{2}{*}{ 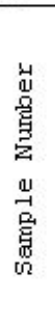 } & \multirow[b]{2}{*}{ 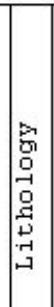 } & \multirow[b]{2}{*}{$\begin{array}{c}\text { Lithologic Description } \\
\text { Comments }\end{array}$} & Field & Screening & Results \\
\hline & & & & & & $\begin{array}{l}\text { VOCs } \\
\text { (ppm) }\end{array}$ & $\begin{array}{l}\text { Alpha } \\
\text { (dpm) }\end{array}$ & $\begin{array}{l}\text { Beta } \\
\text { (dpm) }\end{array}$ \\
\hline
\end{tabular}

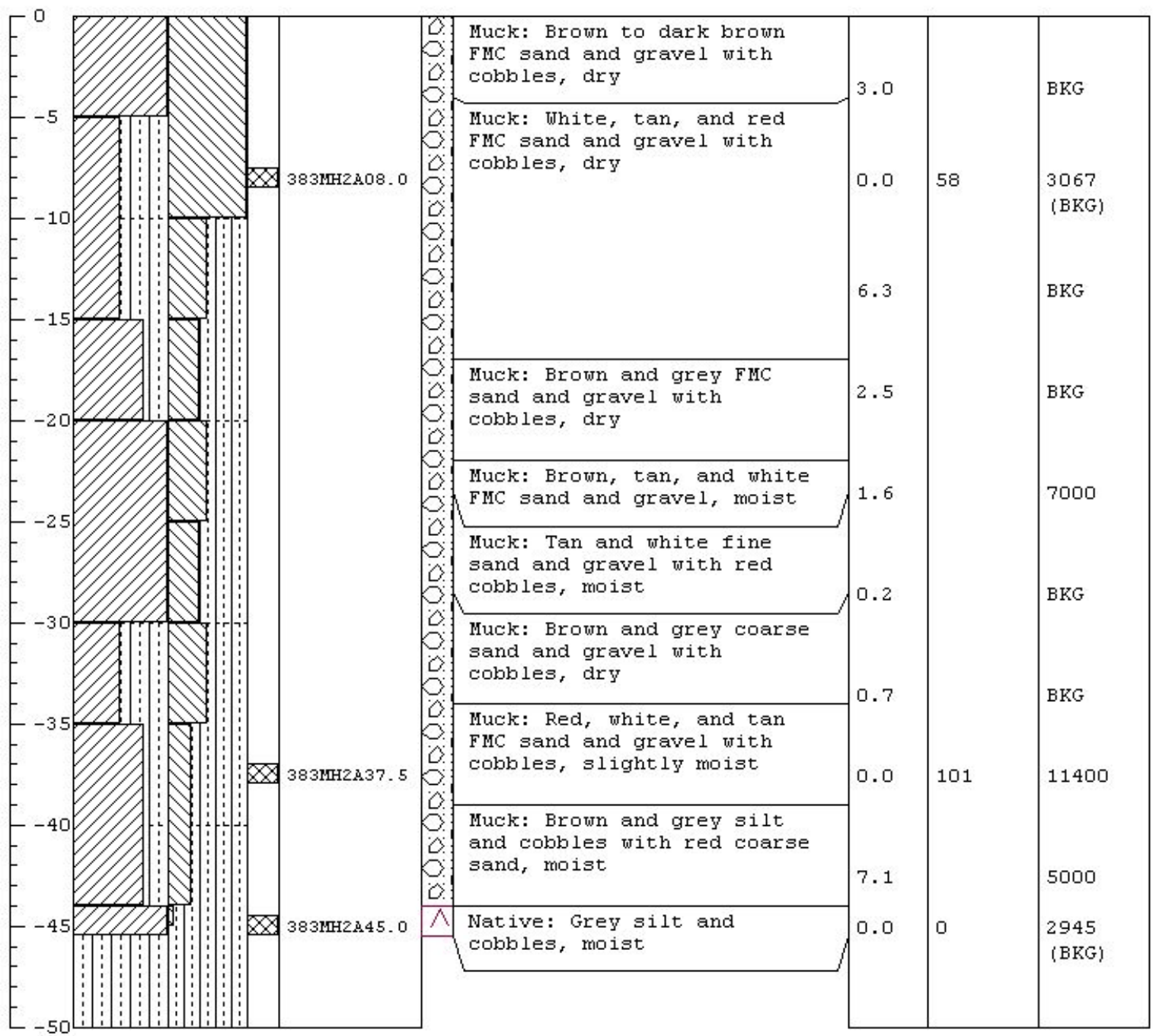


Page 1 of 1

\begin{tabular}{|l|l|}
\hline Project Name: CAU 383 E-Tunnel Sites & Date Started: $5 / 25 / 04$ \\
Project Number: DT04-320 & Date Completed: 5/25/04 \\
Borehole Number: EMHO3 & Elevation: 6131.4 \\
Logged By: M. McLane & Horthing: 887442.1 \\
Drilled By: Boart-Longyear & Easting: 638260.8 \\
Drilling Method: Rotosonic & Total Depth: 61.5 ft.
\end{tabular}

\begin{tabular}{|c|c|c|c|c|c|c|c|c|c|}
\hline \multirow[b]{2}{*}{ 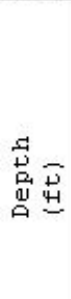 } & \multirow[b]{2}{*}{ 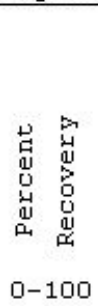 } & \multirow[b]{2}{*}{ 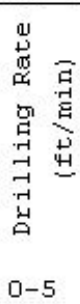 } & \multirow[b]{2}{*}{ 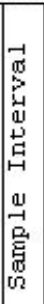 } & \multirow[b]{2}{*}{ 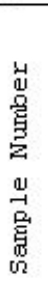 } & \multirow[b]{2}{*}{ 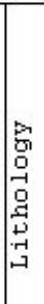 } & \multirow[b]{2}{*}{$\begin{array}{c}\text { Lithologic Description } \\
\text { Comments }\end{array}$} & \multicolumn{3}{|c|}{ Field Screening Results } \\
\hline & & & & & & & VOCs & $\begin{array}{l}\text { Alpha } \\
\text { (dpm) }\end{array}$ & $\begin{array}{l}\text { Beta } \\
\text { (dpm) }\end{array}$ \\
\hline
\end{tabular}

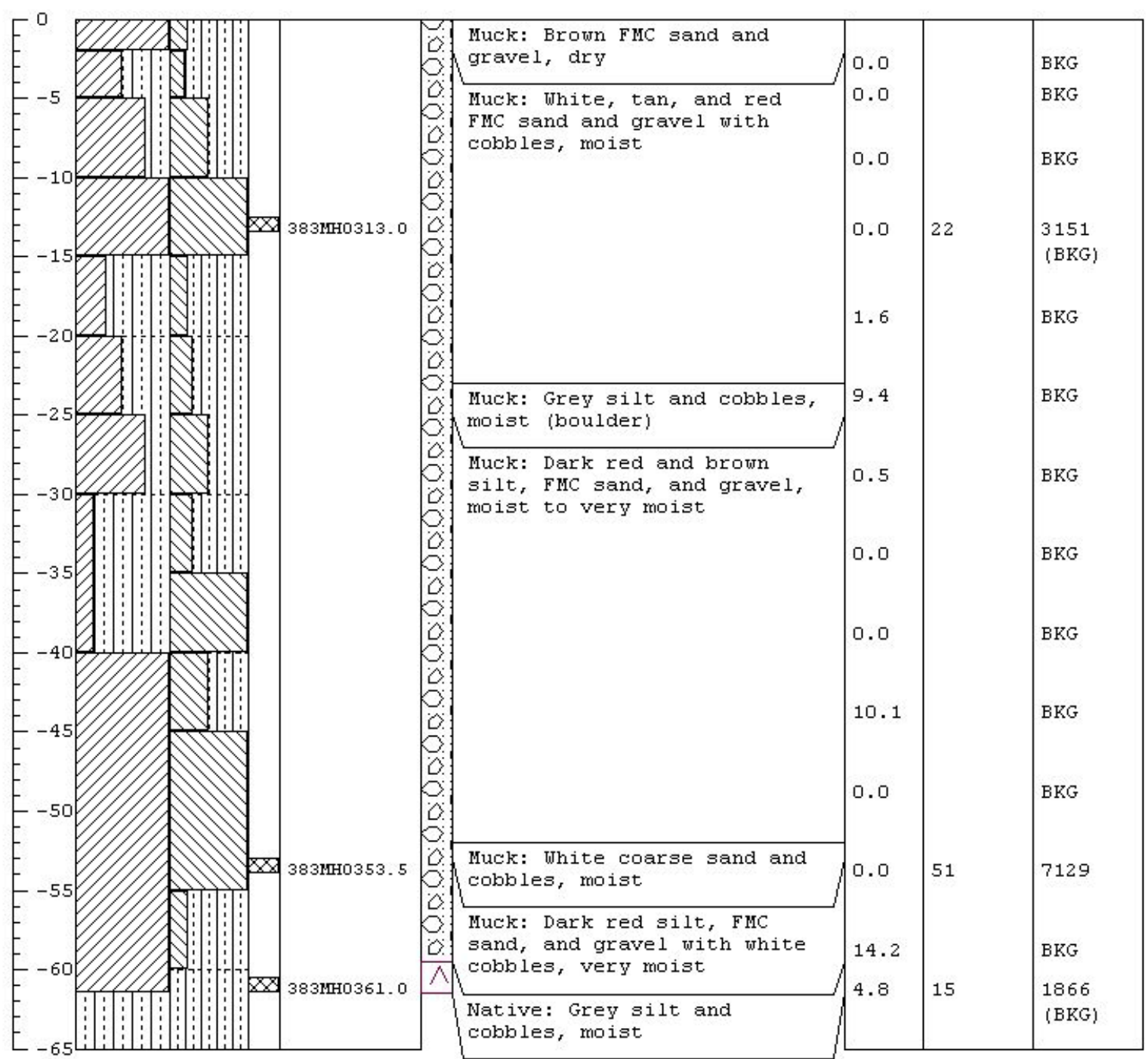


Page 1 of 1

\begin{tabular}{|c|c|c|c|c|c|c|c|c|}
\hline \multicolumn{5}{|c|}{$\begin{array}{l}\text { Project Hame: CAU } 383 \text { E-Tunnel sites } \\
\text { Project Humber: DTO } 4-320 \\
\text { Borehole Humber: EMHO } 4 \\
\text { Logged By: M. MeLane } \\
\text { Drilled By: Boart-Longyear } \\
\text { Drilling Method: Rotosonic }\end{array}$} & \multicolumn{4}{|c|}{$\begin{array}{l}\text { Date Started: } 5 / 22 / 04 \\
\text { Date Completed: } 5 / 22 / 04 \\
\text { Elevation: } 6115.4 \\
\text { Horthing: } 887429.3 \\
\text { Easting: } 638606.1 \\
\text { Total Depth: } 42.0 \mathrm{ft} .\end{array}$} \\
\hline \multirow[b]{2}{*}{ 营吾 } & \multirow[b]{2}{*}{ 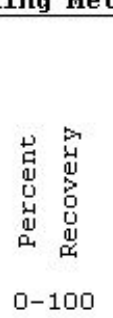 } & \multirow[b]{2}{*}{ 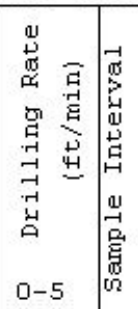 } & \multirow[b]{2}{*}{ 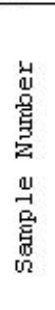 } & \multirow[b]{2}{*}{ 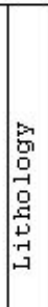 } & \multirow[b]{2}{*}{$\begin{array}{c}\text { Lithologic Description } \\
\text { Comments }\end{array}$} & Field & Screening & Results \\
\hline & & & & & & $\begin{array}{l}\text { VOCs } \\
\text { (ppm) }\end{array}$ & $\begin{array}{l}\text { Alpha } \\
\text { (dpm) }\end{array}$ & $\begin{array}{l}\text { Beta } \\
\text { (dpm) }\end{array}$ \\
\hline
\end{tabular}

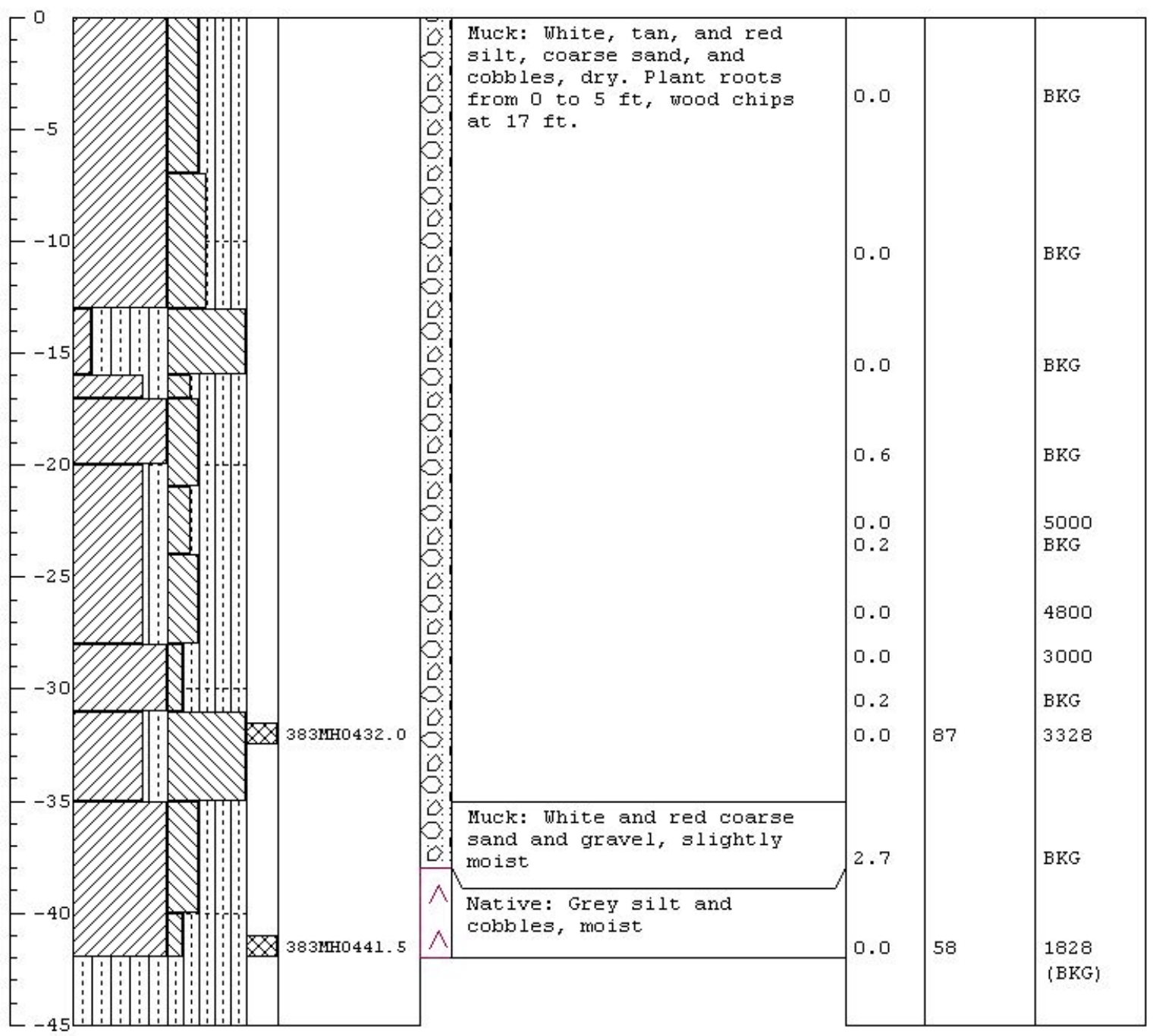


Page 1 of 2

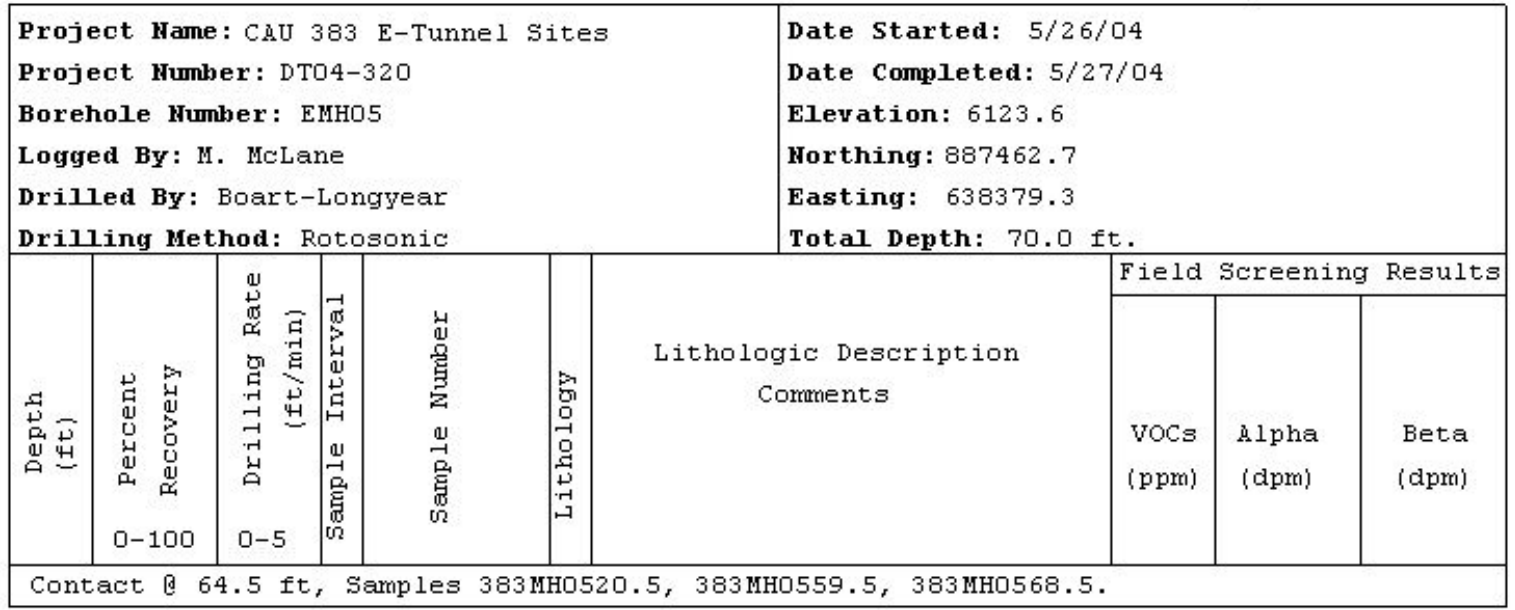

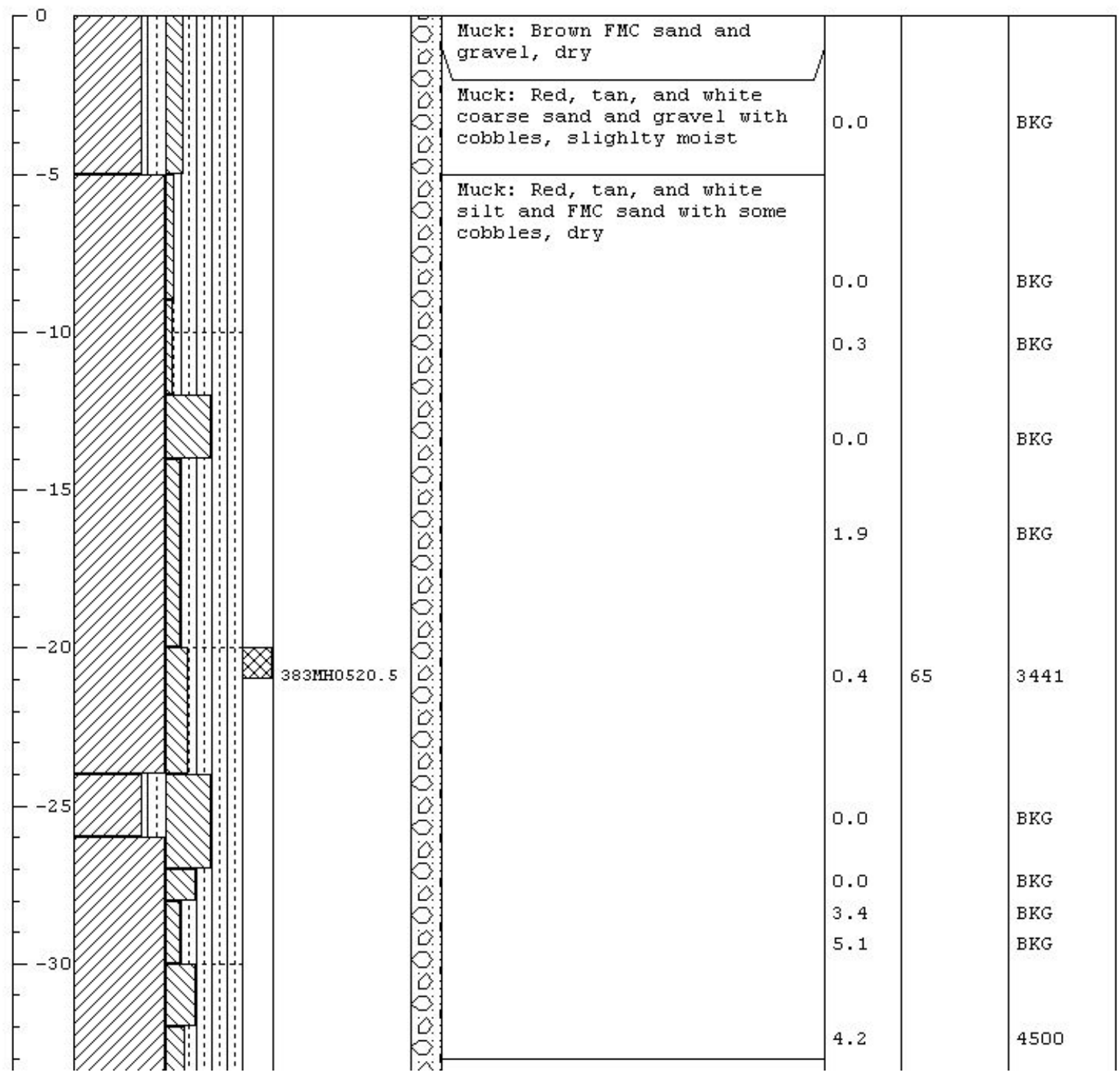


Page 2 of 2

\begin{tabular}{|c|c|c|c|c|c|c|c|c|c|}
\hline \multicolumn{3}{|c|}{ Borehole Number: } & \multicolumn{7}{|c|}{ EMHO5 } \\
\hline \multirow[b]{2}{*}{ 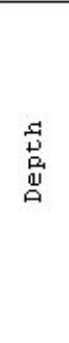 } & \multirow[b]{2}{*}{ 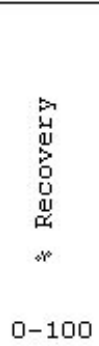 } & \multirow[b]{2}{*}{ 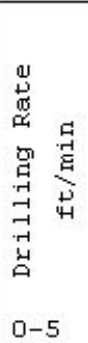 } & \multirow[b]{2}{*}{ 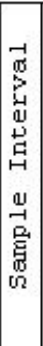 } & \multirow[b]{2}{*}{ 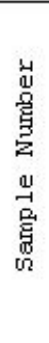 } & \multirow[b]{2}{*}{ 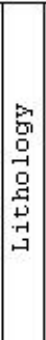 } & \multirow[b]{2}{*}{$\begin{array}{c}\text { Lithologic Description } \\
\text { Comments }\end{array}$} & \multicolumn{3}{|c|}{ Field Screening Results } \\
\hline & & & & & & & $\begin{array}{l}\text { VOCs } \\
\text { (ppm) }\end{array}$ & $\begin{array}{l}\text { Alpha } \\
\text { (dpm) }\end{array}$ & $\begin{array}{l}\text { Beta } \\
\text { (dpm) }\end{array}$ \\
\hline
\end{tabular}

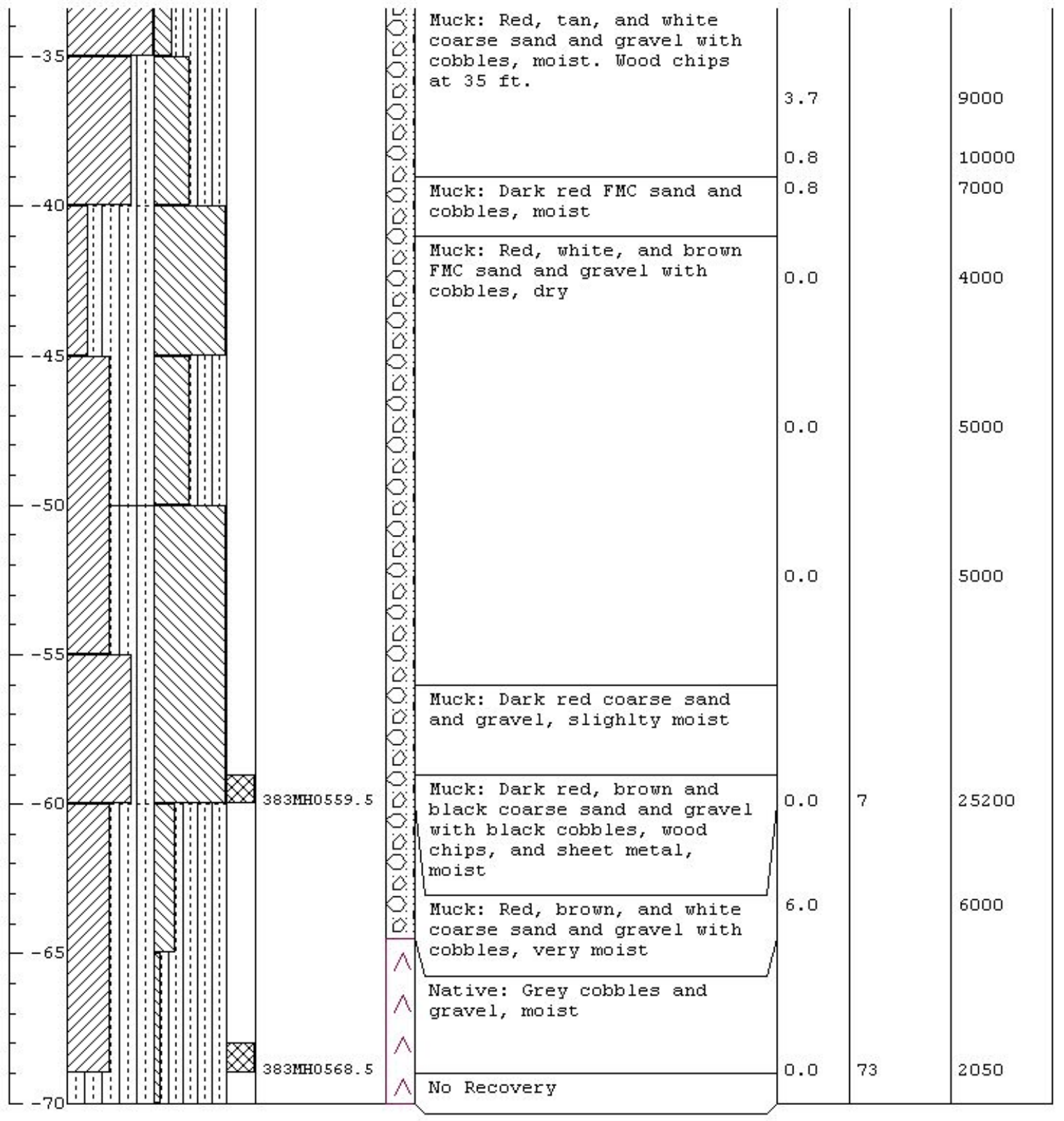


Page 1 of 1

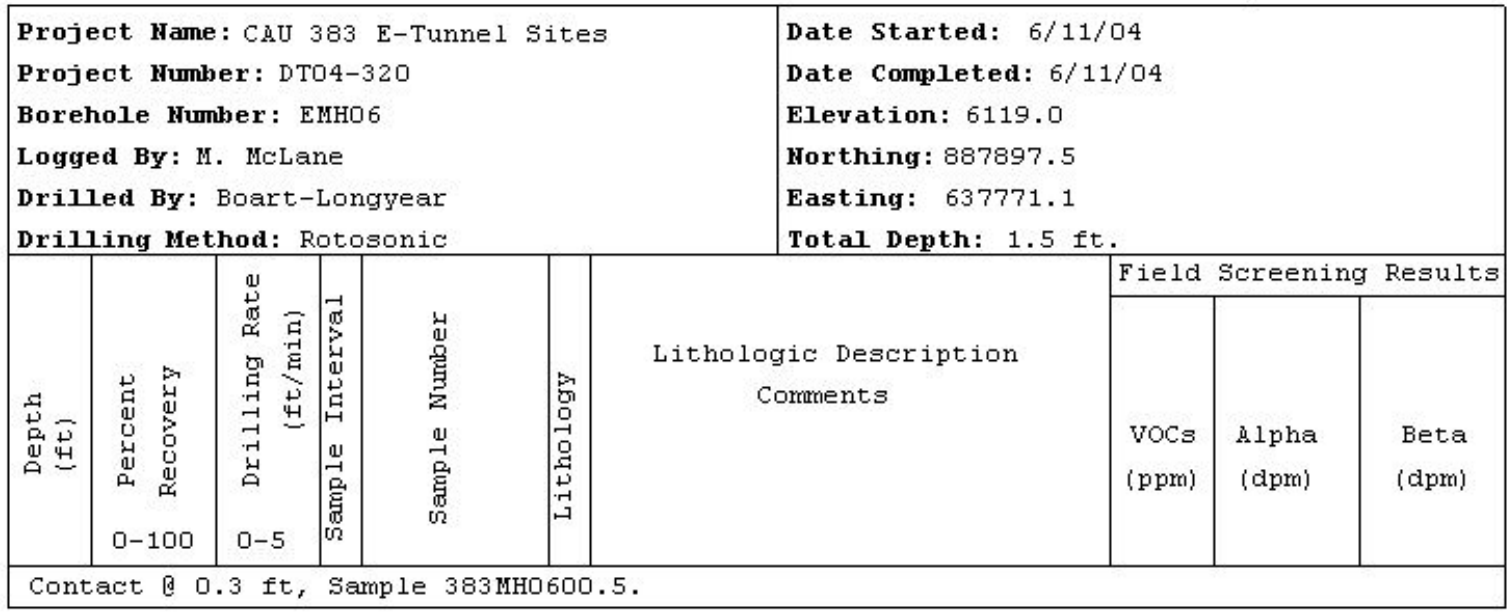

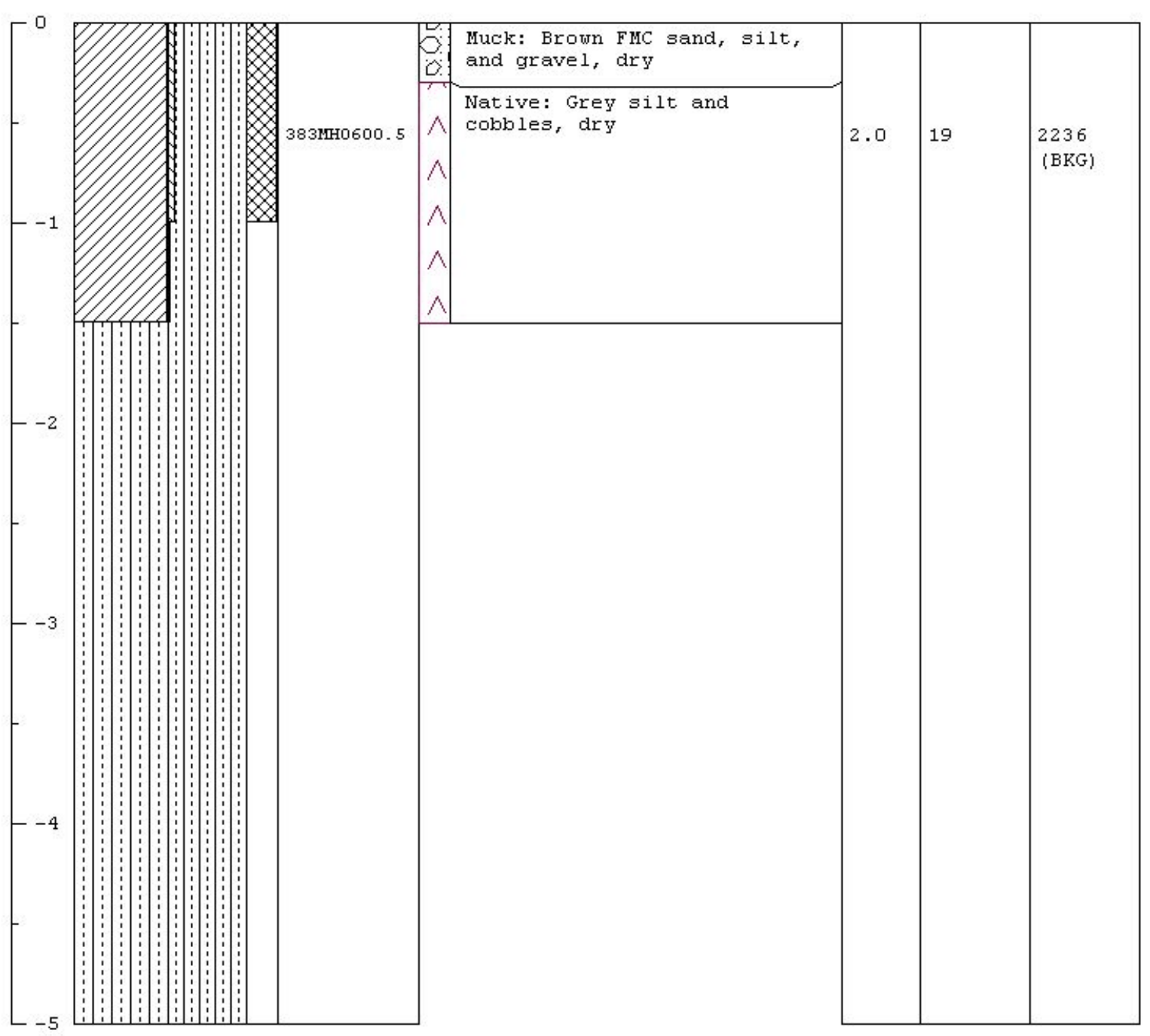


Page 1 of 2

\begin{tabular}{|c|c|c|c|c|c|c|c|c|c|}
\hline \multicolumn{6}{|c|}{$\begin{array}{l}\text { Project Hame: CAU } 383 \text { E-Tunnel sites } \\
\text { Project Humber: DTO4-320 } \\
\text { Borehole Humber: EMHO7 } \\
\text { Logged By: M. McLane } \\
\text { Drilled By: Boart-Longyear } \\
\text { Drilling Method: Rotosonic }\end{array}$} & \multicolumn{4}{|c|}{$\begin{array}{l}\text { Date Started: } 5 / 20 / 04 \\
\text { Date Completed: } 5 / 21 / 04 \\
\text { Elevation: } 6123.5 \\
\text { Northing: } 887475.6 \\
\text { Easting: } 638472.2 \\
\text { Total Depth: } 68 \mathrm{ft} .\end{array}$} \\
\hline \multirow[b]{2}{*}{ 点 } & \multirow[b]{2}{*}{ 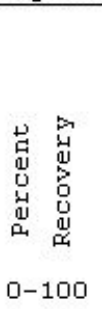 } & \multirow{2}{*}{\multicolumn{2}{|c|}{ 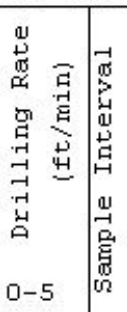 }} & \multirow[b]{2}{*}{ 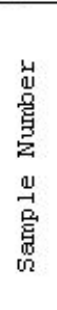 } & \multirow[b]{2}{*}{ 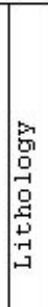 } & \multirow[b]{2}{*}{$\begin{array}{c}\text { Lithologic Description } \\
\text { Comments }\end{array}$} & Field & Screening & Results \\
\hline & & & & & & & $\begin{array}{l}\text { VOCs } \\
\text { (ppm) }\end{array}$ & $\begin{array}{l}\text { Alpha } \\
\text { (dpm) }\end{array}$ & $\begin{array}{l}\text { Beta } \\
\text { (dpm) }\end{array}$ \\
\hline
\end{tabular}

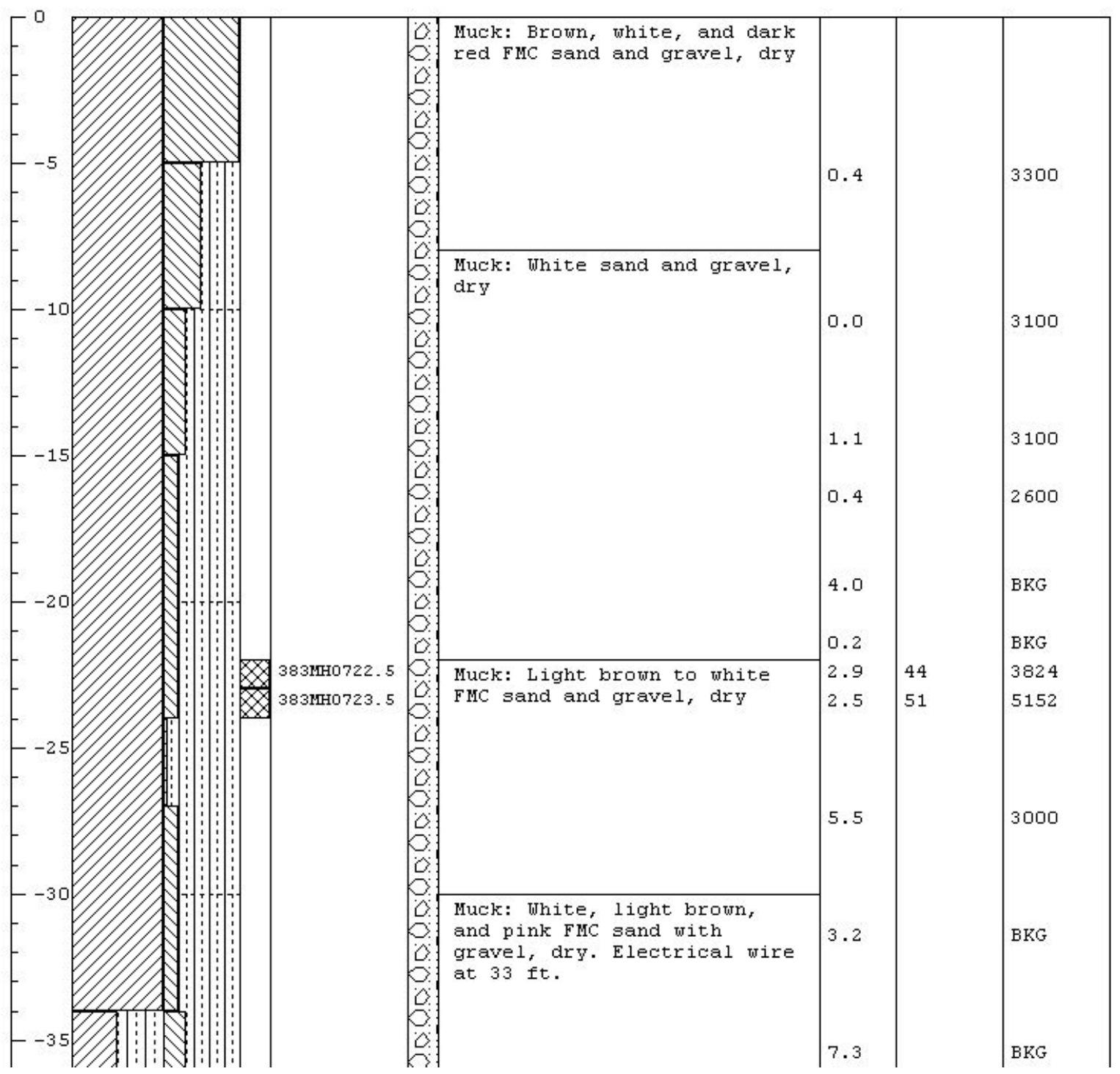




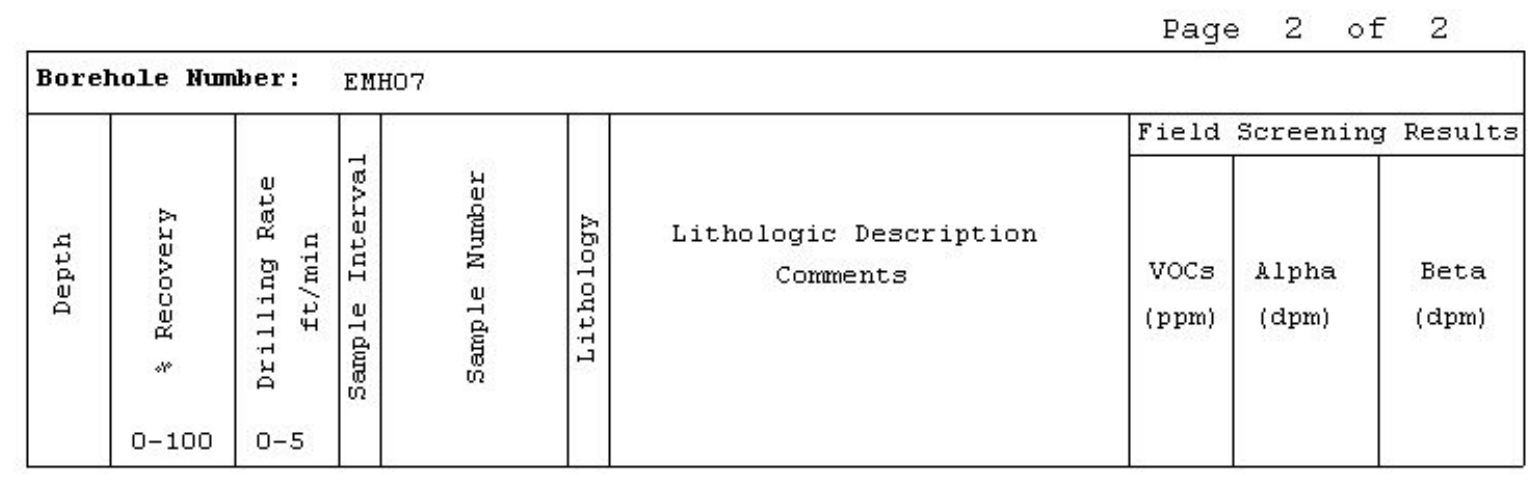

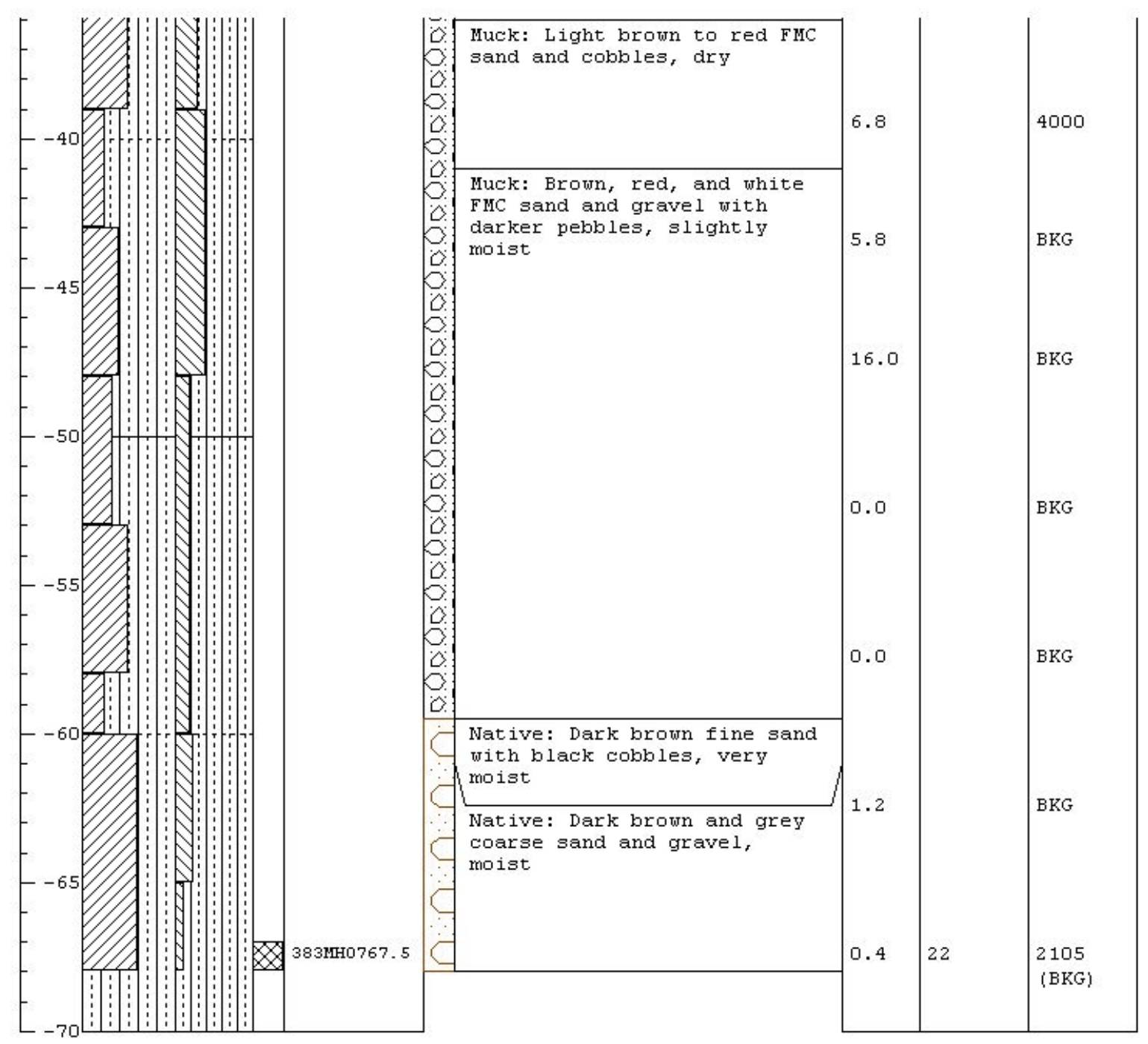


Page 1 of 1

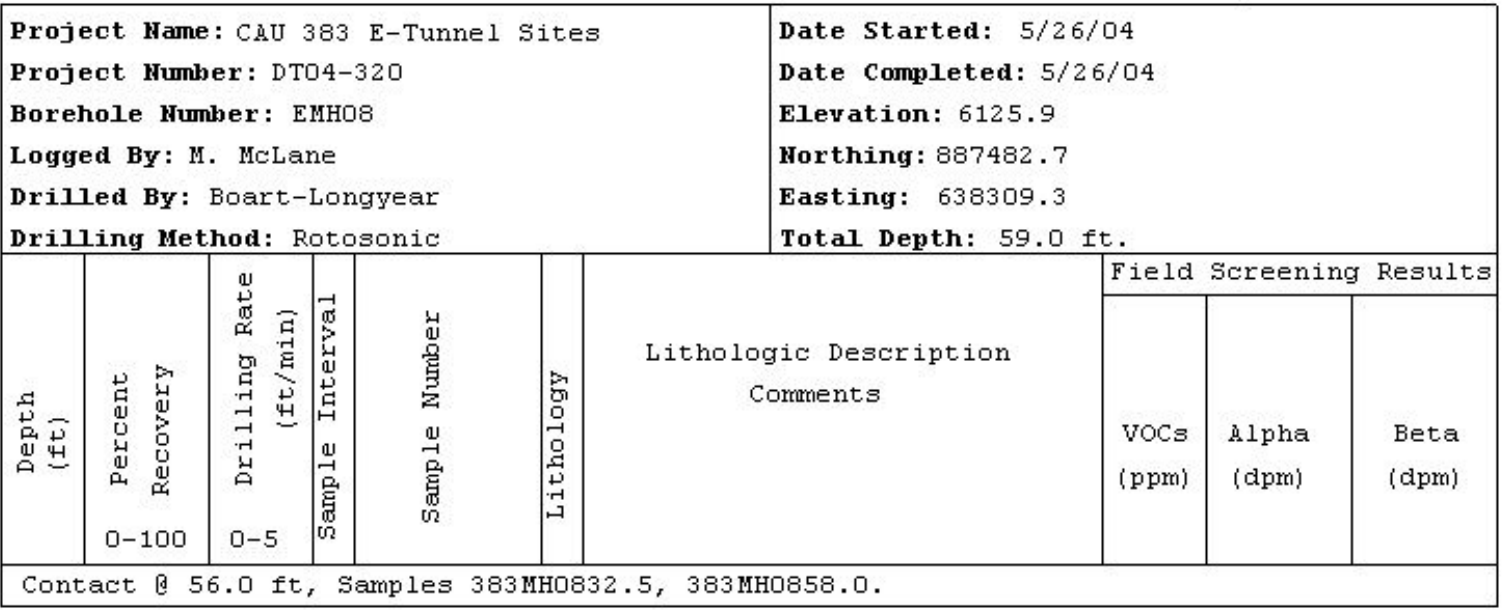

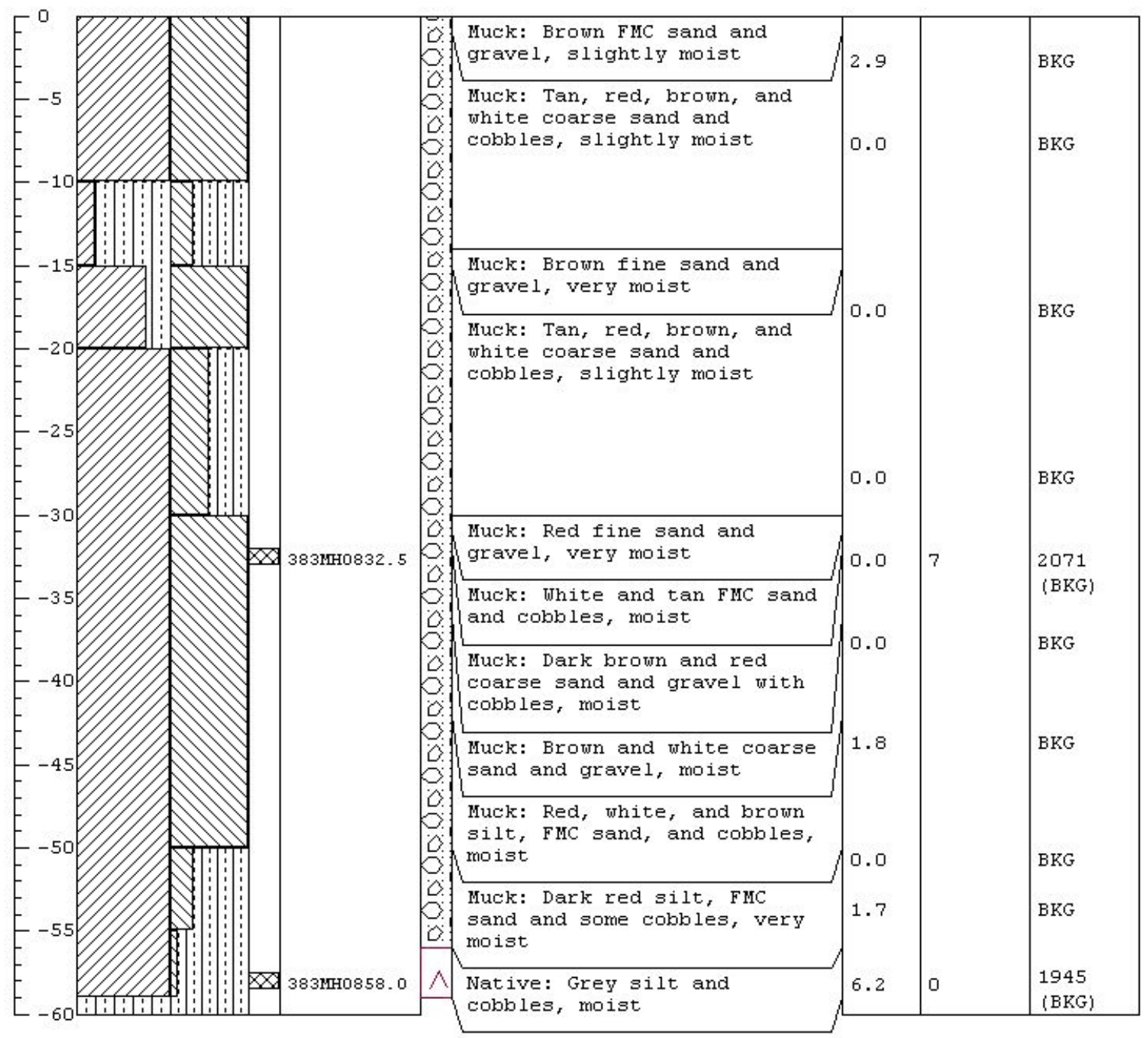


Project Name: CAU 383 E-Tunnel sites

Project Kumber: DTO4-320

Borehole Number: EMHOg

Logged BY: $M$. McLane

Drilled By: Boart-Longyear

Drilling Method: Rotosonic

\begin{tabular}{|c|c|c|c|c|c|c|c|c|c|}
\hline \multirow[b]{2}{*}{ 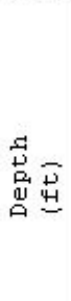 } & \multirow[b]{2}{*}{ 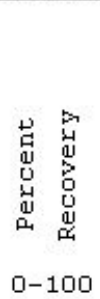 } & \multirow[b]{2}{*}{ 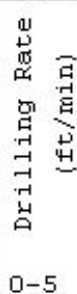 } & \multirow[b]{2}{*}{ 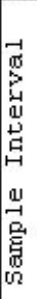 } & \multirow[b]{2}{*}{ 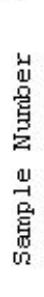 } & \multirow[b]{2}{*}{ 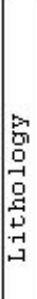 } & \multirow[b]{2}{*}{$\begin{array}{c}\text { Lithologic Description } \\
\text { Comments }\end{array}$} & \multicolumn{3}{|c|}{ Field Screening Results } \\
\hline & & & & & & & $\begin{array}{l}\text { VOCs } \\
\text { (ppm) }\end{array}$ & $\begin{array}{l}\text { Alpha } \\
\text { (dpm) }\end{array}$ & $\begin{array}{l}\text { Beta } \\
\text { (dpm) }\end{array}$ \\
\hline
\end{tabular}

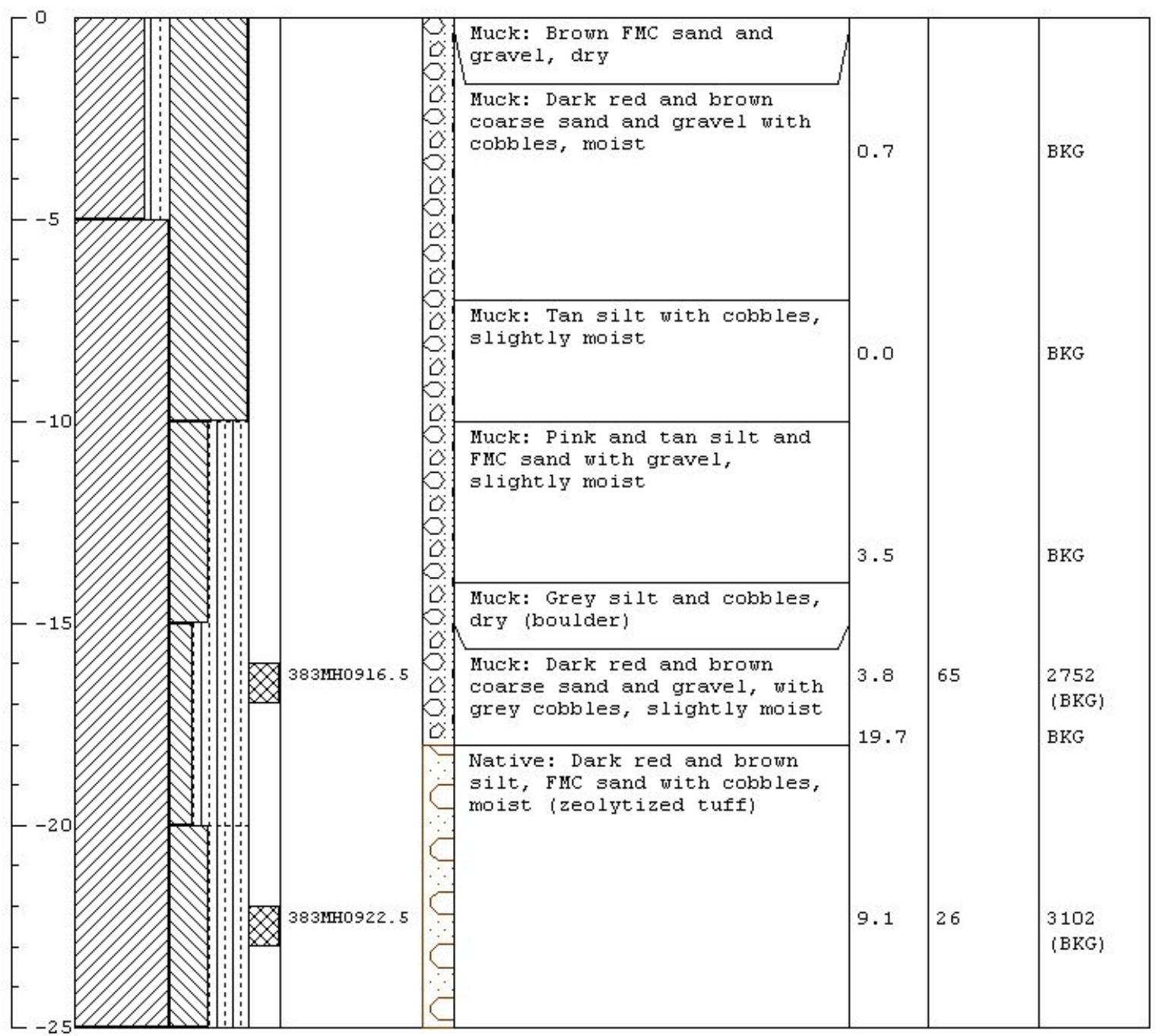


Page 1 of 1

Project Hame: CAU 383 E-Tunnel Sites

Project Number: DT04-320

Borehole Number: EMH10

Logged By: $M$. McLane

Drilled By: Boart-Longyear Drilling Method: Rotosonic

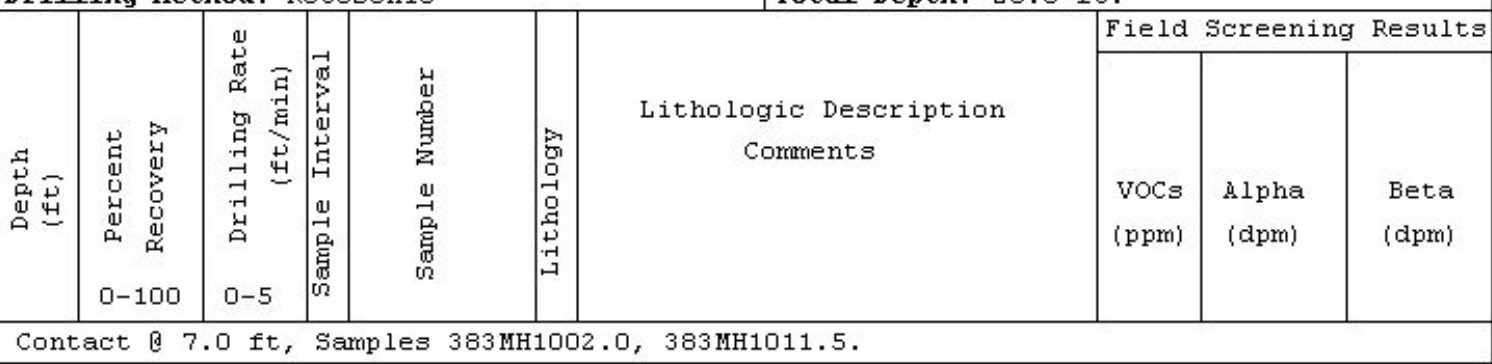

Date Started: $5 / 22 / 04$

Date Completed: $5 / 22 / 04$

Elevation: 6120.8

Horthing: 887395.0

Easting: 638571.8

Total Depth: $20.0 \mathrm{ft}$.

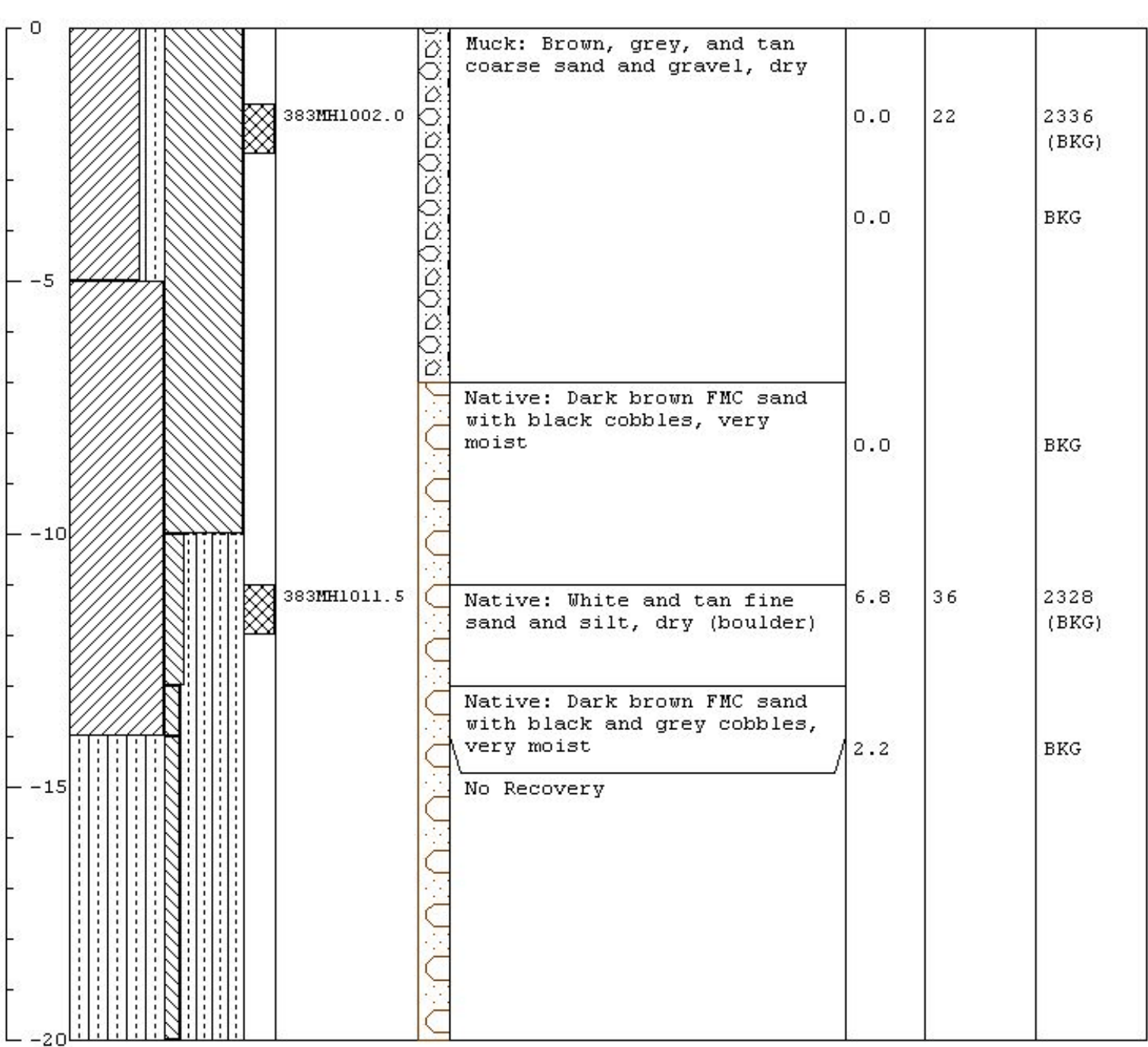


Page 1 of 1

\begin{tabular}{|c|c|c|c|c|c|c|c|c|}
\hline \multicolumn{5}{|c|}{$\begin{array}{l}\text { Project Hame: CAU } 383 \text { E-Tunnel sites } \\
\text { Project Humber: DTO } 4-320 \\
\text { Borehole Humber: EMH } 11 \\
\text { Logged By: M. McLane } \\
\text { Drilled By: Boart-Longyear } \\
\text { Drilling Method: Rotosonic }\end{array}$} & \multicolumn{4}{|c|}{$\begin{array}{l}\text { Date Started: } 6 / 11 / 04 \\
\text { Date Completed: } 6 / 11 / 04 \\
\text { Elevation: } 6116.5 \\
\text { Horthing: } 887858.6 \\
\text { Easting: } 637782.5 \\
\text { Total Depth: } 4.5 \mathrm{ft} .\end{array}$} \\
\hline \multirow[b]{2}{*}{ 窇出 } & \multirow[b]{2}{*}{ 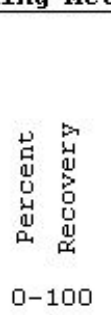 } & \multirow[b]{2}{*}{ 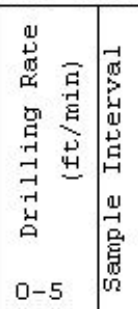 } & \multirow[b]{2}{*}{ 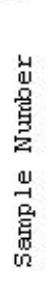 } & \multirow[b]{2}{*}{ 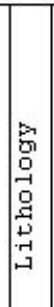 } & \multirow[b]{2}{*}{$\begin{array}{c}\text { Lithologic Description } \\
\text { Comments }\end{array}$} & \multicolumn{3}{|c|}{ Field Screening Results } \\
\hline & & & & & & $\begin{array}{l}\text { VOCs } \\
\text { (ppm) }\end{array}$ & $\begin{array}{l}\text { Alpha } \\
\text { (dpm) }\end{array}$ & $\begin{array}{l}\text { Beta } \\
\text { (dpm) }\end{array}$ \\
\hline
\end{tabular}

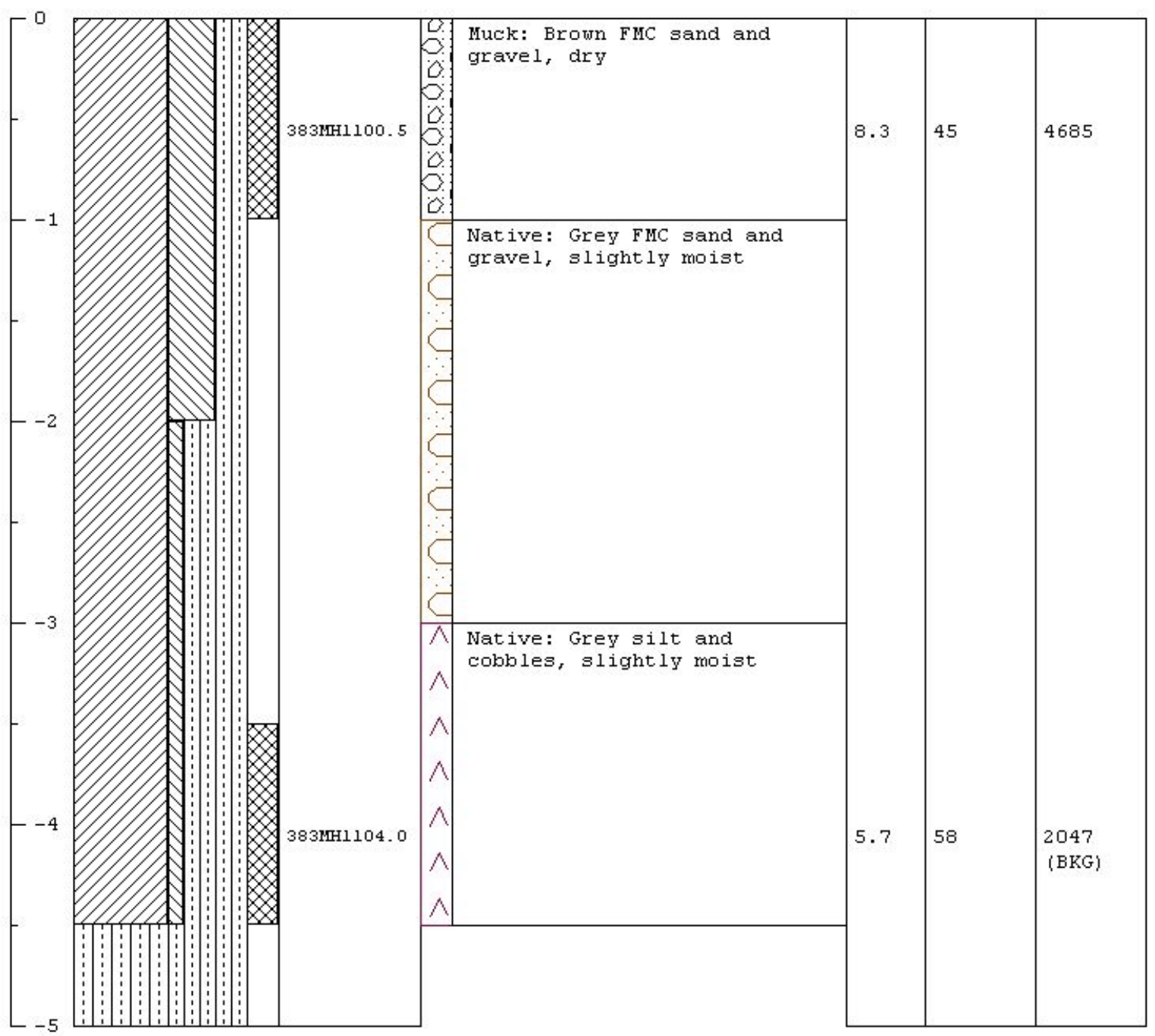


Page 1 of 1

\begin{tabular}{|c|c|c|c|c|c|c|c|c|}
\hline \multicolumn{5}{|c|}{$\begin{array}{l}\text { Project Hame: CAU } 383 \text { E-Tunnel Sites } \\
\text { Project Humber: DTO } 4-320 \\
\text { Borehole Humber: EMH } 12 \\
\text { Logged By: M. McLane } \\
\text { Drilled By: Boart-Longyear } \\
\text { Drilling Method: Rotosonic }\end{array}$} & \multicolumn{4}{|c|}{$\begin{array}{l}\text { Date Started: } 5 / 23 / 04 \\
\text { Date Completed: } 5 / 23 / 04 \\
\text { Elevation: } 6124.1 \\
\text { Northing: } 887374.4 \\
\text { Easting: } 638473.4 \\
\text { Total Depth: } 25.5 \mathrm{ft} .\end{array}$} \\
\hline \multirow[b]{2}{*}{ 秀 } & \multirow[b]{2}{*}{ 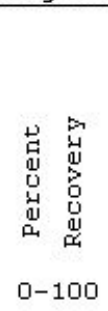 } & \multirow[b]{2}{*}{ 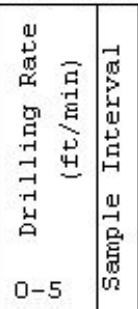 } & \multirow[b]{2}{*}{ 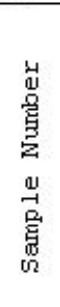 } & \multirow[b]{2}{*}{ 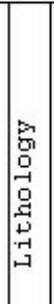 } & \multirow[b]{2}{*}{$\begin{array}{c}\text { Lithologic Description } \\
\text { Comments }\end{array}$} & Field & Screening & Results \\
\hline & & & & & & $\begin{array}{l}\text { VOCs } \\
\text { (ppm) }\end{array}$ & $\begin{array}{l}\text { Alpha } \\
\text { (dpm) }\end{array}$ & $\begin{array}{l}\text { Beta } \\
\text { (dpm) }\end{array}$ \\
\hline
\end{tabular}

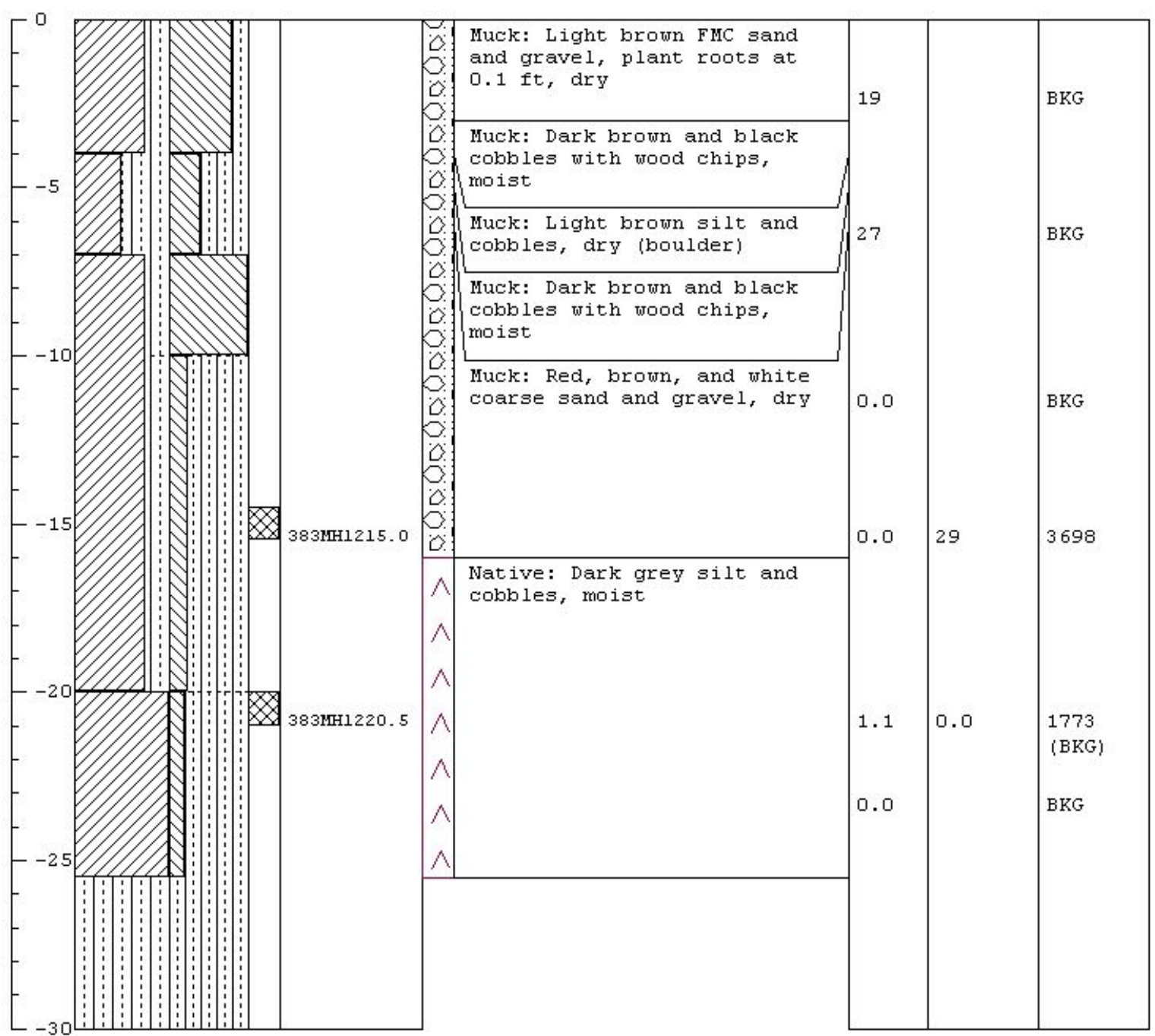


Page 1 of 1

\begin{tabular}{|c|c|c|c|c|c|c|c|c|}
\hline \multicolumn{5}{|c|}{$\begin{array}{l}\text { Project Hame: CAU } 383 \text { E-Tunnel Sites } \\
\text { Project Humber: DTO } 4-320 \\
\text { Borehole Humber: EMH } 13 \\
\text { Logged By: M. McLane } \\
\text { Drilled By: Boart-Longyear } \\
\text { Drilling Method: Rotosonic }\end{array}$} & \multicolumn{4}{|c|}{$\begin{array}{l}\text { Date Started: } 5 / 25 / 04 \\
\text { Date Completed: } 5 / 25 / 04 \\
\text { Elevation: } 6136.5 \\
\text { Horthing: } 887492.0 \\
\text { Easting: } 638209.0 \\
\text { Total Depth: } 21.0 \mathrm{ft} .\end{array}$} \\
\hline \multirow[b]{2}{*}{ 窝 } & \multirow[b]{2}{*}{ 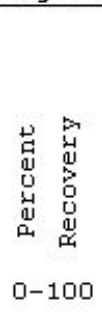 } & \multirow[b]{2}{*}{ 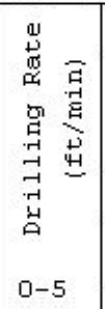 } & \multirow[b]{2}{*}{ 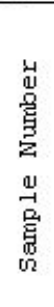 } & \multirow[b]{2}{*}{ 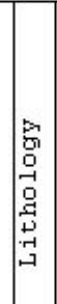 } & \multirow[b]{2}{*}{$\begin{array}{c}\text { Lithologic Description } \\
\text { Corments }\end{array}$} & Field & Screening & Results \\
\hline & & & & & & $\begin{array}{l}\text { VOCs } \\
\text { (ppm) }\end{array}$ & $\begin{array}{l}\text { Alpha } \\
\text { (dpm) }\end{array}$ & $\begin{array}{l}\text { Beta } \\
\text { (dpm) }\end{array}$ \\
\hline
\end{tabular}

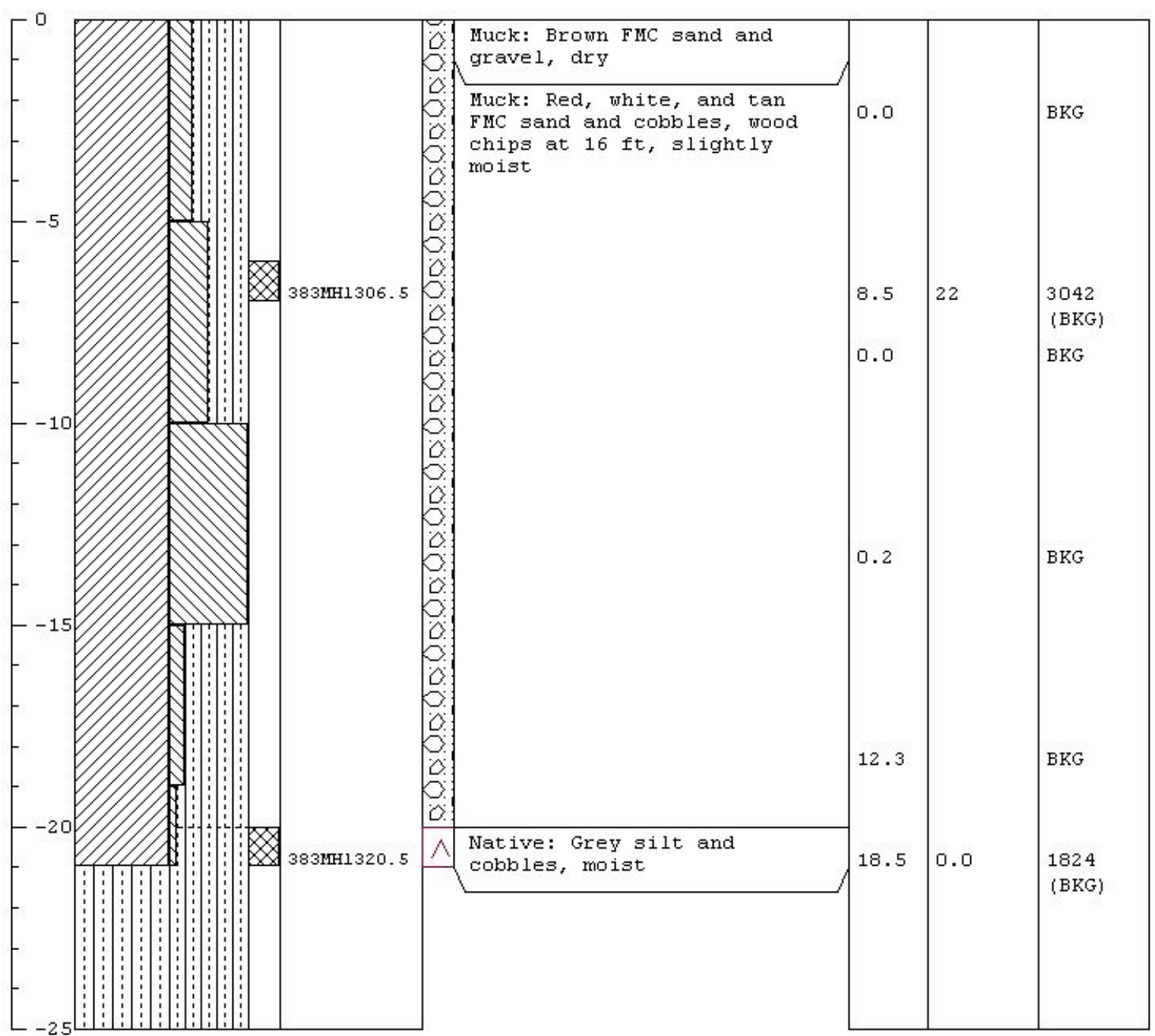


Page 1 of 1

\begin{tabular}{|c|c|c|c|c|c|c|c|c|}
\hline \multicolumn{5}{|c|}{$\begin{array}{l}\text { Project Hame: CAU } 383 \text { E-Tunnel Sites } \\
\text { Project Humber: DTO } 4-320 \\
\text { Borehole Humber: EMH } 14 \\
\text { Logged By: M. McLane } \\
\text { Drilled By: Boart-Longyear } \\
\text { Drilling Method: Rotosonic }\end{array}$} & \multicolumn{4}{|c|}{$\begin{array}{l}\text { Date Started: } 5 / 20 / 04 \\
\text { Date Completed: } 5 / 20 / 04 \\
\text { Elevation: } 6129.7 \\
\text { Horthing: } 887613.0 \\
\text { Easting: } 638322.2 \\
\text { Total Depth: } 22.5 \mathrm{ft} .\end{array}$} \\
\hline \multirow[b]{2}{*}{ 超 } & \multirow[b]{2}{*}{ 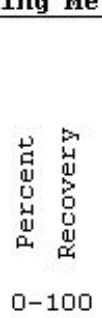 } & \multirow[b]{2}{*}{ 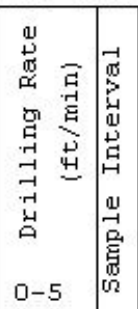 } & \multirow[b]{2}{*}{ 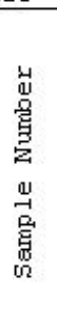 } & \multirow[b]{2}{*}{ 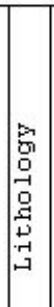 } & \multirow[b]{2}{*}{$\begin{array}{c}\text { Lithologic Description } \\
\text { Comments }\end{array}$} & Field & Screening & Results \\
\hline & & & & & & $\begin{array}{l}\text { VOCs } \\
\text { (ppm) }\end{array}$ & $\begin{array}{l}\text { Alpha } \\
\text { (dpm) }\end{array}$ & $\begin{array}{l}\text { Beta } \\
\text { (dpm) }\end{array}$ \\
\hline
\end{tabular}

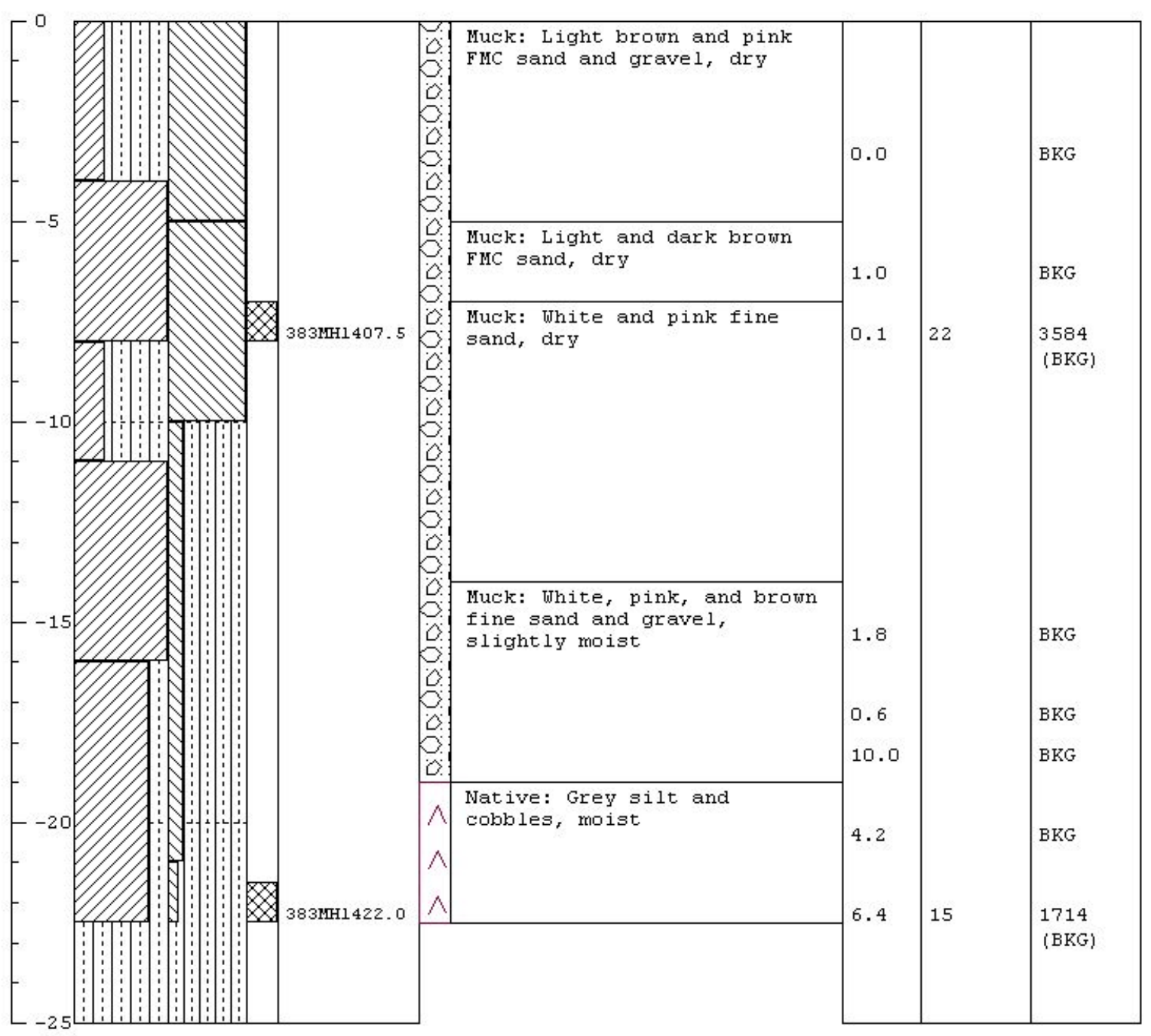


Page 1 of 1

\begin{tabular}{|c|c|c|c|c|c|c|c|c|}
\hline \multicolumn{5}{|c|}{$\begin{array}{l}\text { Project Hame: CAU } 383 \text { E-Tunnel Sites } \\
\text { Project Humber: DTO } 4-320 \\
\text { Borehole Number: EMH } 15 \\
\text { Logged By: M. MeLane } \\
\text { Drilled By: Boart-Longyear } \\
\text { Drilling Method: Rotosonic }\end{array}$} & \multicolumn{4}{|c|}{$\begin{array}{l}\text { Date Started: } 5 / 24 / 04 \\
\text { Date Completed: } 5 / 24 / 04 \\
\text { Elevation: } 6133.6 \\
\text { Horthing: } 887479.0 \\
\text { Easting: } 637912.1 \\
\text { Total Depth: } 4.0 \mathrm{ft} .\end{array}$} \\
\hline \multirow[b]{2}{*}{ 我 } & \multirow[b]{2}{*}{ 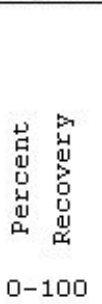 } & \multirow[b]{2}{*}{ 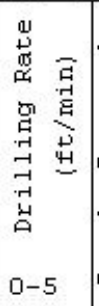 } & \multirow[b]{2}{*}{ 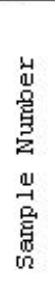 } & \multirow[b]{2}{*}{ 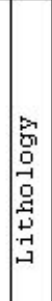 } & \multirow[b]{2}{*}{$\begin{array}{c}\text { Lithologic Description } \\
\text { Comments }\end{array}$} & \multirow[b]{2}{*}{\begin{tabular}{|l} 
Field \\
\\
voCs \\
(ppm)
\end{tabular}} & Screening & Results \\
\hline & & & & & & & $\begin{array}{l}\text { Alpha } \\
\text { (dpm) }\end{array}$ & $\begin{array}{l}\text { Beta } \\
\text { (dpm) }\end{array}$ \\
\hline
\end{tabular}

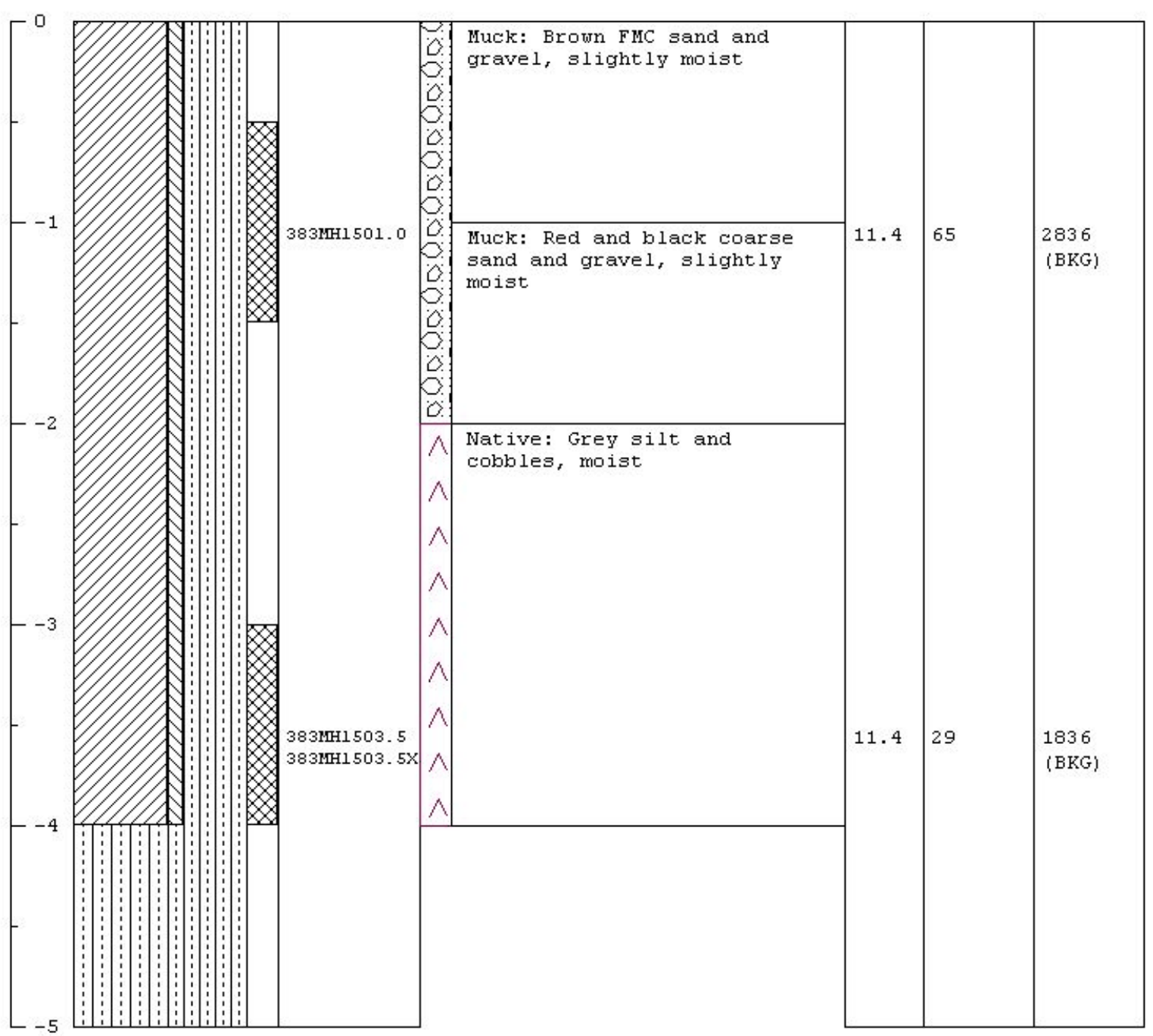


Page 1 of 1

\begin{tabular}{|c|c|c|c|c|c|c|c|c|}
\hline \multicolumn{5}{|c|}{$\begin{array}{l}\text { Project Hame: CAU } 383 \text { E-Tunnel Sites } \\
\text { Project Humber: DTO } 4-320 \\
\text { Borehole Humber: EMH16 } \\
\text { Logged By: M. McLane } \\
\text { Drilled By: Boart-Longyear } \\
\text { Drilling Method: Rotosonic }\end{array}$} & \multicolumn{4}{|c|}{$\begin{array}{l}\text { Date Started: } 6 / 11 / 04 \\
\text { Date Completed: } 6 / 11 / 04 \\
\text { Elevation: } 6127.8 \\
\text { Horthing: } 887726.3 \\
\text { Easting: } 638178.0 \\
\text { Total Depth: } 19.5 \mathrm{ft} .\end{array}$} \\
\hline \multirow[b]{2}{*}{ 空点 } & \multirow[b]{2}{*}{ 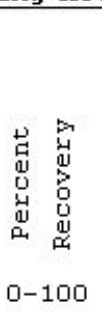 } & \multirow[b]{2}{*}{ 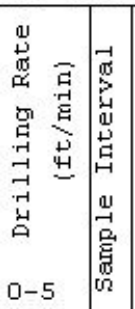 } & \multirow[b]{2}{*}{ 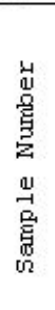 } & \multirow[b]{2}{*}{ 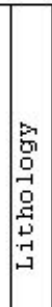 } & \multirow[b]{2}{*}{$\begin{array}{c}\text { Lithologic Description } \\
\text { Comments }\end{array}$} & Field & Screening & Results \\
\hline & & & & & & $\begin{array}{l}\text { VOCs } \\
\text { (ppm) }\end{array}$ & $\begin{array}{l}\text { Alpha } \\
\text { (dpm) }\end{array}$ & $\begin{array}{l}\text { Beta } \\
\text { (dpm) }\end{array}$ \\
\hline
\end{tabular}

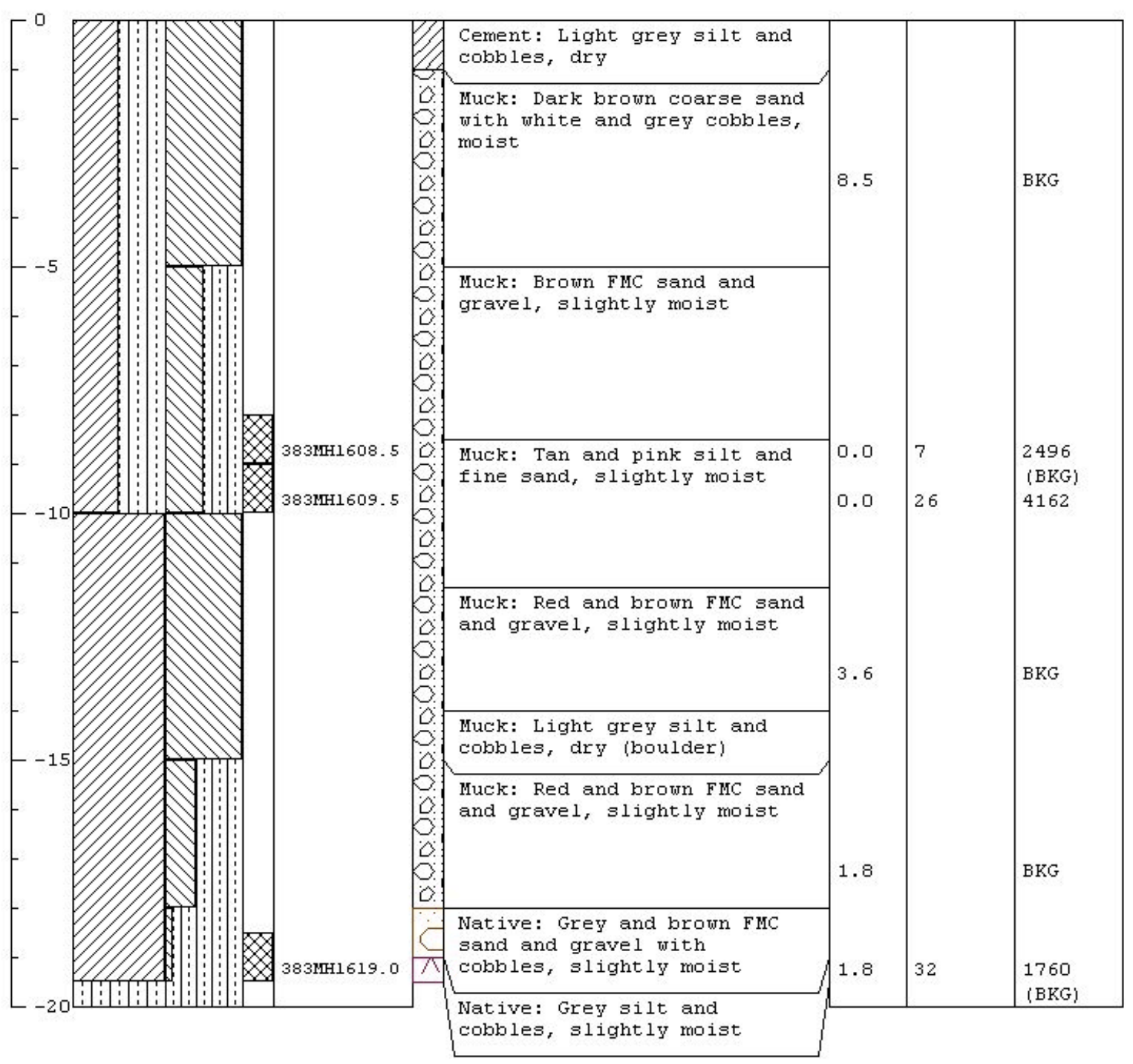


Page 1 of 1

\begin{tabular}{|c|c|c|c|c|c|c|c|c|}
\hline \multicolumn{5}{|c|}{$\begin{array}{l}\text { Project Hame: CAU } 383 \text { E-Tunnel Sites } \\
\text { Project Humber: DTO } 4-320 \\
\text { Borehole Humber: EMH } 17 \\
\text { Logged By: M. McLane } \\
\text { Drilled By: Boart-Longyear } \\
\text { Drilling Method: Rotosonic }\end{array}$} & \multicolumn{4}{|c|}{$\begin{array}{l}\text { Date Started: } 5 / 21 / 04 \\
\text { Date Completed: } 5 / 22 / 04 \\
\text { Elevation: } 6122.8 \\
\text { Horthing: } 887435.9 \\
\text { Easting: } 638653.1 \\
\text { Total Depth: } 36.5 \mathrm{ft} . \\
\end{array}$} \\
\hline \multirow[b]{2}{*}{ 总 } & \multirow[b]{2}{*}{ 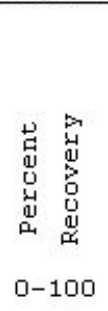 } & \multirow[b]{2}{*}{ 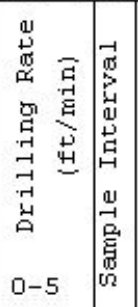 } & \multirow[b]{2}{*}{ 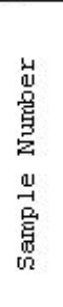 } & \multirow[b]{2}{*}{ 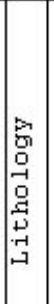 } & \multirow[b]{2}{*}{$\begin{array}{c}\text { Lithologic Description } \\
\text { Comments }\end{array}$} & Field & Screening & Results \\
\hline & & & & & & $\begin{array}{l}\text { VOCs } \\
\text { (ppm) }\end{array}$ & $\begin{array}{l}\text { Alpha } \\
\text { (dpm) }\end{array}$ & $\begin{array}{r}\text { Beta } \\
\text { (dpm) }\end{array}$ \\
\hline
\end{tabular}

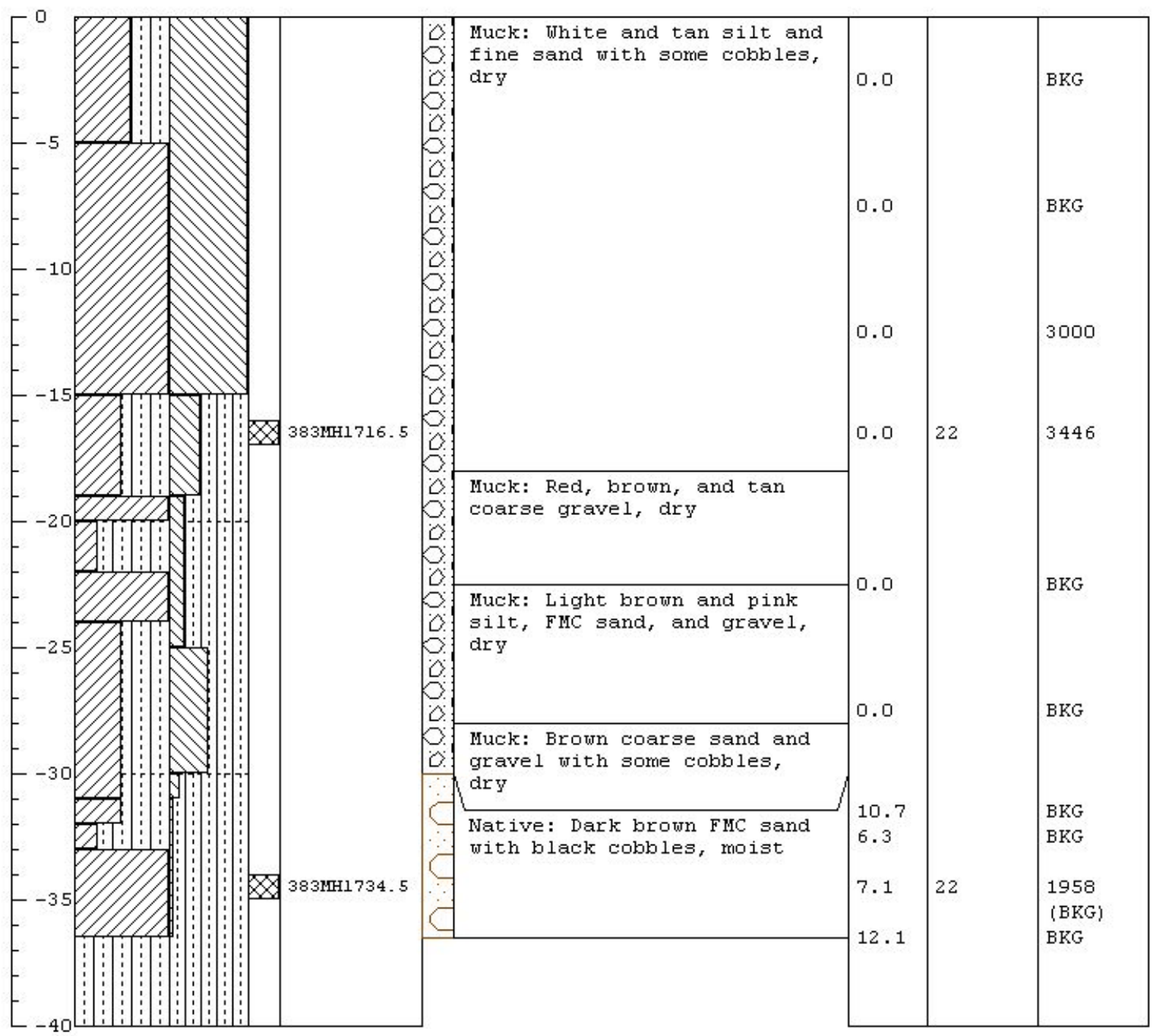


Page 1 of 1

Project Hame: CAU 383 E-Tunnel Sites

Project Humber: DTO $4-320$

Borehole Number: EMH18

Logged By: $M$. McLane

Drilled By: Boart-Longyear

Drilling Method: Rotosonic
Date Started: $5 / 23 / 04$

Date Completed: $5 / 23 / 04$

Elevation : 6129.9

Horthing: 887333.0

Easting: 638343.0

Total Depth: 12.0 ft.

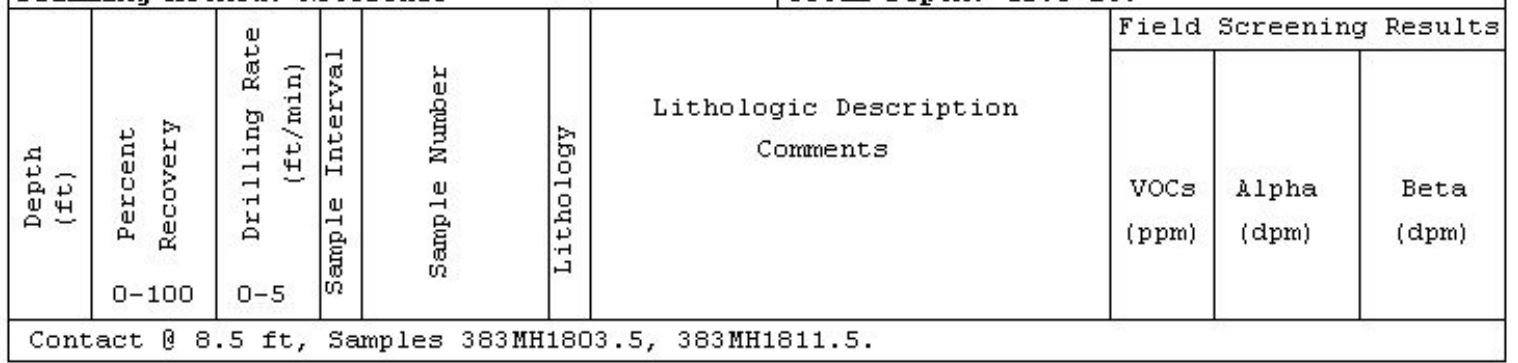


Page 1 of 1

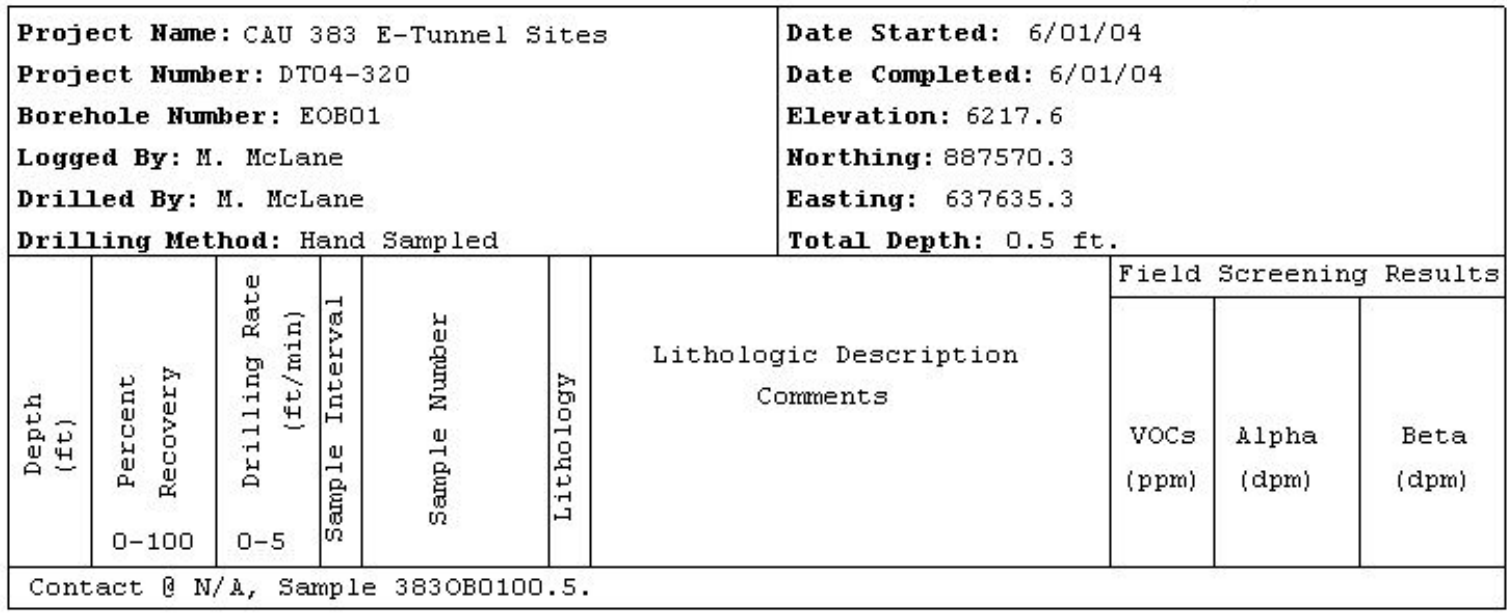

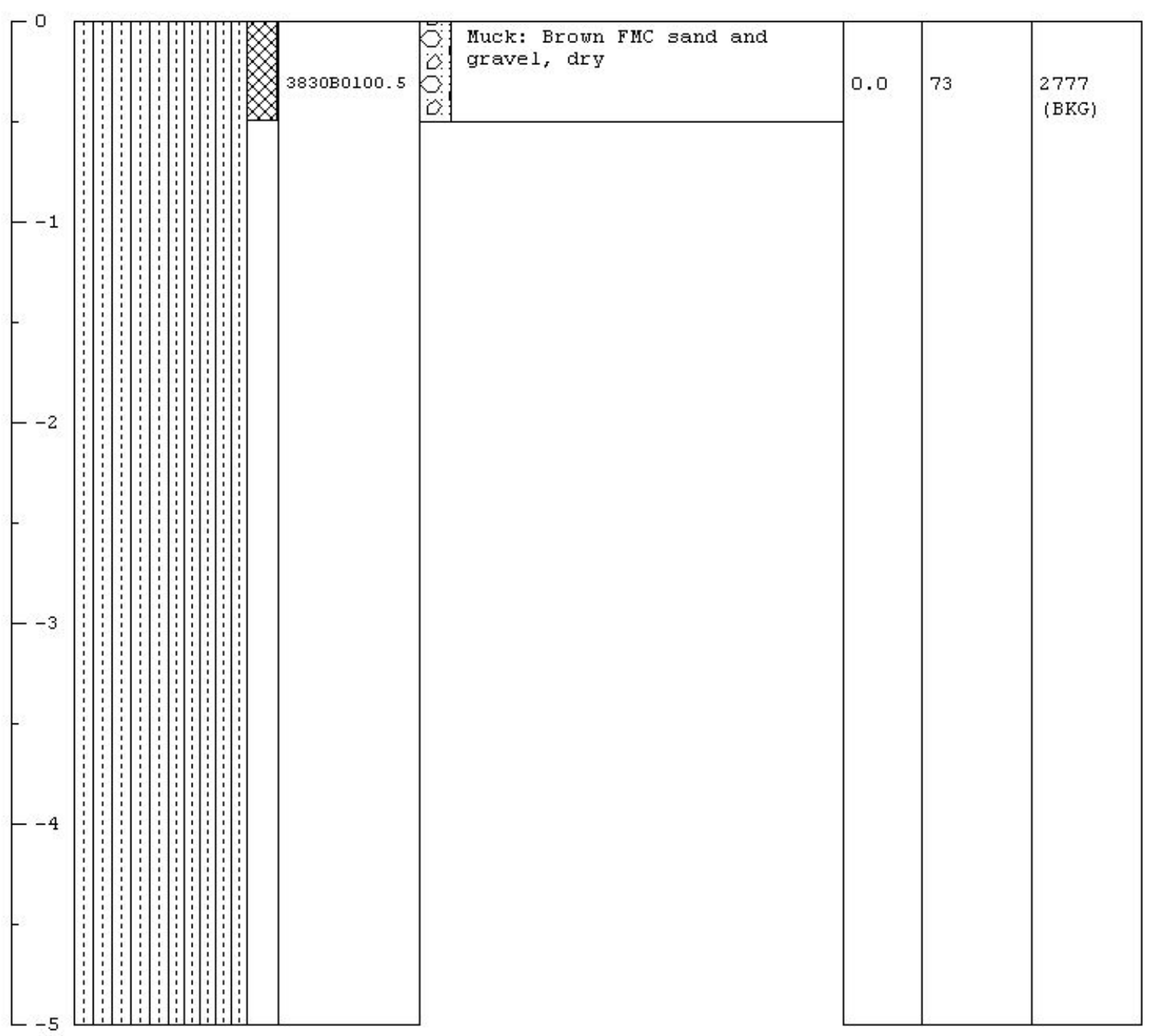


Page 1 of 1

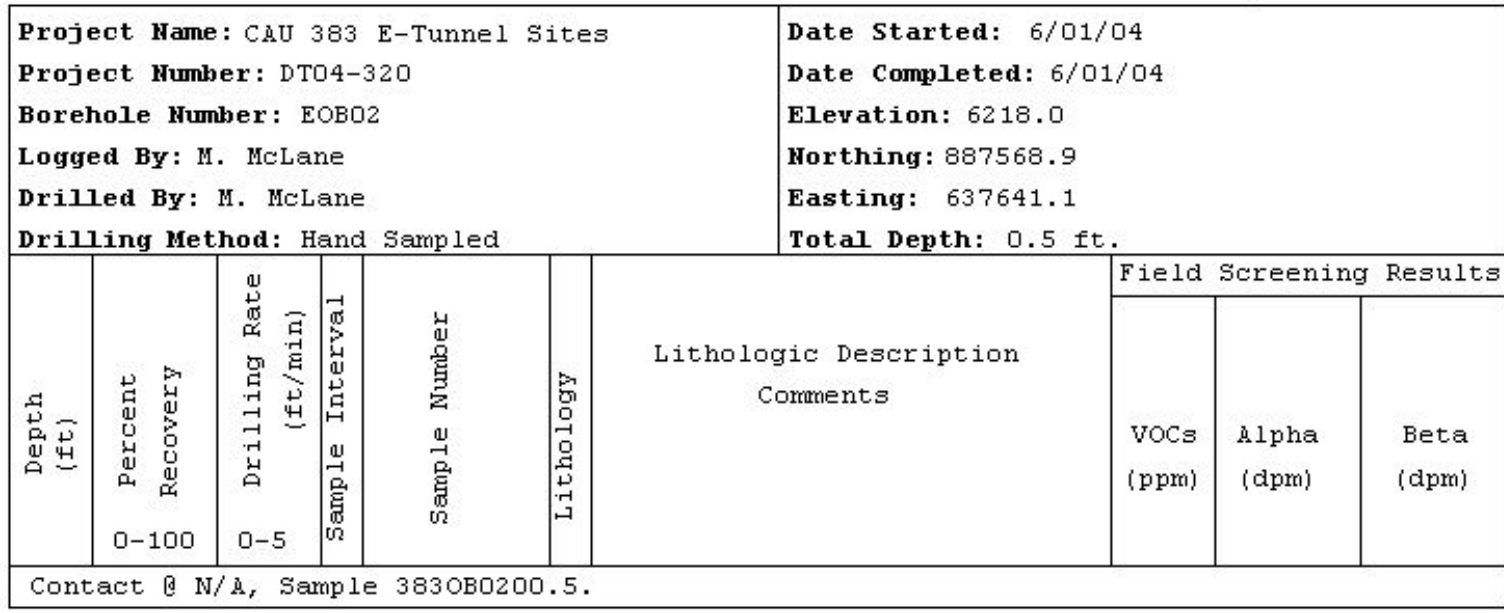

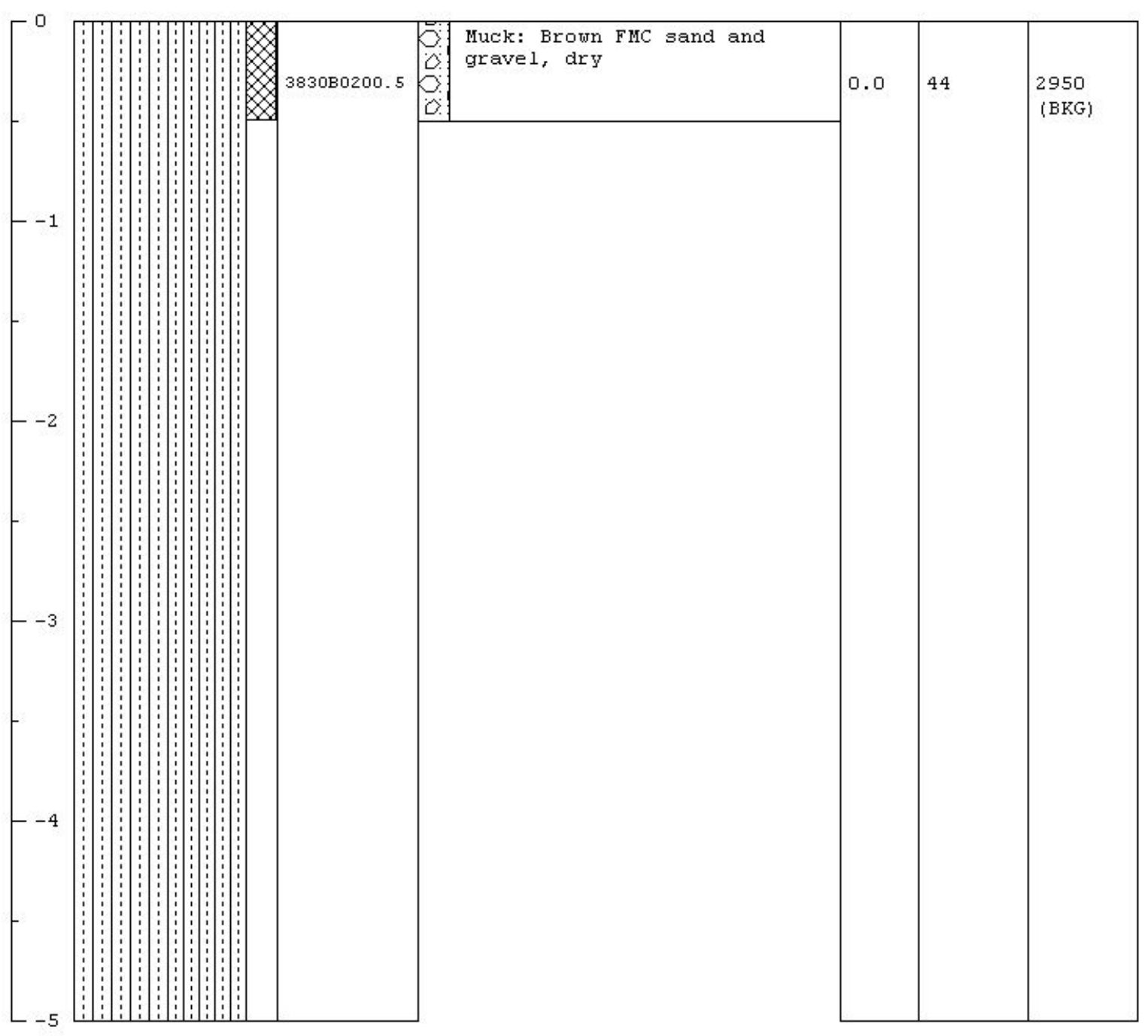


Page 1 of 1

Project Hame: CAU 383 E-Tunnel Sites

Project Kumber: DTO4-320

Borehole Number: EOBO3

Logged BY: $M$. McLane

Drilled By: $M$. McLane

Drilling Method: Hand Sampled
Date Started: $6 / 01 / 04$

Date Completed: $6 / 01 / 04$

Elevation: 6213.9

Horthing : 887531.0

Easting: 637623.9

Total Depth: $0.5 \mathrm{ft}$.

\begin{tabular}{|c|c|c|c|c|c|c|c|c|c|}
\hline \multirow[b]{2}{*}{ 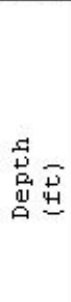 } & \multirow[b]{2}{*}{ 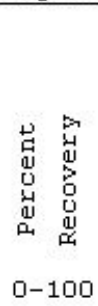 } & \multirow[b]{2}{*}{ 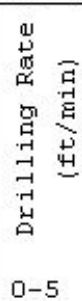 } & \multirow[b]{2}{*}{ 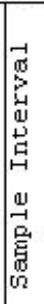 } & \multirow[b]{2}{*}{ 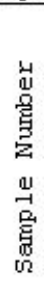 } & \multirow[b]{2}{*}{ 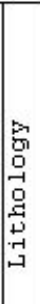 } & \multirow[b]{2}{*}{$\begin{array}{c}\text { Lithologic Description } \\
\text { Comments }\end{array}$} & \multicolumn{3}{|c|}{ Field Screening Results } \\
\hline & & & & & & & $\begin{array}{l}\text { VOCs } \\
\text { (ppm) }\end{array}$ & $\begin{array}{l}\text { Alpha } \\
\text { (dpri) }\end{array}$ & $\begin{array}{l}\text { Beta } \\
\text { (dpm) }\end{array}$ \\
\hline
\end{tabular}

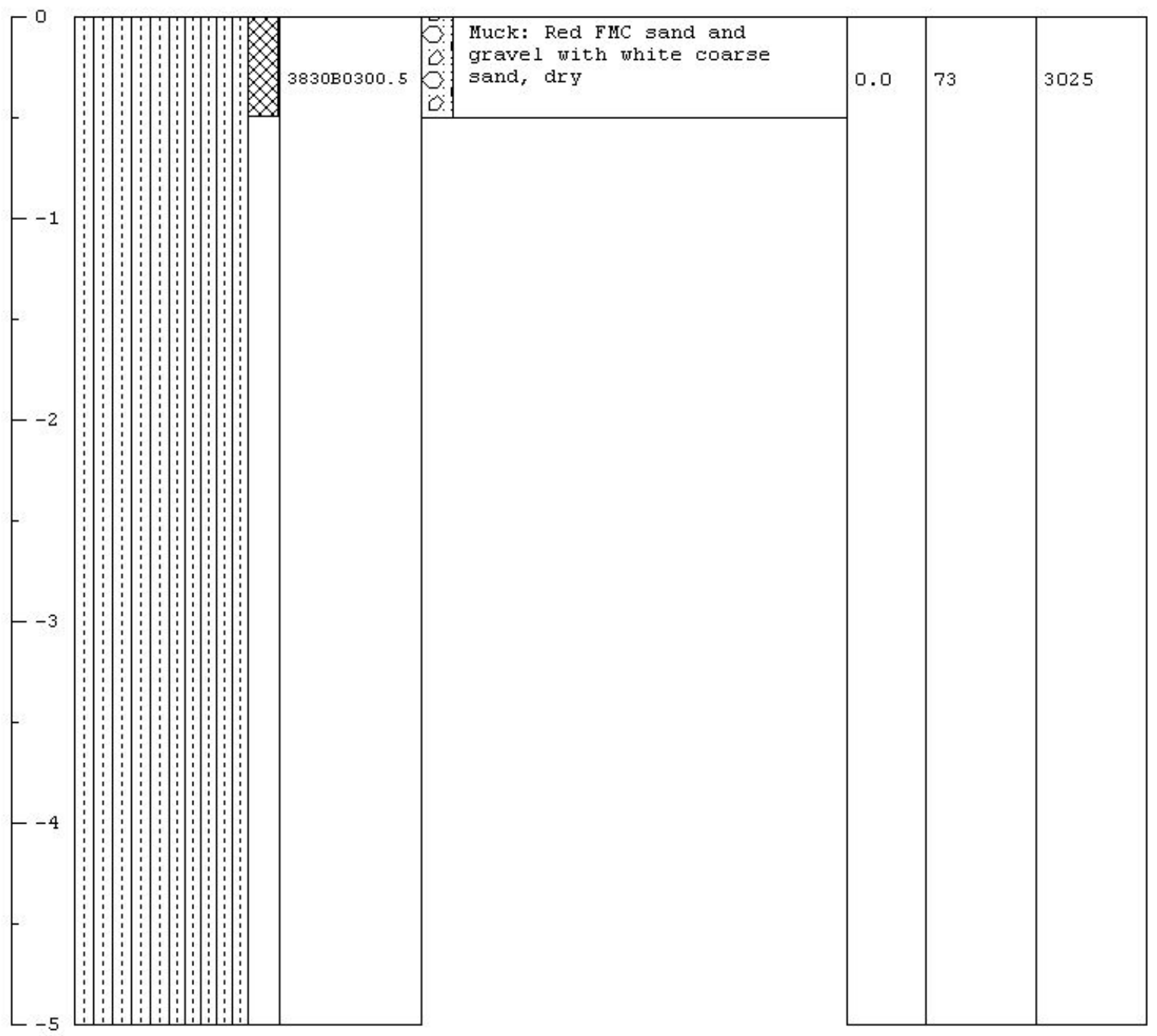


Page 1 of 1

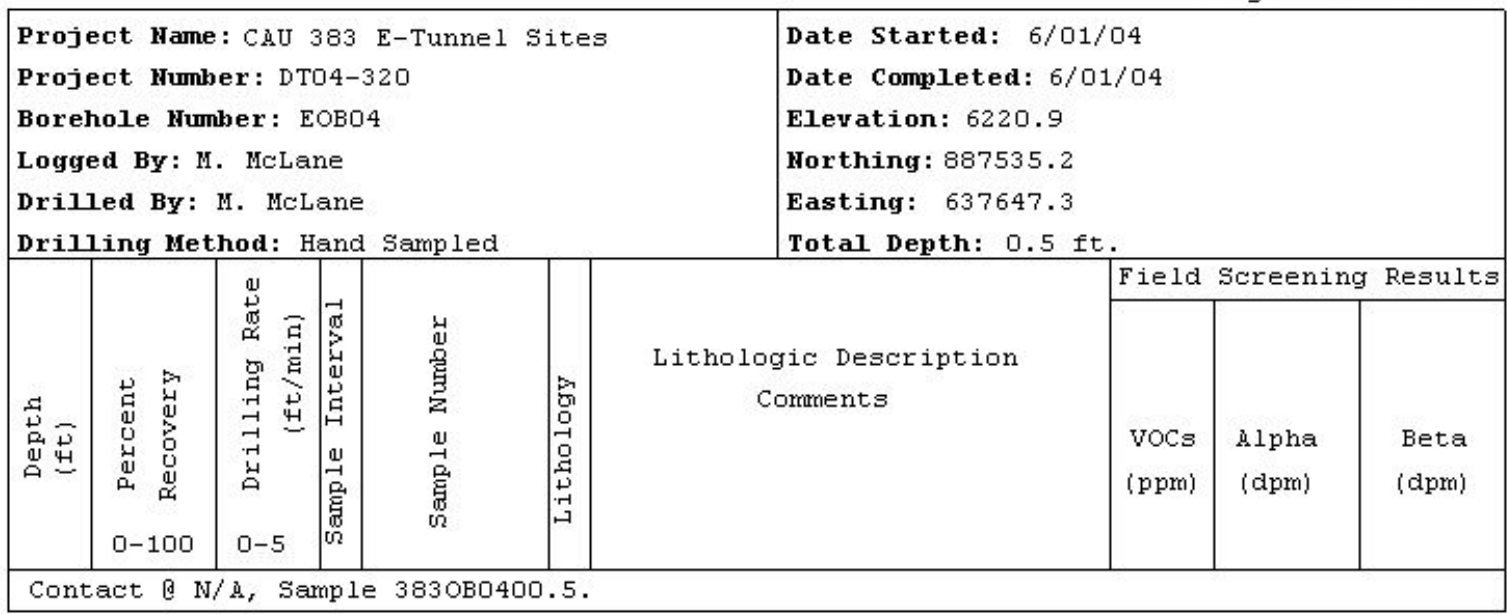

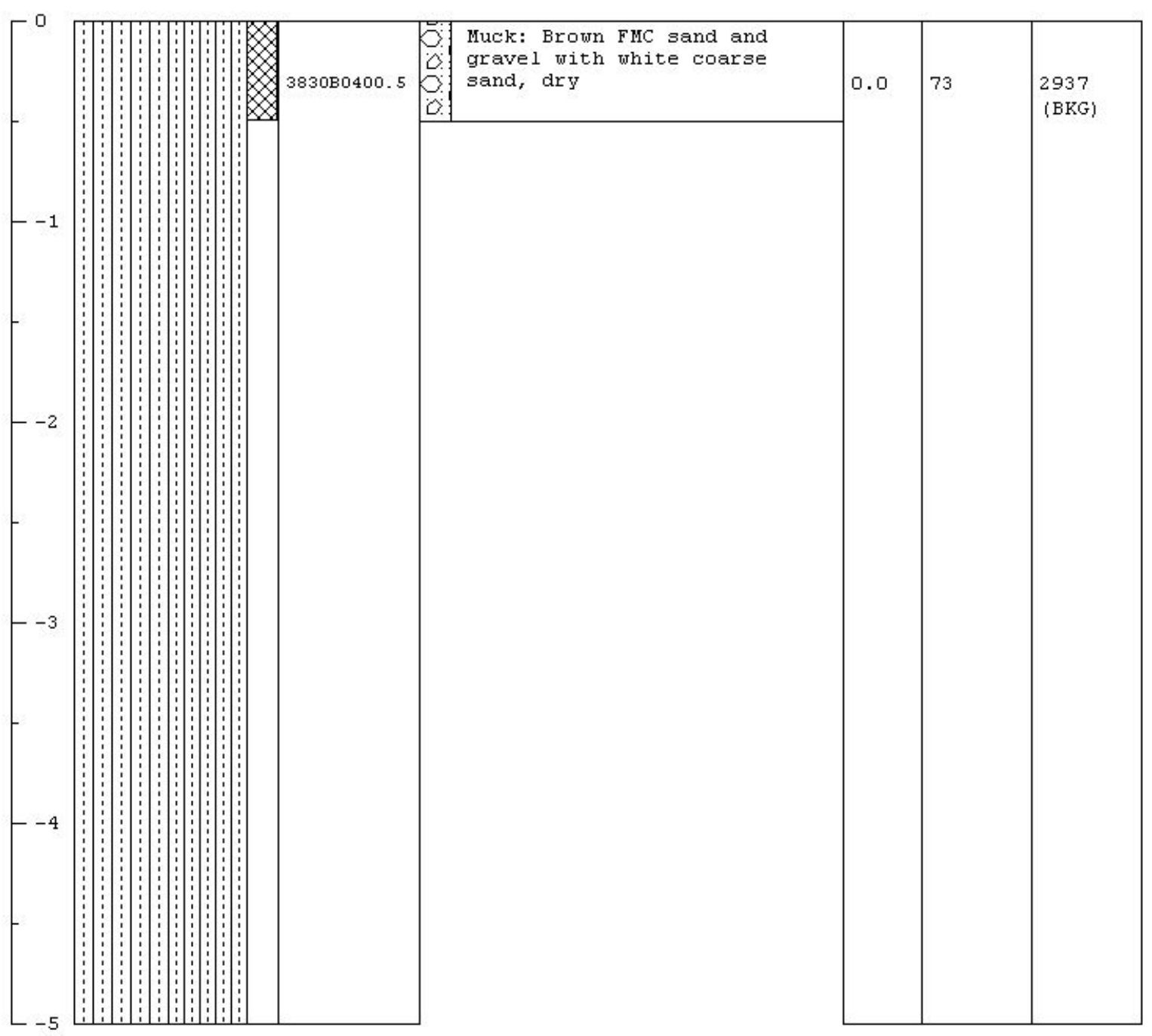


Page 1 of 1

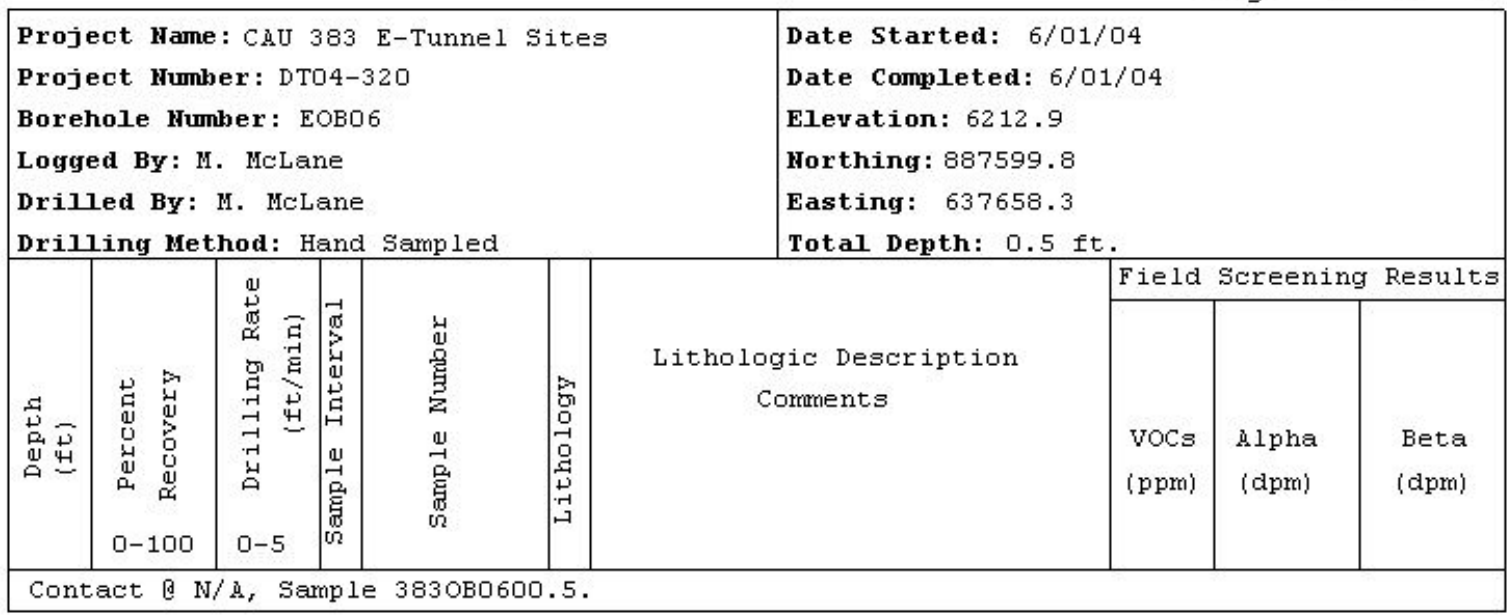

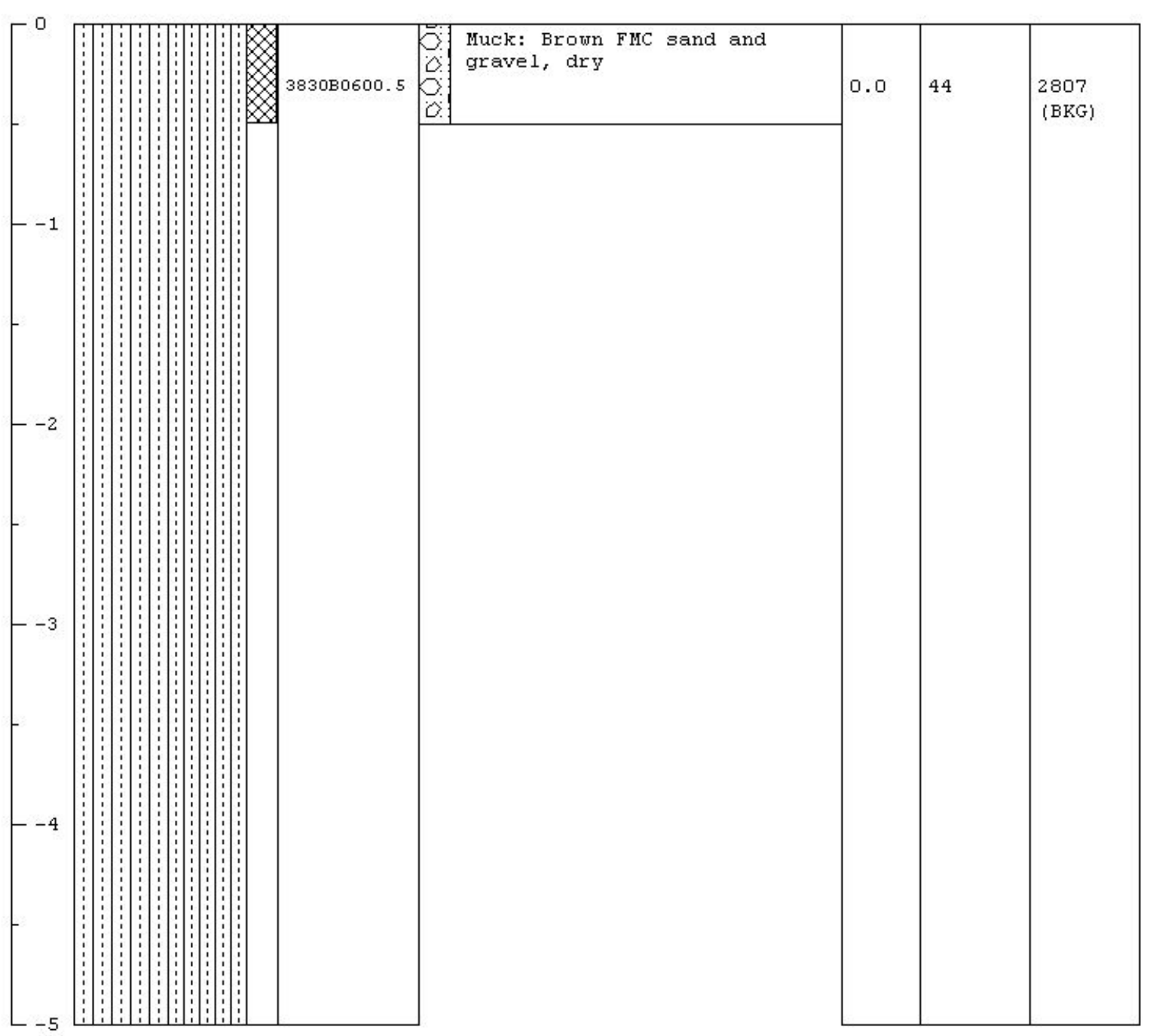


Page 1 of 1

Project Hame: CAU 383 E-Tunnel Sites

Project Number: DT04-320

Borehole Number: EOBO7

Logged By: M. MeLane

Drilled By: M. MeLane

Drilling Method: Hand Sampled
Date Started: $6 / 01 / 04$

Date Completed: $6 / 01 / 04$

Elevation: 6207.4

Horthing: 887598.9

Easting: 637640.6

Total Depth: $0.5 \mathrm{ft}$.

\begin{tabular}{|c|c|c|c|c|c|c|c|c|c|}
\hline \multirow[b]{2}{*}{ 吉 } & \multirow[b]{2}{*}{ 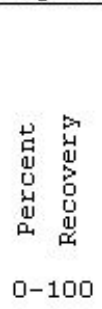 } & \multirow{2}{*}{\multicolumn{2}{|c|}{ 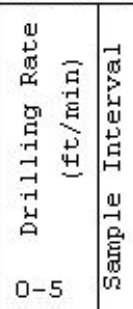 }} & \multirow[b]{2}{*}{ 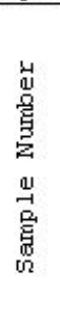 } & \multirow[b]{2}{*}{$\begin{array}{l}3 \\
8 \\
0 \\
9 \\
9 \\
9 \\
.7 \\
-7 \\
-1\end{array}$} & \multirow[b]{2}{*}{$\begin{array}{c}\text { Lithologic Description } \\
\text { Comments }\end{array}$} & \multicolumn{3}{|c|}{ Field Screening Results } \\
\hline & & & & & & & $\begin{array}{l}\text { VOCs } \\
\text { (ppm) }\end{array}$ & $\begin{array}{l}\text { Alpha } \\
\text { (dpm) }\end{array}$ & $\begin{array}{l}\text { Beta } \\
\text { (dpm) }\end{array}$ \\
\hline
\end{tabular}

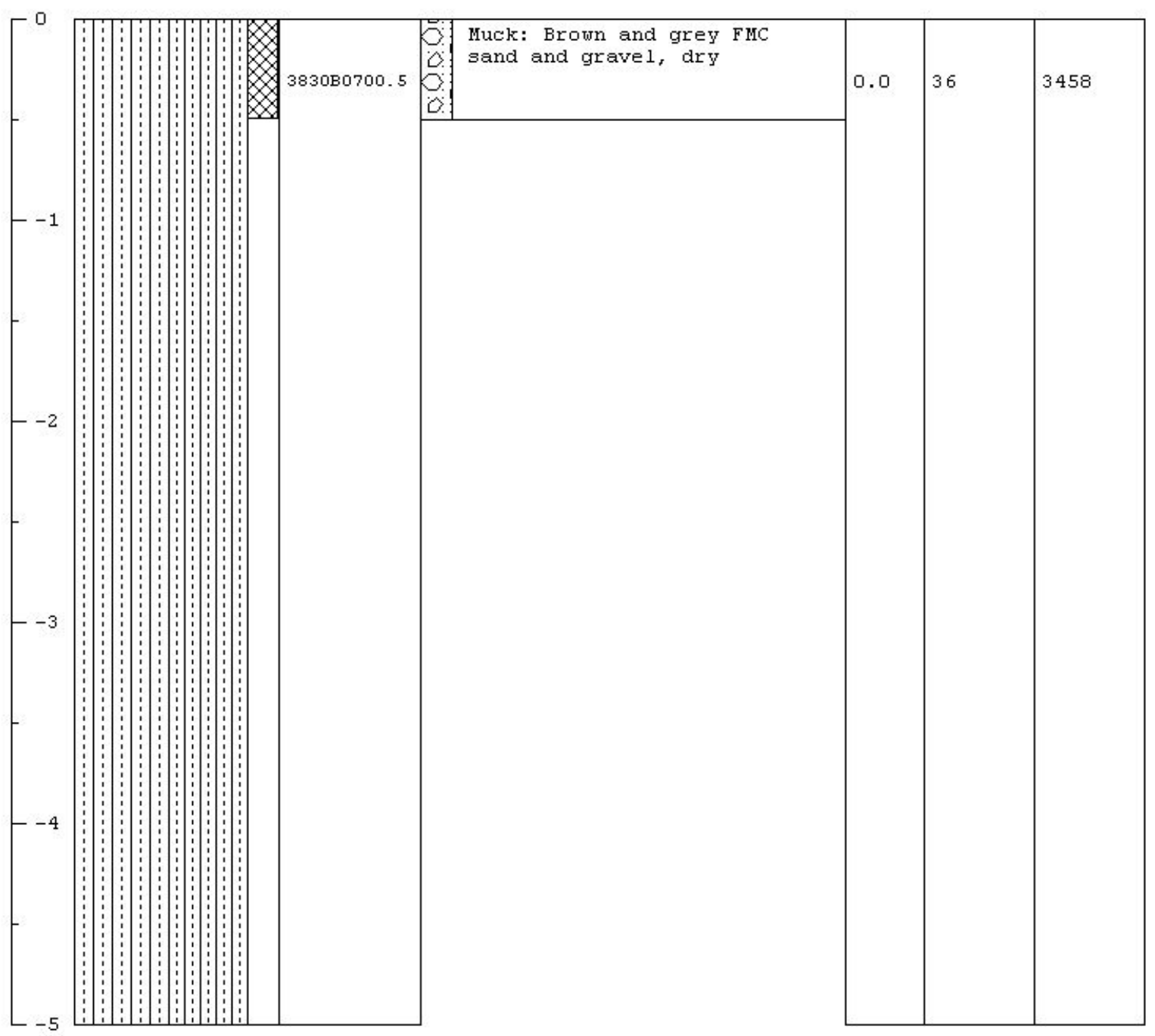


Page 1 of 1

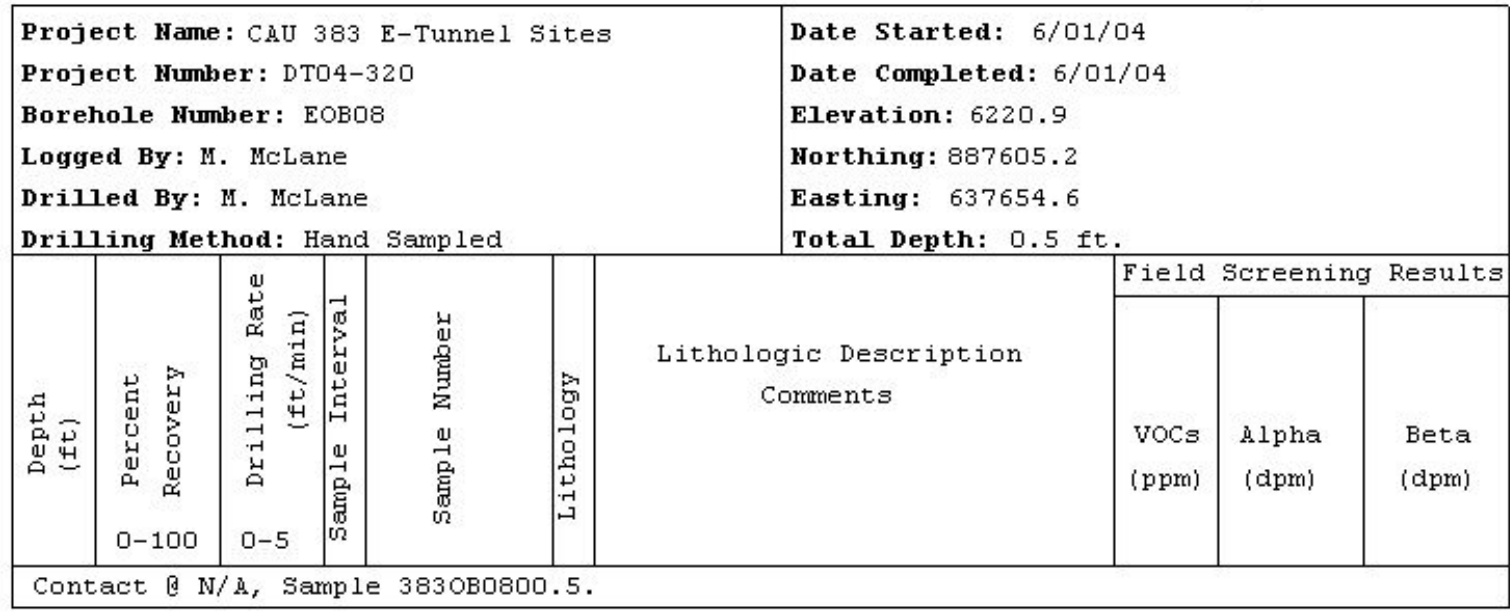

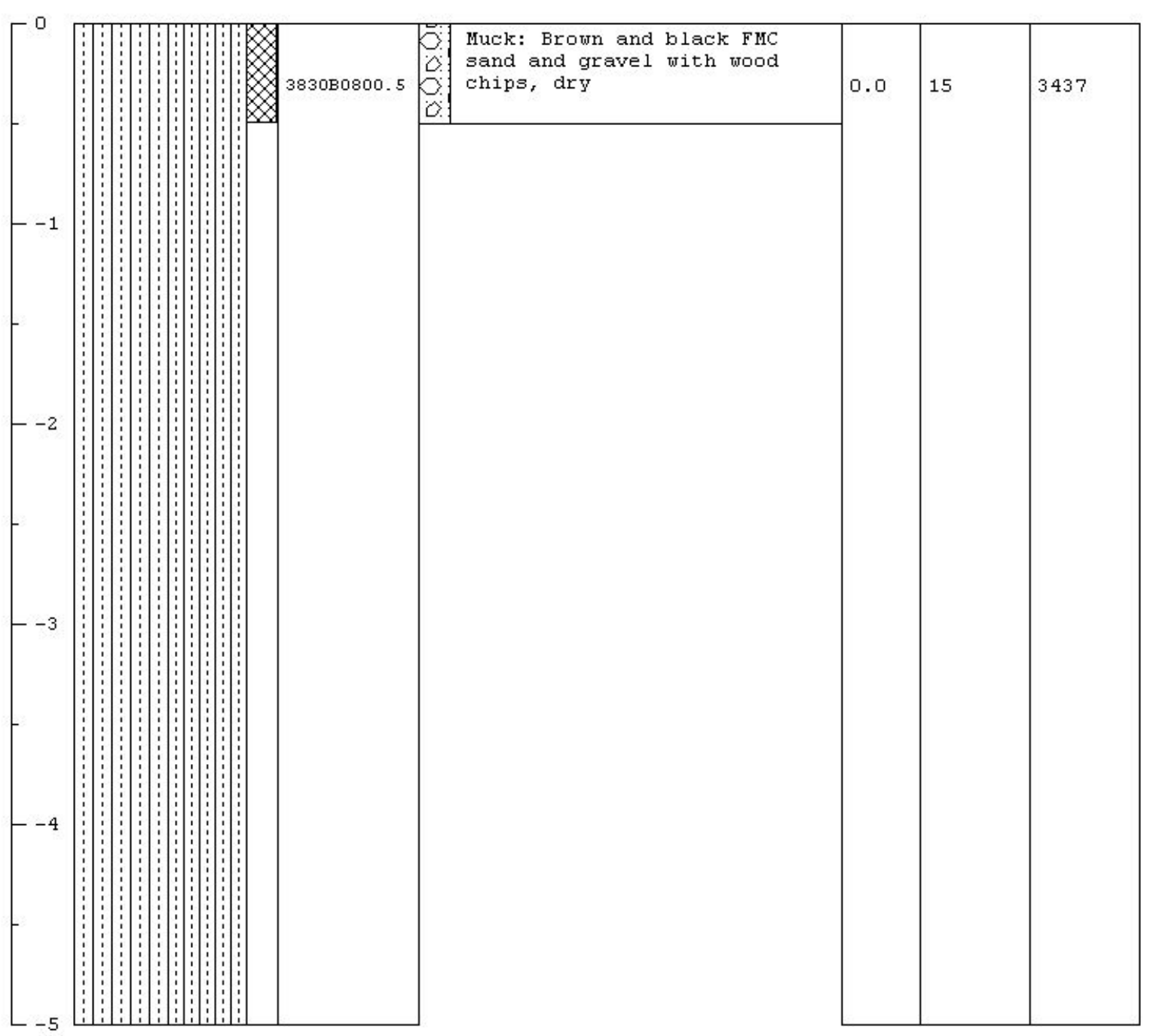


Page 1 of 1

Project Name: CAU 383 E-Tunnel Sites

Project Number: DTO4-320

Borehole Number: EPBO1

Logged By: M. McLane

Drilled By: Boart-Longyear Drilling Method: Rotosonic
Date Started: $6 / 10 / 04$

Date Completed: $6 / 10 / 04$

Elevation: 5959.0

Horthing: 887711.6

Easting: 639006.6

Total Depth: $5.0 \mathrm{ft}$.
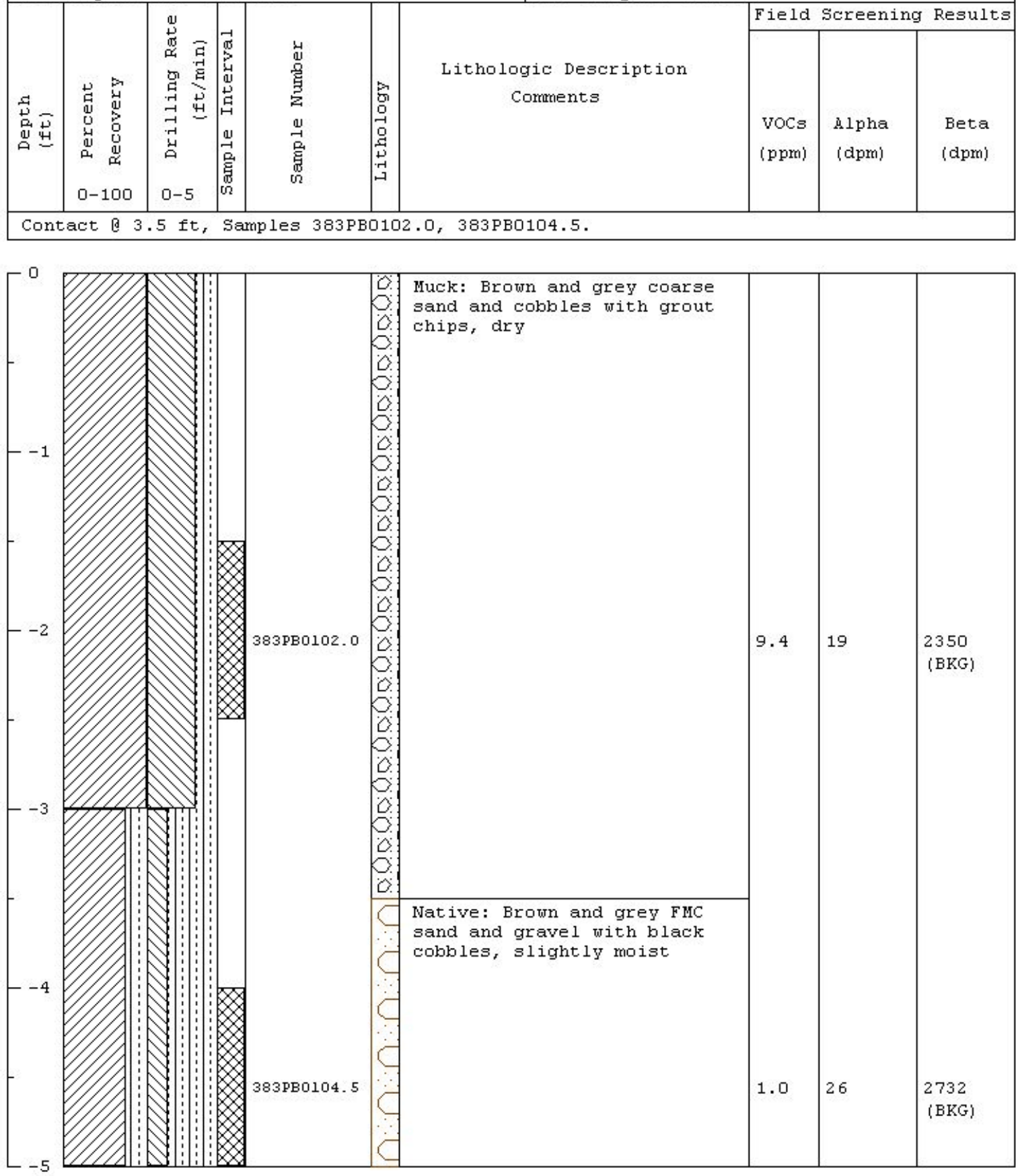
Page 1 of 1

Project Hame: CAU 383 E-Tunnel sites

Project Number: DTO4-320

Borehole Number: EPBO2

Logged By: $M$. McLane

Drilled By: M. McLane

Drilling Method: Hand Sampled
Date Started: $6 / 17 / 04$

Date Completed: $6 / 17 / 04$

Elevation : 5917.5

Northing : 887648.3

Easting: 639275.7

Total Depth: $0.5 \mathrm{ft}$.

\begin{tabular}{|c|c|c|c|c|c|c|c|c|c|}
\hline \multirow[b]{2}{*}{ 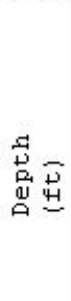 } & \multirow[b]{2}{*}{ 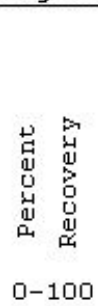 } & \multirow[b]{2}{*}{ 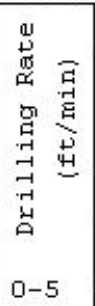 } & \multirow[b]{2}{*}{ 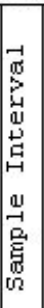 } & \multirow[b]{2}{*}{ 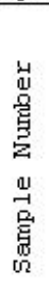 } & \multirow[b]{2}{*}{ 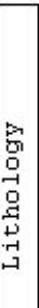 } & \multirow[b]{2}{*}{$\begin{array}{c}\text { Lithologic Description } \\
\text { Comments }\end{array}$} & \multicolumn{3}{|c|}{ Field Screening Results } \\
\hline & & & & & & & $\begin{array}{l}\text { VOCs } \\
\text { (ppm) }\end{array}$ & $\begin{array}{l}\text { Alpha } \\
\text { (dpro) }\end{array}$ & $\begin{array}{l}\text { Beta } \\
\text { (dpm) }\end{array}$ \\
\hline
\end{tabular}

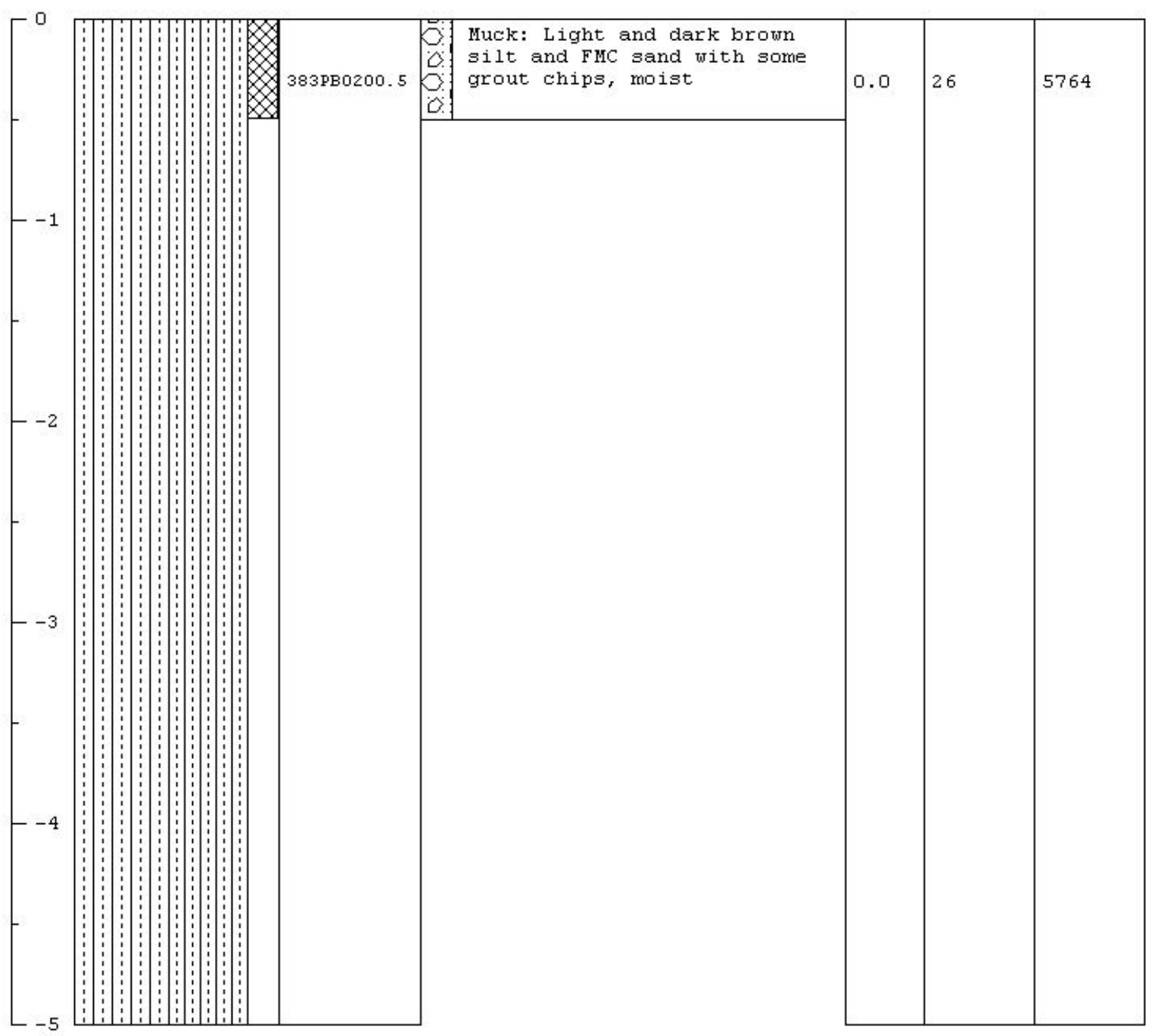


Page 1 of 1

\begin{tabular}{|c|c|c|c|c|c|c|c|c|}
\hline \multicolumn{5}{|c|}{$\begin{array}{l}\text { Project Hame: CAU } 383 \text { E-Tunnel Sites } \\
\text { Project Humber: DTO } 4-320 \\
\text { Borehole Humber: EPBO } 3 \\
\text { Logged By: M. McLane } \\
\text { Drilled By: M. McLane } \\
\text { Drilling Method: Hand Sampled }\end{array}$} & \multicolumn{4}{|c|}{$\begin{array}{l}\text { Date Started: } 6 / 17 / 04 \\
\text { Date Completed: } 6 / 17 / 04 \\
\text { Elevation: } 5927.1 \\
\text { Northing: } 887622.8 \\
\text { Easting: } 639329.0 \\
\text { Total Depth: } 0.5 \mathrm{ft} . \\
\end{array}$} \\
\hline \multirow[b]{2}{*}{ 点 } & \multirow[b]{2}{*}{ 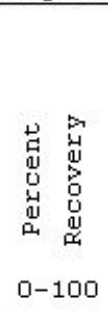 } & \multirow[b]{2}{*}{ 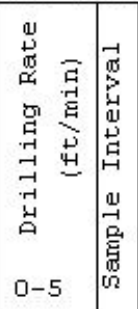 } & \multirow[b]{2}{*}{ 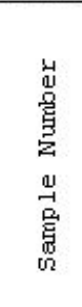 } & \multirow[b]{2}{*}{ 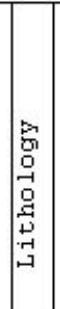 } & \multirow[b]{2}{*}{$\begin{array}{c}\text { Lithologic Description } \\
\text { Comments }\end{array}$} & Field & Screening & Results \\
\hline & & & & & & $\begin{array}{l}\text { VOCs } \\
\text { (ppm) }\end{array}$ & $\begin{array}{l}\text { Alpha } \\
\text { (dpm) }\end{array}$ & $\begin{array}{r}\text { Beta } \\
\text { (dpm) }\end{array}$ \\
\hline
\end{tabular}

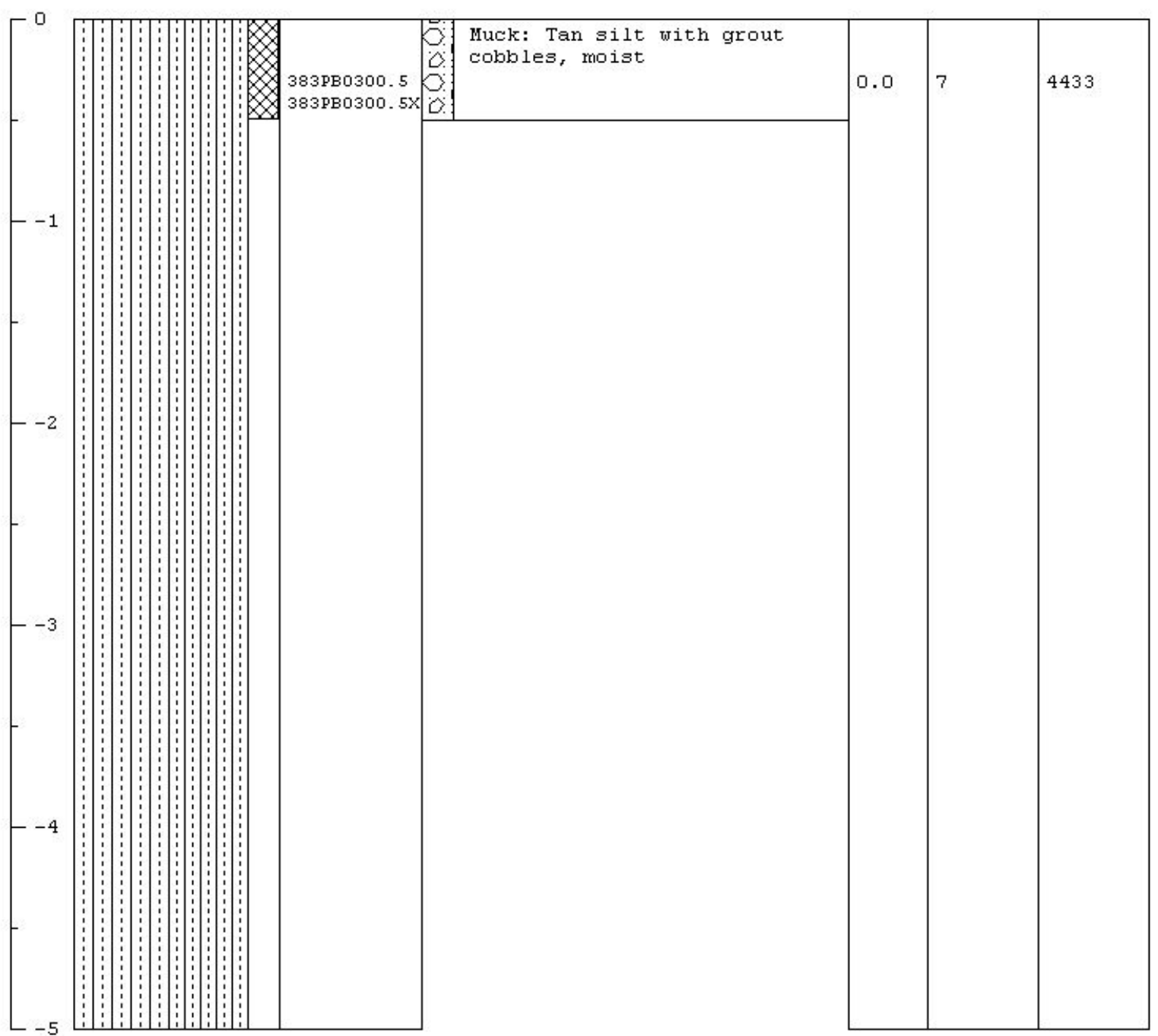


Page 1 of 1

\begin{tabular}{|c|c|c|c|c|c|c|c|c|}
\hline \multicolumn{5}{|c|}{$\begin{array}{l}\text { Project Hame: CAU } 383 \text { E-Tunnel Sites } \\
\text { Project Humber: DTO4-320 } \\
\text { Borehole Humber: EPBO4 } \\
\text { Logged By: M. MeLane } \\
\text { Drilled By: M. MeLane } \\
\text { Drilling Method: Hand Sampled }\end{array}$} & \multicolumn{4}{|c|}{$\begin{array}{l}\text { Date Started: } 6 / 16 / 04 \\
\text { Date Completed: } 6 / 16 / 04 \\
\text { Elevation: } 5942.3 \\
\text { Horthing: } 887624.5 \\
\text { Easting: } 639090.5 \\
\text { Total Depth: } 0.5 \mathrm{ft} . \\
\end{array}$} \\
\hline \multirow[b]{2}{*}{ 点 } & \multirow[b]{2}{*}{ 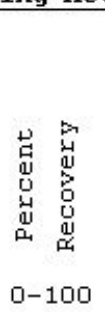 } & \multirow[b]{2}{*}{ 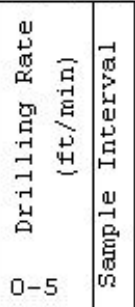 } & \multirow[b]{2}{*}{ 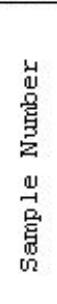 } & \multirow[b]{2}{*}{ 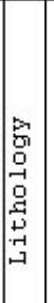 } & \multirow[b]{2}{*}{$\begin{array}{c}\text { Lithologic Description } \\
\text { Comments }\end{array}$} & \multirow{2}{*}{\begin{tabular}{|l} 
Field \\
voCs \\
(ppm)
\end{tabular}} & Screenin & Results \\
\hline & & & & & & & $\begin{array}{l}\text { Alpha } \\
\text { (dpm) }\end{array}$ & $\begin{array}{l}\text { Beta } \\
\text { (dpm) }\end{array}$ \\
\hline
\end{tabular}

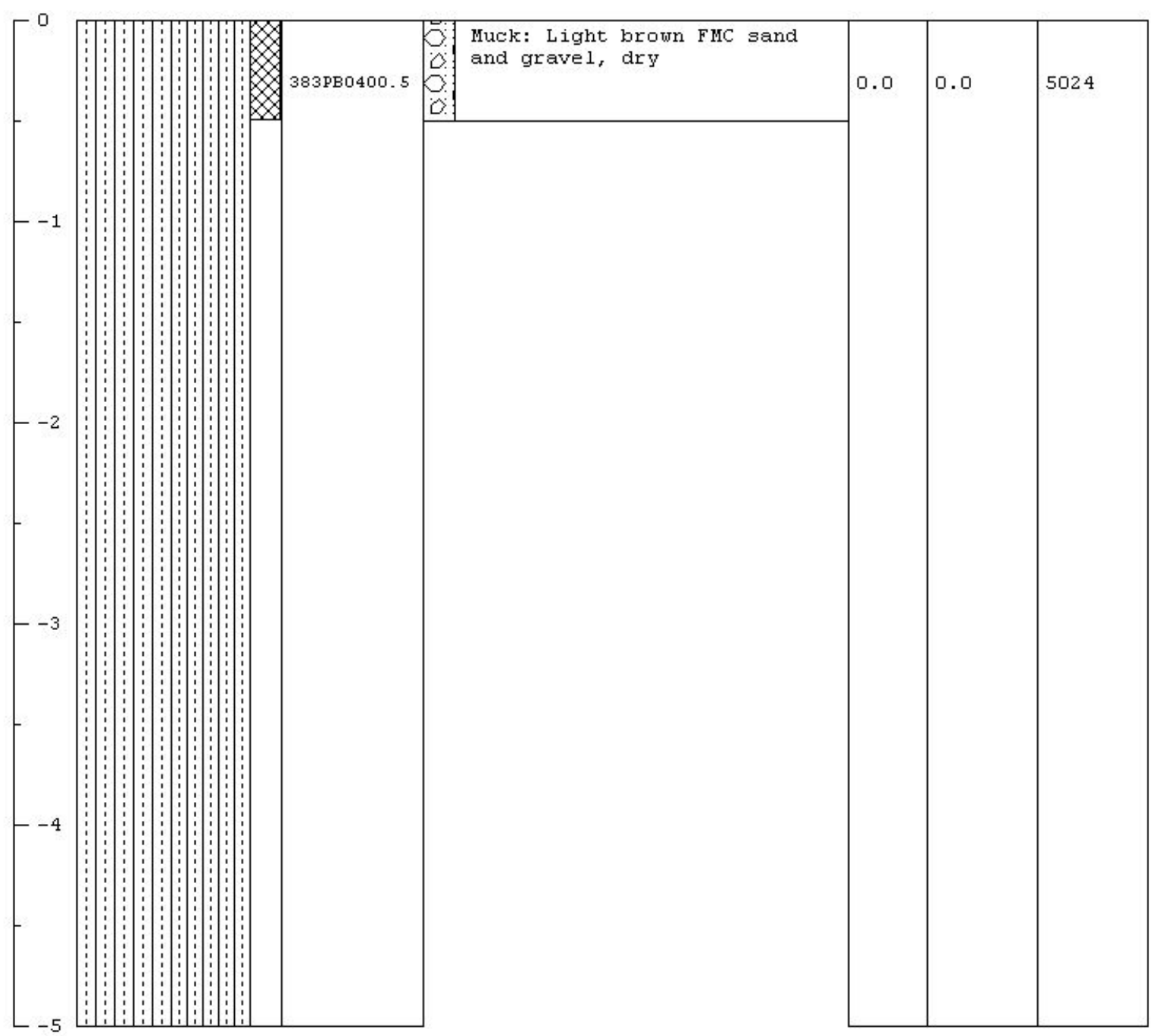


Page 1 of 1

Project Hame: CAU 383 E-Tunnel Sites

Project Humber: DTO4-320

Borehole Nunber: EPBO5

Logged By: M. McLane

Drilled By: M. McLane

Drilling Method: Hand Sampled
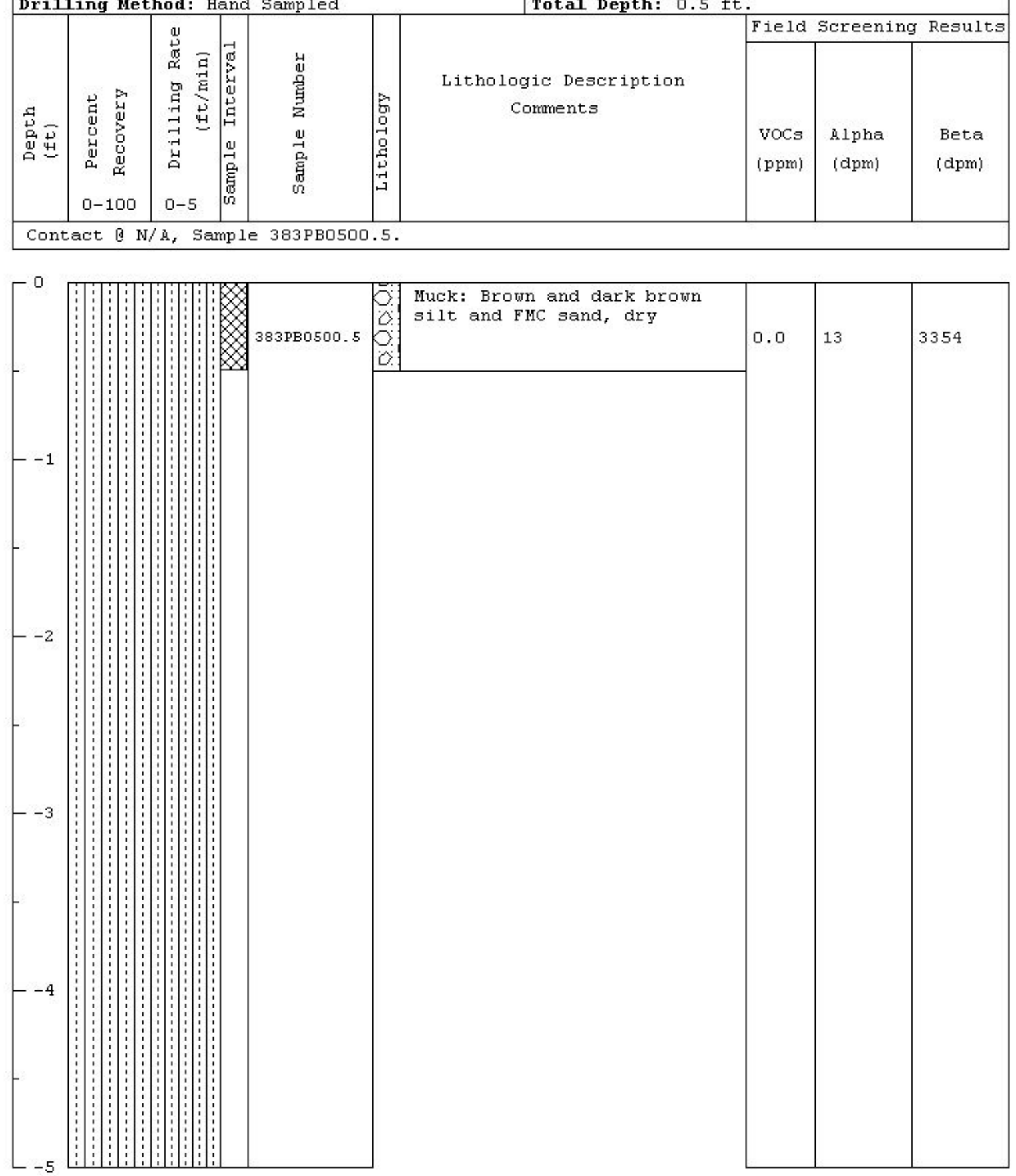
Page 1 of 1

Project Hame: CAU 383 E-Tunnel Sites

Project Humber: DTO4-320

Logged By: M. McLane

Drilled By: Boart-Longyear

Drilling Method: Rotosonic
Borehole Number: EPHO1

Date Started: $6 / 09 / 04$

Date Completed: $6 / 09 / 04$

Elevation: 5954.9

Northing : 887706.3

Easting: 639072.3

Total Depth: $17.0 \mathrm{ft}$.
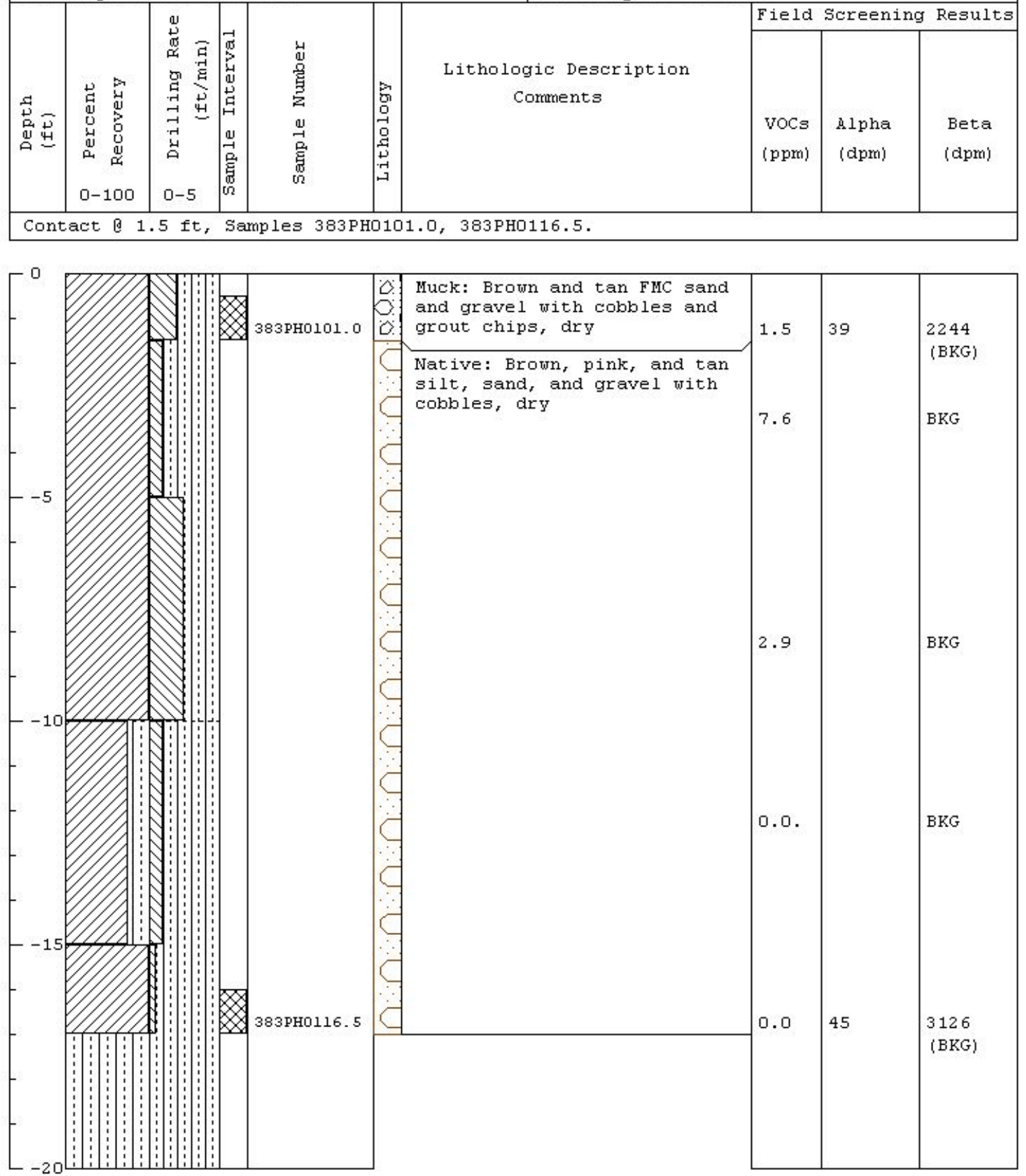
Page 1 of 1

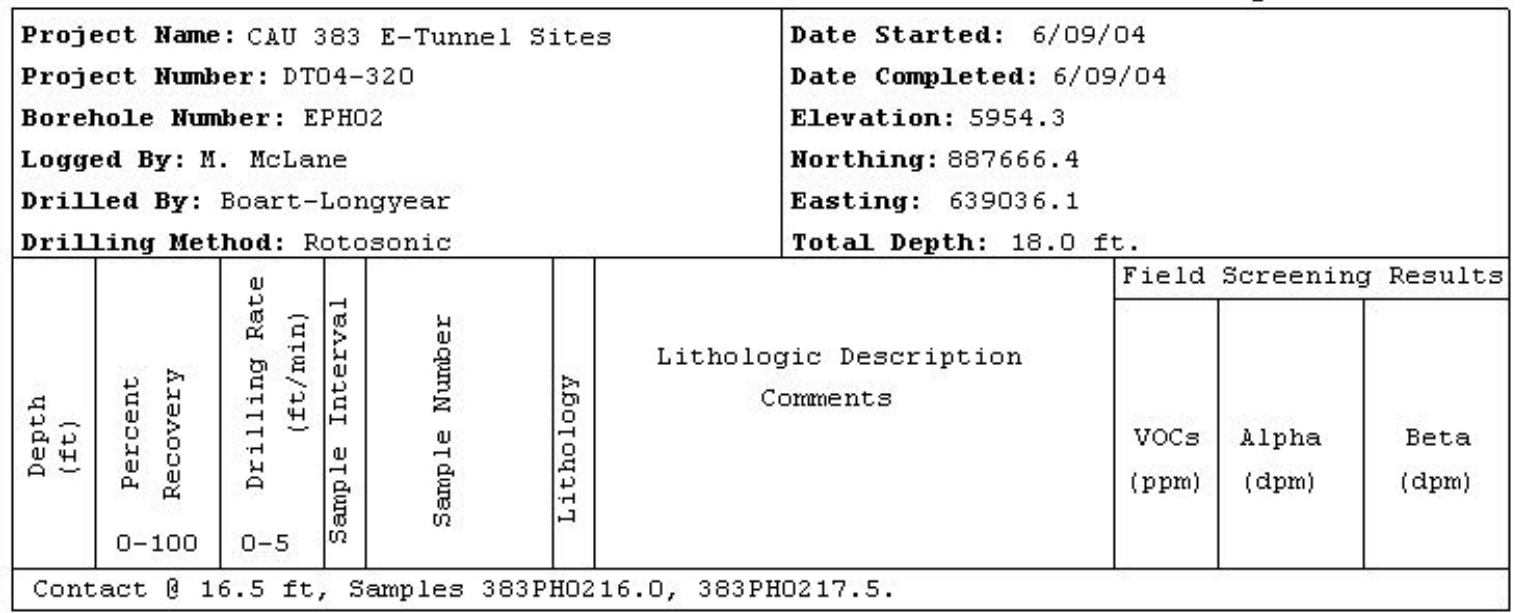

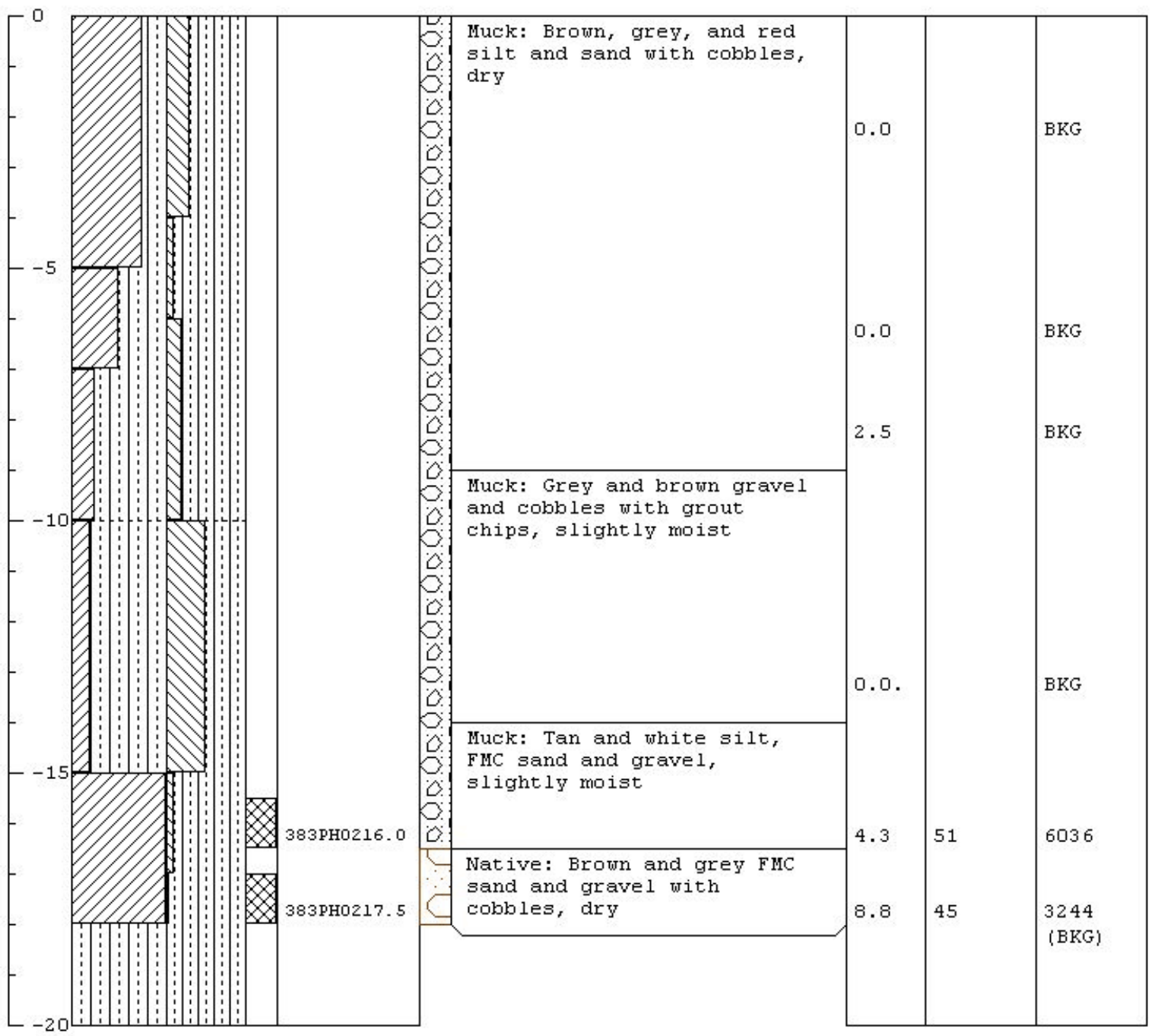


Page 1 of 1

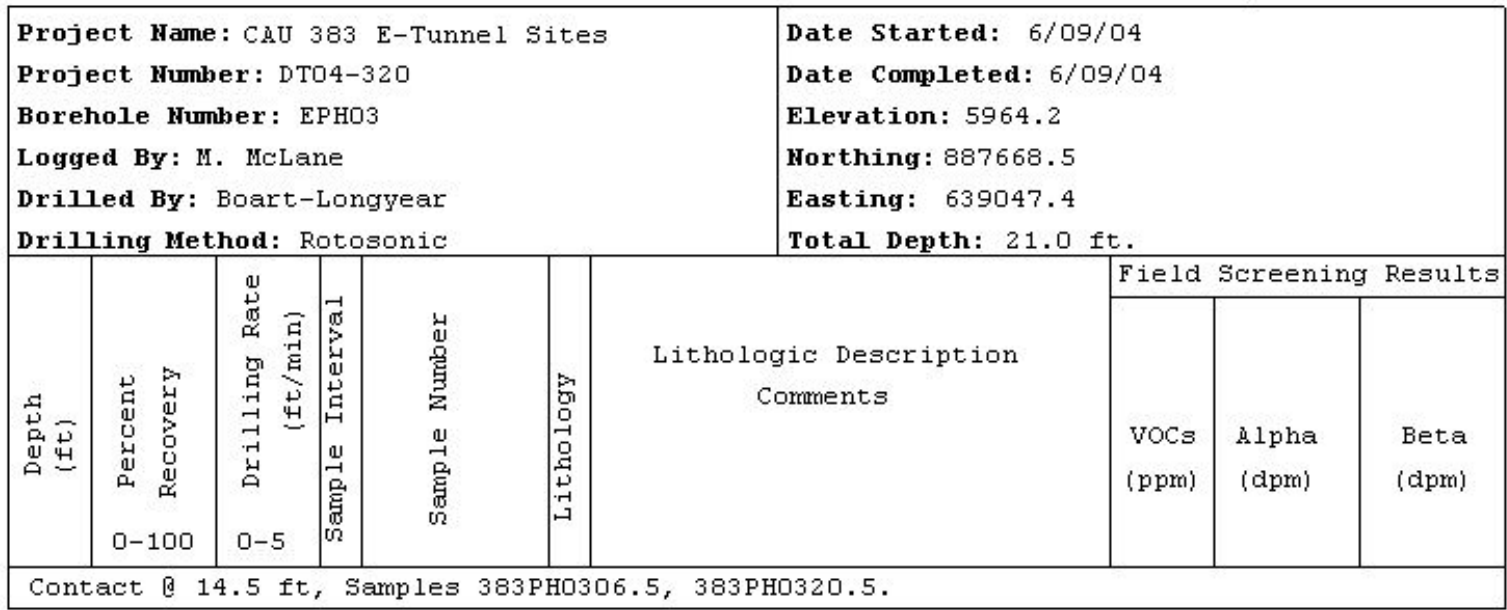

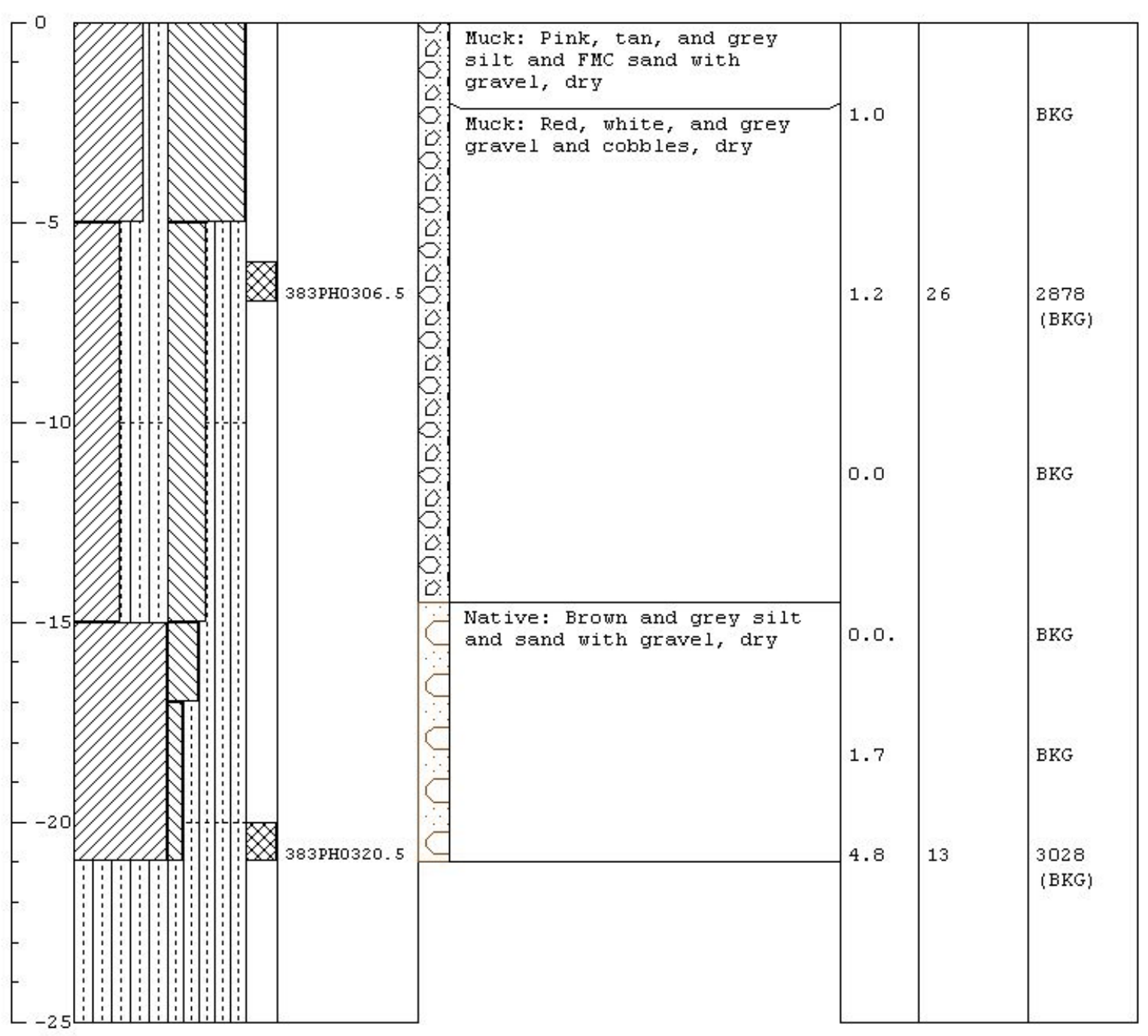


Page 1 of 1

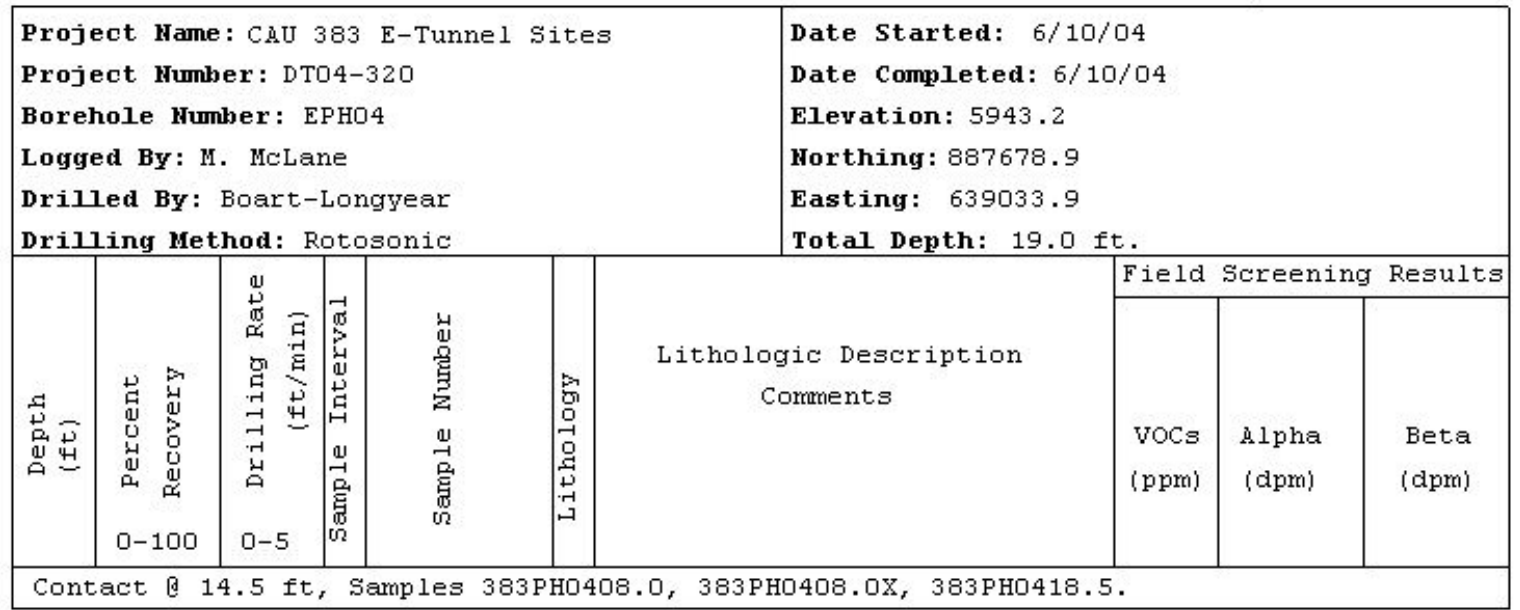

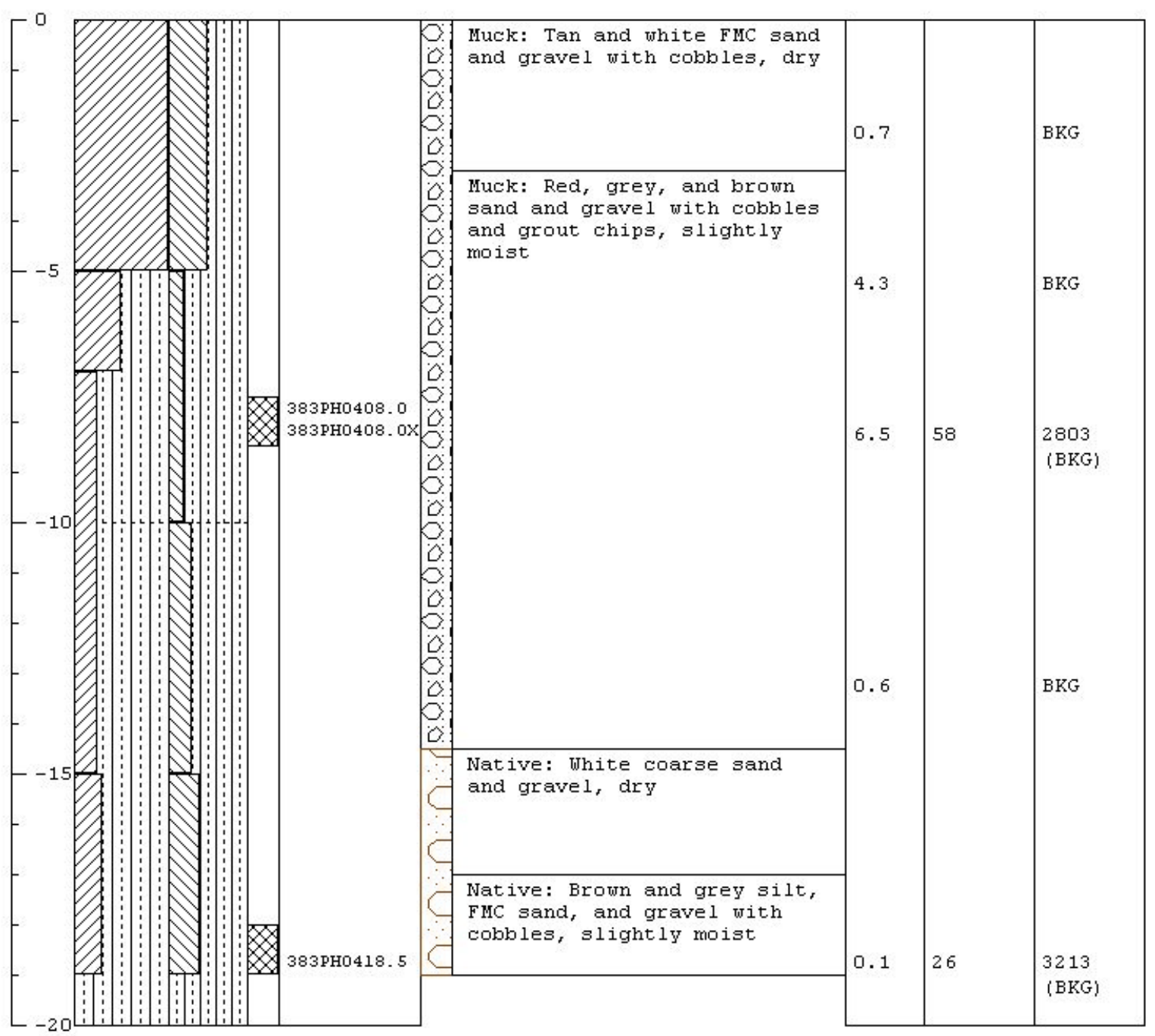


Page 1 of 1

\begin{tabular}{|c|c|c|c|c|c|c|c|c|c|}
\hline \multicolumn{6}{|c|}{$\begin{array}{l}\text { Project Hame: CAU } 383 \text { E-Tunnel Sites } \\
\text { Project Humber: DTO } 4-320 \\
\text { Borehole Humber: ERBO1 } \\
\text { Logged By: M. MeLane } \\
\text { Drilled By: M. MeLane } \\
\text { Drilling Method: Hand Sampled }\end{array}$} & \multicolumn{4}{|c|}{$\begin{array}{l}\text { Date Started: } 6 / 01 / 04 \\
\text { Date Completed: } 6 / 01 / 04 \\
\text { Elevation: } 6122.6 \\
\text { Horthing: } 887807.9 \\
\text { Easting: } 637709.2 \\
\text { Total Depth: } 0.5 \mathrm{ft} . \\
\end{array}$} \\
\hline \multirow[b]{2}{*}{ 㤩 } & \multirow[b]{2}{*}{ 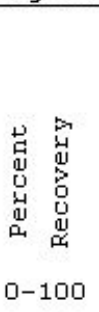 } & \multirow{2}{*}{\multicolumn{2}{|c|}{ 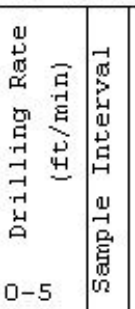 }} & \multirow[b]{2}{*}{ 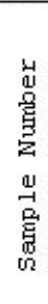 } & \multirow[b]{2}{*}{ 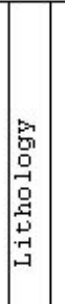 } & \multirow[b]{2}{*}{$\begin{array}{c}\text { Lithologic Description } \\
\text { Comments }\end{array}$} & Field & screening & Results \\
\hline & & & & & & & $\begin{array}{l}\text { VOCs } \\
\text { (ppm) }\end{array}$ & $\begin{array}{l}\text { Alpha } \\
\text { (dpm) }\end{array}$ & $\begin{array}{l}\text { Beta } \\
\text { (dpm) }\end{array}$ \\
\hline
\end{tabular}

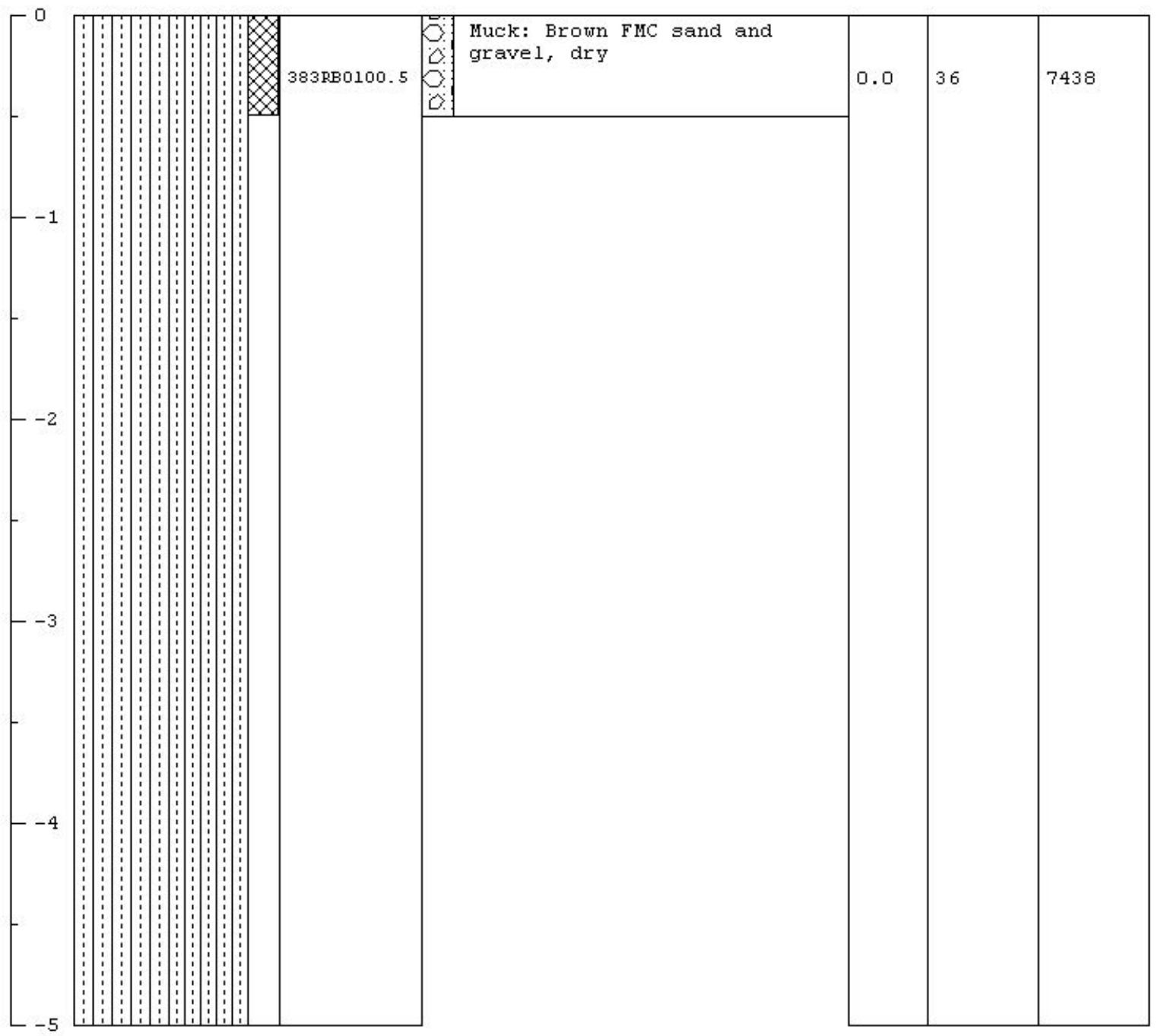


Page 1 of 1

\begin{tabular}{|c|c|c|c|c|c|c|c|c|}
\hline \multicolumn{5}{|c|}{$\begin{array}{l}\text { Project Hame: CAU } 383 \text { E-Tunnel Sites } \\
\text { Project Humber: DTO } 4-320 \\
\text { Borehole Number: ERBO2 } \\
\text { Logged By: M. McLane } \\
\text { Drilled By: M. MeLane } \\
\text { Drilling Method: Hand Sampled }\end{array}$} & \multicolumn{4}{|c|}{$\begin{array}{l}\text { Date Started: } 6 / 01 / 04 \\
\text { Date Completed: } 6 / 01 / 04 \\
\text { Elevation: } 6119.5 \\
\text { Horthing: } 887818.4 \\
\text { Easting: } 637716.4 \\
\text { Total Depth: } 0.5 \mathrm{ft} .\end{array}$} \\
\hline \multirow[b]{2}{*}{ 点 } & \multirow[b]{2}{*}{ 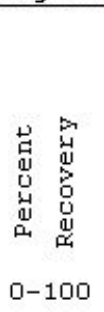 } & \multirow[b]{2}{*}{ 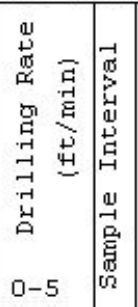 } & \multirow[b]{2}{*}{ 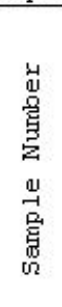 } & \multirow[b]{2}{*}{ 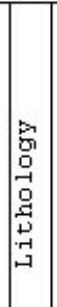 } & \multirow[b]{2}{*}{$\begin{array}{c}\text { Lithologic Description } \\
\text { Comments }\end{array}$} & Field & Screening & Results \\
\hline & & & & & & $\begin{array}{l}\text { VOCs } \\
\text { (ppm) }\end{array}$ & $\begin{array}{l}\text { Alpha } \\
\text { (dpm) }\end{array}$ & $\begin{array}{l}\text { Beta } \\
\text { (dpm) }\end{array}$ \\
\hline
\end{tabular}

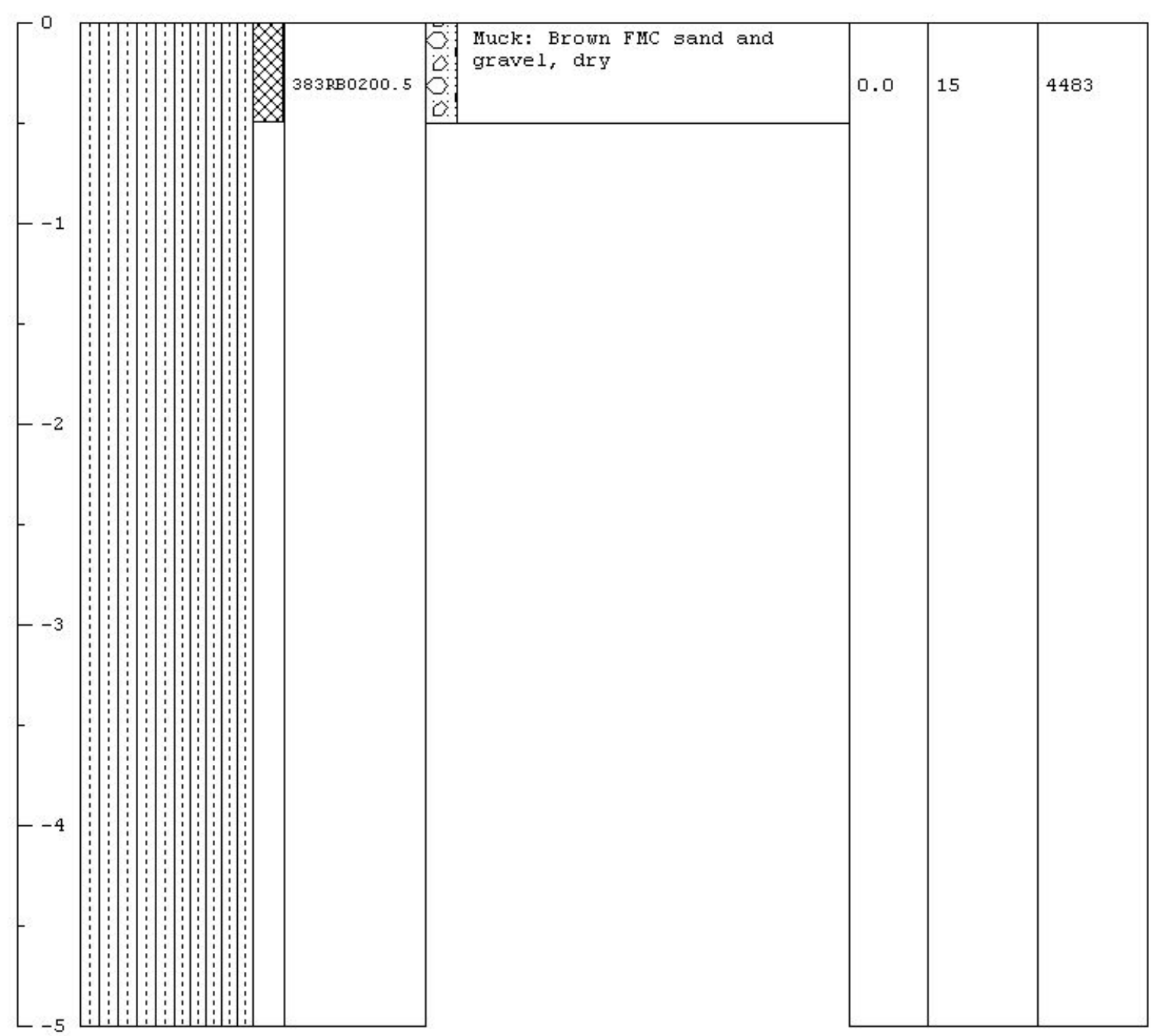




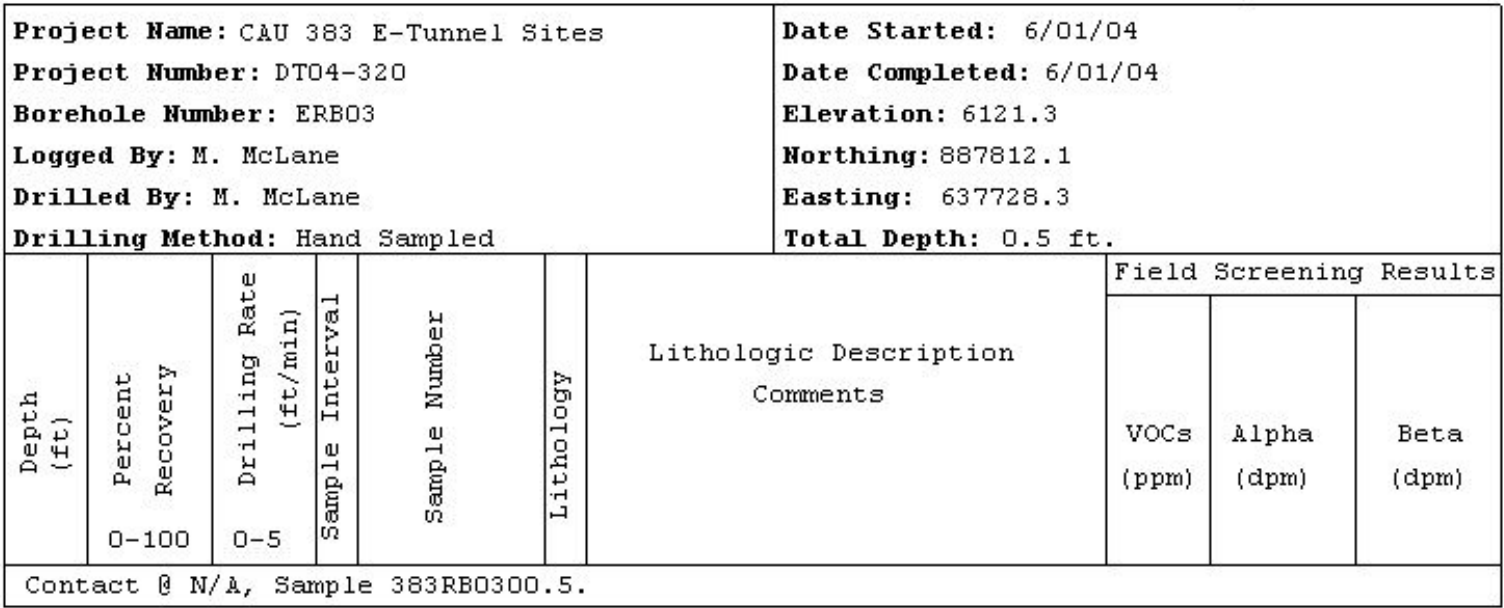

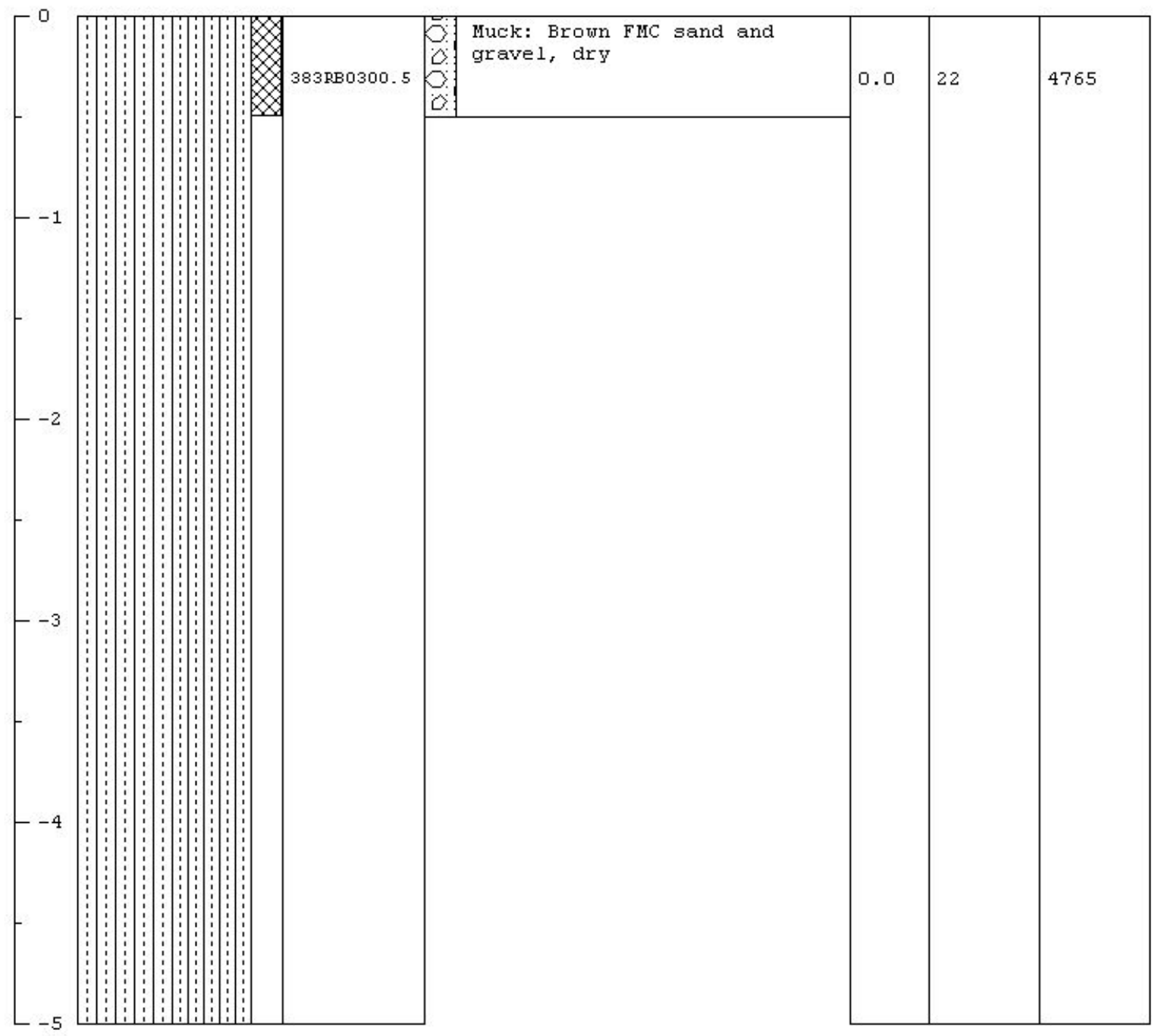


Page 1 of 1

\begin{tabular}{|c|c|c|c|c|c|c|c|c|}
\hline \multicolumn{5}{|c|}{$\begin{array}{l}\text { Project Hame: CAU } 383 \text { E-Tunnel Sites } \\
\text { Project Humber: DT04-320 } \\
\text { Borehole Humber: ERBO4 } \\
\text { Logged By: M. McLane } \\
\text { Drilled By: M. McLane } \\
\text { Drilling Method: Hand Sampled } \\
\end{array}$} & \multicolumn{4}{|c|}{$\begin{array}{l}\text { Date Started: } 6 / 01 / 04 \\
\text { Date Completed: } 6 / 01 / 04 \\
\text { Elevation: } 6118.6 \\
\text { Horthing: } 887795.6 \\
\text { Easting: } 637737.4 \\
\text { Total Depth: } 0.5 \mathrm{ft} .\end{array}$} \\
\hline \multirow[b]{2}{*}{ 营出 } & \multirow[b]{2}{*}{ 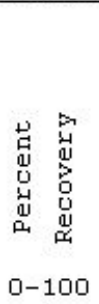 } & \multirow[b]{2}{*}{ 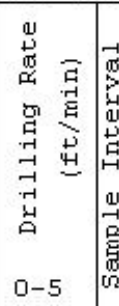 } & \multirow[b]{2}{*}{ 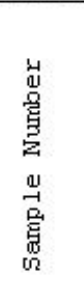 } & \multirow[b]{2}{*}{ 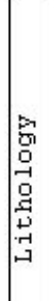 } & \multirow[b]{2}{*}{$\begin{array}{c}\text { Lithologic Description } \\
\text { Comments }\end{array}$} & Field & Screening & Results \\
\hline & & & & & & $\begin{array}{l}\text { VOCs } \\
\text { (ppm) }\end{array}$ & $\begin{array}{l}\text { Alpha } \\
\text { (dpm) }\end{array}$ & $\begin{array}{l}\text { Beta } \\
\text { (dpm) }\end{array}$ \\
\hline
\end{tabular}

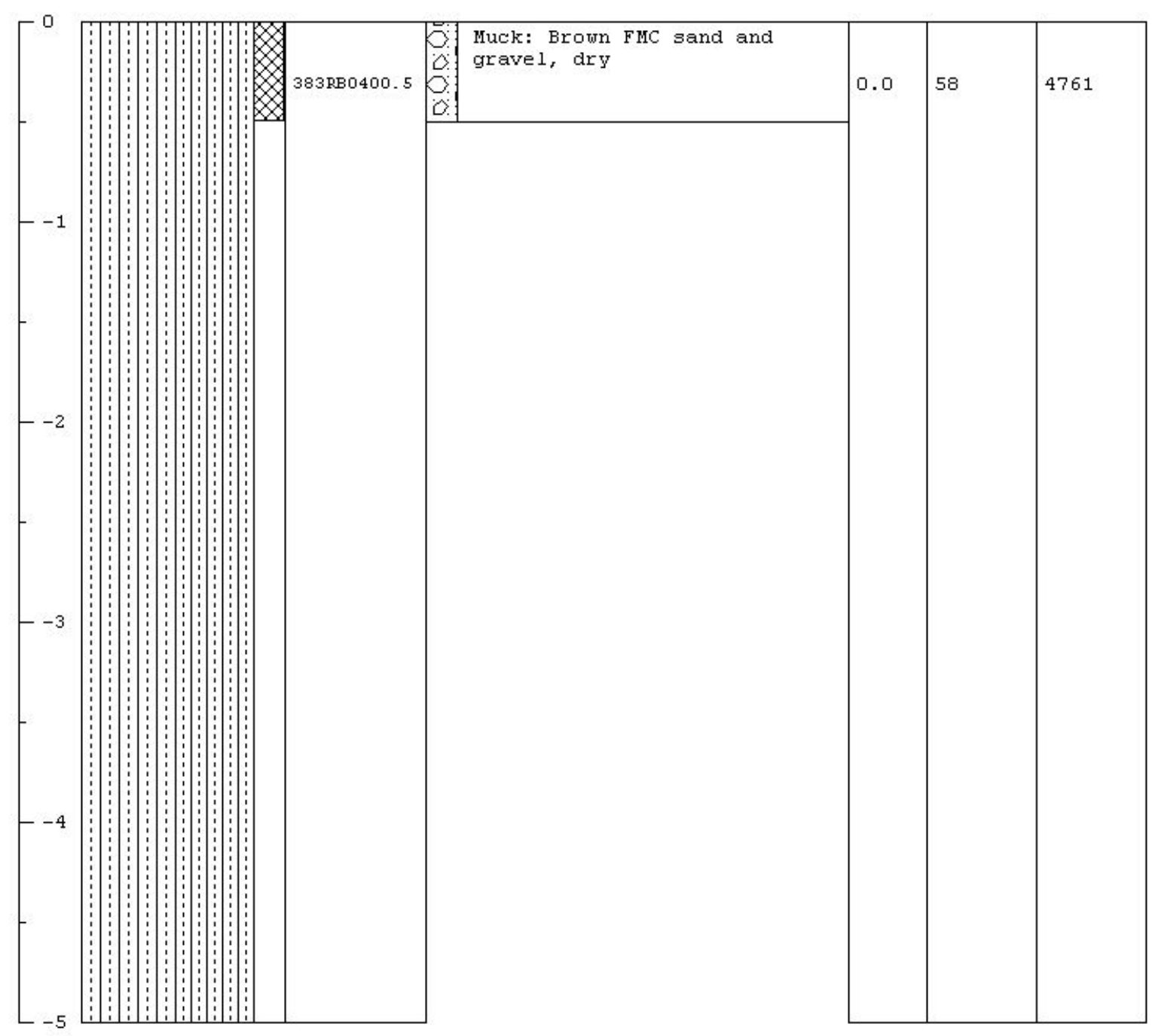




\section{Attachment B}

\section{Cross Sections}

(6 Pages) 


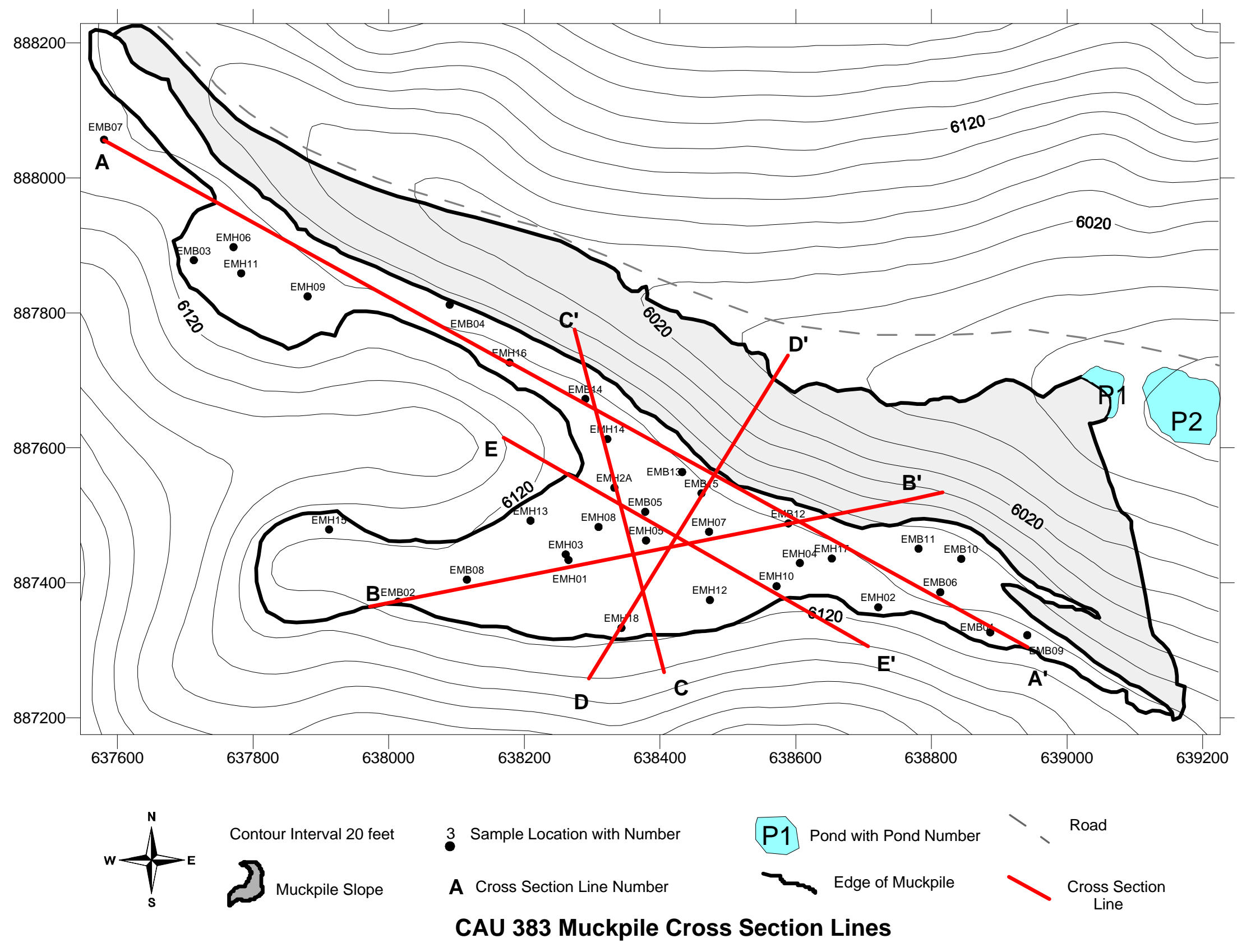

Uncontrolled When Printed 
A

Northwest

Southeast
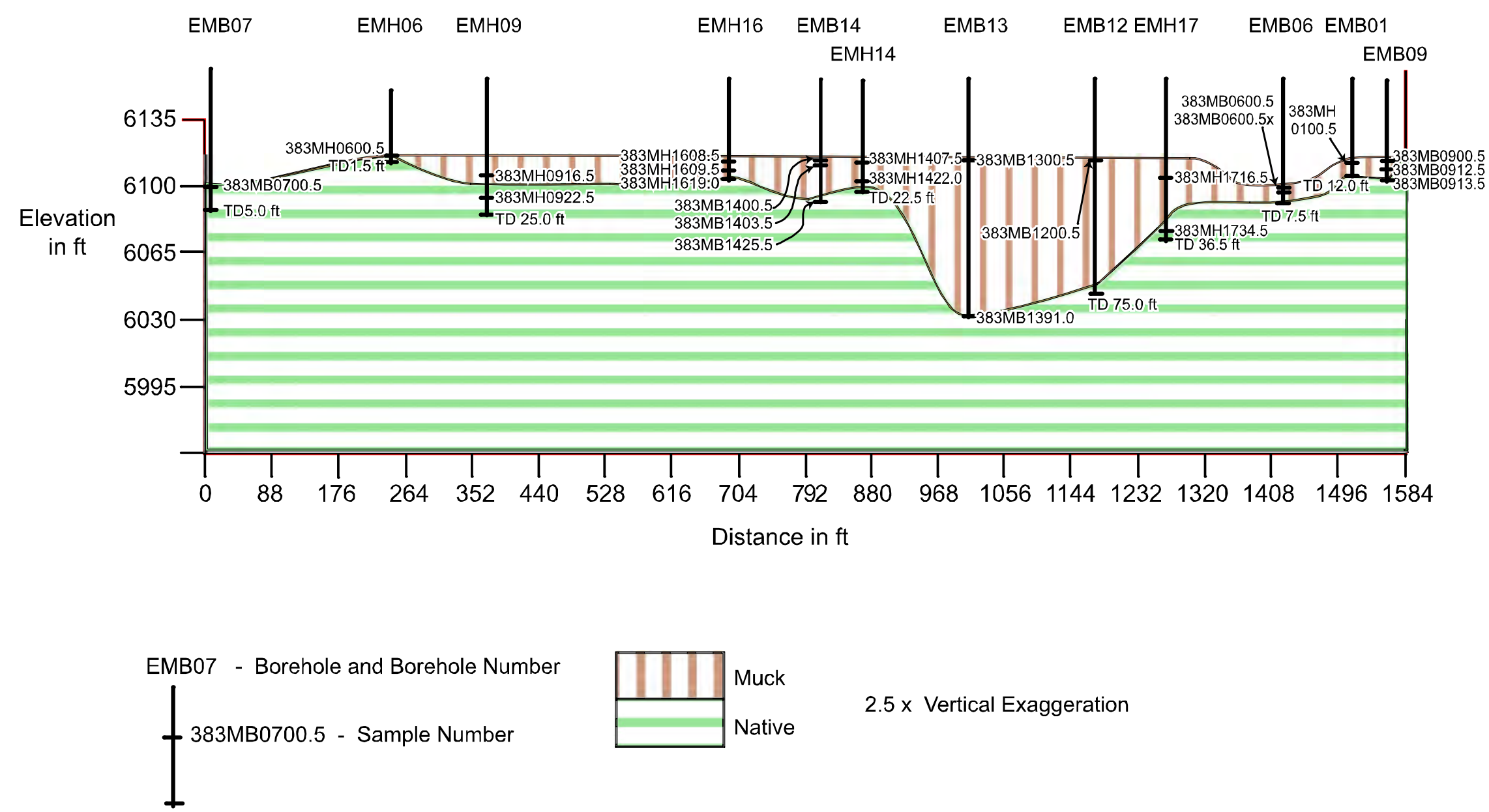

$2.5 \times$ Vertical Exaggeration

Figure B-2 E-Tunnel Muckpile, Northwest to Southeast

Cross Section A - A' 

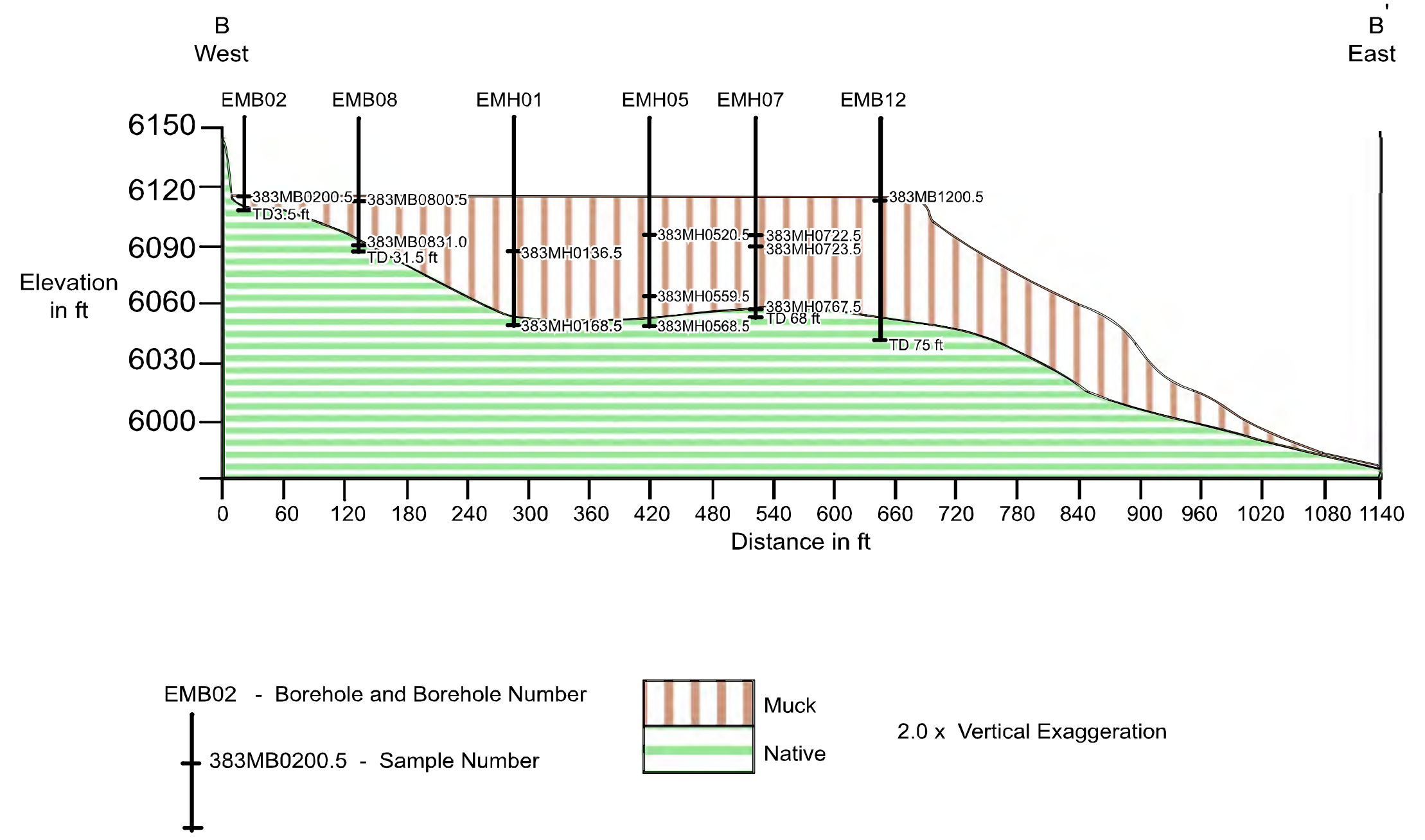

$2.0 \times$ Vertical Exaggeration

Figure B-3 E-Tunnel Muckpile, West to East Cross Section B - B' 
South

North
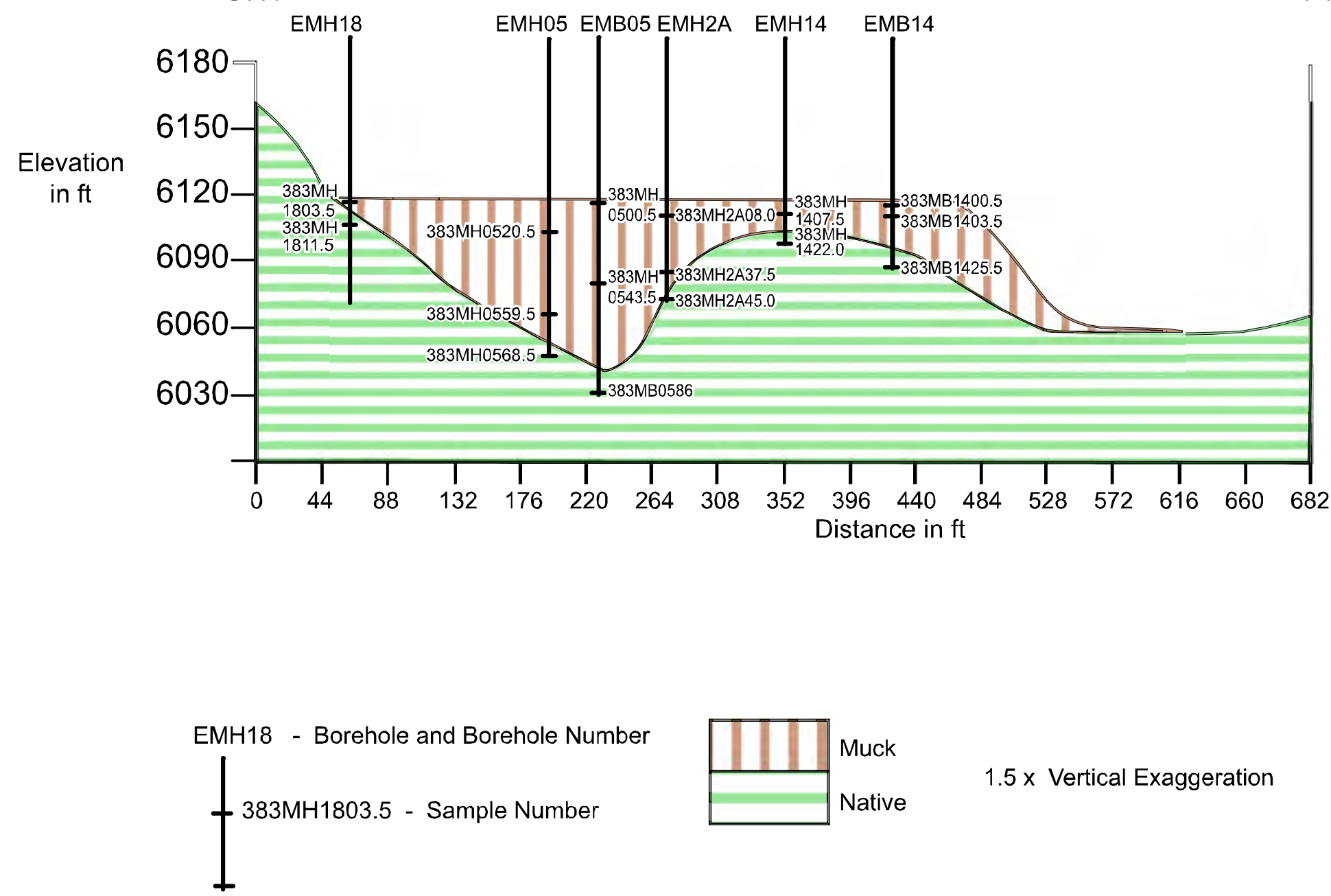

$1.5 \times$ Vertical Exaggeration

Figure B-4 E-Tunnel Muckpile, South to North Cross Section C - C' 


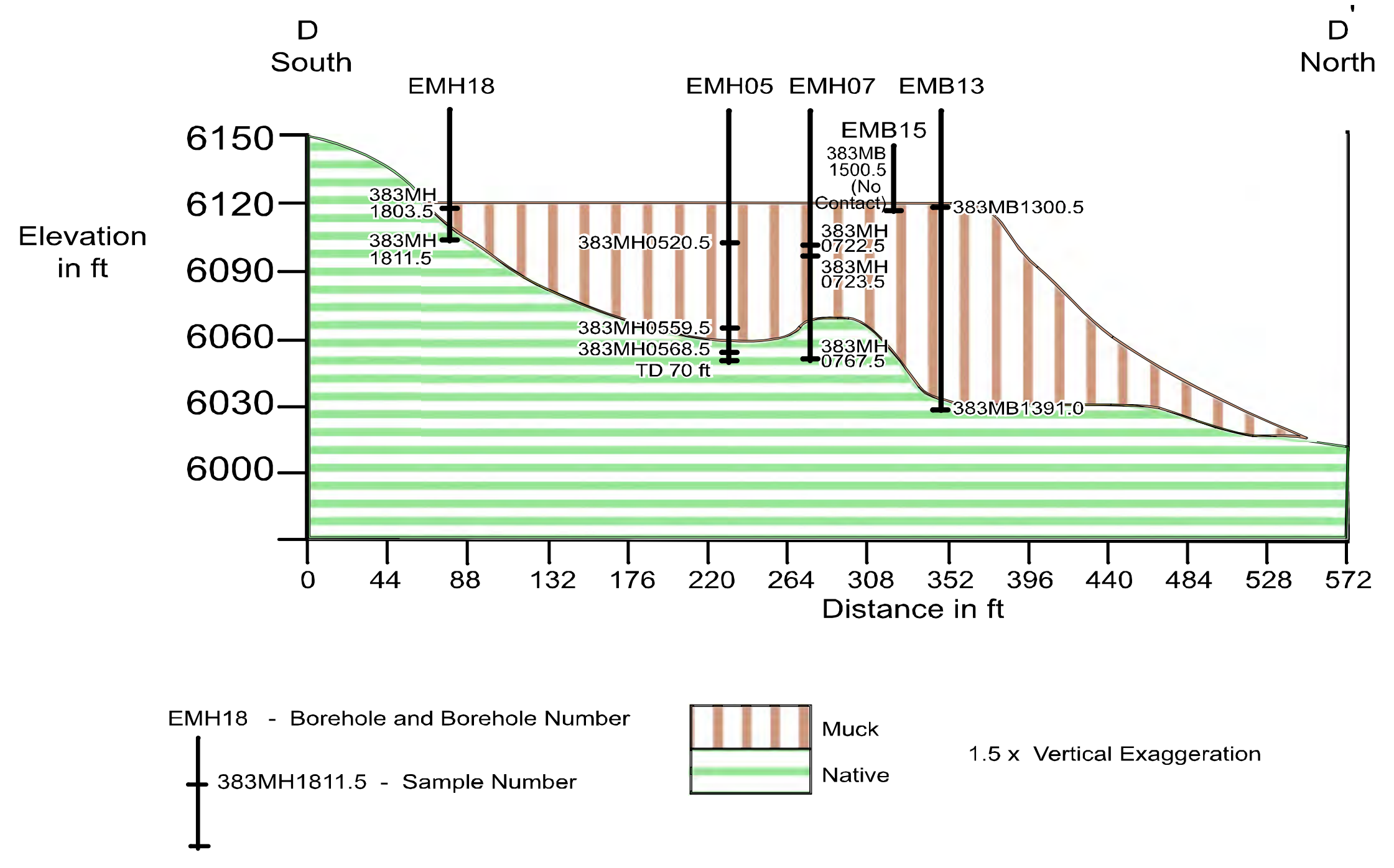

Figure B-5 E-Tunnel Muckpile, South to North Cross Section D - D' 


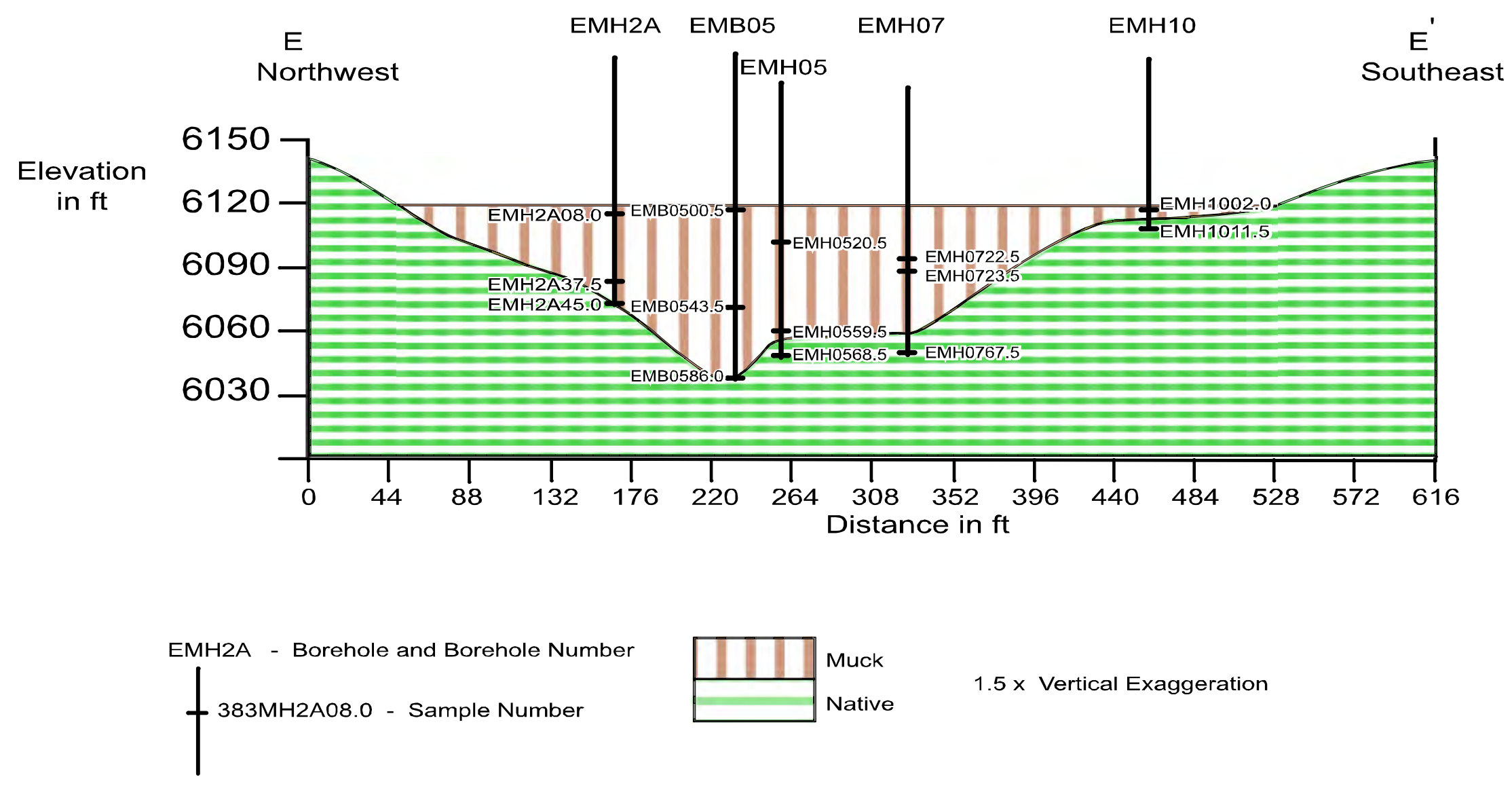

Figure B-6 E-Tunnel Muckpile, Northwest to Southeast Cross Section E - E' 


\section{Attachment C}

\section{Analytical Results Table}

(78 Pages) 


\begin{tabular}{|c|c|c|c|c|c|c|c|c|c|c|}
\hline SAMPLE \# & $\begin{array}{c}\text { SAMPLE } \\
\text { DATE }\end{array}$ & MATRIX & $\begin{array}{c}\text { USER TEST } \\
\text { PANEL }\end{array}$ & $\begin{array}{c}\text { SAMPLE } \\
\text { PURPOSE }\end{array}$ & CAS \# & PARAMETER & RESULT & UNITS & $\mathbf{Q}$ & $\begin{array}{c}\text { DETECT } \\
\text { LIMIT }\end{array}$ \\
\hline 383CW01 & $5 / 23 / 2004$ & water & EPA6010 & SMQC & $7440-38-2$ & Arsenic & 0.0037 & $\mathrm{mg} / \mathrm{L}$ & $\mathrm{B}$ & 0.01 \\
\hline 383CW01 & $5 / 23 / 2004$ & water & EPA6010 & SMQC & $7440-39-3$ & Barium & 0.012 & $\mathrm{mg} / \mathrm{L}$ & $\mathrm{B}$ & 0.1 \\
\hline 383CW01 & $5 / 23 / 2004$ & water & EPA6010 & SMQC & $7440-41-7$ & Beryllium & 0.00006 & $\mathrm{mg} / \mathrm{L}$ & $\mathrm{B}$ & 0.005 \\
\hline 383CW01 & $5 / 23 / 2004$ & water & EPA6010 & SMQC & $7440-47-3$ & Chromium & 0.0017 & $\mathrm{mg} / \mathrm{L}$ & $\mathrm{B}$ & 0.01 \\
\hline 383CW01 & $5 / 23 / 2004$ & water & EPA7470 & SMQC & $7439-97-6$ & Mercury & 0.0000066 & $\mathrm{mg} / \mathrm{L}$ & $\mathrm{B}$ & 0.0002 \\
\hline 383CW01 & $5 / 23 / 2004$ & water & EPA6010 & SMQC & $7440-22-4$ & Silver & 0.0017 & $\mathrm{mg} / \mathrm{L}$ & $\mathrm{B}$ & 0.01 \\
\hline 383CW02 & $5 / 23 / 2004$ & water & EPA8260 & ER & 67-64-1 & Acetone & 22 & $\mu \mathrm{g} / \mathrm{L}$ & & 20 \\
\hline 383CW02 & $5 / 23 / 2004$ & water & EPA6010 & ER & $7440-38-2$ & Arsenic & 0.019 & $\mathrm{mg} / \mathrm{L}$ & & 0.01 \\
\hline 383CW02 & $5 / 23 / 2004$ & water & EPA6010 & ER & 7440-39-3 & Barium & 0.0047 & $\mathrm{mg} / \mathrm{L}$ & $\mathrm{B}$ & 0.1 \\
\hline 383CW02 & $5 / 23 / 2004$ & water & EPA8270 & ER & 117-81-7 & Bis(2-Ethylhexyl)Phthalate & 15 & $\mu \mathrm{g} / \mathrm{L}$ & & 9.5 \\
\hline 383CW02 & $5 / 23 / 2004$ & water & EPA7470 & ER & $7439-97-6$ & Mercury & 0.000007 & $\mathrm{mg} / \mathrm{L}$ & $\mathrm{B}$ & 0.0002 \\
\hline 383CW02 & $5 / 23 / 2004$ & water & EPA6010 & ER & $7440-22-4$ & Silver & 0.0015 & $\mathrm{mg} / \mathrm{L}$ & $\mathrm{B}$ & 0.01 \\
\hline 383CW03 & $5 / 24 / 2004$ & water & EPA8260 & $\mathrm{FB}$ & $67-64-1$ & Acetone & 9.8 & $\mu g / L$ & $\mathrm{~J}$ & 20 \\
\hline 383CW03 & $5 / 24 / 2004$ & water & EPA6010 & FB & $7440-41-7$ & Beryllium & 0.00009 & $\mathrm{mg} / \mathrm{L}$ & $\mathrm{B}$ & 0.005 \\
\hline 383CW03 & $5 / 24 / 2004$ & water & EPA8270 & $\mathrm{FB}$ & 117-81-7 & Bis(2-Ethylhexyl)Phthalate & 5.9 & $\mu g / L$ & $\mathrm{~J}$ & 9.7 \\
\hline 383CW03 & $5 / 24 / 2004$ & water & EPA7470 & FB & $7439-97-6$ & Mercury & 0.0000097 & $\mathrm{mg} / \mathrm{L}$ & $\mathrm{B}$ & 0.0002 \\
\hline 383CW04 & $6 / 1 / 2004$ & water & EPA8270 & FB & $117-81-7$ & Bis(2-Ethylhexyl)Phthalate & 16 & $\mu \mathrm{g} / \mathrm{L}$ & & 11 \\
\hline 383CW04 & $6 / 1 / 2004$ & water & EPA6010 & $\mathrm{FB}$ & $7440-22-4$ & Silver & 0.00068 & $\mathrm{mg} / \mathrm{L}$ & $\mathrm{B}$ & 0.01 \\
\hline 383CW05 & $6 / 1 / 2004$ & water & EPA8260 & ER & 67-64-1 & Acetone & 8.4 & $\mu g / L$ & $\mathrm{~J}$ & 20 \\
\hline 383CW05 & $6 / 1 / 2004$ & water & EPA8270 & ER & 117-81-7 & Bis(2-Ethylhexyl)Phthalate & 17 & $\mu g / L$ & & 9.5 \\
\hline 383CW05 & $6 / 1 / 2004$ & water & EPA6010 & ER & $7782-49-2$ & Selenium & 0.0024 & $\mathrm{mg} / \mathrm{L}$ & $\mathrm{B}$ & 0.005 \\
\hline 383CW06 & $6 / 7 / 2004$ & water & EPA8270 & $\mathrm{FB}$ & 117-81-7 & Bis(2-Ethylhexyl)Phthalate & 8.8 & $\mu g / L$ & $\mathrm{~J}$ & 9.6 \\
\hline 383CW06 & $6 / 7 / 2004$ & water & EPA6010 & FB & $7440-22-4$ & Silver & 0.00094 & $\mathrm{mg} / \mathrm{L}$ & $\mathrm{B}$ & 0.01 \\
\hline 383CW07 & $6 / 7 / 2004$ & water & EPA8260 & ER & $67-64-1$ & Acetone & 6.9 & $\mu g / L$ & $\mathrm{~J}$ & 20 \\
\hline 383CW07 & $6 / 7 / 2004$ & water & EPA6010 & ER & $7440-39-3$ & Barium & 0.0016 & $\mathrm{mg} / \mathrm{L}$ & $\mathrm{B}$ & 0.1 \\
\hline 383CW07 & $6 / 7 / 2004$ & water & EPA8270 & ER & 117-81-7 & Bis(2-Ethylhexyl)Phthalate & 7.4 & $\mu \mathrm{g} / \mathrm{L}$ & $\mathrm{J}$ & 9.6 \\
\hline 383CW07 & $6 / 7 / 2004$ & water & EPA8260 & ER & 74-87-3 & Chloromethane & 0.58 & $\mu \mathrm{g} / \mathrm{L}$ & $\mathrm{J}$ & 10 \\
\hline 383CW08 & $6 / 10 / 2004$ & water & EPA8260 & FB & $67-64-1$ & Acetone & 25 & $\mu \mathrm{g} / \mathrm{L}$ & $\mathrm{B}$ & 20 \\
\hline 383CW08 & $6 / 10 / 2004$ & water & EPA8270 & $\mathrm{FB}$ & 117-81-7 & Bis(2-Ethylhexyl)Phthalate & 6.5 & $\mu g / L$ & $\mathrm{~J}$ & 9.6 \\
\hline 383CW08 & $6 / 10 / 2004$ & water & EPA7470 & FB & 7439-97-6 & Mercury & 0.0000032 & $\mathrm{mg} / \mathrm{L}$ & $\mathrm{B}$ & 0.0002 \\
\hline 383CW09 & $6 / 10 / 2004$ & water & EPA8260 & ER & 67-64-1 & Acetone & 20 & $\mu \mathrm{g} / \mathrm{L}$ & $\mathrm{B}$ & 20 \\
\hline 383CW09 & $6 / 10 / 2004$ & water & EPA8270 & ER & 117-81-7 & Bis(2-Ethylhexyl)Phthalate & 10 & $\mu \mathrm{g} / \mathrm{L}$ & & 9.6 \\
\hline 383CW09 & $6 / 10 / 2004$ & water & EPA6010 & ER & $7440-43-9$ & Cadmium & 0.00018 & $\mathrm{mg} / \mathrm{L}$ & $\mathrm{B}$ & 0.005 \\
\hline 383CW09 & $6 / 10 / 2004$ & water & EPA7470 & ER & $7439-97-6$ & Mercury & 0.0000035 & $\mathrm{mg} / \mathrm{L}$ & $\mathrm{B}$ & 0.0002 \\
\hline 383CW09 & $6 / 10 / 2004$ & water & EPA6010 & ER & $7440-22-4$ & Silver & 0.00066 & $\mathrm{mg} / \mathrm{L}$ & $\mathrm{B}$ & 0.01 \\
\hline
\end{tabular}

Page 1 of 78

Uncontrolled When Printed 


\begin{tabular}{|c|c|c|c|c|c|c|c|c|c|c|}
\hline SAMPLE \# & $\begin{array}{c}\text { SAMPLE } \\
\text { DATE }\end{array}$ & MATRIX & $\begin{array}{l}\text { USER TEST } \\
\text { PANEL }\end{array}$ & $\begin{array}{c}\text { SAMPLE } \\
\text { PURPOSE }\end{array}$ & CAS \# & PARAMETER & RESULT & UNITS & $\mathbf{Q}$ & $\begin{array}{c}\text { DETECT } \\
\text { LIMIT }\end{array}$ \\
\hline 383CW10 & $6 / 12 / 2004$ & water & EPA8270 & FB & $117-81-7$ & Bis(2-Ethylhexyl)Phthalate & 26 & $\mu \mathrm{g} / \mathrm{L}$ & & 9.6 \\
\hline 383CW10 & $6 / 12 / 2004$ & water & EPA8260 & FB & $75-09-2$ & Methylene Chloride & 0.79 & $\mu \mathrm{g} / \mathrm{L}$ & $\mathrm{J}$ & 5 \\
\hline 383CW11 & $6 / 12 / 2004$ & water & EPA6010 & ER & $7440-39-3$ & Barium & 0.005 & $\mathrm{mg} / \mathrm{L}$ & $\mathrm{B}$ & 0.1 \\
\hline 383CW11 & $6 / 12 / 2004$ & water & EPA8270 & ER & 117-81-7 & Bis(2-Ethylhexyl)Phthalate & 42 & $\mu \mathrm{g} / \mathrm{L}$ & & 9.6 \\
\hline 383CW11 & $6 / 12 / 2004$ & water & EPA7470 & ER & $7439-97-6$ & Mercury & 0.0000042 & $\mathrm{mg} / \mathrm{L}$ & $B$ & 0.0002 \\
\hline 383CW11 & $6 / 12 / 2004$ & water & EPA8260 & ER & $75-09-2$ & Methylene Chloride & 0.75 & $\mu \mathrm{g} / \mathrm{L}$ & $\mathrm{J}$ & 5 \\
\hline $383 C W 12$ & $6 / 16 / 2004$ & water & EPA8270 & FB & $117-81-7$ & Bis(2-Ethylhexyl)Phthalate & 6 & $\mu \mathrm{g} / \mathrm{L}$ & $\mathrm{J}$ & 9.5 \\
\hline 383CW12 & $6 / 16 / 2004$ & water & EPA7470 & FB & 7439-97-6 & Mercury & 0.00003 & $\mathrm{mg} / \mathrm{L}$ & B & 0.0002 \\
\hline 383CW12 & $6 / 16 / 2004$ & water & EPA8260 & FB & $75-09-2$ & Methylene Chloride & 1.6 & $\mu \mathrm{g} / \mathrm{L}$ & $\mathrm{J}$ & 5 \\
\hline 383CW13 & $6 / 16 / 2004$ & water & EPA6010 & ER & $7440-39-3$ & Barium & 0.00049 & $\mathrm{mg} / \mathrm{L}$ & $\mathrm{B}$ & 0.1 \\
\hline $383 C W 13$ & $6 / 16 / 2004$ & water & EPA8270 & ER & $117-81-7$ & Bis(2-Ethylhexyl)Phthalate & 24 & $\mu \mathrm{g} / \mathrm{L}$ & & 9.6 \\
\hline 383CW13 & $6 / 16 / 2004$ & water & EPA8260 & ER & $74-87-3$ & Chloromethane & 0.71 & $\mu \mathrm{g} / \mathrm{L}$ & $\mathrm{J}$ & 10 \\
\hline 383CW13 & $6 / 16 / 2004$ & water & EPA7470 & ER & $7439-97-6$ & Mercury & 0.00003 & $\mathrm{mg} / \mathrm{L}$ & $\mathrm{B}$ & 0.0002 \\
\hline $383 C W 13$ & $6 / 16 / 2004$ & water & EPA8260 & ER & $75-09-2$ & Methylene Chloride & 1.4 & $\mu \mathrm{g} / \mathrm{L}$ & J & 5 \\
\hline $383 C W 13$ & $6 / 16 / 2004$ & water & EPA6010 & ER & $7440-22-4$ & Silver & 0.00087 & $\mathrm{mg} / \mathrm{L}$ & $\mathrm{B}$ & 0.01 \\
\hline 383CW14 & $6 / 17 / 2004$ & water & EPA8270 & FB & 117-81-7 & Bis(2-Ethylhexyl)Phthalate & 10 & $\mu \mathrm{g} / \mathrm{L}$ & & 9.5 \\
\hline $383 C W 14$ & $6 / 17 / 2004$ & water & EPA7470 & FB & $7439-97-6$ & Mercury & 0.00003 & $\mathrm{mg} / \mathrm{L}$ & $B$ & 0.0002 \\
\hline 383CW14 & $6 / 17 / 2004$ & water & EPA8260 & FB & $75-09-2$ & Methylene Chloride & 1.5 & $\mu \mathrm{g} / \mathrm{L}$ & $\mathrm{J}$ & 5 \\
\hline 383CW15 & $6 / 17 / 2004$ & water & EPA6010 & ER & $7440-39-3$ & Barium & 0.013 & $\mathrm{mg} / \mathrm{L}$ & $\mathrm{B}$ & 0.1 \\
\hline 383CW15 & $6 / 17 / 2004$ & water & EPA8270 & ER & $117-81-7$ & Bis(2-Ethylhexyl)Phthalate & 18 & $\mu \mathrm{g} / \mathrm{L}$ & & 9.6 \\
\hline 383CW15 & $6 / 17 / 2004$ & water & EPA7470 & ER & $7439-97-6$ & Mercury & 0.00003 & $\mathrm{mg} / \mathrm{L}$ & B & 0.0002 \\
\hline 383CW15 & $6 / 17 / 2004$ & water & EPA8260 & ER & $75-09-2$ & Methylene Chloride & 1.3 & $\mu \mathrm{g} / \mathrm{L}$ & $\mathrm{J}$ & 5 \\
\hline $383 C W 15$ & $6 / 17 / 2004$ & water & EPA6010 & ER & $7440-22-4$ & Silver & 0.00069 & $\mathrm{mg} / \mathrm{L}$ & $B$ & 0.01 \\
\hline 383DH0100.5 & $6 / 7 / 2004$ & soil & HASL300 & REG & 14331-83-0 & Ac-228 & 2.21 & $\mathrm{pCi} / \mathrm{g}$ & G & 0.68 \\
\hline 383DH0100.5 & $6 / 7 / 2004$ & soil & EPA6010 & REG & 7440-38-2 & Arsenic & 2.3 & $\mathrm{mg} / \mathrm{kg}$ & & 1 \\
\hline 383DH0100.5 & $6 / 7 / 2004$ & soil & EPA6010 & REG & $7440-39-3$ & Barium & 750 & $\mathrm{mg} / \mathrm{kg}$ & & 10 \\
\hline 383DH0100.5 & $6 / 7 / 2004$ & soil & EPA6010 & REG & $7440-41-7$ & Beryllium & 1.3 & $\mathrm{mg} / \mathrm{kg}$ & & 0.52 \\
\hline 383DH0100.5 & $6 / 7 / 2004$ & soil & HASL300 & REG & $14733-03-0$ & Bi-214 & 1.24 & $\mathrm{pCi} / \mathrm{g}$ & $\mathrm{G}, \mathrm{J}$ & 0.46 \\
\hline 383DH0100.5 & $6 / 7 / 2004$ & soil & EPA6010 & REG & $7440-43-9$ & Cadmium & 0.14 & $\mathrm{mg} / \mathrm{kg}$ & $B$ & 0.52 \\
\hline 383DH0100.5 & $6 / 7 / 2004$ & soil & EPA6010 & REG & $7440-47-3$ & Chromium & 1.1 & $\mathrm{mg} / \mathrm{kg}$ & & 1 \\
\hline 383DH0100.5 & $6 / 7 / 2004$ & soil & HASL300 & REG & $10045-97-3$ & Cs-137 & 34.4 & $\mathrm{pCi} / \mathrm{g}$ & $\mathrm{G}$ & 0.2 \\
\hline 383DH0100.5 & $6 / 7 / 2004$ & soil & HASL300 & REG & 13966-00-2 & $\mathrm{K}-40$ & 34.5 & $\mathrm{pCi} / \mathrm{g}$ & $\mathrm{G}$ & 1.9 \\
\hline 383DH0100.5 & $6 / 7 / 2004$ & soil & EPA6010 & REG & 7439-92-1 & Lead & 20 & $\mathrm{mg} / \mathrm{kg}$ & & 0.31 \\
\hline 383DH0100.5 & $6 / 7 / 2004$ & soil & EPA8260 & REG & 136777-61-2 & M+P-Xylene & 2.1 & $\mu \mathrm{g} / \mathrm{L}$ & $\mathrm{J}$ & 5.2 \\
\hline 383DH0100.5 & $6 / 7 / 2004$ & soil & EPA7470 & REG & 7439-97-6 & Mercury & 0.01 & $\mathrm{mg} / \mathrm{kg}$ & $\mathrm{B}$ & 0.1 \\
\hline
\end{tabular}

Page 2 of 78

Uncontrolled When Printed 


\begin{tabular}{|c|c|c|c|c|c|c|c|c|c|c|}
\hline SAMPLE \# & $\begin{array}{l}\text { SAMPLE } \\
\text { DATE }\end{array}$ & MATRIX & $\begin{array}{l}\text { USER TEST } \\
\text { PANEL }\end{array}$ & $\begin{array}{c}\text { SAMPLE } \\
\text { PURPOSE }\end{array}$ & CAS \# & PARAMETER & RESULT & UNITS & $\mathbf{Q}$ & $\begin{array}{c}\text { DETECT } \\
\text { LIMIT }\end{array}$ \\
\hline 383DH0100.5 & $6 / 7 / 2004$ & soil & EPA8260 & REG & $75-09-2$ & Methylene Chloride & 0.83 & $\mu \mathrm{g} / \mathrm{L}$ & $\mathrm{J}$ & 5.2 \\
\hline 383DH0100.5 & $6 / 7 / 2004$ & soil & HASL300 & REG & 15092-94-1 & $\mathrm{Pb}-212$ & 2.51 & $\mathrm{pCi} / \mathrm{g}$ & $\mathrm{J}$ & 0.41 \\
\hline 383DH0100.5 & $6 / 7 / 2004$ & soil & HASL300 & REG & $15067-28-4$ & $\mathrm{~Pb}-214$ & 1.41 & $\mathrm{pCi} / \mathrm{g}$ & $\mathrm{G}, \mathrm{J}$ & 0.55 \\
\hline 383DH0100.5 & $6 / 7 / 2004$ & soil & UGTAISOPU & REG & 13981-16-3 & $\mathrm{Pu}-238$ & 0.73 & $\mathrm{pCi} / \mathrm{g}$ & & 0.02 \\
\hline 383DH0100.5 & $6 / 7 / 2004$ & soil & UGTAISOPU & REG & $15117-48-3$ & Pu-239 & 3.03 & $\mathrm{pCi} / \mathrm{g}$ & $\mathrm{J}$ & 0.01 \\
\hline 383DH0100.5 & $6 / 7 / 2004$ & soil & SR7500 & REG & $10098-97-2$ & Sr-90 & 1.88 & $\mathrm{pCi} / \mathrm{g}$ & & 0.24 \\
\hline 383DH0100.5 & $6 / 7 / 2004$ & soil & HASL300 & REG & 14913-50-9 & TI-208 & 0.71 & $\mathrm{pCi} / \mathrm{g}$ & $G$ & 0.22 \\
\hline 383DH0200.5 & $6 / 7 / 2004$ & soil & EPA8260 & REG & $78-93-3$ & 2-Butanone & 10 & $\mu \mathrm{g} / \mathrm{kg}$ & $\mathrm{J}$ & 21 \\
\hline 383DH0200.5 & $6 / 7 / 2004$ & soil & HASL300 & REG & 14331-83-0 & Ac-228 & 2.26 & $\mathrm{pCi} / \mathrm{g}$ & G & 0.71 \\
\hline 383DH0200.5 & $6 / 7 / 2004$ & soil & EPA8260 & REG & 67-64-1 & Acetone & 39 & $\mu \mathrm{g} / \mathrm{kg}$ & & 21 \\
\hline 383DH0200.5 & $6 / 7 / 2004$ & soil & HASL300 & REG & 14596-10-2 & Am-241 & 1.88 & $\mathrm{pCi} / \mathrm{g}$ & $\mathrm{J}$ & 0.48 \\
\hline 383DH0200.5 & $6 / 7 / 2004$ & soil & EPA6010 & REG & $7440-38-2$ & Arsenic & 2.6 & $\mathrm{mg} / \mathrm{kg}$ & & 1 \\
\hline 383DH0200.5 & $6 / 7 / 2004$ & soil & EPA6010 & REG & $7440-39-3$ & Barium & 3100 & $\mathrm{mg} / \mathrm{kg}$ & & 100 \\
\hline 383DH0200.5 & $6 / 7 / 2004$ & soil & EPA6010 & REG & $7440-41-7$ & Beryllium & 1.2 & $\mathrm{mg} / \mathrm{kg}$ & & 0.51 \\
\hline 383DH0200.5 & $6 / 7 / 2004$ & soil & HASL300 & REG & 14733-03-0 & Bi-214 & 1.27 & $\mathrm{pCi} / \mathrm{g}$ & $\mathrm{G}, \mathrm{J}$ & 0.47 \\
\hline 383DH0200.5 & $6 / 7 / 2004$ & soil & EPA6010 & REG & $7440-43-9$ & Cadmium & 0.16 & $\mathrm{mg} / \mathrm{kg}$ & $\mathrm{B}$ & 0.51 \\
\hline 383DH0200.5 & $6 / 7 / 2004$ & soil & EPA6010 & REG & $7440-47-3$ & Chromium & 2.6 & $\mathrm{mg} / \mathrm{kg}$ & & 1 \\
\hline 383DH0200.5 & $6 / 7 / 2004$ & soil & HASL300 & REG & $10045-97-3$ & Cs-137 & 45.8 & $\mathrm{pCi} / \mathrm{g}$ & G & 0.2 \\
\hline 383DH0200.5 & $6 / 7 / 2004$ & soil & EPA8260 & REG & $100-41-4$ & Ethylbenzene & 3.1 & $\mu \mathrm{g} / \mathrm{kg}$ & $\mathrm{J}$ & 5.1 \\
\hline 383DH0200.5 & $6 / 7 / 2004$ & soil & HASL300 & REG & 13966-00-2 & $\mathrm{K}-40$ & 31.8 & $\mathrm{pCi} / \mathrm{g}$ & $G$ & 2.2 \\
\hline 383DH0200.5 & $6 / 7 / 2004$ & soil & EPA6010 & REG & 7439-92-1 & Lead & 15 & $\mathrm{mg} / \mathrm{kg}$ & & 0.31 \\
\hline 383DH0200.5 & $6 / 7 / 2004$ & soil & EPA8260 & REG & 136777-61-2 & M+P-Xylene & 18 & $\mu \mathrm{g} / \mathrm{kg}$ & & 5.1 \\
\hline 383DH0200.5 & $6 / 7 / 2004$ & soil & EPA7470 & REG & 7439-97-6 & Mercury & 0.015 & $\mathrm{mg} / \mathrm{kg}$ & $\mathrm{B}$ & 0.1 \\
\hline 383DH0200.5 & $6 / 7 / 2004$ & soil & EPA8260 & REG & $75-09-2$ & Methylene Chloride & 4 & $\mu \mathrm{g} / \mathrm{kg}$ & $\mathrm{J}$ & 5.1 \\
\hline 383DH0200.5 & $6 / 7 / 2004$ & soil & EPA8260 & REG & $95-47-6$ & O-Xylene & 6.7 & $\mu \mathrm{g} / \mathrm{kg}$ & & 5.1 \\
\hline 383DH0200.5 & $6 / 7 / 2004$ & soil & HASL300 & REG & 15092-94-1 & $\mathrm{Pb}-212$ & 2.42 & $\mathrm{pCi} / \mathrm{g}$ & $\mathrm{J}$ & 0.44 \\
\hline 383DH0200.5 & $6 / 7 / 2004$ & soil & HASL300 & REG & $15067-28-4$ & $\mathrm{~Pb}-214$ & 1.4 & $\mathrm{pCi} / \mathrm{g}$ & $\mathrm{G}, \mathrm{J}$ & 0.58 \\
\hline 383DH0200.5 & $6 / 7 / 2004$ & soil & UGTAISOPU & REG & 13981-16-3 & Pu-238 & 0.156 & $\mathrm{pCi} / \mathrm{g}$ & & 0.029 \\
\hline 383DH0200.5 & $6 / 7 / 2004$ & soil & UGTAISOPU & REG & $15117-48-3$ & Pu-239 & 1.46 & $\mathrm{pCi} / \mathrm{g}$ & $\mathrm{J}$ & 0.02 \\
\hline 383DH0200.5 & $6 / 7 / 2004$ & soil & SR7500 & REG & 10098-97-2 & Sr-90 & 1.23 & $\mathrm{pCi} / \mathrm{g}$ & Y1 & 0.22 \\
\hline 383DH0200.5 & $6 / 7 / 2004$ & soil & EPA8260 & REG & $100-42-5$ & Styrene & 1.2 & $\mu \mathrm{g} / \mathrm{kg}$ & $\mathrm{J}$ & 5.1 \\
\hline 383DH0200.5 & $6 / 7 / 2004$ & soil & HASL300 & REG & 14913-50-9 & TI-208 & 0.7 & $\mathrm{pCi} / \mathrm{g}$ & $\mathrm{G}$ & 0.24 \\
\hline 383DH0200.5 & $6 / 7 / 2004$ & soil & EPA8260 & REG & $108-88-3$ & Toluene & 1.4 & $\mu \mathrm{g} / \mathrm{kg}$ & $\mathrm{J}$ & 5.1 \\
\hline 383DH0300.5 & $6 / 7 / 2004$ & soil & HASL300 & REG & $14331-83-0$ & Ac-228 & 1.91 & $\mathrm{pCi} / \mathrm{g}$ & G & 0.53 \\
\hline 383DH0300.5 & $6 / 7 / 2004$ & soil & EPA8260 & REG & 67-64-1 & Acetone & 18 & $\mu \mathrm{g} / \mathrm{kg}$ & $\mathrm{J}$ & 20 \\
\hline
\end{tabular}

Page 3 of 78

Uncontrolled When Printed 


\begin{tabular}{|c|c|c|c|c|c|c|c|c|c|c|}
\hline SAMPLE \# & $\begin{array}{l}\text { SAMPLE } \\
\text { DATE }\end{array}$ & MATRIX & $\begin{array}{c}\text { USER TEST } \\
\text { PANEL }\end{array}$ & $\begin{array}{c}\text { SAMPLE } \\
\text { PURPOSE }\end{array}$ & CAS \# & PARAMETER & RESULT & UNITS & $\mathbf{Q}$ & $\begin{array}{c}\text { DETECT } \\
\text { LIMIT }\end{array}$ \\
\hline 383DH0300.5 & $6 / 7 / 2004$ & soil & EPA6010 & REG & $7440-38-2$ & Arsenic & 2.7 & $\mathrm{mg} / \mathrm{kg}$ & & 1 \\
\hline 383DH0300.5 & $6 / 7 / 2004$ & soil & EPA6010 & REG & $7440-39-3$ & Barium & 2800 & $\mathrm{mg} / \mathrm{kg}$ & & 100 \\
\hline 383DH0300.5 & $6 / 7 / 2004$ & soil & EPA6010 & REG & $7440-41-7$ & Beryllium & 1.6 & $\mathrm{mg} / \mathrm{kg}$ & & 0.51 \\
\hline 383DH0300.5 & $6 / 7 / 2004$ & soil & HASL300 & REG & $14733-03-0$ & Bi-214 & 1.32 & $\mathrm{pCi} / \mathrm{g}$ & $\mathrm{G}, \mathrm{J}$ & 0.31 \\
\hline 383DH0300.5 & $6 / 7 / 2004$ & soil & EPA6010 & REG & $7440-43-9$ & Cadmium & 0.16 & $\mathrm{mg} / \mathrm{kg}$ & $\mathrm{B}$ & 0.51 \\
\hline 383DH0300.5 & $6 / 7 / 2004$ & soil & EPA6010 & REG & $7440-47-3$ & Chromium & 3.9 & $\mathrm{mg} / \mathrm{kg}$ & & 1 \\
\hline 383DH0300.5 & $6 / 7 / 2004$ & soil & HASL300 & REG & $10045-97-3$ & Cs-137 & 3.72 & $\mathrm{pCi} / \mathrm{g}$ & $G$ & 0.15 \\
\hline 383DH0300.5 & $6 / 7 / 2004$ & soil & EPA8260 & REG & $100-41-4$ & Ethylbenzene & 11 & $\mu \mathrm{g} / \mathrm{kg}$ & & 5.1 \\
\hline 383DH0300.5 & $6 / 7 / 2004$ & soil & HASL300 & REG & 13966-00-2 & $\mathrm{K}-40$ & 27.6 & $\mathrm{pCi} / \mathrm{g}$ & $G$ & 1.5 \\
\hline 383DH0300.5 & $6 / 7 / 2004$ & soil & EPA6010 & REG & $7439-92-1$ & Lead & 17 & $\mathrm{mg} / \mathrm{kg}$ & & 0.31 \\
\hline 383DH0300.5 & $6 / 7 / 2004$ & soil & EPA8260 & REG & 136777-61-2 & M+P-Xylene & 57 & $\mu \mathrm{g} / \mathrm{kg}$ & & 5.1 \\
\hline 383DH0300.5 & $6 / 7 / 2004$ & soil & EPA7470 & REG & 7439-97-6 & Mercury & 0.0088 & $\mathrm{mg} / \mathrm{kg}$ & $\mathrm{B}$ & 0.1 \\
\hline 383DH0300.5 & $6 / 7 / 2004$ & soil & EPA8260 & REG & $75-09-2$ & Methylene Chloride & 3.1 & $\mu \mathrm{g} / \mathrm{kg}$ & $\mathrm{J}$ & 5.1 \\
\hline 383DH0300.5 & $6 / 7 / 2004$ & soil & EPA8260 & REG & $95-47-6$ & O-Xylene & 23 & $\mu \mathrm{g} / \mathrm{kg}$ & & 5.1 \\
\hline 383DH0300.5 & $6 / 7 / 2004$ & soil & HASL300 & REG & 15092-94-1 & $\mathrm{Pb}-212$ & 2.08 & $\mathrm{pCi} / \mathrm{g}$ & $\mathrm{J}$ & 0.25 \\
\hline 383DH0300.5 & $6 / 7 / 2004$ & soil & HASL300 & REG & $15067-28-4$ & $\mathrm{~Pb}-214$ & 1.21 & $\mathrm{pCi} / \mathrm{g}$ & $\mathrm{G}, \mathrm{J}$ & 0.26 \\
\hline 383DH0300.5 & $6 / 7 / 2004$ & soil & UGTAISOPU & REG & $15117-48-3$ & Pu-239 & 0.186 & $\mathrm{pCi} / \mathrm{g}$ & $\mathrm{J}$ & 0.025 \\
\hline 383DH0300.5 & $6 / 7 / 2004$ & soil & EPA8260 & REG & $100-42-5$ & Styrene & 3.6 & $\mu \mathrm{g} / \mathrm{kg}$ & $\mathrm{J}$ & 5.1 \\
\hline 383DH0300.5 & $6 / 7 / 2004$ & soil & HASL300 & REG & $14913-50-9$ & TI-208 & 0.58 & $\mathrm{pCi} / \mathrm{g}$ & G & 0.14 \\
\hline 383DH0300.5 & $6 / 7 / 2004$ & soil & EPA8260 & REG & $108-88-3$ & Toluene & 1.4 & $\mu \mathrm{g} / \mathrm{kg}$ & $\mathrm{J}$ & 5.1 \\
\hline 383DH0400.5 & $6 / 7 / 2004$ & soil & HASL300 & REG & 14331-83-0 & Ac-228 & 1.8 & $\mathrm{pCi} / \mathrm{g}$ & $G$ & 0.56 \\
\hline 383DH0400.5 & $6 / 7 / 2004$ & soil & EPA6010 & REG & $7440-38-2$ & Arsenic & 3.1 & $\mathrm{mg} / \mathrm{kg}$ & & 1 \\
\hline 383DH0400.5 & $6 / 7 / 2004$ & soil & EPA6010 & REG & $7440-39-3$ & Barium & 3300 & $\mathrm{mg} / \mathrm{kg}$ & & 100 \\
\hline 383DH0400.5 & $6 / 7 / 2004$ & soil & EPA6010 & REG & $7440-41-7$ & Beryllium & 1.6 & $\mathrm{mg} / \mathrm{kg}$ & & 0.52 \\
\hline 383DH0400.5 & $6 / 7 / 2004$ & soil & HASL300 & REG & 14733-03-0 & Bi-214 & 1.33 & $\mathrm{pCi} / \mathrm{g}$ & $\mathrm{G}, \mathrm{J}$ & 0.35 \\
\hline 383DH0400.5 & $6 / 7 / 2004$ & soil & EPA8270 & REG & $85-68-7$ & Butyl Benzyl Phthalate & 33 & $\mu \mathrm{g} / \mathrm{kg}$ & $\mathrm{J}$ & 350 \\
\hline 383DH0400.5 & $6 / 7 / 2004$ & soil & EPA6010 & REG & $7440-43-9$ & Cadmium & 0.17 & $\mathrm{mg} / \mathrm{kg}$ & $\mathrm{B}$ & 0.52 \\
\hline 383DH0400.5 & $6 / 7 / 2004$ & soil & EPA6010 & REG & $7440-47-3$ & Chromium & 4.3 & $\mathrm{mg} / \mathrm{kg}$ & & 1 \\
\hline 383DH0400.5 & $6 / 7 / 2004$ & soil & HASL300 & REG & $10045-97-3$ & Cs-137 & 3.86 & $\mathrm{pCi} / \mathrm{g}$ & $\mathrm{G}$ & 0.18 \\
\hline 383DH0400.5 & $6 / 7 / 2004$ & soil & HASL300 & REG & 13966-00-2 & $\mathrm{K}-40$ & 30.8 & $\mathrm{pCi} / \mathrm{g}$ & G & 1.8 \\
\hline 383DH0400.5 & $6 / 7 / 2004$ & soil & EPA6010 & REG & 7439-92-1 & Lead & 17 & $\mathrm{mg} / \mathrm{kg}$ & & 0.31 \\
\hline 383DH0400.5 & $6 / 7 / 2004$ & soil & EPA7470 & REG & $7439-97-6$ & Mercury & 0.012 & $\mathrm{mg} / \mathrm{kg}$ & $\mathrm{B}$ & 0.1 \\
\hline 383DH0400.5 & $6 / 7 / 2004$ & soil & EPA8260 & REG & $75-09-2$ & Methylene Chloride & 1.8 & $\mu \mathrm{g} / \mathrm{kg}$ & $\mathrm{J}$ & 5.2 \\
\hline 383DH0400.5 & $6 / 7 / 2004$ & soil & HASL300 & REG & 15092-94-1 & $\mathrm{Pb}-212$ & 1.78 & $\mathrm{pCi} / \mathrm{g}$ & $\mathrm{J}$ & 0.29 \\
\hline 383DH0400.5 & $6 / 7 / 2004$ & soil & HASL300 & REG & $15067-28-4$ & $\mathrm{~Pb}-214$ & 1.4 & $\mathrm{pCi} / \mathrm{g}$ & $\mathrm{G}, \mathrm{J}$ & 0.3 \\
\hline
\end{tabular}

Page 4 of 78

Uncontrolled When Printed 


\begin{tabular}{|c|c|c|c|c|c|c|c|c|c|c|}
\hline SAMPLE \# & $\begin{array}{l}\text { SAMPLE } \\
\text { DATE }\end{array}$ & MATRIX & $\begin{array}{l}\text { USER TEST } \\
\text { PANEL }\end{array}$ & $\begin{array}{c}\text { SAMPLE } \\
\text { PURPOSE }\end{array}$ & CAS \# & PARAMETER & RESULT & UNITS & $\mathbf{Q}$ & $\begin{array}{l}\text { DETECT } \\
\text { LIMIT }\end{array}$ \\
\hline 383DH0400.5 & $6 / 7 / 2004$ & soil & UGTAISOPU & REG & 13981-16-3 & Pu-238 & 0.063 & $\mathrm{pCi} / \mathrm{g}$ & & 0.009 \\
\hline 383DH0400.5 & $6 / 7 / 2004$ & soil & UGTAISOPU & REG & $15117-48-3$ & Pu-239 & 0.5 & $\mathrm{pCi} / \mathrm{g}$ & $\mathrm{J}$ & 0.02 \\
\hline 383DH0400.5 & $6 / 7 / 2004$ & soil & HASL300 & REG & 14913-50-9 & TI-208 & 0.65 & $\mathrm{pCi} / \mathrm{g}$ & $G$ & 0.17 \\
\hline 383DH0500.5 & $6 / 7 / 2004$ & soil & HASL300 & REG & $14331-83-0$ & Ac-228 & 1.85 & $\mathrm{pCi} / \mathrm{g}$ & $G$ & 0.56 \\
\hline 383DH0500.5 & $6 / 7 / 2004$ & soil & EPA8260 & REG & 67-64-1 & Acetone & 23 & $\mu \mathrm{g} / \mathrm{kg}$ & & 20 \\
\hline 383DH0500.5 & $6 / 7 / 2004$ & soil & EPA6010 & REG & $7440-38-2$ & Arsenic & 3 & $\mathrm{mg} / \mathrm{kg}$ & & 1 \\
\hline 383DH0500.5 & $6 / 7 / 2004$ & soil & EPA6010 & REG & $7440-39-3$ & Barium & 3200 & $\mathrm{mg} / \mathrm{kg}$ & & 100 \\
\hline 383DH0500.5 & $6 / 7 / 2004$ & soil & EPA6010 & REG & $7440-41-7$ & Beryllium & 1.7 & $\mathrm{mg} / \mathrm{kg}$ & & 0.51 \\
\hline 383DH0500.5 & $6 / 7 / 2004$ & soil & HASL300 & REG & $14733-03-0$ & Bi-214 & 1.41 & $\mathrm{pCi} / \mathrm{g}$ & $\mathrm{G}, \mathrm{J}$ & 0.37 \\
\hline 383DH0500.5 & $6 / 7 / 2004$ & soil & EPA8270 & REG & $85-68-7$ & Butyl Benzyl Phthalate & 40 & $\mu \mathrm{g} / \mathrm{kg}$ & $\mathrm{J}$ & 340 \\
\hline 383DH0500.5 & $6 / 7 / 2004$ & soil & EPA6010 & REG & $7440-43-9$ & Cadmium & 0.19 & $\mathrm{mg} / \mathrm{kg}$ & $\mathrm{B}$ & 0.51 \\
\hline 383DH0500.5 & $6 / 7 / 2004$ & soil & EPA6010 & REG & $7440-47-3$ & Chromium & 3.6 & $\mathrm{mg} / \mathrm{kg}$ & & 1 \\
\hline 383DH0500.5 & $6 / 7 / 2004$ & soil & HASL300 & REG & $10045-97-3$ & Cs-137 & 7.21 & $\mathrm{pCi} / \mathrm{g}$ & G & 0.15 \\
\hline 383DH0500.5 & $6 / 7 / 2004$ & soil & EPA8260 & REG & $100-41-4$ & Ethylbenzene & 8.2 & $\mu \mathrm{g} / \mathrm{kg}$ & & 5.1 \\
\hline 383DH0500.5 & $6 / 7 / 2004$ & soil & HASL300 & REG & 13966-00-2 & $\mathrm{K}-40$ & 27.9 & $\mathrm{pCi} / \mathrm{g}$ & $G$ & 2.1 \\
\hline 383DH0500.5 & $6 / 7 / 2004$ & soil & EPA6010 & REG & $7439-92-1$ & Lead & 25 & $\mathrm{mg} / \mathrm{kg}$ & & 0.31 \\
\hline 383DH0500.5 & $6 / 7 / 2004$ & soil & EPA8260 & REG & 136777-61-2 & M+P-Xylene & 42 & $\mu \mathrm{g} / \mathrm{kg}$ & & 5.1 \\
\hline 383DH0500.5 & $6 / 7 / 2004$ & soil & EPA7470 & REG & $7439-97-6$ & Mercury & 0.011 & $\mathrm{mg} / \mathrm{kg}$ & $\mathrm{B}$ & 0.1 \\
\hline 383DH0500.5 & $6 / 7 / 2004$ & soil & EPA8260 & REG & $75-09-2$ & Methylene Chloride & 2.2 & $\mu \mathrm{g} / \mathrm{kg}$ & $\mathrm{J}$ & 5.1 \\
\hline 383DH0500.5 & $6 / 7 / 2004$ & soil & EPA8260 & REG & $95-47-6$ & O-Xylene & 15 & $\mu \mathrm{g} / \mathrm{kg}$ & & 5.1 \\
\hline 383DH0500.5 & $6 / 7 / 2004$ & soil & HASL300 & REG & 15092-94-1 & $\mathrm{Pb}-212$ & 2.26 & $\mathrm{pCi} / \mathrm{g}$ & $\mathrm{J}$ & 0.26 \\
\hline 383DH0500.5 & $6 / 7 / 2004$ & soil & HASL300 & REG & 15067-28-4 & $\mathrm{Pb}-214$ & 1.25 & $\mathrm{pCi} / \mathrm{g}$ & $\mathrm{G}, \mathrm{J}$ & 0.31 \\
\hline 383DH0500.5 & $6 / 7 / 2004$ & soil & UGTAISOPU & REG & 13981-16-3 & Pu-238 & 0.064 & $\mathrm{pCi} / \mathrm{g}$ & & 0.018 \\
\hline 383DH0500.5 & $6 / 7 / 2004$ & soil & UGTAISOPU & REG & $15117-48-3$ & Pu-239 & 0.57 & $\mathrm{pCi} / \mathrm{g}$ & $\mathrm{J}$ & 0.01 \\
\hline 383DH0500.5 & $6 / 7 / 2004$ & soil & SR7500 & REG & $10098-97-2$ & Sr-90 & 0.49 & $\mathrm{pCi} / \mathrm{g}$ & LT & 0.28 \\
\hline 383DH0500.5 & $6 / 7 / 2004$ & soil & EPA8260 & REG & $100-42-5$ & Styrene & 0.99 & $\mu \mathrm{g} / \mathrm{kg}$ & $\mathrm{J}$ & 5.1 \\
\hline 383DH0500.5 & $6 / 7 / 2004$ & soil & HASL300 & REG & 15065-10-8 & Th-234 & 2.4 & $\mathrm{pCi} / \mathrm{g}$ & $\mathrm{J}$ & 2.1 \\
\hline 383DH0500.5 & $6 / 7 / 2004$ & soil & HASL300 & REG & $14913-50-9$ & TI-208 & 0.67 & $\mathrm{pCi} / \mathrm{g}$ & $\mathrm{G}$ & 0.18 \\
\hline 383DH0500.5 & $6 / 7 / 2004$ & soil & EPA8260 & REG & $108-88-3$ & Toluene & 1.6 & $\mu \mathrm{g} / \mathrm{kg}$ & $\mathrm{J}$ & 5.1 \\
\hline 383DH0600.5 & $6 / 7 / 2004$ & soil & HASL300 & REG & 14331-83-0 & Ac-228 & 1.79 & $\mathrm{pCi} / \mathrm{g}$ & $G$ & 0.7 \\
\hline 383DH0600.5 & $6 / 7 / 2004$ & soil & EPA6010 & REG & $7440-38-2$ & Arsenic & 3.5 & $\mathrm{mg} / \mathrm{kg}$ & & 1 \\
\hline 383DH0600.5 & $6 / 7 / 2004$ & soil & EPA6010 & REG & $7440-39-3$ & Barium & 3500 & $\mathrm{mg} / \mathrm{kg}$ & & 100 \\
\hline 383DH0600.5 & $6 / 7 / 2004$ & soil & EPA6010 & REG & $7440-41-7$ & Beryllium & 1.4 & $\mathrm{mg} / \mathrm{kg}$ & & 0.52 \\
\hline 383DH0600.5 & $6 / 7 / 2004$ & soil & HASL300 & REG & $14733-03-0$ & Bi-214 & 0.94 & $\mathrm{pCi} / \mathrm{g}$ & $\mathrm{G}, \mathrm{J}$ & 0.34 \\
\hline 383DH0600.5 & $6 / 7 / 2004$ & soil & EPA8270 & REG & $117-81-7$ & Bis(2-Ethylhexyl)Phthalate & 100 & $\mu \mathrm{g} / \mathrm{kg}$ & $\mathrm{J}$ & 350 \\
\hline
\end{tabular}

Page 5 of 78

Uncontrolled When Printed 


\begin{tabular}{|c|c|c|c|c|c|c|c|c|c|c|}
\hline SAMPLE \# & $\begin{array}{l}\text { SAMPLE } \\
\text { DATE }\end{array}$ & MATRIX & $\begin{array}{c}\text { USER TEST } \\
\text { PANEL }\end{array}$ & $\begin{array}{c}\text { SAMPLE } \\
\text { PURPOSE }\end{array}$ & CAS \# & PARAMETER & RESULT & UNITS & $\mathbf{Q}$ & $\begin{array}{c}\text { DETECT } \\
\text { LIMIT }\end{array}$ \\
\hline 383DH0600.5 & $6 / 7 / 2004$ & soil & EPA6010 & REG & $7440-43-9$ & Cadmium & 0.23 & $\mathrm{mg} / \mathrm{kg}$ & $B$ & 0.52 \\
\hline 383DH0600.5 & $6 / 7 / 2004$ & soil & EPA6010 & REG & $7440-47-3$ & Chromium & 4.1 & $\mathrm{mg} / \mathrm{kg}$ & & 1 \\
\hline 383DH0600.5 & $6 / 7 / 2004$ & soil & HASL300 & REG & $10045-97-3$ & Cs-137 & 18.4 & $\mathrm{pCi} / \mathrm{g}$ & $\mathrm{G}$ & 0.2 \\
\hline 383DH0600.5 & $6 / 7 / 2004$ & soil & EPA8260 & REG & $100-41-4$ & Ethylbenzene & 1.6 & $\mu \mathrm{g} / \mathrm{kg}$ & $\mathrm{J}$ & 5.2 \\
\hline 383DH0600.5 & $6 / 7 / 2004$ & soil & HASL300 & REG & $13966-00-2$ & $\mathrm{~K}-40$ & 26.7 & $\mathrm{pCi} / \mathrm{g}$ & $\mathrm{G}$ & 2.1 \\
\hline 383DH0600.5 & $6 / 7 / 2004$ & soil & EPA6010 & REG & 7439-92-1 & Lead & 22 & $\mathrm{mg} / \mathrm{kg}$ & & 0.31 \\
\hline 383DH0600.5 & $6 / 7 / 2004$ & soil & EPA8260 & REG & 136777-61-2 & M+P-Xylene & 5.9 & $\mu \mathrm{g} / \mathrm{kg}$ & & 5.2 \\
\hline 383DH0600.5 & $6 / 7 / 2004$ & soil & EPA7470 & REG & $7439-97-6$ & Mercury & 0.019 & $\mathrm{mg} / \mathrm{kg}$ & $\mathrm{B}$ & 0.1 \\
\hline 383DH0600.5 & $6 / 7 / 2004$ & soil & EPA8260 & REG & $95-47-6$ & O-Xylene & 1.6 & $\mu \mathrm{g} / \mathrm{kg}$ & $\mathrm{J}$ & 5.2 \\
\hline 383DH0600.5 & $6 / 7 / 2004$ & soil & HASL300 & REG & 15092-94-1 & $\mathrm{Pb}-212$ & 2.54 & $\mathrm{pCi} / \mathrm{g}$ & $\mathrm{J}$ & 0.31 \\
\hline 383DH0600.5 & $6 / 7 / 2004$ & soil & HASL300 & REG & $15067-28-4$ & $\mathrm{~Pb}-214$ & 1.44 & $\mathrm{pCi} / \mathrm{g}$ & $\mathrm{G}, \mathrm{J}$ & 0.42 \\
\hline 383DH0600.5 & $6 / 7 / 2004$ & soil & UGTAISOPU & REG & 13981-16-3 & Pu-238 & 0.133 & $\mathrm{pCi} / \mathrm{g}$ & & 0.009 \\
\hline 383DH0600.5 & $6 / 7 / 2004$ & soil & UGTAISOPU & REG & 15117-48-3 & Pu-239 & 0.8 & $\mathrm{pCi} / \mathrm{g}$ & $\mathrm{J}$ & 0.02 \\
\hline 383DH0600.5 & $6 / 7 / 2004$ & soil & SR7500 & REG & 10098-97-2 & Sr-90 & 0.91 & $\mathrm{pCi} / \mathrm{g}$ & Y1 & 0.25 \\
\hline 383DH0600.5 & $6 / 7 / 2004$ & soil & HASL300 & REG & 14913-50-9 & TI-208 & 0.67 & $\mathrm{pCi} / \mathrm{g}$ & G & 0.19 \\
\hline 383DH0700.5 & $6 / 7 / 2004$ & soil & HASL300 & REG & 14331-83-0 & Ac-228 & 1.73 & $\mathrm{pCi} / \mathrm{g}$ & $\mathrm{G}$ & 0.47 \\
\hline 383DH0700.5 & $6 / 7 / 2004$ & soil & EPA6010 & REG & $7440-38-2$ & Arsenic & 2.7 & $\mathrm{mg} / \mathrm{kg}$ & & 1 \\
\hline 383DH0700.5 & $6 / 7 / 2004$ & soil & EPA6010 & REG & $7440-39-3$ & Barium & 2500 & $\mathrm{mg} / \mathrm{kg}$ & & 100 \\
\hline 383DH0700.5 & $6 / 7 / 2004$ & soil & EPA6010 & REG & $7440-41-7$ & Beryllium & 1.6 & $\mathrm{mg} / \mathrm{kg}$ & & 0.51 \\
\hline 383DH0700.5 & $6 / 7 / 2004$ & soil & HASL300 & REG & $14733-03-0$ & Bi-214 & 1.25 & $\mathrm{pCi} / \mathrm{g}$ & $\mathrm{G}, \mathrm{J}$ & 0.28 \\
\hline 383DH0700.5 & $6 / 7 / 2004$ & soil & EPA6010 & REG & $7440-43-9$ & Cadmium & 0.27 & $\mathrm{mg} / \mathrm{kg}$ & $B$ & 0.51 \\
\hline 383DH0700.5 & $6 / 7 / 2004$ & soil & EPA6010 & REG & $7440-47-3$ & Chromium & 2.8 & $\mathrm{mg} / \mathrm{kg}$ & & 1 \\
\hline 383DH0700.5 & $6 / 7 / 2004$ & soil & HASL300 & REG & $10045-97-3$ & Cs-137 & 2.72 & $\mathrm{pCi} / \mathrm{g}$ & $\mathrm{G}$ & 0.12 \\
\hline 383DH0700.5 & $6 / 7 / 2004$ & soil & HASL300 & REG & $13966-00-2$ & $\mathrm{~K}-40$ & 25.4 & $\mathrm{pCi} / \mathrm{g}$ & G & 1.5 \\
\hline 383DH0700.5 & $6 / 7 / 2004$ & soil & EPA6010 & REG & 7439-92-1 & Lead & 22 & $\mathrm{mg} / \mathrm{kg}$ & & 0.31 \\
\hline 383DH0700.5 & $6 / 7 / 2004$ & soil & EPA8260 & REG & 136777-61-2 & M+P-Xylene & 2.5 & $\mu \mathrm{g} / \mathrm{kg}$ & $\mathrm{J}$ & 5.1 \\
\hline 383DH0700.5 & $6 / 7 / 2004$ & soil & EPA7470 & REG & $7439-97-6$ & Mercury & 0.014 & $\mathrm{mg} / \mathrm{kg}$ & $B$ & 0.1 \\
\hline 383DH0700.5 & $6 / 7 / 2004$ & soil & EPA8260 & REG & $75-09-2$ & Methylene Chloride & 1.6 & $\mu \mathrm{g} / \mathrm{kg}$ & $\mathrm{J}$ & 5.1 \\
\hline 383DH0700.5 & $6 / 7 / 2004$ & soil & HASL300 & REG & 15092-94-1 & $\mathrm{Pb}-212$ & 1.82 & $\mathrm{pCi} / \mathrm{g}$ & $\mathrm{J}$ & 0.23 \\
\hline 383DH0700.5 & $6 / 7 / 2004$ & soil & HASL300 & REG & $15067-28-4$ & $\mathrm{~Pb}-214$ & 1.49 & $\mathrm{pCi} / \mathrm{g}$ & $\mathrm{G}, \mathrm{J}$ & 0.31 \\
\hline 383DH0700.5 & $6 / 7 / 2004$ & soil & UGTAISOPU & REG & $15117-48-3$ & Pu-239 & 0.071 & $\mathrm{pCi} / \mathrm{g}$ & $\mathrm{J}$ & 0.016 \\
\hline 383DH0700.5 & $6 / 7 / 2004$ & soil & HASL300 & REG & $14913-50-9$ & TI-208 & 0.65 & $\mathrm{pCi} / \mathrm{g}$ & $\mathrm{G}$ & 0.13 \\
\hline 383DH0700.5X & $6 / 7 / 2004$ & soil & HASL300 & FD & $14331-83-0$ & Ac-228 & 1.92 & $\mathrm{pCi} / \mathrm{g}$ & G & 0.63 \\
\hline 383DH0700.5X & $6 / 7 / 2004$ & soil & EPA8260 & FD & 67-64-1 & Acetone & 11 & $\mu \mathrm{g} / \mathrm{kg}$ & $\mathrm{J}$ & 20 \\
\hline 383DH0700.5X & $6 / 7 / 2004$ & soil & EPA6010 & FD & $7440-38-2$ & Arsenic & 2.2 & $\mathrm{mg} / \mathrm{kg}$ & & 1 \\
\hline
\end{tabular}

Page 6 of 78

Uncontrolled When Printed 


\begin{tabular}{|c|c|c|c|c|c|c|c|c|c|c|}
\hline SAMPLE \# & $\begin{array}{l}\text { SAMPLE } \\
\text { DATE }\end{array}$ & MATRIX & $\begin{array}{l}\text { USER TEST } \\
\text { PANEL }\end{array}$ & $\begin{array}{c}\text { SAMPLE } \\
\text { PURPOSE }\end{array}$ & CAS \# & PARAMETER & RESULT & UNITS & $\mathbf{Q}$ & $\begin{array}{l}\text { DETECT } \\
\text { LIMIT }\end{array}$ \\
\hline 383DH0700.5X & $6 / 7 / 2004$ & soil & EPA6010 & FD & $7440-39-3$ & Barium & 2500 & $\mathrm{mg} / \mathrm{kg}$ & & 100 \\
\hline 383DH0700.5X & $6 / 7 / 2004$ & soil & EPA6010 & FD & 7440-41-7 & Beryllium & 1.7 & $\mathrm{mg} / \mathrm{kg}$ & & 0.51 \\
\hline 383DH0700.5X & $6 / 7 / 2004$ & soil & HASL300 & FD & 14733-03-0 & Bi-214 & 1.36 & $\mathrm{pCi} / \mathrm{g}$ & $\mathrm{G}, \mathrm{J}$ & 0.23 \\
\hline 383DH0700.5X & $6 / 7 / 2004$ & soil & EPA6010 & FD & $7440-43-9$ & Cadmium & 0.23 & $\mathrm{mg} / \mathrm{kg}$ & B & 0.51 \\
\hline 383DH0700.5X & $6 / 7 / 2004$ & soil & EPA6010 & FD & $7440-47-3$ & Chromium & 2.9 & $\mathrm{mg} / \mathrm{kg}$ & & 1 \\
\hline 383DH0700.5X & $6 / 7 / 2004$ & soil & HASL300 & FD & $10045-97-3$ & Cs-137 & 2.78 & $\mathrm{pCi} / \mathrm{g}$ & $G$ & 0.16 \\
\hline 383DH0700.5X & $6 / 7 / 2004$ & soil & HASL300 & FD & 13966-00-2 & $\mathrm{K}-40$ & 25.9 & $\mathrm{pCi} / \mathrm{g}$ & $G$ & 1.6 \\
\hline 383DH0700.5X & $6 / 7 / 2004$ & soil & EPA6010 & FD & 7439-92-1 & Lead & 18 & $\mathrm{mg} / \mathrm{kg}$ & & 0.31 \\
\hline 383DH0700.5X & $6 / 7 / 2004$ & soil & EPA7470 & FD & 7439-97-6 & Mercury & 0.014 & $\mathrm{mg} / \mathrm{kg}$ & $\mathrm{B}$ & 0.1 \\
\hline 383DH0700.5X & $6 / 7 / 2004$ & soil & EPA8260 & FD & $75-09-2$ & Methylene Chloride & 1.9 & $\mu \mathrm{g} / \mathrm{kg}$ & $\mathrm{J}$ & 5.1 \\
\hline 383DH0700.5X & $6 / 7 / 2004$ & soil & HASL300 & FD & 15092-94-1 & $\mathrm{Pb}-212$ & 2.22 & $\mathrm{pCi} / \mathrm{g}$ & $\mathrm{J}$ & 0.21 \\
\hline 383DH0700.5X & $6 / 7 / 2004$ & soil & HASL300 & FD & $15067-28-4$ & $\mathrm{~Pb}-214$ & 1.2 & $\mathrm{pCi} / \mathrm{g}$ & $\mathrm{G}, \mathrm{J}$ & 0.26 \\
\hline 383DH0700.5X & $6 / 7 / 2004$ & soil & EPA6010 & FD & $7782-49-2$ & Selenium & 0.54 & $\mathrm{mg} / \mathrm{kg}$ & & 0.51 \\
\hline 383DH0700.5X & $6 / 7 / 2004$ & soil & HASL300 & FD & $14913-50-9$ & TI-208 & 0.57 & $\mathrm{pCi} / \mathrm{g}$ & G & 0.14 \\
\hline 383DH0800.5 & $6 / 7 / 2004$ & soil & HASL300 & REG & 14331-83-0 & Ac-228 & 1.72 & $\mathrm{pCi} / \mathrm{g}$ & $G$ & 0.48 \\
\hline 383DH0800.5 & $6 / 7 / 2004$ & soil & EPA6010 & REG & $7440-38-2$ & Arsenic & 4 & $\mathrm{mg} / \mathrm{kg}$ & & 1 \\
\hline 383DH0800.5 & $6 / 7 / 2004$ & soil & EPA6010 & REG & $7440-39-3$ & Barium & 3900 & $\mathrm{mg} / \mathrm{kg}$ & & 100 \\
\hline 383DH0800.5 & $6 / 7 / 2004$ & soil & EPA6010 & REG & $7440-41-7$ & Beryllium & 1.4 & $\mathrm{mg} / \mathrm{kg}$ & & 0.51 \\
\hline 383DH0800.5 & $6 / 7 / 2004$ & soil & HASL300 & REG & $14733-03-0$ & Bi-214 & 1.33 & $\mathrm{pCi} / \mathrm{g}$ & $\mathrm{G}, \mathrm{J}$ & 0.39 \\
\hline 383DH0800.5 & $6 / 7 / 2004$ & soil & EPA8270 & REG & 117-81-7 & Bis(2-Ethylhexyl)Phthalate & 200 & $\mu \mathrm{g} / \mathrm{kg}$ & $\mathrm{J}$ & 340 \\
\hline 383DH0800.5 & $6 / 7 / 2004$ & soil & EPA6010 & REG & $7440-43-9$ & Cadmium & 0.28 & $\mathrm{mg} / \mathrm{kg}$ & $\mathrm{B}$ & 0.51 \\
\hline 383DH0800.5 & $6 / 7 / 2004$ & soil & EPA6010 & REG & $7440-47-3$ & Chromium & 3.9 & $\mathrm{mg} / \mathrm{kg}$ & & 1 \\
\hline 383DH0800.5 & $6 / 7 / 2004$ & soil & HASL300 & REG & $10045-97-3$ & Cs-137 & 12.4 & $\mathrm{pCi} / \mathrm{g}$ & $\mathrm{G}$ & 0.2 \\
\hline 383DH0800.5 & $6 / 7 / 2004$ & soil & HASL300 & REG & 13966-00-2 & $\mathrm{K}-40$ & 30.9 & $\mathrm{pCi} / \mathrm{g}$ & $G$ & 1.6 \\
\hline 383DH0800.5 & $6 / 7 / 2004$ & soil & EPA6010 & REG & $7439-92-1$ & Lead & 22 & $\mathrm{mg} / \mathrm{kg}$ & & 0.31 \\
\hline 383DH0800.5 & $6 / 7 / 2004$ & soil & EPA8260 & REG & 136777-61-2 & M+P-Xylene & 3 & $\mu \mathrm{g} / \mathrm{kg}$ & $\mathrm{J}$ & 5.1 \\
\hline 383DH0800.5 & $6 / 7 / 2004$ & soil & EPA7470 & REG & 7439-97-6 & Mercury & 0.028 & $\mathrm{mg} / \mathrm{kg}$ & $\mathrm{B}$ & 0.1 \\
\hline 383DH0800.5 & $6 / 7 / 2004$ & soil & EPA8260 & REG & $75-09-2$ & Methylene Chloride & 1.9 & $\mu \mathrm{g} / \mathrm{kg}$ & $\mathrm{J}$ & 5.1 \\
\hline 383DH0800.5 & $6 / 7 / 2004$ & soil & HASL300 & REG & 15092-94-1 & $\mathrm{Pb}-212$ & 2.17 & $\mathrm{pCi} / \mathrm{g}$ & $\mathrm{J}$ & 0.31 \\
\hline 383DH0800.5 & $6 / 7 / 2004$ & soil & HASL300 & REG & $15067-28-4$ & $\mathrm{~Pb}-214$ & 1.16 & $\mathrm{pCi} / \mathrm{g}$ & $\mathrm{G}, \mathrm{J}$ & 0.4 \\
\hline 383DH0800.5 & $6 / 7 / 2004$ & soil & UGTAISOPU & REG & 13981-16-3 & Pu-238 & 0.059 & $\mathrm{pCi} / \mathrm{g}$ & & 0.009 \\
\hline 383DH0800.5 & $6 / 7 / 2004$ & soil & UGTAISOPU & REG & $15117-48-3$ & Pu-239 & 1.87 & $\mathrm{pCi} / \mathrm{g}$ & $\mathrm{J}$ & 0.01 \\
\hline 383DH0800.5 & $6 / 7 / 2004$ & soil & SR7500 & REG & 10098-97-2 & Sr-90 & 0.55 & $\mathrm{pCi} / \mathrm{g}$ & Y1 & 0.25 \\
\hline 383DH0800.5 & $6 / 7 / 2004$ & soil & HASL300 & REG & $14913-50-9$ & TI-208 & 0.6 & $\mathrm{pCi} / \mathrm{g}$ & $G$ & 0.17 \\
\hline 383DH0800.5 & $6 / 7 / 2004$ & soil & EPA8260 & REG & $108-88-3$ & Toluene & 1 & $\mu \mathrm{g} / \mathrm{kg}$ & $\mathrm{J}$ & 5.1 \\
\hline
\end{tabular}

Page 7 of 78

Uncontrolled When Printed 


\begin{tabular}{|c|c|c|c|c|c|c|c|c|c|c|}
\hline SAMPLE \# & $\begin{array}{l}\text { SAMPLE } \\
\text { DATE }\end{array}$ & MATRIX & $\begin{array}{l}\text { USER TEST } \\
\text { PANEL }\end{array}$ & $\begin{array}{c}\text { SAMPLE } \\
\text { PURPOSE }\end{array}$ & CAS \# & PARAMETER & RESULT & UNITS & $\mathbf{Q}$ & $\begin{array}{l}\text { DETECT } \\
\text { LIMIT }\end{array}$ \\
\hline 383DH0900.5 & $6 / 7 / 2004$ & soil & HASL300 & REG & 14331-83-0 & Ac-228 & 1.7 & $\mathrm{pCi} / \mathrm{g}$ & $\mathrm{G}$ & 0.69 \\
\hline 383DH0900.5 & $6 / 7 / 2004$ & soil & EPA8260 & REG & 67-64-1 & Acetone & 9.3 & $\mu \mathrm{g} / \mathrm{kg}$ & $\mathrm{J}, \mathrm{B}$ & 21 \\
\hline 383DH0900.5 & $6 / 7 / 2004$ & soil & EPA6010 & REG & $7440-38-2$ & Arsenic & 3.2 & $\mathrm{mg} / \mathrm{kg}$ & & 1 \\
\hline 383DH0900.5 & $6 / 7 / 2004$ & soil & EPA6010 & REG & $7440-39-3$ & Barium & 3500 & $\mathrm{mg} / \mathrm{kg}$ & & 100 \\
\hline 383DH0900.5 & $6 / 7 / 2004$ & soil & EPA6010 & REG & $7440-41-7$ & Beryllium & 1.2 & $\mathrm{mg} / \mathrm{kg}$ & & 0.52 \\
\hline 383DH0900.5 & $6 / 7 / 2004$ & soil & HASL300 & REG & $14733-03-0$ & Bi-214 & 0.86 & $\mathrm{pCi} / \mathrm{g}$ & $\mathrm{G}, \mathrm{J}$ & 0.38 \\
\hline 383DH0900.5 & $6 / 7 / 2004$ & soil & EPA6010 & REG & $7440-43-9$ & Cadmium & 0.21 & $\mathrm{mg} / \mathrm{kg}$ & $\mathrm{B}$ & 0.52 \\
\hline 383DH0900.5 & $6 / 7 / 2004$ & soil & EPA6010 & REG & $7440-47-3$ & Chromium & 2.9 & $\mathrm{mg} / \mathrm{kg}$ & & 1 \\
\hline 383DH0900.5 & $6 / 7 / 2004$ & soil & HASL300 & REG & $10045-97-3$ & Cs-137 & 48.7 & $\mathrm{pCi} / \mathrm{g}$ & G & 0.2 \\
\hline 383DH0900.5 & $6 / 7 / 2004$ & soil & HASL300 & REG & 13966-00-2 & $\mathrm{K}-40$ & 30.3 & $\mathrm{pCi} / \mathrm{g}$ & $G$ & 1.5 \\
\hline 383DH0900.5 & $6 / 7 / 2004$ & soil & EPA6010 & REG & 7439-92-1 & Lead & 52 & $\mathrm{mg} / \mathrm{kg}$ & & 0.31 \\
\hline 383DH0900.5 & $6 / 7 / 2004$ & soil & EPA7470 & REG & 7439-97-6 & Mercury & 0.021 & $\mathrm{mg} / \mathrm{kg}$ & B & 0.1 \\
\hline 383DH0900.5 & $6 / 7 / 2004$ & soil & HASL300 & REG & 15092-94-1 & $\mathrm{Pb}-212$ & 1.71 & $\mathrm{pCi} / \mathrm{g}$ & $\mathrm{J}$ & 0.5 \\
\hline 383DH0900.5 & $6 / 7 / 2004$ & soil & UGTAISOPU & REG & 13981-16-3 & Pu-238 & 0.201 & $\mathrm{pCi} / \mathrm{g}$ & & 0.011 \\
\hline 383DH0900.5 & $6 / 7 / 2004$ & soil & UGTAISOPU & REG & $15117-48-3$ & Pu-239 & 1.17 & $\mathrm{pCi} / \mathrm{g}$ & & 0.01 \\
\hline 383DH0900.5 & $6 / 7 / 2004$ & soil & SR7500 & REG & 10098-97-2 & Sr-90 & 0.92 & $\mathrm{pCi} / \mathrm{g}$ & Y1 & 0.22 \\
\hline 383DH0900.5 & $6 / 7 / 2004$ & soil & HASL300 & REG & 14913-50-9 & TI-208 & 0.71 & $\mathrm{pCi} / \mathrm{g}$ & $G$ & 0.24 \\
\hline 383DH1000.5 & $6 / 7 / 2004$ & soil & HASL300 & REG & 14331-83-0 & Ac-228 & 1.37 & $\mathrm{pCi} / \mathrm{g}$ & $G$ & 0.51 \\
\hline 383DH1000.5 & $6 / 7 / 2004$ & soil & EPA8260 & REG & 67-64-1 & Acetone & 6.2 & $\mu \mathrm{g} / \mathrm{kg}$ & $\mathrm{J}, \mathrm{B}$ & 21 \\
\hline 383DH1000.5 & $6 / 7 / 2004$ & soil & EPA6010 & REG & $7440-38-2$ & Arsenic & 3 & $\mathrm{mg} / \mathrm{kg}$ & & 1 \\
\hline 383DH1000.5 & $6 / 7 / 2004$ & soil & EPA6010 & REG & $7440-39-3$ & Barium & 3800 & $\mathrm{mg} / \mathrm{kg}$ & & 100 \\
\hline 383DH1000.5 & $6 / 7 / 2004$ & soil & EPA6010 & REG & 7440-41-7 & Beryllium & 1.2 & $\mathrm{mg} / \mathrm{kg}$ & & 0.51 \\
\hline 383DH1000.5 & $6 / 7 / 2004$ & soil & HASL300 & REG & $14733-03-0$ & Bi-214 & 0.85 & $\mathrm{pCi} / \mathrm{g}$ & $\mathrm{G}, \mathrm{J}$ & 0.33 \\
\hline 383DH1000.5 & $6 / 7 / 2004$ & soil & EPA6010 & REG & 7440-43-9 & Cadmium & 0.15 & $\mathrm{mg} / \mathrm{kg}$ & B & 0.51 \\
\hline 383DH1000.5 & $6 / 7 / 2004$ & soil & EPA6010 & REG & $7440-47-3$ & Chromium & 5.1 & $\mathrm{mg} / \mathrm{kg}$ & & 1 \\
\hline 383DH1000.5 & $6 / 7 / 2004$ & soil & HASL300 & REG & $10045-97-3$ & Cs-137 & 23.7 & $\mathrm{pCi} / \mathrm{g}$ & G & 0.2 \\
\hline 383DH1000.5 & $6 / 7 / 2004$ & soil & HASL300 & REG & 13966-00-2 & $\mathrm{K}-40$ & 21.6 & $\mathrm{pCi} / \mathrm{g}$ & $G$ & 1.2 \\
\hline 383DH1000.5 & $6 / 7 / 2004$ & soil & EPA6010 & REG & 7439-92-1 & Lead & 22 & $\mathrm{mg} / \mathrm{kg}$ & & 0.31 \\
\hline 383DH1000.5 & $6 / 7 / 2004$ & soil & EPA7470 & REG & $7439-97-6$ & Mercury & 0.027 & $\mathrm{mg} / \mathrm{kg}$ & $\mathrm{B}$ & 0.1 \\
\hline 383DH1000.5 & $6 / 7 / 2004$ & soil & EPA8260 & REG & $75-09-2$ & Methylene Chloride & 3.4 & $\mu \mathrm{g} / \mathrm{kg}$ & $\mathrm{J}$ & 5.1 \\
\hline 383DH1000.5 & $6 / 7 / 2004$ & soil & HASL300 & REG & 15092-94-1 & $\mathrm{Pb}-212$ & 1.57 & $\mathrm{pCi} / \mathrm{g}$ & $\mathrm{J}$ & 0.33 \\
\hline 383DH1000.5 & $6 / 7 / 2004$ & soil & HASL300 & REG & $15067-28-4$ & $\mathrm{~Pb}-214$ & 1.08 & $\mathrm{pCi} / \mathrm{g}$ & $\mathrm{G}, \mathrm{J}$ & 0.47 \\
\hline 383DH1000.5 & $6 / 7 / 2004$ & soil & UGTAISOPU & REG & 13981-16-3 & Pu-238 & 0.176 & $\mathrm{pCi} / \mathrm{g}$ & & 0.009 \\
\hline 383DH1000.5 & $6 / 7 / 2004$ & soil & UGTAISOPU & REG & $15117-48-3$ & Pu-239 & 1.26 & $\mathrm{pCi} / \mathrm{g}$ & & 0.02 \\
\hline 383DH1000.5 & $6 / 7 / 2004$ & soil & SR7500 & REG & 10098-97-2 & Sr-90 & 0.96 & $\mathrm{pCi} / \mathrm{g}$ & & 0.23 \\
\hline
\end{tabular}

Page 8 of 78

Uncontrolled When Printed 


\begin{tabular}{|c|c|c|c|c|c|c|c|c|c|c|}
\hline SAMPLE \# & $\begin{array}{l}\text { SAMPLE } \\
\text { DATE }\end{array}$ & MATRIX & $\begin{array}{l}\text { USER TEST } \\
\text { PANEL }\end{array}$ & $\begin{array}{c}\text { SAMPLE } \\
\text { PURPOSE }\end{array}$ & CAS \# & PARAMETER & RESULT & UNITS & $\mathbf{Q}$ & $\begin{array}{c}\text { DETECT } \\
\text { LIMIT }\end{array}$ \\
\hline 383DH1000.5 & $6 / 7 / 2004$ & soil & HASL300 & REG & $14913-50-9$ & TI-208 & 0.47 & $\mathrm{pCi} / \mathrm{g}$ & G & 0.2 \\
\hline 383DH1100.5 & $6 / 7 / 2004$ & soil & HASL300 & REG & 14331-83-0 & Ac-228 & 1.95 & $\mathrm{pCi} / \mathrm{g}$ & G & 0.46 \\
\hline 383DH1100.5 & $6 / 7 / 2004$ & soil & EPA6010 & REG & $7440-38-2$ & Arsenic & 2.2 & $\mathrm{mg} / \mathrm{kg}$ & & 1 \\
\hline 383DH1100.5 & $6 / 7 / 2004$ & soil & EPA6010 & REG & $7440-39-3$ & Barium & 2900 & $\mathrm{mg} / \mathrm{kg}$ & & 100 \\
\hline 383DH1100.5 & $6 / 7 / 2004$ & soil & EPA6010 & REG & $7440-41-7$ & Beryllium & 1.7 & $\mathrm{mg} / \mathrm{kg}$ & & 0.51 \\
\hline 383DH1100.5 & $6 / 7 / 2004$ & soil & HASL300 & REG & $14733-03-0$ & Bi-214 & 0.98 & $\mathrm{pCi} / \mathrm{g}$ & $\mathrm{G}, \mathrm{J}$ & 0.31 \\
\hline 383DH1100.5 & $6 / 7 / 2004$ & soil & EPA6010 & REG & $7440-43-9$ & Cadmium & 0.13 & $\mathrm{mg} / \mathrm{kg}$ & B & 0.51 \\
\hline 383DH1100.5 & $6 / 7 / 2004$ & soil & EPA6010 & REG & $7440-47-3$ & Chromium & 4.3 & $\mathrm{mg} / \mathrm{kg}$ & & 1 \\
\hline 383DH1100.5 & $6 / 7 / 2004$ & soil & HASL300 & REG & $10045-97-3$ & Cs-137 & 7.52 & $\mathrm{pCi} / \mathrm{g}$ & $\mathrm{G}$ & 0.16 \\
\hline 383DH1100.5 & $6 / 7 / 2004$ & soil & HASL300 & REG & 13966-00-2 & $\mathrm{K}-40$ & 26.3 & $\mathrm{pCi} / \mathrm{g}$ & G & 1.2 \\
\hline 383DH1100.5 & $6 / 7 / 2004$ & soil & EPA6010 & REG & 7439-92-1 & Lead & 23 & $\mathrm{mg} / \mathrm{kg}$ & & 0.31 \\
\hline 383DH1100.5 & $6 / 7 / 2004$ & soil & EPA8260 & REG & 136777-61-2 & M+P-Xylene & 2.1 & $\mu \mathrm{g} / \mathrm{kg}$ & $\mathrm{J}$ & 5.1 \\
\hline 383DH1100.5 & $6 / 7 / 2004$ & soil & EPA7470 & REG & 7439-97-6 & Mercury & 0.014 & $\mathrm{mg} / \mathrm{kg}$ & $\mathrm{B}$ & 0.1 \\
\hline 383DH1100.5 & $6 / 7 / 2004$ & soil & EPA8260 & REG & $75-09-2$ & Methylene Chloride & 2.9 & $\mu \mathrm{g} / \mathrm{kg}$ & $\mathrm{J}$ & 5.1 \\
\hline 383DH1100.5 & $6 / 7 / 2004$ & soil & HASL300 & REG & 15092-94-1 & $\mathrm{Pb}-212$ & 2.23 & $\mathrm{pCi} / \mathrm{g}$ & $\mathrm{J}$ & 0.26 \\
\hline 383DH1100.5 & $6 / 7 / 2004$ & soil & HASL300 & REG & $15067-28-4$ & $\mathrm{~Pb}-214$ & 1.33 & $\mathrm{pCi} / \mathrm{g}$ & $\mathrm{G}, \mathrm{J}$ & 0.32 \\
\hline 383DH1100.5 & $6 / 7 / 2004$ & soil & UGTAISOPU & REG & 13981-16-3 & Pu-238 & 0.048 & $\mathrm{pCi} / \mathrm{g}$ & $\mathrm{LT}$ & 0.01 \\
\hline 383DH1100.5 & $6 / 7 / 2004$ & soil & UGTAISOPU & REG & $15117-48-3$ & Pu-239 & 0.7 & $\mathrm{pCi} / \mathrm{g}$ & & 0.02 \\
\hline 383DH1100.5 & $6 / 7 / 2004$ & soil & HASL300 & REG & 14913-50-9 & TI-208 & 0.66 & $\mathrm{pCi} / \mathrm{g}$ & $\mathrm{G}$ & 0.16 \\
\hline 383DH1200.5 & $6 / 7 / 2004$ & soil & HASL300 & REG & 14331-83-0 & Ac-228 & 1.75 & $\mathrm{pCi} / \mathrm{g}$ & $\mathrm{G}$ & 0.69 \\
\hline 383DH1200.5 & $6 / 7 / 2004$ & soil & EPA8260 & REG & 67-64-1 & Acetone & 6.3 & $\mu \mathrm{g} / \mathrm{kg}$ & $\mathrm{J}, \mathrm{B}$ & 21 \\
\hline 383DH1200.5 & $6 / 7 / 2004$ & soil & EPA6010 & REG & $7440-38-2$ & Arsenic & 3.2 & $\mathrm{mg} / \mathrm{kg}$ & & 1 \\
\hline 383DH1200.5 & $6 / 7 / 2004$ & soil & EPA6010 & REG & $7440-39-3$ & Barium & 3600 & $\mathrm{mg} / \mathrm{kg}$ & & 100 \\
\hline 383DH1200.5 & $6 / 7 / 2004$ & soil & EPA6010 & REG & $7440-41-7$ & Beryllium & 1.5 & $\mathrm{mg} / \mathrm{kg}$ & & 0.52 \\
\hline 383DH1200.5 & $6 / 7 / 2004$ & soil & HASL300 & REG & $14733-03-0$ & Bi-214 & 1.17 & $\mathrm{pCi} / \mathrm{g}$ & $\mathrm{G}, \mathrm{J}$ & 0.54 \\
\hline 383DH1200.5 & $6 / 7 / 2004$ & soil & EPA6010 & REG & $7440-43-9$ & Cadmium & 0.16 & $\mathrm{mg} / \mathrm{kg}$ & $\mathrm{B}$ & 0.52 \\
\hline 383DH1200.5 & $6 / 7 / 2004$ & soil & EPA6010 & REG & $7440-47-3$ & Chromium & 5 & $\mathrm{mg} / \mathrm{kg}$ & & 1 \\
\hline 383DH1200.5 & $6 / 7 / 2004$ & soil & HASL300 & REG & $10045-97-3$ & Cs-137 & 33.8 & $\mathrm{pCi} / \mathrm{g}$ & $\mathrm{G}$ & 0.3 \\
\hline 383DH1200.5 & $6 / 7 / 2004$ & soil & EPA8015 & REG & $68334-30-5$ & Diesel-Range Organics & 2.7 & $\mathrm{mg} / \mathrm{kg}$ & $\mathrm{J}$ & 5.2 \\
\hline 383DH1200.5 & $6 / 7 / 2004$ & soil & HASL300 & REG & $13966-00-2$ & $\mathrm{~K}-40$ & 24.1 & $\mathrm{pCi} / \mathrm{g}$ & $\mathrm{G}$ & 2.3 \\
\hline 383DH1200.5 & $6 / 7 / 2004$ & soil & EPA6010 & REG & 7439-92-1 & Lead & 36 & $\mathrm{mg} / \mathrm{kg}$ & & 0.31 \\
\hline 383DH1200.5 & $6 / 7 / 2004$ & soil & EPA7470 & REG & $7439-97-6$ & Mercury & 0.049 & $\mathrm{mg} / \mathrm{kg}$ & $B$ & 0.1 \\
\hline 383DH1200.5 & $6 / 7 / 2004$ & soil & EPA8260 & REG & $75-09-2$ & Methylene Chloride & 2.5 & $\mu \mathrm{g} / \mathrm{kg}$ & $\mathrm{J}$ & 5.2 \\
\hline 383DH1200.5 & $6 / 7 / 2004$ & soil & HASL300 & REG & 15092-94-1 & $\mathrm{Pb}-212$ & 2.42 & $\mathrm{pCi} / \mathrm{g}$ & $\mathrm{J}$ & 0.45 \\
\hline 383DH1200.5 & $6 / 7 / 2004$ & soil & HASL300 & REG & 15067-28-4 & $\mathrm{Pb}-214$ & 1.31 & $\mathrm{pCi} / \mathrm{g}$ & $\mathrm{G}, \mathrm{J}$ & 0.69 \\
\hline
\end{tabular}

Page 9 of 78

Uncontrolled When Printed 


\begin{tabular}{|c|c|c|c|c|c|c|c|c|c|c|}
\hline SAMPLE \# & $\begin{array}{c}\text { SAMPLE } \\
\text { DATE }\end{array}$ & MATRIX & $\begin{array}{l}\text { USER TEST } \\
\text { PANEL }\end{array}$ & $\begin{array}{l}\text { SAMPLE } \\
\text { PURPOSE }\end{array}$ & CAS \# & PARAMETER & RESULT & UNITS & $\mathbf{Q}$ & $\begin{array}{c}\text { DETECT } \\
\text { LIMIT }\end{array}$ \\
\hline 383DH1200.5 & $6 / 7 / 2004$ & soil & UGTAISOPU & REG & 13981-16-3 & Pu-238 & 0.136 & $\mathrm{pCi} / \mathrm{g}$ & & 0.022 \\
\hline 383DH1200.5 & $6 / 7 / 2004$ & soil & UGTAISOPU & REG & $15117-48-3$ & Pu-239 & 1.54 & $\mathrm{pCi} / \mathrm{g}$ & & 0.02 \\
\hline 383DH1200.5 & $6 / 7 / 2004$ & soil & SR7500 & REG & 10098-97-2 & Sr-90 & 0.97 & $\mathrm{pCi} / \mathrm{g}$ & $\mathrm{Y} 1$ & 0.25 \\
\hline 383DH1200.5 & $6 / 7 / 2004$ & soil & HASL300 & REG & $14913-50-9$ & TI-208 & 0.72 & $\mathrm{pCi} / \mathrm{g}$ & $G$ & 0.25 \\
\hline 383DH1300.5 & $6 / 7 / 2004$ & soil & HASL300 & REG & $14331-83-0$ & Ac-228 & 1.29 & $\mathrm{pCi} / \mathrm{g}$ & $\mathrm{G}$ & 0.42 \\
\hline 383DH1300.5 & $6 / 7 / 2004$ & soil & EPA6010 & REG & $7440-38-2$ & Arsenic & 2.5 & $\mathrm{mg} / \mathrm{kg}$ & & 1 \\
\hline 383DH1300.5 & $6 / 7 / 2004$ & soil & EPA6010 & REG & $7440-39-3$ & Barium & 2900 & $\mathrm{mg} / \mathrm{kg}$ & & 100 \\
\hline 383DH1300.5 & $6 / 7 / 2004$ & soil & EPA6010 & REG & $7440-41-7$ & Beryllium & 1.6 & $\mathrm{mg} / \mathrm{kg}$ & & 0.52 \\
\hline 383DH1300.5 & $6 / 7 / 2004$ & soil & HASL300 & REG & $14733-03-0$ & Bi-214 & 1.1 & $\mathrm{pCi} / \mathrm{g}$ & $\mathrm{G}, \mathrm{J}$ & 0.28 \\
\hline 383DH1300.5 & $6 / 7 / 2004$ & soil & EPA6010 & REG & $7440-43-9$ & Cadmium & 0.11 & $\mathrm{mg} / \mathrm{kg}$ & B & 0.52 \\
\hline 383DH1300.5 & $6 / 7 / 2004$ & soil & EPA6010 & REG & $7440-47-3$ & Chromium & 3.3 & $\mathrm{mg} / \mathrm{kg}$ & & 1 \\
\hline 383DH1300.5 & $6 / 7 / 2004$ & soil & HASL300 & REG & $10045-97-3$ & Cs-137 & 4.87 & $\mathrm{pCi} / \mathrm{g}$ & G & 0.14 \\
\hline 383DH1300.5 & $6 / 7 / 2004$ & soil & HASL300 & REG & $13966-00-2$ & $\mathrm{~K}-40$ & 25.9 & $\mathrm{pCi} / \mathrm{g}$ & $\mathrm{G}$ & 1.1 \\
\hline 383DH1300.5 & $6 / 7 / 2004$ & soil & EPA6010 & REG & $7439-92-1$ & Lead & 22 & $\mathrm{mg} / \mathrm{kg}$ & & 0.31 \\
\hline 383DH1300.5 & $6 / 7 / 2004$ & soil & EPA7470 & REG & $7439-97-6$ & Mercury & 0.018 & $\mathrm{mg} / \mathrm{kg}$ & $B$ & 0.1 \\
\hline 383DH1300.5 & $6 / 7 / 2004$ & soil & EPA8260 & REG & $75-09-2$ & Methylene Chloride & 0.84 & $\mu \mathrm{g} / \mathrm{kg}$ & $\mathrm{J}$ & 5.2 \\
\hline 383DH1300.5 & $6 / 7 / 2004$ & soil & HASL300 & REG & 15092-94-1 & $\mathrm{Pb}-212$ & 2.06 & $\mathrm{pCi} / \mathrm{g}$ & $\mathrm{J}$ & 0.26 \\
\hline 383DH1300.5 & $6 / 7 / 2004$ & soil & HASL300 & REG & $15067-28-4$ & $\mathrm{~Pb}-214$ & 1.37 & $\mathrm{pCi} / \mathrm{g}$ & $\mathrm{G}, \mathrm{J}$ & 0.3 \\
\hline 383DH1300.5 & $6 / 7 / 2004$ & soil & UGTAISOPU & REG & $15117-48-3$ & Pu-239 & 0.268 & $\mathrm{pCi} / \mathrm{g}$ & & 0.018 \\
\hline 383DH1300.5 & $6 / 7 / 2004$ & soil & HASL300 & REG & $14913-50-9$ & TI-208 & 0.48 & $\mathrm{pCi} / \mathrm{g}$ & $\mathrm{G}$ & 0.13 \\
\hline 383DH1400.5 & $6 / 7 / 2004$ & soil & HASL300 & REG & 14331-83-0 & Ac-228 & 2.2 & $\mathrm{pCi} / \mathrm{g}$ & $\mathrm{G}$ & 0.74 \\
\hline 383DH1400.5 & $6 / 7 / 2004$ & soil & EPA8260 & REG & 67-64-1 & Acetone & 8.5 & $\mu \mathrm{g} / \mathrm{kg}$ & $\mathrm{J}, \mathrm{B}$ & 21 \\
\hline 383DH1400.5 & $6 / 7 / 2004$ & soil & EPA6010 & REG & $7440-38-2$ & Arsenic & 3.3 & $\mathrm{mg} / \mathrm{kg}$ & & 1 \\
\hline 383DH1400.5 & $6 / 7 / 2004$ & soil & EPA6010 & REG & $7440-39-3$ & Barium & 3900 & $\mathrm{mg} / \mathrm{kg}$ & & 100 \\
\hline 383DH1400.5 & $6 / 7 / 2004$ & soil & EPA6010 & REG & $7440-41-7$ & Beryllium & 2.1 & $\mathrm{mg} / \mathrm{kg}$ & & 0.52 \\
\hline 383DH1400.5 & $6 / 7 / 2004$ & soil & HASL300 & REG & $14733-03-0$ & Bi-214 & 1.48 & $\mathrm{pCi} / \mathrm{g}$ & $\mathrm{G}, \mathrm{J}$ & 0.62 \\
\hline 383DH1400.5 & $6 / 7 / 2004$ & soil & EPA6010 & REG & $7440-43-9$ & Cadmium & 0.12 & $\mathrm{mg} / \mathrm{kg}$ & $\mathrm{B}$ & 0.52 \\
\hline 383DH1400.5 & $6 / 7 / 2004$ & soil & EPA6010 & REG & $7440-47-3$ & Chromium & 5.2 & $\mathrm{mg} / \mathrm{kg}$ & & 1 \\
\hline 383DH1400.5 & $6 / 7 / 2004$ & soil & HASL300 & REG & $10045-97-3$ & Cs-137 & 68.8 & $\mathrm{pCi} / \mathrm{g}$ & $\mathrm{G}$ & 0.3 \\
\hline 383DH1400.5 & $6 / 7 / 2004$ & soil & EPA8015 & REG & $68334-30-5$ & Diesel-Range Organics & 2.5 & $\mathrm{mg} / \mathrm{kg}$ & $\mathrm{J}$ & 5.2 \\
\hline 383DH1400.5 & $6 / 7 / 2004$ & soil & EPA8260 & REG & $100-41-4$ & Ethylbenzene & 1.2 & $\mu \mathrm{g} / \mathrm{kg}$ & $\mathrm{J}$ & 5.2 \\
\hline 383DH1400.5 & $6 / 7 / 2004$ & soil & HASL300 & REG & $13966-00-2$ & $\mathrm{~K}-40$ & 26.6 & $\mathrm{pCi} / \mathrm{g}$ & $\mathrm{G}$ & 2.5 \\
\hline 383DH1400.5 & $6 / 7 / 2004$ & soil & EPA6010 & REG & 7439-92-1 & Lead & 33 & $\mathrm{mg} / \mathrm{kg}$ & & 0.31 \\
\hline 383DH1400.5 & $6 / 7 / 2004$ & soil & EPA8260 & REG & $136777-61-2$ & M+P-Xylene & 4.8 & $\mu \mathrm{g} / \mathrm{kg}$ & $\mathrm{J}$ & 5.2 \\
\hline 383DH1400.5 & $6 / 7 / 2004$ & soil & EPA7470 & REG & $7439-97-6$ & Mercury & 0.083 & $\mathrm{mg} / \mathrm{kg}$ & $\mathrm{B}$ & 0.1 \\
\hline
\end{tabular}

Page 10 of 78

Uncontrolled When Printed 


\begin{tabular}{|c|c|c|c|c|c|c|c|c|c|c|}
\hline SAMPLE \# & $\begin{array}{l}\text { SAMPLE } \\
\text { DATE }\end{array}$ & MATRIX & $\begin{array}{l}\text { USER TEST } \\
\text { PANEL }\end{array}$ & $\begin{array}{c}\text { SAMPLE } \\
\text { PURPOSE }\end{array}$ & CAS \# & PARAMETER & RESULT & UNITS & $\mathbf{Q}$ & $\begin{array}{l}\text { DETECT } \\
\text { LIMIT }\end{array}$ \\
\hline 383DH1400.5 & $6 / 7 / 2004$ & soil & EPA8260 & REG & $75-09-2$ & Methylene Chloride & 1.2 & $\mu \mathrm{g} / \mathrm{kg}$ & $\mathrm{J}$ & 5.2 \\
\hline 383DH1400.5 & $6 / 7 / 2004$ & soil & EPA8260 & REG & $95-47-6$ & O-Xylene & 1.5 & $\mu \mathrm{g} / \mathrm{kg}$ & $\mathrm{J}$ & 5.2 \\
\hline 383DH1400.5 & $6 / 7 / 2004$ & soil & HASL300 & REG & 15092-94-1 & $\mathrm{Pb}-212$ & 2.03 & $\mathrm{pCi} / \mathrm{g}$ & $\mathrm{J}$ & 0.54 \\
\hline 383DH1400.5 & $6 / 7 / 2004$ & soil & HASL300 & REG & $15067-28-4$ & $\mathrm{~Pb}-214$ & 1.68 & $\mathrm{pCi} / \mathrm{g}$ & $\mathrm{G}, \mathrm{J}$ & 0.9 \\
\hline 383DH1400.5 & $6 / 7 / 2004$ & soil & UGTAISOPU & REG & 13981-16-3 & Pu-238 & 0.68 & $\mathrm{pCi} / \mathrm{g}$ & & 0.02 \\
\hline 383DH1400.5 & $6 / 7 / 2004$ & soil & UGTAISOPU & REG & $15117-48-3$ & Pu-239 & 4.21 & $\mathrm{pCi} / \mathrm{g}$ & & 0.02 \\
\hline 383DH1400.5 & $6 / 7 / 2004$ & soil & SR7500 & REG & $10098-97-2$ & Sr-90 & 1.14 & $\mathrm{pCi} / \mathrm{g}$ & Y1 & 0.25 \\
\hline 383DH1400.5 & $6 / 7 / 2004$ & soil & EPA8260 & REG & $100-42-5$ & Styrene & 1.3 & $\mu \mathrm{g} / \mathrm{kg}$ & $\mathrm{J}$ & 5.2 \\
\hline 383DH1400.5 & $6 / 7 / 2004$ & soil & HASL300 & REG & $14913-50-9$ & TI-208 & 0.87 & $\mathrm{pCi} / \mathrm{g}$ & G & 0.28 \\
\hline 383DH1500.5 & $6 / 7 / 2004$ & soil & HASL300 & REG & 14331-83-0 & Ac-228 & 1.71 & $\mathrm{pCi} / \mathrm{g}$ & $G$ & 0.46 \\
\hline 383DH1500.5 & $6 / 7 / 2004$ & soil & EPA6010 & REG & $7440-38-2$ & Arsenic & 3.4 & $\mathrm{mg} / \mathrm{kg}$ & & 1 \\
\hline 383DH1500.5 & $6 / 7 / 2004$ & soil & EPA6010 & REG & $7440-39-3$ & Barium & 4600 & $\mathrm{mg} / \mathrm{kg}$ & & 100 \\
\hline 383DH1500.5 & $6 / 7 / 2004$ & soil & EPA6010 & REG & $7440-41-7$ & Beryllium & 1.3 & $\mathrm{mg} / \mathrm{kg}$ & & 0.52 \\
\hline 383DH1500.5 & $6 / 7 / 2004$ & soil & HASL300 & REG & 14733-03-0 & Bi-214 & 1.09 & $\mathrm{pCi} / \mathrm{g}$ & $\mathrm{G}, \mathrm{J}$ & 0.31 \\
\hline 383DH1500.5 & $6 / 7 / 2004$ & soil & EPA6010 & REG & 7440-43-9 & Cadmium & 0.19 & $\mathrm{mg} / \mathrm{kg}$ & $B$ & 0.52 \\
\hline 383DH1500.5 & $6 / 7 / 2004$ & soil & EPA6010 & REG & $7440-47-3$ & Chromium & 6 & $\mathrm{mg} / \mathrm{kg}$ & & 1 \\
\hline 383DH1500.5 & $6 / 7 / 2004$ & soil & HASL300 & REG & $10045-97-3$ & Cs-137 & 11.2 & $\mathrm{pCi} / \mathrm{g}$ & G & 0.2 \\
\hline 383DH1500.5 & $6 / 7 / 2004$ & soil & EPA8270 & REG & $206-44-0$ & Fluoranthene & 13 & $\mu \mathrm{g} / \mathrm{kg}$ & $\mathrm{J}$ & 340 \\
\hline 383DH1500.5 & $6 / 7 / 2004$ & soil & HASL300 & REG & 13966-00-2 & $\mathrm{K}-40$ & 23.3 & $\mathrm{pCi} / \mathrm{g}$ & G & 1.3 \\
\hline 383DH1500.5 & $6 / 7 / 2004$ & soil & EPA6010 & REG & 7439-92-1 & Lead & 31 & $\mathrm{mg} / \mathrm{kg}$ & & 0.31 \\
\hline 383DH1500.5 & $6 / 7 / 2004$ & soil & EPA7470 & REG & 7439-97-6 & Mercury & 0.025 & $\mathrm{mg} / \mathrm{kg}$ & B & 0.1 \\
\hline 383DH1500.5 & $6 / 7 / 2004$ & soil & HASL300 & REG & 15092-94-1 & $\mathrm{Pb}-212$ & 1.69 & $\mathrm{pCi} / \mathrm{g}$ & $\mathrm{J}$ & 0.34 \\
\hline 383DH1500.5 & $6 / 7 / 2004$ & soil & HASL300 & REG & $15067-28-4$ & $\mathrm{~Pb}-214$ & 1.22 & $\mathrm{pCi} / \mathrm{g}$ & $\mathrm{G}, \mathrm{J}$ & 0.39 \\
\hline 383DH1500.5 & $6 / 7 / 2004$ & soil & UGTAISOPU & REG & 13981-16-3 & Pu-238 & 0.077 & $\mathrm{pCi} / \mathrm{g}$ & & 0.022 \\
\hline 383DH1500.5 & $6 / 7 / 2004$ & soil & UGTAISOPU & REG & $15117-48-3$ & Pu-239 & 0.57 & $\mathrm{pCi} / \mathrm{g}$ & & 0.02 \\
\hline 383DH1500.5 & $6 / 7 / 2004$ & soil & SR7500 & REG & 10098-97-2 & Sr-90 & 0.53 & $\mathrm{pCi} / \mathrm{g}$ & Y1 & 0.23 \\
\hline 383DH1500.5 & $6 / 7 / 2004$ & soil & HASL300 & REG & 14913-50-9 & TI-208 & 0.59 & $\mathrm{pCi} / \mathrm{g}$ & $G$ & 0.19 \\
\hline 383DH1600.5 & $6 / 7 / 2004$ & soil & HASL300 & REG & 14331-83-0 & Ac-228 & 1.56 & $\mathrm{pCi} / \mathrm{g}$ & $G$ & 0.64 \\
\hline 383DH1600.5 & $6 / 7 / 2004$ & soil & HASL300 & REG & 14682-66-7 & Al-26 & 0.105 & $\mathrm{pCi} / \mathrm{g}$ & $\mathrm{G}, \mathrm{TI}$ & 0.032 \\
\hline 383DH1600.5 & $6 / 7 / 2004$ & soil & EPA6010 & REG & $7440-38-2$ & Arsenic & 3 & $\mathrm{mg} / \mathrm{kg}$ & & 1 \\
\hline 383DH1600.5 & $6 / 7 / 2004$ & soil & EPA6010 & REG & 7440-39-3 & Barium & 4100 & $\mathrm{mg} / \mathrm{kg}$ & & 100 \\
\hline 383DH1600.5 & $6 / 7 / 2004$ & soil & EPA6010 & REG & $7440-41-7$ & Beryllium & 1.4 & $\mathrm{mg} / \mathrm{kg}$ & & 0.52 \\
\hline 383DH1600.5 & $6 / 7 / 2004$ & soil & HASL300 & REG & $14733-03-0$ & Bi-214 & 1.07 & $\mathrm{pCi} / \mathrm{g}$ & $\mathrm{G}, \mathrm{J}$ & 0.41 \\
\hline 383DH1600.5 & $6 / 7 / 2004$ & soil & EPA8270 & REG & $117-81-7$ & Bis(2-Ethylhexyl)Phthalate & 130 & $\mu \mathrm{g} / \mathrm{kg}$ & $\mathrm{J}$ & 350 \\
\hline 383DH1600.5 & $6 / 7 / 2004$ & soil & EPA6010 & REG & 7440-43-9 & Cadmium & 0.17 & $\mathrm{mg} / \mathrm{kg}$ & $\mathrm{B}$ & 0.52 \\
\hline
\end{tabular}

Page 11 of 78

Uncontrolled When Printed 


\begin{tabular}{|c|c|c|c|c|c|c|c|c|c|c|}
\hline SAMPLE \# & $\begin{array}{l}\text { SAMPLE } \\
\text { DATE }\end{array}$ & MATRIX & $\begin{array}{l}\text { USER TEST } \\
\text { PANEL }\end{array}$ & $\begin{array}{c}\text { SAMPLE } \\
\text { PURPOSE }\end{array}$ & CAS \# & PARAMETER & RESULT & UNITS & $\mathbf{Q}$ & $\begin{array}{c}\text { DETECT } \\
\text { LIMIT }\end{array}$ \\
\hline 383DH1600.5 & $6 / 7 / 2004$ & soil & EPA6010 & REG & $7440-47-3$ & Chromium & 4.2 & $\mathrm{mg} / \mathrm{kg}$ & & 1 \\
\hline 383DH1600.5 & $6 / 7 / 2004$ & soil & HASL300 & REG & $10045-97-3$ & Cs-137 & 20.5 & $\mathrm{pCi} / \mathrm{g}$ & $\mathrm{G}$ & 0.2 \\
\hline 383DH1600.5 & $6 / 7 / 2004$ & soil & EPA8270 & REG & $84-74-2$ & Di-N-Butyl Phthalate & 23 & $\mu \mathrm{g} / \mathrm{kg}$ & $\mathrm{J}$ & 350 \\
\hline 383DH1600.5 & $6 / 7 / 2004$ & soil & EPA8260 & REG & $100-41-4$ & Ethylbenzene & 1.5 & $\mu \mathrm{g} / \mathrm{kg}$ & $\mathrm{J}$ & 5.2 \\
\hline 383DH1600.5 & $6 / 7 / 2004$ & soil & HASL300 & REG & $13966-00-2$ & $\mathrm{~K}-40$ & 26.5 & $\mathrm{pCi} / \mathrm{g}$ & G & 1.8 \\
\hline 383DH1600.5 & $6 / 7 / 2004$ & soil & EPA6010 & REG & 7439-92-1 & Lead & 24 & $\mathrm{mg} / \mathrm{kg}$ & & 0.31 \\
\hline 383DH1600.5 & $6 / 7 / 2004$ & soil & EPA8260 & REG & 136777-61-2 & M+P-Xylene & 5.8 & $\mu \mathrm{g} / \mathrm{kg}$ & & 5.2 \\
\hline 383DH1600.5 & $6 / 7 / 2004$ & soil & EPA7470 & REG & $7439-97-6$ & Mercury & 0.038 & $\mathrm{mg} / \mathrm{kg}$ & B & 0.1 \\
\hline 383DH1600.5 & $6 / 7 / 2004$ & soil & EPA8260 & REG & $75-09-2$ & Methylene Chloride & 1 & $\mu \mathrm{g} / \mathrm{kg}$ & $\mathrm{J}$ & 5.2 \\
\hline 383DH1600.5 & $6 / 7 / 2004$ & soil & EPA8260 & REG & $95-47-6$ & O-Xylene & 1.5 & $\mu \mathrm{g} / \mathrm{kg}$ & $\mathrm{J}$ & 5.2 \\
\hline 383DH1600.5 & $6 / 7 / 2004$ & soil & HASL300 & REG & 15092-94-1 & $\mathrm{Pb}-212$ & 1.74 & $\mathrm{pCi} / \mathrm{g}$ & $\mathrm{J}$ & 0.4 \\
\hline 383DH1600.5 & $6 / 7 / 2004$ & soil & HASL300 & REG & $15067-28-4$ & $\mathrm{~Pb}-214$ & 1.32 & $\mathrm{pCi} / \mathrm{g}$ & $\mathrm{G}, \mathrm{J}$ & 0.51 \\
\hline 383DH1600.5 & $6 / 7 / 2004$ & soil & UGTAISOPU & REG & 13981-16-3 & Pu-238 & 0.13 & $\mathrm{pCi} / \mathrm{g}$ & & 0.022 \\
\hline 383DH1600.5 & $6 / 7 / 2004$ & soil & UGTAISOPU & REG & $15117-48-3$ & Pu-239 & 1.08 & $\mathrm{pCi} / \mathrm{g}$ & & 0.01 \\
\hline 383DH1600.5 & $6 / 7 / 2004$ & soil & SR7500 & REG & $10098-97-2$ & Sr-90 & 0.54 & $\mathrm{pCi} / \mathrm{g}$ & Y1 & 0.25 \\
\hline 383DH1600.5 & $6 / 7 / 2004$ & soil & HASL300 & REG & 14913-50-9 & TI-208 & 0.55 & $\mathrm{pCi} / \mathrm{g}$ & $\mathrm{G}$ & 0.19 \\
\hline 383DH1700.5 & $6 / 7 / 2004$ & soil & HASL300 & REG & 14331-83-0 & Ac-228 & 1.8 & $\mathrm{pCi} / \mathrm{g}$ & G & 0.72 \\
\hline 383DH1700.5 & $6 / 7 / 2004$ & soil & EPA6010 & REG & $7440-38-2$ & Arsenic & 3.4 & $\mathrm{mg} / \mathrm{kg}$ & & 1 \\
\hline 383DH1700.5 & $6 / 7 / 2004$ & soil & EPA6010 & REG & $7440-39-3$ & Barium & 2800 & $\mathrm{mg} / \mathrm{kg}$ & & 100 \\
\hline 383DH1700.5 & $6 / 7 / 2004$ & soil & EPA6010 & REG & 7440-41-7 & Beryllium & 1.7 & $\mathrm{mg} / \mathrm{kg}$ & & 0.51 \\
\hline 383DH1700.5 & $6 / 7 / 2004$ & soil & HASL300 & REG & 14733-03-0 & Bi-214 & 1.12 & $\mathrm{pCi} / \mathrm{g}$ & $\mathrm{G}, \mathrm{J}$ & 0.59 \\
\hline 383DH1700.5 & $6 / 7 / 2004$ & soil & EPA6010 & REG & $7440-43-9$ & Cadmium & 0.37 & $\mathrm{mg} / \mathrm{kg}$ & $B$ & 0.51 \\
\hline 383DH1700.5 & $6 / 7 / 2004$ & soil & EPA6010 & REG & $7440-47-3$ & Chromium & 5.8 & $\mathrm{mg} / \mathrm{kg}$ & & 1 \\
\hline 383DH1700.5 & $6 / 7 / 2004$ & soil & HASL300 & REG & $10045-97-3$ & Cs-137 & 68 & $\mathrm{pCi} / \mathrm{g}$ & G & 0.3 \\
\hline 383DH1700.5 & $6 / 7 / 2004$ & soil & HASL300 & REG & 13966-00-2 & $\mathrm{K}-40$ & 19.7 & $\mathrm{pCi} / \mathrm{g}$ & $\mathrm{G}$ & 2.7 \\
\hline 383DH1700.5 & $6 / 7 / 2004$ & soil & EPA6010 & REG & $7439-92-1$ & Lead & 49 & $\mathrm{mg} / \mathrm{kg}$ & & 0.31 \\
\hline 383DH1700.5 & $6 / 7 / 2004$ & soil & EPA7470 & REG & $7439-97-6$ & Mercury & 0.061 & $\mathrm{mg} / \mathrm{kg}$ & $B$ & 0.1 \\
\hline 383DH1700.5 & $6 / 7 / 2004$ & soil & EPA8260 & REG & $75-09-2$ & Methylene Chloride & 1.2 & $\mu \mathrm{g} / \mathrm{kg}$ & $\mathrm{J}$ & 5.1 \\
\hline 383DH1700.5 & $6 / 7 / 2004$ & soil & HASL300 & REG & 15092-94-1 & $\mathrm{Pb}-212$ & 2.21 & $\mathrm{pCi} / \mathrm{g}$ & $\mathrm{J}$ & 0.58 \\
\hline 383DH1700.5 & $6 / 7 / 2004$ & soil & HASL300 & REG & $15067-28-4$ & $\mathrm{~Pb}-214$ & 1.32 & $\mathrm{pCi} / \mathrm{g}$ & $\mathrm{G}, \mathrm{J}$ & 0.84 \\
\hline 383DH1700.5 & $6 / 7 / 2004$ & soil & UGTAISOPU & REG & 13981-16-3 & Pu-238 & 0.41 & $\mathrm{pCi} / \mathrm{g}$ & & 0.03 \\
\hline 383DH1700.5 & $6 / 7 / 2004$ & soil & UGTAISOPU & REG & $15117-48-3$ & Pu-239 & 3.55 & $\mathrm{pCi} / \mathrm{g}$ & & 0.03 \\
\hline 383DH1700.5 & $6 / 7 / 2004$ & soil & EPA6010 & REG & $7782-49-2$ & Selenium & 0.87 & $\mathrm{mg} / \mathrm{kg}$ & & 0.51 \\
\hline 383DH1700.5 & $6 / 7 / 2004$ & soil & SR7500 & REG & 10098-97-2 & Sr-90 & 1.09 & $\mathrm{pCi} / \mathrm{g}$ & Y1 & 0.24 \\
\hline 383DH1700.5 & $6 / 7 / 2004$ & soil & HASL300 & REG & 14913-50-9 & TI-208 & 0.7 & $\mathrm{pCi} / \mathrm{g}$ & $\mathrm{G}$ & 0.32 \\
\hline
\end{tabular}

Page 12 of 78

Uncontrolled When Printed 


\begin{tabular}{|c|c|c|c|c|c|c|c|c|c|c|}
\hline SAMPLE \# & $\begin{array}{l}\text { SAMPLE } \\
\text { DATE }\end{array}$ & MATRIX & $\begin{array}{l}\text { USER TEST } \\
\text { PANEL }\end{array}$ & $\begin{array}{c}\text { SAMPLE } \\
\text { PURPOSE }\end{array}$ & CAS \# & PARAMETER & RESULT & UNITS & $\mathbf{Q}$ & $\begin{array}{c}\text { DETECT } \\
\text { LIMIT }\end{array}$ \\
\hline 383DH1800.5 & $6 / 7 / 2004$ & soil & HASL300 & REG & 14331-83-0 & Ac-228 & 1.17 & $\mathrm{pCi} / \mathrm{g}$ & G & 0.64 \\
\hline 383DH1800.5 & $6 / 7 / 2004$ & soil & EPA6010 & REG & $7440-38-2$ & Arsenic & 3 & $\mathrm{mg} / \mathrm{kg}$ & & 1 \\
\hline 383DH1800.5 & $6 / 7 / 2004$ & soil & EPA6010 & REG & $7440-39-3$ & Barium & 4200 & $\mathrm{mg} / \mathrm{kg}$ & & 100 \\
\hline 383DH1800.5 & $6 / 7 / 2004$ & soil & EPA6010 & REG & $7440-41-7$ & Beryllium & 1.5 & $\mathrm{mg} / \mathrm{kg}$ & & 0.52 \\
\hline 383DH1800.5 & $6 / 7 / 2004$ & soil & HASL300 & REG & 14733-03-0 & Bi-214 & 1.46 & $\mathrm{pCi} / \mathrm{g}$ & $\mathrm{G}, \mathrm{J}$ & 0.37 \\
\hline 383DH1800.5 & $6 / 7 / 2004$ & soil & EPA6010 & REG & $7440-43-9$ & Cadmium & 0.35 & $\mathrm{mg} / \mathrm{kg}$ & B & 0.52 \\
\hline 383DH1800.5 & $6 / 7 / 2004$ & soil & EPA6010 & REG & $7440-47-3$ & Chromium & 5.5 & $\mathrm{mg} / \mathrm{kg}$ & & 1 \\
\hline 383DH1800.5 & $6 / 7 / 2004$ & soil & HASL300 & REG & $10045-97-3$ & Cs-137 & 23.1 & $\mathrm{pCi} / \mathrm{g}$ & $\mathrm{G}$ & 0.2 \\
\hline 383DH1800.5 & $6 / 7 / 2004$ & soil & HASL300 & REG & 13966-00-2 & $\mathrm{K}-40$ & 20.6 & $\mathrm{pCi} / \mathrm{g}$ & $\mathrm{G}$ & 1.3 \\
\hline 383DH1800.5 & $6 / 7 / 2004$ & soil & EPA6010 & REG & 7439-92-1 & Lead & 21 & $\mathrm{mg} / \mathrm{kg}$ & & 0.31 \\
\hline 383DH1800.5 & $6 / 7 / 2004$ & soil & EPA7470 & REG & 7439-97-6 & Mercury & 0.039 & $\mathrm{mg} / \mathrm{kg}$ & $B$ & 0.1 \\
\hline 383DH1800.5 & $6 / 7 / 2004$ & soil & EPA8260 & REG & $75-09-2$ & Methylene Chloride & 1.1 & $\mu \mathrm{g} / \mathrm{kg}$ & $\mathrm{J}$ & 5.2 \\
\hline 383DH1800.5 & $6 / 7 / 2004$ & soil & HASL300 & REG & 15092-94-1 & $\mathrm{Pb}-212$ & 1.69 & $\mathrm{pCi} / \mathrm{g}$ & $\mathrm{J}$ & 0.32 \\
\hline 383DH1800.5 & $6 / 7 / 2004$ & soil & HASL300 & REG & $15067-28-4$ & $\mathrm{~Pb}-214$ & 1.18 & $\mathrm{pCi} / \mathrm{g}$ & $\mathrm{G}, \mathrm{J}$ & 0.52 \\
\hline 383DH1800.5 & $6 / 7 / 2004$ & soil & UGTAISOPU & REG & 13981-16-3 & Pu-238 & 0.177 & $\mathrm{pCi} / \mathrm{g}$ & & 0.023 \\
\hline 383DH1800.5 & $6 / 7 / 2004$ & soil & UGTAISOPU & REG & $15117-48-3$ & Pu-239 & 1.39 & $\mathrm{pCi} / \mathrm{g}$ & & 0.02 \\
\hline 383DH1800.5 & $6 / 7 / 2004$ & soil & SR7500 & REG & 10098-97-2 & Sr-90 & 0.69 & $\mathrm{pCi} / \mathrm{g}$ & Y1 & 0.25 \\
\hline 383DH1800.5 & $6 / 7 / 2004$ & soil & HASL300 & REG & $14913-50-9$ & TI-208 & 0.52 & $\mathrm{pCi} / \mathrm{g}$ & G & 0.21 \\
\hline 383DH1900.5 & $6 / 7 / 2004$ & soil & HASL300 & REG & 14331-83-0 & Ac-228 & 1.82 & $\mathrm{pCi} / \mathrm{g}$ & $\mathrm{G}$ & 0.74 \\
\hline 383DH1900.5 & $6 / 7 / 2004$ & soil & EPA6010 & REG & $7440-38-2$ & Arsenic & 2.7 & $\mathrm{mg} / \mathrm{kg}$ & & 1 \\
\hline 383DH1900.5 & $6 / 7 / 2004$ & soil & EPA6010 & REG & $7440-39-3$ & Barium & 4500 & $\mathrm{mg} / \mathrm{kg}$ & & 100 \\
\hline 383DH1900.5 & $6 / 7 / 2004$ & soil & EPA6010 & REG & $7440-41-7$ & Beryllium & 1.7 & $\mathrm{mg} / \mathrm{kg}$ & & 0.51 \\
\hline 383DH1900.5 & $6 / 7 / 2004$ & soil & HASL300 & REG & $14733-03-0$ & Bi-214 & 1.27 & $\mathrm{pCi} / \mathrm{g}$ & $\mathrm{G}, \mathrm{J}$ & 0.47 \\
\hline 383DH1900.5 & $6 / 7 / 2004$ & soil & EPA8270 & REG & $117-81-7$ & Bis(2-Ethylhexyl)Phthalate & 120 & $\mu \mathrm{g} / \mathrm{kg}$ & $\mathrm{J}$ & 340 \\
\hline 383DH1900.5 & $6 / 7 / 2004$ & soil & EPA6010 & REG & 7440-43-9 & Cadmium & 0.33 & $\mathrm{mg} / \mathrm{kg}$ & $\mathrm{B}$ & 0.51 \\
\hline 383DH1900.5 & $6 / 7 / 2004$ & soil & EPA6010 & REG & $7440-47-3$ & Chromium & 4.6 & $\mathrm{mg} / \mathrm{kg}$ & & 1 \\
\hline 383DH1900.5 & $6 / 7 / 2004$ & soil & HASL300 & REG & $10045-97-3$ & Cs-137 & 42.4 & $\mathrm{pCi} / \mathrm{g}$ & G & 0.3 \\
\hline 383DH1900.5 & $6 / 7 / 2004$ & soil & HASL300 & REG & $13966-00-2$ & $\mathrm{~K}-40$ & 24.1 & $\mathrm{pCi} / \mathrm{g}$ & G & 1.7 \\
\hline 383DH1900.5 & $6 / 7 / 2004$ & soil & EPA6010 & REG & 7439-92-1 & Lead & 25 & $\mathrm{mg} / \mathrm{kg}$ & & 0.31 \\
\hline 383DH1900.5 & $6 / 7 / 2004$ & soil & EPA8260 & REG & 136777-61-2 & M+P-Xylene & 2.4 & $\mu \mathrm{g} / \mathrm{kg}$ & $\mathrm{J}$ & 5.1 \\
\hline 383DH1900.5 & $6 / 7 / 2004$ & soil & EPA7470 & REG & $7439-97-6$ & Mercury & 0.034 & $\mathrm{mg} / \mathrm{kg}$ & $\mathrm{B}$ & 0.1 \\
\hline 383DH1900.5 & $6 / 7 / 2004$ & soil & EPA8260 & REG & $75-09-2$ & Methylene Chloride & 1.2 & $\mu \mathrm{g} / \mathrm{kg}$ & $\mathrm{J}$ & 5.1 \\
\hline 383DH1900.5 & $6 / 7 / 2004$ & soil & EPA8260 & REG & $95-47-6$ & O-Xylene & 1 & $\mu \mathrm{g} / \mathrm{kg}$ & $\mathrm{J}$ & 5.1 \\
\hline 383DH1900.5 & $6 / 7 / 2004$ & soil & HASL300 & REG & 15092-94-1 & $\mathrm{Pb}-212$ & 1.78 & $\mathrm{pCi} / \mathrm{g}$ & $\mathrm{J}$ & 0.47 \\
\hline 383DH1900.5 & $6 / 7 / 2004$ & soil & HASL300 & REG & 15067-28-4 & $\mathrm{Pb}-214$ & 1.3 & $\mathrm{pCi} / \mathrm{g}$ & $\mathrm{G}, \mathrm{J}$ & 0.66 \\
\hline
\end{tabular}

Page 13 of 78

Uncontrolled When Printed 


\begin{tabular}{|c|c|c|c|c|c|c|c|c|c|c|}
\hline SAMPLE \# & $\begin{array}{c}\text { SAMPLE } \\
\text { DATE }\end{array}$ & MATRIX & $\begin{array}{l}\text { USER TEST } \\
\text { PANEL }\end{array}$ & $\begin{array}{c}\text { SAMPLE } \\
\text { PURPOSE }\end{array}$ & CAS \# & PARAMETER & RESULT & UNITS & $\mathbf{Q}$ & $\begin{array}{l}\text { DETECT } \\
\text { LIMIT }\end{array}$ \\
\hline 383DH1900.5 & $6 / 7 / 2004$ & soil & UGTAISOPU & REG & 13981-16-3 & Pu-238 & 0.32 & $\mathrm{pCi} / \mathrm{g}$ & & 0.019 \\
\hline 383DH1900.5 & $6 / 7 / 2004$ & soil & UGTAISOPU & REG & $15117-48-3$ & Pu-239 & 2.28 & $\mathrm{pCi} / \mathrm{g}$ & & 0.01 \\
\hline 383DH1900.5 & $6 / 7 / 2004$ & soil & SR7500 & REG & 10098-97-2 & Sr-90 & 1.14 & $\mathrm{pCi} / \mathrm{g}$ & Y1 & 0.26 \\
\hline 383DH1900.5 & $6 / 7 / 2004$ & soil & HASL300 & REG & $14913-50-9$ & TI-208 & 0.65 & $\mathrm{pCi} / \mathrm{g}$ & $G$ & 0.27 \\
\hline 383DH2000.5 & $6 / 7 / 2004$ & soil & HASL300 & REG & 14331-83-0 & Ac-228 & 1.47 & $\mathrm{pCi} / \mathrm{g}$ & $G$ & 0.59 \\
\hline 383DH2000.5 & $6 / 7 / 2004$ & soil & EPA8260 & REG & 67-64-1 & Acetone & 6.6 & $\mu \mathrm{g} / \mathrm{kg}$ & $\mathrm{J}$ & 21 \\
\hline 383DH2000.5 & $6 / 7 / 2004$ & soil & EPA6010 & REG & $7440-38-2$ & Arsenic & 3.3 & $\mathrm{mg} / \mathrm{kg}$ & & 1 \\
\hline 383DH2000.5 & $6 / 7 / 2004$ & soil & EPA6010 & REG & $7440-39-3$ & Barium & 5300 & $\mathrm{mg} / \mathrm{kg}$ & & 100 \\
\hline 383DH2000.5 & $6 / 7 / 2004$ & soil & EPA6010 & REG & 7440-41-7 & Beryllium & 1.3 & $\mathrm{mg} / \mathrm{kg}$ & & 0.52 \\
\hline 383DH2000.5 & $6 / 7 / 2004$ & soil & HASL300 & REG & $14733-03-0$ & Bi-214 & 1.1 & $\mathrm{pCi} / \mathrm{g}$ & $\mathrm{G}, \mathrm{J}$ & 0.38 \\
\hline 383DH2000.5 & $6 / 7 / 2004$ & soil & EPA6010 & REG & $7440-43-9$ & Cadmium & 0.3 & $\mathrm{mg} / \mathrm{kg}$ & $\mathrm{B}$ & 0.52 \\
\hline 383DH2000.5 & $6 / 7 / 2004$ & soil & EPA6010 & REG & $7440-47-3$ & Chromium & 4.5 & $\mathrm{mg} / \mathrm{kg}$ & & 1 \\
\hline 383DH2000.5 & $6 / 7 / 2004$ & soil & HASL300 & REG & $10045-97-3$ & Cs-137 & 24.1 & $\mathrm{pCi} / \mathrm{g}$ & G & 0.2 \\
\hline 383DH2000.5 & $6 / 7 / 2004$ & soil & HASL300 & REG & 13966-00-2 & $\mathrm{K}-40$ & 22.8 & $\mathrm{pCi} / \mathrm{g}$ & G & 1.4 \\
\hline 383DH2000.5 & $6 / 7 / 2004$ & soil & EPA6010 & REG & 7439-92-1 & Lead & 22 & $\mathrm{mg} / \mathrm{kg}$ & & 0.31 \\
\hline 383DH2000.5 & $6 / 7 / 2004$ & soil & EPA7470 & REG & $7439-97-6$ & Mercury & 0.031 & $\mathrm{mg} / \mathrm{kg}$ & $\mathrm{B}$ & 0.1 \\
\hline 383DH2000.5 & $6 / 7 / 2004$ & soil & HASL300 & REG & 15092-94-1 & $\mathrm{Pb}-212$ & 1.91 & $\mathrm{pCi} / \mathrm{g}$ & $\mathrm{J}$ & 0.33 \\
\hline 383DH2000.5 & $6 / 7 / 2004$ & soil & HASL300 & REG & $15067-28-4$ & $\mathrm{~Pb}-214$ & 1.16 & $\mathrm{pCi} / \mathrm{g}$ & $\mathrm{G}, \mathrm{J}$ & 0.43 \\
\hline 383DH2000.5 & $6 / 7 / 2004$ & soil & UGTAISOPU & REG & 13981-16-3 & Pu-238 & 0.165 & $\mathrm{pCi} / \mathrm{g}$ & & 0.02 \\
\hline 383DH2000.5 & $6 / 7 / 2004$ & soil & UGTAISOPU & REG & $15117-48-3$ & Pu-239 & 1.44 & $\mathrm{pCi} / \mathrm{g}$ & & 0.01 \\
\hline 383DH2000.5 & $6 / 7 / 2004$ & soil & SR7500 & REG & 10098-97-2 & Sr-90 & 0.68 & $\mathrm{pCi} / \mathrm{g}$ & Y1 & 0.22 \\
\hline 383DH2000.5 & $6 / 7 / 2004$ & soil & HASL300 & REG & $14913-50-9$ & TI-208 & 0.47 & $\mathrm{pCi} / \mathrm{g}$ & $G$ & 0.24 \\
\hline 383DH2100.5 & $6 / 15 / 2004$ & soil & HASL300 & REG & $14331-83-0$ & Ac-228 & 1.68 & $\mathrm{pCi} / \mathrm{g}$ & G & 0.65 \\
\hline 383DH2100.5 & $6 / 15 / 2004$ & soil & EPA8260 & REG & 67-64-1 & Acetone & 12 & $\mu \mathrm{g} / \mathrm{kg}$ & $\mathrm{J}$ & 21 \\
\hline 383DH2100.5 & $6 / 15 / 2004$ & soil & EPA6010 & REG & $7440-38-2$ & Arsenic & 3.3 & $\mathrm{mg} / \mathrm{kg}$ & & 1 \\
\hline 383DH2100.5 & $6 / 15 / 2004$ & soil & EPA6010 & REG & $7440-39-3$ & Barium & 5100 & $\mathrm{mg} / \mathrm{kg}$ & & 520 \\
\hline 383DH2100.5 & $6 / 15 / 2004$ & soil & EPA6010 & REG & $7440-41-7$ & Beryllium & 1.6 & $\mathrm{mg} / \mathrm{kg}$ & & 0.52 \\
\hline 383DH2100.5 & $6 / 15 / 2004$ & soil & HASL300 & REG & $14733-03-0$ & $\mathrm{Bi}-214$ & 1.37 & $\mathrm{pCi} / \mathrm{g}$ & $\mathrm{G}, \mathrm{J}$ & 0.6 \\
\hline $383 \mathrm{DH} 2100.5$ & $6 / 15 / 2004$ & soil & EPA8270 & REG & $117-81-7$ & Bis(2-Ethylhexyl)Phthalate & 140 & $\mu \mathrm{g} / \mathrm{kg}$ & $\mathrm{J}$ & 350 \\
\hline $383 \mathrm{DH} 2100.5$ & $6 / 15 / 2004$ & soil & EPA6010 & REG & $7440-43-9$ & Cadmium & 0.27 & $\mathrm{mg} / \mathrm{kg}$ & $\mathrm{B}$ & 0.52 \\
\hline 383DH2100.5 & $6 / 15 / 2004$ & soil & EPA6010 & REG & $7440-47-3$ & Chromium & 4.7 & $\mathrm{mg} / \mathrm{kg}$ & & 1 \\
\hline $383 \mathrm{DH} 2100.5$ & $6 / 15 / 2004$ & soil & HASL300 & REG & $10045-97-3$ & Cs-137 & 78.1 & $\mathrm{pCi} / \mathrm{g}$ & G & 0.3 \\
\hline 383DH2100.5 & $6 / 15 / 2004$ & soil & HASL300 & REG & $13966-00-2$ & $\mathrm{~K}-40$ & 22.9 & $\mathrm{pCi} / \mathrm{g}$ & G & 2.1 \\
\hline $383 \mathrm{DH} 2100.5$ & $6 / 15 / 2004$ & soil & EPA6010 & REG & 7439-92-1 & Lead & 30 & $\mathrm{mg} / \mathrm{kg}$ & & 0.31 \\
\hline 383DH2100.5 & $6 / 15 / 2004$ & soil & EPA7470 & REG & 7439-97-6 & Mercury & 0.049 & $\mathrm{mg} / \mathrm{kg}$ & $\mathrm{B}$ & 0.1 \\
\hline
\end{tabular}

Page 14 of 78

Uncontrolled When Printed 


\begin{tabular}{|c|c|c|c|c|c|c|c|c|c|c|}
\hline SAMPLE \# & $\begin{array}{c}\text { SAMPLE } \\
\text { DATE }\end{array}$ & MATRIX & $\begin{array}{l}\text { USER TEST } \\
\text { PANEL }\end{array}$ & $\begin{array}{c}\text { SAMPLE } \\
\text { PURPOSE }\end{array}$ & CAS \# & PARAMETER & RESULT & UNITS & $\mathbf{Q}$ & $\begin{array}{c}\text { DETECT } \\
\text { LIMIT }\end{array}$ \\
\hline 383DH2100.5 & $6 / 15 / 2004$ & soil & EPA8260 & REG & $75-09-2$ & Methylene Chloride & 1.2 & $\mu \mathrm{g} / \mathrm{kg}$ & $\mathrm{J}, \mathrm{B}$ & 5.2 \\
\hline 383DH2100.5 & $6 / 15 / 2004$ & soil & HASL300 & REG & 15092-94-1 & $\mathrm{Pb}-212$ & 2.16 & $\mathrm{pCi} / \mathrm{g}$ & $\mathrm{J}$ & 0.56 \\
\hline 383DH2100.5 & $6 / 15 / 2004$ & soil & HASL300 & REG & $15067-28-4$ & $\mathrm{~Pb}-214$ & 1.26 & $\mathrm{pCi} / \mathrm{g}$ & $\mathrm{G}, \mathrm{J}$ & 0.66 \\
\hline 383DH2100.5 & $6 / 15 / 2004$ & soil & UGTAISOPU & REG & 13981-16-3 & Pu-238 & 0.341 & $\mathrm{pCi} / \mathrm{g}$ & & 0.021 \\
\hline 383DH2100.5 & $6 / 15 / 2004$ & soil & UGTAISOPU & REG & $15117-48-3$ & Pu-239 & 3.3 & $\mathrm{pCi} / \mathrm{g}$ & & 0.02 \\
\hline 383DH2100.5 & $6 / 15 / 2004$ & soil & EPA6010 & REG & $7782-49-2$ & Selenium & 1.1 & $\mathrm{mg} / \mathrm{kg}$ & & 0.52 \\
\hline 383DH2100.5 & $6 / 15 / 2004$ & soil & SR7500 & REG & $10098-97-2$ & Sr-90 & 1.17 & $\mathrm{pCi} / \mathrm{g}$ & & 0.34 \\
\hline 383DH2100.5 & $6 / 15 / 2004$ & soil & HASL300 & REG & 14913-50-9 & TI-208 & 0.69 & $\mathrm{pCi} / \mathrm{g}$ & G & 0.28 \\
\hline 383DH2200.5 & $6 / 15 / 2004$ & soil & HASL300 & REG & 14331-83-0 & Ac-228 & 1.37 & $\mathrm{pCi} / \mathrm{g}$ & $\mathrm{G}$ & 0.53 \\
\hline 383DH2200.5 & $6 / 15 / 2004$ & soil & EPA6010 & REG & $7440-38-2$ & Arsenic & 3.3 & $\mathrm{mg} / \mathrm{kg}$ & & 1 \\
\hline 383DH2200.5 & $6 / 15 / 2004$ & soil & EPA6010 & REG & $7440-39-3$ & Barium & 5300 & $\mathrm{mg} / \mathrm{kg}$ & & 510 \\
\hline 383DH2200.5 & $6 / 15 / 2004$ & soil & EPA8270 & REG & $56-55-3$ & Benzo(A)Anthracene & 26 & $\mu \mathrm{g} / \mathrm{kg}$ & $\mathrm{J}$ & 340 \\
\hline 383DH2200.5 & $6 / 15 / 2004$ & soil & EPA8270 & REG & $50-32-8$ & Benzo(A)Pyrene & 37 & $\mu \mathrm{g} / \mathrm{kg}$ & $\mathrm{J}$ & 340 \\
\hline 383DH2200.5 & $6 / 15 / 2004$ & soil & EPA8270 & REG & $205-99-2$ & Benzo(B)Fluoranthene & 51 & $\mu \mathrm{g} / \mathrm{kg}$ & $\mathrm{J}$ & 340 \\
\hline 383DH2200.5 & $6 / 15 / 2004$ & soil & EPA8270 & REG & 191-24-2 & Benzo(G,H,I)Perylene & 46 & $\mu \mathrm{g} / \mathrm{kg}$ & $\mathrm{J}$ & 340 \\
\hline 383DH2200.5 & $6 / 15 / 2004$ & soil & EPA8270 & REG & 207-08-9 & Benzo(K)Fluoranthene & 25 & $\mu \mathrm{g} / \mathrm{kg}$ & $\mathrm{J}$ & 340 \\
\hline 383DH2200.5 & $6 / 15 / 2004$ & soil & EPA6010 & REG & $7440-41-7$ & Beryllium & 1.4 & $\mathrm{mg} / \mathrm{kg}$ & & 0.51 \\
\hline 383DH2200.5 & $6 / 15 / 2004$ & soil & HASL300 & REG & 14733-03-0 & Bi-214 & 1.3 & $\mathrm{pCi} / \mathrm{g}$ & $\mathrm{G}, \mathrm{J}$ & 0.42 \\
\hline 383DH2200.5 & $6 / 15 / 2004$ & soil & EPA8270 & REG & 117-81-7 & Bis(2-Ethylhexyl)Phthalate & 2400 & $\mu \mathrm{g} / \mathrm{kg}$ & & 340 \\
\hline 383DH2200.5 & $6 / 15 / 2004$ & soil & EPA6010 & REG & $7440-43-9$ & Cadmium & 0.26 & $\mathrm{mg} / \mathrm{kg}$ & $B$ & 0.51 \\
\hline 383DH2200.5 & $6 / 15 / 2004$ & soil & EPA6010 & REG & $7440-47-3$ & Chromium & 5 & $\mathrm{mg} / \mathrm{kg}$ & & 1 \\
\hline 383DH2200.5 & $6 / 15 / 2004$ & soil & EPA8270 & REG & 218-01-9 & Chrysene & 100 & $\mu \mathrm{g} / \mathrm{kg}$ & $\mathrm{J}$ & 340 \\
\hline 383DH2200.5 & $6 / 15 / 2004$ & soil & HASL300 & REG & $10045-97-3$ & Cs-137 & 29.3 & $\mathrm{pCi} / \mathrm{g}$ & G & 0.2 \\
\hline $383 \mathrm{DH} 2200.5$ & $6 / 15 / 2004$ & soil & EPA8015 & REG & $68334-30-5$ & Diesel-Range Organics & 8.5 & $\mathrm{mg} / \mathrm{kg}$ & $\mathrm{Z}, \mathrm{H}$ & 5.1 \\
\hline 383DH2200.5 & $6 / 15 / 2004$ & soil & EPA8270 & REG & $131-11-3$ & Dimethyl Phthalate & 14 & $\mu \mathrm{g} / \mathrm{kg}$ & $\mathrm{J}$ & 340 \\
\hline 383DH2200.5 & $6 / 15 / 2004$ & soil & EPA8270 & REG & $117-84-0$ & Di-N-Octyl Phthalate & 59 & $\mu \mathrm{g} / \mathrm{kg}$ & $\mathrm{J}$ & 340 \\
\hline 383DH2200.5 & $6 / 15 / 2004$ & soil & EPA8270 & REG & $206-44-0$ & Fluoranthene & 23 & $\mu \mathrm{g} / \mathrm{kg}$ & $\mathrm{J}$ & 340 \\
\hline 383DH2200.5 & $6 / 15 / 2004$ & soil & HASL300 & REG & $13966-00-2$ & $\mathrm{~K}-40$ & 25.6 & $\mathrm{pCi} / \mathrm{g}$ & $\mathrm{G}$ & 1.4 \\
\hline 383DH2200.5 & $6 / 15 / 2004$ & soil & EPA6010 & REG & 7439-92-1 & Lead & 31 & $\mathrm{mg} / \mathrm{kg}$ & & 0.31 \\
\hline 383DH2200.5 & $6 / 15 / 2004$ & soil & EPA7470 & REG & $7439-97-6$ & Mercury & 0.038 & $\mathrm{mg} / \mathrm{kg}$ & $B$ & 0.1 \\
\hline 383DH2200.5 & $6 / 15 / 2004$ & soil & EPA8260 & REG & $75-09-2$ & Methylene Chloride & 1.1 & $\mu \mathrm{g} / \mathrm{kg}$ & $\mathrm{J}, \mathrm{B}$ & 5.1 \\
\hline 383DH2200.5 & $6 / 15 / 2004$ & soil & HASL300 & REG & 15092-94-1 & $\mathrm{Pb}-212$ & 2.09 & $\mathrm{pCi} / \mathrm{g}$ & $\mathrm{J}$ & 0.36 \\
\hline 383DH2200.5 & $6 / 15 / 2004$ & soil & HASL300 & REG & $15067-28-4$ & $\mathrm{~Pb}-214$ & 1.2 & $\mathrm{pCi} / \mathrm{g}$ & $\mathrm{G}, \mathrm{J}$ & 0.55 \\
\hline 383DH2200.5 & $6 / 15 / 2004$ & soil & UGTAISOPU & REG & 13981-16-3 & Pu-238 & 0.226 & $\mathrm{pCi} / \mathrm{g}$ & & 0.011 \\
\hline 383DH2200.5 & $6 / 15 / 2004$ & soil & UGTAISOPU & REG & $15117-48-3$ & Pu-239 & 1.96 & $\mathrm{pCi} / \mathrm{g}$ & & 0.03 \\
\hline
\end{tabular}

Page 15 of 78

Uncontrolled When Printed 


\begin{tabular}{|c|c|c|c|c|c|c|c|c|c|c|}
\hline SAMPLE \# & $\begin{array}{c}\text { SAMPLE } \\
\text { DATE }\end{array}$ & MATRIX & $\begin{array}{c}\text { USER TEST } \\
\text { PANEL }\end{array}$ & $\begin{array}{c}\text { SAMPLE } \\
\text { PURPOSE }\end{array}$ & CAS \# & PARAMETER & RESULT & UNITS & $\mathbf{Q}$ & $\begin{array}{c}\text { DETECT } \\
\text { LIMIT }\end{array}$ \\
\hline 383DH2200.5 & $6 / 15 / 2004$ & soil & EPA8270 & REG & $129-00-0$ & Pyrene & 41 & $\mu \mathrm{g} / \mathrm{kg}$ & $\mathrm{J}$ & 340 \\
\hline 383DH2200.5 & $6 / 15 / 2004$ & soil & EPA6010 & REG & $7782-49-2$ & Selenium & 0.4 & $\mathrm{mg} / \mathrm{kg}$ & $B$ & 0.51 \\
\hline 383DH2200.5 & $6 / 15 / 2004$ & soil & SR7500 & REG & $10098-97-2$ & Sr-90 & 0.82 & $\mathrm{pCi} / \mathrm{g}$ & & 0.22 \\
\hline 383DH2200.5 & $6 / 15 / 2004$ & soil & HASL300 & REG & $14913-50-9$ & TI-208 & 0.62 & $\mathrm{pCi} / \mathrm{g}$ & $\mathrm{G}$ & 0.21 \\
\hline 383DH2200.5X & $6 / 15 / 2004$ & soil & HASL300 & FD & $14331-83-0$ & Ac-228 & 1.52 & $\mathrm{pCi} / \mathrm{g}$ & $\mathrm{G}$ & 0.56 \\
\hline 383DH2200.5X & $6 / 15 / 2004$ & soil & EPA6010 & FD & $7440-38-2$ & Arsenic & 3.7 & $\mathrm{mg} / \mathrm{kg}$ & & 1 \\
\hline 383DH2200.5X & $6 / 15 / 2004$ & soil & EPA6010 & FD & 7440-39-3 & Barium & 5200 & $\mathrm{mg} / \mathrm{kg}$ & & 510 \\
\hline 383DH2200.5X & $6 / 15 / 2004$ & soil & EPA8270 & FD & $56-55-3$ & Benzo(A)Anthracene & 24 & $\mu \mathrm{g} / \mathrm{kg}$ & $\mathrm{J}$ & 340 \\
\hline 383DH2200.5X & $6 / 15 / 2004$ & soil & EPA8270 & FD & $50-32-8$ & Benzo(A)Pyrene & 40 & $\mu \mathrm{g} / \mathrm{kg}$ & $\mathrm{J}$ & 340 \\
\hline 383DH2200.5X & $6 / 15 / 2004$ & soil & EPA8270 & FD & 205-99-2 & Benzo(B)Fluoranthene & 39 & $\mu \mathrm{g} / \mathrm{kg}$ & $\mathrm{J}$ & 340 \\
\hline 383DH2200.5X & $6 / 15 / 2004$ & soil & EPA8270 & FD & $191-24-2$ & Benzo(G,H,I)Perylene & 39 & $\mu \mathrm{g} / \mathrm{kg}$ & $\mathrm{J}$ & 340 \\
\hline 383DH2200.5X & $6 / 15 / 2004$ & soil & EPA8270 & FD & 207-08-9 & Benzo(K)Fluoranthene & 29 & $\mu \mathrm{g} / \mathrm{kg}$ & $\mathrm{J}$ & 340 \\
\hline 383DH2200.5X & $6 / 15 / 2004$ & soil & EPA6010 & FD & $7440-41-7$ & Beryllium & 1.5 & $\mathrm{mg} / \mathrm{kg}$ & & 0.51 \\
\hline 383DH2200.5X & $6 / 15 / 2004$ & soil & HASL300 & FD & $14733-03-0$ & Bi-214 & 1.23 & $\mathrm{pCi} / \mathrm{g}$ & $\mathrm{G}, \mathrm{J}$ & 0.39 \\
\hline 383DH2200.5X & $6 / 15 / 2004$ & soil & EPA8270 & FD & $117-81-7$ & Bis(2-Ethylhexyl)Phthalate & 170 & $\mu \mathrm{g} / \mathrm{kg}$ & $\mathrm{J}$ & 340 \\
\hline 383DH2200.5X & $6 / 15 / 2004$ & soil & EPA6010 & FD & $7440-43-9$ & Cadmium & 0.28 & $\mathrm{mg} / \mathrm{kg}$ & $\mathrm{B}$ & 0.51 \\
\hline 383DH2200.5X & $6 / 15 / 2004$ & soil & EPA6010 & FD & $7440-47-3$ & Chromium & 5.2 & $\mathrm{mg} / \mathrm{kg}$ & & 1 \\
\hline 383DH2200.5X & $6 / 15 / 2004$ & soil & EPA8270 & FD & 218-01-9 & Chrysene & 95 & $\mu \mathrm{g} / \mathrm{kg}$ & $\mathrm{J}$ & 340 \\
\hline 383DH2200.5X & $6 / 15 / 2004$ & soil & HASL300 & FD & $10045-97-3$ & Cs-137 & 28 & $\mathrm{pCi} / \mathrm{g}$ & $\mathrm{G}$ & 0.2 \\
\hline 383DH2200.5X & $6 / 15 / 2004$ & soil & EPA8015 & FD & $68334-30-5$ & Diesel-Range Organics & 6.2 & $\mathrm{mg} / \mathrm{kg}$ & $\mathrm{Z}, \mathrm{H}$ & 5.1 \\
\hline 383DH2200.5X & $6 / 15 / 2004$ & soil & EPA8270 & FD & 206-44-0 & Fluoranthene & 23 & $\mu \mathrm{g} / \mathrm{kg}$ & $\mathrm{J}$ & 340 \\
\hline 383DH2200.5X & $6 / 15 / 2004$ & soil & HASL300 & FD & $13966-00-2$ & $\mathrm{~K}-40$ & 21.8 & $\mathrm{pCi} / \mathrm{g}$ & $\mathrm{G}$ & 1.8 \\
\hline 383DH2200.5X & $6 / 15 / 2004$ & soil & EPA6010 & FD & $7439-92-1$ & Lead & 31 & $\mathrm{mg} / \mathrm{kg}$ & & 0.31 \\
\hline 383DH2200.5X & $6 / 15 / 2004$ & soil & EPA7470 & FD & $7439-97-6$ & Mercury & 0.037 & $\mathrm{mg} / \mathrm{kg}$ & $\mathrm{B}$ & 0.1 \\
\hline 383DH2200.5X & $6 / 15 / 2004$ & soil & EPA8260 & FD & $75-09-2$ & Methylene Chloride & 0.87 & $\mu \mathrm{g} / \mathrm{kg}$ & $\mathrm{J}, \mathrm{B}$ & 5.1 \\
\hline 383DH2200.5X & $6 / 15 / 2004$ & soil & HASL300 & FD & 15092-94-1 & $\mathrm{Pb}-212$ & 1.86 & $\mathrm{pCi} / \mathrm{g}$ & $\mathrm{J}$ & 0.42 \\
\hline 383DH2200.5X & $6 / 15 / 2004$ & soil & HASL300 & FD & $15067-28-4$ & $\mathrm{~Pb}-214$ & 1.05 & $\mathrm{pCi} / \mathrm{g}$ & $\mathrm{G}, \mathrm{J}$ & 0.55 \\
\hline 383DH2200.5X & $6 / 15 / 2004$ & soil & UGTAISOPU & FD & 13981-16-3 & Pu-238 & 0.238 & $\mathrm{pCi} / \mathrm{g}$ & & 0.026 \\
\hline 383DH2200.5X & $6 / 15 / 2004$ & soil & UGTAISOPU & FD & 15117-48-3 & Pu-239 & 1.87 & $\mathrm{pCi} / \mathrm{g}$ & & 0.03 \\
\hline 383DH2200.5X & $6 / 15 / 2004$ & soil & EPA6010 & FD & $7782-49-2$ & Selenium & 0.43 & $\mathrm{mg} / \mathrm{kg}$ & $\mathrm{B}$ & 0.51 \\
\hline 383DH2200.5X & $6 / 15 / 2004$ & soil & SR7500 & FD & 10098-97-2 & Sr-90 & 0.76 & $\mathrm{pCi} / \mathrm{g}$ & & 0.22 \\
\hline 383DH2200.5X & $6 / 15 / 2004$ & soil & HASL300 & FD & $14913-50-9$ & TI-208 & 0.64 & $\mathrm{pCi} / \mathrm{g}$ & $\mathrm{G}$ & 0.19 \\
\hline 383DH2300.5 & $6 / 15 / 2004$ & soil & HASL300 & REG & $14331-83-0$ & Ac-228 & 1.55 & $\mathrm{pCi} / \mathrm{g}$ & G & 0.56 \\
\hline 383DH2300.5 & $6 / 15 / 2004$ & soil & EPA6010 & REG & $7440-38-2$ & Arsenic & 3.7 & $\mathrm{mg} / \mathrm{kg}$ & & 1 \\
\hline 383DH2300.5 & $6 / 15 / 2004$ & soil & EPA6010 & REG & $7440-39-3$ & Barium & 5400 & $\mathrm{mg} / \mathrm{kg}$ & & 510 \\
\hline
\end{tabular}

Page 16 of 78

Uncontrolled When Printed 


\begin{tabular}{|c|c|c|c|c|c|c|c|c|c|c|}
\hline SAMPLE \# & $\begin{array}{c}\text { SAMPLE } \\
\text { DATE }\end{array}$ & MATRIX & $\begin{array}{l}\text { USER TEST } \\
\text { PANEL }\end{array}$ & $\begin{array}{l}\text { SAMPLE } \\
\text { PURPOSE }\end{array}$ & CAS \# & PARAMETER & RESULT & UNITS & $\mathbf{Q}$ & $\begin{array}{c}\text { DETECT } \\
\text { LIMIT }\end{array}$ \\
\hline 383DH2300.5 & $6 / 15 / 2004$ & soil & EPA8270 & REG & $56-55-3$ & Benzo(A)Anthracene & 38 & $\mu \mathrm{g} / \mathrm{kg}$ & $\mathrm{J}$ & 340 \\
\hline 383DH2300.5 & $6 / 15 / 2004$ & soil & EPA8270 & REG & $50-32-8$ & Benzo(A)Pyrene & 38 & $\mu \mathrm{g} / \mathrm{kg}$ & $\mathrm{J}$ & 340 \\
\hline 383DH2300.5 & $6 / 15 / 2004$ & soil & EPA8270 & REG & $205-99-2$ & Benzo(B)Fluoranthene & 61 & $\mu \mathrm{g} / \mathrm{kg}$ & $\mathrm{J}$ & 340 \\
\hline 383DH2300.5 & $6 / 15 / 2004$ & soil & EPA8270 & REG & 207-08-9 & Benzo(K)Fluoranthene & 44 & $\mu \mathrm{g} / \mathrm{kg}$ & $\mathrm{J}$ & 340 \\
\hline 383DH2300.5 & $6 / 15 / 2004$ & soil & EPA6010 & REG & $7440-41-7$ & Beryllium & 1.3 & $\mathrm{mg} / \mathrm{kg}$ & & 0.51 \\
\hline 383DH2300.5 & $6 / 15 / 2004$ & soil & HASL300 & REG & $14733-03-0$ & Bi-214 & 0.92 & $\mathrm{pCi} / \mathrm{g}$ & $\mathrm{G}, \mathrm{J}$ & 0.41 \\
\hline 383DH2300.5 & $6 / 15 / 2004$ & soil & EPA8270 & REG & 117-81-7 & Bis(2-Ethylhexyl)Phthalate & 82 & $\mu \mathrm{g} / \mathrm{kg}$ & $\mathrm{J}$ & 340 \\
\hline 383DH2300.5 & $6 / 15 / 2004$ & soil & EPA6010 & REG & $7440-43-9$ & Cadmium & 0.2 & $\mathrm{mg} / \mathrm{kg}$ & $\mathrm{B}$ & 0.51 \\
\hline 383DH2300.5 & $6 / 15 / 2004$ & soil & EPA6010 & REG & $7440-47-3$ & Chromium & 5.6 & $\mathrm{mg} / \mathrm{kg}$ & & 1 \\
\hline 383DH2300.5 & $6 / 15 / 2004$ & soil & EPA8270 & REG & 218-01-9 & Chrysene & 55 & $\mu \mathrm{g} / \mathrm{kg}$ & $\mathrm{J}$ & 340 \\
\hline 383DH2300.5 & $6 / 15 / 2004$ & soil & HASL300 & REG & $10045-97-3$ & Cs-137 & 37.9 & $\mathrm{pCi} / \mathrm{g}$ & $\mathrm{G}$ & 0.2 \\
\hline 383DH2300.5 & $6 / 15 / 2004$ & soil & EPA8270 & REG & 206-44-0 & Fluoranthene & 33 & $\mu \mathrm{g} / \mathrm{kg}$ & $\mathrm{J}$ & 340 \\
\hline 383DH2300.5 & $6 / 15 / 2004$ & soil & HASL300 & REG & $13966-00-2$ & $\mathrm{~K}-40$ & 21 & $\mathrm{pCi} / \mathrm{g}$ & $\mathrm{G}$ & 1.7 \\
\hline 383DH2300.5 & $6 / 15 / 2004$ & soil & EPA6010 & REG & $7439-92-1$ & Lead & 30 & $\mathrm{mg} / \mathrm{kg}$ & & 0.31 \\
\hline 383DH2300.5 & $6 / 15 / 2004$ & soil & EPA7470 & REG & $7439-97-6$ & Mercury & 0.035 & $\mathrm{mg} / \mathrm{kg}$ & $B$ & 0.1 \\
\hline 383DH2300.5 & $6 / 15 / 2004$ & soil & EPA8260 & REG & $75-09-2$ & Methylene Chloride & 0.91 & $\mu \mathrm{g} / \mathrm{kg}$ & $\mathrm{J}, \mathrm{B}$ & 5.1 \\
\hline 383DH2300.5 & $6 / 15 / 2004$ & soil & HASL300 & REG & 15092-94-1 & $\mathrm{Pb}-212$ & 1.7 & $\mathrm{pCi} / \mathrm{g}$ & $\mathrm{J}$ & 0.38 \\
\hline $383 \mathrm{DH} 2300.5$ & $6 / 15 / 2004$ & soil & HASL300 & REG & $15067-28-4$ & $\mathrm{~Pb}-214$ & 1.19 & $\mathrm{pCi} / \mathrm{g}$ & $\mathrm{G}, \mathrm{J}$ & 0.49 \\
\hline 383DH2300.5 & $6 / 15 / 2004$ & soil & UGTAISOPU & REG & 13981-16-3 & Pu-238 & 0.238 & $\mathrm{pCi} / \mathrm{g}$ & & 0.023 \\
\hline 383DH2300.5 & $6 / 15 / 2004$ & soil & UGTAISOPU & REG & $15117-48-3$ & Pu-239 & 2.7 & $\mathrm{pCi} / \mathrm{g}$ & & 0.02 \\
\hline 383DH2300.5 & $6 / 15 / 2004$ & soil & EPA8270 & REG & $129-00-0$ & Pyrene & 42 & $\mu \mathrm{g} / \mathrm{kg}$ & $\mathrm{J}$ & 340 \\
\hline 383DH2300.5 & $6 / 15 / 2004$ & soil & EPA6010 & REG & $7782-49-2$ & Selenium & 0.7 & $\mathrm{mg} / \mathrm{kg}$ & & 0.51 \\
\hline 383DH2300.5 & $6 / 15 / 2004$ & soil & SR7500 & REG & $10098-97-2$ & Sr-90 & 1.65 & $\mathrm{pCi} / \mathrm{g}$ & & 0.28 \\
\hline 383DH2300.5 & $6 / 15 / 2004$ & soil & HASL300 & REG & $14913-50-9$ & TI-208 & 0.51 & $\mathrm{pCi} / \mathrm{g}$ & $\mathrm{G}$ & 0.2 \\
\hline 383DH2400.5 & $6 / 15 / 2004$ & soil & HASL300 & REG & 14331-83-0 & Ac-228 & 1.29 & $\mathrm{pCi} / \mathrm{g}$ & $\mathrm{G}$ & 0.48 \\
\hline 383DH2400.5 & $6 / 15 / 2004$ & soil & EPA6010 & REG & $7440-38-2$ & Arsenic & 3.3 & $\mathrm{mg} / \mathrm{kg}$ & & 1 \\
\hline 383DH2400.5 & $6 / 15 / 2004$ & soil & EPA6010 & REG & $7440-39-3$ & Barium & 5400 & $\mathrm{mg} / \mathrm{kg}$ & & 520 \\
\hline 383DH2400.5 & $6 / 15 / 2004$ & soil & EPA6010 & REG & $7440-41-7$ & Beryllium & 1.2 & $\mathrm{mg} / \mathrm{kg}$ & & 0.52 \\
\hline 383DH2400.5 & $6 / 15 / 2004$ & soil & HASL300 & REG & $14733-03-0$ & Bi-214 & 0.92 & $\mathrm{pCi} / \mathrm{g}$ & $\mathrm{G}, \mathrm{J}$ & 0.39 \\
\hline 383DH2400.5 & $6 / 15 / 2004$ & soil & EPA6010 & REG & $7440-43-9$ & Cadmium & 0.21 & $\mathrm{mg} / \mathrm{kg}$ & B & 0.52 \\
\hline 383DH2400.5 & $6 / 15 / 2004$ & soil & EPA6010 & REG & $7440-47-3$ & Chromium & 4.6 & $\mathrm{mg} / \mathrm{kg}$ & & 1 \\
\hline 383DH2400.5 & $6 / 15 / 2004$ & soil & HASL300 & REG & $10045-97-3$ & Cs-137 & 32 & $\mathrm{pCi} / \mathrm{g}$ & $\mathrm{G}$ & 0.2 \\
\hline 383DH2400.5 & $6 / 15 / 2004$ & soil & HASL300 & REG & 13966-00-2 & $\mathrm{K}-40$ & 20.1 & $\mathrm{pCi} / \mathrm{g}$ & $\mathrm{G}$ & 1.5 \\
\hline 383DH2400.5 & $6 / 15 / 2004$ & soil & EPA6010 & REG & 7439-92-1 & Lead & 22 & $\mathrm{mg} / \mathrm{kg}$ & & 0.31 \\
\hline 383DH2400.5 & $6 / 15 / 2004$ & soil & EPA7470 & REG & $7439-97-6$ & Mercury & 0.034 & $\mathrm{mg} / \mathrm{kg}$ & $\mathrm{B}$ & 0.1 \\
\hline
\end{tabular}

Page 17 of 78

Uncontrolled When Printed 


\begin{tabular}{|c|c|c|c|c|c|c|c|c|c|c|}
\hline SAMPLE \# & $\begin{array}{c}\text { SAMPLE } \\
\text { DATE }\end{array}$ & MATRIX & $\begin{array}{l}\text { USER TEST } \\
\text { PANEL }\end{array}$ & $\begin{array}{c}\text { SAMPLE } \\
\text { PURPOSE }\end{array}$ & CAS \# & PARAMETER & RESULT & UNITS & $\mathbf{Q}$ & $\begin{array}{l}\text { DETECT } \\
\text { LIMIT }\end{array}$ \\
\hline 383DH2400.5 & $6 / 15 / 2004$ & soil & HASL300 & REG & 15092-94-1 & $\mathrm{Pb}-212$ & 1.76 & $\mathrm{pCi} / \mathrm{g}$ & $\mathrm{J}$ & 0.34 \\
\hline 383DH2400.5 & $6 / 15 / 2004$ & soil & HASL300 & REG & $15067-28-4$ & $\mathrm{~Pb}-214$ & 1.29 & $\mathrm{pCi} / \mathrm{g}$ & $\mathrm{G}, \mathrm{J}$ & 0.62 \\
\hline 383DH2400.5 & $6 / 15 / 2004$ & soil & UGTAISOPU & REG & 13981-16-3 & Pu-238 & 0.316 & $\mathrm{pCi} / \mathrm{g}$ & & 0.01 \\
\hline $383 \mathrm{DH} 2400.5$ & $6 / 15 / 2004$ & soil & UGTAISOPU & REG & $15117-48-3$ & Pu-239 & 2.34 & $\mathrm{pCi} / \mathrm{g}$ & & 0.01 \\
\hline 383DH2400.5 & $6 / 15 / 2004$ & soil & SR7500 & REG & 10098-97-2 & Sr-90 & 1.73 & $\mathrm{pCi} / \mathrm{g}$ & & 0.26 \\
\hline 383DH2400.5 & $6 / 15 / 2004$ & soil & HASL300 & REG & $14913-50-9$ & TI-208 & 0.3 & $\mathrm{pCi} / \mathrm{g}$ & $\mathrm{G}$ & 0.17 \\
\hline 383DH2500.5 & $6 / 15 / 2004$ & soil & HASL300 & REG & 14331-83-0 & Ac-228 & 1.62 & $\mathrm{pCi} / \mathrm{g}$ & $G$ & 0.61 \\
\hline 383DH2500.5 & $6 / 15 / 2004$ & soil & EPA6010 & REG & $7440-38-2$ & Arsenic & 3.3 & $\mathrm{mg} / \mathrm{kg}$ & & 1 \\
\hline 383DH2500.5 & $6 / 15 / 2004$ & soil & EPA6010 & REG & $7440-39-3$ & Barium & 5100 & $\mathrm{mg} / \mathrm{kg}$ & & 520 \\
\hline 383DH2500.5 & $6 / 15 / 2004$ & soil & EPA6010 & REG & $7440-41-7$ & Beryllium & 1.5 & $\mathrm{mg} / \mathrm{kg}$ & & 0.52 \\
\hline 383DH2500.5 & $6 / 15 / 2004$ & soil & HASL300 & REG & $14733-03-0$ & Bi-214 & 1.19 & $\mathrm{pCi} / \mathrm{g}$ & $\mathrm{G}, \mathrm{J}$ & 0.48 \\
\hline 383DH2500.5 & $6 / 15 / 2004$ & soil & EPA6010 & REG & $7440-43-9$ & Cadmium & 0.17 & $\mathrm{mg} / \mathrm{kg}$ & $\mathrm{B}$ & 0.52 \\
\hline 383DH2500.5 & $6 / 15 / 2004$ & soil & EPA6010 & REG & $7440-47-3$ & Chromium & 4.8 & $\mathrm{mg} / \mathrm{kg}$ & & 1 \\
\hline 383DH2500.5 & $6 / 15 / 2004$ & soil & HASL300 & REG & $10045-97-3$ & Cs-137 & 55.6 & $\mathrm{pCi} / \mathrm{g}$ & G & 0.3 \\
\hline $383 \mathrm{DH} 2500.5$ & $6 / 15 / 2004$ & soil & HASL300 & REG & 13966-00-2 & $\mathrm{K}-40$ & 26.3 & $\mathrm{pCi} / \mathrm{g}$ & G & 1.8 \\
\hline $383 \mathrm{DH} 2500.5$ & $6 / 15 / 2004$ & soil & EPA6010 & REG & 7439-92-1 & Lead & 23 & $\mathrm{mg} / \mathrm{kg}$ & & 0.31 \\
\hline 383DH2500.5 & $6 / 15 / 2004$ & soil & EPA7470 & REG & 7439-97-6 & Mercury & 0.052 & $\mathrm{mg} / \mathrm{kg}$ & $\mathrm{B}$ & 0.1 \\
\hline 383DH2500.5 & $6 / 15 / 2004$ & soil & EPA8260 & REG & $75-09-2$ & Methylene Chloride & 0.9 & $\mu \mathrm{g} / \mathrm{kg}$ & $\mathrm{J}, \mathrm{B}$ & 5.2 \\
\hline 383DH2500.5 & $6 / 15 / 2004$ & soil & HASL300 & REG & 15092-94-1 & $\mathrm{Pb}-212$ & 1.78 & $\mathrm{pCi} / \mathrm{g}$ & $\mathrm{J}$ & 0.44 \\
\hline $383 \mathrm{DH} 2500.5$ & $6 / 15 / 2004$ & soil & HASL300 & REG & $15067-28-4$ & $\mathrm{~Pb}-214$ & 1.28 & $\mathrm{pCi} / \mathrm{g}$ & $\mathrm{G}, \mathrm{J}$ & 0.73 \\
\hline $383 \mathrm{DH} 2500.5$ & $6 / 15 / 2004$ & soil & UGTAISOPU & REG & 13981-16-3 & Pu-238 & 0.265 & $\mathrm{pCi} / \mathrm{g}$ & & 0.021 \\
\hline 383DH2500.5 & $6 / 15 / 2004$ & soil & UGTAISOPU & REG & $15117-48-3$ & Pu-239 & 3.1 & $\mathrm{pCi} / \mathrm{g}$ & & 0.02 \\
\hline $383 \mathrm{DH} 2500.5$ & $6 / 15 / 2004$ & soil & EPA6010 & REG & $7782-49-2$ & Selenium & 0.41 & $\mathrm{mg} / \mathrm{kg}$ & $\mathrm{B}$ & 0.52 \\
\hline $383 \mathrm{DH} 2500.5$ & $6 / 15 / 2004$ & soil & SR7500 & REG & $10098-97-2$ & Sr-90 & 1.09 & $\mathrm{pCi} / \mathrm{g}$ & & 0.25 \\
\hline 383DH2500.5 & $6 / 15 / 2004$ & soil & HASL300 & REG & $14913-50-9$ & TI-208 & 0.57 & $\mathrm{pCi} / \mathrm{g}$ & G & 0.3 \\
\hline $383 \mathrm{DH} 2600.5$ & $6 / 15 / 2004$ & soil & HASL300 & REG & 14331-83-0 & Ac-228 & 1.52 & $\mathrm{pCi} / \mathrm{g}$ & $G$ & 0.61 \\
\hline $383 \mathrm{DH} 2600.5$ & $6 / 15 / 2004$ & soil & EPA6010 & REG & $7440-38-2$ & Arsenic & 3.4 & $\mathrm{mg} / \mathrm{kg}$ & & 1 \\
\hline $383 \mathrm{DH} 2600.5$ & $6 / 15 / 2004$ & soil & EPA6010 & REG & $7440-39-3$ & Barium & 6100 & $\mathrm{mg} / \mathrm{kg}$ & & 520 \\
\hline $383 \mathrm{DH} 2600.5$ & $6 / 15 / 2004$ & soil & EPA8270 & REG & $56-55-3$ & Benzo(A)Anthracene & 66 & $\mu \mathrm{g} / \mathrm{kg}$ & $\mathrm{J}$ & 350 \\
\hline $383 \mathrm{DH} 2600.5$ & $6 / 15 / 2004$ & soil & EPA8270 & REG & $50-32-8$ & Benzo(A)Pyrene & 64 & $\mu \mathrm{g} / \mathrm{kg}$ & $\mathrm{J}$ & 350 \\
\hline 383DH2600.5 & $6 / 15 / 2004$ & soil & EPA8270 & REG & 205-99-2 & Benzo(B)Fluoranthene & 62 & $\mu \mathrm{g} / \mathrm{kg}$ & $\mathrm{J}$ & 350 \\
\hline $383 \mathrm{DH} 2600.5$ & $6 / 15 / 2004$ & soil & EPA8270 & REG & 207-08-9 & Benzo(K)Fluoranthene & 57 & $\mu \mathrm{g} / \mathrm{kg}$ & $\mathrm{J}$ & 350 \\
\hline 383DH2600.5 & $6 / 15 / 2004$ & soil & EPA6010 & REG & $7440-41-7$ & Beryllium & 1.5 & $\mathrm{mg} / \mathrm{kg}$ & & 0.52 \\
\hline 383DH2600.5 & $6 / 15 / 2004$ & soil & HASL300 & REG & $14733-03-0$ & Bi-214 & 1.13 & $\mathrm{pCi} / \mathrm{g}$ & $\mathrm{G}, \mathrm{J}$ & 0.47 \\
\hline 383DH2600.5 & $6 / 15 / 2004$ & soil & EPA6010 & REG & 7440-43-9 & Cadmium & 0.25 & $\mathrm{mg} / \mathrm{kg}$ & $\mathrm{B}$ & 0.52 \\
\hline
\end{tabular}

Page 18 of 78

Uncontrolled When Printed 


\begin{tabular}{|c|c|c|c|c|c|c|c|c|c|c|}
\hline SAMPLE \# & $\begin{array}{c}\text { SAMPLE } \\
\text { DATE }\end{array}$ & MATRIX & $\begin{array}{l}\text { USER TEST } \\
\text { PANEL }\end{array}$ & $\begin{array}{c}\text { SAMPLE } \\
\text { PURPOSE }\end{array}$ & CAS \# & PARAMETER & RESULT & UNITS & $\mathbf{Q}$ & $\begin{array}{c}\text { DETECT } \\
\text { LIMIT }\end{array}$ \\
\hline 383DH2600.5 & $6 / 15 / 2004$ & soil & EPA6010 & REG & $7440-47-3$ & Chromium & 5 & $\mathrm{mg} / \mathrm{kg}$ & & 1 \\
\hline 383DH2600.5 & $6 / 15 / 2004$ & soil & EPA8270 & REG & 218-01-9 & Chrysene & 82 & $\mu \mathrm{g} / \mathrm{kg}$ & $\mathrm{J}$ & 350 \\
\hline 383DH2600.5 & $6 / 15 / 2004$ & soil & HASL300 & REG & $10045-97-3$ & Cs-137 & 28.4 & $\mathrm{pCi} / \mathrm{g}$ & $\mathrm{G}$ & 0.2 \\
\hline 383DH2600.5 & $6 / 15 / 2004$ & soil & EPA8015 & REG & $68334-30-5$ & Diesel-Range Organics & 2.5 & $\mathrm{mg} / \mathrm{kg}$ & $\mathrm{J}$ & 5.2 \\
\hline 383DH2600.5 & $6 / 15 / 2004$ & soil & EPA8270 & REG & 206-44-0 & Fluoranthene & 120 & $\mu \mathrm{g} / \mathrm{kg}$ & $\mathrm{J}$ & 350 \\
\hline 383DH2600.5 & $6 / 15 / 2004$ & soil & HASL300 & REG & $13966-00-2$ & $\mathrm{~K}-40$ & 24.8 & $\mathrm{pCi} / \mathrm{g}$ & $\mathrm{G}$ & 1.5 \\
\hline 383DH2600.5 & $6 / 15 / 2004$ & soil & EPA6010 & REG & 7439-92-1 & Lead & 28 & $\mathrm{mg} / \mathrm{kg}$ & & 0.31 \\
\hline 383DH2600.5 & $6 / 15 / 2004$ & soil & EPA7470 & REG & 7439-97-6 & Mercury & 0.038 & $\mathrm{mg} / \mathrm{kg}$ & $B$ & 0.1 \\
\hline 383DH2600.5 & $6 / 15 / 2004$ & soil & EPA8260 & REG & $75-09-2$ & Methylene Chloride & 0.99 & $\mu \mathrm{g} / \mathrm{kg}$ & $\mathrm{J}, \mathrm{B}$ & 5.2 \\
\hline 383DH2600.5 & $6 / 15 / 2004$ & soil & HASL300 & REG & 15092-94-1 & $\mathrm{Pb}-212$ & 2.01 & $\mathrm{pCi} / \mathrm{g}$ & J & 0.34 \\
\hline 383DH2600.5 & $6 / 15 / 2004$ & soil & HASL300 & REG & $15067-28-4$ & $\mathrm{~Pb}-214$ & 1.4 & $\mathrm{pCi} / \mathrm{g}$ & $\mathrm{G}, \mathrm{J}$ & 0.49 \\
\hline 383DH2600.5 & $6 / 15 / 2004$ & soil & EPA8270 & REG & 85-01-8 & Phenanthrene & 49 & $\mu \mathrm{g} / \mathrm{kg}$ & $\mathrm{J}$ & 350 \\
\hline 383DH2600.5 & $6 / 15 / 2004$ & soil & UGTAISOPU & REG & 13981-16-3 & Pu-238 & 0.188 & $\mathrm{pCi} / \mathrm{g}$ & & 0.021 \\
\hline 383DH2600.5 & $6 / 15 / 2004$ & soil & UGTAISOPU & REG & $15117-48-3$ & Pu-239 & 1.82 & $\mathrm{pCi} / \mathrm{g}$ & & 0.01 \\
\hline 383DH2600.5 & $6 / 15 / 2004$ & soil & EPA8270 & REG & $129-00-0$ & Pyrene & 190 & $\mu \mathrm{g} / \mathrm{kg}$ & $\mathrm{J}$ & 350 \\
\hline 383DH2600.5 & $6 / 15 / 2004$ & soil & SR7500 & REG & 10098-97-2 & Sr-90 & 0.93 & $\mathrm{pCi} / \mathrm{g}$ & & 0.25 \\
\hline 383DH2600.5 & $6 / 15 / 2004$ & soil & HASL300 & REG & 14913-50-9 & TI-208 & 0.46 & $\mathrm{pCi} / \mathrm{g}$ & G & 0.19 \\
\hline 383DH2700.5 & $6 / 16 / 2004$ & soil & HASL300 & REG & 14331-83-0 & Ac-228 & 1.66 & $\mathrm{pCi} / \mathrm{g}$ & $\mathrm{G}$ & 0.67 \\
\hline 383DH2700.5 & $6 / 16 / 2004$ & soil & EPA6010 & REG & $7440-38-2$ & Arsenic & 3.4 & $\mathrm{mg} / \mathrm{kg}$ & & 1 \\
\hline 383DH2700.5 & $6 / 16 / 2004$ & soil & EPA6010 & REG & $7440-39-3$ & Barium & 5600 & $\mathrm{mg} / \mathrm{kg}$ & & 520 \\
\hline 383DH2700.5 & $6 / 16 / 2004$ & soil & EPA6010 & REG & $7440-41-7$ & Beryllium & 1.4 & $\mathrm{mg} / \mathrm{kg}$ & & 0.52 \\
\hline 383DH2700.5 & $6 / 16 / 2004$ & soil & HASL300 & REG & 14733-03-0 & Bi-214 & 1.43 & $\mathrm{pCi} / \mathrm{g}$ & $\mathrm{G}, \mathrm{J}$ & 0.36 \\
\hline 383DH2700.5 & $6 / 16 / 2004$ & soil & EPA8270 & REG & 117-81-7 & Bis(2-Ethylhexyl)Phthalate & 100 & $\mu \mathrm{g} / \mathrm{kg}$ & $\mathrm{J}$ & 350 \\
\hline 383DH2700.5 & $6 / 16 / 2004$ & soil & EPA6010 & REG & $7440-43-9$ & Cadmium & 0.2 & $\mathrm{mg} / \mathrm{kg}$ & B & 0.52 \\
\hline 383DH2700.5 & $6 / 16 / 2004$ & soil & EPA6010 & REG & $7440-47-3$ & Chromium & 5.3 & $\mathrm{mg} / \mathrm{kg}$ & & 1 \\
\hline 383DH2700.5 & $6 / 16 / 2004$ & soil & HASL300 & REG & $10045-97-3$ & Cs-137 & 41.7 & $\mathrm{pCi} / \mathrm{g}$ & $\mathrm{G}$ & 0.2 \\
\hline 383DH2700.5 & $6 / 16 / 2004$ & soil & EPA8015 & REG & $68334-30-5$ & Diesel-Range Organics & 1.8 & $\mathrm{mg} / \mathrm{kg}$ & J & 5.2 \\
\hline $383 \mathrm{DH} 2700.5$ & $6 / 16 / 2004$ & soil & EPA8260 & REG & 87-68-3 & Hexachlorobutadiene & 1.6 & $\mu \mathrm{g} / \mathrm{kg}$ & $\mathrm{J}$ & 5.2 \\
\hline 383DH2700.5 & $6 / 16 / 2004$ & soil & HASL300 & REG & $13966-00-2$ & $\mathrm{~K}-40$ & 20.9 & $\mathrm{pCi} / \mathrm{g}$ & $\mathrm{G}$ & 1.7 \\
\hline 383DH2700.5 & $6 / 16 / 2004$ & soil & EPA6010 & REG & 7439-92-1 & Lead & 25 & $\mathrm{mg} / \mathrm{kg}$ & & 0.31 \\
\hline 383DH2700.5 & $6 / 16 / 2004$ & soil & EPA7470 & REG & $7439-97-6$ & Mercury & 0.043 & $\mathrm{mg} / \mathrm{kg}$ & $B$ & 0.1 \\
\hline 383DH2700.5 & $6 / 16 / 2004$ & soil & EPA8260 & REG & $75-09-2$ & Methylene Chloride & 1.1 & $\mu \mathrm{g} / \mathrm{kg}$ & $\mathrm{J}, \mathrm{B}$ & 5.2 \\
\hline 383DH2700.5 & $6 / 16 / 2004$ & soil & HASL300 & REG & 15092-94-1 & $\mathrm{Pb}-212$ & 1.58 & $\mathrm{pCi} / \mathrm{g}$ & $\mathrm{J}$ & 0.38 \\
\hline 383DH2700.5 & $6 / 16 / 2004$ & soil & HASL300 & REG & $15067-28-4$ & $\mathrm{~Pb}-214$ & 1.15 & $\mathrm{pCi} / \mathrm{g}$ & $\mathrm{G}, \mathrm{J}$ & 0.48 \\
\hline 383DH2700.5 & $6 / 16 / 2004$ & soil & UGTAISOPU & REG & 13981-16-3 & $\mathrm{Pu}-238$ & 0.271 & $\mathrm{pCi} / \mathrm{g}$ & & 0.019 \\
\hline
\end{tabular}

Page 19 of 78

Uncontrolled When Printed 


\begin{tabular}{|c|c|c|c|c|c|c|c|c|c|c|}
\hline SAMPLE \# & $\begin{array}{c}\text { SAMPLE } \\
\text { DATE }\end{array}$ & MATRIX & $\begin{array}{l}\text { USER TEST } \\
\text { PANEL }\end{array}$ & $\begin{array}{c}\text { SAMPLE } \\
\text { PURPOSE }\end{array}$ & CAS \# & PARAMETER & RESULT & UNITS & $\mathbf{Q}$ & $\begin{array}{c}\text { DETECT } \\
\text { LIMIT }\end{array}$ \\
\hline 383DH2700.5 & $6 / 16 / 2004$ & soil & UGTAISOPU & REG & $15117-48-3$ & Pu-239 & 3.31 & $\mathrm{pCi} / \mathrm{g}$ & & 0.02 \\
\hline 383DH2700.5 & $6 / 16 / 2004$ & soil & EPA6010 & REG & $7782-49-2$ & Selenium & 0.43 & $\mathrm{mg} / \mathrm{kg}$ & $B$ & 0.52 \\
\hline 383DH2700.5 & $6 / 16 / 2004$ & soil & SR7500 & REG & 10098-97-2 & Sr-90 & 1.55 & $\mathrm{pCi} / \mathrm{g}$ & & 0.25 \\
\hline 383DH2700.5 & $6 / 16 / 2004$ & soil & HASL300 & REG & $14913-50-9$ & TI-208 & 0.51 & $\mathrm{pCi} / \mathrm{g}$ & $G$ & 0.2 \\
\hline 383DH2800.5 & $6 / 16 / 2004$ & soil & HASL300 & REG & 14331-83-0 & Ac-228 & 1.72 & $\mathrm{pCi} / \mathrm{g}$ & G & 0.62 \\
\hline 383DH2800.5 & $6 / 16 / 2004$ & soil & EPA6010 & REG & $7440-38-2$ & Arsenic & 2.8 & $\mathrm{mg} / \mathrm{kg}$ & & 1 \\
\hline 383DH2800.5 & $6 / 16 / 2004$ & soil & EPA6010 & REG & $7440-39-3$ & Barium & 5300 & $\mathrm{mg} / \mathrm{kg}$ & & 520 \\
\hline 383DH2800.5 & $6 / 16 / 2004$ & soil & EPA6010 & REG & 7440-41-7 & Beryllium & 1.7 & $\mathrm{mg} / \mathrm{kg}$ & & 0.52 \\
\hline 383DH2800.5 & $6 / 16 / 2004$ & soil & HASL300 & REG & $14733-03-0$ & Bi-214 & 1.26 & $\mathrm{pCi} / \mathrm{g}$ & $\mathrm{G}, \mathrm{J}$ & 0.36 \\
\hline 383DH2800.5 & $6 / 16 / 2004$ & soil & EPA6010 & REG & $7440-43-9$ & Cadmium & 0.16 & $\mathrm{mg} / \mathrm{kg}$ & $B$ & 0.52 \\
\hline 383DH2800.5 & $6 / 16 / 2004$ & soil & EPA6010 & REG & $7440-47-3$ & Chromium & 4 & $\mathrm{mg} / \mathrm{kg}$ & & 1 \\
\hline 383DH2800.5 & $6 / 16 / 2004$ & soil & HASL300 & REG & $10045-97-3$ & Cs-137 & 14 & $\mathrm{pCi} / \mathrm{g}$ & $\mathrm{G}$ & 0.2 \\
\hline 383DH2800.5 & $6 / 16 / 2004$ & soil & HASL300 & REG & $13966-00-2$ & $\mathrm{~K}-40$ & 27.7 & $\mathrm{pCi} / \mathrm{g}$ & $\mathrm{G}$ & 1.8 \\
\hline 383DH2800.5 & $6 / 16 / 2004$ & soil & EPA6010 & REG & 7439-92-1 & Lead & 27 & $\mathrm{mg} / \mathrm{kg}$ & & 0.31 \\
\hline 383DH2800.5 & $6 / 16 / 2004$ & soil & EPA7470 & REG & $7439-97-6$ & Mercury & 0.023 & $\mathrm{mg} / \mathrm{kg}$ & $B$ & 0.1 \\
\hline 383DH2800.5 & $6 / 16 / 2004$ & soil & EPA8260 & REG & $75-09-2$ & Methylene Chloride & 1.4 & $\mu \mathrm{g} / \mathrm{kg}$ & $\mathrm{J}, \mathrm{B}$ & 5.2 \\
\hline 383DH2800.5 & $6 / 16 / 2004$ & soil & HASL300 & REG & 15092-94-1 & $\mathrm{Pb}-212$ & 2.19 & $\mathrm{pCi} / \mathrm{g}$ & $\mathrm{J}$ & 0.28 \\
\hline 383DH2800.5 & $6 / 16 / 2004$ & soil & HASL300 & REG & $15067-28-4$ & $\mathrm{~Pb}-214$ & 1.4 & $\mathrm{pCi} / \mathrm{g}$ & $\mathrm{G}, \mathrm{J}$ & 0.47 \\
\hline 383DH2800.5 & $6 / 16 / 2004$ & soil & UGTAISOPU & REG & 13981-16-3 & Pu-238 & 0.068 & $\mathrm{pCi} / \mathrm{g}$ & & 0.021 \\
\hline 383DH2800.5 & $6 / 16 / 2004$ & soil & UGTAISOPU & REG & $15117-48-3$ & Pu-239 & 0.93 & $\mathrm{pCi} / \mathrm{g}$ & & 0.01 \\
\hline 383DH2800.5 & $6 / 16 / 2004$ & soil & SR7500 & REG & 10098-97-2 & Sr-90 & 0.45 & $\mathrm{pCi} / \mathrm{g}$ & Y1,L7 & 0.22 \\
\hline 383DH2800.5 & $6 / 16 / 2004$ & soil & HASL300 & REG & 14913-50-9 & TI-208 & 0.67 & $\mathrm{pCi} / \mathrm{g}$ & $\mathrm{G}$ & 0.18 \\
\hline 383DH2900.5 & $6 / 16 / 2004$ & soil & HASL300 & REG & 14331-83-0 & Ac-228 & 1.59 & $\mathrm{pCi} / \mathrm{g}$ & $\mathrm{G}$ & 0.5 \\
\hline 383DH2900.5 & $6 / 16 / 2004$ & soil & EPA8260 & REG & 67-64-1 & Acetone & 7.1 & $\mu \mathrm{g} / \mathrm{kg}$ & $\mathrm{J}$ & 21 \\
\hline 383DH2900.5 & $6 / 16 / 2004$ & soil & EPA6010 & REG & 7440-38-2 & Arsenic & 3.5 & $\mathrm{mg} / \mathrm{kg}$ & & 1 \\
\hline 383DH2900.5 & $6 / 16 / 2004$ & soil & EPA6010 & REG & $7440-39-3$ & Barium & 5900 & $\mathrm{mg} / \mathrm{kg}$ & & 520 \\
\hline 383DH2900.5 & $6 / 16 / 2004$ & soil & EPA6010 & REG & $7440-41-7$ & Beryllium & 1.3 & $\mathrm{mg} / \mathrm{kg}$ & & 0.52 \\
\hline 383DH2900.5 & $6 / 16 / 2004$ & soil & HASL300 & REG & $14733-03-0$ & Bi-214 & 1.12 & $\mathrm{pCi} / \mathrm{g}$ & $\mathrm{G}, \mathrm{J}$ & 0.47 \\
\hline 383DH2900.5 & $6 / 16 / 2004$ & soil & EPA6010 & REG & $7440-43-9$ & Cadmium & 0.2 & $\mathrm{mg} / \mathrm{kg}$ & $B$ & 0.52 \\
\hline 383DH2900.5 & $6 / 16 / 2004$ & soil & EPA6010 & REG & $7440-47-3$ & Chromium & 5.6 & $\mathrm{mg} / \mathrm{kg}$ & & 1 \\
\hline 383DH2900.5 & $6 / 16 / 2004$ & soil & HASL300 & REG & $10045-97-3$ & Cs-137 & 33.1 & $\mathrm{pCi} / \mathrm{g}$ & $\mathrm{G}$ & 0.2 \\
\hline 383DH2900.5 & $6 / 16 / 2004$ & soil & HASL300 & REG & 13966-00-2 & $\mathrm{K}-40$ & 22.1 & $\mathrm{pCi} / \mathrm{g}$ & $\mathrm{G}$ & 2.1 \\
\hline 383DH2900.5 & $6 / 16 / 2004$ & soil & EPA6010 & REG & 7439-92-1 & Lead & 29 & $\mathrm{mg} / \mathrm{kg}$ & & 0.31 \\
\hline 383DH2900.5 & $6 / 16 / 2004$ & soil & EPA7470 & REG & 7439-97-6 & Mercury & 0.042 & $\mathrm{mg} / \mathrm{kg}$ & $B$ & 0.1 \\
\hline 383DH2900.5 & $6 / 16 / 2004$ & soil & EPA8260 & REG & $75-09-2$ & Methylene Chloride & 1.4 & $\mu \mathrm{g} / \mathrm{kg}$ & $\mathrm{J}, \mathrm{B}$ & 5.2 \\
\hline
\end{tabular}

Page 20 of 78

Uncontrolled When Printed 


\begin{tabular}{|c|c|c|c|c|c|c|c|c|c|c|}
\hline SAMPLE \# & $\begin{array}{c}\text { SAMPLE } \\
\text { DATE }\end{array}$ & MATRIX & $\begin{array}{l}\text { USER TEST } \\
\text { PANEL }\end{array}$ & $\begin{array}{c}\text { SAMPLE } \\
\text { PURPOSE }\end{array}$ & CAS \# & PARAMETER & RESULT & UNITS & $\mathbf{Q}$ & $\begin{array}{l}\text { DETECT } \\
\text { LIMIT }\end{array}$ \\
\hline 383DH2900.5 & $6 / 16 / 2004$ & soil & HASL300 & REG & 15092-94-1 & $\mathrm{Pb}-212$ & 1.75 & $\mathrm{pCi} / \mathrm{g}$ & $\mathrm{J}$ & 0.37 \\
\hline 383DH2900.5 & $6 / 16 / 2004$ & soil & HASL300 & REG & $15067-28-4$ & $\mathrm{~Pb}-214$ & 1.06 & $\mathrm{pCi} / \mathrm{g}$ & $\mathrm{G}, \mathrm{J}$ & 0.44 \\
\hline 383DH2900.5 & $6 / 16 / 2004$ & soil & UGTAISOPU & REG & 13981-16-3 & Pu-238 & 0.237 & $\mathrm{pCi} / \mathrm{g}$ & & 0.017 \\
\hline 383DH2900.5 & $6 / 16 / 2004$ & soil & UGTAISOPU & REG & $15117-48-3$ & Pu-239 & 2.14 & $\mathrm{pCi} / \mathrm{g}$ & & 0.02 \\
\hline 383DH2900.5 & $6 / 16 / 2004$ & soil & SR7500 & REG & 10098-97-2 & Sr-90 & 0.76 & $\mathrm{pCi} / \mathrm{g}$ & & 0.25 \\
\hline 383DH2900.5 & $6 / 16 / 2004$ & soil & HASL300 & REG & $14913-50-9$ & TI-208 & 0.47 & $\mathrm{pCi} / \mathrm{g}$ & $\mathrm{G}$ & 0.2 \\
\hline 383DH3000.5 & $6 / 16 / 2004$ & soil & HASL300 & REG & 14331-83-0 & Ac-228 & 1.41 & $\mathrm{pCi} / \mathrm{g}$ & $G$ & 0.56 \\
\hline 383DH3000.5 & $6 / 16 / 2004$ & soil & EPA6010 & REG & $7440-38-2$ & Arsenic & 2.4 & $\mathrm{mg} / \mathrm{kg}$ & & 1 \\
\hline 383DH3000.5 & $6 / 16 / 2004$ & soil & EPA6010 & REG & $7440-39-3$ & Barium & 5100 & $\mathrm{mg} / \mathrm{kg}$ & & 510 \\
\hline 383DH3000.5 & $6 / 16 / 2004$ & soil & EPA6010 & REG & $7440-41-7$ & Beryllium & 1.2 & $\mathrm{mg} / \mathrm{kg}$ & & 0.51 \\
\hline 383DH3000.5 & $6 / 16 / 2004$ & soil & HASL300 & REG & $14733-03-0$ & Bi-214 & 0.89 & $\mathrm{pCi} / \mathrm{g}$ & $\mathrm{G}, \mathrm{J}$ & 0.27 \\
\hline 383DH3000.5 & $6 / 16 / 2004$ & soil & EPA6010 & REG & $7440-43-9$ & Cadmium & 0.21 & $\mathrm{mg} / \mathrm{kg}$ & $\mathrm{B}$ & 0.51 \\
\hline 383DH3000.5 & $6 / 16 / 2004$ & soil & EPA6010 & REG & $7440-47-3$ & Chromium & 5.8 & $\mathrm{mg} / \mathrm{kg}$ & & 1 \\
\hline 383DH3000.5 & $6 / 16 / 2004$ & soil & HASL300 & REG & $10045-97-3$ & Cs-137 & 7.67 & $\mathrm{pCi} / \mathrm{g}$ & $G$ & 0.13 \\
\hline 383DH3000.5 & $6 / 16 / 2004$ & soil & HASL300 & REG & 13966-00-2 & $\mathrm{K}-40$ & 25.6 & $\mathrm{pCi} / \mathrm{g}$ & G & 1.7 \\
\hline 383DH3000.5 & $6 / 16 / 2004$ & soil & EPA6010 & REG & 7439-92-1 & Lead & 20 & $\mathrm{mg} / \mathrm{kg}$ & & 0.31 \\
\hline 383DH3000.5 & $6 / 16 / 2004$ & soil & EPA7470 & REG & 7439-97-6 & Mercury & 0.015 & $\mathrm{mg} / \mathrm{kg}$ & $\mathrm{B}$ & 0.1 \\
\hline 383DH3000.5 & $6 / 16 / 2004$ & soil & EPA8260 & REG & $75-09-2$ & Methylene Chloride & 1.4 & $\mu \mathrm{g} / \mathrm{kg}$ & $\mathrm{J}, \mathrm{B}$ & 5.1 \\
\hline 383DH3000.5 & $6 / 16 / 2004$ & soil & HASL300 & REG & 15092-94-1 & $\mathrm{Pb}-212$ & 1.81 & $\mathrm{pCi} / \mathrm{g}$ & $\mathrm{J}$ & 0.25 \\
\hline 383DH3000.5 & $6 / 16 / 2004$ & soil & HASL300 & REG & $15067-28-4$ & $\mathrm{~Pb}-214$ & 1.22 & $\mathrm{pCi} / \mathrm{g}$ & $\mathrm{G}, \mathrm{J}$ & 0.32 \\
\hline 383DH3000.5 & $6 / 16 / 2004$ & soil & UGTAISOPU & REG & 13981-16-3 & Pu-238 & 0.042 & $\mathrm{pCi} / \mathrm{g}$ & $\mathrm{LT}$ & 0.009 \\
\hline 383DH3000.5 & $6 / 16 / 2004$ & soil & UGTAISOPU & REG & $15117-48-3$ & Pu-239 & 0.286 & $\mathrm{pCi} / \mathrm{g}$ & & 0.022 \\
\hline 383DH3000.5 & $6 / 16 / 2004$ & soil & HASL300 & REG & $14913-50-9$ & TI-208 & 0.55 & $\mathrm{pCi} / \mathrm{g}$ & $G$ & 0.15 \\
\hline 383DH3100.5 & $6 / 16 / 2004$ & soil & HASL300 & REG & 14331-83-0 & Ac-228 & 0.91 & $\mathrm{pCi} / \mathrm{g}$ & G & 0.39 \\
\hline 383DH3100.5 & $6 / 16 / 2004$ & soil & EPA6010 & REG & $7440-38-2$ & Arsenic & 5.3 & $\mathrm{mg} / \mathrm{kg}$ & & 1 \\
\hline 383DH3100.5 & $6 / 16 / 2004$ & soil & EPA6010 & REG & 7440-39-3 & Barium & 6500 & $\mathrm{mg} / \mathrm{kg}$ & & 510 \\
\hline 383DH3100.5 & $6 / 16 / 2004$ & soil & EPA6010 & REG & $7440-41-7$ & Beryllium & 0.77 & $\mathrm{mg} / \mathrm{kg}$ & & 0.51 \\
\hline 383DH3100.5 & $6 / 16 / 2004$ & soil & HASL300 & REG & $14733-03-0$ & Bi-214 & 0.84 & $\mathrm{pCi} / \mathrm{g}$ & $\mathrm{G}, \mathrm{J}$ & 0.28 \\
\hline 383DH3100.5 & $6 / 16 / 2004$ & soil & EPA6010 & REG & $7440-43-9$ & Cadmium & 0.22 & $\mathrm{mg} / \mathrm{kg}$ & $\mathrm{B}$ & 0.51 \\
\hline 383DH3100.5 & $6 / 16 / 2004$ & soil & EPA6010 & REG & $7440-47-3$ & Chromium & 8.4 & $\mathrm{mg} / \mathrm{kg}$ & & 1 \\
\hline 383DH3100.5 & $6 / 16 / 2004$ & soil & HASL300 & REG & $10045-97-3$ & Cs-137 & 8.4 & $\mathrm{pCi} / \mathrm{g}$ & G & 0.1 \\
\hline 383DH3100.5 & $6 / 16 / 2004$ & soil & HASL300 & REG & 13966-00-2 & $\mathrm{K}-40$ & 17.2 & $\mathrm{pCi} / \mathrm{g}$ & $G$ & 1.3 \\
\hline 383DH3100.5 & $6 / 16 / 2004$ & soil & EPA6010 & REG & 7439-92-1 & Lead & 18 & $\mathrm{mg} / \mathrm{kg}$ & & 0.61 \\
\hline 383DH3100.5 & $6 / 16 / 2004$ & soil & EPA7470 & REG & 7439-97-6 & Mercury & 0.021 & $\mathrm{mg} / \mathrm{kg}$ & $B$ & 0.1 \\
\hline 383DH3100.5 & $6 / 16 / 2004$ & soil & EPA8260 & REG & $75-09-2$ & Methylene Chloride & 1.2 & $\mu \mathrm{g} / \mathrm{kg}$ & $\mathrm{J}, \mathrm{B}$ & 5.1 \\
\hline
\end{tabular}

Page 21 of 78

Uncontrolled When Printed 


\begin{tabular}{|c|c|c|c|c|c|c|c|c|c|c|}
\hline SAMPLE \# & $\begin{array}{c}\text { SAMPLE } \\
\text { DATE }\end{array}$ & MATRIX & $\begin{array}{c}\text { USER TEST } \\
\text { PANEL }\end{array}$ & $\begin{array}{c}\text { SAMPLE } \\
\text { PURPOSE }\end{array}$ & CAS \# & PARAMETER & RESULT & UNITS & $\mathbf{Q}$ & $\begin{array}{c}\text { DETECT } \\
\text { LIMIT }\end{array}$ \\
\hline 383DH3100.5 & $6 / 16 / 2004$ & soil & HASL300 & REG & 15092-94-1 & $\mathrm{Pb}-212$ & 0.74 & $\mathrm{pCi} / \mathrm{g}$ & $\mathrm{J}$ & 0.26 \\
\hline 383DH3100.5 & $6 / 16 / 2004$ & soil & HASL300 & REG & $15067-28-4$ & $\mathrm{~Pb}-214$ & 0.87 & $\mathrm{pCi} / \mathrm{g}$ & $\mathrm{G}, \mathrm{J}$ & 0.36 \\
\hline 383DH3100.5 & $6 / 16 / 2004$ & soil & UGTAISOPU & REG & 13981-16-3 & Pu-238 & 0.08 & $\mathrm{pCi} / \mathrm{g}$ & & 0.018 \\
\hline 383DH3100.5 & $6 / 16 / 2004$ & soil & UGTAISOPU & REG & $15117-48-3$ & Pu-239 & 0.51 & $\mathrm{pCi} / \mathrm{g}$ & & 0.01 \\
\hline 383DH3100.5 & $6 / 16 / 2004$ & soil & EPA6010 & REG & $7782-49-2$ & Selenium & 0.37 & $\mathrm{mg} / \mathrm{kg}$ & $\mathrm{B}$ & 0.51 \\
\hline 383DH3200.5 & $6 / 16 / 2004$ & soil & HASL300 & REG & $14331-83-0$ & Ac-228 & 0.7 & $\mathrm{pCi} / \mathrm{g}$ & $\mathrm{G}$ & 0.38 \\
\hline 383DH3200.5 & $6 / 16 / 2004$ & soil & EPA6010 & REG & $7440-38-2$ & Arsenic & 4.6 & $\mathrm{mg} / \mathrm{kg}$ & & 1 \\
\hline 383DH3200.5 & $6 / 16 / 2004$ & soil & EPA6010 & REG & $7440-39-3$ & Barium & 6600 & $\mathrm{mg} / \mathrm{kg}$ & & 510 \\
\hline 383DH3200.5 & $6 / 16 / 2004$ & soil & EPA6010 & REG & $7440-41-7$ & Beryllium & 0.83 & $\mathrm{mg} / \mathrm{kg}$ & & 0.51 \\
\hline 383DH3200.5 & $6 / 16 / 2004$ & soil & HASL300 & REG & $14733-03-0$ & Bi-214 & 1 & $\mathrm{pCi} / \mathrm{g}$ & $\mathrm{G}, \mathrm{J}$ & 0.3 \\
\hline 383DH3200.5 & $6 / 16 / 2004$ & soil & EPA6010 & REG & $7440-43-9$ & Cadmium & 0.23 & $\mathrm{mg} / \mathrm{kg}$ & $\mathrm{B}$ & 0.51 \\
\hline 383DH3200.5 & $6 / 16 / 2004$ & soil & EPA6010 & REG & $7440-47-3$ & Chromium & 9.2 & $\mathrm{mg} / \mathrm{kg}$ & & 1 \\
\hline 383DH3200.5 & $6 / 16 / 2004$ & soil & HASL300 & REG & 10045-97-3 & Cs-137 & 12.7 & $\mathrm{pCi} / \mathrm{g}$ & $\mathrm{G}$ & 0.1 \\
\hline 383DH3200.5 & $6 / 16 / 2004$ & soil & HASL300 & REG & $13966-00-2$ & $\mathrm{~K}-40$ & 17 & $\mathrm{pCi} / \mathrm{g}$ & $\mathrm{G}$ & 1.4 \\
\hline 383DH3200.5 & $6 / 16 / 2004$ & soil & EPA6010 & REG & $7439-92-1$ & Lead & 21 & $\mathrm{mg} / \mathrm{kg}$ & & 0.61 \\
\hline 383DH3200.5 & $6 / 16 / 2004$ & soil & EPA7470 & REG & $7439-97-6$ & Mercury & 0.021 & $\mathrm{mg} / \mathrm{kg}$ & $\mathrm{B}$ & 0.1 \\
\hline 383DH3200.5 & $6 / 16 / 2004$ & soil & EPA8260 & REG & $75-09-2$ & Methylene Chloride & 0.82 & $\mu \mathrm{g} / \mathrm{kg}$ & $\mathrm{J}, \mathrm{B}$ & 5.1 \\
\hline 383DH3200.5 & $6 / 16 / 2004$ & soil & HASL300 & REG & 15092-94-1 & $\mathrm{Pb}-212$ & 0.85 & $\mathrm{pCi} / \mathrm{g}$ & $\mathrm{J}$ & 0.27 \\
\hline 383DH3200.5 & $6 / 16 / 2004$ & soil & HASL300 & REG & $15067-28-4$ & $\mathrm{~Pb}-214$ & 0.8 & $\mathrm{pCi} / \mathrm{g}$ & $\mathrm{G}, \mathrm{J}$ & 0.46 \\
\hline $383 \mathrm{DH} 3200.5$ & $6 / 16 / 2004$ & soil & UGTAISOPU & REG & 13981-16-3 & Pu-238 & 0.058 & $\mathrm{pCi} / \mathrm{g}$ & & 0.021 \\
\hline 383DH3200.5 & $6 / 16 / 2004$ & soil & UGTAISOPU & REG & $15117-48-3$ & Pu-239 & 0.66 & $\mathrm{pCi} / \mathrm{g}$ & & 0.02 \\
\hline 383DH3200.5 & $6 / 16 / 2004$ & soil & EPA6010 & REG & $7782-49-2$ & Selenium & 0.29 & $\mathrm{mg} / \mathrm{kg}$ & $\mathrm{B}$ & 0.51 \\
\hline 383DH3200.5 & $6 / 16 / 2004$ & soil & HASL300 & REG & $14913-50-9$ & TI-208 & 0.41 & $\mathrm{pCi} / \mathrm{g}$ & $\mathrm{G}$ & 0.18 \\
\hline 383DH3300.5 & $6 / 16 / 2004$ & soil & HASL300 & REG & $14331-83-0$ & Ac-228 & 1.9 & $\mathrm{pCi} / \mathrm{g}$ & G & 0.66 \\
\hline 383DH3300.5 & $6 / 16 / 2004$ & soil & EPA6010 & REG & $7440-38-2$ & Arsenic & 3.9 & $\mathrm{mg} / \mathrm{kg}$ & & 1 \\
\hline 383DH3300.5 & $6 / 16 / 2004$ & soil & EPA6010 & REG & $7440-39-3$ & Barium & 5900 & $\mathrm{mg} / \mathrm{kg}$ & & 510 \\
\hline 383DH3300.5 & $6 / 16 / 2004$ & soil & EPA6010 & REG & $7440-41-7$ & Beryllium & 1.4 & $\mathrm{mg} / \mathrm{kg}$ & & 0.51 \\
\hline 383DH3300.5 & $6 / 16 / 2004$ & soil & HASL300 & REG & 14733-03-0 & Bi-214 & 1.06 & $\mathrm{pCi} / \mathrm{g}$ & $\mathrm{G}, \mathrm{J}$ & 0.36 \\
\hline 383DH3300.5 & $6 / 16 / 2004$ & soil & EPA6010 & REG & $7440-43-9$ & Cadmium & 0.26 & $\mathrm{mg} / \mathrm{kg}$ & $\mathrm{B}$ & 0.51 \\
\hline 383DH3300.5 & $6 / 16 / 2004$ & soil & EPA6010 & REG & $7440-47-3$ & Chromium & 5.5 & $\mathrm{mg} / \mathrm{kg}$ & & 1 \\
\hline 383DH3300.5 & $6 / 16 / 2004$ & soil & HASL300 & REG & 10045-97-3 & Cs-137 & 7.5 & $\mathrm{pCi} / \mathrm{g}$ & G & 0.2 \\
\hline 383DH3300.5 & $6 / 16 / 2004$ & soil & EPA8015 & REG & $68334-30-5$ & Diesel-Range Organics & 2.2 & $\mathrm{mg} / \mathrm{kg}$ & $\mathrm{J}$ & 5.1 \\
\hline 383DH3300.5 & $6 / 16 / 2004$ & soil & HASL300 & REG & $13966-00-2$ & $\mathrm{~K}-40$ & 25.3 & $\mathrm{pCi} / \mathrm{g}$ & G & 2 \\
\hline 383DH3300.5 & $6 / 16 / 2004$ & soil & EPA6010 & REG & $7439-92-1$ & Lead & 30 & $\mathrm{mg} / \mathrm{kg}$ & & 0.31 \\
\hline 383DH3300.5 & $6 / 16 / 2004$ & soil & EPA7470 & REG & $7439-97-6$ & Mercury & 0.019 & $\mathrm{mg} / \mathrm{kg}$ & $\mathrm{B}$ & 0.1 \\
\hline
\end{tabular}

Page 22 of 78

Uncontrolled When Printed 


\begin{tabular}{|c|c|c|c|c|c|c|c|c|c|c|}
\hline SAMPLE \# & $\begin{array}{c}\text { SAMPLE } \\
\text { DATE }\end{array}$ & MATRIX & $\begin{array}{l}\text { USER TEST } \\
\text { PANEL }\end{array}$ & $\begin{array}{c}\text { SAMPLE } \\
\text { PURPOSE }\end{array}$ & CAS \# & PARAMETER & RESULT & UNITS & $\mathbf{Q}$ & $\begin{array}{c}\text { DETECT } \\
\text { LIMIT }\end{array}$ \\
\hline 383DH3300.5 & $6 / 16 / 2004$ & soil & EPA8260 & REG & $75-09-2$ & Methylene Chloride & 1.3 & $\mu \mathrm{g} / \mathrm{kg}$ & $\mathrm{J}, \mathrm{B}$ & 5.1 \\
\hline 383DH3300.5 & $6 / 16 / 2004$ & soil & HASL300 & REG & 15092-94-1 & $\mathrm{Pb}-212$ & 1.88 & $\mathrm{pCi} / \mathrm{g}$ & $\mathrm{J}$ & 0.32 \\
\hline 383DH3300.5 & $6 / 16 / 2004$ & soil & HASL300 & REG & $15067-28-4$ & $\mathrm{~Pb}-214$ & 1.26 & $\mathrm{pCi} / \mathrm{g}$ & $\mathrm{G}, \mathrm{J}$ & 0.38 \\
\hline 383DH3300.5 & $6 / 16 / 2004$ & soil & UGTAISOPU & REG & 13981-16-3 & Pu-238 & 0.04 & $\mathrm{pCi} / \mathrm{g}$ & $\mathrm{LT}$ & 0.01 \\
\hline 383DH3300.5 & $6 / 16 / 2004$ & soil & UGTAISOPU & REG & 15117-48-3 & Pu-239 & 0.261 & $\mathrm{pCi} / \mathrm{g}$ & & 0.01 \\
\hline 383DH3300.5 & $6 / 16 / 2004$ & soil & EPA6010 & REG & $7782-49-2$ & Selenium & 0.44 & $\mathrm{mg} / \mathrm{kg}$ & B & 0.51 \\
\hline 383DH3300.5 & $6 / 16 / 2004$ & soil & HASL300 & REG & $14913-50-9$ & TI-208 & 0.47 & $\mathrm{pCi} / \mathrm{g}$ & G & 0.18 \\
\hline 383DH3400.5 & $6 / 10 / 2004$ & soil & HASL300 & REG & 14331-83-0 & Ac-228 & 1.6 & $\mathrm{pCi} / \mathrm{g}$ & G & 0.5 \\
\hline 383DH3400.5 & $6 / 10 / 2004$ & soil & EPA8260 & REG & $67-64-1$ & Acetone & 6.5 & $\mu \mathrm{g} / \mathrm{kg}$ & $\mathrm{J}$ & 21 \\
\hline 383DH3400.5 & $6 / 10 / 2004$ & soil & EPA6010 & REG & $7440-38-2$ & Arsenic & 2.6 & $\mathrm{mg} / \mathrm{kg}$ & & 1.1 \\
\hline 383DH3400.5 & $6 / 10 / 2004$ & soil & EPA6010 & REG & $7440-39-3$ & Barium & 3900 & $\mathrm{mg} / \mathrm{kg}$ & & 110 \\
\hline 383DH3400.5 & $6 / 10 / 2004$ & soil & EPA6010 & REG & $7440-41-7$ & Beryllium & 1 & $\mathrm{mg} / \mathrm{kg}$ & & 0.53 \\
\hline 383DH3400.5 & $6 / 10 / 2004$ & soil & HASL300 & REG & 14733-03-0 & Bi-214 & 1.01 & $\mathrm{pCi} / \mathrm{g}$ & $\mathrm{G}, \mathrm{J}$ & 0.28 \\
\hline 383DH3400.5 & $6 / 10 / 2004$ & soil & EPA6010 & REG & $7440-43-9$ & Cadmium & 0.22 & $\mathrm{mg} / \mathrm{kg}$ & $B$ & 0.53 \\
\hline 383DH3400.5 & $6 / 10 / 2004$ & soil & EPA6010 & REG & $7440-47-3$ & Chromium & 3.9 & $\mathrm{mg} / \mathrm{kg}$ & & 1.1 \\
\hline 383DH3400.5 & $6 / 10 / 2004$ & soil & HASL300 & REG & $10045-97-3$ & Cs-137 & 11.7 & $\mathrm{pCi} / \mathrm{g}$ & G & 0.1 \\
\hline 383DH3400.5 & $6 / 10 / 2004$ & soil & EPA8015 & REG & $68334-30-5$ & Diesel-Range Organics & 51 & $\mathrm{mg} / \mathrm{kg}$ & $\mathrm{Z}, \mathrm{H}$ & 5.3 \\
\hline 383DH3400.5 & $6 / 10 / 2004$ & soil & HASL300 & REG & $13966-00-2$ & $\mathrm{~K}-40$ & 22.8 & $\mathrm{pCi} / \mathrm{g}$ & G & 1.4 \\
\hline 383DH3400.5 & $6 / 10 / 2004$ & soil & EPA6010 & REG & 7439-92-1 & Lead & 14 & $\mathrm{mg} / \mathrm{kg}$ & & 0.32 \\
\hline 383DH3400.5 & $6 / 10 / 2004$ & soil & EPA7470 & REG & 7439-97-6 & Mercury & 0.025 & $\mathrm{mg} / \mathrm{kg}$ & $\mathrm{B}$ & 0.11 \\
\hline 383DH3400.5 & $6 / 10 / 2004$ & soil & HASL300 & REG & 15092-94-1 & $\mathrm{Pb}-212$ & 1.7 & $\mathrm{pCi} / \mathrm{g}$ & $\mathrm{J}$ & 0.3 \\
\hline 383DH3400.5 & $6 / 10 / 2004$ & soil & HASL300 & REG & $15067-28-4$ & $\mathrm{~Pb}-214$ & 1.26 & $\mathrm{pCi} / \mathrm{g}$ & $\mathrm{G}, \mathrm{J}$ & 0.34 \\
\hline 383DH3400.5 & $6 / 10 / 2004$ & soil & UGTAISOPU & REG & 13981-16-3 & Pu-238 & 0.143 & $\mathrm{pCi} / \mathrm{g}$ & & 0.019 \\
\hline 383DH3400.5 & $6 / 10 / 2004$ & soil & UGTAISOPU & REG & $15117-48-3$ & Pu-239 & 1.01 & $\mathrm{pCi} / \mathrm{g}$ & $\mathrm{J}$ & 0.02 \\
\hline 383DH3400.5 & $6 / 10 / 2004$ & soil & SR7500 & REG & 10098-97-2 & Sr-90 & 0.69 & $\mathrm{pCi} / \mathrm{g}$ & & 0.23 \\
\hline 383DH3400.5 & $6 / 10 / 2004$ & soil & HASL300 & REG & 14913-50-9 & TI-208 & 0.47 & $\mathrm{pCi} / \mathrm{g}$ & $\mathrm{G}$ & 0.16 \\
\hline 383DH3500.5 & $6 / 10 / 2004$ & soil & HASL300 & REG & 14331-83-0 & Ac-228 & 1.42 & $\mathrm{pCi} / \mathrm{g}$ & $\mathrm{G}$ & 0.52 \\
\hline 383DH3500.5 & $6 / 10 / 2004$ & soil & EPA8260 & REG & 67-64-1 & Acetone & 6.6 & $\mu \mathrm{g} / \mathrm{kg}$ & $\mathrm{J}$ & 23 \\
\hline 383DH3500.5 & $6 / 10 / 2004$ & soil & EPA6010 & REG & $7440-38-2$ & Arsenic & 4.5 & $\mathrm{mg} / \mathrm{kg}$ & & 1.2 \\
\hline 383DH3500.5 & $6 / 10 / 2004$ & soil & EPA6010 & REG & $7440-39-3$ & Barium & 4600 & $\mathrm{mg} / \mathrm{kg}$ & & 120 \\
\hline 383DH3500.5 & $6 / 10 / 2004$ & soil & EPA6010 & REG & $7440-41-7$ & Beryllium & 0.9 & $\mathrm{mg} / \mathrm{kg}$ & & 0.58 \\
\hline 383DH3500.5 & $6 / 10 / 2004$ & soil & HASL300 & REG & 14733-03-0 & Bi-214 & 0.76 & $\mathrm{pCi} / \mathrm{g}$ & $\mathrm{G}, \mathrm{J}$ & 0.28 \\
\hline 383DH3500.5 & $6 / 10 / 2004$ & soil & EPA6010 & REG & $7440-43-9$ & Cadmium & 0.28 & $\mathrm{mg} / \mathrm{kg}$ & $\mathrm{B}$ & 0.58 \\
\hline 383DH3500.5 & $6 / 10 / 2004$ & soil & EPA6010 & REG & $7440-47-3$ & Chromium & 5.8 & $\mathrm{mg} / \mathrm{kg}$ & & 1.2 \\
\hline 383DH3500.5 & $6 / 10 / 2004$ & soil & HASL300 & REG & $10045-97-3$ & Cs-137 & 5.97 & $\mathrm{pCi} / \mathrm{g}$ & $\mathrm{G}$ & 0.14 \\
\hline
\end{tabular}

Page 23 of 78

Uncontrolled When Printed 


\begin{tabular}{|c|c|c|c|c|c|c|c|c|c|c|}
\hline SAMPLE \# & $\begin{array}{c}\text { SAMPLE } \\
\text { DATE }\end{array}$ & MATRIX & $\begin{array}{l}\text { USER TEST } \\
\text { PANEL }\end{array}$ & $\begin{array}{c}\text { SAMPLE } \\
\text { PURPOSE }\end{array}$ & CAS \# & PARAMETER & RESULT & UNITS & $\mathbf{Q}$ & $\begin{array}{l}\text { DETECT } \\
\text { LIMIT }\end{array}$ \\
\hline 383DH3500.5 & $6 / 10 / 2004$ & soil & EPA8015 & REG & $68334-30-5$ & Diesel-Range Organics & 68 & $\mathrm{mg} / \mathrm{kg}$ & $\mathrm{Z}, \mathrm{H}$ & 5.8 \\
\hline 383DH3500.5 & $6 / 10 / 2004$ & soil & HASL300 & REG & $13966-00-2$ & $\mathrm{~K}-40$ & 17 & $\mathrm{pCi} / \mathrm{g}$ & $\mathrm{G}$ & 1.6 \\
\hline 383DH3500.5 & $6 / 10 / 2004$ & soil & EPA6010 & REG & 7439-92-1 & Lead & 19 & $\mathrm{mg} / \mathrm{kg}$ & & 3.5 \\
\hline 383DH3500.5 & $6 / 10 / 2004$ & soil & EPA7470 & REG & 7439-97-6 & Mercury & 0.026 & $\mathrm{mg} / \mathrm{kg}$ & $\mathrm{B}$ & 0.12 \\
\hline 383DH3500.5 & $6 / 10 / 2004$ & soil & HASL300 & REG & 15092-94-1 & $\mathrm{Pb}-212$ & 1.44 & $\mathrm{pCi} / \mathrm{g}$ & $\mathrm{J}$ & 0.26 \\
\hline 383DH3500.5 & $6 / 10 / 2004$ & soil & HASL300 & REG & $15067-28-4$ & $\mathrm{~Pb}-214$ & 1.14 & $\mathrm{pCi} / \mathrm{g}$ & $\mathrm{G}, \mathrm{J}$ & 0.31 \\
\hline 383DH3500.5 & $6 / 10 / 2004$ & soil & UGTAISOPU & REG & 13981-16-3 & Pu-238 & 0.147 & $\mathrm{pCi} / \mathrm{g}$ & & 0.02 \\
\hline 383DH3500.5 & $6 / 10 / 2004$ & soil & UGTAISOPU & REG & $15117-48-3$ & Pu-239 & 0.51 & $\mathrm{pCi} / \mathrm{g}$ & $\mathrm{J}$ & 0.01 \\
\hline 383DH3500.5 & $6 / 10 / 2004$ & soil & HASL300 & REG & $14913-50-9$ & TI-208 & 0.5 & $\mathrm{pCi} / \mathrm{g}$ & G & 0.13 \\
\hline 383GB0100.5 & $2 / 4 / 2004$ & soil & HASL300 & REG & 14331-83-0 & Ac-228 & 2.14 & $\mathrm{pCi} / \mathrm{g}$ & $G$ & 1.04 \\
\hline 383GB0100.5 & $2 / 4 / 2004$ & soil & EPA6010 & REG & $7440-38-2$ & Arsenic & 4.4 & $\mathrm{mg} / \mathrm{kg}$ & & 1.2 \\
\hline 383GB0100.5 & 2/4/2004 & soil & EPA6010 & REG & $7440-41-7$ & Beryllium & 1.3 & $\mathrm{mg} / \mathrm{kg}$ & & 0.58 \\
\hline 383GB0100.5 & $2 / 4 / 2004$ & soil & HASL300 & REG & $14733-03-0$ & Bi-214 & 1.41 & $\mathrm{pCi} / \mathrm{g}$ & $\mathrm{G}$ & 0.73 \\
\hline 383GB0100.5 & $2 / 4 / 2004$ & soil & HASL300 & REG & $10045-97-3$ & Cs-137 & 0.78 & $\mathrm{pCi} / \mathrm{g}$ & $G$ & 0.31 \\
\hline 383GB0100.5 & $2 / 4 / 2004$ & soil & HASL300 & REG & 13966-00-2 & $\mathrm{K}-40$ & 18.9 & $\mathrm{pCi} / \mathrm{g}$ & G & 4.4 \\
\hline 383GB0100.5 & $2 / 4 / 2004$ & soil & EPA6010 & REG & 7439-92-1 & Lead & 12 & $\mathrm{mg} / \mathrm{kg}$ & & 0.35 \\
\hline 383GB0100.5 & $2 / 4 / 2004$ & soil & HASL300 & REG & 15092-94-1 & $\mathrm{Pb}-212$ & 1.66 & $\mathrm{pCi} / \mathrm{g}$ & $\mathrm{J}$ & 0.52 \\
\hline 383GB0100.5 & $2 / 4 / 2004$ & soil & UGTAISOPU & REG & $15117-48-3$ & Pu-239 & 0.099 & $\mathrm{pCi} / \mathrm{g}$ & $\mathrm{J}$ & 0.009 \\
\hline 383GB0100.5 & $2 / 4 / 2004$ & soil & HASL300 & REG & 14913-50-9 & TI-208 & 0.64 & $\mathrm{pCi} / \mathrm{g}$ & $G$ & 0.27 \\
\hline 383GB0200.5 & $2 / 4 / 2004$ & soil & HASL300 & REG & 14331-83-0 & Ac-228 & 1.85 & $\mathrm{pCi} / \mathrm{g}$ & $\mathrm{TI}, \mathrm{G}$ & 0.85 \\
\hline 383GB0200.5 & $2 / 4 / 2004$ & soil & EPA6010 & REG & $7440-38-2$ & Arsenic & 4.3 & $\mathrm{mg} / \mathrm{kg}$ & & 1.1 \\
\hline 383GB0200.5 & $2 / 4 / 2004$ & soil & EPA6010 & REG & 7440-41-7 & Beryllium & 0.72 & $\mathrm{mg} / \mathrm{kg}$ & & 0.55 \\
\hline 383GB0200.5 & $2 / 4 / 2004$ & soil & HASL300 & REG & $10045-97-3$ & Cs-137 & 4.39 & $\mathrm{pCi} / \mathrm{g}$ & G & 0.23 \\
\hline 383GB0200.5 & $2 / 4 / 2004$ & soil & HASL300 & REG & 13966-00-2 & $\mathrm{K}-40$ & 17.8 & $\mathrm{pCi} / \mathrm{g}$ & G & 2.8 \\
\hline 383GB0200.5 & $2 / 4 / 2004$ & soil & EPA6010 & REG & 7439-92-1 & Lead & 75 & $\mathrm{mg} / \mathrm{kg}$ & & 0.33 \\
\hline 383GB0200.5 & $2 / 4 / 2004$ & soil & HASL300 & REG & 15092-94-1 & $\mathrm{Pb}-212$ & 1.3 & $\mathrm{pCi} / \mathrm{g}$ & $\mathrm{J}$ & 0.42 \\
\hline 383GB0200.5 & $2 / 4 / 2004$ & soil & UGTAISOPU & REG & 13981-16-3 & Pu-238 & 0.032 & $\mathrm{pCi} / \mathrm{g}$ & LT & 0.01 \\
\hline 383GB0200.5 & $2 / 4 / 2004$ & soil & UGTAISOPU & REG & $15117-48-3$ & Pu-239 & 0.318 & $\mathrm{pCi} / \mathrm{g}$ & $\mathrm{J}$ & 0.018 \\
\hline 383GB0200.5 & $2 / 4 / 2004$ & soil & HASL300 & REG & $14913-50-9$ & TI-208 & 0.43 & $\mathrm{pCi} / \mathrm{g}$ & $\mathrm{G}$ & 0.22 \\
\hline 383GB0300.5 & $2 / 4 / 2004$ & soil & EPA6010 & REG & $7440-38-2$ & Arsenic & 3 & $\mathrm{mg} / \mathrm{kg}$ & & 1.1 \\
\hline 383GB0300.5 & $2 / 4 / 2004$ & soil & EPA6010 & REG & 7440-41-7 & Beryllium & 0.79 & $\mathrm{mg} / \mathrm{kg}$ & & 0.57 \\
\hline 383GB0300.5 & $2 / 4 / 2004$ & soil & HASL300 & REG & $13966-00-2$ & $\mathrm{~K}-40$ & 24 & $\mathrm{pCi} / \mathrm{g}$ & $\mathrm{G}$ & 3.1 \\
\hline 383GB0300.5 & $2 / 4 / 2004$ & soil & EPA6010 & REG & 7439-92-1 & Lead & 26 & $\mathrm{mg} / \mathrm{kg}$ & & 0.34 \\
\hline 383GB0300.5 & $2 / 4 / 2004$ & soil & HASL300 & REG & 15092-94-1 & $\mathrm{Pb}-212$ & 1.87 & $\mathrm{pCi} / \mathrm{g}$ & $\mathrm{J}$ & 0.38 \\
\hline 383GB0300.5 & $2 / 4 / 2004$ & soil & HASL300 & REG & 15067-28-4 & $\mathrm{Pb}-214$ & 0.81 & $\mathrm{pCi} / \mathrm{g}$ & $\mathrm{J}$ & 0.43 \\
\hline
\end{tabular}

Page 24 of 78

Uncontrolled When Printed 


\begin{tabular}{|c|c|c|c|c|c|c|c|c|c|c|}
\hline SAMPLE \# & $\begin{array}{l}\text { SAMPLE } \\
\text { DATE }\end{array}$ & MATRIX & $\begin{array}{l}\text { USER TEST } \\
\text { PANEL }\end{array}$ & $\begin{array}{c}\text { SAMPLE } \\
\text { PURPOSE }\end{array}$ & CAS \# & PARAMETER & RESULT & UNITS & $\mathbf{Q}$ & $\begin{array}{c}\text { DETECT } \\
\text { LIMIT }\end{array}$ \\
\hline 383GB0300.5 & $2 / 4 / 2004$ & soil & UGTAISOPU & REG & $15117-48-3$ & Pu-239 & 0.241 & $\mathrm{pCi} / \mathrm{g}$ & $\mathrm{J}$ & 0.018 \\
\hline 383GB0400.5 & $2 / 4 / 2004$ & soil & HASL300 & REG & 14331-83-0 & Ac-228 & 2.09 & $\mathrm{pCi} / \mathrm{g}$ & $\mathrm{G}$ & 1.05 \\
\hline 383GB0400.5 & $2 / 4 / 2004$ & soil & EPA6010 & REG & $7440-38-2$ & Arsenic & 3.8 & $\mathrm{mg} / \mathrm{kg}$ & & 1.1 \\
\hline 383GB0400.5 & $2 / 4 / 2004$ & soil & EPA6010 & REG & 7440-41-7 & Beryllium & 0.82 & $\mathrm{mg} / \mathrm{kg}$ & & 0.57 \\
\hline 383GB0400.5 & $2 / 4 / 2004$ & soil & HASL300 & REG & $10045-97-3$ & Cs-137 & 1.86 & $\mathrm{pCi} / \mathrm{g}$ & $\mathrm{G}$ & 0.27 \\
\hline 383GB0400.5 & $2 / 4 / 2004$ & soil & HASL300 & REG & 13966-00-2 & $\mathrm{K}-40$ & 26 & $\mathrm{pCi} / \mathrm{g}$ & $G$ & 3.2 \\
\hline 383GB0400.5 & $2 / 4 / 2004$ & soil & EPA6010 & REG & 7439-92-1 & Lead & 60 & $\mathrm{mg} / \mathrm{kg}$ & & 0.34 \\
\hline 383GB0400.5 & $2 / 4 / 2004$ & soil & HASL300 & REG & 15092-94-1 & $\mathrm{Pb}-212$ & 1.78 & $\mathrm{pCi} / \mathrm{g}$ & $\mathrm{J}$ & 0.3 \\
\hline 383GB0400.5 & $2 / 4 / 2004$ & soil & UGTAISOPU & REG & $15117-48-3$ & Pu-239 & 0.093 & $\mathrm{pCi} / \mathrm{g}$ & $\mathrm{J}$ & 0.022 \\
\hline 383GB0500.5 & $2 / 4 / 2004$ & soil & HASL300 & REG & 14331-83-0 & Ac-228 & 2.27 & $\mathrm{pCi} / \mathrm{g}$ & $\mathrm{G}$ & 0.55 \\
\hline 383GB0500.5 & $2 / 4 / 2004$ & soil & EPA6010 & REG & 7440-38-2 & Arsenic & 5.3 & $\mathrm{mg} / \mathrm{kg}$ & & 1.1 \\
\hline 383GB0500.5 & $2 / 4 / 2004$ & soil & EPA6010 & REG & $7440-39-3$ & Barium & 130 & $\mathrm{mg} / \mathrm{kg}$ & & 11 \\
\hline 383GB0500.5 & $2 / 4 / 2004$ & soil & EPA6010 & REG & $7440-41-7$ & Beryllium & 1 & $\mathrm{mg} / \mathrm{kg}$ & & 0.57 \\
\hline 383GB0500.5 & $2 / 4 / 2004$ & soil & HASL300 & REG & 14733-03-0 & Bi-214 & 1.24 & $\mathrm{pCi} / \mathrm{g}$ & $\mathrm{G}$ & 0.33 \\
\hline 383GB0500.5 & $2 / 4 / 2004$ & soil & EPA6010 & REG & $7440-47-3$ & Chromium & 8.2 & $\mathrm{mg} / \mathrm{kg}$ & & 1.1 \\
\hline 383GB0500.5 & $2 / 4 / 2004$ & soil & HASL300 & REG & $10045-97-3$ & Cs-137 & 1.27 & $\mathrm{pCi} / \mathrm{g}$ & G & 0.18 \\
\hline 383GB0500.5 & $2 / 4 / 2004$ & soil & HASL300 & REG & 13966-00-2 & $\mathrm{K}-40$ & 30.7 & $\mathrm{pCi} / \mathrm{g}$ & $\mathrm{G}$ & 1.6 \\
\hline 383GB0500.5 & $2 / 4 / 2004$ & soil & EPA6010 & REG & 7439-92-1 & Lead & 15 & $\mathrm{mg} / \mathrm{kg}$ & & 0.34 \\
\hline 383GB0500.5 & $2 / 4 / 2004$ & soil & EPA7470 & REG & 7439-97-6 & Mercury & 0.065 & $\mathrm{mg} / \mathrm{kg}$ & $\mathrm{B}$ & 0.11 \\
\hline 383GB0500.5 & $2 / 4 / 2004$ & soil & HASL300 & REG & 15092-94-1 & $\mathrm{Pb}-212$ & 2.47 & $\mathrm{pCi} / \mathrm{g}$ & $\mathrm{J}$ & 0.24 \\
\hline 383GB0500.5 & $2 / 4 / 2004$ & soil & HASL300 & REG & $15067-28-4$ & $\mathrm{~Pb}-214$ & 1.07 & $\mathrm{pCi} / \mathrm{g}$ & $\mathrm{J}$ & 0.32 \\
\hline 383GB0500.5 & $2 / 4 / 2004$ & soil & UGTAISOPU & REG & 13981-16-3 & Pu-238 & 0.38 & $\mathrm{pCi} / \mathrm{g}$ & & 0.03 \\
\hline 383GB0500.5 & $2 / 4 / 2004$ & soil & UGTAISOPU & REG & $15117-48-3$ & Pu-239 & 1.41 & $\mathrm{pCi} / \mathrm{g}$ & & 0.02 \\
\hline 383GB0500.5 & $2 / 4 / 2004$ & soil & EPA6010 & REG & $7782-49-2$ & Selenium & 0.52 & $\mathrm{mg} / \mathrm{kg}$ & $\mathrm{B}$ & 0.57 \\
\hline 383GB0500.5 & $2 / 4 / 2004$ & soil & HASL300 & REG & 14913-50-9 & TI-208 & 0.83 & $\mathrm{pCi} / \mathrm{g}$ & G & 0.17 \\
\hline 383GB0600.5 & $2 / 4 / 2004$ & soil & HASL300 & REG & 14331-83-0 & Ac-228 & 1.44 & $\mathrm{pCi} / \mathrm{g}$ & $\mathrm{G}$ & 0.53 \\
\hline 383GB0600.5 & $2 / 4 / 2004$ & soil & EPA6010 & REG & $7440-38-2$ & Arsenic & 4 & $\mathrm{mg} / \mathrm{kg}$ & & 1.1 \\
\hline 383GB0600.5 & $2 / 4 / 2004$ & soil & EPA6010 & REG & $7440-39-3$ & Barium & 290 & $\mathrm{mg} / \mathrm{kg}$ & & 11 \\
\hline 383GB0600.5 & $2 / 4 / 2004$ & soil & EPA6010 & REG & $7440-41-7$ & Beryllium & 0.88 & $\mathrm{mg} / \mathrm{kg}$ & & 0.57 \\
\hline 383GB0600.5 & $2 / 4 / 2004$ & soil & HASL300 & REG & $14733-03-0$ & $\mathrm{Bi}-214$ & 0.79 & $\mathrm{pCi} / \mathrm{g}$ & G & 0.29 \\
\hline 383GB0600.5 & $2 / 4 / 2004$ & soil & EPA6010 & REG & $7440-43-9$ & Cadmium & 0.11 & $\mathrm{mg} / \mathrm{kg}$ & $\mathrm{J}$ & 0.57 \\
\hline 383GB0600.5 & $2 / 4 / 2004$ & soil & EPA6010 & REG & $7440-47-3$ & Chromium & 8.4 & $\mathrm{mg} / \mathrm{kg}$ & & 1.1 \\
\hline 383GB0600.5 & $2 / 4 / 2004$ & soil & HASL300 & REG & 10045-97-3 & Cs-137 & 2.7 & $\mathrm{pCi} / \mathrm{g}$ & $\mathrm{G}$ & 0.14 \\
\hline 383GB0600.5 & $2 / 4 / 2004$ & soil & HASL300 & REG & 13966-00-2 & $\mathrm{K}-40$ & 16.3 & $\mathrm{pCi} / \mathrm{g}$ & $\mathrm{G}$ & 1.3 \\
\hline $383 G B 0600.5$ & $2 / 4 / 2004$ & soil & EPA6010 & REG & 7439-92-1 & Lead & 16 & $\mathrm{mg} / \mathrm{kg}$ & & 0.68 \\
\hline
\end{tabular}

Page 25 of 78

Uncontrolled When Printed 


\begin{tabular}{|c|c|c|c|c|c|c|c|c|c|c|}
\hline SAMPLE \# & $\begin{array}{l}\text { SAMPLE } \\
\text { DATE }\end{array}$ & MATRIX & $\begin{array}{c}\text { USER TEST } \\
\text { PANEL }\end{array}$ & $\begin{array}{c}\text { SAMPLE } \\
\text { PURPOSE }\end{array}$ & CAS \# & PARAMETER & RESULT & UNITS & $\mathbf{Q}$ & $\begin{array}{c}\text { DETECT } \\
\text { LIMIT }\end{array}$ \\
\hline 383GB0600.5 & 2/4/2004 & soil & EPA7470 & REG & 7439-97-6 & Mercury & 0.032 & $\mathrm{mg} / \mathrm{kg}$ & $B$ & 0.11 \\
\hline 383GB0600.5 & $2 / 4 / 2004$ & soil & HASL300 & REG & 15092-94-1 & $\mathrm{Pb}-212$ & 1.48 & $\mathrm{pCi} / \mathrm{g}$ & $\mathrm{J}$ & 0.2 \\
\hline 383GB0600.5 & $2 / 4 / 2004$ & soil & HASL300 & REG & 15067-28-4 & $\mathrm{Pb}-214$ & 0.89 & $\mathrm{pCi} / \mathrm{g}$ & $\mathrm{J}$ & 0.28 \\
\hline 383GB0600.5 & $2 / 4 / 2004$ & soil & UGTAISOPU & REG & 13981-16-3 & Pu-238 & 0.71 & $\mathrm{pCi} / \mathrm{g}$ & & 0.02 \\
\hline 383GB0600.5 & $2 / 4 / 2004$ & soil & UGTAISOPU & REG & $15117-48-3$ & Pu-239 & 5.08 & $\mathrm{pCi} / \mathrm{g}$ & & 0.02 \\
\hline 383GB0600.5 & $2 / 4 / 2004$ & soil & EPA6010 & REG & $7782-49-2$ & Selenium & 0.71 & $\mathrm{mg} / \mathrm{kg}$ & & 0.57 \\
\hline 383GB0600.5 & $2 / 4 / 2004$ & soil & HASL300 & REG & $14913-50-9$ & TI-208 & 0.38 & $\mathrm{pCi} / \mathrm{g}$ & $\mathrm{G}$ & 0.13 \\
\hline 383GB0700.5 & $2 / 4 / 2004$ & soil & EPA6010 & REG & $7440-38-2$ & Arsenic & 2.6 & $\mathrm{mg} / \mathrm{kg}$ & & 1.1 \\
\hline 383GB0700.5 & $2 / 4 / 2004$ & soil & EPA6010 & REG & 7440-39-3 & Barium & 170 & $\mathrm{mg} / \mathrm{kg}$ & & 11 \\
\hline 383GB0700.5 & $2 / 4 / 2004$ & soil & HASL300 & REG & $14733-03-0$ & Bi-214 & 0.45 & $\mathrm{pCi} / \mathrm{g}$ & $\mathrm{G}$ & 0.22 \\
\hline 383GB0700.5 & $2 / 4 / 2004$ & soil & EPA6010 & REG & $7440-43-9$ & Cadmium & 0.32 & $\mathrm{mg} / \mathrm{kg}$ & $\mathrm{B}$ & 0.54 \\
\hline 383GB0700.5 & $2 / 4 / 2004$ & soil & EPA6010 & REG & $7440-47-3$ & Chromium & 5 & $\mathrm{mg} / \mathrm{kg}$ & & 1.1 \\
\hline 383GB0700.5 & $2 / 4 / 2004$ & soil & HASL300 & REG & 10045-97-3 & Cs-137 & 0.46 & $\mathrm{pCi} / \mathrm{g}$ & LT,G & 0.1 \\
\hline 383GB0700.5 & $2 / 4 / 2004$ & soil & HASL300 & REG & $13966-00-2$ & $\mathrm{~K}-40$ & 8.8 & $\mathrm{pCi} / \mathrm{g}$ & $\mathrm{G}$ & 0.8 \\
\hline 383GB0700.5 & $2 / 4 / 2004$ & soil & EPA6010 & REG & 7439-92-1 & Lead & 21 & $\mathrm{mg} / \mathrm{kg}$ & & 1.6 \\
\hline 383GB0700.5 & $2 / 4 / 2004$ & soil & EPA7470 & REG & $7439-97-6$ & Mercury & 0.032 & $\mathrm{mg} / \mathrm{kg}$ & $\mathrm{B}$ & 0.11 \\
\hline 383GB0700.5 & $2 / 4 / 2004$ & soil & HASL300 & REG & 15092-94-1 & $\mathrm{Pb}-212$ & 0.72 & $\mathrm{pCi} / \mathrm{g}$ & $\mathrm{J}$ & 0.16 \\
\hline 383GB0700.5 & $2 / 4 / 2004$ & soil & HASL300 & REG & $15067-28-4$ & $\mathrm{~Pb}-214$ & 0.6 & $\mathrm{pCi} / \mathrm{g}$ & $\mathrm{J}$ & 0.21 \\
\hline 383GB0700.5 & $2 / 4 / 2004$ & soil & UGTAISOPU & REG & 13981-16-3 & Pu-238 & 0.088 & $\mathrm{pCi} / \mathrm{g}$ & & 0.018 \\
\hline 383GB0700.5 & $2 / 4 / 2004$ & soil & UGTAISOPU & REG & $15117-48-3$ & Pu-239 & 0.242 & $\mathrm{pCi} / \mathrm{g}$ & & 0.009 \\
\hline 383GB0700.5 & $2 / 4 / 2004$ & soil & HASL300 & REG & $14913-50-9$ & TI-208 & 0.247 & $\mathrm{pCi} / \mathrm{g}$ & $\mathrm{G}$ & 0.113 \\
\hline 383GB0800.5 & $2 / 4 / 2004$ & soil & HASL300 & FD & 14331-83-0 & Ac-228 & 1.36 & $\mathrm{pCi} / \mathrm{g}$ & $\mathrm{G}$ & 0.52 \\
\hline 383GB0800.5 & $2 / 4 / 2004$ & soil & HASL300 & FD & $14596-10-2$ & Am-241 & 0.62 & $\mathrm{pCi} / \mathrm{g}$ & $\mathrm{J}$ & 0.28 \\
\hline 383GB0800.5 & $2 / 4 / 2004$ & soil & EPA6010 & FD & $7440-38-2$ & Arsenic & 4.5 & $\mathrm{mg} / \mathrm{kg}$ & & 1.2 \\
\hline 383GB0800.5 & $2 / 4 / 2004$ & soil & EPA6010 & FD & $7440-39-3$ & Barium & 260 & $\mathrm{mg} / \mathrm{kg}$ & & 12 \\
\hline 383GB0800.5 & $2 / 4 / 2004$ & soil & EPA6010 & FD & $7440-41-7$ & Beryllium & 0.87 & $\mathrm{mg} / \mathrm{kg}$ & & 0.58 \\
\hline 383GB0800.5 & $2 / 4 / 2004$ & soil & HASL300 & FD & $14733-03-0$ & $\mathrm{Bi}-214$ & 0.73 & $\mathrm{pCi} / \mathrm{g}$ & $\mathrm{G}$ & 0.3 \\
\hline 383GB0800.5 & $2 / 4 / 2004$ & soil & EPA6010 & FD & $7440-43-9$ & Cadmium & 0.081 & $\mathrm{mg} / \mathrm{kg}$ & $\mathrm{J}$ & 0.58 \\
\hline 383GB0800.5 & $2 / 4 / 2004$ & soil & EPA6010 & FD & $7440-47-3$ & Chromium & 8.4 & $\mathrm{mg} / \mathrm{kg}$ & & 1.2 \\
\hline 383GB0800.5 & $2 / 4 / 2004$ & soil & HASL300 & FD & $10045-97-3$ & Cs-137 & 2.25 & $\mathrm{pCi} / \mathrm{g}$ & $\mathrm{G}$ & 0.2 \\
\hline 383GB0800.5 & $2 / 4 / 2004$ & soil & HASL300 & FD & 13966-00-2 & $\mathrm{K}-40$ & 12.9 & $\mathrm{pCi} / \mathrm{g}$ & G & 2 \\
\hline 383GB0800.5 & $2 / 4 / 2004$ & soil & EPA6010 & FD & 7439-92-1 & Lead & 17 & $\mathrm{mg} / \mathrm{kg}$ & & 1.7 \\
\hline 383GB0800.5 & $2 / 4 / 2004$ & soil & EPA7470 & FD & $7439-97-6$ & Mercury & 0.035 & $\mathrm{mg} / \mathrm{kg}$ & $\mathrm{B}$ & 0.12 \\
\hline 383GB0800.5 & $2 / 4 / 2004$ & soil & HASL300 & FD & 15092-94-1 & $\mathrm{Pb}-212$ & 1.26 & $\mathrm{pCi} / \mathrm{g}$ & $\mathrm{J}$ & 0.26 \\
\hline 383GB0800.5 & $2 / 4 / 2004$ & soil & HASL300 & FD & 15067-28-4 & $\mathrm{Pb}-214$ & 0.97 & $\mathrm{pCi} / \mathrm{g}$ & $\mathrm{J}$ & 0.36 \\
\hline
\end{tabular}

Page 26 of 78

Uncontrolled When Printed 


\begin{tabular}{|c|c|c|c|c|c|c|c|c|c|c|}
\hline SAMPLE \# & $\begin{array}{c}\text { SAMPLE } \\
\text { DATE }\end{array}$ & MATRIX & $\begin{array}{l}\text { USER TEST } \\
\text { PANEL }\end{array}$ & $\begin{array}{c}\text { SAMPLE } \\
\text { PURPOSE }\end{array}$ & CAS \# & PARAMETER & RESULT & UNITS & $\mathbf{Q}$ & $\begin{array}{l}\text { DETECT } \\
\text { LIMIT }\end{array}$ \\
\hline 383GB0800.5 & $2 / 4 / 2004$ & soil & UGTAISOPU & FD & 13981-16-3 & Pu-238 & 0.71 & $\mathrm{pCi} / \mathrm{g}$ & & 0.01 \\
\hline 383GB0800.5 & $2 / 4 / 2004$ & soil & UGTAISOPU & FD & $15117-48-3$ & Pu-239 & 2.43 & $\mathrm{pCi} / \mathrm{g}$ & & 0.02 \\
\hline 383GB0800.5 & $2 / 4 / 2004$ & soil & HASL300 & FD & $14913-50-9$ & TI-208 & 0.51 & $\mathrm{pCi} / \mathrm{g}$ & G & 0.16 \\
\hline $383 G B 0901$ & $3 / 10 / 2004$ & soil & HASL300 & REG & 14331-83-0 & Ac-228 & 2.27 & $\mathrm{pCi} / \mathrm{g}$ & $G$ & 0.69 \\
\hline 383GB0901 & $3 / 10 / 2004$ & soil & HASL300 & REG & 14596-10-2 & Am-241 & 2.83 & $\mathrm{pCi} / \mathrm{g}$ & $\mathrm{J}$ & 0.65 \\
\hline $383 G B 0901$ & $3 / 10 / 2004$ & soil & HASL300 & REG & $10045-97-3$ & Cs-137 & 180 & $\mathrm{pCi} / \mathrm{g}$ & $G$ & 0 \\
\hline 383GB0901 & $3 / 10 / 2004$ & soil & HASL300 & REG & 13966-00-2 & $\mathrm{K}-40$ & 29.2 & $\mathrm{pCi} / \mathrm{g}$ & $G$ & 2 \\
\hline 383GB0901 & $3 / 10 / 2004$ & soil & HASL300 & REG & 15092-94-1 & $\mathrm{Pb}-212$ & 2.12 & $\mathrm{pCi} / \mathrm{g}$ & $\mathrm{J}$ & 0.74 \\
\hline $383 G B 0901$ & $3 / 10 / 2004$ & soil & UGTAISOPU & REG & 13981-16-3 & Pu-238 & 11.3 & $\mathrm{pCi} / \mathrm{g}$ & & 0 \\
\hline $383 G B 0901$ & $3 / 10 / 2004$ & soil & UGTAISOPU & REG & $15117-48-3$ & Pu-239 & 61 & $\mathrm{pCi} / \mathrm{g}$ & M3 & 0 \\
\hline 383GB0901 & $3 / 10 / 2004$ & soil & SR7500 & REG & 10098-97-2 & Sr-90 & 18.8 & $\mathrm{pCi} / \mathrm{g}$ & & 0.3 \\
\hline 383MB0100.5 & $6 / 12 / 2004$ & soil & EPA6010 & REG & $7440-38-2$ & Arsenic & 0.7 & $\mathrm{mg} / \mathrm{kg}$ & $\mathrm{B}$ & 1 \\
\hline 383MB0100.5 & $6 / 12 / 2004$ & soil & EPA6010 & REG & $7440-39-3$ & Barium & 470 & $\mathrm{mg} / \mathrm{kg}$ & & 10 \\
\hline 383MB0100.5 & $6 / 12 / 2004$ & soil & EPA6010 & REG & $7440-41-7$ & Beryllium & 0.49 & $\mathrm{mg} / \mathrm{kg}$ & $\mathrm{B}$ & 0.51 \\
\hline 383MB0100.5 & $6 / 12 / 2004$ & soil & EPA6010 & REG & 7440-43-9 & Cadmium & 0.099 & $\mathrm{mg} / \mathrm{kg}$ & $B$ & 0.51 \\
\hline 383MB0100.5 & $6 / 12 / 2004$ & soil & EPA6010 & REG & 7440-47-3 & Chromium & 2 & $\mathrm{mg} / \mathrm{kg}$ & & 1 \\
\hline 383MB0100.5 & $6 / 12 / 2004$ & soil & HASL300 & REG & $10045-97-3$ & Cs-137 & 0.94 & $\mathrm{pCi} / \mathrm{g}$ & & 0.05 \\
\hline 383MB0100.5 & $6 / 12 / 2004$ & soil & HASL300 & REG & 13966-00-2 & $\mathrm{K}-40$ & 3.7 & $\mathrm{pCi} / \mathrm{g}$ & & 0.9 \\
\hline 383MB0100.5 & $6 / 12 / 2004$ & soil & EPA6010 & REG & 7439-92-1 & Lead & 3.4 & $\mathrm{mg} / \mathrm{kg}$ & & 1.5 \\
\hline 383MB0100.5 & $6 / 12 / 2004$ & soil & EPA7470 & REG & 7439-97-6 & Mercury & 0.073 & $\mathrm{mg} / \mathrm{kg}$ & $\mathrm{B}$ & 0.1 \\
\hline 383MB0100.5 & $6 / 12 / 2004$ & soil & EPA8260 & REG & $75-09-2$ & Methylene Chloride & 0.95 & $\mu \mathrm{g} / \mathrm{kg}$ & $\mathrm{J}, \mathrm{B}$ & 5.1 \\
\hline $383 \mathrm{MB} 0100.5$ & $6 / 12 / 2004$ & soil & HASL300 & REG & 15092-94-1 & $\mathrm{Pb}-212$ & 0.35 & $\mathrm{pCi} / \mathrm{g}$ & $\mathrm{J}$ & 0.12 \\
\hline 383MB0100.5 & $6 / 12 / 2004$ & soil & HASL300 & REG & $15067-28-4$ & $\mathrm{~Pb}-214$ & 0.3 & $\mathrm{pCi} / \mathrm{g}$ & $\mathrm{J}$ & 0.16 \\
\hline 383MB0100.5 & $6 / 12 / 2004$ & soil & EPA8260 & REG & $99-87-6$ & P-Isopropyltoluene & 1.3 & $\mu \mathrm{g} / \mathrm{kg}$ & $\mathrm{J}$ & 5.1 \\
\hline 383MB0200.5 & $5 / 24 / 2004$ & soil & HASL300 & REG & $14331-83-0$ & Ac-228 & 0.85 & $\mathrm{pCi} / \mathrm{g}$ & G & 0.34 \\
\hline 383MB0200.5 & $5 / 24 / 2004$ & soil & EPA8260 & REG & 67-64-1 & Acetone & 7.9 & $\mu \mathrm{g} / \mathrm{kg}$ & $\mathrm{J}$ & 22 \\
\hline 383MB0200.5 & $5 / 24 / 2004$ & soil & EPA6010 & REG & $7440-38-2$ & Arsenic & 1.9 & $\mathrm{mg} / \mathrm{kg}$ & & 1.1 \\
\hline 383MB0200.5 & $5 / 24 / 2004$ & soil & EPA6010 & REG & $7440-39-3$ & Barium & 910 & $\mathrm{mg} / \mathrm{kg}$ & & 11 \\
\hline $383 \mathrm{MB} 0200.5$ & $5 / 24 / 2004$ & soil & EPA8270 & REG & $56-55-3$ & Benzo(A)Anthracene & 38 & $\mu g / \mathrm{kg}$ & $\mathrm{J}$ & 720 \\
\hline 383MB0200.5 & $5 / 24 / 2004$ & soil & EPA6010 & REG & $7440-41-7$ & Beryllium & 0.47 & $\mathrm{mg} / \mathrm{kg}$ & B & 0.54 \\
\hline 383MB0200.5 & $5 / 24 / 2004$ & soil & HASL300 & REG & $14733-03-0$ & Bi-214 & 0.64 & $\mathrm{pCi} / \mathrm{g}$ & $\mathrm{G}, \mathrm{J}$ & 0.24 \\
\hline 383MB0200.5 & $5 / 24 / 2004$ & soil & EPA8270 & REG & 117-81-7 & Bis(2-Ethylhexyl)Phthalate & 170 & $\mu \mathrm{g} / \mathrm{kg}$ & $\mathrm{J}$ & 720 \\
\hline 383MB0200.5 & $5 / 24 / 2004$ & soil & EPA6010 & REG & 7440-43-9 & Cadmium & 0.74 & $\mathrm{mg} / \mathrm{kg}$ & & 0.54 \\
\hline 383MB0200.5 & $5 / 24 / 2004$ & soil & EPA6010 & REG & $7440-47-3$ & Chromium & 33 & $\mathrm{mg} / \mathrm{kg}$ & & 1.1 \\
\hline 383MB0200.5 & $5 / 24 / 2004$ & soil & HASL300 & REG & $10045-97-3$ & Cs-137 & 18.4 & $\mathrm{pCi} / \mathrm{g}$ & G & 0.1 \\
\hline
\end{tabular}

Page 27 of 78

Uncontrolled When Printed 


\begin{tabular}{|c|c|c|c|c|c|c|c|c|c|c|}
\hline SAMPLE \# & $\begin{array}{c}\text { SAMPLE } \\
\text { DATE }\end{array}$ & MATRIX & $\begin{array}{l}\text { USER TEST } \\
\text { PANEL }\end{array}$ & $\begin{array}{c}\text { SAMPLE } \\
\text { PURPOSE }\end{array}$ & CAS \# & PARAMETER & RESULT & UNITS & $\mathbf{Q}$ & $\begin{array}{l}\text { DETECT } \\
\text { LIMIT }\end{array}$ \\
\hline 383MB0200.5 & $5 / 24 / 2004$ & soil & EPA8015 & REG & $68334-30-5$ & Diesel-Range Organics & 45 & $\mathrm{mg} / \mathrm{kg}$ & $\mathrm{M}, \mathrm{H}$ & 5.4 \\
\hline 383MB0200.5 & $5 / 24 / 2004$ & soil & HASL300 & REG & $13966-00-2$ & $\mathrm{~K}-40$ & 9.8 & $\mathrm{pCi} / \mathrm{g}$ & $G$ & 1.3 \\
\hline 383MB0200.5 & $5 / 24 / 2004$ & soil & EPA6010 & REG & 7439-92-1 & Lead & 39 & $\mathrm{mg} / \mathrm{kg}$ & & 1.6 \\
\hline 383MB0200.5 & $5 / 24 / 2004$ & soil & EPA7470 & REG & 7439-97-6 & Mercury & 0.052 & $\mathrm{mg} / \mathrm{kg}$ & $\mathrm{B}$ & 0.11 \\
\hline 383MB0200.5 & $5 / 24 / 2004$ & soil & EPA8260 & REG & $75-09-2$ & Methylene Chloride & 0.88 & $\mu \mathrm{g} / \mathrm{kg}$ & $\mathrm{J}, \mathrm{B}$ & 5.4 \\
\hline 383MB0200.5 & $5 / 24 / 2004$ & soil & HASL300 & REG & 15092-94-1 & $\mathrm{Pb}-212$ & 0.68 & $\mathrm{pCi} / \mathrm{g}$ & $\mathrm{J}$ & 0.27 \\
\hline 383MB0200.5 & $5 / 24 / 2004$ & soil & HASL300 & REG & $15067-28-4$ & $\mathrm{~Pb}-214$ & 0.71 & $\mathrm{pCi} / \mathrm{g}$ & $\mathrm{G}, \mathrm{J}$ & 0.36 \\
\hline 383MB0200.5 & $5 / 24 / 2004$ & soil & UGTAISOPU & REG & 13981-16-3 & Pu-238 & 0.128 & $\mathrm{pCi} / \mathrm{g}$ & & 0.018 \\
\hline 383MB0200.5 & $5 / 24 / 2004$ & soil & UGTAISOPU & REG & $15117-48-3$ & Pu-239 & 1.33 & $\mathrm{pCi} / \mathrm{g}$ & & 0.02 \\
\hline 383MB0300.5 & $6 / 11 / 2004$ & soil & HASL300 & REG & 14331-83-0 & Ac-228 & 0.5 & $\mathrm{pCi} / \mathrm{g}$ & & 0.39 \\
\hline 383MB0300.5 & $6 / 11 / 2004$ & soil & EPA6010 & REG & $7440-38-2$ & Arsenic & 3.5 & $\mathrm{mg} / \mathrm{kg}$ & & 1 \\
\hline 383MB0300.5 & $6 / 11 / 2004$ & soil & EPA6010 & REG & $7440-39-3$ & Barium & 110 & $\mathrm{mg} / \mathrm{kg}$ & & 10 \\
\hline 383MB0300.5 & $6 / 11 / 2004$ & soil & EPA8270 & REG & $56-55-3$ & Benzo(A)Anthracene & 83 & $\mu \mathrm{g} / \mathrm{kg}$ & $\mathrm{J}$ & 340 \\
\hline 383MB0300.5 & $6 / 11 / 2004$ & soil & EPA8270 & REG & $50-32-8$ & Benzo(A)Pyrene & 78 & $\mu \mathrm{g} / \mathrm{kg}$ & $\mathrm{J}$ & 340 \\
\hline 383MB0300.5 & $6 / 11 / 2004$ & soil & EPA8270 & REG & $205-99-2$ & Benzo(B)Fluoranthene & 140 & $\mu \mathrm{g} / \mathrm{kg}$ & $\mathrm{J}$ & 340 \\
\hline $383 \mathrm{MB} 0300.5$ & $6 / 11 / 2004$ & soil & EPA8270 & REG & 191-24-2 & Benzo(G,H,I)Perylene & 68 & $\mu \mathrm{g} / \mathrm{kg}$ & $\mathrm{J}$ & 340 \\
\hline 383MB0300.5 & $6 / 11 / 2004$ & soil & EPA8270 & REG & $207-08-9$ & Benzo(K)Fluoranthene & 55 & $\mu \mathrm{g} / \mathrm{kg}$ & $\mathrm{J}$ & 340 \\
\hline 383MB0300.5 & $6 / 11 / 2004$ & soil & EPA6010 & REG & $7440-41-7$ & Beryllium & 0.44 & $\mathrm{mg} / \mathrm{kg}$ & $\mathrm{B}$ & 0.52 \\
\hline 383MB0300.5 & $6 / 11 / 2004$ & soil & HASL300 & REG & $14913-49-6$ & $\mathrm{Bi}-212$ & 1.77 & $\mathrm{pCi} / \mathrm{g}$ & $\mathrm{TI}$ & 1.03 \\
\hline 383MB0300.5 & $6 / 11 / 2004$ & soil & HASL300 & REG & $14733-03-0$ & Bi-214 & 0.39 & $\mathrm{pCi} / \mathrm{g}$ & $\mathrm{J}$ & 0.21 \\
\hline 383MB0300.5 & $6 / 11 / 2004$ & soil & EPA6010 & REG & $7440-43-9$ & Cadmium & 0.5 & $\mathrm{mg} / \mathrm{kg}$ & B & 0.52 \\
\hline $383 \mathrm{MB} 0300.5$ & $6 / 11 / 2004$ & soil & EPA6010 & REG & $7440-47-3$ & Chromium & 4.1 & $\mathrm{mg} / \mathrm{kg}$ & & 1 \\
\hline 383MB0300.5 & $6 / 11 / 2004$ & soil & EPA8270 & REG & 218-01-9 & Chrysene & 120 & $\mu \mathrm{g} / \mathrm{kg}$ & $\mathrm{J}$ & 340 \\
\hline 383MB0300.5 & $6 / 11 / 2004$ & soil & HASL300 & REG & $10045-97-3$ & Cs-137 & 24.2 & $\mathrm{pCi} / \mathrm{g}$ & & 0.1 \\
\hline 383MB0300.5 & $6 / 11 / 2004$ & soil & EPA8015 & REG & $68334-30-5$ & Diesel-Range Organics & 3.2 & $\mathrm{mg} / \mathrm{kg}$ & $\mathrm{J}$ & 5.2 \\
\hline 383MB0300.5 & $6 / 11 / 2004$ & soil & EPA8270 & REG & $206-44-0$ & Fluoranthene & 110 & $\mu \mathrm{g} / \mathrm{kg}$ & $\mathrm{J}$ & 340 \\
\hline 383MB0300.5 & $6 / 11 / 2004$ & soil & EPA8270 & REG & $193-39-5$ & Indeno(1,2,3-Cd)Pyrene & 45 & $\mu \mathrm{g} / \mathrm{kg}$ & $\mathrm{J}$ & 340 \\
\hline 383MB0300.5 & $6 / 11 / 2004$ & soil & HASL300 & REG & $13966-00-2$ & $\mathrm{~K}-40$ & 8.4 & $\mathrm{pCi} / \mathrm{g}$ & & 0.7 \\
\hline $383 \mathrm{MB} 0300.5$ & $6 / 11 / 2004$ & soil & EPA6010 & REG & 7439-92-1 & Lead & 18 & $\mathrm{mg} / \mathrm{kg}$ & & 1.6 \\
\hline $383 \mathrm{MB} 0300.5$ & $6 / 11 / 2004$ & soil & EPA7470 & REG & $7439-97-6$ & Mercury & 0.11 & $\mathrm{mg} / \mathrm{kg}$ & & 0.1 \\
\hline $383 \mathrm{MB} 0300.5$ & $6 / 11 / 2004$ & soil & HASL300 & REG & 15092-94-1 & $\mathrm{Pb}-212$ & 0.79 & $\mathrm{pCi} / \mathrm{g}$ & & 0.24 \\
\hline 383MB0300.5 & $6 / 11 / 2004$ & soil & HASL300 & REG & $15067-28-4$ & $\mathrm{~Pb}-214$ & 0.53 & $\mathrm{pCi} / \mathrm{g}$ & $\mathrm{J}$ & 0.33 \\
\hline 383MB0300.5 & $6 / 11 / 2004$ & soil & EPA8270 & REG & 85-01-8 & Phenanthrene & 30 & $\mu \mathrm{g} / \mathrm{kg}$ & $\mathrm{J}$ & 340 \\
\hline 383MB0300.5 & $6 / 11 / 2004$ & soil & UGTAISOPU & REG & 13981-16-3 & Pu-238 & 0.158 & $\mathrm{pCi} / \mathrm{g}$ & & 0.011 \\
\hline 383MB0300.5 & $6 / 11 / 2004$ & soil & UGTAISOPU & REG & 15117-48-3 & Pu-239 & 1.35 & $\mathrm{pCi} / \mathrm{g}$ & & 0.03 \\
\hline
\end{tabular}

Page 28 of 78

Uncontrolled When Printed 


\begin{tabular}{|c|c|c|c|c|c|c|c|c|c|c|}
\hline SAMPLE \# & $\begin{array}{c}\text { SAMPLE } \\
\text { DATE }\end{array}$ & MATRIX & $\begin{array}{l}\text { USER TEST } \\
\text { PANEL }\end{array}$ & $\begin{array}{c}\text { SAMPLE } \\
\text { PURPOSE }\end{array}$ & CAS \# & PARAMETER & RESULT & UNITS & Q & $\begin{array}{c}\text { DETECT } \\
\text { LIMIT }\end{array}$ \\
\hline 383MB0300.5 & $6 / 11 / 2004$ & soil & EPA8270 & REG & $129-00-0$ & Pyrene & 100 & $\mu \mathrm{g} / \mathrm{kg}$ & $\mathrm{J}$ & 340 \\
\hline 383MB0300.5 & $6 / 11 / 2004$ & soil & HASL300 & REG & $14913-50-9$ & TI-208 & 0.131 & $\mathrm{pCi} / \mathrm{g}$ & & 0.117 \\
\hline 383MB0305.5 & $6 / 11 / 2004$ & soil & EPA6010 & REG & $7440-38-2$ & Arsenic & 2.8 & $\mathrm{mg} / \mathrm{kg}$ & & 1 \\
\hline 383MB0305.5 & $6 / 11 / 2004$ & soil & EPA6010 & REG & $7440-39-3$ & Barium & 45 & $\mathrm{mg} / \mathrm{kg}$ & & 10 \\
\hline 383MB0305.5 & $6 / 11 / 2004$ & soil & EPA8270 & REG & $56-55-3$ & Benzo(A)Anthracene & 22 & $\mu \mathrm{g} / \mathrm{kg}$ & $\mathrm{J}$ & 340 \\
\hline 383MB0305.5 & $6 / 11 / 2004$ & soil & EPA8270 & REG & $50-32-8$ & Benzo(A)Pyrene & 16 & $\mu \mathrm{g} / \mathrm{kg}$ & $\mathrm{J}$ & 340 \\
\hline 383MB0305.5 & $6 / 11 / 2004$ & soil & EPA8270 & REG & $205-99-2$ & Benzo(B)Fluoranthene & 28 & $\mu \mathrm{g} / \mathrm{kg}$ & $\mathrm{J}$ & 340 \\
\hline 383MB0305.5 & $6 / 11 / 2004$ & soil & EPA6010 & REG & $7440-41-7$ & Beryllium & 0.3 & $\mathrm{mg} / \mathrm{kg}$ & B & 0.51 \\
\hline 383MB0305.5 & $6 / 11 / 2004$ & soil & HASL300 & REG & $14733-03-0$ & Bi-214 & 0.26 & $\mathrm{pCi} / \mathrm{g}$ & $\mathrm{J}$ & 0.17 \\
\hline 383MB0305.5 & $6 / 11 / 2004$ & soil & EPA6010 & REG & $7440-43-9$ & Cadmium & 0.15 & $\mathrm{mg} / \mathrm{kg}$ & $B$ & 0.51 \\
\hline 383MB0305.5 & $6 / 11 / 2004$ & soil & EPA6010 & REG & $7440-47-3$ & Chromium & 2.8 & $\mathrm{mg} / \mathrm{kg}$ & & 1 \\
\hline 383MB0305.5 & $6 / 11 / 2004$ & soil & EPA8270 & REG & 218-01-9 & Chrysene & 23 & $\mu \mathrm{g} / \mathrm{kg}$ & $\mathrm{J}$ & 340 \\
\hline 383MB0305.5 & $6 / 11 / 2004$ & soil & HASL300 & REG & $10045-97-3$ & Cs-137 & 4.65 & $\mathrm{pCi} / \mathrm{g}$ & & 0.09 \\
\hline 383MB0305.5 & $6 / 11 / 2004$ & soil & EPA8270 & REG & 206-44-0 & Fluoranthene & 23 & $\mu \mathrm{g} / \mathrm{kg}$ & $\mathrm{J}$ & 340 \\
\hline 383MB0305.5 & $6 / 11 / 2004$ & soil & HASL300 & REG & 13966-00-2 & $\mathrm{K}-40$ & 2.5 & $\mathrm{pCi} / \mathrm{g}$ & & 1.08 \\
\hline 383MB0305.5 & $6 / 11 / 2004$ & soil & EPA6010 & REG & 7439-92-1 & Lead & 6.1 & $\mathrm{mg} / \mathrm{kg}$ & & 1.5 \\
\hline 383MB0305.5 & $6 / 11 / 2004$ & soil & EPA7470 & REG & $7439-97-6$ & Mercury & 0.2 & $\mathrm{mg} / \mathrm{kg}$ & & 0.1 \\
\hline 383MB0305.5 & $6 / 11 / 2004$ & soil & HASL300 & REG & 15092-94-1 & $\mathrm{Pb}-212$ & 0.3 & $\mathrm{pCi} / \mathrm{g}$ & & 0.14 \\
\hline 383MB0305.5 & $6 / 11 / 2004$ & soil & HASL300 & REG & $15067-28-4$ & $\mathrm{~Pb}-214$ & 0.33 & $\mathrm{pCi} / \mathrm{g}$ & J & 0.22 \\
\hline 383MB0305.5 & $6 / 11 / 2004$ & soil & UGTAISOPU & REG & 13981-16-3 & Pu-238 & 0.102 & $\mathrm{pCi} / \mathrm{g}$ & & 0.011 \\
\hline 383MB0305.5 & $6 / 11 / 2004$ & soil & UGTAISOPU & REG & $15117-48-3$ & Pu-239 & 0.79 & $\mathrm{pCi} / \mathrm{g}$ & & 0.02 \\
\hline 383MB0305.5 & $6 / 11 / 2004$ & soil & HASL300 & REG & 14913-50-9 & TI-208 & 0.103 & $\mathrm{pCi} / \mathrm{g}$ & & 0.098 \\
\hline 383MB0400.5 & $6 / 1 / 2004$ & soil & HASL300 & REG & 14331-83-0 & Ac-228 & 2.89 & $\mathrm{pCi} / \mathrm{g}$ & $\mathrm{G}, \mathrm{TI}$ & 0.53 \\
\hline 383MB0400.5 & $6 / 1 / 2004$ & soil & HASL300 & REG & $14596-10-2$ & Am-241 & 6.2 & $\mathrm{pCi} / \mathrm{g}$ & $\mathrm{J}$ & 3.1 \\
\hline 383MB0400.5 & $6 / 1 / 2004$ & soil & EPA6010 & REG & 7440-38-2 & Arsenic & 2.9 & $\mathrm{mg} / \mathrm{kg}$ & & 1.1 \\
\hline 383MB0400.5 & $6 / 1 / 2004$ & soil & EPA6010 & REG & $7440-39-3$ & Barium & 440 & $\mathrm{mg} / \mathrm{kg}$ & & 11 \\
\hline 383MB0400.5 & $6 / 1 / 2004$ & soil & EPA8270 & REG & $56-55-3$ & Benzo(A)Anthracene & 90 & $\mu \mathrm{g} / \mathrm{kg}$ & $\mathrm{J}$ & 360 \\
\hline $383 M B 0400.5$ & $6 / 1 / 2004$ & soil & EPA8270 & REG & $50-32-8$ & Benzo(A)Pyrene & 73 & $\mu \mathrm{g} / \mathrm{kg}$ & $\mathrm{J}$ & 360 \\
\hline $383 \mathrm{MB} 0400.5$ & $6 / 1 / 2004$ & soil & EPA8270 & REG & $205-99-2$ & Benzo(B)Fluoranthene & 130 & $\mu \mathrm{g} / \mathrm{kg}$ & $\mathrm{J}$ & 360 \\
\hline 383MB0400.5 & $6 / 1 / 2004$ & soil & EPA8270 & REG & 207-08-9 & Benzo(K)Fluoranthene & 60 & $\mu \mathrm{g} / \mathrm{kg}$ & $\mathrm{J}$ & 360 \\
\hline $383 \mathrm{MB} 0400.5$ & $6 / 1 / 2004$ & soil & EPA6010 & REG & $7440-41-7$ & Beryllium & 0.92 & $\mathrm{mg} / \mathrm{kg}$ & & 0.53 \\
\hline 383MB0400.5 & $6 / 1 / 2004$ & soil & EPA6010 & REG & $7440-43-9$ & Cadmium & 15 & $\mathrm{mg} / \mathrm{kg}$ & & 0.53 \\
\hline 383MB0400.5 & $6 / 1 / 2004$ & soil & EPA6010 & REG & $7440-47-3$ & Chromium & 2.2 & $\mathrm{mg} / \mathrm{kg}$ & & 1.1 \\
\hline 383MB0400.5 & $6 / 1 / 2004$ & soil & EPA8270 & REG & 218-01-9 & Chrysene & 95 & $\mu \mathrm{g} / \mathrm{kg}$ & $\mathrm{J}$ & 360 \\
\hline $383 \mathrm{MB} 0400.5$ & $6 / 1 / 2004$ & soil & HASL300 & REG & 10045-97-3 & Cs-137 & 393 & $\mathrm{pCi} / \mathrm{g}$ & M3,G & 1 \\
\hline
\end{tabular}

Page 29 of 78

Uncontrolled When Printed 


\begin{tabular}{|c|c|c|c|c|c|c|c|c|c|c|}
\hline SAMPLE \# & $\begin{array}{l}\text { SAMPLE } \\
\text { DATE }\end{array}$ & MATRIX & $\begin{array}{l}\text { USER TEST } \\
\text { PANEL }\end{array}$ & $\begin{array}{c}\text { SAMPLE } \\
\text { PURPOSE }\end{array}$ & CAS \# & PARAMETER & RESULT & UNITS & $\mathbf{Q}$ & $\begin{array}{l}\text { DETECT } \\
\text { LIMIT }\end{array}$ \\
\hline 383MB0400.5 & $6 / 1 / 2004$ & soil & EPA8270 & REG & $206-44-0$ & Fluoranthene & 98 & $\mu \mathrm{g} / \mathrm{kg}$ & $\mathrm{J}$ & 360 \\
\hline 383MB0400.5 & $6 / 1 / 2004$ & soil & HASL300 & REG & 13966-00-2 & $\mathrm{K}-40$ & 34.2 & $\mathrm{pCi} / \mathrm{g}$ & G & 1.6 \\
\hline 383MB0400.5 & $6 / 1 / 2004$ & soil & EPA6010 & REG & 7439-92-1 & Lead & 28 & $\mathrm{mg} / \mathrm{kg}$ & & 0.32 \\
\hline 383MB0400.5 & $6 / 1 / 2004$ & soil & HASL300 & REG & 15092-94-1 & $\mathrm{Pb}-212$ & 2.22 & $\mathrm{pCi} / \mathrm{g}$ & $\mathrm{J}$ & 1.07 \\
\hline 383MB0400.5 & $6 / 1 / 2004$ & soil & EPA8270 & REG & 85-01-8 & Phenanthrene & 37 & $\mu \mathrm{g} / \mathrm{kg}$ & $\mathrm{J}$ & 360 \\
\hline 383MB0400.5 & $6 / 1 / 2004$ & soil & UGTAISOPU & REG & 13981-16-3 & Pu-238 & 1.86 & $\mathrm{pCi} / \mathrm{g}$ & & 0.02 \\
\hline 383MB0400.5 & $6 / 1 / 2004$ & soil & UGTAISOPU & REG & $15117-48-3$ & Pu-239 & 17.8 & $\mathrm{pCi} / \mathrm{g}$ & $\mathrm{J}$ & 0 \\
\hline 383MB0400.5 & $6 / 1 / 2004$ & soil & EPA8270 & REG & $129-00-0$ & Pyrene & 91 & $\mu \mathrm{g} / \mathrm{kg}$ & $\mathrm{J}$ & 360 \\
\hline 383MB0400.5 & $6 / 1 / 2004$ & soil & EPA6010 & REG & $7782-49-2$ & Selenium & 0.38 & $\mathrm{mg} / \mathrm{kg}$ & $\mathrm{B}$ & 0.53 \\
\hline 383MB0400.5 & $6 / 1 / 2004$ & soil & SR7500 & REG & 10098-97-2 & Sr-90 & 2.5 & $\mathrm{pCi} / \mathrm{g}$ & Y1 & 0.13 \\
\hline 383MB0500.5 & $6 / 3 / 2004$ & soil & HASL300 & REG & 14331-83-0 & Ac-228 & 2 & $\mathrm{pCi} / \mathrm{g}$ & $G$ & 0.75 \\
\hline 383MB0500.5 & $6 / 3 / 2004$ & soil & EPA6010 & REG & $7440-38-2$ & Arsenic & 3.2 & $\mathrm{mg} / \mathrm{kg}$ & & 1.1 \\
\hline 383MB0500.5 & $6 / 3 / 2004$ & soil & EPA6010 & REG & $7440-39-3$ & Barium & 2500 & $\mathrm{mg} / \mathrm{kg}$ & & 110 \\
\hline 383MB0500.5 & $6 / 3 / 2004$ & soil & EPA6010 & REG & $7440-41-7$ & Beryllium & 1 & $\mathrm{mg} / \mathrm{kg}$ & & 0.56 \\
\hline 383MB0500.5 & $6 / 3 / 2004$ & soil & HASL300 & REG & $14733-03-0$ & Bi-214 & 1.1 & $\mathrm{pCi} / \mathrm{g}$ & $\mathrm{G}, \mathrm{J}$ & 0.45 \\
\hline 383MB0500.5 & $6 / 3 / 2004$ & soil & EPA6010 & REG & 7440-43-9 & Cadmium & 0.2 & $\mathrm{mg} / \mathrm{kg}$ & $\mathrm{B}$ & 0.56 \\
\hline 383MB0500.5 & $6 / 3 / 2004$ & soil & EPA6010 & REG & $7440-47-3$ & Chromium & 3 & $\mathrm{mg} / \mathrm{kg}$ & & 1.1 \\
\hline 383MB0500.5 & $6 / 3 / 2004$ & soil & HASL300 & REG & $10045-97-3$ & Cs-137 & 29.3 & $\mathrm{pCi} / \mathrm{g}$ & G & 0.2 \\
\hline 383MB0500.5 & $6 / 3 / 2004$ & soil & EPA8015 & REG & $68334-30-5$ & Diesel-Range Organics & 160 & $\mathrm{mg} / \mathrm{kg}$ & $\mathrm{Z}, \mathrm{H}$ & 5.6 \\
\hline 383MB0500.5 & $6 / 3 / 2004$ & soil & HASL300 & REG & $13966-00-2$ & $\mathrm{~K}-40$ & 24.6 & $\mathrm{pCi} / \mathrm{g}$ & $\mathrm{G}$ & 1.9 \\
\hline 383MB0500.5 & $6 / 3 / 2004$ & soil & EPA6010 & REG & 7439-92-1 & Lead & 29 & $\mathrm{mg} / \mathrm{kg}$ & & 0.34 \\
\hline 383MB0500.5 & $6 / 3 / 2004$ & soil & EPA7470 & REG & 7439-97-6 & Mercury & 0.016 & $\mathrm{mg} / \mathrm{kg}$ & $\mathrm{B}$ & 0.11 \\
\hline 383MB0500.5 & $6 / 3 / 2004$ & soil & EPA8260 & REG & $75-09-2$ & Methylene Chloride & 1.1 & $\mu \mathrm{g} / \mathrm{kg}$ & $\mathrm{J}$ & 5.6 \\
\hline 383MB0500.5 & $6 / 3 / 2004$ & soil & EPA8260 & REG & $95-47-6$ & O-Xylene & 1.2 & $\mu \mathrm{g} / \mathrm{kg}$ & $\mathrm{J}$ & 5.6 \\
\hline 383MB0500.5 & $6 / 3 / 2004$ & soil & HASL300 & REG & 15092-94-1 & $\mathrm{Pb}-212$ & 2.03 & $\mathrm{pCi} / \mathrm{g}$ & $\mathrm{J}$ & 0.33 \\
\hline 383MB0500.5 & $6 / 3 / 2004$ & soil & HASL300 & REG & $15067-28-4$ & $\mathrm{~Pb}-214$ & 1.37 & $\mathrm{pCi} / \mathrm{g}$ & $\mathrm{G}, \mathrm{J}$ & 0.52 \\
\hline $383 \mathrm{MB} 0500.5$ & $6 / 3 / 2004$ & soil & UGTAISOPU & REG & 13981-16-3 & Pu-238 & 0.191 & $\mathrm{pCi} / \mathrm{g}$ & & 0.011 \\
\hline 383MB0500.5 & $6 / 3 / 2004$ & soil & UGTAISOPU & REG & $15117-48-3$ & Pu-239 & 0.73 & $\mathrm{pCi} / \mathrm{g}$ & $\mathrm{J}$ & 0.02 \\
\hline 383MB0500.5 & $6 / 3 / 2004$ & soil & SR7500 & REG & 10098-97-2 & Sr-90 & 0.51 & $\mathrm{pCi} / \mathrm{g}$ & Y1 & 0.25 \\
\hline $383 \mathrm{MB} 0500.5$ & $6 / 3 / 2004$ & soil & HASL300 & REG & $14913-50-9$ & TI-208 & 0.57 & $\mathrm{pCi} / \mathrm{g}$ & $\mathrm{G}$ & 0.2 \\
\hline 383MB0543.5 & $6 / 3 / 2004$ & soil & HASL300 & REG & 14331-83-0 & Ac-228 & 2.81 & $\mathrm{pCi} / \mathrm{g}$ & $\mathrm{G}$ & 0.78 \\
\hline $383 \mathrm{MB0543.5}$ & $6 / 3 / 2004$ & soil & HASL300 & REG & 14596-10-2 & Am-241 & 28.8 & $\mathrm{pCi} / \mathrm{g}$ & $\mathrm{J}$ & 1.6 \\
\hline 383MB0543.5 & $6 / 3 / 2004$ & soil & EPA6010 & REG & $7440-38-2$ & Arsenic & 2.2 & $\mathrm{mg} / \mathrm{kg}$ & & 1.2 \\
\hline 383MB0543.5 & $6 / 3 / 2004$ & soil & EPA6010 & REG & $7440-39-3$ & Barium & 85 & $\mathrm{mg} / \mathrm{kg}$ & & 12 \\
\hline 383MB0543.5 & $6 / 3 / 2004$ & soil & EPA6010 & REG & 7440-41-7 & Beryllium & 1 & $\mathrm{mg} / \mathrm{kg}$ & & 0.59 \\
\hline
\end{tabular}

Page 30 of 78

Uncontrolled When Printed 


\begin{tabular}{|c|c|c|c|c|c|c|c|c|c|c|}
\hline SAMPLE \# & $\begin{array}{c}\text { SAMPLE } \\
\text { DATE }\end{array}$ & MATRIX & $\begin{array}{l}\text { USER TEST } \\
\text { PANEL }\end{array}$ & $\begin{array}{c}\text { SAMPLE } \\
\text { PURPOSE }\end{array}$ & CAS \# & PARAMETER & RESULT & UNITS & $\mathbf{Q}$ & $\begin{array}{c}\text { DETECT } \\
\text { LIMIT }\end{array}$ \\
\hline 383MB0543.5 & $6 / 3 / 2004$ & soil & EPA6010 & REG & $7440-43-9$ & Cadmium & 0.078 & $\mathrm{mg} / \mathrm{kg}$ & B & 0.59 \\
\hline 383MB0543.5 & $6 / 3 / 2004$ & soil & EPA6010 & REG & $7440-47-3$ & Chromium & 1.1 & $\mathrm{mg} / \mathrm{kg}$ & $\mathrm{B}$ & 1.2 \\
\hline 383MB0543.5 & $6 / 3 / 2004$ & soil & HASL300 & REG & $10045-97-3$ & Cs-137 & 1060 & $\mathrm{pCi} / \mathrm{g}$ & M3,G & 0 \\
\hline 383MB0543.5 & $6 / 3 / 2004$ & soil & HASL300 & REG & $14683-23-9$ & Eu-152 & 2.64 & $\mathrm{pCi} / \mathrm{g}$ & G & 0.81 \\
\hline 383MB0543.5 & $6 / 3 / 2004$ & soil & HASL300 & REG & 13966-00-2 & $\mathrm{K}-40$ & 39.8 & $\mathrm{pCi} / \mathrm{g}$ & $G$ & 1.7 \\
\hline 383MB0543.5 & $6 / 3 / 2004$ & soil & EPA6010 & REG & $7439-92-1$ & Lead & 22 & $\mathrm{mg} / \mathrm{kg}$ & & 0.36 \\
\hline 383MB0543.5 & $6 / 3 / 2004$ & soil & EPA7470 & REG & 7439-97-6 & Mercury & 0.0098 & $\mathrm{mg} / \mathrm{kg}$ & $\mathrm{B}$ & 0.12 \\
\hline 383MB0543.5 & $6 / 3 / 2004$ & soil & EPA8260 & REG & $75-09-2$ & Methylene Chloride & 0.96 & $\mu \mathrm{g} / \mathrm{kg}$ & $\mathrm{J}$ & 5.9 \\
\hline 383MB0543.5 & $6 / 3 / 2004$ & soil & UGTAISOPU & REG & 13981-16-3 & Pu-238 & 15.6 & $\mathrm{pCi} / \mathrm{g}$ & $\mathrm{J}$ & 0 \\
\hline 383MB0543.5 & $6 / 3 / 2004$ & soil & UGTAISOPU & REG & $15117-48-3$ & Pu-239 & 84 & $\mathrm{pCi} / \mathrm{g}$ & $\mathrm{J}$ & 0 \\
\hline 383MB0543.5 & $6 / 3 / 2004$ & soil & SR7500 & REG & 10098-97-2 & Sr-90 & 34.1 & $\mathrm{pCi} / \mathrm{g}$ & & 0.2 \\
\hline 383MB0586.0 & $6 / 3 / 2004$ & soil & EPA8260 & REG & 67-64-1 & Acetone & 8.8 & $\mu \mathrm{g} / \mathrm{kg}$ & $\mathrm{J}$ & 20 \\
\hline 383MB0586.0 & $6 / 3 / 2004$ & soil & EPA6010 & REG & $7440-38-2$ & Arsenic & 0.31 & $\mathrm{mg} / \mathrm{kg}$ & $\mathrm{B}$ & 1 \\
\hline 383MB0586.0 & $6 / 3 / 2004$ & soil & EPA6010 & REG & $7440-39-3$ & Barium & 4.8 & $\mathrm{mg} / \mathrm{kg}$ & $\mathrm{B}$ & 10 \\
\hline 383MB0586.0 & $6 / 3 / 2004$ & soil & EPA6010 & REG & $7440-41-7$ & Beryllium & 0.2 & $\mathrm{mg} / \mathrm{kg}$ & $\mathrm{B}$ & 0.5 \\
\hline 383MB0586.0 & $6 / 3 / 2004$ & soil & EPA6010 & REG & $7440-43-9$ & Cadmium & 0.042 & $\mathrm{mg} / \mathrm{kg}$ & $\mathrm{B}$ & 0.5 \\
\hline 383MB0586.0 & $6 / 3 / 2004$ & soil & EPA6010 & REG & $7440-47-3$ & Chromium & 1.4 & $\mathrm{mg} / \mathrm{kg}$ & & 1 \\
\hline 383MB0586.0 & $6 / 3 / 2004$ & soil & EPA7470 & REG & $7439-97-6$ & Mercury & 0.008 & $\mathrm{mg} / \mathrm{kg}$ & $\mathrm{B}$ & 0.1 \\
\hline 383MB0586.0 & $6 / 3 / 2004$ & soil & EPA8260 & REG & $75-09-2$ & Methylene Chloride & 1.3 & $\mu \mathrm{g} / \mathrm{kg}$ & $\mathrm{J}$ & 5 \\
\hline 383MB0586.0 & $6 / 3 / 2004$ & soil & HASL300 & REG & $15067-28-4$ & $\mathrm{~Pb}-214$ & 0.313 & $\mathrm{pCi} / \mathrm{g}$ & $\mathrm{J}$ & 0.122 \\
\hline 383MB0586.0 & $6 / 3 / 2004$ & soil & EPA6010 & REG & $7440-22-4$ & Silver & 0.19 & $\mathrm{mg} / \mathrm{kg}$ & B & 1 \\
\hline $383 \mathrm{MB} 0600.5$ & $6 / 12 / 2004$ & soil & HASL300 & REG & $14331-83-0$ & Ac-228 & 1.01 & $\mathrm{pCi} / \mathrm{g}$ & $\mathrm{G}$ & 0.51 \\
\hline 383MB0600.5 & $6 / 12 / 2004$ & soil & EPA8260 & REG & 67-64-1 & Acetone & 8.1 & $\mu \mathrm{g} / \mathrm{kg}$ & $\mathrm{J}$ & 24 \\
\hline 383MB0600.5 & $6 / 12 / 2004$ & soil & EPA6010 & REG & $7440-38-2$ & Arsenic & 4.4 & $\mathrm{mg} / \mathrm{kg}$ & & 1.2 \\
\hline 383MB0600.5 & $6 / 12 / 2004$ & soil & EPA6010 & REG & 7440-39-3 & Barium & 7000 & $\mathrm{mg} / \mathrm{kg}$ & & 600 \\
\hline 383MB0600.5 & $6 / 12 / 2004$ & soil & EPA6010 & REG & 7440-41-7 & Beryllium & 0.94 & $\mathrm{mg} / \mathrm{kg}$ & & 0.6 \\
\hline 383MB0600.5 & $6 / 12 / 2004$ & soil & HASL300 & REG & 14733-03-0 & Bi-214 & 1.09 & $\mathrm{pCi} / \mathrm{g}$ & $\mathrm{G}, \mathrm{J}$ & 0.29 \\
\hline 383MB0600.5 & $6 / 12 / 2004$ & soil & EPA6010 & REG & 7440-43-9 & Cadmium & 0.13 & $\mathrm{mg} / \mathrm{kg}$ & $B$ & 0.6 \\
\hline $383 \mathrm{MB} 0600.5$ & $6 / 12 / 2004$ & soil & EPA6010 & REG & $7440-47-3$ & Chromium & 6.8 & $\mathrm{mg} / \mathrm{kg}$ & & 1.2 \\
\hline $383 \mathrm{MB} 0600.5$ & $6 / 12 / 2004$ & soil & HASL300 & REG & $10045-97-3$ & Cs-137 & 0.6 & $\mathrm{pCi} / \mathrm{g}$ & $\mathrm{G}$ & 0.13 \\
\hline $383 \mathrm{MB} 0600.5$ & $6 / 12 / 2004$ & soil & HASL300 & REG & 13966-00-2 & $\mathrm{K}-40$ & 27.7 & $\mathrm{pCi} / \mathrm{g}$ & $\mathrm{G}$ & 1.6 \\
\hline $383 \mathrm{MB} 0600.5$ & $6 / 12 / 2004$ & soil & EPA6010 & REG & 7439-92-1 & Lead & 22 & $\mathrm{mg} / \mathrm{kg}$ & & 0.36 \\
\hline 383MB0600.5 & $6 / 12 / 2004$ & soil & EPA7470 & REG & 7439-97-6 & Mercury & 0.0086 & $\mathrm{mg} / \mathrm{kg}$ & $\mathrm{B}$ & 0.12 \\
\hline 383MB0600.5 & $6 / 12 / 2004$ & soil & EPA8260 & REG & $75-09-2$ & Methylene Chloride & 0.99 & $\mu \mathrm{g} / \mathrm{kg}$ & $\mathrm{J}, \mathrm{B}$ & 6 \\
\hline $383 \mathrm{MB} 0600.5$ & $6 / 12 / 2004$ & soil & HASL300 & REG & 15092-94-1 & $\mathrm{Pb}-212$ & 1.6 & $\mathrm{pCi} / \mathrm{g}$ & $\mathrm{J}$ & 0.29 \\
\hline
\end{tabular}

Page 31 of 78

Uncontrolled When Printed 


\begin{tabular}{|c|c|c|c|c|c|c|c|c|c|c|}
\hline SAMPLE \# & $\begin{array}{c}\text { SAMPLE } \\
\text { DATE }\end{array}$ & MATRIX & $\begin{array}{l}\text { USER TEST } \\
\text { PANEL }\end{array}$ & $\begin{array}{c}\text { SAMPLE } \\
\text { PURPOSE }\end{array}$ & CAS \# & PARAMETER & RESULT & UNITS & $\mathbf{Q}$ & $\begin{array}{c}\text { DETECT } \\
\text { LIMIT }\end{array}$ \\
\hline 383MB0600.5 & $6 / 12 / 2004$ & soil & HASL300 & REG & $15067-28-4$ & $\mathrm{~Pb}-214$ & 1.14 & $\mathrm{pCi} / \mathrm{g}$ & $\mathrm{G}, \mathrm{J}$ & 0.27 \\
\hline 383MB0600.5 & $6 / 12 / 2004$ & soil & UGTAISOPU & REG & $15117-48-3$ & Pu-239 & 0.08 & $\mathrm{pCi} / \mathrm{g}$ & & 0.009 \\
\hline 383MB0600.5 & $6 / 12 / 2004$ & soil & HASL300 & REG & $14913-50-9$ & TI-208 & 0.55 & $\mathrm{pCi} / \mathrm{g}$ & $\mathrm{G}, \mathrm{TI}$ & 0.17 \\
\hline 383MB0600.5X & $6 / 12 / 2004$ & soil & HASL300 & REG & 14331-83-0 & Ac-228 & 1.68 & $\mathrm{pCi} / \mathrm{g}$ & G & 0.73 \\
\hline 383MB0600.5X & $6 / 12 / 2004$ & soil & EPA6010 & REG & $7440-38-2$ & Arsenic & 3.4 & $\mathrm{mg} / \mathrm{kg}$ & & 1.2 \\
\hline 383MB0600.5X & $6 / 12 / 2004$ & soil & EPA6010 & REG & $7440-39-3$ & Barium & 6600 & $\mathrm{mg} / \mathrm{kg}$ & & 590 \\
\hline 383MB0600.5X & $6 / 12 / 2004$ & soil & EPA6010 & REG & $7440-41-7$ & Beryllium & 0.91 & $\mathrm{mg} / \mathrm{kg}$ & & 0.59 \\
\hline 383MB0600.5X & $6 / 12 / 2004$ & soil & HASL300 & REG & $14733-03-0$ & Bi-214 & 1.15 & $\mathrm{pCi} / \mathrm{g}$ & $\mathrm{G}, \mathrm{J}$ & 0.4 \\
\hline 383MB0600.5X & $6 / 12 / 2004$ & soil & EPA8270 & REG & $117-81-7$ & Bis(2-Ethylhexyl)Phthalate & 630 & $\mu \mathrm{g} / \mathrm{kg}$ & & 400 \\
\hline 383MB0600.5X & $6 / 12 / 2004$ & soil & EPA6010 & REG & $7440-43-9$ & Cadmium & 0.12 & $\mathrm{mg} / \mathrm{kg}$ & $\mathrm{B}$ & 0.59 \\
\hline 383MB0600.5X & $6 / 12 / 2004$ & soil & EPA6010 & REG & $7440-47-3$ & Chromium & 5.8 & $\mathrm{mg} / \mathrm{kg}$ & & 1.2 \\
\hline 383MB0600.5X & $6 / 12 / 2004$ & soil & HASL300 & REG & $10045-97-3$ & Cs-137 & 0.67 & $\mathrm{pCi} / \mathrm{g}$ & G & 0.16 \\
\hline 383MB0600.5X & $6 / 12 / 2004$ & soil & HASL300 & REG & $13966-00-2$ & $\mathrm{~K}-40$ & 30 & $\mathrm{pCi} / \mathrm{g}$ & G & 1.7 \\
\hline 383MB0600.5X & $6 / 12 / 2004$ & soil & EPA6010 & REG & 7439-92-1 & Lead & 22 & $\mathrm{mg} / \mathrm{kg}$ & & 0.36 \\
\hline 383MB0600.5X & $6 / 12 / 2004$ & soil & EPA7470 & REG & 7439-97-6 & Mercury & 0.011 & $\mathrm{mg} / \mathrm{kg}$ & $\mathrm{B}$ & 0.12 \\
\hline 383MB0600.5X & $6 / 12 / 2004$ & soil & EPA8260 & REG & $75-09-2$ & Methylene Chloride & 1.1 & $\mu \mathrm{g} / \mathrm{kg}$ & $\mathrm{J}, \mathrm{B}$ & 5.9 \\
\hline 383MB0600.5X & $6 / 12 / 2004$ & soil & HASL300 & REG & 15092-94-1 & $\mathrm{Pb}-212$ & 1.85 & $\mathrm{pCi} / \mathrm{g}$ & $\mathrm{J}$ & 0.24 \\
\hline 383MB0600.5X & $6 / 12 / 2004$ & soil & HASL300 & REG & $15067-28-4$ & $\mathrm{~Pb}-214$ & 1.14 & $\mathrm{pCi} / \mathrm{g}$ & $\mathrm{G}, \mathrm{J}$ & 0.32 \\
\hline 383MB0600.5X & $6 / 12 / 2004$ & soil & UGTAISOPU & REG & $15117-48-3$ & Pu-239 & 0.068 & $\mathrm{pCi} / \mathrm{g}$ & & 0.023 \\
\hline 383MB0600.5X & $6 / 12 / 2004$ & soil & SR7500 & REG & 10098-97-2 & Sr-90 & 0.53 & $\mathrm{pCi} / \mathrm{g}$ & & 0.23 \\
\hline 383MB0600.5X & $6 / 12 / 2004$ & soil & HASL300 & REG & $14913-50-9$ & TI-208 & 0.57 & $\mathrm{pCi} / \mathrm{g}$ & G & 0.17 \\
\hline $383 \mathrm{MB} 0700.5$ & $5 / 19 / 2004$ & soil & HASL300 & REG & $14331-83-0$ & Ac-228 & 0.72 & $\mathrm{pCi} / \mathrm{g}$ & & 0.39 \\
\hline $383 \mathrm{MB} 0700.5$ & $5 / 19 / 2004$ & soil & EPA6010 & REG & $7440-38-2$ & Arsenic & 2.4 & $\mathrm{mg} / \mathrm{kg}$ & & 1 \\
\hline 383MB0700.5 & $5 / 19 / 2004$ & soil & EPA6010 & REG & $7440-39-3$ & Barium & 73 & $\mathrm{mg} / \mathrm{kg}$ & & 10 \\
\hline 383MB0700.5 & $5 / 19 / 2004$ & soil & EPA6010 & REG & 7440-41-7 & Beryllium & 0.5 & $\mathrm{mg} / \mathrm{kg}$ & $\mathrm{B}$ & 0.52 \\
\hline 383MB0700.5 & $5 / 19 / 2004$ & soil & HASL300 & REG & $14733-03-0$ & Bi-214 & 0.63 & $\mathrm{pCi} / \mathrm{g}$ & $\mathrm{J}$ & 0.23 \\
\hline 383MB0700.5 & $5 / 19 / 2004$ & soil & EPA6010 & REG & $7440-43-9$ & Cadmium & 0.065 & $\mathrm{mg} / \mathrm{kg}$ & $\mathrm{B}$ & 0.52 \\
\hline $383 M B 0700.5$ & $5 / 19 / 2004$ & soil & EPA6010 & REG & $7440-47-3$ & Chromium & 3.5 & $\mathrm{mg} / \mathrm{kg}$ & & 1 \\
\hline $383 \mathrm{MB} 0700.5$ & $5 / 19 / 2004$ & soil & HASL300 & REG & $10045-97-3$ & Cs-137 & 0.58 & $\mathrm{pCi} / \mathrm{g}$ & & 0.09 \\
\hline $383 \mathrm{MB} 0700.5$ & $5 / 19 / 2004$ & soil & EPA8270 & REG & 84-74-2 & Di-N-Butyl Phthalate & 26 & $\mu \mathrm{g} / \mathrm{kg}$ & $\mathrm{J}$ & 350 \\
\hline $383 \mathrm{MB} 0700.5$ & $5 / 19 / 2004$ & soil & HASL300 & REG & 13966-00-2 & $\mathrm{K}-40$ & 6.6 & $\mathrm{pCi} / \mathrm{g}$ & & 1.2 \\
\hline $383 \mathrm{MB} 0700.5$ & $5 / 19 / 2004$ & soil & EPA6010 & REG & 7439-92-1 & Lead & 17 & $\mathrm{mg} / \mathrm{kg}$ & & 1.6 \\
\hline 383MB0700.5 & $5 / 19 / 2004$ & soil & EPA7470 & REG & 7439-97-6 & Mercury & 0.11 & $\mathrm{mg} / \mathrm{kg}$ & & 0.1 \\
\hline 383MB0700.5 & $5 / 19 / 2004$ & soil & HASL300 & REG & 15092-94-1 & $\mathrm{Pb}-212$ & 0.68 & $\mathrm{pCi} / \mathrm{g}$ & $\mathrm{J}$ & 0.14 \\
\hline 383MB0700.5 & $5 / 19 / 2004$ & soil & HASL300 & REG & 15067-28-4 & $\mathrm{Pb}-214$ & 0.74 & $\mathrm{pCi} / \mathrm{g}$ & $\mathrm{J}$ & 0.15 \\
\hline
\end{tabular}

Page 32 of 78

Uncontrolled When Printed 


\begin{tabular}{|c|c|c|c|c|c|c|c|c|c|c|}
\hline SAMPLE \# & $\begin{array}{c}\text { SAMPLE } \\
\text { DATE }\end{array}$ & MATRIX & $\begin{array}{c}\text { USER TEST } \\
\text { PANEL }\end{array}$ & $\begin{array}{c}\text { SAMPLE } \\
\text { PURPOSE }\end{array}$ & CAS \# & PARAMETER & RESULT & UNITS & $\mathbf{Q}$ & $\begin{array}{l}\text { DETECT } \\
\text { LIMIT }\end{array}$ \\
\hline 383MB0700.5 & $5 / 19 / 2004$ & soil & UGTAISOPU & REG & 13981-16-3 & Pu-238 & 0.049 & $\mathrm{pCi} / \mathrm{g}$ & $\mathrm{LT}$ & 0.01 \\
\hline 383MB0700.5 & $5 / 19 / 2004$ & soil & UGTAISOPU & REG & $15117-48-3$ & Pu-239 & 0.217 & $\mathrm{pCi} / \mathrm{g}$ & & 0.01 \\
\hline 383MB0700.5 & $5 / 19 / 2004$ & soil & HASL300 & REG & $14913-50-9$ & TI-208 & 0.166 & $\mathrm{pCi} / \mathrm{g}$ & & 0.085 \\
\hline 383MB0800.5 & $5 / 24 / 2004$ & soil & EPA8260 & REG & $95-63-6$ & 1,2,4-Trimethylbenzene & 7.2 & $\mu \mathrm{g} / \mathrm{kg}$ & & 5.9 \\
\hline 383MB0800.5 & $5 / 24 / 2004$ & soil & EPA8260 & REG & $108-67-8$ & 1,3,5-Trimethylbenzene & 7.2 & $\mu \mathrm{g} / \mathrm{kg}$ & & 5.9 \\
\hline 383MB0800.5 & $5 / 24 / 2004$ & soil & HASL300 & REG & 14331-83-0 & Ac-228 & 1.69 & $\mathrm{pCi} / \mathrm{g}$ & G & 0.71 \\
\hline 383MB0800.5 & $5 / 24 / 2004$ & soil & EPA6010 & REG & $7440-38-2$ & Arsenic & 4.1 & $\mathrm{mg} / \mathrm{kg}$ & & 1.2 \\
\hline 383MB0800.5 & $5 / 24 / 2004$ & soil & EPA6010 & REG & $7440-39-3$ & Barium & 5000 & $\mathrm{mg} / \mathrm{kg}$ & & 120 \\
\hline 383MB0800.5 & $5 / 24 / 2004$ & soil & EPA6010 & REG & $7440-41-7$ & Beryllium & 0.77 & $\mathrm{mg} / \mathrm{kg}$ & & 0.59 \\
\hline 383MB0800.5 & $5 / 24 / 2004$ & soil & HASL300 & REG & $14733-03-0$ & Bi-214 & 0.89 & $\mathrm{pCi} / \mathrm{g}$ & $\mathrm{G}, \mathrm{J}$ & 0.31 \\
\hline 383MB0800.5 & $5 / 24 / 2004$ & soil & EPA6010 & REG & 7440-43-9 & Cadmium & 2.1 & $\mathrm{mg} / \mathrm{kg}$ & & 0.59 \\
\hline 383MB0800.5 & $5 / 24 / 2004$ & soil & EPA6010 & REG & $7440-47-3$ & Chromium & 8.6 & $\mathrm{mg} / \mathrm{kg}$ & & 1.2 \\
\hline 383MB0800.5 & $5 / 24 / 2004$ & soil & HASL300 & REG & $10045-97-3$ & Cs-137 & 3.16 & $\mathrm{pCi} / \mathrm{g}$ & G & 0.16 \\
\hline $383 M B 0800.5$ & $5 / 24 / 2004$ & soil & EPA8015 & REG & 68334-30-5 & Diesel-Range Organics & 300 & $\mathrm{mg} / \mathrm{kg}$ & , $\mathrm{H}, \mathrm{M}$ & 5.9 \\
\hline 383MB0800.5 & $5 / 24 / 2004$ & soil & EPA8260 & REG & $100-41-4$ & Ethylbenzene & 120 & $\mu \mathrm{g} / \mathrm{kg}$ & & 5.9 \\
\hline 383MB0800.5 & $5 / 24 / 2004$ & soil & HASL300 & REG & 13966-00-2 & $\mathrm{K}-40$ & 22.2 & $\mathrm{pCi} / \mathrm{g}$ & G & 2 \\
\hline 383MB0800.5 & $5 / 24 / 2004$ & soil & EPA6010 & REG & 7439-92-1 & Lead & 53 & $\mathrm{mg} / \mathrm{kg}$ & & 0.35 \\
\hline 383MB0800.5 & $5 / 24 / 2004$ & soil & EPA8260 & REG & 136777-61-2 & M+P-Xylene & 1500 & $\mu \mathrm{g} / \mathrm{kg}$ & $E$ & 5.9 \\
\hline 383MB0800.5 & $5 / 24 / 2004$ & soil & EPA7470 & REG & 7439-97-6 & Mercury & 0.055 & $\mathrm{mg} / \mathrm{kg}$ & $\mathrm{B}$ & 0.12 \\
\hline 383MB0800.5 & $5 / 24 / 2004$ & soil & EPA8260 & REG & $75-09-2$ & Methylene Chloride & 1.9 & $\mu \mathrm{g} / \mathrm{kg}$ & $\mathrm{J}, \mathrm{B}$ & 5.9 \\
\hline 383MB0800.5 & $5 / 24 / 2004$ & soil & EPA8260 & REG & $103-65-1$ & N-Propylbenzene & 5.9 & $\mu \mathrm{g} / \mathrm{kg}$ & & 5.9 \\
\hline 383MB0800.5 & $5 / 24 / 2004$ & soil & EPA8260 & REG & $95-47-6$ & O-Xylene & 1000 & $\mu \mathrm{g} / \mathrm{kg}$ & $E$ & 5.9 \\
\hline 383MB0800.5 & $5 / 24 / 2004$ & soil & HASL300 & REG & 15092-94-1 & $\mathrm{Pb}-212$ & 1.9 & $\mathrm{pCi} / \mathrm{g}$ & $\mathrm{J}$ & 0.31 \\
\hline $383 \mathrm{MB} 0800.5$ & $5 / 24 / 2004$ & soil & HASL300 & REG & 15067-28-4 & $\mathrm{Pb}-214$ & 0.95 & $\mathrm{pCi} / \mathrm{g}$ & $\mathrm{G}, \mathrm{J}$ & 0.32 \\
\hline 383MB0800.5 & $5 / 24 / 2004$ & soil & UGTAISOPU & REG & 13981-16-3 & Pu-238 & 0.156 & $\mathrm{pCi} / \mathrm{g}$ & & 0.009 \\
\hline 383MB0800.5 & $5 / 24 / 2004$ & soil & UGTAISOPU & REG & $15117-48-3$ & Pu-239 & 0.66 & $\mathrm{pCi} / \mathrm{g}$ & & 0.02 \\
\hline $383 M B 0800.5$ & $5 / 24 / 2004$ & soil & HASL300 & REG & 14913-50-9 & TI-208 & 0.47 & $\mathrm{pCi} / \mathrm{g}$ & G & 0.21 \\
\hline 383MB0800.5RR1 & $5 / 24 / 2004$ & soil & EPA8260 & $\mathrm{RE}$ & $100-41-4$ & Ethylbenzene & 80 & $\mu \mathrm{g} / \mathrm{kg}$ & & 59 \\
\hline 383MB0800.5RR1 & $5 / 24 / 2004$ & soil & EPA8260 & $\mathrm{RE}$ & 136777-61-2 & M+P-Xylene & 1200 & $\mu \mathrm{g} / \mathrm{kg}$ & & 59 \\
\hline 383MB0800.5RR1 & $5 / 24 / 2004$ & soil & EPA8260 & $\mathrm{RE}$ & $75-09-2$ & Methylene Chloride & 9.5 & $\mu \mathrm{g} / \mathrm{kg}$ & $\mathrm{J}, \mathrm{B}$ & 59 \\
\hline 383MB0800.5RR1 & $5 / 24 / 2004$ & soil & EPA8260 & $\mathrm{RE}$ & $95-47-6$ & O-Xylene & 780 & $\mu \mathrm{g} / \mathrm{kg}$ & & 59 \\
\hline 383MB0831.0 & $5 / 24 / 2004$ & soil & EPA8260 & REG & $67-64-1$ & Acetone & 8.9 & $\mu \mathrm{g} / \mathrm{kg}$ & $\mathrm{J}$ & 20 \\
\hline 383MB0831.0 & $5 / 24 / 2004$ & soil & EPA6010 & REG & $7440-38-2$ & Arsenic & 0.73 & $\mathrm{mg} / \mathrm{kg}$ & $\mathrm{B}$ & 1 \\
\hline $383 \mathrm{MB} 0831.0$ & $5 / 24 / 2004$ & soil & EPA6010 & REG & 7440-39-3 & Barium & 7.3 & $\mathrm{mg} / \mathrm{kg}$ & $\mathrm{B}$ & 10 \\
\hline $383 \mathrm{MB} 0831.0$ & $5 / 24 / 2004$ & soil & EPA6010 & REG & $7440-41-7$ & Beryllium & 0.25 & $\mathrm{mg} / \mathrm{kg}$ & $\mathrm{B}$ & 0.51 \\
\hline
\end{tabular}

Page 33 of 78

Uncontrolled When Printed 


\begin{tabular}{|c|c|c|c|c|c|c|c|c|c|c|}
\hline SAMPLE \# & $\begin{array}{c}\text { SAMPLE } \\
\text { DATE }\end{array}$ & MATRIX & $\begin{array}{l}\text { USER TEST } \\
\text { PANEL }\end{array}$ & $\begin{array}{c}\text { SAMPLE } \\
\text { PURPOSE }\end{array}$ & CAS \# & PARAMETER & RESULT & UNITS & $\mathbf{Q}$ & $\begin{array}{l}\text { DETECT } \\
\text { LIMIT }\end{array}$ \\
\hline 383MB0831.0 & $5 / 24 / 2004$ & soil & EPA6010 & REG & $7440-43-9$ & Cadmium & 0.066 & $\mathrm{mg} / \mathrm{kg}$ & $\mathrm{B}$ & 0.51 \\
\hline 383MB0831.0 & $5 / 24 / 2004$ & soil & EPA6010 & REG & $7440-47-3$ & Chromium & 0.94 & $\mathrm{mg} / \mathrm{kg}$ & $\mathrm{B}$ & 1 \\
\hline 383MB0831.0 & $5 / 24 / 2004$ & soil & EPA7470 & REG & $7439-97-6$ & Mercury & 0.094 & $\mathrm{mg} / \mathrm{kg}$ & $\mathrm{B}$ & 0.1 \\
\hline 383MB0831.0 & $5 / 24 / 2004$ & soil & EPA8260 & REG & $75-09-2$ & Methylene Chloride & 0.82 & $\mu \mathrm{g} / \mathrm{kg}$ & $\mathrm{J}, \mathrm{B}$ & 5.1 \\
\hline 383MB0831.0 & $5 / 24 / 2004$ & soil & HASL300 & REG & $15067-28-4$ & $\mathrm{~Pb}-214$ & 0.238 & $\mathrm{pCi} / \mathrm{g}$ & $\mathrm{J}$ & 0.118 \\
\hline 383MB0900.5 & $6 / 12 / 2004$ & soil & HASL300 & REG & $14331-83-0$ & Ac-228 & 2.45 & $\mathrm{pCi} / \mathrm{g}$ & G & 0.65 \\
\hline 383MB0900.5 & $6 / 12 / 2004$ & soil & EPA6010 & REG & $7440-38-2$ & Arsenic & 1.9 & $\mathrm{mg} / \mathrm{kg}$ & & 1.1 \\
\hline 383MB0900.5 & $6 / 12 / 2004$ & soil & EPA6010 & REG & $7440-39-3$ & Barium & 120 & $\mathrm{mg} / \mathrm{kg}$ & & 11 \\
\hline 383MB0900.5 & $6 / 12 / 2004$ & soil & EPA6010 & REG & $7440-41-7$ & Beryllium & 2.4 & $\mathrm{mg} / \mathrm{kg}$ & & 0.57 \\
\hline 383MB0900.5 & $6 / 12 / 2004$ & soil & HASL300 & REG & $14733-03-0$ & Bi-214 & 2.08 & $\mathrm{pCi} / \mathrm{g}$ & $G, J$ & 0.38 \\
\hline 383MB0900.5 & $6 / 12 / 2004$ & soil & EPA6010 & REG & 7440-43-9 & Cadmium & 0.1 & $\mathrm{mg} / \mathrm{kg}$ & $\mathrm{B}$ & 0.57 \\
\hline 383MB0900.5 & $6 / 12 / 2004$ & soil & EPA6010 & REG & $7440-47-3$ & Chromium & 0.89 & $\mathrm{mg} / \mathrm{kg}$ & $\mathrm{B}$ & 1.1 \\
\hline 383MB0900.5 & $6 / 12 / 2004$ & soil & HASL300 & REG & $10045-97-3$ & Cs-137 & 0.27 & $\mathrm{pCi} / \mathrm{g}$ & LT,G & 0.15 \\
\hline 383MB0900.5 & $6 / 12 / 2004$ & soil & HASL300 & REG & 13966-00-2 & $\mathrm{K}-40$ & 30.9 & $\mathrm{pCi} / \mathrm{g}$ & G & 2.1 \\
\hline 383MB0900.5 & $6 / 12 / 2004$ & soil & EPA6010 & REG & 7439-92-1 & Lead & 19 & $\mathrm{mg} / \mathrm{kg}$ & & 0.34 \\
\hline 383MB0900.5 & $6 / 12 / 2004$ & soil & EPA7470 & REG & 7439-97-6 & Mercury & 0.0025 & $\mathrm{mg} / \mathrm{kg}$ & $\mathrm{B}$ & 0.11 \\
\hline 383MB0900.5 & $6 / 12 / 2004$ & soil & EPA8260 & REG & $75-09-2$ & Methylene Chloride & 1.1 & $\mu \mathrm{g} / \mathrm{kg}$ & $\mathrm{J}, \mathrm{B}$ & 5.7 \\
\hline 383MB0900.5 & $6 / 12 / 2004$ & soil & HASL300 & REG & 15092-94-1 & $\mathrm{Pb}-212$ & 3.37 & $\mathrm{pCi} / \mathrm{g}$ & $\mathrm{J}$ & 0.27 \\
\hline 383MB0900.5 & $6 / 12 / 2004$ & soil & HASL300 & REG & $15067-28-4$ & $\mathrm{~Pb}-214$ & 2.08 & $\mathrm{pCi} / \mathrm{g}$ & $\mathrm{G}, \mathrm{J}$ & 0.33 \\
\hline 383MB0900.5 & $6 / 12 / 2004$ & soil & EPA6010 & REG & $7440-22-4$ & Silver & 0.26 & $\mathrm{mg} / \mathrm{kg}$ & $\mathrm{B}$ & 1.1 \\
\hline 383MB0900.5 & $6 / 12 / 2004$ & soil & HASL300 & REG & $14913-50-9$ & TI-208 & 1.02 & $\mathrm{pCi} / \mathrm{g}$ & G & 0.19 \\
\hline 383MB0912.5 & $6 / 12 / 2004$ & soil & HASL300 & REG & $14331-83-0$ & Ac-228 & 1.81 & $\mathrm{pCi} / \mathrm{g}$ & G & 0.56 \\
\hline 383MB0912.5 & $6 / 12 / 2004$ & soil & HASL300 & REG & $14596-10-2$ & Am-241 & 1.39 & $\mathrm{pCi} / \mathrm{g}$ & $\mathrm{J}$ & 0.67 \\
\hline 383MB0912.5 & $6 / 12 / 2004$ & soil & EPA6010 & REG & $7440-38-2$ & Arsenic & 3.1 & $\mathrm{mg} / \mathrm{kg}$ & & 1.1 \\
\hline 383MB0912.5 & $6 / 12 / 2004$ & soil & EPA6010 & REG & 7440-39-3 & Barium & 2100 & $\mathrm{mg} / \mathrm{kg}$ & & 550 \\
\hline 383MB0912.5 & $6 / 12 / 2004$ & soil & EPA6010 & REG & 7440-41-7 & Beryllium & 1.4 & $\mathrm{mg} / \mathrm{kg}$ & & 0.55 \\
\hline 383MB0912.5 & $6 / 12 / 2004$ & soil & HASL300 & REG & 14733-03-0 & Bi-214 & 1.17 & $\mathrm{pCi} / \mathrm{g}$ & $\mathrm{G}, \mathrm{J}$ & 0.51 \\
\hline 383MB0912.5 & $6 / 12 / 2004$ & soil & EPA6010 & REG & 7440-43-9 & Cadmium & 0.26 & $\mathrm{mg} / \mathrm{kg}$ & $B$ & 0.55 \\
\hline 383MB0912.5 & $6 / 12 / 2004$ & soil & EPA6010 & REG & $7440-47-3$ & Chromium & 1.7 & $\mathrm{mg} / \mathrm{kg}$ & & 1.1 \\
\hline 383MB0912.5 & $6 / 12 / 2004$ & soil & HASL300 & REG & $10045-97-3$ & Cs-137 & 135 & $\mathrm{pCi} / \mathrm{g}$ & G & 0 \\
\hline 383MB0912.5 & $6 / 12 / 2004$ & soil & EPA8015 & REG & 68334-30-5 & Diesel-Range Organics & 14 & $\mathrm{mg} / \mathrm{kg}$ & $\mathrm{M}$ & 5.5 \\
\hline 383MB0912.5 & $6 / 12 / 2004$ & soil & HASL300 & REG & $13966-00-2$ & $\mathrm{~K}-40$ & 32 & $\mathrm{pCi} / \mathrm{g}$ & $\mathrm{G}$ & 1.5 \\
\hline 383MB0912.5 & $6 / 12 / 2004$ & soil & EPA6010 & REG & 7439-92-1 & Lead & 120 & $\mathrm{mg} / \mathrm{kg}$ & & 17 \\
\hline 383MB0912.5 & $6 / 12 / 2004$ & soil & EPA7470 & REG & $7439-97-6$ & Mercury & 0.017 & $\mathrm{mg} / \mathrm{kg}$ & $\mathrm{B}$ & 0.11 \\
\hline 383MB0912.5 & $6 / 12 / 2004$ & soil & EPA8260 & REG & $75-09-2$ & Methylene Chloride & 1.2 & $\mu \mathrm{g} / \mathrm{kg}$ & $\mathrm{J}, \mathrm{B}$ & 5.5 \\
\hline
\end{tabular}

Page 34 of 78

Uncontrolled When Printed 


\begin{tabular}{|c|c|c|c|c|c|c|c|c|c|c|}
\hline SAMPLE \# & $\begin{array}{c}\text { SAMPLE } \\
\text { DATE }\end{array}$ & MATRIX & $\begin{array}{c}\text { USER TEST } \\
\text { PANEL }\end{array}$ & $\begin{array}{c}\text { SAMPLE } \\
\text { PURPOSE }\end{array}$ & CAS \# & PARAMETER & RESULT & UNITS & $\mathbf{Q}$ & $\begin{array}{c}\text { DETECT } \\
\text { LIMIT }\end{array}$ \\
\hline 383MB0912.5 & $6 / 12 / 2004$ & soil & HASL300 & REG & 15092-94-1 & $\mathrm{Pb}-212$ & 2.02 & $\mathrm{pCi} / \mathrm{g}$ & $\mathrm{J}$ & 0.65 \\
\hline 383MB0912.5 & $6 / 12 / 2004$ & soil & UGTAISOPU & REG & 13981-16-3 & Pu-238 & 3.32 & $\mathrm{pCi} / \mathrm{g}$ & & 0.01 \\
\hline 383MB0912.5 & $6 / 12 / 2004$ & soil & UGTAISOPU & REG & $15117-48-3$ & Pu-239 & 4.11 & $\mathrm{pCi} / \mathrm{g}$ & & 0.02 \\
\hline 383MB0912.5 & $6 / 12 / 2004$ & soil & EPA6010 & REG & $7782-49-2$ & Selenium & 0.7 & $\mathrm{mg} / \mathrm{kg}$ & & 0.55 \\
\hline 383MB0912.5 & $6 / 12 / 2004$ & soil & SR7500 & REG & 10098-97-2 & Sr-90 & 5.5 & $\mathrm{pCi} / \mathrm{g}$ & $\mathrm{J}$ & 0.2 \\
\hline 383MB0912.5 & $6 / 12 / 2004$ & soil & HASL300 & REG & 14913-50-9 & TI-208 & 0.68 & $\mathrm{pCi} / \mathrm{g}$ & $G$ & 0.39 \\
\hline 383MB0913.5 & $6 / 12 / 2004$ & soil & EPA8260 & REG & 67-64-1 & Acetone & 5.7 & $\mu \mathrm{g} / \mathrm{kg}$ & $\mathrm{J}$ & 20 \\
\hline 383MB0913.5 & $6 / 12 / 2004$ & soil & EPA6010 & REG & $7440-39-3$ & Barium & 14 & $\mathrm{mg} / \mathrm{kg}$ & & 10 \\
\hline 383MB0913.5 & $6 / 12 / 2004$ & soil & EPA6010 & REG & $7440-41-7$ & Beryllium & 0.2 & $\mathrm{mg} / \mathrm{kg}$ & $\mathrm{B}$ & 0.5 \\
\hline 383MB0913.5 & $6 / 12 / 2004$ & soil & EPA6010 & REG & $7440-43-9$ & Cadmium & 0.039 & $\mathrm{mg} / \mathrm{kg}$ & $\mathrm{B}$ & 0.5 \\
\hline 383MB0913.5 & $6 / 12 / 2004$ & soil & EPA6010 & REG & $7440-47-3$ & Chromium & 1.6 & $\mathrm{mg} / \mathrm{kg}$ & & 1 \\
\hline 383MB0913.5 & $6 / 12 / 2004$ & soil & EPA7470 & REG & 7439-97-6 & Mercury & 0.026 & $\mathrm{mg} / \mathrm{kg}$ & $\mathrm{B}$ & 0.1 \\
\hline 383MB0913.5 & $6 / 12 / 2004$ & soil & EPA8260 & REG & $75-09-2$ & Methylene Chloride & 1 & $\mu \mathrm{g} / \mathrm{kg}$ & $\mathrm{J}, \mathrm{B}$ & 5 \\
\hline 383MB0913.5 & $6 / 12 / 2004$ & soil & EPA6010 & REG & $7440-22-4$ & Silver & 0.13 & $\mathrm{mg} / \mathrm{kg}$ & $\mathrm{B}$ & 1 \\
\hline 383MB1000.5 & $6 / 12 / 2004$ & soil & EPA8270 & REG & $91-57-6$ & 2-Methylnaphthalene & 68 & $\mu \mathrm{g} / \mathrm{kg}$ & $\mathrm{J}$ & 380 \\
\hline 383MB1000.5 & $6 / 12 / 2004$ & soil & HASL300 & REG & $14331-83-0$ & Ac-228 & 1.35 & $\mathrm{pCi} / \mathrm{g}$ & G & 0.45 \\
\hline 383MB1000.5 & $6 / 12 / 2004$ & soil & EPA8270 & REG & $83-32-9$ & Acenaphthene & 22 & $\mu \mathrm{g} / \mathrm{kg}$ & $\mathrm{J}$ & 380 \\
\hline 383MB1000.5 & $6 / 12 / 2004$ & soil & EPA8270 & REG & $208-96-8$ & Acenaphthylene & 14 & $\mu \mathrm{g} / \mathrm{kg}$ & $\mathrm{J}$ & 380 \\
\hline 383MB1000.5 & $6 / 12 / 2004$ & soil & EPA8270 & REG & $120-12-7$ & Anthracene & 170 & $\mu \mathrm{g} / \mathrm{kg}$ & $\mathrm{J}$ & 380 \\
\hline 383MB1000.5 & $6 / 12 / 2004$ & soil & EPA6010 & REG & $7440-38-2$ & Arsenic & 4.7 & $\mathrm{mg} / \mathrm{kg}$ & & 1.1 \\
\hline 383MB1000.5 & $6 / 12 / 2004$ & soil & EPA6010 & REG & $7440-39-3$ & Barium & 4900 & $\mathrm{mg} / \mathrm{kg}$ & & 560 \\
\hline 383MB1000.5 & $6 / 12 / 2004$ & soil & EPA8270 & REG & $56-55-3$ & Benzo(A)Anthracene & 1200 & $\mu \mathrm{g} / \mathrm{kg}$ & & 380 \\
\hline 383MB1000.5 & $6 / 12 / 2004$ & soil & EPA8270 & REG & $50-32-8$ & Benzo(A)Pyrene & 1300 & $\mu \mathrm{g} / \mathrm{kg}$ & & 380 \\
\hline 383MB1000.5 & $6 / 12 / 2004$ & soil & EPA8270 & REG & $205-99-2$ & Benzo(B)Fluoranthene & 1700 & $\mu \mathrm{g} / \mathrm{kg}$ & & 380 \\
\hline 383MB1000.5 & $6 / 12 / 2004$ & soil & EPA8270 & REG & $191-24-2$ & Benzo(G,H,I)Perylene & 760 & $\mu \mathrm{g} / \mathrm{kg}$ & & 380 \\
\hline 383MB1000.5 & $6 / 12 / 2004$ & soil & EPA8270 & REG & 207-08-9 & Benzo(K)Fluoranthene & 650 & $\mu \mathrm{g} / \mathrm{kg}$ & & 380 \\
\hline 383MB1000.5 & $6 / 12 / 2004$ & soil & EPA6010 & REG & $7440-41-7$ & Beryllium & 0.94 & $\mathrm{mg} / \mathrm{kg}$ & & 0.56 \\
\hline 383MB1000.5 & $6 / 12 / 2004$ & soil & HASL300 & REG & $14733-03-0$ & Bi-214 & 1.02 & $\mathrm{pCi} / \mathrm{g}$ & $\mathrm{G}, \mathrm{J}$ & 0.24 \\
\hline 383MB1000.5 & $6 / 12 / 2004$ & soil & EPA8270 & REG & 117-81-7 & Bis(2-Ethylhexyl)Phthalate & 130 & $\mu \mathrm{g} / \mathrm{kg}$ & $\mathrm{J}$ & 380 \\
\hline 383MB1000.5 & $6 / 12 / 2004$ & soil & EPA6010 & REG & $7440-43-9$ & Cadmium & 0.34 & $\mathrm{mg} / \mathrm{kg}$ & $\mathrm{B}$ & 0.56 \\
\hline 383MB1000.5 & $6 / 12 / 2004$ & soil & EPA8270 & REG & 86-74-8 & Carbazole & 57 & $\mu \mathrm{g} / \mathrm{kg}$ & $\mathrm{J}$ & 380 \\
\hline 383MB1000.5 & $6 / 12 / 2004$ & soil & EPA6010 & REG & $7440-47-3$ & Chromium & 11 & $\mathrm{mg} / \mathrm{kg}$ & & 1.1 \\
\hline 383MB1000.5 & $6 / 12 / 2004$ & soil & EPA8270 & REG & 218-01-9 & Chrysene & 1000 & $\mu \mathrm{g} / \mathrm{kg}$ & & 380 \\
\hline 383MB1000.5 & $6 / 12 / 2004$ & soil & HASL300 & REG & $10045-97-3$ & Cs-137 & 0.33 & $\mathrm{pCi} / \mathrm{g}$ & $\mathrm{LT}, \mathrm{G}$ & 0.16 \\
\hline 383MB1000.5 & $6 / 12 / 2004$ & soil & EPA8270 & REG & $132-64-9$ & Dibenzofuran & 65 & $\mu \mathrm{g} / \mathrm{kg}$ & $\mathrm{J}$ & 380 \\
\hline
\end{tabular}

Page 35 of 78

Uncontrolled When Printed 


\begin{tabular}{|c|c|c|c|c|c|c|c|c|c|c|}
\hline SAMPLE \# & $\begin{array}{c}\text { SAMPLE } \\
\text { DATE }\end{array}$ & MATRIX & $\begin{array}{l}\text { USER TEST } \\
\text { PANEL }\end{array}$ & $\begin{array}{c}\text { SAMPLE } \\
\text { PURPOSE }\end{array}$ & CAS \# & PARAMETER & RESULT & UNITS & $\mathbf{Q}$ & $\begin{array}{c}\text { DETECT } \\
\text { LIMIT }\end{array}$ \\
\hline 383MB1000.5 & $6 / 12 / 2004$ & soil & EPA8015 & REG & $68334-30-5$ & Diesel-Range Organics & 9.9 & $\mathrm{mg} / \mathrm{kg}$ & $\mathrm{Z}, \mathrm{H}$ & 5.6 \\
\hline 383MB1000.5 & $6 / 12 / 2004$ & soil & EPA8270 & REG & $206-44-0$ & Fluoranthene & 1500 & $\mu \mathrm{g} / \mathrm{kg}$ & & 380 \\
\hline 383MB1000.5 & $6 / 12 / 2004$ & soil & EPA8270 & REG & $86-73-7$ & Fluorene & 16 & $\mu \mathrm{g} / \mathrm{kg}$ & $\mathrm{J}$ & 380 \\
\hline 383MB1000.5 & $6 / 12 / 2004$ & soil & EPA8270 & REG & $193-39-5$ & Indeno(1,2,3-Cd)Pyrene & 630 & $\mu \mathrm{g} / \mathrm{kg}$ & & 380 \\
\hline 383MB1000.5 & $6 / 12 / 2004$ & soil & HASL300 & REG & $13966-00-2$ & $\mathrm{~K}-40$ & 22.3 & $\mathrm{pCi} / \mathrm{g}$ & G & 1 \\
\hline 383MB1000.5 & $6 / 12 / 2004$ & soil & EPA6010 & REG & 7439-92-1 & Lead & 51 & $\mathrm{mg} / \mathrm{kg}$ & & 0.68 \\
\hline 383MB1000.5 & $6 / 12 / 2004$ & soil & EPA7470 & REG & 7439-97-6 & Mercury & 0.047 & $\mathrm{mg} / \mathrm{kg}$ & $B$ & 0.11 \\
\hline 383MB1000.5 & $6 / 12 / 2004$ & soil & EPA8260 & REG & $75-09-2$ & Methylene Chloride & 1.3 & $\mu \mathrm{g} / \mathrm{kg}$ & $\mathrm{J}, \mathrm{B}$ & 5.6 \\
\hline 383MB1000.5 & $6 / 12 / 2004$ & soil & EPA8270 & REG & $91-20-3$ & Naphthalene & 19 & $\mu \mathrm{g} / \mathrm{kg}$ & $\mathrm{J}$ & 380 \\
\hline 383MB1000.5 & $6 / 12 / 2004$ & soil & HASL300 & REG & 15092-94-1 & $\mathrm{Pb}-212$ & 1.5 & $\mathrm{pCi} / \mathrm{g}$ & $\mathrm{J}$ & 0.18 \\
\hline 383MB1000.5 & $6 / 12 / 2004$ & soil & HASL300 & REG & $15067-28-4$ & $\mathrm{~Pb}-214$ & 1.13 & $\mathrm{pCi} / \mathrm{g}$ & $\mathrm{G}, \mathrm{J}$ & 0.27 \\
\hline 383MB1000.5 & $6 / 12 / 2004$ & soil & EPA8270 & REG & 85-01-8 & Phenanthrene & 350 & $\mu \mathrm{g} / \mathrm{kg}$ & $\mathrm{J}$ & 380 \\
\hline 383MB1000.5 & $6 / 12 / 2004$ & soil & UGTAISOPU & REG & $15117-48-3$ & Pu-239 & 0.117 & $\mathrm{pCi} / \mathrm{g}$ & & 0.022 \\
\hline 383MB1000.5 & $6 / 12 / 2004$ & soil & EPA8270 & REG & $129-00-0$ & Pyrene & 1600 & $\mu \mathrm{g} / \mathrm{kg}$ & & 380 \\
\hline 383MB1000.5 & $6 / 12 / 2004$ & soil & EPA6010 & REG & $7782-49-2$ & Selenium & 0.24 & $\mathrm{mg} / \mathrm{kg}$ & $B$ & 0.56 \\
\hline 383МB1000.5 & $6 / 12 / 2004$ & soil & EPA6010 & REG & $7440-22-4$ & Silver & 0.34 & $\mathrm{mg} / \mathrm{kg}$ & $B$ & 1.1 \\
\hline 383MB1000.5 & $6 / 12 / 2004$ & soil & HASL300 & REG & 14913-50-9 & TI-208 & 0.46 & $\mathrm{pCi} / \mathrm{g}$ & G & 0.12 \\
\hline 383MB1100.5 & $6 / 12 / 2004$ & soil & HASL300 & REG & 14331-83-0 & Ac-228 & 1.65 & $\mathrm{pCi} / \mathrm{g}$ & $\mathrm{G}$ & 0.56 \\
\hline 383MB1100.5 & $6 / 12 / 2004$ & soil & EPA6010 & REG & $7440-38-2$ & Arsenic & 4.3 & $\mathrm{mg} / \mathrm{kg}$ & & 1.1 \\
\hline 383MB1100.5 & $6 / 12 / 2004$ & soil & EPA6010 & REG & $7440-39-3$ & Barium & 5300 & $\mathrm{mg} / \mathrm{kg}$ & & 550 \\
\hline 383MB1100.5 & $6 / 12 / 2004$ & soil & EPA6010 & REG & $7440-41-7$ & Beryllium & 1.4 & $\mathrm{mg} / \mathrm{kg}$ & & 0.55 \\
\hline 383MB1100.5 & $6 / 12 / 2004$ & soil & HASL300 & REG & $14733-03-0$ & Bi-214 & 1.3 & $\mathrm{pCi} / \mathrm{g}$ & $\mathrm{G}, \mathrm{J}$ & 0.27 \\
\hline 383MB1100.5 & $6 / 12 / 2004$ & soil & EPA6010 & REG & $7440-43-9$ & Cadmium & 0.23 & $\mathrm{mg} / \mathrm{kg}$ & $\mathrm{B}$ & 0.55 \\
\hline 383MB1100.5 & $6 / 12 / 2004$ & soil & EPA6010 & REG & $7440-47-3$ & Chromium & 5.6 & $\mathrm{mg} / \mathrm{kg}$ & & 1.1 \\
\hline 383MB1100.5 & $6 / 12 / 2004$ & soil & HASL300 & REG & $10045-97-3$ & Cs-137 & 0.87 & $\mathrm{pCi} / \mathrm{g}$ & G & 0.15 \\
\hline 383MB1100.5 & $6 / 12 / 2004$ & soil & EPA8015 & REG & $68334-30-5$ & Diesel-Range Organics & 13 & $\mathrm{mg} / \mathrm{kg}$ & $M$ & 5.5 \\
\hline 383MB1100.5 & $6 / 12 / 2004$ & soil & HASL300 & REG & $13966-00-2$ & $\mathrm{~K}-40$ & 25.6 & $\mathrm{pCi} / \mathrm{g}$ & $\mathrm{G}$ & 1.6 \\
\hline 383MB1100.5 & $6 / 12 / 2004$ & soil & EPA6010 & REG & 7439-92-1 & Lead & 20 & $\mathrm{mg} / \mathrm{kg}$ & & 0.33 \\
\hline 383MB1100.5 & $6 / 12 / 2004$ & soil & EPA7470 & REG & $7439-97-6$ & Mercury & 0.016 & $\mathrm{mg} / \mathrm{kg}$ & $\mathrm{B}$ & 0.11 \\
\hline 383MB1100.5 & $6 / 12 / 2004$ & soil & EPA8260 & REG & $75-09-2$ & Methylene Chloride & 1.1 & $\mu \mathrm{g} / \mathrm{kg}$ & $\mathrm{J}, \mathrm{B}$ & 5.5 \\
\hline 383MB1100.5 & $6 / 12 / 2004$ & soil & HASL300 & REG & 15092-94-1 & $\mathrm{Pb}-212$ & 1.89 & $\mathrm{pCi} / \mathrm{g}$ & $\mathrm{J}$ & 0.24 \\
\hline 383MB1100.5 & $6 / 12 / 2004$ & soil & HASL300 & REG & $15067-28-4$ & $\mathrm{~Pb}-214$ & 1.46 & $\mathrm{pCi} / \mathrm{g}$ & $\mathrm{G}, \mathrm{J}$ & 0.27 \\
\hline 383MB1100.5 & $6 / 12 / 2004$ & soil & EPA6010 & REG & $7782-49-2$ & Selenium & 0.74 & $\mathrm{mg} / \mathrm{kg}$ & & 0.55 \\
\hline 383MB1100.5 & $6 / 12 / 2004$ & soil & HASL300 & REG & $14913-50-9$ & TI-208 & 0.66 & $\mathrm{pCi} / \mathrm{g}$ & G & 0.14 \\
\hline 383MB1114.5 & $6 / 12 / 2004$ & soil & HASL300 & REG & 14331-83-0 & Ac-228 & 1.54 & $\mathrm{pCi} / \mathrm{g}$ & $\mathrm{G}$ & 0.52 \\
\hline
\end{tabular}

Page 36 of 78

Uncontrolled When Printed 


\begin{tabular}{|c|c|c|c|c|c|c|c|c|c|c|}
\hline SAMPLE \# & $\begin{array}{c}\text { SAMPLE } \\
\text { DATE }\end{array}$ & MATRIX & $\begin{array}{l}\text { USER TEST } \\
\text { PANEL }\end{array}$ & $\begin{array}{c}\text { SAMPLE } \\
\text { PURPOSE }\end{array}$ & CAS \# & PARAMETER & RESULT & UNITS & $\mathbf{Q}$ & $\begin{array}{l}\text { DETECT } \\
\text { LIMIT }\end{array}$ \\
\hline 383MB1114.5 & $6 / 12 / 2004$ & soil & EPA6010 & REG & $7440-38-2$ & Arsenic & 2.5 & $\mathrm{mg} / \mathrm{kg}$ & & 1.1 \\
\hline 383MB1114.5 & $6 / 12 / 2004$ & soil & EPA6010 & REG & $7440-39-3$ & Barium & 470 & $\mathrm{mg} / \mathrm{kg}$ & & 11 \\
\hline 383MB1114.5 & $6 / 12 / 2004$ & soil & EPA6010 & REG & $7440-41-7$ & Beryllium & 1.1 & $\mathrm{mg} / \mathrm{kg}$ & & 0.56 \\
\hline 383MB1114.5 & $6 / 12 / 2004$ & soil & HASL300 & REG & $14733-03-0$ & Bi-214 & 1.27 & $\mathrm{pCi} / \mathrm{g}$ & $\mathrm{G}, \mathrm{J}$ & 0.41 \\
\hline 383MB1114.5 & $6 / 12 / 2004$ & soil & EPA6010 & REG & $7440-43-9$ & Cadmium & 0.13 & $\mathrm{mg} / \mathrm{kg}$ & $\mathrm{B}$ & 0.56 \\
\hline 383MB1114.5 & $6 / 12 / 2004$ & soil & EPA6010 & REG & $7440-47-3$ & Chromium & 1.4 & $\mathrm{mg} / \mathrm{kg}$ & & 1.1 \\
\hline 383MB1114.5 & $6 / 12 / 2004$ & soil & HASL300 & REG & $10045-97-3$ & Cs-137 & 37.4 & $\mathrm{pCi} / \mathrm{g}$ & $G$ & 0.2 \\
\hline 383MB1114.5 & $6 / 12 / 2004$ & soil & EPA8015 & REG & $68334-30-5$ & Diesel-Range Organics & 8.8 & $\mathrm{mg} / \mathrm{kg}$ & $\mathrm{M}$ & 5.6 \\
\hline 383MB1114.5 & $6 / 12 / 2004$ & soil & HASL300 & REG & $13966-00-2$ & $\mathrm{~K}-40$ & 37.7 & $\mathrm{pCi} / \mathrm{g}$ & G & 1.5 \\
\hline 383MB1114.5 & $6 / 12 / 2004$ & soil & EPA6010 & REG & 7439-92-1 & Lead & 15 & $\mathrm{mg} / \mathrm{kg}$ & & 0.34 \\
\hline 383MB1114.5 & $6 / 12 / 2004$ & soil & EPA7470 & REG & 7439-97-6 & Mercury & 0.006 & $\mathrm{mg} / \mathrm{kg}$ & $\mathrm{B}$ & 0.11 \\
\hline 383MB1114.5 & $6 / 12 / 2004$ & soil & EPA8260 & REG & $75-09-2$ & Methylene Chloride & 1.2 & $\mu \mathrm{g} / \mathrm{kg}$ & $\mathrm{J}, \mathrm{B}$ & 5.6 \\
\hline 383MB1114.5 & $6 / 12 / 2004$ & soil & HASL300 & REG & 15092-94-1 & $\mathrm{Pb}-212$ & 1.86 & $\mathrm{pCi} / \mathrm{g}$ & $\mathrm{J}$ & 0.39 \\
\hline 383MB1114.5 & $6 / 12 / 2004$ & soil & HASL300 & REG & $15067-28-4$ & $\mathrm{~Pb}-214$ & 1.24 & $\mathrm{pCi} / \mathrm{g}$ & $\mathrm{G}, \mathrm{J}$ & 0.57 \\
\hline 383MB1114.5 & $6 / 12 / 2004$ & soil & UGTAISOPU & REG & $15117-48-3$ & Pu-239 & 0.206 & $\mathrm{pCi} / \mathrm{g}$ & & 0.021 \\
\hline 383MB1114.5 & $6 / 12 / 2004$ & soil & EPA6010 & REG & $7782-49-2$ & Selenium & 0.53 & $\mathrm{mg} / \mathrm{kg}$ & $\mathrm{B}$ & 0.56 \\
\hline 383MB1114.5 & $6 / 12 / 2004$ & soil & SR7500 & REG & 10098-97-2 & Sr-90 & 1.08 & $\mathrm{pCi} / \mathrm{g}$ & Y1 & 0.25 \\
\hline 383MB1114.5 & $6 / 12 / 2004$ & soil & HASL300 & REG & $14913-50-9$ & TI-208 & 0.7 & $\mathrm{pCi} / \mathrm{g}$ & $G$ & 0.21 \\
\hline 383MB1119.5 & $6 / 12 / 2004$ & soil & EPA6010 & REG & $7440-38-2$ & Arsenic & 1.4 & $\mathrm{mg} / \mathrm{kg}$ & & 1 \\
\hline 383MB1119.5 & $6 / 12 / 2004$ & soil & EPA6010 & REG & $7440-39-3$ & Barium & 36 & $\mathrm{mg} / \mathrm{kg}$ & & 10 \\
\hline 383MB1119.5 & $6 / 12 / 2004$ & soil & EPA6010 & REG & $7440-41-7$ & Beryllium & 0.23 & $\mathrm{mg} / \mathrm{kg}$ & B & 0.51 \\
\hline 383MB1119.5 & $6 / 12 / 2004$ & soil & EPA6010 & REG & 7440-43-9 & Cadmium & 0.17 & $\mathrm{mg} / \mathrm{kg}$ & $\mathrm{B}$ & 0.51 \\
\hline 383MB1119.5 & $6 / 12 / 2004$ & soil & EPA6010 & REG & $7440-47-3$ & Chromium & 2.1 & $\mathrm{mg} / \mathrm{kg}$ & & 1 \\
\hline 383MB1119.5 & $6 / 12 / 2004$ & soil & HASL300 & REG & $10045-97-3$ & Cs-137 & 0.121 & $\mathrm{pCi} / \mathrm{g}$ & LT & 0.062 \\
\hline 383MB1119.5 & $6 / 12 / 2004$ & soil & EPA6010 & REG & 7439-92-1 & Lead & 1.1 & $\mathrm{mg} / \mathrm{kg}$ & $\mathrm{B}$ & 1.5 \\
\hline 383MB1119.5 & $6 / 12 / 2004$ & soil & EPA7470 & REG & 7439-97-6 & Mercury & 0.037 & $\mathrm{mg} / \mathrm{kg}$ & $\mathrm{B}$ & 0.1 \\
\hline 383MB1119.5 & $6 / 12 / 2004$ & soil & EPA8260 & REG & $75-09-2$ & Methylene Chloride & 0.97 & $\mu \mathrm{g} / \mathrm{kg}$ & $\mathrm{J}, \mathrm{B}$ & 5.1 \\
\hline 383MB1119.5 & $6 / 12 / 2004$ & soil & HASL300 & REG & $15067-28-4$ & $\mathrm{~Pb}-214$ & 0.38 & $\mathrm{pCi} / \mathrm{g}$ & $\mathrm{J}$ & 0.15 \\
\hline 383MB1119.5 & $6 / 12 / 2004$ & soil & EPA6010 & REG & $7440-22-4$ & Silver & 0.17 & $\mathrm{mg} / \mathrm{kg}$ & $\mathrm{B}$ & 1 \\
\hline 383MB1200.5 & $6 / 13 / 2004$ & soil & HASL300 & REG & 14331-83-0 & Ac-228 & 1.98 & $\mathrm{pCi} / \mathrm{g}$ & $G$ & 0.8 \\
\hline 383MB1200.5 & $6 / 13 / 2004$ & soil & HASL300 & REG & 14682-66-7 & $\mathrm{Al}-26$ & 0.108 & $\mathrm{pCi} / \mathrm{g}$ & $\mathrm{G}, \mathrm{TI}$ & 0.103 \\
\hline 383MB1200.5 & $6 / 13 / 2004$ & soil & EPA6010 & REG & $7440-38-2$ & Arsenic & 2.3 & $\mathrm{mg} / \mathrm{kg}$ & & 1.1 \\
\hline 383MB1200.5 & $6 / 13 / 2004$ & soil & EPA6010 & REG & $7440-39-3$ & Barium & 640 & $\mathrm{mg} / \mathrm{kg}$ & & 11 \\
\hline 383MB1200.5 & $6 / 13 / 2004$ & soil & EPA6010 & REG & 7440-41-7 & Beryllium & 2.4 & $\mathrm{mg} / \mathrm{kg}$ & & 0.56 \\
\hline 383MB1200.5 & $6 / 13 / 2004$ & soil & HASL300 & REG & 14913-49-6 & Bi-212 & 2.5 & $\mathrm{pCi} / \mathrm{g}$ & $\mathrm{G}$ & 2.1 \\
\hline
\end{tabular}

Page 37 of 78

Uncontrolled When Printed 


\begin{tabular}{|c|c|c|c|c|c|c|c|c|c|c|}
\hline SAMPLE \# & $\begin{array}{c}\text { SAMPLE } \\
\text { DATE }\end{array}$ & MATRIX & $\begin{array}{c}\text { USER TEST } \\
\text { PANEL }\end{array}$ & $\begin{array}{c}\text { SAMPLE } \\
\text { PURPOSE }\end{array}$ & CAS \# & PARAMETER & RESULT & UNITS & $\mathbf{Q}$ & $\begin{array}{c}\text { DETECT } \\
\text { LIMIT }\end{array}$ \\
\hline 383MB1200.5 & $6 / 13 / 2004$ & soil & HASL300 & REG & $14733-03-0$ & Bi-214 & 1.84 & $\mathrm{pCi} / \mathrm{g}$ & $\mathrm{G}, \mathrm{J}$ & 0.35 \\
\hline 383MB1200.5 & $6 / 13 / 2004$ & soil & EPA6010 & REG & $7440-43-9$ & Cadmium & 0.17 & $\mathrm{mg} / \mathrm{kg}$ & $\mathrm{B}$ & 0.56 \\
\hline 383MB1200.5 & $6 / 13 / 2004$ & soil & EPA6010 & REG & $7440-47-3$ & Chromium & 2.1 & $\mathrm{mg} / \mathrm{kg}$ & & 1.1 \\
\hline 383MB1200.5 & $6 / 13 / 2004$ & soil & HASL300 & REG & $10045-97-3$ & Cs-137 & 0.23 & $\mathrm{pCi} / \mathrm{g}$ & $\mathrm{LT}, \mathrm{G}$ & 0.14 \\
\hline 383MB1200.5 & $6 / 13 / 2004$ & soil & HASL300 & REG & 13966-00-2 & $\mathrm{K}-40$ & 30.4 & $\mathrm{pCi} / \mathrm{g}$ & G & 2 \\
\hline 383MB1200.5 & $6 / 13 / 2004$ & soil & EPA6010 & REG & 7439-92-1 & Lead & 20 & $\mathrm{mg} / \mathrm{kg}$ & & 0.34 \\
\hline 383MB1200.5 & $6 / 13 / 2004$ & soil & EPA7470 & REG & 7439-97-6 & Mercury & 0.0079 & $\mathrm{mg} / \mathrm{kg}$ & $\mathrm{B}$ & 0.11 \\
\hline 383MB1200.5 & $6 / 13 / 2004$ & soil & HASL300 & REG & 15092-94-1 & $\mathrm{Pb}-212$ & 2.99 & $\mathrm{pCi} / \mathrm{g}$ & G & 0.26 \\
\hline 383MB1200.5 & $6 / 13 / 2004$ & soil & HASL300 & REG & $15067-28-4$ & $\mathrm{~Pb}-214$ & 2.04 & $\mathrm{pCi} / \mathrm{g}$ & $\mathrm{G}, \mathrm{J}$ & 0.31 \\
\hline 383MB1200.5 & $6 / 13 / 2004$ & soil & UGTAISOPU & REG & 13981-16-3 & Pu-238 & 0.017 & $\mathrm{pCi} / \mathrm{g}$ & $\mathrm{LT}$ & 0.009 \\
\hline 383MB1200.5 & $6 / 13 / 2004$ & soil & EPA6010 & REG & $7782-49-2$ & Selenium & 0.36 & $\mathrm{mg} / \mathrm{kg}$ & $\mathrm{B}$ & 0.56 \\
\hline 383MB1200.5 & $6 / 13 / 2004$ & soil & HASL300 & REG & $14913-50-9$ & TI-208 & 0.85 & $\mathrm{pCi} / \mathrm{g}$ & $G$ & 0.16 \\
\hline 383MB1300.5 & $6 / 13 / 2004$ & soil & HASL300 & REG & $14331-83-0$ & Ac-228 & 2.05 & $\mathrm{pCi} / \mathrm{g}$ & $\mathrm{G}$ & 0.58 \\
\hline 383MB1300.5 & $6 / 13 / 2004$ & soil & EPA6010 & REG & $7440-38-2$ & Arsenic & 3.5 & $\mathrm{mg} / \mathrm{kg}$ & & 1.1 \\
\hline 383MB1300.5 & $6 / 13 / 2004$ & soil & EPA6010 & REG & $7440-39-3$ & Barium & 660 & $\mathrm{mg} / \mathrm{kg}$ & & 11 \\
\hline 383MB1300.5 & $6 / 13 / 2004$ & soil & EPA6010 & REG & $7440-41-7$ & Beryllium & 1.1 & $\mathrm{mg} / \mathrm{kg}$ & & 0.56 \\
\hline 383MB1300.5 & $6 / 13 / 2004$ & soil & HASL300 & REG & $14913-49-6$ & Bi-212 & 2.3 & $\mathrm{pCi} / \mathrm{g}$ & G & 2.1 \\
\hline 383MB1300.5 & $6 / 13 / 2004$ & soil & HASL300 & REG & 14733-03-0 & Bi-214 & 1.07 & $\mathrm{pCi} / \mathrm{g}$ & $\mathrm{G}, \mathrm{J}$ & 0.27 \\
\hline 383MB1300.5 & $6 / 13 / 2004$ & soil & EPA6010 & REG & $7440-43-9$ & Cadmium & 0.03 & $\mathrm{mg} / \mathrm{kg}$ & $\mathrm{B}$ & 0.56 \\
\hline 383MB1300.5 & $6 / 13 / 2004$ & soil & EPA6010 & REG & $7440-47-3$ & Chromium & 2 & $\mathrm{mg} / \mathrm{kg}$ & & 1.1 \\
\hline 383MB1300.5 & $6 / 13 / 2004$ & soil & HASL300 & REG & 13966-00-2 & $\mathrm{K}-40$ & 25.7 & $\mathrm{pCi} / \mathrm{g}$ & $G$ & 1.5 \\
\hline 383MB1300.5 & $6 / 13 / 2004$ & soil & EPA6010 & REG & 7439-92-1 & Lead & 13 & $\mathrm{mg} / \mathrm{kg}$ & & 0.33 \\
\hline 383MB1300.5 & $6 / 13 / 2004$ & soil & EPA7470 & REG & 7439-97-6 & Mercury & 0.013 & $\mathrm{mg} / \mathrm{kg}$ & $\mathrm{B}$ & 0.11 \\
\hline 383MB1300.5 & $6 / 13 / 2004$ & soil & HASL300 & REG & 15092-94-1 & $\mathrm{Pb}-212$ & 2.1 & $\mathrm{pCi} / \mathrm{g}$ & $G$ & 0.26 \\
\hline 383MB1300.5 & $6 / 13 / 2004$ & soil & HASL300 & REG & $15067-28-4$ & $\mathrm{~Pb}-214$ & 0.97 & $\mathrm{pCi} / \mathrm{g}$ & $\mathrm{G}, \mathrm{J}$ & 0.28 \\
\hline 383MB1300.5 & $6 / 13 / 2004$ & soil & EPA6010 & REG & $7782-49-2$ & Selenium & 0.27 & $\mathrm{mg} / \mathrm{kg}$ & $\mathrm{B}$ & 0.56 \\
\hline 383MB1300.5 & $6 / 13 / 2004$ & soil & HASL300 & REG & $15065-10-8$ & Th-234 & 2.7 & $\mathrm{pCi} / \mathrm{g}$ & $\mathrm{G}, \mathrm{TI}$ & 2 \\
\hline 383MB1300.5 & $6 / 13 / 2004$ & soil & HASL300 & REG & 14913-50-9 & TI-208 & 0.79 & $\mathrm{pCi} / \mathrm{g}$ & G & 0.15 \\
\hline 383MB1391.0 & $6 / 14 / 2004$ & soil & HASL300 & REG & 14331-83-0 & Ac-228 & 0.71 & $\mathrm{pCi} / \mathrm{g}$ & $\mathrm{G}$ & 0.4 \\
\hline 383MB1391.0 & $6 / 14 / 2004$ & soil & EPA6010 & REG & $7440-38-2$ & Arsenic & 2.3 & $\mathrm{mg} / \mathrm{kg}$ & & 1.1 \\
\hline 383MB1391.0 & $6 / 14 / 2004$ & soil & EPA6010 & REG & $7440-39-3$ & Barium & 67 & $\mathrm{mg} / \mathrm{kg}$ & & 11 \\
\hline 383MB1391.0 & $6 / 14 / 2004$ & soil & EPA6010 & REG & $7440-41-7$ & Beryllium & 0.65 & $\mathrm{mg} / \mathrm{kg}$ & & 0.54 \\
\hline 383MB1391.0 & $6 / 14 / 2004$ & soil & HASL300 & REG & $14733-03-0$ & Bi-214 & 0.65 & $\mathrm{pCi} / \mathrm{g}$ & $G, J$ & 0.2 \\
\hline 383MB1391.0 & $6 / 14 / 2004$ & soil & EPA6010 & REG & 7440-43-9 & Cadmium & 0.092 & $\mathrm{mg} / \mathrm{kg}$ & $\mathrm{B}$ & 0.54 \\
\hline 383MB1391.0 & $6 / 14 / 2004$ & soil & EPA6010 & REG & $7440-47-3$ & Chromium & 5.8 & $\mathrm{mg} / \mathrm{kg}$ & & 1.1 \\
\hline
\end{tabular}

Page 38 of 78

Uncontrolled When Printed 


\begin{tabular}{|c|c|c|c|c|c|c|c|c|c|c|}
\hline SAMPLE \# & $\begin{array}{c}\text { SAMPLE } \\
\text { DATE }\end{array}$ & MATRIX & $\begin{array}{l}\text { USER TEST } \\
\text { PANEL }\end{array}$ & $\begin{array}{c}\text { SAMPLE } \\
\text { PURPOSE }\end{array}$ & CAS \# & PARAMETER & RESULT & UNITS & $\mathbf{Q}$ & $\begin{array}{c}\text { DETECT } \\
\text { LIMIT }\end{array}$ \\
\hline 383MB1391.0 & $6 / 14 / 2004$ & soil & HASL300 & REG & 13966-00-2 & $\mathrm{K}-40$ & 13 & $\mathrm{pCi} / \mathrm{g}$ & G & 1.1 \\
\hline 383MB1391.0 & $6 / 14 / 2004$ & soil & EPA6010 & REG & $7439-92-1$ & Lead & 8.6 & $\mathrm{mg} / \mathrm{kg}$ & & 1.6 \\
\hline 383MB1391.0 & $6 / 14 / 2004$ & soil & EPA7470 & REG & $7439-97-6$ & Mercury & 0.014 & $\mathrm{mg} / \mathrm{kg}$ & $B$ & 0.11 \\
\hline 383MB1391.0 & $6 / 14 / 2004$ & soil & EPA8260 & REG & $75-09-2$ & Methylene Chloride & 0.92 & $\mu \mathrm{g} / \mathrm{kg}$ & $\mathrm{J}, \mathrm{B}$ & 5.4 \\
\hline 383MB1391.0 & $6 / 14 / 2004$ & soil & HASL300 & REG & 15092-94-1 & $\mathrm{Pb}-212$ & 0.8 & $\mathrm{pCi} / \mathrm{g}$ & G & 0.17 \\
\hline 383MB1391.0 & $6 / 14 / 2004$ & soil & HASL300 & REG & $15067-28-4$ & $\mathrm{~Pb}-214$ & 0.76 & $\mathrm{pCi} / \mathrm{g}$ & $\mathrm{G}, \mathrm{J}$ & 0.18 \\
\hline 383MB1391.0 & $6 / 14 / 2004$ & soil & UGTAISOPU & REG & 13981-16-3 & Pu-238 & 0.02 & $\mathrm{pCi} / \mathrm{g}$ & LT & 0.009 \\
\hline 383MB1391.0 & $6 / 14 / 2004$ & soil & UGTAISOPU & REG & $15117-48-3$ & Pu-239 & 0.03 & $\mathrm{pCi} / \mathrm{g}$ & LT & 0.009 \\
\hline 383MB1391.0 & $6 / 14 / 2004$ & soil & EPA6010 & REG & $7440-22-4$ & Silver & 1.6 & $\mathrm{mg} / \mathrm{kg}$ & & 1.1 \\
\hline 383MB1391.0 & $6 / 14 / 2004$ & soil & HASL300 & REG & $14913-50-9$ & TI-208 & 0.183 & $\mathrm{pCi} / \mathrm{g}$ & $\mathrm{G}$ & 0.089 \\
\hline 383MB1400.5 & $6 / 14 / 2004$ & soil & HASL300 & REG & 14331-83-0 & Ac-228 & 0.74 & $\mathrm{pCi} / \mathrm{g}$ & & 0.4 \\
\hline 383MB1400.5 & $6 / 14 / 2004$ & soil & EPA6010 & REG & $7440-38-2$ & Arsenic & 3.4 & $\mathrm{mg} / \mathrm{kg}$ & & 1 \\
\hline 383MB1400.5 & $6 / 14 / 2004$ & soil & EPA6010 & REG & $7440-39-3$ & Barium & 300 & $\mathrm{mg} / \mathrm{kg}$ & & 10 \\
\hline 383MB1400.5 & $6 / 14 / 2004$ & soil & EPA8270 & REG & $56-55-3$ & Benzo(A)Anthracene & 18 & $\mu \mathrm{g} / \mathrm{kg}$ & $\mathrm{J}$ & 340 \\
\hline 383MB1400.5 & $6 / 14 / 2004$ & soil & EPA8270 & REG & $50-32-8$ & Benzo(A)Pyrene & 15 & $\mu \mathrm{g} / \mathrm{kg}$ & $\mathrm{J}$ & 340 \\
\hline $383 \mathrm{MB} 1400.5$ & $6 / 14 / 2004$ & soil & EPA8270 & REG & $205-99-2$ & Benzo(B)Fluoranthene & 27 & $\mu \mathrm{g} / \mathrm{kg}$ & $\mathrm{J}$ & 340 \\
\hline 383MB1400.5 & $6 / 14 / 2004$ & soil & EPA6010 & REG & $7440-41-7$ & Beryllium & 0.53 & $\mathrm{mg} / \mathrm{kg}$ & & 0.52 \\
\hline 383MB1400.5 & $6 / 14 / 2004$ & soil & HASL300 & REG & $14733-03-0$ & Bi-214 & 0.46 & $\mathrm{pCi} / \mathrm{g}$ & $\mathrm{J}$ & 0.29 \\
\hline 383MB1400.5 & $6 / 14 / 2004$ & soil & EPA8270 & REG & $117-81-7$ & Bis(2-Ethylhexyl)Phthalate & 72 & $\mu \mathrm{g} / \mathrm{kg}$ & $\mathrm{J}$ & 340 \\
\hline 383MB1400.5 & $6 / 14 / 2004$ & soil & EPA6010 & REG & $7440-43-9$ & Cadmium & 0.3 & $\mathrm{mg} / \mathrm{kg}$ & $\mathrm{B}$ & 0.52 \\
\hline 383MB1400.5 & $6 / 14 / 2004$ & soil & EPA6010 & REG & $7440-47-3$ & Chromium & 3.4 & $\mathrm{mg} / \mathrm{kg}$ & & 1 \\
\hline 383MB1400.5 & $6 / 14 / 2004$ & soil & EPA8270 & REG & 218-01-9 & Chrysene & 21 & $\mu \mathrm{g} / \mathrm{kg}$ & $\mathrm{J}$ & 340 \\
\hline 383MB1400.5 & $6 / 14 / 2004$ & soil & HASL300 & REG & $10045-97-3$ & Cs-137 & 8.5 & $\mathrm{pCi} / \mathrm{g}$ & & 0.1 \\
\hline 383MB1400.5 & $6 / 14 / 2004$ & soil & EPA8015 & REG & $68334-30-5$ & Diesel-Range Organics & 45 & $\mathrm{mg} / \mathrm{kg}$ & $\mathrm{Z}, \mathrm{H}$ & 5.1 \\
\hline 383MB1400.5 & $6 / 14 / 2004$ & soil & EPA8270 & REG & 206-44-0 & Fluoranthene & 21 & $\mu \mathrm{g} / \mathrm{kg}$ & $\mathrm{J}$ & 340 \\
\hline 383MB1400.5 & $6 / 14 / 2004$ & soil & HASL300 & REG & 13966-00-2 & $\mathrm{K}-40$ & 9.5 & $\mathrm{pCi} / \mathrm{g}$ & & 1.6 \\
\hline 383MB1400.5 & $6 / 14 / 2004$ & soil & EPA6010 & REG & 7439-92-1 & Lead & 20 & $\mathrm{mg} / \mathrm{kg}$ & & 1.5 \\
\hline 383MB1400.5 & $6 / 14 / 2004$ & soil & EPA7470 & REG & $7439-97-6$ & Mercury & 0.21 & $\mathrm{mg} / \mathrm{kg}$ & & 0.1 \\
\hline $383 \mathrm{MB} 1400.5$ & $6 / 14 / 2004$ & soil & EPA8260 & REG & $75-09-2$ & Methylene Chloride & 0.83 & $\mu \mathrm{g} / \mathrm{kg}$ & $\mathrm{J}, \mathrm{B}$ & 5.2 \\
\hline 383MB1400.5 & $6 / 14 / 2004$ & soil & HASL300 & REG & 15092-94-1 & $\mathrm{Pb}-212$ & 0.62 & $\mathrm{pCi} / \mathrm{g}$ & & 0.23 \\
\hline 383MB1400.5 & $6 / 14 / 2004$ & soil & HASL300 & REG & $15067-28-4$ & $\mathrm{~Pb}-214$ & 0.51 & $\mathrm{pCi} / \mathrm{g}$ & $\mathrm{J}$ & 0.27 \\
\hline 383MB1400.5 & $6 / 14 / 2004$ & soil & EPA8260 & REG & $99-87-6$ & P-Isopropyltoluene & 13 & $\mu \mathrm{g} / \mathrm{kg}$ & & 5.2 \\
\hline 383MB1400.5 & $6 / 14 / 2004$ & soil & UGTAISOPU & REG & 13981-16-3 & Pu-238 & 0.88 & $\mathrm{pCi} / \mathrm{g}$ & & 0.02 \\
\hline 383MB1400.5 & $6 / 14 / 2004$ & soil & UGTAISOPU & REG & $15117-48-3$ & Pu-239 & 2.84 & $\mathrm{pCi} / \mathrm{g}$ & & 0.02 \\
\hline 383MB1400.5 & $6 / 14 / 2004$ & soil & SR7500 & REG & 10098-97-2 & Sr-90 & 0.36 & $\mathrm{pCi} / \mathrm{g}$ & Y1,L1 & 0.22 \\
\hline
\end{tabular}

Page 39 of 78

Uncontrolled When Printed 


\begin{tabular}{|c|c|c|c|c|c|c|c|c|c|c|}
\hline SAMPLE \# & $\begin{array}{c}\text { SAMPLE } \\
\text { DATE }\end{array}$ & MATRIX & $\begin{array}{l}\text { USER TEST } \\
\text { PANEL }\end{array}$ & $\begin{array}{c}\text { SAMPLE } \\
\text { PURPOSE }\end{array}$ & CAS \# & PARAMETER & RESULT & UNITS & $\mathbf{Q}$ & $\begin{array}{l}\text { DETECT } \\
\text { LIMIT }\end{array}$ \\
\hline 383MB1400.5 & $6 / 14 / 2004$ & soil & HASL300 & REG & $14913-50-9$ & TI-208 & 0.222 & $\mathrm{pCi} / \mathrm{g}$ & & 0.11 \\
\hline 383MB1403.5 & $6 / 14 / 2004$ & soil & HASL300 & REG & 14331-83-0 & Ac-228 & 1.9 & $\mathrm{pCi} / \mathrm{g}$ & $G$ & 0.52 \\
\hline 383MB1403.5 & $6 / 14 / 2004$ & soil & HASL300 & REG & 14596-10-2 & Am-241 & 1.57 & $\mathrm{pCi} / \mathrm{g}$ & G & 0.7 \\
\hline 383MB1403.5 & $6 / 14 / 2004$ & soil & EPA6010 & REG & $7440-38-2$ & Arsenic & 2.1 & $\mathrm{mg} / \mathrm{kg}$ & & 1.1 \\
\hline 383MB1403.5 & $6 / 14 / 2004$ & soil & EPA6010 & REG & $7440-39-3$ & Barium & 87 & $\mathrm{mg} / \mathrm{kg}$ & & 11 \\
\hline 383MB1403.5 & $6 / 14 / 2004$ & soil & EPA6010 & REG & $7440-41-7$ & Beryllium & 1.3 & $\mathrm{mg} / \mathrm{kg}$ & & 0.57 \\
\hline 383MB1403.5 & $6 / 14 / 2004$ & soil & HASL300 & REG & $14733-03-0$ & Bi-214 & 1.24 & $\mathrm{pCi} / \mathrm{g}$ & $\mathrm{G}, \mathrm{J}$ & 0.79 \\
\hline 383MB1403.5 & $6 / 14 / 2004$ & soil & EPA8270 & REG & $117-81-7$ & Bis(2-Ethylhexyl)Phthalate & 93 & $\mu \mathrm{g} / \mathrm{kg}$ & $\mathrm{J}$ & 380 \\
\hline 383MB1403.5 & $6 / 14 / 2004$ & soil & EPA6010 & REG & $7440-43-9$ & Cadmium & 0.069 & $\mathrm{mg} / \mathrm{kg}$ & $\mathrm{B}$ & 0.57 \\
\hline 383MB1403.5 & $6 / 14 / 2004$ & soil & EPA6010 & REG & $7440-47-3$ & Chromium & 0.71 & $\mathrm{mg} / \mathrm{kg}$ & $\mathrm{B}$ & 1.1 \\
\hline 383MB1403.5 & $6 / 14 / 2004$ & soil & HASL300 & REG & $10045-97-3$ & Cs-137 & 240 & $\mathrm{pCi} / \mathrm{g}$ & G & 0 \\
\hline 383MB1403.5 & $6 / 14 / 2004$ & soil & HASL300 & REG & $13966-00-2$ & $\mathrm{~K}-40$ & 30.7 & $\mathrm{pCi} / \mathrm{g}$ & G & 2.1 \\
\hline 383MB1403.5 & $6 / 14 / 2004$ & soil & EPA6010 & REG & 7439-92-1 & Lead & 27 & $\mathrm{mg} / \mathrm{kg}$ & & 0.34 \\
\hline 383MB1403.5 & $6 / 14 / 2004$ & soil & EPA7470 & REG & $7439-97-6$ & Mercury & 0.017 & $\mathrm{mg} / \mathrm{kg}$ & $\mathrm{B}$ & 0.11 \\
\hline 383MB1403.5 & $6 / 14 / 2004$ & soil & EPA8260 & REG & $75-09-2$ & Methylene Chloride & 0.97 & $\mu \mathrm{g} / \mathrm{kg}$ & $\mathrm{J}, \mathrm{B}$ & 5.7 \\
\hline 383MB1403.5 & $6 / 14 / 2004$ & soil & HASL300 & REG & 15092-94-1 & $\mathrm{Pb}-212$ & 2.15 & $\mathrm{pCi} / \mathrm{g}$ & G & 0.96 \\
\hline 383MB1403.5 & $6 / 14 / 2004$ & soil & HASL300 & REG & $15067-28-4$ & $\mathrm{~Pb}-214$ & 1.49 & $\mathrm{pCi} / \mathrm{g}$ & $\mathrm{G}, \mathrm{J}$ & 1.26 \\
\hline 383MB1403.5 & $6 / 14 / 2004$ & soil & UGTAISOPU & REG & 13981-16-3 & Pu-238 & 1.11 & $\mathrm{pCi} / \mathrm{g}$ & & 0.02 \\
\hline 383MB1403.5 & $6 / 14 / 2004$ & soil & UGTAISOPU & REG & $15117-48-3$ & Pu-239 & 5.34 & $\mathrm{pCi} / \mathrm{g}$ & & 0.02 \\
\hline 383MB1403.5 & $6 / 14 / 2004$ & soil & EPA6010 & REG & $7782-49-2$ & Selenium & 0.3 & $\mathrm{mg} / \mathrm{kg}$ & $\mathrm{B}$ & 0.57 \\
\hline 383MB1403.5 & $6 / 14 / 2004$ & soil & SR7500 & REG & $10098-97-2$ & Sr-90 & 6.9 & $\mathrm{pCi} / \mathrm{g}$ & Y1 & 0.2 \\
\hline 383MB1403.5 & $6 / 14 / 2004$ & soil & HASL300 & REG & $14913-50-9$ & TI-208 & 0.76 & $\mathrm{pCi} / \mathrm{g}$ & $G$ & 0.45 \\
\hline 383MB1425.5 & $6 / 14 / 2004$ & soil & HASL300 & REG & 14331-83-0 & Ac-228 & 1.25 & $\mathrm{pCi} / \mathrm{g}$ & $G$ & 0.53 \\
\hline 383MB1425.5 & $6 / 14 / 2004$ & soil & EPA6010 & REG & $7440-38-2$ & Arsenic & 1.9 & $\mathrm{mg} / \mathrm{kg}$ & & 1.1 \\
\hline 383MB1425.5 & $6 / 14 / 2004$ & soil & EPA6010 & REG & 7440-39-3 & Barium & 30 & $\mathrm{mg} / \mathrm{kg}$ & & 11 \\
\hline 383MB1425.5 & $6 / 14 / 2004$ & soil & EPA6010 & REG & 7440-41-7 & Beryllium & 0.77 & $\mathrm{mg} / \mathrm{kg}$ & & 0.54 \\
\hline $383 \mathrm{MB} 1425.5$ & $6 / 14 / 2004$ & soil & HASL300 & REG & $14733-03-0$ & Bi-214 & 0.7 & $\mathrm{pCi} / \mathrm{g}$ & $\mathrm{G}, \mathrm{J}$ & 0.32 \\
\hline 383MB1425.5 & $6 / 14 / 2004$ & soil & EPA8270 & REG & 117-81-7 & Bis(2-Ethylhexyl)Phthalate & 220 & $\mu \mathrm{g} / \mathrm{kg}$ & $\mathrm{J}$ & 360 \\
\hline 383MB1425.5 & $6 / 14 / 2004$ & soil & EPA6010 & REG & $7440-43-9$ & Cadmium & 0.045 & $\mathrm{mg} / \mathrm{kg}$ & $\mathrm{B}$ & 0.54 \\
\hline 383MB1425.5 & $6 / 14 / 2004$ & soil & EPA6010 & REG & $7440-47-3$ & Chromium & 1.3 & $\mathrm{mg} / \mathrm{kg}$ & & 1.1 \\
\hline 383MB1425.5 & $6 / 14 / 2004$ & soil & HASL300 & REG & $10045-97-3$ & Cs-137 & 3.18 & $\mathrm{pCi} / \mathrm{g}$ & G & 0.14 \\
\hline 383MB1425.5 & $6 / 14 / 2004$ & soil & HASL300 & REG & $13966-00-2$ & $\mathrm{~K}-40$ & 19.6 & $\mathrm{pCi} / \mathrm{g}$ & G & 1.7 \\
\hline 383MB1425.5 & $6 / 14 / 2004$ & soil & EPA6010 & REG & 7439-92-1 & Lead & 95 & $\mathrm{mg} / \mathrm{kg}$ & & 1.6 \\
\hline 383MB1425.5 & $6 / 14 / 2004$ & soil & EPA7470 & REG & 7439-97-6 & Mercury & 0.013 & $\mathrm{mg} / \mathrm{kg}$ & $\mathrm{B}$ & 0.11 \\
\hline 383MB1425.5 & $6 / 14 / 2004$ & soil & EPA8260 & REG & $75-09-2$ & Methylene Chloride & 0.86 & $\mu \mathrm{g} / \mathrm{kg}$ & $\mathrm{J}, \mathrm{B}$ & 5.4 \\
\hline
\end{tabular}

Page 40 of 78

Uncontrolled When Printed 


\begin{tabular}{|c|c|c|c|c|c|c|c|c|c|c|}
\hline SAMPLE \# & $\begin{array}{c}\text { SAMPLE } \\
\text { DATE }\end{array}$ & MATRIX & $\begin{array}{l}\text { USER TEST } \\
\text { PANEL }\end{array}$ & $\begin{array}{c}\text { SAMPLE } \\
\text { PURPOSE }\end{array}$ & CAS \# & PARAMETER & RESULT & UNITS & $\mathbf{Q}$ & $\begin{array}{l}\text { DETECT } \\
\text { LIMIT }\end{array}$ \\
\hline 383MB1425.5 & $6 / 14 / 2004$ & soil & HASL300 & REG & 15092-94-1 & $\mathrm{Pb}-212$ & 1.44 & $\mathrm{pCi} / \mathrm{g}$ & $G$ & 0.26 \\
\hline 383MB1425.5 & $6 / 14 / 2004$ & soil & HASL300 & REG & $15067-28-4$ & $\mathrm{~Pb}-214$ & 0.91 & $\mathrm{pCi} / \mathrm{g}$ & $\mathrm{G}, \mathrm{J}$ & 0.27 \\
\hline 383MB1425.5 & $6 / 14 / 2004$ & soil & UGTAISOPU & REG & 13981-16-3 & Pu-238 & 0.051 & $\mathrm{pCi} / \mathrm{g}$ & & 0.021 \\
\hline 383MB1425.5 & $6 / 14 / 2004$ & soil & UGTAISOPU & REG & $15117-48-3$ & Pu-239 & 0.203 & $\mathrm{pCi} / \mathrm{g}$ & & 0.009 \\
\hline 383MB1425.5 & $6 / 14 / 2004$ & soil & SR7500 & REG & 10098-97-2 & Sr-90 & 0.33 & $\mathrm{pCi} / \mathrm{g}$ & Y1,L7 & 0.25 \\
\hline 383MB1425.5 & $6 / 14 / 2004$ & soil & HASL300 & REG & $14913-50-9$ & TI-208 & 0.48 & $\mathrm{pCi} / \mathrm{g}$ & G & 0.13 \\
\hline 383MB1500.5 & $6 / 17 / 2004$ & soil & HASL300 & REG & 14331-83-0 & Ac-228 & 1.63 & $\mathrm{pCi} / \mathrm{g}$ & G & 0.51 \\
\hline 383MB1500.5 & $6 / 17 / 2004$ & soil & EPA6010 & REG & $7440-38-2$ & Arsenic & 3.2 & $\mathrm{mg} / \mathrm{kg}$ & & 1.1 \\
\hline 383MB1500.5 & $6 / 17 / 2004$ & soil & EPA6010 & REG & $7440-39-3$ & Barium & 3700 & $\mathrm{mg} / \mathrm{kg}$ & & 540 \\
\hline 383MB1500.5 & $6 / 17 / 2004$ & soil & EPA6010 & REG & $7440-41-7$ & Beryllium & 0.73 & $\mathrm{mg} / \mathrm{kg}$ & & 0.54 \\
\hline 383MB1500.5 & $6 / 17 / 2004$ & soil & HASL300 & REG & $14733-03-0$ & Bi-214 & 0.7 & $\mathrm{pCi} / \mathrm{g}$ & $\mathrm{G}, \mathrm{J}$ & 0.32 \\
\hline 383MB1500.5 & $6 / 17 / 2004$ & soil & EPA6010 & REG & $7440-43-9$ & Cadmium & 0.17 & $\mathrm{mg} / \mathrm{kg}$ & $\mathrm{B}$ & 0.54 \\
\hline 383MB1500.5 & $6 / 17 / 2004$ & soil & EPA6010 & REG & $7440-47-3$ & Chromium & 3.7 & $\mathrm{mg} / \mathrm{kg}$ & & 1.1 \\
\hline 383MB1500.5 & $6 / 17 / 2004$ & soil & HASL300 & REG & $10045-97-3$ & Cs-137 & 20.7 & $\mathrm{pCi} / \mathrm{g}$ & G & 0.2 \\
\hline 383MB1500.5 & $6 / 17 / 2004$ & soil & EPA8015 & REG & $68334-30-5$ & Diesel-Range Organics & 19 & $\mathrm{mg} / \mathrm{kg}$ & $\mathrm{Z}, \mathrm{H}$ & 5.4 \\
\hline 383MB1500.5 & $6 / 17 / 2004$ & soil & HASL300 & REG & 13966-00-2 & $\mathrm{K}-40$ & 23.8 & $\mathrm{pCi} / \mathrm{g}$ & $\mathrm{G}$ & 1.3 \\
\hline 383MB1500.5 & $6 / 17 / 2004$ & soil & EPA6010 & REG & 7439-92-1 & Lead & 30 & $\mathrm{mg} / \mathrm{kg}$ & & 0.33 \\
\hline 383MB1500.5 & $6 / 17 / 2004$ & soil & EPA7470 & REG & $7439-97-6$ & Mercury & 0.024 & $\mathrm{mg} / \mathrm{kg}$ & $\mathrm{B}$ & 0.11 \\
\hline 383MB1500.5 & $6 / 17 / 2004$ & soil & EPA8260 & REG & $75-09-2$ & Methylene Chloride & 0.99 & $\mu \mathrm{g} / \mathrm{kg}$ & $\mathrm{J}, \mathrm{B}$ & 5.4 \\
\hline 383MB1500.5 & $6 / 17 / 2004$ & soil & HASL300 & REG & 15092-94-1 & $\mathrm{Pb}-212$ & 1.47 & $\mathrm{pCi} / \mathrm{g}$ & $G$ & 0.28 \\
\hline 383MB1500.5 & $6 / 17 / 2004$ & soil & HASL300 & REG & $15067-28-4$ & $\mathrm{~Pb}-214$ & 0.73 & $\mathrm{pCi} / \mathrm{g}$ & $\mathrm{G}, \mathrm{J}$ & 0.38 \\
\hline 383MB1500.5 & $6 / 17 / 2004$ & soil & UGTAISOPU & REG & 13981-16-3 & Pu-238 & 0.11 & $\mathrm{pCi} / \mathrm{g}$ & & 0.021 \\
\hline 383MB1500.5 & $6 / 17 / 2004$ & soil & UGTAISOPU & REG & $15117-48-3$ & Pu-239 & 1.15 & $\mathrm{pCi} / \mathrm{g}$ & & 0.01 \\
\hline 383MB1500.5 & $6 / 17 / 2004$ & soil & SR7500 & REG & 10098-97-2 & Sr-90 & 0.67 & $\mathrm{pCi} / \mathrm{g}$ & Y1 & 0.24 \\
\hline 383MB1500.5 & $6 / 17 / 2004$ & soil & HASL300 & REG & 14913-50-9 & TI-208 & 0.33 & $\mathrm{pCi} / \mathrm{g}$ & $G$ & 0.19 \\
\hline 383МH0136.5 & $5 / 25 / 2004$ & soil & HASL300 & REG & 14331-83-0 & Ac-228 & 1.67 & $\mathrm{pCi} / \mathrm{g}$ & G & 0.69 \\
\hline 383МH0136.5 & $5 / 25 / 2004$ & soil & EPA8260 & REG & $67-64-1$ & Acetone & 10 & $\mu \mathrm{g} / \mathrm{kg}$ & $\mathrm{J}$ & 24 \\
\hline 383МH0136.5 & $5 / 25 / 2004$ & soil & EPA6010 & REG & $7440-38-2$ & Arsenic & 3.3 & $\mathrm{mg} / \mathrm{kg}$ & & 1.2 \\
\hline $383 \mathrm{MH} 0136.5$ & $5 / 25 / 2004$ & soil & EPA6010 & REG & $7440-39-3$ & Barium & 150 & $\mathrm{mg} / \mathrm{kg}$ & & 12 \\
\hline $383 \mathrm{MH} 0136.5$ & $5 / 25 / 2004$ & soil & EPA6010 & REG & $7440-41-7$ & Beryllium & 1.2 & $\mathrm{mg} / \mathrm{kg}$ & & 0.59 \\
\hline $383 \mathrm{MH} 0136.5$ & $5 / 25 / 2004$ & soil & HASL300 & REG & $14733-03-0$ & Bi-214 & 0.88 & $\mathrm{pCi} / \mathrm{g}$ & $\mathrm{G}, \mathrm{J}$ & 0.39 \\
\hline $383 \mathrm{MH} 0136.5$ & $5 / 25 / 2004$ & soil & EPA6010 & REG & $7440-47-3$ & Chromium & 2.3 & $\mathrm{mg} / \mathrm{kg}$ & & 1.2 \\
\hline 383MH0136.5 & $5 / 25 / 2004$ & soil & HASL300 & REG & $10045-97-3$ & Cs-137 & 4.11 & $\mathrm{pCi} / \mathrm{g}$ & $G$ & 0.2 \\
\hline 383МH0136.5 & $5 / 25 / 2004$ & soil & HASL300 & REG & 13966-00-2 & $\mathrm{K}-40$ & 26.9 & $\mathrm{pCi} / \mathrm{g}$ & $G$ & 2.1 \\
\hline 383MH0136.5 & $5 / 25 / 2004$ & soil & EPA6010 & REG & 7439-92-1 & Lead & 14 & $\mathrm{mg} / \mathrm{kg}$ & & 0.35 \\
\hline
\end{tabular}

Page 41 of 78

Uncontrolled When Printed 


\begin{tabular}{|c|c|c|c|c|c|c|c|c|c|c|}
\hline SAMPLE \# & $\begin{array}{c}\text { SAMPLE } \\
\text { DATE }\end{array}$ & MATRIX & $\begin{array}{c}\text { USER TEST } \\
\text { PANEL }\end{array}$ & $\begin{array}{c}\text { SAMPLE } \\
\text { PURPOSE }\end{array}$ & CAS \# & PARAMETER & RESULT & UNITS & $\mathbf{Q}$ & $\begin{array}{c}\text { DETECT } \\
\text { LIMIT }\end{array}$ \\
\hline 383МН0136.5 & $5 / 25 / 2004$ & soil & EPA7470 & REG & 7439-97-6 & Mercury & 0.079 & $\mathrm{mg} / \mathrm{kg}$ & $B$ & 0.12 \\
\hline 383MH0136.5 & $5 / 25 / 2004$ & soil & HASL300 & REG & 15092-94-1 & $\mathrm{Pb}-212$ & 2.55 & $\mathrm{pCi} / \mathrm{g}$ & $\mathrm{J}$ & 0.38 \\
\hline $383 \mathrm{MH} 0136.5$ & $5 / 25 / 2004$ & soil & HASL300 & REG & 15067-28-4 & $\mathrm{Pb}-214$ & 1.13 & $\mathrm{pCi} / \mathrm{g}$ & $\mathrm{G}, \mathrm{J}$ & 0.4 \\
\hline $383 \mathrm{MH} 0136.5$ & $5 / 25 / 2004$ & soil & UGTAISOPU & REG & $15117-48-3$ & Pu-239 & 0.141 & $\mathrm{pCi} / \mathrm{g}$ & & 0.01 \\
\hline 383МH0136.5 & $5 / 25 / 2004$ & soil & HASL300 & REG & $14913-50-9$ & TI-208 & 0.65 & $\mathrm{pCi} / \mathrm{g}$ & $\mathrm{G}$ & 0.18 \\
\hline 383МH0168.5 & $5 / 25 / 2004$ & soil & EPA6010 & REG & $7440-38-2$ & Arsenic & 0.44 & $\mathrm{mg} / \mathrm{kg}$ & $B$ & 1 \\
\hline 383МH0168.5 & $5 / 25 / 2004$ & soil & EPA6010 & REG & $7440-39-3$ & Barium & 5.3 & $\mathrm{mg} / \mathrm{kg}$ & $\mathrm{B}$ & 10 \\
\hline 383МН0168.5 & $5 / 25 / 2004$ & soil & EPA6010 & REG & $7440-41-7$ & Beryllium & 0.23 & $\mathrm{mg} / \mathrm{kg}$ & $\mathrm{B}$ & 0.52 \\
\hline 383МH0168.5 & $5 / 25 / 2004$ & soil & EPA6010 & REG & $7440-43-9$ & Cadmium & 0.036 & $\mathrm{mg} / \mathrm{kg}$ & $\mathrm{B}$ & 0.52 \\
\hline 383МH0168.5 & $5 / 25 / 2004$ & soil & EPA6010 & REG & $7440-47-3$ & Chromium & 1.3 & $\mathrm{mg} / \mathrm{kg}$ & & 1 \\
\hline 383МH0168.5 & $5 / 25 / 2004$ & soil & EPA7470 & REG & $7439-97-6$ & Mercury & 0.052 & $\mathrm{mg} / \mathrm{kg}$ & $\mathrm{B}$ & 0.1 \\
\hline 383МH0168.5 & $5 / 25 / 2004$ & soil & EPA6010 & REG & $7440-22-4$ & Silver & 0.57 & $\mathrm{mg} / \mathrm{kg}$ & $B$ & 1 \\
\hline 383МH0313.0 & $5 / 25 / 2004$ & soil & HASL300 & REG & 14331-83-0 & Ac-228 & 1.9 & $\mathrm{pCi} / \mathrm{g}$ & $\mathrm{G}$ & 0.73 \\
\hline 383МН0313.0 & $5 / 25 / 2004$ & soil & EPA8260 & REG & 67-64-1 & Acetone & 10 & $\mu \mathrm{g} / \mathrm{kg}$ & $\mathrm{J}$ & 24 \\
\hline 383МН0313.0 & $5 / 25 / 2004$ & soil & EPA6010 & REG & $7440-38-2$ & Arsenic & 1.7 & $\mathrm{mg} / \mathrm{kg}$ & & 1.2 \\
\hline $383 \mathrm{MH} 0313.0$ & $5 / 25 / 2004$ & soil & EPA6010 & REG & $7440-39-3$ & Barium & 250 & $\mathrm{mg} / \mathrm{kg}$ & & 12 \\
\hline 383МH0313.0 & $5 / 25 / 2004$ & soil & EPA6010 & REG & $7440-41-7$ & Beryllium & 1.5 & $\mathrm{mg} / \mathrm{kg}$ & & 0.61 \\
\hline 383МН0313.0 & $5 / 25 / 2004$ & soil & HASL300 & REG & $14733-03-0$ & $\mathrm{Bi}-214$ & 1.27 & $\mathrm{pCi} / \mathrm{g}$ & $\mathrm{G}, \mathrm{J}$ & 0.38 \\
\hline 383МH0313.0 & $5 / 25 / 2004$ & soil & EPA6010 & REG & $7440-43-9$ & Cadmium & 0.045 & $\mathrm{mg} / \mathrm{kg}$ & $\mathrm{B}$ & 0.61 \\
\hline 383МH0313.0 & $5 / 25 / 2004$ & soil & EPA6010 & REG & $7440-47-3$ & Chromium & 0.57 & $\mathrm{mg} / \mathrm{kg}$ & $\mathrm{B}$ & 1.2 \\
\hline 383МН0313.0 & $5 / 25 / 2004$ & soil & HASL300 & REG & $10045-97-3$ & Cs-137 & 0.43 & $\mathrm{pCi} / \mathrm{g}$ & LT,G & 0.17 \\
\hline 383МH0313.0 & $5 / 25 / 2004$ & soil & HASL300 & REG & $13966-00-2$ & $\mathrm{~K}-40$ & 35.9 & $\mathrm{pCi} / \mathrm{g}$ & $\mathrm{G}$ & 1.7 \\
\hline 383МH0313.0 & $5 / 25 / 2004$ & soil & EPA6010 & REG & $7439-92-1$ & Lead & 35 & $\mathrm{mg} / \mathrm{kg}$ & & 0.37 \\
\hline 383МH0313.0 & $5 / 25 / 2004$ & soil & EPA7470 & REG & $7439-97-6$ & Mercury & 0.0037 & $\mathrm{mg} / \mathrm{kg}$ & $B$ & 0.12 \\
\hline 383МH0313.0 & $5 / 25 / 2004$ & soil & HASL300 & REG & 15092-94-1 & $\mathrm{Pb}-212$ & 2.4 & $\mathrm{pCi} / \mathrm{g}$ & $\mathrm{J}$ & 0.26 \\
\hline $383 \mathrm{MH} 0313.0$ & $5 / 25 / 2004$ & soil & HASL300 & REG & $15067-28-4$ & $\mathrm{~Pb}-214$ & 1.32 & $\mathrm{pCi} / \mathrm{g}$ & $\mathrm{G}, \mathrm{J}$ & 0.29 \\
\hline 383МН0313.0 & $5 / 25 / 2004$ & soil & EPA6010 & REG & $7782-49-2$ & Selenium & 0.31 & $\mathrm{mg} / \mathrm{kg}$ & $\mathrm{B}$ & 0.61 \\
\hline 383МH0313.0 & $5 / 25 / 2004$ & soil & HASL300 & REG & 14913-50-9 & TI-208 & 0.81 & $\mathrm{pCi} / \mathrm{g}$ & G & 0.19 \\
\hline $383 \mathrm{MH} 0353.5$ & $5 / 25 / 2004$ & soil & HASL300 & REG & 14331-83-0 & Ac-228 & 2.4 & $\mathrm{pCi} / \mathrm{g}$ & $\mathrm{G}$ & 0.47 \\
\hline 383МH0353.5 & $5 / 25 / 2004$ & soil & HASL300 & REG & $14596-10-2$ & Am-241 & 3.2 & $\mathrm{pCi} / \mathrm{g}$ & $\mathrm{J}$ & 1.8 \\
\hline 383МН0353.5 & $5 / 25 / 2004$ & soil & EPA6010 & REG & $7440-38-2$ & Arsenic & 6 & $\mathrm{mg} / \mathrm{kg}$ & & 1.1 \\
\hline 383МH0353.5 & $5 / 25 / 2004$ & soil & EPA6010 & REG & $7440-39-3$ & Barium & 350 & $\mathrm{mg} / \mathrm{kg}$ & & 11 \\
\hline 383МH0353.5 & $5 / 25 / 2004$ & soil & EPA6010 & REG & $7440-41-7$ & Beryllium & 1.2 & $\mathrm{mg} / \mathrm{kg}$ & & 0.57 \\
\hline 383МН0353.5 & $5 / 25 / 2004$ & soil & HASL300 & REG & $14733-03-0$ & $\mathrm{Bi}-214$ & 0.89 & $\mathrm{pCi} / \mathrm{g}$ & G,J & 0.48 \\
\hline 383МH0353.5 & $5 / 25 / 2004$ & soil & EPA6010 & REG & $7440-43-9$ & Cadmium & 0.079 & $\mathrm{mg} / \mathrm{kg}$ & $\mathrm{B}$ & 0.57 \\
\hline
\end{tabular}

Page 42 of 78

Uncontrolled When Printed 


\begin{tabular}{|c|c|c|c|c|c|c|c|c|c|c|}
\hline SAMPLE \# & $\begin{array}{c}\text { SAMPLE } \\
\text { DATE }\end{array}$ & MATRIX & $\begin{array}{l}\text { USER TEST } \\
\text { PANEL }\end{array}$ & $\begin{array}{c}\text { SAMPLE } \\
\text { PURPOSE }\end{array}$ & CAS \# & PARAMETER & RESULT & UNITS & $\mathbf{Q}$ & $\begin{array}{l}\text { DETECT } \\
\text { LIMIT }\end{array}$ \\
\hline 383МH0353.5 & $5 / 25 / 2004$ & soil & EPA6010 & REG & $7440-47-3$ & Chromium & 2.4 & $\mathrm{mg} / \mathrm{kg}$ & & 1.1 \\
\hline 383МH0353.5 & $5 / 25 / 2004$ & soil & HASL300 & REG & $10045-97-3$ & Cs-137 & 144 & $\mathrm{pCi} / \mathrm{g}$ & G & 0 \\
\hline 383MH0353.5 & $5 / 25 / 2004$ & soil & EPA8015 & REG & 68334-30-5 & Diesel-Range Organics & 3.5 & $\mathrm{mg} / \mathrm{kg}$ & $\mathrm{J}$ & 5.7 \\
\hline $383 \mathrm{MH} 0353.5$ & $5 / 25 / 2004$ & soil & HASL300 & REG & $13966-00-2$ & $\mathrm{~K}-40$ & 28.7 & $\mathrm{pCi} / \mathrm{g}$ & G & 1.3 \\
\hline 383МH0353.5 & $5 / 25 / 2004$ & soil & EPA6010 & REG & 7439-92-1 & Lead & 40 & $\mathrm{mg} / \mathrm{kg}$ & & 1.7 \\
\hline 383МH0353.5 & $5 / 25 / 2004$ & soil & EPA7470 & REG & $7439-97-6$ & Mercury & 0.51 & $\mathrm{mg} / \mathrm{kg}$ & & 0.11 \\
\hline 383МH0353.5 & $5 / 25 / 2004$ & soil & HASL300 & REG & 15092-94-1 & $\mathrm{Pb}-212$ & 2.23 & $\mathrm{pCi} / \mathrm{g}$ & $\mathrm{J}$ & 0.6 \\
\hline 383МH0353.5 & $5 / 25 / 2004$ & soil & UGTAISOPU & REG & 13981-16-3 & Pu-238 & 0.77 & $\mathrm{pCi} / \mathrm{g}$ & & 0.01 \\
\hline 383МH0353.5 & $5 / 25 / 2004$ & soil & UGTAISOPU & REG & $15117-48-3$ & Pu-239 & 7.3 & $\mathrm{pCi} / \mathrm{g}$ & & 0 \\
\hline 383МH0353.5 & $5 / 25 / 2004$ & soil & EPA6010 & REG & $7782-49-2$ & Selenium & 0.67 & $\mathrm{mg} / \mathrm{kg}$ & & 0.57 \\
\hline 383MH0353.5 & $5 / 25 / 2004$ & soil & SR7500 & REG & 10098-97-2 & Sr-90 & 0.94 & $\mathrm{pCi} / \mathrm{g}$ & & 0.22 \\
\hline 383МH0353.5 & $5 / 25 / 2004$ & soil & HASL300 & REG & $14913-50-9$ & TI-208 & 0.78 & $\mathrm{pCi} / \mathrm{g}$ & G & 0.3 \\
\hline 383МH0361.0 & $5 / 25 / 2004$ & soil & EPA6010 & REG & $7440-38-2$ & Arsenic & 0.48 & $\mathrm{mg} / \mathrm{kg}$ & $\mathrm{B}$ & 1 \\
\hline 383МН0361.0 & $5 / 25 / 2004$ & soil & EPA6010 & REG & $7440-39-3$ & Barium & 2.6 & $\mathrm{mg} / \mathrm{kg}$ & $\mathrm{B}$ & 10 \\
\hline 383МН0361.0 & $5 / 25 / 2004$ & soil & EPA6010 & REG & 7440-41-7 & Beryllium & 0.2 & $\mathrm{mg} / \mathrm{kg}$ & $B$ & 0.5 \\
\hline 383МH0361.0 & $5 / 25 / 2004$ & soil & EPA6010 & REG & $7440-47-3$ & Chromium & 1.2 & $\mathrm{mg} / \mathrm{kg}$ & & 1 \\
\hline 383МH0361.0 & $5 / 25 / 2004$ & soil & EPA7470 & REG & 7439-97-6 & Mercury & 0.016 & $\mathrm{mg} / \mathrm{kg}$ & $\mathrm{B}$ & 0.1 \\
\hline 383МH0361.0 & $5 / 25 / 2004$ & soil & EPA6010 & REG & $7440-22-4$ & Silver & 0.23 & $\mathrm{mg} / \mathrm{kg}$ & $\mathrm{B}$ & 1 \\
\hline 383МH0432.0 & $5 / 22 / 2004$ & soil & HASL300 & REG & 14331-83-0 & Ac-228 & 2.18 & $\mathrm{pCi} / \mathrm{g}$ & $G$ & 0.65 \\
\hline 383МH0432.0 & $5 / 22 / 2004$ & soil & EPA6010 & REG & $7440-38-2$ & Arsenic & 2.5 & $\mathrm{mg} / \mathrm{kg}$ & & 1.2 \\
\hline 383МH0432.0 & $5 / 22 / 2004$ & soil & EPA6010 & REG & $7440-39-3$ & Barium & 160 & $\mathrm{mg} / \mathrm{kg}$ & & 12 \\
\hline $383 \mathrm{MH} 0432.0$ & $5 / 22 / 2004$ & soil & EPA6010 & REG & 7440-41-7 & Beryllium & 1.2 & $\mathrm{mg} / \mathrm{kg}$ & & 0.59 \\
\hline 383МH0432.0 & $5 / 22 / 2004$ & soil & HASL300 & REG & $14733-03-0$ & Bi-214 & 1.49 & $\mathrm{pCi} / \mathrm{g}$ & $\mathrm{G}, \mathrm{J}$ & 0.29 \\
\hline 383МH0432.0 & $5 / 22 / 2004$ & soil & EPA6010 & REG & $7440-47-3$ & Chromium & 2.2 & $\mathrm{mg} / \mathrm{kg}$ & & 1.2 \\
\hline $383 \mathrm{MH} 0432.0$ & $5 / 22 / 2004$ & soil & HASL300 & REG & $10045-97-3$ & Cs-137 & 2.96 & $\mathrm{pCi} / \mathrm{g}$ & G & 0.18 \\
\hline 383МH0432.0 & $5 / 22 / 2004$ & soil & HASL300 & REG & 13966-00-2 & $\mathrm{K}-40$ & 36 & $\mathrm{pCi} / \mathrm{g}$ & $G$ & 1.8 \\
\hline 383МH0432.0 & $5 / 22 / 2004$ & soil & EPA6010 & REG & 7439-92-1 & Lead & 15 & $\mathrm{mg} / \mathrm{kg}$ & & 0.36 \\
\hline $383 \mathrm{MH} 0432.0$ & $5 / 22 / 2004$ & soil & EPA7470 & REG & 7439-97-6 & Mercury & 0.0012 & $\mathrm{mg} / \mathrm{kg}$ & $B$ & 0.12 \\
\hline $383 \mathrm{MH} 0432.0$ & $5 / 22 / 2004$ & soil & HASL300 & REG & 15092-94-1 & $\mathrm{Pb}-212$ & 2.68 & $\mathrm{pCi} / \mathrm{g}$ & $\mathrm{J}$ & 0.27 \\
\hline $383 \mathrm{MH} 0432.0$ & $5 / 22 / 2004$ & soil & HASL300 & REG & $15067-28-4$ & $\mathrm{~Pb}-214$ & 1.69 & $\mathrm{pCi} / \mathrm{g}$ & $\mathrm{G}, \mathrm{J}$ & 0.32 \\
\hline $383 \mathrm{MH} 0432.0$ & $5 / 22 / 2004$ & soil & HASL300 & REG & 14913-50-9 & TI-208 & 0.74 & $\mathrm{pCi} / \mathrm{g}$ & $G$ & 0.18 \\
\hline $383 \mathrm{MH} 0441.5$ & $5 / 22 / 2004$ & soil & EPA6010 & REG & $7440-38-2$ & Arsenic & 0.76 & $\mathrm{mg} / \mathrm{kg}$ & $\mathrm{B}$ & 1 \\
\hline 383MH0441.5 & $5 / 22 / 2004$ & soil & EPA6010 & REG & $7440-39-3$ & Barium & 5 & $\mathrm{mg} / \mathrm{kg}$ & $\mathrm{B}$ & 10 \\
\hline 383МH0441.5 & $5 / 22 / 2004$ & soil & EPA6010 & REG & 7440-41-7 & Beryllium & 0.18 & $\mathrm{mg} / \mathrm{kg}$ & $B$ & 0.5 \\
\hline $383 \mathrm{MH} 0441.5$ & $5 / 22 / 2004$ & soil & EPA6010 & REG & 7440-43-9 & Cadmium & 0.46 & $\mathrm{mg} / \mathrm{kg}$ & $\mathrm{B}$ & 0.5 \\
\hline
\end{tabular}

Page 43 of 78

Uncontrolled When Printed 


\begin{tabular}{|c|c|c|c|c|c|c|c|c|c|c|}
\hline SAMPLE \# & $\begin{array}{c}\text { SAMPLE } \\
\text { DATE }\end{array}$ & MATRIX & $\begin{array}{c}\text { USER TEST } \\
\text { PANEL }\end{array}$ & $\begin{array}{c}\text { SAMPLE } \\
\text { PURPOSE }\end{array}$ & CAS \# & PARAMETER & RESULT & UNITS & $\mathbf{Q}$ & $\begin{array}{c}\text { DETECT } \\
\text { LIMIT }\end{array}$ \\
\hline 383МH0441.5 & $5 / 22 / 2004$ & soil & EPA6010 & REG & $7440-47-3$ & Chromium & 1.6 & $\mathrm{mg} / \mathrm{kg}$ & & 1 \\
\hline $383 \mathrm{MH} 0441.5$ & $5 / 22 / 2004$ & soil & EPA6010 & REG & 7439-92-1 & Lead & 56 & $\mathrm{mg} / \mathrm{kg}$ & & 1.5 \\
\hline $383 \mathrm{MH} 0441.5$ & $5 / 22 / 2004$ & soil & EPA7470 & REG & $7439-97-6$ & Mercury & 0.22 & $\mathrm{mg} / \mathrm{kg}$ & & 0.1 \\
\hline $383 \mathrm{MH} 0441.5$ & $5 / 22 / 2004$ & soil & EPA8260 & REG & $75-09-2$ & Methylene Chloride & 0.78 & $\mu \mathrm{g} / \mathrm{kg}$ & $\mathrm{J}, \mathrm{B}$ & 5 \\
\hline $383 \mathrm{MH} 0441.5$ & $5 / 22 / 2004$ & soil & EPA6010 & REG & $7440-22-4$ & Silver & 1.3 & $\mathrm{mg} / \mathrm{kg}$ & & 1 \\
\hline 383МH0520.5 & $5 / 27 / 2004$ & soil & HASL300 & REG & 14331-83-0 & Ac-228 & 2.01 & $\mathrm{pCi} / \mathrm{g}$ & $\mathrm{G}$ & 0.57 \\
\hline 383МH0520.5 & $5 / 27 / 2004$ & soil & EPA8260 & REG & 67-64-1 & Acetone & 7.2 & $\mu \mathrm{g} / \mathrm{kg}$ & $\mathrm{J}$ & 23 \\
\hline 383МH0520.5 & $5 / 27 / 2004$ & soil & EPA6010 & REG & $7440-38-2$ & Arsenic & 2.2 & $\mathrm{mg} / \mathrm{kg}$ & & 1.2 \\
\hline 383МH0520.5 & $5 / 27 / 2004$ & soil & EPA6010 & REG & 7440-39-3 & Barium & 63 & $\mathrm{mg} / \mathrm{kg}$ & & 12 \\
\hline 383МH0520.5 & $5 / 27 / 2004$ & soil & EPA6010 & REG & $7440-41-7$ & Beryllium & 1.2 & $\mathrm{mg} / \mathrm{kg}$ & & 0.59 \\
\hline 383МH0520.5 & $5 / 27 / 2004$ & soil & HASL300 & REG & $14733-03-0$ & $\mathrm{Bi}-214$ & 1.38 & $\mathrm{pCi} / \mathrm{g}$ & $\mathrm{G}, \mathrm{J}$ & 0.33 \\
\hline 383МH0520.5 & $5 / 27 / 2004$ & soil & EPA6010 & REG & $7440-47-3$ & Chromium & 0.73 & $\mathrm{mg} / \mathrm{kg}$ & $B$ & 1.2 \\
\hline 383МH0520.5 & $5 / 27 / 2004$ & soil & HASL300 & REG & 10045-97-3 & Cs-137 & 3.95 & $\mathrm{pCi} / \mathrm{g}$ & $\mathrm{G}$ & 0.18 \\
\hline 383МH0520.5 & $5 / 27 / 2004$ & soil & HASL300 & REG & $13966-00-2$ & $\mathrm{~K}-40$ & 41.6 & $\mathrm{pCi} / \mathrm{g}$ & $\mathrm{G}$ & 1.6 \\
\hline 383МН0520.5 & $5 / 27 / 2004$ & soil & EPA6010 & REG & $7439-92-1$ & Lead & 10 & $\mathrm{mg} / \mathrm{kg}$ & & 0.35 \\
\hline 383МH0520.5 & $5 / 27 / 2004$ & soil & EPA7470 & REG & $7439-97-6$ & Mercury & 0.0014 & $\mathrm{mg} / \mathrm{kg}$ & $\mathrm{B}$ & 0.12 \\
\hline 383МH0520.5 & $5 / 27 / 2004$ & soil & EPA8260 & REG & $75-09-2$ & Methylene Chloride & 1.7 & $\mu \mathrm{g} / \mathrm{kg}$ & $\mathrm{J}, \mathrm{B}$ & 5.9 \\
\hline 383MH0520.5 & $5 / 27 / 2004$ & soil & HASL300 & REG & 15092-94-1 & $\mathrm{Pb}-212$ & 2.48 & $\mathrm{pCi} / \mathrm{g}$ & $\mathrm{J}$ & 0.25 \\
\hline 383MH0520.5 & $5 / 27 / 2004$ & soil & HASL300 & REG & $15067-28-4$ & $\mathrm{~Pb}-214$ & 1.65 & $\mathrm{pCi} / \mathrm{g}$ & $\mathrm{G}, \mathrm{J}$ & 0.34 \\
\hline 383МH0520.5 & $5 / 27 / 2004$ & soil & UGTAISOPU & REG & $15117-48-3$ & Pu-239 & 0.189 & $\mathrm{pCi} / \mathrm{g}$ & & 0.017 \\
\hline 383МH0520.5 & $5 / 27 / 2004$ & soil & HASL300 & REG & $14913-50-9$ & TI-208 & 0.73 & $\mathrm{pCi} / \mathrm{g}$ & $\mathrm{G}$ & 0.14 \\
\hline 383МH0559.5 & $5 / 27 / 2004$ & soil & HASL300 & REG & 14331-83-0 & Ac-228 & 2.27 & $\mathrm{pCi} / \mathrm{g}$ & $\mathrm{G}, \mathrm{TI}$ & 1.01 \\
\hline 383МH0559.5 & $5 / 27 / 2004$ & soil & HASL300 & REG & $14596-10-2$ & Am-241 & 12.8 & $\mathrm{pCi} / \mathrm{g}$ & $\mathrm{J}$ & 4.6 \\
\hline 383МH0559.5 & $5 / 27 / 2004$ & soil & EPA6010 & REG & $7440-38-2$ & Arsenic & 3.6 & $\mathrm{mg} / \mathrm{kg}$ & & 1.3 \\
\hline 383МH0559.5 & $5 / 27 / 2004$ & soil & EPA6010 & REG & $7440-39-3$ & Barium & 56 & $\mathrm{mg} / \mathrm{kg}$ & & 13 \\
\hline 383МH0559.5 & $5 / 27 / 2004$ & soil & EPA8270 & REG & $56-55-3$ & Benzo(A)Anthracene & 140 & $\mu \mathrm{g} / \mathrm{kg}$ & $\mathrm{J}$ & 420 \\
\hline 383МH0559.5 & $5 / 27 / 2004$ & soil & EPA8270 & REG & $50-32-8$ & Benzo(A)Pyrene & 120 & $\mu \mathrm{g} / \mathrm{kg}$ & $\mathrm{J}$ & 420 \\
\hline 383МH0559.5 & $5 / 27 / 2004$ & soil & EPA8270 & REG & $205-99-2$ & Benzo(B)Fluoranthene & 180 & $\mu \mathrm{g} / \mathrm{kg}$ & $\mathrm{J}$ & 420 \\
\hline 383МH0559.5 & $5 / 27 / 2004$ & soil & EPA8270 & REG & $191-24-2$ & Benzo(G,H,I)Perylene & 85 & $\mu \mathrm{g} / \mathrm{kg}$ & $\mathrm{J}$ & 420 \\
\hline 383МH0559.5 & $5 / 27 / 2004$ & soil & EPA8270 & REG & $207-08-9$ & Benzo(K)Fluoranthene & 80 & $\mu \mathrm{g} / \mathrm{kg}$ & $\mathrm{J}$ & 420 \\
\hline 383МH0559.5 & $5 / 27 / 2004$ & soil & EPA6010 & REG & $7440-41-7$ & Beryllium & 1.2 & $\mathrm{mg} / \mathrm{kg}$ & & 0.64 \\
\hline 383МH0559.5 & $5 / 27 / 2004$ & soil & EPA6010 & REG & $7440-47-3$ & Chromium & 2.5 & $\mathrm{mg} / \mathrm{kg}$ & & 1.3 \\
\hline 383МH0559.5 & $5 / 27 / 2004$ & soil & EPA8270 & REG & 218-01-9 & Chrysene & 170 & $\mu \mathrm{g} / \mathrm{kg}$ & $\mathrm{J}$ & 420 \\
\hline 383МН0559.5 & $5 / 27 / 2004$ & soil & HASL300 & REG & $10045-97-3$ & Cs-137 & 471 & $\mathrm{pCi} / \mathrm{g}$ & $\mathrm{M} 3, \mathrm{G}$ & 1 \\
\hline 383МH0559.5 & $5 / 27 / 2004$ & soil & EPA8270 & REG & 206-44-0 & Fluoranthene & 150 & $\mu \mathrm{g} / \mathrm{kg}$ & $\mathrm{J}$ & 420 \\
\hline
\end{tabular}

Page 44 of 78

Uncontrolled When Printed 


\begin{tabular}{|c|c|c|c|c|c|c|c|c|c|c|}
\hline SAMPLE \# & $\begin{array}{c}\text { SAMPLE } \\
\text { DATE }\end{array}$ & MATRIX & $\begin{array}{l}\text { USER TEST } \\
\text { PANEL }\end{array}$ & $\begin{array}{c}\text { SAMPLE } \\
\text { PURPOSE }\end{array}$ & CAS \# & PARAMETER & RESULT & UNITS & $\mathbf{Q}$ & $\begin{array}{c}\text { DETECT } \\
\text { LIMIT }\end{array}$ \\
\hline 383МH0559.5 & $5 / 27 / 2004$ & soil & EPA8270 & REG & $193-39-5$ & Indeno(1,2,3-Cd)Pyrene & 60 & $\mu \mathrm{g} / \mathrm{kg}$ & $\mathrm{J}$ & 420 \\
\hline 383MH0559.5 & $5 / 27 / 2004$ & soil & HASL300 & REG & $13966-00-2$ & $\mathrm{~K}-40$ & 31.2 & $\mathrm{pCi} / \mathrm{g}$ & G & 2.6 \\
\hline 383MH0559.5 & $5 / 27 / 2004$ & soil & EPA6010 & REG & 7439-92-1 & Lead & 20 & $\mathrm{mg} / \mathrm{kg}$ & & 0.38 \\
\hline $383 \mathrm{MH} 0559.5$ & $5 / 27 / 2004$ & soil & EPA7470 & REG & 7439-97-6 & Mercury & 0.013 & $\mathrm{mg} / \mathrm{kg}$ & $\mathrm{B}$ & 0.13 \\
\hline 383МH0559.5 & $5 / 27 / 2004$ & soil & EPA8260 & REG & $75-09-2$ & Methylene Chloride & 2 & $\mu \mathrm{g} / \mathrm{kg}$ & $\mathrm{J}, \mathrm{B}$ & 6.4 \\
\hline 383MH0559.5 & $5 / 27 / 2004$ & soil & EPA8270 & REG & 85-01-8 & Phenanthrene & 52 & $\mu \mathrm{g} / \mathrm{kg}$ & $\mathrm{J}$ & 420 \\
\hline 383МH0559.5 & $5 / 27 / 2004$ & soil & UGTAISOPU & REG & 13981-16-3 & Pu-238 & 5.9 & $\mathrm{pCi} / \mathrm{g}$ & & 0 \\
\hline 383МH0559.5 & $5 / 27 / 2004$ & soil & UGTAISOPU & REG & $15117-48-3$ & Pu-239 & 25.3 & $\mathrm{pCi} / \mathrm{g}$ & & 0 \\
\hline 383МH0559.5 & $5 / 27 / 2004$ & soil & EPA8270 & REG & $129-00-0$ & Pyrene & 310 & $\mu \mathrm{g} / \mathrm{kg}$ & $\mathrm{J}$ & 420 \\
\hline 383МH0559.5 & $5 / 27 / 2004$ & soil & EPA6010 & REG & $7782-49-2$ & Selenium & 0.99 & $\mathrm{mg} / \mathrm{kg}$ & & 0.64 \\
\hline 383МH0559.5 & $5 / 27 / 2004$ & soil & EPA6010 & REG & $7440-22-4$ & Silver & 0.47 & $\mathrm{mg} / \mathrm{kg}$ & $\mathrm{B}$ & 1.3 \\
\hline 383МH0559.5 & $5 / 27 / 2004$ & soil & SR7500 & REG & 10098-97-2 & Sr-90 & 54 & $\mathrm{pCi} / \mathrm{g}$ & & 0 \\
\hline 383МH0568.5 & $5 / 27 / 2004$ & soil & HASL300 & REG & 14331-83-0 & Ac-228 & 0.42 & $\mathrm{pCi} / \mathrm{g}$ & & 0.24 \\
\hline 383МH0568.5 & $5 / 27 / 2004$ & soil & EPA6010 & REG & $7440-38-2$ & Arsenic & 2.4 & $\mathrm{mg} / \mathrm{kg}$ & & 1 \\
\hline 383МH0568.5 & $5 / 27 / 2004$ & soil & EPA6010 & REG & 7440-39-3 & Barium & 29 & $\mathrm{mg} / \mathrm{kg}$ & & 10 \\
\hline 383МH0568.5 & $5 / 27 / 2004$ & soil & EPA6010 & REG & $7440-41-7$ & Beryllium & 0.42 & $\mathrm{mg} / \mathrm{kg}$ & $\mathrm{B}$ & 0.52 \\
\hline 383МH0568.5 & $5 / 27 / 2004$ & soil & HASL300 & REG & 14733-03-0 & Bi-214 & 0.45 & $\mathrm{pCi} / \mathrm{g}$ & $\mathrm{J}$ & 0.17 \\
\hline 383МH0568.5 & $5 / 27 / 2004$ & soil & EPA6010 & REG & $7440-43-9$ & Cadmium & 0.066 & $\mathrm{mg} / \mathrm{kg}$ & $\mathrm{B}$ & 0.52 \\
\hline 383МH0568.5 & $5 / 27 / 2004$ & soil & EPA6010 & REG & $7440-47-3$ & Chromium & 4.2 & $\mathrm{mg} / \mathrm{kg}$ & & 1 \\
\hline 383МH0568.5 & $5 / 27 / 2004$ & soil & HASL300 & REG & $10045-97-3$ & Cs-137 & 0.348 & $\mathrm{pCi} / \mathrm{g}$ & $\mathrm{LT}$ & 0.072 \\
\hline 383МH0568.5 & $5 / 27 / 2004$ & soil & HASL300 & REG & $13966-00-2$ & $\mathrm{~K}-40$ & 3.62 & $\mathrm{pCi} / \mathrm{g}$ & & 0.8 \\
\hline 383МH0568.5 & $5 / 27 / 2004$ & soil & EPA6010 & REG & 7439-92-1 & Lead & 13 & $\mathrm{mg} / \mathrm{kg}$ & & 1.5 \\
\hline 383МH0568.5 & $5 / 27 / 2004$ & soil & EPA7470 & REG & 7439-97-6 & Mercury & 0.22 & $\mathrm{mg} / \mathrm{kg}$ & & 0.1 \\
\hline 383МH0568.5 & $5 / 27 / 2004$ & soil & EPA8260 & REG & $75-09-2$ & Methylene Chloride & 1.4 & $\mu \mathrm{g} / \mathrm{kg}$ & $\mathrm{J}, \mathrm{B}$ & 5.2 \\
\hline 383MH0568.5 & $5 / 27 / 2004$ & soil & HASL300 & REG & 15092-94-1 & $\mathrm{Pb}-212$ & 0.29 & $\mathrm{pCi} / \mathrm{g}$ & $\mathrm{J}$ & 0.14 \\
\hline 383MH0568.5 & $5 / 27 / 2004$ & soil & HASL300 & REG & $15067-28-4$ & $\mathrm{~Pb}-214$ & 0.5 & $\mathrm{pCi} / \mathrm{g}$ & $\mathrm{J}$ & 0.14 \\
\hline 383МH0568.5 & $5 / 27 / 2004$ & soil & EPA6010 & REG & $7440-22-4$ & Silver & 0.5 & $\mathrm{mg} / \mathrm{kg}$ & $\mathrm{B}$ & 1 \\
\hline 383МH0600.5 & $6 / 11 / 2004$ & soil & HASL300 & REG & 14331-83-0 & Ac-228 & 0.43 & $\mathrm{pCi} / \mathrm{g}$ & $\mathrm{TI}$ & 0.31 \\
\hline $383 \mathrm{MH} 0600.5$ & $6 / 11 / 2004$ & soil & EPA6010 & REG & $7440-38-2$ & Arsenic & 1.8 & $\mathrm{mg} / \mathrm{kg}$ & & 1 \\
\hline 383MH0600.5 & $6 / 11 / 2004$ & soil & EPA6010 & REG & $7440-39-3$ & Barium & 370 & $\mathrm{mg} / \mathrm{kg}$ & & 10 \\
\hline 383МH0600.5 & $6 / 11 / 2004$ & soil & EPA6010 & REG & 7440-41-7 & Beryllium & 0.33 & $\mathrm{mg} / \mathrm{kg}$ & $\mathrm{B}$ & 0.51 \\
\hline 383MH0600.5 & $6 / 11 / 2004$ & soil & HASL300 & REG & $14733-03-0$ & Bi-214 & 0.49 & $\mathrm{pCi} / \mathrm{g}$ & $\mathrm{J}$ & 0.18 \\
\hline 383MH0600.5 & $6 / 11 / 2004$ & soil & EPA6010 & REG & 7440-43-9 & Cadmium & 0.44 & $\mathrm{mg} / \mathrm{kg}$ & $\mathrm{B}$ & 0.51 \\
\hline 383MH0600.5 & $6 / 11 / 2004$ & soil & EPA6010 & REG & $7440-47-3$ & Chromium & 2.6 & $\mathrm{mg} / \mathrm{kg}$ & & 1 \\
\hline 383MH0600.5 & $6 / 11 / 2004$ & soil & HASL300 & REG & 10045-97-3 & Cs-137 & 1.65 & $\mathrm{pCi} / \mathrm{g}$ & & 0.08 \\
\hline
\end{tabular}

Page 45 of 78

Uncontrolled When Printed 


\begin{tabular}{|c|c|c|c|c|c|c|c|c|c|c|}
\hline SAMPLE \# & $\begin{array}{c}\text { SAMPLE } \\
\text { DATE }\end{array}$ & MATRIX & $\begin{array}{l}\text { USER TEST } \\
\text { PANEL }\end{array}$ & $\begin{array}{c}\text { SAMPLE } \\
\text { PURPOSE }\end{array}$ & CAS \# & PARAMETER & RESULT & UNITS & $\mathbf{Q}$ & $\begin{array}{c}\text { DETECT } \\
\text { LIMIT }\end{array}$ \\
\hline 383MH0600.5 & $6 / 11 / 2004$ & soil & EPA8015 & REG & $68334-30-5$ & Diesel-Range Organics & 700 & $\mathrm{mg} / \mathrm{kg}$ & $\mathrm{M}$ & 5.1 \\
\hline 383MH0600.5 & $6 / 11 / 2004$ & soil & HASL300 & REG & $13966-00-2$ & $\mathrm{~K}-40$ & 2.68 & $\mathrm{pCi} / \mathrm{g}$ & & 0.91 \\
\hline 383MH0600.5 & $6 / 11 / 2004$ & soil & EPA6010 & REG & 7439-92-1 & Lead & 5.9 & $\mathrm{mg} / \mathrm{kg}$ & & 1.5 \\
\hline $383 \mathrm{MH0600.5}$ & $6 / 11 / 2004$ & soil & EPA7470 & REG & 7439-97-6 & Mercury & 0.067 & $\mathrm{mg} / \mathrm{kg}$ & $\mathrm{B}$ & 0.1 \\
\hline 383MH0600.5 & $6 / 11 / 2004$ & soil & HASL300 & REG & 15092-94-1 & $\mathrm{Pb}-212$ & 0.29 & $\mathrm{pCi} / \mathrm{g}$ & & 0.14 \\
\hline 383MH0600.5 & $6 / 11 / 2004$ & soil & HASL300 & REG & $15067-28-4$ & $\mathrm{~Pb}-214$ & 0.58 & $\mathrm{pCi} / \mathrm{g}$ & $\mathrm{J}$ & 0.14 \\
\hline 383MH0600.5 & $6 / 11 / 2004$ & soil & UGTAISOPU & REG & $15117-48-3$ & Pu-239 & 0.04 & $\mathrm{pCi} / \mathrm{g}$ & LT & 0.02 \\
\hline 383МH0600.5 & $6 / 11 / 2004$ & soil & EPA6010 & REG & $7440-22-4$ & Silver & 0.17 & $\mathrm{mg} / \mathrm{kg}$ & B & 1 \\
\hline 383МH0600.5 & $6 / 11 / 2004$ & soil & SR7500 & REG & 10098-97-2 & Sr-90 & 0.048 & $\mathrm{pCi} / \mathrm{g}$ & $\mathrm{Y} 1, \mathrm{U}$ & 0.182 \\
\hline 383MH0600.5 & $6 / 11 / 2004$ & soil & HASL300 & REG & $14913-50-9$ & TI-208 & 0.093 & $\mathrm{pCi} / \mathrm{g}$ & & 0.075 \\
\hline 383MH0722.5 & $5 / 21 / 2004$ & soil & HASL300 & REG & 14331-83-0 & Ac-228 & 2.1 & $\mathrm{pCi} / \mathrm{g}$ & G & 0.61 \\
\hline 383МH0722.5 & $5 / 21 / 2004$ & soil & EPA8260 & REG & 67-64-1 & Acetone & 8.3 & $\mu \mathrm{g} / \mathrm{kg}$ & $\mathrm{J}$ & 23 \\
\hline $383 \mathrm{MH0722.5}$ & $5 / 21 / 2004$ & soil & EPA6010 & REG & $7440-38-2$ & Arsenic & 2.5 & $\mathrm{mg} / \mathrm{kg}$ & & 1.2 \\
\hline 383МH0722.5 & $5 / 21 / 2004$ & soil & EPA6010 & REG & $7440-39-3$ & Barium & 200 & $\mathrm{mg} / \mathrm{kg}$ & & 12 \\
\hline $383 \mathrm{MH} 0722.5$ & $5 / 21 / 2004$ & soil & EPA6010 & REG & 7440-41-7 & Beryllium & 1.3 & $\mathrm{mg} / \mathrm{kg}$ & & 0.59 \\
\hline $383 \mathrm{MH} 0722.5$ & $5 / 21 / 2004$ & soil & HASL300 & REG & $14733-03-0$ & Bi-214 & 1.38 & $\mathrm{pCi} / \mathrm{g}$ & $\mathrm{G}, \mathrm{J}$ & 0.41 \\
\hline 383МH0722.5 & $5 / 21 / 2004$ & soil & EPA6010 & REG & $7440-47-3$ & Chromium & 2 & $\mathrm{mg} / \mathrm{kg}$ & & 1.2 \\
\hline 383МH0722.5 & $5 / 21 / 2004$ & soil & HASL300 & REG & $10045-97-3$ & Cs-137 & 26.9 & $\mathrm{pCi} / \mathrm{g}$ & G & 0.2 \\
\hline 383МH0722.5 & $5 / 21 / 2004$ & soil & HASL300 & REG & 13966-00-2 & $\mathrm{K}-40$ & 33.2 & $\mathrm{pCi} / \mathrm{g}$ & G & 2.2 \\
\hline 383МH0722.5 & $5 / 21 / 2004$ & soil & EPA6010 & REG & 7439-92-1 & Lead & 12 & $\mathrm{mg} / \mathrm{kg}$ & & 0.35 \\
\hline 383МH0722.5 & $5 / 21 / 2004$ & soil & EPA7470 & REG & 7439-97-6 & Mercury & 0.055 & $\mathrm{mg} / \mathrm{kg}$ & B & 0.12 \\
\hline $383 \mathrm{MH} 0722.5$ & $5 / 21 / 2004$ & soil & HASL300 & REG & 15092-94-1 & $\mathrm{Pb}-212$ & 2.48 & $\mathrm{pCi} / \mathrm{g}$ & $\mathrm{J}$ & 0.37 \\
\hline 383МH0722.5 & $5 / 21 / 2004$ & soil & HASL300 & REG & $15067-28-4$ & $\mathrm{~Pb}-214$ & 1.28 & $\mathrm{pCi} / \mathrm{g}$ & $\mathrm{G}, \mathrm{J}$ & 0.46 \\
\hline 383МH0722.5 & $5 / 21 / 2004$ & soil & UGTAISOPU & REG & $15117-48-3$ & Pu-239 & 0.173 & $\mathrm{pCi} / \mathrm{g}$ & & 0.01 \\
\hline 383MH0722.5 & $5 / 21 / 2004$ & soil & HASL300 & REG & $14913-50-9$ & TI-208 & 0.79 & $\mathrm{pCi} / \mathrm{g}$ & G & 0.24 \\
\hline 383МH0723.5 & $5 / 21 / 2004$ & soil & HASL300 & REG & 14331-83-0 & Ac-228 & 2.01 & $\mathrm{pCi} / \mathrm{g}$ & $G$ & 0.84 \\
\hline 383МH0723.5 & $5 / 21 / 2004$ & soil & EPA8260 & REG & $67-64-1$ & Acetone & 10 & $\mu \mathrm{g} / \mathrm{kg}$ & $\mathrm{J}$ & 23 \\
\hline 383МH0723.5 & $5 / 21 / 2004$ & soil & EPA6010 & REG & $7440-38-2$ & Arsenic & 2.5 & $\mathrm{mg} / \mathrm{kg}$ & & 1.2 \\
\hline 383МH0723.5 & $5 / 21 / 2004$ & soil & EPA6010 & REG & $7440-39-3$ & Barium & 200 & $\mathrm{mg} / \mathrm{kg}$ & & 12 \\
\hline $383 \mathrm{MH} 0723.5$ & $5 / 21 / 2004$ & soil & EPA6010 & REG & $7440-41-7$ & Beryllium & 1.3 & $\mathrm{mg} / \mathrm{kg}$ & & 0.59 \\
\hline $383 \mathrm{MH} 0723.5$ & $5 / 21 / 2004$ & soil & HASL300 & REG & $14733-03-0$ & Bi-214 & 1.32 & $\mathrm{pCi} / \mathrm{g}$ & $\mathrm{G}, \mathrm{J}$ & 0.69 \\
\hline 383МH0723.5 & $5 / 21 / 2004$ & soil & EPA6010 & REG & $7440-47-3$ & Chromium & 1.7 & $\mathrm{mg} / \mathrm{kg}$ & & 1.2 \\
\hline 383MH0723.5 & $5 / 21 / 2004$ & soil & HASL300 & REG & $10045-97-3$ & Cs-137 & 83.7 & $\mathrm{pCi} / \mathrm{g}$ & $G$ & 0.3 \\
\hline 383МH0723.5 & $5 / 21 / 2004$ & soil & HASL300 & REG & 13966-00-2 & $\mathrm{K}-40$ & 40.7 & $\mathrm{pCi} / \mathrm{g}$ & $G$ & 1.8 \\
\hline 383MH0723.5 & $5 / 21 / 2004$ & soil & EPA6010 & REG & 7439-92-1 & Lead & 13 & $\mathrm{mg} / \mathrm{kg}$ & & 0.35 \\
\hline
\end{tabular}

Page 46 of 78

Uncontrolled When Printed 


\begin{tabular}{|c|c|c|c|c|c|c|c|c|c|c|}
\hline SAMPLE \# & $\begin{array}{c}\text { SAMPLE } \\
\text { DATE }\end{array}$ & MATRIX & $\begin{array}{l}\text { USER TEST } \\
\text { PANEL }\end{array}$ & $\begin{array}{c}\text { SAMPLE } \\
\text { PURPOSE }\end{array}$ & CAS \# & PARAMETER & RESULT & UNITS & $\mathbf{Q}$ & $\begin{array}{c}\text { DETECT } \\
\text { LIMIT }\end{array}$ \\
\hline 383МH0723.5 & $5 / 21 / 2004$ & soil & EPA7470 & REG & $7439-97-6$ & Mercury & 0.0021 & $\mathrm{mg} / \mathrm{kg}$ & $\mathrm{B}$ & 0.12 \\
\hline 383MH0723.5 & $5 / 21 / 2004$ & soil & EPA8260 & REG & $75-09-2$ & Methylene Chloride & 0.98 & $\mu \mathrm{g} / \mathrm{kg}$ & $\mathrm{J}, \mathrm{B}$ & 5.9 \\
\hline 383МH0723.5 & $5 / 21 / 2004$ & soil & HASL300 & REG & 15092-94-1 & $\mathrm{Pb}-212$ & 2.48 & $\mathrm{pCi} / \mathrm{g}$ & $\mathrm{J}$ & 0.55 \\
\hline 383МH0723.5 & $5 / 21 / 2004$ & soil & UGTAISOPU & REG & 13981-16-3 & $\mathrm{Pu}-238$ & 0.059 & $\mathrm{pCi} / \mathrm{g}$ & & 0.021 \\
\hline 383МH0723.5 & $5 / 21 / 2004$ & soil & UGTAISOPU & REG & $15117-48-3$ & Pu-239 & 0.262 & $\mathrm{pCi} / \mathrm{g}$ & & 0.009 \\
\hline 383МH0723.5 & $5 / 21 / 2004$ & soil & SR7500 & REG & $10098-97-2$ & Sr-90 & 0.85 & $\mathrm{pCi} / \mathrm{g}$ & & 0.19 \\
\hline 383МH0723.5 & $5 / 21 / 2004$ & soil & HASL300 & REG & $14913-50-9$ & TI-208 & 0.87 & $\mathrm{pCi} / \mathrm{g}$ & $G$ & 0.35 \\
\hline 383МH0767.5 & $5 / 21 / 2004$ & soil & EPA6010 & REG & $7440-38-2$ & Arsenic & 1.1 & $\mathrm{mg} / \mathrm{kg}$ & & 1 \\
\hline 383МH0767.5 & $5 / 21 / 2004$ & soil & EPA6010 & REG & $7440-39-3$ & Barium & 24 & $\mathrm{mg} / \mathrm{kg}$ & & 10 \\
\hline 383МH0767.5 & $5 / 21 / 2004$ & soil & EPA6010 & REG & $7440-41-7$ & Beryllium & 0.28 & $\mathrm{mg} / \mathrm{kg}$ & $\mathrm{B}$ & 0.51 \\
\hline 383MH0767.5 & $5 / 21 / 2004$ & soil & HASL300 & REG & $14733-03-0$ & Bi-214 & 0.27 & $\mathrm{pCi} / \mathrm{g}$ & $\mathrm{J}$ & 0.14 \\
\hline 383МH0767.5 & $5 / 21 / 2004$ & soil & EPA6010 & REG & $7440-43-9$ & Cadmium & 0.046 & $\mathrm{mg} / \mathrm{kg}$ & $\mathrm{B}$ & 0.51 \\
\hline 383МH0767.5 & $5 / 21 / 2004$ & soil & EPA6010 & REG & $7440-47-3$ & Chromium & 2.5 & $\mathrm{mg} / \mathrm{kg}$ & & 1 \\
\hline 383МH0767.5 & $5 / 21 / 2004$ & soil & HASL300 & REG & 13966-00-2 & $\mathrm{K}-40$ & 2.21 & $\mathrm{pCi} / \mathrm{g}$ & & 0.83 \\
\hline 383МН0767.5 & $5 / 21 / 2004$ & soil & EPA6010 & REG & 7439-92-1 & Lead & 8.9 & $\mathrm{mg} / \mathrm{kg}$ & & 1.5 \\
\hline 383МH0767.5 & $5 / 21 / 2004$ & soil & EPA7470 & REG & 7439-97-6 & Mercury & 0.056 & $\mathrm{mg} / \mathrm{kg}$ & $\mathrm{B}$ & 0.1 \\
\hline 383МH0767.5 & $5 / 21 / 2004$ & soil & HASL300 & REG & 15092-94-1 & $\mathrm{Pb}-212$ & 0.212 & $\mathrm{pCi} / \mathrm{g}$ & $\mathrm{J}$ & 0.108 \\
\hline 383МH0767.5 & $5 / 21 / 2004$ & soil & HASL300 & REG & $15067-28-4$ & $\mathrm{~Pb}-214$ & 0.37 & $\mathrm{pCi} / \mathrm{g}$ & $\mathrm{J}$ & 0.14 \\
\hline 383МH0767.5 & $5 / 21 / 2004$ & soil & EPA6010 & REG & $7440-22-4$ & Silver & 0.35 & $\mathrm{mg} / \mathrm{kg}$ & $\mathrm{B}$ & 1 \\
\hline 383МH0832.5 & $5 / 26 / 2004$ & soil & EPA6010 & REG & $7440-38-2$ & Arsenic & 2.7 & $\mathrm{mg} / \mathrm{kg}$ & & 1 \\
\hline 383МH0832.5 & $5 / 26 / 2004$ & soil & EPA6010 & REG & $7440-39-3$ & Barium & 22 & $\mathrm{mg} / \mathrm{kg}$ & & 10 \\
\hline $383 \mathrm{MH} 0832.5$ & $5 / 26 / 2004$ & soil & EPA6010 & REG & 7440-41-7 & Beryllium & 0.42 & $\mathrm{mg} / \mathrm{kg}$ & $\mathrm{B}$ & 0.52 \\
\hline 383МH0832.5 & $5 / 26 / 2004$ & soil & HASL300 & REG & $14733-03-0$ & Bi-214 & 0.56 & $\mathrm{pCi} / \mathrm{g}$ & $\mathrm{G}, \mathrm{J}$ & 0.26 \\
\hline 383МH0832.5 & $5 / 26 / 2004$ & soil & EPA6010 & REG & $7440-47-3$ & Chromium & 2.1 & $\mathrm{mg} / \mathrm{kg}$ & & 1 \\
\hline 383MH0832.5 & $5 / 26 / 2004$ & soil & HASL300 & REG & $10045-97-3$ & Cs-137 & 0.84 & $\mathrm{pCi} / \mathrm{g}$ & G & 0.12 \\
\hline 383МH0832.5 & $5 / 26 / 2004$ & soil & HASL300 & REG & 13966-00-2 & $\mathrm{K}-40$ & 7.1 & $\mathrm{pCi} / \mathrm{g}$ & $G$ & 1.5 \\
\hline 383МH0832.5 & $5 / 26 / 2004$ & soil & EPA6010 & REG & 7439-92-1 & Lead & 6.3 & $\mathrm{mg} / \mathrm{kg}$ & & 1.6 \\
\hline $383 \mathrm{MH} 0832.5$ & $5 / 26 / 2004$ & soil & EPA7470 & REG & 7439-97-6 & Mercury & 0.055 & $\mathrm{mg} / \mathrm{kg}$ & $\mathrm{B}$ & 0.1 \\
\hline $383 \mathrm{MH} 0832.5$ & $5 / 26 / 2004$ & soil & EPA8260 & REG & $75-09-2$ & Methylene Chloride & 1.4 & $\mu g / \mathrm{kg}$ & $\mathrm{J}, \mathrm{B}$ & 5.2 \\
\hline 383МH0832.5 & $5 / 26 / 2004$ & soil & HASL300 & REG & 15092-94-1 & $\mathrm{Pb}-212$ & 0.56 & $\mathrm{pCi} / \mathrm{g}$ & $\mathrm{J}$ & 0.17 \\
\hline $383 \mathrm{MH} 0832.5$ & $5 / 26 / 2004$ & soil & HASL300 & REG & $15067-28-4$ & $\mathrm{~Pb}-214$ & 0.64 & $\mathrm{pCi} / \mathrm{g}$ & $\mathrm{G}, \mathrm{J}$ & 0.21 \\
\hline 383МH0858.0 & $5 / 26 / 2004$ & soil & EPA8260 & REG & 67-64-1 & Acetone & 7.3 & $\mu g / \mathrm{kg}$ & $\mathrm{J}$ & 20 \\
\hline 383MH0858.0 & $5 / 26 / 2004$ & soil & EPA6010 & REG & $7440-38-2$ & Arsenic & 0.68 & $\mathrm{mg} / \mathrm{kg}$ & $\mathrm{B}$ & 1 \\
\hline 383МH0858.0 & $5 / 26 / 2004$ & soil & EPA6010 & REG & $7440-39-3$ & Barium & 3 & $\mathrm{mg} / \mathrm{kg}$ & $B$ & 10 \\
\hline 383МH0858.0 & $5 / 26 / 2004$ & soil & EPA6010 & REG & 7440-41-7 & Beryllium & 0.19 & $\mathrm{mg} / \mathrm{kg}$ & $\mathrm{B}$ & 0.5 \\
\hline
\end{tabular}

Page 47 of 78

Uncontrolled When Printed 


\begin{tabular}{|c|c|c|c|c|c|c|c|c|c|c|}
\hline SAMPLE \# & $\begin{array}{c}\text { SAMPLE } \\
\text { DATE }\end{array}$ & MATRIX & $\begin{array}{l}\text { USER TEST } \\
\text { PANEL }\end{array}$ & $\begin{array}{c}\text { SAMPLE } \\
\text { PURPOSE }\end{array}$ & CAS \# & PARAMETER & RESULT & UNITS & $\mathbf{Q}$ & $\begin{array}{c}\text { DETECT } \\
\text { LIMIT }\end{array}$ \\
\hline 383МH0858.0 & $5 / 26 / 2004$ & soil & EPA6010 & REG & $7440-43-9$ & Cadmium & 0.026 & $\mathrm{mg} / \mathrm{kg}$ & $\mathrm{B}$ & 0.5 \\
\hline 383МH0858.0 & $5 / 26 / 2004$ & soil & EPA6010 & REG & $7440-47-3$ & Chromium & 1.2 & $\mathrm{mg} / \mathrm{kg}$ & & 1 \\
\hline 383МH0858.0 & $5 / 26 / 2004$ & soil & EPA7470 & REG & 7439-97-6 & Mercury & 0.017 & $\mathrm{mg} / \mathrm{kg}$ & $\mathrm{B}$ & 0.1 \\
\hline 383МH0916.5 & $6 / 11 / 2004$ & soil & HASL300 & REG & $14331-83-0$ & Ac-228 & 1.5 & $\mathrm{pCi} / \mathrm{g}$ & $G$ & 0.49 \\
\hline 383МH0916.5 & $6 / 11 / 2004$ & soil & EPA6010 & REG & $7440-38-2$ & Arsenic & 2.4 & $\mathrm{mg} / \mathrm{kg}$ & & 1.1 \\
\hline 383МH0916.5 & $6 / 11 / 2004$ & soil & EPA6010 & REG & $7440-39-3$ & Barium & 81 & $\mathrm{mg} / \mathrm{kg}$ & & 11 \\
\hline 383МH0916.5 & $6 / 11 / 2004$ & soil & EPA6010 & REG & $7440-41-7$ & Beryllium & 0.67 & $\mathrm{mg} / \mathrm{kg}$ & & 0.55 \\
\hline 383МH0916.5 & $6 / 11 / 2004$ & soil & HASL300 & REG & $14733-03-0$ & Bi-214 & 0.71 & $\mathrm{pCi} / \mathrm{g}$ & $\mathrm{G}, \mathrm{J}$ & 0.27 \\
\hline 383MH0916.5 & $6 / 11 / 2004$ & soil & EPA6010 & REG & $7440-47-3$ & Chromium & 4.3 & $\mathrm{mg} / \mathrm{kg}$ & & 1.1 \\
\hline 383МH0916.5 & $6 / 11 / 2004$ & soil & HASL300 & REG & $10045-97-3$ & Cs-137 & 0.15 & $\mathrm{pCi} / \mathrm{g}$ & LT,G & 0.143 \\
\hline 383МH0916.5 & $6 / 11 / 2004$ & soil & HASL300 & REG & 13966-00-2 & $\mathrm{K}-40$ & 27 & $\mathrm{pCi} / \mathrm{g}$ & $\mathrm{G}$ & 1.2 \\
\hline 383МH0916.5 & $6 / 11 / 2004$ & soil & EPA6010 & REG & 7439-92-1 & Lead & 9.6 & $\mathrm{mg} / \mathrm{kg}$ & & 0.33 \\
\hline 383МH0916.5 & $6 / 11 / 2004$ & soil & EPA7470 & REG & $7439-97-6$ & Mercury & 0.088 & $\mathrm{mg} / \mathrm{kg}$ & $\mathrm{B}$ & 0.11 \\
\hline 383МH0916.5 & $6 / 11 / 2004$ & soil & HASL300 & REG & 15092-94-1 & $\mathrm{Pb}-212$ & 1.83 & $\mathrm{pCi} / \mathrm{g}$ & $G$ & 0.18 \\
\hline 383МH0916.5 & $6 / 11 / 2004$ & soil & HASL300 & REG & $15067-28-4$ & $\mathrm{~Pb}-214$ & 0.69 & $\mathrm{pCi} / \mathrm{g}$ & $\mathrm{G}, \mathrm{J}$ & 0.23 \\
\hline 383МH0916.5 & $6 / 11 / 2004$ & soil & UGTAISOPU & REG & $15117-48-3$ & Pu-239 & 0.016 & $\mathrm{pCi} / \mathrm{g}$ & $\mathrm{LT}$ & 0.009 \\
\hline 383МH0916.5 & $6 / 11 / 2004$ & soil & HASL300 & REG & $14913-50-9$ & TI-208 & 0.64 & $\mathrm{pCi} / \mathrm{g}$ & $G$ & 0.11 \\
\hline 383МН0922.5 & $6 / 11 / 2004$ & soil & HASL300 & REG & 14331-83-0 & Ac-228 & 2.14 & $\mathrm{pCi} / \mathrm{g}$ & $G$ & 0.54 \\
\hline 383МH0922.5 & $6 / 11 / 2004$ & soil & EPA6010 & REG & $7440-38-2$ & Arsenic & 3.8 & $\mathrm{mg} / \mathrm{kg}$ & & 1.1 \\
\hline 383МH0922.5 & $6 / 11 / 2004$ & soil & EPA6010 & REG & $7440-39-3$ & Barium & 160 & $\mathrm{mg} / \mathrm{kg}$ & & 11 \\
\hline 383МH0922.5 & $6 / 11 / 2004$ & soil & EPA6010 & REG & $7440-41-7$ & Beryllium & 1 & $\mathrm{mg} / \mathrm{kg}$ & & 0.56 \\
\hline 383МH0922.5 & $6 / 11 / 2004$ & soil & HASL300 & REG & $14733-03-0$ & Bi-214 & 0.92 & $\mathrm{pCi} / \mathrm{g}$ & $\mathrm{G}, \mathrm{J}$ & 0.26 \\
\hline 383МH0922.5 & $6 / 11 / 2004$ & soil & EPA6010 & REG & $7440-47-3$ & Chromium & 4.3 & $\mathrm{mg} / \mathrm{kg}$ & & 1.1 \\
\hline 383МH0922.5 & $6 / 11 / 2004$ & soil & HASL300 & REG & 14391-16-3 & Eu-155 & 0.32 & $\mathrm{pCi} / \mathrm{g}$ & $\mathrm{G}, \mathrm{TI}$ & 0.3 \\
\hline 383MH0922.5 & $6 / 11 / 2004$ & soil & HASL300 & REG & 13966-00-2 & $\mathrm{K}-40$ & 31.7 & $\mathrm{pCi} / \mathrm{g}$ & G & 1.4 \\
\hline 383MH0922.5 & $6 / 11 / 2004$ & soil & EPA6010 & REG & $7439-92-1$ & Lead & 13 & $\mathrm{mg} / \mathrm{kg}$ & & 0.33 \\
\hline 383МH0922.5 & $6 / 11 / 2004$ & soil & EPA7470 & REG & 7439-97-6 & Mercury & 0.07 & $\mathrm{mg} / \mathrm{kg}$ & $\mathrm{B}$ & 0.11 \\
\hline 383МH0922.5 & $6 / 11 / 2004$ & soil & HASL300 & REG & 15092-94-1 & $\mathrm{Pb}-212$ & 2.27 & $\mathrm{pCi} / \mathrm{g}$ & G & 0.23 \\
\hline 383МH0922.5 & $6 / 11 / 2004$ & soil & HASL300 & REG & $15067-28-4$ & $\mathrm{~Pb}-214$ & 0.72 & $\mathrm{pCi} / \mathrm{g}$ & $\mathrm{G}, \mathrm{J}$ & 0.25 \\
\hline $383 \mathrm{MH0922.5}$ & $6 / 11 / 2004$ & soil & EPA6010 & REG & $7782-49-2$ & Selenium & 0.26 & $\mathrm{mg} / \mathrm{kg}$ & $\mathrm{B}$ & 0.56 \\
\hline 383МH0922.5 & $6 / 11 / 2004$ & soil & EPA6010 & REG & $7440-22-4$ & Silver & 0.15 & $\mathrm{mg} / \mathrm{kg}$ & $B$ & 1.1 \\
\hline 383МH0922.5 & $6 / 11 / 2004$ & soil & HASL300 & REG & $15065-10-8$ & Th-234 & 1.8 & $\mathrm{pCi} / \mathrm{g}$ & $\mathrm{G}$ & 1.67 \\
\hline 383МH0922.5 & $6 / 11 / 2004$ & soil & HASL300 & REG & 14913-50-9 & TI-208 & 0.76 & $\mathrm{pCi} / \mathrm{g}$ & $G$ & 0.15 \\
\hline $383 \mathrm{MH} 1002.0$ & $5 / 22 / 2004$ & soil & HASL300 & REG & 14331-83-0 & Ac-228 & 0.7 & $\mathrm{pCi} / \mathrm{g}$ & $G$ & 0.31 \\
\hline 383МH1002.0 & $5 / 22 / 2004$ & soil & EPA6010 & REG & $7440-38-2$ & Arsenic & 3 & $\mathrm{mg} / \mathrm{kg}$ & & 1.1 \\
\hline
\end{tabular}

Page 48 of 78

Uncontrolled When Printed 


\begin{tabular}{|c|c|c|c|c|c|c|c|c|c|c|}
\hline SAMPLE \# & $\begin{array}{c}\text { SAMPLE } \\
\text { DATE }\end{array}$ & MATRIX & $\begin{array}{l}\text { USER TEST } \\
\text { PANEL }\end{array}$ & $\begin{array}{c}\text { SAMPLE } \\
\text { PURPOSE }\end{array}$ & CAS \# & PARAMETER & RESULT & UNITS & $\mathbf{Q}$ & $\begin{array}{l}\text { DETECT } \\
\text { LIMIT }\end{array}$ \\
\hline $383 \mathrm{MH} 1002.0$ & $5 / 22 / 2004$ & soil & EPA6010 & REG & $7440-39-3$ & Barium & 190 & $\mathrm{mg} / \mathrm{kg}$ & & 11 \\
\hline 383МH1002.0 & $5 / 22 / 2004$ & soil & EPA6010 & REG & $7440-41-7$ & Beryllium & 0.78 & $\mathrm{mg} / \mathrm{kg}$ & & 0.53 \\
\hline 383MH1002.0 & $5 / 22 / 2004$ & soil & HASL300 & REG & $14733-03-0$ & Bi-214 & 0.48 & $\mathrm{pCi} / \mathrm{g}$ & $\mathrm{G}, \mathrm{J}$ & 0.18 \\
\hline $383 \mathrm{MH} 1002.0$ & $5 / 22 / 2004$ & soil & EPA6010 & REG & $7440-47-3$ & Chromium & 2.8 & $\mathrm{mg} / \mathrm{kg}$ & & 1.1 \\
\hline $383 \mathrm{MH} 1002.0$ & $5 / 22 / 2004$ & soil & HASL300 & REG & $10045-97-3$ & Cs-137 & 1.04 & $\mathrm{pCi} / \mathrm{g}$ & $G$ & 0.09 \\
\hline 383МH1002.0 & $5 / 22 / 2004$ & soil & HASL300 & REG & 13966-00-2 & $\mathrm{K}-40$ & 13.8 & $\mathrm{pCi} / \mathrm{g}$ & $G$ & 1 \\
\hline $383 \mathrm{MH} 1002.0$ & $5 / 22 / 2004$ & soil & EPA6010 & REG & 7439-92-1 & Lead & 17 & $\mathrm{mg} / \mathrm{kg}$ & & 1.6 \\
\hline 383МH1002.0 & $5 / 22 / 2004$ & soil & EPA7470 & REG & 7439-97-6 & Mercury & 0.056 & $\mathrm{mg} / \mathrm{kg}$ & B & 0.11 \\
\hline 383МH1002.0 & $5 / 22 / 2004$ & soil & HASL300 & REG & 15092-94-1 & $\mathrm{Pb}-212$ & 0.83 & $\mathrm{pCi} / \mathrm{g}$ & $\mathrm{J}$ & 0.15 \\
\hline 383MH1002.0 & $5 / 22 / 2004$ & soil & HASL300 & REG & $15067-28-4$ & $\mathrm{~Pb}-214$ & 0.61 & $\mathrm{pCi} / \mathrm{g}$ & $G, J$ & 0.17 \\
\hline 383MH1002.0 & $5 / 22 / 2004$ & soil & HASL300 & REG & $14913-50-9$ & TI-208 & 0.293 & $\mathrm{pCi} / \mathrm{g}$ & $G$ & 0.078 \\
\hline 383МH1011.5 & $5 / 22 / 2004$ & soil & EPA6010 & REG & $7440-38-2$ & Arsenic & 1.9 & $\mathrm{mg} / \mathrm{kg}$ & & 1.1 \\
\hline 383МH1011.5 & $5 / 22 / 2004$ & soil & EPA6010 & REG & $7440-39-3$ & Barium & 56 & $\mathrm{mg} / \mathrm{kg}$ & & 11 \\
\hline 383МH1011.5 & $5 / 22 / 2004$ & soil & EPA6010 & REG & $7440-41-7$ & Beryllium & 0.47 & $\mathrm{mg} / \mathrm{kg}$ & $\mathrm{B}$ & 0.54 \\
\hline 383МH1011.5 & $5 / 22 / 2004$ & soil & HASL300 & REG & $14733-03-0$ & Bi-214 & 0.67 & $\mathrm{pCi} / \mathrm{g}$ & $\mathrm{G}, \mathrm{J}$ & 0.27 \\
\hline 383МH1011.5 & $5 / 22 / 2004$ & soil & EPA6010 & REG & $7440-47-3$ & Chromium & 3.8 & $\mathrm{mg} / \mathrm{kg}$ & & 1.1 \\
\hline 383МH1011.5 & $5 / 22 / 2004$ & soil & HASL300 & REG & $10045-97-3$ & Cs-137 & 0.42 & $\mathrm{pCi} / \mathrm{g}$ & LT,G & 0.1 \\
\hline 383MH1011.5 & $5 / 22 / 2004$ & soil & HASL300 & REG & 13966-00-2 & $\mathrm{K}-40$ & 5.3 & $\mathrm{pCi} / \mathrm{g}$ & $G$ & 1.5 \\
\hline 383MH1011.5 & $5 / 22 / 2004$ & soil & EPA6010 & REG & 7439-92-1 & Lead & 5.1 & $\mathrm{mg} / \mathrm{kg}$ & & 1.6 \\
\hline 383МH1011.5 & $5 / 22 / 2004$ & soil & EPA7470 & REG & 7439-97-6 & Mercury & 0.073 & $\mathrm{mg} / \mathrm{kg}$ & $\mathrm{B}$ & 0.11 \\
\hline 383МH1011.5 & $5 / 22 / 2004$ & soil & HASL300 & REG & 15092-94-1 & $\mathrm{Pb}-212$ & 0.49 & $\mathrm{pCi} / \mathrm{g}$ & $\mathrm{J}$ & 0.21 \\
\hline 383МH1011.5 & $5 / 22 / 2004$ & soil & HASL300 & REG & 15067-28-4 & $\mathrm{Pb}-214$ & 0.56 & $\mathrm{pCi} / \mathrm{g}$ & $\mathrm{G}, \mathrm{J}$ & 0.25 \\
\hline 383МH1011.5 & $5 / 22 / 2004$ & soil & EPA6010 & REG & $7440-22-4$ & Silver & 0.076 & $\mathrm{mg} / \mathrm{kg}$ & $\mathrm{B}$ & 1.1 \\
\hline 383МH1100.5 & $6 / 11 / 2004$ & soil & HASL300 & REG & 14331-83-0 & Ac-228 & 1.47 & $\mathrm{pCi} / \mathrm{g}$ & G & 0.49 \\
\hline 383MH1100.5 & $6 / 11 / 2004$ & soil & HASL300 & REG & 14596-10-2 & Am-241 & 0.95 & $\mathrm{pCi} / \mathrm{g}$ & $G$ & 0.33 \\
\hline 383MH1100.5 & $6 / 11 / 2004$ & soil & EPA6010 & REG & $7440-38-2$ & Arsenic & 5.7 & $\mathrm{mg} / \mathrm{kg}$ & & 1.1 \\
\hline 383MH1100.5 & $6 / 11 / 2004$ & soil & EPA6010 & REG & $7440-39-3$ & Barium & 210 & $\mathrm{mg} / \mathrm{kg}$ & & 11 \\
\hline $383 \mathrm{MH} 1100.5$ & $6 / 11 / 2004$ & soil & EPA8270 & REG & $56-55-3$ & Benzo(A)Anthracene & 130 & $\mu \mathrm{g} / \mathrm{kg}$ & $\mathrm{J}$ & 350 \\
\hline $383 \mathrm{MH} 1100.5$ & $6 / 11 / 2004$ & soil & EPA8270 & REG & $50-32-8$ & Benzo(A)Pyrene & 110 & $\mu \mathrm{g} / \mathrm{kg}$ & $\mathrm{J}$ & 350 \\
\hline 383MH1100.5 & $6 / 11 / 2004$ & soil & EPA8270 & REG & $205-99-2$ & Benzo(B)Fluoranthene & 210 & $\mu \mathrm{g} / \mathrm{kg}$ & $\mathrm{J}$ & 350 \\
\hline $383 \mathrm{MH} 1100.5$ & $6 / 11 / 2004$ & soil & EPA8270 & REG & 191-24-2 & Benzo(G,H,I)Perylene & 83 & $\mu \mathrm{g} / \mathrm{kg}$ & $\mathrm{J}$ & 350 \\
\hline $383 \mathrm{MH} 1100.5$ & $6 / 11 / 2004$ & soil & EPA8270 & REG & 207-08-9 & Benzo(K)Fluoranthene & 94 & $\mu \mathrm{g} / \mathrm{kg}$ & $\mathrm{J}$ & 350 \\
\hline 383MH1100.5 & $6 / 11 / 2004$ & soil & EPA6010 & REG & 7440-41-7 & Beryllium & 0.71 & $\mathrm{mg} / \mathrm{kg}$ & & 0.53 \\
\hline 383MH1100.5 & $6 / 11 / 2004$ & soil & HASL300 & REG & $14913-49-6$ & Bi-212 & 2.1 & $\mathrm{pCi} / \mathrm{g}$ & $\mathrm{G}, \mathrm{TI}$ & 1.8 \\
\hline 383MH1100.5 & $6 / 11 / 2004$ & soil & HASL300 & REG & $14733-03-0$ & Bi-214 & 0.86 & $\mathrm{pCi} / \mathrm{g}$ & $\mathrm{G}, \mathrm{J}$ & 0.41 \\
\hline
\end{tabular}

Page 49 of 78

Uncontrolled When Printed 


\begin{tabular}{|c|c|c|c|c|c|c|c|c|c|c|}
\hline SAMPLE \# & $\begin{array}{c}\text { SAMPLE } \\
\text { DATE }\end{array}$ & MATRIX & $\begin{array}{l}\text { USER TEST } \\
\text { PANEL }\end{array}$ & $\begin{array}{c}\text { SAMPLE } \\
\text { PURPOSE }\end{array}$ & CAS \# & PARAMETER & RESULT & UNITS & $\mathbf{Q}$ & $\begin{array}{l}\text { DETECT } \\
\text { LIMIT }\end{array}$ \\
\hline $383 \mathrm{MH} 1100.5$ & $6 / 11 / 2004$ & soil & EPA8270 & REG & 117-81-7 & Bis(2-Ethylhexyl)Phthalate & 110 & $\mu \mathrm{g} / \mathrm{kg}$ & $\mathrm{J}$ & 350 \\
\hline 383MH1100.5 & $6 / 11 / 2004$ & soil & EPA8270 & REG & $85-68-7$ & Butyl Benzyl Phthalate & 35 & $\mu \mathrm{g} / \mathrm{kg}$ & $\mathrm{J}$ & 350 \\
\hline $383 \mathrm{MH} 1100.5$ & $6 / 11 / 2004$ & soil & EPA6010 & REG & $7440-43-9$ & Cadmium & 0.35 & $\mathrm{mg} / \mathrm{kg}$ & $\mathrm{B}$ & 0.53 \\
\hline $383 \mathrm{MH} 1100.5$ & $6 / 11 / 2004$ & soil & EPA6010 & REG & $7440-47-3$ & Chromium & 6.9 & $\mathrm{mg} / \mathrm{kg}$ & & 1.1 \\
\hline $383 \mathrm{MH} 1100.5$ & $6 / 11 / 2004$ & soil & EPA8270 & REG & 218-01-9 & Chrysene & 170 & $\mu \mathrm{g} / \mathrm{kg}$ & $\mathrm{J}$ & 350 \\
\hline 383MH1100.5 & $6 / 11 / 2004$ & soil & HASL300 & REG & $10045-97-3$ & Cs-137 & 47.3 & $\mathrm{pCi} / \mathrm{g}$ & G & 0.2 \\
\hline $383 \mathrm{MH} 1100.5$ & $6 / 11 / 2004$ & soil & EPA8015 & REG & $68334-30-5$ & Diesel-Range Organics & 81 & $\mathrm{mg} / \mathrm{kg}$ & $\mathrm{Z}, \mathrm{H}$ & 5.3 \\
\hline $383 \mathrm{MH} 1100.5$ & $6 / 11 / 2004$ & soil & EPA8270 & REG & 84-74-2 & Di-N-Butyl Phthalate & 26 & $\mu \mathrm{g} / \mathrm{kg}$ & $\mathrm{J}$ & 350 \\
\hline 383MH1100.5 & $6 / 11 / 2004$ & soil & EPA8270 & REG & $206-44-0$ & Fluoranthene & 170 & $\mu \mathrm{g} / \mathrm{kg}$ & $\mathrm{J}$ & 350 \\
\hline 383MH1100.5 & $6 / 11 / 2004$ & soil & EPA8270 & REG & $193-39-5$ & Indeno(1,2,3-Cd)Pyrene & 54 & $\mu \mathrm{g} / \mathrm{kg}$ & $\mathrm{J}$ & 350 \\
\hline $383 \mathrm{MH} 1100.5$ & $6 / 11 / 2004$ & soil & HASL300 & REG & $13966-00-2$ & $\mathrm{~K}-40$ & 19.6 & $\mathrm{pCi} / \mathrm{g}$ & G & 1.4 \\
\hline $383 \mathrm{MH} 1100.5$ & $6 / 11 / 2004$ & soil & EPA6010 & REG & 7439-92-1 & Lead & 38 & $\mathrm{mg} / \mathrm{kg}$ & & 1.6 \\
\hline 383MH1100.5 & $6 / 11 / 2004$ & soil & EPA7470 & REG & $7439-97-6$ & Mercury & 0.033 & $\mathrm{mg} / \mathrm{kg}$ & $\mathrm{B}$ & 0.11 \\
\hline $383 \mathrm{MH} 1100.5$ & $6 / 11 / 2004$ & soil & HASL300 & REG & 15092-94-1 & $\mathrm{Pb}-212$ & 1.67 & $\mathrm{pCi} / \mathrm{g}$ & G & 0.38 \\
\hline 383MH1100.5 & $6 / 11 / 2004$ & soil & HASL300 & REG & $15067-28-4$ & $\mathrm{~Pb}-214$ & 0.8 & $\mathrm{pCi} / \mathrm{g}$ & $\mathrm{G}, \mathrm{J}$ & 0.48 \\
\hline 383MH1100.5 & $6 / 11 / 2004$ & soil & EPA8270 & REG & 85-01-8 & Phenanthrene & 56 & $\mu \mathrm{g} / \mathrm{kg}$ & $\mathrm{J}$ & 350 \\
\hline 383MH1100.5 & $6 / 11 / 2004$ & soil & UGTAISOPU & REG & 13981-16-3 & Pu-238 & 0.46 & $\mathrm{pCi} / \mathrm{g}$ & & 0.01 \\
\hline 383MH1100.5 & $6 / 11 / 2004$ & soil & UGTAISOPU & REG & $15117-48-3$ & Pu-239 & 3.04 & $\mathrm{pCi} / \mathrm{g}$ & & 0.03 \\
\hline 383MH1100.5 & $6 / 11 / 2004$ & soil & EPA8270 & REG & $129-00-0$ & Pyrene & 190 & $\mu \mathrm{g} / \mathrm{kg}$ & $\mathrm{J}$ & 350 \\
\hline 383MH1100.5 & $6 / 11 / 2004$ & soil & SR7500 & REG & 10098-97-2 & Sr-90 & 0.62 & $\mathrm{pCi} / \mathrm{g}$ & Y1 & 0.19 \\
\hline 383MH1100.5 & $6 / 11 / 2004$ & soil & HASL300 & REG & $14913-50-9$ & TI-208 & 0.36 & $\mathrm{pCi} / \mathrm{g}$ & $G$ & 0.18 \\
\hline 383MH1104.0 & $6 / 11 / 2004$ & soil & EPA6010 & REG & $7440-38-2$ & Arsenic & 0.69 & $\mathrm{mg} / \mathrm{kg}$ & $\mathrm{B}$ & 1 \\
\hline $383 \mathrm{MH} 1104.0$ & $6 / 11 / 2004$ & soil & EPA6010 & REG & $7440-39-3$ & Barium & 15 & $\mathrm{mg} / \mathrm{kg}$ & & 10 \\
\hline $383 \mathrm{MH} 1104.0$ & $6 / 11 / 2004$ & soil & EPA6010 & REG & $7440-41-7$ & Beryllium & 0.42 & $\mathrm{mg} / \mathrm{kg}$ & $\mathrm{B}$ & 0.51 \\
\hline 383MH1104.0 & $6 / 11 / 2004$ & soil & HASL300 & REG & $14733-03-0$ & Bi-214 & 0.33 & $\mathrm{pCi} / \mathrm{g}$ & $\mathrm{J}$ & 0.22 \\
\hline 383MH1104.0 & $6 / 11 / 2004$ & soil & EPA8270 & REG & 117-81-7 & Bis(2-Ethylhexyl)Phthalate & 84 & $\mu \mathrm{g} / \mathrm{kg}$ & $\mathrm{J}$ & 340 \\
\hline $383 \mathrm{MH} 1104.0$ & $6 / 11 / 2004$ & soil & EPA6010 & REG & $7440-43-9$ & Cadmium & 0.037 & $\mathrm{mg} / \mathrm{kg}$ & $\mathrm{B}$ & 0.51 \\
\hline $383 \mathrm{MH} 1104.0$ & $6 / 11 / 2004$ & soil & EPA6010 & REG & $7440-47-3$ & Chromium & 2.1 & $\mathrm{mg} / \mathrm{kg}$ & & 1 \\
\hline 383МH1104.0 & $6 / 11 / 2004$ & soil & HASL300 & REG & $10045-97-3$ & Cs-137 & 1.83 & $\mathrm{pCi} / \mathrm{g}$ & & 0.1 \\
\hline $383 \mathrm{MH} 1104.0$ & $6 / 11 / 2004$ & soil & EPA6010 & REG & 7439-92-1 & Lead & 4 & $\mathrm{mg} / \mathrm{kg}$ & & 1.5 \\
\hline 383MH1104.0 & $6 / 11 / 2004$ & soil & EPA7470 & REG & 7439-97-6 & Mercury & 0.06 & $\mathrm{mg} / \mathrm{kg}$ & $B$ & 0.1 \\
\hline 383MH1104.0 & $6 / 11 / 2004$ & soil & HASL300 & REG & 15092-94-1 & $\mathrm{Pb}-212$ & 0.212 & $\mathrm{pCi} / \mathrm{g}$ & & 0.134 \\
\hline 383MH1104.0 & $6 / 11 / 2004$ & soil & HASL300 & REG & $15067-28-4$ & $\mathrm{~Pb}-214$ & 0.41 & $\mathrm{pCi} / \mathrm{g}$ & $\mathrm{J}$ & 0.18 \\
\hline 383MH1104.0 & $6 / 11 / 2004$ & soil & UGTAISOPU & REG & $15117-48-3$ & Pu-239 & 0.06 & $\mathrm{pCi} / \mathrm{g}$ & & 0.009 \\
\hline 383МH1104.0 & $6 / 11 / 2004$ & soil & EPA6010 & REG & $7440-22-4$ & Silver & 0.071 & $\mathrm{mg} / \mathrm{kg}$ & $\mathrm{B}$ & 1 \\
\hline
\end{tabular}

Page 50 of 78

Uncontrolled When Printed 


\begin{tabular}{|c|c|c|c|c|c|c|c|c|c|c|}
\hline SAMPLE \# & $\begin{array}{c}\text { SAMPLE } \\
\text { DATE }\end{array}$ & MATRIX & $\begin{array}{l}\text { USER TEST } \\
\text { PANEL }\end{array}$ & $\begin{array}{c}\text { SAMPLE } \\
\text { PURPOSE }\end{array}$ & CAS \# & PARAMETER & RESULT & UNITS & $\mathbf{Q}$ & $\begin{array}{c}\text { DETECT } \\
\text { LIMIT }\end{array}$ \\
\hline $383 \mathrm{MH} 1215.0$ & $5 / 23 / 2004$ & soil & HASL300 & REG & 14331-83-0 & Ac-228 & 1.9 & $\mathrm{pCi} / \mathrm{g}$ & G & 0.49 \\
\hline 383МH1215.0 & $5 / 23 / 2004$ & soil & EPA6010 & REG & $7440-38-2$ & Arsenic & 3.2 & $\mathrm{mg} / \mathrm{kg}$ & & 1.2 \\
\hline 383MH1215.0 & $5 / 23 / 2004$ & soil & EPA6010 & REG & $7440-39-3$ & Barium & 110 & $\mathrm{mg} / \mathrm{kg}$ & & 12 \\
\hline $383 \mathrm{MH} 1215.0$ & $5 / 23 / 2004$ & soil & EPA6010 & REG & 7440-41-7 & Beryllium & 1.1 & $\mathrm{mg} / \mathrm{kg}$ & & 0.58 \\
\hline $383 \mathrm{MH} 1215.0$ & $5 / 23 / 2004$ & soil & HASL300 & REG & 14733-03-0 & Bi-214 & 1.22 & $\mathrm{pCi} / \mathrm{g}$ & $\mathrm{G}, \mathrm{J}$ & 0.32 \\
\hline 383МH1215.0 & $5 / 23 / 2004$ & soil & EPA6010 & REG & $7440-47-3$ & Chromium & 0.98 & $\mathrm{mg} / \mathrm{kg}$ & B & 1.2 \\
\hline $383 \mathrm{MH} 1215.0$ & $5 / 23 / 2004$ & soil & HASL300 & REG & $10045-97-3$ & Cs-137 & 23.5 & $\mathrm{pCi} / \mathrm{g}$ & G & 0.1 \\
\hline 383МH1215.0 & $5 / 23 / 2004$ & soil & HASL300 & REG & $13966-00-2$ & $\mathrm{~K}-40$ & 36.7 & $\mathrm{pCi} / \mathrm{g}$ & G & 1.3 \\
\hline 383МH1215.0 & $5 / 23 / 2004$ & soil & EPA6010 & REG & 7439-92-1 & Lead & 12 & $\mathrm{mg} / \mathrm{kg}$ & & 0.35 \\
\hline 383MH1215.0 & $5 / 23 / 2004$ & soil & EPA7470 & REG & 7439-97-6 & Mercury & 0.011 & $\mathrm{mg} / \mathrm{kg}$ & $B$ & 0.12 \\
\hline $383 \mathrm{MH} 1215.0$ & $5 / 23 / 2004$ & soil & EPA8260 & REG & $75-09-2$ & Methylene Chloride & 0.88 & $\mu \mathrm{g} / \mathrm{kg}$ & $\mathrm{J}, \mathrm{B}$ & 5.8 \\
\hline 383МH1215.0 & $5 / 23 / 2004$ & soil & HASL300 & REG & 15092-94-1 & $\mathrm{Pb}-212$ & 2.72 & $\mathrm{pCi} / \mathrm{g}$ & $\mathrm{J}$ & 0.33 \\
\hline 383МH1215.0 & $5 / 23 / 2004$ & soil & HASL300 & REG & $15067-28-4$ & $\mathrm{~Pb}-214$ & 1.26 & $\mathrm{pCi} / \mathrm{g}$ & $\mathrm{G}, \mathrm{J}$ & 0.35 \\
\hline $383 \mathrm{MH} 1215.0$ & $5 / 23 / 2004$ & soil & UGTAISOPU & REG & 13981-16-3 & Pu-238 & 0.072 & $\mathrm{pCi} / \mathrm{g}$ & & 0.009 \\
\hline $383 \mathrm{MH} 1215.0$ & $5 / 23 / 2004$ & soil & UGTAISOPU & REG & $15117-48-3$ & Pu-239 & 0.74 & $\mathrm{pCi} / \mathrm{g}$ & $\mathrm{J}$ & 0.01 \\
\hline $383 \mathrm{MH} 1215.0$ & $5 / 23 / 2004$ & soil & EPA6010 & REG & $7782-49-2$ & Selenium & 0.78 & $\mathrm{mg} / \mathrm{kg}$ & & 0.58 \\
\hline $383 \mathrm{MH} 1215.0$ & $5 / 23 / 2004$ & soil & HASL300 & REG & $14913-50-9$ & TI-208 & 0.68 & $\mathrm{pCi} / \mathrm{g}$ & G & 0.14 \\
\hline 383MH1220.5 & $5 / 23 / 2004$ & soil & EPA6010 & REG & $7440-38-2$ & Arsenic & 1.5 & $\mathrm{mg} / \mathrm{kg}$ & & 1.1 \\
\hline 383МH1220.5 & $5 / 23 / 2004$ & soil & EPA6010 & REG & $7440-39-3$ & Barium & 8.7 & $\mathrm{mg} / \mathrm{kg}$ & $B$ & 11 \\
\hline 383MH1220.5 & $5 / 23 / 2004$ & soil & EPA6010 & REG & 7440-41-7 & Beryllium & 0.19 & $\mathrm{mg} / \mathrm{kg}$ & $B$ & 0.53 \\
\hline 383МH1220.5 & $5 / 23 / 2004$ & soil & EPA6010 & REG & $7440-43-9$ & Cadmium & 1 & $\mathrm{mg} / \mathrm{kg}$ & & 0.53 \\
\hline 383МH1220.5 & $5 / 23 / 2004$ & soil & EPA6010 & REG & $7440-47-3$ & Chromium & 1 & $\mathrm{mg} / \mathrm{kg}$ & $B$ & 1.1 \\
\hline 383MH1220.5 & $5 / 23 / 2004$ & soil & EPA6010 & REG & 7439-92-1 & Lead & 18 & $\mathrm{mg} / \mathrm{kg}$ & & 1.6 \\
\hline 383МH1220.5 & $5 / 23 / 2004$ & soil & EPA7470 & REG & $7439-97-6$ & Mercury & 0.19 & $\mathrm{mg} / \mathrm{kg}$ & & 0.11 \\
\hline 383MH1220.5 & $5 / 23 / 2004$ & soil & EPA8260 & REG & $75-09-2$ & Methylene Chloride & 0.83 & $\mu \mathrm{g} / \mathrm{kg}$ & $\mathrm{J}, \mathrm{B}$ & 5.3 \\
\hline 383MH1220.5 & $5 / 23 / 2004$ & soil & EPA6010 & REG & $7440-22-4$ & Silver & 0.71 & $\mathrm{mg} / \mathrm{kg}$ & $B$ & 1.1 \\
\hline $383 \mathrm{MH} 1306.5$ & $5 / 25 / 2004$ & soil & HASL300 & REG & 14331-83-0 & Ac-228 & 1.82 & $\mathrm{pCi} / \mathrm{g}$ & G & 0.78 \\
\hline $383 \mathrm{MH} 1306.5$ & $5 / 25 / 2004$ & soil & EPA8260 & REG & 67-64-1 & Acetone & 7.3 & $\mu \mathrm{g} / \mathrm{kg}$ & $\mathrm{J}$ & 24 \\
\hline $383 \mathrm{MH} 1306.5$ & $5 / 25 / 2004$ & soil & EPA6010 & REG & $7440-38-2$ & Arsenic & 1.8 & $\mathrm{mg} / \mathrm{kg}$ & & 1.2 \\
\hline 383МH1306.5 & $5 / 25 / 2004$ & soil & EPA6010 & REG & $7440-39-3$ & Barium & 68 & $\mathrm{mg} / \mathrm{kg}$ & & 12 \\
\hline 383МH1306.5 & $5 / 25 / 2004$ & soil & EPA6010 & REG & $7440-41-7$ & Beryllium & 1.2 & $\mathrm{mg} / \mathrm{kg}$ & & 0.61 \\
\hline $383 \mathrm{MH} 1306.5$ & $5 / 25 / 2004$ & soil & HASL300 & REG & 14733-03-0 & Bi-214 & 1.18 & $\mathrm{pCi} / \mathrm{g}$ & $\mathrm{G}, \mathrm{J}$ & 0.35 \\
\hline 383MH1306.5 & $5 / 25 / 2004$ & soil & EPA6010 & REG & $7440-47-3$ & Chromium & 0.49 & $\mathrm{mg} / \mathrm{kg}$ & B & 1.2 \\
\hline 383MH1306.5 & $5 / 25 / 2004$ & soil & HASL300 & REG & $10045-97-3$ & Cs-137 & 0.57 & $\mathrm{pCi} / \mathrm{g}$ & G & 0.2 \\
\hline 383МH1306.5 & $5 / 25 / 2004$ & soil & HASL300 & REG & $13966-00-2$ & $\mathrm{~K}-40$ & 43.8 & $\mathrm{pCi} / \mathrm{g}$ & $\mathrm{G}$ & 2.7 \\
\hline
\end{tabular}

Page 51 of 78

Uncontrolled When Printed 


\begin{tabular}{|c|c|c|c|c|c|c|c|c|c|c|}
\hline SAMPLE \# & $\begin{array}{c}\text { SAMPLE } \\
\text { DATE }\end{array}$ & MATRIX & $\begin{array}{l}\text { USER TEST } \\
\text { PANEL }\end{array}$ & $\begin{array}{c}\text { SAMPLE } \\
\text { PURPOSE }\end{array}$ & CAS \# & PARAMETER & RESULT & UNITS & $\mathbf{Q}$ & $\begin{array}{l}\text { DETECT } \\
\text { LIMIT }\end{array}$ \\
\hline 383MH1306.5 & $5 / 25 / 2004$ & soil & EPA6010 & REG & 7439-92-1 & Lead & 12 & $\mathrm{mg} / \mathrm{kg}$ & & 0.36 \\
\hline 383MH1306.5 & $5 / 25 / 2004$ & soil & EPA7470 & REG & 7439-97-6 & Mercury & 0.0018 & $\mathrm{mg} / \mathrm{kg}$ & $\mathrm{B}$ & 0.12 \\
\hline 383MH1306.5 & $5 / 25 / 2004$ & soil & HASL300 & REG & 15092-94-1 & $\mathrm{Pb}-212$ & 1.91 & $\mathrm{pCi} / \mathrm{g}$ & $\mathrm{J}$ & 0.33 \\
\hline 383MH1306.5 & $5 / 25 / 2004$ & soil & HASL300 & REG & $15067-28-4$ & $\mathrm{~Pb}-214$ & 1.53 & $\mathrm{pCi} / \mathrm{g}$ & $\mathrm{G}, \mathrm{J}$ & 0.4 \\
\hline $383 \mathrm{MH} 1306.5$ & $5 / 25 / 2004$ & soil & HASL300 & REG & $14913-50-9$ & TI-208 & 0.8 & $\mathrm{pCi} / \mathrm{g}$ & G & 0.22 \\
\hline $383 \mathrm{MH} 1320.5$ & $5 / 25 / 2004$ & soil & EPA8260 & REG & 67-64-1 & Acetone & 10 & $\mu \mathrm{g} / \mathrm{kg}$ & $\mathrm{J}$ & 20 \\
\hline 383MH1320.5 & $5 / 25 / 2004$ & soil & EPA6010 & REG & $7440-39-3$ & Barium & 3.1 & $\mathrm{mg} / \mathrm{kg}$ & $\mathrm{B}$ & 10 \\
\hline 383MH1320.5 & $5 / 25 / 2004$ & soil & EPA6010 & REG & $7440-41-7$ & Beryllium & 0.15 & $\mathrm{mg} / \mathrm{kg}$ & $\mathrm{B}$ & 0.5 \\
\hline 383MH1320.5 & $5 / 25 / 2004$ & soil & EPA6010 & REG & $7440-47-3$ & Chromium & 1.3 & $\mathrm{mg} / \mathrm{kg}$ & & 1 \\
\hline 383MH1320.5 & $5 / 25 / 2004$ & soil & EPA7470 & REG & $7439-97-6$ & Mercury & 0.017 & $\mathrm{mg} / \mathrm{kg}$ & $\mathrm{B}$ & 0.1 \\
\hline 383MH1320.5 & $5 / 25 / 2004$ & soil & EPA6010 & REG & $7440-22-4$ & Silver & 0.19 & $\mathrm{mg} / \mathrm{kg}$ & $\mathrm{B}$ & 1 \\
\hline 383MH1407.5 & $5 / 20 / 2004$ & soil & HASL300 & REG & 14331-83-0 & Ac-228 & 1.84 & $\mathrm{pCi} / \mathrm{g}$ & $G$ & 0.45 \\
\hline 383MH1407.5 & $5 / 20 / 2004$ & soil & EPA6010 & REG & $7440-38-2$ & Arsenic & 3 & $\mathrm{mg} / \mathrm{kg}$ & & 1.1 \\
\hline $383 \mathrm{MH} 1407.5$ & $5 / 20 / 2004$ & soil & EPA6010 & REG & $7440-39-3$ & Barium & 220 & $\mathrm{mg} / \mathrm{kg}$ & & 11 \\
\hline $383 \mathrm{MH} 1407.5$ & $5 / 20 / 2004$ & soil & EPA6010 & REG & $7440-41-7$ & Beryllium & 1.1 & $\mathrm{mg} / \mathrm{kg}$ & & 0.56 \\
\hline 383MH1407.5 & $5 / 20 / 2004$ & soil & HASL300 & REG & $14733-03-0$ & Bi-214 & 1.02 & $\mathrm{pCi} / \mathrm{g}$ & $\mathrm{G}, \mathrm{J}$ & 0.28 \\
\hline 383MH1407.5 & $5 / 20 / 2004$ & soil & EPA6010 & REG & $7440-47-3$ & Chromium & 0.99 & $\mathrm{mg} / \mathrm{kg}$ & $\mathrm{B}$ & 1.1 \\
\hline $383 \mathrm{MH} 1407.5$ & $5 / 20 / 2004$ & soil & HASL300 & REG & $10045-97-3$ & Cs-137 & 10.7 & $\mathrm{pCi} / \mathrm{g}$ & $G$ & 0.1 \\
\hline 383MH1407.5 & $5 / 20 / 2004$ & soil & EPA8015 & REG & $68334-30-5$ & Diesel-Range Organics & 3.5 & $\mathrm{mg} / \mathrm{kg}$ & $\mathrm{J}$ & 5.6 \\
\hline $383 \mathrm{MH} 1407.5$ & $5 / 20 / 2004$ & soil & HASL300 & REG & $13966-00-2$ & $\mathrm{~K}-40$ & 30.6 & $\mathrm{pCi} / \mathrm{g}$ & G & 1.5 \\
\hline $383 \mathrm{MH} 1407.5$ & $5 / 20 / 2004$ & soil & EPA6010 & REG & 7439-92-1 & Lead & 12 & $\mathrm{mg} / \mathrm{kg}$ & & 0.34 \\
\hline 383MH1407.5 & $5 / 20 / 2004$ & soil & EPA7470 & REG & $7439-97-6$ & Mercury & 0.068 & $\mathrm{mg} / \mathrm{kg}$ & $\mathrm{B}$ & 0.11 \\
\hline $383 \mathrm{MH} 1407.5$ & $5 / 20 / 2004$ & soil & EPA8260 & REG & $75-09-2$ & Methylene Chloride & 1.1 & $\mu \mathrm{g} / \mathrm{kg}$ & $\mathrm{J}, \mathrm{B}$ & 5.6 \\
\hline $383 \mathrm{MH} 1407.5$ & $5 / 20 / 2004$ & soil & HASL300 & REG & 15092-94-1 & $\mathrm{Pb}-212$ & 2.35 & $\mathrm{pCi} / \mathrm{g}$ & $\mathrm{J}$ & 0.25 \\
\hline 383MH1407.5 & $5 / 20 / 2004$ & soil & HASL300 & REG & $15067-28-4$ & $\mathrm{~Pb}-214$ & 1.02 & $\mathrm{pCi} / \mathrm{g}$ & $\mathrm{G}, \mathrm{J}$ & 0.3 \\
\hline 383MH1407.5 & $5 / 20 / 2004$ & soil & UGTAISOPU & REG & 13981-16-3 & Pu-238 & 0.141 & $\mathrm{pCi} / \mathrm{g}$ & & 0.018 \\
\hline $383 \mathrm{MH} 1407.5$ & $5 / 20 / 2004$ & soil & UGTAISOPU & REG & $15117-48-3$ & Pu-239 & 1.86 & $\mathrm{pCi} / \mathrm{g}$ & $\mathrm{J}$ & 0.01 \\
\hline $383 \mathrm{MH} 1407.5$ & $5 / 20 / 2004$ & soil & SR7500 & REG & 10098-97-2 & Sr-90 & 0.67 & $\mathrm{pCi} / \mathrm{g}$ & & 0.26 \\
\hline 383MH1407.5 & $5 / 20 / 2004$ & soil & HASL300 & REG & $14913-50-9$ & TI-208 & 0.61 & $\mathrm{pCi} / \mathrm{g}$ & $G$ & 0.14 \\
\hline $383 \mathrm{MH} 1422.0$ & $5 / 20 / 2004$ & soil & EPA8260 & REG & 67-64-1 & Acetone & 18 & $\mu \mathrm{g} / \mathrm{kg}$ & $\mathrm{J}$ & 20 \\
\hline 383MH1422.0 & $5 / 20 / 2004$ & soil & EPA6010 & REG & $7440-38-2$ & Arsenic & 1.1 & $\mathrm{mg} / \mathrm{kg}$ & & 1 \\
\hline $383 \mathrm{MH} 1422.0$ & $5 / 20 / 2004$ & soil & EPA6010 & REG & $7440-39-3$ & Barium & 14 & $\mathrm{mg} / \mathrm{kg}$ & & 10 \\
\hline 383MH1422.0 & $5 / 20 / 2004$ & soil & EPA6010 & REG & $7440-41-7$ & Beryllium & 0.23 & $\mathrm{mg} / \mathrm{kg}$ & $\mathrm{B}$ & 0.51 \\
\hline $383 \mathrm{MH} 1422.0$ & $5 / 20 / 2004$ & soil & EPA6010 & REG & $7440-43-9$ & Cadmium & 0.02 & $\mathrm{mg} / \mathrm{kg}$ & $\mathrm{B}$ & 0.51 \\
\hline $383 \mathrm{MH} 1422.0$ & $5 / 20 / 2004$ & soil & EPA6010 & REG & $7440-47-3$ & Chromium & 1.7 & $\mathrm{mg} / \mathrm{kg}$ & & 1 \\
\hline
\end{tabular}

Page 52 of 78

Uncontrolled When Printed 


\begin{tabular}{|c|c|c|c|c|c|c|c|c|c|c|}
\hline SAMPLE \# & $\begin{array}{c}\text { SAMPLE } \\
\text { DATE }\end{array}$ & MATRIX & $\begin{array}{l}\text { USER TEST } \\
\text { PANEL }\end{array}$ & $\begin{array}{c}\text { SAMPLE } \\
\text { PURPOSE }\end{array}$ & CAS \# & PARAMETER & RESULT & UNITS & $\mathbf{Q}$ & $\begin{array}{l}\text { DETECT } \\
\text { LIMIT }\end{array}$ \\
\hline 383МH1422.0 & $5 / 20 / 2004$ & soil & EPA7470 & REG & $7439-97-6$ & Mercury & 0.023 & $\mathrm{mg} / \mathrm{kg}$ & $\mathrm{B}$ & 0.1 \\
\hline 383МH1422.0 & $5 / 20 / 2004$ & soil & EPA8260 & REG & $75-09-2$ & Methylene Chloride & 0.91 & $\mu \mathrm{g} / \mathrm{kg}$ & $\mathrm{J}, \mathrm{B}$ & 5.1 \\
\hline 383МH1422.0 & $5 / 20 / 2004$ & soil & HASL300 & REG & $15067-28-4$ & $\mathrm{~Pb}-214$ & 0.29 & $\mathrm{pCi} / \mathrm{g}$ & $\mathrm{J}$ & 0.14 \\
\hline 383МH1422.0 & $5 / 20 / 2004$ & soil & EPA6010 & REG & $7440-22-4$ & Silver & 0.11 & $\mathrm{mg} / \mathrm{kg}$ & $\mathrm{B}$ & 1 \\
\hline 383МH1501.0 & $5 / 24 / 2004$ & soil & HASL300 & REG & 14331-83-0 & Ac-228 & 2.02 & $\mathrm{pCi} / \mathrm{g}$ & $G$ & 0.8 \\
\hline 383МH1501.0 & $5 / 24 / 2004$ & soil & EPA6010 & REG & $7440-38-2$ & Arsenic & 3.6 & $\mathrm{mg} / \mathrm{kg}$ & & 1.1 \\
\hline 383МH1501.0 & $5 / 24 / 2004$ & soil & EPA6010 & REG & $7440-39-3$ & Barium & 520 & $\mathrm{mg} / \mathrm{kg}$ & & 11 \\
\hline 383МH1501.0 & $5 / 24 / 2004$ & soil & EPA8270 & REG & $56-55-3$ & Benzo(A)Anthracene & 19 & $\mu \mathrm{g} / \mathrm{kg}$ & $\mathrm{J}$ & 380 \\
\hline 383МH1501.0 & $5 / 24 / 2004$ & soil & EPA6010 & REG & $7440-41-7$ & Beryllium & 0.78 & $\mathrm{mg} / \mathrm{kg}$ & & 0.57 \\
\hline 383МH1501.0 & $5 / 24 / 2004$ & soil & HASL300 & REG & $14733-03-0$ & Bi-214 & 1.29 & $\mathrm{pCi} / \mathrm{g}$ & $\mathrm{G}, \mathrm{J}$ & 0.34 \\
\hline 383МH1501.0 & $5 / 24 / 2004$ & soil & EPA6010 & REG & $7440-47-3$ & Chromium & 4.9 & $\mathrm{mg} / \mathrm{kg}$ & & 1.1 \\
\hline 383МH1501.0 & $5 / 24 / 2004$ & soil & HASL300 & REG & $10045-97-3$ & Cs-137 & 0.55 & $\mathrm{pCi} / \mathrm{g}$ & G & 0.18 \\
\hline 383МH1501.0 & $5 / 24 / 2004$ & soil & EPA8015 & REG & $68334-30-5$ & Diesel-Range Organics & 52 & $\mathrm{mg} / \mathrm{kg}$ & $\mathrm{M}, \mathrm{H}$ & 5.7 \\
\hline 383МH1501.0 & $5 / 24 / 2004$ & soil & HASL300 & REG & 13966-00-2 & $\mathrm{K}-40$ & 31.3 & $\mathrm{pCi} / \mathrm{g}$ & $G$ & 2.1 \\
\hline 383МН1501.0 & $5 / 24 / 2004$ & soil & EPA6010 & REG & 7439-92-1 & Lead & 27 & $\mathrm{mg} / \mathrm{kg}$ & & 0.34 \\
\hline 383МH1501.0 & $5 / 24 / 2004$ & soil & EPA7470 & REG & 7439-97-6 & Mercury & 0.015 & $\mathrm{mg} / \mathrm{kg}$ & $\mathrm{B}$ & 0.11 \\
\hline 383МH1501.0 & $5 / 24 / 2004$ & soil & EPA8260 & REG & $75-09-2$ & Methylene Chloride & 1.7 & $\mu \mathrm{g} / \mathrm{kg}$ & $\mathrm{J}, \mathrm{B}$ & 5.7 \\
\hline 383МH1501.0 & $5 / 24 / 2004$ & soil & HASL300 & REG & 15092-94-1 & $\mathrm{Pb}-212$ & 2.13 & $\mathrm{pCi} / \mathrm{g}$ & $\mathrm{J}$ & 0.24 \\
\hline 383МH1501.0 & $5 / 24 / 2004$ & soil & HASL300 & REG & $15067-28-4$ & $\mathrm{~Pb}-214$ & 1.17 & $\mathrm{pCi} / \mathrm{g}$ & $\mathrm{G}, \mathrm{J}$ & 0.31 \\
\hline 383МH1501.0 & $5 / 24 / 2004$ & soil & HASL300 & REG & $14913-50-9$ & TI-208 & 0.69 & $\mathrm{pCi} / \mathrm{g}$ & $G$ & 0.16 \\
\hline 383МH1503.5 & $5 / 24 / 2004$ & soil & EPA8260 & REG & 67-64-1 & Acetone & 8.2 & $\mu \mathrm{g} / \mathrm{kg}$ & $\mathrm{J}$ & 20 \\
\hline 383МH1503.5 & $5 / 24 / 2004$ & soil & EPA6010 & REG & $7440-38-2$ & Arsenic & 0.46 & $\mathrm{mg} / \mathrm{kg}$ & $\mathrm{B}$ & 1 \\
\hline 383МH1503.5 & $5 / 24 / 2004$ & soil & EPA6010 & REG & $7440-39-3$ & Barium & 3.2 & $\mathrm{mg} / \mathrm{kg}$ & $\mathrm{B}$ & 10 \\
\hline 383МH1503.5 & $5 / 24 / 2004$ & soil & EPA6010 & REG & $7440-41-7$ & Beryllium & 0.16 & $\mathrm{mg} / \mathrm{kg}$ & B & 0.51 \\
\hline 383МH1503.5 & $5 / 24 / 2004$ & soil & HASL300 & REG & $14733-03-0$ & Bi-214 & 0.41 & $\mathrm{pCi} / \mathrm{g}$ & $\mathrm{J}$ & 0.16 \\
\hline 383МH1503.5 & $5 / 24 / 2004$ & soil & EPA6010 & REG & $7440-47-3$ & Chromium & 0.91 & $\mathrm{mg} / \mathrm{kg}$ & $\mathrm{B}$ & 1 \\
\hline 383МH1503.5 & $5 / 24 / 2004$ & soil & EPA8260 & REG & $87-68-3$ & Hexachlorobutadiene & 1.5 & $\mu \mathrm{g} / \mathrm{kg}$ & $\mathrm{J}$ & 5.1 \\
\hline 383МH1503.5 & $5 / 24 / 2004$ & soil & EPA7470 & REG & 7439-97-6 & Mercury & 0.11 & $\mathrm{mg} / \mathrm{kg}$ & & 0.1 \\
\hline 383МH1503.5 & $5 / 24 / 2004$ & soil & EPA8260 & REG & $75-09-2$ & Methylene Chloride & 1.6 & $\mu \mathrm{g} / \mathrm{kg}$ & $\mathrm{J}, \mathrm{B}$ & 5.1 \\
\hline 383МH1503.5 & $5 / 24 / 2004$ & soil & HASL300 & REG & $15067-28-4$ & $\mathrm{~Pb}-214$ & 0.46 & $\mathrm{pCi} / \mathrm{g}$ & $\mathrm{J}$ & 0.14 \\
\hline 383МH1503.5 & $5 / 24 / 2004$ & soil & EPA6010 & REG & $7782-49-2$ & Selenium & 0.29 & $\mathrm{mg} / \mathrm{kg}$ & $\mathrm{B}$ & 0.51 \\
\hline 383МH1503.5X & $5 / 24 / 2004$ & soil & EPA8260 & FD & 67-64-1 & Acetone & 8.3 & $\mu \mathrm{g} / \mathrm{kg}$ & $\mathrm{J}$ & 20 \\
\hline 383МH1503.5X & $5 / 24 / 2004$ & soil & EPA6010 & FD & $7440-38-2$ & Arsenic & 0.59 & $\mathrm{mg} / \mathrm{kg}$ & $\mathrm{B}$ & 1 \\
\hline 383МH1503.5X & $5 / 24 / 2004$ & soil & EPA6010 & FD & $7440-39-3$ & Barium & 3.1 & $\mathrm{mg} / \mathrm{kg}$ & $\mathrm{B}$ & 10 \\
\hline 383МH1503.5X & $5 / 24 / 2004$ & soil & EPA6010 & FD & 7440-41-7 & Beryllium & 0.16 & $\mathrm{mg} / \mathrm{kg}$ & $\mathrm{B}$ & 0.51 \\
\hline
\end{tabular}

Page 53 of 78

Uncontrolled When Printed 


\begin{tabular}{|c|c|c|c|c|c|c|c|c|c|c|}
\hline SAMPLE \# & $\begin{array}{c}\text { SAMPLE } \\
\text { DATE }\end{array}$ & MATRIX & $\begin{array}{l}\text { USER TEST } \\
\text { PANEL }\end{array}$ & $\begin{array}{c}\text { SAMPLE } \\
\text { PURPOSE }\end{array}$ & CAS \# & PARAMETER & RESULT & UNITS & $\mathbf{Q}$ & $\begin{array}{l}\text { DETECT } \\
\text { LIMIT }\end{array}$ \\
\hline 383MH1503.5X & $5 / 24 / 2004$ & soil & HASL300 & FD & $14733-03-0$ & Bi-214 & 0.43 & $\mathrm{pCi} / \mathrm{g}$ & $\mathrm{J}$ & 0.13 \\
\hline 383МH1503.5X & $5 / 24 / 2004$ & soil & EPA6010 & FD & $7440-47-3$ & Chromium & 0.92 & $\mathrm{mg} / \mathrm{kg}$ & B & 1 \\
\hline 383MH1503.5X & $5 / 24 / 2004$ & soil & EPA7470 & FD & $7439-97-6$ & Mercury & 0.13 & $\mathrm{mg} / \mathrm{kg}$ & & 0.1 \\
\hline 383MH1503.5X & $5 / 24 / 2004$ & soil & EPA8260 & FD & $75-09-2$ & Methylene Chloride & 1.6 & $\mu \mathrm{g} / \mathrm{kg}$ & $\mathrm{J}, \mathrm{B}$ & 5.1 \\
\hline 383MH1503.5X & $5 / 24 / 2004$ & soil & HASL300 & FD & $15067-28-4$ & $\mathrm{~Pb}-214$ & 0.47 & $\mathrm{pCi} / \mathrm{g}$ & $\mathrm{J}$ & 0.11 \\
\hline 383MH1503.5X & $5 / 24 / 2004$ & soil & EPA6010 & FD & $7440-22-4$ & Silver & 0.099 & $\mathrm{mg} / \mathrm{kg}$ & $\mathrm{B}$ & 1 \\
\hline 383MH1503.5X & $5 / 24 / 2004$ & soil & EPA8260 & FD & $100-42-5$ & Styrene & 0.89 & $\mu \mathrm{g} / \mathrm{kg}$ & $\mathrm{J}$ & 5.1 \\
\hline 383MH1608.5 & $6 / 11 / 2004$ & soil & HASL300 & REG & 14331-83-0 & Ac-228 & 1.45 & $\mathrm{pCi} / \mathrm{g}$ & G & 0.59 \\
\hline 383МH1608.5 & $6 / 11 / 2004$ & soil & EPA8260 & REG & 67-64-1 & Acetone & 7.5 & $\mu \mathrm{g} / \mathrm{kg}$ & $\mathrm{J}$ & 22 \\
\hline 383MH1608.5 & $6 / 11 / 2004$ & soil & HASL300 & REG & 14596-10-2 & Am-241 & 0.52 & $\mathrm{pCi} / \mathrm{g}$ & $G$ & 0.3 \\
\hline 383MH1608.5 & $6 / 11 / 2004$ & soil & EPA6010 & REG & $7440-38-2$ & Arsenic & 3.6 & $\mathrm{mg} / \mathrm{kg}$ & & 1.1 \\
\hline 383MH1608.5 & $6 / 11 / 2004$ & soil & EPA6010 & REG & $7440-39-3$ & Barium & 64 & $\mathrm{mg} / \mathrm{kg}$ & & 11 \\
\hline 383MH1608.5 & $6 / 11 / 2004$ & soil & EPA6010 & REG & $7440-41-7$ & Beryllium & 0.8 & $\mathrm{mg} / \mathrm{kg}$ & & 0.55 \\
\hline 383MH1608.5 & $6 / 11 / 2004$ & soil & HASL300 & REG & $14913-49-6$ & Bi-212 & 2.2 & $\mathrm{pCi} / \mathrm{g}$ & G & 2 \\
\hline 383MH1608.5 & $6 / 11 / 2004$ & soil & HASL300 & REG & $14733-03-0$ & Bi-214 & 0.8 & $\mathrm{pCi} / \mathrm{g}$ & $\mathrm{G}, \mathrm{J}$ & 0.28 \\
\hline 383MH1608.5 & $6 / 11 / 2004$ & soil & EPA6010 & REG & $7440-43-9$ & Cadmium & 0.091 & $\mathrm{mg} / \mathrm{kg}$ & $\mathrm{B}$ & 0.55 \\
\hline 383MH1608.5 & $6 / 11 / 2004$ & soil & EPA6010 & REG & $7440-47-3$ & Chromium & 2 & $\mathrm{mg} / \mathrm{kg}$ & & 1.1 \\
\hline 383MH1608.5 & $6 / 11 / 2004$ & soil & HASL300 & REG & $10045-97-3$ & Cs-137 & 2.67 & $\mathrm{pCi} / \mathrm{g}$ & $\mathrm{G}$ & 0.12 \\
\hline 383MH1608.5 & $6 / 11 / 2004$ & soil & HASL300 & REG & 13966-00-2 & $\mathrm{K}-40$ & 19.2 & $\mathrm{pCi} / \mathrm{g}$ & $G$ & 1.5 \\
\hline 383MH1608.5 & $6 / 11 / 2004$ & soil & EPA6010 & REG & 7439-92-1 & Lead & 15 & $\mathrm{mg} / \mathrm{kg}$ & & 1.6 \\
\hline 383MH1608.5 & $6 / 11 / 2004$ & soil & EPA7470 & REG & 7439-97-6 & Mercury & 0.089 & $\mathrm{mg} / \mathrm{kg}$ & B & 0.11 \\
\hline 383МH1608.5 & $6 / 11 / 2004$ & soil & HASL300 & REG & 15092-94-1 & $\mathrm{Pb}-212$ & 1.44 & $\mathrm{pCi} / \mathrm{g}$ & $\mathrm{G}$ & 0.22 \\
\hline 383MH1608.5 & $6 / 11 / 2004$ & soil & HASL300 & REG & $15067-28-4$ & $\mathrm{~Pb}-214$ & 0.92 & $\mathrm{pCi} / \mathrm{g}$ & $\mathrm{G}, \mathrm{J}$ & 0.27 \\
\hline 383MH1608.5 & $6 / 11 / 2004$ & soil & UGTAISOPU & REG & 13981-16-3 & Pu-238 & 0.05 & $\mathrm{pCi} / \mathrm{g}$ & & 0.009 \\
\hline 383МH1608.5 & $6 / 11 / 2004$ & soil & UGTAISOPU & REG & $15117-48-3$ & Pu-239 & 2.82 & $\mathrm{pCi} / \mathrm{g}$ & & 0.02 \\
\hline 383MH1608.5 & $6 / 11 / 2004$ & soil & SR7500 & REG & 10098-97-2 & Sr-90 & 0.21 & $\mathrm{pCi} / \mathrm{g}$ & $\mathrm{LT}$ & 0.18 \\
\hline 383MH1608.5 & $6 / 11 / 2004$ & soil & HASL300 & REG & 14913-50-9 & TI-208 & 0.46 & $\mathrm{pCi} / \mathrm{g}$ & $G$ & 0.15 \\
\hline 383MH1609.5 & $6 / 11 / 2004$ & soil & HASL300 & REG & 14331-83-0 & Ac-228 & 2.13 & $\mathrm{pCi} / \mathrm{g}$ & $\mathrm{G}$ & 0.56 \\
\hline 383МH1609.5 & $6 / 11 / 2004$ & soil & EPA6010 & REG & $7440-38-2$ & Arsenic & 2.7 & $\mathrm{mg} / \mathrm{kg}$ & & 1.1 \\
\hline 383MH1609.5 & $6 / 11 / 2004$ & soil & EPA6010 & REG & $7440-39-3$ & Barium & 58 & $\mathrm{mg} / \mathrm{kg}$ & & 11 \\
\hline 383МH1609.5 & $6 / 11 / 2004$ & soil & EPA6010 & REG & 7440-41-7 & Beryllium & 0.68 & $\mathrm{mg} / \mathrm{kg}$ & & 0.56 \\
\hline 383MH1609.5 & $6 / 11 / 2004$ & soil & HASL300 & REG & $14913-49-6$ & Bi-212 & 3.7 & $\mathrm{pCi} / \mathrm{g}$ & $G$ & 2.6 \\
\hline 383MH1609.5 & $6 / 11 / 2004$ & soil & HASL300 & REG & $14733-03-0$ & Bi-214 & 1.02 & $\mathrm{pCi} / \mathrm{g}$ & $\mathrm{G}, \mathrm{J}$ & 0.4 \\
\hline 383МH1609.5 & $6 / 11 / 2004$ & soil & EPA6010 & REG & 7440-43-9 & Cadmium & 0.041 & $\mathrm{mg} / \mathrm{kg}$ & B & 0.56 \\
\hline 383МH1609.5 & $6 / 11 / 2004$ & soil & EPA6010 & REG & 7440-47-3 & Chromium & 0.88 & $\mathrm{mg} / \mathrm{kg}$ & $\mathrm{B}$ & 1.1 \\
\hline
\end{tabular}

Page 54 of 78

Uncontrolled When Printed 


\begin{tabular}{|c|c|c|c|c|c|c|c|c|c|c|}
\hline SAMPLE \# & $\begin{array}{c}\text { SAMPLE } \\
\text { DATE }\end{array}$ & MATRIX & $\begin{array}{l}\text { USER TEST } \\
\text { PANEL }\end{array}$ & $\begin{array}{c}\text { SAMPLE } \\
\text { PURPOSE }\end{array}$ & CAS \# & PARAMETER & RESULT & UNITS & $\mathbf{Q}$ & $\begin{array}{c}\text { DETECT } \\
\text { LIMIT }\end{array}$ \\
\hline 383МH1609.5 & $6 / 11 / 2004$ & soil & HASL300 & REG & $10045-97-3$ & Cs-137 & 34.5 & $\mathrm{pCi} / \mathrm{g}$ & $\mathrm{G}$ & 0.2 \\
\hline 383МH1609.5 & $6 / 11 / 2004$ & soil & HASL300 & REG & 13966-00-2 & $\mathrm{K}-40$ & 34 & $\mathrm{pCi} / \mathrm{g}$ & $G$ & 1.3 \\
\hline 383MH1609.5 & $6 / 11 / 2004$ & soil & EPA6010 & REG & 7439-92-1 & Lead & 9.5 & $\mathrm{mg} / \mathrm{kg}$ & & 0.34 \\
\hline $383 \mathrm{MH} 1609.5$ & $6 / 11 / 2004$ & soil & EPA7470 & REG & 7439-97-6 & Mercury & 0.012 & $\mathrm{mg} / \mathrm{kg}$ & $B$ & 0.11 \\
\hline 383МH1609.5 & $6 / 11 / 2004$ & soil & HASL300 & REG & 15092-94-1 & $\mathrm{Pb}-212$ & 2.26 & $\mathrm{pCi} / \mathrm{g}$ & $G$ & 0.4 \\
\hline 383МH1609.5 & $6 / 11 / 2004$ & soil & HASL300 & REG & $15067-28-4$ & $\mathrm{~Pb}-214$ & 0.96 & $\mathrm{pCi} / \mathrm{g}$ & $\mathrm{G}, \mathrm{J}$ & 0.6 \\
\hline 383МH1609.5 & $6 / 11 / 2004$ & soil & UGTAISOPU & REG & 13981-16-3 & Pu-238 & 0.093 & $\mathrm{pCi} / \mathrm{g}$ & & 0.018 \\
\hline 383МH1609.5 & $6 / 11 / 2004$ & soil & UGTAISOPU & REG & $15117-48-3$ & Pu-239 & 0.5 & $\mathrm{pCi} / \mathrm{g}$ & & 0.01 \\
\hline 383МH1609.5 & $6 / 11 / 2004$ & soil & SR7500 & REG & 10098-97-2 & Sr-90 & 4.2 & $\mathrm{pCi} / \mathrm{g}$ & Y1 & 0.2 \\
\hline 383МH1609.5 & $6 / 11 / 2004$ & soil & HASL300 & REG & $14913-50-9$ & TI-208 & 0.82 & $\mathrm{pCi} / \mathrm{g}$ & $G$ & 0.2 \\
\hline 383MH1619.0 & $6 / 11 / 2004$ & soil & EPA8260 & REG & 67-64-1 & Acetone & 7.7 & $\mu \mathrm{g} / \mathrm{kg}$ & $\mathrm{J}$ & 20 \\
\hline 383МH1619.0 & $6 / 11 / 2004$ & soil & EPA6010 & REG & $7440-38-2$ & Arsenic & 1.6 & $\mathrm{mg} / \mathrm{kg}$ & & 1 \\
\hline 383МH1619.0 & $6 / 11 / 2004$ & soil & EPA6010 & REG & $7440-39-3$ & Barium & 16 & $\mathrm{mg} / \mathrm{kg}$ & & 10 \\
\hline 383МH1619.0 & $6 / 11 / 2004$ & soil & EPA6010 & REG & $7440-41-7$ & Beryllium & 0.27 & $\mathrm{mg} / \mathrm{kg}$ & $\mathrm{B}$ & 0.51 \\
\hline 383МН1619.0 & $6 / 11 / 2004$ & soil & HASL300 & REG & $14733-03-0$ & Bi-214 & 0.35 & $\mathrm{pCi} / \mathrm{g}$ & $\mathrm{J}$ & 0.17 \\
\hline 383МH1619.0 & $6 / 11 / 2004$ & soil & EPA6010 & REG & $7440-43-9$ & Cadmium & 0.085 & $\mathrm{mg} / \mathrm{kg}$ & $\mathrm{B}$ & 0.51 \\
\hline 383МH1619.0 & $6 / 11 / 2004$ & soil & EPA6010 & REG & $7440-47-3$ & Chromium & 2.4 & $\mathrm{mg} / \mathrm{kg}$ & & 1 \\
\hline 383МH1619.0 & $6 / 11 / 2004$ & soil & HASL300 & REG & $10045-97-3$ & Cs-137 & 0.163 & $\mathrm{pCi} / \mathrm{g}$ & $\mathrm{LT}$ & 0.067 \\
\hline 383МH1619.0 & $6 / 11 / 2004$ & soil & HASL300 & REG & 13966-00-2 & $\mathrm{K}-40$ & 2.05 & $\mathrm{pCi} / \mathrm{g}$ & & 0.86 \\
\hline 383МH1619.0 & $6 / 11 / 2004$ & soil & EPA6010 & REG & 7439-92-1 & Lead & 2.7 & $\mathrm{mg} / \mathrm{kg}$ & & 1.5 \\
\hline 383МH1619.0 & $6 / 11 / 2004$ & soil & EPA7470 & REG & 7439-97-6 & Mercury & 0.057 & $\mathrm{mg} / \mathrm{kg}$ & B & 0.1 \\
\hline 383МH1619.0 & $6 / 11 / 2004$ & soil & HASL300 & REG & $15067-28-4$ & $\mathrm{~Pb}-214$ & 0.322 & $\mathrm{pCi} / \mathrm{g}$ & $\mathrm{J}$ & 0.134 \\
\hline 383МH1619.0 & $6 / 11 / 2004$ & soil & UGTAISOPU & REG & 13981-16-3 & Pu-238 & 0.068 & $\mathrm{pCi} / \mathrm{g}$ & & 0.009 \\
\hline 383МH1619.0 & $6 / 11 / 2004$ & soil & UGTAISOPU & REG & $15117-48-3$ & Pu-239 & 0.338 & $\mathrm{pCi} / \mathrm{g}$ & & 0.009 \\
\hline 383МH1619.0 & $6 / 11 / 2004$ & soil & EPA6010 & REG & $7440-22-4$ & Silver & 0.1 & $\mathrm{mg} / \mathrm{kg}$ & $B$ & 1 \\
\hline 383MH1716.5 & $5 / 22 / 2004$ & soil & HASL300 & REG & 14331-83-0 & Ac-228 & 2.24 & $\mathrm{pCi} / \mathrm{g}$ & $G$ & 0.62 \\
\hline 383МH1716.5 & $5 / 22 / 2004$ & soil & EPA6010 & REG & $7440-38-2$ & Arsenic & 2.9 & $\mathrm{mg} / \mathrm{kg}$ & & 1.1 \\
\hline $383 \mathrm{MH} 1716.5$ & $5 / 22 / 2004$ & soil & EPA6010 & REG & $7440-39-3$ & Barium & 86 & $\mathrm{mg} / \mathrm{kg}$ & & 11 \\
\hline $383 \mathrm{MH} 1716.5$ & $5 / 22 / 2004$ & soil & EPA8270 & REG & $56-55-3$ & Benzo(A)Anthracene & 21 & $\mu \mathrm{g} / \mathrm{kg}$ & $\mathrm{J}$ & 380 \\
\hline 383МH1716.5 & $5 / 22 / 2004$ & soil & EPA8270 & REG & $205-99-2$ & Benzo(B)Fluoranthene & 18 & $\mu \mathrm{g} / \mathrm{kg}$ & $\mathrm{J}$ & 380 \\
\hline 383МH1716.5 & $5 / 22 / 2004$ & soil & EPA6010 & REG & 7440-41-7 & Beryllium & 1.3 & $\mathrm{mg} / \mathrm{kg}$ & & 0.57 \\
\hline 383МH1716.5 & $5 / 22 / 2004$ & soil & HASL300 & REG & $14733-03-0$ & Bi-214 & 1.49 & $\mathrm{pCi} / \mathrm{g}$ & $\mathrm{G}, \mathrm{J}$ & 0.33 \\
\hline 383MH1716.5 & $5 / 22 / 2004$ & soil & EPA6010 & REG & 7440-43-9 & Cadmium & 0.031 & $\mathrm{mg} / \mathrm{kg}$ & $\mathrm{B}$ & 0.57 \\
\hline 383MH1716.5 & $5 / 22 / 2004$ & soil & EPA6010 & REG & $7440-47-3$ & Chromium & 0.65 & $\mathrm{mg} / \mathrm{kg}$ & $B$ & 1.1 \\
\hline 383МH1716.5 & $5 / 22 / 2004$ & soil & EPA8270 & REG & 218-01-9 & Chrysene & 21 & $\mu g / \mathrm{kg}$ & $\mathrm{J}$ & 380 \\
\hline
\end{tabular}

Page 55 of 78

Uncontrolled When Printed 


\begin{tabular}{|c|c|c|c|c|c|c|c|c|c|c|}
\hline SAMPLE \# & $\begin{array}{c}\text { SAMPLE } \\
\text { DATE }\end{array}$ & MATRIX & $\begin{array}{l}\text { USER TEST } \\
\text { PANEL }\end{array}$ & $\begin{array}{c}\text { SAMPLE } \\
\text { PURPOSE }\end{array}$ & CAS \# & PARAMETER & RESULT & UNITS & $\mathbf{Q}$ & $\begin{array}{c}\text { DETECT } \\
\text { LIMIT }\end{array}$ \\
\hline 383МH1716.5 & $5 / 22 / 2004$ & soil & HASL300 & REG & $10045-97-3$ & Cs-137 & 6.32 & $\mathrm{pCi} / \mathrm{g}$ & G & 0.15 \\
\hline 383MH1716.5 & $5 / 22 / 2004$ & soil & EPA8270 & REG & $206-44-0$ & Fluoranthene & 22 & $\mu \mathrm{g} / \mathrm{kg}$ & $\mathrm{J}$ & 380 \\
\hline 383MH1716.5 & $5 / 22 / 2004$ & soil & HASL300 & REG & 13966-00-2 & $\mathrm{K}-40$ & 37.2 & $\mathrm{pCi} / \mathrm{g}$ & $\mathrm{G}$ & 1.4 \\
\hline $383 \mathrm{MH} 1716.5$ & $5 / 22 / 2004$ & soil & EPA6010 & REG & 7439-92-1 & Lead & 13 & $\mathrm{mg} / \mathrm{kg}$ & & 0.34 \\
\hline 383МH1716.5 & $5 / 22 / 2004$ & soil & EPA7470 & REG & $7439-97-6$ & Mercury & 0.0029 & $\mathrm{mg} / \mathrm{kg}$ & $B$ & 0.11 \\
\hline 383МH1716.5 & $5 / 22 / 2004$ & soil & EPA8260 & REG & $75-09-2$ & Methylene Chloride & 0.9 & $\mu \mathrm{g} / \mathrm{kg}$ & $\mathrm{J}, \mathrm{B}$ & 5.7 \\
\hline 383МH1716.5 & $5 / 22 / 2004$ & soil & HASL300 & REG & 15092-94-1 & $\mathrm{Pb}-212$ & 1.96 & $\mathrm{pCi} / \mathrm{g}$ & $\mathrm{J}$ & 0.29 \\
\hline 383МH1716.5 & $5 / 22 / 2004$ & soil & HASL300 & REG & $15067-28-4$ & $\mathrm{~Pb}-214$ & 1.43 & $\mathrm{pCi} / \mathrm{g}$ & $\mathrm{G}, \mathrm{J}$ & 0.31 \\
\hline 383МH1716.5 & $5 / 22 / 2004$ & soil & HASL300 & REG & $14913-50-9$ & TI-208 & 0.71 & $\mathrm{pCi} / \mathrm{g}$ & $\mathrm{G}$ & 0.16 \\
\hline 383МH1734.5 & $5 / 22 / 2004$ & soil & EPA8260 & REG & 67-64-1 & Acetone & 6.1 & $\mu \mathrm{g} / \mathrm{kg}$ & J & 21 \\
\hline 383MH1734.5 & $5 / 22 / 2004$ & soil & EPA6010 & REG & $7440-38-2$ & Arsenic & 1.8 & $\mathrm{mg} / \mathrm{kg}$ & & 1 \\
\hline 383МH1734.5 & $5 / 22 / 2004$ & soil & EPA6010 & REG & $7440-39-3$ & Barium & 26 & $\mathrm{mg} / \mathrm{kg}$ & & 10 \\
\hline $383 \mathrm{MH} 1734.5$ & $5 / 22 / 2004$ & soil & EPA6010 & REG & $7440-41-7$ & Beryllium & 0.38 & $\mathrm{mg} / \mathrm{kg}$ & B & 0.52 \\
\hline 383МH1734.5 & $5 / 22 / 2004$ & soil & EPA6010 & REG & $7440-43-9$ & Cadmium & 0.94 & $\mathrm{mg} / \mathrm{kg}$ & & 0.52 \\
\hline 383МH1734.5 & $5 / 22 / 2004$ & soil & EPA6010 & REG & $7440-47-3$ & Chromium & 3.5 & $\mathrm{mg} / \mathrm{kg}$ & & 1 \\
\hline $383 \mathrm{MH} 1734.5$ & $5 / 22 / 2004$ & soil & EPA6010 & REG & 7439-92-1 & Lead & 160 & $\mathrm{mg} / \mathrm{kg}$ & & 1.6 \\
\hline $383 \mathrm{MH} 1734.5$ & $5 / 22 / 2004$ & soil & EPA7470 & REG & $7439-97-6$ & Mercury & 0.33 & $\mathrm{mg} / \mathrm{kg}$ & & 0.1 \\
\hline 383MH1734.5 & $5 / 22 / 2004$ & soil & EPA8260 & REG & $75-09-2$ & Methylene Chloride & 0.9 & $\mu \mathrm{g} / \mathrm{kg}$ & $\mathrm{J}, \mathrm{B}$ & 5.2 \\
\hline 383МH1734.5 & $5 / 22 / 2004$ & soil & HASL300 & REG & $15067-28-4$ & $\mathrm{~Pb}-214$ & 0.3 & $\mathrm{pCi} / \mathrm{g}$ & $\mathrm{G}, \mathrm{J}$ & 0.15 \\
\hline $383 \mathrm{MH} 1734.5$ & $5 / 22 / 2004$ & soil & EPA6010 & REG & $7440-22-4$ & Silver & 2.8 & $\mathrm{mg} / \mathrm{kg}$ & & 1 \\
\hline 383МH1803.5 & $5 / 23 / 2004$ & soil & HASL300 & REG & 14331-83-0 & Ac-228 & 0.92 & $\mathrm{pCi} / \mathrm{g}$ & G & 0.51 \\
\hline 383МH1803.5 & $5 / 23 / 2004$ & soil & EPA6010 & REG & $7440-38-2$ & Arsenic & 2.2 & $\mathrm{mg} / \mathrm{kg}$ & & 1.1 \\
\hline $383 \mathrm{MH} 1803.5$ & $5 / 23 / 2004$ & soil & EPA6010 & REG & $7440-39-3$ & Barium & 75 & $\mathrm{mg} / \mathrm{kg}$ & & 11 \\
\hline 383МH1803.5 & $5 / 23 / 2004$ & soil & EPA6010 & REG & $7440-41-7$ & Beryllium & 0.69 & $\mathrm{mg} / \mathrm{kg}$ & & 0.56 \\
\hline 383MH1803.5 & $5 / 23 / 2004$ & soil & HASL300 & REG & $14733-03-0$ & Bi-214 & 0.8 & $\mathrm{pCi} / \mathrm{g}$ & $\mathrm{G}, \mathrm{J}$ & 0.28 \\
\hline 383MH1803.5 & $5 / 23 / 2004$ & soil & EPA6010 & REG & $7440-43-9$ & Cadmium & 0.27 & $\mathrm{mg} / \mathrm{kg}$ & $\mathrm{B}$ & 0.56 \\
\hline $383 \mathrm{MH} 1803.5$ & $5 / 23 / 2004$ & soil & EPA6010 & REG & $7440-47-3$ & Chromium & 2 & $\mathrm{mg} / \mathrm{kg}$ & & 1.1 \\
\hline $383 \mathrm{MH} 1803.5$ & $5 / 23 / 2004$ & soil & HASL300 & REG & $10045-97-3$ & Cs-137 & 5.14 & $\mathrm{pCi} / \mathrm{g}$ & $\mathrm{G}$ & 0.14 \\
\hline 383МH1803.5 & $5 / 23 / 2004$ & soil & EPA8015 & REG & $68334-30-5$ & Diesel-Range Organics & 5.1 & $\mathrm{mg} / \mathrm{kg}$ & $\mathrm{J}$ & 5.6 \\
\hline $383 \mathrm{MH} 1803.5$ & $5 / 23 / 2004$ & soil & HASL300 & REG & $13966-00-2$ & $\mathrm{~K}-40$ & 16.5 & $\mathrm{pCi} / \mathrm{g}$ & $\mathrm{G}$ & 1 \\
\hline 383МH1803.5 & $5 / 23 / 2004$ & soil & EPA6010 & REG & 7439-92-1 & Lead & 25 & $\mathrm{mg} / \mathrm{kg}$ & & 1.7 \\
\hline 383MH1803.5 & $5 / 23 / 2004$ & soil & EPA7470 & REG & $7439-97-6$ & Mercury & 0.15 & $\mathrm{mg} / \mathrm{kg}$ & & 0.11 \\
\hline 383МH1803.5 & $5 / 23 / 2004$ & soil & EPA8260 & REG & $75-09-2$ & Methylene Chloride & 1.1 & $\mu \mathrm{g} / \mathrm{kg}$ & $\mathrm{J}, \mathrm{B}$ & 5.6 \\
\hline 383MH1803.5 & $5 / 23 / 2004$ & soil & HASL300 & REG & 15092-94-1 & $\mathrm{Pb}-212$ & 0.99 & $\mathrm{pCi} / \mathrm{g}$ & $\mathrm{J}$ & 0.22 \\
\hline 383МH1803.5 & $5 / 23 / 2004$ & soil & HASL300 & REG & 15067-28-4 & $\mathrm{Pb}-214$ & 0.65 & $\mathrm{pCi} / \mathrm{g}$ & $\mathrm{G}, \mathrm{J}$ & 0.32 \\
\hline
\end{tabular}

Page 56 of 78

Uncontrolled When Printed 


\begin{tabular}{|c|c|c|c|c|c|c|c|c|c|c|}
\hline SAMPLE \# & $\begin{array}{c}\text { SAMPLE } \\
\text { DATE }\end{array}$ & MATRIX & $\begin{array}{l}\text { USER TEST } \\
\text { PANEL }\end{array}$ & $\begin{array}{c}\text { SAMPLE } \\
\text { PURPOSE }\end{array}$ & CAS \# & PARAMETER & RESULT & UNITS & $\mathbf{Q}$ & $\begin{array}{l}\text { DETECT } \\
\text { LIMIT }\end{array}$ \\
\hline 383MH1803.5 & $5 / 23 / 2004$ & soil & UGTAISOPU & REG & $15117-48-3$ & Pu-239 & 0.112 & $\mathrm{pCi} / \mathrm{g}$ & $\mathrm{J}$ & 0.017 \\
\hline 383МH1803.5 & $5 / 23 / 2004$ & soil & EPA6010 & REG & $7440-22-4$ & Silver & 0.1 & $\mathrm{mg} / \mathrm{kg}$ & $\mathrm{B}$ & 1.1 \\
\hline 383MH1803.5 & $5 / 23 / 2004$ & soil & HASL300 & REG & 14913-50-9 & TI-208 & 0.32 & $\mathrm{pCi} / \mathrm{g}$ & $G$ & 0.12 \\
\hline 383MH1811.5 & $5 / 23 / 2004$ & soil & EPA8260 & REG & 67-64-1 & Acetone & 8.9 & $\mu \mathrm{g} / \mathrm{kg}$ & $\mathrm{J}$ & 21 \\
\hline 383MH1811.5 & $5 / 23 / 2004$ & soil & EPA6010 & REG & $7440-38-2$ & Arsenic & 1.6 & $\mathrm{mg} / \mathrm{kg}$ & & 1 \\
\hline 383MH1811.5 & $5 / 23 / 2004$ & soil & EPA6010 & REG & $7440-39-3$ & Barium & 34 & $\mathrm{mg} / \mathrm{kg}$ & & 10 \\
\hline 383MH1811.5 & $5 / 23 / 2004$ & soil & EPA6010 & REG & $7440-41-7$ & Beryllium & 0.38 & $\mathrm{mg} / \mathrm{kg}$ & $\mathrm{B}$ & 0.52 \\
\hline 383MH1811.5 & $5 / 23 / 2004$ & soil & HASL300 & REG & $14733-03-0$ & Bi-214 & 0.53 & $\mathrm{pCi} / \mathrm{g}$ & $\mathrm{J}$ & 0.14 \\
\hline 383MH1811.5 & $5 / 23 / 2004$ & soil & EPA6010 & REG & 7440-43-9 & Cadmium & 0.019 & $\mathrm{mg} / \mathrm{kg}$ & $\mathrm{B}$ & 0.52 \\
\hline 383MH1811.5 & $5 / 23 / 2004$ & soil & EPA6010 & REG & $7440-47-3$ & Chromium & 3.5 & $\mathrm{mg} / \mathrm{kg}$ & & 1 \\
\hline 383MH1811.5 & $5 / 23 / 2004$ & soil & HASL300 & REG & $13966-00-2$ & $\mathrm{~K}-40$ & 3.8 & $\mathrm{pCi} / \mathrm{g}$ & & 0.8 \\
\hline $383 \mathrm{MH} 1811.5$ & $5 / 23 / 2004$ & soil & EPA6010 & REG & 7439-92-1 & Lead & 5.4 & $\mathrm{mg} / \mathrm{kg}$ & & 1.5 \\
\hline 383MH1811.5 & $5 / 23 / 2004$ & soil & EPA7470 & REG & $7439-97-6$ & Mercury & 0.13 & $\mathrm{mg} / \mathrm{kg}$ & & 0.1 \\
\hline $383 \mathrm{MH} 1811.5$ & $5 / 23 / 2004$ & soil & EPA8260 & REG & $75-09-2$ & Methylene Chloride & 0.83 & $\mu \mathrm{g} / \mathrm{kg}$ & $\mathrm{J}, \mathrm{B}$ & 5.2 \\
\hline $383 \mathrm{MH} 1811.5$ & $5 / 23 / 2004$ & soil & HASL300 & REG & $15067-28-4$ & $\mathrm{~Pb}-214$ & 0.6 & $\mathrm{pCi} / \mathrm{g}$ & $\mathrm{J}$ & 0.14 \\
\hline 383MH1811.5 & $5 / 23 / 2004$ & soil & EPA6010 & REG & $7440-22-4$ & Silver & 0.17 & $\mathrm{mg} / \mathrm{kg}$ & $\mathrm{B}$ & 1 \\
\hline 383MH1811.5 & $5 / 23 / 2004$ & soil & HASL300 & REG & $14913-50-9$ & TI-208 & 0.125 & $\mathrm{pCi} / \mathrm{g}$ & & 0.066 \\
\hline 383MH2A08.0 & $6 / 8 / 2004$ & soil & HASL300 & REG & 14331-83-0 & Ac-228 & 2.16 & $\mathrm{pCi} / \mathrm{g}$ & G & 0.81 \\
\hline 383MH2A08.0 & $6 / 8 / 2004$ & soil & EPA6010 & REG & $7440-38-2$ & Arsenic & 3.4 & $\mathrm{mg} / \mathrm{kg}$ & & 1.2 \\
\hline $383 \mathrm{MH} 2 \mathrm{~A} 08.0$ & $6 / 8 / 2004$ & soil & EPA6010 & REG & $7440-39-3$ & Barium & 140 & $\mathrm{mg} / \mathrm{kg}$ & & 12 \\
\hline $383 \mathrm{MH} 2 \mathrm{~A} 08.0$ & $6 / 8 / 2004$ & soil & EPA6010 & REG & $7440-41-7$ & Beryllium & 1.1 & $\mathrm{mg} / \mathrm{kg}$ & & 0.61 \\
\hline $383 \mathrm{MH} 2 \mathrm{~A} 08.0$ & $6 / 8 / 2004$ & soil & HASL300 & REG & $14733-03-0$ & Bi-214 & 1.23 & $\mathrm{pCi} / \mathrm{g}$ & $\mathrm{G}, \mathrm{J}$ & 0.4 \\
\hline $383 \mathrm{MH} 2 \mathrm{~A} 08.0$ & $6 / 8 / 2004$ & soil & EPA8270 & REG & 117-81-7 & Bis(2-Ethylhexyl)Phthalate & 170 & $\mu \mathrm{g} / \mathrm{kg}$ & $\mathrm{J}$ & 410 \\
\hline $383 \mathrm{MH} 2 \mathrm{~A} 08.0$ & $6 / 8 / 2004$ & soil & EPA6010 & REG & $7440-43-9$ & Cadmium & 0.09 & $\mathrm{mg} / \mathrm{kg}$ & $\mathrm{B}$ & 0.61 \\
\hline $383 \mathrm{MH} 2 \mathrm{~A} 08.0$ & $6 / 8 / 2004$ & soil & EPA6010 & REG & $7440-47-3$ & Chromium & 1.2 & $\mathrm{mg} / \mathrm{kg}$ & $\mathrm{B}$ & 1.2 \\
\hline 383MH2A08.0 & $6 / 8 / 2004$ & soil & EPA8015 & REG & 68334-30-5 & Diesel-Range Organics & 3.4 & $\mathrm{mg} / \mathrm{kg}$ & $\mathrm{J}$ & 6.1 \\
\hline $383 \mathrm{MH} 2 \mathrm{~A} 08.0$ & $6 / 8 / 2004$ & soil & HASL300 & REG & $13966-00-2$ & $\mathrm{~K}-40$ & 36.7 & $\mathrm{pCi} / \mathrm{g}$ & G & 2 \\
\hline $383 \mathrm{MH} 2 \mathrm{~A} 08.0$ & $6 / 8 / 2004$ & soil & EPA6010 & REG & 7439-92-1 & Lead & 15 & $\mathrm{mg} / \mathrm{kg}$ & & 0.37 \\
\hline $383 \mathrm{MH} 2 \mathrm{~A} 08.0$ & $6 / 8 / 2004$ & soil & EPA7470 & REG & 7439-97-6 & Mercury & 0.0021 & $\mathrm{mg} / \mathrm{kg}$ & $\mathrm{B}$ & 0.12 \\
\hline $383 \mathrm{MH} 2 \mathrm{~A} 08.0$ & $6 / 8 / 2004$ & soil & HASL300 & REG & 15092-94-1 & $\mathrm{Pb}-212$ & 2.52 & $\mathrm{pCi} / \mathrm{g}$ & $\mathrm{J}$ & 0.27 \\
\hline 383MH2A08.0 & $6 / 8 / 2004$ & soil & HASL300 & REG & $15067-28-4$ & $\mathrm{~Pb}-214$ & 1.36 & $\mathrm{pCi} / \mathrm{g}$ & $\mathrm{G}, \mathrm{J}$ & 0.33 \\
\hline 383MH2A08.0 & $6 / 8 / 2004$ & soil & HASL300 & REG & $14913-50-9$ & TI-208 & 0.66 & $\mathrm{pCi} / \mathrm{g}$ & $G$ & 0.18 \\
\hline 383MH2A37.5 & $6 / 8 / 2004$ & soil & HASL300 & REG & 14331-83-0 & Ac-228 & 1.5 & $\mathrm{pCi} / \mathrm{g}$ & $G$ & 0.66 \\
\hline 383MH2A37.5 & $6 / 8 / 2004$ & soil & HASL300 & REG & $14596-10-2$ & Am-241 & 23.5 & $\mathrm{pCi} / \mathrm{g}$ & $\mathrm{J}$ & 2.3 \\
\hline 383MH2A37.5 & $6 / 8 / 2004$ & soil & EPA6010 & REG & $7440-38-2$ & Arsenic & 3.5 & $\mathrm{mg} / \mathrm{kg}$ & & 1.1 \\
\hline
\end{tabular}

Page 57 of 78

Uncontrolled When Printed 


\begin{tabular}{|c|c|c|c|c|c|c|c|c|c|c|}
\hline SAMPLE \# & $\begin{array}{l}\text { SAMPLE } \\
\text { DATE }\end{array}$ & MATRIX & $\begin{array}{l}\text { USER TEST } \\
\text { PANEL }\end{array}$ & $\begin{array}{c}\text { SAMPLE } \\
\text { PURPOSE }\end{array}$ & CAS \# & PARAMETER & RESULT & UNITS & $\mathbf{Q}$ & $\begin{array}{c}\text { DETECT } \\
\text { LIMIT }\end{array}$ \\
\hline 383MH2A37.5 & $6 / 8 / 2004$ & soil & EPA6010 & REG & $7440-39-3$ & Barium & 80 & $\mathrm{mg} / \mathrm{kg}$ & & 11 \\
\hline 383MH2A37.5 & $6 / 8 / 2004$ & soil & EPA8270 & REG & $56-55-3$ & Benzo(A)Anthracene & 85 & $\mu \mathrm{g} / \mathrm{kg}$ & $\mathrm{J}$ & 370 \\
\hline 383MH2A37.5 & $6 / 8 / 2004$ & soil & EPA8270 & REG & $50-32-8$ & Benzo(A)Pyrene & 60 & $\mu \mathrm{g} / \mathrm{kg}$ & $\mathrm{J}$ & 370 \\
\hline 383MH2A37.5 & $6 / 8 / 2004$ & soil & EPA8270 & REG & $205-99-2$ & Benzo(B)Fluoranthene & 140 & $\mu \mathrm{g} / \mathrm{kg}$ & $\mathrm{J}$ & 370 \\
\hline 383MH2A37.5 & $6 / 8 / 2004$ & soil & EPA8270 & REG & 207-08-9 & Benzo(K)Fluoranthene & 34 & $\mu \mathrm{g} / \mathrm{kg}$ & $\mathrm{J}$ & 370 \\
\hline 383MH2A37.5 & $6 / 8 / 2004$ & soil & EPA6010 & REG & $7440-41-7$ & Beryllium & 0.88 & $\mathrm{mg} / \mathrm{kg}$ & & 0.56 \\
\hline 383MH2A37.5 & $6 / 8 / 2004$ & soil & EPA6010 & REG & $7440-43-9$ & Cadmium & 0.26 & $\mathrm{mg} / \mathrm{kg}$ & $B$ & 0.56 \\
\hline 383MH2A37.5 & $6 / 8 / 2004$ & soil & EPA6010 & REG & $7440-47-3$ & Chromium & 3.5 & $\mathrm{mg} / \mathrm{kg}$ & & 1.1 \\
\hline 383MH2A37.5 & $6 / 8 / 2004$ & soil & EPA8270 & REG & 218-01-9 & Chrysene & 130 & $\mu \mathrm{g} / \mathrm{kg}$ & $\mathrm{J}$ & 370 \\
\hline 383MH2A37.5 & $6 / 8 / 2004$ & soil & HASL300 & REG & $10045-97-3$ & Cs-137 & 218 & $\mathrm{pCi} / \mathrm{g}$ & G & 0 \\
\hline 383MH2A37.5 & $6 / 8 / 2004$ & soil & EPA8270 & REG & 206-44-0 & Fluoranthene & 91 & $\mu \mathrm{g} / \mathrm{kg}$ & $\mathrm{J}$ & 370 \\
\hline 383MH2A37.5 & $6 / 8 / 2004$ & soil & EPA8270 & REG & $193-39-5$ & Indeno(1,2,3-Cd)Pyrene & 27 & $\mu \mathrm{g} / \mathrm{kg}$ & $\mathrm{J}$ & 370 \\
\hline 383MH2A37.5 & $6 / 8 / 2004$ & soil & HASL300 & REG & 13966-00-2 & $\mathrm{K}-40$ & 23.1 & $\mathrm{pCi} / \mathrm{g}$ & $\mathrm{G}$ & 1.3 \\
\hline 383MH2A37.5 & $6 / 8 / 2004$ & soil & EPA6010 & REG & 7439-92-1 & Lead & 25 & $\mathrm{mg} / \mathrm{kg}$ & & 1.7 \\
\hline 383MH2A37.5 & $6 / 8 / 2004$ & soil & EPA7470 & REG & 7439-97-6 & Mercury & 0.1 & $\mathrm{mg} / \mathrm{kg}$ & $B$ & 0.11 \\
\hline 383MH2A37.5 & $6 / 8 / 2004$ & soil & HASL300 & REG & 15092-94-1 & $\mathrm{Pb}-212$ & 1.78 & $\mathrm{pCi} / \mathrm{g}$ & $\mathrm{J}$ & 0.68 \\
\hline 383MH2A37.5 & $6 / 8 / 2004$ & soil & EPA8270 & REG & 85-01-8 & Phenanthrene & 34 & $\mu \mathrm{g} / \mathrm{kg}$ & $\mathrm{J}$ & 370 \\
\hline 383MH2A37.5 & 6/8/2004 & soil & UGTAISOPU & REG & 13981-16-3 & Pu-238 & 3.62 & $\mathrm{pCi} / \mathrm{g}$ & Y1 & 0.01 \\
\hline 383MH2A37.5 & $6 / 8 / 2004$ & soil & UGTAISOPU & REG & 15117-48-3 & Pu-239 & 80 & $\mathrm{pCi} / \mathrm{g}$ & $\mathrm{J}$ & 0 \\
\hline 383MH2A37.5 & $6 / 8 / 2004$ & soil & EPA8270 & REG & $129-00-0$ & Pyrene & 93 & $\mu \mathrm{g} / \mathrm{kg}$ & $\mathrm{J}$ & 370 \\
\hline 383MH2A37.5 & $6 / 8 / 2004$ & soil & EPA6010 & REG & $7440-22-4$ & Silver & 1.1 & $\mathrm{mg} / \mathrm{kg}$ & $B$ & 1.1 \\
\hline 383MH2A37.5 & $6 / 8 / 2004$ & soil & SR7500 & REG & 10098-97-2 & Sr-90 & 10.9 & $\mathrm{pCi} / \mathrm{g}$ & & 0.2 \\
\hline 383MH2A45.0 & $6 / 8 / 2004$ & soil & EPA8260 & REG & 67-64-1 & Acetone & 15 & $\mu \mathrm{g} / \mathrm{kg}$ & $\mathrm{J}$ & 21 \\
\hline 383MH2A45.0 & $6 / 8 / 2004$ & soil & HASL300 & REG & 14596-10-2 & Am-241 & 1.1 & $\mathrm{pCi} / \mathrm{g}$ & & 0.25 \\
\hline 383MH2A45.0 & $6 / 8 / 2004$ & soil & EPA6010 & REG & 7440-38-2 & Arsenic & 1.4 & $\mathrm{mg} / \mathrm{kg}$ & & 1 \\
\hline 383MH2A45.0 & $6 / 8 / 2004$ & soil & EPA6010 & REG & $7440-39-3$ & Barium & 40 & $\mathrm{mg} / \mathrm{kg}$ & & 10 \\
\hline 383MH2A45.0 & $6 / 8 / 2004$ & soil & EPA6010 & REG & $7440-41-7$ & Beryllium & 0.53 & $\mathrm{mg} / \mathrm{kg}$ & & 0.52 \\
\hline 383MH2A45.0 & $6 / 8 / 2004$ & soil & HASL300 & REG & $14733-03-0$ & Bi-214 & 0.62 & $\mathrm{pCi} / \mathrm{g}$ & $\mathrm{J}$ & 0.25 \\
\hline 383MH2A45.0 & $6 / 8 / 2004$ & soil & EPA6010 & REG & $7440-43-9$ & Cadmium & 0.16 & $\mathrm{mg} / \mathrm{kg}$ & $\mathrm{B}$ & 0.52 \\
\hline 383MH2A45.0 & $6 / 8 / 2004$ & soil & EPA6010 & REG & $7440-47-3$ & Chromium & 4.3 & $\mathrm{mg} / \mathrm{kg}$ & & 1 \\
\hline 383MH2A45.0 & $6 / 8 / 2004$ & soil & HASL300 & REG & $10045-97-3$ & Cs-137 & 9.8 & $\mathrm{pCi} / \mathrm{g}$ & & 0.1 \\
\hline 383MH2A45.0 & $6 / 8 / 2004$ & soil & HASL300 & REG & $13966-00-2$ & $\mathrm{~K}-40$ & 10.8 & $\mathrm{pCi} / \mathrm{g}$ & & 0.9 \\
\hline 383MH2A45.0 & $6 / 8 / 2004$ & soil & EPA6010 & REG & 7439-92-1 & Lead & 9.5 & $\mathrm{mg} / \mathrm{kg}$ & & 1.6 \\
\hline 383MH2A45.0 & $6 / 8 / 2004$ & soil & EPA7470 & REG & 7439-97-6 & Mercury & 0.043 & $\mathrm{mg} / \mathrm{kg}$ & $B$ & 0.1 \\
\hline 383MH2A45.0 & $6 / 8 / 2004$ & soil & HASL300 & REG & 15092-94-1 & $\mathrm{Pb}-212$ & 0.72 & $\mathrm{pCi} / \mathrm{g}$ & $\mathrm{J}$ & 0.21 \\
\hline
\end{tabular}

Page 58 of 78

Uncontrolled When Printed 


\begin{tabular}{|c|c|c|c|c|c|c|c|c|c|c|}
\hline SAMPLE \# & $\begin{array}{l}\text { SAMPLE } \\
\text { DATE }\end{array}$ & MATRIX & $\begin{array}{l}\text { USER TEST } \\
\text { PANEL }\end{array}$ & $\begin{array}{c}\text { SAMPLE } \\
\text { PURPOSE }\end{array}$ & CAS \# & PARAMETER & RESULT & UNITS & $\mathbf{Q}$ & $\begin{array}{c}\text { DETECT } \\
\text { LIMIT }\end{array}$ \\
\hline 383MH2A45.0 & $6 / 8 / 2004$ & soil & HASL300 & REG & $15067-28-4$ & $\mathrm{~Pb}-214$ & 0.45 & $\mathrm{pCi} / \mathrm{g}$ & $\mathrm{J}$ & 0.24 \\
\hline 383MH2A45.0 & $6 / 8 / 2004$ & soil & UGTAISOPU & REG & 13981-16-3 & Pu-238 & 0.213 & $\mathrm{pCi} / \mathrm{g}$ & & 0.025 \\
\hline 383MH2A45.0 & $6 / 8 / 2004$ & soil & UGTAISOPU & REG & $15117-48-3$ & Pu-239 & 4.55 & $\mathrm{pCi} / \mathrm{g}$ & $\mathrm{J}$ & 0.02 \\
\hline 383MH2A45.0 & $6 / 8 / 2004$ & soil & EPA6010 & REG & $7440-22-4$ & Silver & 0.45 & $\mathrm{mg} / \mathrm{kg}$ & B & 1 \\
\hline 383MH2A45.0 & $6 / 8 / 2004$ & soil & HASL300 & REG & 14913-50-9 & TI-208 & 0.213 & $\mathrm{pCi} / \mathrm{g}$ & & 0.116 \\
\hline $3830 B 0100.5$ & $6 / 1 / 2004$ & soil & EPA8260 & REG & $78-93-3$ & 2-Butanone & 17 & $\mu \mathrm{g} / \mathrm{kg}$ & $\mathrm{J}$ & 21 \\
\hline $3830 B 0100.5$ & $6 / 1 / 2004$ & soil & HASL300 & REG & 14331-83-0 & Ac-228 & 2.21 & $\mathrm{pCi} / \mathrm{g}$ & G & 0.61 \\
\hline $3830 B 0100.5$ & $6 / 1 / 2004$ & soil & EPA8260 & REG & 67-64-1 & Acetone & 72 & $\mu \mathrm{g} / \mathrm{kg}$ & & 21 \\
\hline 383 OB0100.5 & $6 / 1 / 2004$ & soil & EPA6010 & REG & $7440-38-2$ & Arsenic & 2.9 & $\mathrm{mg} / \mathrm{kg}$ & & 1 \\
\hline $3830 B 0100.5$ & $6 / 1 / 2004$ & soil & EPA6010 & REG & $7440-39-3$ & Barium & 180 & $\mathrm{mg} / \mathrm{kg}$ & & 10 \\
\hline 383 OB0100.5 & $6 / 1 / 2004$ & soil & EPA8270 & REG & $56-55-3$ & Benzo(A)Anthracene & 260 & $\mu \mathrm{g} / \mathrm{kg}$ & $\mathrm{J}$ & 1700 \\
\hline 383 OB0100.5 & $6 / 1 / 2004$ & soil & EPA6010 & REG & $7440-41-7$ & Beryllium & 0.64 & $\mathrm{mg} / \mathrm{kg}$ & & 0.51 \\
\hline 383 OB0100.5 & $6 / 1 / 2004$ & soil & HASL300 & REG & 14733-03-0 & Bi-214 & 0.58 & $\mathrm{pCi} / \mathrm{g}$ & $\mathrm{G}, \mathrm{J}$ & 0.29 \\
\hline $3830 B 0100.5$ & $6 / 1 / 2004$ & soil & EPA6010 & REG & $7440-43-9$ & Cadmium & 1.4 & $\mathrm{mg} / \mathrm{kg}$ & & 0.51 \\
\hline 383 OB0100.5 & $6 / 1 / 2004$ & soil & EPA6010 & REG & $7440-47-3$ & Chromium & 6.2 & $\mathrm{mg} / \mathrm{kg}$ & & 1 \\
\hline $3830 B 0100.5$ & $6 / 1 / 2004$ & soil & EPA8270 & REG & 218-01-9 & Chrysene & 590 & $\mu \mathrm{g} / \mathrm{kg}$ & $\mathrm{J}$ & 1700 \\
\hline $3830 B 0100.5$ & $6 / 1 / 2004$ & soil & HASL300 & REG & $10045-97-3$ & Cs-137 & 1.34 & $\mathrm{pCi} / \mathrm{g}$ & G & 0.18 \\
\hline $3830 B 0100.5$ & $6 / 1 / 2004$ & soil & EPA8015 & REG & $68334-30-5$ & Diesel-Range Organics & 3400 & $\mathrm{mg} / \mathrm{kg}$ & $\mathrm{M}, \mathrm{H}$ & 26 \\
\hline $3830 B 0100.5$ & $6 / 1 / 2004$ & soil & EPA8270 & REG & $206-44-0$ & Fluoranthene & 170 & $\mu \mathrm{g} / \mathrm{kg}$ & $\mathrm{J}$ & 1700 \\
\hline $3830 B 0100.5$ & $6 / 1 / 2004$ & soil & HASL300 & REG & $13966-00-2$ & $\mathrm{~K}-40$ & 21.7 & $\mathrm{pCi} / \mathrm{g}$ & G & 1.6 \\
\hline 383 OB0100.5 & $6 / 1 / 2004$ & soil & EPA6010 & REG & 7439-92-1 & Lead & 30 & $\mathrm{mg} / \mathrm{kg}$ & & 0.31 \\
\hline $3830 B 0100.5$ & $6 / 1 / 2004$ & soil & HASL300 & REG & 15092-94-1 & $\mathrm{Pb}-212$ & 2.25 & $\mathrm{pCi} / \mathrm{g}$ & $\mathrm{J}$ & 0.34 \\
\hline $3830 B 0100.5$ & $6 / 1 / 2004$ & soil & HASL300 & REG & $15067-28-4$ & $\mathrm{~Pb}-214$ & 0.86 & $\mathrm{pCi} / \mathrm{g}$ & $\mathrm{G}, \mathrm{J}$ & 0.31 \\
\hline 383 OB0100.5 & $6 / 1 / 2004$ & soil & EPA8270 & REG & 85-01-8 & Phenanthrene & 220 & $\mu \mathrm{g} / \mathrm{kg}$ & $\mathrm{J}$ & 1700 \\
\hline $3830 B 0100.5$ & $6 / 1 / 2004$ & soil & UGTAISOPU & REG & $15117-48-3$ & Pu-239 & 0.213 & $\mathrm{pCi} / \mathrm{g}$ & $\mathrm{J}$ & 0.017 \\
\hline $3830 B 0100.5$ & $6 / 1 / 2004$ & soil & EPA8270 & REG & $129-00-0$ & Pyrene & 2100 & $\mu \mathrm{g} / \mathrm{kg}$ & J & 1700 \\
\hline $3830 B 0100.5$ & $6 / 1 / 2004$ & soil & HASL300 & REG & $14913-50-9$ & TI-208 & 0.68 & $\mathrm{pCi} / \mathrm{g}$ & $\mathrm{G}$ & 0.16 \\
\hline 383OB0100.5RR1 & $6 / 1 / 2004$ & soil & EPA8270 & RE & $56-55-3$ & Benzo(A)Anthracene & 340 & $\mu \mathrm{g} / \mathrm{kg}$ & $\mathrm{J}$ & 3400 \\
\hline 3830B0100.5RR1 & $6 / 1 / 2004$ & soil & EPA8270 & RE & 218-01-9 & Chrysene & 570 & $\mu \mathrm{g} / \mathrm{kg}$ & $\mathrm{J}$ & 3400 \\
\hline 3830B0100.5RR1 & $6 / 1 / 2004$ & soil & EPA8270 & RE & $206-44-0$ & Fluoranthene & 220 & $\mu \mathrm{g} / \mathrm{kg}$ & $\mathrm{J}$ & 3400 \\
\hline 3830B0100.5RR1 & $6 / 1 / 2004$ & soil & EPA8270 & RE & $129-00-0$ & Pyrene & 1400 & $\mu \mathrm{g} / \mathrm{kg}$ & $\mathrm{J}$ & 3400 \\
\hline $3830 B 0200.5$ & $6 / 1 / 2004$ & soil & EPA8260 & REG & 78-93-3 & 2-Butanone & 11 & $\mu \mathrm{g} / \mathrm{kg}$ & $\mathrm{J}$ & 21 \\
\hline $3830 B 0200.5$ & $6 / 1 / 2004$ & soil & HASL300 & REG & 14331-83-0 & Ac-228 & 1.99 & $\mathrm{pCi} / \mathrm{g}$ & G & 0.58 \\
\hline $3830 B 0200.5$ & $6 / 1 / 2004$ & soil & EPA8260 & REG & $67-64-1$ & Acetone & 61 & $\mu \mathrm{g} / \mathrm{kg}$ & & 21 \\
\hline $3830 B 0200.5$ & $6 / 1 / 2004$ & soil & EPA8270 & REG & $120-12-7$ & Anthracene & 86 & $\mu \mathrm{g} / \mathrm{kg}$ & $\mathrm{J}$ & 1700 \\
\hline
\end{tabular}

Page 59 of 78

Uncontrolled When Printed 


\begin{tabular}{|c|c|c|c|c|c|c|c|c|c|c|}
\hline SAMPLE \# & $\begin{array}{l}\text { SAMPLE } \\
\text { DATE }\end{array}$ & MATRIX & $\begin{array}{l}\text { USER TEST } \\
\text { PANEL }\end{array}$ & $\begin{array}{c}\text { SAMPLE } \\
\text { PURPOSE }\end{array}$ & CAS \# & PARAMETER & RESULT & UNITS & $\mathbf{Q}$ & $\begin{array}{c}\text { DETECT } \\
\text { LIMIT }\end{array}$ \\
\hline $3830 B 0200.5$ & $6 / 1 / 2004$ & soil & EPA6010 & REG & $7440-38-2$ & Arsenic & 4.9 & $\mathrm{mg} / \mathrm{kg}$ & & 1 \\
\hline $3830 B 0200.5$ & $6 / 1 / 2004$ & soil & EPA6010 & REG & $7440-39-3$ & Barium & 260 & $\mathrm{mg} / \mathrm{kg}$ & & 10 \\
\hline $3830 B 0200.5$ & $6 / 1 / 2004$ & soil & EPA8270 & REG & $56-55-3$ & Benzo(A)Anthracene & 1400 & $\mu \mathrm{g} / \mathrm{kg}$ & $\mathrm{J}$ & 1700 \\
\hline 3830 B0200.5 & $6 / 1 / 2004$ & soil & EPA6010 & REG & $7440-41-7$ & Beryllium & 0.77 & $\mathrm{mg} / \mathrm{kg}$ & & 0.51 \\
\hline $3830 B 0200.5$ & $6 / 1 / 2004$ & soil & HASL300 & REG & $14733-03-0$ & Bi-214 & 0.79 & $\mathrm{pCi} / \mathrm{g}$ & $\mathrm{G}, \mathrm{J}$ & 0.33 \\
\hline $3830 B 0200.5$ & $6 / 1 / 2004$ & soil & EPA6010 & REG & $7440-43-9$ & Cadmium & 1.4 & $\mathrm{mg} / \mathrm{kg}$ & & 0.51 \\
\hline $3830 B 0200.5$ & $6 / 1 / 2004$ & soil & EPA8270 & REG & 86-74-8 & Carbazole & 160 & $\mu \mathrm{g} / \mathrm{kg}$ & $\mathrm{J}$ & 1700 \\
\hline 383OB0200.5 & $6 / 1 / 2004$ & soil & EPA6010 & REG & $7440-47-3$ & Chromium & 6.7 & $\mathrm{mg} / \mathrm{kg}$ & & 1 \\
\hline $3830 B 0200.5$ & $6 / 1 / 2004$ & soil & EPA8270 & REG & 218-01-9 & Chrysene & 2500 & $\mu \mathrm{g} / \mathrm{kg}$ & $\mathrm{J}$ & 1700 \\
\hline $3830 B 0200.5$ & $6 / 1 / 2004$ & soil & HASL300 & REG & $10045-97-3$ & Cs-137 & 4.1 & $\mathrm{pCi} / \mathrm{g}$ & G & 0.17 \\
\hline $3830 B 0200.5$ & $6 / 1 / 2004$ & soil & EPA8015 & REG & $68334-30-5$ & Diesel-Range Organics & 2500 & $\mathrm{mg} / \mathrm{kg}$ & $\mathrm{M}, \mathrm{H}$ & 26 \\
\hline 383OB0200.5 & $6 / 1 / 2004$ & soil & EPA8270 & REG & $206-44-0$ & Fluoranthene & 1000 & $\mu \mathrm{g} / \mathrm{kg}$ & $\mathrm{J}$ & 1700 \\
\hline $3830 B 0200.5$ & $6 / 1 / 2004$ & soil & HASL300 & REG & $13966-00-2$ & $\mathrm{~K}-40$ & 24.2 & $\mathrm{pCi} / \mathrm{g}$ & G & 1.9 \\
\hline $3830 B 0200.5$ & $6 / 1 / 2004$ & soil & EPA6010 & REG & 7439-92-1 & Lead & 30 & $\mathrm{mg} / \mathrm{kg}$ & & 0.31 \\
\hline $3830 B 0200.5$ & $6 / 1 / 2004$ & soil & HASL300 & REG & 15092-94-1 & $\mathrm{Pb}-212$ & 2.13 & $\mathrm{pCi} / \mathrm{g}$ & $\mathrm{J}$ & 0.37 \\
\hline $3830 B 0200.5$ & $6 / 1 / 2004$ & soil & HASL300 & REG & $15067-28-4$ & $\mathrm{~Pb}-214$ & 0.88 & $\mathrm{pCi} / \mathrm{g}$ & $\mathrm{G}, \mathrm{J}$ & 0.39 \\
\hline $3830 B 0200.5$ & $6 / 1 / 2004$ & soil & EPA8270 & REG & 85-01-8 & Phenanthrene & 1000 & $\mu \mathrm{g} / \mathrm{kg}$ & $\mathrm{J}$ & 1700 \\
\hline $3830 B 0200.5$ & $6 / 1 / 2004$ & soil & EPA8270 & REG & $129-00-0$ & Pyrene & 25000 & $\mu \mathrm{g} / \mathrm{kg}$ & $\mathrm{J}$ & 1700 \\
\hline 383OB0200.5 & $6 / 1 / 2004$ & soil & HASL300 & REG & $14913-50-9$ & TI-208 & 0.88 & $\mathrm{pCi} / \mathrm{g}$ & G & 0.16 \\
\hline 383OB0200.5RR1 & $6 / 1 / 2004$ & soil & EPA8270 & $\mathrm{RE}$ & $56-55-3$ & Benzo(A)Anthracene & 1400 & $\mu \mathrm{g} / \mathrm{kg}$ & $\mathrm{J}$ & 3400 \\
\hline 383OB0200.5RR1 & $6 / 1 / 2004$ & soil & EPA8270 & $\mathrm{RE}$ & $205-99-2$ & Benzo(B)Fluoranthene & 2800 & $\mu \mathrm{g} / \mathrm{kg}$ & $\mathrm{J}$ & 3400 \\
\hline 3830B0200.5RR1 & $6 / 1 / 2004$ & soil & EPA8270 & $\mathrm{RE}$ & 207-08-9 & Benzo(K)Fluoranthene & 1800 & $\mu \mathrm{g} / \mathrm{kg}$ & $\mathrm{J}$ & 3400 \\
\hline 383OB0200.5RR1 & $6 / 1 / 2004$ & soil & EPA8270 & $\mathrm{RE}$ & 218-01-9 & Chrysene & 2400 & $\mu \mathrm{g} / \mathrm{kg}$ & $\mathrm{J}$ & 3400 \\
\hline 383OB0200.5RR1 & $6 / 1 / 2004$ & soil & EPA8270 & $\mathrm{RE}$ & $206-44-0$ & Fluoranthene & 1900 & $\mu \mathrm{g} / \mathrm{kg}$ & $\mathrm{J}$ & 3400 \\
\hline 383OB0200.5RR1 & $6 / 1 / 2004$ & soil & EPA8270 & $\mathrm{RE}$ & 85-01-8 & Phenanthrene & 1100 & $\mu \mathrm{g} / \mathrm{kg}$ & $\mathrm{J}$ & 3400 \\
\hline 3830B0200.5RR1 & $6 / 1 / 2004$ & soil & EPA8270 & $\mathrm{RE}$ & $129-00-0$ & Pyrene & 6400 & $\mu \mathrm{g} / \mathrm{kg}$ & $\mathrm{J}$ & 3400 \\
\hline $3830 B 0300.5$ & $6 / 1 / 2004$ & soil & HASL300 & REG & 14331-83-0 & Ac-228 & 1.96 & $\mathrm{pCi} / \mathrm{g}$ & G & 0.57 \\
\hline $3830 B 0300.5$ & $6 / 1 / 2004$ & soil & EPA8260 & REG & 67-64-1 & Acetone & 7.2 & $\mu \mathrm{g} / \mathrm{kg}$ & $\mathrm{J}, \mathrm{B}$ & 21 \\
\hline $3830 B 0300.5$ & $6 / 1 / 2004$ & soil & EPA6010 & REG & $7440-38-2$ & Arsenic & 4.1 & $\mathrm{mg} / \mathrm{kg}$ & & 1.1 \\
\hline $3830 B 0300.5$ & $6 / 1 / 2004$ & soil & EPA6010 & REG & $7440-39-3$ & Barium & 180 & $\mathrm{mg} / \mathrm{kg}$ & & 11 \\
\hline $3830 B 0300.5$ & $6 / 1 / 2004$ & soil & EPA8270 & REG & $56-55-3$ & Benzo(A)Anthracene & 120 & $\mu \mathrm{g} / \mathrm{kg}$ & $\mathrm{J}$ & 350 \\
\hline $3830 B 0300.5$ & $6 / 1 / 2004$ & soil & EPA8270 & REG & $50-32-8$ & Benzo(A)Pyrene & 230 & $\mu \mathrm{g} / \mathrm{kg}$ & $\mathrm{J}$ & 350 \\
\hline $3830 B 0300.5$ & $6 / 1 / 2004$ & soil & EPA8270 & REG & $205-99-2$ & Benzo(B)Fluoranthene & 510 & $\mu \mathrm{g} / \mathrm{kg}$ & $\mathrm{J}$ & 350 \\
\hline $3830 B 0300.5$ & $6 / 1 / 2004$ & soil & EPA8270 & REG & $191-24-2$ & Benzo(G,H,I)Perylene & 120 & $\mu \mathrm{g} / \mathrm{kg}$ & $\mathrm{J}$ & 350 \\
\hline $3830 B 0300.5$ & $6 / 1 / 2004$ & soil & EPA8270 & REG & 207-08-9 & Benzo(K)Fluoranthene & 220 & $\mu \mathrm{g} / \mathrm{kg}$ & $\mathrm{J}$ & 350 \\
\hline
\end{tabular}

Page 60 of 78

Uncontrolled When Printed 


\begin{tabular}{|c|c|c|c|c|c|c|c|c|c|c|}
\hline SAMPLE \# & $\begin{array}{l}\text { SAMPLE } \\
\text { DATE }\end{array}$ & MATRIX & $\begin{array}{l}\text { USER TEST } \\
\text { PANEL }\end{array}$ & $\begin{array}{c}\text { SAMPLE } \\
\text { PURPOSE }\end{array}$ & CAS \# & PARAMETER & RESULT & UNITS & $\mathbf{Q}$ & $\begin{array}{l}\text { DETECT } \\
\text { LIMIT }\end{array}$ \\
\hline $3830 B 0300.5$ & $6 / 1 / 2004$ & soil & EPA6010 & REG & $7440-41-7$ & Beryllium & 1.1 & $\mathrm{mg} / \mathrm{kg}$ & & 0.53 \\
\hline $3830 B 0300.5$ & $6 / 1 / 2004$ & soil & HASL300 & REG & $14733-03-0$ & Bi-214 & 1.01 & $\mathrm{pCi} / \mathrm{g}$ & $\mathrm{G}, \mathrm{J}$ & 0.29 \\
\hline $3830 B 0300.5$ & $6 / 1 / 2004$ & soil & EPA6010 & REG & $7440-47-3$ & Chromium & 3.9 & $\mathrm{mg} / \mathrm{kg}$ & & 1.1 \\
\hline $3830 B 0300.5$ & $6 / 1 / 2004$ & soil & EPA8270 & REG & 218-01-9 & Chrysene & 340 & $\mu \mathrm{g} / \mathrm{kg}$ & $\mathrm{J}$ & 350 \\
\hline $3830 B 0300.5$ & $6 / 1 / 2004$ & soil & HASL300 & REG & $10045-97-3$ & Cs-137 & 6.13 & $\mathrm{pCi} / \mathrm{g}$ & G & 0.13 \\
\hline $3830 B 0300.5$ & $6 / 1 / 2004$ & soil & EPA8015 & REG & $68334-30-5$ & Diesel-Range Organics & 330 & $\mathrm{mg} / \mathrm{kg}$ & $\mathrm{H}, \mathrm{Z}$ & 5.3 \\
\hline $3830 B 0300.5$ & $6 / 1 / 2004$ & soil & EPA8270 & REG & $206-44-0$ & Fluoranthene & 210 & $\mu g / \mathrm{kg}$ & $\mathrm{J}$ & 350 \\
\hline $3830 B 0300.5$ & $6 / 1 / 2004$ & soil & HASL300 & REG & 13966-00-2 & $\mathrm{K}-40$ & 22 & $\mathrm{pCi} / \mathrm{g}$ & $\mathrm{G}$ & 1.5 \\
\hline $3830 B 0300.5$ & $6 / 1 / 2004$ & soil & EPA6010 & REG & 7439-92-1 & Lead & 20 & $\mathrm{mg} / \mathrm{kg}$ & & 0.32 \\
\hline $3830 B 0300.5$ & $6 / 1 / 2004$ & soil & EPA8270 & REG & $91-20-3$ & Naphthalene & 54 & $\mu \mathrm{g} / \mathrm{kg}$ & $\mathrm{J}$ & 350 \\
\hline $3830 B 0300.5$ & $6 / 1 / 2004$ & soil & HASL300 & REG & 15092-94-1 & $\mathrm{Pb}-212$ & 2.13 & $\mathrm{pCi} / \mathrm{g}$ & $\mathrm{J}$ & 0.24 \\
\hline $3830 B 0300.5$ & $6 / 1 / 2004$ & soil & HASL300 & REG & $15067-28-4$ & $\mathrm{~Pb}-214$ & 0.97 & $\mathrm{pCi} / \mathrm{g}$ & $\mathrm{G}, \mathrm{J}$ & 0.28 \\
\hline $3830 B 0300.5$ & $6 / 1 / 2004$ & soil & EPA8270 & REG & 85-01-8 & Phenanthrene & 65 & $\mu g / \mathrm{kg}$ & $\mathrm{J}$ & 350 \\
\hline $3830 B 0300.5$ & $6 / 1 / 2004$ & soil & UGTAISOPU & REG & $15117-48-3$ & Pu-239 & 0.59 & $\mathrm{pCi} / \mathrm{g}$ & $\mathrm{J}$ & 0.02 \\
\hline $3830 B 0300.5$ & $6 / 1 / 2004$ & soil & EPA8270 & REG & $129-00-0$ & Pyrene & 620 & $\mu \mathrm{g} / \mathrm{kg}$ & $\mathrm{J}$ & 350 \\
\hline $3830 B 0300.5$ & $6 / 1 / 2004$ & soil & HASL300 & REG & $14913-50-9$ & TI-208 & 0.69 & $\mathrm{pCi} / \mathrm{g}$ & G & 0.14 \\
\hline $3830 B 0400.5$ & $6 / 1 / 2004$ & soil & HASL300 & REG & 14331-83-0 & Ac-228 & 2 & $\mathrm{pCi} / \mathrm{g}$ & $G$ & 0.43 \\
\hline $3830 B 0400.5$ & $6 / 1 / 2004$ & soil & EPA6010 & REG & $7440-38-2$ & Arsenic & 5.1 & $\mathrm{mg} / \mathrm{kg}$ & & 1 \\
\hline $3830 B 0400.5$ & $6 / 1 / 2004$ & soil & EPA6010 & REG & $7440-39-3$ & Barium & 300 & $\mathrm{mg} / \mathrm{kg}$ & & 10 \\
\hline $3830 B 0400.5$ & $6 / 1 / 2004$ & soil & EPA8270 & REG & $56-55-3$ & Benzo(A)Anthracene & 110 & $\mu \mathrm{g} / \mathrm{kg}$ & $\mathrm{J}$ & 350 \\
\hline $3830 B 0400.5$ & $6 / 1 / 2004$ & soil & EPA8270 & REG & $50-32-8$ & Benzo(A)Pyrene & 160 & $\mu \mathrm{g} / \mathrm{kg}$ & $\mathrm{J}$ & 350 \\
\hline $3830 B 0400.5$ & $6 / 1 / 2004$ & soil & EPA8270 & REG & 205-99-2 & Benzo(B)Fluoranthene & 310 & $\mu \mathrm{g} / \mathrm{kg}$ & $\mathrm{J}$ & 350 \\
\hline $3830 B 0400.5$ & $6 / 1 / 2004$ & soil & EPA8270 & REG & $191-24-2$ & Benzo(G,H,I)Perylene & 59 & $\mu \mathrm{g} / \mathrm{kg}$ & $\mathrm{J}$ & 350 \\
\hline $3830 B 0400.5$ & $6 / 1 / 2004$ & soil & EPA8270 & REG & 207-08-9 & Benzo(K)Fluoranthene & 140 & $\mu \mathrm{g} / \mathrm{kg}$ & $\mathrm{J}$ & 350 \\
\hline $3830 B 0400.5$ & $6 / 1 / 2004$ & soil & EPA6010 & REG & 7440-41-7 & Beryllium & 0.82 & $\mathrm{mg} / \mathrm{kg}$ & & 0.52 \\
\hline $3830 B 0400.5$ & $6 / 1 / 2004$ & soil & HASL300 & REG & $14733-03-0$ & Bi-214 & 1.04 & $\mathrm{pCi} / \mathrm{g}$ & $\mathrm{G}, \mathrm{J}$ & 0.27 \\
\hline $3830 B 0400.5$ & $6 / 1 / 2004$ & soil & EPA6010 & REG & $7440-47-3$ & Chromium & 5.8 & $\mathrm{mg} / \mathrm{kg}$ & & 1 \\
\hline $3830 B 0400.5$ & $6 / 1 / 2004$ & soil & EPA8270 & REG & 218-01-9 & Chrysene & 230 & $\mu \mathrm{g} / \mathrm{kg}$ & $\mathrm{J}$ & 350 \\
\hline $3830 B 0400.5$ & $6 / 1 / 2004$ & soil & HASL300 & REG & $10045-97-3$ & Cs-137 & 5.97 & $\mathrm{pCi} / \mathrm{g}$ & $\mathrm{G}$ & 0.16 \\
\hline $3830 B 0400.5$ & $6 / 1 / 2004$ & soil & EPA8015 & REG & $68334-30-5$ & Diesel-Range Organics & 63 & $\mathrm{mg} / \mathrm{kg}$ & $\mathrm{M}, \mathrm{H}$ & 5.2 \\
\hline $3830 B 0400.5$ & $6 / 1 / 2004$ & soil & EPA8270 & REG & $206-44-0$ & Fluoranthene & 190 & $\mu \mathrm{g} / \mathrm{kg}$ & $\mathrm{J}$ & 350 \\
\hline $3830 B 0400.5$ & $6 / 1 / 2004$ & soil & EPA8270 & REG & $193-39-5$ & Indeno(1,2,3-Cd)Pyrene & 54 & $\mu \mathrm{g} / \mathrm{kg}$ & $\mathrm{J}$ & 350 \\
\hline $3830 B 0400.5$ & $6 / 1 / 2004$ & soil & HASL300 & REG & $13966-00-2$ & $\mathrm{~K}-40$ & 25 & $\mathrm{pCi} / \mathrm{g}$ & G & 1.5 \\
\hline $3830 B 0400.5$ & $6 / 1 / 2004$ & soil & EPA6010 & REG & $7439-92-1$ & Lead & 30 & $\mathrm{mg} / \mathrm{kg}$ & & 0.31 \\
\hline $3830 B 0400.5$ & $6 / 1 / 2004$ & soil & HASL300 & REG & 15092-94-1 & $\mathrm{Pb}-212$ & 2.48 & $\mathrm{pCi} / \mathrm{g}$ & $\mathrm{J}$ & 0.25 \\
\hline
\end{tabular}

Page 61 of 78

Uncontrolled When Printed 


\begin{tabular}{|c|c|c|c|c|c|c|c|c|c|c|}
\hline SAMPLE \# & $\begin{array}{l}\text { SAMPLE } \\
\text { DATE }\end{array}$ & MATRIX & $\begin{array}{l}\text { USER TEST } \\
\text { PANEL }\end{array}$ & $\begin{array}{c}\text { SAMPLE } \\
\text { PURPOSE }\end{array}$ & CAS \# & PARAMETER & RESULT & UNITS & $\mathbf{Q}$ & $\begin{array}{l}\text { DETECT } \\
\text { LIMIT }\end{array}$ \\
\hline $3830 B 0400.5$ & $6 / 1 / 2004$ & soil & HASL300 & REG & $15067-28-4$ & $\mathrm{~Pb}-214$ & 0.91 & $\mathrm{pCi} / \mathrm{g}$ & $\mathrm{G}, \mathrm{J}$ & 0.34 \\
\hline $3830 B 0400.5$ & $6 / 1 / 2004$ & soil & EPA8270 & REG & 85-01-8 & Phenanthrene & 55 & $\mu \mathrm{g} / \mathrm{kg}$ & $\mathrm{J}$ & 350 \\
\hline $3830 B 0400.5$ & $6 / 1 / 2004$ & soil & UGTAISOPU & REG & $15117-48-3$ & Pu-239 & 0.158 & $\mathrm{pCi} / \mathrm{g}$ & $\mathrm{J}$ & 0.01 \\
\hline $3830 B 0400.5$ & $6 / 1 / 2004$ & soil & EPA8270 & REG & $129-00-0$ & Pyrene & 220 & $\mu \mathrm{g} / \mathrm{kg}$ & $\mathrm{J}$ & 350 \\
\hline $3830 B 0400.5$ & $6 / 1 / 2004$ & soil & HASL300 & REG & $14913-50-9$ & TI-208 & 0.59 & $\mathrm{pCi} / \mathrm{g}$ & G & 0.16 \\
\hline $3830 B 0500.5$ & $6 / 1 / 2004$ & soil & HASL300 & REG & 14331-83-0 & Ac-228 & 2.2 & $\mathrm{pCi} / \mathrm{g}$ & $G$ & 0.66 \\
\hline $3830 B 0500.5$ & $6 / 1 / 2004$ & soil & EPA6010 & REG & $7440-38-2$ & Arsenic & 3.8 & $\mathrm{mg} / \mathrm{kg}$ & & 1.1 \\
\hline $3830 B 0500.5$ & $6 / 1 / 2004$ & soil & EPA6010 & REG & $7440-39-3$ & Barium & 140 & $\mathrm{mg} / \mathrm{kg}$ & & 11 \\
\hline $3830 B 0500.5$ & $6 / 1 / 2004$ & soil & EPA8270 & REG & $56-55-3$ & Benzo(A)Anthracene & 200 & $\mu \mathrm{g} / \mathrm{kg}$ & $\mathrm{J}$ & 350 \\
\hline $3830 B 0500.5$ & $6 / 1 / 2004$ & soil & EPA8270 & REG & $50-32-8$ & Benzo(A)Pyrene & 250 & $\mu \mathrm{g} / \mathrm{kg}$ & $\mathrm{J}$ & 350 \\
\hline $3830 B 0500.5$ & $6 / 1 / 2004$ & soil & EPA8270 & REG & $205-99-2$ & Benzo(B)Fluoranthene & 670 & $\mu \mathrm{g} / \mathrm{kg}$ & $\mathrm{J}$ & 350 \\
\hline $3830 B 0500.5$ & $6 / 1 / 2004$ & soil & EPA8270 & REG & $207-08-9$ & Benzo(K)Fluoranthene & 280 & $\mu \mathrm{g} / \mathrm{kg}$ & $\mathrm{J}$ & 350 \\
\hline $3830 B 0500.5$ & $6 / 1 / 2004$ & soil & EPA6010 & REG & $7440-41-7$ & Beryllium & 1.2 & $\mathrm{mg} / \mathrm{kg}$ & & 0.53 \\
\hline $3830 B 0500.5$ & $6 / 1 / 2004$ & soil & HASL300 & REG & $14733-03-0$ & Bi-214 & 1.01 & $\mathrm{pCi} / \mathrm{g}$ & $\mathrm{G}, \mathrm{J}$ & 0.34 \\
\hline $3830 B 0500.5$ & $6 / 1 / 2004$ & soil & EPA6010 & REG & $7440-47-3$ & Chromium & 2.5 & $\mathrm{mg} / \mathrm{kg}$ & & 1.1 \\
\hline $3830 B 0500.5$ & $6 / 1 / 2004$ & soil & EPA8270 & REG & 218-01-9 & Chrysene & 410 & $\mu \mathrm{g} / \mathrm{kg}$ & & 350 \\
\hline $3830 B 0500.5$ & $6 / 1 / 2004$ & soil & HASL300 & REG & $10045-97-3$ & Cs-137 & 5.65 & $\mathrm{pCi} / \mathrm{g}$ & $G$ & 0.24 \\
\hline $3830 B 0500.5$ & $6 / 1 / 2004$ & soil & EPA8270 & REG & $206-44-0$ & Fluoranthene & 400 & $\mu \mathrm{g} / \mathrm{kg}$ & & 350 \\
\hline 383OB0500.5 & $6 / 1 / 2004$ & soil & HASL300 & REG & 13966-00-2 & $\mathrm{K}-40$ & 24.6 & $\mathrm{pCi} / \mathrm{g}$ & G & 1.9 \\
\hline $3830 B 0500.5$ & $6 / 1 / 2004$ & soil & EPA6010 & REG & 7439-92-1 & Lead & 17 & $\mathrm{mg} / \mathrm{kg}$ & & 0.32 \\
\hline $3830 B 0500.5$ & $6 / 1 / 2004$ & soil & EPA8270 & REG & $91-20-3$ & Naphthalene & 15 & $\mu \mathrm{g} / \mathrm{kg}$ & $\mathrm{J}$ & 350 \\
\hline 383OB0500.5 & $6 / 1 / 2004$ & soil & HASL300 & REG & 15092-94-1 & $\mathrm{Pb}-212$ & 3.1 & $\mathrm{pCi} / \mathrm{g}$ & $\mathrm{J}$ & 0.34 \\
\hline $3830 B 0500.5$ & $6 / 1 / 2004$ & soil & HASL300 & REG & $15067-28-4$ & $\mathrm{~Pb}-214$ & 1.04 & $\mathrm{pCi} / \mathrm{g}$ & $\mathrm{G}, \mathrm{J}$ & 0.4 \\
\hline $3830 B 0500.5$ & $6 / 1 / 2004$ & soil & EPA8270 & REG & 85-01-8 & Phenanthrene & 140 & $\mu \mathrm{g} / \mathrm{kg}$ & $\mathrm{J}$ & 350 \\
\hline 383OB0500.5 & $6 / 1 / 2004$ & soil & UGTAISOPU & REG & $15117-48-3$ & Pu-239 & 0.089 & $\mathrm{pCi} / \mathrm{g}$ & $\mathrm{J}$ & 0.025 \\
\hline $3830 B 0500.5$ & $6 / 1 / 2004$ & soil & EPA8270 & REG & $129-00-0$ & Pyrene & 460 & $\mu \mathrm{g} / \mathrm{kg}$ & & 350 \\
\hline $3830 B 0500.5$ & $6 / 1 / 2004$ & soil & HASL300 & REG & $14913-50-9$ & TI-208 & 0.97 & $\mathrm{pCi} / \mathrm{g}$ & G & 0.18 \\
\hline 3830B0500.5X & $6 / 1 / 2004$ & soil & HASL300 & FD & $14331-83-0$ & Ac-228 & 2.41 & $\mathrm{pCi} / \mathrm{g}$ & $\mathrm{G}$ & 0.57 \\
\hline 3830B0500.5X & $6 / 1 / 2004$ & soil & EPA6010 & FD & $7440-38-2$ & Arsenic & 3.5 & $\mathrm{mg} / \mathrm{kg}$ & & 1.1 \\
\hline 3830B0500.5X & $6 / 1 / 2004$ & soil & EPA6010 & FD & $7440-39-3$ & Barium & 130 & $\mathrm{mg} / \mathrm{kg}$ & & 11 \\
\hline 3830B0500.5X & $6 / 1 / 2004$ & soil & EPA8270 & FD & $56-55-3$ & Benzo(A)Anthracene & 200 & $\mu \mathrm{g} / \mathrm{kg}$ & $\mathrm{J}$ & 360 \\
\hline 3830B0500.5X & $6 / 1 / 2004$ & soil & EPA8270 & FD & $50-32-8$ & Benzo(A)Pyrene & 240 & $\mu \mathrm{g} / \mathrm{kg}$ & $\mathrm{J}$ & 360 \\
\hline 3830B0500.5X & $6 / 1 / 2004$ & soil & EPA8270 & FD & $205-99-2$ & Benzo(B)Fluoranthene & 550 & $\mu \mathrm{g} / \mathrm{kg}$ & $\mathrm{J}$ & 360 \\
\hline 3830B0500.5X & $6 / 1 / 2004$ & soil & EPA8270 & FD & $191-24-2$ & Benzo(G,H,I)Perylene & 81 & $\mu \mathrm{g} / \mathrm{kg}$ & $\mathrm{J}$ & 360 \\
\hline 3830B0500.5X & $6 / 1 / 2004$ & soil & EPA8270 & FD & 207-08-9 & Benzo(K)Fluoranthene & 260 & $\mu \mathrm{g} / \mathrm{kg}$ & $\mathrm{J}$ & 360 \\
\hline
\end{tabular}

Page 62 of 78

Uncontrolled When Printed 


\begin{tabular}{|c|c|c|c|c|c|c|c|c|c|c|}
\hline SAMPLE \# & $\begin{array}{l}\text { SAMPLE } \\
\text { DATE }\end{array}$ & MATRIX & $\begin{array}{l}\text { USER TEST } \\
\text { PANEL }\end{array}$ & $\begin{array}{c}\text { SAMPLE } \\
\text { PURPOSE }\end{array}$ & CAS \# & PARAMETER & RESULT & UNITS & $\mathbf{Q}$ & $\begin{array}{c}\text { DETECT } \\
\text { LIMIT }\end{array}$ \\
\hline 383OB0500.5X & $6 / 1 / 2004$ & soil & EPA6010 & FD & $7440-41-7$ & Beryllium & 1 & $\mathrm{mg} / \mathrm{kg}$ & & 0.54 \\
\hline 3830B0500.5X & $6 / 1 / 2004$ & soil & HASL300 & FD & $14733-03-0$ & Bi-214 & 1.07 & $\mathrm{pCi} / \mathrm{g}$ & $\mathrm{G}, \mathrm{J}$ & 0.36 \\
\hline 3830B0500.5X & $6 / 1 / 2004$ & soil & EPA6010 & FD & $7440-47-3$ & Chromium & 2.5 & $\mathrm{mg} / \mathrm{kg}$ & & 1.1 \\
\hline 383OB0500.5X & $6 / 1 / 2004$ & soil & EPA8270 & FD & 218-01-9 & Chrysene & 410 & $\mu \mathrm{g} / \mathrm{kg}$ & & 360 \\
\hline 383OB0500.5X & $6 / 1 / 2004$ & soil & HASL300 & FD & $10045-97-3$ & Cs-137 & 5 & $\mathrm{pCi} / \mathrm{g}$ & $\mathrm{G}$ & 0.19 \\
\hline 383OB0500.5X & $6 / 1 / 2004$ & soil & EPA8270 & FD & $206-44-0$ & Fluoranthene & 400 & $\mu \mathrm{g} / \mathrm{kg}$ & & 360 \\
\hline 383OB0500.5X & $6 / 1 / 2004$ & soil & EPA8270 & FD & $193-39-5$ & Indeno(1,2,3-Cd)Pyrene & 77 & $\mu \mathrm{g} / \mathrm{kg}$ & $\mathrm{J}$ & 360 \\
\hline 3830B0500.5X & $6 / 1 / 2004$ & soil & HASL300 & FD & 13966-00-2 & $\mathrm{K}-40$ & 24.1 & $\mathrm{pCi} / \mathrm{g}$ & G & 1.9 \\
\hline 383OB0500.5X & $6 / 1 / 2004$ & soil & EPA6010 & FD & 7439-92-1 & Lead & 15 & $\mathrm{mg} / \mathrm{kg}$ & & 0.32 \\
\hline 383OB0500.5X & $6 / 1 / 2004$ & soil & HASL300 & FD & 15092-94-1 & $\mathrm{Pb}-212$ & 2.97 & $\mathrm{pCi} / \mathrm{g}$ & J & 0.24 \\
\hline 383OB0500.5X & $6 / 1 / 2004$ & soil & HASL300 & FD & $15067-28-4$ & $\mathrm{~Pb}-214$ & 1.1 & $\mathrm{pCi} / \mathrm{g}$ & $\mathrm{G}, \mathrm{J}$ & 0.34 \\
\hline 3830B0500.5X & $6 / 1 / 2004$ & soil & EPA8270 & FD & 85-01-8 & Phenanthrene & 140 & $\mu \mathrm{g} / \mathrm{kg}$ & $\mathrm{J}$ & 360 \\
\hline 383OB0500.5X & $6 / 1 / 2004$ & soil & UGTAISOPU & FD & $15117-48-3$ & Pu-239 & 0.157 & $\mathrm{pCi} / \mathrm{g}$ & $\mathrm{J}$ & 0.023 \\
\hline 383OB0500.5X & $6 / 1 / 2004$ & soil & EPA8270 & FD & $129-00-0$ & Pyrene & 510 & $\mu \mathrm{g} / \mathrm{kg}$ & & 360 \\
\hline 3830B0500.5X & $6 / 1 / 2004$ & soil & HASL300 & FD & $14913-50-9$ & TI-208 & 0.81 & $\mathrm{pCi} / \mathrm{g}$ & $\mathrm{G}$ & 0.17 \\
\hline $3830 B 0600.5$ & $6 / 1 / 2004$ & soil & HASL300 & REG & $14331-83-0$ & Ac-228 & 1.87 & $\mathrm{pCi} / \mathrm{g}$ & $\mathrm{G}$ & 0.56 \\
\hline $3830 B 0600.5$ & $6 / 1 / 2004$ & soil & EPA6010 & REG & $7440-38-2$ & Arsenic & 5.8 & $\mathrm{mg} / \mathrm{kg}$ & & 1 \\
\hline $3830 B 0600.5$ & $6 / 1 / 2004$ & soil & EPA6010 & REG & $7440-39-3$ & Barium & 190 & $\mathrm{mg} / \mathrm{kg}$ & & 10 \\
\hline $3830 B 0600.5$ & $6 / 1 / 2004$ & soil & EPA8270 & REG & $56-55-3$ & Benzo(A)Anthracene & 45 & $\mu \mathrm{g} / \mathrm{kg}$ & J & 350 \\
\hline $3830 B 0600.5$ & $6 / 1 / 2004$ & soil & EPA8270 & REG & $50-32-8$ & Benzo(A)Pyrene & 64 & $\mu \mathrm{g} / \mathrm{kg}$ & $\mathrm{J}$ & 350 \\
\hline $3830 B 0600.5$ & $6 / 1 / 2004$ & soil & EPA8270 & REG & $205-99-2$ & Benzo(B)Fluoranthene & 140 & $\mu \mathrm{g} / \mathrm{kg}$ & $\mathrm{J}$ & 350 \\
\hline $3830 B 0600.5$ & $6 / 1 / 2004$ & soil & EPA8270 & REG & 207-08-9 & Benzo(K)Fluoranthene & 45 & $\mu \mathrm{g} / \mathrm{kg}$ & $\mathrm{J}$ & 350 \\
\hline $3830 B 0600.5$ & $6 / 1 / 2004$ & soil & EPA6010 & REG & $7440-41-7$ & Beryllium & 0.77 & $\mathrm{mg} / \mathrm{kg}$ & & 0.52 \\
\hline $3830 B 0600.5$ & $6 / 1 / 2004$ & soil & HASL300 & REG & $14733-03-0$ & Bi-214 & 0.98 & $\mathrm{pCi} / \mathrm{g}$ & $\mathrm{G}, \mathrm{J}$ & 0.27 \\
\hline $3830 B 0600.5$ & $6 / 1 / 2004$ & soil & EPA6010 & REG & $7440-47-3$ & Chromium & 8.3 & $\mathrm{mg} / \mathrm{kg}$ & & 1 \\
\hline $3830 B 0600.5$ & $6 / 1 / 2004$ & soil & EPA8270 & REG & 218-01-9 & Chrysene & 110 & $\mu \mathrm{g} / \mathrm{kg}$ & $\mathrm{J}$ & 350 \\
\hline $3830 B 0600.5$ & $6 / 1 / 2004$ & soil & HASL300 & REG & $10045-97-3$ & Cs-137 & 0.53 & $\mathrm{pCi} / \mathrm{g}$ & G & 0.12 \\
\hline $3830 B 0600.5$ & $6 / 1 / 2004$ & soil & EPA8270 & REG & 206-44-0 & Fluoranthene & 93 & $\mu \mathrm{g} / \mathrm{kg}$ & $\mathrm{J}$ & 350 \\
\hline $3830 B 0600.5$ & $6 / 1 / 2004$ & soil & HASL300 & REG & $13966-00-2$ & $\mathrm{~K}-40$ & 22.7 & $\mathrm{pCi} / \mathrm{g}$ & $\mathrm{G}$ & 1.5 \\
\hline $3830 B 0600.5$ & $6 / 1 / 2004$ & soil & EPA6010 & REG & 7439-92-1 & Lead & 12 & $\mathrm{mg} / \mathrm{kg}$ & & 0.31 \\
\hline $3830 B 0600.5$ & $6 / 1 / 2004$ & soil & HASL300 & REG & 15092-94-1 & $\mathrm{Pb}-212$ & 2.12 & $\mathrm{pCi} / \mathrm{g}$ & $\mathrm{J}$ & 0.2 \\
\hline $3830 B 0600.5$ & $6 / 1 / 2004$ & soil & HASL300 & REG & $15067-28-4$ & $\mathrm{~Pb}-214$ & 1.06 & $\mathrm{pCi} / \mathrm{g}$ & $\mathrm{G}, \mathrm{J}$ & 0.24 \\
\hline $3830 B 0600.5$ & $6 / 1 / 2004$ & soil & EPA8270 & REG & $85-01-8$ & Phenanthrene & 32 & $\mu \mathrm{g} / \mathrm{kg}$ & $\mathrm{J}$ & 350 \\
\hline $3830 B 0600.5$ & $6 / 1 / 2004$ & soil & EPA8270 & REG & $129-00-0$ & Pyrene & 75 & $\mu \mathrm{g} / \mathrm{kg}$ & $\mathrm{J}$ & 350 \\
\hline $3830 B 0600.5$ & $6 / 1 / 2004$ & soil & HASL300 & REG & 14913-50-9 & TI-208 & 0.59 & $\mathrm{pCi} / \mathrm{g}$ & $\mathrm{G}$ & 0.13 \\
\hline
\end{tabular}

Page 63 of 78

Uncontrolled When Printed 


\begin{tabular}{|c|c|c|c|c|c|c|c|c|c|c|}
\hline SAMPLE \# & $\begin{array}{l}\text { SAMPLE } \\
\text { DATE }\end{array}$ & MATRIX & $\begin{array}{l}\text { USER TEST } \\
\text { PANEL }\end{array}$ & $\begin{array}{c}\text { SAMPLE } \\
\text { PURPOSE }\end{array}$ & CAS \# & PARAMETER & RESULT & UNITS & $\mathbf{Q}$ & $\begin{array}{l}\text { DETECT } \\
\text { LIMIT }\end{array}$ \\
\hline $3830 B 0700.5$ & $6 / 1 / 2004$ & soil & HASL300 & REG & 14331-83-0 & Ac-228 & 2.14 & $\mathrm{pCi} / \mathrm{g}$ & $\mathrm{G}$ & 0.54 \\
\hline $3830 B 0700.5$ & $6 / 1 / 2004$ & soil & EPA8270 & REG & $83-32-9$ & Acenaphthene & 39 & $\mu \mathrm{g} / \mathrm{kg}$ & $\mathrm{J}$ & 700 \\
\hline $3830 B 0700.5$ & $6 / 1 / 2004$ & soil & EPA8270 & REG & $120-12-7$ & Anthracene & 150 & $\mu \mathrm{g} / \mathrm{kg}$ & $\mathrm{J}$ & 700 \\
\hline $3830 B 0700.5$ & $6 / 1 / 2004$ & soil & EPA6010 & REG & $7440-38-2$ & Arsenic & 6.7 & $\mathrm{mg} / \mathrm{kg}$ & & 1 \\
\hline $3830 B 0700.5$ & $6 / 1 / 2004$ & soil & EPA6010 & REG & $7440-39-3$ & Barium & 250 & $\mathrm{mg} / \mathrm{kg}$ & & 10 \\
\hline $3830 B 0700.5$ & $6 / 1 / 2004$ & soil & EPA8270 & REG & $56-55-3$ & Benzo(A)Anthracene & 4300 & $\mu \mathrm{g} / \mathrm{kg}$ & $\mathrm{J}$ & 700 \\
\hline $3830 B 0700.5$ & $6 / 1 / 2004$ & soil & EPA8270 & REG & $50-32-8$ & Benzo(A)Pyrene & 4300 & $\mu \mathrm{g} / \mathrm{kg}$ & $\mathrm{J}$ & 700 \\
\hline $3830 B 0700.5$ & $6 / 1 / 2004$ & soil & EPA8270 & REG & $205-99-2$ & Benzo(B)Fluoranthene & 8200 & $\mu \mathrm{g} / \mathrm{kg}$ & $\mathrm{J}$ & 700 \\
\hline $3830 B 0700.5$ & $6 / 1 / 2004$ & soil & EPA8270 & REG & $191-24-2$ & Benzo(G,H,I)Perylene & 7000 & $\mu \mathrm{g} / \mathrm{kg}$ & $\mathrm{J}$ & 700 \\
\hline $3830 B 0700.5$ & $6 / 1 / 2004$ & soil & EPA8270 & REG & $207-08-9$ & Benzo(K)Fluoranthene & 3200 & $\mu \mathrm{g} / \mathrm{kg}$ & $\mathrm{J}$ & 700 \\
\hline $3830 B 0700.5$ & $6 / 1 / 2004$ & soil & EPA6010 & REG & $7440-41-7$ & Beryllium & 1 & $\mathrm{mg} / \mathrm{kg}$ & & 0.52 \\
\hline $3830 B 0700.5$ & $6 / 1 / 2004$ & soil & HASL300 & REG & $14733-03-0$ & Bi-214 & 0.92 & $\mathrm{pCi} / \mathrm{g}$ & $\mathrm{G}, \mathrm{J}$ & 0.35 \\
\hline $3830 B 0700.5$ & $6 / 1 / 2004$ & soil & EPA8270 & REG & 117-81-7 & Bis(2-Ethylhexyl)Phthalate & 1000 & $\mu \mathrm{g} / \mathrm{kg}$ & $\mathrm{J}$ & 700 \\
\hline $3830 B 0700.5$ & $6 / 1 / 2004$ & soil & EPA6010 & REG & $7440-43-9$ & Cadmium & 1.1 & $\mathrm{mg} / \mathrm{kg}$ & & 1 \\
\hline $3830 B 0700.5$ & $6 / 1 / 2004$ & soil & EPA8270 & REG & 86-74-8 & Carbazole & 290 & $\mu \mathrm{g} / \mathrm{kg}$ & $\mathrm{J}$ & 700 \\
\hline $3830 B 0700.5$ & $6 / 1 / 2004$ & soil & EPA6010 & REG & $7440-47-3$ & Chromium & 10 & $\mathrm{mg} / \mathrm{kg}$ & & 1 \\
\hline $3830 B 0700.5$ & $6 / 1 / 2004$ & soil & EPA8270 & REG & 218-01-9 & Chrysene & 5300 & $\mu \mathrm{g} / \mathrm{kg}$ & $\mathrm{J}$ & 700 \\
\hline $3830 B 0700.5$ & $6 / 1 / 2004$ & soil & HASL300 & REG & $10045-97-3$ & Cs-137 & 20.5 & $\mathrm{pCi} / \mathrm{g}$ & G & 0.2 \\
\hline $3830 B 0700.5$ & $6 / 1 / 2004$ & soil & EPA8270 & REG & $53-70-3$ & Dibenzo(A,H)Anthracene & 2100 & $\mu \mathrm{g} / \mathrm{kg}$ & $\mathrm{J}$ & 700 \\
\hline $3830 B 0700.5$ & $6 / 1 / 2004$ & soil & EPA8015 & REG & $68334-30-5$ & Diesel-Range Organics & 120 & $\mathrm{mg} / \mathrm{kg}$ & $\mathrm{H}, \mathrm{Z}$ & 5.2 \\
\hline $3830 B 0700.5$ & $6 / 1 / 2004$ & soil & EPA8270 & REG & $206-44-0$ & Fluoranthene & 3800 & $\mu \mathrm{g} / \mathrm{kg}$ & & 700 \\
\hline $3830 B 0700.5$ & $6 / 1 / 2004$ & soil & EPA8270 & REG & 86-73-7 & Fluorene & 31 & $\mu \mathrm{g} / \mathrm{kg}$ & $\mathrm{J}$ & 700 \\
\hline $3830 B 0700.5$ & $6 / 1 / 2004$ & soil & EPA8270 & REG & $193-39-5$ & Indeno(1,2,3-Cd)Pyrene & 5000 & $\mu \mathrm{g} / \mathrm{kg}$ & $\mathrm{J}$ & 700 \\
\hline $3830 B 0700.5$ & $6 / 1 / 2004$ & soil & HASL300 & REG & $13966-00-2$ & $\mathrm{~K}-40$ & 19.8 & $\mathrm{pCi} / \mathrm{g}$ & G & 1.2 \\
\hline $3830 B 0700.5$ & $6 / 1 / 2004$ & soil & EPA6010 & REG & 7439-92-1 & Lead & 31 & $\mathrm{mg} / \mathrm{kg}$ & & 0.63 \\
\hline $3830 B 0700.5$ & $6 / 1 / 2004$ & soil & EPA8270 & REG & 91-20-3 & Naphthalene & 32 & $\mu \mathrm{g} / \mathrm{kg}$ & $\mathrm{J}$ & 700 \\
\hline $3830 B 0700.5$ & $6 / 1 / 2004$ & soil & HASL300 & REG & 15092-94-1 & $\mathrm{Pb}-212$ & 2.32 & $\mathrm{pCi} / \mathrm{g}$ & $\mathrm{J}$ & 0.36 \\
\hline $3830 B 0700.5$ & $6 / 1 / 2004$ & soil & EPA8270 & REG & 85-01-8 & Phenanthrene & 1200 & $\mu \mathrm{g} / \mathrm{kg}$ & & 700 \\
\hline $3830 B 0700.5$ & $6 / 1 / 2004$ & soil & UGTAISOPU & REG & $15117-48-3$ & Pu-239 & 0.051 & $\mathrm{pCi} / \mathrm{g}$ & $\mathrm{J}$ & 0.018 \\
\hline $3830 B 0700.5$ & $6 / 1 / 2004$ & soil & EPA8270 & REG & $129-00-0$ & Pyrene & 26000 & $\mu \mathrm{g} / \mathrm{kg}$ & $\mathrm{J}$ & 700 \\
\hline $3830 B 0700.5$ & $6 / 1 / 2004$ & soil & HASL300 & REG & $14913-50-9$ & TI-208 & 0.82 & $\mathrm{pCi} / \mathrm{g}$ & G & 0.19 \\
\hline 3830B0700.5RR1 & $6 / 1 / 2004$ & soil & EPA8270 & $\mathrm{RE}$ & $120-12-7$ & Anthracene & 160 & $\mu \mathrm{g} / \mathrm{kg}$ & $\mathrm{J}$ & 2100 \\
\hline 3830B0700.5RR1 & $6 / 1 / 2004$ & soil & EPA8270 & $\mathrm{RE}$ & $56-55-3$ & Benzo(A)Anthracene & 4800 & $\mu \mathrm{g} / \mathrm{kg}$ & $\mathrm{J}$ & 2100 \\
\hline 3830B0700.5RR1 & $6 / 1 / 2004$ & soil & EPA8270 & $\mathrm{RE}$ & $50-32-8$ & Benzo(A)Pyrene & 4800 & $\mu \mathrm{g} / \mathrm{kg}$ & $\mathrm{J}$ & 2100 \\
\hline 3830B0700.5RR1 & $6 / 1 / 2004$ & soil & EPA8270 & $\mathrm{RE}$ & 205-99-2 & Benzo(B)Fluoranthene & 9600 & $\mu \mathrm{g} / \mathrm{kg}$ & $\mathrm{J}$ & 2100 \\
\hline
\end{tabular}

Page 64 of 78

Uncontrolled When Printed 


\begin{tabular}{|c|c|c|c|c|c|c|c|c|c|c|}
\hline SAMPLE \# & $\begin{array}{l}\text { SAMPLE } \\
\text { DATE }\end{array}$ & MATRIX & $\begin{array}{l}\text { USER TEST } \\
\text { PANEL }\end{array}$ & $\begin{array}{c}\text { SAMPLE } \\
\text { PURPOSE }\end{array}$ & CAS \# & PARAMETER & RESULT & UNITS & $\mathbf{Q}$ & $\begin{array}{c}\text { DETECT } \\
\text { LIMIT }\end{array}$ \\
\hline 383OB0700.5RR1 & $6 / 1 / 2004$ & soil & EPA8270 & $\mathrm{RE}$ & $191-24-2$ & Benzo(G,H,I)Perylene & 2100 & $\mu \mathrm{g} / \mathrm{kg}$ & $\mathrm{J}$ & 2100 \\
\hline 3830B0700.5RR1 & $6 / 1 / 2004$ & soil & EPA8270 & RE & 207-08-9 & Benzo(K)Fluoranthene & 5500 & $\mu \mathrm{g} / \mathrm{kg}$ & $\mathrm{J}$ & 2100 \\
\hline 3830B0700.5RR1 & $6 / 1 / 2004$ & soil & EPA8270 & RE & $117-81-7$ & Bis(2-Ethylhexyl)Phthalate & 710 & $\mu \mathrm{g} / \mathrm{kg}$ & $\mathrm{J}$ & 2100 \\
\hline 3830B0700.5RR1 & $6 / 1 / 2004$ & soil & EPA8270 & RE & 86-74-8 & Carbazole & 290 & $\mu \mathrm{g} / \mathrm{kg}$ & $\mathrm{J}$ & 2100 \\
\hline 383OB0700.5RR1 & $6 / 1 / 2004$ & soil & EPA8270 & $\mathrm{RE}$ & 218-01-9 & Chrysene & 5800 & $\mu \mathrm{g} / \mathrm{kg}$ & $\mathrm{J}$ & 2100 \\
\hline 3830B0700.5RR1 & $6 / 1 / 2004$ & soil & EPA8270 & $\mathrm{RE}$ & $206-44-0$ & Fluoranthene & 5100 & $\mu \mathrm{g} / \mathrm{kg}$ & & 2100 \\
\hline 383OB0700.5RR1 & $6 / 1 / 2004$ & soil & EPA8270 & $\mathrm{RE}$ & $193-39-5$ & Indeno(1,2,3-Cd)Pyrene & 1900 & $\mu \mathrm{g} / \mathrm{kg}$ & $\mathrm{J}$ & 2100 \\
\hline 383OB0700.5RR1 & $6 / 1 / 2004$ & soil & EPA8270 & $\mathrm{RE}$ & 85-01-8 & Phenanthrene & 1300 & $\mu \mathrm{g} / \mathrm{kg}$ & $\mathrm{J}$ & 2100 \\
\hline 3830B0700.5RR1 & $6 / 1 / 2004$ & soil & EPA8270 & $\mathrm{RE}$ & $129-00-0$ & Pyrene & 15000 & $\mu \mathrm{g} / \mathrm{kg}$ & $\mathrm{J}$ & 2100 \\
\hline $3830 B 0800.5$ & $6 / 1 / 2004$ & soil & HASL300 & REG & $14331-83-0$ & Ac-228 & 1.27 & $\mathrm{pCi} / \mathrm{g}$ & $G$ & 0.63 \\
\hline $3830 B 0800.5$ & $6 / 1 / 2004$ & soil & EPA8270 & REG & $83-32-9$ & Acenaphthene & 93 & $\mu \mathrm{g} / \mathrm{kg}$ & $\mathrm{J}$ & 1400 \\
\hline $3830 B 0800.5$ & $6 / 1 / 2004$ & soil & EPA8270 & REG & $120-12-7$ & Anthracene & 470 & $\mu \mathrm{g} / \mathrm{kg}$ & $\mathrm{J}$ & 1400 \\
\hline $3830 B 0800.5$ & $6 / 1 / 2004$ & soil & EPA6010 & REG & $7440-38-2$ & Arsenic & 7.1 & $\mathrm{mg} / \mathrm{kg}$ & & 1 \\
\hline $3830 B 0800.5$ & $6 / 1 / 2004$ & soil & EPA6010 & REG & $7440-39-3$ & Barium & 220 & $\mathrm{mg} / \mathrm{kg}$ & & 10 \\
\hline $3830 B 0800.5$ & $6 / 1 / 2004$ & soil & EPA8270 & REG & $56-55-3$ & Benzo(A)Anthracene & 17000 & $\mu \mathrm{g} / \mathrm{kg}$ & $\mathrm{J}$ & 1400 \\
\hline $3830 B 0800.5$ & $6 / 1 / 2004$ & soil & EPA8270 & REG & $50-32-8$ & Benzo(A)Pyrene & 20000 & $\mu \mathrm{g} / \mathrm{kg}$ & $\mathrm{J}$ & 1400 \\
\hline $3830 B 0800.5$ & $6 / 1 / 2004$ & soil & EPA8270 & REG & $205-99-2$ & Benzo(B)Fluoranthene & 37000 & $\mu \mathrm{g} / \mathrm{kg}$ & $\mathrm{J}$ & 1400 \\
\hline $3830 B 0800.5$ & $6 / 1 / 2004$ & soil & EPA8270 & REG & 191-24-2 & Benzo(G,H,I)Perylene & 32000 & $\mu \mathrm{g} / \mathrm{kg}$ & $\mathrm{J}$ & 1400 \\
\hline $3830 B 0800.5$ & $6 / 1 / 2004$ & soil & EPA8270 & REG & 207-08-9 & Benzo(K)Fluoranthene & 10000 & $\mu \mathrm{g} / \mathrm{kg}$ & $\mathrm{J}$ & 1400 \\
\hline $3830 B 0800.5$ & $6 / 1 / 2004$ & soil & EPA6010 & REG & $7440-41-7$ & Beryllium & 0.77 & $\mathrm{mg} / \mathrm{kg}$ & & 0.52 \\
\hline $3830 B 0800.5$ & $6 / 1 / 2004$ & soil & HASL300 & REG & $14733-03-0$ & Bi-214 & 1.1 & $\mathrm{pCi} / \mathrm{g}$ & $\mathrm{G}, \mathrm{J}$ & 0.28 \\
\hline $3830 B 0800.5$ & $6 / 1 / 2004$ & soil & EPA8270 & REG & 117-81-7 & Bis(2-Ethylhexyl)Phthalate & 4000 & $\mu \mathrm{g} / \mathrm{kg}$ & $\mathrm{J}$ & 1400 \\
\hline $3830 B 0800.5$ & $6 / 1 / 2004$ & soil & EPA6010 & REG & $7440-43-9$ & Cadmium & 0.58 & $\mathrm{mg} / \mathrm{kg}$ & & 0.52 \\
\hline $3830 B 0800.5$ & $6 / 1 / 2004$ & soil & EPA8270 & REG & 86-74-8 & Carbazole & 790 & $\mu \mathrm{g} / \mathrm{kg}$ & $\mathrm{J}$ & 1400 \\
\hline $3830 B 0800.5$ & $6 / 1 / 2004$ & soil & EPA6010 & REG & $7440-47-3$ & Chromium & 7.7 & $\mathrm{mg} / \mathrm{kg}$ & & 1 \\
\hline $3830 B 0800.5$ & $6 / 1 / 2004$ & soil & EPA8270 & REG & 218-01-9 & Chrysene & 21000 & $\mu g / \mathrm{kg}$ & $\mathrm{J}$ & 1400 \\
\hline $3830 B 0800.5$ & $6 / 1 / 2004$ & soil & HASL300 & REG & $10045-97-3$ & Cs-137 & 13.6 & $\mathrm{pCi} / \mathrm{g}$ & G & 0.2 \\
\hline $3830 B 0800.5$ & $6 / 1 / 2004$ & soil & EPA8270 & REG & $53-70-3$ & Dibenzo(A,H)Anthracene & 10000 & $\mu \mathrm{g} / \mathrm{kg}$ & $\mathrm{J}$ & 1400 \\
\hline $3830 B 0800.5$ & $6 / 1 / 2004$ & soil & EPA8015 & REG & $68334-30-5$ & Diesel-Range Organics & 490 & $\mathrm{mg} / \mathrm{kg}$ & $\mathrm{H}, \mathrm{Z}$ & 5.2 \\
\hline $3830 B 0800.5$ & $6 / 1 / 2004$ & soil & EPA8270 & REG & $206-44-0$ & Fluoranthene & 10000 & $\mu \mathrm{g} / \mathrm{kg}$ & & 1400 \\
\hline $3830 B 0800.5$ & $6 / 1 / 2004$ & soil & EPA8270 & REG & 86-73-7 & Fluorene & 68 & $\mu \mathrm{g} / \mathrm{kg}$ & $\mathrm{J}$ & 1400 \\
\hline $3830 B 0800.5$ & $6 / 1 / 2004$ & soil & EPA8270 & REG & $193-39-5$ & Indeno(1,2,3-Cd)Pyrene & 22000 & $\mu \mathrm{g} / \mathrm{kg}$ & $\mathrm{J}$ & 1400 \\
\hline $3830 B 0800.5$ & $6 / 1 / 2004$ & soil & HASL300 & REG & $13966-00-2$ & $\mathrm{~K}-40$ & 22.4 & $\mathrm{pCi} / \mathrm{g}$ & G & 1.8 \\
\hline $3830 B 0800.5$ & $6 / 1 / 2004$ & soil & EPA6010 & REG & $7439-92-1$ & Lead & 25 & $\mathrm{mg} / \mathrm{kg}$ & & 0.31 \\
\hline $3830 B 0800.5$ & $6 / 1 / 2004$ & soil & EPA8270 & REG & 91-20-3 & Naphthalene & 56 & $\mu \mathrm{g} / \mathrm{kg}$ & $\mathrm{J}$ & 1400 \\
\hline
\end{tabular}

Page 65 of 78

Uncontrolled When Printed 


\begin{tabular}{|c|c|c|c|c|c|c|c|c|c|c|}
\hline SAMPLE \# & $\begin{array}{c}\text { SAMPLE } \\
\text { DATE }\end{array}$ & MATRIX & $\begin{array}{l}\text { USER TEST } \\
\text { PANEL }\end{array}$ & $\begin{array}{c}\text { SAMPLE } \\
\text { PURPOSE }\end{array}$ & CAS \# & PARAMETER & RESULT & UNITS & $\mathbf{Q}$ & $\begin{array}{l}\text { DETECT } \\
\text { LIMIT }\end{array}$ \\
\hline $3830 B 0800.5$ & $6 / 1 / 2004$ & soil & HASL300 & REG & 15092-94-1 & $\mathrm{Pb}-212$ & 1.83 & $\mathrm{pCi} / \mathrm{g}$ & $\mathrm{J}$ & 0.34 \\
\hline $3830 B 0800.5$ & $6 / 1 / 2004$ & soil & HASL300 & REG & $15067-28-4$ & $\mathrm{~Pb}-214$ & 0.9 & $\mathrm{pCi} / \mathrm{g}$ & $\mathrm{G}, \mathrm{J}$ & 0.41 \\
\hline $3830 B 0800.5$ & $6 / 1 / 2004$ & soil & EPA8270 & REG & 85-01-8 & Phenanthrene & 2500 & $\mu \mathrm{g} / \mathrm{kg}$ & & 1400 \\
\hline $3830 B 0800.5$ & $6 / 1 / 2004$ & soil & UGTAISOPU & REG & $15117-48-3$ & Pu-239 & 1.04 & $\mathrm{pCi} / \mathrm{g}$ & $\mathrm{J}$ & 0.02 \\
\hline $3830 B 0800.5$ & $6 / 1 / 2004$ & soil & EPA8270 & REG & $129-00-0$ & Pyrene & 89000 & $\mu \mathrm{g} / \mathrm{kg}$ & $\mathrm{J}$ & 1400 \\
\hline $3830 B 0800.5$ & $6 / 1 / 2004$ & soil & EPA6010 & REG & $7782-49-2$ & Selenium & 1.2 & $\mathrm{mg} / \mathrm{kg}$ & & 0.52 \\
\hline $3830 B 0800.5$ & $6 / 1 / 2004$ & soil & HASL300 & REG & $14913-50-9$ & TI-208 & 0.54 & $\mathrm{pCi} / \mathrm{g}$ & $G$ & 0.18 \\
\hline 383OB0800.5RR1 & $6 / 1 / 2004$ & soil & EPA8270 & RE & $56-55-3$ & Benzo(A)Anthracene & 18000 & $\mu \mathrm{g} / \mathrm{kg}$ & $\mathrm{J}$ & 6900 \\
\hline 383OB0800.5RR1 & $6 / 1 / 2004$ & soil & EPA8270 & $\mathrm{RE}$ & $50-32-8$ & Benzo(A)Pyrene & 23000 & $\mu \mathrm{g} / \mathrm{kg}$ & $\mathrm{J}$ & 6900 \\
\hline 3830B0800.5RR1 & $6 / 1 / 2004$ & soil & EPA8270 & $\mathrm{RE}$ & $205-99-2$ & Benzo(B)Fluoranthene & 43000 & $\mu \mathrm{g} / \mathrm{kg}$ & $\mathrm{J}$ & 6900 \\
\hline 3830B0800.5RR1 & $6 / 1 / 2004$ & soil & EPA8270 & $\mathrm{RE}$ & $191-24-2$ & Benzo(G,H,I)Perylene & 16000 & $\mu \mathrm{g} / \mathrm{kg}$ & $\mathrm{J}$ & 6900 \\
\hline 3830B0800.5RR1 & $6 / 1 / 2004$ & soil & EPA8270 & RE & 207-08-9 & Benzo(K)Fluoranthene & 19000 & $\mu \mathrm{g} / \mathrm{kg}$ & $\mathrm{J}$ & 6900 \\
\hline 383OB0800.5RR1 & $6 / 1 / 2004$ & soil & EPA8270 & $\mathrm{RE}$ & 117-81-7 & Bis(2-Ethylhexyl)Phthalate & 3900 & $\mu \mathrm{g} / \mathrm{kg}$ & $\mathrm{J}$ & 6900 \\
\hline 383OB0800.5RR1 & $6 / 1 / 2004$ & soil & EPA8270 & $\mathrm{RE}$ & 218-01-9 & Chrysene & 23000 & $\mu \mathrm{g} / \mathrm{kg}$ & $\mathrm{J}$ & 6900 \\
\hline 3830B0800.5RR1 & $6 / 1 / 2004$ & soil & EPA8270 & $\mathrm{RE}$ & $206-44-0$ & Fluoranthene & 13000 & $\mu \mathrm{g} / \mathrm{kg}$ & $\mathrm{J}$ & 6900 \\
\hline 3830B0800.5RR1 & $6 / 1 / 2004$ & soil & EPA8270 & $\mathrm{RE}$ & 193-39-5 & Indeno(1,2,3-Cd)Pyrene & 12000 & $\mu \mathrm{g} / \mathrm{kg}$ & $\mathrm{J}$ & 6900 \\
\hline 383OB0800.5RR1 & $6 / 1 / 2004$ & soil & EPA8270 & $\mathrm{RE}$ & $129-00-0$ & Pyrene & 64000 & $\mu \mathrm{g} / \mathrm{kg}$ & $\mathrm{J}$ & 6900 \\
\hline 383PB0102.0 & $6 / 10 / 2004$ & soil & EPA8260 & REG & 67-64-1 & Acetone & 11 & $\mu \mathrm{g} / \mathrm{kg}$ & $\mathrm{J}$ & 22 \\
\hline 383PB0102.0 & $6 / 10 / 2004$ & soil & EPA6010 & REG & $7440-38-2$ & Arsenic & 1 & $\mathrm{mg} / \mathrm{kg}$ & $\mathrm{B}$ & 1.1 \\
\hline 383PB0102.0 & $6 / 10 / 2004$ & soil & EPA6010 & REG & $7440-39-3$ & Barium & 4900 & $\mathrm{mg} / \mathrm{kg}$ & & 110 \\
\hline 383РВ0102.0 & $6 / 10 / 2004$ & soil & EPA6010 & REG & 7440-41-7 & Beryllium & 0.19 & $\mathrm{mg} / \mathrm{kg}$ & B & 0.54 \\
\hline 383РВ0102.0 & $6 / 10 / 2004$ & soil & EPA6010 & REG & 7440-43-9 & Cadmium & 0.16 & $\mathrm{mg} / \mathrm{kg}$ & $\mathrm{B}$ & 0.54 \\
\hline 383РВ0102.0 & $6 / 10 / 2004$ & soil & EPA6010 & REG & $7440-47-3$ & Chromium & 4.2 & $\mathrm{mg} / \mathrm{kg}$ & & 1.1 \\
\hline 383РВ0102.0 & $6 / 10 / 2004$ & soil & HASL300 & REG & 13966-00-2 & $\mathrm{K}-40$ & 17.8 & $\mathrm{pCi} / \mathrm{g}$ & G & 1.3 \\
\hline 383PB0102.0 & $6 / 10 / 2004$ & soil & EPA6010 & REG & 7439-92-1 & Lead & 5.3 & $\mathrm{mg} / \mathrm{kg}$ & & 0.33 \\
\hline 383РВ0102.0 & $6 / 10 / 2004$ & soil & EPA7470 & REG & 7439-97-6 & Mercury & 0.0016 & $\mathrm{mg} / \mathrm{kg}$ & $B$ & 0.11 \\
\hline 383РВ0102.0 & $6 / 10 / 2004$ & soil & HASL300 & REG & 15092-94-1 & $\mathrm{Pb}-212$ & 0.27 & $\mathrm{pCi} / \mathrm{g}$ & $G$ & 0.15 \\
\hline 383РВ0102.0 & $6 / 10 / 2004$ & soil & EPA6010 & REG & $7440-22-4$ & Silver & 0.077 & $\mathrm{mg} / \mathrm{kg}$ & $B$ & 1.1 \\
\hline 383PB0104.5 & $6 / 10 / 2004$ & soil & HASL300 & REG & 14331-83-0 & Ac-228 & 1.67 & $\mathrm{pCi} / \mathrm{g}$ & $\mathrm{G}$ & 0.55 \\
\hline 383PB0104.5 & $6 / 10 / 2004$ & soil & EPA8260 & REG & 67-64-1 & Acetone & 10 & $\mu \mathrm{g} / \mathrm{kg}$ & $\mathrm{J}$ & 21 \\
\hline 383PB0104.5 & $6 / 10 / 2004$ & soil & EPA6010 & REG & $7440-38-2$ & Arsenic & 2.9 & $\mathrm{mg} / \mathrm{kg}$ & & 1.1 \\
\hline 383PB0104.5 & $6 / 10 / 2004$ & soil & EPA6010 & REG & 7440-39-3 & Barium & 120 & $\mathrm{mg} / \mathrm{kg}$ & & 11 \\
\hline 383PB0104.5 & $6 / 10 / 2004$ & soil & EPA6010 & REG & 7440-41-7 & Beryllium & 0.71 & $\mathrm{mg} / \mathrm{kg}$ & & 0.53 \\
\hline 383PB0104.5 & $6 / 10 / 2004$ & soil & HASL300 & REG & 14733-03-0 & Bi-214 & 0.96 & $\mathrm{pCi} / \mathrm{g}$ & $\mathrm{G}, \mathrm{J}$ & 0.28 \\
\hline 383PB0104.5 & $6 / 10 / 2004$ & soil & EPA6010 & REG & 7440-43-9 & Cadmium & 0.17 & $\mathrm{mg} / \mathrm{kg}$ & $\mathrm{B}$ & 0.53 \\
\hline
\end{tabular}

Page 66 of 78

Uncontrolled When Printed 


\begin{tabular}{|c|c|c|c|c|c|c|c|c|c|c|}
\hline SAMPLE \# & $\begin{array}{c}\text { SAMPLE } \\
\text { DATE }\end{array}$ & MATRIX & $\begin{array}{l}\text { USER TEST } \\
\text { PANEL }\end{array}$ & $\begin{array}{c}\text { SAMPLE } \\
\text { PURPOSE }\end{array}$ & CAS \# & PARAMETER & RESULT & UNITS & $\mathbf{Q}$ & $\begin{array}{l}\text { DETECT } \\
\text { LIMIT }\end{array}$ \\
\hline 383PB0104.5 & $6 / 10 / 2004$ & soil & EPA6010 & REG & $7440-47-3$ & Chromium & 2.7 & $\mathrm{mg} / \mathrm{kg}$ & & 1.1 \\
\hline 383PB0104.5 & $6 / 10 / 2004$ & soil & HASL300 & REG & 13966-00-2 & $\mathrm{K}-40$ & 26.2 & $\mathrm{pCi} / \mathrm{g}$ & G & 1.2 \\
\hline 383PB0104.5 & $6 / 10 / 2004$ & soil & EPA6010 & REG & 7439-92-1 & Lead & 5.7 & $\mathrm{mg} / \mathrm{kg}$ & & 0.64 \\
\hline 383PB0104.5 & $6 / 10 / 2004$ & soil & EPA7470 & REG & 7439-97-6 & Mercury & 0.022 & $\mathrm{mg} / \mathrm{kg}$ & $B$ & 0.11 \\
\hline 383PB0104.5 & $6 / 10 / 2004$ & soil & HASL300 & REG & 15092-94-1 & $\mathrm{Pb}-212$ & 1.87 & $\mathrm{pCi} / \mathrm{g}$ & $\mathrm{J}$ & 0.16 \\
\hline 383РВ0104.5 & $6 / 10 / 2004$ & soil & HASL300 & REG & $15067-28-4$ & $\mathrm{~Pb}-214$ & 1.08 & $\mathrm{pCi} / \mathrm{g}$ & $\mathrm{G}, \mathrm{J}$ & 0.22 \\
\hline 383PB0104.5 & $6 / 10 / 2004$ & soil & HASL300 & REG & $14913-50-9$ & TI-208 & 0.59 & $\mathrm{pCi} / \mathrm{g}$ & G & 0.11 \\
\hline 383PB0200.5 & $6 / 17 / 2004$ & soil & HASL300 & REG & 14331-83-0 & Ac-228 & 1.84 & $\mathrm{pCi} / \mathrm{g}$ & $\mathrm{G}$ & 0.54 \\
\hline 383PB0200.5 & $6 / 17 / 2004$ & soil & EPA6010 & REG & $7440-38-2$ & Arsenic & 3 & $\mathrm{mg} / \mathrm{kg}$ & & 1.1 \\
\hline 383PB0200.5 & $6 / 17 / 2004$ & soil & EPA6010 & REG & $7440-39-3$ & Barium & 5200 & $\mathrm{mg} / \mathrm{kg}$ & & 550 \\
\hline 383PB0200.5 & $6 / 17 / 2004$ & soil & EPA6010 & REG & 7440-41-7 & Beryllium & 1.3 & $\mathrm{mg} / \mathrm{kg}$ & & 0.55 \\
\hline 383РВ0200.5 & $6 / 17 / 2004$ & soil & HASL300 & REG & $14733-03-0$ & Bi-214 & 1.58 & $\mathrm{pCi} / \mathrm{g}$ & $\mathrm{G}, \mathrm{J}$ & 0.66 \\
\hline 383PB0200.5 & $6 / 17 / 2004$ & soil & EPA6010 & REG & 7440-43-9 & Cadmium & 0.16 & $\mathrm{mg} / \mathrm{kg}$ & $B$ & 0.55 \\
\hline 383PB0200.5 & $6 / 17 / 2004$ & soil & EPA6010 & REG & $7440-47-3$ & Chromium & 4.9 & $\mathrm{mg} / \mathrm{kg}$ & & 1.1 \\
\hline 383PB0200.5 & $6 / 17 / 2004$ & soil & HASL300 & REG & $10045-97-3$ & Cs-137 & 91 & $\mathrm{pCi} / \mathrm{g}$ & G & 0 \\
\hline 383PB0200.5 & $6 / 17 / 2004$ & soil & EPA8015 & REG & 68334-30-5 & Diesel-Range Organics & 5.8 & $\mathrm{mg} / \mathrm{kg}$ & $\mathrm{Z}, \mathrm{H}$ & 5.5 \\
\hline 383PB0200.5 & $6 / 17 / 2004$ & soil & HASL300 & REG & $13966-00-2$ & $\mathrm{~K}-40$ & 22 & $\mathrm{pCi} / \mathrm{g}$ & G & 1.6 \\
\hline 383PB0200.5 & $6 / 17 / 2004$ & soil & EPA6010 & REG & 7439-92-1 & Lead & 21 & $\mathrm{mg} / \mathrm{kg}$ & & 0.33 \\
\hline 383PB0200.5 & $6 / 17 / 2004$ & soil & EPA7470 & REG & $7439-97-6$ & Mercury & 0.047 & $\mathrm{mg} / \mathrm{kg}$ & $\mathrm{B}$ & 0.11 \\
\hline 383PB0200.5 & $6 / 17 / 2004$ & soil & EPA8260 & REG & $75-09-2$ & Methylene Chloride & 0.97 & $\mu \mathrm{g} / \mathrm{kg}$ & $\mathrm{J}, \mathrm{B}$ & 5.5 \\
\hline 383PB0200.5 & $6 / 17 / 2004$ & soil & HASL300 & REG & 15092-94-1 & $\mathrm{Pb}-212$ & 2.18 & $\mathrm{pCi} / \mathrm{g}$ & G & 0.5 \\
\hline 383PB0200.5 & $6 / 17 / 2004$ & soil & HASL300 & REG & $15067-28-4$ & $\mathrm{~Pb}-214$ & 1.5 & $\mathrm{pCi} / \mathrm{g}$ & $\mathrm{G}, \mathrm{J}$ & 0.89 \\
\hline 383PB0200.5 & $6 / 17 / 2004$ & soil & UGTAISOPU & REG & 13981-16-3 & Pu-238 & 0.271 & $\mathrm{pCi} / \mathrm{g}$ & & 0.021 \\
\hline 383PB0200.5 & $6 / 17 / 2004$ & soil & UGTAISOPU & REG & $15117-48-3$ & Pu-239 & 2.65 & $\mathrm{pCi} / \mathrm{g}$ & & 0.02 \\
\hline 383PB0200.5 & $6 / 17 / 2004$ & soil & SR7500 & REG & 10098-97-2 & Sr-90 & 1.38 & $\mathrm{pCi} / \mathrm{g}$ & Y1 & 0.24 \\
\hline 383PB0200.5 & $6 / 17 / 2004$ & soil & HASL300 & REG & 14913-50-9 & TI-208 & 0.57 & $\mathrm{pCi} / \mathrm{g}$ & $G$ & 0.3 \\
\hline 383РВ0300.5 & $6 / 17 / 2004$ & soil & HASL300 & REG & 14331-83-0 & Ac-228 & 1.98 & $\mathrm{pCi} / \mathrm{g}$ & $G$ & 0.85 \\
\hline 383PB0300.5 & $6 / 17 / 2004$ & soil & HASL300 & REG & 14596-10-2 & Am-241 & 0.72 & $\mathrm{pCi} / \mathrm{g}$ & $G$ & 0.5 \\
\hline 383PB0300.5 & $6 / 17 / 2004$ & soil & EPA6010 & REG & $7440-38-2$ & Arsenic & 3.6 & $\mathrm{mg} / \mathrm{kg}$ & & 1.2 \\
\hline 383РВ0300.5 & $6 / 17 / 2004$ & soil & EPA6010 & REG & $7440-39-3$ & Barium & 6300 & $\mathrm{mg} / \mathrm{kg}$ & & 590 \\
\hline 383PB0300.5 & $6 / 17 / 2004$ & soil & EPA8270 & REG & $56-55-3$ & Benzo(A)Anthracene & 21 & $\mu \mathrm{g} / \mathrm{kg}$ & $\mathrm{J}$ & 390 \\
\hline 383PB0300.5 & $6 / 17 / 2004$ & soil & EPA8270 & REG & $50-32-8$ & Benzo(A)Pyrene & 18 & $\mu \mathrm{g} / \mathrm{kg}$ & $\mathrm{J}$ & 390 \\
\hline 383РВ0300.5 & $6 / 17 / 2004$ & soil & EPA8270 & REG & $205-99-2$ & Benzo(B)Fluoranthene & 31 & $\mu \mathrm{g} / \mathrm{kg}$ & $\mathrm{J}$ & 390 \\
\hline 383PB0300.5 & $6 / 17 / 2004$ & soil & EPA6010 & REG & 7440-41-7 & Beryllium & 1.6 & $\mathrm{mg} / \mathrm{kg}$ & & 0.59 \\
\hline 383PB0300.5 & $6 / 17 / 2004$ & soil & HASL300 & REG & $14733-03-0$ & Bi-214 & 1.1 & $\mathrm{pCi} / \mathrm{g}$ & $\mathrm{G}, \mathrm{J}$ & 0.72 \\
\hline
\end{tabular}

Page 67 of 78

Uncontrolled When Printed 


\begin{tabular}{|c|c|c|c|c|c|c|c|c|c|c|}
\hline SAMPLE \# & $\begin{array}{c}\text { SAMPLE } \\
\text { DATE }\end{array}$ & MATRIX & $\begin{array}{l}\text { USER TEST } \\
\text { PANEL }\end{array}$ & $\begin{array}{c}\text { SAMPLE } \\
\text { PURPOSE }\end{array}$ & CAS \# & PARAMETER & RESULT & UNITS & $\mathbf{Q}$ & $\begin{array}{c}\text { DETECT } \\
\text { LIMIT }\end{array}$ \\
\hline 383PB0300.5 & $6 / 17 / 2004$ & soil & EPA8270 & REG & $117-81-7$ & Bis(2-Ethylhexyl)Phthalate & 85 & $\mu \mathrm{g} / \mathrm{kg}$ & $\mathrm{J}$ & 390 \\
\hline 383PB0300.5 & $6 / 17 / 2004$ & soil & EPA6010 & REG & $7440-43-9$ & Cadmium & 0.34 & $\mathrm{mg} / \mathrm{kg}$ & $\mathrm{B}$ & 0.59 \\
\hline 383PB0300.5 & $6 / 17 / 2004$ & soil & EPA6010 & REG & $7440-47-3$ & Chromium & 8.7 & $\mathrm{mg} / \mathrm{kg}$ & & 1.2 \\
\hline 383PB0300.5 & $6 / 17 / 2004$ & soil & EPA8270 & REG & 218-01-9 & Chrysene & 30 & $\mu \mathrm{g} / \mathrm{kg}$ & $\mathrm{J}$ & 390 \\
\hline 383PB0300.5 & $6 / 17 / 2004$ & soil & HASL300 & REG & $10045-97-3$ & Cs-137 & 56.3 & $\mathrm{pCi} / \mathrm{g}$ & G & 0.3 \\
\hline 383PB0300.5 & $6 / 17 / 2004$ & soil & EPA8015 & REG & $68334-30-5$ & Diesel-Range Organics & 17 & $\mathrm{mg} / \mathrm{kg}$ & $\mathrm{Z}, \mathrm{H}$ & 5.9 \\
\hline 383PB0300.5 & $6 / 17 / 2004$ & soil & EPA8270 & REG & 206-44-0 & Fluoranthene & 25 & $\mu \mathrm{g} / \mathrm{kg}$ & $\mathrm{J}$ & 390 \\
\hline 383РВ0300.5 & $6 / 17 / 2004$ & soil & HASL300 & REG & 13966-00-2 & $\mathrm{K}-40$ & 23.3 & $\mathrm{pCi} / \mathrm{g}$ & $G$ & 2.6 \\
\hline 383РВ0300.5 & $6 / 17 / 2004$ & soil & EPA6010 & REG & 7439-92-1 & Lead & 38 & $\mathrm{mg} / \mathrm{kg}$ & & 0.71 \\
\hline 383РВ0300.5 & $6 / 17 / 2004$ & soil & EPA7470 & REG & 7439-97-6 & Mercury & 0.048 & $\mathrm{mg} / \mathrm{kg}$ & $B$ & 0.12 \\
\hline 383PB0300.5 & $6 / 17 / 2004$ & soil & HASL300 & REG & 15092-94-1 & $\mathrm{Pb}-212$ & 1.4 & $\mathrm{pCi} / \mathrm{g}$ & $G$ & 0.58 \\
\hline 383РВ0300.5 & $6 / 17 / 2004$ & soil & HASL300 & REG & $15067-28-4$ & $\mathrm{~Pb}-214$ & 1.11 & $\mathrm{pCi} / \mathrm{g}$ & $\mathrm{G}, \mathrm{J}$ & 0.92 \\
\hline 383PВ0300.5 & $6 / 17 / 2004$ & soil & UGTAISOPU & REG & 13981-16-3 & Pu-238 & 0.348 & $\mathrm{pCi} / \mathrm{g}$ & & 0.017 \\
\hline 383PB0300.5 & $6 / 17 / 2004$ & soil & UGTAISOPU & REG & $15117-48-3$ & Pu-239 & 4.49 & $\mathrm{pCi} / \mathrm{g}$ & & 0.02 \\
\hline 383РВ0300.5 & $6 / 17 / 2004$ & soil & EPA8270 & REG & $129-00-0$ & Pyrene & 50 & $\mu \mathrm{g} / \mathrm{kg}$ & $\mathrm{J}$ & 390 \\
\hline 383РВ0300.5 & $6 / 17 / 2004$ & soil & EPA6010 & REG & $7782-49-2$ & Selenium & 0.53 & $\mathrm{mg} / \mathrm{kg}$ & $B$ & 0.59 \\
\hline 383PВ0300.5 & $6 / 17 / 2004$ & soil & SR7500 & REG & 10098-97-2 & Sr-90 & 3.58 & $\mathrm{pCi} / \mathrm{g}$ & & 0.24 \\
\hline 383PB0300.5 & $6 / 17 / 2004$ & soil & HASL300 & REG & $14913-50-9$ & TI-208 & 0.63 & $\mathrm{pCi} / \mathrm{g}$ & $G$ & 0.38 \\
\hline 383PB0300.5X & $6 / 17 / 2004$ & soil & HASL300 & FD & $14331-83-0$ & Ac-228 & 1.88 & $\mathrm{pCi} / \mathrm{g}$ & G & 0.74 \\
\hline 383PB0300.5X & $6 / 17 / 2004$ & soil & EPA6010 & FD & $7440-38-2$ & Arsenic & 3.8 & $\mathrm{mg} / \mathrm{kg}$ & & 1.2 \\
\hline 383PB0300.5X & $6 / 17 / 2004$ & soil & EPA6010 & FD & $7440-39-3$ & Barium & 6500 & $\mathrm{mg} / \mathrm{kg}$ & & 580 \\
\hline 383PB0300.5X & $6 / 17 / 2004$ & soil & EPA8270 & FD & $56-55-3$ & Benzo(A)Anthracene & 21 & $\mu \mathrm{g} / \mathrm{kg}$ & $\mathrm{J}$ & 380 \\
\hline 383PB0300.5X & $6 / 17 / 2004$ & soil & EPA8270 & FD & $50-32-8$ & Benzo(A)Pyrene & 19 & $\mu \mathrm{g} / \mathrm{kg}$ & $\mathrm{J}$ & 380 \\
\hline 383PB0300.5X & $6 / 17 / 2004$ & soil & EPA8270 & FD & $205-99-2$ & Benzo(B)Fluoranthene & 26 & $\mu \mathrm{g} / \mathrm{kg}$ & $\mathrm{J}$ & 380 \\
\hline 383РB0300.5X & $6 / 17 / 2004$ & soil & EPA6010 & FD & $7440-41-7$ & Beryllium & 1.5 & $\mathrm{mg} / \mathrm{kg}$ & & 0.58 \\
\hline 383PB0300.5X & $6 / 17 / 2004$ & soil & HASL300 & FD & $14733-03-0$ & Bi-214 & 1.2 & $\mathrm{pCi} / \mathrm{g}$ & $\mathrm{G}, \mathrm{J}$ & 0.59 \\
\hline 383PB0300.5X & $6 / 17 / 2004$ & soil & EPA8270 & FD & 117-81-7 & Bis(2-Ethylhexyl)Phthalate & 90 & $\mu \mathrm{g} / \mathrm{kg}$ & $\mathrm{J}$ & 380 \\
\hline 383PB0300.5X & $6 / 17 / 2004$ & soil & EPA6010 & FD & $7440-43-9$ & Cadmium & 0.35 & $\mathrm{mg} / \mathrm{kg}$ & $\mathrm{B}$ & 0.58 \\
\hline 383PB0300.5X & $6 / 17 / 2004$ & soil & EPA6010 & FD & $7440-47-3$ & Chromium & 8.5 & $\mathrm{mg} / \mathrm{kg}$ & & 1.2 \\
\hline 383PB0300.5X & $6 / 17 / 2004$ & soil & EPA8270 & FD & 218-01-9 & Chrysene & 29 & $\mu \mathrm{g} / \mathrm{kg}$ & $\mathrm{J}$ & 380 \\
\hline 383PB0300.5X & $6 / 17 / 2004$ & soil & HASL300 & FD & $10045-97-3$ & Cs-137 & 60.9 & $\mathrm{pCi} / \mathrm{g}$ & G & 0.3 \\
\hline 383PB0300.5X & $6 / 17 / 2004$ & soil & EPA8015 & FD & $68334-30-5$ & Diesel-Range Organics & 22 & $\mathrm{mg} / \mathrm{kg}$ & $\mathrm{Z}, \mathrm{H}$ & 5.8 \\
\hline 383PB0300.5X & $6 / 17 / 2004$ & soil & EPA8270 & FD & 84-66-2 & Diethyl Phthalate & 89 & $\mu \mathrm{g} / \mathrm{kg}$ & $\mathrm{J}$ & 380 \\
\hline 383PB0300.5X & $6 / 17 / 2004$ & soil & EPA8270 & FD & $206-44-0$ & Fluoranthene & 23 & $\mu \mathrm{g} / \mathrm{kg}$ & $\mathrm{J}$ & 380 \\
\hline 383PB0300.5X & $6 / 17 / 2004$ & soil & HASL300 & FD & $13966-00-2$ & $\mathrm{~K}-40$ & 22.8 & $\mathrm{pCi} / \mathrm{g}$ & G & 2.3 \\
\hline
\end{tabular}

Page 68 of 78

Uncontrolled When Printed 


\begin{tabular}{|c|c|c|c|c|c|c|c|c|c|c|}
\hline SAMPLE \# & $\begin{array}{c}\text { SAMPLE } \\
\text { DATE }\end{array}$ & MATRIX & $\begin{array}{l}\text { USER TEST } \\
\text { PANEL }\end{array}$ & $\begin{array}{c}\text { SAMPLE } \\
\text { PURPOSE }\end{array}$ & CAS \# & PARAMETER & RESULT & UNITS & $\mathbf{Q}$ & $\begin{array}{c}\text { DETECT } \\
\text { LIMIT }\end{array}$ \\
\hline 383PB0300.5X & $6 / 17 / 2004$ & soil & EPA6010 & FD & 7439-92-1 & Lead & 38 & $\mathrm{mg} / \mathrm{kg}$ & & 0.7 \\
\hline 383PB0300.5X & $6 / 17 / 2004$ & soil & EPA7470 & FD & 7439-97-6 & Mercury & 0.045 & $\mathrm{mg} / \mathrm{kg}$ & $\mathrm{B}$ & 0.12 \\
\hline 383PB0300.5X & $6 / 17 / 2004$ & soil & HASL300 & FD & 15092-94-1 & $\mathrm{Pb}-212$ & 2.03 & $\mathrm{pCi} / \mathrm{g}$ & $G$ & 0.53 \\
\hline 383РВ0300.5X & $6 / 17 / 2004$ & soil & HASL300 & FD & $15067-28-4$ & $\mathrm{~Pb}-214$ & 1.21 & $\mathrm{pCi} / \mathrm{g}$ & $\mathrm{G}, \mathrm{J}$ & 0.68 \\
\hline 383PB0300.5X & $6 / 17 / 2004$ & soil & EPA8270 & FD & 85-01-8 & Phenanthrene & 18 & $\mu \mathrm{g} / \mathrm{kg}$ & $\mathrm{J}$ & 380 \\
\hline 383РВ0300.5X & $6 / 17 / 2004$ & soil & UGTAISOPU & FD & 13981-16-3 & Pu-238 & 0.361 & $\mathrm{pCi} / \mathrm{g}$ & & 0.017 \\
\hline 383PB0300.5X & $6 / 17 / 2004$ & soil & UGTAISOPU & FD & $15117-48-3$ & Pu-239 & 4.48 & $\mathrm{pCi} / \mathrm{g}$ & & 0.01 \\
\hline 383PB0300.5X & $6 / 17 / 2004$ & soil & EPA8270 & FD & $129-00-0$ & Pyrene & 51 & $\mu \mathrm{g} / \mathrm{kg}$ & $\mathrm{J}$ & 380 \\
\hline 383PB0300.5X & $6 / 17 / 2004$ & soil & SR7500 & FD & 10098-97-2 & Sr-90 & 3.62 & $\mathrm{pCi} / \mathrm{g}$ & $\mathrm{Y} 1$ & 0.23 \\
\hline 383РВ0300.5X & $6 / 17 / 2004$ & soil & HASL300 & FD & $14913-50-9$ & TI-208 & 0.6 & $\mathrm{pCi} / \mathrm{g}$ & G & 0.36 \\
\hline 383PB0400.5 & $6 / 16 / 2004$ & soil & HASL300 & REG & 14331-83-0 & Ac-228 & 1.48 & $\mathrm{pCi} / \mathrm{g}$ & $\mathrm{G}$ & 0.67 \\
\hline 383PB0400.5 & $6 / 16 / 2004$ & soil & EPA6010 & REG & $7440-38-2$ & Arsenic & 2.5 & $\mathrm{mg} / \mathrm{kg}$ & & 1 \\
\hline 383PB0400.5 & $6 / 16 / 2004$ & soil & EPA6010 & REG & $7440-39-3$ & Barium & 4900 & $\mathrm{mg} / \mathrm{kg}$ & & 520 \\
\hline 383PB0400.5 & $6 / 16 / 2004$ & soil & EPA6010 & REG & $7440-41-7$ & Beryllium & 1.6 & $\mathrm{mg} / \mathrm{kg}$ & & 0.52 \\
\hline 383PB0400.5 & $6 / 16 / 2004$ & soil & HASL300 & REG & 14733-03-0 & Bi-214 & 0.95 & $\mathrm{pCi} / \mathrm{g}$ & $\mathrm{G}, \mathrm{J}$ & 0.45 \\
\hline 383PB0400.5 & $6 / 16 / 2004$ & soil & EPA6010 & REG & $7440-43-9$ & Cadmium & 0.19 & $\mathrm{mg} / \mathrm{kg}$ & $\mathrm{B}$ & 0.52 \\
\hline 383PB0400.5 & $6 / 16 / 2004$ & soil & EPA6010 & REG & $7440-47-3$ & Chromium & 4 & $\mathrm{mg} / \mathrm{kg}$ & & 1 \\
\hline 383PB0400.5 & $6 / 16 / 2004$ & soil & HASL300 & REG & 10045-97-3 & Cs-137 & 46.9 & $\mathrm{pCi} / \mathrm{g}$ & $G$ & 0.2 \\
\hline 383PB0400.5 & $6 / 16 / 2004$ & soil & EPA8260 & REG & $100-41-4$ & Ethylbenzene & 1.6 & $\mu \mathrm{g} / \mathrm{kg}$ & $\mathrm{J}$ & 5.2 \\
\hline 383PB0400.5 & $6 / 16 / 2004$ & soil & EPA8270 & REG & $206-44-0$ & Fluoranthene & 20 & $\mu \mathrm{g} / \mathrm{kg}$ & $\mathrm{J}$ & 350 \\
\hline 383PB0400.5 & $6 / 16 / 2004$ & soil & HASL300 & REG & 13966-00-2 & $\mathrm{K}-40$ & 31.7 & $\mathrm{pCi} / \mathrm{g}$ & $G$ & 1.7 \\
\hline 383PB0400.5 & $6 / 16 / 2004$ & soil & EPA6010 & REG & 7439-92-1 & Lead & 30 & $\mathrm{mg} / \mathrm{kg}$ & & 0.31 \\
\hline 383PB0400.5 & $6 / 16 / 2004$ & soil & EPA8260 & REG & 136777-61-2 & M+P-Xylene & 8.3 & $\mu \mathrm{g} / \mathrm{kg}$ & & 5.2 \\
\hline 383PB0400.5 & $6 / 16 / 2004$ & soil & EPA7470 & REG & 7439-97-6 & Mercury & 0.027 & $\mathrm{mg} / \mathrm{kg}$ & $\mathrm{B}$ & 0.1 \\
\hline 383PB0400.5 & $6 / 16 / 2004$ & soil & EPA8260 & REG & $75-09-2$ & Methylene Chloride & 1.2 & $\mu \mathrm{g} / \mathrm{kg}$ & $\mathrm{J}, \mathrm{B}$ & 5.2 \\
\hline 383PB0400.5 & $6 / 16 / 2004$ & soil & EPA8260 & REG & $95-47-6$ & O-Xylene & 3.3 & $\mu \mathrm{g} / \mathrm{kg}$ & $\mathrm{J}$ & 5.2 \\
\hline 383PB0400.5 & $6 / 16 / 2004$ & soil & HASL300 & REG & 15092-94-1 & $\mathrm{Pb}-212$ & 2.04 & $\mathrm{pCi} / \mathrm{g}$ & $\mathrm{J}$ & 0.48 \\
\hline 383PB0400.5 & $6 / 16 / 2004$ & soil & HASL300 & REG & $15067-28-4$ & $\mathrm{~Pb}-214$ & 1.53 & $\mathrm{pCi} / \mathrm{g}$ & $\mathrm{G}, \mathrm{J}$ & 0.73 \\
\hline 383PB0400.5 & $6 / 16 / 2004$ & soil & UGTAISOPU & REG & 13981-16-3 & Pu-238 & 0.42 & $\mathrm{pCi} / \mathrm{g}$ & & 0.02 \\
\hline 383PB0400.5 & $6 / 16 / 2004$ & soil & UGTAISOPU & REG & $15117-48-3$ & Pu-239 & 3.25 & $\mathrm{pCi} / \mathrm{g}$ & & 0.01 \\
\hline 383РB0400.5 & $6 / 16 / 2004$ & soil & SR7500 & REG & 10098-97-2 & Sr-90 & 0.76 & $\mathrm{pCi} / \mathrm{g}$ & & 0.29 \\
\hline 383PB0400.5 & $6 / 16 / 2004$ & soil & HASL300 & REG & $14913-50-9$ & TI-208 & 0.51 & $\mathrm{pCi} / \mathrm{g}$ & $\mathrm{G}$ & 0.23 \\
\hline 383PB0500.5 & $6 / 16 / 2004$ & soil & HASL300 & REG & 14331-83-0 & Ac-228 & 1.79 & $\mathrm{pCi} / \mathrm{g}$ & $G$ & 0.46 \\
\hline 383PB0500.5 & $6 / 16 / 2004$ & soil & EPA6010 & REG & $7440-38-2$ & Arsenic & 2.1 & $\mathrm{mg} / \mathrm{kg}$ & & 1 \\
\hline 383PB0500.5 & $6 / 16 / 2004$ & soil & EPA6010 & REG & $7440-39-3$ & Barium & 550 & $\mathrm{mg} / \mathrm{kg}$ & & 10 \\
\hline
\end{tabular}

Page 69 of 78

Uncontrolled When Printed 


\begin{tabular}{|c|c|c|c|c|c|c|c|c|c|c|}
\hline SAMPLE \# & $\begin{array}{c}\text { SAMPLE } \\
\text { DATE }\end{array}$ & MATRIX & $\begin{array}{c}\text { USER TEST } \\
\text { PANEL }\end{array}$ & $\begin{array}{c}\text { SAMPLE } \\
\text { PURPOSE }\end{array}$ & CAS \# & PARAMETER & RESULT & UNITS & $\mathbf{Q}$ & $\begin{array}{c}\text { DETECT } \\
\text { LIMIT }\end{array}$ \\
\hline 383PB0500.5 & $6 / 16 / 2004$ & soil & EPA6010 & REG & $7440-41-7$ & Beryllium & 0.81 & $\mathrm{mg} / \mathrm{kg}$ & & 0.51 \\
\hline 383PB0500.5 & $6 / 16 / 2004$ & soil & HASL300 & REG & $14733-03-0$ & Bi-214 & 1.06 & $\mathrm{pCi} / \mathrm{g}$ & $\mathrm{G}, \mathrm{J}$ & 0.26 \\
\hline 383PB0500.5 & $6 / 16 / 2004$ & soil & EPA6010 & REG & $7440-43-9$ & Cadmium & 0.052 & $\mathrm{mg} / \mathrm{kg}$ & $\mathrm{B}$ & 0.51 \\
\hline 383РВ0500.5 & $6 / 16 / 2004$ & soil & EPA6010 & REG & $7440-47-3$ & Chromium & 2.5 & $\mathrm{mg} / \mathrm{kg}$ & & 1 \\
\hline 383PB0500.5 & $6 / 16 / 2004$ & soil & HASL300 & REG & $10045-97-3$ & Cs-137 & 0.86 & $\mathrm{pCi} / \mathrm{g}$ & G & 0.13 \\
\hline 383PB0500.5 & $6 / 16 / 2004$ & soil & EPA8015 & REG & $68334-30-5$ & Diesel-Range Organics & 100 & $\mathrm{mg} / \mathrm{kg}$ & $\mathrm{Z}, \mathrm{H}$ & 5.1 \\
\hline 383PB0500.5 & $6 / 16 / 2004$ & soil & HASL300 & REG & $13966-00-2$ & $\mathrm{~K}-40$ & 30.5 & $\mathrm{pCi} / \mathrm{g}$ & G & 1.4 \\
\hline 383PB0500.5 & $6 / 16 / 2004$ & soil & EPA6010 & REG & 7439-92-1 & Lead & 15 & $\mathrm{mg} / \mathrm{kg}$ & & 0.31 \\
\hline 383РВ0500.5 & $6 / 16 / 2004$ & soil & EPA7470 & REG & 7439-97-6 & Mercury & 0.013 & $\mathrm{mg} / \mathrm{kg}$ & $\mathrm{B}$ & 0.1 \\
\hline 383РВ0500.5 & $6 / 16 / 2004$ & soil & EPA8260 & REG & $75-09-2$ & Methylene Chloride & 0.95 & $\mu \mathrm{g} / \mathrm{kg}$ & $\mathrm{J}, \mathrm{B}$ & 5.1 \\
\hline 383PB0500.5 & $6 / 16 / 2004$ & soil & HASL300 & REG & 15092-94-1 & $\mathrm{Pb}-212$ & 2.21 & $\mathrm{pCi} / \mathrm{g}$ & $\mathrm{J}$ & 0.2 \\
\hline 383PB0500.5 & $6 / 16 / 2004$ & soil & HASL300 & REG & $15067-28-4$ & $\mathrm{~Pb}-214$ & 1.09 & $\mathrm{pCi} / \mathrm{g}$ & $\mathrm{G}, \mathrm{J}$ & 0.23 \\
\hline 383PB0500.5 & $6 / 16 / 2004$ & soil & UGTAISOPU & REG & $15117-48-3$ & Pu-239 & 0.318 & $\mathrm{pCi} / \mathrm{g}$ & & 0.011 \\
\hline 383PB0500.5 & $6 / 16 / 2004$ & soil & EPA6010 & REG & $7782-49-2$ & Selenium & 0.65 & $\mathrm{mg} / \mathrm{kg}$ & & 0.51 \\
\hline 383PB0500.5 & $6 / 16 / 2004$ & soil & HASL300 & REG & 14913-50-9 & TI-208 & 0.65 & $\mathrm{pCi} / \mathrm{g}$ & $G$ & 0.12 \\
\hline 383РН0101.0 & 6/9/2004 & soil & EPA8260 & REG & $67-64-1$ & Acetone & 14 & $\mu \mathrm{g} / \mathrm{kg}$ & $\mathrm{J}$ & 38 \\
\hline 383РН0101.0 & $6 / 9 / 2004$ & soil & EPA6010 & REG & $7440-38-2$ & Arsenic & 14 & $\mathrm{mg} / \mathrm{kg}$ & & 1.9 \\
\hline 383РН0101.0 & $6 / 9 / 2004$ & soil & EPA6010 & REG & $7440-39-3$ & Barium & 6300 & $\mathrm{mg} / \mathrm{kg}$ & & 95 \\
\hline 383РН0101.0 & $6 / 9 / 2004$ & soil & EPA6010 & REG & $7440-41-7$ & Beryllium & 1.1 & $\mathrm{mg} / \mathrm{kg}$ & & 0.95 \\
\hline 383РН0101.0 & $6 / 9 / 2004$ & soil & HASL300 & REG & $14733-03-0$ & Bi-214 & 1 & $\mathrm{pCi} / \mathrm{g}$ & $\mathrm{G}, \mathrm{J}$ & 0.56 \\
\hline 383РН0101.0 & $6 / 9 / 2004$ & soil & EPA6010 & REG & $7440-43-9$ & Cadmium & 0.39 & $\mathrm{mg} / \mathrm{kg}$ & $\mathrm{B}$ & 0.95 \\
\hline 383РН0101.0 & $6 / 9 / 2004$ & soil & EPA6010 & REG & $7440-47-3$ & Chromium & 12 & $\mathrm{mg} / \mathrm{kg}$ & & 1.9 \\
\hline 383РН0101.0 & $6 / 9 / 2004$ & soil & HASL300 & REG & $10045-97-3$ & Cs-137 & 19 & $\mathrm{pCi} / \mathrm{g}$ & G & 0.3 \\
\hline 383РН0101.0 & $6 / 9 / 2004$ & soil & EPA8015 & REG & $68334-30-5$ & Diesel-Range Organics & 21 & $\mathrm{mg} / \mathrm{kg}$ & $\mathrm{Z}, \mathrm{H}$ & 9.5 \\
\hline 383РH0101.0 & $6 / 9 / 2004$ & soil & HASL300 & REG & $13966-00-2$ & $\mathrm{~K}-40$ & 8.1 & $\mathrm{pCi} / \mathrm{g}$ & $G$ & 3.2 \\
\hline 383РН0101.0 & $6 / 9 / 2004$ & soil & EPA6010 & REG & $7439-92-1$ & Lead & 19 & $\mathrm{mg} / \mathrm{kg}$ & & 2.8 \\
\hline 383PH0101.0 & $6 / 9 / 2004$ & soil & EPA7470 & REG & 7439-97-6 & Mercury & 0.046 & $\mathrm{mg} / \mathrm{kg}$ & $\mathrm{B}$ & 0.19 \\
\hline 383РН0101.0 & $6 / 9 / 2004$ & soil & HASL300 & REG & 15092-94-1 & $\mathrm{Pb}-212$ & 1.29 & $\mathrm{pCi} / \mathrm{g}$ & $\mathrm{J}$ & 0.49 \\
\hline 383РН0101.0 & $6 / 9 / 2004$ & soil & HASL300 & REG & $15067-28-4$ & $\mathrm{~Pb}-214$ & 1.25 & $\mathrm{pCi} / \mathrm{g}$ & $\mathrm{G}, \mathrm{J}$ & 0.53 \\
\hline 383РН0101.0 & $6 / 9 / 2004$ & soil & UGTAISOPU & REG & $15117-48-3$ & Pu-239 & 0.305 & $\mathrm{pCi} / \mathrm{g}$ & $\mathrm{J}$ & 0.01 \\
\hline 383РН0101.0 & $6 / 9 / 2004$ & soil & SR7500 & REG & 10098-97-2 & Sr-90 & 1.33 & $\mathrm{pCi} / \mathrm{g}$ & & 0.23 \\
\hline 383РН0101.0 & $6 / 9 / 2004$ & soil & HASL300 & REG & $14913-50-9$ & TI-208 & 0.61 & $\mathrm{pCi} / \mathrm{g}$ & $\mathrm{G}$ & 0.27 \\
\hline 383РН0116.5 & $6 / 9 / 2004$ & soil & HASL300 & REG & 14331-83-0 & Ac-228 & 2.4 & $\mathrm{pCi} / \mathrm{g}$ & $G$ & 0.49 \\
\hline 383РН0116.5 & $6 / 9 / 2004$ & soil & EPA8260 & REG & 67-64-1 & Acetone & 7.2 & $\mu \mathrm{g} / \mathrm{kg}$ & $\mathrm{J}$ & 21 \\
\hline 383РН0116.5 & $6 / 9 / 2004$ & soil & EPA6010 & REG & $7440-38-2$ & Arsenic & 2 & $\mathrm{mg} / \mathrm{kg}$ & & 1 \\
\hline
\end{tabular}

Page 70 of 78

Uncontrolled When Printed 


\begin{tabular}{|c|c|c|c|c|c|c|c|c|c|c|}
\hline SAMPLE \# & $\begin{array}{l}\text { SAMPLE } \\
\text { DATE }\end{array}$ & MATRIX & $\begin{array}{l}\text { USER TEST } \\
\text { PANEL }\end{array}$ & $\begin{array}{c}\text { SAMPLE } \\
\text { PURPOSE }\end{array}$ & CAS \# & PARAMETER & RESULT & UNITS & $\mathbf{Q}$ & $\begin{array}{c}\text { DETECT } \\
\text { LIMIT }\end{array}$ \\
\hline 383РН0116.5 & $6 / 9 / 2004$ & soil & EPA6010 & REG & $7440-39-3$ & Barium & 71 & $\mathrm{mg} / \mathrm{kg}$ & & 10 \\
\hline 383РН0116.5 & $6 / 9 / 2004$ & soil & EPA6010 & REG & $7440-41-7$ & Beryllium & 0.65 & $\mathrm{mg} / \mathrm{kg}$ & & 0.52 \\
\hline 383РН0116.5 & $6 / 9 / 2004$ & soil & HASL300 & REG & $14733-03-0$ & Bi-214 & 1.46 & $\mathrm{pCi} / \mathrm{g}$ & $\mathrm{G}, \mathrm{J}$ & 0.24 \\
\hline 383РН0116.5 & $6 / 9 / 2004$ & soil & EPA6010 & REG & $7440-43-9$ & Cadmium & 0.11 & $\mathrm{mg} / \mathrm{kg}$ & B & 0.52 \\
\hline 383РН0116.5 & $6 / 9 / 2004$ & soil & EPA6010 & REG & $7440-47-3$ & Chromium & 1.7 & $\mathrm{mg} / \mathrm{kg}$ & & 1 \\
\hline 383РН0116.5 & $6 / 9 / 2004$ & soil & HASL300 & REG & 13966-00-2 & $\mathrm{K}-40$ & 29.9 & $\mathrm{pCi} / \mathrm{g}$ & $\mathrm{G}$ & 1.6 \\
\hline 383PH0116.5 & $6 / 9 / 2004$ & soil & EPA6010 & REG & 7439-92-1 & Lead & 7.2 & $\mathrm{mg} / \mathrm{kg}$ & & 0.31 \\
\hline 383РН0116.5 & 6/9/2004 & soil & EPA7470 & REG & 7439-97-6 & Mercury & 0.015 & $\mathrm{mg} / \mathrm{kg}$ & $B$ & 0.1 \\
\hline 383РН0116.5 & $6 / 9 / 2004$ & soil & HASL300 & REG & 15092-94-1 & $\mathrm{Pb}-212$ & 2.51 & $\mathrm{pCi} / \mathrm{g}$ & $\mathrm{J}$ & 0.21 \\
\hline 383РН0116.5 & $6 / 9 / 2004$ & soil & HASL300 & REG & $15067-28-4$ & $\mathrm{~Pb}-214$ & 1.61 & $\mathrm{pCi} / \mathrm{g}$ & $\mathrm{G}, \mathrm{J}$ & 0.25 \\
\hline 383РН0116.5 & $6 / 9 / 2004$ & soil & HASL300 & REG & $14913-50-9$ & TI-208 & 1.05 & $\mathrm{pCi} / \mathrm{g}$ & G & 0.12 \\
\hline 383РН0216.0 & $6 / 9 / 2004$ & soil & HASL300 & REG & $14331-83-0$ & Ac-228 & 2.31 & $\mathrm{pCi} / \mathrm{g}$ & $\mathrm{G}$ & 0.72 \\
\hline 383РH0216.0 & $6 / 9 / 2004$ & soil & EPA6010 & REG & $7440-38-2$ & Arsenic & 2.3 & $\mathrm{mg} / \mathrm{kg}$ & & 1.2 \\
\hline 383PH0216.0 & $6 / 9 / 2004$ & soil & EPA6010 & REG & $7440-39-3$ & Barium & 320 & $\mathrm{mg} / \mathrm{kg}$ & & 12 \\
\hline 383РH0216.0 & $6 / 9 / 2004$ & soil & EPA8270 & REG & $56-55-3$ & Benzo(A)Anthracene & 26 & $\mu \mathrm{g} / \mathrm{kg}$ & $\mathrm{J}$ & 390 \\
\hline 383РH0216.0 & $6 / 9 / 2004$ & soil & EPA8270 & REG & $50-32-8$ & Benzo(A)Pyrene & 28 & $\mu \mathrm{g} / \mathrm{kg}$ & $\mathrm{J}$ & 390 \\
\hline 383PH0216.0 & $6 / 9 / 2004$ & soil & EPA8270 & REG & 205-99-2 & Benzo(B)Fluoranthene & 59 & $\mu \mathrm{g} / \mathrm{kg}$ & $\mathrm{J}$ & 390 \\
\hline 383РН0216.0 & $6 / 9 / 2004$ & soil & EPA8270 & REG & 207-08-9 & Benzo(K)Fluoranthene & 31 & $\mu \mathrm{g} / \mathrm{kg}$ & $\mathrm{J}$ & 390 \\
\hline 383РH0216.0 & $6 / 9 / 2004$ & soil & EPA6010 & REG & $7440-41-7$ & Beryllium & 1.3 & $\mathrm{mg} / \mathrm{kg}$ & & 0.58 \\
\hline 383PH0216.0 & $6 / 9 / 2004$ & soil & HASL300 & REG & $14733-03-0$ & Bi-214 & 1.16 & $\mathrm{pCi} / \mathrm{g}$ & $\mathrm{G}, \mathrm{J}$ & 0.59 \\
\hline 383РH0216.0 & $6 / 9 / 2004$ & soil & EPA8270 & REG & $117-81-7$ & Bis(2-Ethylhexyl)Phthalate & 330 & $\mu \mathrm{g} / \mathrm{kg}$ & $\mathrm{J}$ & 390 \\
\hline 383PH0216.0 & $6 / 9 / 2004$ & soil & EPA6010 & REG & $7440-43-9$ & Cadmium & 0.31 & $\mathrm{mg} / \mathrm{kg}$ & $\mathrm{B}$ & 0.58 \\
\hline 383PH0216.0 & $6 / 9 / 2004$ & soil & EPA6010 & REG & $7440-47-3$ & Chromium & 1.4 & $\mathrm{mg} / \mathrm{kg}$ & & 1.2 \\
\hline 383РH0216.0 & $6 / 9 / 2004$ & soil & EPA8270 & REG & 218-01-9 & Chrysene & 66 & $\mu \mathrm{g} / \mathrm{kg}$ & $\mathrm{J}$ & 390 \\
\hline 383РH0216.0 & $6 / 9 / 2004$ & soil & HASL300 & REG & $10045-97-3$ & Cs-137 & 142 & $\mathrm{pCi} / \mathrm{g}$ & G & 0 \\
\hline 383РH0216.0 & $6 / 9 / 2004$ & soil & EPA8015 & REG & $68334-30-5$ & Diesel-Range Organics & 330 & $\mathrm{mg} / \mathrm{kg}$ & $\mathrm{M}$ & 5.8 \\
\hline 383PH0216.0 & $6 / 9 / 2004$ & soil & EPA8270 & REG & 206-44-0 & Fluoranthene & 31 & $\mu \mathrm{g} / \mathrm{kg}$ & $\mathrm{J}$ & 390 \\
\hline 383РH0216.0 & $6 / 9 / 2004$ & soil & HASL300 & REG & $13966-00-2$ & $\mathrm{~K}-40$ & 33.7 & $\mathrm{pCi} / \mathrm{g}$ & $\mathrm{G}$ & 1.9 \\
\hline 383РH0216.0 & $6 / 9 / 2004$ & soil & EPA6010 & REG & 7439-92-1 & Lead & 12 & $\mathrm{mg} / \mathrm{kg}$ & & 0.35 \\
\hline 383PH0216.0 & $6 / 9 / 2004$ & soil & EPA7470 & REG & 7439-97-6 & Mercury & 0.027 & $\mathrm{mg} / \mathrm{kg}$ & $\mathrm{B}$ & 0.12 \\
\hline 383РH0216.0 & $6 / 9 / 2004$ & soil & HASL300 & REG & 15092-94-1 & $\mathrm{Pb}-212$ & 2.73 & $\mathrm{pCi} / \mathrm{g}$ & $\mathrm{J}$ & 0.62 \\
\hline 383PH0216.0 & $6 / 9 / 2004$ & soil & UGTAISOPU & REG & 13981-16-3 & Pu-238 & 0.74 & $\mathrm{pCi} / \mathrm{g}$ & & 0.02 \\
\hline 383РН0216.0 & $6 / 9 / 2004$ & soil & UGTAISOPU & REG & $15117-48-3$ & Pu-239 & 5.37 & $\mathrm{pCi} / \mathrm{g}$ & $\mathrm{J}$ & 0.01 \\
\hline 383РН0216.0 & $6 / 9 / 2004$ & soil & EPA8270 & REG & $129-00-0$ & Pyrene & 63 & $\mu \mathrm{g} / \mathrm{kg}$ & $\mathrm{J}$ & 390 \\
\hline 383РН0216.0 & $6 / 9 / 2004$ & soil & EPA6010 & REG & $7782-49-2$ & Selenium & 0.42 & $\mathrm{mg} / \mathrm{kg}$ & $\mathrm{B}$ & 0.58 \\
\hline
\end{tabular}

Page 71 of 78

Uncontrolled When Printed 


\begin{tabular}{|c|c|c|c|c|c|c|c|c|c|c|}
\hline SAMPLE \# & $\begin{array}{l}\text { SAMPLE } \\
\text { DATE }\end{array}$ & MATRIX & $\begin{array}{l}\text { USER TEST } \\
\text { PANEL }\end{array}$ & $\begin{array}{c}\text { SAMPLE } \\
\text { PURPOSE }\end{array}$ & CAS \# & PARAMETER & RESULT & UNITS & $\mathbf{Q}$ & $\begin{array}{c}\text { DETECT } \\
\text { LIMIT }\end{array}$ \\
\hline 383PH0216.0 & $6 / 9 / 2004$ & soil & SR7500 & REG & 10098-97-2 & Sr-90 & 2.84 & $\mathrm{pCi} / \mathrm{g}$ & & 0.25 \\
\hline 383РН0216.0 & $6 / 9 / 2004$ & soil & HASL300 & REG & $14913-50-9$ & TI-208 & 0.78 & $\mathrm{pCi} / \mathrm{g}$ & $G$ & 0.33 \\
\hline 383РН0217.5 & $6 / 9 / 2004$ & soil & HASL300 & REG & 14331-83-0 & Ac-228 & 2.27 & $\mathrm{pCi} / \mathrm{g}$ & G & 0.6 \\
\hline 383PH0217.5 & $6 / 9 / 2004$ & soil & EPA8260 & REG & 67-64-1 & Acetone & 25 & $\mu \mathrm{g} / \mathrm{kg}$ & & 21 \\
\hline 383PH0217.5 & $6 / 9 / 2004$ & soil & EPA6010 & REG & $7440-38-2$ & Arsenic & 1.7 & $\mathrm{mg} / \mathrm{kg}$ & & 1.1 \\
\hline 383РH0217.5 & $6 / 9 / 2004$ & soil & EPA6010 & REG & $7440-39-3$ & Barium & 3200 & $\mathrm{mg} / \mathrm{kg}$ & & 110 \\
\hline 383PH0217.5 & $6 / 9 / 2004$ & soil & EPA6010 & REG & $7440-41-7$ & Beryllium & 0.69 & $\mathrm{mg} / \mathrm{kg}$ & & 0.53 \\
\hline 383РН0217.5 & $6 / 9 / 2004$ & soil & HASL300 & REG & $14733-03-0$ & Bi-214 & 1.49 & $\mathrm{pCi} / \mathrm{g}$ & $\mathrm{G}, \mathrm{J}$ & 0.31 \\
\hline 383PH0217.5 & $6 / 9 / 2004$ & soil & EPA6010 & REG & $7440-43-9$ & Cadmium & 0.12 & $\mathrm{mg} / \mathrm{kg}$ & $\mathrm{B}$ & 0.53 \\
\hline 383PH0217.5 & $6 / 9 / 2004$ & soil & EPA6010 & REG & $7440-47-3$ & Chromium & 3.3 & $\mathrm{mg} / \mathrm{kg}$ & & 1.1 \\
\hline 383РН0217.5 & $6 / 9 / 2004$ & soil & HASL300 & REG & $10045-97-3$ & Cs-137 & 4.34 & $\mathrm{pCi} / \mathrm{g}$ & $\mathrm{G}$ & 0.17 \\
\hline 383РН0217.5 & $6 / 9 / 2004$ & soil & HASL300 & REG & 13966-00-2 & $\mathrm{K}-40$ & 33.2 & $\mathrm{pCi} / \mathrm{g}$ & $\mathrm{G}$ & 1.4 \\
\hline 383PH0217.5 & $6 / 9 / 2004$ & soil & EPA6010 & REG & 7439-92-1 & Lead & 12 & $\mathrm{mg} / \mathrm{kg}$ & & 0.32 \\
\hline 383PH0217.5 & $6 / 9 / 2004$ & soil & EPA7470 & REG & 7439-97-6 & Mercury & 0.011 & $\mathrm{mg} / \mathrm{kg}$ & $\mathrm{B}$ & 0.11 \\
\hline 383РH0217.5 & $6 / 9 / 2004$ & soil & HASL300 & REG & 15092-94-1 & $\mathrm{Pb}-212$ & 2.4 & $\mathrm{pCi} / \mathrm{g}$ & $\mathrm{J}$ & 0.22 \\
\hline 383PH0217.5 & $6 / 9 / 2004$ & soil & HASL300 & REG & 15067-28-4 & $\mathrm{Pb}-214$ & 1.42 & $\mathrm{pCi} / \mathrm{g}$ & $\mathrm{G}, \mathrm{J}$ & 0.31 \\
\hline 383PH0217.5 & $6 / 9 / 2004$ & soil & UGTAISOPU & REG & $15117-48-3$ & Pu-239 & 0.154 & $\mathrm{pCi} / \mathrm{g}$ & $\mathrm{J}$ & 0.02 \\
\hline 383РH0217.5 & $6 / 9 / 2004$ & soil & EPA6010 & REG & $7440-22-4$ & Silver & 0.48 & $\mathrm{mg} / \mathrm{kg}$ & $\mathrm{B}$ & 1.1 \\
\hline 383PH0217.5 & $6 / 9 / 2004$ & soil & HASL300 & REG & 14913-50-9 & TI-208 & 0.8 & $\mathrm{pCi} / \mathrm{g}$ & $G$ & 0.16 \\
\hline 383РH0306.5 & $6 / 9 / 2004$ & soil & HASL300 & REG & 14331-83-0 & Ac-228 & 2.18 & $\mathrm{pCi} / \mathrm{g}$ & $G$ & 0.58 \\
\hline 383РН0306.5 & $6 / 9 / 2004$ & soil & EPA8260 & REG & 67-64-1 & Acetone & 7.2 & $\mu \mathrm{g} / \mathrm{kg}$ & $\mathrm{J}$ & 23 \\
\hline 383РН0306.5 & $6 / 9 / 2004$ & soil & EPA6010 & REG & $7440-38-2$ & Arsenic & 2 & $\mathrm{mg} / \mathrm{kg}$ & & 1.1 \\
\hline 383РН0306.5 & $6 / 9 / 2004$ & soil & EPA6010 & REG & $7440-39-3$ & Barium & 4000 & $\mathrm{mg} / \mathrm{kg}$ & & 110 \\
\hline 383РН0306.5 & $6 / 9 / 2004$ & soil & EPA6010 & REG & 7440-41-7 & Beryllium & 2 & $\mathrm{mg} / \mathrm{kg}$ & & 0.57 \\
\hline 383РН0306.5 & $6 / 9 / 2004$ & soil & HASL300 & REG & $14733-03-0$ & Bi-214 & 1.72 & $\mathrm{pCi} / \mathrm{g}$ & $\mathrm{G}, \mathrm{J}$ & 0.33 \\
\hline 383РН0306.5 & $6 / 9 / 2004$ & soil & EPA6010 & REG & $7440-43-9$ & Cadmium & 0.24 & $\mathrm{mg} / \mathrm{kg}$ & $B$ & 0.57 \\
\hline 383РН0306.5 & $6 / 9 / 2004$ & soil & EPA6010 & REG & $7440-47-3$ & Chromium & 3.4 & $\mathrm{mg} / \mathrm{kg}$ & & 1.1 \\
\hline 383РH0306.5 & $6 / 9 / 2004$ & soil & HASL300 & REG & $10045-97-3$ & Cs-137 & 1.33 & $\mathrm{pCi} / \mathrm{g}$ & G & 0.14 \\
\hline 383РН0306.5 & $6 / 9 / 2004$ & soil & EPA8015 & REG & $68334-30-5$ & Diesel-Range Organics & 1.9 & $\mathrm{mg} / \mathrm{kg}$ & $\mathrm{J}$ & 5.7 \\
\hline 383РH0306.5 & $6 / 9 / 2004$ & soil & HASL300 & REG & $13966-00-2$ & $\mathrm{~K}-40$ & 29 & $\mathrm{pCi} / \mathrm{g}$ & $\mathrm{G}$ & 1.2 \\
\hline 383РH0306.5 & $6 / 9 / 2004$ & soil & EPA6010 & REG & 7439-92-1 & Lead & 22 & $\mathrm{mg} / \mathrm{kg}$ & & 0.34 \\
\hline 383РH0306.5 & $6 / 9 / 2004$ & soil & EPA7470 & REG & 7439-97-6 & Mercury & 0.011 & $\mathrm{mg} / \mathrm{kg}$ & $\mathrm{B}$ & 0.11 \\
\hline 383РH0306.5 & $6 / 9 / 2004$ & soil & HASL300 & REG & 15092-94-1 & $\mathrm{Pb}-212$ & 2.56 & $\mathrm{pCi} / \mathrm{g}$ & $\mathrm{J}$ & 0.25 \\
\hline 383РН0306.5 & $6 / 9 / 2004$ & soil & HASL300 & REG & $15067-28-4$ & $\mathrm{~Pb}-214$ & 1.74 & $\mathrm{pCi} / \mathrm{g}$ & $\mathrm{G}, \mathrm{J}$ & 0.3 \\
\hline 383РН0306.5 & $6 / 9 / 2004$ & soil & EPA6010 & REG & 7782-49-2 & Selenium & 0.31 & $\mathrm{mg} / \mathrm{kg}$ & $\mathrm{B}$ & 0.57 \\
\hline
\end{tabular}

Page 72 of 78

Uncontrolled When Printed 


\begin{tabular}{|c|c|c|c|c|c|c|c|c|c|c|}
\hline SAMPLE \# & $\begin{array}{c}\text { SAMPLE } \\
\text { DATE }\end{array}$ & MATRIX & $\begin{array}{l}\text { USER TEST } \\
\text { PANEL }\end{array}$ & $\begin{array}{c}\text { SAMPLE } \\
\text { PURPOSE }\end{array}$ & CAS \# & PARAMETER & RESULT & UNITS & $\mathbf{Q}$ & $\begin{array}{l}\text { DETECT } \\
\text { LIMIT }\end{array}$ \\
\hline 383РН0306.5 & $6 / 9 / 2004$ & soil & HASL300 & REG & $14913-50-9$ & TI-208 & 0.74 & $\mathrm{pCi} / \mathrm{g}$ & $\mathrm{G}$ & 0.12 \\
\hline 383РН0320.5 & $6 / 9 / 2004$ & soil & HASL300 & REG & 14331-83-0 & Ac-228 & 2.3 & $\mathrm{pCi} / \mathrm{g}$ & $G$ & 0.51 \\
\hline 383РН0320.5 & $6 / 9 / 2004$ & soil & EPA8260 & REG & 67-64-1 & Acetone & 7.9 & $\mu \mathrm{g} / \mathrm{kg}$ & $\mathrm{J}$ & 22 \\
\hline 383РН0320.5 & $6 / 9 / 2004$ & soil & EPA6010 & REG & $7440-38-2$ & Arsenic & 1.8 & $\mathrm{mg} / \mathrm{kg}$ & & 1.1 \\
\hline 383РH0320.5 & $6 / 9 / 2004$ & soil & EPA6010 & REG & $7440-39-3$ & Barium & 260 & $\mathrm{mg} / \mathrm{kg}$ & & 11 \\
\hline 383РН0320.5 & $6 / 9 / 2004$ & soil & EPA6010 & REG & $7440-41-7$ & Beryllium & 1 & $\mathrm{mg} / \mathrm{kg}$ & & 0.54 \\
\hline 383РH0320.5 & $6 / 9 / 2004$ & soil & HASL300 & REG & $14733-03-0$ & Bi-214 & 1.36 & $\mathrm{pCi} / \mathrm{g}$ & $\mathrm{G}, \mathrm{J}$ & 0.28 \\
\hline 383РН0320.5 & $6 / 9 / 2004$ & soil & EPA6010 & REG & $7440-43-9$ & Cadmium & 0.092 & $\mathrm{mg} / \mathrm{kg}$ & $\mathrm{B}$ & 0.54 \\
\hline 383РН0320.5 & $6 / 9 / 2004$ & soil & EPA6010 & REG & $7440-47-3$ & Chromium & 3.4 & $\mathrm{mg} / \mathrm{kg}$ & & 1.1 \\
\hline 383РН0320.5 & $6 / 9 / 2004$ & soil & HASL300 & REG & $10045-97-3$ & Cs-137 & 0.33 & $\mathrm{pCi} / \mathrm{g}$ & LT,G & 0.14 \\
\hline 383РН0320.5 & $6 / 9 / 2004$ & soil & HASL300 & REG & 13966-00-2 & $\mathrm{K}-40$ & 29.8 & $\mathrm{pCi} / \mathrm{g}$ & $\mathrm{G}$ & 1.5 \\
\hline 383РН0320.5 & $6 / 9 / 2004$ & soil & EPA6010 & REG & 7439-92-1 & Lead & 10 & $\mathrm{mg} / \mathrm{kg}$ & & 0.32 \\
\hline 383РН0320.5 & $6 / 9 / 2004$ & soil & EPA7470 & REG & 7439-97-6 & Mercury & 0.12 & $\mathrm{mg} / \mathrm{kg}$ & & 0.11 \\
\hline 383РН0320.5 & $6 / 9 / 2004$ & soil & HASL300 & REG & 15092-94-1 & $\mathrm{Pb}-212$ & 2.76 & $\mathrm{pCi} / \mathrm{g}$ & $\mathrm{J}$ & 0.22 \\
\hline 383РН0320.5 & $6 / 9 / 2004$ & soil & HASL300 & REG & $15067-28-4$ & $\mathrm{~Pb}-214$ & 1.6 & $\mathrm{pCi} / \mathrm{g}$ & $\mathrm{G}, \mathrm{J}$ & 0.27 \\
\hline 383РН0320.5 & $6 / 9 / 2004$ & soil & HASL300 & REG & $14913-50-9$ & TI-208 & 0.73 & $\mathrm{pCi} / \mathrm{g}$ & $\mathrm{G}$ & 0.14 \\
\hline 383РН0408.0 & $6 / 10 / 2004$ & soil & HASL300 & REG & 14331-83-0 & Ac-228 & 2.02 & $\mathrm{pCi} / \mathrm{g}$ & G & 0.55 \\
\hline 383РН0408.0 & $6 / 10 / 2004$ & soil & EPA6010 & REG & $7440-38-2$ & Arsenic & 2.4 & $\mathrm{mg} / \mathrm{kg}$ & & 1.1 \\
\hline 383РН0408.0 & $6 / 10 / 2004$ & soil & EPA6010 & REG & $7440-39-3$ & Barium & 4900 & $\mathrm{mg} / \mathrm{kg}$ & & 110 \\
\hline 383РН0408.0 & $6 / 10 / 2004$ & soil & EPA6010 & REG & $7440-41-7$ & Beryllium & 1.9 & $\mathrm{mg} / \mathrm{kg}$ & & 0.55 \\
\hline 383РН0408.0 & $6 / 10 / 2004$ & soil & HASL300 & REG & 14733-03-0 & Bi-214 & 1.53 & $\mathrm{pCi} / \mathrm{g}$ & $\mathrm{G}, \mathrm{J}$ & 0.23 \\
\hline 383PH0408.0 & $6 / 10 / 2004$ & soil & EPA6010 & REG & $7440-43-9$ & Cadmium & 0.27 & $\mathrm{mg} / \mathrm{kg}$ & $\mathrm{B}$ & 0.55 \\
\hline 383PH0408.0 & $6 / 10 / 2004$ & soil & EPA6010 & REG & $7440-47-3$ & Chromium & 3.4 & $\mathrm{mg} / \mathrm{kg}$ & & 1.1 \\
\hline 383PH0408.0 & $6 / 10 / 2004$ & soil & HASL300 & REG & $10045-97-3$ & Cs-137 & 0.78 & $\mathrm{pCi} / \mathrm{g}$ & G & 0.15 \\
\hline 383PH0408.0 & $6 / 10 / 2004$ & soil & EPA8015 & REG & 68334-30-5 & Diesel-Range Organics & 7.5 & $\mathrm{mg} / \mathrm{kg}$ & $\mathrm{Z}, \mathrm{H}$ & 5.5 \\
\hline 383PH0408.0 & $6 / 10 / 2004$ & soil & HASL300 & REG & 13966-00-2 & $\mathrm{K}-40$ & 26.6 & $\mathrm{pCi} / \mathrm{g}$ & G & 1.3 \\
\hline 383PH0408.0 & $6 / 10 / 2004$ & soil & EPA6010 & REG & 7439-92-1 & Lead & 24 & $\mathrm{mg} / \mathrm{kg}$ & & 0.33 \\
\hline 383РН0408.0 & $6 / 10 / 2004$ & soil & EPA7470 & REG & $7439-97-6$ & Mercury & 0.007 & $\mathrm{mg} / \mathrm{kg}$ & $\mathrm{B}$ & 0.11 \\
\hline 383РН0408.0 & $6 / 10 / 2004$ & soil & HASL300 & REG & 15092-94-1 & $\mathrm{Pb}-212$ & 2.42 & $\mathrm{pCi} / \mathrm{g}$ & $\mathrm{J}$ & 0.23 \\
\hline 383PH0408.0 & $6 / 10 / 2004$ & soil & HASL300 & REG & $15067-28-4$ & $\mathrm{~Pb}-214$ & 1.73 & $\mathrm{pCi} / \mathrm{g}$ & $\mathrm{G}, \mathrm{J}$ & 0.29 \\
\hline 383РН0408.0 & $6 / 10 / 2004$ & soil & HASL300 & REG & $14913-50-9$ & TI-208 & 0.8 & $\mathrm{pCi} / \mathrm{g}$ & G & 0.15 \\
\hline 383РH0408.0X & $6 / 10 / 2004$ & soil & HASL300 & FD & $14331-83-0$ & Ac-228 & 2.27 & $\mathrm{pCi} / \mathrm{g}$ & $\mathrm{G}$ & 0.57 \\
\hline 383РH0408.0X & $6 / 10 / 2004$ & soil & EPA6010 & FD & $7440-38-2$ & Arsenic & 2.3 & $\mathrm{mg} / \mathrm{kg}$ & & 1.1 \\
\hline 383РH0408.0X & $6 / 10 / 2004$ & soil & EPA6010 & FD & $7440-39-3$ & Barium & 5200 & $\mathrm{mg} / \mathrm{kg}$ & & 110 \\
\hline 383РH0408.0X & $6 / 10 / 2004$ & soil & EPA6010 & FD & $7440-41-7$ & Beryllium & 2 & $\mathrm{mg} / \mathrm{kg}$ & & 0.55 \\
\hline
\end{tabular}

Page 73 of 78

Uncontrolled When Printed 


\begin{tabular}{|c|c|c|c|c|c|c|c|c|c|c|}
\hline SAMPLE \# & $\begin{array}{c}\text { SAMPLE } \\
\text { DATE }\end{array}$ & MATRIX & $\begin{array}{l}\text { USER TEST } \\
\text { PANEL }\end{array}$ & $\begin{array}{c}\text { SAMPLE } \\
\text { PURPOSE }\end{array}$ & CAS \# & PARAMETER & RESULT & UNITS & $\mathbf{Q}$ & $\begin{array}{c}\text { DETECT } \\
\text { LIMIT }\end{array}$ \\
\hline 383PH0408.0X & $6 / 10 / 2004$ & soil & HASL300 & FD & $14733-03-0$ & Bi-214 & 1.62 & $\mathrm{pCi} / \mathrm{g}$ & $\mathrm{G}, \mathrm{J}$ & 0.31 \\
\hline 383PH0408.0X & $6 / 10 / 2004$ & soil & EPA6010 & FD & $7440-43-9$ & Cadmium & 0.27 & $\mathrm{mg} / \mathrm{kg}$ & $B$ & 0.55 \\
\hline 383РН0408.0X & $6 / 10 / 2004$ & soil & EPA6010 & FD & $7440-47-3$ & Chromium & 3.6 & $\mathrm{mg} / \mathrm{kg}$ & & 1.1 \\
\hline 383PH0408.0X & $6 / 10 / 2004$ & soil & HASL300 & FD & $10045-97-3$ & Cs-137 & 0.76 & $\mathrm{pCi} / \mathrm{g}$ & G & 0.15 \\
\hline 383PH0408.0X & $6 / 10 / 2004$ & soil & EPA8015 & FD & $68334-30-5$ & Diesel-Range Organics & 4.7 & $\mathrm{mg} / \mathrm{kg}$ & $\mathrm{J}$ & 5.5 \\
\hline 383РH0408.0X & $6 / 10 / 2004$ & soil & HASL300 & FD & $13966-00-2$ & $\mathrm{~K}-40$ & 28.5 & $\mathrm{pCi} / \mathrm{g}$ & $\mathrm{G}$ & 1.5 \\
\hline 383PH0408.0X & $6 / 10 / 2004$ & soil & EPA6010 & FD & 7439-92-1 & Lead & 23 & $\mathrm{mg} / \mathrm{kg}$ & & 0.33 \\
\hline 383РH0408.0X & $6 / 10 / 2004$ & soil & EPA7470 & FD & 7439-97-6 & Mercury & 0.007 & $\mathrm{mg} / \mathrm{kg}$ & $B$ & 0.11 \\
\hline 383РН0408.0X & $6 / 10 / 2004$ & soil & HASL300 & FD & 15092-94-1 & $\mathrm{Pb}-212$ & 2.54 & $\mathrm{pCi} / \mathrm{g}$ & $\mathrm{J}$ & 0.22 \\
\hline 383РН0408.0X & $6 / 10 / 2004$ & soil & HASL300 & FD & $15067-28-4$ & $\mathrm{~Pb}-214$ & 1.81 & $\mathrm{pCi} / \mathrm{g}$ & $\mathrm{G}, \mathrm{J}$ & 0.28 \\
\hline 383PH0408.0X & $6 / 10 / 2004$ & soil & HASL300 & FD & $14913-50-9$ & TI-208 & 0.87 & $\mathrm{pCi} / \mathrm{g}$ & G & 0.14 \\
\hline 383РН0418.5 & $6 / 10 / 2004$ & soil & HASL300 & REG & 14331-83-0 & Ac-228 & 2.19 & $\mathrm{pCi} / \mathrm{g}$ & $\mathrm{G}$ & 0.39 \\
\hline 383РH0418.5 & $6 / 10 / 2004$ & soil & EPA8260 & REG & 67-64-1 & Acetone & 6.7 & $\mu \mathrm{g} / \mathrm{kg}$ & $\mathrm{J}$ & 21 \\
\hline 383PH0418.5 & $6 / 10 / 2004$ & soil & EPA6010 & REG & $7440-38-2$ & Arsenic & 2.1 & $\mathrm{mg} / \mathrm{kg}$ & & 1.1 \\
\hline 383РH0418.5 & $6 / 10 / 2004$ & soil & EPA6010 & REG & $7440-39-3$ & Barium & 590 & $\mathrm{mg} / \mathrm{kg}$ & & 11 \\
\hline 383PH0418.5 & $6 / 10 / 2004$ & soil & EPA6010 & REG & $7440-41-7$ & Beryllium & 0.74 & $\mathrm{mg} / \mathrm{kg}$ & & 0.53 \\
\hline 383PH0418.5 & $6 / 10 / 2004$ & soil & HASL300 & REG & $14733-03-0$ & Bi-214 & 1.4 & $\mathrm{pCi} / \mathrm{g}$ & $\mathrm{G}, \mathrm{J}$ & 0.27 \\
\hline 383PH0418.5 & $6 / 10 / 2004$ & soil & EPA6010 & REG & $7440-43-9$ & Cadmium & 0.12 & $\mathrm{mg} / \mathrm{kg}$ & B & 0.53 \\
\hline 383PH0418.5 & $6 / 10 / 2004$ & soil & EPA6010 & REG & $7440-47-3$ & Chromium & 1.7 & $\mathrm{mg} / \mathrm{kg}$ & & 1.1 \\
\hline 383PH0418.5 & $6 / 10 / 2004$ & soil & EPA8260 & REG & $87-68-3$ & Hexachlorobutadiene & 1.7 & $\mu \mathrm{g} / \mathrm{kg}$ & $\mathrm{J}$ & 5.3 \\
\hline 383PH0418.5 & $6 / 10 / 2004$ & soil & HASL300 & REG & $13966-00-2$ & $\mathrm{~K}-40$ & 31.7 & $\mathrm{pCi} / \mathrm{g}$ & G & 1.4 \\
\hline 383PH0418.5 & $6 / 10 / 2004$ & soil & EPA6010 & REG & 7439-92-1 & Lead & 6.4 & $\mathrm{mg} / \mathrm{kg}$ & & 0.32 \\
\hline 383PH0418.5 & $6 / 10 / 2004$ & soil & EPA7470 & REG & 7439-97-6 & Mercury & 0.017 & $\mathrm{mg} / \mathrm{kg}$ & $B$ & 0.11 \\
\hline 383PH0418.5 & $6 / 10 / 2004$ & soil & HASL300 & REG & 15092-94-1 & $\mathrm{Pb}-212$ & 2.68 & $\mathrm{pCi} / \mathrm{g}$ & $\mathrm{J}$ & 0.22 \\
\hline 383PH0418.5 & $6 / 10 / 2004$ & soil & HASL300 & REG & $15067-28-4$ & $\mathrm{~Pb}-214$ & 1.33 & $\mathrm{pCi} / \mathrm{g}$ & $\mathrm{G}, \mathrm{J}$ & 0.3 \\
\hline 383PH0418.5 & $6 / 10 / 2004$ & soil & HASL300 & REG & $14913-50-9$ & TI-208 & 0.83 & $\mathrm{pCi} / \mathrm{g}$ & G & 0.14 \\
\hline 383RB0100.5 & $6 / 1 / 2004$ & soil & HASL300 & REG & 14331-83-0 & Ac-228 & 2.9 & $\mathrm{pCi} / \mathrm{g}$ & $\mathrm{G}$ & 0.48 \\
\hline 383RB0100.5 & $6 / 1 / 2004$ & soil & EPA6010 & REG & $7440-38-2$ & Arsenic & 6.2 & $\mathrm{mg} / \mathrm{kg}$ & & 1.1 \\
\hline 383RB0100.5 & $6 / 1 / 2004$ & soil & EPA6010 & REG & $7440-39-3$ & Barium & 140 & $\mathrm{mg} / \mathrm{kg}$ & & 11 \\
\hline 383RB0100.5 & $6 / 1 / 2004$ & soil & EPA8270 & REG & $56-55-3$ & Benzo(A)Anthracene & 40 & $\mu \mathrm{g} / \mathrm{kg}$ & $\mathrm{J}$ & 370 \\
\hline 383RB0100.5 & $6 / 1 / 2004$ & soil & EPA8270 & REG & $50-32-8$ & Benzo(A)Pyrene & 46 & $\mu \mathrm{g} / \mathrm{kg}$ & $\mathrm{J}$ & 370 \\
\hline 383RB0100.5 & $6 / 1 / 2004$ & soil & EPA8270 & REG & $205-99-2$ & Benzo(B)Fluoranthene & 96 & $\mu \mathrm{g} / \mathrm{kg}$ & $\mathrm{J}$ & 370 \\
\hline 383RB0100.5 & $6 / 1 / 2004$ & soil & EPA8270 & REG & $207-08-9$ & Benzo(K)Fluoranthene & 45 & $\mu \mathrm{g} / \mathrm{kg}$ & $\mathrm{J}$ & 370 \\
\hline 383RB0100.5 & $6 / 1 / 2004$ & soil & EPA6010 & REG & $7440-41-7$ & Beryllium & 1.7 & $\mathrm{mg} / \mathrm{kg}$ & & 0.55 \\
\hline 383RB0100.5 & $6 / 1 / 2004$ & soil & HASL300 & REG & 14913-49-6 & $\mathrm{Bi}-212$ & 3.2 & $\mathrm{pCi} / \mathrm{g}$ & $\mathrm{G}$ & 1.9 \\
\hline
\end{tabular}

Page 74 of 78

Uncontrolled When Printed 


\begin{tabular}{|c|c|c|c|c|c|c|c|c|c|c|}
\hline SAMPLE \# & $\begin{array}{l}\text { SAMPLE } \\
\text { DATE }\end{array}$ & MATRIX & $\begin{array}{l}\text { USER TEST } \\
\text { PANEL }\end{array}$ & $\begin{array}{c}\text { SAMPLE } \\
\text { PURPOSE }\end{array}$ & CAS \# & PARAMETER & RESULT & UNITS & $\mathbf{Q}$ & $\begin{array}{c}\text { DETECT } \\
\text { LIMIT }\end{array}$ \\
\hline 383RB0100.5 & $6 / 1 / 2004$ & soil & EPA8270 & REG & $117-81-7$ & Bis(2-Ethylhexyl)Phthalate & 260 & $\mu \mathrm{g} / \mathrm{kg}$ & $\mathrm{J}$ & 370 \\
\hline 383RB0100.5 & $6 / 1 / 2004$ & soil & EPA8270 & REG & $85-68-7$ & Butyl Benzyl Phthalate & 780 & $\mu \mathrm{g} / \mathrm{kg}$ & & 370 \\
\hline 383RB0100.5 & $6 / 1 / 2004$ & soil & EPA6010 & REG & $7440-43-9$ & Cadmium & 0.56 & $\mathrm{mg} / \mathrm{kg}$ & & 0.55 \\
\hline 383RB0100.5 & $6 / 1 / 2004$ & soil & EPA6010 & REG & $7440-47-3$ & Chromium & 3.2 & $\mathrm{mg} / \mathrm{kg}$ & & 1.1 \\
\hline 383RB0100.5 & $6 / 1 / 2004$ & soil & EPA8270 & REG & 218-01-9 & Chrysene & 74 & $\mu \mathrm{g} / \mathrm{kg}$ & $\mathrm{J}$ & 370 \\
\hline 383RB0100.5 & $6 / 1 / 2004$ & soil & HASL300 & REG & $10045-97-3$ & Cs-137 & 114 & $\mathrm{pCi} / \mathrm{g}$ & G & 0 \\
\hline 383RB0100.5 & $6 / 1 / 2004$ & soil & EPA8015 & REG & $68334-30-5$ & Diesel-Range Organics & 9.6 & $\mathrm{mg} / \mathrm{kg}$ & $\mathrm{Z}, \mathrm{H}$ & 5.5 \\
\hline 383RB0100.5 & $6 / 1 / 2004$ & soil & EPA8270 & REG & 84-74-2 & Di-N-Butyl Phthalate & 4500 & $\mu \mathrm{g} / \mathrm{kg}$ & $E$ & 370 \\
\hline 383RB0100.5 & $6 / 1 / 2004$ & soil & EPA8270 & REG & $206-44-0$ & Fluoranthene & 91 & $\mu \mathrm{g} / \mathrm{kg}$ & $\mathrm{J}$ & 370 \\
\hline 383RB0100.5 & $6 / 1 / 2004$ & soil & HASL300 & REG & $13966-00-2$ & $\mathrm{~K}-40$ & 27.8 & $\mathrm{pCi} / \mathrm{g}$ & $G$ & 1.4 \\
\hline 383RB0100.5 & $6 / 1 / 2004$ & soil & EPA6010 & REG & 7439-92-1 & Lead & 18 & $\mathrm{mg} / \mathrm{kg}$ & & 0.33 \\
\hline 383RB0100.5 & $6 / 1 / 2004$ & soil & EPA7470 & REG & $7439-97-6$ & Mercury & 0.16 & $\mathrm{mg} / \mathrm{kg}$ & & 0.11 \\
\hline 383RB0100.5 & $6 / 1 / 2004$ & soil & HASL300 & REG & 15092-94-1 & $\mathrm{Pb}-212$ & 2.96 & $\mathrm{pCi} / \mathrm{g}$ & $\mathrm{J}$ & 0.52 \\
\hline 383RB0100.5 & $6 / 1 / 2004$ & soil & EPA8270 & REG & 85-01-8 & Phenanthrene & 42 & $\mu \mathrm{g} / \mathrm{kg}$ & $\mathrm{J}$ & 370 \\
\hline 383RB0100.5 & $6 / 1 / 2004$ & soil & UGTAISOPU & REG & $15117-48-3$ & Pu-239 & 0.166 & $\mathrm{pCi} / \mathrm{g}$ & $\mathrm{J}$ & 0.018 \\
\hline 383RB0100.5 & $6 / 1 / 2004$ & soil & EPA8270 & REG & $129-00-0$ & Pyrene & 93 & $\mu \mathrm{g} / \mathrm{kg}$ & $\mathrm{J}$ & 370 \\
\hline 383RB0100.5 & $6 / 1 / 2004$ & soil & HASL300 & REG & 15623-47-9 & Th-227 & 4.1 & $\mathrm{pCi} / \mathrm{g}$ & $\mathrm{J}$ & 2.3 \\
\hline 383RB0100.5 & $6 / 1 / 2004$ & soil & HASL300 & REG & $15065-10-8$ & Th-234 & 7.2 & $\mathrm{pCi} / \mathrm{g}$ & $\mathrm{G}, \mathrm{TI}$ & 4 \\
\hline 383RB0100.5 & $6 / 1 / 2004$ & soil & HASL300 & REG & 14913-50-9 & TI-208 & 0.85 & $\mathrm{pCi} / \mathrm{g}$ & G & 0.25 \\
\hline 383RB0100.5RR1 & $6 / 1 / 2004$ & soil & EPA8270 & $\mathrm{RE}$ & $56-55-3$ & Benzo(A)Anthracene & 60 & $\mu \mathrm{g} / \mathrm{kg}$ & $\mathrm{J}$ & 740 \\
\hline 383RB0100.5RR1 & $6 / 1 / 2004$ & soil & EPA8270 & $\mathrm{RE}$ & $50-32-8$ & Benzo(A)Pyrene & 39 & $\mu \mathrm{g} / \mathrm{kg}$ & $\mathrm{J}$ & 740 \\
\hline 383RB0100.5RR1 & $6 / 1 / 2004$ & soil & EPA8270 & $\mathrm{RE}$ & 205-99-2 & Benzo(B)Fluoranthene & 84 & $\mu \mathrm{g} / \mathrm{kg}$ & $\mathrm{J}$ & 740 \\
\hline 383RB0100.5RR1 & $6 / 1 / 2004$ & soil & EPA8270 & $\mathrm{RE}$ & 117-81-7 & Bis(2-Ethylhexyl)Phthalate & 230 & $\mu \mathrm{g} / \mathrm{kg}$ & $\mathrm{J}$ & 740 \\
\hline 383RB0100.5RR1 & $6 / 1 / 2004$ & soil & EPA8270 & $\mathrm{RE}$ & $85-68-7$ & Butyl Benzyl Phthalate & 630 & $\mu \mathrm{g} / \mathrm{kg}$ & $\mathrm{J}$ & 740 \\
\hline 383RB0100.5RR1 & $6 / 1 / 2004$ & soil & EPA8270 & $\mathrm{RE}$ & 218-01-9 & Chrysene & 63 & $\mu \mathrm{g} / \mathrm{kg}$ & $\mathrm{J}$ & 740 \\
\hline 383RB0100.5RR1 & $6 / 1 / 2004$ & soil & EPA8270 & $\mathrm{RE}$ & 84-74-2 & Di-N-Butyl Phthalate & 5100 & $\mu g / \mathrm{kg}$ & & 740 \\
\hline 383RB0100.5RR1 & $6 / 1 / 2004$ & soil & EPA8270 & $\mathrm{RE}$ & $206-44-0$ & Fluoranthene & 86 & $\mu \mathrm{g} / \mathrm{kg}$ & $\mathrm{J}$ & 740 \\
\hline 383RB0100.5RR1 & $6 / 1 / 2004$ & soil & EPA8270 & $\mathrm{RE}$ & 85-01-8 & Phenanthrene & 43 & $\mu \mathrm{g} / \mathrm{kg}$ & $\mathrm{J}$ & 740 \\
\hline 383RB0200.5 & $6 / 1 / 2004$ & soil & HASL300 & REG & 14331-83-0 & Ac-228 & 1.64 & $\mathrm{pCi} / \mathrm{g}$ & $\mathrm{G}$ & 0.5 \\
\hline 383RB0200.5 & $6 / 1 / 2004$ & soil & EPA6010 & REG & $7440-38-2$ & Arsenic & 7.1 & $\mathrm{mg} / \mathrm{kg}$ & & 1 \\
\hline 383RB0200.5 & $6 / 1 / 2004$ & soil & EPA6010 & REG & $7440-39-3$ & Barium & 180 & $\mathrm{mg} / \mathrm{kg}$ & & 10 \\
\hline 383RB0200.5 & $6 / 1 / 2004$ & soil & EPA8270 & REG & $56-55-3$ & Benzo(A)Anthracene & 56 & $\mu g / \mathrm{kg}$ & $\mathrm{J}$ & 340 \\
\hline 383RB0200.5 & $6 / 1 / 2004$ & soil & EPA8270 & REG & $50-32-8$ & Benzo(A)Pyrene & 52 & $\mu \mathrm{g} / \mathrm{kg}$ & $\mathrm{J}$ & 340 \\
\hline 383RB0200.5 & $6 / 1 / 2004$ & soil & EPA8270 & REG & $205-99-2$ & Benzo(B)Fluoranthene & 120 & $\mu \mathrm{g} / \mathrm{kg}$ & $\mathrm{J}$ & 340 \\
\hline 383RB0200.5 & $6 / 1 / 2004$ & soil & EPA8270 & REG & 207-08-9 & Benzo(K)Fluoranthene & 41 & $\mu \mathrm{g} / \mathrm{kg}$ & $\mathrm{J}$ & 340 \\
\hline
\end{tabular}

Page 75 of 78

Uncontrolled When Printed 


\begin{tabular}{|c|c|c|c|c|c|c|c|c|c|c|}
\hline SAMPLE \# & $\begin{array}{l}\text { SAMPLE } \\
\text { DATE }\end{array}$ & MATRIX & $\begin{array}{l}\text { USER TEST } \\
\text { PANEL }\end{array}$ & $\begin{array}{c}\text { SAMPLE } \\
\text { PURPOSE }\end{array}$ & CAS \# & PARAMETER & RESULT & UNITS & Q & $\begin{array}{c}\text { DETECT } \\
\text { LIMIT }\end{array}$ \\
\hline 383RB0200.5 & $6 / 1 / 2004$ & soil & EPA6010 & REG & $7440-41-7$ & Beryllium & 0.86 & $\mathrm{mg} / \mathrm{kg}$ & & 0.52 \\
\hline 383RB0200.5 & $6 / 1 / 2004$ & soil & HASL300 & REG & $14733-03-0$ & Bi-214 & 1.12 & $\mathrm{pCi} / \mathrm{g}$ & $\mathrm{G}, \mathrm{J}$ & 0.29 \\
\hline 383RB0200.5 & $6 / 1 / 2004$ & soil & EPA8270 & REG & $117-81-7$ & Bis(2-Ethylhexyl)Phthalate & 540 & $\mu \mathrm{g} / \mathrm{kg}$ & $\mathrm{J}$ & 340 \\
\hline 383RB0200.5 & $6 / 1 / 2004$ & soil & EPA6010 & REG & $7440-47-3$ & Chromium & 9.1 & $\mathrm{mg} / \mathrm{kg}$ & & 1 \\
\hline 383RB0200.5 & $6 / 1 / 2004$ & soil & EPA8270 & REG & 218-01-9 & Chrysene & 84 & $\mu \mathrm{g} / \mathrm{kg}$ & J & 340 \\
\hline 383RB0200.5 & $6 / 1 / 2004$ & soil & HASL300 & REG & $10045-97-3$ & Cs-137 & 7.63 & $\mathrm{pCi} / \mathrm{g}$ & $\mathrm{G}$ & 0.15 \\
\hline 383RB0200.5 & $6 / 1 / 2004$ & soil & EPA8270 & REG & 84-74-2 & Di-N-Butyl Phthalate & 81 & $\mu \mathrm{g} / \mathrm{kg}$ & $\mathrm{J}$ & 340 \\
\hline 383RB0200.5 & $6 / 1 / 2004$ & soil & EPA8270 & REG & $206-44-0$ & Fluoranthene & 96 & $\mu \mathrm{g} / \mathrm{kg}$ & $\mathrm{J}$ & 340 \\
\hline 383RB0200.5 & $6 / 1 / 2004$ & soil & HASL300 & REG & $13966-00-2$ & $\mathrm{~K}-40$ & 24.3 & $\mathrm{pCi} / \mathrm{g}$ & $\mathrm{G}$ & 1.6 \\
\hline 383RB0200.5 & $6 / 1 / 2004$ & soil & EPA6010 & REG & 7439-92-1 & Lead & 18 & $\mathrm{mg} / \mathrm{kg}$ & & 0.31 \\
\hline 383RB0200.5 & $6 / 1 / 2004$ & soil & HASL300 & REG & 15092-94-1 & $\mathrm{Pb}-212$ & 1.59 & $\mathrm{pCi} / \mathrm{g}$ & $\mathrm{J}$ & 0.24 \\
\hline 383RB0200.5 & $6 / 1 / 2004$ & soil & HASL300 & REG & $15067-28-4$ & $\mathrm{~Pb}-214$ & 0.96 & $\mathrm{pCi} / \mathrm{g}$ & $\mathrm{G}, \mathrm{J}$ & 0.33 \\
\hline 383RB0200.5 & $6 / 1 / 2004$ & soil & EPA8270 & REG & 85-01-8 & Phenanthrene & 38 & $\mu \mathrm{g} / \mathrm{kg}$ & $\mathrm{J}$ & 340 \\
\hline 383RB0200.5 & $6 / 1 / 2004$ & soil & UGTAISOPU & REG & 13981-16-3 & Pu-238 & 0.076 & $\mathrm{pCi} / \mathrm{g}$ & & 0.01 \\
\hline 383RB0200.5 & $6 / 1 / 2004$ & soil & UGTAISOPU & REG & $15117-48-3$ & Pu-239 & 2.09 & $\mathrm{pCi} / \mathrm{g}$ & $\mathrm{J}$ & 0.01 \\
\hline 383RB0200.5 & $6 / 1 / 2004$ & soil & EPA8270 & REG & $129-00-0$ & Pyrene & 73 & $\mu \mathrm{g} / \mathrm{kg}$ & $\mathrm{J}$ & 340 \\
\hline 383RB0200.5 & $6 / 1 / 2004$ & soil & HASL300 & REG & 14913-50-9 & TI-208 & 0.61 & $\mathrm{pCi} / \mathrm{g}$ & G & 0.14 \\
\hline 383RB0300.5 & $6 / 1 / 2004$ & soil & HASL300 & REG & 14331-83-0 & Ac-228 & 1.26 & $\mathrm{pCi} / \mathrm{g}$ & $G$ & 0.36 \\
\hline 383RB0300.5 & $6 / 1 / 2004$ & soil & EPA6010 & REG & 7440-38-2 & Arsenic & 5.3 & $\mathrm{mg} / \mathrm{kg}$ & & 1 \\
\hline 383RB0300.5 & $6 / 1 / 2004$ & soil & EPA6010 & REG & $7440-39-3$ & Barium & 260 & $\mathrm{mg} / \mathrm{kg}$ & & 10 \\
\hline 383RB0300.5 & $6 / 1 / 2004$ & soil & EPA8270 & REG & $56-55-3$ & Benzo(A)Anthracene & 170 & $\mu \mathrm{g} / \mathrm{kg}$ & $\mathrm{J}$ & 340 \\
\hline 383RB0300.5 & $6 / 1 / 2004$ & soil & EPA8270 & REG & $50-32-8$ & Benzo(A)Pyrene & 200 & $\mu \mathrm{g} / \mathrm{kg}$ & $\mathrm{J}$ & 340 \\
\hline 383RB0300.5 & $6 / 1 / 2004$ & soil & EPA8270 & REG & $205-99-2$ & Benzo(B)Fluoranthene & 460 & $\mu \mathrm{g} / \mathrm{kg}$ & $\mathrm{J}$ & 340 \\
\hline 383RB0300.5 & $6 / 1 / 2004$ & soil & EPA8270 & REG & 207-08-9 & Benzo(K)Fluoranthene & 230 & $\mu \mathrm{g} / \mathrm{kg}$ & $\mathrm{J}$ & 340 \\
\hline 383RB0300.5 & $6 / 1 / 2004$ & soil & EPA6010 & REG & 7440-41-7 & Beryllium & 0.84 & $\mathrm{mg} / \mathrm{kg}$ & & 0.52 \\
\hline 383RB0300.5 & $6 / 1 / 2004$ & soil & HASL300 & REG & $14733-03-0$ & Bi-214 & 0.87 & $\mathrm{pCi} / \mathrm{g}$ & $\mathrm{G}, \mathrm{J}$ & 0.29 \\
\hline 383RB0300.5 & $6 / 1 / 2004$ & soil & EPA8270 & REG & $117-81-7$ & Bis(2-Ethylhexyl)Phthalate & 1200 & $\mu \mathrm{g} / \mathrm{kg}$ & J & 340 \\
\hline 383RB0300.5 & $6 / 1 / 2004$ & soil & EPA8270 & REG & 85-68-7 & Butyl Benzyl Phthalate & 23 & $\mu \mathrm{g} / \mathrm{kg}$ & $\mathrm{J}$ & 340 \\
\hline 383RB0300.5 & $6 / 1 / 2004$ & soil & EPA6010 & REG & $7440-43-9$ & Cadmium & 0.57 & $\mathrm{mg} / \mathrm{kg}$ & & 0.52 \\
\hline 383RB0300.5 & $6 / 1 / 2004$ & soil & EPA6010 & REG & $7440-47-3$ & Chromium & 8.7 & $\mathrm{mg} / \mathrm{kg}$ & & 1 \\
\hline 383RB0300.5 & $6 / 1 / 2004$ & soil & EPA8270 & REG & 218-01-9 & Chrysene & 270 & $\mu \mathrm{g} / \mathrm{kg}$ & $\mathrm{J}$ & 340 \\
\hline 383RB0300.5 & $6 / 1 / 2004$ & soil & HASL300 & REG & $10045-97-3$ & Cs-137 & 23.4 & $\mathrm{pCi} / \mathrm{g}$ & $\mathrm{G}$ & 0.1 \\
\hline 383RB0300.5 & $6 / 1 / 2004$ & soil & EPA8015 & REG & $68334-30-5$ & Diesel-Range Organics & 3 & $\mathrm{mg} / \mathrm{kg}$ & $\mathrm{J}$ & 5.2 \\
\hline 383RB0300.5 & $6 / 1 / 2004$ & soil & EPA8270 & REG & 84-74-2 & Di-N-Butyl Phthalate & 79 & $\mu \mathrm{g} / \mathrm{kg}$ & $\mathrm{J}$ & 340 \\
\hline 383RB0300.5 & $6 / 1 / 2004$ & soil & EPA8270 & REG & 206-44-0 & Fluoranthene & 280 & $\mu \mathrm{g} / \mathrm{kg}$ & $\mathrm{J}$ & 340 \\
\hline
\end{tabular}

Page 76 of 78

Uncontrolled When Printed 


\begin{tabular}{|c|c|c|c|c|c|c|c|c|c|c|}
\hline SAMPLE \# & $\begin{array}{l}\text { SAMPLE } \\
\text { DATE }\end{array}$ & MATRIX & $\begin{array}{l}\text { USER TEST } \\
\text { PANEL }\end{array}$ & $\begin{array}{c}\text { SAMPLE } \\
\text { PURPOSE }\end{array}$ & CAS \# & PARAMETER & RESULT & UNITS & $\mathbf{Q}$ & $\begin{array}{l}\text { DETECT } \\
\text { LIMIT }\end{array}$ \\
\hline 383RB0300.5 & $6 / 1 / 2004$ & soil & HASL300 & REG & 13966-00-2 & $\mathrm{K}-40$ & 21.4 & $\mathrm{pCi} / \mathrm{g}$ & $\mathrm{G}$ & 1.2 \\
\hline 383RB0300.5 & $6 / 1 / 2004$ & soil & EPA6010 & REG & 7439-92-1 & Lead & 61 & $\mathrm{mg} / \mathrm{kg}$ & & 0.31 \\
\hline 383RB0300.5 & $6 / 1 / 2004$ & soil & HASL300 & REG & 15092-94-1 & $\mathrm{Pb}-212$ & 1.52 & $\mathrm{pCi} / \mathrm{g}$ & $\mathrm{J}$ & 0.26 \\
\hline 383RB0300.5 & $6 / 1 / 2004$ & soil & HASL300 & REG & 15067-28-4 & $\mathrm{Pb}-214$ & 0.93 & $\mathrm{pCi} / \mathrm{g}$ & $\mathrm{G}, \mathrm{J}$ & 0.32 \\
\hline 383RB0300.5 & $6 / 1 / 2004$ & soil & EPA8270 & REG & 85-01-8 & Phenanthrene & 79 & $\mu \mathrm{g} / \mathrm{kg}$ & $\mathrm{J}$ & 340 \\
\hline 383RB0300.5 & $6 / 1 / 2004$ & soil & UGTAISOPU & REG & 13981-16-3 & Pu-238 & 0.151 & $\mathrm{pCi} / \mathrm{g}$ & & 0.023 \\
\hline 383RB0300.5 & $6 / 1 / 2004$ & soil & UGTAISOPU & REG & $15117-48-3$ & Pu-239 & 0.85 & $\mathrm{pCi} / \mathrm{g}$ & $\mathrm{J}$ & 0.02 \\
\hline 383RB0300.5 & $6 / 1 / 2004$ & soil & EPA8270 & REG & $129-00-0$ & Pyrene & 360 & $\mu \mathrm{g} / \mathrm{kg}$ & & 340 \\
\hline 383RB0300.5 & $6 / 1 / 2004$ & soil & HASL300 & REG & 14913-50-9 & TI-208 & 0.47 & $\mathrm{pCi} / \mathrm{g}$ & $\mathrm{G}$ & 0.14 \\
\hline 383RB0400.5 & $6 / 1 / 2004$ & soil & HASL300 & REG & 14331-83-0 & Ac-228 & 2.46 & $\mathrm{pCi} / \mathrm{g}$ & G & 0.43 \\
\hline 383RB0400.5 & $6 / 1 / 2004$ & soil & EPA6010 & REG & $7440-38-2$ & Arsenic & 4.5 & $\mathrm{mg} / \mathrm{kg}$ & & 1.1 \\
\hline 383RB0400.5 & $6 / 1 / 2004$ & soil & EPA6010 & REG & $7440-39-3$ & Barium & 190 & $\mathrm{mg} / \mathrm{kg}$ & & 11 \\
\hline 383RB0400.5 & $6 / 1 / 2004$ & soil & EPA8270 & REG & $56-55-3$ & Benzo(A)Anthracene & 140 & $\mu \mathrm{g} / \mathrm{kg}$ & $\mathrm{J}$ & 360 \\
\hline 383RB0400.5 & $6 / 1 / 2004$ & soil & EPA8270 & REG & $50-32-8$ & Benzo(A)Pyrene & 230 & $\mu \mathrm{g} / \mathrm{kg}$ & $\mathrm{J}$ & 360 \\
\hline 383RB0400.5 & $6 / 1 / 2004$ & soil & EPA8270 & REG & $205-99-2$ & Benzo(B)Fluoranthene & 620 & $\mu \mathrm{g} / \mathrm{kg}$ & & 360 \\
\hline 383RB0400.5 & $6 / 1 / 2004$ & soil & EPA8270 & REG & 191-24-2 & Benzo(G,H,I)Perylene & 90 & $\mu \mathrm{g} / \mathrm{kg}$ & $\mathrm{J}$ & 360 \\
\hline 383RB0400.5 & $6 / 1 / 2004$ & soil & EPA8270 & REG & 207-08-9 & Benzo(K)Fluoranthene & 170 & $\mu \mathrm{g} / \mathrm{kg}$ & $\mathrm{J}$ & 360 \\
\hline 383RB0400.5 & $6 / 1 / 2004$ & soil & EPA6010 & REG & 7440-41-7 & Beryllium & 1.1 & $\mathrm{mg} / \mathrm{kg}$ & & 0.54 \\
\hline 383RB0400.5 & $6 / 1 / 2004$ & soil & HASL300 & REG & $14733-03-0$ & Bi-214 & 0.7 & $\mathrm{pCi} / \mathrm{g}$ & $\mathrm{G}, \mathrm{J}$ & 0.31 \\
\hline 383RB0400.5 & $6 / 1 / 2004$ & soil & EPA8270 & REG & 117-81-7 & Bis(2-Ethylhexyl)Phthalate & 76 & $\mu \mathrm{g} / \mathrm{kg}$ & $\mathrm{J}$ & 360 \\
\hline 383RB0400.5 & $6 / 1 / 2004$ & soil & EPA8270 & REG & 86-74-8 & Carbazole & 23 & $\mu \mathrm{g} / \mathrm{kg}$ & $\mathrm{J}$ & 360 \\
\hline 383RB0400.5 & $6 / 1 / 2004$ & soil & EPA6010 & REG & $7440-47-3$ & Chromium & 4.9 & $\mathrm{mg} / \mathrm{kg}$ & & 1.1 \\
\hline 383RB0400.5 & $6 / 1 / 2004$ & soil & EPA8270 & REG & 218-01-9 & Chrysene & 480 & $\mu \mathrm{g} / \mathrm{kg}$ & & 360 \\
\hline 383RB0400.5 & $6 / 1 / 2004$ & soil & HASL300 & REG & 10045-97-3 & Cs-137 & 7.06 & $\mathrm{pCi} / \mathrm{g}$ & $G$ & 0.13 \\
\hline 383RB0400.5 & $6 / 1 / 2004$ & soil & EPA8270 & REG & $53-70-3$ & Dibenzo $(\mathrm{A}, \mathrm{H})$ Anthracene & 27 & $\mu \mathrm{g} / \mathrm{kg}$ & $\mathrm{J}$ & 360 \\
\hline 383RB0400.5 & $6 / 1 / 2004$ & soil & EPA8015 & REG & $68334-30-5$ & Diesel-Range Organics & 15 & $\mathrm{mg} / \mathrm{kg}$ & $\mathrm{Z}, \mathrm{H}$ & 5.4 \\
\hline 383RB0400.5 & $6 / 1 / 2004$ & soil & EPA8270 & REG & 84-74-2 & Di-N-Butyl Phthalate & 45 & $\mu \mathrm{g} / \mathrm{kg}$ & $\mathrm{J}$ & 360 \\
\hline 383RB0400.5 & $6 / 1 / 2004$ & soil & EPA8270 & REG & $206-44-0$ & Fluoranthene & 510 & $\mu \mathrm{g} / \mathrm{kg}$ & & 360 \\
\hline 383RB0400.5 & $6 / 1 / 2004$ & soil & EPA8270 & REG & $193-39-5$ & Indeno(1,2,3-Cd)Pyrene & 80 & $\mu \mathrm{g} / \mathrm{kg}$ & $\mathrm{J}$ & 360 \\
\hline 383RB0400.5 & $6 / 1 / 2004$ & soil & HASL300 & REG & 13966-00-2 & $\mathrm{K}-40$ & 27.3 & $\mathrm{pCi} / \mathrm{g}$ & $\mathrm{G}$ & 1.4 \\
\hline 383RB0400.5 & $6 / 1 / 2004$ & soil & EPA6010 & REG & $7439-92-1$ & Lead & 22 & $\mathrm{mg} / \mathrm{kg}$ & & 0.32 \\
\hline 383RB0400.5 & $6 / 1 / 2004$ & soil & EPA8270 & REG & $91-20-3$ & Naphthalene & 18 & $\mu \mathrm{g} / \mathrm{kg}$ & $\mathrm{J}$ & 360 \\
\hline 383RB0400.5 & $6 / 1 / 2004$ & soil & HASL300 & REG & 15092-94-1 & $\mathrm{Pb}-212$ & 2.44 & $\mathrm{pCi} / \mathrm{g}$ & $\mathrm{J}$ & 0.24 \\
\hline 383RB0400.5 & $6 / 1 / 2004$ & soil & HASL300 & REG & $15067-28-4$ & $\mathrm{~Pb}-214$ & 1.04 & $\mathrm{pCi} / \mathrm{g}$ & $\mathrm{G}, \mathrm{J}$ & 0.26 \\
\hline 383RB0400.5 & $6 / 1 / 2004$ & soil & EPA8270 & REG & 85-01-8 & Phenanthrene & 210 & $\mu \mathrm{g} / \mathrm{kg}$ & $\mathrm{J}$ & 360 \\
\hline
\end{tabular}

Page 77 of 78

Uncontrolled When Printed 


\begin{tabular}{|c|c|c|c|c|c|c|c|c|c|c|}
\hline SAMPLE \# & $\begin{array}{c}\text { SAMPLE } \\
\text { DATE }\end{array}$ & MATRIX & $\begin{array}{c}\text { USER TEST } \\
\text { PANEL }\end{array}$ & $\begin{array}{c}\text { SAMPLE } \\
\text { PURPOSE }\end{array}$ & CAS \# & PARAMETER & RESULT & UNITS & $\mathbf{Q}$ & $\begin{array}{c}\text { DETECT } \\
\text { LIMIT }\end{array}$ \\
\hline 383RB0400.5 & $6 / 1 / 2004$ & soil & UGTAISOPU & REG & 13981-16-3 & Pu-238 & 0.232 & $\mathrm{pCi} / \mathrm{g}$ & & 0.03 \\
\hline 383RB0400.5 & $6 / 1 / 2004$ & soil & UGTAISOPU & REG & $15117-48-3$ & Pu-239 & 1.15 & $\mathrm{pCi} / \mathrm{g}$ & $\mathrm{J}$ & 0.02 \\
\hline 383RB0400.5 & $6 / 1 / 2004$ & soil & EPA8270 & REG & $129-00-0$ & Pyrene & 430 & $\mu \mathrm{g} / \mathrm{kg}$ & & 360 \\
\hline 383RB0400.5 & $6 / 1 / 2004$ & soil & HASL300 & REG & $14913-50-9$ & TI-208 & 0.74 & $\mathrm{pCi} / \mathrm{g}$ & $\mathrm{G}$ & 0.12 \\
\hline 383TR10 & $6 / 3 / 2004$ & water & EPA8260 & TB & $75-09-2$ & Methylene Chloride & 0.97 & $\mu \mathrm{g} / \mathrm{L}$ & $\mathrm{J}, \mathrm{B}$ & 5 \\
\hline 383TR10 & $6 / 3 / 2004$ & water & EPA8260 & TB & $108-88-3$ & Toluene & 1.3 & $\mu \mathrm{g} / \mathrm{L}$ & $\mathrm{J}$ & 5 \\
\hline 383TR11 & $6 / 7 / 2004$ & water & EPA8260 & TB & $75-09-2$ & Methylene Chloride & 0.87 & $\mu g / L$ & $\mathrm{~J}, \mathrm{~B}$ & 5 \\
\hline 383TR13 & $6 / 8 / 2004$ & water & EPA8260 & TB & 67-64-1 & Acetone & 12 & $\mu \mathrm{g} / \mathrm{L}$ & $\mathrm{J}, \mathrm{B}$ & 20 \\
\hline 383TR13 & $6 / 8 / 2004$ & water & EPA8260 & TB & $75-09-2$ & Methylene Chloride & 0.85 & $\mu \mathrm{g} / \mathrm{L}$ & $\mathrm{J}, \mathrm{B}$ & 5 \\
\hline 383TR14 & $6 / 9 / 2004$ & water & EPA8260 & TB & 67-64-1 & Acetone & 13 & $\mu g / L$ & $\mathrm{~J}, \mathrm{~B}$ & 20 \\
\hline 383TR14 & $6 / 9 / 2004$ & water & EPA8260 & TB & $75-09-2$ & Methylene Chloride & 5.8 & $\mu \mathrm{g} / \mathrm{L}$ & $\mathrm{B}$ & 5 \\
\hline 383TR15 & $6 / 10 / 2004$ & water & EPA8260 & TB & 67-64-1 & Acetone & 13 & $\mu \mathrm{g} / \mathrm{L}$ & $\mathrm{J}, \mathrm{B}$ & 20 \\
\hline 383TR15 & $6 / 10 / 2004$ & water & EPA8260 & TB & $75-09-2$ & Methylene Chloride & 15 & $\mu g / L$ & $\mathrm{~B}$ & 5 \\
\hline 383TR16 & $6 / 11 / 2004$ & water & EPA8260 & TB & $75-09-2$ & Methylene Chloride & 2.3 & $\mu \mathrm{g} / \mathrm{L}$ & $\mathrm{J}$ & 5 \\
\hline 383TR16 & $6 / 11 / 2004$ & water & EPA8260 & TB & $108-88-3$ & Toluene & 1.1 & $\mu \mathrm{g} / \mathrm{L}$ & $\mathrm{J}$ & 5 \\
\hline 383TR17 & $6 / 12 / 2004$ & water & EPA8260 & TB & $75-09-2$ & Methylene Chloride & 0.96 & $\mu g / L$ & $\mathrm{~J}$ & 5 \\
\hline 383TR17 & $6 / 12 / 2004$ & water & EPA8260 & TB & $108-88-3$ & Toluene & 0.61 & $\mu \mathrm{g} / \mathrm{L}$ & $\mathrm{J}$ & 5 \\
\hline 383TR18 & $6 / 13 / 2004$ & water & EPA8260 & TB & $75-09-2$ & Methylene Chloride & 2 & $\mu g / L$ & $\mathrm{~J}$ & 5 \\
\hline 383TR19 & $6 / 14 / 2004$ & water & EPA8260 & TB & $75-09-2$ & Methylene Chloride & 1.3 & $\mu \mathrm{g} / \mathrm{L}$ & $\mathrm{J}$ & 5 \\
\hline 383TR19 & $6 / 14 / 2004$ & water & EPA8260 & TB & $108-88-3$ & Toluene & 0.78 & $\mu \mathrm{g} / \mathrm{L}$ & $\mathrm{J}$ & 5 \\
\hline 383TR20 & $6 / 15 / 2004$ & water & EPA8260 & TB & $75-09-2$ & Methylene Chloride & 1.5 & $\mu \mathrm{g} / \mathrm{L}$ & $\mathrm{J}$ & 5 \\
\hline 383TR21 & $6 / 16 / 2004$ & water & EPA8260 & TB & $75-09-2$ & Methylene Chloride & 1.4 & $\mu \mathrm{g} / \mathrm{L}$ & $\mathrm{J}$ & 5 \\
\hline 383TR22 & $6 / 17 / 2004$ & water & EPA8260 & TB & $75-09-2$ & Methylene Chloride & 1.3 & $\mu \mathrm{g} / \mathrm{L}$ & $\mathrm{J}$ & 5 \\
\hline \multicolumn{11}{|c|}{ An X suffix indicates a duplicate sample. } \\
\hline An RR1 suffix ind & tes the lab & nducted a & re-analysis at & a higher dilt & ution due to & gher than expected & & & & \\
\hline
\end{tabular}

Page 78 of 78

Uncontrolled When Printed 
Appendix B

\section{Data Quality Objective Process for \\ CAU 383, Area 12 E-Tunnel Sites}




\section{B.1.0 Seven-Step DQO Process Characterization of CAU 383 (Area 12 E-Tunnel Sites)}

The DQO process is a planning approach based on the scientific method that is used to ensure that data collected during site characterization will provide sufficient and reliable information to identify, evaluate, and technically defend potentially viable corrective actions (i.e., no further action, closure in place, or clean closure). The existing information about the nature and extent of contamination at the three CASs in CAU 383 and associated sites is insufficient to evaluate and select preferred corrective actions. The CAU 383 investigation will be based on DQOs agreed to by representatives of NDEP and DTRA.

Three CASs comprise CAU 383:

- CAS 12-06-06, Muckpile

- CAS 12-25-02, Oil Spill

- CAS 12-28-02, Radioactive Material

In addition, the CAI will evaluate the ponds downgradient of the Muckpile, the drainage between the Muckpile and ponds, and the adjacent blower pad.

\section{B.1.1 Step 1 - State the Problem}

This step identifies the DQO planning team members, states the problem that has initiated the CAU 383 investigation, and develops the CSM.

\section{B.1.1.1 Planning Team Members}

The DQO planning team consists of representatives from NDEP, DTRA, NNSA/NSO, and the environmental engineering contractor. The primary decision-makers are NDEP, DTRA, and NNSA/NSO representatives. Table B.1-1 lists representatives from each organization who attended the September 16, 2003, meeting held to present and discuss the DQOs for CAU 383. 
Table B.1-1

DQO Meeting Participants

\begin{tabular}{||c|c||}
\hline Participant & Affiliation \\
\hline \hline Tiffany Lantow & DTRA \\
\hline Wayne Griffin & BN/DTRA \\
\hline Greg Raab & NDEP \\
\hline Steve Mergenmeier & Shaw/Quality Assurance \\
\hline Stacey Alderson & Shaw/Health Physicist \\
\hline Rick Deshler & Shaw/Project Manager \\
\hline David Schrock & Shaw/Waste Management \\
\hline Brian Hoenes & SNJV \\
\hline Richard Marty & SNJV \\
\hline Barb Deshler & Shaw/Technical \\
\hline
\end{tabular}

\section{B.1.1.2 State the Problem}

It is unknown whether COPCs exceeding PALs are present in or beneath the E-Tunnel sites, including the Muckpile CAS, the ponds, the drainage between the Muckpile and the ponds, the Oil Spill CAS, the Radioactive Material CAS, and/or the blower pad. It is also unknown whether COPCs have migrated from these sites. If contaminants are present in concentrations that exceed the PALs, they may present a risk to human health and/or the environment and have the potential for migrating off site. If contaminants are present, their nature and extent need to be determined and their risk to human health and/or the environment evaluated.

\section{B.1.1.3 Conceptual Site Model}

A CSM has been developed to describe potential exposure pathways from possible contaminant sources in the Area $12 \mathrm{E}$-Tunnel Sites. If the CSM is proven incorrect by the results of the environmental sampling, NDEP will be notified and the CAI rescoped. The following statements are assumptions and/or facts that were considered in developing the model:

- The Muckpile and ponds were placed on a thin veneer of alluvium overlying the tuffs of the Tunnel Formation and the Older Tunnel Beds and the slope facies carbonate rocks.

- The E-Tunnel Muckpile consists primarily of mining debris (rock) generated during the excavation phase of shaft construction; therefore, the bulk of the Muckpile is assumed to be uncontaminated material. The post-test portion, which includes disturbed geologic materials and construction/re-entry debris, comprises a small fraction of the Muckpile. 
- The thickness of the Muckpile ranges from less than $5 \mathrm{ft}$ at the west end of the Muckpile to approximately $100 \mathrm{ft}$ at the north side of the Muckpile. The Muckpile is approximately $200 \mathrm{ft}$ wide and 1,200 ft long in a northwest-southeast direction. The Muckpile is estimated to contain $356,000 \mathrm{yd}^{3}$ of material.

- The drive-over and walk-over radiological surveys identified two areas with elevated radiological readings on the Muckpile: one area along the road by the blower pad, and one by the electrical substation by the blower pad.

- Arsenic is found in the native soils and may also be found in the Muckpile (NBMG, 1998). In addition, the Muckpile may also contain very small volumes of RCRA-regulated hazardous constituents and beryllium. The possible distribution of these COPCs is not known.

- The Muckpile does not have a liner at its base; however, it probably received very little free liquid waste. Although specific permeability data are lacking, low precipitation and high evaporation rates in this region would tend to limit downward leaching of contaminants within the Muckpile.

- Past surface activities of concern include equipment maintenance and storage of equipment and petroleum products. Releases to the Muckpile from surface activities may be locally significant, but vertical infiltration of contaminants is probably limited to less than $5 \mathrm{ft}$.

- There are six ponds 50 to $100 \mathrm{ft}$ wide, extending a distance of approximately $1,200 \mathrm{ft}$ down canyon in an easterly direction from the Muckpile.

- This characterization will include Ponds 1, 2, and 3, which extend a distance of approximately $350 \mathrm{ft}$ down canyon from the Muckpile.

- $\quad$ Pond 1 is mostly covered by the Muckpile.

- Pond 2 is currently receiving E-Tunnel effluent. It will be allowed to dry out before it is characterized.

- $\quad$ Pond 3 is dry.

- The material in the ponds consists of fine-grained sediments that settled out of construction and groundwater from the E-Tunnel workings.

- The thickness of the material in the ponds is unknown.

- The most likely pathway for migration of contaminants away from the Muckpile is in stormwater runoff. Migration might also result when precipitation infiltrates into the thin, peripheral portions of the Muckpile and enters the native material and/or drains 
downgradient along the contact between the fill and the underlying native alluvium or bedrock.

- Possible future uses of the Muckpile may include activities that might intrude no more than $7 \mathrm{ft}$ into the Muckpile.

- The blower pad is located on the hill between the two portals and still has some equipment on it. Most of the pad is not accessible to the drill rig.

- The Oil Spill is located on the blower pad.

- The Radioactive Material area is a small fenced area at the entrance to the E-Tunnel Main portal.

The CSM was developed using these assumptions. It was postulated that the majority of the Muckpile does not contain COPCs, and if any COPCs are present, they are probably isolated. Because there are no records concerning the precise subsurface distribution of fill materials, the location of buried COPCs cannot be predicted. Operational knowledge and surface radiological surveys can be used to increase the probability of finding impacted areas of the Muckpile. The areas most likely to be affected are the areas where petroleum compounds were used for tunnel equipment maintenance activities, possibly resulting in releases to the surface and shallow subsurface (0 to $5 \mathrm{ft}$ ) soils. These releases, if present, are expected to have limited lateral and vertical extent. It is also possible, but unlikely, that the native soil beneath the Muckpile and ponds has been impacted by downward migration of COPCs. Figure B.1-1 is a generalized drawing of the CSM.

\section{B.1.2 Step 2 - Identify the Decision}

This step develops the decision statement.

\section{B.1.2.1 Develop the Decision Statement}

Are there concentrations of COPCs present in the Muckpile, Oil Spill or Radioactive Material CASs; ponds; or blower pad that exceed the action levels and, if so, do they pose a threat to human health and/or the environment? 


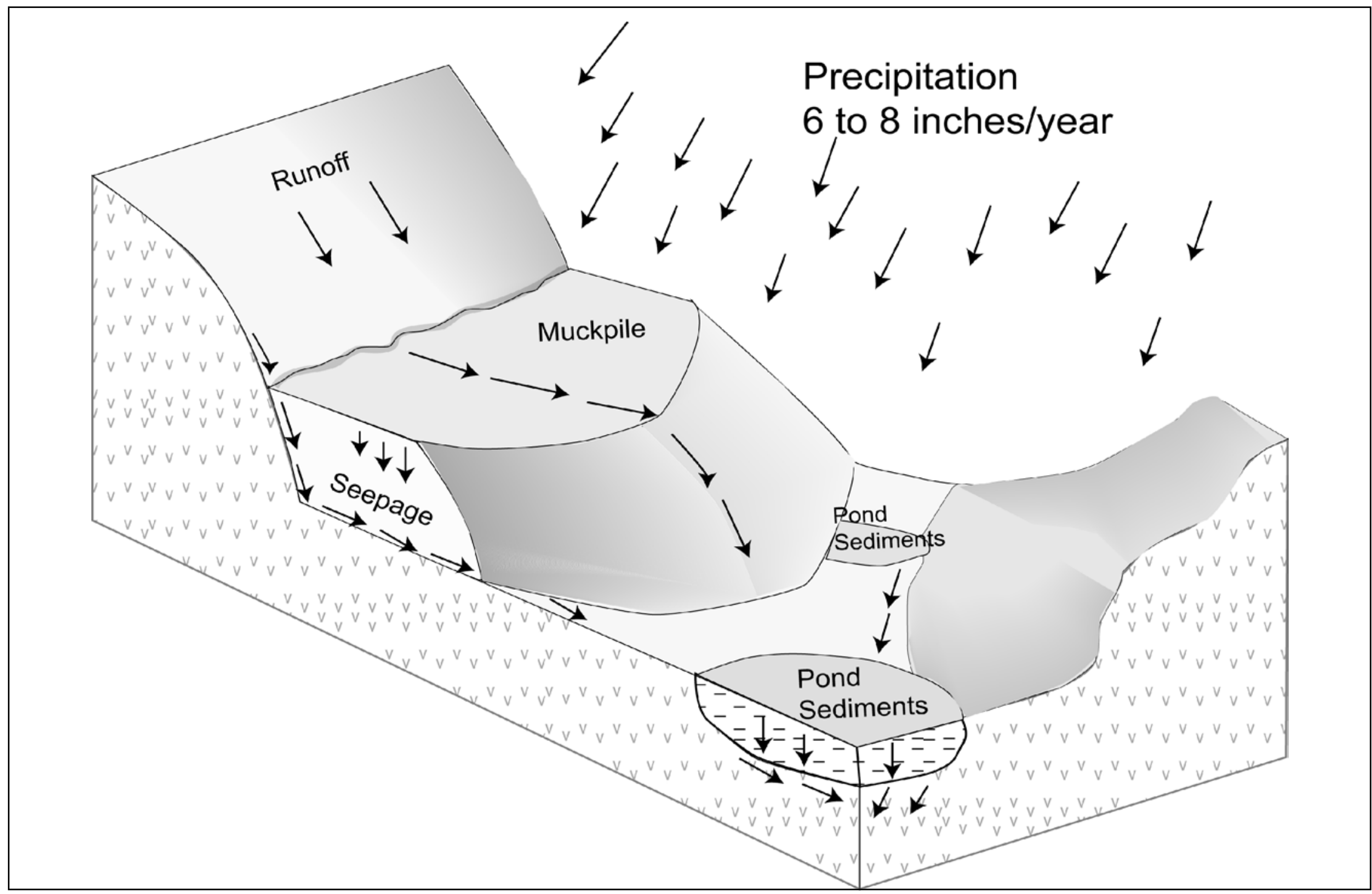

Figure B.1-1

Generalized Conceptual Site Model for CAU 383 


\section{B.1.3 Step 3 - Identify Inputs to the Decision}

This step identifies the information needed, determines sources for information, determines the basis for establishing the action level, and identifies sampling and analysis methods that can meet the data requirements.

\section{B.1.3.1 Information Needs and Information Sources}

Inputs to the decision are centered on the sampling approach, which depends foremost on a reasonable CSM. The model provides a basis for development of the approach and ultimately the course of action that will be taken for the site. In turn, the CSM is tested and confirmed by the results of the sampling. The sampling will consist of collecting random and biased environmental samples from the Muckpile, the ponds, the native materials beneath the Muckpiles and ponds, and the drainages below the Muckpile and ponds. In addition, the areas identified as CAS 12-25-02, Oil Spill, and CAS 12-28-02, Radioactive Material, will be sampled separately.

Table B.1-2 lists the information needs, the source of information for each need, the proposed methods to collect the data, and the QA/QC data type. The data type is determined by the intended use of the resulting data in decision making. Data types are discussed in the following text.

\section{B.1.3.1.1 Quantitative Data}

Quantitative data directly measure the quantity or amount of a characteristic or component within the population of interest. These data require the highest level of QA/QC in collection and measurement systems because the intended use of the data is to resolve primary decisions (i.e., rejecting or accepting the null hypothesis) and/or verifying that closure standards have been met. Laboratory analytical data are generally considered quantitative.

\section{B.1.3.1.2 Semiquantitative Data}

Semiquantitative data indirectly measure the quantity or amount of a characteristic or component. Inferences are drawn about the quantity or amount of a characteristic or component because a correlation has been shown to exist between the indirect measurement and the results from a quantitative measurement. The QA/QC requirements on semiquantitative collection and measurement systems are high, but may not be as rigorous as a quantitative measurement system. Semiquantitative data contribute to decision making but are not used alone to resolve primary decisions. 
Field-screening data are generally considered semiquantitative. The data are often used to guide investigations toward quantitative data collection.

Table B.1-2

Information Needs to Resolve the Decision

(Page 1 of 2)

\begin{tabular}{|c|c|c|c|}
\hline $\begin{array}{l}\text { Information } \\
\text { Need }\end{array}$ & Information Source & Collection Method & Data Type \\
\hline $\begin{array}{l}\text { Identify the } \\
\text { COPCs. }\end{array}$ & $\begin{array}{l}\text { - Results of historical data } \\
\text { review } \\
\text { - Results of the walk-over } \\
\text { and/or drive-over radiological } \\
\text { survey }\end{array}$ & $\begin{array}{l}\text { - Review the source terms for all } \\
\text { tests that could have contributed } \\
\text { contaminants to the Muckpile. } \\
\text { - Review historical data for } \\
\text { chemicals that may have been } \\
\text { used on site. } \\
\text { - Review historical data for potential } \\
\text { release information. }\end{array}$ & Qualitative \\
\hline $\begin{array}{l}\text { Are any COPCs } \\
\text { present in the } \\
\text { Muckpile, Oil } \\
\text { Spill, or } \\
\text { Radioactive } \\
\text { Material CASs; } \\
\text { ponds; blower } \\
\text { pad; or drainage? }\end{array}$ & $\begin{array}{l}\text { Analytical results of soil } \\
\text { samples }\end{array}$ & $\begin{array}{l}\text { - Collect soil samples at random } \\
\text { locations and at random depths in } \\
\text { the Muckpile, ponds, and drainage } \\
\text { utilizing a rotosonic drill rig. } \\
\text { - Collect near-surface biased soil } \\
\text { samples from the Oil Spill and } \\
\text { Radioactive Material area using } \\
\text { the rotosonic drill rig or hand tools. } \\
\text { - Send samples to an off-site } \\
\text { laboratory to be analyzed for } \\
\text { chemical and radiological } \\
\text { constituents. }\end{array}$ & Quantitative \\
\hline $\begin{array}{l}\text { Are any COPCs } \\
\text { present in the } \\
\text { native material } \\
\text { beneath the } \\
\text { Muckpile, Oil } \\
\text { Spill, or } \\
\text { Radioactive } \\
\text { Material CASs; } \\
\text { ponds, or blower } \\
\text { pad? }\end{array}$ & $\begin{array}{l}\text { Analytical results of soil } \\
\text { samples }\end{array}$ & $\begin{array}{l}\text { - Collect soil samples of native } \\
\text { material at various locations } \\
\text { immediately beneath the Muckpile } \\
\text { and ponds utilizing a rotosonic drill } \\
\text { rig. } \\
\text { - Send all samples to an off-site } \\
\text { laboratory to be analyzed for } \\
\text { chemical and radiological } \\
\text { constituents. }\end{array}$ & Quantitative \\
\hline $\begin{array}{l}\text { If COPCs are } \\
\text { present in or } \\
\text { beneath the } \\
\text { Muckpile, Oil } \\
\text { Spill, or } \\
\text { Radioactive } \\
\text { Material CASs; } \\
\text { ponds, or blower } \\
\text { pad, are the } \\
\text { concentrations } \\
\text { high enough to } \\
\text { exceed PALs? }\end{array}$ & $\begin{array}{l}\text { - Analytical results of soil } \\
\text { samples collected with the } \\
\text { drill rig } \\
\text { - U.S. Environmental } \\
\text { Protection Agency Region } 9 \\
\text { Preliminary Remediation } \\
\text { Goals (PRGs) (EPA, 2002) } \\
\text { - Nevada Administrative Code } \\
445.2272 \text { (NAC, 2005) } \\
\text { - Individual isotope and metals } \\
\text { concentrations in background } \\
\text { soil samples } \\
\text { - Individual isotope and metals } \\
\text { concentrations from } \\
\text { published sources }\end{array}$ & $\begin{array}{l}\text { - Compare the analytical results } \\
\text { from the off-site laboratory for the } \\
\text { chemical and radiological data to } \\
\text { the PALs taking into account that } \\
\text { the site is an industrial facility in a } \\
\text { nuclear and high explosives test } \\
\text { zone. }\end{array}$ & Quantitative \\
\hline
\end{tabular}


Table B.1-2

Information Needs to Resolve the Decision

(Page 2 of 2)

\begin{tabular}{|c|c|c|c|}
\hline $\begin{array}{c}\text { Information } \\
\text { Need }\end{array}$ & Information Source & Collection Method & Data Type \\
\hline $\begin{array}{l}\text { If COPCs are } \\
\text { present in } \\
\text { concentrations } \\
\text { that exceed the } \\
\text { PALs, what is } \\
\text { their extent? }\end{array}$ & $\begin{array}{l}\text { - Analytical results of soil } \\
\text { samples } \\
\text { - Results of the walk-over and } \\
\text { drive-over radiological survey } \\
\text { - Field-screening results } \\
\text { collected with the Electra } \\
\text { - Analytical results from soil } \\
\text { samples run on the on-site } \\
\text { hyperpure Germanium } \\
\text { (HPGe) (gamma counter) }\end{array}$ & $\begin{array}{l}\text { - The walk-over and/or drive-over } \\
\text { survey will be used to determine } \\
\text { the lateral extent of any } \\
\text { contamination. } \\
\text { - Boreholes will be continued until } \\
\text { two consecutive drilling intervals } \\
\text { are below the field-screening } \\
\text { levels as determined from Electra } \\
\text { and HPGe results (unless refusal } \\
\text { is encountered) to define the } \\
\text { vertical extent. }\end{array}$ & Quantitative \\
\hline $\begin{array}{l}\text { If COPCs are } \\
\text { present in } \\
\text { concentrations } \\
\text { that exceed PALs, } \\
\text { is there a } \\
\text { potential for } \\
\text { migration? }\end{array}$ & $\begin{array}{l}\text { - Analytical results of soil } \\
\text { samples } \\
\text { - Results of the walk-over and } \\
\text { drive-over radiological survey } \\
\text { - Stability and drainage } \\
\text { surveys }\end{array}$ & $\begin{array}{l}\text { - Assess existing data for usability } \\
\text { and collect geotechnical samples if } \\
\text { needed. } \\
\text { - Evaluate the results of the surveys } \\
\text { to determine the stability of the } \\
\text { Muckpile. }\end{array}$ & Semiquantitative \\
\hline
\end{tabular}

\section{B.1.3.1.3 Qualitative Data}

Qualitative data identify or describe the characteristics or components of the population of interest. The QA/QC requirements are the least rigorous on data collection methods and measurement systems. The intended use of the data is for information purposes, to refine CSMs, and to guide investigations rather than resolve primary decisions. This measurement of quality is typically assigned to historical information and data where QA/QC may be highly variable or not known. Professional judgment is often used to generate qualitative data.

\section{B.1.3.1.4 Determine the Basis for the Preliminary Action Levels}

Laboratory analytical results for soils will be compared to the following PALs to evaluate whether COPCs are present at levels that may pose an unacceptable risk to human health and/or the environment:

- $100 \mathrm{mg} / \mathrm{kg}$ TPH in soil, per NAC 445A.2272, “Contamination of Soils: Establishment of Action Levels” (NAC, 2005)

- Background concentrations for metals when natural background exceeds the PRG, as is often the case with arsenic. Background is considered the highest measurement from soil samples taken as local background or from soil samples collected by the Nevada Bureau of Mines and Geology throughout the Nellis Air Force Range (NBMG, 1998; Moore, 1999). 
- EPA Region 9 Preliminary Remediation Goals (EPA, 2002) for industrial soils, as based on future use of the site in a Nuclear and High Explosive Test Zone (DOE/NV, 1996a and $\mathrm{b}$ and 1998).

- The PALs for radionuclides are isotope-specific and defined as the higher of the maximum concentration for that isotope found in samples from undisturbed background locations in the vicinity of the Nevada Test Site (McArthur and Miller, 1989; US Ecology and AtlanTech, Inc., 1992; Black and Townsend, 1996), from any of the three background samples collected during the investigation, or the POC as specified in the Nevada Test Site Performance Objective for Certification of Nonradioactive Hazardous Waste (POC) (BN, 1995).

\section{B.1.3.1.5 Potential Sampling Techniques and Appropriate Analytical Methods}

\section{B.1.3.1.5.1 Radiological Surveys}

Radiological surveys will be used to help determine the presence and lateral extent of radiological contamination. Radiological surveys will follow standard procedures. Further information is provided in Section B.1.7.1.

\section{B.1.3.1.5.2 Soil Sampling}

Rotosonic drilling and hand tools will be used to collect the soil samples. Soil sample collection and handling activities will follow standard procedures. Section 6.0 of the CAIP lists analytical methods and laboratory requirements (e.g., detection limits, precision, and accuracy) for the investigation. Sample volumes are laboratory- and method-specific and will be determined in accordance with laboratory requirements. Specific analyses required for the disposal of IDW are identified in Section 5.0 of the CAIP.

To ensure that laboratory analyses are sufficient to detect contamination in soil samples at concentrations exceeding the minimum reporting limit, chemical and/or radiological parameters of interest have been selected for the CAU. The chemical and radiological parameters are listed in Table B.1-3. 
Table B.1-3

Analyses to Be Performed

\begin{tabular}{|c|c|c|c|c|c|c|c|}
\hline \multirow[b]{2}{*}{ Analyses } & \multicolumn{7}{|c|}{ Sample Type } \\
\hline & 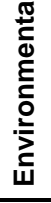 & $\begin{array}{l}\text { 쥬 } \\
\frac{.0}{0} \\
\overline{0}\end{array}$ & 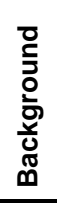 & 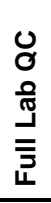 & 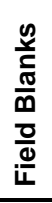 & 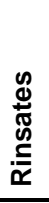 & 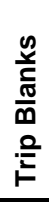 \\
\hline \multicolumn{8}{|l|}{ Organics } \\
\hline VOCs & $x$ & $x$ & & $x$ & $x$ & $x$ & $x$ \\
\hline SVOCS & $x$ & $X$ & & $x$ & $x$ & $x$ & \\
\hline TPH-DRO & $x$ & $X$ & & $x$ & $x$ & $x$ & \\
\hline \multicolumn{8}{|l|}{ Metals } \\
\hline Total RCRA Metals & $x$ & $X$ & $x$ & $x$ & $x$ & $x$ & \\
\hline Beryllium & $x$ & $\mathrm{X}$ & $x$ & $x$ & $x$ & $x$ & \\
\hline \multicolumn{8}{|l|}{ Radionuclides } \\
\hline Gamma Spectrometry & $x$ & $x$ & $x$ & & $x$ & $x$ & \\
\hline Isotopic Plutonium & $X$ & $\mathrm{X}$ & 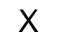 & $x$ & $x$ & $x$ & \\
\hline Total Strontium ${ }^{\mathrm{a}, \mathrm{b}}$ & $x$ & $X$ & $x$ & $x$ & $x$ & $x$ & \\
\hline Tritium (pond samples only) & $\mathrm{X}$ & $\mathrm{X}$ & & & $x$ & $x$ & \\
\hline
\end{tabular}

a Total strontium uses a stable strontium carrier as opposed to a radioactive tracer.

${ }^{\mathrm{b}}$ Although total strontium is measured, the results are reported as $\mathrm{Sr}-90$ because $\mathrm{Sr}-89$ has too short a half life to be present.

The VOCs and SVOCs expected to be analyzed for in the investigation are listed in

Tables B.1-4 and B.1-5, respectively.

\section{B.1.4 Step 4 - Define the Boundaries of the Study}

The purpose of this step is to define the target population of interest, specify the spatial and temporal features of the population that are pertinent for decision making, determine practical constraints on data collection, and define the scale of decision making relevant to target populations. 
Table B.1-4

Proposed VOCs for Analysis

\begin{tabular}{|c|c|c|}
\hline 1,1,1-Trichloroethane & 2-Chlorotoluene & Ethylbenzene \\
\hline 1,1,2-Trichloroethane & 4-Methyl-2-pentanone & Iodomethane \\
\hline 1,1,1,2-Tetrachloroethane & Acetone & Isopropylbenzene \\
\hline 1,1,2,2-Tetrachloroethane & Benzene & Methyl tertiary butyl ether \\
\hline 1,2,3-Trichloropropane & Bromobenzene & Methylene chloride \\
\hline 1,2,4-Trimethyl-benzene & Bromochloromethane & n-Butylbenzene \\
\hline 1,2-Dibromo-3-chloropropane & Bromodichloromethane & n-Propyl benzene \\
\hline 1,2-Dibromoethane & Bromoform & Sec-butyl benzene \\
\hline 1,1-Dichloroethane & Bromomethane & Styrene \\
\hline 1,1-Dichloroethene & Carbon disulfide & Tert-butyl benzene \\
\hline cis-1,2-Dichloroethene & Carbon tetrachloride & Tetrachloroethene \\
\hline trans-1,2-Dichloroethene & Chlorobenzene & Toluene \\
\hline 1,2-Dichloroethane & Chloroethane & Trichloroethene \\
\hline 1,2-Dichloropropane & Chloroform & Trichlorofluoromethane \\
\hline 1,3,5-Trimethylbenzene & Chloromethane & Trichlorotrifluoroethane \\
\hline cis-1,3-Dichloropropene & Dibromochloromethane & Vinyl acetate \\
\hline trans-1,3-Dichloropropene & Dibromomethane & Vinyl chloride \\
\hline 2-Butanone & Dichlorodifluoromethane & Xylene \\
\hline
\end{tabular}

Table B.1-5

Proposed SVOCs for Analysis

\begin{tabular}{|c|c|c|}
\hline 1,2,4-Trichlorobenzene* & Acenaphthylene & Di-n-butyl phthalate \\
\hline 1,2-Dichlorobenzene* & Aniline & Di-n-octyl phthalate \\
\hline 1,3-Dichlorobenzene* & Anthracene & Fluoranthene \\
\hline 1,4-Dichlorobenzene & Benzo(a)anthracene & Fluorene \\
\hline 2,4,5-Trichlorophenol & Benzo(a)pyrene & Hexachlorobenzene \\
\hline 2,4,6-Trichlorophenol & Benzo(b)fluoranthene & Hexachlorobutadiene* \\
\hline 2,4-Dichlorophenol & Benzo(g,h,i)perylene & Hexachlorocyclopentadiene \\
\hline 2,4-Dimethylphenol & Benzo(k)fluoranthene & Hexachloroethane \\
\hline 2,4-Dinitrophenol & Benzoic acid & Indeno(1,2,3-cd)pyrene \\
\hline 2,4-Dinitrotoluene & Benzyl alcohol & Isophorone \\
\hline 2,6-Dinitrotoluene & Bis(2-chloroethoxy) methane & Naphthalene* \\
\hline 2-Chloronaphthalene & Bis(2-chloroethyl)ether & Nitrobenzene \\
\hline 2-Chlorophenol & Bis(2-chloroisopropyl)ether & N-Nitroso-di-n-propylamine \\
\hline 2-Methylphenol & Bis(2-ethylhexyl) phthalate & N-Nitrosodimethylamine \\
\hline 2-Nitroaniline & Butyl benzyl phthalate & N-Nitrosodiphenylamine \\
\hline 3,3'-Dichlorobenzidine & Carbazole & Pentachlorophenol \\
\hline 4-Bromophenyl phenyl ether & Chrysene & Phenanthrene \\
\hline 4-Chloroaniline & Dibenzo(a,h)anthracene & Phenol \\
\hline 4-Methylphenol & Dibenzofuran & Pyrene \\
\hline 4-Nitrophenol & Diethyl phthalate & Pyridine \\
\hline Acenaphthene & Dimethyl phthalate & \\
\hline
\end{tabular}

* May be reported with VOCs 


\section{B.1.4.1 Define the Target Population}

The target populations for the characterization are:

a. The material in the Muckpile, Oil Spill, and Radioactive Material area, and ponds

b. The native material underlying the Muckpile, Oil Spill, and Radioactive Material area, and ponds to a depth of no more than $5 \mathrm{ft}$

c. The muck underlying the Radioactive Material CAS near the portal

\section{B.1.4.2 Identify the Spatial and Temporal Boundaries}

The horizontal boundaries of the study area are the edges of the Muckpile, Oil Spill, and ponds to the extent that elevated radiological readings were identified by the drive-over and/or walk-

over surveys. The vertical boundary is 2 to $5 \mathrm{ft}$ below the bottom of the Muckpile and ponds or until drill refusal (defined as requiring more than 15 minutes to drill $1 \mathrm{ft}$ ). The Radioactive Material is located within the spatial boundaries of the Muckpile. The study area also includes undisturbed background sample locations outside, but adjacent to, the Muckpile boundaries. There are no temporal constraints on the characterization.

\section{B.1.4.3 Identify Practical Constraints on the Characterization}

The practical constraints on the investigation are the capability of the drill to penetrate the Muckpile and native material, the ability to get the drill onto the sample locations, and the weather, all of which can impact the field work.

\section{B.1.4.4 Define the Scale of the Decision Making}

The scale of decision making is defined as the Muckpile, consisting of two CASs as one unit, the ponds as a separate unit, the drainage between the Muckpile and ponds as a separate unit, and the blower pad (including the Oil Spill) on the bench above the Muckpile as a fourth unit. Dividing the units in this manner allows the corrective actions to be tailored to the different media that may be contaminated.

\section{B.1.5 Step 5 - Develop a Decision Rule}

This step integrates outputs from the previous steps into a decision rule (“If..., then...”) statement. This rule describes the conditions under which possible alternative actions would be chosen. 


\section{B.1.5.1 Specify the Population Parameter}

The population parameter is the maximum observed concentration of each COPC within the target population. For radiological surveys, the maximum observed concentration of each COPC will be the population parameter. If sampling is performed to support the radiological survey results, the maximum observed concentration of each COPC identified in the sample will be the population parameter. Radiological sampling results will supersede radiological survey results.

\section{B.1.5.2 Choose an Action Level}

Action levels are defined in Section B.1.3.1.4.

\section{B.1.5.3 Measurement and Analysis Methods}

Radiological surveys and soil sampling and analysis, identified in Sections B.1.7.1 and B.1.7.2, will be used to identify the presence and location of COPCs.

The measurement and analysis methods referenced in the Industrial Sites QAPP (NNSA/NV, 2002) are capable of achieving the expected range of values to resolve the Primary Decision. The detection limit of the measurement method to be used must be less than the action level for each COPC unless otherwise specified in the CAIP.

\section{B.1.5.4 Decision Rule}

The following decision rules are applicable to the CAU 383 investigation and will be used to guide the investigation and data evaluation:

- If laboratory results for the soil samples indicate the presence of COPCs above the PALs, then a CADD will be prepared.

- If the laboratory results for the soil samples do not indicate the presence of COPCs above the PALs, then a CADD or CADD/CR will be prepared.

The PALs are defined in Section B.1.3.1.4.

\section{B.1.6 Step 6 - Specify the Tolerable Limits on the Decision Errors}

Only validated analytical results (quantitative data) will be used to determine whether COPCs are present, unless otherwise stated. The baseline condition, or null hypothesis, assumed for this site is that COPCs above the action levels are present in the Muckpile. The alternate hypothesis is that COPCs above the action levels are not present in the Muckpile. Based on these 
hypotheses, two types of decision errors are possible, false positive and false negative. This CAIP has been designed to minimize both types of errors.

\section{B.1.6.1 False Positive Decision Error}

The consequences of a false positive are: (1) the corrective action could be needlessly expanded to encompass a greater quantity of media than is necessary, and (2) media incorrectly judged to be contaminated could be treated as regulated waste rather than unregulated waste. Both of these consequences could lead to increased corrective action and waste disposal costs.

\section{B.1.6.2 False Negative Decision Error}

The consequences of a false negative are: (1) regulated contaminants might not be appropriately addressed by the corrective action, (2) contaminated media could be wrongly classified as non contaminated, and (3) contaminated media might be disposed of improperly. These consequences could result in unacceptable risks to human health and the environment and potential fines from regulatory agencies.

\section{B.1.6.3 Statistical Model}

A statistical analysis was conducted to determine the answer to two questions. The first question is, were sufficient samples taken to ensure a 90 percent confidence level in the mean COPC concentration? The second question is, does the mean concentration exceed the regulatory threshold $(\mathrm{RT})$ ?

The EPA has defined RTs for many chemicals and metals in soil. However, RTs for radionuclides in soil have not been promulgated for sites such as CAU 383, nor have they been defined for the radionuclide COPCs likely to be found at NNSA/NSO and/or DTRA sites. Therefore, the radionuclide RTs used for this analysis are the established radionuclide-specific background concentration or the "rad added” screening levels of the POC (BN, 1995), whichever is greater.

If chemical COPCs are found at the site, the RT values used to calculate whether sufficient samples have been taken will be the PALs as defined in Section 3.3 of the CAIP. For TPH, the PAL is $100 \mathrm{mg} / \mathrm{kg}$ in soil, and for RCRA-regulated contaminants, the EPA Region 9 PRGs for Industrial Soils (EPA, 2002) are the PALs.

To estimate the minimum number of samples required to characterize the E-Tunnel sites, the Cs-137 results from earlier investigations were used to provide input into equation 8 in SW-846 
Chapter 9 (EPA, 1998). The Cs-137 results were used because Cs-137 is found in more of the tunnel samples than any other contaminant. The number of samples required to ensure a 90 percent confidence level was calculated using only the samples with Cs-137 concentrations exceeding the minimum detectable concentration (MDC).

\section{Testing for the Number of Required Samples}

The radionuclide analytical data from previous Muckpile, drainage, and pond characterizations were used to estimate the number of samples needed to characterize the E-Tunnel Muckpile, drainage, and ponds because there are no analytical data available from the E-Tunnel sites on which to make a determination. After the characterization is completed the procedure described in SW-846 Chapter 9 will again be used to confirm that sufficient samples were collected to characterize the site at the 90 percent confidence level (EPA, 1998).

\section{Calculation of the Number of Samples}

Determine the number of samples required to obtain a 90 percent confidence level for the E-Tunnel Muckpile, ponds, and drainage using the Cs-137 data.

$$
\mathrm{n}=\mathrm{t}_{.20}{ }^{2} \times \mathrm{s}^{2} /(\mathrm{RT}-0)^{2}
$$

Required number of samples for the Muckpile:

$$
\begin{aligned}
& \mathrm{n}=\quad \begin{array}{l}
\text { minimum number of samples to ensure a } 90 \text { percent confidence level in the calculated mean } \\
\text { concentration in the COPC }
\end{array} \\
& \mathrm{t}_{.20}{ }^{2}=\begin{array}{l}
\text { 1.680, the square of } 1.296 \text {, the } \mathrm{t}_{.20} \text { value for } 60 \text { degrees of freedom, } 90 \text { percent confidence } \\
\text { level, Table } 9-2 \text { of SW-846 (EPA, 1998) }
\end{array} \\
& \mathrm{s}^{2}=\quad \text { variance of the Cs-137 concentration in the positive Muckpile samples, } 271,961 \mathrm{pCi} / \mathrm{g} \\
& \mathrm{RT}=3 \mathrm{pCi} / \mathrm{g} \\
& 0=\quad \text { mean Cs-137 concentration in the positive Muckpile soil samples, } 228.6 \mathrm{pCi} / \mathrm{g} \\
& \mathrm{n}=\quad \mathrm{t}_{.20}{ }^{2} \times \mathrm{s}^{2} /(\mathrm{RT}-0)^{2}=(1.680 \times 271,961) /(3-228.6)^{2}=8.98 \text { samples }
\end{aligned}
$$


Required number of samples for the ponds:

$\mathrm{n}=$ minimum number of samples to ensure a 90 percent confidence level in the calculated mean concentration in the COPC

$\mathrm{t}_{.20}{ }^{2}=1.698$, the square of 1.303, the $\mathrm{t}_{.20}$ value for 40 degrees of freedom, 90 percent confidence level, Table 9-2 of SW-846 (EPA, 1998)

$s^{2}=$ variance of the Cs-137 concentration in the positive pond samples, 20,064 pCi/g

$\mathrm{RT}=3 \mathrm{pCi} / \mathrm{g}$

$0=$ mean Cs-137 concentration in the positive pond soil samples, $69.32 \mathrm{pCi} / \mathrm{g}$

$\mathrm{n}=\mathrm{t}_{.20}{ }^{2} \times \mathrm{s}^{2} /(\mathrm{RT}-0)^{2}=(1.798 \times 20,064) /(3-69.32)^{2}=7.75$ samples

Required number of samples for the drainage:

$\mathrm{n}=$ minimum number of samples to ensure a 90 percent confidence level in the calculated mean concentration in the COPC

$\mathrm{t}_{.20}{ }^{2}=1.6796$, the square of 1.296 , the $\mathrm{t}_{.20}$ value for 90 degrees of freedom, 90 percent confidence level, Table 9-2 of SW-846 (EPA, 1998)

$\mathrm{s}^{2}=\quad$ variance of the Cs-137 concentration in the positive ravine samples, 2,962 $\mathrm{pCi} / \mathrm{g}$

$\mathrm{RT}=3 \mathrm{pCi} / \mathrm{g}$

$0=$ mean Cs-137 concentration in the positive drainage soil samples, $20.02 \mathrm{pCi} / \mathrm{g}$

$\mathrm{n}=\mathrm{t}_{.20}{ }^{2} \times \mathrm{s}^{2} /(\mathrm{RT}-0)^{2}=(1.6796 \times 2,962) /(3-20.02)^{2}=17.17$ samples

In addition to the calculated number of required samples, decision performance curve graphs were prepared using the same data from the previous Muckpile, pond, and drainage characterizations. The number of samples was calculated using a simple random sampling strategy. The action level (the lower limit on the graph) was set at the POC (BN, 1995). The upper bound of the gray area was set at the action level plus one standard deviation of the data from the previous investigations. 


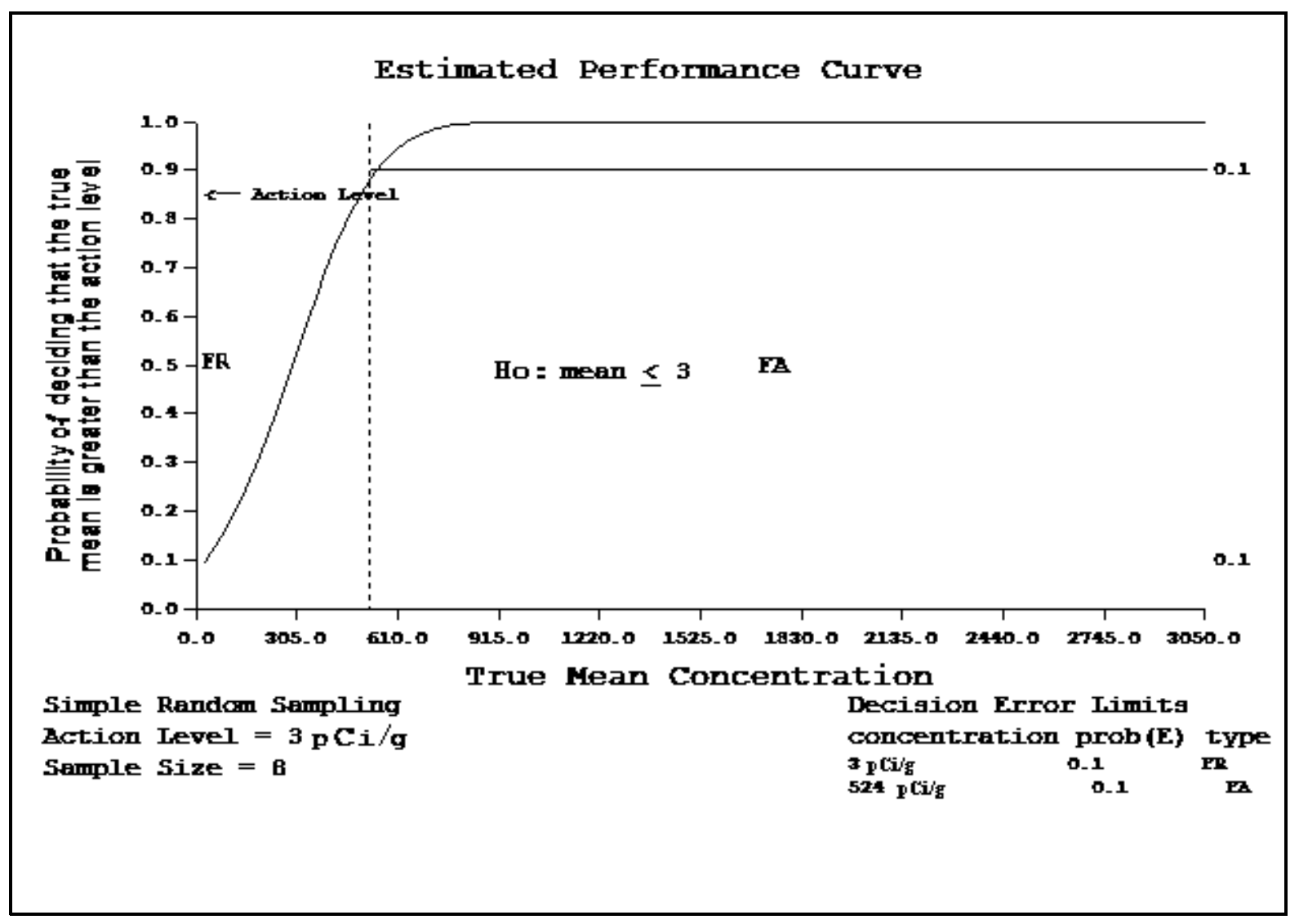

Figure B.1-2

Decision Performance Curve for Cesium-137 in Muckpiles from Previous Investigations

The graphs are presented in Figures B.1-2, B.1-3, and B.1-4. The decision performance curves are in agreement with the results from the 90 percent confidence level calculations.

The number of samples required to characterize the Muckpile, ponds, and drainage were multiplied by a factor of safety of 2 to provide additional assurance that the 90 percent confidence level would be reached.

$$
\begin{aligned}
& \mathrm{n}+(\text { Muckpile) }=8.98 \text { samples x } 2=17.96 \text { samples (round up) }=18 \text { samples } \\
& \mathrm{n}+\text { (ponds) } \quad=\quad 7.75 \text { samples } \mathrm{x} 2=15.5 \text { samples (round up) }=16 \text { samples } \\
& \mathrm{n}+(\text { drainage })=17.17 \text { samples } \times 2=34.34 \text { samples (round up) }=35 \text { samples }
\end{aligned}
$$




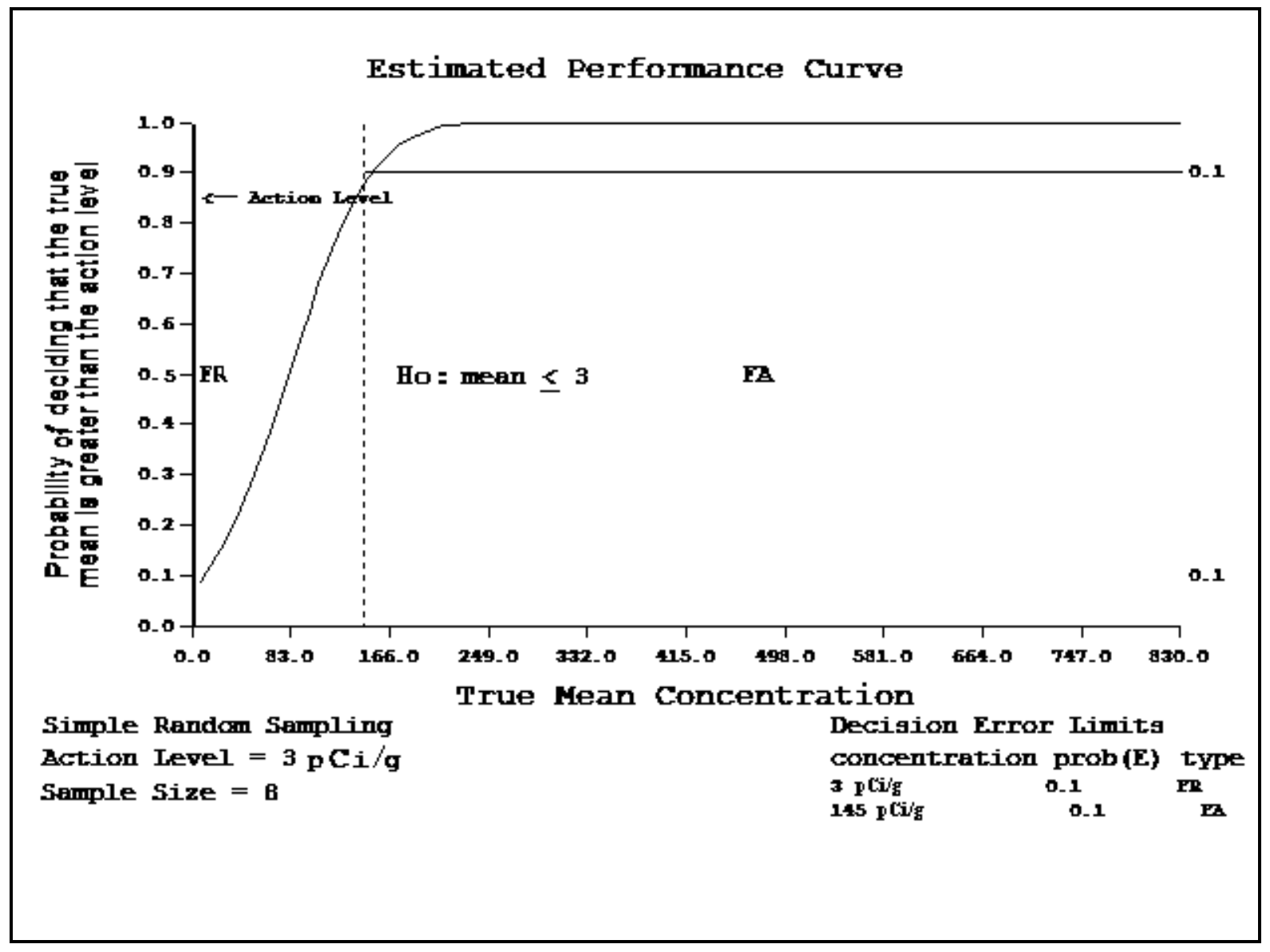

Figure B.1-3

Decision Performance Curve for Cesium-137 in Ponds from Previous Investigation

The sampling program for CAU 383 was designed to provide sufficient data to allow a statistical determination of whether enough samples were collected to adequately characterize the site. This determination will be made using the procedures described in Chapter 9 of the EPA publication SW-846, Test Methods for Evaluating Solid Waste (EPA, 1998). The mean concentration (or activity) and standard deviation of the Cs-137 concentration in the Muckpile, ponds, and drainage soils will be used to calculate the number of samples necessary to make the determination with a 90 percent confidence level.

\section{B.1.6.4 Quality Assurance/Quality Control}

Radiological survey instruments will be calibrated in accordance with the manufacturer's instructions, and periodic calibrations will be performed in accordance with approved procedures. 


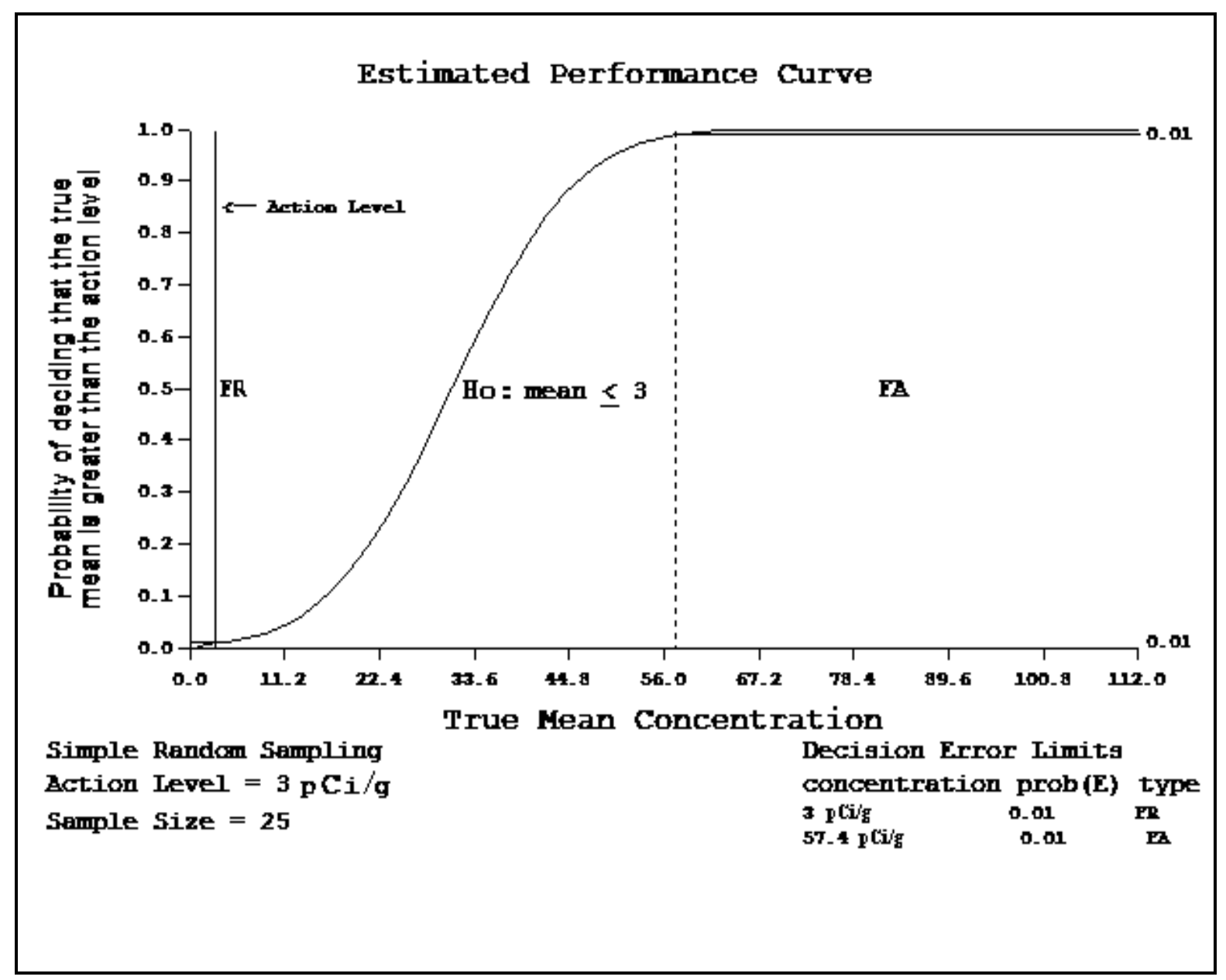

Figure B.1-4

Decision Performance Curve for Cesium-137 in Drainages from Previous Investigations

Quality control samples will be collected as required by established procedures. The required QC samples include:

- Trip blanks (one per sample cooler containing VOC environmental samples)

- Equipment blanks (one per sampling event for each type of decontamination procedure)

- Source blanks (one per source of water for decontamination and 1 from the water tank the first time it is filled); one sample can fill both requirements.

- Field duplicates (minimum of one per matrix per 20 environmental samples)

- Field blanks (minimum of one per 20 environmental samples); additional samples may be collected at the discretion of the SS if conditions change. 
- $\quad$ Matrix spike/matrix spike duplicate (minimum of one per matrix per 20 environmental samples), not needed for some radioanalytical measurements (e.g., gamma spectrometry)

Additional QC samples may be submitted based on site conditions.

Data Quality Indicators of precision, accuracy, comparability, completeness, and representativeness are defined in the Industrial Sites QAPP (NNSA/NV, 2002). Site-specific DQIs are discussed in more detail in Section 6.0 of the CAIP.

\section{B.1.7 Step 7 - Optimize the Design}

The site characterization will consist of collecting both random and biased soil samples from the Muckpile, ponds, and the drainage downgradient from the Muckpiles and ponds. The sampling program has been optimized by conducting preliminary drive-over and walk-over radiological surveys to help guide the biased sampling, by determining the location and number of samples to collect, and by determining which parameters to analyze for. The COPCs for CAU 383 listed in Section B.1.3.1.5.2 are TPH-DRO, VOCs, SVOCs, total RCRA metals, and radionuclides. All environmental samples will be analyzed for these parameters with the exception of the background samples, which will be analyzed for radionuclides and RCRA metals only. Sample locations identified on the surface of the Muckpile are biased to reflect areas where preliminary radiological surveys identified elevated radiological readings, there is visible staining, or work processes may have impacted the Muckpile surface soils.

\section{B.1.7.1 Radiological Survey Methodologies and Instruments}

Radiological surveys were conducted at CAU 383 to define the presence and lateral extent of radiological contaminants.

A combination of walk-over surveys using hand-held instruments and drive-over surveys using a vehicle-mounted detector were performed at CAU 383. Hand-held radiological survey instruments such as the NE Technology Electra, Eberline E-600, TSA-PRM-470B, and Bicron mRem, or equivalent were be used and the Dual Large-Area Plastic Scintillator with a TSA-VMR-3 controller was used as the vehicle-mounted instrument.

Additional equipment and software used in the radiological data collection and processing include a Trimble global positioning system receiver and associated laptop computers used to log and process the walk-over and drive-over radiological data. The mapping program Surfer was used to plot data on site maps. 


\section{B.1.7.2 Intrusive Investigation}

Intrusive investigations will be conducted at CAU 383 to determine whether COPCs are present. Samples will be collected from predetermined random and biased locations. Selection of biased locations will be based on the results of the radiological surveys, a review of the operational history, and visual inspection of the CAU.

Borehole and sampling locations on the Muckpile (Figure B.1-5) and the drainage (Figure B.1-6) were identified using a simple random sampling strategy. Borehole and sampling locations in the ponds (Figure B.1-7) were identified using a stratified random sampling strategy. Additional locations were identified to be used in the event that not all of the identified locations can be utilized. Table B.1-6 shows the random intrusive investigation locations, as well as biased locations that have been identified. The Muckpile, the drainage, and the ponds are considered as separate units because different historical data were used to determine the number of samples needed to adequately characterize each area.

Rotosonic drilling will be the primary tool used to collect the soil samples for laboratory analysis to determine whether COPCs are present in the Muckpile and ponds. Boreholes will be drilled $5 \mathrm{ft}$ into the native material if it is alluvium, $2 \mathrm{ft}$ if it is bedrock, or until refusal. If the bottom interval of the hole exceeds the field-screening levels, the hole will be continued at 2-ft intervals until there are two consecutive clean intervals or until drill refusal. If a drilling location cannot be accessed by the drill rig, hand augers or similar hand tools will be used to collect the soil samples.

The sampling strategies for each site are shown in Figures B.1-8 through B.1-12. 


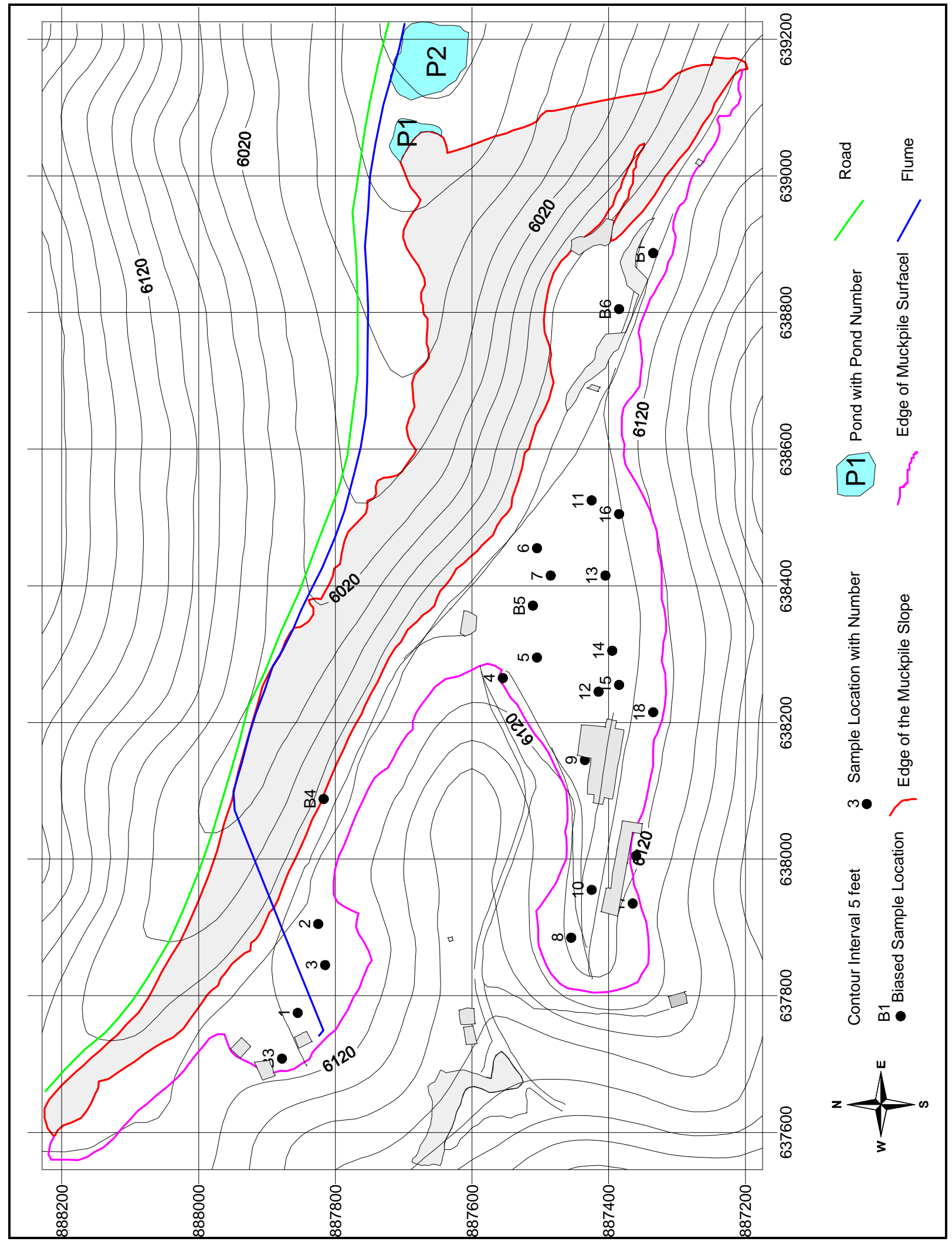

Figure B.1-5

Borehole and Sampling Locations on the Muckpile 


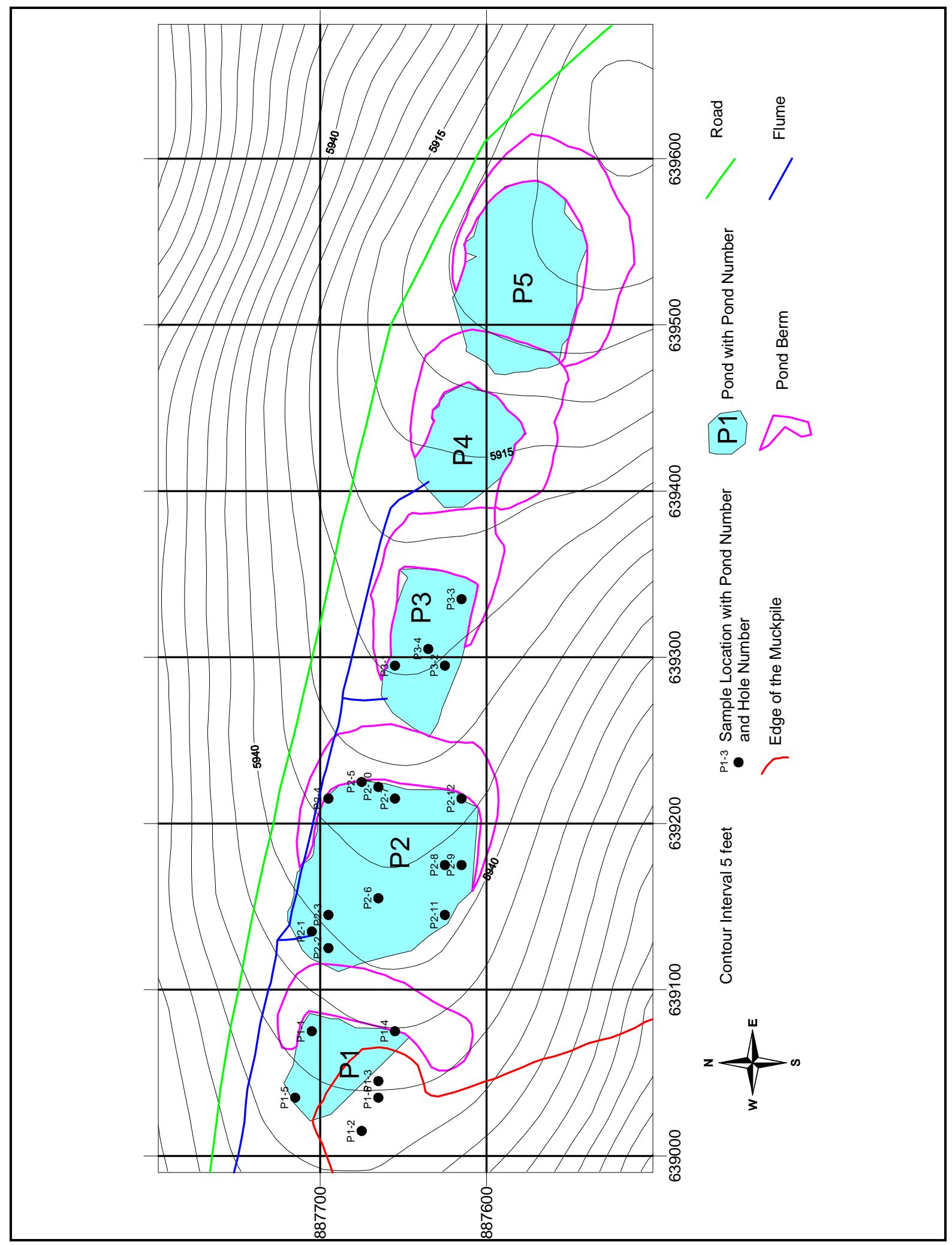

Figure B.1-6

Borehole and Sampling Locations in the Ponds 


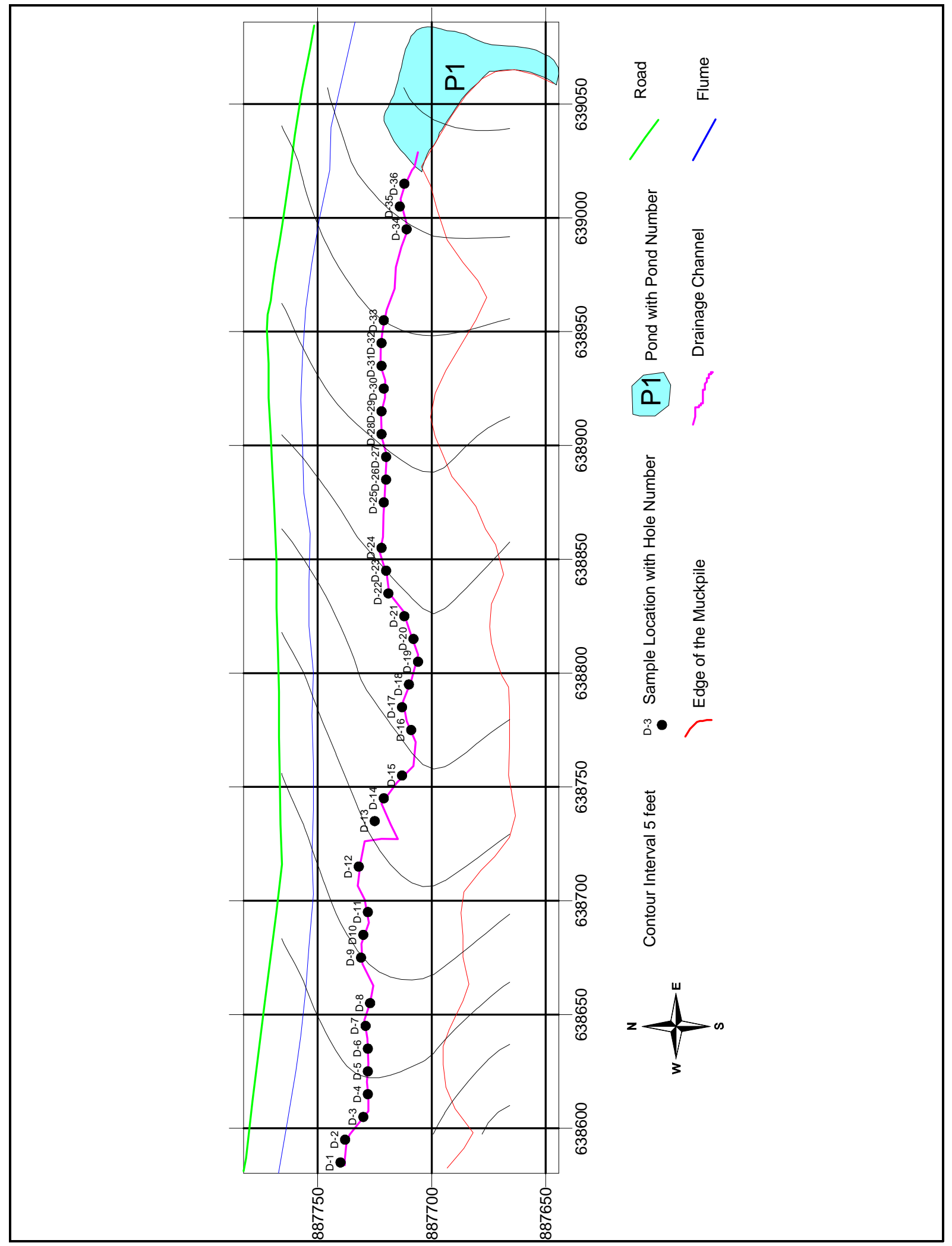

Figure B.1-7

Borehole and Sampling Locations in the Drainage 
Table B.1-6

Coordinates for Intrusive Investigation Locations

(Page 1 of 2)

\begin{tabular}{|c|c|c|c|c|c|}
\hline \multicolumn{6}{|c|}{ Muckpile Sampling Locations } \\
\hline Location & Easting & Northing & Location & Easting & Northing \\
\hline 1 & 637775 & 887855 & 11 & 638525 & 887425 \\
\hline 2 & 637905 & 887825 & 12 & 638245 & 887415 \\
\hline 3 & 637845 & 887815 & 13 & 638415 & 887405 \\
\hline A1 (alternate) & 737715 & 887875 & 14 & 638305 & 887395 \\
\hline A2 (alternate) & 637855 & 887825 & 15 & 638255 & 887385 \\
\hline A3 (alternate) & 637895 & 887775 & 16 & 638505 & 887385 \\
\hline 4 & 638265 & 887555 & 17 & 637935 & 887365 \\
\hline 5 & 638295 & 887505 & 18 & 638215 & 887335 \\
\hline 6 & 638455 & 887505 & A4 (alternate) & 638295 & 887645 \\
\hline 7 & 638415 & 887485 & A5 (alternate) & 638115 & 887455 \\
\hline 8 & 637885 & 887455 & A6 (alternate) & 638015 & 887425 \\
\hline 9 & 638145 & 887435 & A7 (alternate) & 638395 & 887415 \\
\hline 10 & 637955 & 887425 & A8 (alternate) & 638405 & 887335 \\
\hline \multicolumn{6}{|c|}{ Pond Sampling Locations } \\
\hline Location & Easting & Northing & Location & Easting & Northing \\
\hline P1-1 & 639075 & 887705 & P2-6 & 887655 & 887665 \\
\hline P1-2 & 639015 & 887675 & P2-7 & 639215 & 887655 \\
\hline P1-3 & 639045 & 887665 & P2-8 & 639175 & 887625 \\
\hline P1-4 & 639075 & 887655 & P2-9 & 639175 & 887615 \\
\hline P1-5 (alternate) & 639035 & 887715 & P2-10 (alternate) & 639222 & 887665 \\
\hline P1-6 (alternate) & 639035 & 887665 & P2-11 (alternate) & 639145 & 887625 \\
\hline P2-1 & 639135 & 887705 & P2-12 (alternate) & 639215 & 887615 \\
\hline P2-2 & 639125 & 887695 & P3-1 & 639295 & 887655 \\
\hline P2-3 & 639145 & 887695 & P3-2 & 639295 & 887625 \\
\hline P2-4 & 639215 & 887695 & P3-3 & 639335 & 887615 \\
\hline P2-5 & 639225 & 887675 & P3-4 (alternate) & 639305 & 887635 \\
\hline \multicolumn{6}{|c|}{ Drainage Sampling Locations } \\
\hline Location & Easting & Northing & Location & Easting & Northing \\
\hline D-1 & 638585 & 887740 & D-19 & 638805 & 887706 \\
\hline D-2 & 638595 & 887738 & D-20 & 638815 & 887708 \\
\hline
\end{tabular}


Table B.1-6

Coordinates for Intrusive Investigation Locations (Page 2 of 2)

\begin{tabular}{|c|c|c|c|c|c|}
\hline \multicolumn{6}{|c|}{ Drainage Sampling Locations } \\
\hline Location & Easting & Northing & Location & Easting & Northing \\
\hline D-3 & 638605 & 887730 & D-21 & 638825 & 887712 \\
\hline D-4 & 638615 & 887728 & D-22 & 638835 & 887719 \\
\hline D-5 & 638625 & 887728 & D-23 & 638845 & 887720 \\
\hline D-6 & 638635 & 887728 & D-24 & 638855 & 887722 \\
\hline D-8 & 638655 & 887727 & D-26 & 638885 & 887720 \\
\hline D-9 & 638675 & 887731 & D-27 & 638895 & 887720 \\
\hline D-10 & 638685 & 887730 & D-28 & 638905 & 887722 \\
\hline D-11 & 638695 & 887728 & D-29 & 638915 & 887722 \\
\hline D-12 & 638715 & 887732 & D-30 & 638925 & 887721 \\
\hline D-10 & 638685 & 887730 & D-28 & 638905 & 887722 \\
\hline D-11 & 638695 & 887728 & D-29 & 638915 & 887722 \\
\hline D-12 & 638715 & 887732 & D-30 & 638925 & 887721 \\
\hline D-13 & 638735 & 887725 & D-31 & 638935 & 887722 \\
\hline D-14 & 638745 & 887721 & D-32 & 638945 & 887722 \\
\hline D-15 & 638755 & 887713 & D-33 & 638955 & 887721 \\
\hline D-16 & 638775 & 887709 & D-34 & 638995 & 887711 \\
\hline D-17 & 638785 & 887713 & D-35 & 639005 & 887714 \\
\hline D-18 & 638795 & 887710 & D-36 & 639015 & 887712 \\
\hline
\end{tabular}




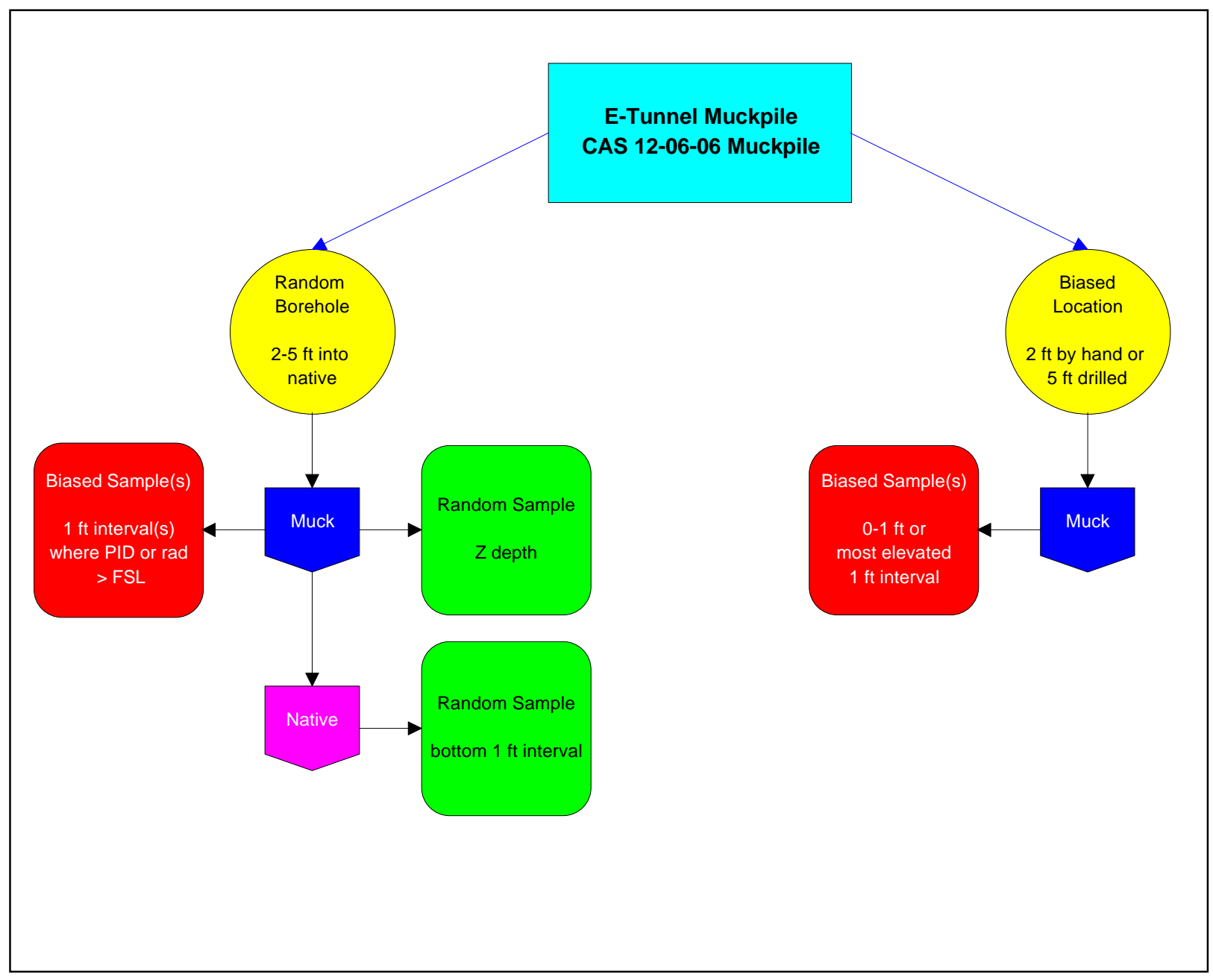

Figure B.1-8

Sampling Strategy for the Muckpile 


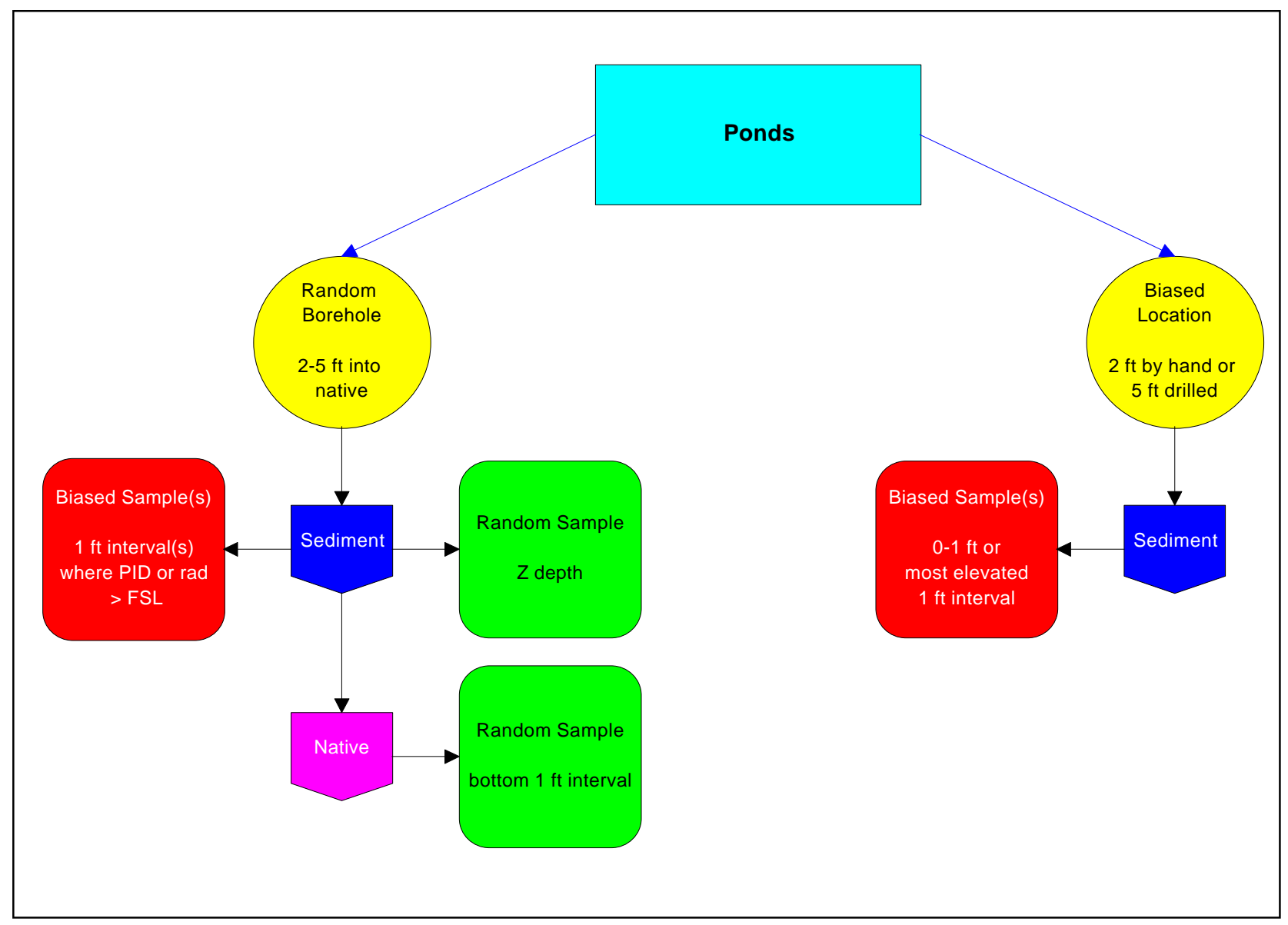

Figure B.1-9

Sampling Strategy for the Ponds 


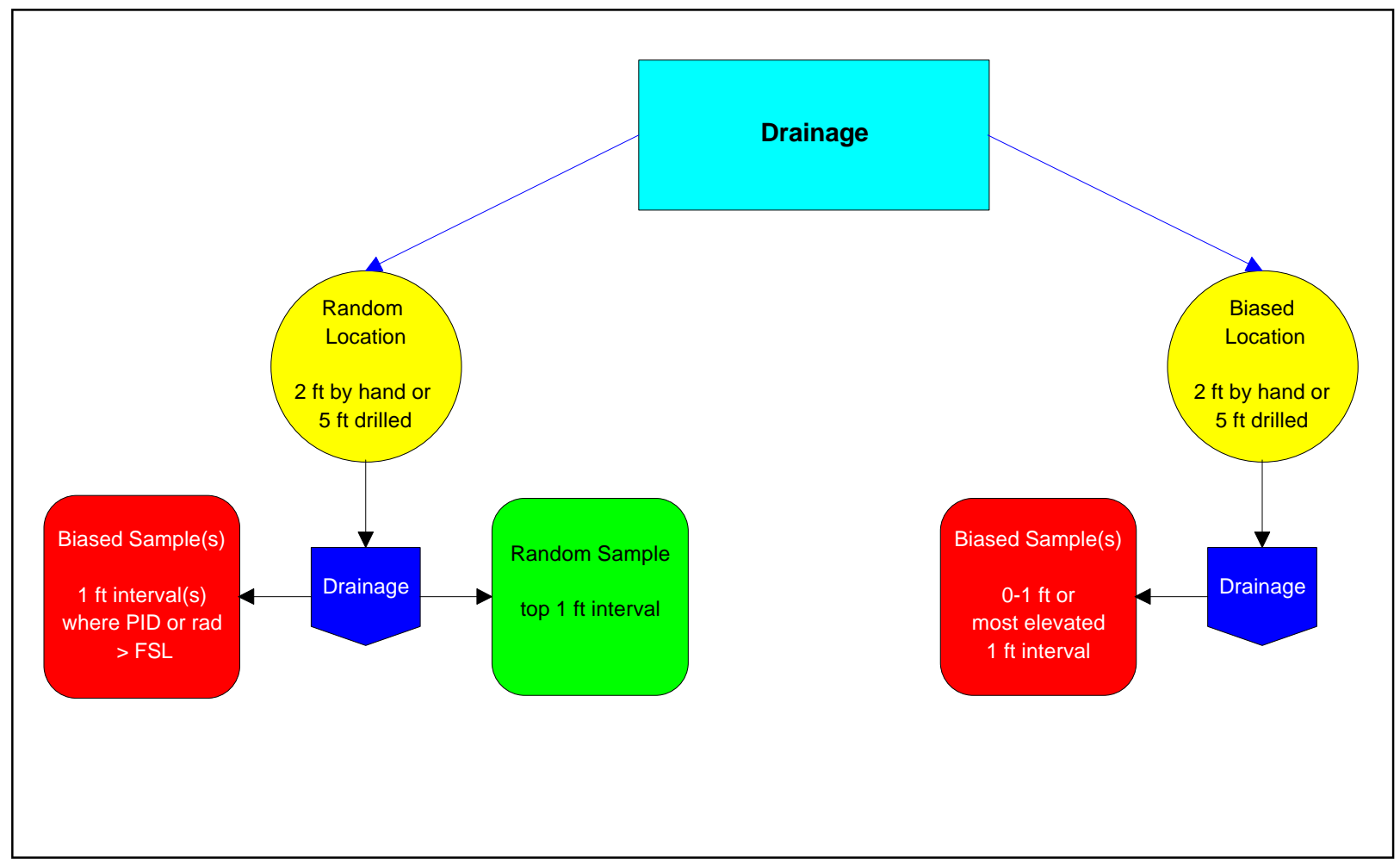

Figure B.1-10

Sampling Strategy for the Drainage 


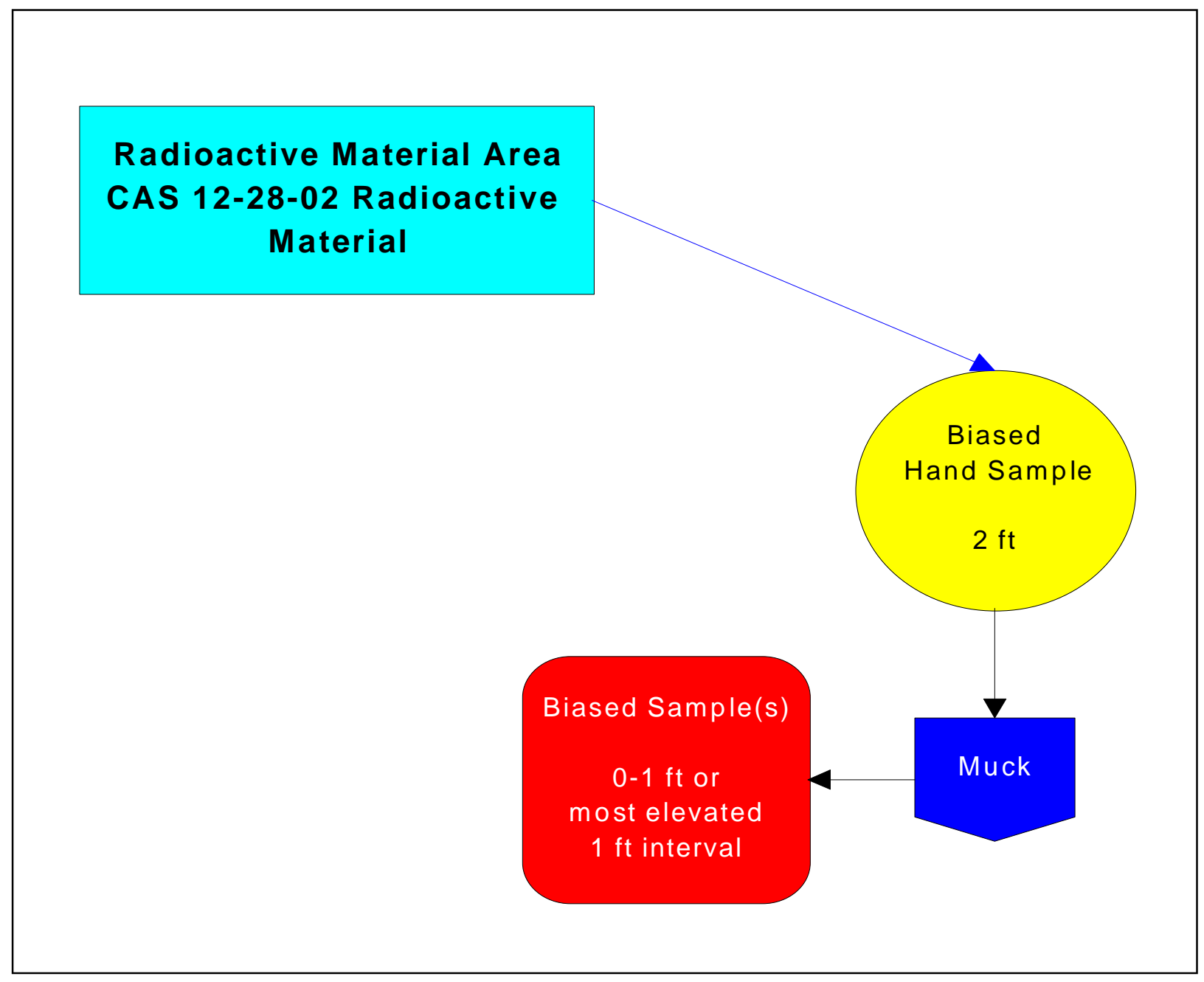

Figure B.1-11

Sampling Strategy for the Radioactive Material Area 


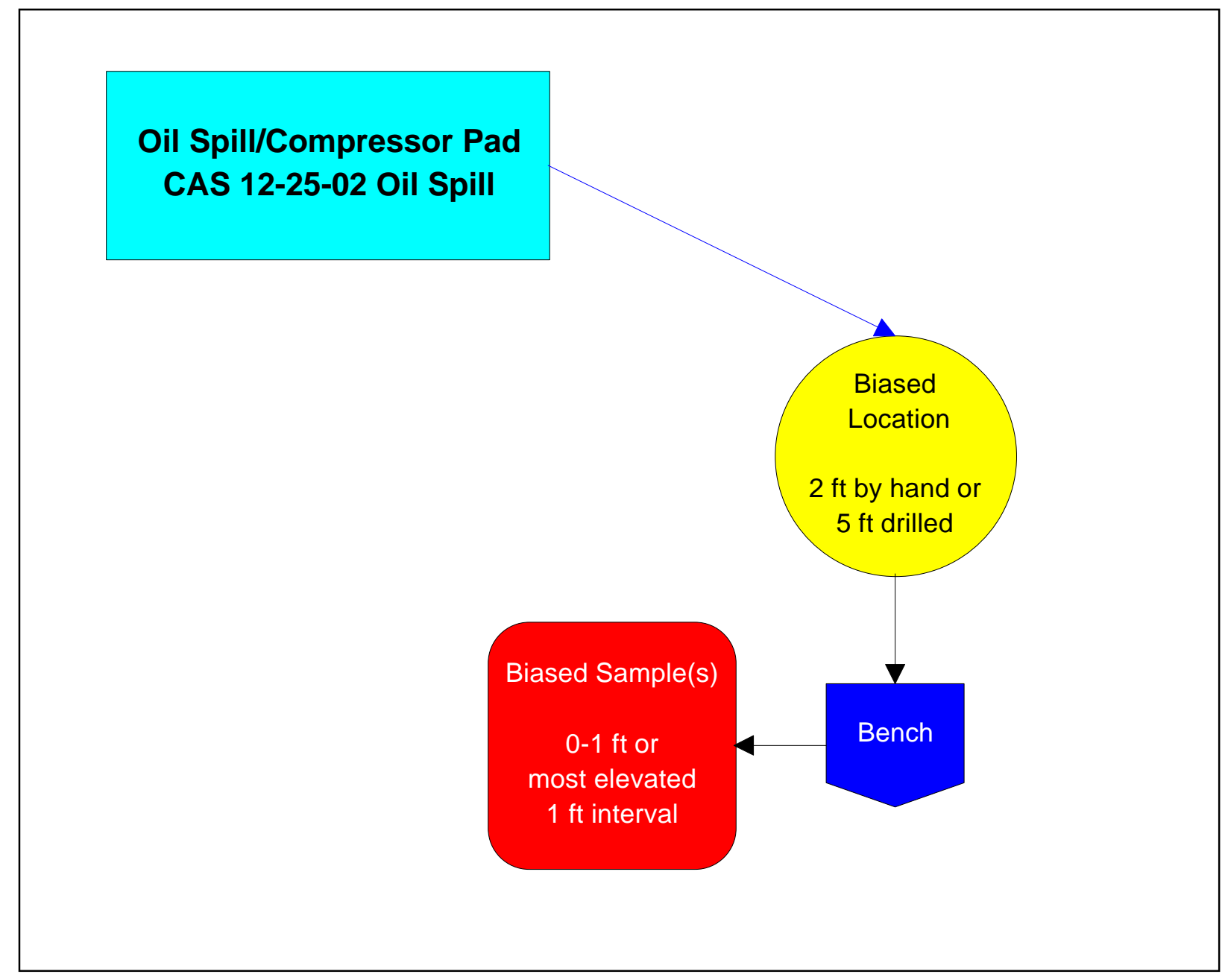

Figure B.1-12

Sampling Strategy for the Oil Spill 


\section{B.2.0 References}

BN, see Bechtel Nevada.

Bechtel Nevada. 1995. Nevada Test Site Performance Objective for Certification of Nonradioactive Hazardous Waste. G-E11/96.01, Rev. 0. Las Vegas, NV

Black, S.C., and Y.E. Townsend. 1996. U.S. Department of Energy, Nevada Operations Office Environmental Data Report for the Nevada Test Site - 1994. Las Vegas, NV.

DOE/NV, see U.S. Department of Energy, Nevada Operations Office.

EPA, see U.S. Environmental Protection Agency.

McArthur, R.D., and F.L. Miller, Jr. 1989. Offsite Radiation Exposure Review Project (ORERP), Phase II Soils Programs, DOE/NV/10384-23. Las Vegas, NV: Desert Research Institute.

Moore, J., Science Applications International Corporation. 1999. Memorandum to M. Todd (SAIC) entitled, "Background Concentrations for NTS and TTR Soil Samples.” Las Vegas, NV.

NAC, see Nevada Administrative Code.

NBMG, see Nevada Bureau of Mines and Geology.

NNSA/NV, see U.S. Department of Energy, National Nuclear Security Administration Nevada Operations Office.

Nevada Administrative Code. 2005. NAC 445A.2272, "Water Controls.” Carson City, NV.

Nevada Bureau of Mines and Geology. 1998. Mineral and Energy Resource Assessment of the Nellis Air Force Range, Open File Report 98-1. Las Vegas, NV.

U.S. Department of Energy, Nevada Operations Office. 1996a. Final Environmental Impact Statement for the Nevada Test Site and Off-site Locations in the State of Nevada, Volume 1, Chapters 1-9. Las Vegas, NV.

U.S. Department of Energy, Nevada Operations Office. 1996b. Radiological Effluents Released from U.S. Continental Tests 1961 through 1992, DOE/NV-317, Rev. 1. Las Vegas, NV

U.S. Department of Energy, Nevada Operations Office. 1998. Nevada Test Site Resource Management Plan, DOE/NV-518. Las Vegas, NV 
U.S. Department of Energy, National Nuclear Security Administration Nevada Operations Office. 2002. Industrial Sites Quality Assurance Project Plan, Nevada Test Site, Nevada, NNSA/NV--372, Rev. 3. Las Vegas, NV.

US Ecology and Atlan-Tech, Inc. 1991. Environmental Monitoring Report for the Proposed Ward Valley, California, Low-Level Radioactive Waste (LLRW) Facility, Vols. 1 and 2, Version 2. Rosewell, GA: California Department of Health, Environmental Management Branch.

U.S. Environmental Protection Agency. 1998. Test Methods for Evaluating Solid Waste, Physical/Chemical Methods, SW-846, $3^{\text {rd }}$ Edition, CD-ROM PB97-501928GEI. Washington, DC.

U.S. Environmental Protection Agency. 2002. Region 9 Preliminary Remediation Goals $(P R G s)$. San Francisco, CA. 


\section{Appendix C}

\section{Data Assessment}

Uncontrolled When Printed 


\section{C.1.0 Data Assessment}

The DQA process is the scientific evaluation of the investigation results to determine whether the Data Quality Objectives DQO criteria established in the CAU 383 CAIP were met and if the DQO decisions can be answered at the desired level of confidence. The DQO process ensures that the right type, quality, and quantity of data will be available to support the resolution of the decisions at an appropriate level of confidence. Using both the DQO and DQA processes helps to ensure that the DQO decisions are sound and defensible.

The DQA involves five steps that begin with a review of the DQOs and end with an answer to the DQO decisions. The five steps are briefly summarized below.

Step 1: Review the DQOs and Sampling Design - Review the DQO process to provide context for analyzing the data. State the primary statistical hypotheses; confirm the limits on the decision errors for committing false rejection (Type I) or false acceptance (Type II) decision errors; and review any special features, potential problems, or deviations to the sampling design.

Step 2: Conduct a Preliminary Data Review - The preliminary data review involves reviewing QA reports and inspecting the data both numerically and graphically, validating and verifying the data to ensure that the measurement systems performed in accordance with the criteria specified, and using the validated data to determine whether the quality of the data is satisfactory.

Step 3: Select the Test - Select the test based on the population of interest, population parameter, and the hypotheses. Identify the key underlying assumptions that could cause a change in one of the DQO questions.

Step 4: Verify the Assumptions - Perform tests of assumptions. If data are missing or are censored, determine the impact on the DQO decision error.

Step 5: Draw Conclusions from the Data - Perform the calculations required for the test. 


\section{C.1.1 Review the DQOs and Sampling Design}

This section contains a review of the DQO process presented in Appendix A of the CAU 383 CAIP and Appendix B of this document. The DQO decisions are presented with the DQO provisions for limiting false negative or false positive decision errors. Special features, potential problems, or any deviations from the sampling design are also presented.

\section{C.1.1.1 Review DQOs}

The decision statement as presented in the CAU 383 CAIP: “Are there concentrations of COPCs present in the muckpile, ponds, oil spill or radioactive material CASs, or blower pad which exceed the action levels and, if so, do they pose a threat to human health and/or the environment?"

Decision Rules:

- If laboratory results for the soil samples indicate the presence of COPCs above the PALs, then a CADD will be prepared.

- If laboratory results for the soil samples do not indicate the presence of COPCs above the PALs, then a CADD or CADD/CR will be prepared.

Population Parameter: The mean plus one standard deviation of each analyte with observed results.

\section{DQO Provisions to Limit False Negative Decision Error}

A false negative decision error (where consequences are more severe) was controlled by meeting the following criteria:

1. Having a high degree of confidence that the combination of random and biased sampling strategies will identify COCs if present in the CASs.

2. Having a high degree of confidence that analyses conducted will be sufficient to detect any COCs present in the samples.

3. Having a high degree of confidence that the data is of sufficient quality and completeness. 


\section{Criterion 1:}

The following methods (stipulated in the CAU 383 DQOs [DTRA, 2003]) were used in selecting the sample locations:

- Random locations based on methods described in Chapter 4 of Statistical Methods for Environmental Pollution Monitoring (Gilbert, 1987).

- Selection of biased sampling locations associated with professional judgment, site knowledge, and preliminary radiological walk-over and drive-over surveys.

This provides a high degree of confidence that sampling will detect any COCs that may be present.

\section{Criterion 2:}

All samples were analyzed using the analytical methods listed in Table 3-1 of the CAIP and for the chemical and radiological parameters listed in Table A.1-3 of the CAIP. Table C.1-1 provides a reconciliation of samples analyzed to the planned analytical program.

Samples were analyzed for all of the analytical methods specified in the CAIP

Sample results were assessed against the DQI of sensitivity as defined in the Industrial Sites QAPP (NNSA/NV, 2002). The sensitivity acceptance criteria defined in the CAIP is that analytical detection limits will be less than the corresponding action level. This goal was not achieved for the chemical analyses listed in Table C.1-2. All radiological analytes met the sensitivity goal. Results not meeting the sensitivity goal were not used in making DQO decisions and will therefore be considered as rejected data. 
Table C.1-1

CAU 383 Number of Samples Submitted per Analyte

\begin{tabular}{|c|c|c|c|c|c|c|c|c|}
\hline \multirow[b]{2}{*}{$\begin{array}{c}\text { CAS } \\
\text { or } \\
\text { Location }\end{array}$} & \multicolumn{8}{|c|}{ ANALYTES } \\
\hline & $\begin{array}{l}\text { O্ } \\
\text { O্ }\end{array}$ & 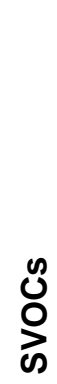 & 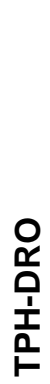 & $\begin{array}{l}\frac{\omega}{\pi} \\
\frac{\pi}{0}\end{array}$ & 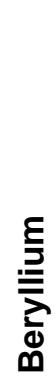 & 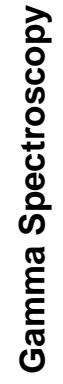 & 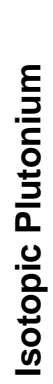 & 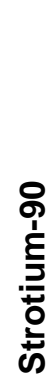 \\
\hline $12-06-06$ & 68 & 68 & 68 & 68 & 68 & 68 & 68 & 68 \\
\hline $12-25-02$ & 9 & 9 & 9 & 9 & 9 & 9 & 9 & 9 \\
\hline $12-28-02$ & 4 & 4 & 4 & 4 & 4 & 4 & 4 & 4 \\
\hline Drainage & 37 & 37 & 37 & 37 & 37 & 37 & 37 & 37 \\
\hline Ponds & 16 & 16 & 16 & 16 & 16 & 16 & 16 & 16 \\
\hline
\end{tabular}

Table C.1-2

Chemical Analytes Failing Sensitivity Criteria for CAU 383

\begin{tabular}{||c|c|c|c|c||}
\hline \hline Sample Number & Parameter & $\begin{array}{c}\text { Result } \\
(\mathbf{F g} / \mathbf{k g})\end{array}$ & $\begin{array}{c}\text { Detection Limit } \\
(\mathbf{F g} / \mathbf{k g})\end{array}$ & $\begin{array}{c}\text { 2004 Industrial } \\
\text { PRG (Fg/kg) }\end{array}$ \\
\hline \hline 383OB0800.5RR1 & Benzo(A)Pyrene & 23,000 & 250 & 210 \\
\hline 383OB0100.5RR1 & Dibenzo(A,H)Anthracene & 3,400 & 250 & 210 \\
\hline 383OB0200.5RR1 & Dibenzo(A,H)Anthracene & 3,400 & 250 & 210 \\
\hline 383OB0800.5RR1 & Dibenzo(A,H)Anthracene & 6,900 & 500 & 210 \\
\hline 383OB0800.5RR1 & N-Nitroso-Di-N-Propylamine & 6,900 & 290 & 250 \\
\hline 383OB0100.5 & N-Nitrosodimethylamine & 1,700 & 73 & 34 \\
\hline 383OB0800.5RR1 & N-Nitrosodimethylamine & 3,400 & 150 & 34 \\
\hline 383OB0200.5 & N-Nitrosodimethylamine & 1,700 & 73 & 34 \\
\hline 383OB0200.5RR1 & N-Nitrosodimethylamine & 3,400 & 150 & 34 \\
\hline 383OB0700.5RR1 & N-Nitrosodimethylamine & 2,100 & 89 & 34 \\
\hline 383OB0800.5 & N-Nitrosodimethylamine & 1,400 & 59 & 34 \\
\hline 383OB0800.5RR1 & N-Nitrosodimethylamine & 6,900 & 290 & 34 \\
\hline
\end{tabular}

$\mathrm{Fg} / \mathrm{kg}=$ Micrograms per kilogram 


\section{Criterion 3:}

To satisfy the third criterion, the entire dataset, as well as individual sample results, were assessed against the acceptance criteria for the DQIs of precision, accuracy, comparability, completeness, and representativeness, as defined in the Industrial Sites QAPP (NNSA/NV, 2002). The DQI acceptance criteria are presented in Table 6-1 of the CAIP. As presented in the following sections these goals were met for each DQI.

\section{Precision}

The duplicate precision is evaluated using the relative percent difference (RPD) or normalized difference. For the purpose of determining the data precision of chemical analyses, the RPD between duplicate analyses was calculated. For radionuclides, the RPD was not calculated unless both the sample and its duplicate had concentrations of the target radionuclide exceeding five times their MDC. Otherwise radionuclide, duplicate results were evaluated using the normalized difference. Table C.1-3 provides the chemical and radiological precision analysis results for all constituents that were qualified for precision. The chemical analytes qualified for precision were lead and barium. Radionuclides qualified for precision were Th-227 and Pu-239.

Table C.1-3

Precision Measurements

\begin{tabular}{||c|c|c|c|c|c||}
\hline Parameter & CAS Number & $\begin{array}{c}\text { User Test } \\
\text { Panel }\end{array}$ & $\begin{array}{c}\text { Number of } \\
\text { Analyses } \\
\text { Qualified }\end{array}$ & $\begin{array}{c}\text { Number of } \\
\text { Measurements } \\
\text { Performed }\end{array}$ & $\begin{array}{c}\text { Percent within } \\
\text { Criteria }\end{array}$ \\
\hline \hline Lead & $7439-92-1$ & EPA6010B & 9 & 134 & 93.3 \\
\hline Barium & $7440-39-3$ & EPA6010B & 15 & 130 & 8.5 \\
\hline Thorium-227 & $15623-47-9$ & HASL300 & 13 & 135 & 90.4 \\
\hline Plutonium-239 & $15117-48-3$ & UGTAISOPU & 66 & 135 & 51.1 \\
\hline
\end{tabular}

CAS $=$ Chemical Abstract Number

EPA = U.S. Environmental Protection Agency, SW 846 methods (EPA, 1999 and 2002) HASL $=$ Health and Safety Laboratory

As shown in Table C.1-3, the precision rate for lead, barium, and thorium were above the CAIP acceptance criterion of 80 percent; however, Pu-239 was below the acceptance criterion. The precision rate for all of the other constituents is 100 percent. The precision rate for $\mathrm{Pu}-239$ was 51.1 percent; however, because the Muckpile has concentrations of $\mathrm{Pu}-239$ above the FAL, a false negative will not impact the decision and will not significantly increase the potential risk to 
human health or the environment. Because all of the other constituents exceed the acceptance criteria for precision, the dataset is determined to be acceptable for the DQI of precision.

\section{$\underline{\text { Accuracy }}$}

For the purpose of determining data accuracy of sample analyses, environmental soil samples were evaluated and incorporated into the accuracy calculation. The results qualified for accuracy were associated with matrix spike recoveries that were outside control limits and could potentially be reported at concentrations lower or higher than actual concentration. Table C.1-4 provides the chemical accuracy analysis results for all constituents qualified for accuracy. Accuracy rates are above the performance criterion of 80 percent specified in the CAIP for all parameters.

Table C.1-4

Accuracy Measurements

\begin{tabular}{||c|c|c|c|c|c||}
\hline Parameter & CAS Number & $\begin{array}{c}\text { User Test } \\
\text { Panel }\end{array}$ & $\begin{array}{c}\text { Number of } \\
\text { Analyses } \\
\text { Qualified }\end{array}$ & $\begin{array}{c}\text { Number of } \\
\text { Measurements } \\
\text { Performed }\end{array}$ & $\begin{array}{c}\text { Percent } \\
\text { within } \\
\text { Criteria }\end{array}$ \\
\hline \hline 1,2,4-Trimethylbenzene & $95-63-6$ & EPA8260 & 1 & 127 & 99.2 \\
\hline $1,3,5-$ Trimethylbenzene & $108-67-8$ & EPA8260 & 1 & 127 & 99.2 \\
\hline Acetone & $67-64-1$ & EPA8260 & 1 & 127 & 99.2 \\
\hline Benzene & $71-43-2$ & EPA8260 & 1 & 127 & 99.2 \\
\hline Ethylbenzene & $100-41-4$ & EPA8260 & 1 & 127 & 99.2 \\
\hline Hexachlorobutadiene & $87-68-3$ & EPA8270 & 1 & 132 & 99.2 \\
\hline Hexachlorobutadiene & $87-68-3$ & EPA8260 & 1 & 127 & 99.2 \\
\hline M+P-Xylene & $136777-61-2$ & EPA8260 & 1 & 127 & 99.2 \\
\hline Mercury & $7439-97-6$ & EPA6010B & 1 & 130 & 99.2 \\
\hline N-Propylbenzene & $103-65-1$ & EPA8260 & 1 & 127 & 99.2 \\
\hline O-Xylene & $95-47-6$ & EPA8260 & 1 & 127 & 99.2 \\
\hline Toluene & $108-88-3$ & EPA8260 & 1 & 127 & 99.2 \\
\hline Methylene Chloride & $75-09-2$ & EPA8260 & 2 & 127 & 98.4 \\
\hline Trichloroethene & $79-01-6$ & EPA8260 & 3 & 127 & 97.6 \\
\hline Chlorobenzene & $108-90-7$ & EPA8260 & 7 & 127 & 94.5 \\
\hline Lead & $7439-92-1$ & EPA6010B & 9 & 134 & 93.3 \\
\hline Barium & $7440-39-3$ & EPA6010B & 15 & 130 & 88.5 \\
\hline
\end{tabular}

As the accuracy rate for all other constituents exceed the acceptance criteria for accuracy, the dataset is determined to be acceptable for the DQI of accuracy. 


\section{Representativeness}

The DQO process as identified in Appendix A of the CAU 383 CAIP (DTRA, 2003) was used to address sampling and analytical requirements for CAU 383. During this process, appropriate locations were selected that enabled the samples collected to be representative of the population parameters identified in the DQO (random locations and biased locations that were most likely to encounter contamination). The sampling locations identified in the Criterion 1 discussion meet these criteria. Therefore, the analytical data acquired during the CAU 383 CAI are considered to be representative of the population parameters.

\section{$\underline{\text { Comparability }}$}

Field sampling, as described in the CAU 383 CAIP (DTRA, 2003), was performed and documented in accordance with approved procedures that are comparable to standard industry practices. Approved analytical methods and procedures were used to analyze, report, and validate the data. These are comparable to other methods used not only in industry and government practices, but most importantly are comparable to other investigations conducted at the NTS. Therefore, project datasets are considered comparable to other datasets generated using these same standardized DOE procedures, thereby meeting the DQO requirements. Also, standard, approved field and analytical methods ensure that data were appropriate for comparison to the investigation action levels specified in the CAIP.

\section{Completeness}

The CAU 383 CAIP (DTRA, 2003) defines acceptable criteria for completeness to be 80 percent of CAS-specific non-critical analytes identified in the CAIP having valid results and 100 percent of critical analytes having valid results. Also, the dataset must be sufficiently complete to be able to make the DQO decisions. Critical analytes for CAU 383 are the COCs identified from previously investigated NTS muckpiles (arsenic, lead, TPH-DRO, Pu-239, Cs-137, and Co-60).

Rejected data (either qualified as rejected or data that failed the criterion of sensitivity) were not used in the resolution of DQO decisions and are not counted toward meeting the completeness acceptance criterion. Table C.1-5 shows the data rejected because of completeness. Because none of the rejected data area critical analytes and all of the qualified data exceed the 80 percent criteria for completeness the dataset is considered complete for purposes of making the DQO decisions. 
Table C.1-5

Rejected Measurements for Completeness

\begin{tabular}{||c|c|c|c|c|c||}
\hline Parameter & $\begin{array}{c}\text { CAS } \\
\text { Number }\end{array}$ & $\begin{array}{c}\text { User Test } \\
\text { Panel }\end{array}$ & $\begin{array}{c}\text { Number of } \\
\text { Analyses } \\
\text { Qualified }\end{array}$ & $\begin{array}{c}\text { Number of } \\
\text { Measurements } \\
\text { Performed }\end{array}$ & $\begin{array}{c}\text { Percent } \\
\text { within } \\
\text { Criteria }\end{array}$ \\
\hline \hline Benzo(A)Anthracene & $56-55-3$ & EPA8270 & 2 & 132 & 98.5 \\
\hline Chrysene & $218-01-9$ & EPA8270 & 4 & 132 & 97 \\
\hline Pyrene & $129-00-0$ & EPA8270 & 4 & 132 & 97 \\
\hline Bis(2-Ethylhexyl)Phthalate & $117-81-7$ & EPA8270 & 6 & 132 & 95.5 \\
\hline 3,3'-Dichlorobenzidine & $91-94-1$ & EPA8270 & 9 & 132 & 93.2 \\
\hline Butylbenzylphthalate & $85-68-7$ & EPA8270 & 9 & 132 & 93.2 \\
\hline Di-N-Octylphthalate & $117-84-0$ & EPA8270 & 9 & 132 & 93.2 \\
\hline Benzo(B)Fluoranthene & $205-99-2$ & EPA8270 & 18 & 132 & 86.4 \\
\hline Benzo(K)Fluoranthene & $207-08-9$ & EPA8270 & 18 & 132 & 86.4 \\
\hline Benzo(A)Pyrene & $50-32-8$ & EPA8270 & 19 & 132 & 85.6 \\
\hline Benzo(G,H,I)Perylene & $191-24-2$ & EPA8270 & 21 & 132 & 84.1 \\
\hline Ideno(1,2,3-CD)Pyrene & $193-39-5$ & EPA8270 & 22 & 132 & 83.3 \\
\hline Dibenzo(A,H)Anthracene & $53-70-3$ & EPA68270 & 24 & 132 & 81.8 \\
\hline \hline
\end{tabular}

\section{DQO Provisions to Limit False Positive Decision Error}

The false positive decision error was controlled by assessing the potential for false positive analytical results. Quality assurance/QC samples such as field blanks, trip blanks, laboratory control samples, and method blanks were used to determine whether a false positive analytical result may have occurred. Of the $52 \mathrm{QA} / \mathrm{QC}$ samples submitted, no false positive analytical results were detected.

Proper decontamination of sampling equipment, and the use of certified clean sampling equipment and containers, minimized the potential for cross contamination that could lead to a false positive analytical result.

\section{C.1.1.2 Sampling Design}

The CAIP made the following commitments for sampling:

1. Random sampling will be conducted at CAS 12-06-06, in the drainage below the Muckpile, and in Ponds 1, 2, and 3. 
Result: All of the random locations identified by the simple random sampling method defined in Chapter 4 of Statistical Methods for Environmental Pollution Monitoring (Gilbert, 1987) were collected and analyzed. This included 40 samples from CAS 12-06-06 (the Muckpile), 35 from the drainage, and eight from the ponds.

2. Biased locations will be selected at CAS 12-06-06, CAS 12-25-02, CAS 12-28-02, and locations that are most likely to contain contaminated soil.

Result: Eight biased soil samples were collected at CAS 12-25-02, four were collected at CAS 12-28-02, 26 were collected at CAS 12-25-02, and six were collected in the ponds. The locations were identified through walk-over radiological surveys, visual inspection of the surface, or process knowledge.

\section{C.1.2 Conduct a Preliminary Data Review}

A preliminary data review was conducted by reviewing QA reports and inspecting the data. The contract analytical laboratories generate a QA non-conformance report when data quality do not meet contractual requirements. All data received from the analytical laboratories met contractual requirements, and no QA non-conformance reports were generated. Data were validated and verified to ensure that the measurement systems performed in accordance with the criteria specified. The validated dataset quality was found to be satisfactory.

\section{C.1.3 Select the Test}

The CAIP (DTRA, 2003) committed to using the procedure described in Chapter 9 of the EPA SW-846 Method (EPA, 1996) to answer two questions: 1) were enough samples collected to ensure a 90 percent confidence level in the mean COPC concentration, and 2) does the mean concentration exceed the regulatory threshold?

Because of the change in closure strategy agreed to by NDEP, DTRA, and NNSA/NSO, the regulatory threshold is now the risk based FAL instead of the PALs discussed in the CAIP. Comparing the average concentration of the most prevalent contaminants to their PAL and, if they exceed the PAL, comparing them to their respective FALs will also be used to help answer the questions. 


\section{C.1.4 SW-846 Evaluation}

To answer the first question described in equation (8) of Table 9-1 in SW 846 was used. To answer the second question, equation (6) of Table 9-1 in SW-846 was used (EPA, 1996). Only random samples were used for this evaluation. Cesium-137 was chosen for conducting the calculations because it was the most prevalent contaminant found during the characterization. Both of these equations are presented and explained in Appendix B of this CADD/CR. The PAL screening level for Cs-137 is $7.3 \mathrm{pCi} / \mathrm{g}$. The values used for the calculations and the results are presented in Table C.1-6.

Based on the results of the calculations, an adequate number of samples were collected to meet the 90 percent confidence level that enough samples had been collected to characterize the sites except in the ponds. In comparing the 90 percent confidence level to the PAL, the confidence level exceeds the PAL in the Muckpile, the drainage, and the ponds. Because the PAL is exceeded in the ponds by almost 13 times, the fact that not enough samples were collected at that site has less importance. It would be more significant if the 90 percent confidence level were close to the PAL. 
Table C.1-6

SW-846 Evaluation of the Number of Samples and Comparison of $90 \%$ Confidence Level with the PAL

\begin{tabular}{||l|c|c|c|c|c||}
\hline Variable & Pond Native & $\begin{array}{c}\text { Pond } \\
\text { Sediment }\end{array}$ & Drainage & Muckpile Native & $\begin{array}{c}\text { Muckpile } \\
\text { Muck }\end{array}$ \\
\hline \hline $\mathrm{T}_{.20}$ & 1.638 & 1.638 & 1.307 & 1.333 & 1.323 \\
\hline $\mathrm{T}_{\cdot 20}{ }^{2}$ & 2.683 & 2.683 & 1.708 & 1.777 & 1.750 \\
\hline $\mathrm{S}^{2}$ & 3.208 & 3469.040 & 402.249 & 4.980 & 11280.912 \\
\hline $\mathrm{RT}$ for Cs-137 & 7.3 & 7.3 & 7.3 & 7.3 & 7.3 \\
\hline Avg (BarX) & 1.241 & 40.778 & 25.978 & 0.846 & 49.480 \\
\hline $\mathrm{n}$ collected & 4 & 4 & 35 & 18 & 22 \\
\hline $\mathrm{n}$ needed* & "1" & 8.305 & 1.970 & $1 "$ & 11.098 \\
\hline Confidence Interval & 1.467 & 48.238 & 4.431 & 0.701 & 29.959 \\
\hline $90 \%$ Confidence & 2.707 & 89.015 & 30.409 & 1.547 & 79.439 \\
\hline $90 \%$ Confidence>7.3pCi/g & no & yes & yes & no & yes \\
\hline
\end{tabular}

Note: Only random samples were used in this evaluation.

* - Minimum samples needed are actually 0.234 and 0.212 , respectively.

\section{C.1.5 Verify the Assumptions}

The results of the investigation support the assumptions identified in the CAU 383 DQOs and Table C.1-7.

\section{Table C.1-7 \\ Key Assumptions}

(Page 1 of 2)

\begin{tabular}{|c|c|}
\hline \multirow[t]{2}{*}{ Exposure Scenario } & $\begin{array}{l}\text { Exposure to contaminants is limited to industrial site workers, } \\
\text { construction/remediation workers and military personnel conducting training. } \\
\text { Exposure could occur through ingestion, inhalation, external exposure, or } \\
\text { dermal contact. }\end{array}$ \\
\hline & $\begin{array}{l}\text { The investigation did not reveal any potential exposures that were not identified } \\
\text { in the CSM. }\end{array}$ \\
\hline \multirow[t]{2}{*}{ Affected Media } & $\begin{array}{l}\text { Surface and subsurface soils in and below the Muckpile. Contamination of } \\
\text { perched, deep, and regional groundwater is not a concern. }\end{array}$ \\
\hline & $\begin{array}{l}\text { The investigation results did not identify any affected media that was not } \\
\text { identified in the CSM. }\end{array}$ \\
\hline
\end{tabular}




\section{Table C.1-7 \\ Key Assumptions}

(Page 2 of 2)

\begin{tabular}{|c|c|}
\hline \multirow[t]{2}{*}{$\begin{array}{l}\text { Location of Contamination } \\
\text { Release Points }\end{array}$} & $\begin{array}{l}\text { The Muckpile, compressor pad, Radioactive Material area, drainage, and ponds } \\
\text { may contain small volumes of RCRA-regulated constituents in addition to } \\
\text { radiological constituents. }\end{array}$ \\
\hline & $\begin{array}{l}\text { The investigation results confirmed this and did not reveal any potential } \\
\text { releases off the Muckpile. }\end{array}$ \\
\hline \multirow[t]{2}{*}{ Transport Mechanisms } & $\begin{array}{l}\text { Surface transport may occur as a result of storm water runoff. Contamination } \\
\text { may migrate through the Muckpile into the native material as a result of } \\
\text { rainwater infiltration. }\end{array}$ \\
\hline & The investigation results did not confirm any transport mechanisms \\
\hline \multirow[t]{2}{*}{ Preferential Pathways } & Percolation of precipitation through the soils of the Muckpile. \\
\hline & The investigation did not identify any contaminant migration. \\
\hline \multirow[t]{2}{*}{$\begin{array}{l}\text { Lateral and Vertical Extent of } \\
\text { Contamination }\end{array}$} & $\begin{array}{l}\text { Contamination could be locally significant, but vertical infiltration of } \\
\text { contaminants is probably limited to less than } 5 \mathrm{ft} \text {. }\end{array}$ \\
\hline & The investigation results confirmed this. \\
\hline Groundwater Impacts & There are no groundwater impacts. \\
\hline \multirow[t]{2}{*}{ Future Land Use } & Nonresidential, zoned for nuclear and high explosives tests. \\
\hline & $\begin{array}{l}\text { The investigation results did not reveal any future land uses other than those } \\
\text { identified in the CSM. }\end{array}$ \\
\hline
\end{tabular}

\section{C.1.6 Results}

This section resolves the DQO decision for CAU 383.

\section{C.1.6.1 Decision Rules for CAU 383}

Decision Rule: If laboratory results for the soil samples indicate the presence of COPCs above the PALs, then a CADD will be prepared.

Result: Because the site was characterized and a CADD was prepared, the DOE and NDEP came to an agreement that a risk based approach could be used for characterizing the muckpiles. Because of this decision, NDEP agreed to allow existing muckpile CADDs that had been approved by NDEP to be re-evaluated using the risk-based approach. Using the risk-based approach FALs were exceeded for Am-241, Cs-137, Sr-90, Pu-238, and Pu-239. 
Decision Rule: If laboratory results for the soil samples do not indicate the presence of COPCs above the PALs, then a CADD or CADD/CR will be prepared.

Result: Because of the change in the strategy for closing the muckpiles, a CADD/CR will be prepared that will proposed use restrictions for controlling the contaminated soils. 


\section{C.2.0 References}

DTRA, see Defense Threat Reduction Agency.

Defense Threat Reduction Agency. 2003. Corrective Action Investigation Plan for Corrective Action Unit 383: Area 12 E Tunnel Sites, Nevada Test Site, Nevada, Rev. 0. February.

EPA, see U.S. Environmental Protection Agency.

Gilbert, R.O. 1987. Statistical Methods for Environmental Pollution Monitoring, Chapter 4. New York, NY: Van Nostrand Reinhold.

NNSA/NV, see U.S. Department of Energy, National Nuclear Security Administration Nevada Operations Office.

U.S. Department of Energy, National Nuclear Security Administration Nevada Operations Office. 2002. Industrial Sites Quality Assurance Project Plan, Nevada Test Site, Nevada, DOE/NV--372, Rev. 3. Las Vegas, NV.

U.S. Environmental Protection Agency. 1996. Test Methods for Evaluating Solid Waste, Physical/Chemical Methods, SW-846 CD ROM PB97-501928GEI, which contains updates for 1986, 1992, 1994, and 1996. Washington, DC.

U.S. Environmental Protection Agency. 1999. Test Methods for Evaluating Solid Waste, Physical/Chemical Methods, SW-846. $3^{\text {rd }}$ Edition. Washington, DC.

U.S. Environmental Protection Agency. 2002. Region 9 Preliminary Remediation Goals (PRGs). San Francisco, CA 


\section{Appendix D}

\section{Risk Assessment for CAU 383}




\section{D.1.0 Risk-Based Corrective Action Process}

This section contains documentation of the ASTM Method E 1739-95 (ASTM, 1995) risk-based corrective action process as applied to CAU 383. The ASTM Method E 1739-95 defines three tiers or levels in evaluating DQO decisions involving increasingly more sophisticated analyses.

- Tier 1 - Sample results from source areas (highest concentrations) compared to the PALs based on generic (non-site-specific) conditions.

- $\quad$ Tier 2 - Sample results from exposure points compared to SSTLs calculated using site-specific inputs and Tier I formulas (from the ASTM procedure).

- $\quad$ Tier 3 - Sample results from exposure points compared to SSTLs and points of compliance calculated using chemical fate/transport and probabilistic modeling.

The risk based corrective action decision process stipulated in ASTM Method E 1739-95 is summarized in Figure D.1-1.

\section{D.1.1 Scenario}

Corrective Action Unit 383 consists of the three CASs and two associated areas listed below.

- CAS 12-06-06, Muckpile

- CAS 12-25-02, Oil Spill

- CAS 12-28-02, Radioactive Material

- The drainage below the Muckpile

- The first three ponds below the Muckpile

Corrective Action Site 12-06-06 consists of the E-Tunnel Muckpile. E-Tunnel was mined horizontally into bedded ash flow tuffs underlying Rainier Mesa. It was used for nine nuclear 


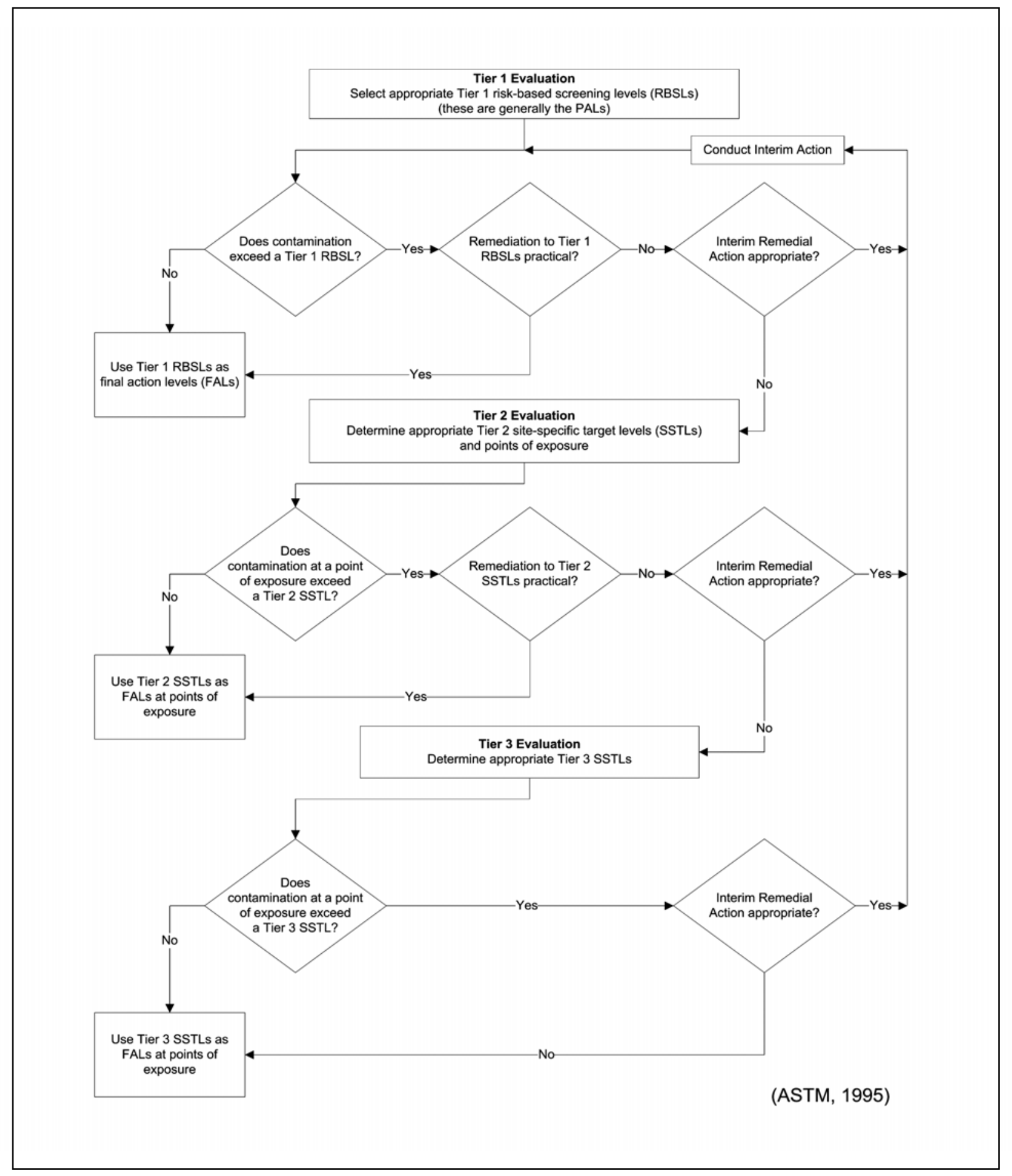

Figure D.1-1

ASTM Method E 1739-95 Risk-Based Corrective Action Decision Process 
weapons effects tests between 1958 and 1977. The Muckpile consists of approximately 355,000 $\mathrm{yd}^{3}$ of material mined from the tunnel. The surface elevation at the top of the Muckpile is about 6,120 ft above mean sea level. The Muckpile was deposited in an easterly trend in front of the tunnel portals. The Muckpile is approximately $200 \mathrm{ft}$ across and 1,200 ft long in an east-west direction. The thickness of the Muckpile ranges from less than $5 \mathrm{ft}$ at the west end to approximately $100 \mathrm{ft}$ at the northeast side.

Corrective Action Site 12-28-02 (Radioactive Material) is located at the E-Tunnel Main portal and is part of the Muckpile.

Corrective Action Site 12-25-02 (Oil Spill) is located on the blower pad, which is on a bench approximately $100 \mathrm{ft}$ above the E-Tunnel south portal. The pad was constructed in the hillside and is about $100 \mathrm{ft}$ long north to south and $50 \mathrm{ft}$ wide. Fill on the pad varies in thickness from less than $1 \mathrm{ft}$ next to the slope to about $10 \mathrm{ft}$ on the north edge of the pad.

The drainage that was included in this investigation trends easterly along the base of the Muckpile. It includes approximately $750 \mathrm{ft}$ of the drainage above the uppermost pond.

The first three ponds below the Muckpile received discharge water from E-Tunnel. Pond 1 is almost covered by the Muckpile. Ponds 2 and 3 did not have standing water but were too muddy to drive the drill rig on. Pond 2 is round and approximately $115 \mathrm{ft}$ in diameter, and Pond 3 is $100 \mathrm{ft}$ long by $50 \mathrm{ft}$ wide. Ponds 4, 5, and 6 were not sampled because they are still active and receiving discharge water from E-Tunnel.

\section{D.1.2 Site Assessment}

The CAI at CAU 383 involved walk-over and drive-over radiological surveys, soil sampling with a rotosonic drill rig, and soil sampling with hand tools. The investigation results identified both chemical and radiological COCs that exceeded the PALs as defined in the CAIP (DTRA, 2003) in all of the CASs and areas that were characterized. The maximum concentration of the COCs identified at each site and their corresponding PALs are presented in Tables D.1-1 (chemical results) and D.1-2 (radiological results). 


\section{Table D.1-1 \\ Maximum Reported Chemical Values for Tier 1 Comparison} (Page 1 of 6 )

\begin{tabular}{|c|c|c|c|c|}
\hline Contaminant of Concern & CAS Number & Sample Number & $\begin{array}{l}\text { Results } \\
\text { (mg/kg) }\end{array}$ & $\begin{array}{c}\mathrm{PAL}^{1} \\
(\mathrm{mg} / \mathrm{kg})\end{array}$ \\
\hline \multicolumn{5}{|c|}{ Muckpile Muck } \\
\hline Arsenic & $7440-38-2$ & 383МН0353.5 & 6 & 23 \\
\hline Barium & $7740-39-3$ & 383МВ0600.5 & 7,000 & 67,000 \\
\hline Beryllium & $7440-41-7$ & 383MB1200.5 & 2.4 & 1,900 \\
\hline Cadmium & $7440-43-9$ & 383МB0400.5 & 15 & 450 \\
\hline Chromium & $7440-47-3$ & 383МВ0200.5 & 33 & 450 \\
\hline Lead & $7439-92-1$ & 383МВ0912.5 & 120 & 800 \\
\hline Selenium & $7782-49-2$ & 383МВ0559.5 & 0.99 & 310 \\
\hline Silver & $7440-22-4$ & $383 \mathrm{MH} 2 \mathrm{~A} 37.5$ & 1.1 & 5,100 \\
\hline Mercury & $7439-97-6$ & 383МН0353.5 & 0.51 & 5,100 \\
\hline Diesel-Range Organics $^{2}$ & $68334-30-5$ & 383 MB0800.5 & 300 & 100 \\
\hline 1,2,4-Trimethylbenzene & $95-63-6$ & $383 M B 0800.5$ & 0.0072 & 170 \\
\hline 1,3,5-Trimethybenzene & $108-67-8$ & 383 MB0800.5 & 0.0072 & 70 \\
\hline Acetone & $67-64-1$ & 383MH0723.5 & 0.01 & 54,000 \\
\hline Ethylbenzene & $100-41-4$ & 383МВ0800.5 & 0.12 & 400 \\
\hline M+P-Xylene & $136777-61-2$ & 383МВ0800.5 & 1.5 & 420 \\
\hline Methylene Chloride & $75-09-2$ & 383МB0586.0 & 0.0013 & 21 \\
\hline O-Xylene & $95-47-6$ & 383МВ0800.5 & 1 & 420 \\
\hline Acenaphthene & $83-32-9$ & 383 MB1000.5 & 0.022 & 29,000 \\
\hline Anthracene & $120-12-7$ & $383 \mathrm{MB} 1000.5$ & 0.17 & 100,000 \\
\hline Benzo(A)Anthracene & $56-55-3$ & $383 \mathrm{MB} 1000.5$ & 1.2 & 2.1 \\
\hline Benzo(A)Pyrene & $50-32-8$ & 383 MB1000.5 & 1.3 & 0.21 \\
\hline Benzo(B)Fluoranthene & $205-99-2$ & $383 \mathrm{MB} 1000.5$ & 1.7 & 2.1 \\
\hline Benzo(K)Fluoranthene & $207-08-9$ & $383 M B 1000.5$ & 0.65 & 21 \\
\hline Bis(2-Ethylhexyl)Phthalate & $117-81-7$ & $383 \mathrm{MH} 2 \mathrm{~A} 08.0$ & 0.17 & 120 \\
\hline Butyl Benzyl Phthalate & $85-68-7$ & $383 \mathrm{MH} 1100.5$ & 0.035 & 100,000 \\
\hline Carbazole & $86-74-8$ & $383 \mathrm{MB} 1000.5$ & 0.057 & 86 \\
\hline Chrysene & $218-01-9$ & $383 \mathrm{MB} 1000.5$ & 1 & 210 \\
\hline Dibenzofuran & $132-64-9$ & $383 \mathrm{MB} 1000.5$ & 0.065 & 1,600 \\
\hline Fluoranthene & $206-44-0$ & 383MB1000.5 & 1.5 & 22,000 \\
\hline
\end{tabular}




\section{Table D.1-1 \\ Maximum Reported Chemical Values for Tier 1 Comparison} (Page 2 of 6 )

\begin{tabular}{|c|c|c|c|c|}
\hline Contaminant of Concern & CAS Number & Sample Number & $\begin{array}{l}\text { Results } \\
\text { (mg/kg) }\end{array}$ & $\begin{array}{c}\mathrm{PAL}^{1} \\
(\mathrm{mg} / \mathrm{kg})\end{array}$ \\
\hline \multicolumn{5}{|c|}{ Muckpile Native } \\
\hline Fluorene & $86-73-7$ & 383MB1000.5 & 0.016 & 26,000 \\
\hline Ideno(1,2,3-Cd)Pyrene & $193-39-5$ & $383 M B 1000.5$ & 0.63 & 2.1 \\
\hline Naphthalene & $91-20-3$ & 383MB1000.5 & 0.019 & 190 \\
\hline Pyrene & $129-00-0$ & 383MB1000.5 & 1.6 & 29,000 \\
\hline Arsenic & $7440-38-2$ & 383MH0922.5 & 3.8 & 23 \\
\hline Barium & $7740-39-3$ & $383 \mathrm{MH} 0600.5$ & 370 & 67,000 \\
\hline Beryllium & $7440-41-7$ & 383MH0922.5 & 1 & 1,900 \\
\hline Cadmium & $7440-43-9$ & $383 \mathrm{MH} 122.5$ & 1 & 450 \\
\hline Chromium & $7440-47-3$ & $383 \mathrm{MH} 1391.0$ & 5.8 & 450 \\
\hline Lead & $7439-92-1$ & 383МH1734.5 & 160 & 800 \\
\hline Selenium & $7782-49-2$ & 383MH1503.5 & 0.29 & 310 \\
\hline Silver & $7440-22-4$ & $383 \mathrm{MH} 1734.5$ & 2.8 & 5,100 \\
\hline Mercury & $7439-97-6$ & $383 \mathrm{MH} 1734.5$ & 0.33 & 5,100 \\
\hline Diesel-Range Organics $^{2}$ & $68334-30-5$ & 383МH0600.5 & 700 & 100 \\
\hline Acetone & $67-64-1$ & $383 \mathrm{MH} 1422.0$ & 0.018 & 54,000 \\
\hline Hexachlorobutadiene & $87-68-3$ & $383 \mathrm{MH} 1503.5$ & 0.0015 & 22 \\
\hline Methylene Chloride & $75-09-2$ & 383MH1503.5 & 0.0016 & 21 \\
\hline Benzo(A)Anthracene & $56-55-3$ & 383MB0305.5 & 0.022 & 2.1 \\
\hline Benzo(A)Pyrene & $50-32-8$ & $383 M B 0305.5$ & 0.016 & 0.21 \\
\hline Benzo(B)Fluoranthene & $205-99-2$ & 383MB0305.5 & 0.028 & 2.1 \\
\hline Bis(2-Ethylhexyl)Phthalate & $117-81-7$ & 383MB1425.5 & 0.22 & 120 \\
\hline Chrysene & $218-01-9$ & $383 M B 0305.5$ & 0.023 & 210 \\
\hline Fluoranthene & $206-44-0$ & 383 MB0305.5 & 0.023 & 22,000 \\
\hline \multicolumn{5}{|c|}{ Pond Sediment } \\
\hline Arsenic & $7440-38-2$ & 383РН0101.0 & 14 & 23 \\
\hline Barium & $7740-39-3$ & 383РН0101.0 & 6,300 & 67,000 \\
\hline Beryllium & $7440-41-7$ & $383 \mathrm{PH} 0306.5$ & 2 & 1,900 \\
\hline Cadmium & $7440-43-9$ & 383РН0300.5 & 0.34 & 450 \\
\hline Chromium & $7440-47-3$ & 383РН0101.0 & 12 & 450 \\
\hline
\end{tabular}


Table D.1-1

Maximum Reported Chemical Values for Tier 1 Comparison (Page 3 of 6)

\begin{tabular}{|c|c|c|c|c|}
\hline Contaminant of Concern & CAS Number & Sample Number & $\begin{array}{l}\text { Results } \\
(\mathrm{mg} / \mathrm{kg})\end{array}$ & $\begin{array}{c}\mathrm{PAL}^{1} \\
(\mathrm{mg} / \mathrm{kg})\end{array}$ \\
\hline Lead & $7439-92-1$ & 383РВ0300.5 & 38 & 800 \\
\hline Silver & $7440-22-4$ & 383РВ0102.0 & 0.077 & 5,100 \\
\hline Mercury & $7439-97-6$ & 383РВ0300.5 & 0.048 & 5,100 \\
\hline Diesel-Range Organics $^{2}$ & $68334-30-5$ & $383 \mathrm{PH} 0216.0$ & 330 & 100 \\
\hline Acetone & $67-64-1$ & $383 \mathrm{PH} 0101.0$ & 0.014 & 54,000 \\
\hline Ethylbenzene & $100-41-4$ & 383PB0400.5 & 0.0016 & 400 \\
\hline M+P-Xylene & $136777-61-2$ & 383PB0400.5 & 0.0083 & 420 \\
\hline O-Xylene & $95-47-6$ & 383РВ0400.5 & 0.0033 & 420 \\
\hline Benzo(A)Anthracene & $56-55-3$ & 383РН0216.0 & 0.026 & 2.1 \\
\hline Benzo(A)Pyrene & $50-32-8$ & $383 \mathrm{PH} 0216.0$ & 0.028 & 0.21 \\
\hline Benzo(B)Fluoranthene & $205-99-2$ & $383 \mathrm{PH} 0216.0$ & 0.059 & 2.1 \\
\hline Benzo(K)Fluoranthene & $207-08-9$ & $383 \mathrm{PH} 0216.0$ & 0.031 & 21 \\
\hline Bis(2-Ethylhexyl)Phthalate & $117-81-7$ & $383 \mathrm{PH} 0216.0$ & 0.33 & 120 \\
\hline Chrysene & $218-01-9$ & $383 \mathrm{PH} 0216.0$ & 0.066 & 210 \\
\hline Fluoranthene & $206-44-0$ & $383 \mathrm{PH} 0216.0$ & 0.031 & 22,000 \\
\hline Pyrene & $129-00-0$ & $383 \mathrm{PH} 0216.0$ & 0.063 & 29,000 \\
\hline \multicolumn{5}{|c|}{ Pond Native } \\
\hline Arsenic & $7440-38-2$ & 383РВ0104.5 & 2.9 & 23 \\
\hline Barium & $7740-39-3$ & $383 \mathrm{PH} 0217.5$ & 3,200 & 67,000 \\
\hline Beryllium & $7440-41-7$ & $383 \mathrm{PH} 0320.5$ & 1 & 1,900 \\
\hline Cadmium & $7440-43-9$ & 383РВ0104.5 & 0.17 & 450 \\
\hline Chromium & $7440-47-3$ & $383 \mathrm{PH} 0320.5$ & 3.4 & 450 \\
\hline Lead & $7439-92-1$ & $383 \mathrm{PH} 0217.5$ & 12 & 800 \\
\hline Silver & $7440-22-4$ & $383 \mathrm{PH} 0217.5$ & 0.48 & 5,100 \\
\hline Mercury & $7439-97-6$ & $383 \mathrm{PH} 0320.5$ & 0.12 & 5,100 \\
\hline Acetone & $67-64-1$ & 383РН0217.5 & 0.025 & 54,000 \\
\hline Hexachlorobutadiene & $87-68-3$ & 383РН0418.5 & 0.0017 & 22 \\
\hline
\end{tabular}


Table D.1-1

Maximum Reported Chemical Values for Tier 1 Comparison (Page 4 of 6 )

\begin{tabular}{|c|c|c|c|c|}
\hline Contaminant of Concern & CAS Number & Sample Number & $\begin{array}{l}\text { Results } \\
\text { (mg/kg) }\end{array}$ & $\begin{array}{c}\mathrm{PAL}^{1} \\
(\mathrm{mg} / \mathrm{kg})\end{array}$ \\
\hline \multicolumn{5}{|c|}{ Oil Spill } \\
\hline Arsenic & $7440-38-2$ & $3830 \mathrm{OB} 0800.5$ & 7.1 & 23 \\
\hline Barium & $7740-39-3$ & $3830 B 0400.5$ & 300 & 67,000 \\
\hline Beryllium & $7440-41-7$ & $3830 B 0500.5$ & 1.2 & 1,900 \\
\hline Cadmium & $7440-43-9$ & 383 OB0200.5 & 1.4 & 450 \\
\hline Chromium & $7440-47-3$ & $3830 B 0700.5$ & 10 & 450 \\
\hline Lead & $7439-92-1$ & $3830 B 0700.5$ & 31 & 800 \\
\hline Selenium & $7782-49-2$ & $3830 B 0800.5$ & 1.2 & 310 \\
\hline Diesel-Range Organics ${ }^{2}$ & $68334-30-5$ & $3830 B 0100.5$ & 3,400 & 100 \\
\hline Acetone & $67-64-1$ & $3830 B 0100.5$ & 0.072 & 54,000 \\
\hline Acenaphthene & $83-32-9$ & $3830 B 0800.5$ & 0.093 & 29,000 \\
\hline Anthracene & $120-12-7$ & $3830 B 0800.5$ & 0.47 & 100,000 \\
\hline Benzo(A)Anthracene & $56-55-3$ & $3830 B 0800.5$ & 17 & 2.1 \\
\hline Benzo(A)Pyrene & $50-32-8$ & 383 OB0800.5 & 20 & 0.21 \\
\hline Benzo(B)Fluoranthene & $205-99-2$ & $3830 B 0800.5$ & 37 & 2.1 \\
\hline Benzo(K)Fluoranthene & $207-08-9$ & $3830 B 0800.5$ & 10 & 21 \\
\hline Bis(2-Ethylhexyl)Phthalate & $117-81-7$ & $3830 B 0800.5$ & 4 & 120 \\
\hline 2-Butanone & $78-93-3$ & $3830 B 0100.5$ & 0.017 & 110,000 \\
\hline Carbazole & $86-74-8$ & $3830 B 0800.5$ & 0.79 & 86 \\
\hline Chrysene & $218-01-9$ & $3830 B 0800.5$ & 21 & 210 \\
\hline Dibenzo $(A, H)$ Anthracene & $53-70-3$ & $3830 B 0800.5$ & 10 & 0.21 \\
\hline Fluoranthene & $206-44-0$ & $3830 B 0800.5$ & 10 & 22,000 \\
\hline Fluorene & $86-73-7$ & $3830 B 0800.5$ & 0.068 & 26,000 \\
\hline Ideno(1,2,3-Cd)Pyrene & $193-39-5$ & $3830 B 0800.5$ & 22 & 2.1 \\
\hline Naphthalene & $91-20-3$ & $3830 B 0800.5$ & 0.056 & 190 \\
\hline Pyrene & $129-00-0$ & $3830 B 0800.5$ & 89 & 29,000 \\
\hline \multicolumn{5}{|c|}{ Radioactive Material } \\
\hline Arsenic & $7440-38-2$ & 383RB0200.5 & 7.1 & 23 \\
\hline Barium & $7740-39-3$ & 383RB0300.5 & 260 & 67,000 \\
\hline Beryllium & $7440-41-7$ & 383RB0100.5 & 1.7 & 1,900 \\
\hline Cadmium & $7440-43-9$ & 383RB0300.5 & 0.57 & 450 \\
\hline
\end{tabular}




\section{Table D.1-1 \\ Maximum Reported Chemical Values for Tier 1 Comparison} (Page 5 of 6 )

\begin{tabular}{|c|c|c|c|c|}
\hline Contaminant of Concern & CAS Number & Sample Number & $\begin{array}{l}\text { Results } \\
(\mathrm{mg} / \mathrm{kg})\end{array}$ & $\begin{array}{c}\mathrm{PAL}^{1} \\
(\mathrm{mg} / \mathrm{kg})\end{array}$ \\
\hline Lead & $7439-92-1$ & 383RB0300.5 & 61 & 800 \\
\hline Mercury & $7439-97-6$ & 383RB0100.5 & 0.16 & 5,100 \\
\hline Diesel-Range Organics $^{2}$ & $68334-30-5$ & 383RB0400.5 & 15 & 100 \\
\hline Benzo(A)Anthracene & $56-55-3$ & 383RB0300.5 & 0.17 & 2.1 \\
\hline Benzo(A)Pyrene & $50-32-8$ & 383RB0400.5 & 0.23 & 0.21 \\
\hline Benzo(B)Fluoranthene & $205-99-2$ & 383RB0400.5 & 0.62 & 2.1 \\
\hline Benzo(K)Fluoranthene & 207-08-9 & 383RB0300.5 & 0.23 & 21 \\
\hline Bis(2-Ethylhexyl)Phthalate & $117-81-7$ & 383RB0300.5 & 1.2 & 120 \\
\hline Butyl Benzyl Phthalate & $85-68-7$ & 383RB0100.5 & 0.78 & 100,000 \\
\hline Carbazole & $86-74-8$ & 383RB0400.5 & 0.023 & 86 \\
\hline Chrysene & $218-01-9$ & 383RB0400.5 & 0.48 & 210 \\
\hline Dibenzo $(A, H)$ Anthracene & $53-70-3$ & 383RB0400.5 & 0.027 & 21 \\
\hline Di-N-Butyl-Phthalate & $84-74-2$ & 383RB0100.5 & 4.5 & 62,000 \\
\hline Fluoranthene & $206-44-0$ & 383RB0400.5 & 0.51 & 22,000 \\
\hline Ideno(1,2,3-Cd)Pyrene & $193-39-5$ & 383RB0400.5 & 0.08 & 2.1 \\
\hline Naphthalene & $91-20-3$ & 383RB0400.5 & 0.018 & 190 \\
\hline Pyrene & $129-00-0$ & 383RB0400.5 & 0.43 & 29,000 \\
\hline \multicolumn{5}{|c|}{ Drainage } \\
\hline Arsenic & $7440-38-2$ & $383 \mathrm{DH} 3100.5$ & 5.3 & 23 \\
\hline Barium & $7740-39-3$ & $383 \mathrm{DH} 3200.5$ & 6,600 & 67,000 \\
\hline Beryllium & $7440-41-7$ & $383 \mathrm{DH} 1400.5$ & 2.1 & 1,900 \\
\hline Chromium & $7440-47-3$ & 383DH3200.5 & 9.2 & 450 \\
\hline Lead & $7439-92-1$ & 383DH0900.5 & 52 & 800 \\
\hline Mercury & $7439-97-6$ & 383DH1400.5 & 0.083 & 5,100 \\
\hline Diesel-Range Organics $^{2}$ & $68334-30-5$ & 383DH3500.5 & 68 & 100 \\
\hline Acetone & $67-64-1$ & 383DH0200.5 & 0.039 & 54,000 \\
\hline 2-Butanone & $78-93-3$ & $383 \mathrm{DH} 0200.5$ & 0.01 & 110,000 \\
\hline Ethylbenzene & $100-41-4$ & 383DH0300.5 & 0.011 & 400 \\
\hline Hexachlorobutadiene & $87-68-3$ & $383 \mathrm{DH} 2700.5$ & 0.0016 & 22 \\
\hline M+P-Xylene & $136777-61-2$ & 383DH0300.5 & 0.057 & 420 \\
\hline
\end{tabular}


Table D.1-1

Maximum Reported Chemical Values for Tier 1 Comparison (Page 6 of 6)

\begin{tabular}{|c|c|c|c|c|}
\hline Contaminant of Concern & CAS Number & Sample Number & $\begin{array}{l}\text { Results } \\
(\mathrm{mg} / \mathrm{kg})\end{array}$ & $\begin{array}{c}\mathrm{PAL}^{1} \\
(\mathrm{mg} / \mathrm{kg}) \\
\end{array}$ \\
\hline Methylene Chloride & $75-09-2$ & $383 \mathrm{DH} 0200.5$ & 0.004 & 21 \\
\hline O-Xylene & $95-47-6$ & 383DH0300.5 & 0.023 & 420 \\
\hline Styrene & $100-42-5$ & 383DH0300.5 & 0.0036 & 1,700 \\
\hline Toluene & $108-88-3$ & 383DH0500.5 & 0.0016 & 520 \\
\hline Benzo(A)Anthracene & $56-55-3$ & $383 \mathrm{DH} 2600.5$ & 0.066 & 2.1 \\
\hline Benzo(A)Pyrene & $50-32-8$ & $383 \mathrm{DH} 2600.5$ & 0.064 & 0.21 \\
\hline Benzo(B)Fluoranthene & $205-99-2$ & $383 \mathrm{DH} 2600.5$ & 0.062 & 2.1 \\
\hline Benzo(K)Fluoranthene & 207-08-9 & $383 \mathrm{DH} 2600.5$ & 0.057 & 21 \\
\hline Bis(2-Ethylhexyl)Phthalate & $117-81-7$ & $383 \mathrm{DH} 2200.5$ & 2.4 & 120 \\
\hline Butyl Benzyl Phthalate & $85-68-7$ & 383DH0500.5 & 0.04 & 100,000 \\
\hline Chrysene & $218-01-9$ & $383 \mathrm{DH} 2200.5$ & 0.1 & 210 \\
\hline Dimethyl Phthalate & $131-11-3$ & $383 \mathrm{DH} 2200.5$ & 0.014 & 100,000 \\
\hline Di-N-Octyl Phthalate & $117-84-0$ & 383DH2200.5 & 0.059 & 25,000 \\
\hline Fluoranthene & $206-44-0$ & $383 \mathrm{DH} 2600.5$ & 0.12 & 22,000 \\
\hline Pyrene & $129-00-0$ & $383 \mathrm{DH} 2600.5$ & 0.19 & 29,000 \\
\hline
\end{tabular}

RR1 - Indicates the sample was reanalyzed at a higher dilution due to higher-than-expected results.

${ }^{1}$ PALs (as per the CAU 383 CAIP)

${ }^{2}$ Diesel-range organics (i.e., petroleum hydrocarbons) level is defined by NAC 445.2272 (NAC, 2005b).

Table D.1-2

Maximum Reported Radiological Values for Tier 1 Comparison (Page 1 of 4)

\begin{tabular}{||c|c|c|c|c||}
\hline $\begin{array}{c}\text { Contaminant of } \\
\text { Concern }\end{array}$ & CAS Number & Sample Number & $\begin{array}{c}\text { Results } \\
(\mathbf{p C i} / \mathbf{g})\end{array}$ & $\begin{array}{c}\text { PAL }^{1} \\
(\mathbf{p C i} / \mathbf{g})\end{array}$ \\
\hline \hline \multicolumn{5}{|c|}{ Muckpile Muck } \\
\hline \hline Actinium-228 & $14331-83-0$ & $383 \mathrm{MB} 0400.5$ & 2.89 & 5 \\
\hline Americium-241 & $14596-10-2$ & $383 \mathrm{MB} 0543.5$ & 28.8 & 7.62 \\
\hline Bismuth-214 & $14733-03-0$ & $383 \mathrm{MB} 0900.5$ & 2.08 & 5 \\
\hline Cesium-137 & $10045-97-3$ & $383 \mathrm{MB} 0543.5$ & 1,060 & 7.3 \\
\hline Europium-152 & $14683-23-9$ & 383 MB0543.5 & 2.64 & 5.7 \\
\hline
\end{tabular}


Table D.1-2

Maximum Reported Radiological Values for Tier 1 Comparison (Page 2 of 4)

\begin{tabular}{|c|c|c|c|c|}
\hline $\begin{array}{c}\text { Contaminant of } \\
\text { Concern }\end{array}$ & CAS Number & Sample Number & $\begin{array}{l}\text { Results } \\
\text { (pCi/g) }\end{array}$ & $\begin{array}{c}\mathrm{PAL}^{1} \\
(\mathrm{pCi} / \mathrm{g})\end{array}$ \\
\hline Lead-212 & $15092-94-1$ & 383МВ0900.5 & 3.37 & 5 \\
\hline Lead-214 & $15067-28-4$ & 383МВ0900.5 & 2.08 & 5 \\
\hline Thallium-208 & $14913-50-9$ & 383 MB0900.5 & 1.02 & 5 \\
\hline Strontium-90 & $10098-97-2$ & 383MH0559.5 & 54 & 503 \\
\hline Plutonium-238 & $13981-16-3$ & $383 \mathrm{MB} 0543.5$ & 15.6 & 7.78 \\
\hline Plutonium-239 & $15117-48-3$ & $383 \mathrm{MB} 0543.5$ & 84 & 7.62 \\
\hline \multicolumn{5}{|c|}{ Muckpile Native } \\
\hline Actinium-228 & $14331-83-0$ & $383 \mathrm{MH} 0922.5$ & 2.14 & 5 \\
\hline Americium-241 & $14596-10-2$ & $383 \mathrm{MH} 2 \mathrm{~A} 45.0$ & 1.1 & 7.62 \\
\hline Bismuth-214 & $14733-03-0$ & 383МH0922.5 & 0.92 & 5 \\
\hline Cesium-137 & $10045-97-3$ & 383MH2A45.0 & 9.8 & 7.3 \\
\hline Europium-155 & $14391-16-3$ & $383 \mathrm{MH} 0922.5$ & 0.32 & 1.35 \\
\hline Lead-212 & $15092-94-1$ & $383 \mathrm{MH} 0922.5$ & 2.27 & 5 \\
\hline Lead-214 & $15067-28-4$ & $383 M B 1425.5$ & 0.91 & 5 \\
\hline Thorium-234 & $15065-10-8$ & $383 \mathrm{MH} 0922.5$ & 1.8 & 5 \\
\hline Thallium-208 & $14913-50-9$ & $383 \mathrm{MH} 0922.5$ & 0.76 & 5 \\
\hline Strontium-90 & $10098-97-2$ & $383 M B 1425.5$ & 0.33 & 503 \\
\hline Plutonium-238 & $13981-16-3$ & $383 \mathrm{MH} 2 \mathrm{~A} 45.0$ & 0.213 & 7.78 \\
\hline Plutonium-239 & $15117-48-3$ & $383 \mathrm{MH} 2 \mathrm{~A} 45.0$ & 4.55 & 7.62 \\
\hline \multicolumn{5}{|c|}{ Pond Sediment } \\
\hline Actinium-228 & $14331-83-0$ & 383PH0216.0 & 2.31 & 5 \\
\hline Bismuth-214 & $14733-03-0$ & 383РН0306.5 & 1.72 & 5 \\
\hline Cesium-137 & $10045-97-3$ & 383PH0216.0 & 142 & 7.3 \\
\hline Lead-212 & $15092-94-1$ & $383 \mathrm{PH} 0216.0$ & 2.73 & 5 \\
\hline Lead-214 & $15067-28-4$ & $383 \mathrm{PH} 0306.5$ & 1.74 & 5 \\
\hline Thallium-208 & $14913-50-9$ & 383РН0408.0 & 0.8 & 5 \\
\hline
\end{tabular}


Table D.1-2

Maximum Reported Radiological Values for Tier 1 Comparison (Page 3 of 4)

\begin{tabular}{|c|c|c|c|c|}
\hline $\begin{array}{c}\text { Contaminant of } \\
\text { Concern }\end{array}$ & CAS Number & Sample Number & $\begin{array}{l}\text { Results } \\
(\mathrm{pCi} / \mathrm{g})\end{array}$ & $\begin{array}{c}\mathrm{PAL}^{1} \\
(\mathrm{pCi} / \mathrm{g})\end{array}$ \\
\hline Strontium-90 & $10098-97-2$ & 383РН0300.5 & 3.58 & 503 \\
\hline Plutonium-238 & 13981-16-3 & 383РН0216.0 & 0.74 & 7.78 \\
\hline Plutonium-239 & $15117-48-3$ & 383РН0101.0 & 0.305 & 7.62 \\
\hline \multicolumn{5}{|c|}{ Pond Native } \\
\hline Actinium-228 & $14331-83-0$ & 383РН0116.5 & 2.4 & 5 \\
\hline Bismuth-214 & $14733-03-0$ & 383РН0217.5 & 1.49 & 5 \\
\hline Cesium-137 & $10045-97-3$ & 383РН0217.5 & 4.34 & 7.3 \\
\hline Lead-212 & 15092-94-1 & 383РН0320.5 & 2.76 & 5 \\
\hline Lead-214 & $15067-28-4$ & 383РН0116.5 & 1.81 & 5 \\
\hline Thallium-208 & $14913-50-9$ & 383РН0116.5 & 1.05 & 5 \\
\hline Plutonium-239 & $15117-48-3$ & $383 \mathrm{PH} 0217.5$ & 0.154 & 7.62 \\
\hline \multicolumn{5}{|c|}{ Drainage } \\
\hline Actinium-228 & 14331-83-0 & 383DH0200.5 & 2.26 & 5 \\
\hline Aluminum-26 & $14682-66-7$ & 383DH1600.5 & 0.105 & 2.32 \\
\hline Americium-241 & $14596-10-2$ & 383DH0200.5 & 1.88 & 7.62 \\
\hline Bismuth-214 & $14733-03-0$ & $383 \mathrm{DH} 1400.5$ & 1.48 & 5 \\
\hline Cesium-137 & $10045-97-3$ & $383 \mathrm{DH} 2100.5$ & 78.1 & 7.3 \\
\hline Lead-212 & $15092-94-1$ & 383DH0600.5 & 2.54 & 5 \\
\hline Lead-214 & $15067-28-4$ & 383DH1400.5 & 1.68 & 5 \\
\hline Thorium-234 & $15065-10-8$ & 383DH0500.5 & 2.4 & 5 \\
\hline Thallium-208 & $14913-50-9$ & $383 \mathrm{DH} 1400.5$ & 0.87 & 5 \\
\hline Strontium-90 & $10098-97-2$ & 383DH0100.5 & 1.88 & 503 \\
\hline Plutonium-238 & $13981-16-3$ & 383DH0100.5 & 0.73 & 7.78 \\
\hline Plutonium-239 & $15117-48-3$ & $383 \mathrm{DH} 1400.5$ & 4.21 & 7.62 \\
\hline \multicolumn{5}{|c|}{ Oil Spill } \\
\hline Actinium-228 & $14331-83-0$ & 3830В0100.5 & 2.21 & 5 \\
\hline Bismuth-214 & $14733-03-0$ & $3830 B 0800.5$ & 1.1 & 5 \\
\hline Cesium-137 & $10045-97-3$ & 3830 O0700.5 & 20.5 & 7.3 \\
\hline
\end{tabular}


Table D.1-2

Page D-12 of D-26

\section{Maximum Reported Radiological Values for Tier 1 Comparison}

(Page 4 of 4)

\begin{tabular}{|c|c|c|c|c|}
\hline $\begin{array}{c}\text { Contaminant of } \\
\text { Concern }\end{array}$ & CAS Number & Sample Number & $\begin{array}{l}\text { Results } \\
\text { (pCi/g) }\end{array}$ & $\begin{array}{c}\mathrm{PAL}^{1} \\
(\mathrm{pCi} / \mathrm{g}) \\
\end{array}$ \\
\hline \multicolumn{5}{|c|}{ Radioactive Material } \\
\hline Lead-212 & $15092-94-1$ & 3830 OB0500.5 & 3.1 & 5 \\
\hline Lead-214 & $15067-28-4$ & 383OB0600.5 & 1.06 & 5 \\
\hline Thallium-208 & $14913-50-9$ & $3830 \mathrm{OB} 0500.5$ & 0.97 & 5 \\
\hline Plutonium-239 & $15117-48-3$ & 383 ОВ0800.5 & 1.04 & 7.62 \\
\hline Actinium-228 & $14331-83-0$ & 383RB0100.5 & 2.9 & 5 \\
\hline Bismuth-212 & $14913-49-6$ & 383RB0100.5 & 3.2 & 5 \\
\hline Bismuth-214 & $14733-03-0$ & 383RB0200.5 & 1.12 & 5 \\
\hline Cesium-137 & $10045-97-3$ & 383RB0100.5 & 114 & 7.3 \\
\hline Lead-212 & $15092-94-1$ & 383RB0100.5 & 2.96 & 5 \\
\hline Lead-214 & $15067-28-4$ & 383RB0400.5 & 1.04 & 5 \\
\hline Thorium-227 & $15065-47-9$ & 383RB0100.5 & 4.1 & 5 \\
\hline Thorium-234 & $15065-10-8$ & 383RB0100.5 & 7.2 & 5 \\
\hline Thallium-208 & $14913-50-9$ & 383RB0100.5 & 0.85 & 5 \\
\hline Plutonium-238 & $13981-16-3$ & 383RB0400.5 & 0.232 & 7.78 \\
\hline Plutonium-239 & $15117-48-3$ & 383RB0200.5 & 2.09 & 7.62 \\
\hline
\end{tabular}

${ }^{1}$ PALs used as action levels (The PALs for radiological contaminants are based on background or the National Council of Radiation Protection and Measurement (NCRP) Report No. 129 recommended screening limits for construction, commercial, and industrial land use scenario (NCRP, 1999) scaled from 25- to 15-millirem-per-year dose and the generic guidelines for residual concentration of radionuclides in DOE Order 5400.5 (DOE, 1993).

\section{D.1.3 Site Classification and Initial Response Action}

The four major site classifications listed in Table 3 of the ASTM standard are: (1) immediate threat to human health, safety, and/or the environment; (2) short-term (0 to 2 years) threat to human health, safety, and/or the environment; (3) long-term (greater than 2 years) threat to human health, safety, and/or the environment; (4) no demonstrated long-term threats (ASTM, 1995).

Based on the CAI, none of the CASs or associated areas present a threat to human health, safety, and/or the environment; therefore, no interim response actions are necessary at these sites. The CAI demonstrated that the contamination present at CAU 383 is limited to the points of release: the Muckpile, the ponds, the drainage, and the compressor/blower pad. The results further showed that there has been no migration into the subsurface and only minor downgradient 
migration in storm runoff. The storm runoff is constrained by the drainage and the ponds. Analytical results from the native material show only a single instance of radiological contamination in the underlying native material. The sample from the muck $7 \mathrm{ft}$ above the native sample contained the same contaminants at a higher concentration, and there was drill refusal $1.5 \mathrm{ft}$ below the contact so it was not possible to get a sample that was not impacted by the muck. Based on this evidence, the contamination in the native material is most likely the result of slough in the borehole and not migration of contaminants from the muck to the native material. A detailed discussion of the nature and extent of contamination is presented in Appendix A of this report. Based on this information, these sites are determined to be Classification 3 as defined by ASTM Method E 1739-95. At all of the sites, COCs were identified that may pose long-term threats to human health or the environment.

\section{D.1.4 Development of Tier 1 Look-Up Table of Risk-Based Screening Level Selection}

Tier 1 action levels have been defined as the PALs established during the DQO process. The PALs are a tabulation of chemical-specific (but not site-specific) screening levels based on the type of media (soil) and potential exposure scenarios (industrial). These are very conservative estimates of risk, are preliminary in nature, and are used as action levels for site screening purposes. Although the PALs are not intended to be used as FALs, a FAL may be defined as the Tier 1 action level (i.e., PAL) if individual constituent analytical results are below the corresponding Tier 1 action level. The FAL may also be established as the Tier I action level if individual constituent analytical results exceed the corresponding Tier 1 action level value and implementation of a corrective action based on the final action level is practical. The PALs are defined as:

- The EPA Region 9 Risk-Based PRGs for Industrial Soils (2002).

- Background concentrations for RCRA metals will be evaluated when natural background exceeds the PAL, as is often the case with arsenic. Background is considered to be the mean plus two times the standard deviation of the mean based on data published in Mineral and Energy Resource Assessment of the Nellis Air Force Range (NBMG, 1998; Moore, 1999).

- Concentrations for TPH-DRO above $100 \mathrm{mg} / \mathrm{kg}$ per NAC 445A.2272 (NAC, 2005b). 
- $\quad$ For COPCs without established PRGs, a protocol similar to EPA Region 9 will be used to establish an action level; otherwise, an established PRG from another EPA region may be chosen.

- When the CAIP was written, the PALs for radionuclides were isotope-specific and defined as the higher of the maximum concentration for that isotope found in samples from undisturbed background locations in the vicinity of the Nevada Test Site (McArthur and Miller, 1989; US Ecology and Atlan-Tech, 1992; Black and Townsend, 1996), from any of the three background samples collected during the investigation, or as specified in the Nevada Test Site Performance Objective for Certification of Nonradioactive Hazardous Waste (BN, 1995).

Since the corrective action was completed, the radiological PALs have been changed through agreement with NDEP as follows: The PALs for radioactive contaminants are based on the NCRP Report No. 129 recommended screening limits for construction, commercial, and industrial land-use scenarios (NCRP, 1999) scaled to 25 millirem per year dose constraint (Appenzeller-Wing, 2004) and the generic guidelines for residual concentration of radionuclides in DOE Order 5400.5 (DOE, 1993).

The PALs were developed based on an industrial scenario. Because CAU 383 in Area 12 is not assigned any work stations and is considered to be in remote or occasional use areas, the use of industrial-land-use-based PALs is conservative. The Tier 1 look-up table is defined as the PAL concentrations or activities defined in the CAIP.

\section{D.1.5 Exposure Pathway Evaluation}

The DQOs stated that site workers would only be exposed to COCs through oral ingestion, inhalation, or dermal contact (absorption) due to exposure to potentially contaminated media (i.e., soil) at the CASs. The results of the CAI showed that all COCs identified in CAU 383 are localized near the release points and have not migrated laterally or vertically. Because the contaminants were only identified in the soil of the Muckpile, the pad, and the drainage and the sediments in the ponds, the only potential exposure pathway would be through worker contact with the contaminated soil. The lack of migration demonstrated by the analytical results, elapsed time since the suspected release, and the depth to groundwater supports the selection and evaluation of only the surface and shallow subsurface contact as the complete exposure pathway. Groundwater is not considered to be an exposure mechanisms or pathway. 


\section{D.1.6 Comparison of Site Conditions with Tier 1 Risk-Based Screening Levels}

All analytical results for CAU 383 were less than corresponding Tier 1 action levels (i.e., PALs) except for those listed in Table D.1-3.

Table D.1-3

COPCs Detected Above Preliminary Action Levels

\begin{tabular}{|c|c|c|c|c|c|c|c|c|c|c|c|c|}
\hline & 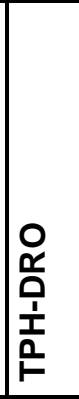 & 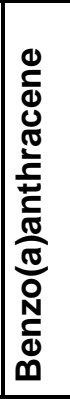 & 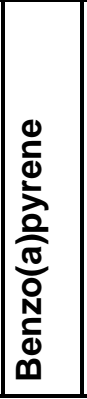 & 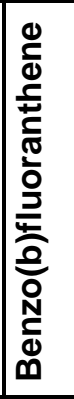 & 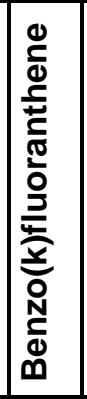 & 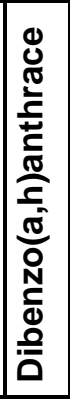 & 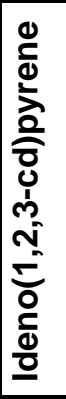 & 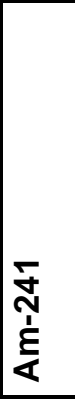 & 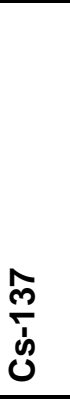 & $\begin{array}{l}8 \\
\frac{9}{1} \\
\overline{1}\end{array}$ & 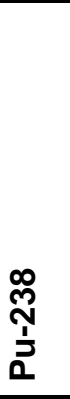 & 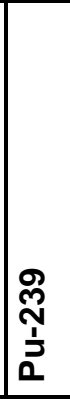 \\
\hline CAS 12-06-06 Muckpile Muck & $\mathrm{x}$ & & & & & & & $\mathrm{x}$ & $x$ & $\mathrm{x}$ & $x$ & $x$ \\
\hline CAS 12-06-06 Muckpile Native & $\mathrm{x}$ & & & & & & & & $x$ & & & \\
\hline Drainage & & & & & & & & & $\mathrm{x}$ & & & \\
\hline Pond Sediment & $\mathrm{x}$ & & & & & & & & $x$ & & & \\
\hline CAS 12-25-02 Oil Spill & $x$ & $x$ & $\mathrm{x}$ & $x$ & $\mathrm{x}$ & $x$ & $x$ & & $x$ & & & \\
\hline CAS 12-28-02 Radioactive Material & & & $\mathrm{x}$ & & & & & & $x$ & & & \\
\hline
\end{tabular}

\section{D.1.7 Evaluation of Tier 1 Results}

For all constituents at CAU 383 not listed in Section D.1.6, the FALs were established as the Tier 1 risk-based screening levels. It was determined that no further action is required for these constituents at CAU 383.

It was determined by DTRA and NNSA/NSO that remediation of the remaining constituents listed in Table D.1-3 is not practical. Therefore, a Tier 2 SSTLs will be calculated for those constituents at each CAS. 


\section{D.1.8 Tier 1 Remedial Action Evaluation}

TPH-DRO Fvaluation

Remediation to Tier 1 action levels would be difficult and expensive while potentially not providing a significant risk reduction. It would also present an increased risk to workers operating heavy equipment on the Muckpile. Therefore, it was determined to assess the risk to human health posed by the hazardous constituents of TPH-DRO at CAU 383 under a Tier 2 evaluation before establishing FALs for TPH-DRO constituents or implementing a corrective action.

\section{Chemical Evaluation}

Remediation to Tier 1 action levels for benzo(a)anthracene, benzo(a)pyrene, benzo(b)fluoranthene, benzo(k)fluoranthene, dibenzo(a,h)anthracene, and ideno(1,2,3-cd)pyrene would be difficult and expensive while potentially not providing a significant risk reduction. Therefore, the risk to human health posed by the chemicals that exceeded the Tier 1 action levels at CAU 383 needs to be assessed under a Tier 2 evaluation before establishing FALs for these chemicals or implementing a corrective action.

\section{Radionuclide Evaluation}

Actions to remediate Am-241, Cs-137, Pu-238, and Pu-239 to Tier 1 action levels would be difficult, expensive, and increase the risk to site workers while potentially not providing a significant risk reduction. Therefore, these radionuclides were moved to a Tier 2 evaluation before establishing FALs or implementing a corrective action.

\section{D.1.9 Tier 2 Evaluation}

No additional data were needed to complete a Tier 2 evaluation.

\section{D.1.10 Development of Tier 2 Table of SSTLS}

Evaluation of TPH-DRO SSTLS

The ASTM Method E 1739-95 stipulates that risk evaluations for TPH-DRO contamination be calculated and evaluated based on the risk posed by the potentially hazardous constituents of TPH-DRO (ASTM, 1999). Section 6.4.3, "Use of Total Petroleum Hydrocarbon Measurements,” of ASTM Method E 1739-95 states: “TPH-d should not be used for risk assessment because the general measure of TPH-d provides insufficient information about the amounts of individual chemical(s) of concern present” (see also Sections X1.5.4 and X1.42 of 
Method E 1739-95). Therefore, the individual potentially hazardous constituents in TPH-DRO were compared to corresponding Tier 2 SSTLs to evaluate the need for corrective action at CAU 383. Although Tier 2 SSTLs are generally calculated using site-specific inputs and general risk formulas, the Tier 2 SSTLs selected for the hazardous constituents of TPH-DRO (except for benzo(a)anthracene) are the EPA Region 9 PRGs (EPA, 2002). These SSTLs and the maximum reported level for each diesel constituent per CAS are presented in Table D.1-4. For an explanation of the benzo(a)anthracene SSTL, see the following text.

Table D.1-4

Tier 2 SSTLs and CAU 383 Results for Hazardous Constituents of Diesel

\begin{tabular}{|c|c|c|c|c|c|}
\hline \multirow{2}{*}{ CAS No. } & \multirow{2}{*}{ Common Name } & \multirow{2}{*}{$\begin{array}{l}\text { SSTL } \\
\text { (mg/kg) }\end{array}$} & \multicolumn{3}{|c|}{ Maximum Reported Value (mg/kg) } \\
\hline & & & $12-06-06^{1}$ & $12-25-02$ & $12-28-02$ \\
\hline $108-67-8$ & 1,3,5-Trimethylbenzene & 687 & 0.0072 & ND & ND \\
\hline $91-57-6$ & 2-Methylnaphthalene & 175,000 & ND & ND & ND \\
\hline $56-55-3$ & Benzo(a)anthracene & $147^{2}$ & 1.2 & 18 & 0.17 \\
\hline $71-43-2$ & Benzene & 122 & ND & ND & ND \\
\hline $50-32-8$ & Benzo(a)pyrene & 15 & 1.3 & 23 & 0.23 \\
\hline $100-41-4$ & Ethylbenzene & 1,210 & 0.12 & ND & ND \\
\hline $91-20-3$ & Naphthalene & 2,300 & 0.019 & 0.056 & 0.018 \\
\hline $108-88-3$ & Toluene & 40,200 & ND & ND & ND \\
\hline $1330-20-7$ & Total Xylene & 9,900 & 2.5 & ND & ND \\
\hline $104-51-8$ & N-Butylbenzene & 240 & ND & ND & ND \\
\hline $103-65-1$ & N-Propylbenzene & 240 & ND & ND & ND \\
\hline
\end{tabular}

${ }^{1}$ Includes the drainage and ponds

${ }^{2}$ SSTL calculated as described in the Evaluation of Chemical SSTLs Section.

\section{Evaluation of Chemical SSTLs}

The Tier 2 evaluation consisted of calculating Tier 2 SSTLs using site-specific inputs to standard risk equations for chemical contaminants. The SSTLs were calculated using equations which are compliant with the Risk Assessment Guidance for Superfund Part B procedures and were extracted from the Risk Assessment Information System (ORNL, 2004) located online at: http://risk.lsd.ornl.gov/cgi-bin/prg/PRG search. This website provides an online menu-driven environmental risk assessment system that among other things will calculate PRGs based on 
site-specific parameters. The calculated SSTLs and the maximum reported level for each chemical constituent per CAS are presented in Table D.1-5. The CAS-specific FALs established for these chemical constituents are the SSTLs listed in Table D.1-5.

Table D.1-5

Tier 2 SSTLs and CAU 383 Results for Chemical Constituents

\begin{tabular}{||c|c|c|c|c|c||}
\hline \multirow{2}{*}{ CAS No. } & \multirow{2}{*}{ Common Name } & \multirow{2}{*}{$\begin{array}{c}\text { SSTL } \\
(\mathbf{m g} / \mathbf{k g})\end{array}$} & \multicolumn{3}{|c|}{ Maximum Reported Value (mg/kg) } \\
\cline { 4 - 7 } & & $\mathbf{1 2 - 0 6 - 0 6}$ & $\mathbf{1 2 - 2 5 - 0 2}$ & $\mathbf{1 2 - 2 8 - 0 2}$ \\
\hline \hline $56-55-3$ & Benzo(a)anthracene & $147^{2}$ & 1.2 & 18 & 0.17 \\
\hline $50-32-8$ & Benzo(a)pyrene & 15 & 1.3 & 23 & 0.23 \\
\hline $205-99-2$ & Benzo(B)fluoranthene & 150 & 1.7 & 43 & 0.62 \\
\hline $207-08-9$ & Benzo(K)fluoranthene & 1,500 & 0.65 & 19 & 0.23 \\
\hline $53-70-3$ & Dibenzo(a,h)anthracene & 15 & ND & 10 & 0.027 \\
\hline $193-39-5$ & Ideno(1,2,3-Cd)pyrene & 150 & 0.63 & 22 & 0.08 \\
\hline
\end{tabular}

${ }^{1}$ Includes the drainage and ponds

${ }^{2}$ SSTL calculated under the Occasional Use scenario as described in the Evaluation of Chemical SSTLs Section.

The site-specific input parameters used for this calculation were the "NTS standard" parameter values listed in Table D.1-6 for the Occasional Use Area exposure scenario. The Occasional Use Area scenario addresses exposure to industrial workers exposed daily to contaminants in soil during an average work day. This scenario assumes that this is only an occasional work area for the worker who will be on the site for a very limited time during his career (10 days per year, 8 hours per day, for 5 years). The criteria for this scenario are that there are no active powered buildings with toilets present at the site for the shelter and comfort of the worker. The worker exposures are limited to working hours and do not include contributions from ingestion of drinking water, plant foods, meat, or fish taken from the immediate area. 
Table D.1-6

Specific Input Parameters with Proposed NTS Standard Values

\begin{tabular}{|c|c|c|c|c|c|c|}
\hline \multirow{2}{*}{ Parameter } & \multirow{2}{*}{ Definition } & \multirow{2}{*}{ Units } & \multirow{2}{*}{$\begin{array}{c}\text { EPA } \\
\text { Region } 9 \\
\text { Default } \\
\text { Value }\end{array}$} & \multicolumn{3}{|c|}{ Exposure Scenario } \\
\hline & & & & $\begin{array}{c}\text { Industrial } \\
\text { Area }\end{array}$ & $\begin{array}{c}\text { Remote } \\
\text { Work Area }\end{array}$ & $\begin{array}{l}\text { Occasiona } \\
\text { I Use Area }\end{array}$ \\
\hline ED & Exposure duration & $\mathrm{yr}$ & 25 & 25 & 25 & $5^{a}$ \\
\hline EF & Exposure frequency & day/yr & 250 & 250 & $42^{b}$ & $10^{c}$ \\
\hline IR & Soil ingestion rate & kg/day & 0.0001 & $0.00005^{a}$ & $0.00005^{d}$ & $0.00005^{d}$ \\
\hline foc & Fraction of organic carbon in soil & $g / g$ & 0.006 & $0.001^{b}$ & $0.001^{b}$ & $0.001^{b}$ \\
\hline$?_{\mathrm{w}}$ & Water-filled soil porosity & $\mathrm{cm}^{3} / \mathrm{cm}^{3}$ & 0.15 & $0.1^{\mathrm{c}}$ & $0.1^{c}$ & $0.1^{\mathrm{c}}$ \\
\hline $\mathrm{V}$ & Fraction of vegetative cover & unitless & 0.5 & $0.1^{d}$ & $0.1^{\mathrm{d}}$ & $0.1^{d}$ \\
\hline TR & $\begin{array}{l}\text { Target excess individual lifetime } \\
\text { cancer risk }\end{array}$ & unitless & 1E-06 & $1 \mathrm{E}-06$ & 1E-05 & 1E-05 \\
\hline
\end{tabular}

Rational for non-default input values:

aEPA Exposure Factors Handbook (EPA/600/P-95/002Fa) (EPA, 1997)

${ }^{b}$ Estimated value based on reduced total organic carbon content of NTS soils

'Estimated value based on reduced soil moisture content of NTS soils

${ }^{\mathrm{d} E s t i m a t e d}$ value based on reduced vegetative cover at NTS

$\mathrm{cm}^{3} / \mathrm{cm}^{3}=$ Cubic centimeters per cubic centimeter

day/yr = Days per year

$\mathrm{g} / \mathrm{g}=$ Grams per gram

$\mathrm{kg} /$ day $=$ Kilograms per day

$\mathrm{yr}=$ Year

\section{Evaluation of Radiological Constituent SSTLs}

The Tier 2 evaluation consisted of evaluating the mixture of all radionuclides detected at each CAS to develop Tier 2 action levels for the radionuclides that exceeded Tier 1 levels. The CAS specific Tier 2 action levels were calculated using the RESRAD code (version 6.22) and site-specific parameters. The RESRAD calculations were based on continued use of the site under the Occasional Use Area scenario, assuming that a site worker will be on site for 10 days per year, 8 hours a day for 5 years. A more detailed discussion of the RESRAD code, site-specific parameters used, and the printed RESRAD outputs is provided in Attachment A of this Appendix. These SSTLs and the maximum reported level for each radiological constituent per CAS are presented in Table D.1-7. 
Table D.1-7

Tier 2 SSTLs and CAU 383 Results for Radiological Constituents

\begin{tabular}{||c|c|c|c|c|c|c|c|c|c||}
\hline \multirow{2}{*}{$\begin{array}{c}\text { Common } \\
\text { Name }\end{array}$} & \multicolumn{3}{|c|}{$\mathbf{1 2 - 0 6 - 0 6} \mathbf{1}$ (pCi/g) } & \multicolumn{3}{c|}{$\mathbf{1 2 - 2 5 - 0 2 ~ ( p C i / g ) ~}$} & \multicolumn{3}{|c|}{ 12-28-02 (pCi/g) } \\
\cline { 2 - 12 } & SSTL & $\begin{array}{c}\text { Maximum } \\
\text { Result }\end{array}$ & $\begin{array}{c}\text { Site } \\
\text { Average }\end{array}$ & SSTL & $\begin{array}{c}\text { Maximum } \\
\text { Result }\end{array}$ & $\begin{array}{c}\text { Site } \\
\text { Average }\end{array}$ & SSTL & $\begin{array}{c}\text { Maximum } \\
\text { Result }\end{array}$ & $\begin{array}{c}\text { Site } \\
\text { Average }\end{array}$ \\
\hline \hline Americium-241 & 7.32 & 28.8 & 1.03 & N/A & ND & N/A & N/A & ND & N/A \\
\hline Cesium-137 & 269.56 & 1,060 & 35.72 & 275 & 20.5 & 6.98 & 275.2 & 114 & 38 \\
\hline Plutonium-238 & 3.97 & 15.6 & 0.44 & N/A & ND & N/A & 2370 & 0.232 & 0.116 \\
\hline Plutonium-239 & 21.36 & 84 & 3.04 & 215 & 1.04 & 0.257 & 2152 & 2.09 & 1.064 \\
\hline \hline
\end{tabular}

${ }^{1}$ Includes the drainage and ponds

Although all detected radionuclides at a CAS are used in the sum-of-fractions calculation and a unique Tier 2 action level is developed for all radionuclides, only the five radionuclides that initially exceeded Tier 1 levels have a Tier 2-based FAL. The CAS-specific FALs established for these radionuclides are the SSTLs listed in Table D.1-7.

\section{D.1.11 Comparison of Site Conditions with Tier 2 FALs}

The Tier 2 action levels are typically compared to individual sample results from reasonable points of exposure (as opposed to the source areas as is done in Tier 1) on a point-by-point basis. Points of exposure are defined as those locations or areas at which an individual or population may come in contact with a COC originating from a CAS. For CAU 383, the Tier 2 action levels were compared to maximum constituent concentrations from each sample location and to the average concentration for the entire site.

A comparison of the maximum concentration of the hazardous constituents of TPH-DRO and the chemical constituents identified above the Tier 1 action levels was conducted against the CAS-specific Tier 2 FALs as shown on Tables D.1-4 and D.1-5. All analytical results for potentially hazardous constituents in TPH-DRO were below the FALs for all three CASs, the drainage, and the ponds. All of the chemical constituents were below the CAS-specific FALs in the Muckpile (CAS 12-06-06 including the drainage and ponds) and the Radioactive Material (CAS 12-28-02). All of the chemical constituents were below the CAS-specific FALs in the Oil Spill (CAS 12-25-02) except for benzo(a)pyrene. 
A comparison between the maximum concentration of the radionuclides identified above Tier 1 action levels (Am-241, Cs-137, Pu-238, and Pu-239) was conducted against the CAS-specific Tier 2-based FALs listed in Table D.1-7. All four isotopes are above Tier 2-based FALs in the Muckpile (CAS 12-06-06) but not in the drainage or the ponds. None of the isotopes exceed the CAS-specific FALs for the Oil Spill (CAS 12-25-02) or the Radioactive Material (12-28-02).

\section{D.1.12 Tier 2 Remedial Action Evaluation}

Based on the Tier 2 evaluation of the TPH-DRO hazardous constituents, the chemical constituents, and the radiological constituents, CAS 12-06-06 is contaminated with radiological constituents and CAS 12-25-02 is contaminated with benzo(a)pyrene. These CASs pose an unacceptable risk. Corrective Action Site 12-28-02, the drainage, and the ponds do not have radiological or chemical concentrations above the FALs; therefore, they are not considered to be contaminated. Therefore, the close in place corrective action with use restrictions is recommended for CAU 383.

As all contaminant FALs were established as Tier 1 or Tier 2 action levels, a Tier 3 evaluation was considered unnecessary. 


\section{D.2.0 Regulatory Basis}

The FFACO Part III, Section III.3 (FFACO, 1996) stipulates conformance with Chapter 445 of the NAC (NAC, 2005a). Section NAC 445A.227 lists the factors to be considered in determining whether corrective action is required.

Section NAC 445A.227 states:

1. Except as otherwise provided in NAC 445A.22715, the Director may require an owner or operator to take corrective action if the release of a hazardous substance, hazardous waste, or a regulated substance contaminates soil and the level of contamination exceeds the action level established for the soil pursuant to NAC 445A.2272.

2. In determining whether corrective action is required, the Director shall consider:

(a) The depth of any groundwater

(b) The distance to irrigation wells or wells for drinking water.

(c) The type of soil that is contaminated.

(d) The annual precipitation.

(e) The type of waste or substance that was released.

(f) The extent of the contamination.

(g) The present and potential use for the land.

(h) The preferred routes of migration.

(i) The location of structures or impediments.

(j) The potential for a hazard related to fire, vapor, or explosion.

(k) Any other information specifically related to the site that the director determines is appropriate.

For a site where it is determined that corrective action is required (the corrective action process applies to all FFACO sites), Section NAC 445A.22705 (NAC, 2005c) stipulates a process to determine the necessary remediation standards (or FALs) based on an evaluation of the risk the site poses to public health and the environment. 
Section NAC 445A.22705 states:

1. Except as otherwise provided in NAC 445A.22715, if an owner or operator is required to take corrective action pursuant to NAC 445A.227, the owner or operator may conduct an evaluation of the site, based on the risk it poses to public health and the environment, to determine the necessary remediation standards or to establish that corrective action is not necessary. Such an evaluation must be conducted using Method E 1739-95, adopted by the ASTM, as it exists on October 3, 1996, or an equivalent method approved by the Division.

2. The Division shall determine whether an evaluation complies with the requirements of Method E 1739-95, or an equivalent method of testing approved by the Division. The Division may reject, require revisions be made to, or withdraw its concurrence with the evaluation at any time after the completion of the evaluation for the following reasons:

(a) The evaluation does not comply with the applicable requirements for conducting the evaluation.

(b) Conditions at the site have changed.

(c) New information or previously unidentified information that would alter the results of the evaluation becomes available and demonstrates that the release may have a detrimental impact on public health or the environment.

Therefore, in compliance with Section NAC 445A.22705 (NAC, 2005c), NNSA/NSO will "conduct an evaluation of the site, based on the risk it poses to public health and the environment, to determine the necessary remediation standards or to establish that corrective action is not necessary” using ASTM Method E 1739-95. 


\section{D.3.0 Recommendations}

Organic, inorganic, and radiological constituents detected in environmental samples during the CAI were evaluated against FALs to determine the nature and extent of COCs for CAU 383. Assessment of the data generated from investigation activities indicates that FALs were exceeded for radionuclides in three subsurface samples from three locations in CAS 12-06-06 and for benzo(a)pyrene in one surface sample in CAS 12-25-02.

As COCs were identified above corresponding FALs in CASs 12-06-06, 12-25-02, and CAS 12-28-02, it was determined through discussions with NDEP, DTRA, and NNSA/NSO that a closure in place with use restrictions is proposed for all of the CASs.

No further corrective action is necessary. 


\section{D.4.0 References}

ASTM, see American Society for Testing and Materials.

American Society for Testing and Materials. 1995. Standard Guide for Risk-Based Corrective Action Applied at Petroleum Release Sites/American Society for Testing and Materials, ASTM E 1739-95 (Reapproved 2002). Philadelphia, PA.

Appenzeller-Wing, J., U.S. Department of Energy, National Nuclear Security Administration Nevada Site Office. 2004. Letter to T.A. Maize (NDEP) entitled "Submittal of Proposed Radiological Preliminary Action Levels (PALs) for the Industrial Sites Project,” 15 January. Las Vegas, NV.

BN, see Bechtel Nevada.

Bechtel Nevada. 1995. Nevada Test Site Performance Objective for Certification of Nonradioactive Hazardous Waste. G-E11/96.01, Rev. 0. Las Vegas, NV.

Black, S.C., and Y.E. Townsend. 1996. U.S. Department of Energy, Nevada Operations Office Environmental Data Report for the Nevada Test Site - 1994. Las Vegas, NV.

DOE, see U.S. Department of Energy.

DTRA, see Defense Threat Reduction Agency.

Defense Threat Reduction Agency. 2003. Corrective Action Investigation Plan for Corrective Action Unit 383: Area 12 E-Tunnel Sites, Nevada Test Site, Rev. 0. Prepared by SNJV. Las Vegas, NV.

EPA, see U.S. Environmental Protection Agency.

FFACO, see Federal Facility Agreement and Consent Order.

Federal Facility Agreement and Consent Order. 1996 (as amended). Agreed to by the State of Nevada, the U.S. Department of Energy, and the U.S. Department of Defense.

Las Vegas, NV.

McArthur, R.D., and F.L. Miller, Jr. 1989. Offsite Radiation Exposure Review Project, Phase II Soils Programs, DOE/NV/10384-23. Las Vegas, NV: Desert Research Institute.

Moore, J., Science Applications International Corporation. 1999. Memorandum to M. Todd (SAIC) entitled, "Background Concentrations for NTS and TTR Soil Samples.”

Las Vegas, NV. 
NAC, see Nevada Administrative Code.

NBMG, see Nevada Bureau of Mines and Geology.

NCRP, see National Council on Radiation Protection and Measurements.

National Council on Radiation Protection and Measurements. 1999. Recommended Screening Limits for Contaminated Surface Soil and Review of Factors Relevant to Site-Specific Studies, Report No. 129. Bethesda, MD.

Nevada Administrative Code. 2005a. NAC 445A, "Water Controls.” Carson City, NV.

Nevada Administrative Code. 2005b. NAC 445A.2272, "Contamination of Soil: Establishment of Action Levels.” Carson City, NV.

Nevada Administrative Code. 2005c. NAC 445A.22705, "Contamination of Soil: Evaluation of Site by Owner or Operator; Review of Evaluation by Division.” Carson City, NV.

Nevada Bureau of Mines and Geology. 1998. Mineral and Energy Resource Assessment of the Nellis Air Force Range, Clark, Lincoln, and Nye Counties, Nevada, Open File Report 98-1. Reno, NV.

ORNL, see Oak Ridge National Laboratory.

Oak Ridge National Laboratory. 2004. Risk Assessment Information System. As accessed at http://risk.lsd.ornl.gov/cgi-bin/prg/PRG_search on 25 April 2006.

U.S. Department of Energy. 1993. DOE Order 5400.5 Change 2, "Radiation Protection of the Public and Environment.” Washington, DC.

US Ecology and Atlan-Tech, Inc. 1991. Environmental Monitoring Report for the Proposed Ward Valley, California, Low-Level Radioactive Waste (LLRW) Facility, Vols. 1 and 2, Version 2. Rosewell, GA: California Department of Health, Environmental Management Branch.

U.S. Environmental Protection Agency. 1997. Exposure Factors Handbook, EPA/600/P-95/002Fa. Washington, DC: Office of Research and Development.

U.S. Environmental Protection Agency. 2002. Region 9 Preliminary Remediation Goals (PRGs). San Francisco, CA. 


\section{Attachment A}

\section{RESRAD Report}


Derivation of Residual Radioactive Material Guidelines for Radionuclides in Soil at Corrective Action Unit (CAU) 383, Area 12 E-Tunnel Sites, Nevada Test Site, Nevada

May 2006

Prepared by:

Stoller-Navarro Joint Venture, 7710 W. Cheyenne, Las Vegas, Nevada 89193

Work sponsored by United States Department of Energy, National Nuclear Security Administration Nevada Operations Office, Environmental Restoration Division,

Las Vegas, Nevada 


\section{Derivation of Residual Radioactive Material Guidelines for Radionuclides in Soil at Corrective Action Unit (CAU) 383, Area 12 E-Tunnel Sites, Nevada Test Site, Nevada}

\subsection{Introduction}

The U.S. Department of Energy (DOE), the U.S. Department of Defense (DoD) (through the Defense Threat Reduction Agency [DTRA]), and the National Nuclear Security Administration Nevada Site Office (NNSA/NSO) Environmental Restoration Division have numerous sites impacted from the development, testing, and production of nuclear weapons. These impacts can take the form of chemical and/or radiological contaminants. Similar to its approach for chemical contamination, DoD and NNSA/NSO are committed to properly evaluating, radiologically characterizing, and where appropriate, remediating these sites to ensure the doses to radiation workers and members of the public are maintained as-low-as-reasonably achievable (ALARA), at a minimum, below the primary dose limits as stated in DOE Order 5400.5 (DOE, 1993).

To accomplish this, the potential for residual radioactive contamination in soils must be evaluated to determine the status of compliance with the requirements of DOE Order 5400.5 (DOE, 1993). The DOE Order 5400.5 requires that: "The Authorized Limits shall be established to (1) provide that, at a minimum, the basic dose limits ... will not be exceeded, or (2) be consistent with applicable generic guidelines.” Because generic guidelines have not been established for volumetric residual radioactivity for the radionuclides of concern at CAU 383 land areas, Authorized Limits or final action levels (FALs) were derived using the Residual Radioactivity (RESRAD) model and computer code (Yu et al., 2001). The goal of this effort was to produce Authorized Limits, in units of picocuries per gram (pCi/g) in soil above background, for CAU 383 that would result in radiation doses less than $25 \mathrm{mrem}$ per year (mrem/y) to an industrial worker at the site.

To develop the FALs, a "realistic" yet conservative radiation dose analysis was conducted using approved exposure scenarios and site-specific data to determine the translation between surface soil concentrations and individual radiation doses. For this analysis, site-specific data included soil sampling results obtained during site investigation activities at CAU 383, and meteorological data obtained from the Air Resources Laboratory (ARL)/Special Operations and Research Division (SORD). This report provides the radiation dose modeling analysis supporting the technical derivation of the Authorized Limits for CAU 383, Area 12 E-Tunnel Sites, Nevada Test Site (NTS), Nevada. This report also defines the radionuclides considered and approved exposure scenarios for the NTS, identifies the applicable exposure pathways and key input data or assumptions, presents the radiation doses for unit concentrations of radionuclides in soil, and establishes the FALs for selected land parcels at CAU 383. 


\subsection{Facility Description}

Corrective Action Unit 383, Area 12 E-Tunnel Sites, consists of three Corrective Action Sites (CASs) located in Area 12. E-Tunnel was mined horizontally into the southeastern side of Rainier Mesa, penetrating a zeolytized bedded tuff. The E-Tunnel Muckpile (CAS 12-06-06) consists of material mined out of the tunnel. The surface elevation at the top of the muckpile is about 6,100 feet (ft) above sea level. The muckpile was deposited in an easterly trend in front of the tunnel portals. The muckpile is approximately $200 \mathrm{ft}$ across and 1,200 ft long in an east-west direction. The thickness of the muckpile ranges from less than $5 \mathrm{ft}$ at the west end to approximately $100 \mathrm{ft}$ at the northeast side.

Corrective Action Site 12-28-02 (Radioactive Material) is located at the E-Tunnel main portal and is part of the muckpile.

Corrective Action Site 12-25-02 (Oil Spill) is located on the blower pad, which is on a bench approximately $100 \mathrm{ft}$ above the E-Tunnel south portal. The pad was constructed in the hillside and is about $100 \mathrm{ft}$ long north to south and $50 \mathrm{ft}$ wide. Fill on the pad varies in thickness from less than $1 \mathrm{ft}$ next to the slope to about $10 \mathrm{ft}$ on the north edge of the pad.

These sites were used during underground nuclear testing at the E-Tunnel from the late 1950s to the late 1970s. The site has mostly been inactive since that period. In addition to the three CASs, ponds 1, 2, and 3 and the drainage between the muckpile and ponds have been included in this site investigation. The CASs and impacted areas investigated during the CAU 383 Corrective Action Investigation (CAI) are:

- CAS 12-06-06, Muckpile and the down slope area (drainage and ponds)

- CAS 12-25-02, Oil Spill

- CAS 12-28-02, Radioactive Material

Each CAS is fully described in the Corrective Action Investigation Plan (CAIP) for CAU 383. To facilitate the development of FALs, land areas included within the CAU 383 CAI were grouped into the following land parcels:

- CAS 12-06-06, Muckpile

o Muckpile

o Drainage

0 Ponds

- CAS 12-25-02, Oil Spill

- CAS 12-28-02, Radioactive Material

\subsection{Operational History}

The following sections provide a brief summary of the operational history for CAU 383. 
E-Tunnel was used for nuclear weapons tests between 1958 and 1977. Reentry mining generated rock debris and construction waste such as cabling, scrap metal, and cementitious mixtures, which could have contained low levels of radioactivity. The materials excavated during mining of the tunnel and the reentry mining debris were generally hauled outside the tunnel to the edge of the muckpile, dumped over the edge, and then leveled. Nine nuclear tests were conducted in E-Tunnel. The E-Tunnel Muckpile is comprised of mining, construction, and reentry debris from the tests.

\subsection{Release Information}

Past surface activities of concern in CAS 12-06-06 include equipment maintenance and storage of equipment and petroleum products. Release to muckpile from surface activities may be locally significant, but vertical infiltration of contaminants is probably limited to less than five ft from the release point. Corective Action Site 12-28-02 is part of CAS 12-06-06. At CAS 12-25-02 there is a visible oil stain. This is the only evidence found of a potential release at this CAS. Release mechanisms, migration pathways, exposure pathways, and exposure points are described in the CAU 383 CAIP.

\subsection{Site Investigation Activities}

\subsection{Site Investigation Plans}

Corrective action investigation activities were performed as set forth in the CAU 383 CAIP (DTRA, 2003) from May 19 through June 24, 2004. The objectives of the CAU 383 CAIP were to address the decision statements in the project-specific Data Quality Objectives (DQOs) by:

- Characterizing the muckpile material from the surface to the native material interface.

- Characterizing the native materials underlying the muckpile (from 2 to $5 \mathrm{ft}$ below the muckpile or until drill refusal) for COPCs.

- Characterizing the soils in the drainage between the muckpile and the first pond.

- Characterizing the pond material in the first three ponds from the surface to the native material interface.

- Characterizing the native materials underlying the first three ponds (from 2 to 5 ft below the pond or until drill refusal) for COPCs.

- Characterizing the soils at the blower pad where the oil stain is located.

- Characterizing the soils in the Radioactive Material area.

- Establishing background levels of radioactivity and metals in the native soils surrounding the muckpile.

The DQO process is a seven-step strategic planning approach based upon the scientific method used to plan data collection activities for CAU 383, Area 12 E-Tunnel Sites, Nevada Test Site. The DQOs are designed to ensure that data collected will provide sufficient and reliable information to identify, evaluate and technically defend the 
recommended corrective actions (i.e., no further action, closure in place, or clean closure).

The primary objective of the investigation was to provide sufficient information and data to develop appropriate corrective action alternatives for each CAS in CAU 383. This objective was achieved by identifying the nature and extent, both horizontal and vertical of COCs (i.e., COPCs at concentrations above action levels).

The investigation strategy was developed by representatives of NDEP and DTRA, in accordance with U.S. Environmental Protection Agency (EPA) Guidance for Quality Assurance Project Plans, EPA QA/G-5 (EPA, 2002a) and Guidance for the Data Quality Objectives Process, EPA QA/G-4 (EPA, 2000b). The investigation strategy also identifies and references the associated EPA Quality System Documents entitled Data Quality Objectives for Hazardous Waste Site Investigation, EPA QA/G-4HW (EPA, 2000a), and Guidance on Choosing a Sampling Design for Environmental Data Collection, EPA QA/G-5S (EPA, 2002b), upon which the DQO process is based. The CAU 383 CAIP contains a detailed description of the investigation strategy and the DQO process.

\subsection{Summary of Specific Site Investigation Activities}

This section provides a brief description of work activities conducted to support the investigation of radioactive contamination at CAU 383.

\section{Radiological Surveys}

Prior to commencing drilling and sampling, walk-over and drive-over radiological surveys were conducted to identify surface and near-surface areas with elevated readings. The results of the surveys were used to guide the investigation and provide for site worker safety, focusing on the identification of "hot-spot" areas.

Sampling of the Muckpile and Underlying Native Material Thirty-four locations were drilled or hand sampled on the Muckpile to characterize the muck and the native material under the Muckpile. A total of 1,039.5 ft were drilled in 32 boreholes ranging from 1.5 to $91.5 \mathrm{ft}$ deep. Two locations were sampled by hand to 6 inches deep. Up to three samples were collected from each location. One location was drilled but not sampled. During the characterization, 66 environmental samples were collected. These included 41 muck samples and 25 native material samples. All samples were sent to an approved off-site analytical laboratory for analysis.

Sampling of the Drainage

Thirty-five locations were drilled or hand sampled in the drainage to characterize the site. The two boreholes were each drilled to five ft deep. Thirty-three locations were hand sampled to 6 inches deep. One sample was collected from each location for a total of 35 environmental samples. 
Sampling of the Pond Sediments and Underlying Native Material

Nine locations were drilled or hand sampled in Ponds One, Two, and Three to characterize the sediment and the native material under the sediments. In Pond One, a total of $80 \mathrm{ft}$ was drilled in five boreholes ranging in depth from 18.0 to $21.0 \mathrm{ft}$. Due to soil wetness in Pond Two and Three, only two locations were sampled in each pond; all four locations were hand sampled to 6 inches deep. During the characterization, 14 environmental samples were collected. These included nine sediment samples and five native material samples. All samples were sent to an approved off-site analytical laboratory for analysis.

Sampling of Oil Spill (CAS 12-25-02)

Eight locations were hand sampled to 6 inches deep in the Oil Spill to characterize the site. One sample was collected from each location for a total of eight environmental samples. All samples were sent to an approved off-site analytical laboratory for analysis.

Sampling of the Radioactive Material Area (RMA) (CAS 12-28-02)

Four locations were hand sampled to 6 inches deep in the RMA to characterize the site. One sample was collected from each location for a total of four environmental samples. All samples were sent to an approved off-site analytical laboratory for analysis.

Background and preliminary soil samples were collected at locations on and around the Muckpile. Three background samples were hand-collected at three undisturbed locations around the Muckpile. Preliminary samples were hand-colleted at five biased locations on the Muckpile. All samples were sent to an approved off-site analytical laboratory for analysis.

\subsection{Sampling Locations}

To achieve the objective of identifying the nature and extent of the COCs, a combined random and judgmental (non-probabilistic) sampling scheme was used for selecting sample locations and evaluating analytical results. Judgmental sampling allows the selection of sample locations that target the populations of interest (defined in the DQOs). Sampling points for each CAS were selected based on the approach described in the CAIP, which included interpretation of existing engineering drawings, aerial and land photographs, interviews with former site employees, information obtained during site visits, and site-specific biasing factors. The planned sample locations are discussed the CAIP. Some planned locations were modified slightly from planned positions due to field conditions and observations. Sample locations were staked, labeled appropriately, and surveyed with a global positioning system (GPS) instrument. Appendix A of the CAU 383 CADD/CR contains a detailed description of the actual sample locations. 


\subsection{Site Investigation Sample Results}

The RESRAD calculations are based on validated analytical soil sample results obtained during site investigation activities and other applicable information specified in the CAIP. The RESRAD calculations are based upon the value of the maximum radionuclide concentration, as specified in the CAU 383 CAIP. The RESRAD calculations of the downslope area (i.e., drainage and ponds) were performed for the contaminants of concern (COC) present in the CAU 383 muckpile using the maximum radionuclide concentrations obtained from the downslope area soil sample results. Appendix A of the CAU 383 CADD/CR contains a detailed description of the sample results, analytical parameters, and laboratory methods used to analyze the soil samples. The following section provides a summary of the radiological concentrations from samples taken at CAU 383.

The maximum principal radionuclide concentrations (including background) detected at the CASs included:

Muckpile, CAS 12-06-06

- Muck

$\begin{array}{llll}\text { o } & \text { Americium (Am)-241 } & 28.8 & \mathrm{pCi} / \mathrm{g} \\ \text { o } & \text { Cesium (Cs) -137 } & 1060 & \mathrm{pCi} / \mathrm{g} \\ \text { o } & \text { Europium (Eu)-152 } & 2.64 & \mathrm{pCi} / \mathrm{g} \\ \mathrm{o} & \text { Eu-155 } & 0.32 & \mathrm{pCi} / \mathrm{g} \\ \mathrm{o} & \text { Strontium (Sr) }-90 & 34.1 & \mathrm{pCi} / \mathrm{g} \\ \text { o } & \text { Plutonium (Pu) -238 } & 15.6 & \mathrm{pCi} / \mathrm{g} \\ \text { o } & \text { Pu-239 } & 84 & \mathrm{pCi} / \mathrm{g}\end{array}$

- Drainage

$\begin{array}{llll}\text { o } & \text { Am-241 } & 1.88 & \mathrm{pCi} / \mathrm{g} \\ \text { o } & \mathrm{Cs}-137 & 78.1 & \mathrm{pCi} / \mathrm{g} \\ \text { o } & \mathrm{Sr}-90 & 1.88 & \mathrm{pCi} / \mathrm{g} \\ \text { o } & \mathrm{Pu}-238 & 0.73 & \mathrm{pCi} / \mathrm{g} \\ \text { o } & \mathrm{Pu}-239 & 4.21 & \mathrm{pCi} / \mathrm{g}\end{array}$

- Pond

$\begin{array}{llll}\text { o } & \text { Am-241 } & 0.72 & \mathrm{pCi} / \mathrm{g} \\ \text { o } & \mathrm{Cs}-137 & 91 & \mathrm{pCi} / \mathrm{g} \\ \text { o } & \mathrm{Sr}-90 & 3.62 & \mathrm{pCi} / \mathrm{g} \\ \text { o } & \mathrm{Pu}-238 & 0.74 & \mathrm{pCi} / \mathrm{g} \\ \text { o } & \mathrm{Pu}-239 & 5.37 & \mathrm{pCi} / \mathrm{g}\end{array}$

Oil Spill, CAS 12-25-02
- Cs-137
20.5
$\mathrm{pCi} / \mathrm{g}$
- $\mathrm{Pu}-239$
$1.04 \mathrm{pCi} / \mathrm{g}$

Radioactive Material, CAS 12-28-02
- Cs-137
$114 \mathrm{pCi} / \mathrm{g}$
- $\mathrm{Pu}-238$
$0.232 \mathrm{pCi} / \mathrm{g}$ 
- $\quad$ Pu-239 $2.09 \mathrm{pCi} / \mathrm{g}$

The maximum radionuclide concentration values were used to perform the RESRAD calculations. 


\subsection{Initial Concentrations for Principal Radionuclides}

Principal radionuclides are defined as radionuclides with a half-life greater than six months. The decay products of any principal radionuclide down to, but not including, the next principal radionuclide in its decay chain are defined as associated radionuclides. RESRAD assumes that a principal radionuclide is in secular equilibrium with its associated radionuclides at the point of exposure. Therefore, associated radionuclides and radionuclides with half-lives less than six months are not input into the RESRAD calculations.

\subsection{Authorized Values for Initial Concentrations of Principal Radionuclides}

The authorized exposure scenarios specify that value of the arithmetic mean plus the 95 percent UCL obtained from site-specific sampling results be entered as the principal radionuclide concentrations for RESRAD calculates. The sample results for all samples with radionuclide concentrations above the MDC within the land parcels are entered into the EPA software application ProUCL version 3.0. The ProUCL software is used to calculate the 95 percent UCL for principal radionuclide concentrations based on the distribution of the unknown mean.

For instances where the ProUCL software determined that there was not enough data to calculate the 95 percent UCL for a specific radionuclide, the maximum concentration from the sample dataset was used as the initial concentration for that radionuclide.

\subsection{Authorized Values Initial Concentrations of Principal Radionuclides for Area Averaging/Hot-Spot Scenarios}

The DOE Order 5400.5 (DOE, 1993) states: "Residual concentrations of radioactive material in soil are defined as those in excess of background concentrations averaged over an area of $100 \mathrm{~m}^{2 \text { ” }}(5400.5$, IV, 4.a.). DOE Order 5400.5 also states: "If the average concentration of any surface or below-surface area less than or equal to $25 \mathrm{~m}^{2}$, exceeds the limit or guideline by a factor of $(100 / \mathrm{A})^{0.5}$, [where $\mathrm{A}$ is the area (in square meters) of the region in which concentrations are elevated], limits for "hot-spots" shall also be developed and applied" (5400.5, IV, 4.a.(1)). DOE G 441.1-XX (DOE 2002) discusses the rationale for the hotspot criterion in Section 5.2.2.

The purpose of the hot-spot criterion is to ensure that applying the homogeneous criteria, in which the concentrations of residual radioactive material are averaged over a $100 \mathrm{~m}^{2}$ area, does not result in the release of small areas that, because of averaging, contain unacceptably high concentrations of residual radioactive material. The hot-spot criterion is used to supplement Authorized Limits for larger areas and is intended to prevent excessive exposures from a small, contaminated area that is within a larger area that meets the basic Authorized Limits. Thus, it is intended for use in areas where the residual radioactive material concentrations are not uniform. Also, the above hotspot criterion was derived conservatively, assuming the Authorized Limits were based on a dose constraint of $25 \mathrm{mrem} / \mathrm{y}$ and selected to ensure unlikely exposure conditions would not cause the primary dose limit (100 mrem/y) to be exceeded. The authorized exposure scenarios specify that the value of the maximum concentration of principal radionuclides obtained from site-specific sampling results be entered as the principal radionuclide concentrations for RESRAD hot-spot calculations. The authorized area parameters for RESRAD hot-spot calculations are $1 \mathrm{~m}^{2}, 10 \mathrm{~m}^{2}$, and $100 \mathrm{~m}^{2}$ contamination areas. 


\subsection{Initial Concentrations of Principal Radionuclide for CAU 383}

These maximum radionuclide concentration values were used to perform the RESRAD calculations.

The initial radionuclide concentrations used for the three RESRAD calculations are those listed in Table 7-2 for each CAS and location.

\subsection{Inhomogeneous Contamination and Initial Radionuclide Concentrations}

A contaminated zone is inhomogeneous if it contains a contaminated region within which the concentration of a radionuclide exceeds three times the average for the contaminated zone. RESRAD uses a mathematical construct that assumes uniform distribution of radionuclides within a volume. However, RESRAD recognizes that radiological contamination is inhomogeneous in nature and provides detailed guidance for applying inhomogeneous criteria (hot spot criteria, sum of fractions rule, etc.). The RESRAD User Manual states that the inhomogeneous release criteria are generally more realistic and hence less restrictive than the homogeneous release criteria. This shows that the approved initial radionuclide concentration values (i.e., arithmetic mean plus 95 percent UCL or the maximum radionuclide concentration from the sample dataset) will result in more restrictive release criteria. The arithmetic mean plus the 95 percent UCL are used for the initial concentrations of principal radionuclides when the sample results are obtained using a random sampling method. The maximum radionuclide concentration values are used for the initial concentrations of principal radionuclides when the sample results are obtained using a non-random (e.g., bias or judgmental sampling) sampling method.

RESRAD states that a statistical approach should always be considered as a first priority regarding the estimation of soil concentrations, as cited in the Data Collection Handbook to Support Modeling Impacts of Radioactive Material in Soil (Yu et al., 1993). The 95 percent UCL represents a value that has a 5 percent chance that the actual mean of the dataset would exceed it. The 95 percent UCL is computed using the EPA code ProUCL. The code calculates the 95 percent UCL based on the distribution of the dataset (e.g., normal, log-normal, gamma, non-parametric, etc.).

The ProUCL software has been developed to compute an appropriate 95 percent UCL of the unknown population mean to support exposure assessment and cleanup decisions for EPA projects. A 95 percent UCL of the unknown population arithmetic mean is often used to:

- Estimate the EPC term,

- Determine the attainment of cleanup standards,

- Estimate background level mean contaminant concentrations, or

- Compare the soil concentrations with site-specific soil screening levels.

It is important to compute a reliable, conservative, and stable 95 percent UCL of the population mean using the available data. The 95 percent UCL should approximately provide the 95 percent coverage for the unknown population mean.

The EPA has recommended that the maximum value of the dataset be used for the initial EPC term when the 95 percent UCL exceeds the maximum (EPA RAGS Document, 1992). However, if the 
maximum value of the dataset is used, then most of the statistical data associated with the distribution of the dataset is ignored (except for the maximum). Therefore, by using the mean plus the 95 percent UCL the statistical data associated with the dataset is retained and the value approaches or exceeds the maximum value of the dataset as recommended by EPA. 


\subsection{Authorized RESRAD Exposure Pathways and Scenarios}

This section describes the input parameters, exposures scenarios, and guidance for calculating sitespecific radiological remediation levels for projects using the RESRAD computer code, as agreed to by NNSA/NSO, Stoller-Navarro Joint Venture (SNJV), Bechtel Nevada (BN), and NDEP.

\subsection{Guidance for RESRAD Calculations}

The guidance in this section was developed by NNSA/NSO, SNJV, BN, and NDEP and is only applicable to soils containing residual radioactive material. This guidance does not apply to structures, facilities, equipment, and building materials containing contaminated surfaces or volume contamination. The primary dose limit for any member of the public is 100-mrem total effective dose equivalent (TEDE) in a year. This limit applies to the sum of internal and external doses resulting from all modes of exposure to all radiation sources other than background radiation and doses received as a patient from medical sources as required by DOE 5400.5, II.1.a.(3)(a) (DOE, 1993). The dose constraint is defined as one quarter of the dose limit (i.e., 25-mrem) and will be applied to ensure that in a 1,000-year period the maximally exposed individual does not exceed the dose constraint in any single year. The requirements of Chapter IV of DOE 5400.5 Chapter IV will not specifically apply if NNSA/NSO chooses to continue to own and actively control access or use of the site. However, the radiation protection requirements in the other sections of DOE 5400.5 will apply to NNSA/NSO owned and maintained sites.

Due to the large spatial variability in background amongst sites, the "above background criterion" will be defined as the concentration of a specific radionuclide in soil that equals or exceeds its corresponding PAL. The source data for these radionuclide specific PALs are taken directly from NCRP Report No. 129 Table 2.1, Construction, Commercial, Industrial land-use scenario column for a 25-mrem dose constraint (NCRP, 1999). The generic guidelines for residual concentrations of Radium (Ra)-226, Ra-228, Thorium (Th)-230, and Th-232 are found in Chapter IV of DOE Order 5400.5, Change 2 "Radiation Protection of the Public and Environment."

Background radiation refers to the local area and includes:

- Concentration of naturally occurring radionuclides.

- Cosmic radiation.

- Radionuclides of anthropogenic origin that have been globally dispersed and are present at low concentrations such as fallout from nuclear weapons. (Note: This is not the case at the NTS because the historical aspects of the NTS, e.g., above- and below-ground testing, and other operations resulted in dispersion of radionuclides locally.)

Due to the impracticality of determining "true" background, a dose constraint with no background subtraction will be used (i.e., a dose constraint not in excess of background). The use of the dose constraint with no background subtraction is a far more conservative and sensitive approach because it does not deal with the uncertainty of natural background. 


\subsection{Description of Approved Scenarios}

Based on the future land use as identified in the Nevada Test Site Resource Management Plan (DOE/NV, 1998), the following two exposure scenarios have been identified as "actual" and "likely" use scenarios. Stoller-Navarro Joint Venture has approval to use two scenarios (Scenario A and Scenario B) for use with the RESRAD code (NDEP, 2004). Both scenarios consider radiation exposures to the critical population group via the following pathways:

- Direct exposure to external radiation from the contaminated soil.

- Internal dose from inhalation of airborne radionuclides.

- Internal dose from ingestion of contaminated soil.

The two scenarios vary the parameters associated with the future land use of the site but use the same dose constraint parameter of $25 \mathrm{mrem} / \mathrm{yr}$. Scenario A is approved for sites in Mercury or within $500 \mathrm{ft}$ of an active building. Scenario B is approved for all other sites. Scenarios A and B are briefly described below.

For Scenario A, the future land use assumes continued industrial use of the site. This scenario addresses long-term exposure received by industrial workers exposed daily to residual levels of radionuclides in soil during an average workday outdoors on site (EPA, 1991). Scenario A parameters are based on the following:

- A worker will be outdoors at the site for a total of 2,000 hr per year (hr/yr) (250 days per year, 8 hours per day) for a duration of 25 years.

- Indoor fraction time is zero, which means that the worker is outside being exposed for the entire workday.

- The outdoor time fraction is 0.228 and is calculated by dividing the total work hours at the site per year (2,000 hr/yr) by the total number of hours in a year $(8,760 \mathrm{hr} / \mathrm{yr})$.

- Worker exposures are limited to working hours and do not include contributions from ingestion of drinking water, plant foods, meat, or fish taken from the immediate area.

For Scenario B, the future land use assumes land use restrictions with a low occupancy factor and lighter work activities at the site. The assumptions for Scenario B include the following:

- A worker will be at the site and outdoors for a total of $335 \mathrm{hr} / \mathrm{yr}$ for a duration of 25 years.

- The indoor fraction time is zero

- The outdoor time fraction is 0.038 , which is calculated by dividing the total work hours at the site per year (335 hr/yr) by the total number of hours in a year $(8,760 \mathrm{hr} / \mathrm{yr})$.

- The worker exposures are limited to working hours and do not include contributions from ingestion of drinking water, plant foods, meat or fish taken from the immediate area.

When Scenario B is selected, a Use Restriction will be included at closure that will state the use scenario and the requirement for an occupant agency or entity to re-evaluate the closure if site use changes to fit the parameters of Scenario A.

Table 6-1 lists the pathways considered for Scenarios A and B. 
Table 6-1. Summary of Pathways Considered for Scenarios A and B

\begin{tabular}{|c|c|c|}
\hline Pathway & Scenario A & Scenario B \\
\hline \hline External exposure & Yes & Yes \\
\hline Particulate inhalation & Yes & Yes \\
\hline Radon inhalation & No & No \\
\hline Ingestion of soil & Yes & Yes \\
\hline Ingestion of produce from on-site garden & No & No \\
\hline Ingestion of meat from on-site livestock & No & No \\
\hline Ingestion of milk from on-site livestock & No & No \\
\hline Ingestion of fish from on-site pond & No & No \\
\hline Ingestion of water from on-site well & No & No \\
\hline
\end{tabular}

\subsection{RESRAD Parameters}

The RESRAD User's Manual states that: "The RESRAD default parameter values were carefully selected and are realistic, although conservative, parameter values. (In most cases, use of these values will not result in underestimation of the dose or risk.) Site-specific parameters should be used whenever possible. Therefore, use of default values that significantly overestimate the dose or risk for a particular site is discouraged" (Yu et al., 2001).

Table 6-2 lists all of the RESRAD default values along with the site-specific RESRAD parameters approved for use with Scenarios A and B. A reference or reason is provided for parameters that require site-specific input. 
Table 6-2. Approved RESRAD Parameters

\begin{tabular}{|c|c|c|c|c|c|}
\hline Parameter & Units & Scenario A & Scenario B & Defaults & Reference/Rationale \\
\hline Dose Conversion Factors & & & & & Use FGR 13 Morbidity \\
\hline \multicolumn{6}{|l|}{ R02 Exposure Pathways } \\
\hline Pathway 1- External Gamma & & Active & Active & & \\
\hline Pathway 2- Inhalation & & Active & Active & & \\
\hline Pathway 3- Plant Ingestion & & Suppressed & Suppressed & & \\
\hline Pathway 4- Meat Ingestion & & Suppressed & Suppressed & & \\
\hline Pathway 5- Milk Ingestion & & Suppressed & Suppressed & & \\
\hline Pathway 6- Aquatic Foods & & Suppressed & Suppressed & & \\
\hline Pathway 7- Drinking Water & & Suppressed & Suppressed & & \\
\hline Pathway 8- Soil Ingestion & & Active & Active & & \\
\hline Pathway 9- Radon & & Suppressed & Suppressed & & \\
\hline
\end{tabular}

\begin{tabular}{|c|c|c|c|c|c|}
\hline \multicolumn{6}{|l|}{ R011 Contaminated Zone } \\
\hline Area of CZ & $\mathrm{m}^{2}$ & Site Specific & Site Specific & $1.000 \mathrm{E}+04$ & $\begin{array}{l}\text { Maximum area of contamination out to two successive sample } \\
\text { intervals below PALs. ( } 15 \mathrm{ft} \text { intervals laterally) }\end{array}$ \\
\hline Thickness of CZ & $\mathrm{m}$ & Site Specific & Site Specific & $2.000 \mathrm{E}+00$ & $\begin{array}{l}\text { Maximum identified depth plus two successive intervals below } \\
\text { PALs as identified during the site characterization. ( } 5 \mathrm{ft} \text {. } \\
\text { intervals vertically) }\end{array}$ \\
\hline Length Parallel to Aquifer Flow & $\mathrm{m}$ & not used & not used & $1.000 \mathrm{E}+02$ & Not used with the above pathway selection \\
\hline Radiation Dose Limit & $\mathrm{mrem} / \mathrm{yr}$ & 25 & 25 & $2.5 \mathrm{E}+001$ & RESRAD Default (DOE, 1993) \\
\hline Elapsed Time Since Placement of Material & yr & 0.0 & 0.0 & 0.0 & RESRAD Default \\
\hline \multicolumn{6}{|l|}{ R012 Initial Principal Radionuclide } \\
\hline $\begin{array}{l}\text { Site Specific Parent Radionuclide with half-life } \\
\text { greater than } 180 \text { days, does not include } \\
\text { naturally occurring and primordial } \\
\text { radionuclides }\end{array}$ & $\mathrm{pCi} / \mathrm{g}$ & Site Specific & Site Specific & 0.0 & The arithmetic mean plus the 95\% UCL for the site. \\
\hline
\end{tabular}


Table 6-2. Approved RESRAD Parameters

\begin{tabular}{|c|c|c|c|c|c|}
\hline Parameter & Units & Scenario A & Scenario B & Defaults & Reference/Rationale \\
\hline \multicolumn{6}{|c|}{ R013 Cover and Contaminated Zone Hydrological Data } \\
\hline Cover Depth & $\mathrm{m}$ & Site Specific & Site Specific & 0.0 & The minimum depth as identified during the site characterization \\
\hline Density of Cover Material & $\mathrm{g} / \mathrm{cm}^{3}$ & 1.5 & 1.5 & 1.5 & RESRAD Default unless site data significantly different \\
\hline Cover Depth Erosion Rate & $\mathrm{m} / \mathrm{yr}$ & $1.000 \mathrm{E}-03$ & $1.000 \mathrm{E}-03$ & $1.000 \mathrm{E}-03$ & RESRAD Default unless site data significantly different \\
\hline Density of Contaminated Zone & $\mathrm{g} / \mathrm{cm}^{3}$ & 1.5 & 1.5 & 1.5 & RESRAD Default unless site data significantly different \\
\hline Contamination Zone Erosion Rate & $\mathrm{m} / \mathrm{yr}$ & $1.000 \mathrm{E}-03$ & $1.000 \mathrm{E}-03$ & $1.000 \mathrm{E}-03$ & RESRAD Default unless site data significantly different \\
\hline Contaminated Zone Total Porosity & - & 4.000E-01 & 4.000E-01 & $4.000 \mathrm{E}-01$ & RESRAD Default unless site data significantly different \\
\hline Contaminated Zone Field Capacity & - & $2.000 \mathrm{E}-01$ & $2.000 \mathrm{E}-01$ & $2.000 \mathrm{E}-01$ & RESRAD Default unless site data significantly different \\
\hline Contaminated Zone Hydraulic Conductivity & $\mathrm{m} / \mathrm{yr}$ & $1.000 \mathrm{E}+01$ & $1.000 \mathrm{E}+01$ & $1.000 \mathrm{E}+01$ & RESRAD Default unless site data significantly different \\
\hline Contaminated Zone b Parameter & - & $5.300 \mathrm{E}+00$ & $5.300 \mathrm{E}+00$ & $5.300 \mathrm{E}+00$ & RESRAD Default unless site data significantly different \\
\hline Average Annual Wind Speed & $\mathrm{m} / \mathrm{sec}$ & Site Specific & Site Specific & $2.000 \mathrm{E}+00$ & $\begin{array}{l}\text { Data from Air Resources Laboratory } \\
\text { http://www.sord.nv.doe.gov/arlsord-1.htm }\end{array}$ \\
\hline Humidity in Air & $\mathrm{g} / \mathrm{m}^{3}$ & not used & not used & $8.000 \mathrm{E}+00$ & Not used with the above pathway selection \\
\hline Evapotranspiration Coefficient & - & $5.000 \mathrm{E}-01$ & $5.000 \mathrm{E}-01$ & $5.000 \mathrm{E}-01$ & $\begin{array}{l}\text { RESRAD Default not significant due to lack of groundwater } \\
\text { pathway }\end{array}$ \\
\hline Precipitation & $\mathrm{m} / \mathrm{yr}$ & Site Specific & Site Specific & $1.000 \mathrm{E}+00$ & $\begin{array}{l}\text { Data from Air Resources Laboratory } \\
\text { http://www.sord.nv.doe.gov/arlsord-1.htm }\end{array}$ \\
\hline Irrigation & $\mathrm{m} / \mathrm{yr}$ & 0 & 0 & $2.000 \mathrm{E}-01$ & Assumes no artificial supply of water to soil \\
\hline Irrigation Mode & - & overhead & overhead & overhead & RESRAD Default \\
\hline Runoff Coefficient & - & $4.000 \mathrm{E}-01$ & $4.000 \mathrm{E}-01$ & $2.000 \mathrm{E}-01$ & $\begin{array}{l}\text { Open Sandy Loam 30\% impervious Table } 10.1 \text { (Yu, et. al., } \\
\text { 1993) }\end{array}$ \\
\hline Watershed Area for Nearby Stream or Pond & $\mathrm{m}^{2}$ & not used & not used & $1.000 \mathrm{E}+06$ & Not used with the above pathway selection \\
\hline Accuracy for Water/Soil Computations & - & not used & not used & $1.000 \mathrm{E}-03$ & Not used with the above pathway selection \\
\hline
\end{tabular}


Table 6-2. Approved RESRAD Parameters

\begin{tabular}{|c|c|c|c|c|c|}
\hline Parameter & Units & Scenario A & Scenario B & Defaults & Reference/Rationale \\
\hline \multicolumn{6}{|l|}{ R014 Saturated Zone Hydrological Data } \\
\hline Density of Saturated Zone & $\mathrm{g} / \mathrm{cm}^{3}$ & not used & not used & $1.500 \mathrm{E}+00$ & Not used with the above pathway selection \\
\hline Saturated Zone Total Porosity & - & not used & not used & $4.000 \mathrm{E}-01$ & Not used with the above pathway selection \\
\hline Saturated Zone Effective Porosity & - & not used & not used & $2.000 \mathrm{E}-01$ & Not used with the above pathway selection \\
\hline Saturated Zone Field Capacity & - & not used & not used & $2.000 \mathrm{E}-01$ & Not used with the above pathway selection \\
\hline Saturated Zone Hydraulic Conductivity & $\mathrm{m} / \mathrm{yr}$ & not used & not used & $1.000 \mathrm{E}+02$ & Not used with the above pathway selection \\
\hline Saturated Zone Hydraulic Gradient & - & not used & not used & $2.000 \mathrm{E}-02$ & Not used with the above pathway selection \\
\hline Saturated Zone b Parameter & - & not used & not used & $5.300 \mathrm{E}+00$ & Not used with the above pathway selection \\
\hline Water Table Drop Rate & $\mathrm{m} / \mathrm{yr}$ & not used & not used & $1.000 \mathrm{E}-03$ & Not used with the above pathway selection \\
\hline Well Pump Intake Depth & $\mathrm{m}$ & not used & not used & $1.000 \mathrm{E}+01$ & Not used with the above pathway selection \\
\hline Model: Nondispersion or Mass-Balance & - & ND & ND & ND & RESRAD Default \\
\hline Well Pumping Rate & $\mathrm{m}^{3} / \mathrm{yr}$ & not used & not used & $2.500 \mathrm{E}+02$ & Not used with the above pathway selection \\
\hline \multicolumn{6}{|c|}{ R015 Uncontaminated and Unsaturated Strata Hydrological Data } \\
\hline Number of Unsaturated Zone Strata & - & not used & not used & 1 & Not used with the above pathway selection \\
\hline Thickness & $\mathrm{m}$ & not used & not used & $4.000 \mathrm{E}+00$ & Not used with the above pathway selection \\
\hline Soil Density & $\mathrm{g} / \mathrm{cm}^{3}$ & not used & not used & $1.500 \mathrm{E}+00$ & Not used with the above pathway selection \\
\hline Total Porosity & - & not used & not used & $4.000 \mathrm{E}-01$ & Not used with the above pathway selection \\
\hline Effective Porosity & - & not used & not used & $2.000 \mathrm{E}-01$ & Not used with the above pathway selection \\
\hline Field Capacity & - & not used & not used & $2.000 \mathrm{E}-01$ & Not used with the above pathway selection \\
\hline Soil-specific b Parameter & - & not used & not used & $5.300 \mathrm{E}+00$ & Not used with the above pathway selection \\
\hline Hydraulic Conductivity & $\mathrm{m} / \mathrm{yr}$ & not used & not used & $1.000 \mathrm{E}+01$ & Not used with the above pathway selection \\
\hline
\end{tabular}


Table 6-2. Approved RESRAD Parameters

\begin{tabular}{|c|c|c|c|c|c|}
\hline Parameter & Units & Scenario A & Scenario B & Defaults & Reference/Rationale \\
\hline \multicolumn{6}{|c|}{ R016 Distribution Coefficients and Leach Rates } \\
\hline Contaminated Zone $\mathrm{K}_{\mathrm{d}}$ (all Zones) & $\mathrm{cm}^{3} / \mathrm{g}$ & & & & RESRAD Defaults \\
\hline Saturated Leach Rate & /yr & 0.0 & 0.0 & 0.0 & Not used \\
\hline Solubility Constant & - & 0.0 & 0.0 & 0.0 & Not used \\
\hline \multicolumn{6}{|l|}{ R017 Inhalation and External Gamma } \\
\hline Inhalation Rate & $\mathrm{m}^{3} / \mathrm{yr}$ & $8.400 \mathrm{E}+03$ & $1.230 \mathrm{E}+04$ & $8.400 \mathrm{E}+03$ & $\begin{array}{l}\text { RESRAD Default and for an individual performing outdoor } \\
\text { activities, a typical activity mix can consist of } 37 \% \text { at a moderate } \\
\text { activity level, } 28 \% \text { at both resting and light activity levels, and } \\
7 \% \text { at a heavy activity level, which results in a } 1.4 \mathrm{~m} 3 / \mathrm{h}(12,300 \\
\text { m3/yr) inhalation rate. } \\
\text { (Yu, et. al., 1993) }\end{array}$ \\
\hline Mass Loading for Inhalation & $\mathrm{g} / \mathrm{m}^{3}$ & $6.00 \mathrm{E}-04$ & $6.00 \mathrm{E}-04$ & $1 \mathrm{E}-04$ & $\begin{array}{l}\text { The estimated mass loading for construction activities. (Yu, et. } \\
\text { al., 1993) }\end{array}$ \\
\hline Exposure Duration & yr & 25 & 25 & 30 & Standard for Industrial/Commercial Scenario \\
\hline Shielding Factor Inhalation & - & 1 & 1 & 0.4 & Assumes no indoor time fraction. \\
\hline Shielding Factor External Gamma & - & 1 & 1 & 0.7 & Assumes no indoor time fraction. \\
\hline Fraction of Time Spent Indoors & - & 0.0 & 0.0 & 0.5 & Assumes no indoor time fraction. \\
\hline Fraction of Time Spent Outdoors & - & 0.228 & 0.038 & 0.25 & $\begin{array}{l}\text { Based on Industrial/Commercial use scenarios for standard } \\
\text { occupancy and low occupancy. }\end{array}$ \\
\hline Shape Factor & - & 1.0 & 1.0 & 1.0 & RESRAD Default \\
\hline
\end{tabular}


Table 6-2. Approved RESRAD Parameters

\begin{tabular}{|c|c|c|c|c|c|}
\hline Parameter & Units & Scenario A & Scenario B & Defaults & Reference/Rationale \\
\hline \multicolumn{6}{|c|}{ R018 Ingestion Pathway Data, Dietary Parameters } \\
\hline Fruits, Vegetables, and Grain Consumption & $\mathrm{kg} / \mathrm{yr}$ & not used & not used & $1.600 \mathrm{E}+02$ & Not used with the above pathway selection \\
\hline Leafy Vegetable Consumption & $\mathrm{kg} / \mathrm{yr}$ & not used & not used & $1.400 \mathrm{E}+01$ & Not used with the above pathway selection \\
\hline Milk Consumption & $\mathrm{L} / \mathrm{yr}$ & not used & not used & $9.200 \mathrm{E}+01$ & Not used with the above pathway selection \\
\hline Meat and Poultry Consumption & $\mathrm{kg} / \mathrm{yr}$ & not used & not used & $6.300 \mathrm{E}+01$ & Not used with the above pathway selection \\
\hline Fish Consumption & $\mathrm{kg} / \mathrm{yr}$ & not used & not used & $5.400 \mathrm{E}+00$ & Not used with the above pathway selection \\
\hline Other Seafood Consumption & $\mathrm{kg} / \mathrm{yr}$ & not used & not used & $9.000 \mathrm{E}-01$ & Not used with the above pathway selection \\
\hline Soil Ingestion Rate & $\mathrm{g} / \mathrm{yr}$ & $1.752 \mathrm{E}+02$ & $1.752 \mathrm{E}+02$ & 36.5 & 480 mg/day (EPA, 1991) \\
\hline Drinking Water Intake & $\mathrm{L} / \mathrm{yr}$ & not used & not used & $5.100 \mathrm{E}+02$ & Not used with the above pathway selection \\
\hline Drinking Water Contaminated Fraction & - & not used & not used & $1.000 \mathrm{E}+00$ & Not used with the above pathway selection \\
\hline Household Water Contaminated Fraction & - & not used & not used & $1.000 \mathrm{E}+00$ & Not used with the above pathway selection \\
\hline Livestock Water Contaminated Fraction & - & not used & not used & $1.000 \mathrm{E}+00$ & Not used with the above pathway selection \\
\hline Irrigation Water Contaminated Fraction & - & not used & not used & $1.000 \mathrm{E}+00$ & Not used with the above pathway selection \\
\hline Aquatic Food Contamination Fraction & - & not used & not used & $5.000 \mathrm{E}-01$ & Not used with the above pathway selection \\
\hline Plant Food Contamination Fraction & - & not used & not used & -1 & Not used with the above pathway selection \\
\hline Meat Contamination Fraction & - & not used & not used & -1 & Not used with the above pathway selection \\
\hline Milk Contamination Fraction & - & not used & not used & -1 & Not used with the above pathway selection \\
\hline \multicolumn{6}{|l|}{ R019 Ingestion Pathway Data, Nondietary } \\
\hline Livestock Fodder Intake for Meat & kg/day & not used & not used & $6.800 \mathrm{E}+01$ & Not used with the above pathway selection \\
\hline Livestock Fodder Intake for Milk & kg/day & not used & not used & $5.500 \mathrm{E}+01$ & Not used with the above pathway selection \\
\hline Livestock Water Intake for Meat & L/day & not used & not used & $5.000 \mathrm{E}+01$ & Not used with the above pathway selection \\
\hline Livestock Water Intake for Milk & L/day & not used & not used & $1.600 \mathrm{E}+02$ & Not used with the above pathway selection \\
\hline
\end{tabular}


Table 6-2. Approved RESRAD Parameters

\begin{tabular}{|c|c|c|c|c|c|}
\hline Parameter & Units & Scenario A & Scenario B & Defaults & Reference/Rationale \\
\hline Livestock Soil Intake & $\mathrm{kg} / \mathrm{day}$ & not used & not used & $5.000 \mathrm{E}-01$ & Not used with the above pathway selection \\
\hline Mass Loading for Foliar Deposition & $\mathrm{g} / \mathrm{m}^{3}$ & not used & not used & $1.000 \mathrm{E}-04$ & Not used with the above pathway selection \\
\hline Depth of Soil Mixing layer & $\mathrm{m}$ & not used & not used & $1.500 \mathrm{E}-01$ & Not used with the above pathway selection \\
\hline Depth of Roots & $\mathrm{m}$ & not used & not used & $9.000 \mathrm{E}-01$ & Not used with the above pathway selection \\
\hline Drinking Water Fraction from Groundwater & - & not used & not used & $1.000 \mathrm{E}+00$ & Not used with the above pathway selection \\
\hline Household Water Fraction from Groundwater & - & not used & not used & $1.000 \mathrm{E}+00$ & Not used with the above pathway selection \\
\hline Livestock Water Fraction from Groundwater & - & not used & not used & $1.000 \mathrm{E}+00$ & Not used with the above pathway selection \\
\hline Irrigation Fraction from Groundwater & - & not used & not used & $1.000 \mathrm{E}+00$ & Not used with the above pathway selection \\
\hline \multicolumn{6}{|l|}{ R021 Radon } \\
\hline Radon Parameters Not Used & & & & & Not used with the above pathway selection \\
\hline
\end{tabular}




\subsection{Residual Radioactive Material Guidelines}

The residual radioactive material guideline represents the concentration of residual radioactive material that can remain in place and still allow use of that area without radiological restrictions. Using site-specific parameters and sample analysis results, the radioactive material guideline, $\mathrm{G}$, can be calculated for a given dose limit of $\mathrm{H}_{\mathrm{EL}}$ for an individual as follows;

$$
\mathrm{G}=\mathrm{H}_{\mathrm{EL}} / \mathrm{DSR} \text {, }
$$

where DSR is the total dose/source concentration ratio. The dose limit $\mathrm{H}_{\mathrm{EL}}$, used to derive the residual radioactive material guideline is $25 \mathrm{mrem} / \mathrm{yr}$.

Single radionuclide guidelines are calculated for individual radionuclides such that the annual dose to industrial/construction workers at the site should not exceed an annual dose limitation of $25 \mathrm{mrem} / \mathrm{yr}$. Sites contaminated with two or more radionuclides (i.e., a mixture of radionuclides) require further evaluation to ensure that collective exposures from individual radionuclides do not exceed the $25 \mathrm{mrem} / \mathrm{yr}$ annual dose constraint. This evaluation is performed using a sum of the fractions method. The initial soil concentration of each radionuclide is divided by the single radionuclide guideline for that radionuclide to produce a ratio. These ratios are then summed. If the sum is less than or equal to unity, then the collective annual dose from all radionuclides at the site should not exceed the $25 \mathrm{mrem} / \mathrm{yr}$ annual dose constraint. If the sum does exceed unity, the annual dose to industrial/construction workers could exceed the $25 \mathrm{mrem} / \mathrm{yr}$ dose constraint, even if the concentrations of residual radionuclides at the site are below the single radionuclide guideline values. For sites where the sum of the ratios exceeds unity, residual radioactive material guidelines for mixtures of radionuclides are calculated such that the following equation is satisfied;

$$
\overline{\mathrm{M}}=\sum_{\mathrm{i}} \overline{\mathrm{S}}_{\mathrm{i}}(0) / \mathrm{G}_{\mathrm{i}}\left(\mathrm{t}_{\mathrm{m}}\right) \leq 1
$$

Where: $\quad \mathrm{M}(\mathrm{bar}) \quad=\quad$ average mixture sum (dimensionless)

$$
\mathrm{S}_{\mathrm{i}} \text { (bar)naught }=\quad \text { initial concentration of the ith principal radionuclide }
$$
averaged over an area determined by scenario activities

$\mathrm{G}_{\mathrm{i}}\left(\mathrm{t}_{\mathrm{m}}\right) \quad=\quad$ single radionuclide soil concentration guideline for the ith principal radionuclide at time $\mathrm{t}$ maximum.

For a site where the sum of the ratios does not exceed unity, the residual radioactive guidelines for single radionuclides are the radionuclide concentrations to be used as the FAL. For sites where the sum of the ratios exceeds unity, the residual radioactive guidelines for mixtures of radionuclides are mathematically adjusted so that the above equation is satisfied. Those adjusted values are then used as the FAL. 


\subsection{RESRAD Calculations for CAS 12-06-06, Muckpile and the Down Slope Area (Drainage and Pond)}

This section discuses the RESRAD calculations and results for CAS 12-06-06, Muckpile and the down slope area.

\subsection{Selection of RESRAD Exposure Scenario}

Scenario B was selected as the exposure scenario for the CAS 12-06-06 because of the remote location of the site. Because Scenario B parameters will be used for these calculations, a Use Restriction will be implemented at closure that will state the use scenario and the requirement for an occupant agency or entity to re-evaluate the closure if site use changes to fit the parameters of Scenario A.

\subsection{User Input Parameters}

The RESRAD default parameters that were modified for the calculations performed for CAU 383 in this report and the site-specific values entered are presented in Table 7-1, RESRAD Parameter Input Values for CAU 383. A complete list of the RESRAD default parameters and the parameters used for CAU 383 is provided in Table A.1 of Attachment A.

\subsection{Radionuclide Concentrations and Dose Estimates}

The maximum dose results from RESRAD calculations for the areas of Muck (98.31 mrem/yr), Drainage (2.35 mrem/yr), and Pond (8.33 mrem/yr) are evaluated and the most conservative results, i.e., Muck) is selected to represent this CAS. The detailed RESRAD results for all three portions of this CAS are provided in Exhibit 1, RESRAD Summary Report: CAU 383 CAS 1206-06.

Uncertainty in the derivation of dose estimates and dose/source contribution ratios comes from the distribution of possible input parameter values, as well as uncertainty in the conceptual model used to represent the site. The pathway contributions to the total annual dose at time zero are almost all (98.45 percent) for external exposure, 1.20 percent for inhalation, and 0.34 percent for soil ingestion pathways. Therefore, uncertainties in the following parameters: Erosion rates, thickness of contaminated zone, occupancy factors, and wind speed have the greatest significance on the model predictions.

The maximum dose contributions and total dose/source concentration ratios for the muckpile under Scenario B parameters have been predicted to occur at time zero. The calculated maximum dose contributions for all considered pathways are presented in Table 7-3, Maximum Dose Contributions for CAS 12-06-06 Using Scenario B. Figure 7-1. CAU 383 CAS 12-06-06 Scenario B: Dose Rate Per Year All Radionuclides Summed, All Pathways Summed, shows that at time zero, the TEDE to industrial/construction workers for the considered pathways is 98.31 $\mathrm{mrem} / \mathrm{yr}$ which is above the $25 \mathrm{mrem} / \mathrm{yr}$ dose constraint. 
Figure 7-2. CAU 383 CAS 12-06-06 Scenario B: Annual Dose All Radionuclides Summed, Component Pathways, shows the breakdown of the total dose into the component pathways. Together, Table 7-2 and Figures 1 and 2 show that the dose from Cs-137 at time zero is 96.30 mrem/year and drops to zero after the 300-year time interval. The result also shows that the annual dose from external radiation (almost all from Cs-137) at time zero is $96.26 \mathrm{mrem} / \mathrm{year}$ and is reduced to zero within 300 years.

Because Cs-137 has a half-life of 30 years, the concentration of Cs-137 at this site will not decay to a level below the $25 \mathrm{mrem} / \mathrm{yr}$ constraint through the radioactive decay processes within the first 50-year time interval (the annual dose at year 50 is $25.41 \mathrm{mrem}$ ). Site remediation and/or controls that reduce workers exposures and minimize the spread of radioactive contamination into uncontaminated areas are recommended for this site.

\subsection{Residual Radioactive Material Guidelines for CAS 12-06-06}

The sum of the ratios for CAS 12-06-06 exceeded unity. Residual radioactive guidelines for mixtures of radionuclides were calculated for this CAS. Table 7-4, presents the calculations results for deriving guidelines for mixtures radionuclides for this CAS. The residual radioactive material guidelines for single radionuclides and mixture radionuclides are listed in Table 7-4, Residual Radioactive Material Guidelines for Single Radionuclides and Radionuclide Mixtures. The FALs for the CAS 12-06-06 scenario are the residual radioactive material guideline values for mixture radionuclides. 


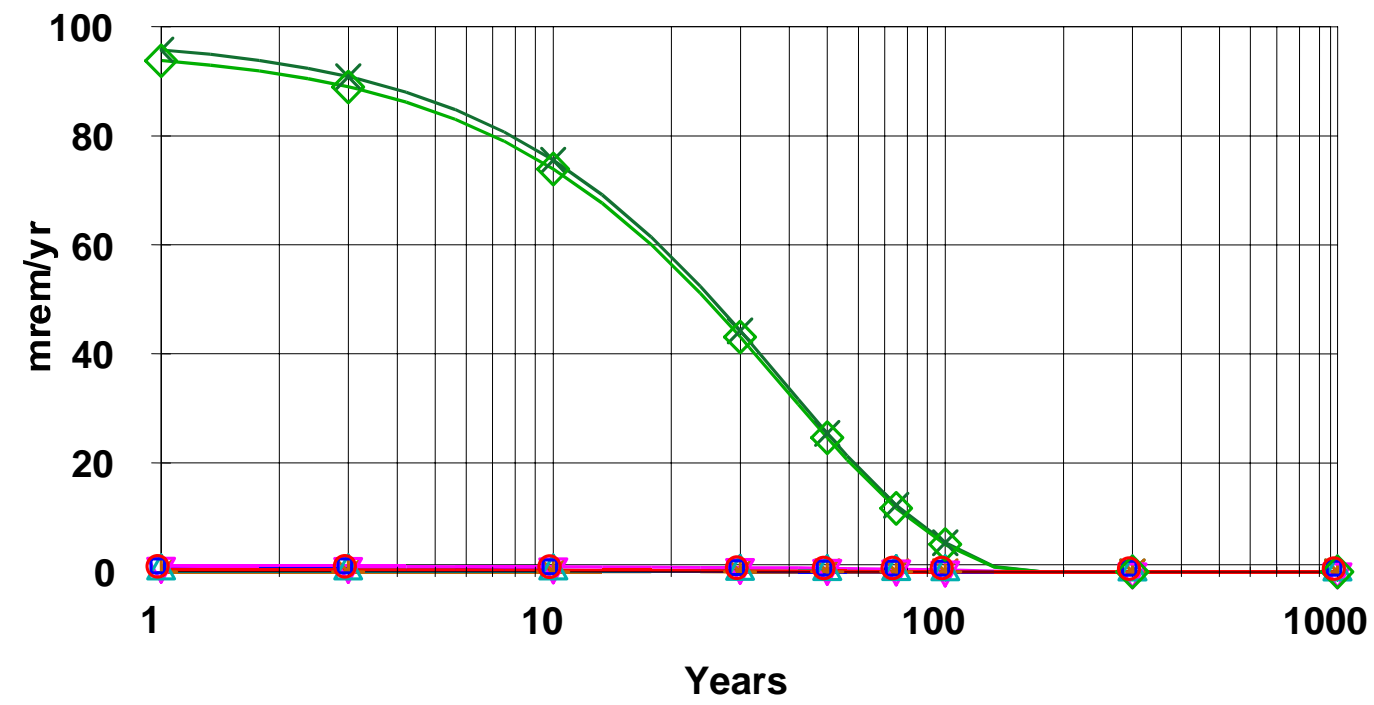

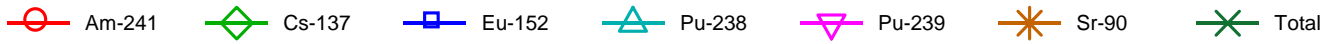

Figure 7-1. CAU 383 CAS 12-06-06, Muckpile, Scenario B: Dose Rate Per Year, All Radionuclides Summed, All Pathways Summed 

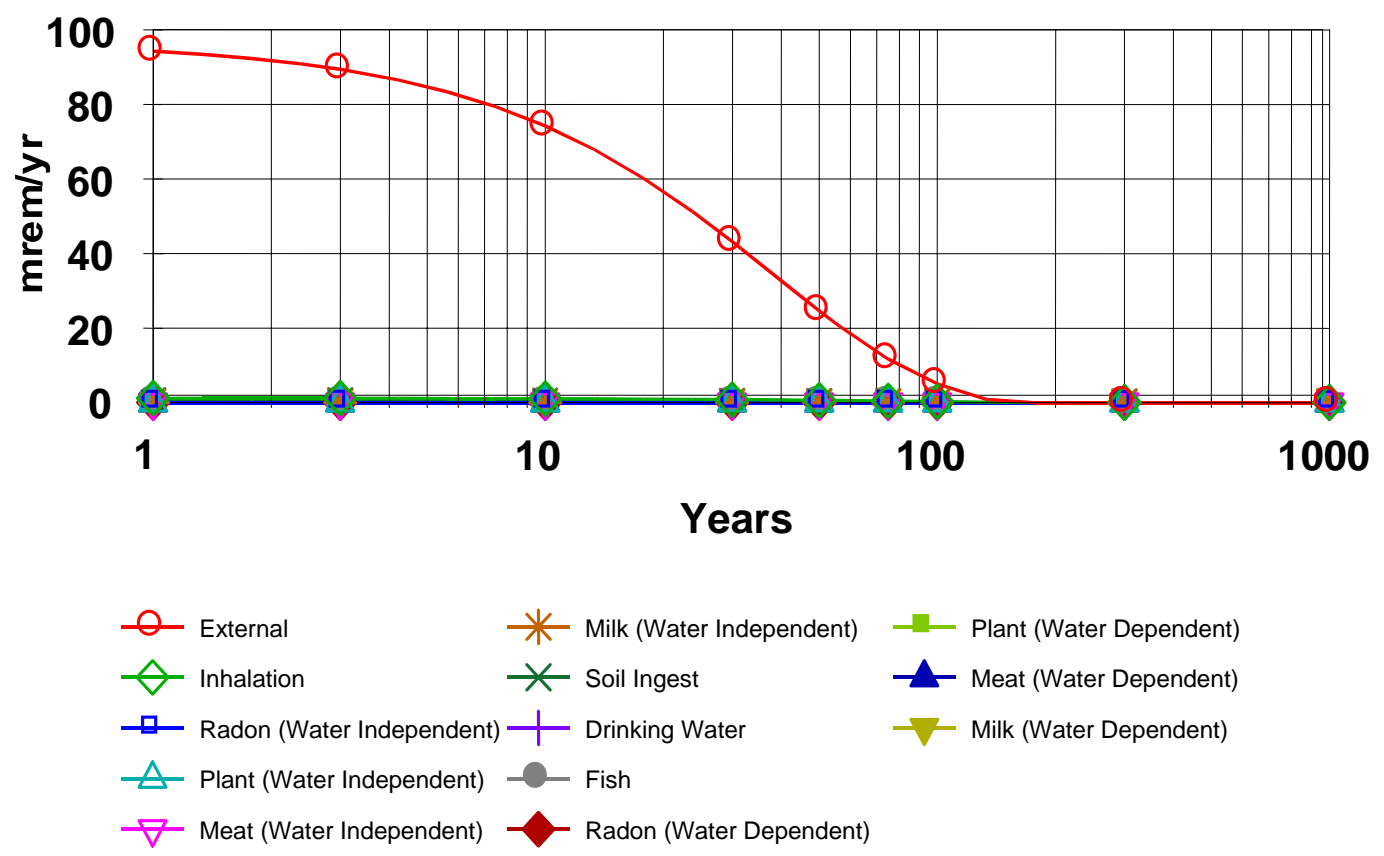

Figure 7-2. CAU 383 CAS 12-06-06, Muckpile, Scenario B: Annual Dose, All Radionuclides Summed, Component Pathways 
Table 7-2. Principal Radionuclide Concentrations Used for CAU 383 REARAD Calculations (pCi/g)

\begin{tabular}{|c|c|c|c|c|c|}
\hline & \multicolumn{3}{|c|}{ CAS 12-06-06 } & \multirow{2}{*}{ CAS 12-25-02 } & CAS 12-28-02 \\
\hline & Muck & Drainage & Pond & NA & NA \\
\hline Americium-241 & 28.8 & 1.88 & 0.72 & 20.5 & 114 \\
\hline Cicium-137 & 1060 & 25.41 & 91 & NA & NA \\
\hline Europiam-152 & 2.64 & NA & NA & NA & NA \\
\hline Strotium-90 & 34.1 & 0.97 & 3.62 & NA & 0.232 \\
\hline Plutonium-238 & 15.6 & 0.2 & 0.361 & 1.04 & 2.09 \\
\hline
\end{tabular}

Table 7-3. Maximum Dose Contributions for CAS 12-06-06* Using Scenario B (occurs at $\mathrm{t}=0)(\mathrm{mrem} / \mathrm{yr})$

\begin{tabular}{|c|c|c|c|c|c|c|c|c|}
\hline \multirow{2}{*}{ Radionuclide } & \multicolumn{2}{|c|}{ Ground } & \multicolumn{2}{|c|}{ Inhalation } & \multicolumn{2}{|c|}{ Soil } & \multicolumn{2}{|c|}{ Total } \\
\hline & $\begin{array}{l}\text { Annual } \\
\text { Dose }\end{array}$ & Fraction & $\begin{array}{l}\text { Annual } \\
\text { Dose }\end{array}$ & Fraction & $\begin{array}{c}\text { Annual } \\
\text { Dose }\end{array}$ & Fraction & $\begin{array}{l}\text { Annual } \\
\text { Dose }\end{array}$ & Fraction \\
\hline Americium-241 & 3.935E-02 & 0.0004 & 2.729E-01 & 0.0028 & 6.876E-02 & 0.0007 & 3.810E-01 & 0.0039 \\
\hline Cesium-137 & $9.626 \mathrm{E}+01$ & 0.9791 & 7.217E-04 & 0.0000 & 3.476E-02 & 0.0004 & 9.630E-01 & 0.9795 \\
\hline Europium-152 & 4.688E-01 & 0.0048 & 1.227E-05 & 0.0000 & 1.106E-05 & 0.0000 & 4.688E-01 & 0.0048 \\
\hline Plutonium-238 & 8.080E-05 & 0.0000 & 1.315E-01 & 0.0013 & 3.299E-02 & 0.0003 & 1.646E-01 & 0.0017 \\
\hline Plutonium-239 & 7.545E-04 & 0.0000 & 7.780E-01 & 0.0079 & 1.973E-02 & 0.0020 & 9.760E-01 & 0.0099 \\
\hline Strotium-90 & 2.338E-02 & 0.0002 & 9.452E-04 & 0.0000 & 3.392E-03 & 0.0000 & 2.772E-02 & 0.0003 \\
\hline Total & $9.679 \mathrm{E}+01$ & 0.9845 & 1.184E-01 & 0.0120 & 3.372E-01 & 0.0034 & $9.831 E+01$ & 1.0000 \\
\hline
\end{tabular}

*The conservative results from Muck data are used as representative for this CAS. 
Table 7-4. CAU 383 CAS 12-06-06 Sum of Fractions and Proportional Scaling

\begin{tabular}{|c|c|c|c|c|c|c|}
\hline Radionuclide & $\begin{array}{c}\text { Initial } \\
\text { Radionuclide } \\
\text { Concentration } \\
\text { (pCi/g) }\end{array}$ & $\begin{array}{c}\text { Contribution } \\
\%\end{array}$ & $\begin{array}{c}\text { Single } \\
\text { Radionuclide } \\
\text { Guidelines } \\
\text { (pCi/g) }\end{array}$ & $\begin{array}{c}\text { Ratio for } \\
\text { Single } \\
\text { Radionuclide } \\
\text { Guideline }\end{array}$ & $\begin{array}{c}\text { Mixture } \\
\text { Radionuclides } \\
\text { Guidelines }\end{array}$ & $\begin{array}{c}\text { Ratio for } \\
\text { Mixture } \\
\text { Radionuclide } \\
\text { Guidelines }\end{array}$ \\
\hline Americium-241 & 28.8 & 2.35 & 1890 & 0.02 & 7.32 & 0.00 \\
\hline Cesium-137 & 1060 & 86.52 & 275.2 & 3.85 & 269.56 & 0.98 \\
\hline Europium-152 & 2.64 & 0.22 & 140.8 & 0.02 & 0.67 & 0.00 \\
\hline Plutonium-238 & 15.6 & 1.27 & 2370 & 0.01 & 3.97 & 0.00 \\
\hline Plutonium-239 & 84 & 6.86 & 2152 & 0.04 & 21.36 & 0.01 \\
\hline Strotium-90 & 34.1 & 2.78 & 30760 & 0.00 & 8.67 & 0.00 \\
\hline Total & 1225.14 & & & & 311.55 & 1.00 \\
\hline
\end{tabular}


Table 7-5. Residual Radioactive Material Guidelines for Single Radionuclides and Radionuclide Mixtures*

\begin{tabular}{|l|c|c|c|c|}
\hline \multirow{2}{*}{ Radionuclides } & \multicolumn{2}{|c|}{$\begin{array}{c}\text { CAS 12-06-06 } \\
\text { Guidelines (pCi/g) }\end{array}$} & $\begin{array}{c}\text { CAS 12-25-02 } \\
\text { Guidelines (pCi/g) }\end{array}$ & $\begin{array}{c}\text { CAS 12-28-02 } \\
\text { Guidelines (pCi/g) }\end{array}$ \\
\cline { 2 - 5 } & $\begin{array}{c}\text { *Single } \\
\text { Radionuclide }\end{array}$ & $\begin{array}{c}\text { *Mixture } \\
\text { Radionuclide }\end{array}$ & *Single Radionuclide & *Single Radionuclide \\
\hline Amercium-241 & 1890 & 7.32 & - & - \\
\hline Cesium-137 & 275.2 & 269.56 & 275.2 & 275.2 \\
\hline Europium-152 & 140.8 & 0.67 & - & - \\
\hline Plutonium-238 & 2370 & 3.97 & - & 2370 \\
\hline Plutonium-239 & 2152 & 21.36 & 2152 & - \\
\hline Strontium-90 & 30760 & 8.67 & - & 2152 \\
\hline
\end{tabular}

*Single radionuclide guidelines apply to areas uniformly contaminated with a single radionuclide. The mixture radionuclide guidelines apply to areas uniformly contaminated with a mixture of radionuclides. The FALs for all three land parcels are the radionuclide guidelines for mixture radionuclides (i.e., Mixture Radionuclide columns). 
Table 7-1. RESRAD Parameters Input Values for CAU 383 (Page 1 of 2)

\begin{tabular}{|c|c|c|c|c|c|c|}
\hline Parameter & Units & CAS 12-06-06 & CAS 12-25-02 & CAS 12 28-02 & Defaults & Reference/Rationale \\
\hline \multicolumn{7}{|c|}{ R011 Contaminated Zone } \\
\hline Area of CZ & $\mathrm{m}^{2}$ & $1.000 \mathrm{E}+02$ & $1.000 \mathrm{E}+02$ & $1.000 \mathrm{E}+02$ & $1.000 \mathrm{E}+04$ & Estimated using the site boundary \\
\hline Thickness of CZ & $\mathrm{m}$ & 1.500E-01 & 1.500E-01 & 1.500E-01 & $2.000 \mathrm{E}+00$ & Top layer of the contamination soil \\
\hline \multicolumn{7}{|c|}{ R012 Initial Principal Radionuclide } \\
\hline Principal radionuclides & $\mathrm{pCi} / \mathrm{g}$ & See Table 7.2 & See Table 7.2 & See Table 7.2 & 0.0 & $\begin{array}{l}\text { Initial concentrations are the maximum } \\
\text { concentrations from sample results: } \\
\text { maximum for biased sample or average for } \\
\text { random sample. }\end{array}$ \\
\hline \multicolumn{7}{|c|}{ R013 Cover and Contaminated Zone Hydrological Data } \\
\hline Average Annual Wind Speed & $\mathrm{m} / \mathrm{sec}$ & 3.4 & 3.4 & 3.4 & $2.000 \mathrm{E}+00$ & Data from Air Resource Laboratory (2005) \\
\hline Precipitation & $\mathrm{m} / \mathrm{yr}$ & 3.260E-01 & 3.260E-01 & 3.260E-01 & $1.000 \mathrm{E}+00$ & Data from Air Resources Laboratory \\
\hline Runoff Coefficient & - & 4.000E-01 & 4.000E-01 & 4.000E-01 & 2.000E-01 & $\begin{array}{l}\text { Open Sandy Loam 30\% impervious Table } \\
10.1 \text { (Yu, et al., 1993) }\end{array}$ \\
\hline \multicolumn{7}{|c|}{ R017 Inhalation and External Gamma } \\
\hline Inhalation Rate & $\mathrm{m}^{3} / \mathrm{yr}$ & $1.230 \mathrm{E}+04$ & $1.230 \mathrm{E}+04$ & $1.230 \mathrm{E}+04$ & $8.400 \mathrm{E}+03$ & $\begin{array}{l}\text { RESRAD Default and for an individual } \\
\text { performing outdoor activities, a typical } \\
\text { activity mix can consist of } 37 \% \text { at a } \\
\text { moderate activity level, } 28 \% \text { at both resting } \\
\text { and light activity levels, and } 7 \% \text { at a heavy } \\
\text { activity level, which results in a } 1.4 \mathrm{~m}^{3} / \mathrm{h} \\
\left(12,300 \mathrm{~m}^{3} / \mathrm{yr} \text { ) inhalation rate. (Yu, et al., }\right. \\
1993)\end{array}$ \\
\hline Mass Loading for Inhalation & $\mathrm{g} / \mathrm{m}^{3}$ & $6.00 \mathrm{E}-04$ & $6.00 \mathrm{E}-04$ & $6.00 \mathrm{E}-04$ & 1E-04 & $\begin{array}{l}\text { The estimated mass loading for } \\
\text { construction activities. (Yu, et al., 1993) }\end{array}$ \\
\hline Exposure Duration & $\mathrm{yr}$ & 25 & 25 & 25 & 30 & $\begin{array}{l}\text { Standard for Industrial/Commercial } \\
\text { Scenario }\end{array}$ \\
\hline Shielding Factor Inhalation & - & 1.0 & 1.0 & 1.0 & 0.4 & Assumes no indoor time fraction \\
\hline
\end{tabular}


Table 7-1. RESRAD Parameters Input Values for CAU 383 (Page 1 of 2)

\section{Parameter}

Shielding Factor External

Gamma

Fraction of Time Spent Indoors

Fraction of Time Spent Outdoors

Soil Ingestion Rate
Units

CAS 12-06-06

1.0

0.0

0.038

$1.752 \mathrm{E}+02$

$\mathrm{g} / \mathrm{yr}$
CAS $12-25-02$

1.0

0.0

0.038

$1.752 \mathrm{E}+02$
CAS 12 28-02

1.0

0.0

0.038
1.752E+02
Defaults

0.7

0.5

0.25

36.5
Reference/Rationale

Assumes no indoor time fraction

Assumes no indoor time fraction

Scenario specific based on Industrial/ Commercial Use Scenarios for standard occupancy and low occupancy.

EPA, 1991; 480 mg/day 
$\mathrm{cm}^{3} / \mathrm{g}=$ Cubic centimeters per gram $\mathrm{g} / \mathrm{cm}^{3}=$ Grams per cubic centimeter $\mathrm{g} / \mathrm{m}^{3}=$ Grams per cubic meter

$\mathrm{g} / \mathrm{yr}=$ Grams per year

$\mathrm{kg} /$ day $=$ Kilograms per day

$\mathrm{kg} / \mathrm{yr}=$ Kilograms per year

L/day $=$ Liters per day

$\mathrm{L} / \mathrm{yr}=\mathrm{Liters}$ per year

$\mathrm{m}=$ Meter

$\mathrm{m}^{2}=$ Square meter

$\mathrm{m}^{2}=$ Square meter

$\mathrm{m} / \mathrm{sec}=$ Meters per second

$\mathrm{m} / \mathrm{yr}=$ Meters per year

$\mathrm{m}^{3} / \mathrm{h}=$ Cubic meters per hour

$\mathrm{m}^{3} / \mathrm{yr}=$ Cubic meters per year

$\mathrm{mrem} / \mathrm{yr}=$ Millirem per year

N/A = Not applicable

$\mathrm{pCi} / \mathrm{g}=$ Picocuries per gram

$\mathrm{yr}=$ Year

/yr = Per year

UCL = Upper confidence level 


\subsection{RESRAD Calculations for CAS 12-25-02, Oil Spill}

This section discuses the RESRAD calculations and results for CAS 12-25-02, Oil Spill.

\subsection{Selection of RESRAD Exposure Scenario}

Scenario B was selected as the exposure scenario for the CAS 12-25-02 because of its remote location. Because Scenario B parameters will be used for these calculations, a Use Restriction will be implemented at closure that will state the use scenario and the requirement for an occupant agency or entity to re-evaluate the closure if site use changes to fit the parameters of Scenario A.

\subsection{User Input Parameters}

The RESRAD default parameters that were altered for the calculations in this report and the sitespecific values entered are presented in Table 7-1, RESRAD Parameters Input Values for CAU 383. A complete list of the RESRAD default parameters and the parameters used for CAU 383 is provided in Table A.1 of Attachment A.

\subsection{Radionuclide Concentrations and Dose Estimates for CAS 12-25-02}

Uncertainty in the derivation of dose estimates and dose/source contribution ratios comes from the distribution of possible input parameter values, as well as uncertainty in the conceptual model used to represent the site. The pathway contributions to the total annual dose at time zero are 99.32 percent for external exposure, 0.51 percent for inhalation, and 0.17 percent for soil ingestion pathways. Therefore, uncertainties in the following parameters: Erosion rates, thickness of contaminated zone, occupancy factors, and wind speed have the greatest significance on the model predictions. The RESRAD results for this CAS do not include the effects of soil migration (contaminated or clean) into the site from the adjacent land areas. The input parameters entered into the RESRAD code for this analysis are listed in Exhibit 2, RESRAD Summary Report: CAU 383 CAS 12-25-02.

The maximum dose contributions and total dose/source concentration ratios for CAS 12-25-02 under Scenario B parameters have been predicted to occur at time zero. The calculated maximum dose contributions for all considered pathways are presented in Table 8-1, Maximum Dose Contributions for CAS 12-25-02 Using Scenario B. and Figure 8-1. CAU 383 CAS 12-2502 Scenario B: Dose Rate Per Year All Radionuclides Summed, All Pathways Summed, shows that at time zero, the TEDE to industrial/construction workers for the considered pathways is $1.87 \mathrm{mrem} / \mathrm{yr}$, which is below the $25 \mathrm{mrem} / \mathrm{yr}$ dose constraint.

Figure 8-2. CAU 383 CAS 12-25-02 Scenario B: Annual Dose All Radionuclides Summed, Component Pathways, shows the breakdown of the total dose into the component pathways. Together, Table 8-1 and Figures 8-1 and 8-2 show that at time zero, the annual dose from Pu-239 is $1.21 \mathrm{E}-02 \mathrm{mrem} / \mathrm{yr}(0.64$ percent of the total dose), and Cs-137 is $1.86 \mathrm{mrem} / \mathrm{yr}$ (99.36 percent of the total dose). 
This result also shows that the annual dose at time zero for external radiation is $1.86 \mathrm{mrem} / \mathrm{year}$, for inhalation 9.65E-03 mrem/yr, and for ingestion of soil 2.12E-03 mrem/yr. The calculated annual dose to industrial/construction workers for CAS 12-25-02 will fall to zero within 300 years.

As best management practices, site remediation and/or controls that reduce workers exposures and minimize the spread of radioactive contamination into uncontaminated areas are recommended for this site. As contamination from adjacent land areas erodes, the radionuclide concentrations within this land parcel are potentially to increase over time. However, there is insufficient data to derive dose estimates for contamination in such a case. It is recommended that controls be implemented to minimize the spread of contamination from the muckpiles into uncontaminated areas and to prevent further contamination of CAS 12-25-02.

\subsection{Residual Radioactive Material Guidelines for CAS 12-25-02}

The sum of the ratios for CAS 12-25-02 did not exceed unity. Residual radioactive guidelines for mixtures of radionuclides were not calculated for this CAS. Table 8-2, presents the calculations results for deriving guidelines for single radionuclides for this CAS. The residual radioactive material guidelines for single radionuclides are listed in Table 7-3, Residual Radioactive Material Guidelines for Single Radionuclides and Radionuclide Mixtures. The FALs for the CAS 12-25-02 scenario are the residual radioactive material guideline values for single radionuclides 
Table 8-1. Maximum Dose Contributions for CAS 12-25-02 Using Scenario B (occurs at $\mathrm{t}=0)(\mathrm{mrem} / \mathrm{yr})$

\begin{tabular}{|l|c|c|c|c|c|c|c|c|}
\hline \multirow{2}{*}{ Radionuclide } & \multicolumn{2}{|c|}{ Ground } & \multicolumn{2}{c|}{ Inhalation } & \multicolumn{2}{c|}{ Soil } & \multicolumn{2}{c|}{ Total } \\
\cline { 2 - 9 } & $\begin{array}{c}\text { Annual } \\
\text { Dose }\end{array}$ & Fraction & $\begin{array}{c}\text { Annual } \\
\text { Dose }\end{array}$ & Fraction & $\begin{array}{c}\text { Annual } \\
\text { Dose }\end{array}$ & Fraction & $\begin{array}{c}\text { Annual } \\
\text { Dose }\end{array}$ & Fraction \\
\hline \hline Cesium-137 & $1.862 \mathrm{E}+00$ & 0.9932 & $1.396 \mathrm{E}-05$ & 0.0000 & $6.723 \mathrm{E}-04$ & 0.0004 & $1.862 \mathrm{E}+00$ & 0.9936 \\
\hline Plutonium-239 & $9.341 \mathrm{E}-06$ & 0.0000 & $9.646 \mathrm{E}-03$ & 0.0051 & $2.443 \mathrm{E}-03$ & 0.0013 & $1.208 \mathrm{E}-02$ & 0.0064 \\
\hline Total & $1.862 \mathrm{E}+00$ & 0.9932 & $9.646 \mathrm{E}-03$ & 0.0051 & $3.115 \mathrm{E}-03$ & 0.0017 & $1.874 \mathrm{E}+00$ & 1.0000 \\
\hline
\end{tabular}

Table 8-2. CAU 383 CAS 12-25-02 Sum of Fractions and Proportional Scaling

\begin{tabular}{|c|c|c|c|c|}
\hline Radionuclide & $\begin{array}{c}\text { Initial } \\
\text { Radionuclide } \\
\text { Concentration } \\
\text { (pCi/g) }\end{array}$ & Contribution & $\begin{array}{c}\text { Single } \\
\text { Radionuclide } \\
\text { Guidelines } \\
\text { (pCi/g) }\end{array}$ & $\begin{array}{c}\text { Ratio for Single } \\
\text { Radionuclide } \\
\text { Guideline }\end{array}$ \\
\hline Cesium-137 & 20.5 & 0.95 & 275.2 & 0.07 \\
\hline $\begin{array}{c}\text { Plutonium- } \\
239\end{array}$ & 1.04 & 0.05 & 2152 & 0.00 \\
\hline Total & 21.54 & & & 0.07 \\
\hline
\end{tabular}




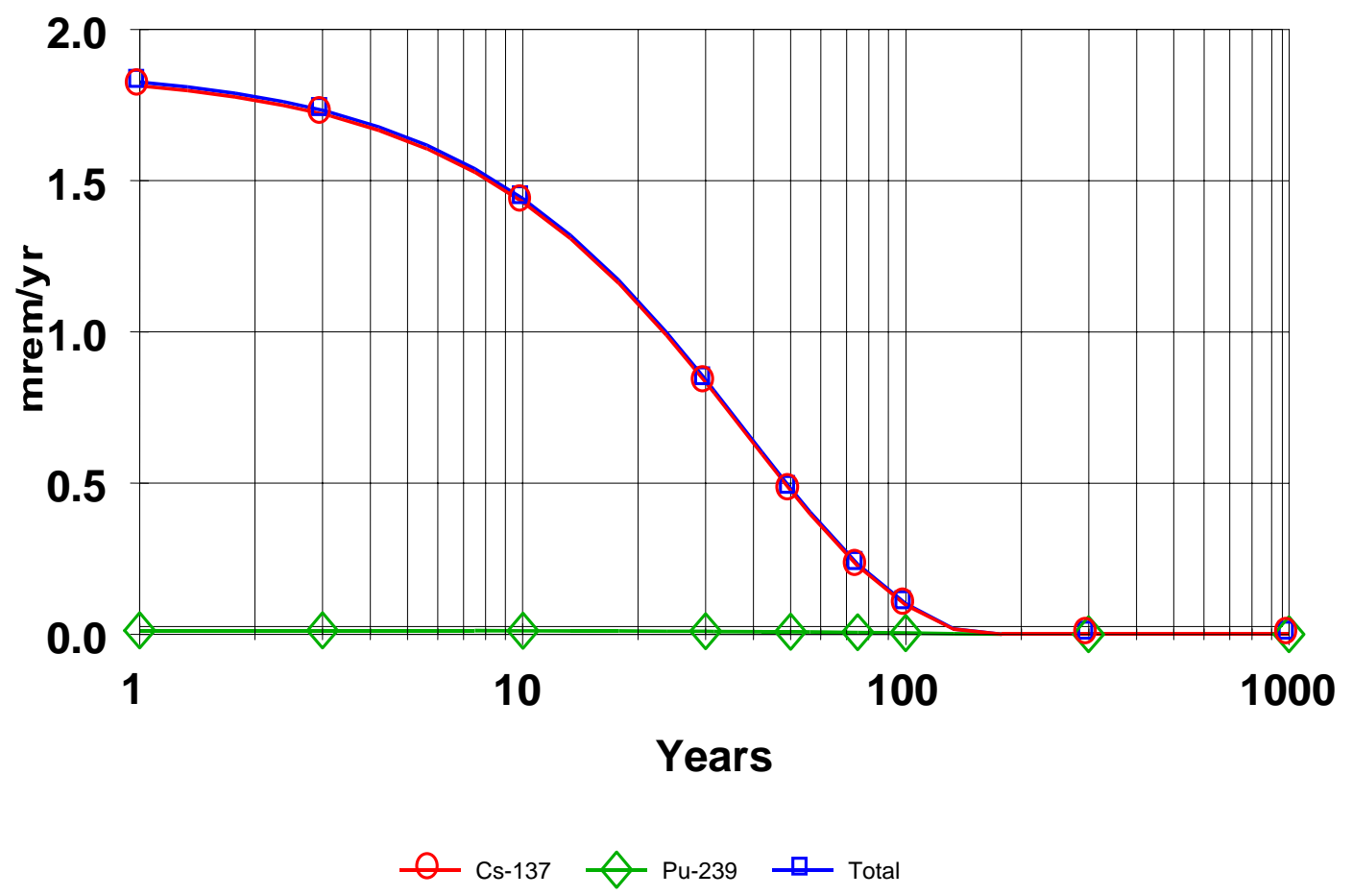

Figure 8-1. CAU 383 CAS 12-25-02, Oil Spill, Scenario B: Dose Rate Per Year, All Radionuclides Summed, All Pathways Summed 

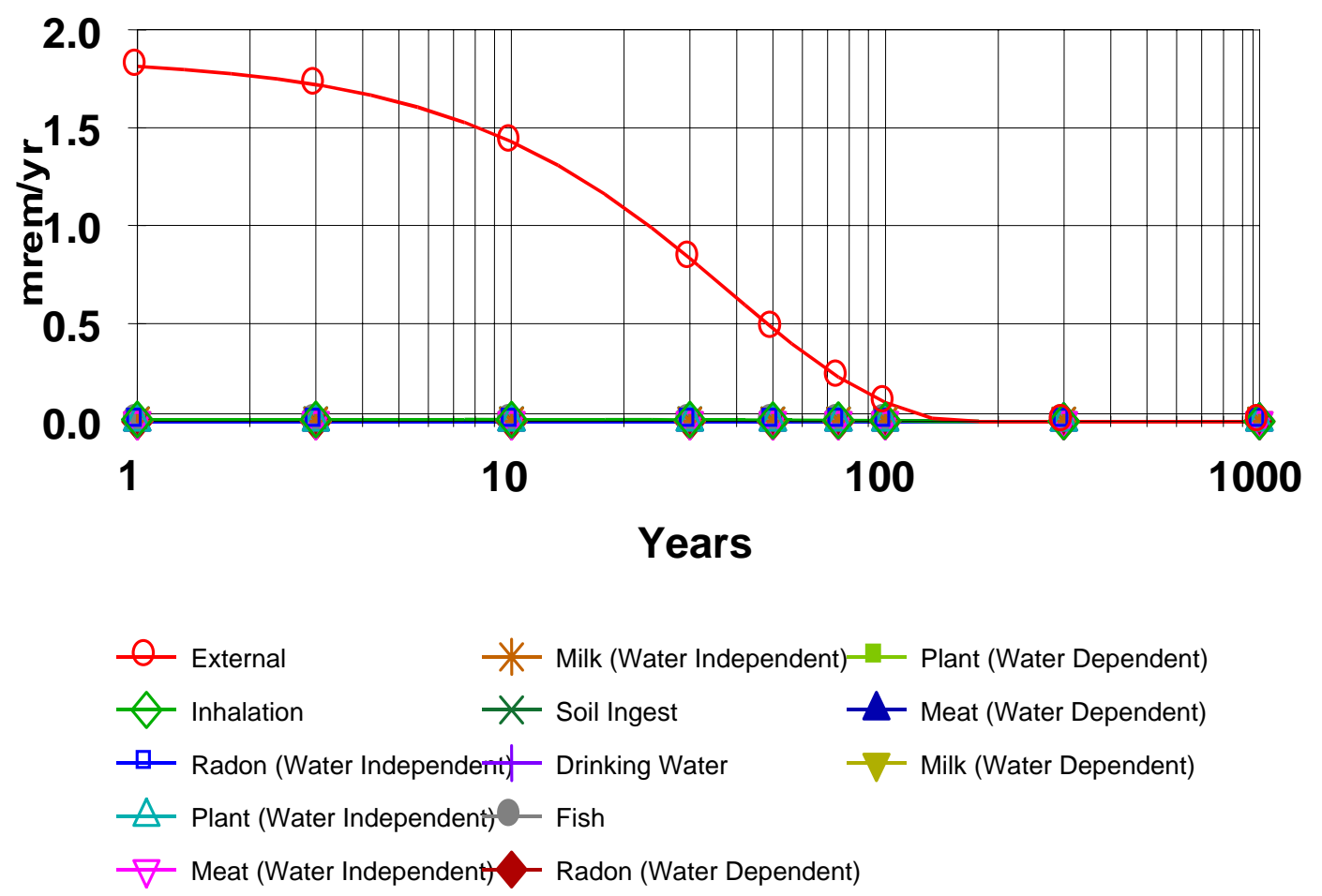

Figure 8-2. CAU 383 CAS 12-25-02, Oil Spill, Scenario B: Annual Dose, All Radionuclides Summed, Component Pathways 


\subsection{RESRAD Calculations for CAS 12-28-02, Radioactive Material Area}

This section discuses the RESRAD calculations and results for the CAS 12-28-02, Radioactive Material Area.

\subsection{Selection of RESRAD Exposure Scenario}

Scenario B was selected as the exposure scenario for the CAS 12-28-02 because of the remote location of the site. Because Scenario B parameters will be used for these calculations, a Use Restriction will be implemented at closure that will state the use scenario and the requirement for an occupant agency or entity to re-evaluate the closure if site use changes to fit the parameters of Scenario A.

\subsection{User Input Parameters}

The RESRAD default parameters that were altered for the calculations in this report and the sitespecific values entered are presented in Table 7-1 RESRAD Parameters Input Values for CAU 383. A complete list of the RESRAD default parameters and the parameters used for CAU 383 is provided in Table A.1 of Attachment A.

\subsection{Radionuclide Concentrations and Dose Estimates for CAS 12-28-02}

The RESRAD results for this CAS are only slightly different from the results for CAS 12-06-06. This evaluation applied the radionuclide concentrations for the muckpiles all of the areas investigated under this CAU. These land areas include both the CAS 12-06-06 and CAS 12-2502 areas.

Uncertainty in the derivation of dose estimates and dose/source contribution ratios comes from the distribution of possible input parameter values, as well as uncertainty in the conceptual model used to represent the site. The pathway contributions to the total annual dose at time zero are 99.71 percent for external exposure, 0.21 percent for inhalation, and 0.09 percent for soil ingestion pathways. Therefore, uncertainties in the following parameters: Erosion rates, thickness of contaminated zone, occupancy factors, and wind speed have the greatest significance on the model predictions. This CAS is located within the CAS-12-06-06 Muckpile area. However, the migration of radionuclides from muckpile material into this CAS is not considered in the calculations. The detailed results for this RESRAD exposure scenario are provided in Exhibit 3, RESRAD Summary Report: CAU 383 CAS 12-28-02.

The maximum dose contributions and total dose/source concentration ratios for CAS 12-28-02 using Scenario B parameters have been predicted to occur at time zero. The calculated maximum dose contributions for all considered pathways are presented in Table 9-1, Maximum Dose Contributions for CAS 12-28-02 Using Scenario B and Figure 9-1. The CAU 383 CAS 1228-02 Scenario B: Dose Rate Per Year All Radionuclides Summed, All Pathways Summed, shows that at time zero, the TEDE to industrial/construction workers for the considered pathways is $10.38 \mathrm{mrem} / \mathrm{yr}$ and that the annual dose rate drops to zero at the 300 -year interval. 
Figure 9-2. CAU 383 CAS 12-28-02 Scenario B: Annual Dose All Radionuclides Nuclides

Summed, Component Pathways shows the breakdown of the total does into the component pathways. Together, Table 9-1 and Figures 9-1 and 9-2 show that the dose from Cs-137 at time zero is $10.36 \mathrm{mrem} /$ year and only drops to zero after the 300 -year time interval. This result also shows that the annual dose from external radiation (almost all from Cs-137) at time zero is 10.35 $\mathrm{mrem} / \mathrm{year}$ and is reduced to zero mrem/yr within 300 years.

Because Cs-137 has a half-life of 30 years, the concentration of CS-137 at this site will not decay to a minute level through the radioactive decay processes within the 300-year time interval. As a best management practice, site remediation or controls that reduce worker exposures and minimize the spread of radioactive contamination into uncontaminated areas is recommended for this site.

\subsection{Residual Radioactive Material Guidelines for CAS 12-28-02}

The sum of the ratios for CAS 12-28-02 did not exceed unity. Residual radioactive guidelines for mixtures of radionuclides were not calculated for this CAS. Table 9-2, presents the calculation results for deriving guidelines for single radionuclides for this CAS. The residual radioactive material guidelines for single radionuclides are listed in Table 7-3, Residual Radioactive Material Guidelines for Single Radionuclides and Radionuclide Mixtures. The FALs for the CAS 12-28-02 scenario are the residual radioactive material guideline values for single radionuclides. 
Table 9-1. Maximum Dose Contributions for CAS 12-28-02 Using Scenario B (occurs at $\mathrm{t}=0)(\mathrm{mrem} / \mathrm{yr})$

\begin{tabular}{|c|c|c|c|c|c|c|c|c||}
\hline \multirow{2}{*}{ Radionuclide } & \multicolumn{2}{|c|}{ Ground } & \multicolumn{2}{c|}{ Inhalation } & \multicolumn{2}{c|}{ Soil } & \multicolumn{3}{c|}{ Total } \\
\cline { 2 - 9 } & $\begin{array}{c}\text { Annual } \\
\text { Dose }\end{array}$ & Fraction & $\begin{array}{c}\text { Annual } \\
\text { Dose }\end{array}$ & Fraction & $\begin{array}{c}\text { Annual } \\
\text { Dose }\end{array}$ & Fraction & $\begin{array}{c}\text { Annual } \\
\text { Dose }\end{array}$ & Fraction \\
\hline Cesium-137 & $1.035 \mathrm{E}+01$ & 0.9971 & $7.761 \mathrm{E}-05$ & 0.0000 & $3.739 \mathrm{E}-03$ & 0.0004 & $1.036 \mathrm{E}+01$ & 0.9974 \\
\hline Plutonium-238 & $1.202 \mathrm{E}-06$ & 0.0000 & $1.956 \mathrm{E}-03$ & 0.0002 & $4.906 \mathrm{E}-04$ & 0.0000 & $2.447 \mathrm{E}-03$ & 0.0002 \\
\hline Plutonium-239 & $1.877 \mathrm{E}-05$ & 0.0000 & $1.936 \mathrm{E}-02$ & 0.0019 & $4.909 \mathrm{E}-03$ & 0.0005 & $2.428 \mathrm{E}-02$ & 0.0023 \\
\hline Total & $1.035 \mathrm{E}+01$ & 0.9971 & $2.139 \mathrm{E}-02$ & 0.0021 & $9.138 \mathrm{E}-03$ & 0.0009 & $1.038 \mathrm{E}+01$ & 1.0000 \\
\hline
\end{tabular}

Table 9-2. CAU 383 CAS 12-28-02 Sum of Fractions and Proportional Scaling

\begin{tabular}{|c|c|c|c|c|}
\hline Radionuclide & $\begin{array}{c}\text { Initial } \\
\text { Radionuclide } \\
\text { Concentration } \\
\text { (pCi/g) }\end{array}$ & Contribution & $\begin{array}{c}\text { Single } \\
\text { Radionuclide } \\
\text { Guidelines (pCi/g) }\end{array}$ & $\begin{array}{c}\text { Ratio for Single } \\
\text { Radionuclide } \\
\text { Guideline }\end{array}$ \\
\hline Cesium-137 & 114 & 0.98 & 275.2 & 0.41 \\
\hline Plutonium-238 & 0.23 & 0.00 & 2370 & 0.00 \\
\hline Plutonium-239 & 2.09 & 0.02 & 2152 & 0.00 \\
\hline Total & 116.32 & & & 0.41 \\
\hline
\end{tabular}




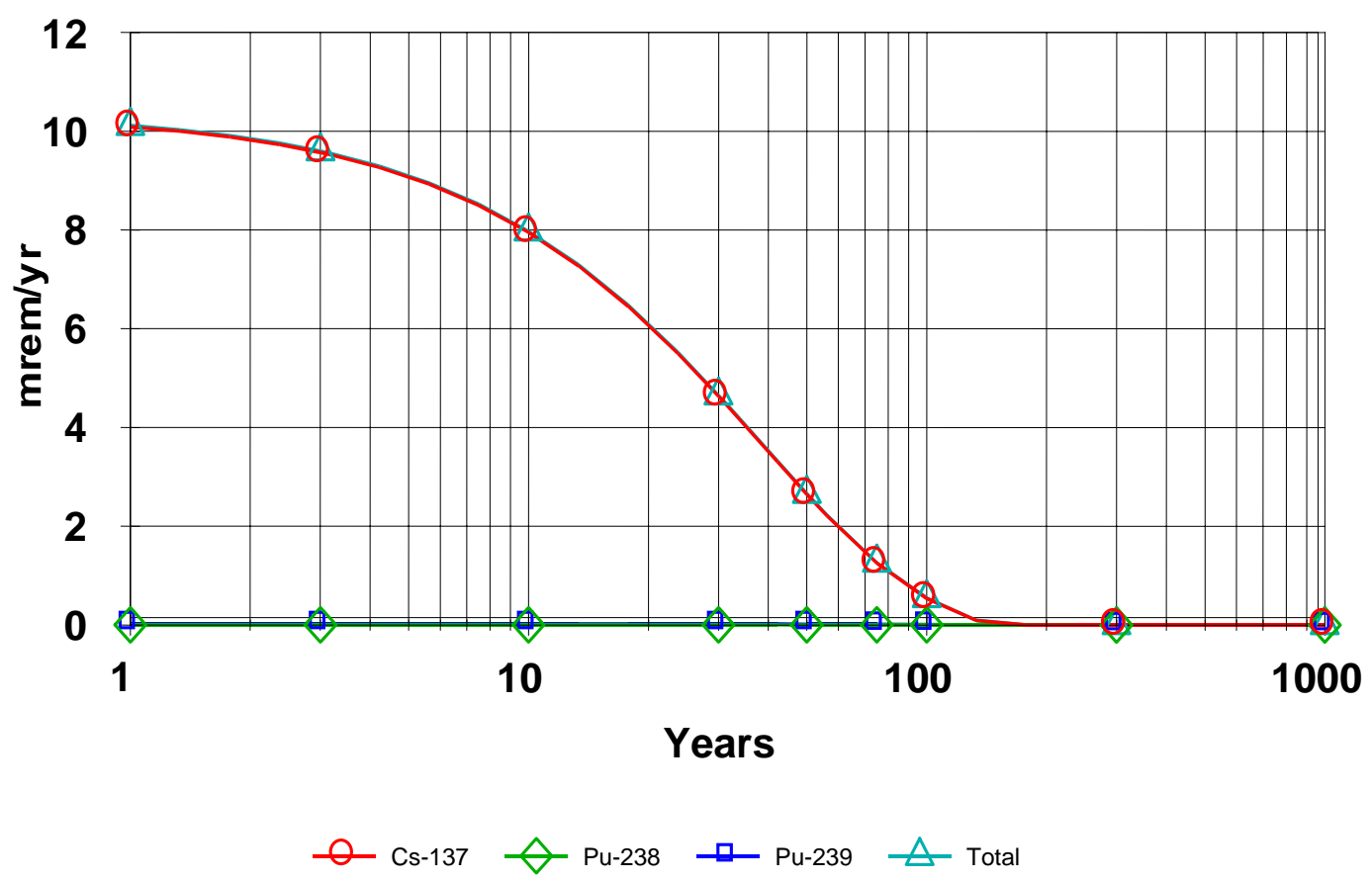

Figure 9-1. CAU 383 CAS 12-28-02, Radioactive Material Area, Scenario B: Dose Per Year, All Radionuclides Summed, All Pathways Summed 

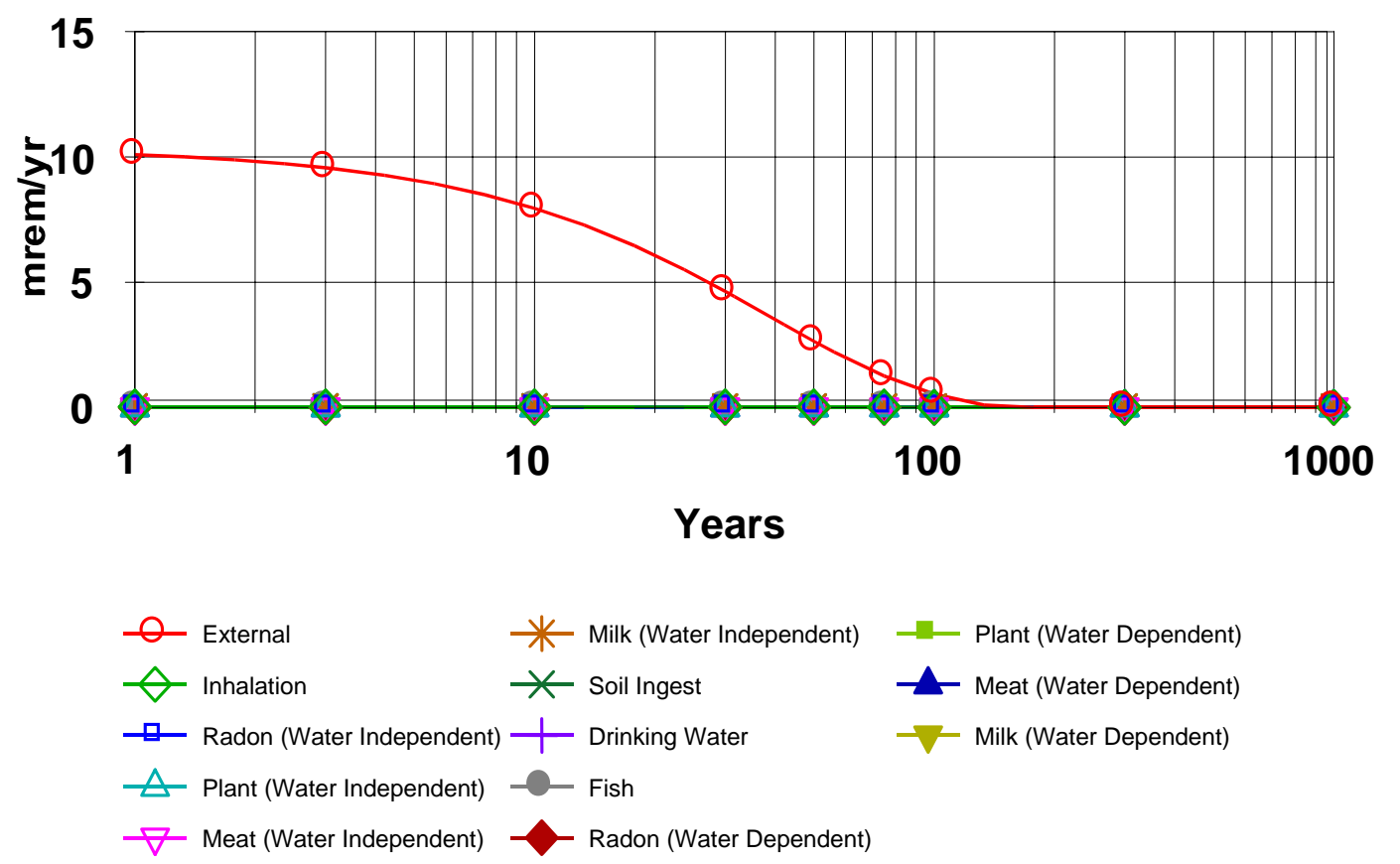

Figure 9-2. CAU 383 CAS 12-28-02, Radioactive Material Area, Scenario B: Annual Dose, All Radionuclides Summed, Component Pathways 


\section{References:}

AEC, see Atomic Energy Commission.

ARL, see Air Resources Laboratory.

Air Resources Laboratory. 2005. “Climatological Information and Data.” As accessed at http://www.sord.nv.doe.gov.

Atomic Energy Commission. 1958. "Press Release, Subject: 2,000 Pounds of Conventional High Explosives will be Detonated Within the Next Few Days in a Chamber Leading Off the Tunnel in Which the Rainier Deep Underground Event," NTA Accession Number NV0323213. Las Vegas, NV: Nuclear Testing Archive.

CFR, see Code of Federal Regulations.

Code of Federal Regulations. 2003a. Title 40 CFR Parts 260 - 282, "Hazardous Waste Management.” Washington, DC: U.S. Government Printing Office.

Code of Federal Regulations. 2003b. Title 40 CFR 761, "Polychlorinated Biphenyls (PCBs) Manufacturing, Processing, Distribution in Commerce and Prohibitions.” Washington, DC: U.S. Government Printing Office.

Defense Threat Reduction Agency. 2003. Corrective Action Investigation Plan for Corrective Action Unit 383: Area 12 E-Tunnel Sites, Nevada Test Site, Nevada, Rev. 0, DOE/NV--939. Las Vegas, NV.

DOE, see U.S. Department of Energy.

DOE/NV, see U.S. Department of Energy, Nevada Operations Office.

EPA, see U.S. Environmental Protection Agency.

Holmes \& Narver. 1959a. Operation Hardtack, Phase II Completion Report, July. Prepared for the U.S. Atomic Energy Commission. Las Vegas, NV.

Holmes \& Narver. 1959b. Engineering drawing entitled, “Generator Station Plot Plan.” Los Angeles, CA.

Holmes \& Narver, Inc. 1966. Aerial photograph N-53_009 showing Area 12 U12b-Tunnel, 24 September. Mercury, NV: Archives and Records Center.

Lawrence, A., Office of Environmental Policy \& Guidance. 2002. Memorandum to Distribution entitled, "Radiation Risk Estimation from Total Effective Dose Equivalents (TEDEs)," (EH-412-2002-1), 9 August. 
NCRP, see National Council on Radiation Protection and Measurements.

NDEP, see Nevada Division of Environmental Protection.

NNSA/NV, see U.S. Department of Energy, National Nuclear Security Administration Nevada Operations Office.

National Council on Radiation Protection and Measurements. 1999. Recommended Screening Limits for Contaminated Surface Soil and Review of Factors Relevant to Site-Specific Studies/National Council on Radiation Protection and Measurements, NCRP Report No. 129. Bethesda, MD.

Nevada Division of Environmental Protection. 2004. Review of Industrial Sites Project Document “Guidance for Calculating Industrial Sites Project Remediation Goals for Radionuclides in Soil Using the Residual Radiation (RESRAD) Computer Code.”

U.S. Department of Energy. 1993. Radiation Protection of the Public and the Environment, DOE 5400.5. Washington, DC.

U.S. Department of Energy. 2002. Draft Implementation Guide, Control and Release of Property with Residual Radioactive Material, for use with DOE 5400.5, Radiation Protection of the Public and the Environment, DOE G 441.1-XX. Washington, DC.

U.S. Department of Energy, Nevada Operation Office. 1996. Final Environmental Impact Statement for the Nevada Test Site and Off-Site Locations in the State of Nevada, DOE/EIS 0243. Las Vegas, NV.

U.S. Department of Energy, Nevada Operations Office. 1998. Nevada Test Site Resource Management Plan. DOE/NV--518, Las Vegas, NV.

U.S. Environmental Protection Agency. 1991. Human Health Evaluation Manual Supplemental Guidance: "Standard Default Exposure Factors.” OSWER Directive 9285.6-03.

U.S. Environmental Protection Agency. 1992. Guidance on Risk Managers and Risk Assessors. Memorandum from F.H. Habicht, Deputy Administrator, Washington, D.C.

U.S. Environmental Protection Agency. 2000a. Data Quality Objectives Process for Hazardous Waste Site Investigations, EPA QA/G-4HW. Washington, D.C.

U.S. Environmental Protection Agency. 2000b. Guidance for the Data Quality Objectives Process, EPA QA/G-4. Washington, DC.

U.S. Environmental Protection Agency. 2002a. Guidance for Quality Assurance Project Plans, EPA QA/G-5, EPA/240/R-02/009. Washington, D.C. 
U.S. Environmental Protection Agency. 2002b. Guidance on Choosing a Sampling Design for Environmental Data Collection, EPA QA/G-5S. Washington, D.C.

Yu C., Loureiro C., Cheng C. C., Jones L. G., Wang Y. Y., Chia Y. P., and Faillace E. 1993. Data Collection Handbook To Support Modeling the Impacts of Radioactive Material in Soil, ANL/EAIS-8. Chicago, IL: Environmental Assessment Division, Argonne National Laboratory.

Yu C., A.J. Zielen, J.J Cheng, D.J. LePoire., E. Gnanapragasam, S. Kamboj, J. Arnish, A. Wallo III, W.A. Williams, and H. Peterson. 2001. User's Manual for RESRAD Version 6, ANL/EAD-4, Argonne National Laboratory, Environmental Assessment Division, Argonne, IL. 


\section{Attachment A}

\section{RESRAD Parameters Used for Analysis of CAU 383 Sites}

The parametric values used in the RESRAD code for the analysis of the CAU 383 site are listed in Table A.1. Some parameters are site specific, while other values are default RESRAD values. The dose conversion factors used for inhalation and ingestion were the default FGR 13 morbidity values and correspond to the guidance and recommendations per the August 9, 2002, memorandum from A. Lawrence, Office of Environmental Policy \& Guidance, to Distribution, titled "Radiation Risk Estimation from Total Effective Dose Equivalents (TEDEs)” (EH-412-2002-1) (Lawrence, 2002). 
Table A.1 RESRAD Parameters (Page 1 of 6)

\begin{tabular}{|c|c|c|c|c|}
\hline Parameter & Units & CAU 383 & Defaults & Reference/Rationale \\
\hline \multicolumn{5}{|l|}{ R011 Contaminated Zone } \\
\hline Area of CZ & $\mathrm{m}^{2}$ & $1.00 \mathrm{E}+02$ & $1.000 \mathrm{E}+04$ & $10 \times 10 \mathrm{~m}$ hot spot \\
\hline Thickness of CZ & $\mathrm{m}$ & $1.200 \mathrm{E}+00$ & $2.000 \mathrm{E}+00$ & $\begin{array}{l}\text { Maximum depth from contaminated } \\
\text { samples }\end{array}$ \\
\hline Length Parallel to Aquifer Flow & $\mathrm{m}$ & not used & $1.000 \mathrm{E}+02$ & Not Used \\
\hline Radiation Dose Limit & $\mathrm{mrem} / \mathrm{yr}$ & $2.5 \mathrm{E}+001$ & $2.5 \mathrm{E}+001$ & RESRAD Default (Yu, et al., 1993) \\
\hline Elapsed Time Since Placement of Material & $\mathrm{yr}$ & 0.0 & 0.0 & RESRAD Default \\
\hline \multicolumn{5}{|l|}{ R012 Initial Principal Radionuclide } \\
\hline Principal radionuclides & $\mathrm{pCi} / \mathrm{g}$ & See Table 7.2 & 0.0 & Site-specific \\
\hline
\end{tabular}


Table A.1 RESRAD Parameters (Page 2 of 6 )

\begin{tabular}{|c|c|c|c|c|}
\hline Parameter & Units & CAU 383 & Defaults & Reference/Rationale \\
\hline \multicolumn{5}{|c|}{ R013 Cover and Contaminated Zone Hydrological Data } \\
\hline Cover Depth & $\mathrm{m}$ & 0.0 & 0.0 & No Cover Assumed \\
\hline Density of Cover Material & $\mathrm{g} / \mathrm{cm}^{3}$ & not used & 1.5 & No Cover Assumed \\
\hline Cover Depth Erosion Rate & $\mathrm{m} / \mathrm{yr}$ & not used & 1.000E-03 & No Cover Assumed \\
\hline Density of Contaminated Zone & $\mathrm{g} / \mathrm{cm}^{3}$ & 1.5 & 1.5 & RESRAD Default \\
\hline Contamination Zone Erosion Rate & $\mathrm{m} / \mathrm{yr}$ & 1.000E-03 & $1.000 \mathrm{E}-03$ & RESRAD Default \\
\hline Contaminated Zone Total Porosity & - & 4.000E-01 & 4.000E-01 & RESRAD Default \\
\hline Contaminated Zone Field Capacity & - & 2.000E-01 & 2.000E-01 & RESRAD Default \\
\hline Contaminated Zone Hydraulic Conductivity & $\mathrm{m} / \mathrm{yr}$ & $1.000 \mathrm{E}+01$ & $1.000 \mathrm{E}+01$ & RESRAD Default \\
\hline Contaminated Zone b Parameter & - & $5.300 \mathrm{E}+00$ & $5.300 \mathrm{E}+00$ & RESRAD Default \\
\hline Average Annual Wind Speed & $\mathrm{m} / \mathrm{sec}$ & 3.4 & $2.000 \mathrm{E}+00$ & Data from Air Resource Laboratory (2005) \\
\hline Humidity in Air & $\mathrm{g} / \mathrm{m}^{3}$ & not used & $8.000 \mathrm{E}+00$ & Not used \\
\hline Evapotranspiration Coefficient & - & 5.000E-01 & 5.000E-01 & RESRAD Default \\
\hline Precipitation & $\mathrm{m} / \mathrm{yr}$ & 3.260E-01 & $1.000 \mathrm{E}+00$ & Data from Air Resources Laboratory \\
\hline Irrigation & $\mathrm{m} / \mathrm{yr}$ & 2.000E-01 & 2.000E-01 & RESRAD Default \\
\hline Irrigation Mode & - & overhead & overhead & RESRAD Default \\
\hline Runoff Coefficient & - & 4.000E-01 & 2.000E-01 & $\begin{array}{l}\text { Open Sandy Loam 30\% impervious Table } \\
10.1 \text { (Yu, et al., 1993) }\end{array}$ \\
\hline Watershed Area for Nearby Stream or Pond & $\mathrm{m}^{2}$ & not used & $1.000 \mathrm{E}+06$ & Not used \\
\hline Accuracy for Water/Soil Computations & - & not used & 1.000E-03 & Not used \\
\hline
\end{tabular}


Table A.1 RESRAD Parameters (Page 3 of 6)

\begin{tabular}{|c|c|c|c|c|}
\hline Parameter & Units & CAU 383 & Defaults & Reference/Rationale \\
\hline \multicolumn{5}{|l|}{ R014 Saturated Zone Hydrological Data } \\
\hline Density of Saturated Zone & $\mathrm{g} / \mathrm{cm}^{3}$ & not used & $1.500 \mathrm{E}+00$ & Not used \\
\hline Saturated Zone Total Porosity & - & not used & 4.000E-01 & Not used \\
\hline Saturated Zone Effective Porosity & - & not used & 2.000E-01 & Not used \\
\hline Saturated Zone Field Capacity & - & not used & 2.000E-01 & Not used \\
\hline Saturated Zone Hydraulic Conductivity & $\mathrm{m} / \mathrm{yr}$ & not used & $1.000 \mathrm{E}+02$ & Not used \\
\hline Saturated Zone Hydraulic Gradient & - & not used & 2.000E-02 & Not used \\
\hline Saturated Zone b Parameter & - & not used & $5.300 \mathrm{E}+00$ & Not used \\
\hline Water Table Drop Rate & $\mathrm{m} / \mathrm{yr}$ & not used & 1.000E-03 & Not used \\
\hline Well Pump Intake Depth & $\mathrm{m}$ & not used & 1.000E+01 & Not used \\
\hline Model: Nondispersion or Mass-Balance & - & not used & ND & Not used \\
\hline Well Pumping Rate & $\mathrm{m}^{3} / \mathrm{yr}$ & not used & $2.500 \mathrm{E}+02$ & Not used \\
\hline \multicolumn{5}{|c|}{ R015 Uncontaminated and Unsaturated Strata Hydrological Data } \\
\hline Number of Unsaturated Zone Strata & - & not used & 1 & Not used \\
\hline Thickness & $\mathrm{m}$ & not used & $4.000 \mathrm{E}+00$ & Not used \\
\hline Soil Density & $\mathrm{g} / \mathrm{cm}^{3}$ & not used & $1.500 \mathrm{E}+00$ & Not used \\
\hline Total Porosity & - & not used & 4.000E-01 & Not used \\
\hline Effective Porosity & - & not used & 2.000E-01 & Not used \\
\hline Field Capacity & - & not used & 2.000E-01 & Not used \\
\hline Soil-specific b Parameter & - & not used & $5.300 E+00$ & Not used \\
\hline Hydraulic Conductivity & $\mathrm{m} / \mathrm{yr}$ & not used & $1.000 \mathrm{E}+01$ & Not used \\
\hline
\end{tabular}


Table A.1 RESRAD Parameters (Page 4 of 6)

\begin{tabular}{|c|c|c|c|c|}
\hline Parameter & Units & CAU 383 & Defaults & Reference/Rationale \\
\hline \multicolumn{5}{|l|}{ R016 Distribution Coefficients and Leach Rates } \\
\hline Contaminated Zone $\mathrm{K}_{\mathrm{d}}$ (all Zones) & $\mathrm{cm}^{3} / \mathrm{g}$ & & & RESRAD Default \\
\hline Saturated Leach Rate & /yr & 0.0 & 0.0 & Not used \\
\hline Solubility Constant & - & 0.0 & 0.0 & Not used \\
\hline \multicolumn{5}{|l|}{ R017 Inhalation and External Gamma } \\
\hline Inhalation Rate & $\mathrm{m}^{3} / \mathrm{yr}$ & $1.230 \mathrm{E}+04$ & $8.400 \mathrm{E}+03$ & $\begin{array}{l}\text { RESRAD Default and for an individual } \\
\text { performing outdoor activities, a typical activity } \\
\text { mix can consist of } 37 \% \text { at a moderate activity } \\
\text { level, } 28 \% \text { at both resting and light activity } \\
\text { levels, and } 7 \% \text { at a heavy activity level, which } \\
\text { results in a } 1.4 \mathrm{~m}^{3} / \mathrm{h}\left(12,300 \mathrm{~m}^{3} / \mathrm{yr} \text { ) inhalation }\right. \\
\text { rate. ( } \mathrm{Yu} \text {, et al., } 1993)\end{array}$ \\
\hline Mass Loading for Inhalation & $\mathrm{g} / \mathrm{m}^{3}$ & $6.00 \mathrm{E}-04$ & $1 \mathrm{E}-04$ & $\begin{array}{l}\text { The estimated mass loading for construction } \\
\text { activities. (Yu, et al., 1993) }\end{array}$ \\
\hline Exposure Duration & $\mathrm{yr}$ & 25 & 30 & Standard for Industrial/Commercial Scenario \\
\hline Shielding Factor Inhalation & - & 1.0 & 0.4 & Assumes no indoor time fraction \\
\hline Shielding Factor External Gamma & - & 1.0 & 0.7 & Assumes no indoor time fraction \\
\hline Fraction of Time Spent Indoors & - & 0.0 & 0.5 & Assumes no indoor time fraction \\
\hline Fraction of Time Spent Outdoors & - & 0.038 & 0.25 & $\begin{array}{l}\text { Scenario specific based on Industrial/ } \\
\text { Commercial Use Scenarios for standard } \\
\text { occupancy and low occupancy. }\end{array}$ \\
\hline Shape Factor & - & 1.0 & 1.0 & RESRAD Default \\
\hline \multicolumn{5}{|c|}{ R018 Ingestion Pathway Data, Dietary Parameters } \\
\hline Fruits, Vegetables, and Grain Consumption & $\mathrm{kg} / \mathrm{yr}$ & not used & $1.600 \mathrm{E}+02$ & Not used \\
\hline Leafy Vegetable Consumption & $\mathrm{kg} / \mathrm{yr}$ & not used & $1.400 \mathrm{E}+01$ & Not used \\
\hline Milk Consumption & L/yr & not used & $9.200 \mathrm{E}+01$ & Not used \\
\hline Meat and Poultry Consumption & $\mathrm{kg} / \mathrm{yr}$ & not used & $6.300 \mathrm{E}+01$ & Not used \\
\hline
\end{tabular}


Table A.1 RESRAD Parameters (Page 5 of 6 )

\begin{tabular}{|c|c|c|c|c|}
\hline Parameter & Units & CAU 383 & Defaults & Reference/Rationale \\
\hline Fish Consumption & $\mathrm{kg} / \mathrm{yr}$ & not used & $5.400 E+00$ & Not used \\
\hline Other Seafood Consumption & $\mathrm{kg} / \mathrm{yr}$ & not used & 9.000E-01 & Not used \\
\hline Soil Ingestion Rate & $\mathrm{g} / \mathrm{yr}$ & $1.752 \mathrm{E}+02$ & 36.5 & EPA, 1991; 480 mg/day \\
\hline Drinking Water Intake & L/yr & not used & $5.100 \mathrm{E}+02$ & Not used \\
\hline Drinking Water Contaminated Fraction & - & not used & $1.000 \mathrm{E}+00$ & Not used \\
\hline Household Water Contaminated Fraction & - & not used & $1.000 \mathrm{E}+00$ & Not used \\
\hline Livestock Water Contaminated Fraction & - & not used & $1.000 \mathrm{E}+00$ & Not used \\
\hline Irrigation Water Contaminated Fraction & - & not used & $1.000 \mathrm{E}+00$ & Not used \\
\hline Aquatic Food Contamination Fraction & - & not used & 5.000E-01 & Not used \\
\hline Plant Food Contamination Fraction & - & not used & -1 & Not used \\
\hline Meat Contamination Fraction & - & not used & -1 & Not used \\
\hline Milk Contamination Fraction & - & not used & -1 & Not used \\
\hline \multicolumn{5}{|l|}{ R019 Ingestion Pathway Data, Nondietary } \\
\hline Livestock Fodder Intake for Meat & kg/day & not used & $6.800 E+01$ & Not used \\
\hline Livestock Fodder Intake for Milk & kg/day & not used & $5.500 \mathrm{E}+01$ & Not used \\
\hline Livestock Water Intake for Meat & L/day & not used & $5.000 \mathrm{E}+01$ & Not used \\
\hline Livestock Water Intake for Milk & L/day & not used & $1.600 \mathrm{E}+02$ & Not used \\
\hline Livestock Soil Intake & kg/day & not used & 5.000E-01 & Not used \\
\hline Mass Loading for Foliar Deposition & $\mathrm{g} / \mathrm{m}^{3}$ & not used & 1.000E-04 & Not used \\
\hline Depth of Soil Mixing Layer & $\mathrm{m}$ & 1.500E-01 & 1.500E-01 & RESRAD Default \\
\hline Depth of Roots & $\mathrm{m}$ & not used & 9.000E-01 & Not used \\
\hline
\end{tabular}


Table A.1 RESRAD Parameters (Page 6 of 6)

\begin{tabular}{|c|c|c|c|c|}
\hline Parameter & Units & CAU 383 & Defaults & Reference/Rationale \\
\hline Drinking Water Fraction from Groundwater & - & not used & $1.000 \mathrm{E}+00$ & Not used \\
\hline $\begin{array}{l}\text { Household Water Fraction from } \\
\text { Groundwater }\end{array}$ & - & not used & $1.000 \mathrm{E}+00$ & Not used \\
\hline Livestock Water Fraction from Groundwater & - & not used & $1.000 \mathrm{E}+00$ & Not used \\
\hline Irrigation Fraction from Groundwater & - & not used & $1.000 \mathrm{E}+00$ & Not used \\
\hline \multicolumn{5}{|l|}{ R021 Radon } \\
\hline Radon Parameters Not Used & & & & Not used \\
\hline
\end{tabular}

$\mathrm{cm}^{3} / \mathrm{g}=$ Cubic centimeters per gram

$\mathrm{g} / \mathrm{cm}^{3}=$ Grams per cubic centimeter

$\mathrm{g} / \mathrm{m}^{3}=$ Grams per cubic meter

$\mathrm{g} / \mathrm{yr}=\mathrm{Grams}$ per year

$\mathrm{kg} /$ day = Kilograms per day

$\mathrm{kg} / \mathrm{yr}=$ Kilograms per year

L/day = Liters per day

L/yr = Liters per year

$\mathrm{m}=$ Meter

$\mathrm{m}^{2}=$ Square meter

$\mathrm{m} / \mathrm{sec}=$ Meters per second $\mathrm{m} / \mathrm{yr}=$ Meters per year

$\mathrm{m}^{3} / \mathrm{h}=$ Cubic meters per hour

$\mathrm{m}^{3} / \mathrm{yr}=$ Cubic meters per year

mg.day = Milligrams per day

$\mathrm{mrem} / \mathrm{yr}=$ Millirem per year

$\mathrm{N} / \mathrm{A}=$ Not applicable

$\mathrm{pCi} / \mathrm{g}=$ Picocuries per gram

$\mathrm{yr}=$ Year

/yr $=$ Per year

$\mathrm{UCL}=$ Upper confidence level 


\section{Exhibit 1}

\section{RESRAD Summary Report: CAU 383 CAS 12-06-06}

Muck: 27 Pages

Drainage: 26 pages

Ponds: 26 Pages 
Table of Contents

Part I: Mixture Sums and Single Radionuclide Guidelines

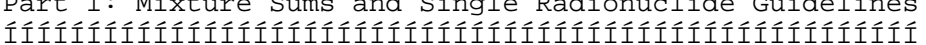

Dose Conversion Factor (and Related) Parameter Summary ... Site-Specific Parameter Summary ..................... Summary of Pathway Selections ........................ 12 Contaminated Zone and Total Dose Summary ............... 13 Total Dose Components

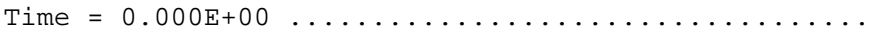

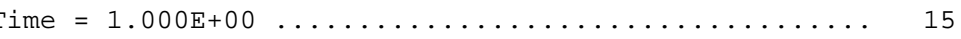

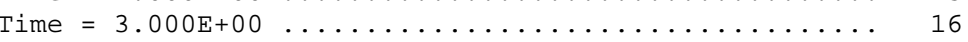

Time $=1.000 \mathrm{E}+01 \ldots \ldots \ldots \ldots \ldots \ldots \ldots \ldots \ldots \ldots \ldots \ldots \ldots, 17$

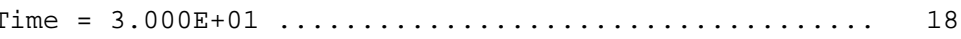

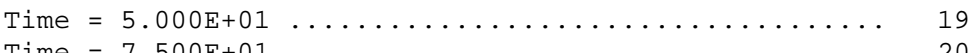

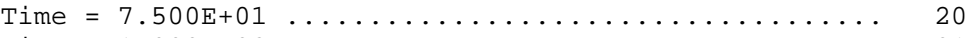

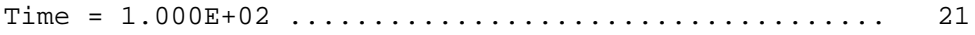

Time $=3.000 \mathrm{E}+02 \ldots \ldots \ldots \ldots \ldots \ldots \ldots \ldots \ldots \ldots \ldots \ldots, 22$

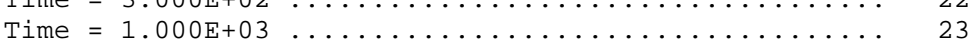

Dose/Source Ratios Summed over All Pathways ........... 24

Single Radionuclide Soil Guidelines ............... 24

Dose Per Nuclide Summed over All Pathways ............. 26

Soil Concentration Per Nuclide .................. 27 
Dose Conversion Factor (and Related) Parameter Summary File: FGR 13 MORBIDITY

$0 \quad 3$

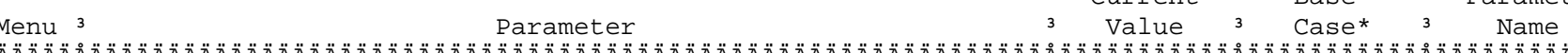

${ }_{3}$ Current ${ }^{3}$ Base ${ }_{3}$ Parameter

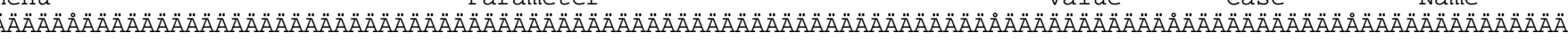

B-1 3 Dose conversion factors for inhalation, $\mathrm{mrem} / \mathrm{pCi}$ :

B-1 3 AC $-227+D$

B-1 3 Am-241

B-1 3 CS $-137+D$

B-1 3 Eu-152

$\mathrm{B}-1 \quad 3 \mathrm{Gd}-152$

$\mathrm{B}-1 \quad 3 \mathrm{~Np}-237+\mathrm{D}$

$\mathrm{B}-1$ - $3 \mathrm{~Pa}-231$

$\mathrm{B}-1 \quad 3 \mathrm{~Pb}-210+\mathrm{D}$

B-1 3 Pu-238

B-1 3 Pu-239

B-1 3 Ra-226+D

B-1 3 Sr $-90+D$

B-1 3 Th-229+D

B-1 3 Th -230

B-1 3 U-233

$B-1 \quad 3 \quad U-234$

B-1 3 U-235+D

D-1 3 Dose conversion factors for ingestion, $\mathrm{mrem} / \mathrm{pCi}$ :

D-1 3 AC - 227+D

D-1 3 Am-241

D-1 3 Cs $-137+D$

D-1 $3 \mathrm{Eu}-152$

D-1 3 Gd-152

D-1 $3 \mathrm{~Np}-237+\mathrm{D}$

D-1 $3 \mathrm{~Pa}-231$

$\mathrm{D}-1 \quad 3 \mathrm{~Pb}-210+\mathrm{D}$

$\mathrm{D}-1 \quad 3 \mathrm{Pu}-238$

D-1 3 Pu-239

D-1 3 Ra-226+D

D-1 3 Sr $-90+D$

$D-1 \quad 3$ Th $-229+D$

$\begin{array}{lll}\mathrm{D}-1 & 3 & \mathrm{Th}-230 \\ \mathrm{D}-1 & 3 & \mathrm{U}-233\end{array}$

D-1 3 U -234

D-1 3 U-235+D

-34 3 Food transfer factors:

D-34 3 AC-227+D, plant/soil concentration ratio, dimensionless

D-34 3 AC-227+D, beef/livestock-intake ratio, (pCi/kg)/(pCi/d)

$\mathrm{D}-34{ }^{3} \mathrm{AC}-227+\mathrm{D}$, milk/livestock-intake ratio, (pCi/L)/(pCi/d)

D- $344^{3}$

D-34 3 Am-241 , plant/soil concentration ratio, dimensionless

$\mathrm{D}-343 \mathrm{Am}-241$, beef/livestock-intake ratio, $(\mathrm{pCi} / \mathrm{kg}) /(\mathrm{pCi} / \mathrm{d})$

$36.724 \mathrm{E}+00^{3}{ }^{3} 6.700 \mathrm{E}+00^{3}{ }^{3} \operatorname{DCF} 2\left(\begin{array}{l}1 \\ 3\end{array}\right)$

3 4.440E-01 3 4.440E-01 3 DCF2( 2 )

3.190E-05 3 3.190E-05 3 DCF2( 3 )

$32.210 E-044^{3} 2.210 E-04{ }^{3}$ DCF2( 4$)$

3 2.430E-01 3 2.430E-01 3 DCF2 ( 6)

3 5.400E-01 3 5.400E-01 3 DCF2( 7$)$

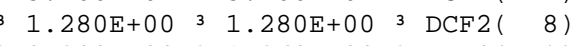

2.320E-02 3 1.360E-02 3 DCF2( 9$)$

$3.920 E-011^{3}$ 3.920E-01 3 DCF2 ( 10)

3 4.290E-01 3 4.290E-01 3 DCF2( 12$)$

3 8.594E-03 3 8.580E-03 3 DCF2( 13 )

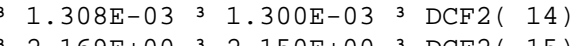

$2.169 \mathrm{E}+00{ }^{3} \quad 2.150 \mathrm{E}+00$ DCF2( 15$)$

3 3.260E-01 3 3.260E-01 3 DCF2( 16$)$

$\begin{array}{lllll}1.350 E-01 & 3 & 1.350 E-01 & 3 & \text { DCF2 }(17) \\ 1.320 E-01 & 3 & 1.320 E-01 & 3 & \text { DCF2 }(18)\end{array}$

3 $1.230 \mathrm{E}-01$ 3 $1.230 \mathrm{E}-01$ 3 DCF2( 19$)$

3 (n)

1.480E-02 3 1.410E-02 3 DCF3( 1$)$

$3.640 \mathrm{E}-03 \quad 3 \quad 3.640 \mathrm{E}-03$ 3 DCF3 $(2)$

$5.000 \mathrm{E}-053 \mathrm{5}$. $500 \mathrm{E}-055^{3} \mathrm{DCF} 3(3)$

(

1.

$1.610 E-04$ 1.610E-04 DCF3( 6)

$4.444 \mathrm{E}-03$ 3 $4.440 \mathrm{E}-03$ 3 DCF3( 7$)$

1.060E-02 3 1.060E-02 3 DCF3( 8)

3 7.276E-03 3 5.370E-03 3 DCF3(9)

$3.200 \mathrm{E}-033^{3} 3.200 \mathrm{E}-03{ }^{3}$ DCF3( 10$)$

3 3.540E-03 3 3.540E-03 3 DCF3( 12$)$

$1.321 E-03$ 3 $1.320 \mathrm{E}-03 \quad 3$ DCF3( 13$)$

3 1.528E-04 3 1.420E- $04{ }^{3}$ DCF3( 14$)$

3 4.027E-03 3 3.530E-03 3 DCF3( 15)

5.480E-04 3 5.480E-04 3 DCF3( 16$)$

$32.890 \mathrm{E}-044^{3} 2.890 \mathrm{E}-04$ 3 $\mathrm{DCF} 3(17)$

$32.830 E-043^{3}$ 2.830E-04 3 DCF3( 18$)$

2.673E-04 3 2.660E-04 3 DCF3( 19)

3 (3)

$32.500 \mathrm{E}-03 \quad 3 \quad 2.500 \mathrm{E}-03 \quad 3 \operatorname{RTF}(1,1)$

$32.000 \mathrm{E}-053$ 2.000E-05 3 RTF $(1,2)$

2.000E-05 $32.000 \mathrm{E}-053 \operatorname{RTF}(1,3)$

1. $000 \mathrm{E}-03 \quad 3 \quad 1.000 \mathrm{E}-03 \quad 3 \operatorname{RTF}(2,1)$

3 5.000E-05 3 5.000E-05 3 RTF $(2,2)$

3 2.00९E-๑6 3 2.000E-06 $3 \operatorname{RTF}(2,3)$

4. $000 \mathrm{E}-02 \quad 3 \quad 4.000 \mathrm{E}-023 \mathrm{RTF}(3,1)$

$3.000 \mathrm{C}-02$ 3 $3.000 \mathrm{E}-023 \mathrm{RTF}(3,1)$

3 3.

3 8.

D $-137+\mathrm{D}$, plant/soil concentration ratio, dimension

D-34 3 Cs-137+D, beef/livestock-intake ratio, $(\mathrm{pCi} / \mathrm{kg}) /(\mathrm{pCi} / \mathrm{d})$

$\mathrm{D}-343$

Uncontrolled When Printed 
Dose Conversion Factor (and Related) Parameter Summary (continued)

$0^{3}{ }^{3}$

Menu 3

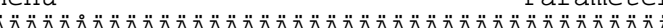

$\mathrm{D}-344^{3} \mathrm{Eu}-152$

$\mathrm{D}-34^{3} \mathrm{Eu}-152$

$\mathrm{D}-344^{3} \mathrm{Eu}-152$

$\mathrm{D}-344^{3}$

D-34 3 Gd-152

D-34 3 Gd-152

D-34 3 Gd-152

D- 343

D-34 3 Np-237+D

$\mathrm{D}-343 \mathrm{~Np}-237+\mathrm{D}$

D-34 $3 \mathrm{~Np}-237+\mathrm{D}$

D-34 3

D-34 3 Pa-231

$\mathrm{D}-34 \quad 3 \mathrm{~Pa}-231$

D-34 3 Pa-231

$\begin{array}{ll}D-34 & \\ D-34 & 3\end{array}$

D-34 3 Pb-210+D

$\mathrm{D}-343 \mathrm{~Pb}-210+\mathrm{D}$

D-34 $3 \mathrm{~Pb}-210+\mathrm{D}$

D-34 3

D-34 3 Pu-238

D-34 3 Pu-238

D-34 3 Pu-238

$\begin{array}{ll}D-34 & 3 \\ D-34 & 3\end{array}$

D-34 3 Pu-239

$\mathrm{D}-34 \quad \mathrm{Pu}-239$

D-34 3 Pu-239

D-34 3

D-34 3 Ra-226+D

$\mathrm{D}-343 \mathrm{Ra}-226+\mathrm{D}$

D-34 3 Ra-226+D

D -343

D-34 $3 \mathrm{Sr}-90+\mathrm{D}$

$\mathrm{D}-34$ 3 $\mathrm{Sr}-90+\mathrm{D}$

D-34 3 Sr $-90+D$

D-34 3

D-34 3 Th-229+D

D-34 3 Th-229+D

D-34 3 Th-229+D

D $-344^{3}$

D-34 3 Th-230

D-34 3 Th-230

D-34 3 Th -230

D-34 3

D-34 3 U-233

$\mathrm{D}-34 \quad 3 \quad \mathrm{U}-233$

$\mathrm{D}-34 \quad 3 \mathrm{U}-233$

D -343

$\mathrm{D}-34 \quad 3 \quad \mathrm{U}-234$

D $-34 \quad 3 \quad \mathrm{U}-234$

$\mathrm{D}-34 \quad 3 \quad \mathrm{U}-234$

\section{Parameter}

File: FGR 13 MORBIDITY

3 Current 3 Base ${ }_{3}$ Parameter

3 Value 3 Case* ${ }^{*}$ Name

plant/soil con

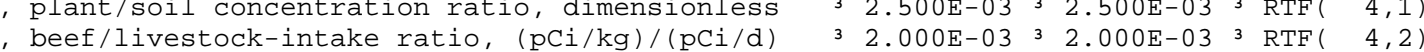

, milk/livestock-intake ratio, $(\mathrm{pCi} / \mathrm{L}) /(\mathrm{pCi} / \mathrm{d})$

plant/soil concentration ratio, dimensionless beef/livestock-intake ratio, $(\mathrm{pCi} / \mathrm{kg}) /(\mathrm{pCi} / \mathrm{d})$

milk/livestock-intake ratio, $(\mathrm{pCi} / \mathrm{L}) /(\mathrm{pCi} / \mathrm{d})$

plant/soil concentration ratio, dimensionless beef/livestock-intake ratio, $(\mathrm{pCi} / \mathrm{kg}) /(\mathrm{pCi} / \mathrm{d})$ milk/livestock-intake ratio, $(\mathrm{pCi} / \mathrm{L}) /(\mathrm{pCi} / \mathrm{d})$

plant/soil concentration ratio, dimensionless beef/livestock-intake ratio, $(\mathrm{pCi} / \mathrm{kg}) /(\mathrm{pCi} / \mathrm{d})$

milk/livestock-intake ratio, $(\mathrm{pCi} / \mathrm{L}) /(\mathrm{pCi} / \mathrm{d})$

plant/soil concentration ratio, dimensionless beef/livestock-intake ratio, $(\mathrm{pCi} / \mathrm{kg}) /(\mathrm{pCi} / \mathrm{d})$ $\mathrm{milk} /$ livestock-intake ratio, $(\mathrm{pCi} / \mathrm{L}) /(\mathrm{pCi} / \mathrm{d})$

, plant/soil concentration ratio, dimensionless beef/livestock-intake ratio, $(\mathrm{pCi} / \mathrm{kg}) /(\mathrm{pCi} / \mathrm{d})$ $\mathrm{milk} /$ livestock-intake ratio, $(\mathrm{pCi} / \mathrm{L}) /(\mathrm{pCi} / \mathrm{d})$

, plant/soil concentration ratio, dimensionless beef/livestock-intake ratio, ( $\mathrm{pCi} / \mathrm{kg}) /(\mathrm{pCi} / \mathrm{d})$ $\mathrm{milk} /$ livestock-intake ratio, $(\mathrm{pCi} / \mathrm{L}) /(\mathrm{pCi} / \mathrm{d})$

plant/soil concentration ratio, dimensionless beef/livestock-intake ratio, $(\mathrm{pCi} / \mathrm{kg}) /(\mathrm{pCi} / \mathrm{d})$ milk/livestock-intake ratio, $(\mathrm{pCi} / \mathrm{L}) /(\mathrm{pCi} / \mathrm{d})$

plant/soil concentration ratio, dimensionless beef/livestock-intake ratio, $(\mathrm{pCi} / \mathrm{kg}) /(\mathrm{pCi} / \mathrm{d})$ milk/livestock-intake ratio, $(\mathrm{pCi} / \mathrm{L}) /(\mathrm{pCi} / \mathrm{d})$

plant/soil concentration ratio, dimensionless beef/livestock-intake ratio, $(\mathrm{pCi} / \mathrm{kg}) /(\mathrm{pCi} / \mathrm{d})$ $\mathrm{milk} /$ livestock-intake ratio, $(\mathrm{pCi} / \mathrm{L}) /(\mathrm{pCi} / \mathrm{d})$

, plant/soil concentration ratio, dimensionless beef/livestock-intake ratio, ( $\mathrm{pCi} / \mathrm{kg}) /(\mathrm{pCi} / \mathrm{d})$ $\mathrm{milk} /$ livestock-intake ratio, $(\mathrm{pCi} / \mathrm{L}) /(\mathrm{pCi} / \mathrm{d})$

, plant/soil concentration ratio, dimensionless beef/livestock-intake ratio, $(\mathrm{pCi} / \mathrm{kg}) /(\mathrm{pCi} / \mathrm{d})$ milk/livestock-intake ratio, $(\mathrm{pCi} / \mathrm{L}) /(\mathrm{pCi} / \mathrm{d})$

plant/soil concentration ratio, dimensionless beef/livestock-intake ratio, $(\mathrm{pCi} / \mathrm{kg}) /(\mathrm{pCi} / \mathrm{d})$ milk/livestock-intake ratio, $(\mathrm{pCi} / \mathrm{L}) /(\mathrm{pCi} / \mathrm{d})$
2. $000 \mathrm{E}-03$ 3 $2.000 \mathrm{E}-03$ 3 $\operatorname{RTF}(4,2)$

5.000E-05 35 5.000E-05 3 RTF ( 4,3$)$

2.500E-03 3 2.500E-03 3 RTF $(6,1)$

2.000E-05 3 2.000E-05 3 RTF $(6,3)$

2. $000 \mathrm{E}-02 \quad 3 \quad 2.000 \mathrm{E}-02 \quad 3 \operatorname{RTF}(7,1)$

1. $000 \mathrm{E}-03 \quad 3 \quad 1.000 \mathrm{~B}-03$ 3 $\operatorname{RTF}(7,2)$

5.000E-06 3 5.000E-06 $3 \operatorname{RTF}(7,3)$

1. $000 \mathrm{E}-02 \quad 3 \quad 1.000 \mathrm{E}-02{ }^{3} \operatorname{RTF}(8,1)$

$5.000 \mathrm{E}-03 \quad 3 \quad 5,000 \mathrm{E}-03 \quad 3 \operatorname{RTF}(8,2)$

5. $5.000 \mathrm{E}-06$ 3 5.000E-06 $3 \operatorname{RTF}(8,3)$

3 . 3

3 1. $000 \mathrm{E}-023$ 1.000E-02 $3 \operatorname{RTF}(9,1)$

3 8.000E-๑4 3 8.000E-๑4 3 RTF $(9,2)$

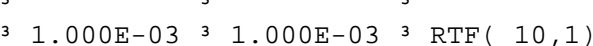

$1.000 \mathrm{E}-0431.000 \mathrm{~B}-04 \quad 3 \mathrm{RTF}(10,2)$

$1.000 \mathrm{E}-06$ 3 $1.000 \mathrm{E}-06$ 3 $\operatorname{RTF}(10,3)$

31 1. $000 \mathrm{E}-03 \quad 3 \quad 1.000 \mathrm{E}-033 \operatorname{RTF}(12,1)$

3 1. $000 \mathrm{E}-04$ 3 $1.000 \mathrm{E}-043 \operatorname{RTF}(12,2)$

3 1. $000 \mathrm{E}-06$ 3 $1.000 \mathrm{E}-063 \operatorname{RTF}(12,3)$

3 4. $000 \mathrm{E}-02 \quad 3 \quad 4.000 \mathrm{E}-023 \operatorname{RTF}(13,1)$

1. $000 \mathrm{E}-03$ 3 $1,000 \mathrm{E}-03$ 3 $\operatorname{RTF}(13,2)$

1. 1. 000E-03 $31.000 \mathrm{E}-033 \operatorname{RTF}(13,3)$

3 i. 3

3 3. $000 \mathrm{E}-013 \quad 3.000 \mathrm{Q}-013 \operatorname{RTF}(14,1)$

3 8.000E-03 3 8.000E-03 $3 \operatorname{RTF}(14,2)$

$32.000 \mathrm{E}-03 \quad 3 \quad 2.000 \mathrm{E}-03 \quad 3 \operatorname{RTF}(14,3)$

3 1. $000 \mathrm{E}-03$ 3 $1.000 \mathrm{E}-033 \operatorname{RTF}(15,1)$

1.000E-04 3 1.000E-04 3 RTF $(15,2)$

3 5. 000E-06 3 5. 000E-06 3 RTF( 15,3$)$

3 3

1. $000 \mathrm{E}-03 \quad 3 \quad 1.000 \mathrm{E}-033 \operatorname{RTF}(16,1)$

3 1. $000 \mathrm{E}-04$ 3 $1.000 \mathrm{E}-043 \operatorname{RTF}(16,2)$

$32.500 \mathrm{E}-03 \quad 3 \quad 2.500 \mathrm{E}-03 \quad 3 \operatorname{RTF}(17,1)$

$3 \quad 3.400 \mathrm{E}-04 \quad 3 \quad 3,400 \mathrm{E}-043 \operatorname{RTF}(17,2)$

3 6. $000 \mathrm{E}-04{ }^{3}$ 6.000E-04 3 RTF( 17,3$)$

2.500E-03 $3 \quad 2.500 \mathrm{E}-03 \quad 3 \operatorname{RTF}(18,1)$

3 3. $400 \mathrm{E}-04$ 3 $3.400 \mathrm{E}-04$ 3 $\operatorname{RTF}(18,2)$

$36.000 \mathrm{E}-0436.000 \mathrm{3}-043 \operatorname{RTF}(18,3)$
2. $000 \mathrm{E}-03 \quad 3 \quad 2.000 \mathrm{E}-03 \quad 3 \mathrm{RTF}(6,2)$

3 3. 000E-04 3 3.000E-04 $3 \operatorname{RTF}(9,3)$

3 5. $500 \mathrm{E}-06$ 3 $5.000 \mathrm{E}-06$ 3 $\operatorname{RTF}(16,3)$ 
Dose Conversion Factor (and Related) Parameter Summary (continued)

$0_{\text {Menu }}^{3}{ }^{3}$

Menu ${ }^{3}$

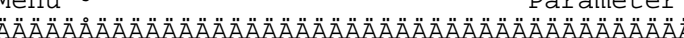

D- 34 - 3 U

$\mathrm{D}-34 \quad 3 \quad \mathrm{U}-235+\mathrm{D}$

$D-34 \quad 3 \quad U-235+D$

\section{plant/soil concentration} File: FGR 13 MORBIDITY

D-5 3 Bioaccumulation factors, fresh water, L/kg:

$D-5 \quad 3$ AC-227+D, fish

D-5 3 AC-227+D , crustacea and mollusks

D-5 3

D-5 3 Am-241, fish

D-5 3 Am-241 ', crustacea and mollusks

D-5 3

D-5 3 Cs $-137+D$, fish

D-5 3 Cs $-137+D$, crustacea and mollusks

D $-5 \quad 3$

D-5

D-5 3 Eu-152, fish

D-5 3 Eu-152 , crustacea and mollusks

D-5 3

D-5 3 Gd-152 , fish

D-5 3 Gd-152 , crustacea and mollusks

$D-5 \quad 3$

$\mathrm{D}-5 \quad 3 \mathrm{~Np}-237+\mathrm{D}$, fish

D-5 $3 \mathrm{~Np}-237+\mathrm{D}$, crustacea and mollusks

D $-5 \quad 3$

D-5

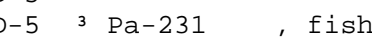

D-5 3 Pa-231 ', crustacea and mollusks

$-5 \quad 3$

D-5 $3 \mathrm{~Pb}-210+\mathrm{D}$, fish

D-5 $3 \mathrm{~Pb}-210+\mathrm{D}$, crustacea and mollusks

$\mathrm{D}-5 \quad 3$

D-5 3 Pu-238, fish

D-5 3 Pu-238, crustacea and mollusks

D-5 3

D-5 3 Pu-239

D-5 3

Ra-226+D, fish

D-5 3 Ra-226+D, crustacea and mollusks

D-5 3

D-5 3 Sr-90+D, fish

D-5 3 Sr-90+D , crustacea and mollusks

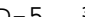

D-5 3 Th-229+D, fish

D-5 3 Th-229+D ', crustacea and mollusks

D-5 3

D-5 3 Th-230 , fish

D-5 3 Th-230, crustacea and mollusks

D -53

D-5 3 U-233 , fish

D-5 3 U-233 , crustacea and mollusks

3 Current 3 Base ${ }_{3}^{3}$ Parameter 3 Value 3 Case* ${ }^{*}$ Name

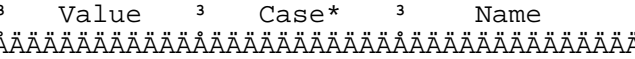

$\begin{aligned} & 2.500 \mathrm{E}-03 \\ & 3\end{aligned} 2.500 \mathrm{E}-03 \quad 3 \operatorname{RTF}(19,1)$

3. 400E-04 3 3.400E-04 $3 \operatorname{RTF}(19,2)$

$6.000 \mathrm{E}-044^{3}$ 6.000E-04 ${ }^{3} \operatorname{RTF}(19,3)$

3

$1.500 \mathrm{E}+01 \quad 3 \quad 1.500 \mathrm{E}+01 \quad 3 \quad \operatorname{BIOFAC}(1,1)$

$1.000 \mathrm{E}+03 \quad 3 \quad 1.000 \mathrm{E}+033 \operatorname{BIOFAC}(1,2)$

$3 \quad 3.000 \mathrm{E}+01 \quad 3 \quad 3.000 \mathrm{E}+013 \operatorname{BIOFAC}(2,1)$

1. $000 \mathrm{E}+03$ 3 3 1. $000 \mathrm{E}+03$ 3 $\mathrm{BIOFAC}(2,2)$

$32.000 \mathrm{E}+03 \quad 3 \quad 2.000 \mathrm{E}+03 \quad 3 \operatorname{BIOFAC}(3,1)$

$31.000 \mathrm{E}+02$ 3 $1.000 \mathrm{E}+023 \operatorname{BIOFAC}(3,2)$

5. $000 \mathrm{E}+0135.000 \mathrm{E}+013 \operatorname{BIOFAC}(4,1)$

3 1. $000 \mathrm{E}+03$ 3 $1.000 \mathrm{E}+03$ 3 $\operatorname{BIOFAC}(4,2)$

$2.500 \mathrm{E}+01 \quad 3 \quad 2.500 \mathrm{E}+013 \operatorname{BIOFAC}(6,1)$

$31.000 \mathrm{E}+03 \quad 3 \quad 1.000 \mathrm{E}+03$ 3 $\operatorname{BIOFAC}(6,2)$

$3 \quad 3.000 \mathrm{E}+0133.000 \mathrm{E}+013 \operatorname{BIOFAC}(7,1)$

3 4. $000 \mathrm{E}+023 \quad 4.000 \mathrm{E}+02{ }^{3} \operatorname{BIOFAC}(7,2)$

$3 \quad 1.000 \mathrm{E}+0131.000 \mathrm{E}+013 \operatorname{BIOFAC}(8,1)$

$\begin{aligned} & 1.100 \mathrm{E}+02 \\ & 3\end{aligned} 1.100 \mathrm{E}+023 \operatorname{BIOFAC}(8,2)$

$3 \quad 3.000 \mathrm{E}+02 \quad 3 \quad 3.000 \mathrm{E}+023 \operatorname{BIOFAC}(9,1)$

1. $000 \mathrm{E}+02 \quad 3 \quad 1.000 \mathrm{E}+\odot 23 \operatorname{BIOFAC}(9,2)$

$3.000 \mathrm{E}+0133.000 \mathrm{E}+013 \mathrm{BIOFAC}(10,1)$

1. $00 \odot \mathrm{E}+022^{3} 1.00 \odot \mathrm{E}+023 \operatorname{BIOFAC}(10,2)$

$3.000 \mathrm{E}+01 \quad 3 \quad 3.000 \mathrm{E}+013 \operatorname{BIOFAC}(12,1)$

$1.000 \mathrm{E}+02$ 3 $1.000 \mathrm{E}+023 \operatorname{BIOFAC}(12,2)$

$5.000 \mathrm{E}+013 \quad 5.000 \mathrm{E}+013$ BIOFAC $(13,1)$

$2.500 \mathrm{E}+02 \quad 3 \quad 2.500 \mathrm{E}+02 \quad 3 \operatorname{BIOFAC}(13,2)$

$6.000 \mathrm{E}+013 \quad 6.000 \mathrm{E}+013 \operatorname{BIOFAC}(14,1)$

1. $000 \mathrm{E}+02 \quad 3 \quad 1.000 \mathrm{E}+\odot 23 \operatorname{BIOFAC}(14,2)$

1. $000 \mathrm{E}+02 \quad 3 \quad 1.000 \mathrm{E}+023 \operatorname{BIOFAC}(15,1)$

$35.000 \mathrm{E}+\odot 2$ 3 $5.000 \mathrm{E}+\odot 2$ 3 $\operatorname{BIOFAC}(15,2)$

$31.000 \mathrm{E}+02 \quad 3 \quad 1.000 \mathrm{E}+023 \operatorname{BIOFAC}(16,1)$

$35.000 \mathrm{E}+\odot 235.000 \mathrm{3}+\odot 23 \operatorname{BIOFAC}(16,2)$

1. $000 \mathrm{E}+013 \quad 1.000 \mathrm{E}+013 \operatorname{BIOFAC}(17,1)$

6. $600 \mathrm{E}+0136.000 \mathrm{1}+013 \mathrm{BIOFAC}(17,2)$ 
Dose Conversion Factor (and Related) Parameter Summary (continued) File: FGR 13 MORBIDITY

\section{$\odot{ }^{3}$}

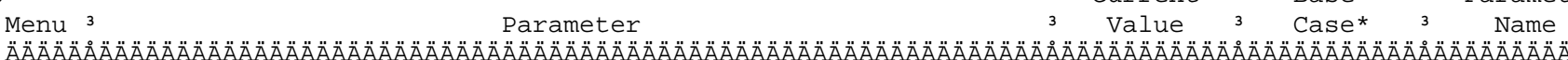

3 Current ${ }^{3}$ Base ${ }^{3}$ Parameter

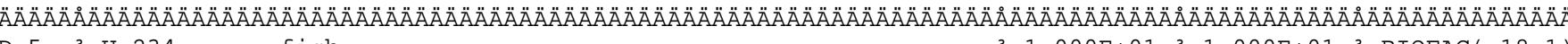

D-5 $3 \mathrm{U}-234$, fish $31.000 \mathrm{E}+0131.000 \mathrm{E}+013 \mathrm{BIOFAC}(18,1)$

$\mathrm{D}-5{ }^{3} \mathrm{U}-234$, crustacea and mollusks, ${ }^{3} 6.000 \mathrm{E}+01 \mathbf{3}^{3}$ 6. $600 \mathrm{E}+01 \mathrm{3}^{3} \mathrm{BIOFAC}(18,2)$

$\mathrm{D}-5$ 3

D-5 3 U-235+D, fish

1. $1.00 \mathrm{E}+0131.000 \mathrm{E}+011^{3} \operatorname{BIOFAC}(19,1)$

$\mathrm{D}-5{ }^{3} \mathrm{U}-235+\mathrm{D}$, crustacea and mollusks, ${ }^{3} 6.000 \mathrm{E}+01 \mathrm{3}^{3}$ 6.000E+01 3 BIOFAC( 19,2$)$

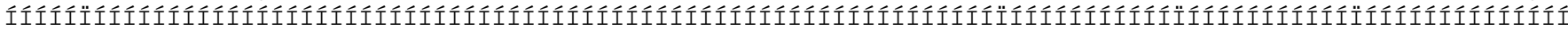

*Base Case means Default.Lib w/o Associate Nuclide contributions. 
Site-Specific Parameter Summary

Used by RESRAD 3 Parameter

Menu ${ }^{3}$

Parameter

3 Input

3

1.000E+O2 3 1.

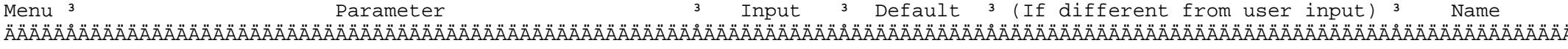

R011 3 Area of contaminated zone $(\mathrm{m} * * 2)$

R011 3 Thickness of contaminated zone $(\mathrm{m})$

R011 3 Length parallel to aquifer flow $(\mathrm{m})$

R011 3 Basic radiation dose limit (mrem/yr)

R011 3 Time since placement of material (yr)

R011 3 Times for calculations ( $\mathrm{yr}$ )

R011 3 Times for calculations ( $\mathrm{yr}$ )

R011 3 Times for calculations (yr)

R011 3 Times for calculations ( $\mathrm{yr}$ )

R011 3 Times for calculations ( $\mathrm{yr}$ )

R011 3 Times for calculations ( $\mathrm{yr}$ )

R011 3 Times for calculations ( $y r$ )

R011 3 Times for calculations ( $\mathrm{yr}$ )

R011 3 Times for calculations ( $y r$ )

R012 3 Initial principal radionuclide ( $\mathrm{pCi} / \mathrm{g})$ : $\mathrm{Am}-241$

R012 3 Initial principal radionuclide (pCi/g):

R012 3 Initial principal radionuclide ( $\mathrm{Ci} / \mathrm{g}$ ):

012 Initial principal radionuclide (pCi/g): Eu-152

R012 Initial principal radionuclide (pci/g):

Initial principal radionuclide (pCi/g): $\mathrm{Pu}-239$

$1.500 \mathrm{E}-01^{3} 2.000 \mathrm{E}+00$

not used $31.000 \mathrm{E}+02$

$2.500 \mathrm{E}+011^{3} \quad 3.000 \mathrm{E}+01$

$0.000 \mathrm{E}+0 \odot$ 3 $0.000 \mathrm{E}+0 \odot$

$1.000 \mathrm{E}+00 \quad 3 \quad 1.000 \mathrm{E}+00$

$3.000 \mathrm{E}+00$ 3 3 3. $000 \mathrm{E}+00$

1. $000 \mathrm{O}+01$ 3 $1.000 \mathrm{H}+1$

$1.000 \mathrm{CO}$ ( $1.00 \mathrm{C}+01$

$3.000+01$ 1. $3.000 \mathrm{E}+01$

$5.000 \mathrm{E}+01$ 1 $1.000 \mathrm{E}+02$

$7.500 \mathrm{E}+011^{3} 3.000 \mathrm{E}+02$

$1.000 \mathrm{E}+022^{3} 1.000 \mathrm{E}+03$

$3.000 \mathrm{E}+02$ 3 $0.000 \mathrm{E}+00$

$\begin{array}{llll}1.000 \mathrm{E}+03 & 3 & 0.000 \mathrm{E}+00 & 3 \\ & 3 & & 3\end{array}$

2. $880 \mathrm{E}+013 \quad 3 \quad 0.000 \mathrm{E}+00^{3}$

$1.06 \odot E+03 \quad 3 \quad 0.00 \odot \mathrm{E}+0 \Theta^{3}$

$2.640 \mathrm{E}+0 \odot \quad 3 \quad 0.0 \odot \odot \mathrm{E}+0 \Theta^{3}$

$1.560 \mathrm{E}+01 \quad 3 \quad 0.000 \mathrm{E}+00$

$8.400 \mathrm{E}+01$ 3 $0.000 \mathrm{E}+0 \odot$

$\mathrm{Sr}-90 \quad 3 \quad 3.410 \mathrm{E}+01 \quad 3 \quad 0.000 \mathrm{E}+00$

R012 3 Concentration in groundwater (pCi/L): Am-241 3 not used $30.000 \mathrm{E}+00$

R012 3 Concentration in groundwater (pCi/L): $\mathrm{Cs}-1373$ not used $30.000 \mathrm{E}+00$

R012 3 Concentration in groundwater ( $\mathrm{PCi} / \mathrm{L}$ ): $\mathrm{Eu}-1523$ not used 3 $0.000 \mathrm{E}+00$

R012 concentration in groundwater

R012 3 Concentration in groundwater ( $\mathrm{pCi} / \mathrm{L})$ : $\mathrm{Pu}-238{ }^{3}$ not used $30.000 \mathrm{E}+00$

R012 3 Concentration in groundwater (pCi/L): Pu-239 3 not used $30.000 \mathrm{E}+00$

$\mathrm{R} 0123$ Concentration in groundwater (pCi/L): $\mathrm{Sr}-90$

R013 3 Cover depth $(\mathrm{m})$

R013 3 Density of cover material $\left(\mathrm{g} / \mathrm{cm}^{*} 3\right)$

R013 3 Cover depth erosion rate $(\mathrm{m} / \mathrm{yr}$ )

R013 3 Density of contaminated zone $\left(\mathrm{g} / \mathrm{cm}^{* *} 3\right)$

R013 3 Contaminated zone erosion rate (m/yr)

R013 3 Contaminated zone total porosity

R013 3 Contaminated zone field capacity

not used 3 $९ .000 \mathrm{E}+0{ }_{3}$

$0.000 \mathrm{E}+00 \quad 3 \quad 0.000 \mathrm{E}+00$

3 not used $31.500 E+00^{3}$

not used $31.000 \mathrm{E}-03$

$1.500 \mathrm{E}+00 \quad 3 \quad 1.500 \mathrm{E}+00^{3}$

$1.000 \mathrm{E}-03$ 3 $1.000 \mathrm{E}-03$

$4.000 E-01344.000 E-011^{3}$

.

$1.000 \mathrm{E}+01$ 3 $1.000 \mathrm{E}+\odot 1$

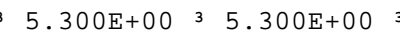

$3.400 \mathrm{E}+0 \mathrm{O}^{3} \quad 2.000 \mathrm{E}+00$

not used 3 8.000E+00

$5.000 \mathrm{E}-013 \quad 5.000 \mathrm{E}-01$

$3.200 E-01{ }^{3} 1.000 E+00{ }^{3}$

(.).

$0.000 E+00$ 2.000E- 01

overhead 3 overhead

4.000E-01 3 2.000E-01

R013 3 Irrigation (m/yr)

$\begin{array}{lll}\mathrm{R} 013 & 3 & \text { Irrigation mode } \\ \mathrm{R} 013 & 3 & \text { Runoff coefficien }\end{array}$

$\begin{array}{llll}\mathrm{R} 013 & 3 & \text { Watershed area for nearby stream or pond }\left(m^{* *} 2\right) & 3 \\ \text { R013 not used } & 3 & 1.000 \mathrm{E}+06 \\ \text { Accuracy for water/soil computations } & 3 \text { not used } & 3.000 \mathrm{E}-03\end{array}$

R013 3 Accuracy for water/soil computations 3

3 not used $31.500 \mathrm{E}+00$

R014 3 Density of saturated zone $\left(\mathrm{g} / \mathrm{cm}^{* *} 3\right)$

R014 3 Saturated zone total porosity

R014 3 Saturated zone effective porosity

3 not used 4 .

3 not used $32.000 \mathrm{E}-01$

$\begin{array}{ll}-- & 3 \text { AREA } \\ -\cdot- & 3 \text { THICKO }\end{array}$

3 LCZPAQ

3 BRDL

3 TI

$3 \mathrm{~T}(2)$

$3 \mathrm{~T}(3)$

$3 \mathrm{~T}(4)$

-. 3 T( 5$)$

-. 3 T( 6$)$

$3 \mathrm{~T}(7)$

$\begin{array}{ll}3 & T( \\ 3 & 8 \\ 3 & T(9) \\ 3 & T(10)\end{array}$

$--$

$-\cdot$

$-\cdot-$

$3 \mathrm{~S} 1(4)$

$3 \mathrm{~S} 1(10)$

$\begin{array}{ll}3 & \mathrm{~S} 1(12) \\ 3 & \mathrm{~S} 1(14)\end{array}$

$3 \mathrm{~W} 1(\mathrm{2})$

3 W1 ( 3$)$

$--$

${ }^{3} \mathrm{~W} 1(\mathrm{r})$

3 W1 (10)

$\begin{array}{ll}-.- & 3 \mathrm{~W} 1(12) \\ -. & 3 \mathrm{~W} 1(14)\end{array}$

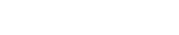

3 DENSCV

3 VCV

-- 3 DENSCZ

- - 3 VCZ

- - 3 TPCZ

-. 3 FCCZ

$-\cdot-$

$\begin{array}{ll}3 \text { BCZ } \\ -\ldots & 3 \text { WIND }\end{array}$

3 HUMID

$-.-$

-. 3 RI

3 IDITCH

3 RUNOFF

- . 3 WAREA

3

3 DENSAQ

- - 3 TPSZ

$\begin{array}{ll}3 & \text { EPSZ } \\ 3 & \text { FCSZ }\end{array}$ 
Site-Specific Parameter Summary (continued)

Parameter

3 Default

3 Used by RESRAD

3 Parameter

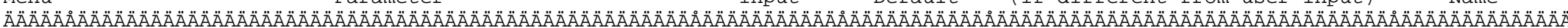

R014 3 Saturated zone hydraulic conductivity (m/yr)

not used $31.000 \mathrm{E}+02$

R014 3 Saturated zone hydraulic gradient

R014 3 Saturated zone b parameter

R014 3 Water table drop rate $(\mathrm{m} / \mathrm{yr})$

R014 3 Well pump intake depth ( $\mathrm{m}$ below water table)

R014 3 Model: Nondispersion (ND) or Mass-Balance (MB)

not use

not used $35.300 \mathrm{E}+\odot \odot$

not used 3 1.000E-03

not used $31.000 \mathrm{E}+013$

not used 3 ND

R014 ${ }^{3}$ Well pumping rate $\left(m^{* *} 3 / \mathrm{yr}\right)$

not used $32.500 \mathrm{E}+02$

R015 3 Number of unsaturated zone strata

R015 3 Unsat. zone 1, thickness (m)

R015 3 Unsat. zone 1 , soil density $\left(\mathrm{g} / \mathrm{cm}^{*} 3\right)$

R015 3 Unsat. zone 1 , total porosity

R015 3 Unsat. zone 1, effective porosity

R015 3 Unsat. zone 1, field capacity

R015 3 Unsat. zone 1 , soil-specific b parameter

R015 3 Unsat. zone 1, hydraulic conductivity (m/yr)

R016 3 Distribution coefficients for Am-241

R016 3 Contaminated zone $\left(\mathrm{cm}^{* *} 3 / \mathrm{g}\right)$

R016 3 Unsaturated zone $1\left(\mathrm{~cm}^{* *} 3 / \mathrm{g}\right)$

R016 3 Saturated zone $\left(\mathrm{cm}^{* *} 3 / \mathrm{g}\right)$

R016 3 Leach rate (/yr)

R016 3 Solubility constant

R016 3 Distribution coefficients for Cs -137

R016 3 Contaminated zone $\left(\mathrm{cm}^{* *} 3 / \mathrm{g}\right)$

R016 3 Unsaturated zone $1\left(\mathrm{~cm}^{*} * 3 / \mathrm{g}\right)$

R016 3 Saturated zone $\left(\mathrm{cm}^{* *} 3 / \mathrm{g}\right)$

R016 3 Leach rate (/yr)

R016 3 Solubility constant

R016 3 Distribution coefficients for Eu-152

R016 3 Contaminated zone $\left(\mathrm{cm}^{* *} 3 / \mathrm{g}\right)$

R016 3 Unsaturated zone $1(\mathrm{~cm} * * 3 / \mathrm{g})$

R016 3 Saturated zone $\left(\mathrm{cm}^{* *} 3 / \mathrm{g}\right)$

R016 3 Leach rate (/yr)

R016 3 Solubility constant

R016 3 Distribution coefficients for $\mathrm{Pu}-238$

R016 3 Contaminated zone $\left(\mathrm{cm}^{* *} 3 / \mathrm{g}\right)$

R016 3 Unsaturated zone $1\left(\mathrm{~cm}^{* *} 3 / \mathrm{g}\right)$

R016 3 Saturated zone $\left(\mathrm{cm}^{* *} 3 / \mathrm{g}\right)$

R016 3 Leach rate (/yr)

R016 3 Solubility constant

R016 3 Distribution coefficients for Pu-239

R016 3 Contaminated zone $\left(\mathrm{cm}^{* *} 3 / \mathrm{g}\right)$

R016 3 Unsaturated zone $1\left(\mathrm{~cm}^{* *} 3 / \mathrm{g}\right)$

R016 3 Saturated zone $\left(\mathrm{cm}^{* *} 3 / \mathrm{g}\right)$

R016 3 Leach rate (/yr)

R016 3 Solubility constant

not used 31

not used $34.000 \mathrm{E}+00$

not used $31.500 \mathrm{E}+00$

not used 3 4.000E-01

not used $32.000 \mathrm{E}-01$

not used 32 2.000E-01

3 not used $35.300 \mathrm{E}+00$

not used 3 1.000E+01

$2.000 \mathrm{E}+01 \quad 3 \quad 2.000 \mathrm{E}+01$

3 not used $32.000 E+01$

not used $32.000 \mathrm{E}+01$

$0.000 \mathrm{E}+00 \quad 3 \quad 0.000 \mathrm{E}+00$

$0.000 \mathrm{E}+0 \odot^{3} \quad 0.000 \mathrm{E}+\Theta \Theta^{3}$

$4.600 \mathrm{E}+03 \quad 3 \quad 4.600 \mathrm{E}+03$

not used 3 4.600E+03

not used 3 4.600E +03

$0.000 \mathrm{E}+00 \quad 3 \quad 0.000 \mathrm{E}+00$

$\odot .000 \mathrm{E}+0 \odot$ 3 $0.000 \mathrm{E}+0 \odot$

- $.000 \mathrm{E}+00^{3}-1.000 \mathrm{~B}+00^{3}$

not used $3-1.000 \mathrm{E}+00$

not used $3-1.000 \mathrm{E}+00$

$\odot .00 \odot E+\odot \odot \quad 3 \quad 0.000 \mathrm{E}+\odot \odot$

$\odot .000 \mathrm{E}+00{ }^{3} \quad 0.000 \mathrm{E}+\Theta \odot$

$2.000 \mathrm{E}+03 \quad 3 \quad 2.000 \mathrm{E}+03$

not used $32.000 \mathrm{E}+03$

not used $32.000 \mathrm{E}+03$

$0.00 \odot E+\odot \odot \quad 3 \quad 0.00 \odot E+\odot \odot$

०.

$2.000 \mathrm{E}+03 \quad 3 \quad 2.000 \mathrm{E}+03$

not used $32.000 \mathrm{E}+03$

not used $32.000 \mathrm{E}+03$

$0.000 \mathrm{E}+0 \odot \quad 3 \quad 0.00 \Theta \mathrm{E}+0 \odot$

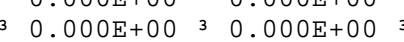

- - $\quad 3 \mathrm{HCSZ}$

3 BSZ

-. 3 VWT

3 DWIBWT

3 MODEL

$--$

- . 3 NS

- - $3 \mathrm{H}(1)$

3 DENSUZ(1)

3 EPUZ (1)

${ }^{3}$ FCUZ (1)

3 BUZ(1)

$\operatorname{HCUZ}(1)$

$\operatorname{DCNUCC}(2)$

DCNUCU( 2,1)

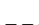

$2.153 E-02$

not used

ALEACH ( 2)

SOLUBK( 2)

DCNUCC ( 3$)$

$\operatorname{DCNUCU}(3,1)$

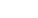

$9.449 \mathrm{E}-05$

not used

ALEACH ( 3 )

SOLUBK( 3 )

$8.249 \mathrm{E}+02$

$-$

$5.268 \mathrm{E}-04$

not used

DCNUCC ( 4)

DCNUCU $(4,1)$

DCNUCS $(4)$

ALEACH ( 4$)$

$\operatorname{DCNUCC}(10)$

$-$

$-$

$2.173 \mathrm{E}-\mathrm{O}$

$\operatorname{DCNUCU}(10,1)$

3 DCNUCS (10)

3 ALEACH (10)

not used

$-$

$2.173 \mathrm{E}-04$

not used
$3 \operatorname{DCNUCC}(12)$

$\operatorname{DCNUCU}(12,1)$

3 DCNUCS (12)

$\begin{array}{ll}3 & \operatorname{ALEACH}(12) \\ 3 & \operatorname{SOLUBK}(12)\end{array}$ 
Site-Specific Parameter Summary (continued)

$0^{3}{ }^{3}$

Parameter

User

Used by RESRAD

Used by RESRAD 3 Parameter

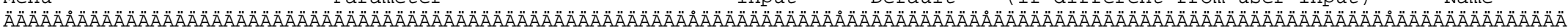

R016 3 Distribution coefficients for Sr-90

R016 3 Contaminated zone $\left(\mathrm{cm}^{* *} 3 / \mathrm{g}\right)$

R016 3 Unsaturated zone $1\left(\mathrm{~cm}^{* *} 3 / \mathrm{g}\right)$

R016 3 Saturated zone $\left(\mathrm{cm}^{* *} 3 / \mathrm{g}\right)$

R016 3 Leach rate (/yr)

R016 3 Solubility constant

R016 3 Distribution coefficients for daughter AC-227

R016 3 Contaminated zone $\left(\mathrm{cm}^{* *} 3 / \mathrm{g}\right)$

R016 3 Unsaturated zone $1\left(\mathrm{~cm}^{* *} 3 / \mathrm{g}\right)$

R016 3 Saturated zone $\left(\mathrm{cm}^{* *} 3 / \mathrm{g}\right)$

R016 3 Leach rate (/yr)

R016 ${ }^{3} \quad$ Solubility constant

R016 3 Distribution coefficients for daughter Gd-152

R016 3 Contaminated zone $\left(\mathrm{cm}^{* *} 3 / \mathrm{g}\right)$

R016 3 Unsaturated zone $1(\mathrm{~cm} * * 3 / \mathrm{g})$

R016 3 Saturated zone $\left(\mathrm{cm}^{* *} 3 / \mathrm{g}\right)$

R016 3 Leach rate (/yr)

R016 ${ }^{3}$ Solubility constant

R016 3 Distribution coefficients for daughter Np-237

R016 3 Contaminated zone $\left(\mathrm{cm}^{* *} 3 / \mathrm{g}\right)$

R016 3 Unsaturated zone $1\left(\mathrm{~cm}^{* *} 3 / \mathrm{g}\right)$

R016 3 Saturated zone $\left(\mathrm{cm}^{* *} 3 / \mathrm{g}\right)$

R016 3 Leach rate (/yr)

R016 3 Solubility constant

R016 3 Distribution coefficients for daughter Pa-231

R016 3 Contaminated zone $\left(\mathrm{cm}^{* *} 3 / \mathrm{g}\right)$

R016 3 Unsaturated zone $1\left(\mathrm{~cm}^{* *} 3 / \mathrm{g}\right)$

R016 3 Saturated zone $\left(\mathrm{cm}^{* *} 3 / \mathrm{g}\right)$

R016 3 Leach rate (/yr)

R016 3 Solubility constant

R016 3 Distribution coefficients for daughter $\mathrm{Pb}-210$

R016 3 Contaminated zone $\left(\mathrm{cm}^{* *} 3 / \mathrm{g}\right)$

R016 3 Unsaturated zone $1\left(\mathrm{~cm}^{* *} 3 / \mathrm{g}\right)$

R016 3 Saturated zone $\left(\mathrm{cm}^{* *} 3 / \mathrm{g}\right)$

R016 3 Leach rate (/yr)

R016 3 Solubility constant

R016 3 Distribution coefficients for daughter Ra-226

R016 3 Contaminated zone $\left(\mathrm{cm}^{* *} 3 / \mathrm{g}\right)$

R016 3 Unsaturated zone $1(\mathrm{~cm} * * 3 / \mathrm{g})$

R016 3 Saturated zone $\left(\mathrm{cm}^{* *} 3 / \mathrm{g}\right)$

R016 3 Leach rate (/yr)

33

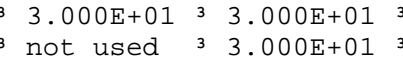

not used $33.000 E+01$

$0.000 \mathrm{E}+00^{3} \quad 0.000 \mathrm{E}+00^{3}$

$0.000 \mathrm{E}+\Theta \Theta^{3} \quad 0.000 \mathrm{E}+\Theta \Theta^{3}$

$2.000 \mathrm{E}+01 \quad 3 \quad 2.000 \mathrm{E}+01$

not used 3 2. $000 \mathrm{E}+01$

not used $32.000 \mathrm{E}+01$

$0.000 \mathrm{E}+00 \quad 3 \quad 0.000 \mathrm{E}+00$

$0.000 \mathrm{E}+00 \quad 3 \quad 0.000 \mathrm{E}+00$

3

$-1.000 \mathrm{E}+00^{3}-1.000 \mathrm{E}+00$

not used $3-1.000 \mathrm{E}+00$

not used $3-1.00 \odot E+\odot \odot$
not used $3-1.000 E+\odot \odot$

$30.00 \odot \mathrm{E}+\odot \odot$ 3 $\odot .00 \odot \mathrm{E}+\odot \odot$

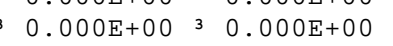

$-1.000 \mathrm{E}+00 \quad 3-1.000 \mathrm{E}+0 \odot$

not used $3-1.000 E+00$

not used 3

not used $-1.000 \mathrm{E}+0 \odot$

$\odot . \odot \odot \odot E+\odot \odot$ $0 . \odot \odot \odot E+\odot \odot$

$\odot . \odot \odot \odot E+\odot \odot \quad 3 \quad 0 . \odot \odot \odot E+\odot \odot$

$35.000 \mathrm{E}+01 \quad 3 \quad 5.000 \mathrm{E}+01$

not used $35.000 \mathrm{E}+01$

not used $35.000 \mathrm{E}+01$

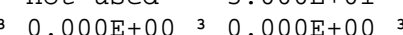

๑.

$1.000 \mathrm{E}+02 \quad 3 \quad 1.000 \mathrm{E}+02$

3 not used $31.000 \mathrm{E}+02$

3 not used 3 1.000E+02

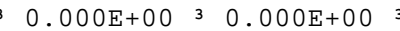

$\odot .00 \odot E+\odot \odot \quad 3 \quad \odot . \odot \odot \odot E+\odot \odot$

$7.000 \mathrm{E}+01 \quad 3 \quad 7.000 \mathrm{E}+01$

not used 3 7.000E+01

not used 3 7.000E +01

$0.000 \mathrm{E}+003 \quad 0.000 \mathrm{E}+0 \odot$

๑.

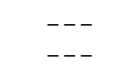

- -

$1.440 \mathrm{E}-02$

not used

$--$

- - -

$2.153 \mathrm{E}-02$

not used

8. $249 \mathrm{E}+02$

- -

$5.268 \mathrm{E}-\mathrm{-} 4$

not used

2. $574 \mathrm{E}+02$

- -

$1.687 \mathrm{E}-03$

not used

$-$

$-\cdots$

$8.660 \mathrm{E}-03$

not used

- -

$-$

4. $338 \mathrm{E}-03$

not used

$-$

(1)

$6.193 \mathrm{E}-03$

not used
DCNUCC (14)

$\operatorname{DCNUCU}(14,1)$

DCNUCS $(14)$

ALEACH (14)

$\operatorname{DCNUCC}(1)$

$\operatorname{DCNUCU}(1,1)$

DCNUCS ( 1)

ALEACH ( 1)

$\operatorname{DCNUCC}(6)$

$\operatorname{DCNUCU}(6,1)$

DCNUCS( 6 )

ALEACH ( 6)
SOLUBK( 6)

DCNUCC ( 7)

$\operatorname{DCNUCU}(7,1)$

DCNUCS( 7 )

ALEACH ( 7$)$

SOLUBK( 7$)$

DCNUCC ( 8)

$\operatorname{DCNUCU}(8,1)$

DCNUCS( 8 )

ALEACH( 8 )

SOLUBK( 8 )

DCNUCC ( 9 )

3 DCNUCU $(9,1)$

DCNUCS( 9)

ALEACH $(9)$

SOLUBK( 9)

$\operatorname{DCNUCC}(13)$

$3 \operatorname{DCNUCU}(13,1)$

3 DCNUCS(13)

3 ALEACH (13)

3 SOLUBK(13) 
Site-Specific Parameter Summary (continued)

Menu 3

3 User

Used by RESRAD

3 Parameter

3 Input 3 Default 3 (If different from user input) 3 Name

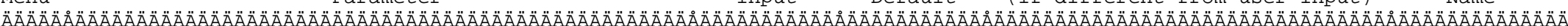

R016 3 Distribution coefficients for daughter Th-229

R016 3 Contaminated zone $\left(\mathrm{cm}^{* *} 3 / \mathrm{g}\right)$

R016 3 Unsaturated zone $1\left(\mathrm{~cm}^{* *} 3 / \mathrm{g}\right)$

R016 3 Saturated zone $\left(\mathrm{cm}^{* *} 3 / \mathrm{g}\right)$

R016 3 Leach rate (/yr)

R016 3 Solubility constant

R016 3 Distribution coefficients for daughter Th-230

R016 3 Contaminated zone $\left(\mathrm{cm}^{* *} 3 / \mathrm{g}\right)$

R016 3 Unsaturated zone $1\left(\mathrm{~cm}^{* *} 3 / \mathrm{g}\right)$

R016 3 Saturated zone $\left(\mathrm{cm}^{* *} 3 / \mathrm{g}\right)$

R016 3 Leach rate (/yr)

R016 ${ }^{3}$ Solubility constant

R016 3 Distribution coefficients for daughter U-233

R016 3 Contaminated zone $\left(\mathrm{cm}^{* *} 3 / \mathrm{g}\right)$

R016 3 Unsaturated zone $1(\mathrm{~cm} * * 3 / \mathrm{g})$

R016 3 Saturated zone $\left(\mathrm{cm}^{* *} 3 / \mathrm{g}\right)$

R016 3 Leach rate (/yr)

R016 ${ }^{3}$ Solubility constant

R016 3 Distribution coefficients for daughter U-234

R016 3 Contaminated zone $\left(\mathrm{cm}^{* *} 3 / \mathrm{g}\right)$

R016 3 Unsaturated zone $1\left(\mathrm{~cm}^{* *} 3 / \mathrm{g}\right)$

R016 3 Saturated zone $\left(\mathrm{cm}^{* *} 3 / \mathrm{g}\right)$

R016 3 Leach rate (/yr)

R016 3 Solubility constant

R016 3 Distribution coefficients for daughter U-235

R016 3 Contaminated zone $\left(\mathrm{cm}^{* *} 3 / \mathrm{g}\right)$

R016 3 Unsaturated zone $1\left(\mathrm{~cm}^{* *} 3 / \mathrm{g}\right)$

R016 3 Saturated zone $\left(\mathrm{cm}^{* *} 3 / \mathrm{g}\right)$

R016 3 Leach rate (/yr)

R016 3 Solubility constant

R017 3 Inhalation rate $\left(\mathrm{m}^{* *} 3 / \mathrm{yr}\right)$

R017 3 Mass loading for inhalation $(\mathrm{g} / \mathrm{m} * * 3)$

R017 3 Exposure duration

R017 3 Shielding factor, inhalation

R017 3 Shielding factor, external gamma

R017 3 Fraction of time' spent indoors

R017 3 Fraction of time spent outdoors (on site)

$6.000 \mathrm{E}+04 \quad 3 \quad 6.000 \mathrm{E}+04$

not used $36.000 \mathrm{E}+04$

not used 3 6.000E+O4

$0.000 \mathrm{E}+0 \odot$ उ $0.000 \mathrm{E}+\Theta \odot$

$0.000 \mathrm{E}+\Theta \Theta^{3} \quad 0.000 \mathrm{E}+\Theta \Theta^{3}$

$36.000 \mathrm{E}+\odot 4 \quad 3 \quad 6.000 \mathrm{E}+\odot 4$

not used 3 6.000E+O4

not used $36.000 \mathrm{E}+\Theta 4$

$\begin{array}{llll}3 & 0.000 \mathrm{E}+00 & 3 & 0.000 \mathrm{E}+00^{3}\end{array}$

$0.000 \mathrm{E}+00^{3} \quad 0.000 \mathrm{E}+00^{3}$

5. $\odot \odot \odot \mathrm{E}+01 \quad 3 \quad 5.0 \odot \odot \mathrm{E}+01$

not used $35.000 \mathrm{E}+01$

$\begin{array}{lll}\text { not used } & 3 & 5.000 \mathrm{E}+01 \\ \text { not used } & 3 & 5.000 \mathrm{E}+01\end{array}$

$\odot .000 \mathrm{E}+\odot \odot 3 \quad \odot .000 \mathrm{E}+\odot \odot$

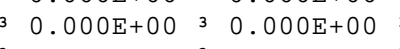

$5.000 \mathrm{E}+01 \quad 3 \quad 5.000 \mathrm{E}+01$

not used $35.000 E+01$

not used $5.000 \mathrm{C}+01$

not Used $5.000 \mathrm{E}+01$

$0.000 \mathrm{E}+00$ 3 $0.000 \mathrm{E}+00$

$\odot . \odot \odot \odot E+\odot \odot \quad 3 \quad \odot . \odot \odot \odot E+\odot \odot$

3. $5.000 \mathrm{E}+01 \quad 3 \quad 5.000 \mathrm{E}+01$

not used 3 5. $000 \mathrm{E}+01$

not used $35.000 \mathrm{E}+01$

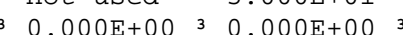

$\odot .00 \odot E+\odot \odot \quad 3 \quad \odot .00 \odot E+\odot \odot 3$

$1.230 \mathrm{E}+04 \quad 3 \quad 8.400 \mathrm{E}+03$

6.000E-04 3 1.000E- 04

$2.500 \mathrm{E}+0133.000 \mathrm{E}+01$

$1.000 \mathrm{E}+003 \quad 4.000 \mathrm{E}-01$

$1.000 \mathrm{E}+00$ 3 $7.000 \mathrm{E}-01$

$0.000 \mathrm{E}+00 \quad 3 \quad 5.000 \mathrm{E}-01$

3. 800E-02 3 2.500E- 01

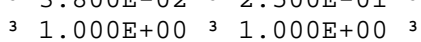

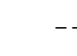

$--$

7. 244E- 06

not used

$--$

$-$

$7.244 \mathrm{E}-06$

not used

- - -

8. $660 \mathrm{E}-03$

not used

- - -

$-$

$8.660 \mathrm{E}-03$

not used

$--$

$-\ldots$

$8.660 \mathrm{E}-03$

not used

$--$

$--$

$--$

$-n^{-1}$

$-$

$>0$ shows circular AREA
$\operatorname{DCNUCC}(15)$

$\operatorname{DCNUCU}(15,1)$

DCNUCS $(15)$

3 ALEACH(15)

SOLUBK(15)

$\operatorname{DCNUCC}(16)$

$\operatorname{DCNUCU}(16,1)$

DCNUCS (16)

ALEACH (16)

SOLUBK(16)

$\operatorname{DCNUCC}(17)$

$\operatorname{DCNUCU}(17,1)$

DCNUCS $(17)$

ALEACH (17)

SOLUBK (17)

DCNUCC (18)

$\operatorname{DCNUCU}(18,1)$

DCNUCS (18)

ALEACH (18)

SOLUBK (18)

DCNUCC (19)

$\operatorname{DCNUCU}(19,1)$

DCNUCS $(19)$

3 ALEACH (19)

3 SOLUBK(19)

INHALR

3 MLINH

3 ED

3 SHF3

3 SHF1

3 FIND

$\begin{array}{ll}3 & \text { FOT } \\ 3 & \text { FS }\end{array}$ 
Site-Specific Parameter Summary (continued)

Menu ${ }^{3}$

Unput 3 Default 3 (If different from use

ÄÄÄÄÄÄÄÄÄÄÄÄÄÄÄÄÄÄÄÄÄÄÄÄÄÄÄÄÄÄÄÄÄÄÄÄÄÄÄÄÄÄÄÄÄÄÄÄÄÄÄÄÄ

Used by RESRAD 3 Parameter

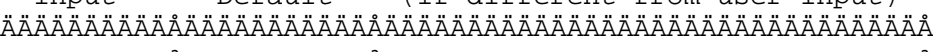

R017 3 Radii of shape factor array (used if FS = -1):

$\mathrm{R} 017^{3}$ Outer annular radius $(\mathrm{m})$, ring 1 :

R017 3 Outer annular radius (m), ring 2:

R017 3 Outer annular radius (m), ring 3 :

R017 3 Outer annular radius $(\mathrm{m})$, ring 4:

R017 3 Outer annular radius $(\mathrm{m})$, ring 5:

$\mathrm{R} 017^{3}$ Outer annular radius $(\mathrm{m})$, ring 6 :

$\mathrm{R} 017^{3}$ Outer annular radius $(\mathrm{m})$, ring 7 :

R017 3 Outer annular radius $(\mathrm{m})$, ring 8:

R017 3 Outer annular radius $(\mathrm{m})$, ring 9:

R017 3 Outer annular radius $(\mathrm{m})$, ring 10:

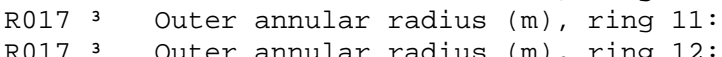

R017 3 Fractions of annular areas within AREA

R017 3 Ring 1

$\begin{array}{llll}\mathrm{R} 017 & 3 & \text { Ring } & 1 \\ \mathrm{R} 017 & 3 & \text { Ring } & 2\end{array}$

$\begin{array}{llll}R 017 & 3 & \text { Ring } & 2 \\ R 017 & 3 & \text { Ring } & 3\end{array}$

$\begin{array}{llll}R 017 & 3 & \text { Ring } & 4\end{array}$

R017 3 Ring 5

$\begin{array}{llll}R 017 & 3 & \text { Ring } & 6 \\ R 017 & 3 & \text { Ring } & 7\end{array}$

R017 3 Ring 8

R017 3 Ring 9

not used $35.000 \mathrm{E}+01^{3}$

not used $3 \quad 7.071 \mathrm{E}+01$

not used 3 ०. $000 \mathrm{E}+00$

not used $30.000 \mathrm{E}+00$

3 not used $30.000 \mathrm{E}+0 \odot^{3}$

3 not used $30.000 \mathrm{E}+00^{3}$

not used 3 ०. $000 \mathrm{E}+0 \odot$

not used 3 ०. $000 \mathrm{E}+0 \Theta^{3}$

not used $3 \quad 0.000 \mathrm{E}+0 \odot$

not used $30.000 \mathrm{E}+\Theta \odot$

not used 3 ०.000E+00

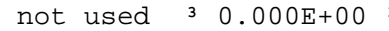

3

not used $3 \quad 1.000 \mathrm{E}+00$

not used 3 2.732E-01

not used $30.000 \mathrm{E}+0{ }^{3}$

not used $30.000 \mathrm{E}+\odot \odot^{3}$

not used $30.00 \odot E+\odot \odot$

3 not used $30.000 \mathrm{E}+00^{3}$

3 not used $30.000 \mathrm{E}+\Theta \Theta^{3}$

3 not used 3 ๑. $\odot \odot \odot E+\Theta \odot$

3 not used 3 O. $000 \mathrm{E}+00^{3}$

3 not used $30.000 E+00$

not used $30.000 \mathrm{E}+00^{3}$

not used $30.000 \mathrm{E}+\odot \odot^{3}$

R017 3 Ring 12

R018 3 Fruits, vegetables and grain consumption (kg/yr) R018 3 Leafy vegetable consumption ( $\mathrm{kg} / \mathrm{yr}$ )

R018 3 Milk consumption ( $\mathrm{L} / \mathrm{yr}$ )

R018 3 Meat and poultry consumption ( $\mathrm{kg} / \mathrm{yr}$ )

R018 3 Fish consumption ( $\mathrm{kg} / \mathrm{yr}$ )

R018 3 Other seafood consumption ( $k g / y r)$

R018 3 Soil ingestion rate (g/yr)

R018 3 Drinking water intake (L/yr)

R018 3 Contamination fraction of drinking water

R018 3 Contamination fraction of household water

R018 3 Contamination fraction of livestock water

R018 3 Contamination fraction of irrigation water

R018 3 Contamination fraction of aquatic food

R018 3 Contamination fraction of plant food

R018 3 Contamination fraction of meat

R018 3 Contamination fraction of milk

R019 3 Livestock fodder intake for meat ( $\mathrm{kg} /$ day) R019 3 Livestock fodder intake for milk ( $\mathrm{kg} / \mathrm{day}$ )

R019 3 Livestock water intake for meat (L/day)

R019 3 Livestock water intake for milk (L/day)

R019 3 Livestock soil intake ( $\mathrm{kg} /$ day)

R019 3 Mass loading for foliar deposition $\left(\mathrm{g} / \mathrm{m}^{* *} 3\right)$
3 not used

3 not used

3 not used

not used 3 6.300E+01

not used $35.400 E+00$

not used 3 .

$\begin{array}{lll}1.752 \mathrm{E}+02 & 3 & 3.650 \mathrm{E}+01 \\ 3\end{array}$

not used $35.100 \mathrm{E}+02$

not used $31.000 \mathrm{E}+00$

not used 3 1.000E+००

not used $31.000 \mathrm{E}+00$

not used $31.000 \mathrm{E}+0 \mathrm{O}^{3}$

not used 3 5.000E-01

not used $3-1$

not used $3-1$

not used 3 6.800E+01

not used $35.500 \mathrm{E}+01$

not used $31.00 \mathrm{E}+01$

3 not used 3 . $500 \mathrm{E}+02$

3 not used 3 1. $10 \odot \odot \mathrm{E}-\odot 4$
$--$

$-$

$-\cdots$

$-\cdot$

$-$

$-$

$-$

$-$

$-$

$--$

$-$

$--$

$-$

$--$

$-$

$-$

$---$

$---$

$-$

$-$

$--$

$--$

$--$

$--$

$---$

$---$

$--$

$-\cdot$

$-\cdot$

$--$

$x^{-1}$

$-\cdot$

$-\ldots$

$--$

$--$
ÄÄÄÄÄÄÄÄÄÄÄÄ

RAD_SHAPE ( 1$)$

3 RAD_SHAPE ( 2)

3 RAD_SHAPE ( 3 )

3 RAD_SHAPE ( 4$)$

3 RAD_SHAPE( 5 )

3 RAD_SHAPE $(6)$

3 RAD_SHAPE $(7)$

3 RAD_SHAPE ( 8)

3 RAD_SHAPE ( 9$)$

3 RAD_SHAPE (10)

3 RAD_SHAPE (11)

FRACA( 1)

3 FRACA ( 2)

3 FRACA ( 3 )

FRACA( 4)

FRACA( 5)

FRACA ( 6)

3 FRACA ( 7)

FRACA ( 8)

FRACA( 9)

3 FRACA (10)

3 FRACA (11)

3 FRACA (12)

DIET(1)

3 DIET (3)

DIET (4)

DIET (5)

$3 \operatorname{DIET}(6)$

3 SOIL

3 DWI

3 FDW

3 FHHW

3 FLW

3 FIRW

3 FPLANT

3 FMEAT

3 FMILK

3 LFI5

3 LFI6

3 LWI5

3 LWI6

3 MLFD 
Site-Specific Parameter Summary (continued)

$0^{3}{ }^{3}$

Parameter

3 User

3 Input 3 Default 3 (If different from user input) 3 Name

3 Used by RESRAD

3 Parameter

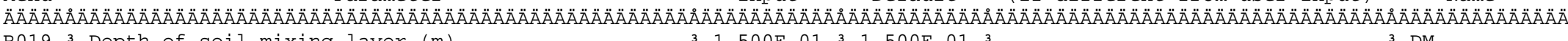

R019 3 Depth of soil mixing layer (m) $31.500 \mathrm{E}-0131.500 \mathrm{E}-013$

R019 3 Depth of roots $(\mathrm{m}) \quad 3$ not used $39.000 \mathrm{E}-01$

R019 3 Drinking water fraction from ground water

R019 3 Household water fraction from ground water

R019 3 Livestock water fraction from ground water

not used $31.000 \mathrm{E}+00$

3 not used $31.000 \mathrm{E}+0 \odot$

R019 3 Irrigation fraction from ground water

not used $31.000 \mathrm{E}+00$

not used $31.000 \mathrm{E}+00^{3}$

R19B 3 Wet weight crop yield for Non-Leafy $\left(\mathrm{kg} / \mathrm{m}^{* *} 2\right)$

R19B 3 Wet weight crop yield for Leafy $(\mathrm{kg} / \mathrm{m} * * 2)$

R19B 3 Wet weight crop yield for Fodder $\left(\mathrm{kg} / \mathrm{m}^{* *} 2\right)$

not used $37.000 \mathrm{E}-01$

3 not used $31.500 \mathrm{E}+00$

not used 3 1.100E+00

not used $31.700 \mathrm{E}-01$

R19B 3 Growing Season for Non-Leafy (years)

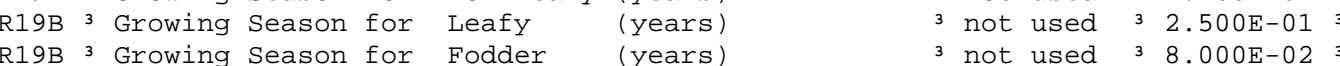

R19B 3 Translocation Factor for Non-Leafy 3 not used $31.000 \mathrm{E}-01$

R19B 3 Translocation Factor for Leafy 3 not used $31.000 \mathrm{E}+00^{3}$

R19B 3 Translocation Factor for Fodder 3 not used $31.000 \mathrm{E}+00^{3}$

R19B 3 Dry Foliar Interception Fraction for Non-Leafy 3 not used $32.500 \mathrm{E}-013$

R19B 3 Dry Foliar Interception Fraction for Leafy 3 not used $32.500 \mathrm{E}-011^{3}$

R19B 3 Dry Foliar Interception Fraction for Fodder 3 not used $32.500 \mathrm{E}-01$

R19B 3 Wet Foliar Interception Fraction for Non-Leafy 3 not used $32.500 \mathrm{E}-01^{3}$

R19B 3 Wet Foliar Interception Fraction for Leafy 3 not used $32.500 \mathrm{E}-01$

R19B 3 Wet Foliar Interception Fraction for Fodder

R19B 3 Weathering Removal Constant for Vegetation

C14 3 C-12 concentration in water $\left(\mathrm{g} / \mathrm{cm}^{* *} 3\right)$

C14 3 C-12 concentration in contaminated soil $(\mathrm{g} / \mathrm{g})$

C14 3 Fraction of vegetation carbon from soil

C14 3 Fraction of vegetation carbon from air

C14 3 C-14 evasion layer thickness in soil $(\mathrm{m})$

C14 3 C-14 evasion flux rate from soil (1/sec)

C14 3 C-12 evasion flux rate from soil ( $1 / \mathrm{sec})$

C14 3 Fraction of grain in beef cattle feed

C14 3 Fraction of grain in milk cow feed

3 not used $32.500 \mathrm{E}-013$

3 not used

$32.000 \mathrm{E}+01$

3 not used $32.000 \mathrm{E}-05$

not used $33.000 \mathrm{E}-02$

not used $32.000 \mathrm{E}-02$

not used 3 9.800E- 01

not used 3 3.000E-01

3 not used 3 7.000E- 07

not used 3 1. $000 \mathrm{E}-10$

not used 3 \&.000E-01

3 not used 3 2.000E-01

C14 3 DCF correction factor for gaseous forms of C14 3 not used $30.000 \mathrm{E}+00^{3}$

STOR 3 Storage times of contaminated foodstuffs (days): 3

STOR 3 Fruits, non-leafy vegetables, and grain

STOR 3 Leafy vegetables

STOR 3 Milk

STOR 3 Meat and poultry

STOR 3 Fish

STOR 3 Crustacea and mollusks

STOR 3 Well water

STOR 3 Surface water

STOR 3 Livestock fodder

R021 3 Thickness of building foundation ( $m$ )

RO21 3 Bulk density of building foundation $\left(\mathrm{g} / \mathrm{cm}^{* *} 3\right)$

RO21 3 Total porosity of the cover material

(a)

$1.400 \mathrm{E}+01 \quad 3 \quad 1.400 \mathrm{E}+01$

$31.000 \mathrm{E}+00$ 3 $1.000 \mathrm{E}+00$

$1.000 \mathrm{E}+00 \quad 3 \quad 1.000 \mathrm{E}+00$

2. $000 \mathrm{E}+01 \quad 3 \quad 2.000 \mathrm{E}+01$

$7.000 \mathrm{E}+00^{3} \mathrm{3} .000 \mathrm{E}+01 \mathrm{O}^{3}$

$37.0000+00^{3} 7.000 E+00$

$1.000 \mathrm{E}+00$ 3 $1.000 \mathrm{E}+0 \mathrm{O}^{3}$

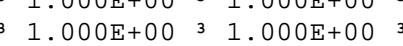

$4.500 E+013 \quad 4.500 E+013$

3 not used $31.500 \mathrm{E}-01$

3 not used 3 2, $400 \mathrm{E}+00^{3}$

3 not used $34.000 \mathrm{C}-01$

3 not used 3 . $1.000 \mathrm{C}-01$

Ro21 3 Total porosity of the building foundation

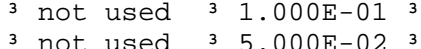

- - $\quad 3$ DM

3 FGWDW

3 FGWIR

$-\cdot-$

$-$

$\begin{array}{ll}3 & \mathrm{TE}(2) \\ 3 & \mathrm{TE}(3)\end{array}$

3 TIV (1)

$3 \operatorname{TIV}(2)$

3 TIV $(3)$

$3 \operatorname{RDRY}(1)$

$3 \operatorname{RDRY}(2)$

$3 \operatorname{RDRY}(3)$

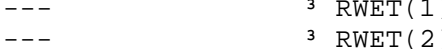

3 RWET (3)

$---$

C12WTR

$--$

- -

$--$

$--$

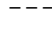

$--$

$-\cdot$

$-$

$--$

$--$

$-$

$--$

$---$

$-\cdot$

$-$

- -

- -

$---$

$--$
C12CZ

CSOIL

3 CAIR

DMC

3 EVSN

REVSN

AVFG5

3 CO2F

STOR_T(1)

3 STOR_T(2)

3 STOR_T( 3 )

3 STOR_T(4)

3 STOR_T(5)

3 STOR_T( 6)

STOR_T( 7)

$\begin{array}{ll}3 & \text { STOR_T( } 8) \\ 3 & \text { STOR_T (9) }\end{array}$

3 FLOOR1

3 DENSFL

3 TPCV

3 PH2OCV 
Site-Specific Parameter Summary (continued)

Menu 3 Menu 3 Parameter 3 Input 3 Default 3 (If different from user input) 3 Name Used by RESRAD Used by RESRAD 3 Parameter

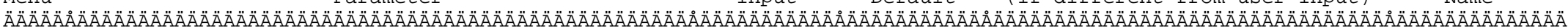
RO21 3 Volumetric water content of the foundation R021 3 Diffusion coefficient for radon gas $(\mathrm{m} / \mathrm{sec})$ : R०21 3 in cover material

Re21 3 in foundation material

RO21 3 in contaminated zone soil

R021 3 Radon vertical dimension of mixing $(\mathrm{m})$

R021 3 Average building air exchange rate $(1 / \mathrm{hr}$ )

R021 3 Height of the building (room) (m)

RO21 3 Building interior area factor

R०21 3 Building depth below ground surface $(\mathrm{m})$

R०21 3 Emanating power of $\mathrm{Rn}-222$ gas

not used $33.000 \mathrm{E}-02$

not used

3 not used

2. $000 \mathrm{E}-066^{3}$

3 not used $33.000 \mathrm{E}-07^{3}$

3 not used $32.000 E-063$

3 not used $32.000 E+00$

not used $35.000 \mathrm{E}-01$

not used $32.500 E+00^{3}$

not used 3 .

not used 3 - $1.000 \mathrm{C}+00^{3}$

not used $3-1.000 \mathrm{E}+00$

not used 3 2.500E-01

not used 3 1.500E-01

R०21 3 Emanating power of Rn-220 gas

TITL 3 Number of graphical time points

TITL 3 Maximum number of integration points for dose

TITL 3 Maxin

\begin{tabular}{|c|c|c|}
\hline-- & $\begin{array}{l}3 \\
3\end{array}$ & PH2OFL \\
\hline$\ldots$ & 3 & DIFCV \\
\hline$\ldots$ & 3 & DIFFL \\
\hline$\ldots$ & 3 & DIFCZ \\
\hline-- & 3 & HMIX \\
\hline$-\ldots$ & 3 & REXG \\
\hline - - - & 3 & HRM \\
\hline-- & 3 & FAI \\
\hline- & 3 & DMFL \\
\hline - & 3 & EMANA (1) \\
\hline$-\ldots$ & $\begin{array}{l}3 \\
3\end{array}$ & EMANA (2) \\
\hline- & 3 & NPTS \\
\hline - & 3 & LYMAX \\
\hline - & 3 & KYMAX \\
\hline
\end{tabular}

Summary of Pathway Selections

Pathway 3 User Selection

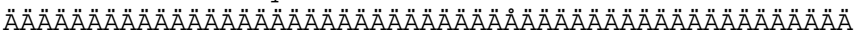

1 - - external gamma ${ }_{3}$ active

2 - - inhalation (w/o radon) ${ }^{3}$ active

3 - - plant ingestion 3 suppressed

4 - - meat ingestion 3 suppressed

5 -- milk ingestion 3 suppressed

6 - - aquatic foods 3 suppressed

7 -- drinking water 3 suppressed

8 - - soil ingestion 3 active

9 -- radon 33 suppressed

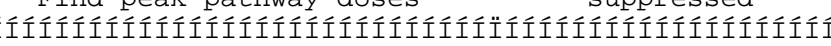


Contaminated Zone Dimensions AAAAAÄÄÄÄÄÄÄÄÄÄÄÄÄÄÄÄÄÄÄÄÄÄÄÄ

Area: 100.00 square meters

0.15 meters

Cover Depth: 0.00 meters

Initial Soil concentrations, $\mathrm{pCi} / \mathrm{g}$

ÄÄÄÄÄÄÄÄÄÄÄÄÄÄÄÄÄÄÄÄÄÄÄÄÄÄÄÄÄÄÄÄÄÄ
Am-241
2. $880 \mathrm{E}+01$
Cs -137
$1.060 \mathrm{E}+03$
Eu-152
$2.640 \mathrm{E}+00$
$\mathrm{Pu}-238$
$1.560 \mathrm{E}+01$
$\mathrm{Pu}-239$
8. $400 \mathrm{E}+01$
$\mathrm{Sr}-90$
$3.410 \mathrm{E}+01$

Total Dose TDOSE(t), mrem/yr

Basic Radiation Dose Limit $=2.500 \mathrm{E}+01 \mathrm{mrem} / \mathrm{yr}$

Total Mixture Sum $\mathrm{m}(\mathrm{t})=$ Fraction of Basic Dose Limit Received at Time $(\mathrm{t})$

Ӓ̈̈̈̈̈̈̈̈̈̈̈̈̈̈̈̈̈̈̈̈̈̈̈̈̈̈̈̈̈̈̈̈̈̈̈̈̈̈̈̈̈̈̈̈̈̈̈̈̈̈̈̈̈̈̈̈̈̈̈̈̈̈̈̈̈̈̈̈̈̈̈̈̈̈̈̈̈̈̈̈̈̈̈̈̈̈̈̈̈̈̈̈̈̈̈̈̈̈̈̈̈̈̈̈̈̈̈̈̈̈̈̈̈̈̈̈̈̈̈̈̈̈̈

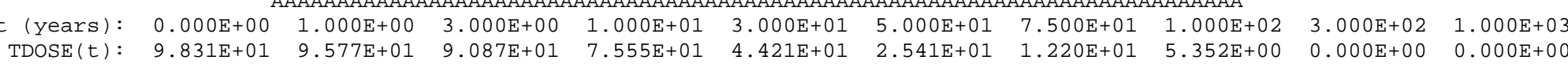

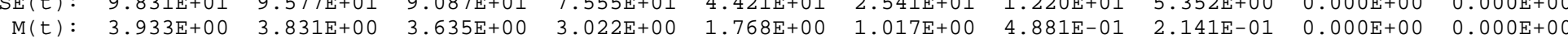

OMaximum TDOSE $(\mathrm{t}): 9.831 \mathrm{E}+01 \mathrm{mrem} / \mathrm{yr}$ at $\mathrm{t}=0.000 \mathrm{E}+00$ years 
Total Dose Contributions TDOSE( $i, p, t)$ for Individual Radionuclides (i) and Pathways ( $p$ )

As mrem/yr and Fraction of Total Dose At $t=0.000 \mathrm{E}+00$ years

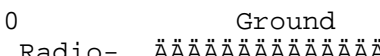
Inhalation water Independent Pathways (Inhalation excludes radon)

$$
\text { Radon }
$$

Plant

Meat

Milk

Soil

Radio- ÄÄAAAAAAAAAAAAA
Nuclide mrem/yr fract
ÄAÄAAA AÄÄ̈ÄA ÄÄÄA ÄÄÄÄÄÄÄ ÄÄÄÄÄÄÄÄÄ ÄÄÄÄÄA Am-241 3.935E- 02 ० $0.00 \odot 4$ Cs-137 9.626E+01 0.9791 $\mathrm{Eu}-152 \quad 4.688 \mathrm{E}-01 \quad 0.0048$ Pu-238 8.080E-05 0.0000 $\mathrm{Pu}-239$ 7.545E-04 0.0000 $\begin{array}{lll}\mathrm{Sr}-90 & 2.338 \mathrm{E}-02 & 0.0002\end{array}$ Íííííí íííííííi ííííi

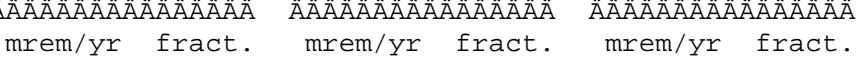
AAAAÄÄÄÄÄÄÄÄÄA mrem/yr fract. mrem/yr fract ÄÄÄÄÄÄÄÄÄ ÄÄÄÄÄÄ ÄÄÄÄÄÄÄÄÄ ÄÄÄÄÄÄ

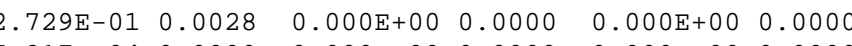
$\begin{array}{llllll}7.217 \mathrm{E}-04 & 0.0000 & 0.000 \mathrm{E}+00 & 0.0000 & 0.000 \mathrm{E}+00 & 0.0000\end{array}$ $\begin{array}{llllll}1.227 \mathrm{E}-05 & 0.0000 & 0.000 \mathrm{E}+0 \odot & 0.000 \odot & 0.000 \mathrm{E}+0 \odot & 0.000 \odot\end{array}$ $\begin{array}{llllll}1.315 E-01 & 0.0013 & 0.000 E+00 & 0.0000 & 0.000 E+00 & 0.0000\end{array}$ $\begin{array}{llllll}7.780 \mathrm{E}-01 & 0.0079 & 0.000 \mathrm{E}+0 \odot & 0.000 \odot & 0.000 \mathrm{E}+0 \odot & 0.000\end{array}$

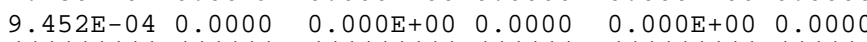
IIIIIIíi ííííi ííííííí ííííi ííííííí íííít $\odot$

Total Dose Contributions $\operatorname{TDOSE}(i, p, t)$ for Individual Radionuclides (i) and Pathways (p) As $\mathrm{mrem} / \mathrm{yr}$ and Fraction of Total Dose At $t=0.000 \mathrm{E}+0 \odot$ years Water Dependent Pathways Water

Radio - ÄÄÄÄÄÄÄÄÄÄÄÄÄÄÄÄ Nuclide mrem/yr fract. ÄÄÄÄÄÄÄ ÄÄÄÄÄÄÄÄÄÄ ÄÄÄÄÄÄ Am-241 $0.000 \mathrm{E}+\odot \odot \quad 0.000 \odot$ CS $-137 \quad 0.000 \mathrm{E}+00 \quad 0.0000$ Eu-152 $0.000 \mathrm{E}+00 \quad 0.0000$ $\mathrm{Pu}-238 \quad 0.000 \mathrm{E}+00 \quad 0.0000$ $\mathrm{Pu}-239 \quad 0.000 \mathrm{E}+00 \quad 0.0000$ Sr-9० $0.000 \mathrm{E}+00 \quad 0.0000$ íííííi íííííííi íííít Total $0.000 \mathrm{E}+00 \quad 0.0000$ * Sum of all water independent and 0.0000 Fish

\begin{abstract}
Radon
\end{abstract}

$$
\text { Plant }
$$

mrem/yr fract. mrem/yr fract. $0.000 \mathrm{E}+00 \quad 0.0000$ $\odot .000 \mathrm{E}+000.0000$ $0.000 \mathrm{E}+0 \odot \quad 0.000 \odot$ $0.000 \mathrm{E}+0 \odot \quad 0.0000$ $0.000 \mathrm{C}+00$ 0.0000 $\odot .0 \odot \odot E+\odot \odot \odot . \diamond \odot \odot \odot$ $\begin{array}{llll}0.000 \mathrm{E}+0 \odot & 0.000 \odot & 0.000 \mathrm{E}+\odot \odot & 0.00 \odot \odot\end{array}$ pathways ÍÍííííí ííííí

\section{A} ÄÄÄÄÄÄÄÄÄ ÄÄÄÄÄ $\odot .000 \mathrm{E}+0 \odot \quad 0.000 \odot$ $0.000 \mathrm{E}+0 \odot$ ०. 0000 $0.000 \mathrm{E}+000.0000$ $\odot .000 \mathrm{E}+00$ ००.0००० $\odot .000 \mathrm{E}+0 \odot \quad 0.0000$ $0.000 \mathrm{E}+\odot \odot \quad 0.0000$
Meat ÄÄÄÄÄÄÄÄÄÄÄÄÄÄÄ mrem/yr fract. ÄÄÄÄÄÄÄÄ ÄÄÄÄÄÄ $\odot .0 \odot \odot E+\odot \odot \quad \odot .0 \odot \odot \odot$ $0.000 \mathrm{E}+000.0000$ $\odot .0 \odot \odot E+\odot \odot \quad \odot . \odot \odot \odot \odot$ $\odot .000 \mathrm{E}+00 \quad 0.0000$ $\odot .000 \mathrm{E}+0 \odot \quad 0.000 \odot$ $0.000 \mathrm{E}+000.0000$ íííííííí ííííi $\odot .000 \mathrm{E}+0 \odot \quad 0.0000$
ÄÄÄÄÄÄÄÄÄÄÄÄÄÄ mrem/yr fract. ÄÄÄÄÄÄÄÄA ÄÄÄÄÄÄ $\odot .000 \mathrm{E}+0 \odot \quad 0.000 \odot$ $\odot .000 \mathrm{E}+000.0000$ $\odot .000 \mathrm{E}+0 \odot \quad 0.000 \odot$ $0.000 \mathrm{E}+000.0000$ $0.000 \mathrm{E}+000.00000$ $0.000 \mathrm{E}+000.0000$ Íííííííi ííííí $0.000 \mathrm{E}+000.0000$
Milk

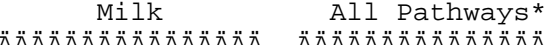
mrem/yr fract. mrem/yr fract. ÄÄÄÄÄÄÄÄÄ ÄÄÄÄÄÄ ÄÄÄÄÄÄÄÄÄ ÄÄÄÄÄÄ $0.000 \mathrm{E}+00$ 0.00०० $3.810 \mathrm{E}-01 \quad 0.0039$

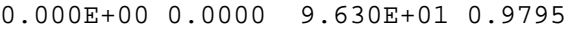

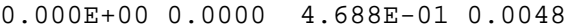
$\odot .000 \mathrm{E}+0 \odot \quad 0.000 \odot \quad 1.646 \mathrm{E}-01 \quad 0.0017$ $0.000 \mathrm{E}+00 \quad 0.0000 \quad 9.760 \mathrm{E}-01 \quad 0.0099$ $0.000 \mathrm{E}+00 \quad 0.0000 \quad 2.772 \mathrm{E}-02 \quad 0.0003$ íííííííi ííííí íííííííi ííííí $\odot .000 \mathrm{E}+0 \odot$ $0.000 \odot \quad 9.831 \mathrm{E}+01 \quad 1.000 \odot$ 
Total Dose Contributions TDOSE( $i, p, t)$ for Individual Radionuclides (i) and Pathways ( $p$ )

As mrem/yr and Fraction of Total Dose At $t=1.000 \mathrm{E}+00$ years

Water Independent Pathways (Inhalation excludes radon)

Total Dose Contributions $\operatorname{TDOSE}(i, p, t)$ for Individual Radionuclides (i) and Pathways (p) As $\mathrm{mrem} / \mathrm{yr}$ and Fraction of Total Dose At $t=1.000 \mathrm{E}+0 \odot$ years

$$
\begin{array}{r}
\text { As mrem/yr and Fraction of Total Dose At } t \\
\text { Water Dependent Pathways }
\end{array}
$$
Fish

$$
\text { Radon }
$$

Radio- ÄÄÄÄÄÄÄÄÄÄÄÄÄÄÄÄ Nuclide $\mathrm{mrem} / \mathrm{yr}$ fract ÄÄÄÄÄÄÄ ÄÄÄÄÄÄÄÄÄ ÄÄÄÄÄ̈ $\begin{array}{lll}\mathrm{Am}-241 & 0.00 \odot \mathrm{E}+\odot \odot & \odot .0 \odot \odot \odot \\ \mathrm{CS}-137 & \odot .00 \odot \mathrm{E}+\odot \odot & \odot .00 \odot \odot\end{array}$ $\mathrm{Eu}-152 \quad 0.000 \mathrm{E}+00 \quad 0.0000$ $\mathrm{Pu}-238 \quad 0.000 \mathrm{E}+00 \quad 0.0000$ $\mathrm{Pu}-239 \quad 0.000 \mathrm{E}+00 \quad 0.0000$ $\begin{array}{lll}\mathrm{Su}-90 & 0.000 \mathrm{E}+0 \odot & 0.0000\end{array}$ Íííííi ííííííí íííít

Total $0.000 \mathrm{E}+\odot \odot \odot .0 \odot \odot \bullet$ mrem/yr fract. mrem/yr fract. mrem/yr fract.

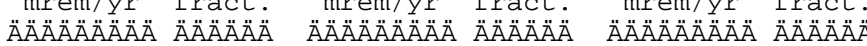

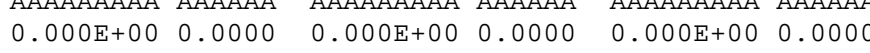
$\begin{array}{llllll}0.000 \mathrm{E}+00 & 0.0000 & 0.000 \mathrm{E}+00 & 0.0000 & 0.000 \mathrm{E}+0 \odot & 0.0000\end{array}$ $\begin{array}{llllll}0.000 E+00 & 0.0000 & 0.000 \mathrm{E}+00 & 0.0000 & 0.000 \mathrm{E}+00 & 0.0000\end{array}$

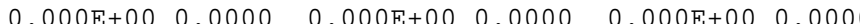
$0.000 E+00$ 0.0000 $0.000 E+000.0000 \quad 0.000 E+000.0000$

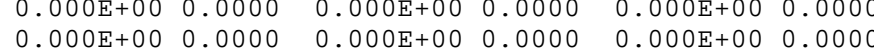

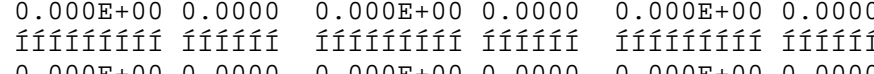
$\odot *$ Sum of all water independent and dependent pathways.
Milk

\section{ÄÄÄÄÄÄÄÄÄÄÄÄÄÄÄ} $\mathrm{mrem} / \mathrm{yr}$ fract $0.000 \mathrm{O}+00$ 0.000९ $0.000 \mathrm{E}+00 \quad 0.0000$ $0.000 \mathrm{E}+00 \quad 0.0000$ $\odot .000 \mathrm{E}+00$ ๑. 0000 $0.000 \mathrm{O}$ $0.000 \mathrm{E}+0 \odot$ 0.0000 ÍÍÍÍÍÍI ÍÍÍÍÍ $0.000 \mathrm{E}+000.0000$

\section{ÄÄÄÄÄÄÄÄÄÄÄÄÄÄÄÄ} mrem/yr fract. ÄÄÄÄÄÄÄÄÄ ÄÄÄÄÄÄ $\odot .000 \mathrm{E}+00 \quad 0.0000$ $\odot .000 \mathrm{E}+0 \odot \quad 0.000 \odot$ $\odot .000 \mathrm{E}+0 \odot \quad 0.0000$ $0.000 \mathrm{E}+0 \odot \quad 0.000 \odot$ $0.000 \mathrm{E}+00 \quad 0.0000$ $0.000 \mathrm{E}+00 \quad 0.0000$ ííííííí ííííí $\odot .000 \mathrm{E}+000.0000$ AАÄÄÄÄÄÄÄÄÄÄÄÄÄ mrem/yr fract. ÄÄÄÄÄÄÄÄÄ ÄÄÄÄÄÄ $\begin{array}{lll}0.674 \mathrm{E}-02 & 0.0007\end{array}$ $3.374 \mathrm{E}-02 \quad 0.0004$ $1.042 \mathrm{E}-05 \quad 0.0000$ 1. $3.243 E-03$ 0.000

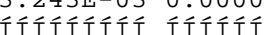
3.322E-01 0.0035
All Pathways* ÄÄÄÄÄÄÄÄÄÄÄÄÄÄÄ mrem/yr fract. ÄÄÄÄÄÄÄÄÄ ÄÄÄÄÄÄ 3.701E-01 0.0039 $9.380 \mathrm{E}+01 \quad 0.9794$ 4. 433E-01 0.0046 $1.621 \mathrm{E}-01 \quad 0.0017$ $9.692 \mathrm{E}-01 \quad 0.0101$ $2.659 \mathrm{E}-02 \quad 0.0003$ Ííííííí ííííí $9.577 \mathrm{E}+011.0000$ 
Total Dose Contributions TDOSE( $i, p, t)$ for Individual Radionuclides (i) and Pathways ( $p$ )

As mrem/yr and Fraction of Total Dose At $t=3.000 \mathrm{E}+00$ years

Water Independent Pathways (Inhalation excludes radon)

Total Dose Contributions $\operatorname{TDOSE}(i, p, t)$ for Individual Radionuclides (i) and Pathways (p) As $\mathrm{mrem} / \mathrm{yr}$ and Fraction of Total Dose At $t=3.000 \mathrm{E}+\odot \odot$ years

$$
\begin{array}{r}
\text { As mrem/yr and Fraction of Total Dose At } t \\
\text { Water Dependent Pathways }
\end{array}
$$
Fish

$$
\text { Radon }
$$

$$
\text { Plant }
$$

Meat

Milk

Radio- ÄÄÄÄÄÄÄÄÄÄÄÄÄÄA ÄÄÄÄÄÄÄÄÄÄÄÄÄÄÄ ÄÄÄÄÄÄÄÄÄÄÄÄÄÄ mrem/yr fract. ÄÄÄÄÄÄÄ ÄÄÄÄÄÄÄÄÄ ÄÄÄÄÄÄ

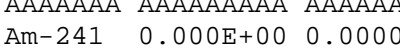
CS-137 $0.000 \mathrm{E}+\odot \odot \quad \odot . \odot \odot \odot \odot$ Eu-152 $0.000 \mathrm{E}+00 \quad 0.0000$ $\mathrm{Pu}-238 \quad 0.000 \mathrm{E}+00 \quad 0.0000$ Pu-239 $0.000 \mathrm{E}+00 \quad 0.0000$ $\begin{array}{lll}\mathrm{Pu}-239 & 0.000 \mathrm{E}+\odot \odot & 0.0000 \\ \mathrm{Sr}-900 \mathrm{E}+0 \odot & 0.0000\end{array}$

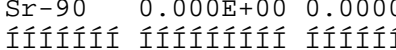
AAAAAAAA AÄ̈̈̈̈̈̈ $0.000 \mathrm{E}+\odot \odot \quad 0.000 \odot$ $\odot .000 \mathrm{E}+0 \odot$ $\odot .00 \odot \odot$ $0.000 \mathrm{E}+0 \odot$ O 90000 $0.000 \mathrm{E}+0 \odot \quad 0.0000$ mrem/yr fract. mrem/yr fract. D.

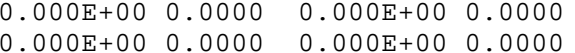
$\odot .000 \mathrm{E}+0 \odot \quad 0.000 \odot \quad 0.000 \mathrm{E}+0 \odot \quad 0.000 \odot$ $\odot .000 \mathrm{C}+00$ - 0.0000 $0.000 \mathrm{E}+00 \quad 0.0000$ $0.000 \mathrm{E}+00 \quad 0.0000 \quad 0.000 \mathrm{E}+00 \quad 0.0000$

Total 0 $\odot *$ Sum of all water independent and dependent pathways. $0.000 \mathrm{E}+000.0000$ $\odot .000 \mathrm{E}+0 \odot \quad 0.0000$ $0.000 \mathrm{E}+00 \quad 0.0000$ ÍÍÍÍÍÍÍ ÍÍÍÍÍ $0.000 \mathrm{E}+00$ ๑. $000 \odot$ ÄÄÄÄÄÄÄÄÄÄÄÄÄÄÄĂ $\mathrm{mrem} / \mathrm{yr}$ fract ÄÄÄÄÄÄÄÄ ÄÄÄÄÄA $\begin{array}{ll}0.000 \mathrm{E}+0 \odot & 0.0000 \\ \odot .000 \mathrm{E}+0 \odot & 0.0000\end{array}$ $\odot .000 \mathrm{E}+0 \odot \quad 0.000 \odot$ $\odot .000 \mathrm{E}+\odot \odot \quad \odot .000 \odot$ $\odot .000 \mathrm{E}+0 \odot \quad 0.0000$ $\odot . \odot \odot \odot E+\odot \odot \quad \odot . \diamond \odot \odot \odot$ ÍÍÍííííi Ííííít $\bullet .000 \mathrm{E}+00$ 0.00०० ÄÄÄÄÄÄÄÄÄÄÄÄÄÄÄÄÄ mrem/yr fract. ÄÄÄÄÄÄÄÄÄ ÄÄÄÄÄÄ $\odot .000 \mathrm{E}+00$ ० $0.000 \odot$

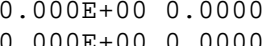
$0.000 \mathrm{E}+00 \quad 0.0000$ $\odot .000 \mathrm{E}+00 \quad 0.0000$ $0.000 \mathrm{E}+00 \quad 0.0000$

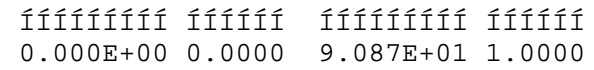

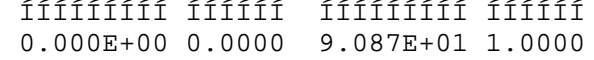

All Pathways* ÄÄÄÄÄÄÄÄÄÄÄÄÄÄÄ mrem/yr fract. ÄÄÄÄÄÄÄÄÄ ÄÄÄÄÄÄ $3.491 \mathrm{E}-010.0038$ $8.899 E+01 \quad 0.9793$ $3.964 \mathrm{E}-01 \quad 0.0044$ $1.574 \mathrm{E}-010.0017$ 9.557E-01 $\odot .0105$ 9.557 E 010.0105 IIIIíííí ííííí 9.087E+01 1.0000 
Total Dose Contributions TDOSE( $i, p, t)$ for Individual Radionuclides (i) and Pathways ( $p$ )

As mrem/yr and Fraction of Total Dose At $t=1.000 \mathrm{E}+01$ years

Water Independent Pathways (Inhalation excludes radon)

Total Dose Contributions $\operatorname{TDOSE}(i, p, t)$ for Individual Radionuclides (i) and Pathways $(p)$ As $\mathrm{mrem} / \mathrm{yr}$ and Fraction of Total Dose At $t=1.000 \mathrm{E}+01$ years

$$
\begin{array}{r}
\text { As mrem/yr and Fraction of Total Dose At } t \\
\text { Water Dependent Pathways }
\end{array}
$$
Fish Radon

Radio - ÄÄÄÄÄÄÄÄÄÄÄÄÄÄÄÄ Nuclide mrem/yr fract. ÄÄÄÄÄÄÄ ÄÄÄÄÄÄÄÄÄ ÄÄÄÄÄÄ Am-241 $0.000 \mathrm{E}+0 \odot \quad 0.000 \odot$ CS $-137 \quad 0.000 \mathrm{E}+00$ - 0.0000 $\mathrm{Pu}-238 \quad 0.000 \mathrm{E}+000.0000$ $\mathrm{Pu}-239 \quad 0.000 \mathrm{E}+00 \quad 0.0000$ $\mathrm{Sr}-90 \quad 0.000 \mathrm{E}+0 \odot \quad 0.000 \odot$ Íííííi ííííííí íííít

Total $0.00 \odot \mathrm{E}+\odot \odot \odot .0 \odot \odot)^{\circ}$

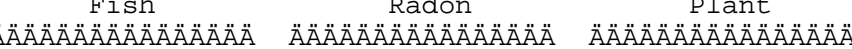
mrem/yr fract. mrem/yr fract. mrem/yr fract. ÄÄÄÄÄÄÄÄÄ ÄÄÄÄÄÄ ÄÄÄÄÄÄÄÄÄ ÄÄÄÄÄÄ ÄÄÄÄÄÄÄÄÄ ÄÄÄÄÄ̈

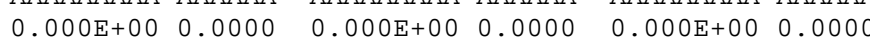
$\begin{array}{llllll}0.000 \mathrm{E}+00 & 0.0000 & 0.000 \mathrm{E}+0 \odot & 0.0000 & 0.000 \mathrm{E}+0 \odot & 0.0000\end{array}$ $\begin{array}{llllll}0.000 \mathrm{E}+00 & 0.0000 & 0.000 \mathrm{E}+00 & 0.0000 & 0.000 \mathrm{E}+00 & 0.0000\end{array}$

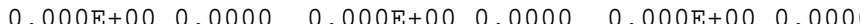
$0.000 \mathrm{E}+00$ 0.0000 $0.000 \mathrm{E}+000.0000 \quad 0.000 \mathrm{E}+000.0000$

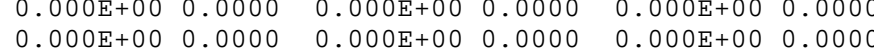

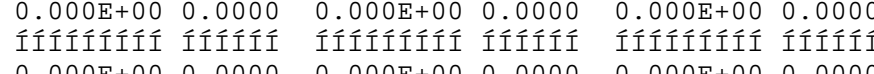
$\odot$ *Sum of all water independent and dependent pathways.

Milk

\section{ÄÄÄÄÄÄÄÄÄÄÄÄÄÄÄ} mrem/yr fract $0.000 \mathrm{E}+00 \quad 0.0000$ $0.000 \mathrm{E}+00 \quad 0.0000$ $0.000 \mathrm{E}+00 \quad 0.0000$ $0.000 \mathrm{E}+00$ - $\odot .0000$ $0.0005+00$ (0.000 $0.000 \mathrm{E}+00$ 0. 0.000 ÍÍÍÍÍÍI ÍÍÍÍÍ $0.000 \mathrm{E}+000.0000$

\section{ÄÄÄÄÄÄÄÄÄÄÄÄÄÄÄÄ} mrem/yr fract. ÄÄÄÄÄÄÄÄÄ ÄÄÄÄÄÄ $0.000 \mathrm{E}+00 \quad 0.0000$ $0.000 \mathrm{E}+000.0000$ $\odot .000 \mathrm{E}+0 \odot$ ०. 0.0000 $0.000 \mathrm{E}+000.0000$ $0.000 \mathrm{E}+00 \quad 0.0000$ $0.000 \mathrm{E}+000.0000$ íííííííi ííííí $0.000 \mathrm{E}+00 \quad 0.0000$ AАAAÄÄÄÄÄÄÄÄÄÄÄ mrem/yr fract. ÄÄÄÄÄÄÄÄ ÄÄÄÄÄÄ $5.091 \mathrm{E}-02 \quad 0.0007$ $2.572 \mathrm{E}-02 \quad 0.0003$ $6.103 \mathrm{E}-06 \quad 0.0000$ 1.836E 01 0.0024 $2.160 \mathrm{0}-03$ 0.0000

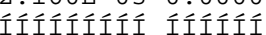
2.908E-01 0.0038
All Pathways* ÄÄÄÄÄÄÄÄÄÄÄÄÄÄÄ mrem/yr fract. ÄÄÄÄÄÄÄÄÄ ÄÄÄÄÄÄ 2.842E-01 0.0038 $\begin{array}{lll}7.393 \mathrm{E}+01 & 0.9786\end{array}$ $2.676 \mathrm{E}-01 \quad 0.0035$ 1. 416E-01 0.0019 $9.085 \mathrm{E}-01 \quad 0.0120$ $1.829 \mathrm{E}-02 \quad 0.0002$ Ííííííí ÍÍííí $7.555 \mathrm{E}+011.0000$ 
Total Dose Contributions TDOSE( $i, p, t)$ for Individual Radionuclides (i) and Pathways ( $p$ )

As mrem/yr and Fraction of Total Dose At $t=3.000 \mathrm{E}+01$ years

Water Independent Pathways (Inhalation excludes radon)

Total Dose Contributions $\operatorname{TDOSE}(i, p, t)$ for Individual Radionuclides (i) and Pathways $(p)$ As $\mathrm{mrem} / \mathrm{yr}$ and Fraction of Total Dose At $t=3.000 \mathrm{E}+01$ years

$$
\begin{array}{r}
\text { As mrem/yr and Fraction of Total Dose At } t \\
\text { Water Dependent Pathways }
\end{array}
$$

\section{Water}

$$
\text { Fish }
$$$$
\text { Radon }
$$$$
\text { Plant }
$$

Radio- ÄÄÄÄÄÄÄÄÄÄÄÄÄÄÄA
Nuclide mrem/yr fract Nuclide mrem/yr fract.
ÄÄÄÄÄÄÄ ÄÄÄÄÄÄÄÄÄ ÄÄÄÄÄÄ

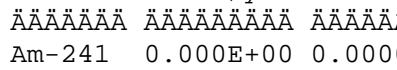
CS-137 $0.000 \mathrm{E}+\odot \odot \quad \odot . \odot \odot \odot \odot$ Eu-152 $0.000 \mathrm{E}+\odot \odot \quad \odot .0 \odot \odot \odot$ Pu-238 $0.000 \mathrm{E}+00 \quad 0.0000$ $\mathrm{Pu}-239 \quad 0.000 \mathrm{E}+00 \quad 0.0000$ $\begin{array}{lll}\mathrm{Pu}-239 & 0.000 \mathrm{E}+\odot \odot & 0.0000 \\ \mathrm{Sr}-90 & 0.000 \mathrm{E}+0 \odot & 0.0000\end{array}$ íííííi ííííííí íííít ÄÄÄÄÄÄÄÄÄÄÄÄÄÄÄÄ ÄÄÄÄÄÄÄÄÄÄÄÄÄÄÄ mrem/yr fract. mrem/yr fract. ÄÄÄÄÄÄÄÄÄ ÄÄÄÄÄÄ $\odot . \odot \odot \odot E+\odot \odot ~ \odot . \odot \odot \odot \odot ~$ $\odot .00 \odot E+\odot \odot$ $\odot .00 \odot \odot$ $0.000 \mathrm{E}+0 \odot$ O 90000 $\odot .000 \mathrm{E}+0 \odot \odot .000 \odot$ $\odot .0 \odot \odot \mathrm{E}+\odot \odot$ $\odot . \odot \odot \odot \odot$ Íííííííi ííííi Total $0.000 \mathrm{E}+00 \quad 0.0000$ 0 *Sum of all water independent $0.000 \mathrm{E}+000.0000$ АÄÄÄÄÄÄÄÄÄ̈̈̈̈̈̈

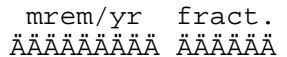

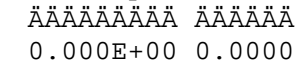
$\begin{array}{ll}0.000 \mathrm{E}+00 & 0.0000 \\ 0.000 \mathrm{E}+00 & 0.0000\end{array}$ $0.000 \mathrm{E}+0 \odot \quad 0.0000$ $0.000 \mathrm{E}+00 \quad 0.0000$ $\odot .000 \mathrm{E}+\odot \odot \odot .00 \odot 0$

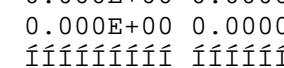
$0.000 \mathrm{E}+000.0000$
Meat ÄÄÄÄÄÄÄÄÄÄÄÄÄÄÄÄ mrem/yr fract. ÄÄÄÄÄÄÄÄ ÄÄÄÄÄA $\begin{array}{ll}0.000 \mathrm{E}+00 & 0.0000 \\ 0.000 \mathrm{E}+00 & 0.0000\end{array}$ $0.000 \mathrm{E}+00 \quad 0.0000$ $\odot .000 \mathrm{E}+\odot \odot \quad \odot .000 \odot$ $0.000 \mathrm{E}+0 \odot \quad 0.0000$ $0.000 \mathrm{E}+000.0000$ Íííííííi ííííi $0.000 \mathrm{E}+000.0000$
Milk

All Pathways* mrem/y r fract. ÄÄÄÄÄÄÄÄÄ ÄÄÄÄÄÄ Ä̈̈̈̈̈̈̈̈̈̈̈̈̈̈̈ ÄÄÄÄÄÄ̈

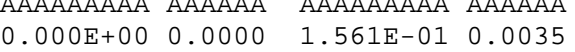
$0.000 \mathrm{E}+00 \quad 0.0000 \quad 4.308 \mathrm{E}+01 \quad 0.9745$ $0.000 \mathrm{E}+00 \quad 0.0000 \quad 8.615 \mathrm{E}-02 \quad 0.0019$ $\odot .000 \mathrm{E}+00 \quad 0.0000 \quad 1.031 \mathrm{E}-01 \quad 0.0023$ $0.000 \mathrm{E}+00 \quad 0.0000 \quad 7.745 \mathrm{E}-010.0175$ $0.000 \mathrm{E}+00 \quad 0.0000 \quad 7.891 \mathrm{E}-03 \quad 0.0002$ Ííííííí ííííi Ííííííí ííííi $0.000 \mathrm{E}+000.0000 \quad 4.421 \mathrm{E}+01 \quad 1.0000$ 
Total Dose Contributions TDOSE( $i, p, t)$ for Individual Radionuclides (i) and Pathways ( $p$ )

As mrem/yr and Fraction of Total Dose At $t=5.000 \mathrm{E}+01$ years

Water Independent Pathways (Inhalation excludes radon)

Total Dose Contributions TDOSE(i,p,t) for Individual Radionuclides (i) and Pathways ( $p$ ) As $\mathrm{mrem} / \mathrm{yr}$ and Fraction of Total Dose At $t=5.000 \mathrm{E}+01$ years Fish Radon Water Dependent Pathways \\ Radio Ä̈̈̈̈̈̈Äter} Nuclide mrem/yr fract. ÄÄÄÄÄÄÄ ÄÄÄÄÄÄÄÄÄ ÄÄÄÄÄ̈̈ Am-241 $0.000 \mathrm{E}+00 \quad 0.0000$ CS $-137 \quad 0.000 \mathrm{E}+00$ - 0.0000 Pu-238 $9.000 \mathrm{E}+00$ - 0.0000 $\mathrm{Pu}-239 \quad 0.000 \mathrm{E}+00 \quad 0.0000$ $\mathrm{Sr}-90 \quad 0.000 \mathrm{E}+0 \odot \quad 0.000 \odot$ Íííííi ííííííí íííít

Total $0.000 \mathrm{E}+\odot \odot \odot, 0 \odot \odot$

$$
\text { Plant }
$$

Meat ÄӒÄÄÄÄÄÄÄÄ̈̈ÄÄÄÄÄÄ mrem/yr fract. mrem/yr fract. mrem/yr fract. mrem/yr fract. ÄÄÄÄÄÄÄÄ ÄÄÄÄÄÄ ÄÄÄÄÄÄÄÄÄ ÄÄÄÄÄÄ ÄÄÄÄÄÄÄÄÄ ÄÄÄÄÄÄ

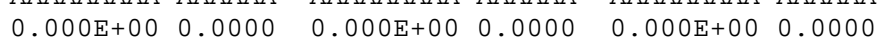

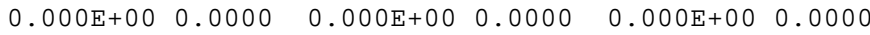
$\begin{array}{llllll}0.000 E+00 & 0.0000 & 0.000 E+00 & 0.0000 & 0.000 E+00 & 0.000 \odot\end{array}$

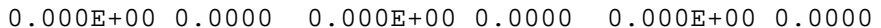
$0.000 \mathrm{E}+00$ 0.0000 $0.000 \mathrm{E}+000.0000 \quad 0.000 \mathrm{E}+000.0000$

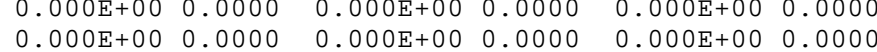

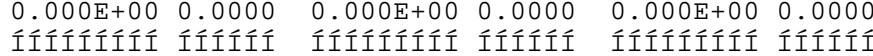
$\odot *$ Sum of all water independent and dependent pathways. $0.000 \mathrm{E}+000.00000$ mrem/yr frac $0.000 \mathrm{E}+000.0000$ $0.000 \mathrm{E}+00$ - 0.0000 $0.000 \mathrm{E}+000.0000$ $0.000 \mathrm{E}+000.0000$

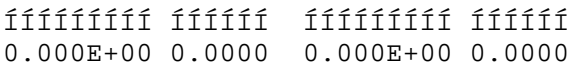
$0.000 \mathrm{E}+000.0000 \quad 0.000 \mathrm{E}+00 \quad 0.0000$
$0.000 \mathrm{E}+000.0000 \quad 0.000 \mathrm{E}+000.0000$
АÄÄÄÄÄÄÄÄÄÄÄÄÄÄÄ mrem/yr fract. АAАAАAАA ÄAAAÄ $1.440 \mathrm{E}-02 \quad 0.0006$ $.253 \mathrm{E}-03 \quad 0.0003$ $5.325 \mathrm{E}-07 \quad 0.0000$ 1. $207 \mathrm{E}-010.0051$ $3.3435-040.0000$ ÍÍítítiti ittití $1.663 E-01$ 0.0065 8.384E-02 0.0033 $\begin{array}{cccc}0.000 \mathrm{E}+00 & 0.0000 & 2.458 \mathrm{E}+01 & 0.9674 \\ 0.000 \mathrm{C}+00 & 0.0000 & 2.715 \mathrm{E}-02 & 0.0011\end{array}$ $\odot . \odot \odot \odot E+\odot \odot \quad \odot .0 \odot \odot \odot \quad 7.315 \mathrm{E}-\odot 2 \quad 0.0011$ $0.000 \mathrm{E}+00 \quad 0.0000 \quad 6.418 \mathrm{E}-01 \quad 0.0253$ $\odot .000 \mathrm{E}+00 \quad 0.0000 \quad 3.341 \mathrm{E}-03 \quad 0.0001$ Iíííííi ííííí ííííííí ííííi $2.541 \mathrm{E}+011.0000$ 
Total Dose Contributions TDOSE( $i, p, t)$ for Individual Radionuclides (i) and Pathways ( $p$ )

As mrem/yr and Fraction of Total Dose At $t=7.500 \mathrm{E}+01$ years

Ground Inhalation water Independent Pathways (Inhalation excludes radon)

\section{Radio - ÄÄÄÄÄÄÄÄÄÄÄÄÄÄÄ}

Radon

Plant

Meat

Milk

Soil

Radio- AAAAAAAAAAAAAA
Nuclide mrem/yr fract ÄÄÄÄÄÄÄ ÄÄÄÄÄÄÄÄÄ ÄÄÄÄÄA $\begin{array}{lll}\mathrm{Am}-241 & 6.791 \mathrm{E}-03 & 0.0006 \\ \mathrm{Cs}-137 & 1.163 \mathrm{E}+01 & 0.9533\end{array}$ EU-152 6.124E- -03 \% 0.0005 $\mathrm{Pu}-238 \quad 4.281 \mathrm{E}-05 \quad 0.0000$ $\mathrm{Pu}-2395.923 \mathrm{E}-04 \quad 0.0000$ Sr-90 $9.704 \mathrm{E}-04 \quad 0.0001$ Ííííí Ííííííí íííít Total 1.165E+01 0.9545 mrem $\begin{array}{llllll}2.399 \mathrm{E}-02 & 0.0020 & 0.000 \mathrm{E}+00 & 0.0000 & 0.000 \mathrm{E}+00 & 0.0000\end{array}$ $\begin{array}{llllll}6.313 \mathrm{E}-05 & 0.0000 & 0.000 \mathrm{E}+00 & 0.0000 & 0.000 \mathrm{E}+00 & 0.0000\end{array}$ $1.190 \mathrm{E}-07 \quad 0.0000 \quad 0.000 \mathrm{E}+00$ - $0.0000 \quad 0.000 \mathrm{E}+00 \quad 0.0000$ $3.565 \mathrm{E}-02 \quad 0.0029 \quad 0.000 \mathrm{E}+00$ - 0.0000 0.000E+00 0.0000

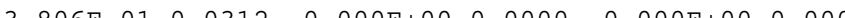

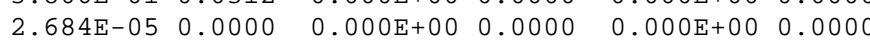
Íííííííí ííííí íííííííí íííííi íííííííi ííííi $\odot$

Total Dose Contributions $\operatorname{TDOSE}(i, p, t)$ for Individual Radionuclides (i) and Pathways $(p)$

As mrem/yr and Fraction of Total Dose At $t=7.500 \mathrm{E}+01$ years Water Fish Radon

Radio - ÄÄÄÄÄÄÄÄÄÄÄÄÄÄÄ Nuclide mrem/yr fract. ÄÄÄÄÄÄÄ ÄÄÄÄÄÄÄÄÄÄ ÄÄÄÄÄÄ Am-241 $\quad 0.000 \mathrm{E}+0 \odot \quad 0.0000$ CS $-137 \quad 0.000 \mathrm{E}+00$ ० 0.0000 $\mathrm{Eu}-152 \quad 0.000 \mathrm{E}+00 \quad 0.0000$ $\mathrm{Pu}-2390.000 \mathrm{E}+0 \odot \quad 0.000 \odot$ $\begin{array}{lll}\mathrm{Pu}-239 & 0.000 \mathrm{E}+\odot \odot & 0.0000 \\ \mathrm{IIT} & 0.000 \mathrm{E}+00 & 0.0000\end{array}$

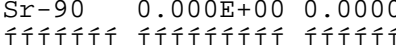

Total $0.000 \mathrm{E}+00 \quad 0.0000$

* Sum of all water Water Dependent Pathways

Aล̈ÄÄÄÄÄÄÄÄÄÄÄÄÄA mrem/yr fract.
ÄÄÄÄÄÄÄÄ ÄÄÄÄÄÄ 0.000E+0○ 0.0000 $0.000 E+00 \quad 0.0000$ $0.000 \mathrm{E}+0 \odot \quad 0.000 \odot$ $0.000 \mathrm{E}+0 \odot \quad 0.0000$ $0.000 \mathrm{E}+00 \quad 0.0000$ $0.000 \mathrm{E}+00 \quad 0.0000$ Iíííííí ííííi ÄÄÄÄÄÄÄÄÄÄÄÄÄÄÄÄ mrem/yr fract. ÄÄÄÄÄÄÄÄ ÄÄÄÄÄÄ $\odot .000 \mathrm{E}+0 \odot \quad 0.000 \odot$ $\odot .000 \mathrm{E}+00 \quad 0.0000$ $\odot .000 \mathrm{E}+000.0000$ $0.000 \mathrm{E}+000.0000$ $0.000 \mathrm{E}+00 \quad 0.0000$ $0.000 \mathrm{E}+00 \quad 0.0000$ Íííííííi Ííííí $\odot .000 \mathrm{E}+000.0000$ ӒÄÄÄÄÄÄÄÄÄÄÄÄÄÄÄÄ mrem/yr fract. ÄÄÄÄÄÄÄÄÄ ÄÄÄÄÄÄ $6.045 \mathrm{E}-03 \quad 0.0005$ $3.041 E-03 \quad 0.0002$ $1.072 \mathrm{E}-07 \quad 0.0000$ $8.944 \mathrm{E}-03 \quad 0.0007$ $9.652 \mathrm{E}-02 \quad 0.0079$ 9.632E-05 0.0000 Íííítíí́ Ííííí $\begin{array}{lll}1.146 \mathrm{E}-01 & 0.0094\end{array}$

ÄÄÄÄÄÄÄÄÄÄÄÄÄÄÄÄ ÄÄÄÄÄÄÄÄÄÄÄÄÄÄÄÄ ÄÄÄÄÄÄÄÄÄÄÄÄÄÄÄÄ

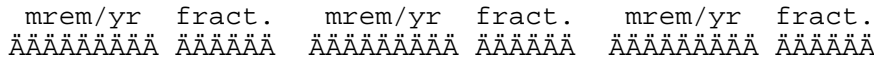

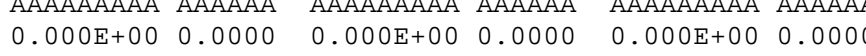

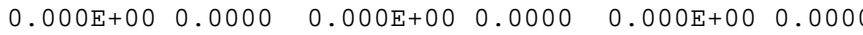
$\begin{array}{llllll}0.000 \mathrm{E}+00 & 0.0000 & 0.000 \mathrm{E}+00 & 0.0000 & 0.000 \mathrm{E}+00 & 0.0000\end{array}$ $\begin{array}{llllll}0.000 \mathrm{E}+00 & 0.0000 & 0.000 \mathrm{E}+00 & 0.0000 & 0.000 \mathrm{E}+0 \odot & 0.0000\end{array}$ $\begin{array}{llllll}0.000 E+0 \odot & 0.000 \odot & 0.000 E+00 & 0.0000 & 0.000 E+0 \odot & 0.0000\end{array}$

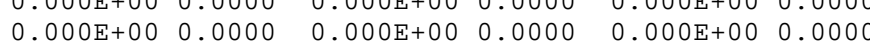

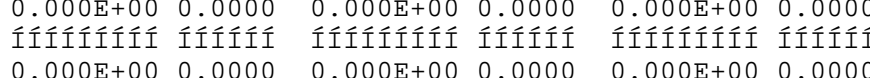
Meat Milk

All Pathways*

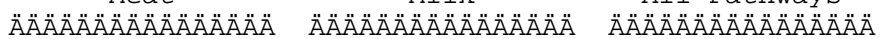
mrem/yr fract. mrem/yr fract. mrem/yr fract. ÄÄÄÄÄÄÄÄÄ ÄÄÄÄÄÄ ÄÄÄÄÄÄÄÄÄ ÄÄÄÄÄÄ ÄÄÄÄÄÄÄÄÄ ÄÄÄÄÄÄ $0.000 \mathrm{E}+00 \quad 0.0000 \quad 0.000 \mathrm{E}+00 \quad 0.0000 \quad 3.683 \mathrm{E}-02 \quad 0.0030$ $\begin{array}{lllllll}0.00 \odot E+\odot \odot ~ & 0.000 \odot & 0.000 E+00 & 0.000 \odot & 1.164 E+01 & 0.9536\end{array}$ $\begin{array}{llllll}0.000 \mathrm{E}+00 & 0.0000 & 0.000 \mathrm{E}+00 & 0.0000 & 6.124 \mathrm{E}-03 & 0.0005\end{array}$ $\begin{array}{llllll}0.000 \mathrm{E}+0 \odot & 0.000 \odot & 0.000 \mathrm{E}+\odot \odot & 0.000 \odot & 4.464 \mathrm{E}-02 & 0.0037\end{array}$

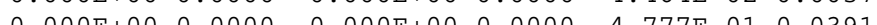

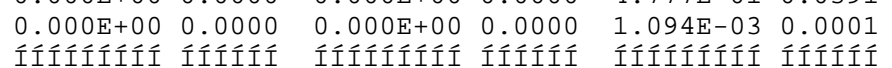

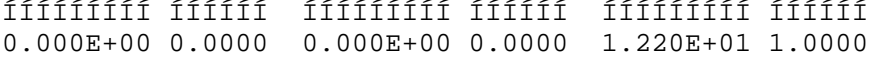
$\odot *$ Sum of all water independent and dependent pathways. 
Total Dose Contributions TDOSE( $i, p, t)$ for Individual Radionuclides (i) and Pathways ( $p$ )

As mrem/yr and Fraction of Total Dose At $t=1.000 \mathrm{E}+02$ years

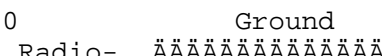
Inhalation water Independent Pathways (Inhalation excludes radon)

$$
\text { Radon }
$$

Plant

Meat

Milk

Soil

Radio- AAAAAAAAAAAAAA
Nuclide mrem/yr fract
AिÄÄÄ ÄÄÄÄÄÄ ÄÄÄÄ ÄÄÄÄÄÄÄ ÄÄÄÄÄÄÄÄÄ ÄÄÄÄÄA Am-241 3.588E- 03 ० 0.0007 Cs-137 4.994E+00 0.9332 Eu-152 1.258E-03 0.0002 Pu-238 3.375E-05 0.0000 $\mathrm{Pu}-239$ 4.946E-04 0.0001 $\mathrm{Sr}-90 \quad 2.950 \mathrm{E}-04 \quad 0.0001$ íííííi ííííííí íííít Total mrem/yr fract

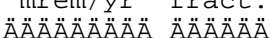
AAAAÄÄÄÄÄÄÄÄÄ ÄÄÄÄÄÄÄÄÄÄÄÄÄÄÄ ААAАAАÄÄÄÄÄÄÄÄ $\begin{array}{llllll}8.942 \mathrm{E}-03 & 0.0017 & 0.000 \mathrm{E}+00 & 0.0000 & 0.000 \mathrm{E}+00 & 0.0000\end{array}$ $2.348 \mathrm{E}-05 \quad 0.0000 \quad 0.000 \mathrm{E}+00 \quad 0.0000 \quad 0.000 \mathrm{E}+00 \quad 0.0000$ $2.127 \mathrm{E}-08 \quad 0.0000 \quad 0.000 \mathrm{E}+0 \odot \quad 0.0000 \quad 0.000 \mathrm{E}+0 \odot \quad 0.0000$ $1.934 \mathrm{E}-020.00360 .000 \mathrm{E}+000.0000 \quad 0.000 \mathrm{E}+000.0000$ $2.513 E-010.0470$ 0.000E+00 0.0000 0.000E+00 0.0000

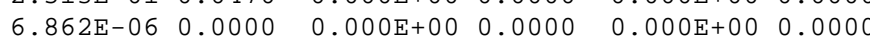

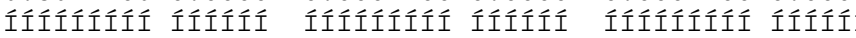
$\odot$

Total Dose Contributions $\operatorname{TDOSE}(i, p, t)$ for Individual Radionuclides (i) and Pathways $(p)$ As $\mathrm{mrem} / \mathrm{yr}$ and Fraction of Total Dose At $t=1.000 \mathrm{E}+02$ years Water Dependent Pathways Water Fish Radon

Radio - ÄÄÄÄÄÄÄÄÄÄÄÄÄÄÄ Nuclide mrem/yr fract. ÄÄÄÄÄÄÄ ÄÄÄÄÄÄÄÄÄÄ ÄÄÄÄÄÄ Am-241 $0.000 \mathrm{E}+00 \quad 0.0000$ CS $-137 \quad 0.000 \mathrm{E}+00 \quad 0.0000$ $\mathrm{Eu}-152 \quad 0.000 \mathrm{E}+00 \quad 0.0000$ $\mathrm{Pu}-2390.000 \mathrm{E}+0 \odot \quad \odot .00 \odot \odot$ $\begin{array}{lll}\mathrm{Pu}-239 & 0.000 \mathrm{E}+0 \odot & 0.0000 \\ \mathrm{IIIIIII} & 0.000 \mathrm{E}+0 \odot & 0.0000\end{array}$

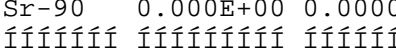

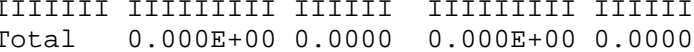

$$
\text { Plant }
$$

Meat ÄÄÄÄÄÄÄÄÄÄÄÄÄÄ mrem/yr fract. ÄÄÄÄÄÄÄÄ ÄÄÄÄÄA $\odot .0 \odot \odot E+\odot \odot \quad \odot .0 \odot \odot \odot$ $0.000 \mathrm{E}+00 \quad 0.0000$ $\odot .0 \odot \odot E+\odot \odot \quad \odot .0 \odot \odot \odot$ $\odot .000 \mathrm{E}+00 \quad 0.0000$ $\odot .000 \mathrm{E}+00 \quad 0.0000$ $0.000 \mathrm{E}+0 \odot \quad 0.0000$ ÍÍÍÍÍÍÍ ÍÍÍÍÍ $0.000 \mathrm{E}+00 \quad 0.0000$
ÄÄÄÄÄÄÄÄÄÄÄÄÄ mrem/yr fract. ÄÄÄÄÄÄÄÄA ÄÄÄÄÄÄ $0.000 \mathrm{E}+0 \odot$ 0.0000 $\odot .000 \mathrm{E}+000.0000$

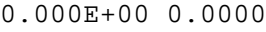
$0.000 \mathrm{E}+000.0000$ $0.000 \mathrm{E}+000.0000$ $0.000 \mathrm{E}+000.0000$ Íííííííí ííííí $0.000 \mathrm{E}+000.0000$ AAAÄÄÄÄÄÄÄÄÄÄÄ ÄÄÄÄÄÄÄÄÄÄÄÄÄÄÄ ÄÄÄÄÄÄÄÄAAAAAA
mrem/yr fract. mrem/yr fract. mrem/yr fract.

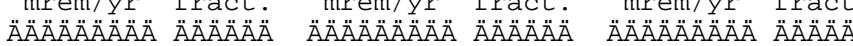

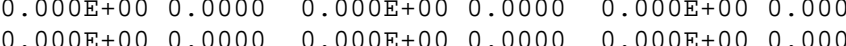
$0.000 \mathrm{E}+00 \quad 0.0000 \quad 0.000 \mathrm{E}+00 \quad 0.0000 \quad 0.000 \mathrm{E}+00 \quad 0.0000$ $\begin{array}{llllll}0.000 E+00 & 0.0000 & 0.000 E+00 & 0.0000 & 0.000 E+00 & 0.0000\end{array}$

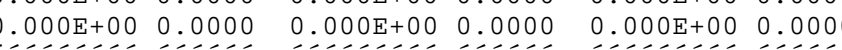
IIÍíílíi IIIIfi $0.000 \mathrm{E}+00 \quad 0.0000$
Milk

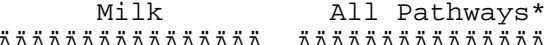
mrem/yr fract. mrem/yr fract. ÄÄÄÄÄÄÄÄÄ ÄÄÄÄÄÄ ÄÄÄÄÄÄÄÄÄ ÄÄÄÄÄÄ $0.000 \mathrm{E}+00$ 0.0000 1.478E-02 0.0028 $0.000 \mathrm{E}+00 \quad 0.0000 \quad 4.996 \mathrm{E}+00 \quad 0.9335$ $0.000 \mathrm{E}+00 \quad 0.0000 \quad 1.258 \mathrm{E}-03 \quad 0.0002$ $\begin{array}{lllll}0.000 \mathrm{E}+00 & 0.0000 & 2.422 \mathrm{E}-02 & 0.0045\end{array}$ $0.000 \mathrm{E}+00 \quad 0.0000 \quad 3.156 \mathrm{E}-01 \quad 0.0590$ $0.000 \mathrm{E}+00 \quad 0.0000 \quad 3.265 \mathrm{E}-04 \quad 0.0001$ Ííííííí ííííi ííííííi ííííi $\odot .000 \mathrm{E}+0 \odot \quad 0.000 \odot \quad 5.352 \mathrm{E}+0 \odot 1.000 \odot$ 
Total Dose Contributions TDOSE( $i, p, t)$ for Individual Radionuclides (i) and Pathways ( $p$ )

As $\mathrm{mrem} / \mathrm{yr}$ and Fraction of Total Dose At $t=3.000 \mathrm{E}+02$ years

Ground Inhalation
In
A water Independent Pathways (Inhalation excludes radon)

Radio - ÄÄÄÄÄÄÄÄÄÄÄÄÄÄÄ

$$
\text { Radon }
$$

Plant

Meat

Milk

Soil

Radio- ÄÄÄÄÄÄÄÄÄÄAAAA
Nuclide mrem/yr fract
ÄÄÄÄÄ Ä̈ÄÄÄÄ ÄÄÄA ÄÄÄÄÄÄÄ Ä Ä̈̈ÄÄÄÄÄÄÄ ÄÄÄÄÄÄ Am-241 $0.0 \odot \odot \mathrm{E}+\odot \odot \quad \odot . \odot \odot \odot \odot$ CS $-137 \quad 0.000 \mathrm{E}+00 \quad 0.0000$ Eu-152 $0.000 \mathrm{E}+0 \odot \quad 0.000 \odot$ $\mathrm{PU}-238 \quad 0.000 \mathrm{E}+00 \quad 0.0000$ $\mathrm{Pu}-2390.000 \mathrm{E}+00 \quad 0.0000$ $\begin{array}{lll}\mathrm{Pu}-239 & 0.000 \mathrm{E}+0 \odot & 0.000 \odot \\ \mathrm{Sr}-90 & 0.000 \mathrm{E}+0 \odot & 0.0000\end{array}$

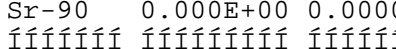
Total $0.000 \mathrm{E}+0 \odot 0.0000$

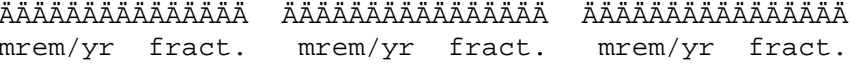
AลАAAAÄÄÄÄÄÄÄA mrem/yr fract. mrem/yr fract. ÄÄÄÄÄÄÄÄÄ ÄÄÄÄÄÄ ÄÄÄÄÄÄÄÄÄ ÄÄÄÄÄÄ $\begin{array}{llllll}0.000 \mathrm{E}+00 & 0.0000 & 0.000 \mathrm{E}+00 & 0.0000 & 0.000 \mathrm{E}+00 & 0.0000\end{array}$ $\begin{array}{llllll}0.000 \mathrm{E}+00 & 0.0000 & 0.000 \mathrm{E}+00 & 0.0000 & 0.000 \mathrm{E}+00 & 0.0000\end{array}$

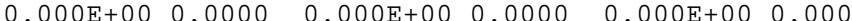
$0.000 E+00$ O. 0000 O. $000 E+00$ 0.0000 $0.000 E+00$ 0.0000 $0.000 E+00$ 0.0000 $0.000 E+00$ 0.0000 $0.000 E+000.0000$

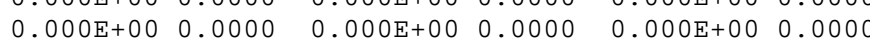
Íííííííí ííííí íííííííi íííííi íííííííi íííít

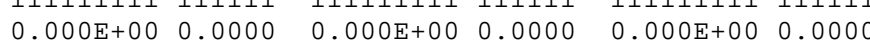
mrem/yr fract ÄÄÄÄÄÄÄÄÄ ÄÄÄÄÄÄ $0.000 \mathrm{E}+00$ 0.000९ $\odot .000 \mathrm{E}+00$ ०.0000 $0.000 \mathrm{E}+0 \odot \quad 0.0000$ $\odot .000 \mathrm{E}+0 \odot \quad 0.0000$ $0.000 \mathrm{E}+0 \odot \quad 0.0000$ $0.000 \mathrm{E}+000.0000$ $\bullet .000 \mathrm{E}+0 \odot$ 0.0000 ÄÄÄÄÄÄÄÄÄÄÄÄÄÄÄÄ mrem/yr fract. ÄÄÄÄÄÄÄÄÄ ÄÄÄÄÄÄ $\odot .000 \mathrm{E}+\odot \odot \quad 0.000 \odot$ $0.000 \mathrm{E}+000.0000$ $\odot .000 \mathrm{E}+000.0000$ $0.000 \mathrm{E}+000.0000$ $0.000 \mathrm{E}+000.0000$ $0.000 \mathrm{E}+000.0000$ Íííííííí Íííííi Total Dose Contributions $\operatorname{TDOSE}(i, p, t)$ for Individual Radionuclides ( $i$ ) and Pathways (p) As $\mathrm{mrem} / \mathrm{yr}$ and Fraction of Total Dose At $t=3.000 \mathrm{E}+02$ years Water Dependent Pathways Water Fish Radon

$$
\text { Plant }
$$

Meat

Milk

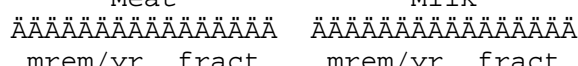
ÄÄÄÄÄÄÄÄÄ ÄÄÄÄÄÄA Nuclide mrem/yr fract ÄÄÄÄÄÄÄ Ä ÄÄ̈̈̈̈̈̈̈̈̈̈̈̈̈̈ ÄÄÄÄÄ̈̈̈ Am-241 $0.000 \mathrm{E}+00 \quad 0.0000$ CS $-137 \quad 0.000 \mathrm{E}+00$ ० 0.0000 Eu-152 $0.000 \mathrm{E}+00 \quad 0.0000$ $\mathrm{Pu}-238 \quad 0.000 \mathrm{E}+00 \quad 0.0000$ $\mathrm{Pu}-239 \quad 0.000 \mathrm{E}+00 \quad 0.0000$ $\begin{array}{lll}\mathrm{Sr}-90 & 0.000 \mathrm{E}+0 \odot & 0.0000\end{array}$

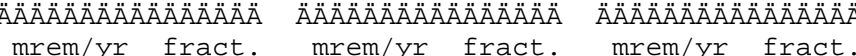

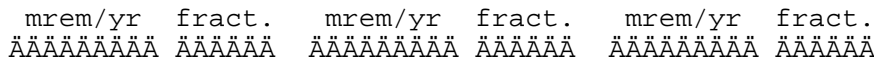

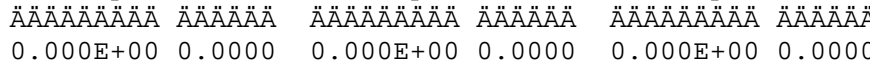

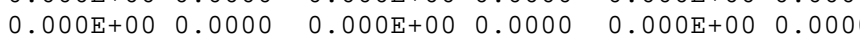

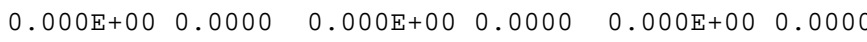
$\begin{array}{llllll}0.000 \mathrm{E}+00 & 0.0000 & 0.000 \mathrm{E}+00 & 0.0000 & 0.000 \mathrm{E}+0 \odot & 0.0000\end{array}$

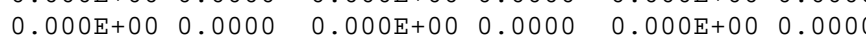

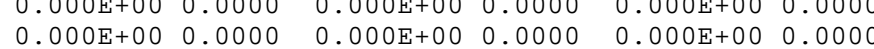

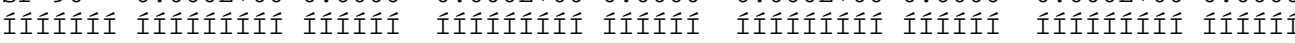

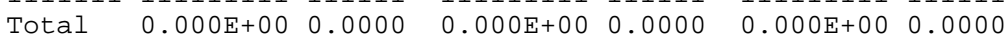
ÍÍÍÍÍÍÍ I ÍÍÍÍ ÄÄÄÄÄÄÄÄÄ ÄÄÄÄÄA $\odot .000 \mathrm{E}+\odot \odot \odot .000 \odot$ $0.000 \mathrm{E}+000.0000$ $0.000+000.0000$ $\odot .000 \mathrm{E}+0 \odot$ ०. $\odot .0000$ $0.000 \mathrm{E}+000.0000$ Íííííííi ííííí $\odot *$ Sum of all water independent and dependent pathways. $\begin{array}{cccc}0.000 \mathrm{E}+0 \odot & 0.000 \odot & 0.000 \mathrm{E}+00 & 0.0000 \\ 0.00 \mathrm{E}+0 \odot & 0.0000\end{array}$ $\odot .000 E+00 \quad 0.0000 \quad 0.000 E+00 \quad 0.0000$

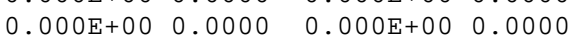

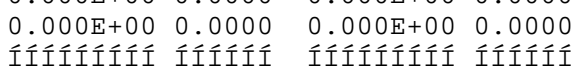

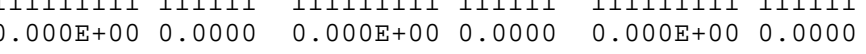


Total Dose Contributions TDOSE( $i, p, t)$ for Individual Radionuclides (i) and Pathways ( $p$ )

As $\mathrm{mrem} / \mathrm{yr}$ and Fraction of Total Dose At $t=1.000 \mathrm{E}+03$ years

Ground Inhalation
In
A water Independent Pathways (Inhalation excludes radon)

Radio - ÄÄÄÄÄÄÄÄÄÄÄÄÄÄÄ

$$
\text { Radon }
$$

Plant

Meat

Milk

Soil

Radio- ÄÄÄÄÄÄÄÄÄÄAAAA
Nuclide mrem/yr fract
ÄÄÄÄÄ Ä̈ÄÄÄÄ ÄÄÄÄ ÄÄÄÄÄÄÄ Ä Ä̈̈ÄÄÄÄÄÄÄ ÄÄÄÄÄÄ

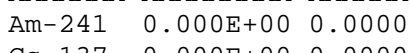
CS $-137 \quad 0.000 \mathrm{E}+00 \quad 0.0000$ Eu-152 $0.000 \mathrm{E}+0 \odot \quad 0.000 \odot$ $\mathrm{PU}-238 \quad 0.000 \mathrm{E}+00 \quad 0.0000$ $\mathrm{Pu}-2390.000 \mathrm{E}+00 \quad 0.0000$ $\begin{array}{lll}\mathrm{Pu}-239 & 0.00 \odot \mathrm{E}+\odot \odot & \odot .0000 \\ \mathrm{Sr}-90 & 0.000 \mathrm{E}+0 \odot & 0.0000\end{array}$ íííííi ííííííí íííít Total $0.000 \mathrm{E}+000.0000$

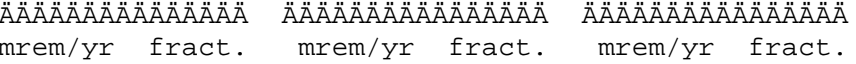
МลAAAAÄÄÄÄÄÄÄA mrem/yr fract. mrem/yr fract.

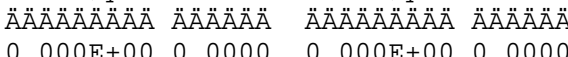
$\begin{array}{llllll}0.000 \mathrm{E}+0 \odot & 0.000 \odot & 0.000 \mathrm{E}+0 \odot & 0.000 \odot & 0.000 \mathrm{E}+0 \odot & 0.000\end{array}$ $\begin{array}{llllll}0.000 \mathrm{E}+00 & 0.0000 & 0.000 \mathrm{E}+00 & 0.0000 & 0.000 \mathrm{E}+00 & 0.0000\end{array}$

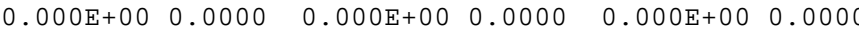
$\begin{array}{llllll}0.000 E+00 & 0.0000 & 0.000 E+00 & 0.0000 & 0.000 E+00 & 0.0000\end{array}$

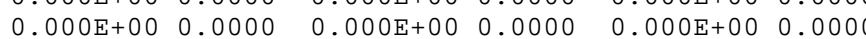

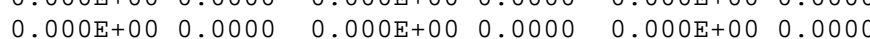
Íííííííi ííííi ííííííí ííííi ííííííí íííít mrem/yr fract ÄÄÄÄÄÄÄÄÄ ÄÄÄÄÄÄ $0.000 \mathrm{E}+00 \quad 0.0000$ $\odot .000 \mathrm{E}+0 \odot \quad 0.0000$ $\odot .000 \mathrm{E}+0 \odot \quad 0.0000$ $0.000 \mathrm{E}+00 \quad 0.0000$ $0.000 \mathrm{E}+000.0000$ ÍÍÍÍÍÍí ÍÍÍíí $0.000 \mathrm{E}+0 \odot \odot .0000$ $0.000 \mathrm{E}+000.0000$ $0.000 \mathrm{E}+000.0000$

$0.000 \mathrm{E}+000.0000$ ÄÄÄÄÄÄÄÄÄÄÄÄÄÄÄÄÄ mrem/yr fract. ÄÄÄÄÄÄÄÄÄ ÄÄÄÄÄ̈̈̈ $\odot .000 \mathrm{E}+0 \odot \quad 0.0000$ $0.000 \mathrm{E}+000.0000$ $\odot .000 \mathrm{E}+000.0000$ $0.000 \mathrm{E}+00 \quad 0.0000$ $\odot .000 \mathrm{E}+00$ ० $\odot .000 \odot$ $0.000 \mathrm{E}+000.0000$ IIÍÍÍÍÍÍ ÍÍÍÍÍ Total Dose Contributions TDOSE( $i, p, t)$ for Individual Radionuclides (i) and Pathways (p) As $\mathrm{mrem} / \mathrm{yr}$ and Fraction of Total Dose At $t=1.000 \mathrm{E}+03$ years Water Dependent Pathways Water Radon

Radio - ÄÄÄÄÄÄÄÄÄÄÄÄÄÄÄÄ Nuclide mrem/yr fract. ÄÄÄÄÄÄÄ ÄÄÄÄÄÄÄÄÄ ÄÄÄÄÄÄ Am-241 $0.000 \mathrm{E}+0 \odot \quad 0.000 \odot$ $\begin{array}{lll}\text { CS }-137 & 0.000 \mathrm{E}+00 & 0.0000 \\ \mathrm{EH}-152 & 0.000 \mathrm{E}+00 & 0.0000\end{array}$ $\mathrm{Eu}-152 \quad 0.000 \mathrm{E}+00 \quad 0.0000$ $\mathrm{Pu}-239 \quad 0.000 \mathrm{E}+0 \odot \quad 0.0000$ $\begin{array}{lll}\mathrm{Pu}-239 & 0.00 \odot \mathrm{E}+\odot \odot & 0.0 \odot \odot \odot \\ \mathrm{Sr}-90 & 0.000 \mathrm{E}+0 \odot & 0.000 \odot\end{array}$

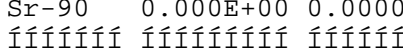

Total $0.000 \mathrm{E}+000.0000$

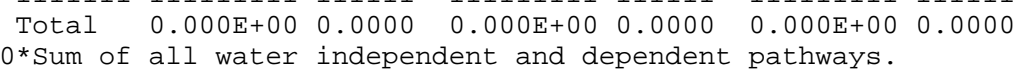

$$
\text { Plant }
$$

Meat

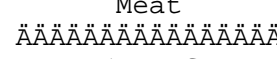
mrem/yr fract. ÄÄÄÄÄÄÄÄÄ ÄÄÄÄÄÄ $\odot . \odot \odot \odot E+\odot \odot$ $\odot .00 \odot \odot$ $\odot .000 \mathrm{E}+00$ ०. 0.0000 $\odot .0 \odot \odot E+\odot \odot \quad \odot . \odot \odot \odot \odot$ $\odot .000 \mathrm{E}+00 \quad 0.0000$ $\odot .000 \mathrm{E}+00 \quad 0.0000$

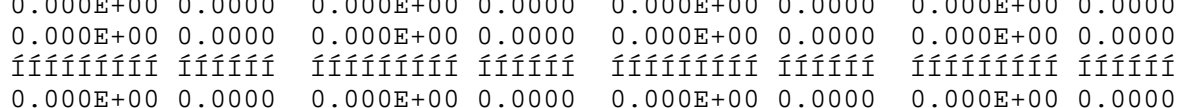

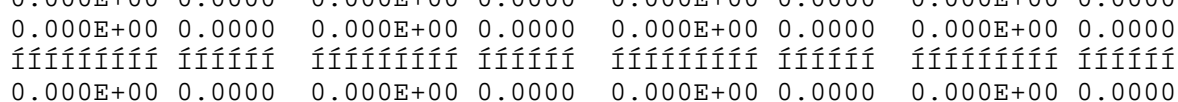

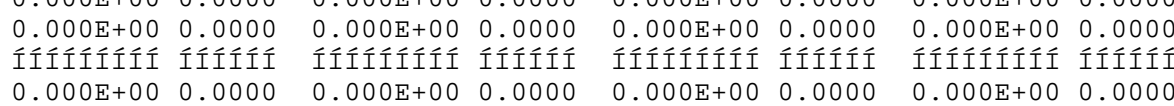

Milk

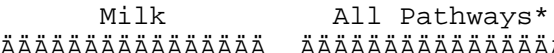
mrem/yr fract. mrem/yr fract. ÄÄÄÄÄÄÄÄÄ ÄÄÄÄÄÄ ÄÄÄÄÄÄÄÄÄ ÄÄÄÄÄÄ

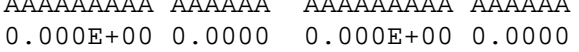

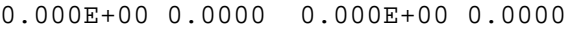
$0.000 E+00 \quad 0.0000 \quad 0.000 E+00 \quad 0.0000$ $\odot .00 \odot E+\odot \odot \quad \odot .00 \odot \odot \quad \odot .00 \odot E+\odot \odot \quad \odot .0 \odot \odot \odot$ $0.000 E+00 \quad 0.0000 \quad 0.000 E+00 \quad 0.0000$ $0.000 E+00 \quad 0.0000 \quad 0.000 E+00 \quad 0.0000$ Iííííííi ííííi ííííííí ííííi

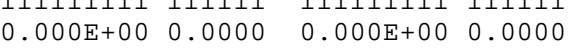

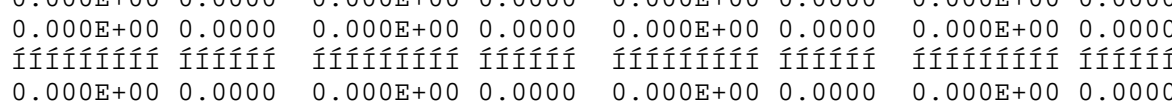

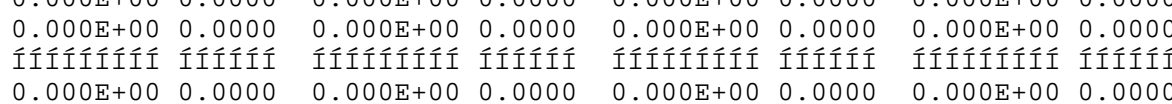

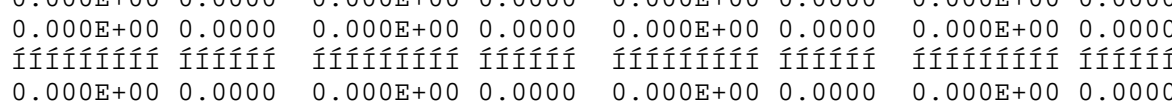
$\odot$ Sum of all water independent and dependent pathways. 
๑ Parent Product Thread (i) (j) Fraction ÄÄÄÄÄÄÄÄÄÄ ÄÄÄÄÄÄÄÄÄÄ ÄÄÄÄÄÄÄÄ $\begin{array}{lll}\mathrm{Am}-241 & \mathrm{Am}-241 & 1.000 \mathrm{E}+00\end{array}$ Am-241 $\quad \mathrm{Np}-237+\mathrm{D} \quad 1.000 \mathrm{E}+\Theta$ Am-241 U-233 $1.000 \mathrm{E}+00$

Am-241 Th-229+D 1. $1.00 \mathrm{E}+00$ Am-241 äDSR $(j)$ OCs $-137+D \quad$ Cs $-137+D$ OEu-152 Eu-152 OEU-152

Eu-152 Eu-152 OPu-238

๑Pu-238

$\mathrm{Pu}-238$

$\mathrm{Pu}-238$

$\mathrm{Pu}-238$

Pu-238

$\mathrm{Pu}-238$

०Pu-239

$\mathrm{Pu}-239$

$\mathrm{Pu}-239$

$\mathrm{Pu}-239$

$\mathrm{Pu}-239$

OSr-90+D

Eu-152

Gd-152

$\operatorname{äDSR}(j)$

$\mathrm{Pu}-238$

$\mathrm{Pu}-238$

$\mathrm{U}-234$

Th- 230

$\mathrm{Ra}-226+\mathrm{D}$

$\mathrm{Pb}-210+\mathrm{D}$

äDSR ( $j$ )

$\mathrm{Pu}-239$

$\mathrm{U}-235+\mathrm{D}$

$\mathrm{Pa}-231$

AC-227+D

äDSR $(j)$

$1.000 \mathrm{E}+00$

7. 208E- -1

$2.792 \mathrm{E}-01$
$2.792 \mathrm{E}-01$

1. $840 \mathrm{E}-09$

$1.000 \mathrm{E}+00$

$1.000 \mathrm{E}+00$

1. $000 \mathrm{E}+\odot$

1. $000 \mathrm{E}+\odot \circ$

1. $000 \mathrm{E}+\odot 0$

1. $000 \mathrm{E}+0 \odot$

1. $000 \mathrm{E}+00$

$1.000 \mathrm{E}+00$

$1.000 \mathrm{E}+00$

Source Ratios Summed Over All Pathways

Parent and Progeny Principal Radionuclide Contributions Indicated

$\operatorname{DSR}(j, \mathrm{t})$ At Time in Years $(\mathrm{mrem} / \mathrm{yr}) /(\mathrm{pCi} / \mathrm{g})$

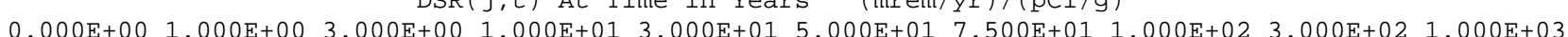

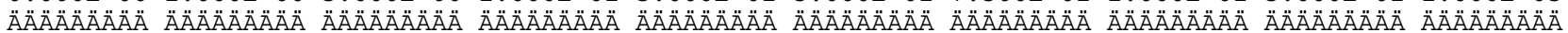

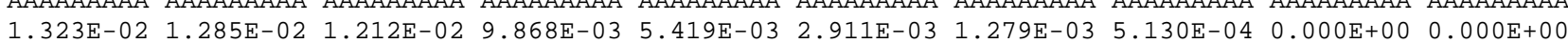

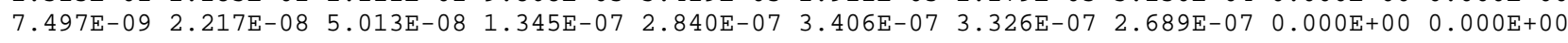

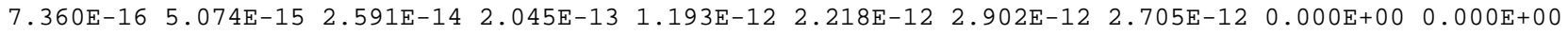
$5.229 \mathrm{E}-19$ 7.756E-18 8.824E-17 2.135E-15 3.988E-14 1.362E-13 3.072E-13 4.483E-13 0.000E+00 $0.000 \mathrm{E}+00$

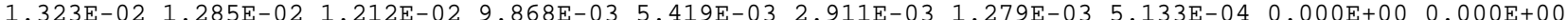
$9.085 \mathrm{E}-02$ 8.849E-02 8.395E-02 6.975E-02 4.064E-02 2.319E-02 1.098E-02 4.713E-03 $0.000 \mathrm{E}+00 \quad 0.000 \mathrm{E}+00$

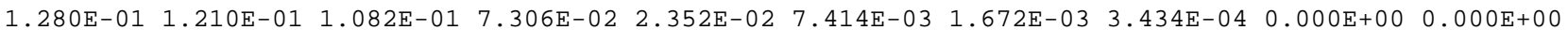

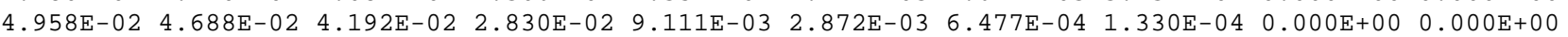

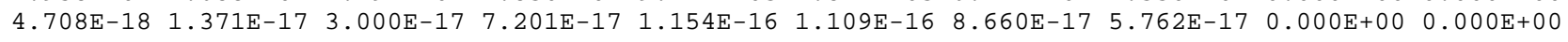

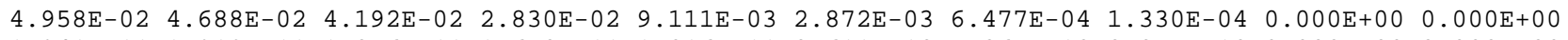

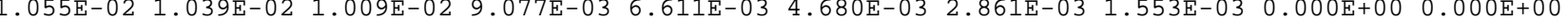

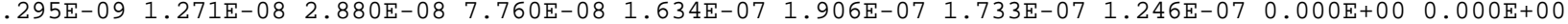

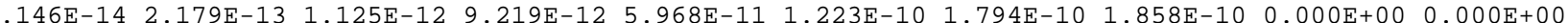
$\begin{array}{llllllllll}1.298 \mathrm{E}-16 & 1.932 \mathrm{E}-15 & 2.213 \mathrm{E}-14 & 5.489 \mathrm{E}-13 & 1.096 \mathrm{E}-11 & 3.975 \mathrm{E}-11 & 9.598 \mathrm{E}-11 & 1.491 \mathrm{E}-10 & 0.000 \mathrm{E}+00 & 0.000 \mathrm{E}+00\end{array}$ $1.561 \mathrm{E}-20 \quad 4.763 \mathrm{E}-19 \quad 1.159 \mathrm{E}-17 \quad 7.973 \mathrm{E}-16 \quad 3.885 \mathrm{E}-14 \quad 1.969 \mathrm{E}-13 \quad 5.743 \mathrm{E}-13 \quad 9.526 \mathrm{E}-13 \quad 0.000 \mathrm{E}+00 \quad 0.000 \mathrm{E}+00$

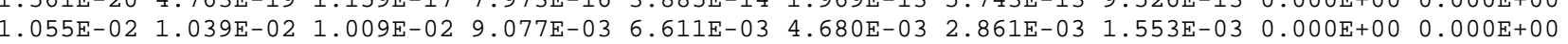
1.162E-02 1.154E-02 1.138E-02 1.082E-02 9.220E-03 7.640E-03 5.687E-03 3.757E-03 $0.000 \mathrm{E}+00 \quad 0.000 \mathrm{E}+00$

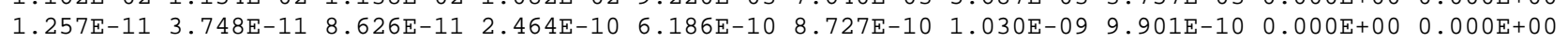
1.387E-16 9.613E-16 4.963E-15 4.078E-14 2.660E-13 $5.506 \mathrm{E}-13 \quad 8.215 \mathrm{E}-13 \quad 8.717 \mathrm{E}-13 \quad 0.000 \mathrm{E}+00 \quad 0.000 \mathrm{E}+00$

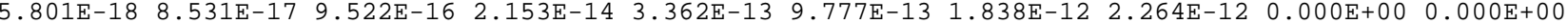
1.162E-02 1.154E-02 1.138E-02 1.082E-02 9.220E-03 7.640E-03 5.687E-03 3.757E-03 $0.000 \mathrm{E}+00 \quad 0.000 \mathrm{E}+00$ 128E-04 $7.798 \mathrm{E}-04 \quad 7.177 \mathrm{E}-04 \quad 5.365 \mathrm{E}-04 \quad 2.314 \mathrm{E}-04 \quad 9.799 \mathrm{E}-05$ 3. $207 \mathrm{E}-05 \quad 9.574 \mathrm{E}-06$ - $0.000 \mathrm{E}+00 \quad 0.000 \mathrm{E}+00$

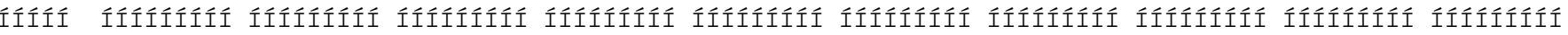
$\odot$

Single Radionuclide Soil Guidelines $G(i, t)$ in $\mathrm{pCi} / \mathrm{g}$

Basic Radiation Dose Limit $=2.500 \mathrm{E}+01 \mathrm{mrem} / \mathrm{yr}$

onuclide

\begin{tabular}{|c|c|}
\hline & .000 \\
\hline 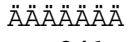 & ÄÄÄÄÄÄÄ \\
\hline $\mathrm{Am}-241$ & $1.890 \mathrm{E}+$ \\
\hline Cs -137 & $2.752 \mathrm{E}$ \\
\hline Eu-152 & $1.408 \mathrm{E}$ \\
\hline Pu-238 & $2.370 \mathrm{E}$ \\
\hline Pu-239 & $2.152 \mathrm{E}$ \\
\hline $\mathrm{Sr}-90$ & $3.076 \mathrm{E}$ \\
\hline ÍÍÍÍÍÍ & ÍÍÍÍÍ \\
\hline
\end{tabular}
${ }^{*}$ At specific activity limit

\begin{abstract}
1. $\odot \odot \odot E+\odot \odot$ ÄÄÄÄÄÄÄÄÄ 1. $945 \mathrm{E}+03$ $2.825 \mathrm{E}+02$ $1.489 \mathrm{E}+02$ $2.405 \mathrm{E}+03$ 2. $167 \mathrm{E}+03$ 3. $206 \mathrm{E}+04$
\end{abstract}

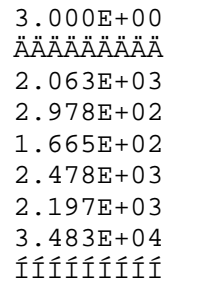

1. $\odot \odot \odot E+\odot 1$ AAAAAAAAA $2.533 \mathrm{E}+03$ $3.584 \mathrm{E}+02$ 2. $467 \mathrm{E}+02$ 2. $754 \mathrm{E}+03$ 2. $311 \mathrm{E}+03$ $4.660 \mathrm{E}+04$

\begin{tabular}{|c|c|c|c|}
\hline 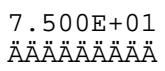 & 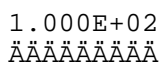 & 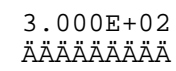 & 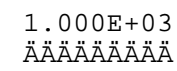 \\
\hline 1. $955 \mathrm{E}+04$ & $4.870 E+04$ & *3. 431E+12 & $* 3.431 \mathrm{E}+12$ \\
\hline $2.277 \mathrm{E}+03$ & $5.305 E+03$ & $\star 8.704 \mathrm{E}+13$ & $* 8.704 \mathrm{E}+13$ \\
\hline $1.078 \mathrm{E}+04$ & $5.247 E+04$ & ${ }^{*} 1.765 \mathrm{E}+14$ & ${ }^{*} 1.765 \mathrm{E}+14$ \\
\hline $8.737 \mathrm{E}+03$ & $1.610 \mathrm{E}+04$ & $* 1.712 \mathrm{E}+13$ & $* 1.712 \mathrm{E}+13$ \\
\hline $4.396 \mathrm{E}+03$ & $6.655 E+03$ & ${ }^{*} 6.214 \mathrm{E}+10$ & $* 6.214 \mathrm{E}+10$ \\
\hline 7.795 & $2.611 \mathrm{E}+$ & *1.365E+14 & $5 E+14$ \\
\hline Í́f & Íííííl & Íííííííí & Ííííííít \\
\hline
\end{tabular}


Summed Dose/Source Ratios DSR( $i, \mathrm{t})$ in (mrem/yr)/(pCi/g)

and Single Radionuclide Soil Guidelines $\mathrm{G}(\mathrm{i}, \mathrm{t})$ in $\mathrm{pCi} / \mathrm{g}$

at $\operatorname{tmin}=$ time of minimum single radionuclide soil guideline

and at $\mathrm{tmax}=\mathrm{time}$ of maximum total dose $=0.000 \mathrm{E}+0 \odot$ years

\begin{tabular}{|c|c|c|c|c|c|c|}
\hline 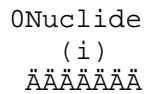 & $\begin{array}{c}\text { Initial } \\
(p C i / g) \\
\text { ÄÄÄÄÄÄÄÄ }\end{array}$ & 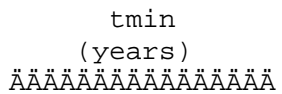 & $\operatorname{DSR}(i, \operatorname{tmin})$ & 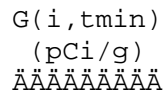 & $\operatorname{DSR}(i, \operatorname{tmax})$ & $\begin{array}{l}\mathrm{G}(\mathrm{i}, \mathrm{tmax}) \\
(\mathrm{pC} i / \mathrm{g}) \\
\mathrm{A} A \ddot{A} A \ddot{A} A \ddot{A} A\end{array}$ \\
\hline Am-241 & $2.880 \mathrm{E}+01$ & $\odot .000 \mathrm{E}+\odot \odot$ & $1.323 \mathrm{E}-02$ & $1.890 \mathrm{E}+03$ & $1.323 \mathrm{E}-02$ & $1.890 \mathrm{E}+03$ \\
\hline Cs -137 & 1. $060 \mathrm{E}+03$ & $\odot .00 \odot \mathrm{E}+\odot \odot$ & $9.085 E-02$ & $2.752 \mathrm{E}+02$ & $9.085 E-02$ & $2.152 t+6$ \\
\hline Eu-152 & $2.640 \mathrm{E}+0 \odot$ & $\odot . \odot \odot \odot E+\odot \odot$ & $1.776 \mathrm{E}-01$ & $1.408 \mathrm{E}+02$ & $1.776 \mathrm{E}-01$ & $1.408 \mathrm{E}+02$ \\
\hline$u-238$ & $1.560 \mathrm{E}+01$ & $\odot . \odot \odot \odot E+\odot \odot$ & 1. $055 \mathrm{E}-02$ & $2.370 \mathrm{E}+03$ & E- 02 & $2.370 \mathrm{E}+03$ \\
\hline Pu-239 & $8.400 \mathrm{E}+01$ & $\odot . \odot \odot \odot E+\odot \odot$ & $1.162 \mathrm{E}-02$ & $E+03$ & $2 E-02$ & $2 \mathrm{E}+03$ \\
\hline $\operatorname{Sr}-90$ & $3.410 \mathrm{E}+01$ & $\odot .00 \odot \mathrm{E}+\odot \odot$ & $8.128 \mathrm{E}-04$ & $3.076 E+04$ & $8.128 E-04$ & $3.076 \mathrm{E}+\odot$ \\
\hline f́f́ín & Ííííííííi & Ííííííííííííííí & Íííííííín & Íííííííí & Ííííííííí & Íííííín \\
\hline
\end{tabular}


onuclide Parent THF(i) (j) (i) Am-241 AmAAA AAAAAAAAA Am-241 Am-241 1.000E+00 ANp-237 Am-241 1.000E+O० OU-233 Am-241 1.000E+00 ०Th-229 Am-241 1. $000 \mathrm{E}+\odot 0$ ๑CS -137 Cs -137 1. $000 \mathrm{E}+\odot \Theta$ OEU-152 Eu-152 $7.208 E-01$ EU-152 EU-15 Eu-152 Eu-152 2.792E-01 Eu-152 äDOSE(j)

OGd-152 Eu-152 2.792E-01 OPu-238 Pu-238 1.840E- 09 $\mathrm{Pu}-238$ Pu-238 1.000E+०0 $\mathrm{Pu}-238$ äDOSE $(j)$

๑U-234 PU-238 1. $000 \mathrm{E}+0 \odot$ OTh-230 Pu-238 $1.000 \mathrm{E}+00$ OTh -230 $\odot$ Ra-226 Pu-238 $1.000 \mathrm{E}+00$ OPb-210 $\mathrm{Pu}-238 \quad 1.000 \mathrm{E}+00$ ๑Pu-239 Pu-239 1.00०E +00 OU-235 Pu-239 1.000E+०0

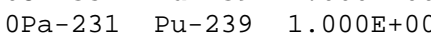
OAC-227 Pu-239 1. $000 \mathrm{E}+00$ OSr-90 Sr-90 $1.000 \mathrm{E}+00$ IIITíí ítííít ítitítít
Individual Nuclide Dose Summed Over All Pathways

Parent Nuclide and Branch Fraction Indicated

$$
\operatorname{DOSE}(j, t), \mathrm{mrem} / \mathrm{yr}
$$

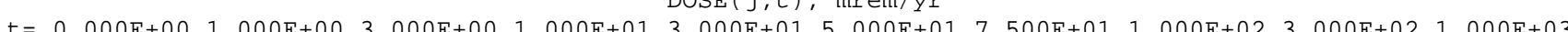

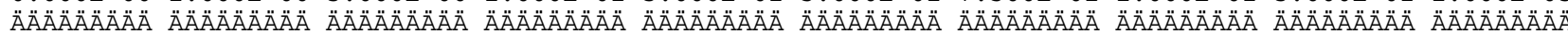

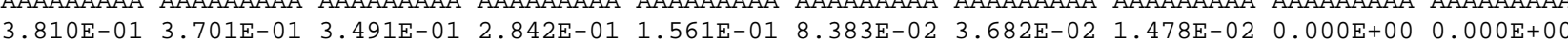

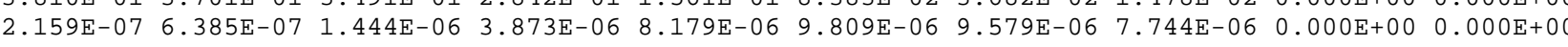
2.120E-14 1.461E-13 7.461E-13 5.890E-12 3.437E-11 6.387E-11 8.359E-11 $7.790 \mathrm{E}-11 \quad 0.000 \mathrm{E}+00 \quad 0.000 \mathrm{E}+00$ 1.506E-17 2.234E-16 2.541E-15 6.149E-14 1.148E-12 3.922E-12 8.848E-12 1.291E-11 0.000E+00 $0.000 \mathrm{E}+00$ $\begin{array}{lllllllllll}9.630 \mathrm{E}+01 & 9.380 \mathrm{E}+01 & 8.899 \mathrm{E}+01 & 7.393 \mathrm{E}+01 & 4.308 \mathrm{E}+01 & 2.458 \mathrm{E}+01 & 1.164 \mathrm{E}+01 & 4.996 \mathrm{E}+00 & 0.000 \mathrm{E}+00 & 0.000 \mathrm{E}+00\end{array}$

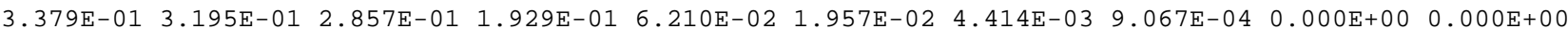
$\begin{array}{lllll}1.309 \mathrm{E}-01 & 1.238 \mathrm{E}-01 & 1.107 \mathrm{E}-01 & 7.471 \mathrm{E}-02 & 2\end{array}$

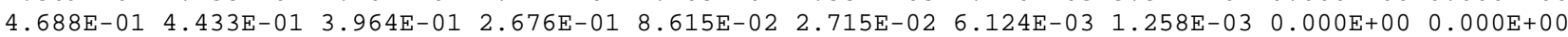

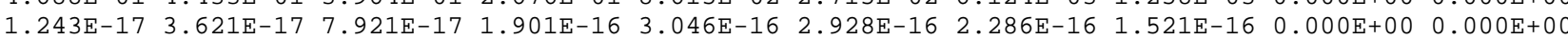

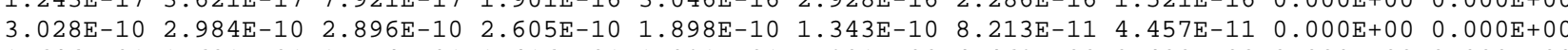

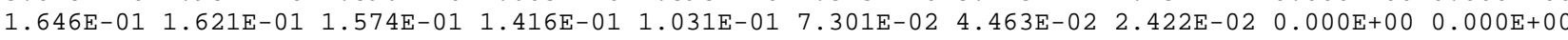

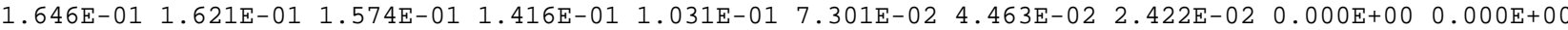
6.701E-08 1.983E-07 4.492E-07 1.211E-06 2.549E-06 2.974E-06 2.703E-06 $1.943 \mathrm{E}-06 \quad 0.000 \mathrm{E}+00 \quad 0.000 \mathrm{E}+00$ 4.908E-13 3.399E-12 1.754E-11 1.438E-10 9.310E-10 1.908E-09 2.799E-๑9 2.898E-09 $0.000 \mathrm{E}+00 \quad 0.000 \mathrm{E}+00$

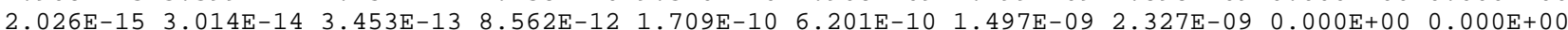
$2.435 \mathrm{E}-19$ 7.430E-18 $1.808 \mathrm{E}-16 \quad 1.244 \mathrm{E}-14 \quad 6.060 \mathrm{E}-13 \quad 3.071 \mathrm{E}-12 \quad 8.959 \mathrm{E}-12$ 1.486E-11 $0.000 \mathrm{E}+00 \quad 0.000 \mathrm{E}+00$

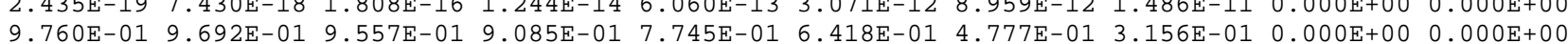

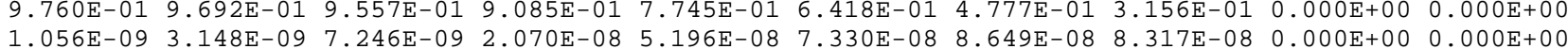
$\begin{array}{lllllllll}1.165 \mathrm{E}-14 & 8.075 \mathrm{E}-14 & 4.169 \mathrm{E}-13 & 3.425 \mathrm{E}-12 & 2.234 \mathrm{E}-11 & 4.625 \mathrm{E}-11 & 6.901 \mathrm{E}-11 & 7.322 \mathrm{E}-11 & 0.00 \odot \mathrm{E}+\odot \odot \quad 0.00 \odot \mathrm{E}+\Theta \odot\end{array}$ 4.873E-16 7.166E-15 7.998E-14 1.809E-12 2.824E-11 8.213E-11 1.544E-10 1.902E-10 0.000E+00 $0.000 \mathrm{E}+00$ 2.772E-02 2.659E-02 2.447E-02 1.829E-02 7.891E-03 3.341E-03 1.094E-03 3.265E-04 $0.000 \mathrm{E}+00 \quad 0.000 \mathrm{E}+0 \odot$

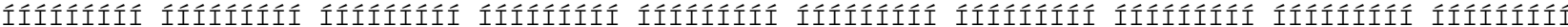

THF $(i)$ is the thread fraction of the parent nuclide. 
oNuclide Parent THF(i) ( j) (i) Am-241 AMAAAA AAAAAAAAA Am-241 Am-241 1.000E+OO ONp-237 Am-241 $1.000 \mathrm{E}+00$ OU-233 Am-241 1.000E+00 OTh-229 Am-241 1.000E+๑९ ๑CS -137 Cs -137 1. $000 \mathrm{E}+\odot \Theta$ थEu-152 Eu-152 7.208E-01 $\begin{array}{lll} & \end{array}$ $\begin{array}{lll}E u-152 & E u-152 & 2.792 E-01 \\ E u-152 & \text { äS }(j): & \end{array}$ Eu-152 äs $(j)$

OGd-152 Eu-152 2.792E-01 OPu-238 Pu-238 1.840E- 99 $\mathrm{Pu}-238$ Pu-238 1.000E+@० $\mathrm{Pu}-238$ äS $(j)$ :

OU-234 Pu-238 1.000E+०๑ ๑Th-230 Pu-238 $1.000 \mathrm{E}+00$ ORa -226 Pu $2381.000 E+00$ $\odot \mathrm{Ra}-226 \quad \mathrm{Pu}-238 \quad 1.000 \mathrm{E}+00$ OPb-210 $\mathrm{Pu}-238 \quad 1.000 \mathrm{E}+00$ OPu-239 Pu-239 1.000E+०० OU-235 Pu-239 1.000E +00

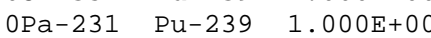
OAC-227 Pu-239 1. $200 \mathrm{E}+00$ OSr-90 Sr-90 $1.000 \mathrm{E}+00$ IIIIííi íííííi ííííííít

THF(i) is the thread fraction ORESCALC. EXE execution time =
Individual Nuclide Soil Concentration Parent Nuclide and Branch Fraction Indicated

$$
\mathrm{S}(j, \mathrm{t}), \mathrm{pCi} / \mathrm{g}
$$

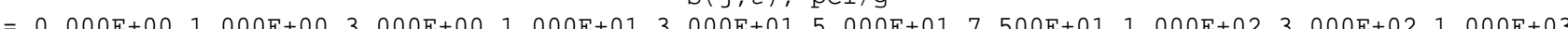

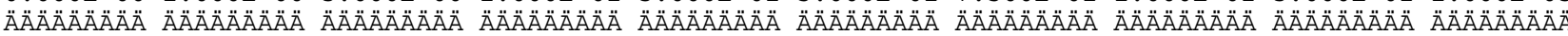

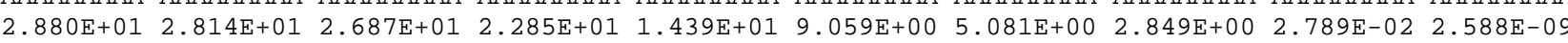

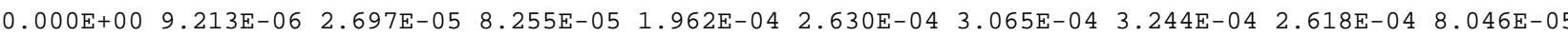

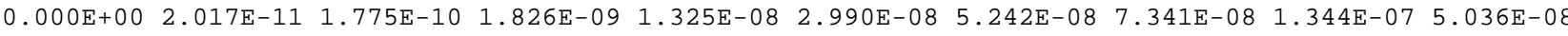

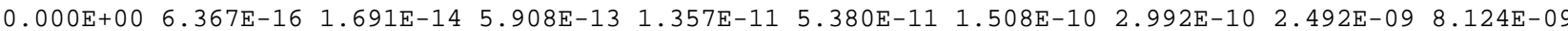

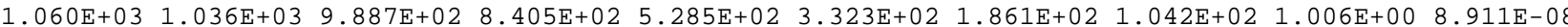

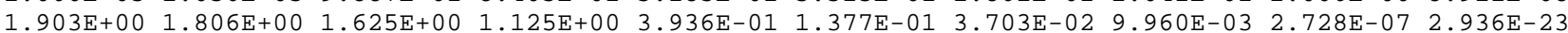

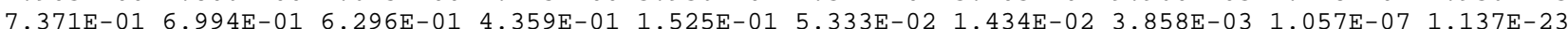

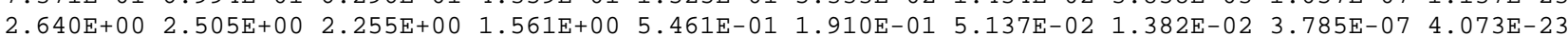
$0.000 \mathrm{E}+00 \quad 4.607 \mathrm{E}-15 \quad 1.312 \mathrm{E}-14 \quad 3.669 \mathrm{E}-14 \quad 7.073 \mathrm{E}-14 \quad 8.203 \mathrm{E}-14 \quad 8.568 \mathrm{E}-14 \quad 8.583 \mathrm{E}-14 \quad 7.768 \mathrm{E}-14 \quad 5.372 \mathrm{E}-14$ $2.870 \mathrm{E}-08$ 2.847E-08 $2.801 \mathrm{E}-08$ 2.647E-08 $2.250 \mathrm{E}-08$ 1.913E-08 $1.562 \mathrm{E}-08$ 1.275E-08 $2.514 \mathrm{E}-09$ 8.563E-12

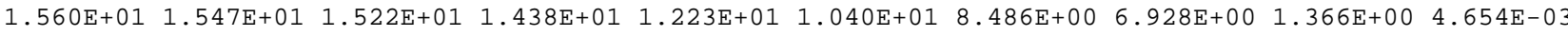

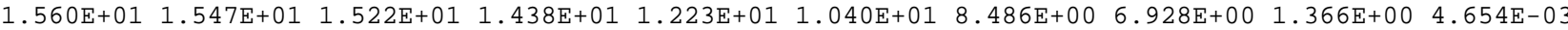
$0.000 \mathrm{E}+00$ 4.386E-05 1.294E-04 4.067E-04 1.032E-03 1.454E-03 1.768E-03 1.911E-03 1.072E-03 1.017E-05 $0.000 \mathrm{E}+00$ 1.979E-10 1.762E-09 $1.883 \mathrm{E}-08$ 1.517E-07 3.780E-07 7.441E-@7 $1.160 \mathrm{E}-06$ 4.042E-06 5.579E-06

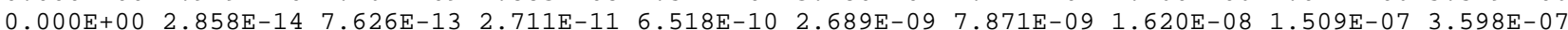

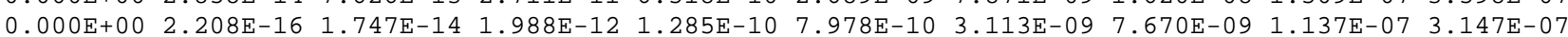
$\begin{array}{llllllllllll}8.40 \Theta \mathrm{E}+01 & 8.398 \mathrm{E}+01 & 8.394 \mathrm{E}+01 & 8.379 \mathrm{E}+01 & 8.338 \mathrm{E}+01 & 8.297 \mathrm{E}+01 & 8.246 \mathrm{E}+01 & 8.196 \mathrm{E}+01 & 7.802 \mathrm{E}+01 & 6.567 \mathrm{E}+01\end{array}$

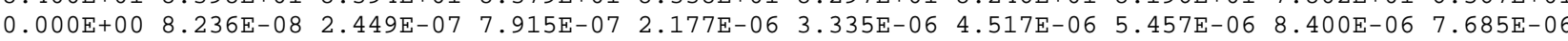

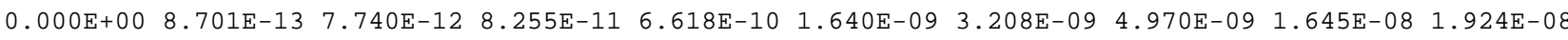
$0.000 \mathrm{E}+00$ 9.124E-15 2.379E-13 7.811E-12 1.527E-10 5.263E-10 1.268E-09 2.211E-09 9.377E-09 1.153E-08 $\begin{array}{llllllllll}3.410 \mathrm{E}+01 & 3.282 \mathrm{E}+01 & 3.041 \mathrm{E}+01 & 2.327 \mathrm{E}+01 & 1.084 \mathrm{E}+01 & 5.049 \mathrm{E}+00 & 1.943 \mathrm{E}+00 & 7.477 \mathrm{E}-01 & 3.594 \mathrm{E}-04 & 8.756 \mathrm{E}-16\end{array}$

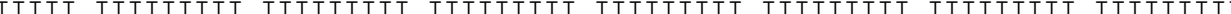

\section{the parent nuclide.}

1.48 seconds 
1RESRAD, Version 6.3 T« Limit $=180$ days Summary : CAU 383 Drainage

Table of Contents

ÄÄÄÄÄÄÄÄÄÄÄÄÄÄÄÄÄ

Part I: Mixture Sums and Single Radionuclide Guidelines

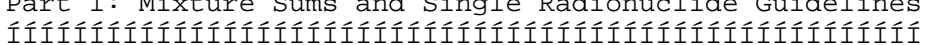

Dose Conversion Factor (and Related) Parameter Summary ... Site-Specific Parameter Summary ..................... 5 Summary of Pathway Selections ..................... 11 Contaminated Zone and Total Dose Summary ............... 12 Total Dose Components

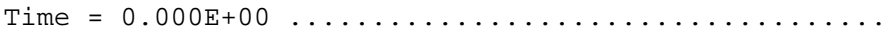

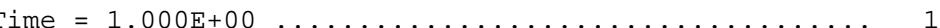

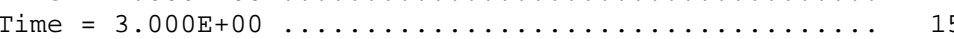

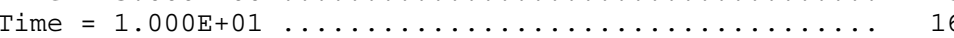

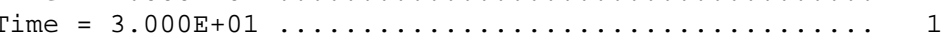

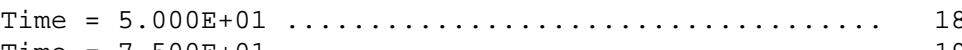

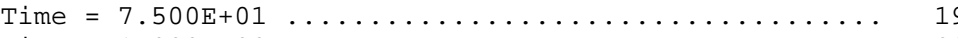

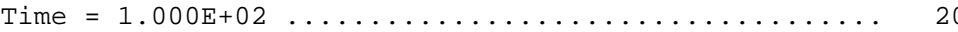

Time $=3.000 \mathrm{E}+02 \ldots \ldots \ldots \ldots \ldots \ldots \ldots \ldots \ldots \ldots \ldots \ldots, 21$

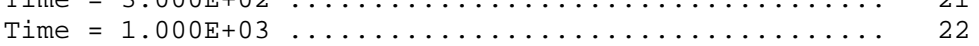

Dose/Source Ratios summed Over All Pathways ............. 23

Single Radionuclide Soil Guidelines ................. 23

Dose Per Nuclide Summed over All Pathways $\ldots \ldots \ldots \ldots \ldots \ldots \ldots$

Soil Concentration Per Nuclide ................... 26

\section{Uncontrolled When Printed}


Dose Conversion Factor (and Related) Parameter Summary File: FGR 13 MORBIDITY

$\odot \quad 3$

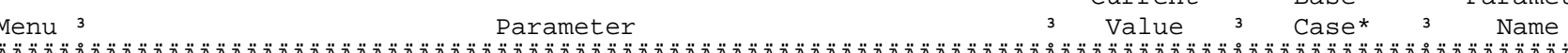

${ }^{3}$ Current ${ }^{3}$ Base ${ }^{3}$ Parameter

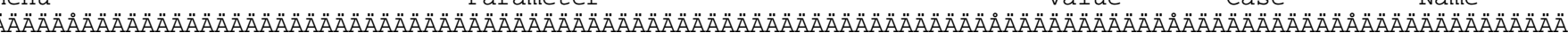

B-1 3 Dose conversion factors for inhalation, $\mathrm{mrem} / \mathrm{pCi}$ :

$B-1 \quad 3 \quad A C-227+D$

$B-1 \quad 3$ Am-241

B-1 3 Cs $-137+D$

$\mathrm{B}-1 \quad 3 \mathrm{~Np}-237+\mathrm{D}$

$\mathrm{B}-1 \quad 3 \mathrm{~Pa}-231$

$\mathrm{B}-1 \quad 3 \mathrm{~Pb}-210+\mathrm{D}$

$\mathrm{B}-1 \quad 3 \mathrm{Pu}-238$

B-1 3 Pu-239

B-1 3 Ra $-226+D$

B-1 3 Sr $-90+D$

B-1 3 Th-229+D

B-1 3 Th-230

$B-1 \quad 3 \quad U-233$

$\mathrm{B}-1 \quad 3 \quad \mathrm{U}-234$

$\mathrm{B}-1 \quad 3 \mathrm{U}-235+\mathrm{D}$

D-1 3 Dose conversion factors for ingestion, $\mathrm{mrem} / \mathrm{pCi}$ :

$D-1 \quad 3 \quad A C-227+D$

$D-1 \quad 3 \quad A m-241$

D-1 3 Cs $-137+D$

$D-1 \quad 3 \quad N P-237+D$

$\mathrm{D}-1-3 \mathrm{~Pa}-231$

$\mathrm{D}-1 \quad 3 \mathrm{~Pb}-210+\mathrm{D}$

$\begin{array}{lll}\mathrm{D}-1 & 3 & \mathrm{~Pb}-210+\mathrm{D} \\ \mathrm{D}-1 & 3 & \mathrm{Pu}-238\end{array}$

$\begin{array}{lll}\mathrm{D}-1 & 3 & \mathrm{Pu}-238 \\ \mathrm{D}-1 & 3 & \mathrm{Pu}-239\end{array}$

D-1 3 Ra-226+D

D-1 3 Sr $-90+D$

D-1 3 Th-229+D

D-1 3 Th -230

D-1 3 U-233

D-1 3 U -234

D-1 3 U $-235+D$

D-1 3 U $\begin{array}{lll}3 & -235+D \\ & 3 & \end{array}$

D-34 3 Food transfer factors:

D-34 3 Ac-227+D, plant/soil concentration ratio, dimensionless

$\mathrm{D}-344^{3} \mathrm{AC}-227+\mathrm{D}$, beef/livestock-intake ratio, $(\mathrm{pCi} / \mathrm{kg}) /(\mathrm{pCi} / \mathrm{d})$

$\mathrm{D}-343 \mathrm{AC}-227+\mathrm{D}, \mathrm{milk} /$ livestock-intake ratio, $(\mathrm{pCi} / \mathrm{L}) /(\mathrm{pCi} / \mathrm{d})$

D-34 3

D-34 3 Am-241

D-34 3 Am-241

D-34 3 Am-241

D- $34{ }^{3}$

Cs-137+D, plant/soil concentration ratio, dimensionless

$\mathrm{D}-343 \mathrm{Cs}-137+\mathrm{D}$, beef/livestock-intake ratio, (pCi/ $/ \mathrm{kg}) /(\mathrm{pCi} / \mathrm{d})$

D-34 3 Cs $-137+D$

plant/soil concentration ratio, dimensionless , beef/livestock-intake ratio, $(\mathrm{pCi} / \mathrm{kg}) /(\mathrm{pCi} / \mathrm{d})$

$6.724 \mathrm{E}+00^{3} \quad{ }^{3} \quad 6.700 \mathrm{E}+00^{3}{ }^{3} \mathrm{DCF} 2\left(\begin{array}{ll}1 \\ 4\end{array}\right)$

4.440E-01 3 4.440E-01 3 DCF2( 2 )

3 3.190E-05 3 3.190E-05 3 DCF2( 3$)$

$5.400 \mathrm{E}-011^{3} \quad 5.400 \mathrm{E}-01 \quad 3 \mathrm{DCF} 2(4)$

$31.280 E+\Theta \odot \quad 3 \quad 1.280 E+\Theta \odot 3$ DCF2( 5$)$

2.320E-02 3 1.360E-02 3 DCF2 ( 6)

3 3.920E-01 3 3.920E-01 3 DCF2( 7$)$

4.290E-01 3 4.290E-01 3 DCF2( 9)

8.594E-03 3 8.580E-03 3 DCF2 ( 10)

3 1.308E-03 3 1.300E-03 3 DCF2( 11$)$

$2.169 \mathrm{E}+00$ 3 $2.150 \mathrm{E}+00$ 3 $\mathrm{DCF} 2(\mathrm{12})$

$3.260 E-01 \quad 3 \quad 3.260 E-01{ }^{3}$ DCF2 ( 13$)$

$1.350 \mathrm{E}-0131.350 \mathrm{E}-013 \mathrm{DCF} 2(14)$

$1.320 \mathrm{E}-0131.320 \mathrm{E}-01{ }^{3}$ DCF2 ( 15$)$

1.230 E- 01 3 1.230E-01 3 DCF2( 16$)$

33

1. 480E-02 3 1.410E-02 3 DCF3( 1$)$

$3.640 \mathrm{E}-03$ 3 $3.640 \mathrm{E}-03$ 3 DCF3( 2$)$

3 5.000E-05 3 5.000E-05 3 DCF3( 3 )

3 4.444E-03 3 4.440E-03 3 DCF3( 4)

1.060E-02 3 1.060E-02 3 DCF3( 5$)$

$37.276 \mathrm{E}-03$ 3 $5.370 \mathrm{E}-03$ 3 DCF3( 6)

3.200E-03 3 3.200E-03 3 DCF3( 7$)$

3.540E-03 3 3.540E-03 3 DCF3( 9)

1.321E-03 3 1.320E-03 3 DCF3( 10$)$

$31.528 \mathrm{E}-043$ 1.420E- $04{ }^{3}$ DCF3( 11$)$

4.027E-03 3 3.530E-03 3 DCF3( 12)

$35.480 \mathrm{E}-04{ }^{3}$ 5.480E-04 3 DCF3( 13$)$

$2.890 \mathrm{E}-04$ 3 $2.890 \mathrm{E}-04{ }^{3}$ DCF3( 14$)$

$32.830 E-04 \quad 3 \quad 2.830 E-04 \quad 3$ DCF3( 15$)$

2.673E-04 3 2.660E-04 3 DCF3( 16$)$

3.3

3 2.500E-03 3 2.500E-03 $3 \operatorname{RTF}(1,1)$

2.000E-05 3 2.000E-05 3 RTF $(1,2)$

2.000E-05 3 2.000E-05 $3 \operatorname{RTF}(1,3)$

3

1. 000E-03 3 1. $000 \mathrm{E}-03 \quad 3 \operatorname{RTF}(2,1)$

3 5. 000E-05 3 5. 000E-05 3 RTF $(2,2)$

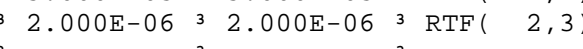

4. $000 \mathrm{E}-02$ 3 4.000E-02 $3 \operatorname{RTF}(3,1)$

3 3. 000E-02 3 3. 000E-02 3 RTF( 3,2$)$

3 8.00९E-๑3 3 8.000E-03 $3 \operatorname{RTF}(3,3)$

2. $\odot \odot \odot E-02 \quad 3 \quad 2.0 \odot \odot E-02 \quad 3$ RTF $(4,1)$

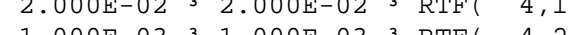

3 1.000-03 1 .

3
$5.000 \mathrm{E}-06$

$\mathrm{D}-343 \mathrm{~Np}-237+\mathrm{D}$, beef/livestock-intake ratio, (pCi/kg)/(pCi/d)

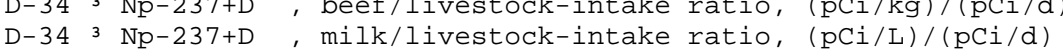

D-34 3

Uncontrolled When Printed 
Dose Conversion Factor (and Related) Parameter Summary (continued)

Menu ${ }^{3}$

Menu 3

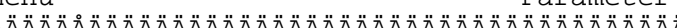

$\mathrm{D}-344^{3} \mathrm{~Pa}-231$

$\mathrm{D}-34{ }^{3} \mathrm{~Pa}-231$

$\mathrm{D}-34{ }^{3} \mathrm{~Pa}-231$

$\mathrm{D}-344^{3}$

$\mathrm{D}-343 \mathrm{~Pb}-210+\mathrm{D}$

D-34 $3 \mathrm{~Pb}-210+\mathrm{D}$

$\mathrm{D}-343 \mathrm{~Pb}-210+\mathrm{D}$

D-34 3

$\mathrm{D}-34 \quad 3 \mathrm{Pu}-238$

$\mathrm{D}-34 \quad 3 \mathrm{Pu}-238$

$\mathrm{D}-34{ }^{3} \mathrm{Pu}-238$

D $-34^{3}$

D-34 3 Pu-239

$\mathrm{D}-34 \quad 3$ Pu-239

D- -34 3 $\mathrm{Pu}-239$

$D-34$ 3

D-34 3 Ra-226+D

$\mathrm{D}-343 \mathrm{Ra}-226+\mathrm{D}$

D-34 3 Ra-226+D

D-34 3

D-34 $3 \mathrm{Sr}-90+\mathrm{D}$

D-34 3 Sr $-90+D$

D-34 3 Sr-90+D

D-34 3

D-34 3 Th-229+D

D-34 3 Th-229+D

D-34 3 Th-229+D

D-34 3

D-34 3 Th -230

D-34 3 Th -230

D -343 Th -230

D-34 3

D- $-34 \quad 3 \quad U-233$

$\mathrm{D}-34 \quad 3 \quad \mathrm{U}-233$

D-34 3 U-233

$\mathrm{D}-34{ }^{3}$

D-34 3 U-234

D-34 $3 \quad \mathrm{U}-234$

D-34 3 U-234

D-34 3

D-34 3 U $-235+D$

D-34 3 U $-235+D$

$\mathrm{D}-343 \mathrm{U}-235+\mathrm{D}$

D-5 3 Bioaccum

D-5 3 AC-227+D

D -5

D-5

3 Am-241

D-5 3 Am-241

D-5 3

\section{Parameter}

File: FGR 13 MORBIDITY

3 Current 3 Base ${ }_{3}$ Parameter

\section{Value ${ }^{3}$ Case $^{*}{ }^{3}$ Name}

plant/soil concentration ratio, di

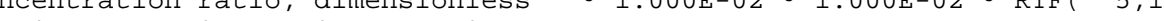

, beef/livestock-intake ratio, $(\mathrm{pCi} / \mathrm{kg}) /(\mathrm{pCi} / \mathrm{d})$

5. $000 \mathrm{E}-03$ 3 $5.000 \mathrm{E}-03$ 3 3 RTF $(5,2)$

5.000E-06 3 5.000E-06 $3 \operatorname{RTF}(5,3)$

3 1. $000 \mathrm{E}-02 \quad 3 \quad 1.000 \mathrm{E}-023 \mathrm{RTF}(6,1)$

3 8.

$3.000 \mathrm{E}-04 \quad 3 \quad 3.000 \mathrm{E}-043 \operatorname{RTF}(6,3)$ beef/livestock-intake ratio, $(\mathrm{pCi} / \mathrm{kg}) /(\mathrm{pCi} / \mathrm{d})$

milk/livestock-intake ratio, $(\mathrm{pCi} / \mathrm{L}) /(\mathrm{pCi} / \mathrm{d})$

, plant/soil concentration ratio, dimensionless beef/livestock-intake ratio, $(\mathrm{pCi} / \mathrm{kg}) /(\mathrm{pCi} / \mathrm{d})$ $\mathrm{milk} /$ livestock-intake ratio, $(\mathrm{pCi} / \mathrm{L}) /(\mathrm{pCi} / \mathrm{d})$

plant/soil concentration ratio, dimensionless beef/livestock-intake ratio, $(\mathrm{pCi} / \mathrm{kg}) /(\mathrm{pCi} / \mathrm{d})$

milk/livestock-intake ratio, $(\mathrm{pCi} / \mathrm{L}) /(\mathrm{pCi} / \mathrm{d})$

plant/soil concentration ratio, dimensionless beef/livestock-intake ratio, ( $\mathrm{pCi} / \mathrm{kg}) /(\mathrm{pCi} / \mathrm{d})$ $\mathrm{milk} /$ livestock-intake ratio, $(\mathrm{pCi} / \mathrm{L}) /(\mathrm{pCi} / \mathrm{d})$

, plant/soil concentration ratio, dimensionless beef/livestock-intake ratio, $(\mathrm{pCi} / \mathrm{kg}) /(\mathrm{pCi} / \mathrm{d})$ $\mathrm{milk} /$ livestock-intake ratio, $(\mathrm{pCi} / \mathrm{L}) /(\mathrm{pCi} / \mathrm{d})$

plant/soil concentration ratio, dimensionless beef/livestock-intake ratio, $(\mathrm{pCi} / \mathrm{kg}) /(\mathrm{pCi} / \mathrm{d})$ $\mathrm{milk} /$ livestock-intake ratio, $(\mathrm{pCi} / \mathrm{L}) /(\mathrm{pCi} / \mathrm{d})$

, plant/soil concentration ratio, dimensionless beef/livestock-intake ratio, $(\mathrm{pCi} / \mathrm{kg}) /(\mathrm{pCi} / \mathrm{d})$ $\mathrm{milk} /$ livestock-intake ratio, $(\mathrm{pCi} / \mathrm{L}) /(\mathrm{pCi} / \mathrm{d})$

plant/soil concentration ratio, dimensionless beef/livestock-intake ratio, $(\mathrm{pCi} / \mathrm{kg}) /(\mathrm{pCi} / \mathrm{d})$ $\mathrm{milk} /$ livestock-intake ratio, $(\mathrm{pCi} / \mathrm{L}) /(\mathrm{pCi} / \mathrm{d})$

plant/soil concentration ratio, dimensionless beef/livestock-intake ratio, $(\mathrm{pCi} / \mathrm{kg}) /(\mathrm{pCi} / \mathrm{d})$

$\mathrm{milk} /$ livestock-intake ratio, $(\mathrm{pCi} / \mathrm{L}) /(\mathrm{pCi} / \mathrm{d})$

plant/soil concentration ratio, dimensionless beef/livestock-intake ratio, ( $\mathrm{pCi} / \mathrm{kg}) /(\mathrm{pCi} / \mathrm{d})$ $\mathrm{milk} /$ livestock-intake ratio, $(\mathrm{pCi} / \mathrm{L}) /(\mathrm{pCi} / \mathrm{d})$

1. $000 \mathrm{E}-03 \quad 3 \quad 1.000 \mathrm{E}-03 \quad 3 \operatorname{RTF}(7,1)$

1.000E-04 3 1.000E-04 3 RTF $(7,2)$

1. $000 \mathrm{E}-066^{3} 1$ 1. $00 \odot \mathrm{E}-06{ }^{3} \operatorname{RTF}(7,3)$

1. $000 \mathrm{E}-03 \quad 3 \quad 1.000 \mathrm{E}-03 \quad 3 \operatorname{RTF}(9,1)$

1. $0000 \mathrm{E}-0431.000 \mathrm{3}-043 \mathrm{RTF}(2,2)$

1. $000 \mathrm{E}-06 \quad 3 \quad 1.000 \mathrm{E}-063 \operatorname{RTF}(9,3)$

3

4. $400 \mathrm{E}-022^{3}$ 4.000E-02 $3 \operatorname{RTF}(10,1)$

1. $1.000 \mathrm{E}-03 \quad 3 \quad 1.000 \mathrm{E}-033 \operatorname{RTF}(10,2)$

1. $1.000 \mathrm{E}-03$ 3 1.000E-03 $3 \operatorname{RTF}(10,3)$

3 3. $000 \mathrm{E}-0133.000 \mathrm{3}-013 \operatorname{RTF}(11,1)$

$8,000 \mathrm{E}-03$ 3 $8,000 \mathrm{E}-03$ 3 RTF $(11,2)$

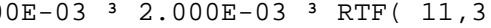

$1.000 \mathrm{E}-03 \quad 3 \quad 1.000 \mathrm{E}-03 \quad 3 \operatorname{RTF}(12,1)$

3 1. $000 \mathrm{E}-043$ 1.000E-04 $3 \operatorname{RTF}(12,2)$

5. 5.000E-06 3 5. 000E-06 $3 \operatorname{RTF}(12,3)$

3 1. $000 \mathrm{E}-03 \quad 3 \quad 1.000 \mathrm{E}-033 \operatorname{RTF}(13,1)$

$1,000 \mathrm{C}-04 \quad 3 \quad 1,000 \mathrm{C}-04 \quad 3 \operatorname{RTF}(13,2)$

5 5. 000E-06 3 5.000E-06 3 RTF( 13,3$)$

$2.500 \mathrm{E}-03 \quad 3 \quad 2.500 \mathrm{E}-03 \quad 3 \operatorname{RTF}(14,1)$

3 3.400E-04 3 $3.400 \mathrm{E}-043 \operatorname{RTF}(14,2)$

3 6.00९E-04 3 6.000E-04 $3 \operatorname{RTF}(14,3)$

3 2.500E-03 3 2.500E-03 $3 \operatorname{RTF}(15,1)$

$3.400 \mathrm{E}-04 \quad 3 \quad 3.400 \mathrm{E}-04 \quad 3 \operatorname{RTF}(15,2)$

3 6. 000E-04 3 6.000E-04 ${ }^{3} \operatorname{RTF}(15,3)$

2.500E-03 $3 \quad 2.500 \mathrm{E}-03 \quad 3 \operatorname{RTF}(16,1)$

3.400E- 04 3 $3.400 \mathrm{E}-04$ 3 $\operatorname{RTF}(16,2)$

$36.000 \mathrm{E}-04$ 3 $6.000 \mathrm{E}-04{ }^{3} \operatorname{RTF}(16,3)$

$31.500 \mathrm{E}+0131.500 \mathrm{E}+013 \operatorname{BIOFAC}(1,1)$

$1.000 \mathrm{E}+0331.000 \mathrm{3}+033 \operatorname{BIOFAC}(1,2)$

$3.0 \odot \odot \mathrm{E}+01 \quad 3 \quad 3.0 \odot \odot \mathrm{E}+01 \quad 3 \quad \mathrm{BIOFAC}(2,1)$

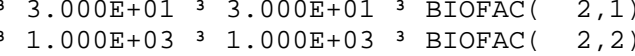


Dose Conversion Factor (and Related) Parameter Summary (continued)

$\odot \quad 3$ File: FGR 13 MORBIDITY

\section{Menu 3}

Parameter

3 Current 3 Base 3 Parameter

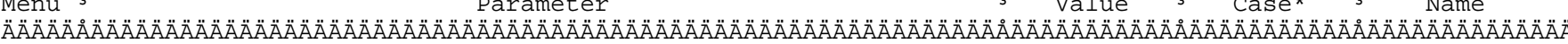

D-5 3 Cs-137+D, fish $32.000 \mathrm{D}+03$ 3 $2.000 \mathrm{E}+03$ 3 BIOFAC ( 3,1$)$

D-5 3 Cs $-137+D$, crustacea and mollusks

D - 53

D-5 3 Np-237+D , fish

D-5 3 Np-237+D, crustacea and mollusks

D $-5 \quad 3$

D-5 3 Pa-231 fish

D-5 3 Pa-231, ' crustacea and mollusks

D-5 3

D-5 3 Pb-210+D, fish

D-5 $3 \mathrm{~Pb}-210+\mathrm{D}$, crustacea and mollusks

D-5 3

D-5 3 Pu-238, fish

D-5 3 Pu-238, crustacea and mollusks

D $-5 \quad 3$

D-5 3 Pu-239, fish

D-5 3 Pu-239 , crustacea and mollusks

$-5 \quad 3$

D-5 3 Ra-226+D , fish

D-5 3 Ra-226+D, crustacea and mollusks

D -53

D-5 3 Sr-90+D , fish

D-5 3 Sr-90+D, crustacea and mollusks

D-5 3 Th-229+D, fish

D-5 3 Th-229+D ', crustacea and mollusks

D-5 3

D-5 3 Th-230 , fish

D-5 3 Th-230, crustacea and mollusks

D $-5 \quad 3$

D-5 3 U-233 , fish

D-5 3 U-233 , crustacea and mollusks

D $-5 \quad 3$

D-5 3 U-234 , fish

D-5 3 U-234 , crustacea and mollusks

$D-5 \quad 3 \quad U-235+D$, fish

$\begin{array}{lllll}3 & 1.000 \mathrm{E}+02 & 3 & 1.000 \mathrm{E}+02 & 3 \\ 3 & & & & \end{array}$

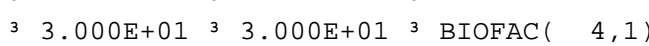

3 4. $000 \mathrm{E}+022^{3}$ 4. $000 \mathrm{E}+\odot 2{ }^{3} \operatorname{BIOFAC}(4,2)$

$31.000 \mathrm{E}+013 \quad 1.000 \mathrm{E}+013 \operatorname{BIOFAC}(5,1)$

$31.100 \mathrm{E}+0231.100 \mathrm{E}+023 \operatorname{BIOFAC}(5,2)$

$33.000 \mathrm{E}+0233.000 \mathrm{E}+023 \operatorname{BIOFAC}(6,1)$

1. $1.000 \mathrm{E}+0231.000 \mathrm{E}+023 \operatorname{BIOFAC}(6,2)$

$3 \quad 3.000 \mathrm{E}+01 \quad 3 \quad 3.000 \mathrm{E}+013 \operatorname{BIOFAC}(7,1)$

1. $000 \mathrm{E}+023$ 1. $300 \mathrm{E}+02{ }^{3} \operatorname{BIOFAC}(7,2)$

$33.000 \mathrm{E}+0133.000 \mathrm{~B}+013 \mathrm{BIOFAC}(9,1)$

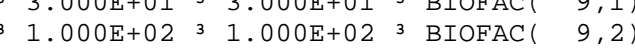

$35.000 \mathrm{E}+0135.000 \mathrm{E}+013 \operatorname{BIOFAC}(10,1)$

$2.500 \mathrm{E}+02 \quad 3 \quad 2.500 \mathrm{E}+023 \operatorname{BIOFAC}(10,2)$

$36.000 \mathrm{E}+013 \quad 6.000 \mathrm{E}+013 \operatorname{BIOFAC}(11,1)$

$31.000 \mathrm{E}+0231.000 \mathrm{E}+023 \operatorname{BIOFAC}(11,2)$

$1.000 \mathrm{E}$

1. $000 \mathrm{E}+\odot 2 \quad 3 \quad 1.000 \mathrm{E}+\odot 23 \operatorname{BIOFAC}(12,1)$

$5.000 \mathrm{E}+02$ 3 $5.000 \mathrm{E}+02$ 3 $\operatorname{BIOFAC}(12,2)$

$31.000 \mathrm{E}+023 \quad 1.000 \mathrm{E}+023 \operatorname{BIOFAC}(13,1)$

$35.000 \mathrm{E}+\odot 235.000 \mathrm{3}+\odot 23 \operatorname{BIOFAC}(13,2)$

1. $000 \mathrm{E}+0131.000 \mathrm{E}+013 \mathrm{BIOFAC}(14,1)$

6. 000E+01 3 6. $600 \mathrm{E}+013 \operatorname{BIOFAC}(14,2)$

1. $000 \mathrm{E}+01 \quad 3 \quad 1.000 \mathrm{E}+013 \operatorname{BIOFAC}(15,1)$

3 $6.000 \mathrm{E}+013$ 6. $6.000 \mathrm{E}+01$ 3 $\operatorname{BIOFAC}(15,2)$

$31.000 \mathrm{E}+0131.000 \mathrm{~B}+013 \operatorname{BIOFAC}(16,1)$

D-5 3 U-235+D, crustacea and mollusks

$36.000 \mathrm{E}+01^{3} \quad 6.000 \mathrm{E}+011^{3} \operatorname{BIOFAC}(16,2)$

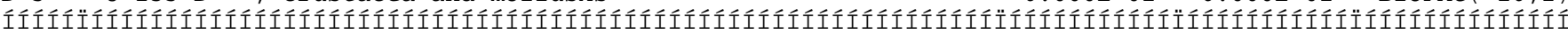

*Base Case means Default. Lib W/o Associate Nuclide contributions. 
Site-Specific Parameter Summary

3 Used by RESRAD
3 (If different from user input)
(If

3 Parameter

Menu 3

Parameter

3 User

3 Default

Name

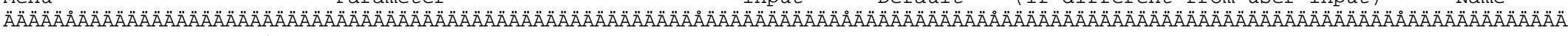

R011 3 Area of contaminated zone $\left(m^{* *} 2\right)$

R011 3 Thickness of contaminated zone $(\mathrm{m})$

R011 3 Length parallel to aquifer flow $(\mathrm{m})$

R011 3 Basic radiation dose limit (mrem/yr)

R011 3 Time since placement of material ( $\mathrm{yr}$ )

1. $000 \mathrm{E}+02 \quad 3 \quad 1.000 \mathrm{E}+04$

R011 3 Times for calculations ( $\mathrm{yr}$ )

R011 3 Times for calculations $(y r)$

R011 3 Times for calculations ( $y r$ )

R011 3 Times for calculations ( $\mathrm{yr}$ )

R011 3 Times for calculations $(\mathrm{yr})$

R011 3 Times for calculations ( $\mathrm{yr}$ )

R011 3 Times for calculations ( $y r$ )

R011 3 Times for calculations ( $y r$ )

R011 3 Times for calculations ( $y r$ )

3

R012 3 Initial principal radionuclide ( $\mathrm{pCi} / \mathrm{g}$ )

$\mathrm{R} 0123$ Initial principal radionuclide ( $\mathrm{pCi} / \mathrm{g}$ ):

R012 3 Initial principal radionuclide ( $\mathrm{PC} / \mathrm{g}):$

0012 3 Initial principal radionuclide (pci/g):

$1.500 \mathrm{E}-0132.000 \mathrm{E}+00$

not used $31.000 \mathrm{E}+02$

$2.500 \mathrm{E}+011^{3} \quad 3.000 \mathrm{E}+01$

$0.000 \mathrm{E}+0 \odot$ 3 $0.000 \mathrm{E}+0 \odot$

$1.000 \mathrm{E}+00 \quad 3 \quad 1.000 \mathrm{E}+00$

$3.000 \mathrm{E}+00$ 3 $3.000 \mathrm{E}+00$

1. $1.00 \mathrm{E}+013 \quad 1.000 \mathrm{E}+01$

3. $000 \mathrm{E}+01$ 3 3.000E+01

$5.000 \mathrm{E}+01$ 3 $1.000 \mathrm{E}+02$

7.500E+01 3 $3.000 \mathrm{E}+02$

$1.000 \mathrm{E}+0231.000 \mathrm{E}+03$

$3.000 \mathrm{E}+02$ 3 $0.000 \mathrm{E}+00$

$1.000 \mathrm{E}+03$ 3 $0.000 \mathrm{E}+003$

$1.880 \mathrm{E}+0 \odot 3 \quad 0.000 \mathrm{E}+\odot \odot$

$2.541 \mathrm{E}+01 \quad 3 \quad 0.000 \mathrm{E}+00$

2. $000 \mathrm{E}-013 \quad 0.000 \mathrm{E}+0 \odot$

$1.520 \mathrm{E}+00$ 3 $0.000 \mathrm{E}+00$

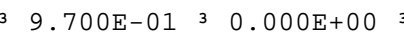

R012 3 Concentration in groundwater (pCi/L): Am-241 3 not used $30.000 \mathrm{E}+00$

R012 3 Concentration in groundwater ( $\mathrm{pCi} / \mathrm{L}): \mathrm{Cs}-1373$ not used $30.000 \mathrm{E}+00$

R012 3 Concentration in groundwater (pCi/L): Pu-238 3 not used $30.000 \mathrm{E}+00$

R012 3 Concentration in groundwater ( $\mathrm{PCi} / \mathrm{L}$ ): $\mathrm{Pu}-2393$ not used $30.000 \mathrm{E}+00$

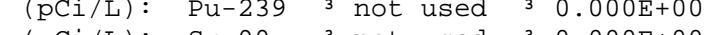

R012 3 Concentration in groundwater $(\mathrm{pCi} / \mathrm{L}): \mathrm{Sr}-90$

R013 3 Cover depth (m)

R013 3 Density of cover material $\left(\mathrm{g} / \mathrm{cm}^{* *} 3\right)$

R013 3 Cover depth erosion rate ( $\mathrm{m} / \mathrm{yr}$ )

R013 3 Density of contaminated zone $(\mathrm{g} / \mathrm{cm} * * 3)$

R013 3 Contaminated zone erosion rate $(\mathrm{m} / \mathrm{yr})$

R013 3 Contaminated zone total porosity

R013 3 Contaminated zone field capacity

not used 3 $\quad 0.000 \mathrm{E}+0 \Theta^{3}$

$0.000 \mathrm{E}+00 \quad 3 \quad 0.000 \mathrm{E}+00$

3 not used $31.500 \mathrm{E}+\Theta \Theta^{3}$

not used $31.000 \mathrm{E}-03$

$1.500 \mathrm{E}+00 \quad 3 \quad 1.500 \mathrm{E}+00$

$1.000 \mathrm{E}-03$ 3 $1.000 \mathrm{E}-033^{3}$

4.000E-01 3 4.000E-01

2. $000 \mathrm{E}-01 \quad 3 \quad 2,000 \mathrm{E}-01$

R013 3 Contaminated zone hydraulic conductivity (m/yr) $3{ }_{1} .000 \mathrm{E}+01{ }^{3} 1.000 \mathrm{E}+01$

$\mathrm{R} 013$ 3 Contaminated zone b parameter $35.300 \mathrm{E}+00^{3} \quad 5.300 \mathrm{E}+00$

R013 3 Average annual wind speed (m/sec) $33.400 \mathrm{E}+00^{3} 2.000 \mathrm{E}+00^{3}$

R013 3 Humidity in air $(\mathrm{g} / \mathrm{m} * \star 3)$

R013 3 Evapotranspiration coefficient

not used 3 $8.000 \mathrm{E}+00$

5.000E-01 3 5.000E-01

$3.260 \mathrm{E}-0131.000 \mathrm{E}+00$

$0.000 \mathrm{E}+00{ }^{3} \quad 2.000 \mathrm{E}-01$

R013 3 Precipitation ( $\mathrm{m} / \mathrm{yr}$ )

R013 3 Irrigation ( $\mathrm{m} / \mathrm{yr}$

R013 3 Irrigation mode

$3 \quad 4.000 \mathrm{E}-013{ }^{3} 2.00 \odot \mathrm{E}-01$

R013 3 Watershed area for nearby stream or pond $\left(m^{* * 2)} 3\right.$ not used $31.000 \mathrm{E}+06$

R013 3 Accuracy for water/soil computations

R014 3 Density of saturated zone $\left(\mathrm{g} / \mathrm{cm}^{* *} 3\right)$

R014 3 Saturated zone total porosity

not used $31.000 \mathrm{E}-03$

3 not used $31.500 \mathrm{E}+00$

3 not used 3 4.000E- 01

3 not used 3 2.000E- 01

R014 3 Saturated zone effective porosity

R014 3 Saturated zone field capacity

3 not used 32 2. $000 \mathrm{E}-\odot 1^{3}$

R014 3 Saturated zone hydraulic conductivity $(\mathrm{m} / \mathrm{yr}) \quad 3$ not used $31.000 \mathrm{E}+02^{3}$

R014 3 Saturated zone hydraulic gradient

3 not used 3 1.000E+O2 3

$--$

- -

$-$

$-\cdot$

$+-$

$-$

$--$

$-\cdot$

$--$

3 THICKO

3 LCZPAQ

3 BRDL

3 T ( 2)

$3 \mathrm{~T}(3)$

$3 \mathrm{~T}(4)$

$\begin{array}{lll}T & T(5)\end{array}$

$3 \mathrm{~T}(6)$

$3 \mathrm{~T}(7)$

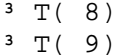

$3 \mathrm{~T}(10)$

$--$

S1( 2)

$-\cdot$

3 S1 ( 3)

$3 \mathrm{~S} 1(7)$

3 S1 ( 9)

3 S1(11)

3 W1( 2)

3 W1 $(7)$

3 W1 9 ( 9)

3 W1(11)

COVERO

3 DENSCV

3 VCV

3 DENSCZ

$3 \mathrm{VCZ}$

$\begin{array}{lll}3 & \text { TPCZ } \\ 3 & \text { FCCZ }\end{array}$

3 FCCZ

$3 \mathrm{HCCZ}$

$3 \mathrm{BCZ}$

3 WIND

3 HUMID

3 EVAPTR

3 PRECIP

3 RI

3 IDITCH

RUNOFF

3 WAREA

3 EPS

-. 3 EPS

- - 3 DENSAQ

-.- 3 TPSZ

-. - 3 EPSZ

-.- $3 \mathrm{FCSZ}$

3 HCSZ

3 HGWT 
$\odot \quad 3$

Site-Specific Parameter Summary (continued)

Menu 3

3 User

3 Default

Used by RESRAD 3 Parameter

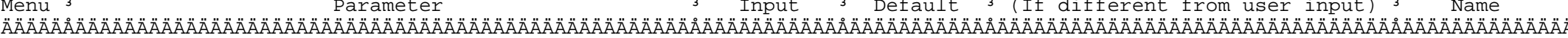
R014 3 Saturated ZOnEA D PäAAAÄA

3
not used $35.300 \mathrm{E}+00$
3

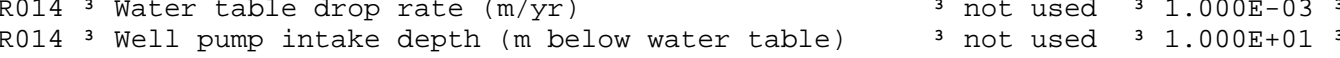

R014 3 Model: Nondispersion (ND) or Mass-Balance (MB)

not used $3 \mathrm{ND}$

not used $32.500 \mathrm{E}+02^{3}$

3

R015 3 Number of unsaturated zone strata

R015 3 Unsat. zone 1, thickness (m)

R015 3 Unsat. zone 1 , soil density $\left(\mathrm{g} / \mathrm{cm}^{*} * 3\right)$

R015 3 Unsat. zone 1 , total porosity

R015 3 Unsat. zone 1, effective porosity

R015 3 Unsat. zone 1, field capacity

R015 3 Unsat. zone 1, soil-specific b parameter

R015 3 Unsat. zone 1 , hydraulic conductivity (m/yr)

R016 3 Distribution coefficients for Am-241

R016 3 Contaminated zone $\left(\mathrm{cm}^{* *} 3 / \mathrm{g}\right)$

R016 3 Unsaturated zone $1\left(\mathrm{~cm}^{* *} 3 / \mathrm{g}\right)$

R016 3 Saturated zone $\left(\mathrm{cm}^{* *} 3 / \mathrm{g}\right)$

R016 3 Leach rate (/yr)

R016 3 Solubility constant

R016 3 Distribution coefficients for Cs -137

R016 3 Contaminated zone $\left(\mathrm{cm}^{* *} 3 / \mathrm{g}\right)$

R016 3 Unsaturated zone $1\left(\mathrm{~cm}^{* *} 3 / \mathrm{g}\right)$

R016 3 Saturated zone $\left(\mathrm{cm}^{* *} 3 / \mathrm{g}\right)$

R016 3 Leach rate (/yr)

R016 3 Solubility constant

R016 3 Distribution coefficients for $\mathrm{Pu}-238$

R016 3 Contaminated zone $\left(\mathrm{cm}^{* *} 3 / \mathrm{g}\right)$

R016 3 Unsaturated zone $1\left(\mathrm{~cm}^{* *} 3 / \mathrm{g}\right)$

R016 3 Saturated zone $\left(\mathrm{cm}^{* *} 3 / \mathrm{g}\right)$

R016 3 Leach rate (/yr)

R016 3 Solubility constant

R016 3 Distribution coefficients for $\mathrm{Pu}-239$

R016 3 Contaminated zone $\left(\mathrm{cm}^{* *} 3 / \mathrm{g}\right)$

R016 3 Unsaturated zone $1\left(\mathrm{~cm}^{* *} 3 / \mathrm{g}\right)$

R016 3 Saturated zone $\left(\mathrm{cm}^{* *} 3 / \mathrm{g}\right)$

R016 3 Leach rate (/yr)

RO16 3 Solubility constant

R016 3 Distribution coefficients for Sr-90

R016 3 Contaminated zone $\left(\mathrm{cm}^{* *} 3 / \mathrm{g}\right)$

R016 3 Unsaturated zone $1(\mathrm{~cm} * * 3 / \mathrm{g})$

R016 3 Saturated zone $\left(\mathrm{cm}^{* *} 3 / \mathrm{g}\right)$

R016 3 Leach rate (/yr)

R016 3 Solubility constant 
Menu 3 Parameter 3 Input 3 Default 3 (If different from user input) 3

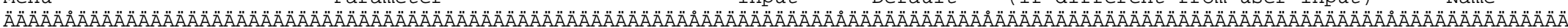

R016 3 Distribution coefficients for daughter Ac-227

R016 3 Contaminated zone $\left(\mathrm{cm}^{* *} 3 / \mathrm{g}\right)$

R016 3 Unsaturated zone $1(\mathrm{~cm} * * 3 / \mathrm{g})$

R016 3 Saturated zone $\left(\mathrm{cm}^{* *} 3 / \mathrm{g}\right)$

R016 3 Leach rate (/yr)

R016 3 Solubility constant

R016 3 Distribution coefficients for daughter Np-237

R016 3 Contaminated zone $\left(\mathrm{cm}^{* *} 3 / \mathrm{g}\right)$

R016 3 Unsaturated zone $1\left(\mathrm{~cm}^{* *} 3 / \mathrm{g}\right)$

R016 3 Saturated zone $\left(\mathrm{cm}^{* *} 3 / \mathrm{g}\right)$

R016 3 Leach rate (/yr)

R016 ${ }^{3}$ Solubility constant

R016 3 Distribution coefficients for daughter Pa-231

R016 3 Contaminated zone $\left(\mathrm{cm}^{* *} 3 / \mathrm{g}\right)$

R016 3 Unsaturated zone $1(\mathrm{~cm} * * 3 / \mathrm{g})$

R016 3 Saturated zone $\left(\mathrm{cm}^{* *} 3 / \mathrm{g}\right)$

R016 3 Leach rate (/yr)

R016 ${ }^{3}$ Solubility constant

R016 3 Distribution coefficients for daughter Pb-210

R016 3 Contaminated zone $\left(\mathrm{cm}^{* *} 3 / \mathrm{g}\right)$

R016 3 Unsaturated zone $1\left(\mathrm{~cm}^{* *} 3 / \mathrm{g}\right)$

R016 3 Saturated zone $\left(\mathrm{cm}^{* *} 3 / \mathrm{g}\right)$

R016 3 Leach rate (/yr)

R016 3 Solubility constant

R016 3 Distribution coefficients for daughter Ra-226

R016 3 Contaminated zone $\left(\mathrm{cm}^{* *} 3 / \mathrm{g}\right)$

R016 3 Unsaturated zone $1\left(\mathrm{~cm}^{* *} 3 / \mathrm{g}\right)$

R016 3 Saturated zone $\left(\mathrm{cm}^{* *} 3 / \mathrm{g}\right)$

R016 3 Leach rate (/yr)

R016 ${ }^{3}$ Solubility constant

R016 3 Distribution coefficients for daughter Th-229

R016 3 Contaminated zone $\left(\mathrm{cm}^{* *} 3 / \mathrm{g}\right)$

R016 3 Unsaturated zone $1\left(\mathrm{~cm}^{* *} 3 / \mathrm{g}\right)$

R016 3 Saturated zone $\left(\mathrm{cm}^{* *} 3 / \mathrm{g}\right)$

R016 3 Leach rate (/yr)

R016 3 Solubility constant

R016 3 Distribution coefficients for daughter Th-230

R016 3 Contaminated zone $\left(\mathrm{cm}^{* *} 3 / \mathrm{g}\right)$

R016 3 Unsaturated zone $1(\mathrm{~cm} * * 3 / \mathrm{g})$

R016 3 Saturated zone $\left(\mathrm{cm}^{* *} 3 / \mathrm{g}\right)$

R016 3 Leach rate (/yr)

3
3
$2.000 E+013$

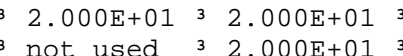

not used $32.000 \mathrm{E}+01$

$0.000 \mathrm{E}+00{ }^{3} \quad 0.000 \mathrm{E}+00$

$0.000 \mathrm{E}+\Theta \Theta^{3} \quad 0.000 \mathrm{E}+\Theta \Theta^{3}$

$3-1.000 \mathrm{E}+0 \odot{ }^{3}-1.000 \mathrm{E}+\odot \odot$

not used $3-1.000 \mathrm{E}+0 \odot$

not used $3-1.000 \mathrm{E}+00$

$0.000 \mathrm{E}+00 \quad 3 \quad 0.000 \mathrm{E}+00$

$\odot . \odot \odot \odot E+\odot \odot \quad 3 \quad \odot . \odot \odot \odot E+\odot \odot{ }^{3}$

5. $00 \odot \mathrm{E}+013 \quad 5.000 \mathrm{E}+01$

3 not used 3 5.000E+01

not used 3 5.000E+01

$3 \quad \odot .00 \odot \mathrm{E}+\odot \odot \quad 3 \quad \odot .0 \odot \odot \mathrm{E}+\odot \odot$

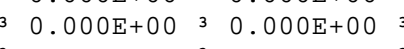

$1.000 \mathrm{E}+02 \quad 3 \quad 1.000 \mathrm{E}+02$

not used 3 1. $1.000 \mathrm{E}+02$

not used $1.000 \mathrm{E}+02$

not used $1.000 \mathrm{E}+02$

$\odot . \odot \odot \odot E+\odot \odot$ उ $0 . \odot \odot \odot E+\odot \odot$

$\odot .00 \odot E+\odot \odot \quad 3 \quad 0.00 \odot E+\odot \odot$

$37.000 \mathrm{E}+01 \quad 3 \quad 7.000 \mathrm{E}+01$

not used $37.000 \mathrm{E}+01$

not used $37.000 \mathrm{E}+01$

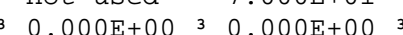

$0.000 \mathrm{E}+0 \odot$ з $\odot .00 \odot \mathrm{E}+\odot \odot$

$6.000 \mathrm{E}+04 \quad 3 \quad 6.000 \mathrm{E}+04$

3 not used $36.000 \mathrm{E}+04$

3 not used 3 6.000E+O4

$0.000 \mathrm{E}+003 \quad 0.000 \mathrm{E}+0 \odot$

$\odot .00 \odot E+\odot \odot \quad 3 \quad 0.00 \odot E+\odot \odot$

$6.000 \mathrm{E}+04 \quad 3 \quad 6.000 \mathrm{E}+04$

not used 3 6.000E+04

3 not used 3 6.000E $+04{ }^{3}$

$0.000 \mathrm{E}+003 \quad 0.000 \mathrm{E}+0 \odot$

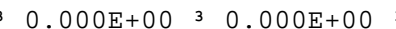

\begin{tabular}{|c|c|c|c|}
\hline & & & \\
\hline-- & 3 & DCNUCC ( & 1) \\
\hline$-\ldots$ & 3 & DCNUCU( & $1,1)$ \\
\hline-- & 3 & DCNUCS( & 1) \\
\hline $2.153 \mathrm{E}-02$ & 3 & ALEACH ( & 1) \\
\hline not used & 3 & SOLUBK( & 1) \\
\hline & 3 & & \\
\hline & 3 & & \\
\hline $2.574 \mathrm{E}+02$ & 3 & DCNUCC ( & 4) \\
\hline- & 3 & DCNUCU( & $4,1)$ \\
\hline - - - & 3 & DCNUCS( & 4) \\
\hline 1.687E- 03 & 3 & ALEACH ( & 4) \\
\hline not used & 3 & SOLUBK( & 4) \\
\hline & 3 & & \\
\hline & 3 & & \\
\hline - - - & 3 & DCNUCC ( & 5) \\
\hline-- & 3 & DCNUCU( & $5,1)$ \\
\hline- & 3 & DCNUCS( & 5) \\
\hline $8.660 \mathrm{E}-03$ & 3 & ALEACH ( & 5) \\
\hline not used & 3 & SOLUBK( & 5) \\
\hline & 3 & & \\
\hline & 3 & & \\
\hline - - - & 3 & DCNUCC ( & 6) \\
\hline$-\ldots$ & 3 & DCNUCU( & $6,1)$ \\
\hline-- & 3 & DCNUCS( & 6) \\
\hline $4.338 E-03$ & 3 & ALEACH ( & 6) \\
\hline not used & 3 & SOLUBK( & 6) \\
\hline & 3 & & \\
\hline & 3 & & \\
\hline-- & 3 & DCNUCC & 10) \\
\hline - - - & 3 & DCNUCU( & $10,1)$ \\
\hline$-\ldots$ & 3 & DCNUCS & 10) \\
\hline $6.193 \mathrm{E}-03$ & 3 & ALEACH & 10) \\
\hline not used & 3 & SOLUBK( & 10) \\
\hline & $\begin{array}{l}3 \\
3\end{array}$ & & \\
\hline - . - & 3 & DCNUCC & 12) \\
\hline$-\ldots$ & 3 & DCNUCU & $12,1)$ \\
\hline - - - & 3 & DCNUCS & 12) \\
\hline $7.244 \mathrm{E}-06$ & 3 & ALEACH & 12) \\
\hline not used & 3 & SOLUBK & 12) \\
\hline & $\begin{array}{l}3 \\
3\end{array}$ & & \\
\hline - - - & 3 & DCNUCC & 13) \\
\hline - - - & 3 & DCNUCU & $13,1)$ \\
\hline-- & 3 & DCNUCS & 13) \\
\hline $7.244 \mathrm{E}-06$ & 3 & ALEACH & 13) \\
\hline not used & 3 & SOLUB & 13) \\
\hline
\end{tabular}


Site-Specific Parameter Summary (continued)

Menu ${ }^{3}$

3 User

3 Default

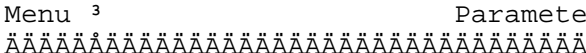

3 Input 3 Default

Used by RESRAD ${ }^{3}$ Parameter

R016 3 Distribution coefficients for daughter U-233

R016 3 Contaminated zone $\left(\mathrm{cm}^{* *} 3 / \mathrm{g}\right)$

R016 3 Unsaturated zone $1\left(\mathrm{~cm}^{* *} 3 / \mathrm{g}\right)$

R016 3 Saturated zone $\left(\mathrm{cm}^{\star *} 3 / \mathrm{g}\right)$

R016 3 Leach rate (/yr)

R016 3 Solubility constant

R016 3 Distribution coefficients for daughter U-234

$\begin{array}{lll}5.000 \mathrm{E}+01 & 3 & 5.000 \mathrm{E}+01\end{array}$

$\begin{array}{llll}3 & 5.000 \mathrm{E}+01^{3} & 5.000 \mathrm{E}+01^{3} \\ 3 & \text { not used } 3 & 5.000 \mathrm{E}+01 & 3\end{array}$

not used $35.000 \mathrm{E}+01$

$0.000 \mathrm{E}+0 \odot \quad 3 \quad 0.000 \mathrm{E}+0 \odot$

$0.000 \mathrm{E}+\odot \Theta^{3} \quad 0.000 \mathrm{E}+0 \Theta^{3}$

R016 3 Contaminated zone $\left(\mathrm{cm}^{* *} 3 / \mathrm{g}\right)$

R016 3 Unsaturated zone $1\left(\mathrm{~cm}^{* *} 3 / \mathrm{g}\right)$

R016 3 Saturated zone $\left(\mathrm{cm}^{* *} 3 / \mathrm{g}\right)$

R016 3 Leach rate (/yr)

R016 3 Solubility constant

R016 3 Distribution coefficients for daughter U-235

R016 3 Contaminated zone $\left(\mathrm{cm}^{* *} 3 / \mathrm{g}\right)$

R016 3 Unsaturated zone $1\left(\mathrm{~cm}^{* *} 3 / \mathrm{g}\right)$

R016 3 Saturated zone $\left(\mathrm{cm}^{* *} 3 / \mathrm{g}\right)$

$\mathrm{R} 016^{3}$ Leach rate (/yr)

R016 3 Solubility constant

R017 3 Inhalation rate ( $\left.m^{* *} 3 / y r\right)$

R017 3 Mass loading for inhalation $(\mathrm{g} / \mathrm{m} * * 3)$

R017 3 Exposure duration

R017 3 Shielding factor, inhalation

R017 3 Shielding factor, external gamma

R017 3 Fraction of time spent indoors

R017 3 Fraction of time spent outdoors (on site)

R017 3 Shape factor flag, external gamma

$35.000 \mathrm{E}+01 \quad 3 \quad 5.000 \mathrm{E}+01$

not used 3 5.000E+01

not used $35.000 E+01$

$\begin{array}{lll}0.000 \mathrm{E}+00 & 3 & 0.000 \mathrm{E}+00^{3}\end{array}$

$0.000 \mathrm{E}+00^{3} \quad 0.000 \mathrm{E}+0 \odot^{3}$$$
3
$$

$5.000 \mathrm{E}+01 \quad 3 \quad 5.000 \mathrm{E}+01$ not used 3 5. $000 \mathrm{E}+01$ not used 3 s. $5.000 \mathrm{E}+01$

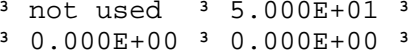

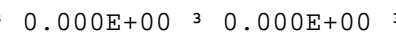

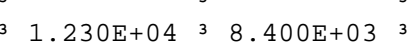

6. $6000 \mathrm{E}-044^{3} 1.000 \mathrm{E}-04{ }^{3}$

$32.500 E+013 \quad 3.000 E+013$

$1.000 \mathrm{E}+00$ 3 $4.000 \mathrm{E}-013$

$1.000 \mathrm{E}+00$ 3 $\quad 7.000 \mathrm{E}-013$

$0.000 \mathrm{E}+00$ 3 5. $5000 \mathrm{E}-01$

3 3.800E-02 32 2.500E-01 3

$1.000 \mathrm{E}+00 \quad 3 \quad 1.000 \mathrm{E}+003$

R017 Radii of shape factor array (used if FS = -1):

R017 3 Outer annular radius $(\mathrm{m})$, ring 1 :

$\mathrm{R} 0173$ Outer annular radius $(\mathrm{m})$, ring 2 :

R017 3 Outer annular radius $(\mathrm{m})$, ring 3 :

R017 3 Outer annular radius $(\mathrm{m})$, ring 4:

R017 3 Outer annular radius ( $m)$, ring 5:

R017 3 Outer annular radius $(\mathrm{m})$, ring 6 :

R017 3 Outer annular radius $(\mathrm{m})$, ring 7 :

R017 3 Outer annular radius $(\mathrm{m})$, ring 8:

R017 3 Outer annular radius (m), ring

R017 3 Outer annular radius (I), ring 10:

$\begin{array}{llll}\mathrm{R} 017 & 3 & \text { Outer annular radius } \\ \mathrm{R} 017 & 3 & \text { Outer annular radius }(\mathrm{m}), & \text {, ring 12: }\end{array}$ not used $35.000 \mathrm{E}+01$

not used $3 \quad 7.071 \mathrm{E}+013$

not used $3 \quad 0.000 \mathrm{E}+00^{3}$

not used $3 \quad 0.000 \mathrm{E}+\odot \odot^{3}$

not used $3 \quad 0.000 \mathrm{E}+0{ }^{3}$

not used $30.000 \mathrm{E}+\odot \Theta^{3}$

not used $30.000 \mathrm{E}+00$

not used $30.000 \mathrm{E}+0 \odot$

3 not used $30.000 \mathrm{E}+0 \odot$

3 not used $30.000 \mathrm{E}+\odot \Theta^{3}$

3 not used $30.000 \mathrm{E}+0 \odot^{3}$

3 not used $30.000 \mathrm{E}+\odot \odot^{3}$

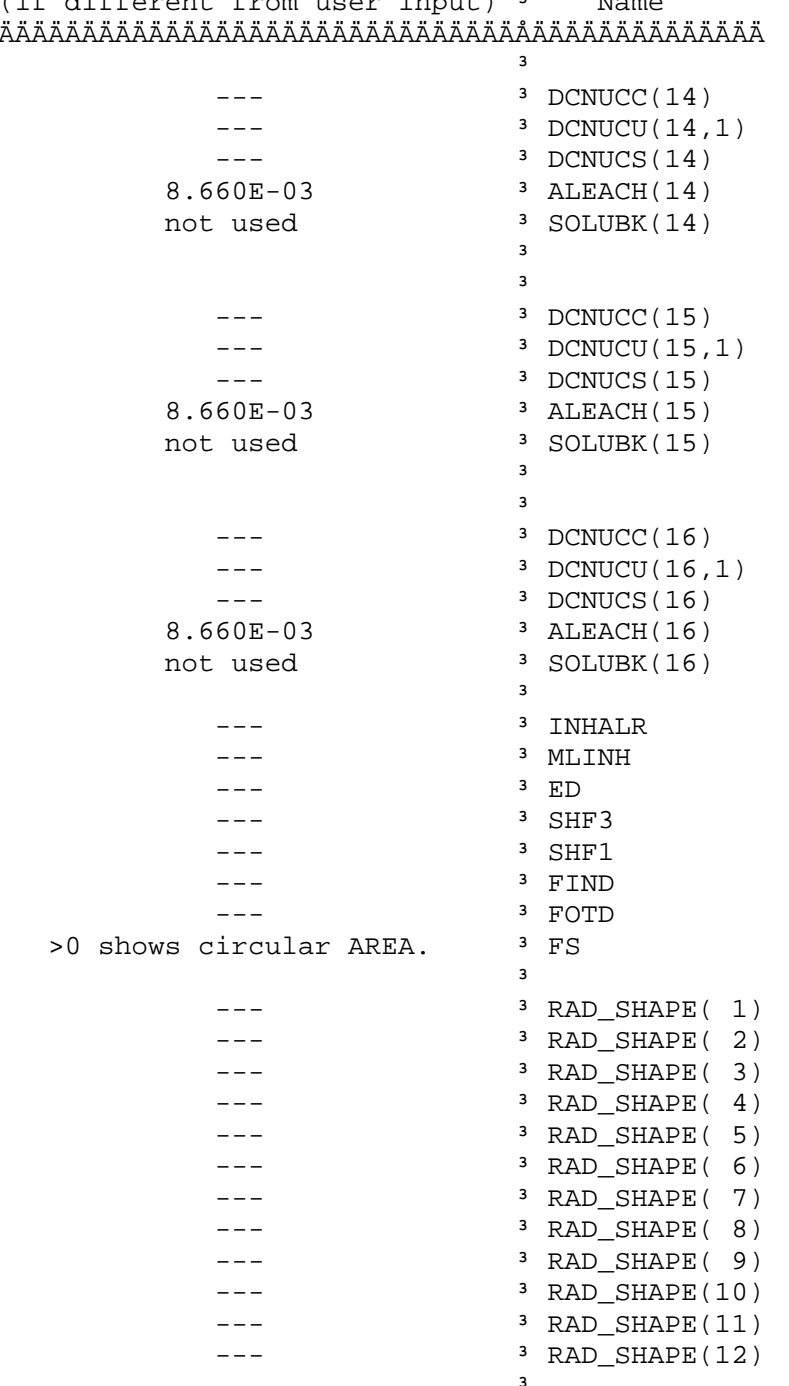


Site-Specific Parameter Summary (continued)

Menu ${ }^{3}$

3 User

3 Input 3 Default 3 (If different from user input) 3 Name

3 Used by RESRAD

by RESRAD 3 Parameter

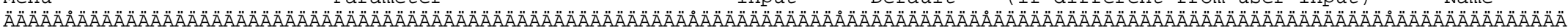

R017 3 Fractions of annular areas within AREA:

$\begin{array}{lllr}R 017 & 3 & \text { Ring } & 1 \\ R 017 & 3 & \text { Ring } & 2 \\ R 017 & 3 & \text { Ring } & 3 \\ R 017 & 3 & \text { Ring } & 4 \\ R 017 & 3 & \text { Ring } & 5 \\ \text { R017 } & 3 & \text { Ring } & 6 \\ R 017 & 3 & \text { Ring } & 7 \\ R 017 & 3 & \text { Ring } & 8 \\ R 017 & 3 & \text { Ring } & 9 \\ R 017 & 3 & \text { Ring } 10 \\ R 017 & 3 & \text { Ring } 11 \\ R 017 & 3 & \text { Ring } 12\end{array}$

not used $31.000 E+00$ 3

not used $32.732 \mathrm{E}-01$

not used $30.000 \mathrm{E}+00$

not used $30.000 \mathrm{E}+0 \odot$

not used $30.000 \mathrm{E}+0 \odot$

3 not used 3 \% $000 \mathrm{E}+00^{3}$

not used $30.000 \mathrm{E}+00^{3}$

3 not used $30.000 \mathrm{E}+00^{3}$

not used 3 ०. $\odot \odot \odot E+\odot \odot$

not used $30.0 \odot \odot E+\odot \odot$

not used $30.000 \mathrm{E}+\Theta \odot$

not used 3 O. $000 \mathrm{E}+00$

3

R018 3 Fruits, vegetables and grain consumption ( $\mathrm{kg} / \mathrm{yr}$ )

R018 3 Leafy vegetable consumption ( $\mathrm{kg} / \mathrm{yr}$ )

R018 3 Milk consumption ( $\mathrm{L} / \mathrm{yr}$ )

R018 3 Meat and poultry consumption ( $\mathrm{kg} / \mathrm{yr}$ )

R018 3 Fish consumption ( $\mathrm{kg} / \mathrm{yr}$ )

R018 3 Other seafood consumption ( $\mathrm{kg} / \mathrm{yr}$ )

R018 3 Soil ingestion rate $(\mathrm{g} / \mathrm{yr}$ )

R018 3 Drinking water intake (L/yr)

R018 3 Contamination fraction of drinking water

R018 3 Contamination fraction of household water

R018 3 Contamination fraction of livestock water

R018 3 Contamination fraction of irrigation water

R018 3 Contamination fraction of aquatic food

R018 3 Contamination fraction of plant food

R018 3 Contamination fraction of meat

R018 3 Contamination fraction of milk

$$
3
$$

R019 3 Livestock fodder intake for meat ( $\mathrm{kg} /$ day)

R019 3 Livestock fodder intake for milk ( $\mathrm{kg} /$ day)

R019 3 Livestock water intake for meat (L/day)

R019 3 Livestock water intake for milk (L/day)

R019 3 Livestock soil intake ( $\mathrm{kg} /$ day)

R019 3 Mass loading for foliar deposition $(\mathrm{g} / \mathrm{m} * * 3)$

R019 3 Depth of soil mixing layer $(\mathrm{m})$

R019 3 Depth of roots (m)

R019 3 Drinking water fraction from ground water

R019 3 Household water fraction from ground water

R019 3 Livestock water fraction from ground water

R019 3 Irrigation fraction from ground water

R19B 3 Wet weight crop yield for Non-Leafy $\left(\mathrm{kg} / \mathrm{m}^{* *} 2\right)$

R19B 3 Wet weight crop yield for Leafy $\left(\mathrm{kg} / \mathrm{m}^{* *} 2\right)$

R19B 3 Wet weight crop yield for Fodder $\left(\mathrm{kg} / \mathrm{m}^{* *} 2\right)$

R19B 3 Growing Season for Non-Leafy (years)

R19B 3 Growing Season for Leafy (years)

R19B 3 Growing Season for Fodder (years)

R19B 3 Translocation Factor for Non-Leafy
3 not used $31.600 \mathrm{E}+02$

3 not used $31.400 E+01^{3}$

not used $3 \quad 6.300 \mathrm{E}+01^{3}$

not used $35.400 \mathrm{E}+0{ }^{3}$

not used 3 9.000E- 01

$1.752 \mathrm{E}+02 \quad 3 \quad 3.650 \mathrm{E}+01$

3 not used 3 5.100E+02

not used 3 1.000E+00 3

not used $31.000 \mathrm{E}+00^{3}$

not used $31.000 \mathrm{E}+0{ }^{3}$

not used 3 1.000E+00

not used 3 5.000E-01

not used $3-1$

not used $3-1$

not used $3-1$

not used $36.800 \mathrm{E}+01$

not used 3 1.600E+O2 3

not used 3 5.000E-01

not used $31.000 \mathrm{E}-04$

$1.500 \mathrm{E}-0131.500 \mathrm{E}-01$

not used 3 9.00คE- 01

3 not used $31.000 E+00$

not used 31 . 1 OOQE+OQ

3 not used 31 .

not used $31.000 \mathrm{E}+0 \odot^{3}$

not used 3 7.000E-01

3 not used $31.500 \mathrm{E}+00$

3 not used $31.100 E+00$

3 not used $31.700 \mathrm{E}-01$

3 not used 32 500E-01

3 not used 3 8.000E-02

3 not used 3 1.000E-01 3
3 not used $39.200 \mathrm{E}+01$

not used 3 5.500E+01

not used $3 \quad 5.000 \mathrm{E}+01$ $-\cdot-$

- - -

- -

$-$

$-$

$-$

$-$

$-$

$-$.

$-\cdot$

- . -

$--$

- -

$--$

- - -

$-\cdot$

$--$

- . -

$--$

$---$

- -

- - -

- .

$-\cdot$

$--$

- -

- -

$--$

$-$.

$-$.

$--$

$-\cdot-$

- - -

$--$

$-$.

- -

$--$

$--$
$\operatorname{FRACA}(1)$

FRACA( 2)

FRACA( 3 )

3 FRACA ( 4)

FRACA ( 6)

FRACA ( 6)

FRACA ( 7)

FRACA ( 9)

FRACA (10)

FRACA (11)

DIET(1)

3 DIET (2)

$3 \operatorname{DIET}(3)$

3 DIET(4)

3 DIET(5)

3 SOIL

3 DWI

3 FDW

3 FHHW

3 FLW

$\begin{array}{ll}3 & \mathrm{FIRW} \\ 3 & \mathrm{FRO}\end{array}$

3 FR9

$\begin{array}{ll}3 & \text { FPLANT } \\ 3 & \text { FMEAT }\end{array}$

3 FMEAT

3 FMILK

3 LFI5

3 LFI6

3 LWI5

3 LWI6

3 LSI

3 MLFD

3 DM

3 DROOT

3 FGWDW

3 FGWHH

3 FGWLW

3 FGWIR

$3 \mathrm{YV}(1)$

$3 \mathrm{YV}(2)$

3 YV(3)

3 TE (1)

3 TE (2)

3 TE (3)

Uncontrolled When Printed 
Site-Specific Parameter Summary (continued)

Menu $33 \quad$ Parameter 3 Input 3 Default 3 (If different from user input) 3 Name

3 User

Menu $33 \quad$ Parameter 3 Input 3 Default 3 (If different from user input) 3 Name

3 Used by RESRAD

Used by RESRAD 3 Parameter

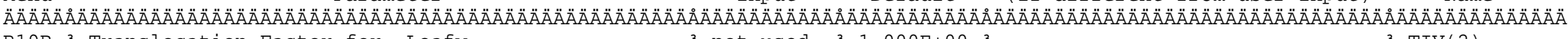

R19B 3 Translocation Factor for Leafy 3 not used 3 1.000E+00 $3 \quad$-. -

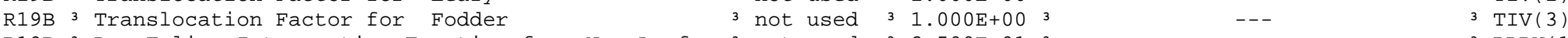

R19B 3 Dry Foliar Interception Fraction for Non-Leafy 3 not used $32.500 \mathrm{E}-01^{3} \quad$-.-

R19B 3 Dry Foliar Interception Fraction for Leafy 3 not used 3 2.500E-01

R19B 3 Dry Foliar Interception Fraction for Fodder 3 not used 3 2.500E-01

R19B 3 wet Foliar Interception Fraction for Non-Leafy ${ }^{3}$ not used $32.500 \mathrm{E}-01^{3}$

R19B 3 Wet Foliar Interception Fraction for Leafy 3 not used $32.500 \mathrm{E}-01$

R19B 3 Wet Foliar Interception Fraction for Fodder 3 not used $32.500 \mathrm{E}-01$

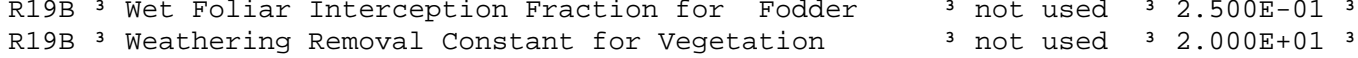

C14 3 C-12 concentration in water $\left(\mathrm{g} / \mathrm{cm}^{* *} 3\right)$

C14 ${ }^{3}$ C-12 concentration in contaminated soil $(\mathrm{g} / \mathrm{g})$

C14 3 Fraction of vegetation carbon from soil

C14 3 Fraction of vegetation carbon from air

C14 3 C-14 evasion layer thickness in soil (m)

C14 3 C-14 evasion flux rate from soil ( $1 / \mathrm{sec})$

C14 3 C-12 evasion flux rate from soil (1/sec)

C14 3 Fraction of grain in beef cattle feed

C14 3 Fraction of grain in milk cow feed

3 not used $32.000 E+013$

not used $32.000 \mathrm{E}-05$

not used 3 3.000E-02

not used 3 2.000E-02

not used 3 9.800E-01

not used $3 \quad 3.000 \mathrm{E}-01$

not used $37.000 \mathrm{C}-07$

not used $37.000 \mathrm{C}-07$

not used 1 . $000 \mathrm{E}-10$

not used 3 . 8.0

not used $32.000 \mathrm{E}-01$

DCF correction factor for gaseous forms of $\mathrm{C}_{14}$

not used

$\odot .000 \mathrm{E}+00^{3}$

STOR 3 Storage times of contaminated foodstuffs (days): 3

STOR 3 Fruits, non-leafy vegetables, and grain

STOR 3 Leafy vegetables

$\begin{array}{lll}\text { STOR } & 3 & \text { Leafy } \\ \text { STOR } & 3 & \text { Milk }\end{array}$

STOR 3 Meat and poultry

STOR ${ }^{3}$ Fish

STOR 3 Crustacea and mollusks

STOR 3 Well water

STOR 3 Surface water

STOR 3 Livestock fodder

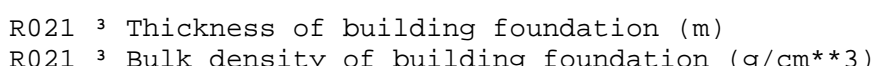

RO21 3 Total porosity of the cover material

R०21 3 Total porosity of the building foundation

R021 3 Volumetric water content of the cover material

R021 3 Volumetric water content of the foundation

R021 3 Diffusion coefficient for radon gas $(\mathrm{m} / \mathrm{sec})$ :

R०21 3 in cover material

$R_{021} 3$ in foundation material

RO21 3 in contaminated zone soil

R०21 3 Radon vertical dimension of mixing $(\mathrm{m})$

RO21 3 Average building air exchange rate $(1 / \mathrm{hr})$

R021 3 Height of the building (room) (m)

R021 3 Building interior area factor

R021 3 Building depth below ground surface $(\mathrm{m})$

R021 3 Emanating power of $\mathrm{Rn}-222$ gas

R021 3 Emanating power of $\mathrm{Rn}-220$ gas

TITL 3 Number of graphical time points

TITL 3 Maximum number of integration points for dose

3 1. $400 \mathrm{E}+01$ 3 $1.400 \mathrm{E}+01$

$1.000 E+00^{3} 1.400 E+01$

1. $000 E+00$ 3 $1.000 E+00$ 3

$1.000 E+00$ 3 $1.000 \mathrm{E}+\odot \odot$

$2.000 \mathrm{E}+01$ 3 $2.000 \mathrm{E}+01$

$7.000 \mathrm{E}+\odot \odot$ 3 $7.000 \mathrm{E}+0 \odot$

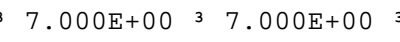

$1.000 \mathrm{E}+00$ 3 $1.000 \mathrm{E}+0 \odot$

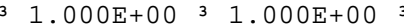

4.500E+01 3 4.500E+01 3

3

not used 3 1.500E-01

not used $32.400 \mathrm{E}+00^{3}$

not used 3 4.000E-01

not used $31.000 \mathrm{E}-01$

not used 3 5.000E-02

not used 3 3.000E- 023

not used 32 . $000 \mathrm{E}-06$

not used 3 3. 3 .

not used $33.000 \mathrm{E}-07$

not used $2.000 \mathrm{E}-06$

$\begin{array}{lll}\text { not used } & 3 & 2.000 \mathrm{E}+00 \\ \text { not used } & 3 & 5.000 \mathrm{E}-01\end{array}$

not used $32.500 E+00$

not used $30.000 \mathrm{E}+00$

3 not used $3-1.000 \mathrm{E}+00^{3}$

3 not used 3 2.500E-01

3 not used 3 1.500E-01 3

$\begin{array}{llll}32 & 3 & \ldots & 3 \\ 17 & 3 & -\ldots & \end{array}$

Uncontrolled When Printed 
1RESRAD, Version $6.3 \quad$ T« Limit $=180$ days Summary : CAU 383 Drainage
05/11/2006 10:08 Page 11

File: CAU 383 Drainage. RAD

Site-Specific Parameter Summary (continued)

Menu 3

$$
\text { User } 3 \text { Used by RESRAD }
$$

3 Parameter

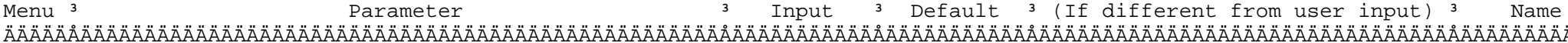

TITL 3 Maximum number of integration points for risk 3257

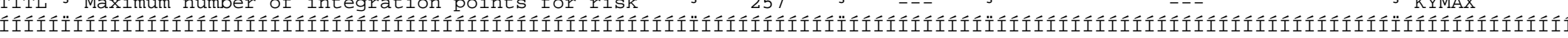

Summary of Pathway Selections

Pathway User Selection

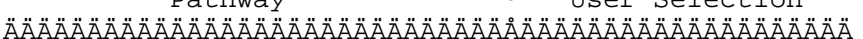

1 - - external gamma 3

2 - - inhalation (w/o radon) $)^{3} \quad$ active

3 -- plant ingestion 3 suppressed

4 -- meat ingestion 3 suppressed

5 -- milk ingestion 3 suppressed

6 . - aquatic foods 3 suppressed

7 -. drinking water 3 suppressed

- 3 suppressed

$\begin{array}{llc}8 \text { - - soil ingestion } & 3 & \text { active } \\ 9 & 3 & \text { suppressed }\end{array}$

Find peak pathway doses 3 suppressed

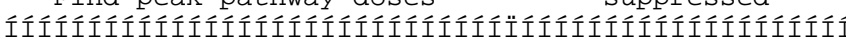


1RESRAD, Version $6.3 \quad$ T« Limit $=180$ days Summary : CAU 383 Drainage

Contaminated Zone Dimensions ÄÄÄÄÄÄÄÄÄÄÄÄÄÄÄÄÄÄÄÄÄÄÄÄÄÄÄÄ

$$
\text { Area: } \quad 100.00 \text { square meter }
$$

$\begin{array}{rr}\text { Area: } & 100.00 \\ \text { Thickness: } & 0.15 \text { meters }\end{array}$

Cover Depth: 0.00 meters
05/11/2006 10:08 Page 12

File: CAU 383 Drainage. RAD

Initial Soil Concentrations, pci/g

ÄÄÄÄÄÄÄÄÄÄÄÄÄÄÄÄÄÄÄÄÄÄÄÄÄÄÄÄÄÄÄÄ

$$
\begin{array}{ll}
\mathrm{Am}-241 & 1.880 \mathrm{E}+0 \odot \\
\mathrm{Cs}-137 & 2.541 \mathrm{E}+01 \\
\mathrm{Pu}-238 & 2.000 \mathrm{E}-01 \\
\mathrm{Pu}-239 & 1.520 \mathrm{E}+\odot \odot \\
\mathrm{Sr}-9 \odot & 9.700 \mathrm{E}-01
\end{array}
$$

Total Dose TDOSE(t), mrem/yr

Basic Radiation Dose Limit $=2.500 \mathrm{E}+01 \mathrm{mrem} / \mathrm{yr}$

Total Mixture Sum $\mathrm{m}(\mathrm{t})=$ Fraction of Basic Dose Limit Received at Time $(\mathrm{t})$

A

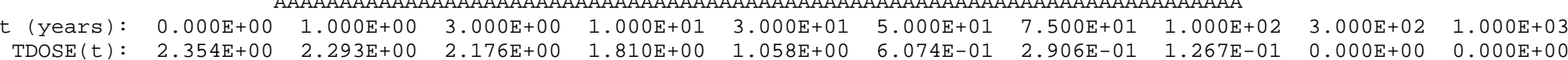

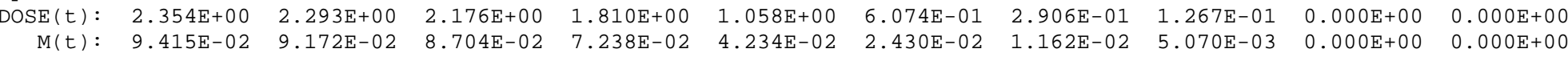

OMaximum TDOSE $(\mathrm{t}): \quad 2.354 \mathrm{E}+00 \mathrm{mrem} / \mathrm{yr}$ at $\mathrm{t}=0.000 \mathrm{E}+\odot \odot$ years 
Total Dose Contributions $\operatorname{TDOSE}(i, p, t)$ for Individual Radionuclides (i) and Pathways (p)

As mrem/yr and Fraction of Total Dose At $t=0.000 \mathrm{E}+00$ years

Water Independent Pathways (Inhalation excludes radon)

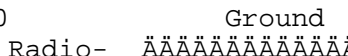

Inhalation

Radon

Plant

Meat

Milk

Soil

Radio- ÄAÄAAAAAAAAA
Nuclide mrem/yr fract

ÄÄÄÄÄÄÄ Ä ÄÄÄÄÄÄÄÄÄ ÄÄÄÄÄÄ

\begin{tabular}{lll}
$\mathrm{Am}-241$ & $2.569 \mathrm{E}-03$ & 0.0011 \\
\hline
\end{tabular}

Cs $-137 \quad 2.308 \mathrm{E}+00 \quad 0.9803$

$\begin{array}{lll}\mathrm{Pu}-238 & 1.036 \mathrm{E}-06 & 0.000 \\ \mathrm{Pu}-239 & 1.365 \mathrm{E}-05 & 0.0000\end{array}$

$\begin{array}{lll}\mathrm{Pu}-239 & 1.365 \mathrm{E}-05 & \odot .000 \odot \\ \mathrm{Sr}-90 & 6.650 \mathrm{E}-04 & 0.0003\end{array}$

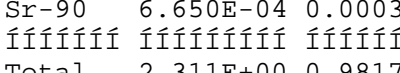

$\odot$

mrem $r$ fract

AAAAAÄÄÄÄÄÄÄÄA

AAÄÄÄÄÄÄÄÄÄÄÄÄ

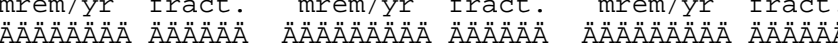

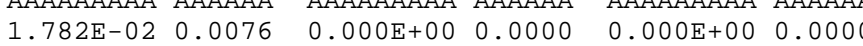

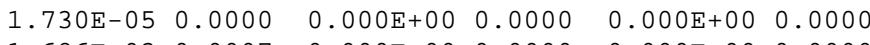

$\begin{array}{llllll}1.686 \mathrm{E}-03 & 0.0007 & 0.000 \mathrm{E}+00 & 0.0000 & 0.000 \mathrm{E}+00 & 0.0000\end{array}$

ÄÄÄÄÄÄÄÄÄ ÄÄÄÄÄÄ

ÄÄÄÄÄÄÄÄÄ ÄÄÄÄÄÄ

$\odot .000 \mathrm{E}+0 \odot \quad 0.0000$

1. $408 \mathrm{E}-02$ - $\odot .060$

(0.00E+00 $0.0000 \quad 0.000 \mathrm{E}+\odot \odot \quad 0.0000$

$0.000 E+00$ - $0.0000 \quad 0.000 E+00$ 0.0000

$0.000 \mathrm{E}+00 \quad 0.0000$

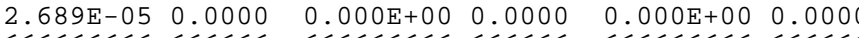

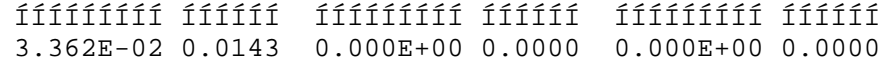

$0.000 \mathrm{E}+00$ 0 0000

$0.000 \mathrm{E}+000.0000$

Íííííííi íííít

Íííííííi ÍÍííí

Total Dose Contributions $\operatorname{TdOSE}(i, p, t)$ for Individual Radionuclides (i) and Pathways $(p)$ As mrem/yr and Fraction of Total Dose At $t=0.000 \mathrm{E}+00$ years

Water Dependent Pathways Fish

Radon

$$
\text { Plant }
$$

Meat

Milk

\section{ÄÄÄÄÄÄÄÄÄÄÄÄÄÄÄ̈}

mrem/yr fract.

$\odot .000 \mathrm{Q}+0 \odot$ $\odot .0000$

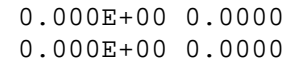

$\odot .000 \mathrm{E}+0 \odot \quad 0.0000$

$\odot .000 \mathrm{E}+0 \odot \quad 0.000 \odot$

$0.000 E+00 \quad 0.0000$

Íííííííí ííííi

$\odot .000 \mathrm{E}+00 \quad 0.0000$

$0.000 \mathrm{E}+0 \odot$ - 0.0000

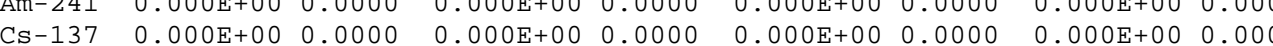

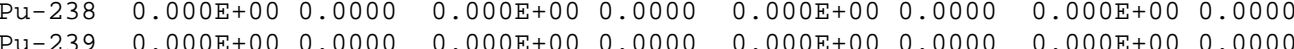

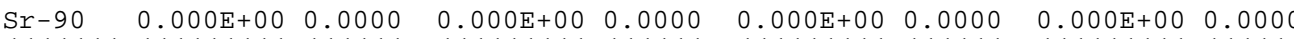

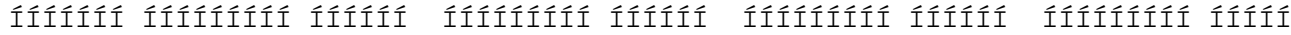

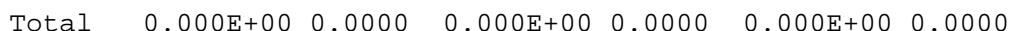

$\odot *$ Sum of all water independent and dependent pathways.

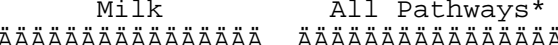
mrem/yr fract. mrem/yr fract. ÄÄÄÄÄÄÄÄÄ ÄÄÄÄÄÄ ÄÄÄÄÄÄÄÄÄ ÄÄÄÄÄÄ $0.000 \mathrm{E}+000.0000 \quad 2.487 \mathrm{E}-020.0106$ $\odot . \odot \odot \odot E+\odot \odot \quad \odot . \odot \odot \odot \odot \quad 2.308 \mathrm{E}+\odot \odot \quad 0.9807$ $0.000 \mathrm{E}+000.0000 \quad 2.110 \mathrm{E}-03 \quad 0.00009$ $0.000 \mathrm{E}+000.0000 \quad 1.766 \mathrm{E}-02 \quad 0.0075$ $0.000 \mathrm{E}+000.0000 \quad 7.884 \mathrm{E}-04 \quad 0.00003$ IIIIííííi ííííí íííííííi ííííí

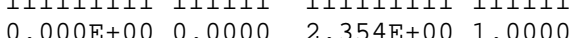


Total Dose Contributions $\operatorname{TDOSE}(i, p, t)$ for Individual Radionuclides (i) and Pathways (p)

As mrem/yr and Fraction of Total Dose At $t=1.000 \mathrm{E}+00$ years

Water Independent Pathways (Inhalation excludes radon)

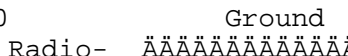
Inhalation

Radon

Plant

Meat

Milk

Soil

Radio-
Nuclide mrem/yr fract

ÄÄÄÄÄÄÄ Ä̈̈̈̈̈̈̈̈̈̈̈̈̈̈̈ ÄÄÄÄ̈̈̈ AÄÄÄÄÄÄÄÄÄÄÄÄÄÄÄ Ä̈̈̈̈̈̈̈̈̈̈̈̈̈̈̈̈̈̈̈̈̈̈̈̈ ÄÄÄÄÄÄÄÄÄÄÄÄÄ AAAÄÄÄÄÄÄÄÄÄÄÄ

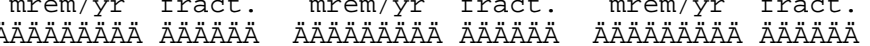

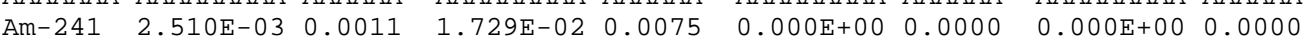
Cs-137 2.248E+00 0.9802 $\mathrm{Pu}-238 \quad 1.027 \mathrm{E}-06 \quad 0.0000$

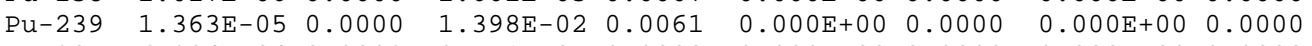
$\begin{array}{llllll}1.679 E-05 & 0.0000 & 0.000 E+00 & 0.0000 & 0.000 E+00 & 0.0000\end{array}$ $1.661 \mathrm{E}-03 \quad 0.0007 \quad 0.000 \mathrm{E}+00 \quad 0.0000 \quad 0.000 \mathrm{E}+000.0000$

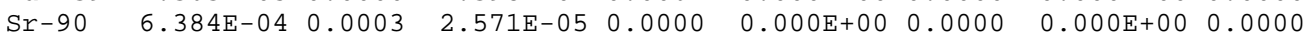
íííííi íííííííi ííííi $\odot$

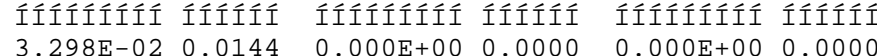
mrem/yr fract ÄӒÄÄÄÄÄÄ ÄÄÄÄÄÀ $0.000 \mathrm{E}+000.0000$ $0.000 \mathrm{E}+0 \circ 0.0000$ $\odot .000 \mathrm{E}+0 \odot \quad 0.000 \odot$ $0.000+00.000$ Íííííííi ííííí Total Dose Contributions TDOSE $(i, p, t)$ for Individual Radionuclides (i) and Pathways $(p)$ As $\mathrm{mrem} / \mathrm{yr}$ and Fraction of Total Dose At $t=1.000 \mathrm{E}+0 \odot$ years Water Dependent Pathways Fish

\begin{abstract}
Radon
\end{abstract} Plant Meat ÄÄÄÄÄÄÄÄÄÄÄÄÄÄA mrem/yr fract. $\odot .0 \odot \odot E+\odot \odot \quad \odot .0 \odot \odot \odot$ $0.000 \mathrm{E}+0 \odot \quad 0.0000$ $\odot .000 \mathrm{E}+000.0000$ $0.000 \mathrm{E}+000.0000$ íííííííi ííííí $0.000 \mathrm{E}+000.0000$ ÄÄÄAAAAAAAAAA ÄÄÄÄÄÄÄÄÄ ÄÄÄÄÄÄ
ÄÄÄÄÄÄÄÄÄÄÄÄÄÄ mrem/yr fract. ÄÄÄÄÄÄÄÄÄ ÄÄÄÄÄÄ $0.000 \mathrm{E}+00 \quad 0.0000$ $\odot .000 \mathrm{E}+00 \quad 0.0000$ $0.000 E+000.0000$ $0.000 \mathrm{E}+00 \quad 0.0000$ íííííííí íííííi $0.000 \mathrm{E}+00 \quad 0.0000$

Radio - ÄÄÄÄÄÄÄÄÄÄÄÄÄÄ Nuclide mrem/yr fract. $\begin{array}{lll}\text { Am-241 } & 0.000 \mathrm{E}+00 & 0.0000\end{array}$ $\mathrm{CS}-137 \quad 0.000 \mathrm{E}+00 \quad 0.0000$ $\begin{array}{lll}\mathrm{Pu}-238 & 0.000 \mathrm{E}+00 & 0.0000 \\ \mathrm{Pu}-239 & 0.000 \mathrm{E}+00 & 0.0000\end{array}$ Sr-90 $0.000 \mathrm{E}+00 \quad 0.0000$

\section{ÄÄÄÄÄÄÄÄÄÄÄÄÄÄ} AิÄÄÄÄÄÄÄÄÄÄÄÄÄÄ

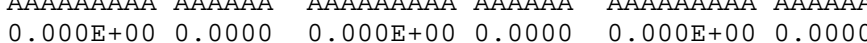

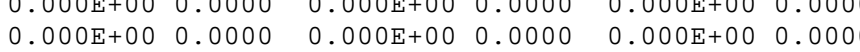

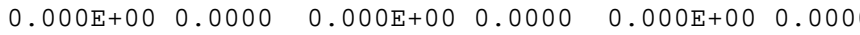
(1)

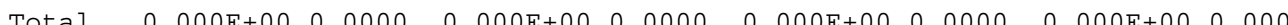

$\odot$ * Sum of all water independent and dependent pathways.

Milk

\section{ÄÄÄÄÄÄÄÄÄÄÄÄÄÄÄ} mrem/yr fract. ÄÄÄÄÄÄÄÄÄ ÄÄÄÄÄÄ $0.000 \mathrm{E}+00 \quad 0.0000$ $\odot .000 \mathrm{E}+00 \quad 0.0000$ $\odot .000 \mathrm{E}+000.0000$ $0.000 \mathrm{E}+00 \quad 0.0000$ $0.000 \mathrm{E}+000.0000$ Ííííííí ííííí $\odot .000 \mathrm{E}+0 \odot \quad 0.000 \odot$
АAАÄÄÄÄÄÄÄÄÄÄÄÄ $\begin{array}{ll}m r e m / y r & \text { fract. } \\ \text { ÄÄÄÄÄÄÄÄÄ } & \ddot{A ̈ A ̈ A ̈ A ̈ A ̈ A ̈ ~}\end{array}$ 4. 357E- 03 ๑. 0019 $8.088 \mathrm{E}-04 \quad 0.0004$ $4.167 \mathrm{E}-04 \quad 0.0002$ $3.545 \mathrm{E}-03 \quad 0.0015$ $9.226 \mathrm{E}-050.0000$ Íííííííi ííííí $9.220 \mathrm{E}-030.0040$ 
Total Dose Contributions $\operatorname{TDOSE}(i, p, t)$ for Individual Radionuclides (i) and Pathways (p)

As mrem/yr and Fraction of Total Dose At $t=3.000 \mathrm{E}+00$ years

Water Independent Pathways (Inhalation excludes radon)

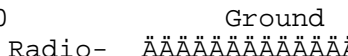
Inhalation

Radon

Plant

Meat

Milk

Soil

Radio-
Nuclide mrem/yr fract

ÄÄÄÄÄÄÄ Ä̈̈̈̈̈̈̈̈̈̈̈̈̈̈̈ ÄÄÄÄÄ̈ AÄÄÄÄÄÄÄÄÄÄÄÄÄÄÄ Ä̈̈̈̈̈̈̈̈̈̈̈̈̈̈̈̈̈̈̈̈̈̈̈̈ ÄÄÄÄÄÄÄÄÄÄÄÄÄ АAАÄÄÄÄÄÄÄÄÄÄÄ

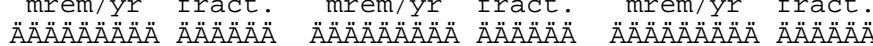

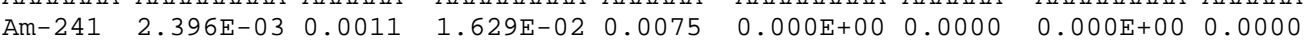
CS-137 2.132E+00 $\odot .9800$ $\mathrm{Pu}-238 \quad 1.011 \mathrm{E}-06 \quad 0.0000$

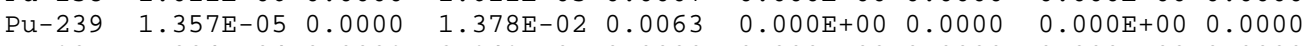

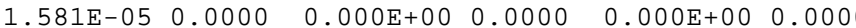
$1.612 \mathrm{E}-03 \quad 0.0007 \quad 0.000 \mathrm{E}+00 \quad 0.0000 \quad 0.000 \mathrm{E}+0 \odot \quad 0.0000$

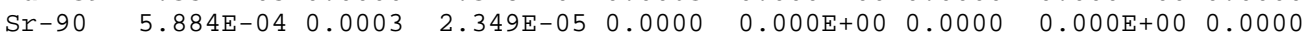

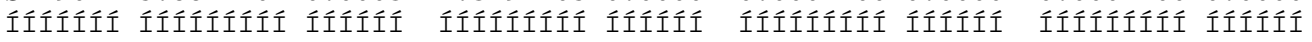
0

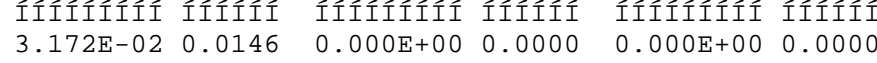
mrem/yr fract ÄÄÄÄÄÄÄÄÄ ÄÄÄÄÄÄ ÄÄÄÄÄÄÄÄÄÄÄÄÄÄÄÄ mrem/yr fract. ÄÄÄÄÄÄÄÄÄ ÄÄÄÄÄ̈̈ $\begin{array}{llll}\odot .000 \mathrm{E}+0 \odot & 0.000 \odot & 0.000 \mathrm{E}+0 \odot & 0.000 \odot\end{array}$ $\odot .00 \odot E+\odot \odot \quad 0.00 \odot \odot$ $0.000 \mathrm{E}+00$ 0. 0000 $0.000 \mathrm{E}+000.0000$ $\odot .000 \mathrm{E}+0 \odot \quad 0.000 \odot$

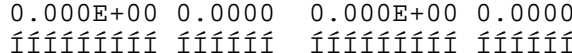

Total Dose Contributions TDOSE $(i, p, t)$ for Individual Radionuclides (i) and Pathways ( $p$ ) As $\mathrm{mrem} / \mathrm{yr}$ and Fraction of Total Dose At $t=3.000 \mathrm{E}+00$ years Water Dependent Pathways Fish

\begin{abstract}
Radon
\end{abstract}

$$
\text { Plant }
$$

Meat

\section{ÄÄÄÄÄÄÄÄÄÄÄÄÄÄÄÄÄ} mrem/yr fract. Nuclide mrem/yr fract ÄÄÄÄÄÄÄ ÄÄÄÄÄÄÄÄÄ ÄÄÄÄÄ $\begin{array}{lll}\mathrm{Am}-241 & 0.000 \mathrm{E}+0 \odot & 0.000 \odot \\ \mathrm{Cs}-137 & 0.000 \mathrm{E}+0 \odot & 0.000 \odot\end{array}$

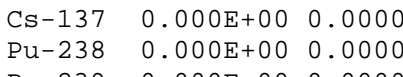
$\mathrm{Pu}-2390.000 \mathrm{E}+00 \quad 0.0000$ $\begin{array}{lll}\mathrm{Sr}-90 & 0.000 \mathrm{E}+00 & 0.0000\end{array}$

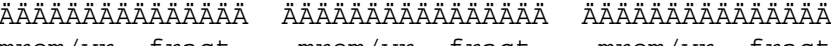
mrem/yr fract. mrem/yr fract. mrem/yr fract

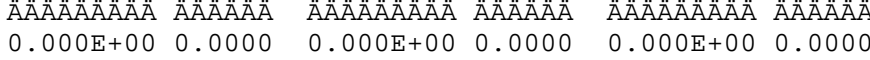

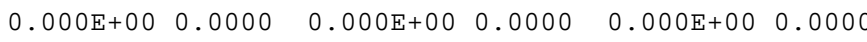
$\begin{array}{llllll}0.000 E+00 & 0.0000 & 0.000 E+00 & 0.0000 & 0.000 E+0 \odot & 0.0000\end{array}$ $\begin{array}{llllll}0.000 \mathrm{E}+00 & 0.0000 & 0.000 \mathrm{E}+00 & 0.0000 & 0.000 \mathrm{E}+00 & 0.0000\end{array}$

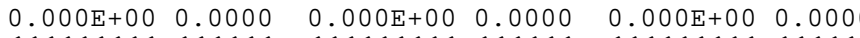

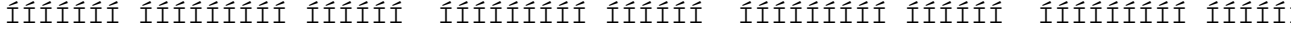

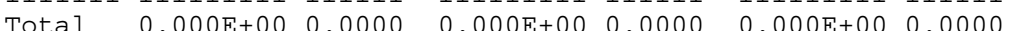
$0.000 \mathrm{E}+0 \odot$ ० 0.0000 $\odot .000 \mathrm{E}+00 \bigcirc .0000$ $\odot .000 \mathrm{E}+00 \quad 0.0000$ $0.000 \mathrm{E}+00 \quad 0.0000$ $0.000 \mathrm{E}+000.0000$ $0.000 \mathrm{E}+000.0000$ Íííííííi ííííi $0.000 \mathrm{E}+000.0000$
ÍÍÍÍÍÍÍ ÍÍÍÍÍ́

$\odot *$ Sum of all water independent and dependent pathways. 
Total Dose Contributions TDOSE( $i, p, t)$ for Individual Radionuclides ( $i$ ) and Pathways ( $p$ )

As mrem/yr and Fraction of Total Dose At $t=1.000 \mathrm{E}+01$ years water Independent Pathways (Inhalation excludes radon)

\section{Ground Inhalation

$$
\text { Plant }
$$$$
\text { Meat }
$$

Radio - ÄÄÄÄÄÄÄÄÄÄÄÄÄÄĂ Nuclide $\mathrm{mrem} / \mathrm{yr}$ fract ÄÄÄÄÄÄÄ ÄÄÄÄÄÄÄÄÄ ÄÄÄÄÄÄ Am-241 2.038E-03 0.0011 CS-137 1.772E+๑๑ 0.9790 $\mathrm{Pu}-238 \quad 9.542 \mathrm{E}-07 \quad 0.0000$ $\mathrm{Pu}-239$ 1.337E-05 0.0000 $\begin{array}{lll}\mathrm{Pu}-90 & 4.418 \mathrm{E}-04 & 0.0002\end{array}$ íííííi ííííííí íííít $\odot$

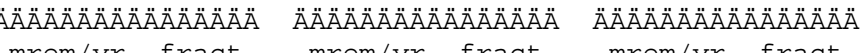
$\mathrm{mrem} / \mathrm{yr}$ fract.

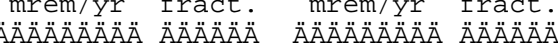
$\mathrm{mrem} / \mathrm{yr}$ fract ÄÄÄÄÄÄÄÄ ÄÄÄÄÄÄ

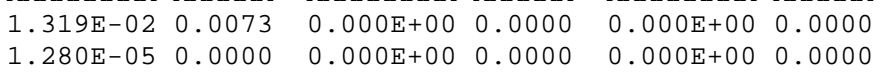
$\begin{array}{llllll}1.450 E-03 & 0.0008 & 0.000 E+00 & 0.0000 & 0.000 E+00 & 0.0000\end{array}$

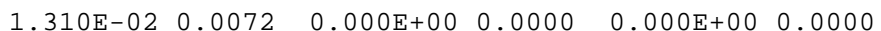
$\begin{array}{llllll}1.712 \mathrm{E}-05 & 0.000 \odot & 0.000 \mathrm{E}+00 & 0.000 \odot & 0.000 \mathrm{E}+0 \odot & 0.0000\end{array}$ Íííííííi ííííí íííííííi ííííí íííííííi ííííi

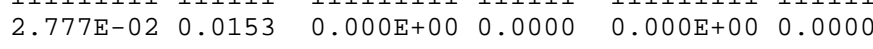
mrem/yr fract. ÄÄÄÄÄÄÄÄ ÄÄÄÄÄÄ $0.000 \mathrm{E}+00 \quad 0.0000$ $0.000 \mathrm{E}+00 \quad 0.0000$ $0.000 \mathrm{E}+000.0000$ $\odot .000 E+0 \odot \quad 0.0000$ $0.000 E+00 \quad 0.0000$ Ííííííí ííííi $0.000 \mathrm{E}+000.0000$
ÄÄÄÄÄÄÄÄÄÄÄÄÄÄ mrem/yr fract. ÄÄÄÄÄÄÄÄ ÄÄÄÄÄÄ $0.000 \mathrm{E}+0 \odot \quad 0.000 \odot$ $0.000 \mathrm{E}+00 \quad 0.0000$ $\odot .000 \mathrm{E}+00 \quad 0.0000$ $0.000 \mathrm{E}+00 \quad 0.0000$ $0.000 \mathrm{E}+000.0000$ IIIIIIIII IIIIII $\odot .000 \mathrm{E}+00 \quad 0.0000$
Soil AAÄAAAAÄÄÄÄÄÄÄÄ mrem/yr fract ÄÄÄÄÄÄÄÄÄ ÄÄÄÄÄÄ $3.323 \mathrm{E}-03 \quad 0.0018$ $6.166 \mathrm{E}-040.0003$ $3.639 \mathrm{E}-04 \quad 0.0002$ $3.323 \mathrm{E}-03 \quad 0.0018$ $6.145 \mathrm{E}-05 \quad 0.0000$ Íííííííi ííííí $7.688 \mathrm{E}-03 \quad 0.0042$

Total Dose Contributions $\operatorname{TDOSE}(i, p, t)$ for Individual Radionuclides ( $i$ ) and Pathways (p) As $\mathrm{mrem} / \mathrm{yr}$ and Fraction of Total Dose At $t=1.000 \mathrm{E}+01$ years water Dependent Pathways Fish

\begin{abstract}
Radon
\end{abstract}

$$
\text { Plant }
$$

Meat

\section{ÄÄÄÄÄÄÄÄÄÄÄÄÄÄÄÄÄ} mrem/yr fract A. $000 \mathrm{~A}+00$ 0.0000

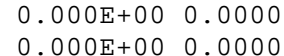
$0.000 E+00 \quad 0.0000$ $0.000 \mathrm{E}+0 \odot \quad 0.0000$ $0.000 \mathrm{E}+00 \quad 0.0000$ Íííííííí ííííi

$0.000 \mathrm{E}+00 \quad 0.0000$
Milk

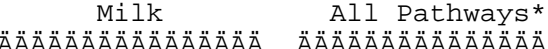
mrem/yr fract. ÄÄÄÄÄÄÄÄÄ ÄÄÄÄÄÄ ÄÄÄÄÄÄÄÄÄ ÄÄÄÄÄÄ $0.000 \mathrm{E}+00$ 0.000९ $1.855 \mathrm{E}-02 \quad 0.0103$ $\odot .0 \odot \odot \mathrm{E}+0 \odot \quad \odot .00 \odot \odot \quad 1.772 \mathrm{E}+\odot \odot \quad 0.9794$ $0.000 \mathrm{E}+000.0000 \quad 1.815 \mathrm{E}-03 \quad 0.0010$ $\odot .000 \mathrm{E}+0 \odot \quad 0.0000 \quad 1.644 \mathrm{E}-02 \quad 0.0091$ $0.000 \mathrm{E}+00 \quad 0.0000 \quad 5.204 \mathrm{E}-04 \quad 0.0003$ íííííííi ííííí íííííííi ííííí $\odot .00 \odot E+\odot \odot \quad 0.00 \odot \odot \quad 1.810 \mathrm{E}+0 \odot 1.000 \odot$

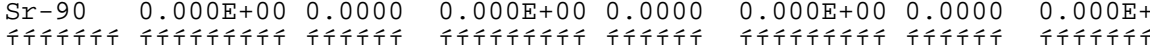

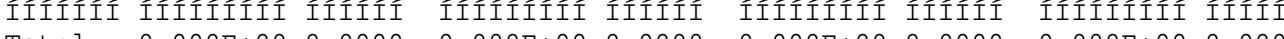
$\odot *$ Sum of all water independent and dependent pathways. 
Total Dose Contributions TDOSE( $i, p, t)$ for Individual Radionuclides ( $i$ ) and Pathways ( $p$ )

As mrem/yr and Fraction of Total Dose At $t=3.000 \mathrm{E}+01$ years

Water Independent Pathways (Inhalation excludes radon)

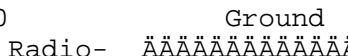

Inhalation

Radon

Plant

Meat

Milk

Soil

Radio-
Nuclide mrem/yr fract.

ÄÄÄ̈̈̈̈̈̈̈ Ӓ̈̈̈̈̈̈̈̈̈̈̈̈̈̈̈ ÄÄÄÄ̈̈̈

Am-241 $1.282 \mathrm{E}-03 \quad 0.001$

CS-137 1.032E+00 0.9754

$\mathrm{Pu}-238 \quad 8.085 \mathrm{E}-07 \quad 0.0000$

$\mathrm{Pu}-239$ 1.273E-05 0.0000

$\mathrm{Sr}-90 \quad 1.931 \mathrm{E}-04 \quad 0.0002$

Íííííi ííííííí ííííí Aล̈ÄÄÄÄÄÄÄÄÄÄÄÄÄÄÄ Ä̈̈̈̈̈̈ÄÄ̈̈̈̈̈̈̈̈̈̈̈̈̈̈̈̈̈̈ ÄÄÄÄÄÄÄÄÄÄÄÄÄ AAAÄÄÄÄÄÄÄÄÄÄÄ

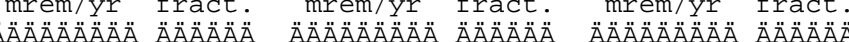

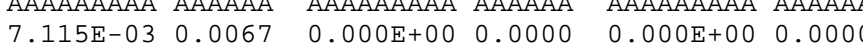
$\begin{array}{llllll}6.895 \mathrm{E}-06 & 0.0000 & 0.000 \mathrm{E}+0 \odot & 0.0000 & 0.000 \mathrm{E}+00 & 0.0000\end{array}$ $\begin{array}{lllllll}1.056 \mathrm{E}-03 & 0.0010 & 0.000 \mathrm{E}+00 & 0.0000 & 0.000 \mathrm{E}+00 & 0.0000\end{array}$ $1.117 \mathrm{E}-020.0106 \quad 0.000 \mathrm{E}+000.0000 \quad 0.000 \mathrm{E}+000.0000$ $6.832 E-00.0000$ 0.000E+00 0.0000 0.000

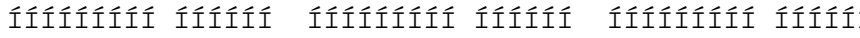
$\odot$

Total Dose Contributions $\operatorname{TDOSE}(i, p, t)$ for Individual Radionuclides ( $i$ ) and Pathways ( $p$ ) As $\mathrm{mrem} / \mathrm{yr}$ and Fraction of Total Dose At $t=3.000 \mathrm{E}+01$ years

$$
\text { Water Dependent Pathways }
$$
mrem/yr fract. ÄÄÄÄÄÄÄÄÄ ÄÄÄÄÄÄ $0.000 \mathrm{E}+000.0000$ $\odot .000 \mathrm{E}+0 \odot \quad 0.0000$ $0.000 \mathrm{E}+000.0000$ $0.000 \mathrm{E}+000.0000$ $0.000 \mathrm{E}+00$ - 0.0000 $0.000 \mathrm{E}+00$ 0 $0000 \quad 0.000 \mathrm{E}+00$ 0.0000 Ííííííí ííííi ííííííí ííííi $0.000 \mathrm{E}+0 \odot 0.0000$ Radon Plant Meat

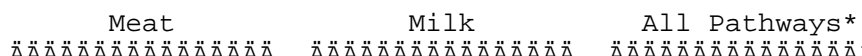
mremA mrem/yr fract. mrem/yr fract. mrem/yr fract.

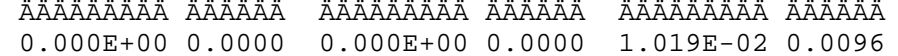
$\begin{array}{llllll}0.000 \mathrm{E}+0 \odot & 0.000 \odot & 0.000 \mathrm{E}+\odot \odot & 0.000 \odot & 1.033 \mathrm{E}+\odot \odot & 0.9757\end{array}$ $\begin{array}{llllll}0.000 \mathrm{E}+0 \odot & 0.000 \odot & 0.000 \mathrm{E}+00 & 0.000 \odot & 1.322 \mathrm{E}-03 & 0.0012\end{array}$ $\begin{array}{llllll}0.000 \mathrm{E}+00 & 0.0000 & 0.000 \mathrm{E}+00 & 0.0000 & 1.401 \mathrm{E}-02 & 0.0132\end{array}$ $\begin{array}{llllll}0.000 E+00 & 0.0000 & 0.000 E+00 & 0.0000 & 2.245 E-04 & 0.0002\end{array}$ ííííííí ííííí ííííííi ííííi ííííííi ííííi

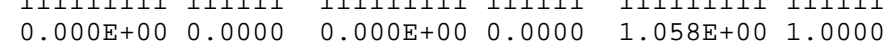

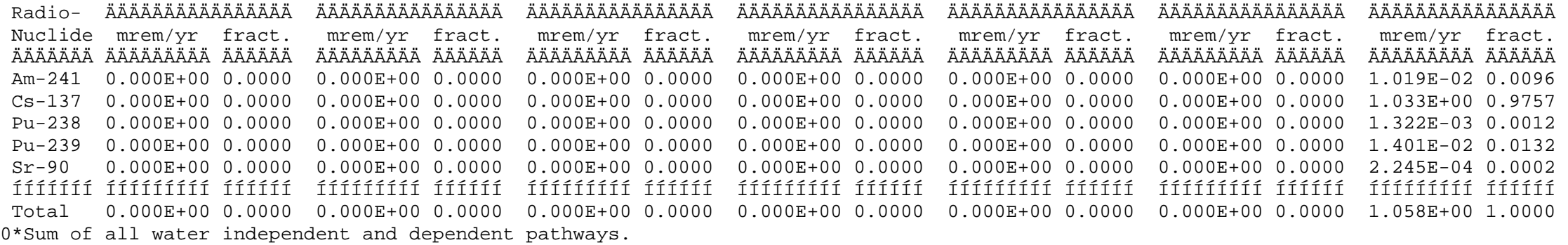
$\odot$ * Sum of all water independent and dependent pathways. 
Total Dose Contributions $\operatorname{TDOSE}(i, p, t)$ for Individual Radionuclides (i) and Pathways (p)

As mrem/yr and Fraction of Total Dose At $t=5.000 \mathrm{E}+01$ years

water Independent Pathways (Inhalation excludes radon)

Ground Inhalation

$$
\text { Radon }
$$

Plant

Meat

Milk

Soil

Radio- ÄÄÄÄÄÄÄÄÄÄÄÄÄÄÄA Nuclide mrem/yr fract
$\ddot{A ̈ A ̈ A ̈ A ̈ A ̈ A ̈ ~ A ̈ A ̈ A ̈ A ̈ A ̈ A ̈ A ̈ A ̈ ~ A ̈ A ̈ A ̈ A ̈ A ̈ A ~}$ ÄÄÄÄÄÄÄ ÄÄÄÄÄÄÄÄÄ ÄÄÄÄÄA $\begin{array}{lll}\mathrm{Am}-241 & 8.037 \mathrm{E}-04 & 0.0013 \\ \mathrm{C}-137 & 5.892 \mathrm{E}-01 & 0.9699\end{array}$ $\mathrm{PU}-238 \quad 6.830 \mathrm{E}-07 \quad 0.0000$ $\mathrm{Pu}-239$ 1.196E-05 0.0000 $\mathrm{Sr}-90 \quad 8.289 \mathrm{E}-05 \quad 0.0001$ Íííííi ííííííí ííííí Aล̈ÄÄÄÄÄÄÄÄÄÄÄÄÄÄÄ A АAАAลÄÄÄÄÄÄÄÄÄÄÄ

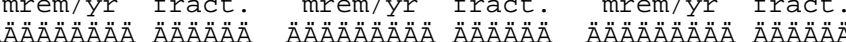

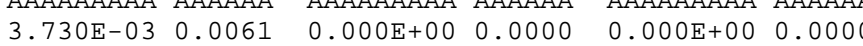
$\begin{array}{llllll}3.610 \mathrm{E}-06 & 0.0000 & 0.000 \mathrm{E}+00 & 0.0000 & 0.000 \mathrm{E}+00 & 0.0000\end{array}$ $\begin{array}{llllll}7.478 \mathrm{E}-04 & 0.0012 & 0.000 \mathrm{E}+00 & 0.0000 & 0.000 \mathrm{E}+00 & 0.0000\end{array}$ $9.255 \mathrm{E}-03 \quad 0.0152 \quad 0.000 \mathrm{E}+00-0.0000 \quad 0.000 \mathrm{E}+000.0000$ $2.6505-06$ 0.0000 $0.000 E+$

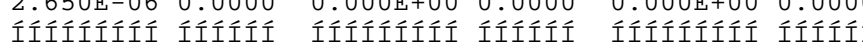
$\odot$

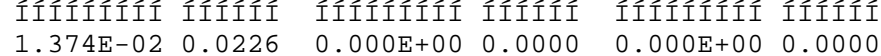
mrem/yr fract ÄÄÄÄÄÄÄÄÄ ÄÄÄÄÄÄ ÄÄÄÄÄÄÄÄÄÄÄÄÄÄÄÄ mrem/yr fract. ÄÄÄÄÄÄÄÄ ÄÄÄÄÄÄ $\odot .000 \mathrm{E}+0 \odot \quad 0.0000$ $0.000 \mathrm{E}+00 \quad 0.0000$ $\odot .000 \mathrm{E}+000.00000$ $\odot .000 E+00 \quad 0.0000$ $\begin{array}{lllll}.000 \mathrm{E}+0 \odot & 0.0000 & 0.000 \mathrm{E}+0 \odot & 0.0000\end{array}$ $0.000 \mathrm{E}+00$ - 00000 $0.000 \mathrm{C}+00$ 0 0000 IIIIIIIIÍi íííi

Total Dose Contributions TDOSE $(i, p, t)$ for Individual Radionuclides ( $i$ ) and Pathways ( $p$ ) As $\mathrm{mrem} / \mathrm{yr}$ and Fraction of Total Dose At $t=5.000 \mathrm{E}+01$ years Water Dependent Pathways Fish Radon

$$
\text { Plant }
$$

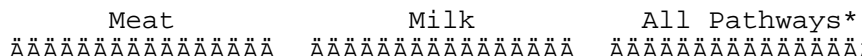

Meat
Milk

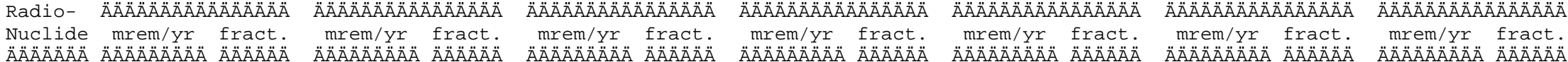

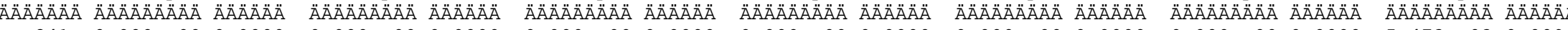

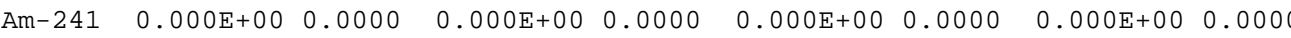

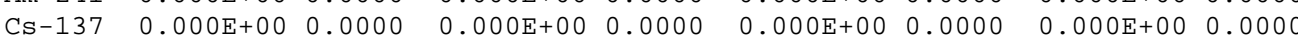

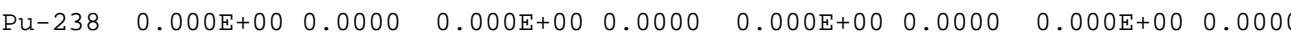

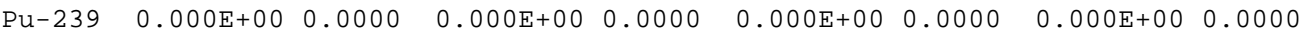

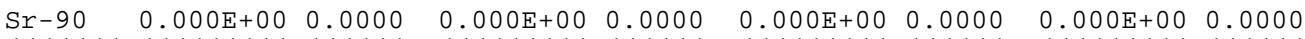

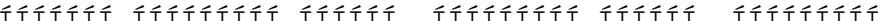

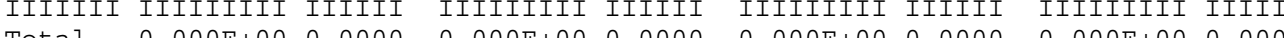
$\odot$ Sum of all water independent and dependent pathways. $0.000 \mathrm{E}+00 \quad 0.0000 \quad 0.000 \mathrm{E}+00 \quad 0.0000 \quad 5.893 \mathrm{E}-01 \quad 0.9702$ $0.000 \mathrm{E}+00 \quad 0.0000 \quad 0.000 \mathrm{E}+00 \quad 0.0000 \quad 9.360 \mathrm{E}-04 \quad 0.0015$ $0.000 \mathrm{E}+00 \quad 0.0000 \quad 0.000 \mathrm{E}+00 \quad 0.0000 \quad 1.161 \mathrm{E}-02 \quad 0.0191$

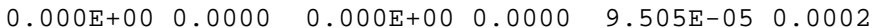
T́t́títít

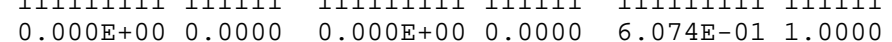


Total Dose Contributions $\operatorname{TDOSE}(i, p, t)$ for Individual Radionuclides (i) and Pathways (p)

As mrem/yr and Fraction of Total Dose At $t=7.500 \mathrm{E}+01$ years

Water Independent Pathways (Inhalation excludes radon)

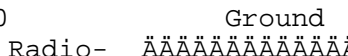
Inhalation

Radon

Plant

Meat

Milk

Soil

Radio-
Nuclide mrem/yr fract

ÄÄÄÄÄÄÄ Ä̈̈̈̈̈̈̈̈̈̈̈̈̈̈̈ ÄÄÄÄ̈̈̈ AÄÄÄÄÄÄÄÄÄÄÄÄÄÄÄ Ä̈̈̈̈̈̈̈̈̈̈̈̈̈̈̈̈̈̈̈̈̈̈̈̈ ÄÄÄÄÄÄÄÄÄÄÄÄÄ AAAÄÄÄÄÄÄÄÄÄÄÄ

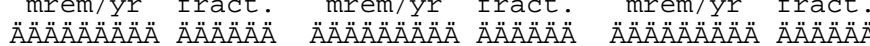

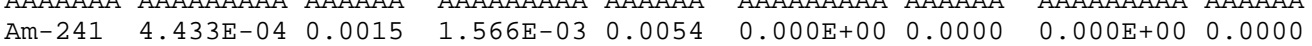
Cs-137 2.789E-01 0.9596 $\mathrm{Pu}-238 \quad 5.488 \mathrm{E}-07 \quad 0.0000$

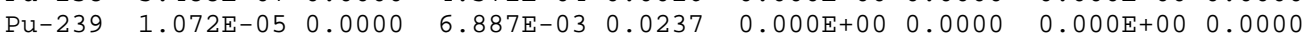

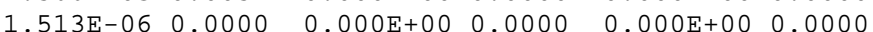

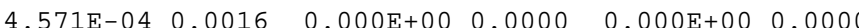

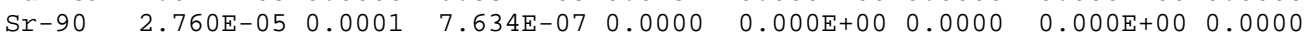

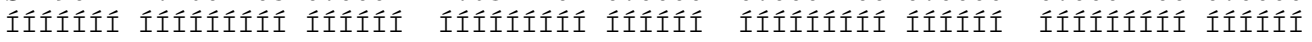
Tot

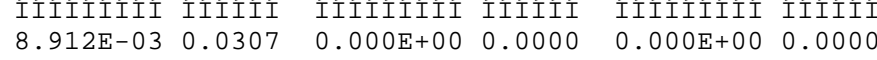
mrem/yr fract ÄÄÄÄÄÄÄÄÄ ÄÄÄÄÄÄ $0.000 \mathrm{E}+00$ - 0.0000 $0.000 \mathrm{E}+00$ - 9.0000 $\odot .000 \mathrm{E}+0 \odot \quad 0.0000$ $0.000 \mathrm{E}+000.0000$ ííííííí ííííí Total Dose Contributions TDOSE $(i, p, t)$ for Individual Radionuclides ( $i$ ) and Pathways ( $p$ ) As $\mathrm{mrem} / \mathrm{yr}$ and Fraction of Total Dose At $t=7.500 \mathrm{E}+01$ years Water Dependent Pathways Fish Radon

$$
\text { Plant }
$$
Milk A ÄÄÄÄÄÄÄÄÄÄÄÄÄÄ ÄÄÄÄÄÄÄÄÄÄÄÄÄÄÄÄ mrem/yr fract. mrem/yr fract. ÄÄÄÄÄÄÄÄÄ ÄÄÄÄÄÄ ÄÄÄÄÄÄÄÄÄ ÄÄÄÄÄÄ $0.000 \mathrm{E}+00 \quad 0.0000 \quad 3.946 \mathrm{E}-04 \quad 0.0014$ $0.000 \mathrm{E}+00 \quad 0.0000 \quad 7.289 \mathrm{E}-05 \quad 0.0003$ $0.000 E+00 \quad 0.0000 \quad 1.147 E-04 \quad 0.0004$ $0.000 \mathrm{E}+00 \quad 0.0000 \quad 1.746 \mathrm{E}-03 \quad 0.0060$ $\begin{array}{lllll}0.000 \mathrm{E}+00 & 0.0000 & 2.740 \mathrm{E}-06 & 0.0000\end{array}$

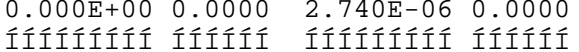
Nuclide mrem/yr fract. mrem/yr fract. mrem/yr fract. mrem/yr fract.

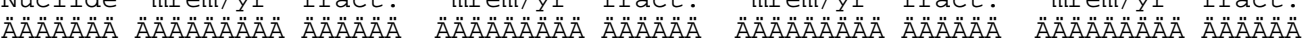

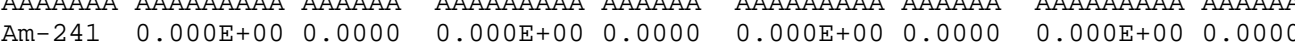

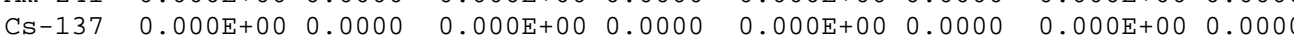

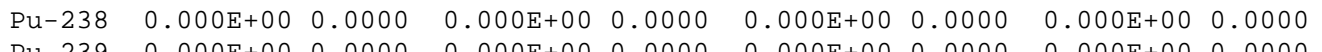

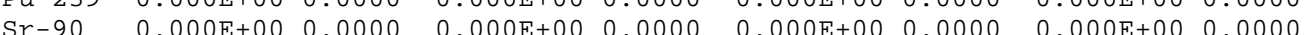
Ĺtítí ́f́títítí Ifilit lifififi $\odot$ Sum of all water independent and dependent pathways.

Meat mrem/yr fract. ÄÄÄÄÄÄÄÄÄ ÄÄÄÄÄÄ $0.000 \mathrm{E}+00$ 0.0000 $0.000 \mathrm{E}+000.0000$ $0.000 \mathrm{E}+000.0000$ $0.000 \mathrm{E}+000.0000$ $0.000 \mathrm{E}+000.0000$ Íííííííi ííííi $0.000 \mathrm{E}+000.0000$
Milk

ÄÄÄÄÄÄÄÄÄÄÄÄÄÄ̈̈̈̈ mrem/yr fract. ÄÄÄÄÄÄÄÄÄ ÄÄÄÄÄÄ $0.000 \mathrm{E}+00 \quad 0.0000$ $\odot .000 \mathrm{E}+0 \odot \quad 0.0000$ $\odot .000 \mathrm{E}+0 \odot \quad 0.0000$ $0.000 \mathrm{E}+00 \quad 0.0000$ $0.000 \mathrm{E}+000.0000$ Íííííííi ííííí $0.000 \mathrm{E}+000.0000$ 
Total Dose Contributions $\operatorname{TDOSE}(i, p, t)$ for Individual Radionuclides (i) and Pathways (p)

As mrem/yr and Fraction of Total Dose At $t=1.000 \mathrm{E}+02$ years

Ground water Independent Pathways (Inhalation excludes radon)

\section{Inhalation \\ Radio- ÄÄÄÄÄÄÄÄÄÄÄÄÄÄÄ \\ Meat$$
\text { Plant }
$$$$
\text { Radon }
$$
Nuclide mrem/yr fract. ÄÄÄÄÄÄÄ ÄÄÄÄÄÄÄÄÄ ÄÄÄÄÄ Am-241 $2.342 \mathrm{E}-04 \quad 0.0018$ Cs-137 1.197E-01 0.9446 Pu-238 4.327E-07 0.0000 Pu-239 8.949E-06 0.0001 Sr-90 8.391E-06 0.0001 Íííííí íííííííí ííííi Tot
ÄÄÄÄÄÄÄÄÄÄÄÄÄÄÄ mrem/yr fract. $0.000 \mathrm{E}+0 \odot \quad 0.0000$ $0.000 \mathrm{E}+00 \quad 0.0000$ $\odot .000 \mathrm{E}+0 \odot \odot .000 \odot$ $\odot .000 \mathrm{E}+00 \quad 0.000 \odot$ $0.000 \mathrm{E}+000.0000$ Íííííííi ííííi $0.000 \mathrm{E}+000.0000$ mrem/yr fract. ÄÄÄÄÄÄÄ ÄÄÄÄÄ $0.000 \mathrm{E}+00 \quad 0.0000$ $0.000 \mathrm{E}+00 \quad 0.0000$ $\odot .000 \mathrm{E}+0 \odot \quad 0.000 \odot$ $0.000 E+00 \quad 0.0000$ $0.000 \mathrm{E}+00 \quad 0.0000$ ííííííí ííííi $0.000 \mathrm{E}+0 \odot \odot .0000$
Milk ÄÄÄÄÄÄÄÄÄÄÄÄÄÄÄÄ mrem/yr fract ÄÄÄÄÄÄÄÄ ÄÄÄÄÄÄ $\odot .000 \mathrm{E}+0 \odot \quad 0.000 \odot$ $\odot .000 \mathrm{E}+00 \quad 0.000 \odot$ $\odot .000 \mathrm{E}+000.0000$ $0.000 \mathrm{E}+000.0000$ $0.000 \mathrm{E}+00$ - 0.0000 IIIIIIIIII IIIIII $\odot .000 \mathrm{E}+000.0000$

Total Dose Contributions TDOSE $(i, p, t)$ for Individual Radionuclides (i) and Pathways ( $p$ ) As $\mathrm{mrem} / \mathrm{yr}$ and Fraction of Total Dose At $t=1.000 \mathrm{E}+02$ years

$$
\text { Water Dependent Pathways }
$$
Water Fish

$$
\text { Radon }
$$

$$
\text { Plant }
$$

Meat

\section{Milk}

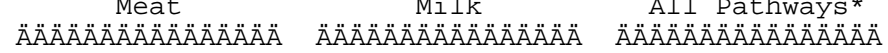
mrem/yr fract. mrem/yr fract. mrem/yr fract. ÄÄÄÄÄÄÄÄÄ ÄÄÄÄÄÄ ÄÄÄÄÄÄÄÄÄ ÄÄÄÄÄÄ ÄÄÄÄÄÄÄÄÄ ÄÄÄÄÄÄ $\begin{array}{llllll}0.000 \mathrm{E}+00 & 0.0000 & 0.000 \mathrm{E}+00 & 0.0000 & 9.650 \mathrm{E}-04 & 0.0076\end{array}$ $\begin{array}{llllll}0.000 \mathrm{E}+0 \odot & 0.000 \odot & 0.000 \mathrm{E}+\odot \odot & 0.000 \odot & 1.198 \mathrm{E}-01 & 0.9448\end{array}$ $\begin{array}{llllll}0.000 \mathrm{E}+00 & 0.0000 & 0.000 \mathrm{E}+00 & 0.0000 & 3.105 \mathrm{E}-04 & 0.0025\end{array}$ $\begin{array}{llllll}0.000 \mathrm{E}+00 & 0.0000 & 0.000 \mathrm{E}+00 & 0.0000 & 5.710 \mathrm{E}-03 & 0.0450\end{array}$ $\begin{array}{llllll}0.000 E+00 & 0.0000 & 0.000 E+00 & 0.0000 & 9.287 E-06 & 0.0001\end{array}$

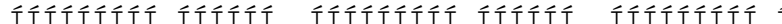

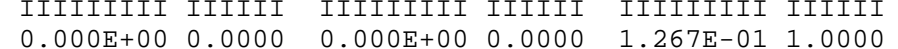

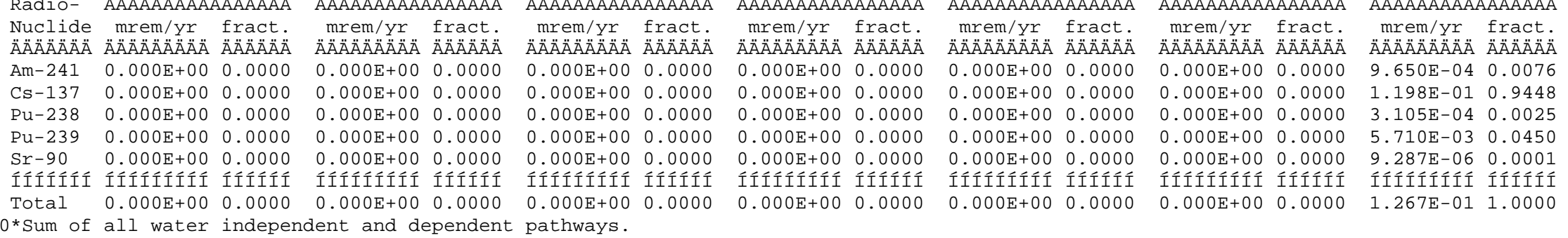
$\odot$ * Sum of all water independent and dependent pathways. 
Total Dose Contributions $\operatorname{TDOSE}(i, p, t)$ for Individual Radionuclides (i) and Pathways (p)

As mrem/yr and Fraction of Total Dose At $t=3.000 \mathrm{E}+02$ years

Water Independent Pathways (Inhalation excludes radon)

Ground Inhalation

$$
\text { Radon }
$$

Plant

Meat

Milk

Soil

Radio- ÄÄÄÄÄÄÄÄÄÄÄÄÄÄÄ

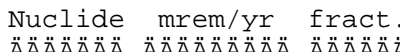
ÄÄÄÄÄÄÄ ÄÄÄÄÄÄÄÄÄ ÄÄÄÄÄ̈
Am-241 $0.000 E+0 \odot$ $0.00 \odot \bullet$ $\begin{array}{lll}\mathrm{Am}-241 & 0.00 \odot \mathrm{E}+0 \odot & 0.0 \odot \odot \odot \\ \mathrm{CS}-137 & 0.00 \odot \mathrm{E}+0 \odot & 0.0 \odot \odot \odot\end{array}$ $\mathrm{Pu}-238 \quad 0.000 \mathrm{E}+00 \quad 0.0000$ Pu-239 $0.000 \mathrm{E}+00 \quad 0.0000$ $\begin{array}{ccc}\mathrm{Sr}-90 & 0.000 \mathrm{E}+0 \odot \quad 0.0000\end{array}$ Ííííí ííííííí íííít Total $0.000 \mathrm{E}+000.0000$ АÄÄÄÄÄÄÄÄÄÄÄÄÄÄÄ ÄӒÄÄÄÄÄÄÄÄÄÄÄÄ ÄÄÄÄÄÄÄÄÄÄÄÄÄ AАAAÄÄÄÄÄÄÄÄÄÄ ÄÄÄÄÄÄÄÄÄ ÄÄÄÄÄÄ $\begin{array}{llllll}0.000 \mathrm{E}+00 & 0.0000 & 0.000 \mathrm{E}+00 & 0.0000 & 0.000 \mathrm{E}+00 & 0.0000\end{array}$

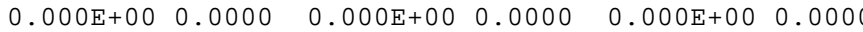

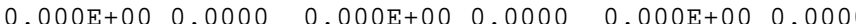
$0.000 \mathrm{E}+00 \quad 0.0000 \quad 0.000 \mathrm{E}+00 \quad 0.0000 \quad 0.000 \mathrm{E}+00 \quad 0.0000$

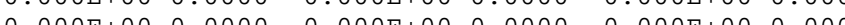

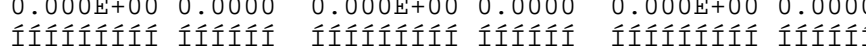
$\odot$

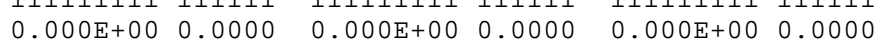
mrem/yr fract ÄÄÄÄÄÄÄÄÄ ÄÄÄÄÄÄ $0.000 \mathrm{E}+00$ 0.000९ $\odot .000 \mathrm{E}+00 \quad 0.0000$ $0.000 \mathrm{E}+00 \quad 0.0000$ $0.000 \mathrm{E}+00 \quad 0.0000$ $0.000 \mathrm{E}+000.0000$ Íííííííi ííííí Total Dose Contributions TDOSE $(i, p, t)$ for Individual Radionuclides ( $i$ ) and Pathways ( $p$ ) As $\mathrm{mrem} / \mathrm{yr}$ and Fraction of Total Dose At $t=3.000 \mathrm{E}+02$ years

$$
\text { Water Dependent Pathways }
$$

$$
\text { Fish }
$$$$
\text { Radon }
$$

$$
\text { Plant }
$$

Meat

Milk

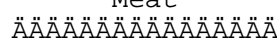
mrem/yr fract. Nuclide mrem/yr fract

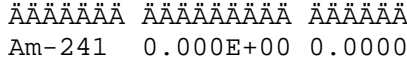
$\begin{array}{lll}\mathrm{Am}-241 & 0.000 \mathrm{E}+00 & 0.000 \odot \\ \mathrm{CS}-137 & 0.000 \mathrm{E}+0 \odot & 0.000 \odot\end{array}$ $\mathrm{Pu}-2380.000 \mathrm{E}+00 \quad 0.0000$ Pu-239 $0.000 \mathrm{E}+0 \odot \quad 0.000 \odot$

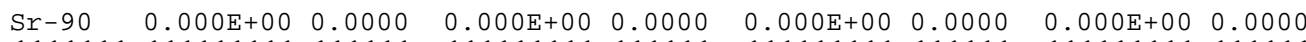
mrem/yr fract. mrem/yr fract. mrem/yr fract. ÄÄÄÄÄÄÄA ÄÄÄÄÄ ÄÄÄÄÄÄÄÄ ÄÄÄÄÄA ÄÄÄÄÄÄÄÄ ÄÄÄÄÄ

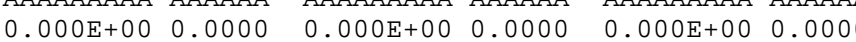

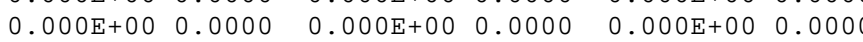

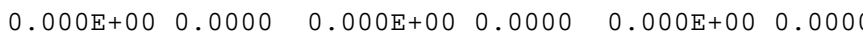

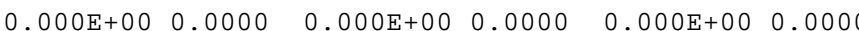

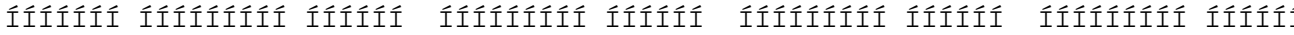
$\begin{array}{lllllllllll}\text { Total } & 0.000 \mathrm{E}+00 & 0.0000 & 0.000 \mathrm{E}+00 & 0.0000 & 0.000 \mathrm{E}+00 & 0.0000 & 0.000 \mathrm{E}+00 & 0.0000 & 0.000 \mathrm{E}+00 & 0.0000\end{array}$ $\odot$ * Sum of all water independent and dependent pathways.

\section{$0.000 \mathrm{E}+00 \quad 0.0000$} $0.000 \mathrm{E}+00 \quad 0.0000$ $0.000 \mathrm{E}+00 \quad 0.0000$ $0.000 \mathrm{E}+000.0000$

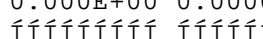

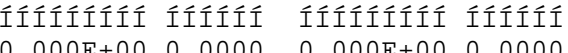

ÄÄÄÄÄÄÄÄÄÄÄÄÄÄÄÄ ÄÄÄÄÄÄÄÄÄÄÄÄÄÄÄÄ mrem/yr fract. mrem/yr fract. АÄÄÄÄÄÄÄÄ ÄÄÄÄÄÄ ÄÄÄÄÄÄÄÄÄ ÄÄÄÄÄÄ $\begin{array}{llll}0.000 \mathrm{E}+00 & 0.0000 & 0.000 \mathrm{E}+00 & 0.0000 \\ 0.000 \mathrm{C}+00 & 0.0000 & 0.000 \mathrm{E}+00 & 0.0000\end{array}$ $\odot .000 \mathrm{E}+0 \odot \quad 0.000 \odot \quad 0.00 \odot \mathrm{E}+\odot \odot \quad \odot .0 \odot \odot \odot$ (.,000E+00 0.0000 0.000E+00 0.0000 $\begin{array}{lllll}0.000 \mathrm{E}+00 & 0.0000 & 0.000 \mathrm{E}+00 & 0.0000\end{array}$

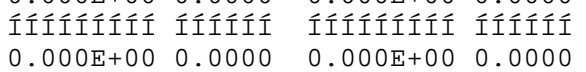
任任ííííííí

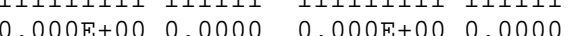
$\mathrm{mrem} / \mathrm{yr}$ fract. mrem/yr fract. ÄAAAA AAAAAAAA AAAAAA 0.000E+00 0.0000 $\odot .0 \odot \odot E+\odot \odot \quad \odot . \odot \odot \odot \odot \quad \odot . \odot \odot \odot E+\odot \odot \quad \odot . \odot \odot \odot \odot$

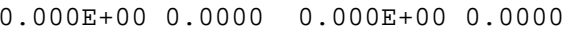
$0.000 \mathrm{E}+00 \quad 0.0000 \quad 0.000 \mathrm{E}+00 \quad 0.0000$ 
Total Dose Contributions $\operatorname{TDOSE}(i, p, t)$ for Individual Radionuclides (i) and Pathways (p)

As mrem/yr and Fraction of Total Dose At $t=1.000 \mathrm{E}+03$ years

Water Independent Pathways (Inhalation excludes radon)

Ground Inhalation

$$
\text { Radon }
$$

Plant

Meat

Milk

Soil

Radio - ӒÄÄÄÄÄÄÄÄÄÄÄÄÄÄ Nuclide mrem/yr fract.
ÄÄÄÄÄÄ Ä̈̈̈̈̈̈̈̈̈̈̈̈ ÄÄÄÄÄ ÄÄÄÄÄÄÄ ÄÄÄÄÄÄÄÄÄ ÄÄÄÄÄÄ $\begin{array}{lll}\mathrm{Am}-241 & 0.000 \mathrm{E}+\odot \odot & 0.000 \odot \\ \mathrm{CS}-137 & 0.0 \odot \odot \mathrm{E}+\odot \odot & \odot .0 \odot \odot \odot\end{array}$ $\mathrm{Pu}-238 \quad 0.000 \mathrm{E}+000.0000$ Pu-239 $0.000 \mathrm{E}+00 \quad 0.0000$ $\mathrm{Sr}-90 \quad 0.000 \mathrm{E}+00$ - 0.0000 Ííííí ííííííí íííít Total $0.000 \mathrm{E}+000.0000$ АÄÄÄÄÄÄÄÄÄÄÄÄÄÄÄ ÄÄÄÄÄÄÄÄÄÄÄÄÄÄ ÄÄÄÄÄÄÄÄÄÄÄÄÄ AАAAÄÄÄÄÄÄÄÄÄÄ ÄÄÄÄÄ̈̈̈̈ ÄÄÄÄÄÄ $\begin{array}{llllll}0.000 \mathrm{E}+00 & 0.0000 & 0.000 \mathrm{E}+00 & 0.0000 & 0.000 \mathrm{E}+00 & 0.0000\end{array}$

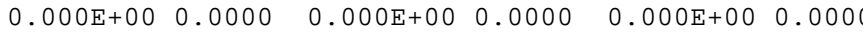

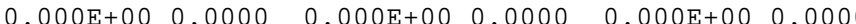
$0.000 E+00 \quad 0.0000 \quad 0.000 E+00 \quad 0.0000 \quad 0.000 E+00 \quad 0.0000$ $0.000 \mathrm{C}+00$ 0.0000

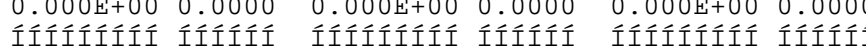
$\odot$

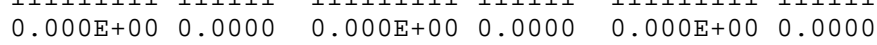
mrem/yr fract ÄÄÄÄÄÄÄÄ̈ ÄÄÄÄÄÄ $0.000 \mathrm{E}+00$ 0.000९ $\odot .000 \mathrm{E}+000.0000$ $0.000 \mathrm{E}+000.0000$ $0.000 \mathrm{E}+00 \quad 0.0000$ $0.000 \mathrm{E}+000.0000$ Íííííííi ííííí Total Dose Contributions TDOSE $(i, p, t)$ for Individual Radionuclides ( $i$ ) and Pathways ( $p$ ) As $\mathrm{mrem} / \mathrm{yr}$ and Fraction of Total Dose At $t=1.000 \mathrm{E}+03$ years

$$
\text { Water Dependent Pathways }
$$$$
\text { Fish }
$$$$
\text { Radon }
$$

$$
\text { Plant }
$$

Meat

Milk

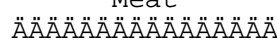
$\mathrm{mrem} / \mathrm{yr}$ fract. Nuclide mrem/yr fract

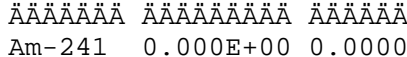
$\begin{array}{lll}\mathrm{Am}-241 & 0.000 \mathrm{E}+0 \odot & 0.000 \odot \\ \mathrm{Cs}-137 & 0.000 \mathrm{E}+0 \odot & 0.000 \odot\end{array}$ $\mathrm{Pu}-238 \quad 0.000 \mathrm{E}+00 \quad 0.0000$ Pu-239 $0.000 \mathrm{E}+0 \odot \quad 0.000 \odot$

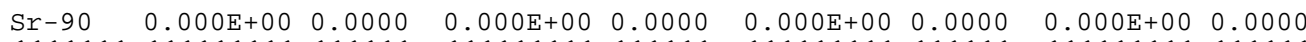
mrem/yr fract. mrem/yr fract. mrem/yr fract. ÄÄÄÄÄÄÄ ÄÄÄÄÄ ÄÄÄÄÄÄÄÄ ÄÄÄÄÄA ÄÄÄÄÄÄÄÄ ÄÄÄÄÄ

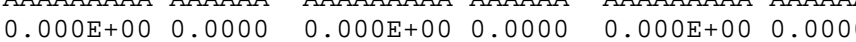

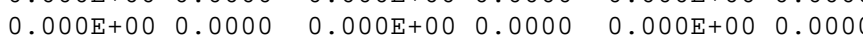
$\begin{array}{llllll}0.000 E+00 & 0.0000 & 0.000 E+00 & 0.0000 & 0.000 E+00 & 0.0000\end{array}$

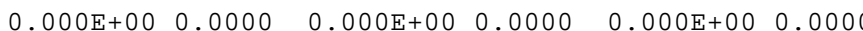

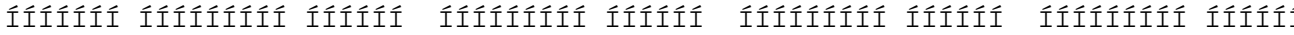
$\begin{array}{lllllllllll}\text { Total } & 0.000 \mathrm{E}+00 & 0.0000 & 0.000 \mathrm{E}+00 & 0.0000 & 0.000 \mathrm{E}+00 & 0.0000 & 0.000 \mathrm{E}+00 & 0.0000 & 0.000 \mathrm{E}+00 & 0.0000\end{array}$ $\odot$ Sum of all water independent and dependent pathways.

\section{$0.000 \mathrm{E}+00 \quad 0.0000$} $0.000 \mathrm{E}+00 \quad 0.0000$ $0.000 \mathrm{E}+00 \quad 0.0000$ $0.000 \mathrm{E}+000.0000$

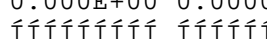

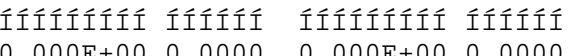

ÄÄÄÄÄÄÄÄÄÄÄÄÄÄÄÄ ÄÄÄÄÄÄÄÄÄÄÄÄÄÄÄÄ mrem/yr fract. mrem/yr fract. Ä̈ÄÄÄÄÄÄÄ ÄÄÄÄÄÄ ÄÄÄÄÄÄÄÄÄ ÄÄÄÄÄÄ $\begin{array}{llll}0.000 \mathrm{E}+00 & 0.0000 & 0.000 \mathrm{E}+00 & 0.0000 \\ 0.000 \mathrm{C}+00 & 0.0000 & 0.000 \mathrm{E}+00 & 0.0000\end{array}$ $\odot .000 \mathrm{E}+0 \odot \quad 0.000 \odot \quad 0.00 \odot \mathrm{E}+\odot \odot \quad \odot . \odot \odot \odot \odot$ (.,

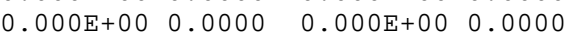

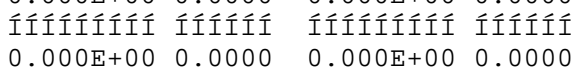
任任ííííííi

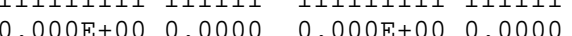
$\mathrm{mrem} / \mathrm{yr}$ fract. mrem/yr fract. ÄAAAA AAAAAAAA AAAAAA (1) $\odot .0 \odot \odot E+\odot \odot \quad \odot . \odot \odot \odot \odot \quad \odot . \odot \odot \odot E+\odot \odot \quad \odot . \odot \odot \odot \odot$

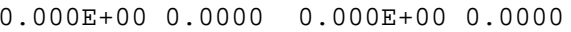
$0.000 \mathrm{E}+00 \quad 0.0000 \quad 0.000 \mathrm{E}+00 \quad 0.0000$ 
Parent and Progeny Principal Radionuclide Contribution

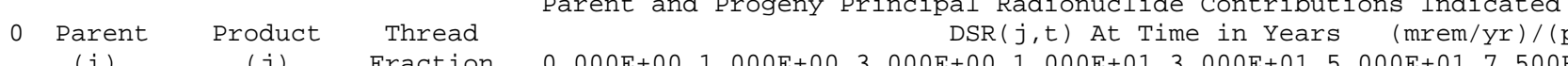

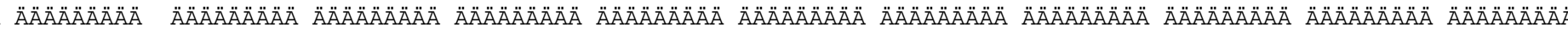

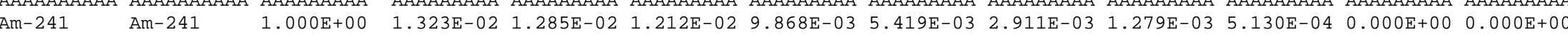

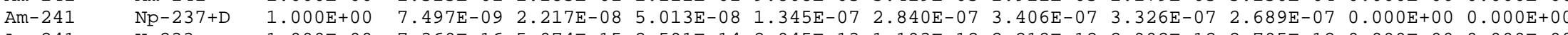

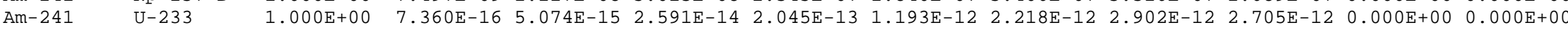

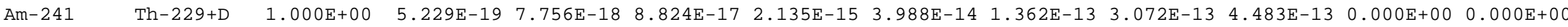
Am-241 äDSR $(j)$

OCS $-137+\mathrm{D}$

๑Pu-238

०Pu-238 Pu-238

$\mathrm{Pu}-238 \quad \mathrm{U}-234$

$\mathrm{Pu}-238$ Th-230

$\begin{array}{ll}\mathrm{Pu}-238 & \mathrm{Ra}-226+\mathrm{D} \\ \mathrm{Pu}-238 & \mathrm{~Pb}-210+\mathrm{D}\end{array}$

$\mathrm{Pu}-238 \quad$ äDR $(j)$

คPu-239 Pu-239

$\mathrm{PU}-239$

$\mathrm{U}-235+\mathrm{D}$

$\begin{array}{ll}\mathrm{Pu}-239 & \mathrm{~Pa}-231 \\ \mathrm{Pu}-239 & \mathrm{Ac}-227+\mathrm{D}\end{array}$

$\begin{array}{ll}\mathrm{Pu}-239 & \mathrm{AC}-227+\mathrm{P} \\ \mathrm{Pu}-239 & \mathrm{a} D S R(j)\end{array}$

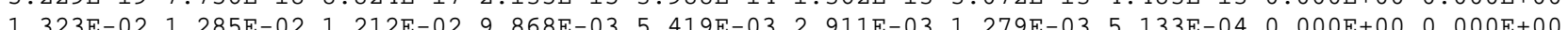

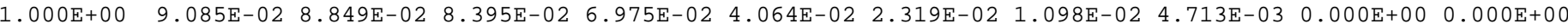

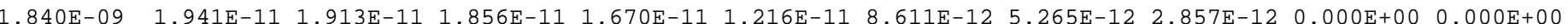

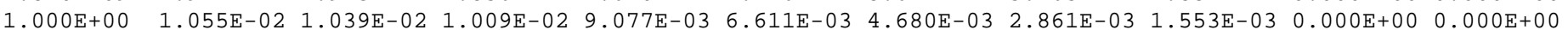

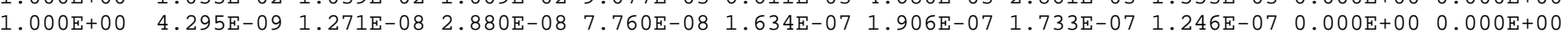

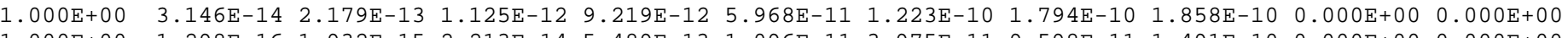

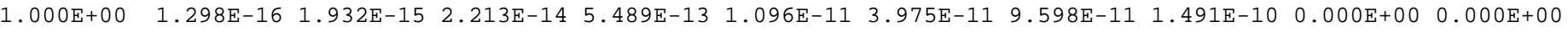

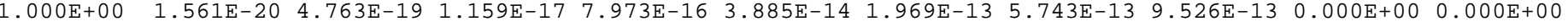

1.055E-02 1.039E-02 1.009E-02 9.077E-03 6.611E-03 4.680E-03 2.861E-03 $1.553 \mathrm{E}-03 \quad 0.000 \mathrm{E}+00 \quad 0.000 \mathrm{E}+00$

$1.000 \mathrm{E}+00 \quad 1.162 \mathrm{E}-02$ 1.154E-02 1.138E-02 1.082E-02 9.220E-03 7.640E-03 5.687E-03 3.757E-03 $0.000 \mathrm{E}+00 \quad 0.000 \mathrm{E}+00$

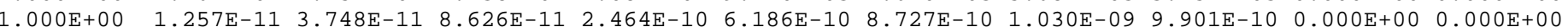

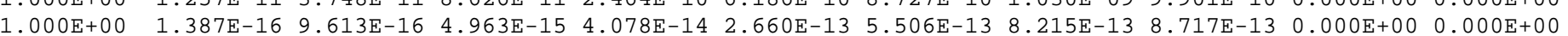

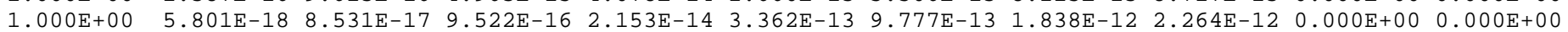

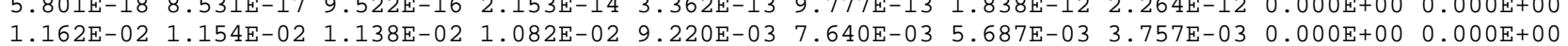

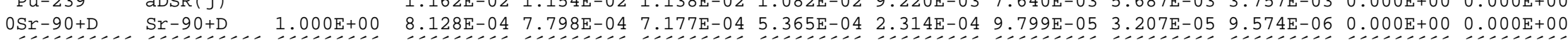

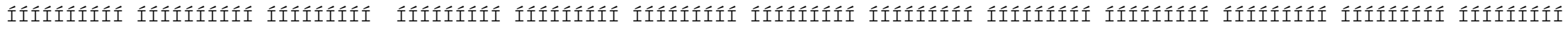

\section{ONuclide}

ÄÄÄÄÄÄÄ

Am- 241

Cs -137

$\mathrm{Pu}-238$

$\mathrm{Pu}-239$

$\mathrm{Sr}-90$

Íííííí

$=0.000 \mathrm{E}+\odot \odot$

Äล̈ล̈ÄÄÄล̈ÄÄ $1.890 \mathrm{E}+03$

$2.752 \mathrm{E}+02$

2. $370 \mathrm{E}+03$

$2.152 \mathrm{E}+03$

3. $076 \mathrm{E}+04$

Ííííííí

${ }^{*}$ At specific activity limit
Single Radionuclide Soil Guidelines $\mathrm{G}(i, \mathrm{t})$ in $\mathrm{pCi} / \mathrm{g}$ Basic Radiation Dose Limit $=2.500 \mathrm{E}+01 \mathrm{mrem} / \mathrm{yr}$

$.000 \mathrm{E}+\odot \odot$

3. $\odot \odot \odot E+\odot \odot \quad 1.000 \mathrm{E}+01$

ÄÄÄÄÄÄÄÄÄ

ÄÄÄÄÄÄÄÄ

3. $000 \mathrm{E}+01$

ÄÄÄÄÄÄÄÄÄ

5. $000 \mathrm{E}+01$

ÄÄÄÄÄÄÄÄÄA

$7.500 \mathrm{E}+01$

$1.945 \mathrm{E}+03$

2. $978 \mathrm{E}+02$

$3.584 \mathrm{E}+02$

$1.5878+03$

$.825 \mathrm{E}+02$
$2.405 \mathrm{E}+03$

$2.478 \mathrm{E}+03$

$2.754 \mathrm{E}+03$

1. $078 \mathrm{E}+03$

$5.342 \mathrm{E}+03$

$167 \mathrm{E}+03 \quad 2.197 \mathrm{E}+03$

$3.483 \mathrm{E}+04 \quad 2.311 \mathrm{E}+03$

Íííííííi Iííííííí

$1.080 \mathrm{E}+05 \quad 2.551 \mathrm{E}+05$

Íííííííi
1. $955 \mathrm{E}+0$

$2.277 \mathrm{E}+03$

$8.737 \mathrm{E}+03$

$4.396 \mathrm{E}+03$

$7.795 \mathrm{E}+05$

Íííííííí

\begin{tabular}{|c|c|c|}
\hline $\begin{array}{l}\odot E+\odot 2 \\
\ddot{A ̈ A ̈ A ̈ A ̈ A ̈ ~}\end{array}$ & 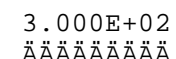 & 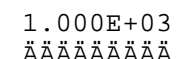 \\
\hline $\begin{array}{l}\text { AAAAAAAAA } \\
4.870 \mathrm{E}+\odot 4\end{array}$ & * $3.431 \mathrm{E}+12$ & $\star 3.431 \mathrm{E}+12$ \\
\hline 5.30 & $* 8.704 \mathrm{E}+13$ & $4 E+13$ \\
\hline .61 & *1.712E+13 & $* 1.712 \mathrm{E}+13$ \\
\hline $.655 E+03$ & ${ }^{*} 6.214 \mathrm{E}+10$ & ${ }^{*} 6.214 \mathrm{E}+10$ \\
\hline 0 & ${ }^{*} 1.365 E+14$ & ${ }^{*} 1.365 \mathrm{E}+14$ \\
\hline Ííííííí & Ííííííííí & Íííííííí \\
\hline
\end{tabular}


1RESRAD, Version 6.3 T« Limit $=180$ days Summary : CAU 383 Drainage
05/11/2006 10:08 Page 24

File: CAU 383 Drainage. RAD

Summed Dose/Source Ratios DSR(i,t) in (mrem/yr)/(pCi/g)

and Single Radionuclide Soil Guidelines $\mathrm{G}(\mathrm{i}, \mathrm{t})$ in $\mathrm{pCi} / \mathrm{g}$

at $\mathrm{tmin}=$ time of minimum single radionuclide soil guideline

and at $\operatorname{tmax}=$ time of maximum total dose $=0.000 \mathrm{E}+0 \odot$ years

\begin{tabular}{|c|c|c|c|c|c|c|}
\hline uclide & $\begin{array}{l}\text { Initial } \\
(p C i / g)\end{array}$ & $\begin{array}{c}\text { tmin } \\
\text { (years) }\end{array}$ & $\operatorname{DSR}(i, \operatorname{tmin})$ & $\begin{array}{l}\mathrm{G}(\mathrm{i}, \mathrm{tmin}) \\
(\mathrm{pCi} \mathrm{i} / \mathrm{g})\end{array}$ & $\operatorname{DSR}(i, \operatorname{tmax})$ & $\mathrm{G}(i, \operatorname{tmax})$ \\
\hline & & ÄÄÄÄÄÄÄÄÄÄÄÄ & ÄÄÄÄÄÄÄÄÄÄ & 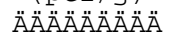 & ÄÄÄÄÄÄÄÄÄÄ & \\
\hline$A m-241$ & $1.880 \mathrm{E}+0 \odot$ & $\odot .000 \mathrm{E}+\odot \odot$ & $E-02$ & $1.890 \mathrm{E}+03$ & 02 & $1.890 \mathrm{E}$ \\
\hline Cs -137 & $2.541 \mathrm{E}+01$ & $\odot . \odot \odot \odot E+\odot \odot$ & $9.085 E-02$ & $2.752 \mathrm{E}+\Theta 2$ & $9.085 E-02$ & $2.152 \mathrm{E}+$ \\
\hline Pu-238 & 2. $000 \mathrm{E}-01$ & $\odot . \odot \odot \odot E+\odot \odot$ & 1. $055 E-02$ & $2.370 E+03$ & 1. $055 E-02$ & $2.370 \mathrm{E}+\mathrm{C}$ \\
\hline & $=+\Theta \odot$ & $E+\odot \odot$ & -02 & $E+03$ & -02 & $2.152 \mathrm{E}+\Theta$ \\
\hline & $E-01$ & $\odot . \odot \odot \odot E+\odot \odot$ & -04 & 3. & 04 & \\
\hline & 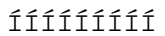 & Ííííííííííl & Éííi & & Íííííííí & Í \\
\hline
\end{tabular}


Individual Nuclide Dose Summed Over All Pathways

Parent Nuclide and Branch Fraction Indicated

ONuclide Parent THF(i) (j) (i) (j) Am-241 A AMAAAA AAAAAA AAAAAAAA ONp-237 Am-241 1. $1.000 \mathrm{E}+00$ OU-233 Am-241 1.000E+00 ๑Th-229 Am-241 1.000E+@० ๑Cs -137 Cs $-137 \quad 1.000 \mathrm{E}+00$ OPu-238 Pu-238 $1.840 E-09$ Pu-238 Pu$1.000 \mathrm{E}+0 \odot$ Pu-238 äDOSE $(j)$

○U-234 PU-238 1.000E+๑0 ๑Th-230 Pu-238 1.000E+०0 ORa-226 Pu-238 1. $1.00 \mathrm{E}+\odot \odot$ $\odot \mathrm{Pb}-210 \quad \mathrm{Pu}-238 \quad 1.000 \mathrm{E}+\odot \odot$ ๑Pu-239 Pu-239 1.000E $+\odot \odot$ OU-235 Pu-239 $1.000 \mathrm{E}+00$ $\odot \mathrm{Pa}-231 \mathrm{Pu}-239$ 1. $000 \mathrm{E}+\odot \odot$ $\odot \mathrm{Pa}-231$ Pu-239 $1.000 \mathrm{E}+00$ $\begin{array}{lll}\text { PAC-227 } & \mathrm{Pu}-239 & 1.000 \mathrm{E}+00 \\ \odot \mathrm{Sr}-90 & \mathrm{Sr}-90 & 1.000 \mathrm{E}+0 \odot\end{array}$ Íííííi íííííi ííííííít DOSE $(j$, Indicated

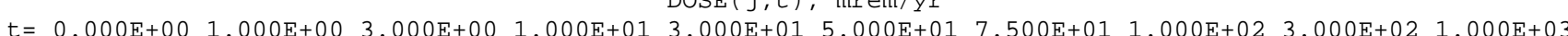

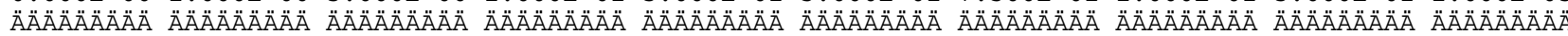

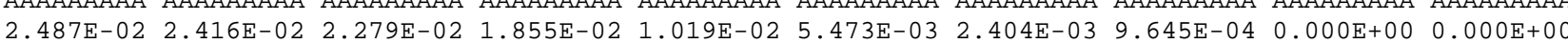

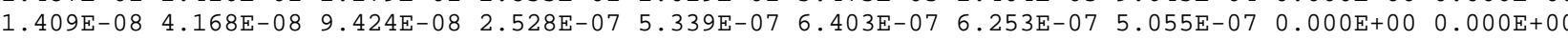

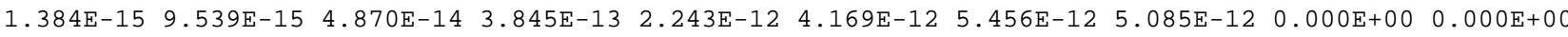
$9.830 \mathrm{E}-19$ 1.458E-17 1.659E-16 4.014E-15 7.497E-14 2.560E-13 5.775E-13 8.428E-13 0.000E+00 $0.000 \mathrm{E}+00$

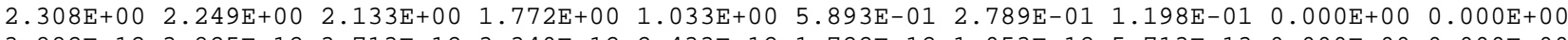

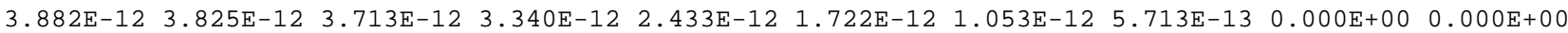
$2.110 \mathrm{E}-03 \quad 2.079 \mathrm{E}-03 \quad 2.018 \mathrm{E}-03 \quad 1.815 \mathrm{E}-03 \quad 1.322 \mathrm{E}-03 \quad 9.360 \mathrm{E}-04 \quad 5.722 \mathrm{E}-04 \quad 3.105 \mathrm{E}-04 \quad 0.000 \mathrm{E}+0 \odot \quad 0.000 \mathrm{E}+00$

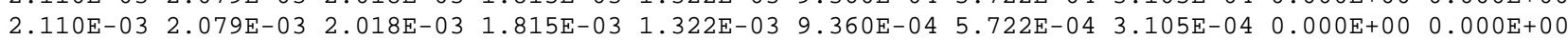

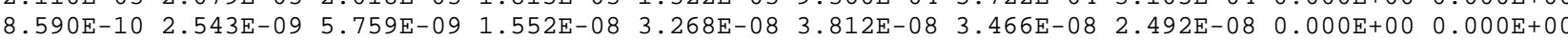

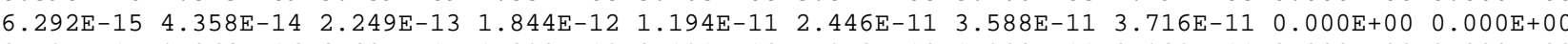
2.597E-17 3.864E-16 $4.427 \mathrm{E}-15$ 1.098E-13 $2.191 \mathrm{E}-12 \quad 7.950 \mathrm{E}-12$ 1.920E-11 $2.983 \mathrm{E}-11 \quad 0.000 \mathrm{E}+00 \quad 0.000 \mathrm{E}+00$ 3.122E-21 9.525E-20 2.318E-18 1.595E-16 $7.769 \mathrm{E}-15 \quad 3.937 \mathrm{E}-14 \quad 1.149 \mathrm{E}-13 \quad 1.905 \mathrm{E}-13 \quad 0.000 \mathrm{E}+000.000 \mathrm{E}+00$ 1.766E-02 1.754E-02 1.729E-02 1.644E-02 1.401E-02 1.161E-02 8.644E-03 5.710E-03 $0.000 \mathrm{E}+00 \quad 0.000 \mathrm{E}+00$ 1.911E-11 5.697E-11 1.311E-10 3.746E-10 9.402E-10 1.326E-09 1.565E-09 1.505E-09 $0.000 \mathrm{E}+00 \quad 0.000 \mathrm{E}+00$ $2.109 \mathrm{E}-16 \quad 1.461 \mathrm{E}-15 \quad 7.544 \mathrm{E}-15 \quad 6.198 \mathrm{E}-14 \quad 4.043 \mathrm{E}-13 \quad 8.369 \mathrm{E}-13 \quad 1.249 \mathrm{E}-12 \quad 1.325 \mathrm{E}-12 \quad 0.000 \mathrm{E}+00 \quad 0.000 \mathrm{E}+00$ 8.817E-18 1.297E-16 1.447E-15 3.273E-14 5.110E-13 $1.486 \mathrm{E}-12 \quad 2.794 \mathrm{E}-12 \quad 3.442 \mathrm{E}-12 \quad 0.000 \mathrm{E}+00 \quad 0.000 \mathrm{E}+00$ 7.884E-04 7.564E-04 6.962E-04 5.204E-04 2.245E-04 9.505E-05 3.111E-05 9.287E-06 $0.000 \mathrm{E}+00 \quad 0.000 \mathrm{E}+00$

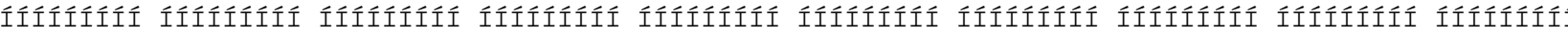
$\operatorname{THF}(i)$ is the thread fraction of the parent nuclide. 
Individual Nuclide Soil Concentration Parent Nuclide and Branch Fraction Indicated

ONuclide Parent THF(i) (j) (i) स्̈ Am-241 AM-241 Am-241 1.00OE+OO ONp-237 Am-241 1. $1.000 \mathrm{E}+00$ OU-233 Am-241 1.000E+00 ๑Th-229 Am-241 1.000E +०९ ๑Cs -137 Cs $-137 \quad 1.000 \mathrm{E}+00$ OPu-238 Pu-238 $1.840 \mathrm{E}-09$ PU-238 PU-238 $1.000 \mathrm{C}+00$ $\mathrm{Pu}-238$ äs $(j):$ $\begin{array}{ccc}\mathrm{Pu}-238 & \mathrm{äS}(\mathrm{j}): & \\ \mathrm{OU}-234 & \mathrm{Pu}-238 & 1.000 \mathrm{E}+00\end{array}$ $\begin{array}{lll}\odot \mathrm{U}-234 & \mathrm{Pu}-238 & 1.000 \mathrm{E}+\odot \odot \\ \text { ○Th-230 } & \mathrm{Pu}-238 & 1.000 \mathrm{E}+0 \odot\end{array}$ $\odot \mathrm{Ra}-226 \quad \mathrm{Pu}-238 \quad 1.000 \mathrm{E}+00$ $\odot \mathrm{Pb}-210 \quad \mathrm{Pu}-238 \quad 1.000 \mathrm{E}+\Theta 0$ ๑Pu-239 Pu-239 1.000E $+\odot \odot$ OU-235 PU-239 $1.000 \mathrm{E}+00$ $\odot \mathrm{Pa}-231 \quad \mathrm{Pu}-239 \quad 1.000 \mathrm{E}+\odot \odot$ $\odot \mathrm{AC}-227 \quad \mathrm{Pu}-239 \quad 1.000 \mathrm{E}+00$ $0 \mathrm{Sr}-90 \mathrm{Sr}-90 \quad 1.000 \mathrm{E}+00$ Íííííí íííííí íííííííi $\operatorname{THF}(i)$ is the thread fraction ORESCALC.EXE execution time =

$$
\mathrm{S}(j, \mathrm{t}), \mathrm{pCi} / \mathrm{g}
$$

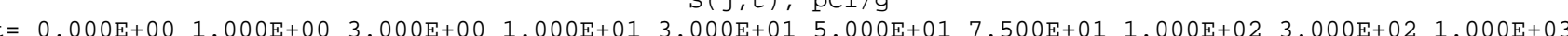

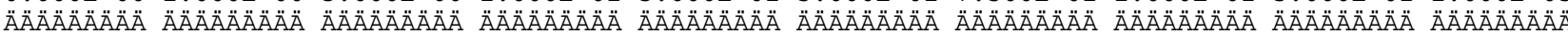

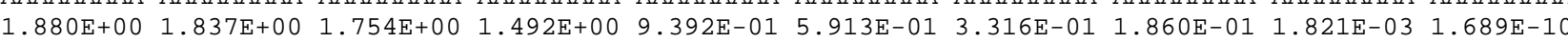
$0.000 \mathrm{E}+00$ 6.014E-07 $1.760 \mathrm{E}-06$ 5.389E-06 $1.281 \mathrm{E}-05$ 1.717E-05 2.001E-05 $2.118 \mathrm{E}-05$ 1.709E-05 5.252E-06

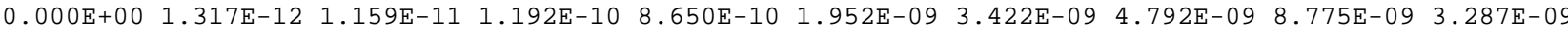
$0.000 \mathrm{E}+00$ 4.156E-17 1.104E-15 3.857E-14 8.861E-13 3.512E-12 9.841E-12 $1.953 \mathrm{E}-11$ 1.627E-10 $5.303 \mathrm{E}-10$

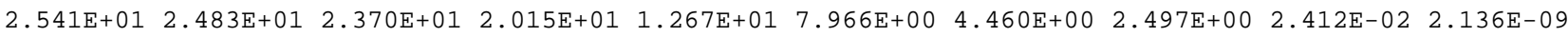

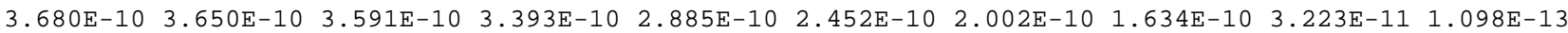

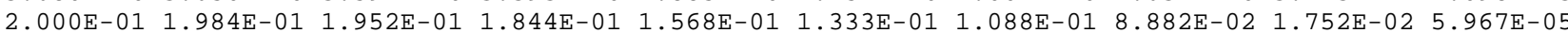
2. $.0 \odot \mathrm{E}-01$ 1.984E-01 $1.952 \mathrm{E}-01$ 1.844E-01 $1.568 \mathrm{E}-01$ 1.333E-01 $1.088 \mathrm{E}-01$ 8.882E-02 $1.752 \mathrm{E}-02$ 5.967E-05

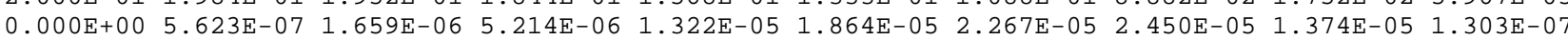
$\odot .000 \mathrm{E}+0 \odot$ 2.538E-12 2.259E-11 2.414E-10 $1.945 \mathrm{E}-09$ 4.846E-09 9.540E-09 $1.488 \mathrm{E}-08$ 5.182E-08 $7.153 \mathrm{E}-08$ $0.000 \mathrm{E}+00 \quad 3.664 \mathrm{E}-16 \quad 9.777 \mathrm{E}-15 \quad 3.476 \mathrm{E}-13 \quad 8.356 \mathrm{E}-12 \quad 3.448 \mathrm{E}-11 \quad 1.009 \mathrm{E}-10 \quad 2.077 \mathrm{E}-10$ 1.935E-09 $\quad$ 1.613E-09 $0.000 \mathrm{E}+00$ 2.830E-18 2.239E-16 2.549E-14 1.647E-12 1.023E-11 3.992E-11 9.834E-11 $1.457 \mathrm{E}-09$ 4.035E-09

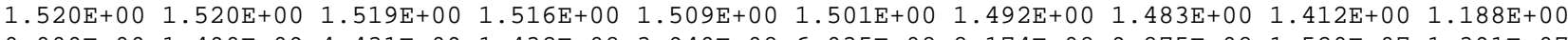
$0.000 \mathrm{E}+00$ 1.490E-09 4.431E-09 1.432E-08 3.940E-08 6.035E-08 8.174E-08 9.875E-08 $1.520 \mathrm{E}-07$ 1.391E-07 $\odot .000 \mathrm{E}+0 \odot$ 1.574E-14 $1.401 \mathrm{E}-13$ 1.494E-12 $1.198 \mathrm{E}-11 \quad 2.968 \mathrm{E}-11 \quad 5.805 \mathrm{E}-11 \quad 8.993 \mathrm{E}-11 \quad 2.977 \mathrm{E}-10 \quad 3.482 \mathrm{E}-10$ $0.00 \odot \mathrm{E}+0 \odot$ 1.651E-16 $4.304 \mathrm{E}-15$ 1.413E-13 $2.764 \mathrm{E}-12 \quad 9.524 \mathrm{E}-12 \quad 2.295 \mathrm{E}-11$ 4.000E-11 $1.697 \mathrm{E}-10 \quad 2.086 \mathrm{E}-10$

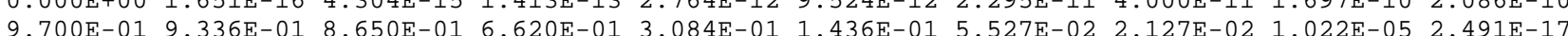

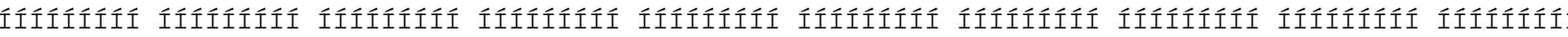
of the parent nuclide.

1.43 seconds 
Table of Contents

ÄÄÄÄÄÄÄÄÄÄÄÄÄÄÄÄÄ

Part I: Mixture Sums and Single Radionuclide Guidelines

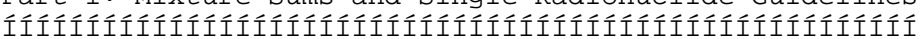

Dose Conversion Factor (and Related) Parameter Summary ... Site-Specific Parameter Summary $\ldots \ldots \ldots \ldots \ldots \ldots \ldots \ldots$ Summary of Pathway Selections $\ldots \ldots \ldots \ldots \ldots \ldots \ldots \ldots \ldots$ Contaminated Zone and Total Dose Summary

Total Dose Components

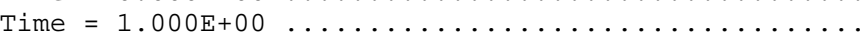

Time $=1.000 \mathrm{E}+01$

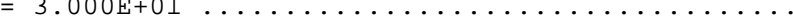

Time $=5.000 \mathrm{E}+01$

Time $=7.500 \mathrm{E}+01$

Time $=3.000 \mathrm{E}+02$

Time $=1,00 \ominus \mathrm{E}+03$

Single Radionuclide Soil Guidelines 
Dose Conversion Factor (and Related) Parameter Summary File: FGR 13 MORBIDITY

$\odot \quad 3$ Menu 3 Parameter

3 Current ${ }_{3}^{3}$ Base ${ }_{3}$ Parameter

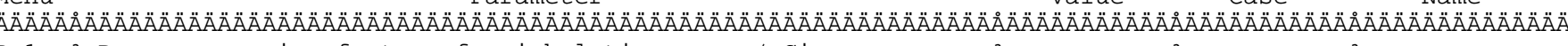
B-1 3 Dose conversion factors for inhalation, $\mathrm{mrem} / \mathrm{pCi}$

B-1 3 AC-227+D

$B-1 \quad 3$ Am-241

$B-1 \quad 3$ CS $-137+D$

$\mathrm{B}-1 \quad 3 \mathrm{~Np}-237+\mathrm{D}$

$\mathrm{B}-1 \quad 3 \mathrm{~Pa}-231$

$\mathrm{B}-1 \quad 3 \mathrm{~Pb}-210+\mathrm{D}$

B-1 3 Pu-238

$\mathrm{B}-1 \quad 3 \quad \mathrm{Pu}-239$

$\mathrm{B}-1 \quad 3 \mathrm{Ra}-226+\mathrm{D}$

B-1 3 Sr $-90+D$

B-1 3 Th-229+D

B-1 3 Th-230

B-1 3 U-233

$\mathrm{B}-1 \quad 3 \quad \mathrm{U}-234$

B-1 3 U-235+D

3

D-1 3 Dose conversion factors for ingestion, $\mathrm{mrem} / \mathrm{pCi}$ :

D-1 3 AC $-227+D$

D-1 3 Am-241

D-1 3 Cs $-137+D$

$\mathrm{D}-1 \quad 3 \mathrm{NP}-237+\mathrm{D}$

$\mathrm{D}-1$ (1 $3 \mathrm{~Np}-237+\mathrm{Pa}-231$

$\mathrm{D}-1 \quad 3 \mathrm{~Pb}-210+\mathrm{D}$

$\mathrm{D}-1 \quad 3 \mathrm{Pu}-238$

D-1 3 Pu-239

D-1 3 Ra-226+D

D-1 3 Sr $-90+D$

D-1 3 Th-229+D

D-1 3 Th -230

D-1 3 U -233

D $-1 \quad 3 \quad U-234$

D-1 3 U-235+D

3

D-34 3 Food transfer factors:

D-34 3 AC-227+D, plant/soil concentration ratio, dimensionless $\mathrm{D}-343 \mathrm{AC}-227+\mathrm{D}$, beef/livestock-intake ratio, $(\mathrm{pCi} / \mathrm{kg}) /(\mathrm{pCi} / \mathrm{d})$ $\mathrm{D}-34{ }^{3} \mathrm{AC}-227+\mathrm{D}$, milk/livestock-intake ratio, $(\mathrm{pCi} / \mathrm{L}) /(\mathrm{pCi} / \mathrm{d})$

D-34 3

D-34 3 Am-241, plant/soil concentration ratio, dimensionless

D-34 3 Am-241

$\mathrm{D}-34 \quad 3 \mathrm{Am}-241$

D-34 3

D-34 3 Cs-137+D, plant/soil concentration ratio, dimensionless $\mathrm{D}-34{ }^{3} \mathrm{Cs}-137+\mathrm{D}$, beef/livestock-intake ratio, $(\mathrm{pCi} / \mathrm{kg}) /(\mathrm{pCi} / \mathrm{d})$

D $-34 \quad 3$ CS $-137+D$

D-34 3

$\mathrm{D}-34 \quad 3 \mathrm{NP}-237+\mathrm{D}$, plant/soil concentration ratio, dimensionless

$\mathrm{D}-343^{3} \mathrm{~Np}-237+\mathrm{D}$, , beef/livestock-intake ratio, $(\mathrm{pCi} / \mathrm{kg}) /(\mathrm{pCi} / \mathrm{d})$

$\mathrm{D}-34$ $3 \mathrm{~Np}-237+\mathrm{D}$, milk/livestock-intake ratio, $(\mathrm{pCi} / \mathrm{L}) /(\mathrm{pCi} / \mathrm{d})$

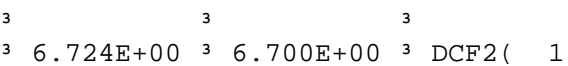

3 4.440E-01 3 4.440E-01 3 DCF2( 2$)$

$3.190 \mathrm{E}-05$ 3 $3.190 \mathrm{E}-05$ 3 DCF2( 3$)$

$5.400 E-01305,400 E-013$ DCF2 ( 4$)$

$1.280 E+000^{3} 1.280 E+00{ }^{3}$ DCF2 $(5)$

3 $2.320 \mathrm{E}-02$ 3 $1.360 \mathrm{E}-02$ 3 DCF2 $(6)$

3 3.920E-01 3 3.920E-01 3 DCF2( 7$)$

3 4.290E-01 3 4.290E-01 3 DCF2( 9)

3 8.594E-03 3 8.580E-03 3 DCF2( 10$)$

$1.308 \mathrm{E}-03$ 3 $1.300 \mathrm{E}-03$ 3 DCF2( 11$)$

$32.169 \mathrm{E}+00$ 3 $2.150 \mathrm{E}+00$ 3 DCF2( 12$)$

3 3.260E-01 3 3.260E-01 3 DCF2( 13 )

3 1.350E-01 3 1.350E-01 3 DCF2( 14$)$

3 1.320E-01 3 1.320E-01 3 DCF2( 15$)$

31.230 E-01 3 1.230E-01 3 DCF2( 16$)$

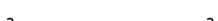

1.480E-02 3 1.410E-02 3 DCF3( 1$)$

$3.640 \mathrm{E}-03 \quad 3 \quad 3.640 \mathrm{E}-03$ 3 DCF3( 2$)$

$35.000 \mathrm{E}-05 \quad 3 \quad 5.000 \mathrm{E}-053 \mathrm{DCF}(3)$

$34.444 E-03 \quad 3 \quad 4.440 E-03$ 3 DCF3 ( 4$)$

1. $1.060 \mathrm{E}-02$ 3 $1.060 \mathrm{O}-02$ 3 $\mathrm{DCF} 3(5)$

$1.060 \mathrm{E}-02$ 3 $1.060 \mathrm{E}-02$ 3 DCF3( 5$)$

7.276E-03 $3.370 E-03$ aCF3( 6$)$

$3.200 \mathrm{E}-03$ 3 $3.200 \mathrm{E}-03$ 3 DCF3 ( 7)

3.540E-03 3 3.540E-03 3 DCF3(9)

3 1.321E-03 3 1.320E-03 3 DCF3( 10$)$

1.528E-04 3 1.420E-04 3 DCF3( 11$)$

3 4.027E-03 3 3.530E-03 3 DCF3( 12)

$35.480 \mathrm{E}-04 \quad 3 \quad 5.480 \mathrm{E}-04{ }^{3}$ DCF3( 13$)$

$32.890 E-04 \quad 3 \quad 2.890 E-04 \quad 3$ DCF3( 14$)$

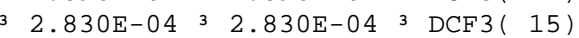

$2.673 E-0432.660 E-043$ DCF3( 16$)$

33

2.500E-03 $3 \quad 2.500 \mathrm{E}-03 \quad 3 \operatorname{RTF}(1,1)$

2. $000 \mathrm{E}-05$ 3 $2.000 \mathrm{E}-053 \mathrm{RTF}(1,2)$

2. $0000 \mathrm{E}-05 \quad 3 \quad 2.000 \mathrm{E}-053 \operatorname{RTF}(1,3)$

3

3 1. $000 \mathrm{E}-03 \quad 3 \quad 1.000 \mathrm{E}-03 \quad 3 \mathrm{RTF}(2,1)$

3 5. 000E-05 3 5. $000 \mathrm{E}-053 \mathrm{RTF}(2,2)$

3 2. $2000-06 \quad 3 \quad 2.000 \mathrm{E}-06$ 3 $3 \operatorname{RTF}(2,3)$

3 4. $000 \mathrm{E}-023$ 4.000E-02 $3 \operatorname{RTF}(3,1)$

3 3. $300 \mathrm{E}-02$ 3 $3.000 \mathrm{E}-02$ 3 RTF $(3,2)$

8.000E-03 3 8.000E-03 3 RTF( 3,3$)$

$3 \quad 3$

2.000E-02 $3 \quad 2.000 \mathrm{E}-023 \operatorname{RTF}(4,1)$

3 1. $000 \mathrm{E}-03$ 3 $1.000 \mathrm{E}-033 \operatorname{RTF}(4,2)$

$35.000 \mathrm{E}-06$ 3 $5.000 \mathrm{E}-063 \operatorname{RTF}(4,3)$ 
Dose Conversion Factor (and Related) Parameter Summary (continued)

File: FGR 13 MORBIDITY

${ }^{3}$ File: FGR 13 MORBIDITY

\section{Menu 3 Parameter}

3 Current ${ }_{3}^{3}$ Base ${ }_{3}$ Parameter

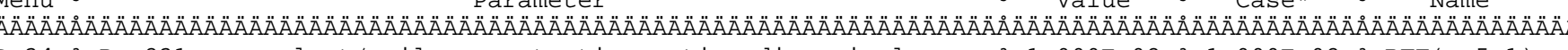

$\mathrm{D}-34{ }^{3} \mathrm{~Pa}-231$

$\mathrm{D}-34{ }^{3} \mathrm{~Pa}-231$

$\mathrm{D}-34{ }^{3} \mathrm{~Pa}-231$

$\mathrm{D}-34{ }^{3}$

$\mathrm{D}-343 \mathrm{~Pb}-210+\mathrm{D}$

plant/soil concentration ratio, dimensionless

3 1. $000 \mathrm{E}-0231.000 \mathrm{2}-023 \mathrm{RTF}(5,1)$

milk/livestock-intake ratio, $(\mathrm{pCi} / \mathrm{L}) /(\mathrm{pCi} / \mathrm{d})$

( $5.000 \mathrm{E}-03$ 3 RTF $(5,2)$

plant/soil concentration ratio, dimensionless beef/livestock-intake ratio, $(\mathrm{pCi} / \mathrm{kg}) /(\mathrm{pCi} / \mathrm{d})$

$\mathrm{D}-343^{3} \mathrm{~Pb}-210+\mathrm{D}$, milk/livestock-intake ratio, (pCi/L)/(pCi/d)

$\mathrm{D}-34{ }^{3}$

D-34 3 Pu-238

$\mathrm{D}-34 \quad 3 \mathrm{Pu}-238$

$\mathrm{D}-344^{3} \mathrm{Pu}-238$

D-34 3

$\mathrm{D}-344^{3} \mathrm{Pu}-239$

D-34 3 Pu -239

D-34 3 Pu-239

$\mathrm{D}-34 \mathrm{D}^{3} \mathrm{Pu}-239$

D-34 3 Ra-226+D

$\mathrm{D}-34{ }^{3} \mathrm{Ra}-226+\mathrm{D}$

$\mathrm{D}-34{ }^{3} \mathrm{Ra}-226+\mathrm{D}$

D-34 3

D-34 3 Sr $-90+D$

, plant/soil concentration ratio, dimensionless

, beef/livestock-intake ratio, $(\mathrm{pCi} / \mathrm{kg}) /(\mathrm{pCi} / \mathrm{d})$

, milk/livestock-intake ratio, $(\mathrm{pCi} / \mathrm{L}) /(\mathrm{pCi} / \mathrm{d})$

, plant/soil concentration ratio, dimensionless - beef/livestock-intake ratio, $(\mathrm{pCi} / \mathrm{kg}) /(\mathrm{pCi} / \mathrm{d})$

$\mathrm{milk} / \mathrm{livestock}$-intake ratio, $(\mathrm{pCi} / \mathrm{L}) /(\mathrm{pCi} / \mathrm{d})$

plant/soil concentration ratio, dimensionless beef/livestock-intake ratio, $(\mathrm{pCi} / \mathrm{kg}) /(\mathrm{pCi} / \mathrm{d})$ milk/livestock-intake ratio, $(\mathrm{pCi} / \mathrm{L}) /(\mathrm{pCi} / \mathrm{d})$

$D-343 \mathrm{Sr}-90+\mathrm{D}$

D $-343 \mathrm{Sr}-90+\mathrm{D}$

D-34 3

D-34 3 Th-229+D

D-34 3 Th-229+D

D-34 3 Th-229+D

D-34 3

D-34 3 Th-230

D-34 3 Th-230

D -34 - 34

$\mathrm{D}-34 \quad 3 \quad \mathrm{U}-233$

$\mathrm{D}-34$ 3 $\mathrm{U}-233$

$\mathrm{D}-34$ - 3 U-233

$\mathrm{D}-344^{3}$

$\mathrm{D}-34 \quad 3 \quad \mathrm{U}-234$

$\mathrm{D}-34 \quad 3 \quad \mathrm{U}-234$

D-34 3 U-234

D-34 3

$D-34 \quad 3 \quad U-235+D$

$\mathrm{D}-34 \quad 3 \quad \mathrm{U}-235+\mathrm{D}$

plant/soil concentration ratio, dimensionless beef/livestock-intake ratio, $(\mathrm{pCi} / \mathrm{kg}) /(\mathrm{pCi} / \mathrm{d})$

milk/livestock-intake ratio, $(\mathrm{pCi} / \mathrm{L}) /(\mathrm{pCi} / \mathrm{d})$

plant/soil concentration ratio, dimensionless beef/livestock-intake ratio, $(\mathrm{pCi} / \mathrm{kg}) /(\mathrm{pCi} / \mathrm{d})$ milk/livestock-intake ratio, $(\mathrm{pCi} / \mathrm{L}) /(\mathrm{pCi} / \mathrm{d})$

plant/soil concentration ratio, dimensionless beef/livestock-intake ratio, $(\mathrm{pCi} / \mathrm{kg}) /(\mathrm{pCi} / \mathrm{d})$

$\mathrm{milk} /$ livestock-intake ratio, $(\mathrm{pCi} / \mathrm{L}) /(\mathrm{pCi} / \mathrm{d})$

plant/soil concentration ratio, dimensionless beef/livestock-intake ratio, $(\mathrm{pCi} / \mathrm{kg}) /(\mathrm{pCi} / \mathrm{d})$ , milk/livestock-intake ratio, $(\mathrm{pCi} / \mathrm{L}) /(\mathrm{pCi} / \mathrm{d})$

plant/soil concentration ratio, dimensionless beef/livestock-intake ratio, $(\mathrm{pCi} / \mathrm{kg}) /(\mathrm{pCi} / \mathrm{d})$

$\mathrm{milk} /$ livestock-intake ratio, $(\mathrm{pCi} / \mathrm{L}) /(\mathrm{pCi} / \mathrm{d})$

plant/soil concentration ratio, dimensionless beef/livestock-intake ratio, $(\mathrm{pCi} / \mathrm{kg}) /(\mathrm{pCi} / \mathrm{d})$

, milk/livestock-intake ratio, $(\mathrm{pCi} / \mathrm{L}) /(\mathrm{pCi} / \mathrm{d})$

3 1. $000 \mathrm{E}-02 \quad 3 \quad 1.000 \mathrm{E}-023 \operatorname{RTF}(6,1)$

8. 000 - 04 3 8. $8000 \mathrm{E}-043 \mathrm{RTF}(6,2)$

3.

3 . 3 .

1.000E-03 3 1.000E-03 $3 \operatorname{RTF}(7,1)$

3 1. $000 \mathrm{E}-044^{3}$ 1. $000 \mathrm{E}-04{ }^{3} \operatorname{RTF}(7,2)$

1.000E-06 3 1.000E-06 3 RTF $(7,3)$

3 1. $000 \mathrm{E}-03 \quad 3 \quad 1.000 \mathrm{E}-03 \quad 3 \operatorname{RTF}(9,1)$

3 1. $000 \mathrm{E}-04$ 3 1.000E-04 3 RTF $(9,2)$

3 1. $1.00 \mathrm{E}-06$ 3 1. $1.00 \mathrm{E}-063 \operatorname{RTF}(9,3)$

33

4.000E-02 3 4. 4.000E-02 $3 \operatorname{RTF}(10,1)$

3 1. 000E-03 3 1.000E-03 $3 \operatorname{RTF}(10,2)$

1.000E-03 3 1.000E-03 $3 \operatorname{RTF}(10,3)$

3 3. $000 \mathrm{E}-013 \quad 3.000 \mathrm{1}-013 \operatorname{RTF}(11,1)$

8.000E-03 3 8.000E-03 3 RTF $(11,2)$

$2.000 \mathrm{E}-03 \quad 3 \quad 2.000 \mathrm{E}-03 \quad 3 \operatorname{RTF}(11,3)$

3 1. $000 \mathrm{E}-043$ 1. $3000 \mathrm{E}-043 \operatorname{RTF}(12,2)$

5.000E-06 3 5.000อE-06 3 RTF $(12,3)$

3 1. 000E-03 3 1.000E-03 $3 \operatorname{RTF}(13,1)$

$1.000 \mathrm{C}-043$ 1. $1.000 \mathrm{E}-043 \mathrm{RTF}(13,1)$

5. $000 \mathrm{E}-06 \quad 3 \quad 5.000 \mathrm{E}-06 \quad 3 \operatorname{RTF}(13,3)$

2.500E- $03 \quad 3 \quad 2.500 \mathrm{E}-03 \quad 3 \operatorname{RTF}(14,1)$

3.400E-๑4 3 $3.400 \mathrm{E}-043 \operatorname{RTF}(14,2)$

6.000E-04 3 6.000E-04 3 RTF( 14,3$)$

2.500E-๑3 $3 \quad 2.500 \mathrm{E}-03 \quad 3 \operatorname{RTF}(15,1)$

$3.400 E-04 \quad 3 \quad 3,400 E-04 \quad 3 \quad \operatorname{RTF}(15,2)$

6.000E-04 3 6.000E-04 $3 \operatorname{RTF}(15,3)$

3 2.500E-03 3 2.500E-03 $3 \operatorname{RTF}(16,1)$

3.400E-04 $33.400 \mathrm{E}-043 \operatorname{RTF}(16,2)$

6. ๑०९E-๑4 3 6. $6.00 E-\odot 43 \operatorname{RTF}(16,3)$

D-5 3 Bioaccumulation factors, fresh water, $\mathrm{L} / \mathrm{kg}$ :

D-5 3 AC-227+D fish

D-5 3 AC-227+D, crustacea and mollusks

$\begin{array}{lll}D-5 & 3 \\ D-5 & 3\end{array}$

$D-5$ - 35

D-5 3 Am-241

, crusta

D-5 3

, crustacea and mollusks

$1.500 \mathrm{E}+0131.500 \mathrm{E}+013 \operatorname{BIOFAC}(1,1)$

$1.000 \mathrm{E}+03 \quad 3 \quad 1.000 \mathrm{E}+03 \quad 3 \operatorname{BIOFAC}(1,2)$

3 3. $000 \mathrm{E}+01 \quad 3 \quad 3.000 \mathrm{E}+013 \operatorname{BIOFAC}(2,1)$

$1.000 \mathrm{E}+03$ 3 $1.000 \mathrm{E}+03$ 3 $\operatorname{BIOFAC}(2,2)$ 
Dose Conversion Factor (and Related) Parameter Summary (continued)

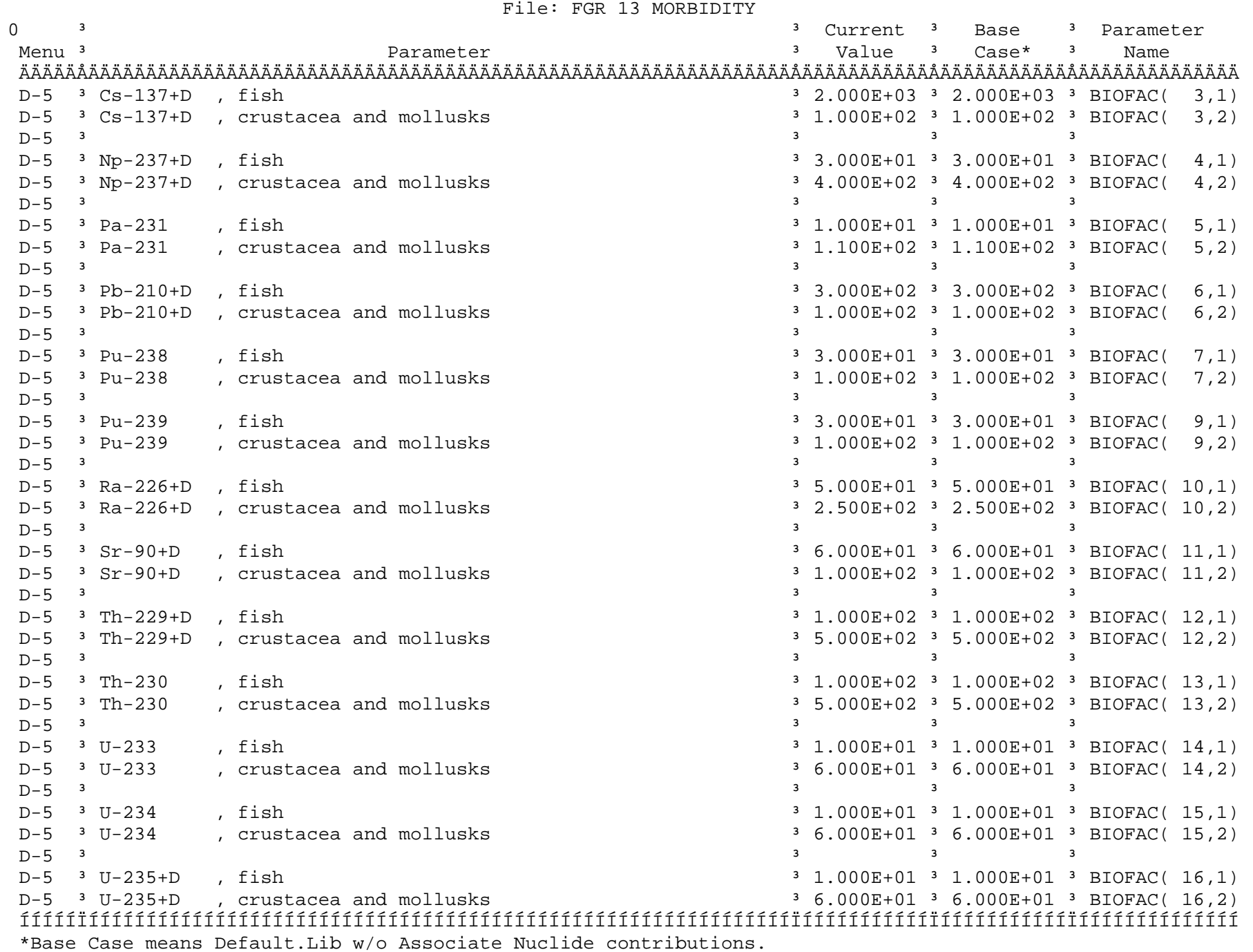


$\odot \quad 3$

Site-Specific Parameter Summary

$3 \quad$ Used by RESRAD

3 Parameter

Menu 3 Parameter 3 Input 3 Default 3 (If different from user input) 3 Name

R011 3 Area of contaminated zone $\left(\mathrm{m}^{* *} 2\right.$

R011 3 Thickness of contaminated zone $(\mathrm{m})$

R011 3 Length parallel to aquifer flow $(\mathrm{m})$

R011 3 Basic radiation dose limit (mrem/yr)

R011 3 Time since placement of material ( $\mathrm{yr}$ )

R011 3 Times for calculations ( $y r$ )

R011 3 Times for calculations ( $y r$ )

R011 3 Times for calculations ( $y r$ )

R011 3 Times for calculations (yr)

R011 3 Times for calculations ( $y r$ )

R011 3 Times for calculations (yr)

R011 3 Times for calculations ( $y r$ )

R011 3 Times for calculations ( $y r$ )

R011 3 Times for calculations ( $y r$ )

R012 3 Initial principal radionuclide ( $\mathrm{pCi} / \mathrm{g})$ : Am-241

R012 3 Initial principal radionuclide (pCi/g): $\mathrm{Cs}-137$

principal radionuclide (pCi/g):

R012 3 Initial principal radionuclide ( $\mathrm{pCi} / \mathrm{g})$ : $\mathrm{Pu}-239$

$1.000 \mathrm{E}+02 \quad 3 \quad 1.000 \mathrm{E}+\odot 4$

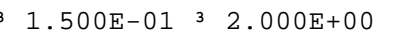

3 not used $31.000 \mathrm{E}+02$

$\begin{array}{llll}3 & 2.500 \mathrm{E}+01 & 3 & 3.000 \mathrm{E}+01 \\ 3\end{array}$

3 ०. $0.000 \mathrm{E}+00 \quad 3 \quad 0.000 \mathrm{E}+\odot \odot$

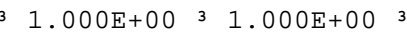

$3 \quad 3.000 \mathrm{E}+00 \quad 3 \quad 3.000 \mathrm{E}+00 \quad 3$

1. $000 \mathrm{E}+01$ 3 $1.000 \mathrm{E}+01$

$3.000 \mathrm{E}+01$ 3 3.000E+01

$5.000 \mathrm{E}+0131.000 \mathrm{E}+02$

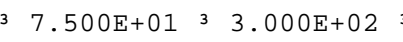

1.000E+02 3 1.000E+03

$3.000 \mathrm{E}+02 \quad 3 \quad 0.000 \mathrm{E}+00$

$1.000 \mathrm{E}+03$ 3 $0.000 \mathrm{E}+00$

7. 200E- $01 \quad 3 \quad 0.000 \mathrm{E}+0 \odot$

$9.100 \mathrm{E}+01 \quad 3 \quad 0.000 \mathrm{E}+00$

$3.610 \mathrm{E}-01 \quad 3 \quad 0.000 \mathrm{E}+0 \odot$

$4.490 \mathrm{E}+0 \odot \quad 3 \quad 0.000 \mathrm{E}+0 \odot$

$3.620 \mathrm{E}+00 \quad 3 \quad 0.000 \mathrm{E}+00$

R012 3 Concentration in groundwater (pCi/L): Am-241 3 not used $30.000 \mathrm{E}+00$

R012 3 Concentration in groundwater ( $\mathrm{pCi} / \mathrm{L}): \mathrm{Cs}-1373$ not used $30.000 \mathrm{E}+00$

(pCi/L): Pu-238 3 not used 3 $0.000 \mathrm{E}+00$

(PCi/L): Pu-230 3 not used 3 $0.000 E+00$

$\mathrm{R} 0123$ Concentration in groundwater (pCi/L): $\mathrm{Sr}-90$

R013 3 Cover depth (m)

R013 3 Density of cover material $\left(\mathrm{g} / \mathrm{cm}^{* *} 3\right)$

R013 3 Cover depth erosion rate (m/yr)

R013 3 Density of contaminated zone $\left(\mathrm{g} / \mathrm{cm}^{* *} 3\right)$

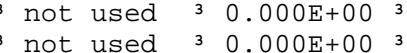

$0.000 \mathrm{E}+00 \quad 3 \quad 0.000 \mathrm{E}+00$

3 not used 3 1.500E+00

not used $31.000 \mathrm{E}-03$

$1.500 \mathrm{E}+00 \quad 3 \quad 1.500 \mathrm{E}+00$

$1.000 \mathrm{E}-03 \quad 3 \quad 1.000 \mathrm{E}-03$

4.000E-01 3 4.000E-01

2. $00 \odot \mathrm{E}-01 \quad 3 \quad 2.000 \mathrm{E}-01$

R013 3 Contaminated zone total porosity

R013 3 Contaminated zone field capacity

$1.000 \mathrm{E}+01$ 3 $1.000 \mathrm{E}+01$

$35.300 \mathrm{E}+00$ 3 $5.300 \mathrm{E}+00$

$3.400 \mathrm{E}+00$ 3 $2.000 \mathrm{E}+0 \odot$

not used 3 8.000E+00

3 5. 000E-01 3 5.000E-01

3 3. $260 \mathrm{E}-01$ 3 $1.000 \mathrm{E}+00$

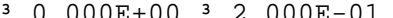

3 overhead 3 .

3. $4.000 \mathrm{C}-01$ 3 $2.000 \mathrm{E}-01$

3 not used $31.000 \mathrm{E}+06$

not used 31 1.000E-03

3 not used $31.500 \mathrm{E}+00^{3}$

not used $3 \quad 4.000 \mathrm{E}-01$

not used $32.000 \mathrm{E}-01$

not used $32.000 \mathrm{C}-01$

not used $32.000 \mathrm{E}-01$

$\begin{array}{ll}3 \text { not used } & 1.000 \mathrm{E}+\odot 2 \\ \text { not } & 2.000 \mathrm{E}-02\end{array}$

\begin{tabular}{|c|c|c|}
\hline$-\ldots$ & 3 & AREA \\
\hline - - - & 3 & THICKO \\
\hline - - - & 3 & LCZPAQ \\
\hline - . - & 3 & BRDL \\
\hline - - - & 3 & TI \\
\hline - - - & 3 & $\mathrm{~T}(\mathrm{2})$ \\
\hline - - - & 3 & $\mathrm{~T}(3)$ \\
\hline - - - & 3 & $\mathrm{~T}(4)$ \\
\hline - - - & 3 & $\mathrm{~T}(5)$ \\
\hline - - - & 3 & $\mathrm{~T}(6)$ \\
\hline-- & 3 & $\mathrm{~T}(7)$ \\
\hline-- & 3 & $\mathrm{~T}(8)$ \\
\hline - & 3 & $\mathrm{~T}(9)$ \\
\hline - - - & $\begin{array}{l}3 \\
3\end{array}$ & $\mathrm{~T}(10)$ \\
\hline - - - & 3 & $\mathrm{~S} 1(2)$ \\
\hline - - - & 3 & $\mathrm{~S} 1(3)$ \\
\hline - - - & 3 & S1( 7$)$ \\
\hline - - - & 3 & $\mathrm{~S} 1(9)$ \\
\hline - - - & 3 & S1(11) \\
\hline - - - & 3 & W1( 2) \\
\hline - - - & 3 & W1( 3$)$ \\
\hline - - - & 3 & W1 ( 7) \\
\hline-- & 3 & W1( 9) \\
\hline - - - & $\begin{array}{l}3 \\
3\end{array}$ & W1(11) \\
\hline - . - & 3 & COVER \\
\hline - - & 3 & DENSCV \\
\hline - - - & 3 & VCV \\
\hline - - - & 3 & DENSCZ \\
\hline - - - & 3 & VCZ \\
\hline - - & 3 & TPCZ \\
\hline - - - & 3 & FCCZ \\
\hline - - - & 3 & $\mathrm{HCCZ}$ \\
\hline- & 3 & $\mathrm{BCZ}$ \\
\hline- & 3 & WIND \\
\hline - - & 3 & HUMID \\
\hline - - & 3 & EVAPTR \\
\hline- & 3 & PRECIP \\
\hline- & 3 & RI \\
\hline - - & 3 & IDITCH \\
\hline - - & 3 & RUNOFF \\
\hline- & 3 & WAREA \\
\hline- & $\begin{array}{l}3 \\
3\end{array}$ & EPS \\
\hline- & 3 & DENSAQ \\
\hline 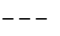 & 3 & TPSZ \\
\hline & 3 & EPSZ \\
\hline- & 3 & FCSZ \\
\hline- & 3 & HCSZ \\
\hline & 3 & HGWT \\
\hline
\end{tabular}

R014 3 Saturated zone hydraulic conductivity (m/yr)

R014 3 Saturated zone hydraulic gradient 
$\odot \quad 3$

Site-Specific Parameter Summary (continued)

Menu 3 Parameter $\quad 3$ Input 3 Default 3 (If different from user input) 3 Name

$$
3 \text { User }
$$

Menu 3 Parameter 3 Input 3 Default 3 (If different from user input) 3 Name

Menu 3 Parameter 3 Input 3 Default 3 (If different from user input) 3 Name

Menu 3 Parameter 3 Us Input 3 Default 3 (If different from user input) 3 Par Name

Used by RESRAD

3 Parameter

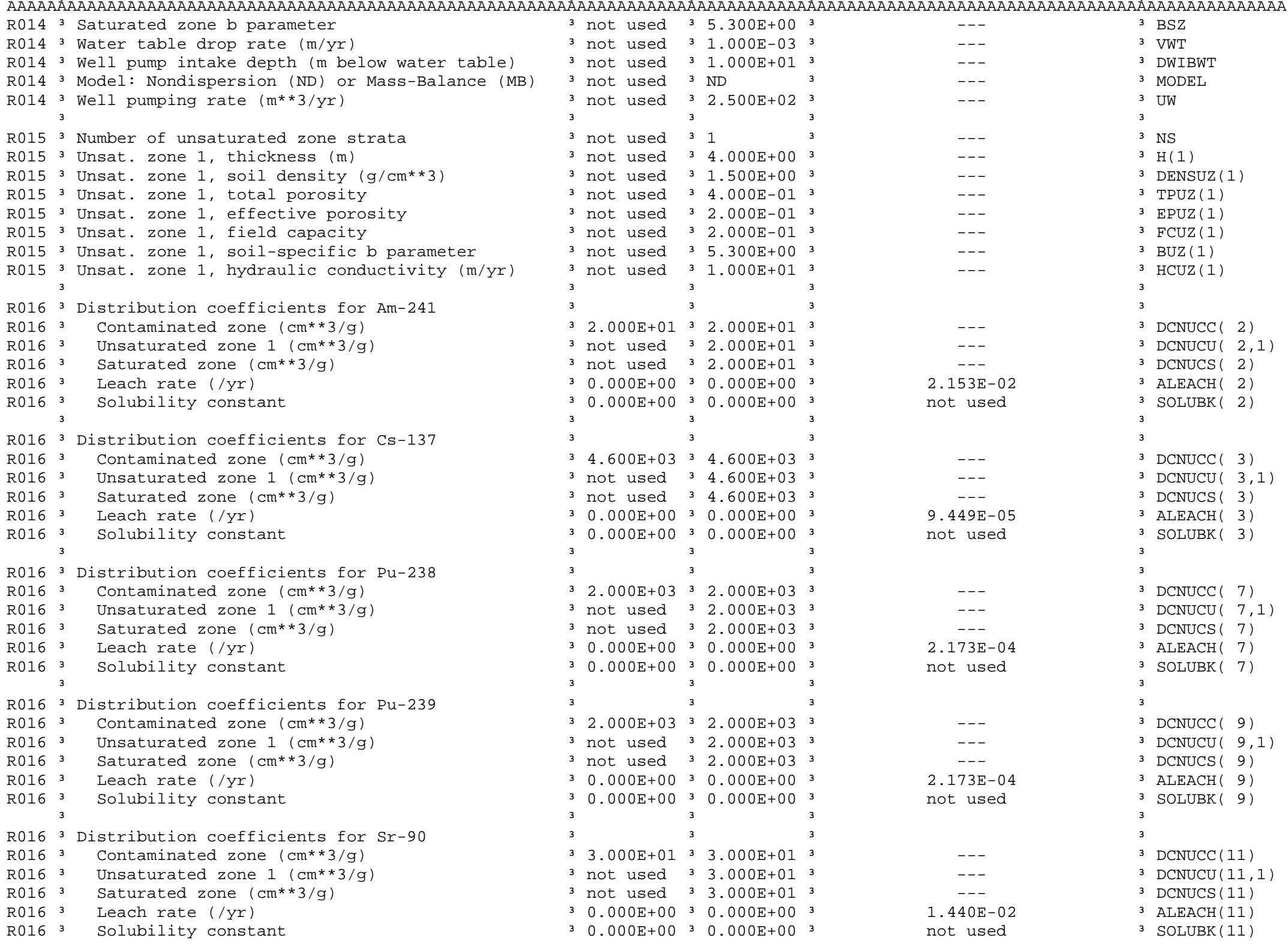


$\odot \quad 3$

Site-Specific Parameter Summary (continued)

Menu 3

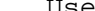

3 User

Default

Used by RESRAD

3 Parameter

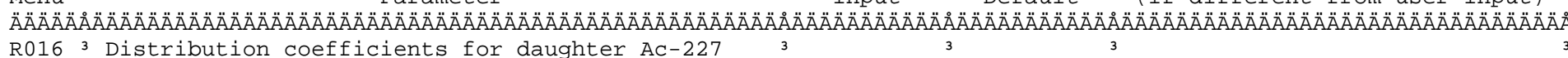

R016

R016 Contaminated zone $\left(\mathrm{cm}^{* *} 3 / \mathrm{g}\right)$

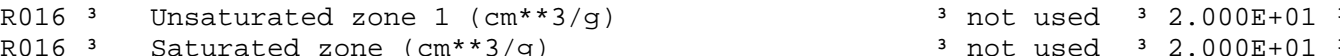

R016 3 Saturated zone $\left(\mathrm{cm}^{* *} 3 / \mathrm{g}\right)$

2. $000 \mathrm{E}+01 \quad 3 \quad 2.000 \mathrm{E}+01$

R016 3 Leach rate (/yr)

R016 3 Solubility constant

R016 3 Distribution coefficients for daughter Np-237

R016 3 Contaminated zone $\left(\mathrm{cm}^{* *} 3 / \mathrm{g}\right)$

R016 3 Unsaturated zone $1\left(\mathrm{~cm}^{* *} 3 / \mathrm{g}\right)$

R016 3 Saturated zone $\left(\mathrm{cm}^{* *} 3 / \mathrm{g}\right)$

R016 3 Leach rate (/yr)

R016 3 Solubility constant

R016 3 Distribution coefficients for daughter Pa-231

R016 3 Contaminated zone $\left(\mathrm{cm}^{* *} 3 / \mathrm{g}\right)$

R016 3 Unsaturated zone $1\left(\mathrm{~cm}^{* *} 3 / \mathrm{g}\right)$

RO16 3 Saturated zone $\left(\mathrm{cm}^{* *} 3 / \mathrm{g}\right)$

$\mathrm{R} 016$ Leach rate (/yr)

R016 3 Solubility constant

R016 3 Distribution coefficients for daughter Pb-210

R016 3 Contaminated zone $\left(\mathrm{cm}^{* *} 3 / \mathrm{g}\right)$

R016 3 Unsaturated zone $1\left(\mathrm{~cm}^{* *} 3 / \mathrm{g}\right)$

R016 3 Unsaturated zone $1\left(\mathrm{~cm}^{* *} 3 / \mathrm{g}\right.$
$\mathrm{R} 016 \quad 3 \quad$ Saturated zone $\left(\mathrm{cm}^{* *} 3 / \mathrm{g}\right)$

R016 3 Leach rate (/yr)

R016 3 Solubility constant

R016 3 Distribution coefficients for daughter Ra-226

R016 3 Contaminated zone $\left(\mathrm{cm}^{* *} 3 / \mathrm{g}\right)$

R016 3 Unsaturated zone $1\left(\mathrm{~cm}^{* *} 3 / \mathrm{g}\right)$

R016 3 Saturated zone $\left(\mathrm{cm}^{* *} 3 / \mathrm{g}\right)$

R016 3 Leach rate (/yr)

R016 3 Solubility constant

R016 3 Distribution coefficients for daughter Th-229

R016 3 Contaminated zone $\left(\mathrm{cm}^{* *} 3 / \mathrm{g}\right)$

R016 3 Unsaturated zone $1\left(\mathrm{~cm}^{* *} 3 / \mathrm{g}\right)$

R016 3 Saturated zone $\left(\mathrm{cm}^{* *} 3 / \mathrm{g}\right)$

R016 3 Leach rate (/yr)

R016 3 Solubility constant

R016 3 Distribution coefficients for daughter Th-230

R016 3 Contaminated zone $\left(\mathrm{cm}^{* *} 3 / \mathrm{g}\right)$

R016 3 Unsaturated zone $1\left(\mathrm{~cm}^{* *} 3 / \mathrm{g}\right)$

R016 3 Saturated zone $\left(\mathrm{cm}^{* *} 3 / \mathrm{g}\right)$

R016 3 Leach rate (/yr)

not used $32.000 \mathrm{E}+01$

$0.000 \mathrm{E}+00 \quad 3 \quad 0.000 \mathrm{E}+00$

$0.000 \mathrm{E}+0 \Theta^{3} \quad 0.000 \mathrm{E}+0 \Theta^{3}$

$3-1.000 \mathrm{E}+0 \odot^{3}-1.000 \mathrm{E}+0 \odot^{3}$

not used $3-1.000 \mathrm{E}+0 \odot^{3}$

3 not used $3-1.000 \mathrm{E}+00^{3}$

$0.000 \mathrm{E}+00 \quad 3 \quad 0.000 \mathrm{E}+00$

$0.000 \mathrm{E}+0 \odot{ }^{3} \quad 0.000 \mathrm{E}+0 \odot^{3}$

3 5. $000 \mathrm{E}+013 \quad 5.000 \mathrm{E}+01$

not used $3 \quad 5.000 \mathrm{E}+01$

not used $35.000 \mathrm{E}+01$

๑.

$\odot .000 \mathrm{E}+0 \odot{ }^{3} \quad 0.000 \mathrm{E}+\odot \odot$

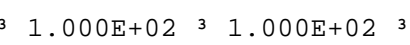

3 not used $31.000 \mathrm{E}+02$

not used $31.000 \mathrm{E}+02$

$\odot .00 \odot \mathrm{E}+\odot \odot$ з $\odot .00 \odot \mathrm{E}+\odot \odot{ }^{3}$

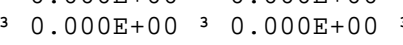

$37.000 \mathrm{E}+01 \quad 3 \quad 7.000 \mathrm{E}+01$

3 not used 3 7.000E+01 3

3 not used $37.000 \mathrm{E}+013$

$\begin{array}{llll}3 & 0.000 \mathrm{E}+0 \odot & 3 & 0.000 \mathrm{E}+\odot \odot \quad 3\end{array}$

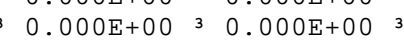

3 $6.000 \mathrm{E}+04 \quad 3 \quad 6.000 \mathrm{E}+04$

3 not used 3 6.000E +04

3 not used 3 6. $000 \mathrm{E}+04$

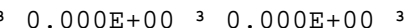

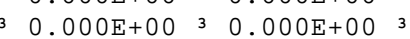

$6.000 \mathrm{E}+04 \quad 3 \quad 6.0000 \mathrm{E}+04$

3 not used 3 6.000E+04 3

3 not used 3 6.000E+04

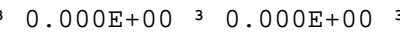

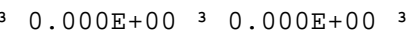

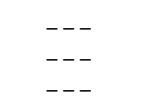

$2.153 \mathrm{E}-02$

not used

2. $574 \mathrm{E}+\odot 2$

-.-

$1.687 \mathrm{E}-03$

not used

$--$

$--$

8. $660 \mathrm{E}-03$

not used

$--$

$-\cdot$

4. $338 \mathrm{E}-03$

not used

$-$

$-$

$6.193 \mathrm{E}-03$

not used

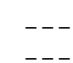

$-$

$7.244 \mathrm{E}-06$

not used

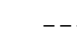

$-$

$--$

$.244 \mathrm{E}-06$

not used
$\operatorname{DCNUCC}(1)$

$\operatorname{DCNUCU}(1,1)$

DCNUCS $(1)$

SOLUBK( 1$)$

DCNUCC ( 4$)$

DCNUCU ( 4,1)

DCNUCS( 4 )

ALEACH ( 4$)$

SOLUBK( 4$)$

DCNUCC ( 5)

$\operatorname{DCNUCU}(5,1)$

DCNUCS( 5 )

ALEACH ( 5)

$\operatorname{DCNUCC}(6)$

$\operatorname{DCNUCU}(6,1)$

DCNUCS( 6)

ALEACH ( 6)

SOLUBK( 6 )

$\operatorname{DCNUCC}(10)$

$\operatorname{DCNUCU}(10,1)$

DCNUCS $(10)$

ALEACH $(10)$

SOLUBK (10)

3 DCNUCC (12)

$\operatorname{DCNUCU}(12,1)$

$\operatorname{DCNUCS}(12)$

ALEACH (12)

SOLUBK(12)

$\operatorname{DCNUCC}(13)$

$\operatorname{DCNUCU}(13,1)$

DCNUCS $(13)$

3 SOLUBK(13) 
Site-Specific Parameter Summary (continued)$$
\text { User }
$$

3 Default

3

Used by RESRAD

Parameter

3 User

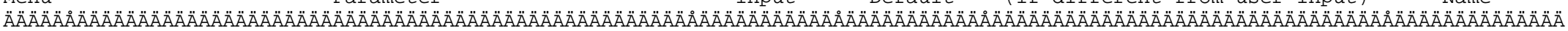

If different from user input)

Parameter

R016 3 Distribution coefficients for daughter U-233

R016 3 Contaminated zone $\left(\mathrm{cm}^{* *} 3 / \mathrm{g}\right)$

R016 3 Unsaturated zone $1\left(\mathrm{~cm}^{* *} 3 / \mathrm{g}\right)$

R016 3 Saturated zone $\left(\mathrm{cm}^{* *} 3 / \mathrm{g}\right)$

R016 3 Leach rate (/yr)

R016 3 Solubility constant

R016 3 Distribution coefficients for daughter U-234

R016 3 Contaminated zone $\left(\mathrm{cm}^{* *} 3 / \mathrm{g}\right)$

R016 3 Unsaturated zone $1\left(\mathrm{~cm}^{* *} 3 / \mathrm{g}\right)$

R016 3 Saturated zone $\left(\mathrm{cm}^{* *} 3 / \mathrm{g}\right)$

R016 3 Leach rate (/yr)

R016 ${ }^{3} \quad$ Solubility constant

R016 3 Distribution coefficients for daughter U-235

R016 3 Contaminated zone $\left(\mathrm{cm}^{* *} 3 / \mathrm{g}\right)$

R016 3 Unsaturated zone $1\left(\mathrm{~cm}^{* *} 3 / \mathrm{g}\right)$

RO16 3 Saturated zone $\left(\mathrm{cm}^{* *} 3 / \mathrm{g}\right)$

R016 3 Leach rate (/yr)

R016 3 Solubility constant

R017 3 Inhalation rate $\left(m^{* *} 3 / \mathrm{yr}\right)$

R017 3 Mass loading for inhalation $(\mathrm{g} / \mathrm{m} * * 3)$

R017 3 Exposure duration

R017 3 Shielding factor, inhalation

R017 3 Shielding factor, external gamma

R017 3 Fraction of time spent indoors

R017 3 Fraction of time spent outdoors (on site)

R017 3 Shape factor flag, external gamma

R017 3 Radii of shape factor array (used if FS = -1):

R017 3 Outer annular radius $(\mathrm{m})$, ring 1 :

$\mathrm{R} 017^{3}$ Outer annular radius $(\mathrm{m})$, ring 2 :

R017 3 Outer annular radius $(\mathrm{m})$, ring 3 :

$\mathrm{R} 017^{3}$ Outer annular radius $(\mathrm{m})$, ring 4 :

$\mathrm{R} 017^{3}$ Outer annular radius $(\mathrm{m})$, ring 5:

$\begin{array}{llll}\mathrm{R} 017 & 3 & \text { Outer annular radius }(\mathrm{m}) \text {, ring } 6 \text { : } \\ \mathrm{R} 017 & 3 & \text { Outer annular radius }(\mathrm{m}) \text {, ring } & 7 \text { : }\end{array}$

R017 3 Outer annular radius $(\mathrm{m})$, ring 8:

$\mathrm{R} 017$ Outer annular radius $(\mathrm{m})$, ring 9:

R017 3 Outer annular radius (m), ring 10:

$\mathrm{R} 017^{3}$ Outer annular radius (m), ring 11:

$\mathrm{R} 0173$ Outer annular radius $(\mathrm{m})$, r ring 12 :

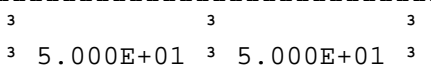

3 not used $35.000 \mathrm{E}+013$

3 not used $35.000 \mathrm{E}+01^{3}$

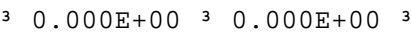

$30.000 \mathrm{E}+00^{3} \quad 0.000 \mathrm{E}+\Theta \Theta^{3}$

5. $000 \mathrm{E}+01 \quad 3 \quad 5.000 \mathrm{E}+01$

not used $35.000 \mathrm{E}+01$

not used $35.000 \mathrm{E}+01$

3 $0.000 \mathrm{E}+00 \quad 3 \quad 0.000 \mathrm{E}+00^{3}$

$0.000 E+00 \quad 3 \quad 0.000 E+00 \quad 3$

$5.000 \mathrm{E}+01 \quad 3 \quad 5.000 \mathrm{E}+01$

not used $35.00 \odot E+01$

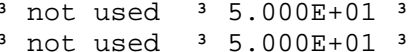

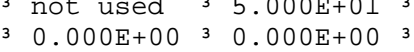

$\odot .000 \mathrm{E}+0 \odot \quad 3 \quad 0.000 \mathrm{E}+\odot \odot$

3 $1.230 \mathrm{E}+04 \quad 3 \quad 8.400 \mathrm{E}+033$

6.000E-04 3 1.000E-04 3

$2.500 \mathrm{E}+01 \quad 3 \quad 3.000 \mathrm{E}+01$

$1.000 \mathrm{E}+00$ 3 4.000E-01

$1.000 \mathrm{E}+0 \odot$ 3 $7.000 \mathrm{E}-01$

$3 \quad 0.000 \mathrm{E}+003$ 5. $3.000 \mathrm{E}-013$

3 3.800E-02 3 2.500E-01

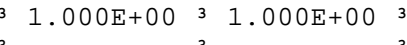

3

not used 3 5.000E+01

3 not used $37.071 \mathrm{E}+013$

3 not used $3 \quad 0.000 \mathrm{E}+0 \Theta^{3}$

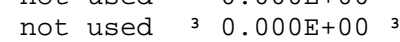

3 not used $30.000 \mathrm{E}+0{ }^{3}$

not used 3 ०.000E+००

3 not used $30.000 \mathrm{E}+0 \odot$

3 not used $30.000 \mathrm{E}+00^{3}$

3 not used $30.000 \mathrm{E}+0 \Theta^{3}$

3 not used $30.000 \mathrm{E}+0 \odot^{3}$

3 not used $3 \quad 0.000 \mathrm{E}+\odot \odot^{3}$

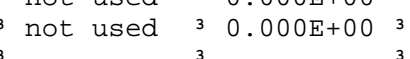

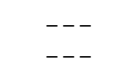

$--$

$8.660 \mathrm{E}-03$

not used

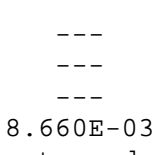

not used

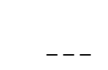

$-$

8. $660 \mathrm{E}-03$

not used

- - -

$--$

$-\cdot$

$--$

- - -

$>0$ shows circular AREA.

$-\cdot$

$-\cdot$

$---$

$--$

$--$

$---$

$--$

$--$

$--$
DCNUCC $(14)$

DCNUCS $(14)$

SOLUBK(14)

$\operatorname{DCNUCC}(15)$

$\operatorname{DCNUCU}(15,1)$

DCNUCS (15)

ALEACH (15)

SOLUBK(15)

DCNUCC (16)

$\operatorname{DCNUCU}(16,1)$

$\operatorname{DCNUCS}(16)$

ALEACH (16)

3 SOLUBK(16)

INHALR

MLINH

ED

SHF3

SHF1

FIND

FOTD

3 FS

RAD_SHAPE( 1$)$

RAD_SHAPE ( 2)

RAD_SHAPE( 3 )

RAD_SHAPE ( 4$)$

RAD_SHAPE ( 5$)$

RAD_SHAPE ( 6$)$

RAD_SHAPE( 7 )

RAD_SHAPE ( 8)

RAD_SHAPE (10)

3 RAD_SHAPE $(12)$
$\operatorname{DCNUCU}(14,1)$

RAD_SHAPE ( 9$)$ 
$\odot \quad 3$

Site-Specific Parameter Summary (continued)

Menu 3 Parameter 3 Input 3 Default 3 (If different from user input) 3 Name

User

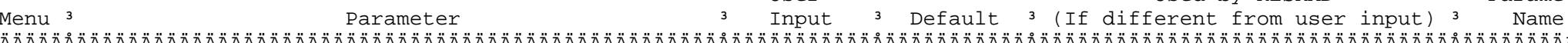

Used by RESRAD

3 Parameter

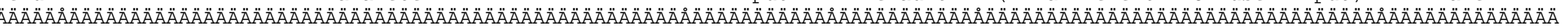
R017 3 Fractions of annular areas within AREA:

$\begin{array}{rrrr}\mathrm{R} 017 & 3 & \text { Ring } & 1 \\ \mathrm{R} 017 & 3 & \text { Ring } & 2 \\ \mathrm{R} 017 & 3 & \text { Ring } & 3 \\ \mathrm{R} 017 & 3 & \text { Ring } & 4 \\ \mathrm{R} 017 & 3 & \text { Ring } & 5 \\ \mathrm{R} 017 & 3 & \text { Ring } & 6 \\ \mathrm{R} 017 & 3 & \text { Ring } & 7 \\ \mathrm{R} 017 & 3 & \text { Ring } & 8 \\ \mathrm{R} 017 & 3 & \text { Ring } & 9 \\ \mathrm{R} 017 & 3 & \text { Ring } & 10 \\ \mathrm{R} 017 & 3 & \text { Ring } & 11 \\ \mathrm{R} 017 & 3 & \text { Ring } & 12\end{array}$

R018 3 Fruits, vegetables and grain consumption (kg/yr)

R018 3 Leafy vegetable consumption ( $\mathrm{kg} / \mathrm{yr}$ )

R018 3 Milk consumption ( $\mathrm{L} / \mathrm{yr}$ )

R018 3 Meat and poultry consumption ( $\mathrm{kg} / \mathrm{yr}$ )

R018 3 Fish consumption ( $\mathrm{kg} / \mathrm{yr}$ )

R018 3 Other seafood consumption ( $\mathrm{kg} / \mathrm{yr}$ )

R018 3 Soil ingestion rate (g/yr)

R018 3 Drinking water intake (L/yr)

R018 3 Contamination fraction of drinking water

R018 3 Contamination fraction of household water

R018 3 Contamination fraction of livestock water

R018 3 Contamination fraction of irrigation water

R018 3 Contamination fraction of aquatic food

R018 3 Contamination fraction of plant food

R018 3 Contamination fraction of meat

R018 3 Contamination fraction of milk

R019 3 Livestock fodder intake for meat ( $\mathrm{kg} /$ day)

R019 3 Livestock fodder intake for milk ( $\mathrm{kg} /$ day)

R019 3 Livestock water intake for meat (L/day)

R019 3 Livestock water intake for milk (L/day)

R019 3 Livestock soil intake ( $\mathrm{kg} /$ day)

R019 3 Mass loading for foliar deposition $(\mathrm{g} / \mathrm{m} * * 3)$

R019 3 Depth of soil mixing layer $(\mathrm{m})$

R019 3 Depth of roots $(\mathrm{m})$

R019 3 Drinking water fraction from ground water

R019 3 Household water fraction from ground water R019 3 Livestock water fraction from ground water R019 3 Irrigation fraction from ground water 3

R19B 3 Wet weight crop yield for Non-Leafy $\left(\mathrm{kg} / \mathrm{m}^{* *} 2\right)$

R19B 3 Wet weight crop yield for Leafy $\left(\mathrm{kg} / \mathrm{m}^{* *} 2\right)$

R19B 3 wet weight crop yield for Fodder

R19B 3 Growing Season for Non-Leafy (years)

R19B 3 Growing Season for Leafy (years)

R19B 3 Growing Season for Fodder (years)

R19B 3 Translocation Factor for Non-Leafy not used $31.000 \mathrm{E}+00$

3 not used $32.732 \mathrm{E}-01$

3 not used $30.000 \mathrm{E}+0 \odot$

3 not used $30.000 \mathrm{E}+\odot \Theta^{3}$

3 not used $30.000 \mathrm{E}+\Theta \Theta^{3}$

3 not used $30.000 \mathrm{E}+0 \odot^{3}$

3 not used $30.000 \mathrm{E}+00^{3}$

not used $30.00 \odot \mathrm{E}+00^{3}$

3 not used $30.000 \mathrm{E}+\odot \odot$

3 not used $30.000 \mathrm{E}+\odot \odot$

3 not used $30.000 \mathrm{E}+\odot \odot$ 3

\begin{tabular}{|c|c|c|}
\hline- & 3 & $\operatorname{FRACA}(1$ \\
\hline-- & 3 & FRACA $(2$ \\
\hline-- & 3 & FRACA ( \\
\hline-- & 3 & FRACA ( \\
\hline - - - & 3 & FRACA $(5$ \\
\hline$\cdots$ & 3 & FRACA $(6$ \\
\hline$\ldots$ & 3 & FRACA $(7$ \\
\hline- & 3 & FRACA ( 8 \\
\hline- & 3 & FRACA $(9$ \\
\hline - & 3 & FRACA ( 10 \\
\hline & 3 & FRACA (11 \\
\hline - & $\begin{array}{l}3 \\
3\end{array}$ & FRACA (12 \\
\hline- & 3 & $\operatorname{DIET}(1)$ \\
\hline- & 3 & DIET (2) \\
\hline- & 3 & $\operatorname{DIET}(3)$ \\
\hline - & 3 & DIET (4) \\
\hline- & 3 & DIET (5) \\
\hline 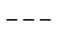 & 3 & $\operatorname{DIET}(6)$ \\
\hline- & 3 & SOIL \\
\hline - & 3 & DWI \\
\hline - & 3 & FDW \\
\hline - & 3 & FHHW \\
\hline & 3 & FLW \\
\hline- & 3 & FIRW \\
\hline-- & 3 & FR9 \\
\hline- & 3 & FPLANT \\
\hline- & 3 & FMEAT \\
\hline-- & 3 & FMILK \\
\hline & 3 & \\
\hline- & 3 & LFI5 \\
\hline- & 3 & LFI6 \\
\hline & 3 & LWI5 \\
\hline & 3 & LWI6 \\
\hline- & 3 & LSI \\
\hline- & 3 & MLFD \\
\hline & 3 & DM \\
\hline- & 3 & DROOT \\
\hline - & 3 & FGWDW \\
\hline & 3 & FGWHH \\
\hline & 3 & FGWLW \\
\hline- & 3 & FGWIR \\
\hline & 3 & \\
\hline-- & 3 & $Y V(1)$ \\
\hline & 3 & YV (2) \\
\hline & 3 & $Y V(3)$ \\
\hline- & 3 & $\operatorname{TE}(1)$ \\
\hline & 3 & TE (2) \\
\hline & 3 & TE(3) \\
\hline & 3 & $\operatorname{TIV}(1)$ \\
\hline
\end{tabular}


Default

Used by RESRAD 3 Parameter R19B 3 rran

Translocation Factor for Leafy

R19B 3 Translocation Factor for Fodder 3 not used $31.000 \mathrm{E}+00$

R19B 3 Dry Foliar Interception Fraction for Non-Leafy 3 not used $32.500 \mathrm{E}-01^{3}$

R19B 3 Dry Foliar Interception Fraction for Leafy 3 not used $32.500 \mathrm{E}-01$

R19B 3 Dry Foliar Interception Fraction for Fodder 3 not used $32.500 \mathrm{E}-01$

R19B 3 Wet Foliar Interception Fraction for Non-Leafy 3 not used $32.500 \mathrm{E}-01^{3}$

R19B 3 Wet Foliar Interception Fraction for Leafy 3 not used $32.500 \mathrm{E}-01^{3}$

$\mathrm{R} 19 \mathrm{~B} 3$ Wet Foliar Interception Fraction for Fodder 3 not used $32.500 \mathrm{E}-01^{3}$

R19B ${ }^{3}$ Weathering Renoval Constant for Vegetation

not used $32.000 \mathrm{E}+01$ 3

C14 3 C-12 concentration in water $(\mathrm{g} / \mathrm{cm} * * 3)$

C14 3 C-12 concentration in contaminated soil $(\mathrm{g} / \mathrm{g})$

C14 3 Fraction of vegetation carbon from soil

C14 3 Fraction of vegetation carbon from air

C14 3 C-14 evasion layer thickness in soil (m)

C14 3 C-14 evasion flux rate from soil ( $1 / \mathrm{sec})$

C14 3 C-12 evasion flux rate from soil (1/sec)

C14 3 Fraction of grain in beef cattle fee

C14 3 Fraction of grain in milk cow feed

DCF correction factor for gaseous forms of C14 3

STOR 3 Storage times of contaminated foodstuffs (days): STOR 3 Fruits, non-leafy vegetables, and grain $\begin{array}{lllll}3 & 1.400 \mathrm{E}+01 & 3 & 1.400 \mathrm{E}+01 & 3\end{array}$

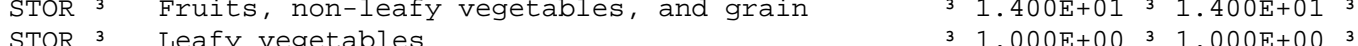

STOR 3 Leafy vegetables

STOR 3 Milk

STOR 3 Meat and poultry

STOR ${ }^{3}$ Fish

STOR 3 Crustacea and mollusks

3 not used 32 2.000E- 05

3 not used 3 3. $3000 \mathrm{E}-02$

not used $32.000 \mathrm{E}-02$

not used 33 . $300 \mathrm{E}-01$

not used 3 7.000E- -07

not used 3 1.000E-10

not used $38.000 \mathrm{E}-01$

not used $32.000 \mathrm{E}-01$

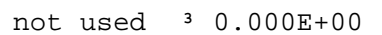

$1.000 \mathrm{E}+003 \quad 1.000 \mathrm{E}+003$

$2.000 \mathrm{E}+011^{3} 2.000 \mathrm{E}+01$

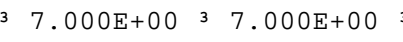

$7.000 \mathrm{E}+00$ 3 $7.000 \mathrm{E}+00$

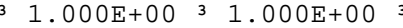

3 1. $000 \mathrm{E}+00 \quad 3 \quad 1.000 \mathrm{E}+00$

$4.500 \mathrm{E}+01 \quad 3 \quad 4.500 \mathrm{E}+013$

STOR 3 Surface water

STOR 3 Livestock fodder

R021 3 Thickness of building foundation $(\mathrm{m})$

RO21 3 Bulk density of building foundation $\left(\mathrm{g} / \mathrm{cm}^{* *} 3\right)$

R021 3 Total porosity of the cover material

3 not used 3 (.500E- $01{ }^{3}$

not used ${ }^{3} 1.500 \mathrm{E}-01$

not used $32.400 \mathrm{E}+\odot \odot$

3 not used 3 4.000E- 01

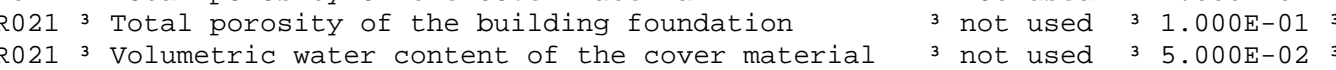

R021 3 Volumetric water content of the foundation 3 not used $33.000 \mathrm{E}-02^{3}$

R021 3 Diffusion coefficient for radon gas $(\mathrm{m} / \mathrm{sec})$ :

$\mathrm{R} 021^{3}$ in cover material

$\mathrm{R} 021$ in foundation material

$\mathrm{R} 021^{3}$ in contaminated zone soil

R021 3 Radon vertical dimension of mixing $(m)$

RO21 3 Average building air exchange rate $(1 / \mathrm{hr})$

R021 3 Height of the building (room) (m)

R021 3 Building interior area factor

3 not used $32.000 \mathrm{E}-06$

not used $33.000 \mathrm{E}-07$

not used 3 2.000E-06

not used $32.000 \mathrm{E}+0 \mathrm{O}^{3}$

not used 3 5.000E-01

not used $32.500 \mathrm{E}+00$

3 not used $30.000 \mathrm{E}+00$

3 not used $3-1.000 \mathrm{E}+00$

3 not used $32.500 \mathrm{E}-011^{3}$

R०21 3 Building depth below ground

$\mathrm{R} 0213$ Emanating power of $\mathrm{Rn}-220$ gas

$\mathrm{R} \odot 21{ }_{3}^{3}$

TITL 3 Number of graphical time points

TITL 3 Maximum number of integration points for dose

$\begin{array}{ll}3 & 1.500 \mathrm{E}-01 \\ 3\end{array}$

$\begin{array}{llll}32 & 3 & - & 3 \\ 17 & 3 & -- & 3\end{array}$

\begin{tabular}{|c|c|c|}
\hline-- & 3 & $\operatorname{TIV}(2)$ \\
\hline.- & 3 & $\operatorname{TIV}(3)$ \\
\hline-- & 3 & $\operatorname{RDRY}(1)$ \\
\hline-- & 3 & $\operatorname{RDRY}(2)$ \\
\hline - - & 3 & RDRY (3) \\
\hline-- & 3 & RWET (1) \\
\hline-- & 3 & RWET (2) \\
\hline-- & 3 & RWET ( 3 ) \\
\hline-- & 3 & WLAM \\
\hline & 3 & \\
\hline$\cdots$ & 3 & C12WTR \\
\hline- & 3 & C12CZ \\
\hline - - & 3 & CSOIL \\
\hline-- & 3 & CAIR \\
\hline-- & 3 & DMC \\
\hline- & 3 & EVSN \\
\hline - - & 3 & REVSN \\
\hline - & 3 & AVFG4 \\
\hline- & 3 & AVFG5 \\
\hline-- & 3 & $\mathrm{CO} 2 \mathrm{~F}$ \\
\hline & $\begin{array}{l}3 \\
3\end{array}$ & \\
\hline - - & 3 & STOR_T(1) \\
\hline-- & 3 & STOR_T(2) \\
\hline-- & 3 & STOR_T(3) \\
\hline- & 3 & STOR_T(4) \\
\hline--- & 3 & STOR_T (5) \\
\hline- & 3 & STOR_T(6) \\
\hline- & 3 & STOR_T(7) \\
\hline - & 3 & STOR_T(8) \\
\hline - & $\begin{array}{l}3 \\
3\end{array}$ & STOR_T(9) \\
\hline - & 3 & FL00R1 \\
\hline-- & 3 & DENSFL \\
\hline- & 3 & TPCV \\
\hline - & 3 & TPFL \\
\hline - & 3 & PH2OCV \\
\hline-- & 3 & PH20FL \\
\hline & 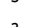 & \\
\hline- & 3 & DIFCV \\
\hline 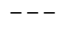 & 3 & DIFFL \\
\hline - & 3 & DIFCZ \\
\hline - & 3 & HMIX \\
\hline- & 3 & REXG \\
\hline - & 3 & HRM \\
\hline - & 3 & FAI \\
\hline- & 3 & DMFL \\
\hline- & 3 & EMANA (1) \\
\hline & 3 & EMANA(2) \\
\hline & 3 & \\
\hline & 3 & NPTS \\
\hline & 3 & LYM \\
\hline
\end{tabular}


Site-Specific Parameter Summary (continued)

Menu ${ }^{3}$

$$
3 \text { User }{ }_{3} \quad \text { Used by RESRAD }
$$

3 Parameter

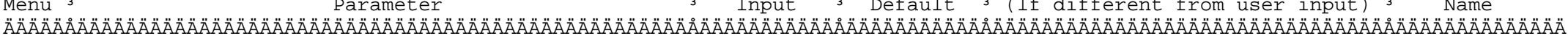
TITL 3 Maximum number of integration points for risk $3_{3} 257 \quad 3_{3}$

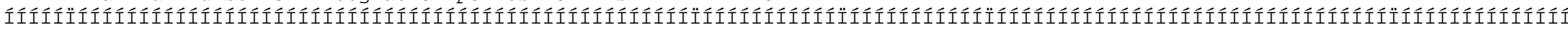

\begin{tabular}{|c|c|c|c|c|}
\hline \multicolumn{4}{|c|}{ 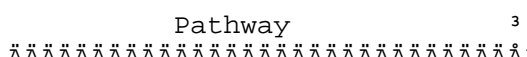 } & \\
\hline $\begin{array}{c}\text { AAAA- } \\
1\end{array}$ & $\begin{array}{l}\text { AAAAAAAAAAAAAAAAAAAAAAAAAAAAmma } \\
\text { - - external qamma }\end{array}$ & & & \\
\hline 2 & - inhalation (w/o & radon) & & active \\
\hline 3 & -- plant ingestion & & 3 & suppressed \\
\hline 4 & - - meat ingestion & & 3 & supp \\
\hline 5 & -- milk ingestion & & 3 & supp \\
\hline 6 & - - aquatic foods & & 3 & suppressed \\
\hline 7 & - - drinking water & & 3 & suppressed \\
\hline 8 & - - soil ingestion & & 3 & active \\
\hline 9 & - - radon & & 3 & suppressed \\
\hline & & & 3 & suppressed \\
\hline
\end{tabular}

Summary of Pathway Selections 
Contaminated Zone Dimensions

ÄÄÄÄÄÄÄÄÄÄÄÄÄÄÄÄÄÄÄÄÄÄÄÄÄÄÄÄ

Area: 100.00 square meters

Thickness: $\quad 0.15$ meters

Cover Depth: $\quad 0.00$ meters

$\odot$

Initial Soil concentrations, pCi/g

ÄÄÄÄÄÄÄÄÄÄÄÄÄÄÄÄÄÄÄÄÄÄÄÄÄÄÄÄÄÄÄÄÄĂ

Am-241 7.200E-01

Cs-137 9.100

3. $620 \mathrm{E}+0 \odot$

Total Dose TDOSE $(t), m r e m / y r$

Basic Radiation Dose Limit $=2.500 \mathrm{E}+01 \mathrm{mrem} / \mathrm{yr}$

Total Mixture Sum $M(t)=$ Fraction of Basic Dose Limit Received at Time $(t)$

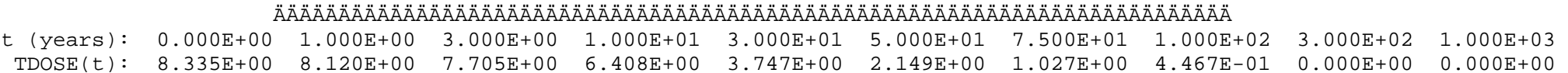

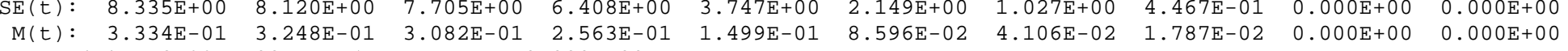
๑Maximum TDOSE $(\mathrm{t}): \quad 8.335 \mathrm{E}+\odot \odot \mathrm{mrem} / \mathrm{yr}$ at $\mathrm{t}=0 . \odot \odot \odot \mathrm{E}+\odot \odot$ years 
Total Dose Contributions TDOSE( $i, p, t)$ for Individual Radionuclides ( $i$ ) and Pathways ( $p$ ) $P l a n t$
ÄÄÄÄÄÄÄÄÄÄÄÄÄÄÄÄ

$\mathrm{Milk}$

Soil

Radio- ÄÄÄÄÄÄÄÄÄÄÄÄÄÄÄÄ

mrem/yr fract. ÄÄÄÄÄÄÄÄÄÄÄÄÄÄÄÄ ӒÄÄÄÄÄÄÄÄÄÄÄÄÄÄÄ ÄÄÄÄÄÄÄÄÄÄÄÄÄÄÄÄ mrem/yr fract. ÄÄÄÄÄÄÄ̈ ÄÄÄÄÄÄÄÄÄÄ ÄÄÄÄÄÄ̈ Am-241 9.837E-04 0.0001 Cs-137 8.264E+0๑ 0.9914 $\begin{array}{lll}\mathrm{Pu}-238 & 1.870 \mathrm{E}-06 & 0.0000 \\ \mathrm{Pu}-239 & 4.033 \mathrm{E}-05 & 0.0000\end{array}$ $\begin{array}{lll}\mathrm{Pu}-239 & 4.033 \mathrm{E}-05 & \odot .00 \odot \\ \mathrm{Sr}-90 & 2.482 \mathrm{E}-03 & 0.0003\end{array}$

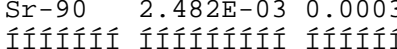
$\begin{array}{lll}\text { Total } & 8.267 \mathrm{E}+00 & 0.9918\end{array}$

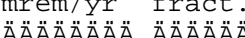
A

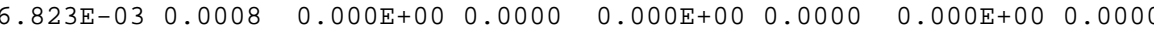
$\begin{array}{llllll}196 \mathrm{E}-05 & 0.0000 & 0.000 \mathrm{E}+00 & 0.0000 & 0.000 \mathrm{E}+00 & 0.0000\end{array}$ $3.043 \mathrm{E}-03 \quad 0.0004$ $4.158 \mathrm{E}-02 \quad 0.0050$

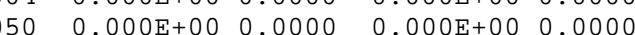

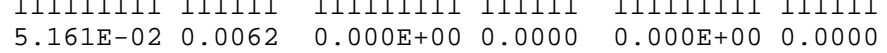
$0.000 \mathrm{E}+000.0000$ $0.000 \mathrm{E}+000.0000$ $\odot .000 \mathrm{E}+00$ ๑. 0.000 $0.000 \mathrm{E}+00 \quad 0.0000$ $0.000 \mathrm{E}+000.0000$ $0.000 \mathrm{E}+00 \quad 0.0000$ $0.000 \mathrm{E}+0 \odot$ 0.000๑

$0.000 \mathrm{E}+000.0000$

Total Dose Contributions TDOSE $(i, p, t)$ for Individual Radionuclides (i) and Pathways ( $p$ ) As mrem/yr and Fraction of Total Dose At $t=0.000 \mathrm{E}+0 \odot$ years Fish Radon Water Dependent Pathways Radio - ÄÄÄÄÄÄÄÄÄÄÄÄÄÄÄÄ Nuclide mrem/yr fract. ÄÄÄÄÄÄÄÄ ÄÄÄÄÄÄÄÄÄÄ ÄÄÄÄÄÄ Am-241 $0.000 \mathrm{E}+00 \quad 0.0000$ Cs-137 $0.000 \mathrm{E}+000.0000$ $\mathrm{Pu}-238 \quad 0.00 \odot \mathrm{E}+0 \odot \quad \odot .000 \odot$ Pu-239 $0.000 \mathrm{E}+000.0000$ Sr-90 $0.000 \mathrm{E}+00 \quad 0.0000$ íííííi íííííííi íííít $\odot *$ Sum of all water independent and dependent pathways.
$0.00 \mathrm{E}+00.120$ ÍÍÍÍÍÍÍí ÍÍÍÍí

$0.000 \mathrm{E}+000.0000$
Plant
Meat

ÄÄÄÄÄÄÄÄÄÄÄÄÄÄÄ

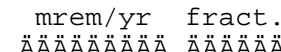
ÄÄÄÄÄÄÄÄÄ ÄÄÄÄÄA $0.000 \mathrm{E}+0 \odot \quad 0.000 \odot$ $0.000 \mathrm{E}+0 \odot \quad 0.0000$ $0.000 \mathrm{E}+000.0000$ $0.000 \mathrm{E}+0 \odot \quad 0.0000$ $0.000 E+00 \quad 0.0000$ Íííííííí ííííít $0.000 \mathrm{E}+000.0000$
Milk

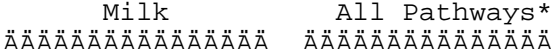
mrem/yr fract

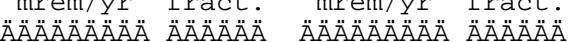
$0.000 \mathrm{E}+0 \odot \quad 0.000 \odot \quad 9.526 \mathrm{E}-03 \quad 0.0011$

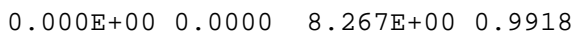
$0.000 \mathrm{E}+00 \quad 0.0000 \quad 3.808 \mathrm{E}-03 \quad 0.0005$ $0.000 \mathrm{E}+00 \quad 0.0000 \quad 5.217 \mathrm{E}-02 \quad 0.0063$ $\begin{array}{llll}0.000 E+00 & 0.0000 & 2.942 E-03 & 0.0004\end{array}$ Íííííííi ííííí íííííííi ííííí $\begin{array}{llll}0.000 \mathrm{E}+00 & 0.0000 & 8.335 \mathrm{E}+00 & 1.0000\end{array}$ 
Total Dose Contributions TDOSE( $i, p, t)$ for Individual Radionuclides ( $i$ ) and Pathways ( $p$ )

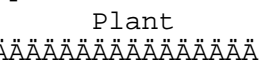

$\mathrm{Milk}$

Soil

Radio - ÄÄÄÄÄÄÄÄÄÄÄÄÄÄÄÄ

mrem/yr fract.

ÄÄÄÄÄÄÄÄÄÄÄÄÄÄÄÄ АAลAลÄÄÄÄÄÄÄÄÄÄÄA ÄÄÄÄÄÄÄÄ̈̈̈ ÄÄÄÄÄÄÄ ÄÄÄÄÄÄÄÄÄÄ ÄÄÄÄÄÄ Am-241 9.612E-04 0.0001 CS-137 8.050E+00 0.9913 $\mathrm{Pu}-238 \quad 1.855 \mathrm{E}-06 \quad 0.0000$ Ӓ̈ÄÄÄÄÄÄÄ ÄÄÄÄÄ

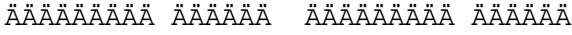
$\begin{array}{lllllll}0.000 \mathrm{E}+00 & 0.0000 & 0.000 \mathrm{E}+00 & 0.0000 & 0.000 \mathrm{E}+00 & 0.0000\end{array}$ $\begin{array}{llllll}0.013 \mathrm{E}-05 & 0.0000 & 0.000 \mathrm{E}+00 & 0.0000 & 0.000 \mathrm{E}+00 & 0.0000\end{array}$

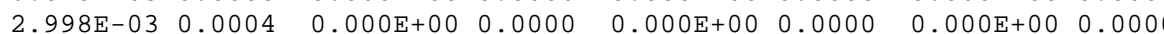
$\mathrm{Pu}-239$ 4.025E-05 0.0000 $\begin{array}{lll}\mathrm{Sr}-90 & 2.383 \mathrm{E}-03 & 0.0003 \\ \text { Ít́títi }\end{array}$ $\begin{array}{ll}\text { IIIIII IIIIIII IIIII } \\ \text { Total } & 8.053 \mathrm{E}+000.9917\end{array}$ 0 $\begin{array}{llllll}.130 \mathrm{E}-02 & 0.0051 & 0.000 \mathrm{E}+0 \odot & 0.000 \odot & 0.000 \mathrm{E}+00 & 0.0000\end{array}$

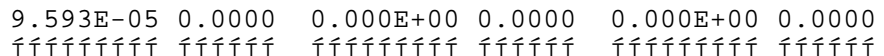

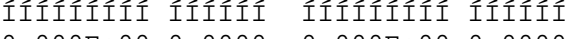
0.000 $.000+000.0000$ OUE Total Dose Contributions TDOSE(i,p,t) for Individual Radionuclides (i) and Pathways $(p)$ As $\mathrm{mrem} / \mathrm{yr}$ and Fraction of Total Dose At $t=1.000 \mathrm{E}+00$ years

$$
\begin{array}{r}
\text { As mrem/yr and Fraction Dependent Pathways } \\
\text { Water }
\end{array}
$$
Water Fish Radon Plant Meat ӒӒÄÄÄÄÄÄÄÄÄÄÄÄÄ̈

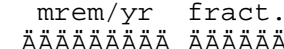
ÄÄÄÄÄÄÄÄÄ ÄÄÄÄÄ̈ $\begin{array}{ll}0.000 E+0 \odot & 0.000 \odot \\ 0.000 E+0 \odot & 0.000 \odot\end{array}$ $0.000 \mathrm{E}+000.0000$ $\odot .00 \odot E+\odot \odot \quad \odot .0 \odot \odot \odot$ $0.000 \mathrm{E}+0 \odot \quad 0.000 \odot$ $0.000 \mathrm{E}+000.0000$ Íííííííí ííííít $0.000 \mathrm{E}+000.0000$ АÄÄÄÄÄÄÄÄÄÄÄÄÄÄÄ ÄÄÄÄÄÄÄÄÄÄÄÄÄÄÄÄ ÄÄÄÄÄÄÄÄÄÄÄÄÄÄÄ ÄÄÄÄÄÄÄ ÄÄÄÄÄ Ä

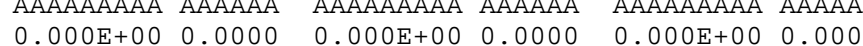
$\begin{array}{lllll}0.00 \odot E+\odot \odot ~ & 0.000 \odot & 0.00 \odot E+0 \odot & 0.000 \odot & 0.00 \odot \mathrm{E}+\odot \odot\end{array}$ $0.000 E+00 \quad 0.0000 \quad 0.000 E+00 \quad 0.0000 \quad 0.000 E+00$ mrem/yr fract. $0.000 \mathrm{E}+0 \odot$ O. 0000 $0.000 \mathrm{E}+00 \quad 0.0000$ $\odot .000 \mathrm{E}+00 \quad 0.0000$ $0.000 \mathrm{E}+00 \quad 0.0000$ $0.000 \mathrm{E}+000.0000$ ÍÍÍÍÍÍÍ ÍÍÍÍí Radio- ÄÄÄÄÄÄÄÄÄÄÄÄÄÄÄÄ $\begin{array}{lll}A m-241 & 0.000 \mathrm{E}+00 & 0.0000\end{array}$ CS-137 $0.000 \mathrm{E}+00$ - 0.0000 Pu-239 $0.000 \mathrm{E}+00 \quad 0.0000$

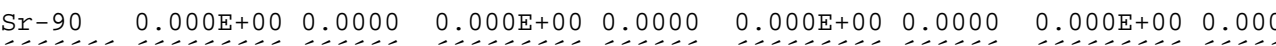

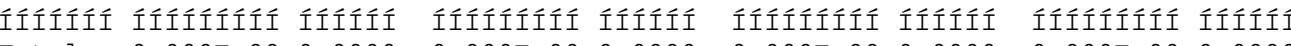
$\odot *$ Sum of all water independent and dependent pathways.

Milk

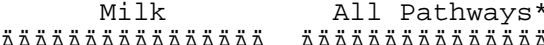
mrem/yr fract. mrem/yr fract. ÄÄÄÄÄÄÄÄ ÄÄÄÄÄÄ ÄÄÄÄÄÄÄÄÄ ÄÄÄÄÄÄ ๑. $000 \mathrm{E}+0 \odot$ $0.0000 \quad 9.252 \mathrm{E}-03 \quad 0.0011$ $\begin{array}{llll}0.000 \mathrm{E}+00 & 0.0000 & 8.053 \mathrm{E}+00 & 0.9917\end{array}$ $0.000 \mathrm{E}+00 \quad 0.0000 \quad 3.752 \mathrm{E}-03 \quad 0.0005$ $0.000 \mathrm{E}+00 \quad 0.0000 \quad 5.181 \mathrm{E}-02 \quad 0.0064$ $\begin{array}{llll}0.000 E+00 & 0.0000 & 2.823 E-03 & 0.0003\end{array}$ Ííííííí ííííí Iííííííi ííííi $\begin{array}{lll}0.000 \mathrm{E}+0 \odot & 0.000 \odot \quad 8.120 \mathrm{E}+0 \odot & 1.000 \odot\end{array}$ 
Total Dose Contributions TDOSE( $i, p, t)$ for Individual Radionuclides ( $i$ ) and Pathways ( $p$ ) Plant
ÄÄÄÄÄÄÄÄÄÄÄÄÄÄÄÄ

$\mathrm{Milk}$

Soil

Radio - ÄÄÄÄÄÄÄÄÄÄÄÄÄÄÄÄ АÄÄÄÄÄÄÄÄÄÄÄÄÄÄÄÄ ÄÄÄÄÄÄÄÄÄÄÄÄÄÄÄÄÄ ӒӒÄÄÄÄÄÄÄÄÄÄÄÄÄÄÄ ÄӒÄÄÄÄÄÄÄ̈̈̈̈̈̈̈̈̈̈̈̈̈ mrem/yr fract. ÄÄÄÄÄÄÄ Ä $\begin{array}{lll}\mathrm{Am}-241 & 9.178 \mathrm{E}-04 \quad 0.0001\end{array}$ CS-137 7.637E+00 0.9911 $\begin{array}{lll}\mathrm{Pu}-238 & 1.824 \mathrm{E}-06 & 0.0000 \\ \mathrm{Pu}-239 & 4.008 \mathrm{E}-05 & 0.0000\end{array}$

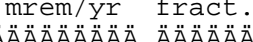
АลАÄÄÄÄÄÄ ÄÄÄÄÄÄ ÄÄÄÄÄÄÄÄÄ ÄÄÄÄÄÄ

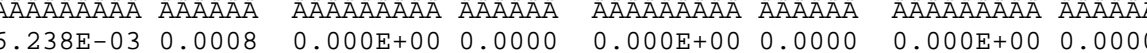

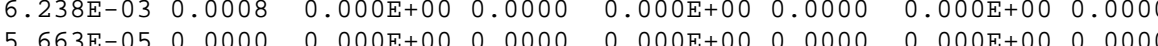
$0.000 E+00$ 0.0000 $0.000 E+00$ 0.0000 $4.072 E-02$. 0053 0.000E+00 0.0000 0.000E+00 0.0000

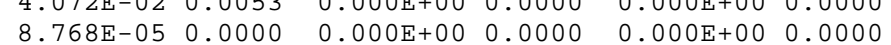
$\mathrm{Sr}-90 \quad 2.196 \mathrm{E}-03 \quad 0.0003$ Íííííi ííííííí ííííi $0^{\text {Total }} 7.640 \mathrm{E}+0 \odot \quad 0.9915$

Íííílíi ííííi Iííííííi ííííi
$0.000 E+00$ 0.0000 $0.000 E+00$ ( $\odot .0 \odot \odot E+\odot \odot$ $\odot .0 \odot \odot \odot$ $\odot .000 \mathrm{E}+00 \quad 0.0000$ $0.000 \mathrm{E}+00 \quad 0.0000$ $\odot .000 E+00 \quad 0.0000$ $0.000 \mathrm{E}+00 \quad 0.0000 \quad 0.000 \mathrm{E}+00 \quad 0.0000$

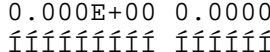

Total Dose Contributions $\operatorname{TDOSE}(i, p, t)$ for Individual Radionuclides (i) and Pathways $(p)$ As mrem/yr and Fraction of Total Dose At $t=3.000 \mathrm{E}+00$ years Water Dependent Pathways Fish Radon

Radio - ÄÄÄÄÄÄÄÄÄÄÄÄÄÄÄÄ Nuclide mrem/yr fract ÄÄÄÄÄÄÄ ÄÄÄÄÄÄÄÄÄ ÄÄÄÄÄ̈̈ $\begin{array}{lll}\mathrm{Am}-241 & 0.000 \mathrm{E}+0 \odot & 0.00 \odot \\ \mathrm{CS}-137 & 0.000 \mathrm{E}+0 \odot & 0.000 \odot\end{array}$ $\mathrm{Pu}-238 \quad \odot .0 \odot \odot \mathrm{E}+\odot \odot \odot . \odot \odot \odot \odot$ $\begin{array}{lll}\mathrm{Pu}-239 & 0.000 \mathrm{E}+00 & 0.0000\end{array}$ $\begin{array}{lll}\mathrm{Sr}-90 & 0.000 \mathrm{E}+00 & 0.0000\end{array}$ Íííííi ííííííí íííít

IÍííííííi ííííín * Sum of all water independent and dependent pathways.
Plant
Meat

ÄÄÄÄÄÄÄÄÄÄÄÄÄÄA

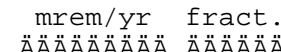
ÄÄÄÄÄÄÄÄÄ ÄÄÄÄÄA $0.000 \mathrm{E}+0 \odot$ $0.000 \odot$ $0.000 \mathrm{E}+000.0000$ $0.000 \mathrm{E}+0 \odot \quad 0.0000$ $0.000 \mathrm{E}+00 \quad 0.0000$ $0.000 E+00 \quad 0.0000$ Íííííííi ííííí

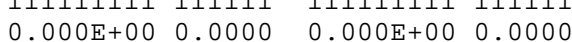

Milk

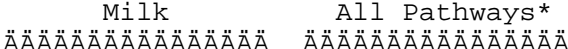
mrem/yr fract

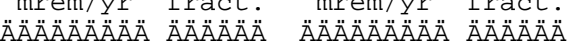
$0.000 \mathrm{E}+0 \odot \quad 0.000 \odot \quad 8.727 \mathrm{E}-03 \quad 0.0011$ $\begin{array}{lllll}0.000 E+00 & 0.0000 & 7.639 E+00 & 0.9914\end{array}$ $0.000 \mathrm{E}+00 \quad 0.0000 \quad 3.642 \mathrm{E}-03 \quad 0.0005$ $\odot .000 \mathrm{E}+0 \odot \quad 0.000 \odot \quad 5.109 \mathrm{E}-02 \quad 0.0066$ $\odot .000 \mathrm{E}+0 \odot \quad 0.0000 \quad 2.598 \mathrm{E}-03 \quad 0.0003$ Íííííííi ííííí íííííííi ííííí

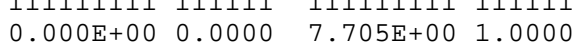


Total Dose Contributions TDOSE( $i, p, t)$ for Individual Radionuclides ( $i$ ) and Pathways ( $p$ ) Plant
ÄÄÄÄÄÄÄÄÄÄÄÄÄÄÄÄ

$\mathrm{Milk}$

Soil

Radio - ÄÄÄÄÄÄÄÄÄÄÄÄÄÄÄÄ АÄÄÄÄÄÄÄÄÄÄÄÄÄÄÄÄ ÄÄÄÄÄÄÄÄÄÄÄÄÄÄÄÄÄ Ӓ̈̈ÄÄÄÄÄÄÄÄÄÄÄÄÄÄÄ ÄÄÄÄÄÄÄÄÄÄÄÄÄÄÄÄ mrem/yr fract. $m r e m / y r$
Aract. ÄÄÄÄÄÄÄ ÄÄÄÄÄÄÄÄÄ ÄÄÄÄÄÄ $\begin{array}{lll}\text { Am-241 } & 7.805 \mathrm{E}-04 & 0.0001\end{array}$ Cs-137 6.345E+0๑ $\odot .9901$ $\begin{array}{lll}\mathrm{Pu}-238 & 1.722 \mathrm{E}-06 & 0.0000 \\ \mathrm{Pu}-239 & 3.950 \mathrm{E}-05 & 0.0000\end{array}$ $\begin{array}{lll}\mathrm{Pu}-239 & 3.950 \mathrm{E}-05 & 0.000 \odot \\ \mathrm{Sr}-90 & 1.649 \mathrm{E}-03 & 0.0003\end{array}$

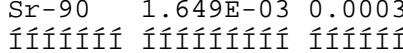

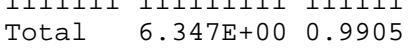

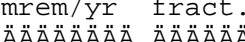
$\begin{array}{llllllll}5.052 \mathrm{E}-03 & 0.0008 & 0.000 \mathrm{E}+00 & 0.0000 & 0.000 \mathrm{E}+00 & 0.0000 & 0.000 \mathrm{E}+00 & 0.0000\end{array}$

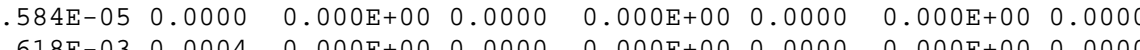
. $\begin{array}{llllll}3.871 E-02 & 0.0060 & 0.000 E+00 & 0.0000 & 0.000 \mathrm{E}+0 \odot & 0.0000\end{array}$

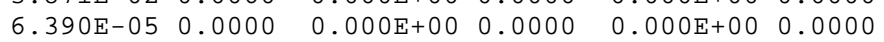
Iilííííi ííííí íííííííi ííííí íííííííi ííííí $0.000 \mathrm{E}+000.0000$ $0.000 \mathrm{E}+00$ 0.0000 Ít́́t $\odot$

Total Dose Contributions TDOSE( $i, p, t)$ for Individual Radionuclides (i) and Pathways ( $p$ ) As mrem/yr and Fraction of Total Dose At $t=1.000 E+01$ years

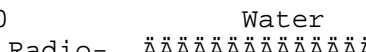
Fish Radon Water Dependent Pathways vuclide mrem/yr fract.

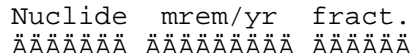

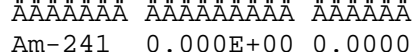
$\begin{array}{lll}\mathrm{Am}-241 & 0.000 \mathrm{E}+0 \odot & 0.00 \odot \\ \mathrm{Cs}-137 & 0.00 \odot \mathrm{E}+\odot \odot & 0.00 \odot \odot\end{array}$ $\mathrm{Pu}-238 \quad 0.000 \mathrm{E}+0 \odot \quad \odot .000 \odot$ Pu-239 $0.000 \mathrm{E}+00 \quad 0.0000$ Sr-90 $0.000 \mathrm{E}+00 \quad 0.0000$ íííííi íííííííi ííííí Total $0.000 \mathrm{t}$ (1) $\odot *$ Sum of all water independent and dependent pathways.

mrem/yr fract. mrem/yr fract. mrem/yr fract. Ӓ̈̈ӒÄÄÄÄÄ ÄÄÄÄÄÄ Ä ÄÄÄÄÄÄÄÄÄ ÄÄÄÄÄÄ ÄÄÄÄÄÄÄÄÄ̈ $\begin{array}{llllll}0.000 \mathrm{E}+00 & 0.0000 & 0.000 \mathrm{E}+00 & 0.0000 & 0.000 \mathrm{E}+00 & 0.0000\end{array}$

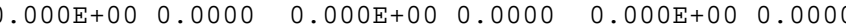

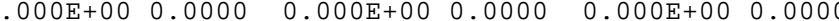
$0.000 \mathrm{E}+00$ (0. 0000 $0.000 \mathrm{E}+00$ 0 0000 .
Plant
Meat

ÄÄÄÄÄÄÄÄÄÄÄÄÄÄ̈

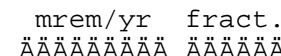
ÄÄÄÄÄ̈̈̈̈̈̈̈̈ ÄÄÄÄÄ̈ $0.000 \mathrm{E}+0 \odot$ 0.0000 $0.000 \mathrm{E}+0 \odot \quad 0.0000$ $0.000 \mathrm{E}+0 \odot \quad 0.0000$ $0.000 \mathrm{E}+0 \odot \quad 0.0000$ $0.000 E+00 \quad 0.0000$ Íííííííí ííííít

$0.000 \mathrm{E}+00 \quad 0.0000 \quad 0.000 \mathrm{E}+000.0000$

$0.000 \mathrm{E}+000.0000 \quad 0.000 \mathrm{E}+000.0000$
$0.000 \mathrm{E}+00 \quad 0.0000$ $0.000 \mathrm{E}+0 \odot \quad 0.0000$ $\odot .000 \mathrm{E}+00 \quad 0.0000$ $\odot .000 \mathrm{E}+0 \odot \quad 0.0000$ $0.000 \mathrm{E}+00 \quad 0.0000$ Íííííííí ííííí $0.000 \mathrm{E}+0 \odot 0.000 \odot$

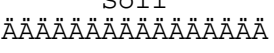
$\mathrm{mrem} / \mathrm{yr}$ fract. ÄÄÄÄÄÄÄÄÄ ÄÄÄÄÄÄ $1.273 \mathrm{E}-03 \quad 0.0002$ $2.208 \mathrm{E}-03 \quad 0.0003$ $6.568 \mathrm{E}-04 \odot .0001$ $9.816 \mathrm{E}-03 \quad 0.0015$ $2.293 E-04 \quad 0.0000$ Íííííííi ííííí $1.418 \mathrm{E}-02 \quad 0.0022$ Milk

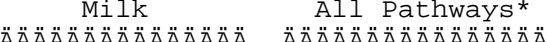
mrem/yr fract

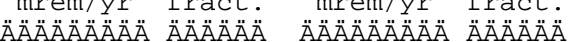
$0.000 \mathrm{E}+0 \odot \quad 0.000 \odot \quad 7.105 \mathrm{E}-03 \quad 0.0011$ $\begin{array}{lllll}0.000 \mathrm{E}+00 & 0.0000 & 6.347 \mathrm{E}+00 & 0.9905\end{array}$ $0.000 \mathrm{E}+00 \quad 0.0000 \quad 3.277 \mathrm{E}-03 \quad 0.0005$ $0.000 \mathrm{E}+00 \quad 0.0000 \quad 4.856 \mathrm{E}-02 \quad 0.0076$ $0.000 \mathrm{E}+00$ 0.0000 1 942E-03 0.0003 c.0utú $\odot .000 \mathrm{E}+\odot \odot$ $\odot . \odot \odot \odot \odot \quad 6.408 \mathrm{E}+0 \odot 1.00 \odot \odot$ 
Total Dose Contributions TDOSE( $i, p, t)$ for Individual Radionuclides ( $i$ ) and Pathways ( $p$ )

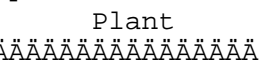

$\mathrm{Milk}$

Soil

Radio - ÄÄÄÄÄÄÄÄÄÄÄÄÄÄÄÄ АÄÄÄÄÄÄÄÄÄÄÄÄÄÄÄÄ ÄÄÄÄÄÄÄÄÄÄÄÄÄÄÄÄÄ АÄÄÄÄÄÄÄÄÄÄÄÄÄÄA

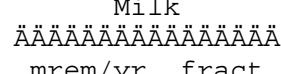
mrem/yr fract. ÄÄÄÄÄÄÄ ÄÄÄÄÄÄÄÄÄ ÄÄÄÄÄÄ̈
Am-241 $\mathrm{Cs}-137 \quad 3.697 \mathrm{E}+\odot \odot \quad \odot .9867$ $\mathrm{Pu}-238 \quad 1.459 \mathrm{E}-06 \quad 0.0000$ Ӓ̈̈̈̈̈̈̈̈̈̈̈̈̈̈̈ ÄÄÄÄÄÄ \begin{tabular}{lllllll}
$2.725 E-03$ & 0.0007 & $0.000 \mathrm{E}+00$ & 0.0000 & $0.000 \mathrm{E}+00$ & 0.0000 & $0.000 \mathrm{~A}+00$ \\
\hline
\end{tabular}

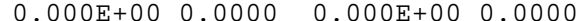
$1.907 \mathrm{E}-03 \quad 0.0005$ $\mathrm{Pu}-239 \quad 3.762 \mathrm{E}-05 \quad 0.0000$ Sr-90 7.207E-04 0.0002 Íííííí íííííííi ííííí Tot

$0.000 \mathrm{E}+00 \quad 0.0000 \quad 0.000 \mathrm{E}+00 \quad 0.0000$

$0.000 \mathrm{E}+00 \quad 0.0000$

$\odot .000 \mathrm{E}+00 \quad 0.0000$

$\odot .000 \mathrm{E}+000.0000$

$\odot .000 \mathrm{E}+00 \quad 0.0000$

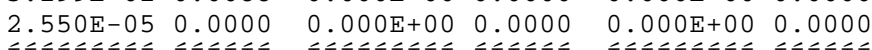

$0.000 \mathrm{E}+000.0000$

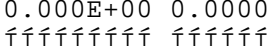

$0.000 \mathrm{E}+000.0000$

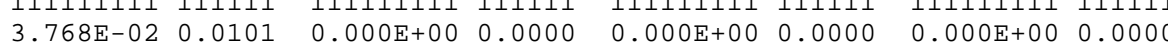

$0.000 \mathrm{E}+000.0000$

$0.000 \mathrm{E}+00 \quad 0.0000$

Total Dose Contributions $\operatorname{TDOSE}(i, p, t)$ for Individual Radionuclides (i) and Pathways $(p)$ As $\mathrm{mrem} / \mathrm{yr}$ and Fraction of Total Dose At $t=3.000 \mathrm{E}+01$ years

$$
\begin{array}{r}
\text { As mrem/yr and Fraction of Total Dose At } t \\
\text { Water Dependent Pathways }
\end{array}
$$
Fish Radon

Radio- ÄÄÄÄÄÄÄÄÄÄÄÄÄÄÄ Nuclide mrem/yr fract. ÄÄÄÄÄÄÄ ÄÄÄÄÄÄÄÄÄ ÄÄÄÄÄ̈̈ $\begin{array}{lll}\mathrm{Am}-241 & 0.000 \mathrm{E}+0 \odot & \odot .000 \odot \\ \mathrm{CS}-137 & 0.00 \odot \mathrm{E}+0 \odot & 0.00 \odot \odot\end{array}$ $\mathrm{Pu}-238 \quad 0.000 \mathrm{E}+00 \quad 0.0000$ Pu-239 $0.000 \mathrm{E}+00 \quad 0.0000$ Sr-90 $0.000 \mathrm{E}+00 \quad 0.0000$ Íííííi ííííííí íííít

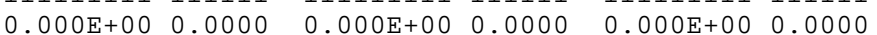
$0.000+000.0000$ 1IIIIIII IIIÍI

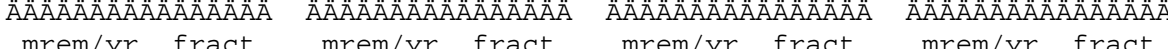
Ä̈AAAAAA AAAAA $\begin{array}{llllll}0.000 E+0 \odot & 0.000 \odot & 0.000 \mathrm{E}+0 \odot & 0.0000 & 0.000 \mathrm{E}+0 \odot & 0.000 \odot\end{array}$

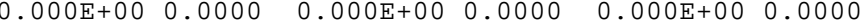

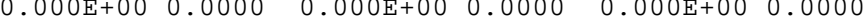

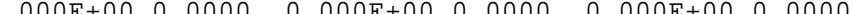
$\odot *$ Sum of all water independent and dependent pathways. ÄÄÄÄÄÄÄÄ $0.000 \mathrm{E}+000.0000$
Meat

Milk

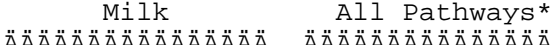
mrem/r r ract ÄÄÄÄÄÄÄÄÄ ÄÄÄÄÄÄ ÄÄÄÄÄÄÄÄÄ ÄÄÄÄÄÄ $0.000 \mathrm{E}+0 \odot$ $0.0000 \quad 3.902 \mathrm{E}-03 \quad 0.0010$ $\begin{array}{lllll}0.000 \mathrm{E}+0 \odot & 0.0000 & 3.699 \mathrm{E}+\odot \odot & 0.9870\end{array}$ $0.000 \mathrm{E}+00 \quad 0.0000 \quad 2.387 \mathrm{E}-03 \quad 0.0006$ $0.000 \mathrm{E}+00 \quad 0.0000 \quad 4.140 \mathrm{E}-02 \quad 0.0110$ $0.000 \mathrm{E}+00$ 0.0000 $8.377 \mathrm{E}-040.0002$

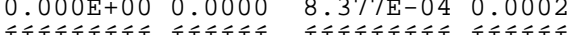

$0.000 \mathrm{E}+000.0000 \quad 3.747 \mathrm{E}+001.0000$ АÄÄÄÄÄÄÄÄÄÄÄÄÄÄ

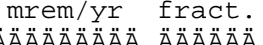
$0.865 \mathrm{E}-04 \quad 0.0002$ $0.151 E-0.0022$ íííííííi ííííí 
Total Dose Contributions TDOSE( $i, p, t)$ for Individual Radionuclides ( $i$ ) and Pathways ( $p$ )

$\mathrm{Milk}$

Soil

Radio- ÄÄÄÄÄÄÄÄÄÄÄÄÄÄÄÄA АÄÄÄÄÄÄÄÄÄÄÄÄÄÄÄ ÄÄÄÄÄÄÄÄÄÄÄÄÄÄÄÄÄ ÄÄÄÄÄÄÄÄÄÄÄÄÄÄÄÄÄ Ӓ̈̈ÄÄÄÄÄÄÄÄÄÄÄÄÄÄÄ ÄÄÄÄÄÄÄÄÄÄÄÄÄÄÄÄ mrem/yr fract. mrem/yr fract. ÄÄÄÄÄÄÄÄ ÄÄÄÄÄÄÄÄÄÄ ÄÄÄÄÄÄ̈̈ $\begin{array}{lll}\mathrm{Am}-241 & 3.078 \mathrm{E}-04 & 0.0001\end{array}$ $\begin{array}{lll}\mathrm{CS}-137 & 2.110 \mathrm{E}+00 & 0.9818\end{array}$ $\mathrm{Pu}-238$ 1.233E- 06 0.0000 Ӓ̈̈̈̈̈̈̈̈̈̈̈̈̈̈̈ Ӓ ӒÄÄÄÄ̈̈ AÄAAAAÄÄ̈ ÄÄÄÄÄÄ ÄÄÄÄÄÄÄÄÄ ÄÄÄÄÄÄ $\begin{array}{lllllll}1.428 \mathrm{E}-03 & 0.0007 & 0.000 \mathrm{E}+00 & 0.0000 & 0.000 \mathrm{E}+00 & 0.0000 & 0.000 \mathrm{~A} A \mathrm{~A} \text { AAAAAA }\end{array}$ (. $1.350 \mathrm{E}-03$ 0. $0006 \quad 0.000 \mathrm{E}+00$ - 0.0000 - $0.000 \mathrm{E}+00$ - 0.0000 $2.734 \mathrm{E}-02$ 0.0127 $9.889 \mathrm{E}-06 \quad 0.000 \odot$ $\mathrm{Pu}-239 \quad 3.534 \mathrm{E}-05 \quad 0.0000$

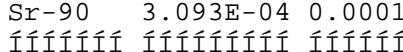
$\begin{array}{lll}\text { Total } & 2.111 \mathrm{E}+00 & 0.9821\end{array}$

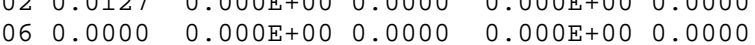

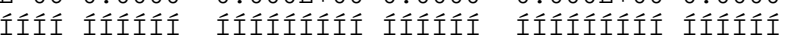
$0.000 \mathrm{E}+00$ - 0.0000 $0.000 \mathrm{E}+00$ $0.000 \mathrm{E}+00$ 0.0000 ÍÍÍÍÍÍ ÍÍÍÍI $\odot$

Total Dose Contributions TDOSE( $i, p, t)$ for Individual Radionuclides (i) and Pathways ( $p$ ) As mrem/yr and Fraction of Total Dose At $t=5.000 E+01$ years Water Dependent Pathways Fish Radon Plant Meat

Milk ÄÄÄÄÄÄÄÄÄÄÄÄÄÄÄ̈ $\begin{aligned} m r e m / y r & \text { fract. } \\ \text { ÄÄÄÄÄÄÄÄ } & \text { ÄÄÄÄÄÄ }\end{aligned}$ ÄÄÄÄÄÄÄÄÄ ÄÄÄÄÄÄ

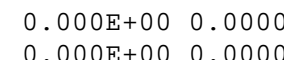
$0.000 \mathrm{E}+00 \quad 0.0000$ $0.000 E+00 \quad 0.0000$ $0.000 \mathrm{E}+00$ - 0.0000 ÍÍÍÍíííí Ííííít $0.000 \mathrm{E}+000.0000$

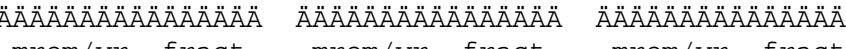

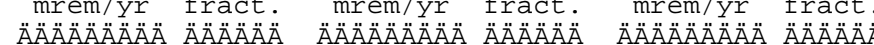

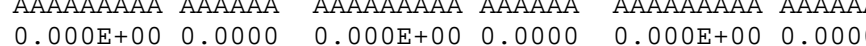

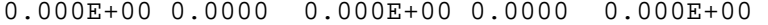

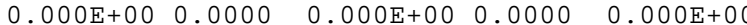
6.227E-04 0.0003 $\odot .000 \mathrm{E}+00 \quad 0.0000 \quad 3.386 \mathrm{E}-04 \quad 0.0002$ $0.000 \mathrm{E}+00 \quad 0.0000 \quad 6.933 \mathrm{E}-03 \quad 0.0032$

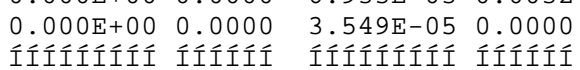
Radio- ÄÄÄÄÄÄÄÄÄÄÄÄÄÄÄA Am-241 $0.000 \mathrm{E}+00 \quad 0.0000$ CS-137 $0.000 \mathrm{E}+00 \quad 0.0000$ $\mathrm{Pu}-2390.000 \mathrm{E}+00 \quad 0.0000$

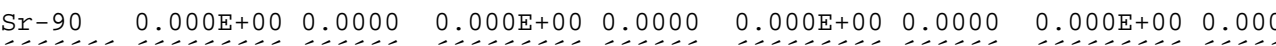

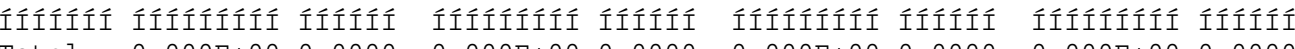
$\odot *$ Sum of all water independent and dependent pathways.

ÄÄÄÄÄÄÄ̈ÄÄÄÄÄÄÄĂ $\mathrm{mrem} / \mathrm{yr}$ fract. $0.000 \mathrm{E}+000.0000$ $\odot .000 \mathrm{E}+0 \odot \quad 0.0000$ $0.000 \mathrm{E}+000.00000$ $0.000 \mathrm{E}+00 \quad 0.0000$ $0.000 E+00 \quad 0.0000$ Ííííííí Ííííí $0.000 \mathrm{E}+0 \odot \quad 0.000 \odot$ 
Total Dose Contributions TDOSE( $i, p, t)$ for Individual Radionuclides ( $i$ ) and Pathways ( $p$ ) Plant
ÄÄÄÄÄÄÄÄÄÄÄÄÄÄÄÄ

$\mathrm{Milk}$

Soil

Radio - ÄÄÄÄÄÄÄÄÄÄÄÄÄÄÄÄ АÄÄÄÄÄÄÄÄÄÄÄÄÄÄÄ ÄÄÄÄÄÄÄÄÄÄÄÄÄÄÄÄÄ АÄÄÄÄÄÄÄÄÄÄÄÄÄÄA ÄӒÄÄÄÄÄÄÄ̈̈̈̈̈̈̈̈̈̈̈̈̈ mrem/yr fract. ÄÄÄÄÄÄÄÄ ÄÄÄÄÄÄÄÄÄÄ ÄÄÄÄÄÄ̈ Am-241 $1.698 \mathrm{E}-04 \quad 0.0002$ Cs-137 9.987E-01 0.9729 Pu-238 9.906E- $07 \quad 0.0000$ 范 АลАAÄÄÄÄÄ ÄÄÄÄÄÄ ÄÄÄÄÄÄÄÄÄ ÄÄÄÄÄÄ

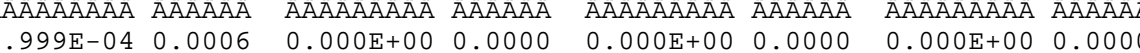

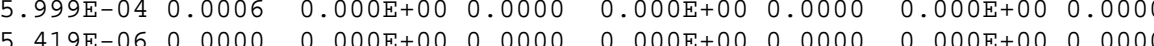
$0.000 E+00$ 0.0000 $0.000 E+000.0000$ 2.849E- 06 0.0000 Pu-239 3.166E-05 0.0000 $\begin{array}{lll}\mathrm{Sr}-90 & 1.030 \mathrm{E}-04 & 0.0001\end{array}$ $\begin{array}{lll}\text { IIIIII IIIIIIII IIIIII } \\ \text { Total } & 9.990 \mathrm{E}-01 & 0.9731\end{array}$ $\odot$

$0.000 \mathrm{E}+000.0000$

$0.000 \mathrm{E}+00 \quad 0.0000$

$\odot .000 \mathrm{E}+0 \odot$ ๑. $000 \odot$

$0.000 \mathrm{E}+0 \odot \quad 0.0000$

$0.000 \mathrm{E}+00 \quad 0.0000$

$0.000 \mathrm{E}+000.0000$

Ííílití ííííi ííííííí ííííi ííííííí íííít

$0.000 \mathrm{E}+00 \quad 0.0000$

$0.000 \mathrm{E}+00 \quad 0.0000$

$0.000 \mathrm{E}+00 \quad 0.0000 \quad 0.000 \mathrm{E}+00 \quad 0.0000$

$.000 \mathrm{E}+00 \quad 0.0000$

Total Dose Contributions $\operatorname{TDOSE}(i, p, t)$ for Individual Radionuclides (i) and Pathways $(p)$ As $\mathrm{mrem} / \mathrm{yr}$ and Fraction of Total Dose At $t=7.500 \mathrm{E}+01$ years Fish Radon Water Dependent Pathways

Radio - ÄÄÄÄÄÄÄÄÄÄÄÄÄÄA Nuclide mrem/yr fract ÄÄÄÄÄÄÄ ÄÄÄÄÄÄÄÄÄ ÄÄÄÄÄ̈ Am-241 $0.000 \mathrm{E}+00$ ๑. 0.0000 CS-137 $0.000 \mathrm{E}+000.0000$ $\begin{array}{lll}\mathrm{Pu}-238 & 0.000 \mathrm{E}+00 & 0.0000 \\ \mathrm{Pu}-239 & 0.000 \mathrm{E}+00 & 0.0000\end{array}$ Sr $-90 \quad 0.000 \mathrm{E}+00 \quad 0.0000$

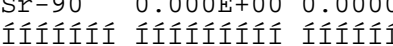
Total $\odot *$ Sum of all water independent and dependent pathways. $\odot ., 0 \odot \mathrm{E}+0 \mathrm{O}^{2}$ Íííííííí íííííi

$0.000 \mathrm{E}+00 \quad 0.0000$
Plant
Meat

ÄÄÄÄÄÄÄÄÄÄÄÄÄÄA mrem/yr fract.
ÄÄÄÄÄ̈̈̈̈̈̈̈ ÄÄÄÄÄÄ ÄÄÄÄÄ̈̈̈̈̈̈̈̈ ÄÄÄÄÄ̈ $0.000 \mathrm{E}+0 \odot \quad 0.000 \odot$ $0.000 \mathrm{E}+0 \odot \quad 0.0000$ $0.000 \mathrm{E}+000.0000$ $0.000 \mathrm{E}+0 \odot \quad 0.0000$ $0.000 E+00 \quad 0.0000$ Íííííííí ííííít $0.000 \mathrm{E}+000.0000$
Milk

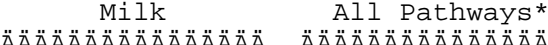
mrem/yr fract. mrem/yr fract.

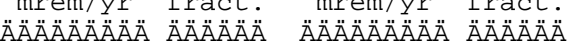
0.000E+00 0.0000 9.208E-04 0.0009 $\begin{array}{lllll}0.000 \mathrm{E}+0 \odot & 0.000 \odot \quad 9.989 \mathrm{E}-01 & 0.9731\end{array}$ $0.000 \mathrm{E}+00 \quad 0.0000 \quad 1.033 \mathrm{E}-03 \quad 0.0010$ $0.000 \mathrm{E}+00 \quad 0.0000 \quad 2.553 \mathrm{E}-02 \quad 0.0249$ $0.000 \mathrm{E}+00$ 0.0000 $1.51 \mathrm{E}-040.0001$ 1.útio

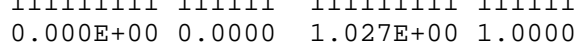


Total Dose Contributions TDOSE( $i, p, t)$ for Individual Radionuclides ( $i$ ) and Pathways ( $p$ )

$\mathrm{Milk}$

Soil

Radio- ÄÄÄÄÄÄÄÄÄÄÄÄÄÄÄ
Nuclide mrem/yr fract

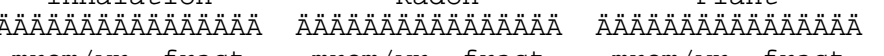

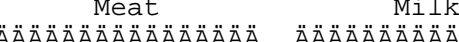
ÄÄÄÄÄÄÄÄÄÄÄÄÄÄÄ mrem/yr fract mrem/yr fract.

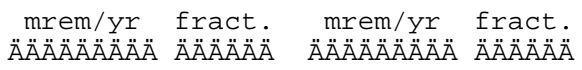

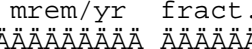
ÄÄÄÄÄÄÄ ÄÄÄÄÄÄÄÄÄ ÄÄÄÄÄÄ $\mathrm{Am}-241 \quad 8.971 \mathrm{E}-05 \quad 0.0002$ $\mathrm{Pu}-238 \quad 7.810 \mathrm{E}-07 \quad 0.0000$ mrem/yr fract Ӓ̈ӒÄÄÄÄÄÄ ÄÄÄÄA

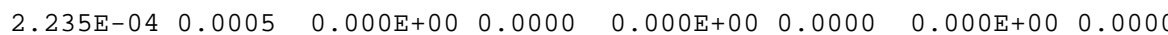

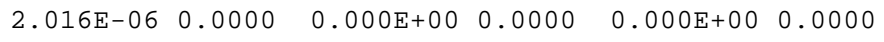
$\begin{array}{llllll}4.475 \mathrm{E}-04 & 0.0010 & 0.000 \mathrm{E}+00 & 0.0000 & 0.000 \mathrm{E}+00 & 0.0000\end{array}$ $\odot .000 \mathrm{E}+000.0000$ $\odot .000 \mathrm{E}+00 \quad 0.0000$ $\odot .000 \mathrm{E}+\odot \odot$ 0.0000 $0.000 \mathrm{E}+00 \quad 0.0000$ $\odot .000 \mathrm{E}+00$ ๑. .0000 $\mathrm{Pu}-239 \quad 2.644 \mathrm{E}-05 \quad 0.0001$ $\mathrm{Sr}-90 \quad 3.132 \mathrm{E}-05 \quad 0.0001$ Ííííí ííííííi ííííí $\begin{array}{llllll}1.343 \mathrm{E}-02 & 0.0301 & 0.000 \mathrm{E}+00 & 0.0000 & 0.000 \mathrm{E}+00 & 0.0000\end{array}$

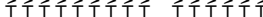

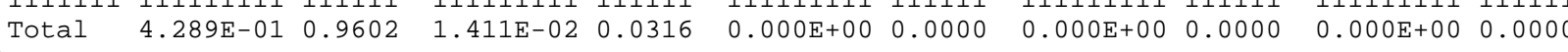
$0.000 \mathrm{E}+000.0000$ $0.000 \mathrm{E}+00 \quad 0.0000$ Total Dose Contributions $\operatorname{TDOSE}(i, p, t)$ for Individual Radionuclides (i) and Pathways $(p)$ As $\mathrm{mrem} / \mathrm{yr}$ and Fraction of Total Dose At $t=1.000 \mathrm{E}+02$ years Water Dependent Pathways Water Fish Radon

Radio - ÄÄÄÄÄÄÄÄÄÄÄÄÄÄÄÄ Nuclide mrem/yr fract. ÄÄÄÄÄÄÄ Ä ÄÄÄÄÄÄÄÄÄ ÄÄÄÄÄÄ Am-241 $0.000 \mathrm{E}+00 \quad 0.0000$ CS-137 $0.000 \mathrm{E}+00 \quad 0.0000$ $\mathrm{Pu}-238 \quad 0.000 \mathrm{E}+\odot \odot \quad \odot .000 \odot$ Pu-239 $0.000 \mathrm{E}+00 \quad 0.0000$ Sr-90 $0.000 \mathrm{E}+00 \quad 0.0000$ íííííi íííííííi íííít

Total $\odot *$ Sum of all water independent and dependent pathways.
Plant
Meat

ÄÄÄÄÄÄÄÄÄÄÄÄÄÄÄ

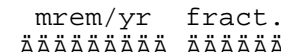
ÄÄÄÄÄÄÄÄÄ ÄÄÄÄÄÄ $0.000 \mathrm{E}+0 \odot \quad 0.000 \odot$ $0.000 \mathrm{E}+00 \quad 0.0000$ $0.000 \mathrm{E}+00 \quad 0.0000$ $0.000 \mathrm{E}+0 \odot \quad 0.000 \odot$ $0.000 E+00 \quad 0.0000$ Íííííííí ííííít

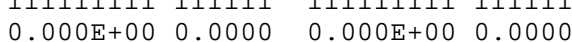

$\odot .000 \mathrm{E}+00$ Íííííííí íííííi

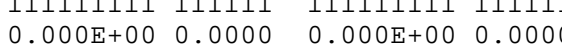

Milk

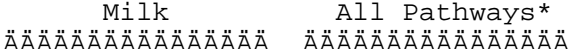
mremar fraA AAAAAAAAAAAAAAAAAA

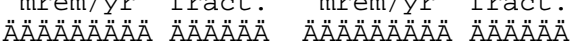
$0.000 \mathrm{E}+0 \odot$ $0.0000 \quad 3.696 \mathrm{E}-04 \quad 0.0008$ $0.000 \mathrm{E}+00 \quad 0.0000 \quad 4.289 \mathrm{E}-01 \quad 0.9601$ $0.000 \mathrm{E}+00 \quad 0.0000 \quad 5.605 \mathrm{E}-04 \quad 0.0013$ $0.000 \mathrm{E}+0 \odot \quad 0.0000 \quad 1.687 \mathrm{E}-02 \quad 0.0378$ $\odot .000 \mathrm{E}+00 \quad 0.0000 \quad 3.466 \mathrm{E}-05 \quad 0.0001$ Íííííííi ííííí íííííííi ííííí $\odot .000 \mathrm{E}+0 \odot$ $0.000 \odot$ 4.467E-01 $1.000 \odot$ 
Total Dose Contributions TDOSE $(i, p, t)$ for Individual Radionuclides (i) and Pathways ( $p$ ) Plant
ÄÄÄÄÄÄÄÄÄÄÄÄÄÄÄÄ

$\mathrm{Milk}$

AAAAÄÄÄÄÄÄÄÄÄA
mrem/yr fract. ÄÄÄÄÄÄÄÄÄÄÄÄÄÄÄÄ АÄÄÄÄÄÄÄÄÄÄÄÄÄÄA ÄӒÄÄÄÄÄÄÄ̈̈̈̈̈̈̈̈̈̈̈̈̈ mrem/yr fract. ÄÄÄÄÄÄÄ ÄÄÄÄÄÄÄÄÄ ÄÄÄÄÄÄ

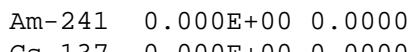
Cs-137 $0.000 \mathrm{E}+0 \odot \quad 0.0000$ $\begin{array}{lll}\mathrm{Pu}-238 & 0.000 \mathrm{E}+00 & 0.000 \\ \mathrm{Pu}-239 & 0.000 \mathrm{E}+0 \odot & 0.0000\end{array}$ ÄÄÄÄÄÄÄÄÄ ÄÄÄÄÄÄ $0.000 \mathrm{E}+000.0000 \quad 0.000 \mathrm{E}+00 \quad 0.0000$ 0.000AAA AAAAAA 年 $0.000 \mathrm{E}+00 \quad 0.0000 \quad 0.000 \mathrm{E}+00$ - $0.0000 \quad 0.000 \mathrm{E}+000.0000$ $0.000 \mathrm{E}+000.0000$ Sr-90 $0.000 \mathrm{E}+00 \quad 0.0000$ íííííi íííííííi íííít $\odot$ $\odot .000 \mathrm{E}+00$ ०. 0.0000 $0.000 \mathrm{E}+00 \quad 0.0000$ $0.000 \mathrm{E}+00 \quad 0.0000$ $0.000 \mathrm{E}+000.0000$ $0.000 \mathrm{E}+000.0000$ $0.000 \mathrm{E}+000.0000$ IIIIIIIÍí ÍÍíít $0.000 \mathrm{E}+00 \quad 0.0000$ $0.000 \mathrm{E}+00 \quad 0.0000$ Total $0.000 \mathrm{E}+\odot \odot \odot .000 \odot$

$0.000 \mathrm{E}+000.0000$

Total Dose Contributions $\operatorname{TDOSE}(i, p, t)$ for Individual Radionuclides (i) and Pathways (p) As mrem/yr and Fraction of Total Dose At $t=3.000 E+02$ years Fish Radon Water Dependent Pathways Radio - ÄÄÄÄÄÄÄÄÄÄÄÄÄÄÄÄ Nuclide mrem/yr fract ÄÄÄÄÄÄÄ ÄÄÄÄÄÄÄÄÄ ÄÄÄÄÄA Am-241 $0.000 \mathrm{E}+00$ - 0.0000 $\begin{array}{lll}\mathrm{CS}-137 & 0.000 \mathrm{E}+00 & 0.0000 \\ \mathrm{PU}-238 & 0.000 \mathrm{E}+0 \odot & 0.000 \odot\end{array}$ $\begin{array}{lll}\mathrm{Pu}-238 & 0.000 \mathrm{E}+00 & 0.0000 \\ \mathrm{Pu}-239 & 0.000 \mathrm{E}+00 & 0.0000\end{array}$ $\begin{array}{ccc}\mathrm{Sr}-90 & 0.000 \mathrm{E}+00 & 0.0000\end{array}$ Íííííi íííííííi Ííííí Total $\odot *$ Sum of all water independent and dependent pathways. $\odot, 000 \mathrm{E}+00$ Íííííííí íííííi

$0.000 \mathrm{E}+00 \quad 0.0000$
Plant
Meat

ÄÄÄÄÄÄÄÄÄÄÄÄÄÄA

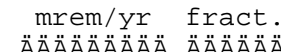
ÄÄÄÄÄÄÄÄÄ ÄÄÄÄÄÄ $0.000 \mathrm{E}+0 \odot \quad 0.000 \odot$ $0.000 \mathrm{E}+00 \quad 0.0000$ $0.000 \mathrm{E}+00 \quad 0.0000$ $0.000 \mathrm{E}+0 \odot \quad 0.000 \odot$ $0.000 E+00 \quad 0.0000$ Íííííííí ííííít $0.000 \mathrm{E}+000.0000$
Milk

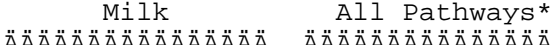
mrem/yr fract

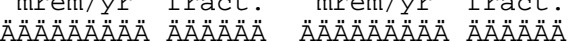

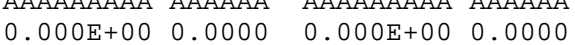
$\begin{array}{lllll}0.000 \mathrm{E}+0 \odot & 0.000 \odot & 0.000 \mathrm{E}+0 \odot & 0.000 \odot\end{array}$ $0.000 \mathrm{E}+0 \odot \quad 0.0000 \quad 0.000 \mathrm{E}+0 \odot \quad 0.00 \odot \odot$ $\begin{array}{lllll}0.000 E+00 & 0.0000 & 0.000 E+00 & 0.0000\end{array}$ $\begin{array}{llll}0.000 E+00 & 0.0000 & 0.000 \mathrm{E}+00 & 0.0000\end{array}$ Íííííííi ííííí íííííííi ííííí

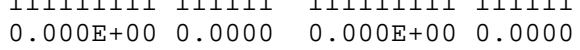


Total Dose Contributions TDOSE( $i, p, t)$ for Individual Radionuclides ( $i$ ) and Pathways ( $p$ ) Plant
ÄÄÄÄÄÄÄÄÄÄÄÄÄÄÄÄ

$\mathrm{Milk}$

mrem/yr fract. ÄÄÄÄÄÄÄÄÄÄÄÄÄÄÄÄ ӒÄӒÄÄÄÄÄÄÄÄÄÄÄÄÄ ÄÄÄÄÄÄÄÄÄÄÄÄÄÄÄÄ mrem/yr fract. ÄÄÄÄÄÄÄ ÄÄÄÄÄÄÄÄÄ ÄÄÄÄÄÄ

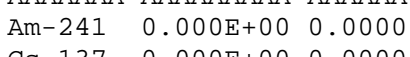
CS-137 $0.00 \odot \mathrm{E}+\odot \odot \quad 0.00 \odot \odot$ $\begin{array}{lll}\mathrm{Pu}-238 & 0.000 \mathrm{E}+0 \odot & 0.000 \\ \mathrm{Pu}-239 & 0.000 \mathrm{E}+0 \odot & 0.0000\end{array}$ ÄÄÄÄÄÄÄÄÄ ÄÄÄÄÄÄ $0.000 \mathrm{E}+000.0000 \quad 0.000 \mathrm{E}+00 \quad 0.0000 \quad 0.000 \mathrm{C}+00 \mathrm{~A}$ (1) $0.000 \mathrm{E}+00$ 0. $0000 \quad 0.000 \mathrm{E}+00$ - $0.0000 \quad 0.000 \mathrm{E}+000.0000$ $0.000 \mathrm{E}+00 \quad 0.0000$ Sr-90 $0.000 \mathrm{E}+00 \quad 0.0000$ íííííi ííííííí íííít $\odot$ $\odot .000 \mathrm{E}+00$ ०. 0.0000 $0.000 \mathrm{E}+00 \quad 0.0000$ $0.000 \mathrm{E}+00 \quad 0.0000$ $0.000 \mathrm{E}+000.0000$ $0.000 \mathrm{E}+000.0000$ $0.000 \mathrm{E}+000.0000$ IIIIIIIÍííííít $0.000 \mathrm{E}+00 \quad 0.0000$ $0.000 \mathrm{E}+00 \quad 0.0000$ Total $\odot .000 \mathrm{E}+\odot \odot \odot .0 \odot \odot \odot$ $0.000 \mathrm{E}+000.0000$

Total Dose Contributions $\operatorname{TDOSE}(i, p, t)$ for Individual Radionuclides (i) and Pathways $(p)$ As mrem/yr and Fraction of Total Dose At $t=1.000 \mathrm{E}+03$ years Water Dependent Pathways Fish Radon

Radio - ÄÄÄÄÄÄÄÄÄÄÄÄÄÄÄÄ Nuclide mrem/yr fract ÄÄÄÄÄÄÄ ÄÄÄÄÄÄÄÄÄ ÄÄÄÄÄ̈̈ $\begin{array}{lll}\mathrm{Am}-241 & 0.000 \mathrm{E}+0 \odot & \odot .0 \odot \odot \odot ~ \\ \mathrm{CS}-137 & 0.000 \mathrm{E}+0 \odot & 0.000 \odot\end{array}$ $\mathrm{Pu}-238 \quad \odot .00 \odot \mathrm{E}+\odot \odot \quad \odot . \odot \odot \odot \odot$ Pu-239 $0.000 \mathrm{E}+00 \quad 0.0000$ Sr-90 $0.000 \mathrm{E}+00 \quad 0.0000$ Íííííi ííííííí íííít

Total $\odot *$ Sum of all water independent and dependent pathways.
Plant
Meat

ÄÄÄÄÄÄÄÄÄÄÄÄÄÄ̈

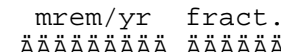
ÄÄÄÄÄÄÄÄÄ ÄÄ̈̈̈̈̈̈̈̈ $0.000 \mathrm{E}+0 \odot \quad 0.000 \odot$ $0.000 \mathrm{E}+0 \odot \quad 0.000 \odot$ $0.000 \mathrm{E}+00 \quad 0.0000$ $0.000 E+00 \quad 0.0000$ $0.000 \mathrm{E}+000.0000$ Íííííííí ííííít $0.000 \mathrm{E}+000.0000$
Milk

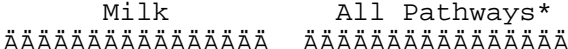
mrem/yr fract

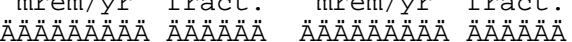

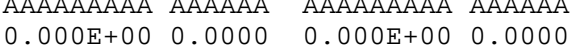
$\begin{array}{lllll}0.000 E+00 & 0.0000 & 0.000 E+00 & 0.0000\end{array}$ $0.000 \mathrm{E}+0 \odot \quad 0.0000 \quad 0.000 \mathrm{E}+0 \odot \quad 0.00 \odot \odot$ $\begin{array}{lllll}0.000 E+00 & 0.0000 & 0.000 E+00 & 0.0000\end{array}$ $\begin{array}{llll}0.000 E+00 & 0.0000 & 0.000 \mathrm{E}+00 & 0.0000\end{array}$ Ííííííí Ííííi ííííííi ííííi

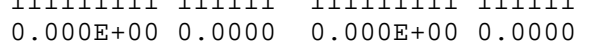


Parent and Dose/Source Ratios Summed Over All Pathways

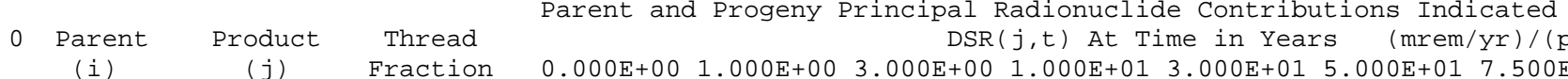

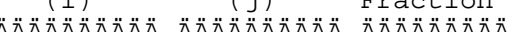

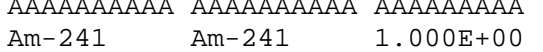

$\begin{array}{lll}A m-241 & \mathrm{~Np}-237+\mathrm{D} & 1.000 \mathrm{E}+00 \\ \mathrm{Am}-241 & \mathrm{U}-233 & 1.000 \mathrm{E}+00\end{array}$

Am-241

Am- 241

○Cs $-137+\mathrm{D}$

OPU -238

๑Pu-238

$\mathrm{Pu}-238$
$\mathrm{Pu}-238$

$\mathrm{Pu}-238$

$\mathrm{Pu}-238$

$\mathrm{Pu}-238$

$\mathrm{Pu}-238$
Pि -239

$\mathrm{Pu}-239$

Pu-239

Pu-239

$\mathrm{Pu}-239$

OSr $-90+\mathrm{D}$

$1.000 \mathrm{E}+00$

äDSR $(j)$

Cs-137+D

$\mathrm{Pu}-238$

$\mathrm{Pu}-238$

U-234

Th -230

$\mathrm{Ra}-226+\mathrm{D}$
$\mathrm{Pb}-210+\mathrm{D}$

$\operatorname{äDSR}(j)$

$\mathrm{Pu}-239$

$\mathrm{U}-235+\mathrm{D}$

$\mathrm{Pa}-231$
$\mathrm{AC}-227+\mathrm{D}$

äDSR $(j)$

1. $\odot \odot \odot E+\odot \odot$

$1.000 \mathrm{E}+\odot \odot$

1. 840E- 09

1. $.0 \odot \mathrm{E}+\odot \odot$
$1.0 \odot \odot \mathrm{E}+\odot \odot$

1. $\odot \odot \odot \mathrm{E}+\odot \odot$

$1.000 \mathrm{E}+00$

1. $\odot \odot \odot \mathrm{E}+\odot \odot$

$1.000 \mathrm{E}+00$

$1 . \odot \odot \odot E+\odot \odot$

$1.000 \mathrm{E}+0 \odot$

$\mathrm{Sr}-90+\mathrm{D}$

1. $000 \mathrm{E}+02 \quad 3.000 \mathrm{E}+02 \quad 1.000 \mathrm{E}+03$

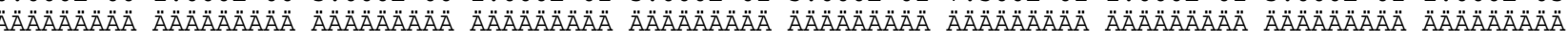

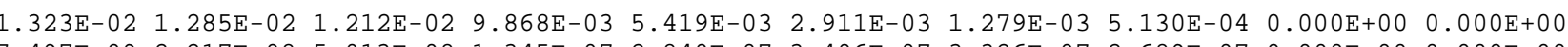

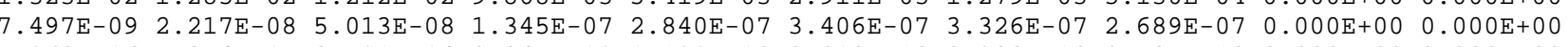

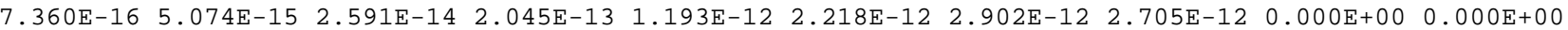
$\begin{array}{lllllll}2 & & \end{array}$

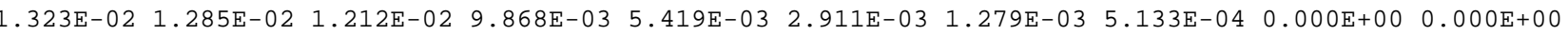

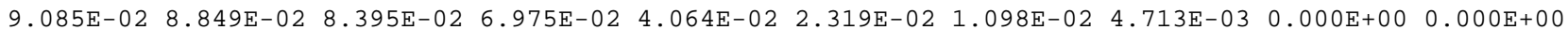

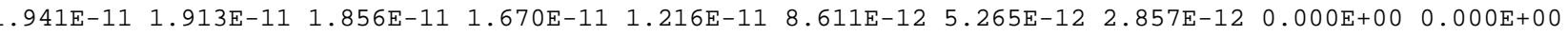

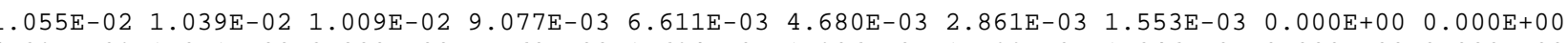

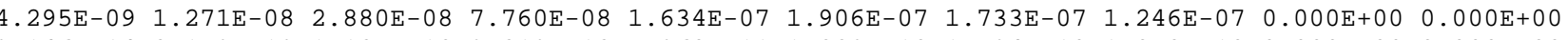
3.146E-14 2.179E-13 1.125E-12 9.219E-12 5.968E-11 1.223E-10 1.794E-10 1.858E-10 $0.000 \mathrm{E}+00 \quad 0.000 \mathrm{E}+00$

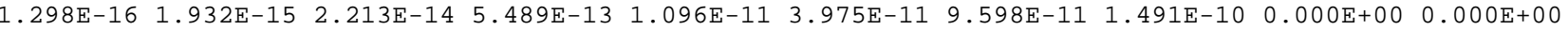

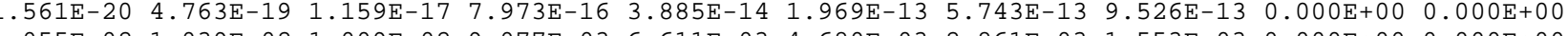

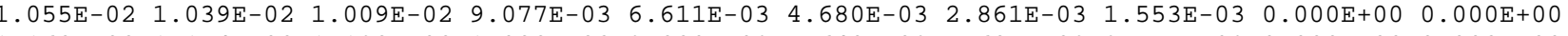
1.162E-๑2 1.154E-02 1.138E-๑2 $1.082 \mathrm{E}-02 \quad 9.220 \mathrm{E}-03 \quad 7.640 \mathrm{E}-03 \quad 5.687 \mathrm{E}-03 \quad 3.757 \mathrm{E}-03 \quad 0.000 \mathrm{E}+00 \quad 0.000 \mathrm{E}+00$ $\begin{array}{llllllllll}1.257 \mathrm{E}-11 & 3.748 \mathrm{E}-11 & 8.626 \mathrm{E}-11 & 2.464 \mathrm{E}-10 & 6.186 \mathrm{E}-10 & 8.727 \mathrm{E}-10 & 1.030 \mathrm{E}-09 & 9.901 \mathrm{E}-10 & 0.000 \mathrm{E}+00 & 0.000 \mathrm{E}+00\end{array}$

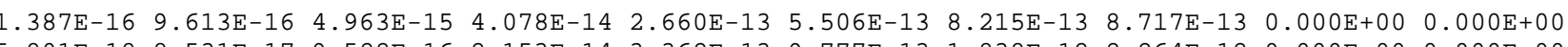

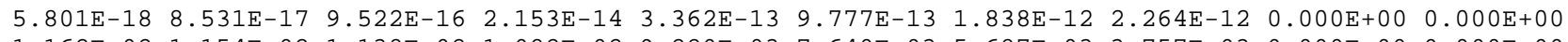
1.162E-02 1.154E-02 1.138E-02 1.082E-02 9.220E-03 7.640E-03 5.687E-03 3.757E-03 $0.000 \mathrm{E}+00 \quad 0.000 \mathrm{E}+00$

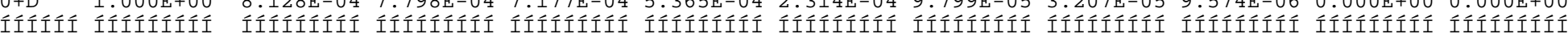

Single Radionuclide Soil Guidelines $\mathrm{G}(\mathrm{i}, \mathrm{t})$ in $\mathrm{pCi} / \mathrm{g}$ Basic Radiation Dose Limit $=2.500 \mathrm{E}+01 \mathrm{mrem} / \mathrm{yr}$

onuclide

\begin{tabular}{|c|c|c|}
\hline & $t=0.000 E+\odot \odot$ & 1.00 \\
\hline & ÄÄÄÄÄÄÄÄÄ & $\ddot{A} A ̈ A ̈ A ̈ A ̈ A ̈ A ̈$ \\
\hline Am-241 & $1.890 \mathrm{E}+03$ & $1.945 \mathrm{E}+\odot 3$ \\
\hline-137 & $2.752 \mathrm{E}+\odot 2$ & $2.825 E+02$ \\
\hline 238 & $2.370 \mathrm{E}+03$ & $2.405 \mathrm{E}+03$ \\
\hline-239 & $2.152 \mathrm{E}+03$ & $2.167 \mathrm{E}+03$ \\
\hline & $3.076 \mathrm{E}+04$ & $3.206 \mathrm{E}+\odot 4$ \\
\hline & Íííííííí & Ííííííít \\
\hline
\end{tabular}

\section{3. $0 \odot \odot E+\odot \odot$ \\ 1. $000 \mathrm{E}+01$} ÄÄÄÄÄÄÄÄÄ

2. $063 \mathrm{E}+03$

2. $978 \mathrm{E}+02$

2. $478 \mathrm{E}+03$

2. $197 \mathrm{E}+03$

3. $483 \mathrm{E}+04$ Ííííííí ÄÄÄÄÄÄÄÄÄÄ $3.584 \mathrm{E}+02$ $3.584 \mathrm{E}+02$ 2. $754 \mathrm{E}+03$ $2.311 \mathrm{E}+03$
$4.660 \mathrm{E}+04$ íííííííi $2.533 \mathrm{E}+03$

\section{$3.000 E+01$} ÄÄÄÄÄÄÄÄĂ 4. $613 \mathrm{E}+03$ $6.151 \mathrm{E}+02$ $3.782 \mathrm{E}+03$ $3.782 \mathrm{E}+03$ 2. $711 \mathrm{E}+03$ I.
5. $000 \mathrm{E}+01$ Äล̈ÄÄÄÄÄÄĂ $8.587 \mathrm{E}+03$ $1.078 \mathrm{E}+03$ 5. $342 \mathrm{E}+03$ $5.342 \mathrm{E}+03$ $2.551 \mathrm{E}+05$ ÍÍÍÍÍí
$7.500 \mathrm{E}+01$
ÄÄÄÄÄÄÄÄA
$1.955 \mathrm{E}+04$
$2.277 \mathrm{E}+03$
$8.737 \mathrm{E}+03$
$4.396 \mathrm{E}+03$
$7.795 \mathrm{E}+05$
ÍÍÍÍÍÍÍÍ
1. $000 \mathrm{E}+02$ ÄÄÄÄÄÄÄÄ $4.870 E+04$ $5.305 \mathrm{E}+03$ $1.610 \mathrm{E}+04$ $6.655 \mathrm{E}+03$ $2.611 \mathrm{E}+06$ Íííííííí
3. $000 \mathrm{E}+02$ Äล̈ล̈ล̈ล̈Äล̈Äล̈ *3. 431E+12 *8. $704 \mathrm{E}+13$ *1. $712 \mathrm{E}+13$ $1.365 \mathrm{E}+14$ íííííííi
1. $000 \mathrm{E}+03$ ÄÄÄÄÄÄÄÄÄ *3. $431 \mathrm{E}+12$ $\star 8.704 \mathrm{E}+13$ $* 1.712 \mathrm{E}+13$ $* 1.365 \mathrm{E}+14$ Íííííííi 
Summed Dose/Source Ratios DSR(i,t) in (mrem/yr)/(pCi/g)

and Single Radionuclide Soil Guidelines $G(i, t)$ in $\mathrm{pCi} / \mathrm{g}$ at $\mathrm{tmin}=\mathrm{time}$ of minimum single radionuclide soil guideline and at $\operatorname{tmax}=$ time of maximum total dose $=0.000 \mathrm{E}+00$ years

\begin{tabular}{|c|c|c|c|c|c|c|}
\hline $\begin{array}{l}\text { Juclide } \\
\text { (i) }\end{array}$ & $\begin{array}{l}\text { Initial } \\
\text { (pci/g) }\end{array}$ & $\begin{array}{c}\text { tmin } \\
\text { (years) }\end{array}$ & $\operatorname{DSR}(i, \operatorname{tmin})$ & $\begin{array}{c}\mathrm{G}(\mathrm{i}, \mathrm{tmin}) \\
(\mathrm{pCi} / \mathrm{g})\end{array}$ & $\operatorname{DSR}(i, \operatorname{tmax})$ & $\mathrm{G}(i, \operatorname{tmax})$ \\
\hline $\mathrm{Am}-2$ & 7.2 & 0 & & & & \\
\hline Cs -137 & 9.1 & $\odot . \odot \odot \odot E+\odot \odot$ & & 2.75 & -02 & re \\
\hline Pu-238 & 3.616 & $\odot .00 \odot \mathrm{E}$ & & 2.37 & 02 & 2.3 \\
\hline & & & & & & \\
\hline & & 0.0 & & 3. & & \\
\hline$x+4$ - & Ííí & Íííííi & IIIIIIIII & Ííí & Ííííííí & Ííííí \\
\hline
\end{tabular}


1RESRAD, Version 6.3

Summary : CAU 383 Pond

$\mathrm{T} \ll$ Limit $=180$ days

05/11/2006 11:16 Page 25

File: CAU 383 Ponds.RAD onuclide Parent THF(i) (j) (i)

ÄÄÄÄÄÄÄ ÄÄÄÄÄÄÄ ÄÄÄÄÄÄÄÄÄ Am-241 Am-241 1.000E+O९ ONp-237 Am-241 1.000E+O० OU-233 Am-241 1.000E +00

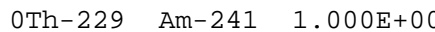
OCS -137 CS $-137 \quad 1.000 \mathrm{E}+00$ OPU -238 Pu-238 $1.840 E-09$ Pu-238 Pu-238 $1.840 \mathrm{E}-09$ $\begin{array}{ll}\mathrm{Pu}-238 & \mathrm{Pu}-238 \text { 1.000E+00 } \\ \mathrm{Pu}-238 & \text { äDOSE }(\mathrm{j})\end{array}$ Pu-238 äDOSE $(j)$

OU-234 PU-238 $1.000 \mathrm{E}+00$ OTh-230 Pu-238 $1.000 \mathrm{E}+00$

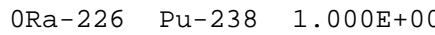
$\odot \mathrm{Pb}-210 \quad \mathrm{Pu}-238 \quad 1.000 \mathrm{E}+00$ $\odot P u-239$ Pu-239 $1.000 E+00$ OU-235 PU $2391.000 E+00$ OU -235 Pu-239 $1.000 \mathrm{E}+00$ OPa-231 $\mathrm{Pu}-239 \quad 1.000 \mathrm{E}+00$ $\odot \mathrm{AC}-227 \quad \mathrm{Pu}-239 \quad 1.000 \mathrm{E}+00$

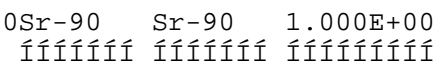

Individual Nuclide Dose Summed Over All Pathways

Parent Nuclide and Branch Fraction Indicated

$\operatorname{DOSE}(j, \mathrm{t}), \mathrm{mrem} / \mathrm{yr}$

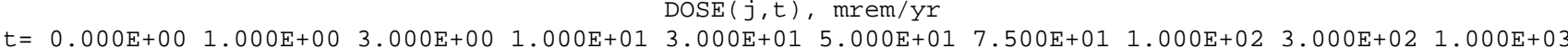

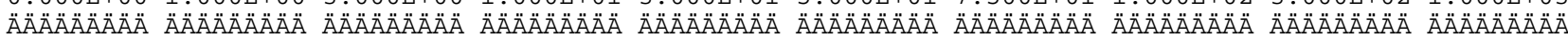

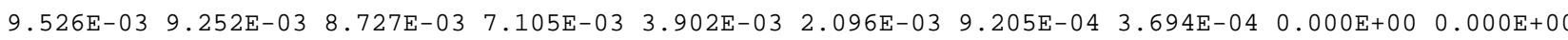

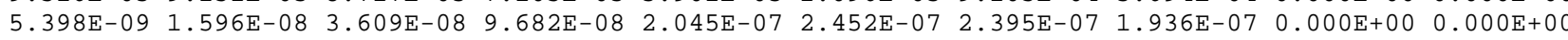
5.299E-16 3.653E-15 1.865E-14 1.472E-13 8.592E-13 1.597E-12 2.090E-12 1.948E-12 0.000E+00 $0.000 \mathrm{E}+00$ 3.765E-19 5.585E-18 $6.353 \mathrm{E}-17$ 1.537E-15 2.871E-14 $9.805 \mathrm{E}-14 \quad 2.212 \mathrm{E}-13 \quad 3.228 \mathrm{E}-13 \quad 0.000 \mathrm{E}+00 \quad 0.000 \mathrm{E}+00$

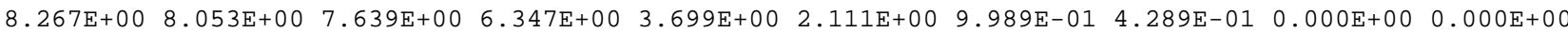
7. $007 \mathrm{E}-12 \quad 6.904 \mathrm{E}-12 \quad 6.702 \mathrm{E}-12 \quad 6.029 \mathrm{E}-12 \quad 4.391 \mathrm{E}-12 \quad 3.109 \mathrm{E}-12 \quad 1.901 \mathrm{E}-12 \quad 1.031 \mathrm{E}-12 \quad 0.000 \mathrm{E}+00 \quad 0.000 \mathrm{E}+00$

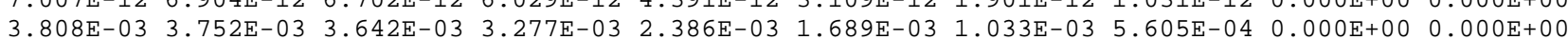

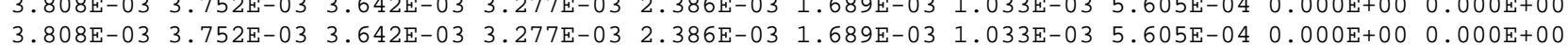

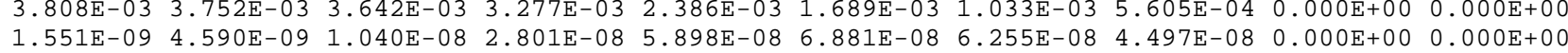

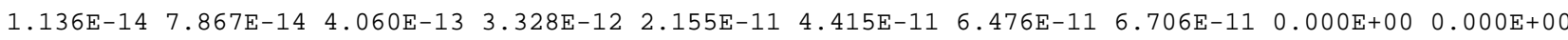
4.687E-17 6.975E-16 7.990E-15 1.981E-13 3.955E-12 1.435E-11 3.465E-11 5.384E-11 $0.000 \mathrm{E}+00 \quad 0.000 \mathrm{E}+0 \odot$ 5.634E-21 1.719E-19 4.183E-18 2.878E-16 1.402E-14 7.107E-14 2.073E-13 3.439E-13 0.000E+00 $0.000 \mathrm{E}+00$ 5.217E-02 5.181E-02 5.109E-02 4.856E-02 4.140E-02 3.431E-02 2.553E-02 1.687E-๑2 $0.000 E+00 \quad 0.000 \mathrm{E}+00$

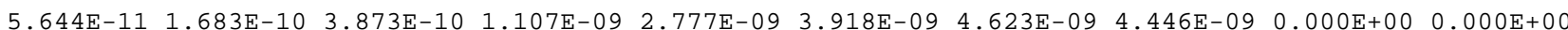

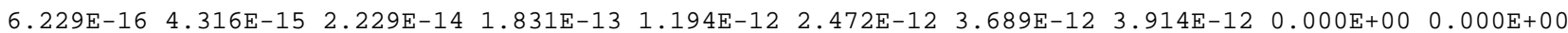

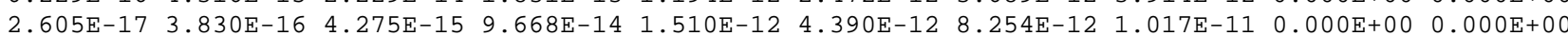

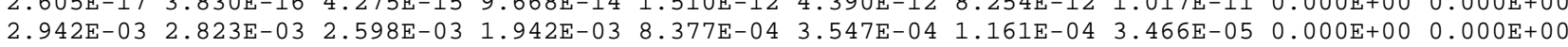

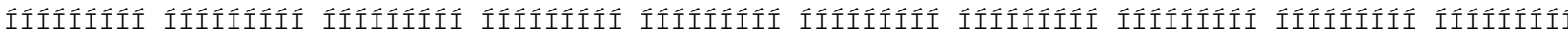


1RESRAD, Version 6.3

Summary : CAU 383 Pond

$\mathrm{T} \ll$ Limit $=180$ days

05/11/2006 11:16 Page 26

File: CAU 383 Ponds. RAD
ONuclide Parent THF(i) (j) (i)

ÄÄÄÄÄÄÄ ÄÄÄÄÄÄÄ ÄÄÄÄÄÄÄÄÄ Am-241 Am-241 1.000E+०๑ ONp-237 Am-241 1.000E+O० OU-233 Am-241 1.000E $+0 \odot$ OTh-229 Am-241 1.000E+०० OCS -137 CS $-137 \quad 1.000 \mathrm{E}+00$ OCS -137 Cs $-1371.00 \mathrm{P})$ $\begin{array}{lll}\mathrm{PU}-238 & \mathrm{Pu}-238 & 1.840 \mathrm{E}-09\end{array}$ $\begin{array}{lll}\mathrm{Pu}-238 & \mathrm{Pu}-238 & 1.000 \mathrm{E}+00 \\ \mathrm{Pu}-238 & \text { äS }(\mathrm{j}): & \end{array}$ Pu-238 äS $(j)$ :

०U-234 PU-238 1.000E+०० OTh-230 Pu-238 1.000E+00 ORa-226 Pu-238 1.000E+00 OPb-210 Pu-238 1.000E +00 $\odot \mathrm{Pu}-239 \mathrm{Pu}-239 \quad 1.000 \mathrm{E}+00$ OU -235 Pu-239 $1.000 E+00$ OU-235 PU-239 $1.000 \mathrm{E}+00$ $\odot \mathrm{Pa}-231 \quad \mathrm{Pu}-239 \quad 1.000 \mathrm{E}+00$ OAC-227 Pu-239 1.000E+०० $\odot \mathrm{Sr}-9 \odot \quad \mathrm{Sr}-9 \odot \quad 1.00 \odot \mathrm{E}+\Theta \odot$ IIÍííi Iíííí ííííííít THF(i) is the thread fraction
Individual Nuclide Soil Concentration

Parent Nuclide and Branch Fraction Indicated

$$
\mathrm{S}(j, \mathrm{t}), \mathrm{pCi} / \mathrm{g}
$$

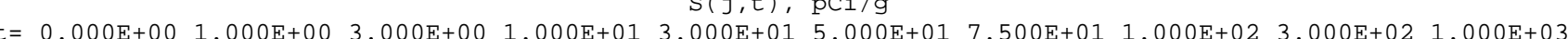
ÄÄÄÄÄÄÄÄ Ä ÄÄÄÄÄÄÄÄ ÄÄÄÄÄÄÄÄ 7.200E-01 7.035E-01 6.717E-01 5.713E-01 3.597E-01 $2.265 E-01$ 1.270E-01 7.123E-02 6.973E-04 6.470E-11

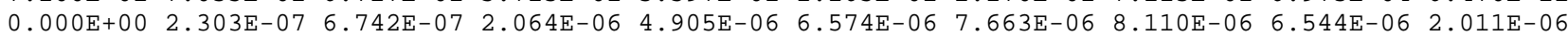
$0.000 \mathrm{E}+00$ 5.043E-13 4.439E-12 4.565E-11 3.313E-10 7.475E-10 $1.311 \mathrm{E}-09$ 1.835E-09 $3.361 \mathrm{E}-09$ 1.259E-09 $0.000 \mathrm{E}+00$ 1.592E-17 $4.226 \mathrm{E}-16 \quad 1.477 \mathrm{E}-14 \quad 3.393 \mathrm{E}-13 \quad 1.345 \mathrm{E}-12 \quad 3.769 \mathrm{E}-12 \quad 7.481 \mathrm{E}-12 \quad 6.231 \mathrm{E}-11 \quad 2.031 \mathrm{E}-10$ $\begin{array}{llllllllll}9.100 \mathrm{E}+01 & 8.891 \mathrm{E}+01 & 8.488 \mathrm{E}+01 & 7.216 \mathrm{E}+01 & 4.537 \mathrm{E}+01 & 2.853 \mathrm{E}+01 & 1.597 \mathrm{E}+01 & 8.943 \mathrm{E}+00 & 8.638 \mathrm{E}-02 & 7.650 \mathrm{E}-09\end{array}$

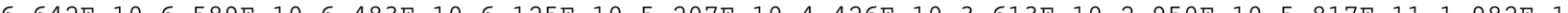

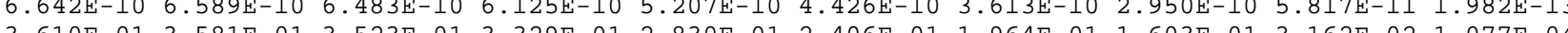
$3.610 \mathrm{E}-013.581 \mathrm{E}-013.523 \mathrm{E}-013.329 \mathrm{E}-012.830 \mathrm{E}-012.406 \mathrm{E}-01$ 1.964E-01 $1.603 \mathrm{E}-01$ 3.162E-02 $1.077 \mathrm{E}-04$ $3.610 E-013.581 E$ $0.000 \mathrm{E}+00$ 1.015E-06 2.994E-06 9.411E-06 2.387E-05 3.364E-05 4.091E-05 4.423E-05 2.480E-05 2.353E-07 $0.000 \mathrm{E}+00$ 4.581E-12 4.077E-11 4.356E-10 3.511E-09 $\quad 8.747 \mathrm{E}-09$ 1.722E-08 2.685E-08 $9.354 \mathrm{E}-08$ 1.291E-07 $0.000 \mathrm{E}+00$ 6.613E-16 $1.765 \mathrm{E}-14 \quad 6.274 \mathrm{E}-13$ 1.508E-11 $6.223 \mathrm{E}-11$ 1.821E-10 $3.750 \mathrm{E}-10$ 3.493E-09 $\quad 8.326 \mathrm{E}-09$ $0.000 \mathrm{E}+00$ 5.109E-18 $\quad 4.042 \mathrm{E}-16$ 4.601E-14 2.973E-12 $1.846 \mathrm{E}-11 \quad 7.205 \mathrm{E}-11$ 1.775E-10 2.630E-09 $7.283 \mathrm{E}-09$

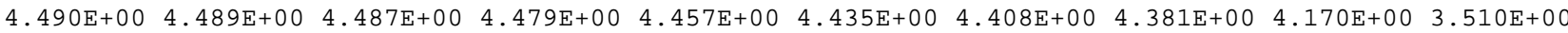

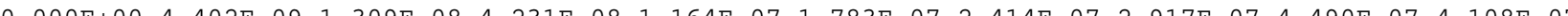

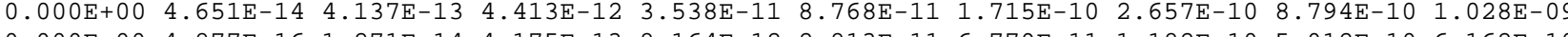
$0.000 \mathrm{E}+00$ 4.877E-16 1.271E-14 4.175E-13 8.164E-12 $2.813 \mathrm{E}-11$ 6.779E-11 1.182E-10 5.012E-10 6.162E-10

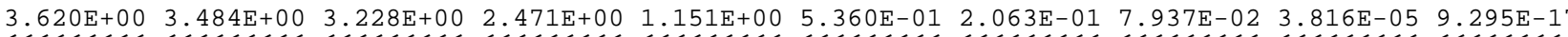

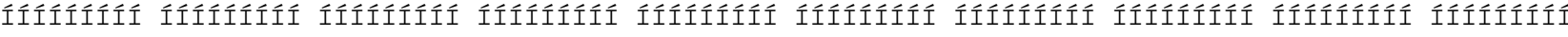
the parent nuclide.

1.41 seconds 


\section{Exhibit 2}

\section{RESRAD Summary Report: CAU 383 CAS 12-25-02}

Oil Spill: 20 Pages 
1RESRAD, Version 6.3 T« Limit $=180$ days

Summary : CAU 383 Oil spill

Table of Contents

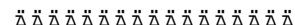

Part I: Mixture sums and Single Radionuclide Guidelines

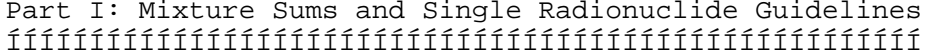

Dose Conversion Factor (and Related) Parameter Summary ... Site-Specific Parameter Summary $\ldots \ldots \ldots \ldots \ldots \ldots \ldots \ldots$ Summary of Pathway Selections

Contaminated Zone and Total Dose Summary

Total Dose Components

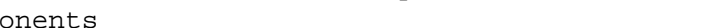

Time $=0.000 \mathrm{E}+00$

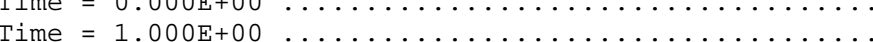

Time $=3.000 \mathrm{E}+00$

Time $=1.000 \mathrm{E}+01$

Time $=5.000 \mathrm{E}+01$

Time $=7.500 \mathrm{E}+01$

Time $=1.000 \mathrm{E}+02$

Time $=3.000 \mathrm{E}+02$

Time $=1.000 \mathrm{E}+03$

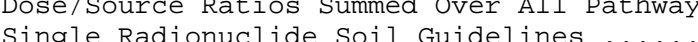


Dose Conversion Factor (and Related) Parameter Summary File: FGR 13 MORBIDITY

$\odot \quad 3$

Menu 3 Parameter 3 value 3 Case* 3 Name

3 Current 3 Base 3 Parameter

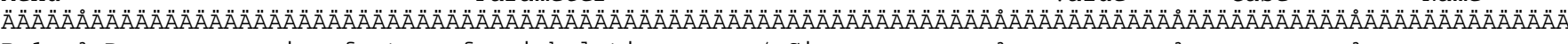

B-1 3 Dose conversion factors for inhalation, $\mathrm{mrem} / \mathrm{pCi}$

B-1 3 AC-227+D

$B-1 \quad 3$ CS $-137+D$

$\mathrm{B}-1 \quad 3 \quad \mathrm{~Pa}-231$

$\mathrm{B}-1 \quad 3 \mathrm{Pu}-239$

B-1 3 U-235+D

D-1 3 Dose conversion factors for ingestion, $\mathrm{mrem} / \mathrm{pCi}$ :

D-1 3 AC $-227+D$

D-1 3 CS $-137+D$

$\mathrm{D}-1 \quad 3 \mathrm{~Pa}-231$

D-1 13 Pu-239

D-1 3 U-235+D

D-34 3 Food transfer factors:

D-34 3 AC-227+D, plant/soil concentration ratio, dimensionless

$\mathrm{D}-343 \mathrm{AC}-227+\mathrm{D}$, beef/livestock-intake ratio, ( $\mathrm{pCi} / \mathrm{kg}) /(\mathrm{pCi} / \mathrm{d})$

$\mathrm{D}-343 \mathrm{AC}-227+\mathrm{D}, \mathrm{milk} /$ livestock-intake ratio, (pCi/L)/(pCi/d)

D-34 3

D-34 3 Cs-137+D , plant/soil concentration ratio, dimensionless

$\mathrm{D}-343 \mathrm{Cs}-137+\mathrm{D}$, beef/livestock-intake ratio, $(\mathrm{pCi} / \mathrm{kg}) /(\mathrm{pCi} / \mathrm{d})$

$\mathrm{D}-343 \mathrm{Cs}-137+\mathrm{D}$, milk/livestock-intake ratio, $(\mathrm{pCi} / \mathrm{L}) /(\mathrm{pCi} / \mathrm{d})$

$\mathrm{D}-344^{3}$

D-34 3 Pa-231

$\mathrm{D}-34 \quad \mathrm{~Pa}-231$

$\mathrm{D}-344^{3} \mathrm{~Pa}-231$

D-34 3

D-34 3 Pu-239

D-34 3 Pu-239

D 34 Pu 239

milk/livestock-intake ratio, ( $\mathrm{pCi} / \mathrm{L}) /(\mathrm{pCi} / \mathrm{d})$

, plant/soil concentration ratio, dimensionless , milk/livestock-intake ratio, $(\mathrm{pCi} / \mathrm{L}) /(\mathrm{pCi} / \mathrm{d})$

$\begin{array}{lr}3 & 3 \\ 3 & 6.724 \mathrm{E}+00^{3}\end{array}$

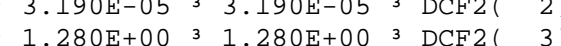

$4.290 \mathrm{E}-01301.290 \mathrm{E}-013 \mathrm{DCF} 2(-4)$

$\begin{array}{l}1.230 E-01 \\ 3\end{array} 1.230 E-013$ DCF2( 5$)$

3 1.230E-01 ${ }_{3}^{3} 1.230 \mathrm{E}-01{ }_{3}^{3}$

1.480E-02 3 1.410E-02 3 DCF3( 1$)$

$5.000 \mathrm{E}-05$ 3 $5.000 \mathrm{E}-05{ }^{3} \mathrm{DCF} 3(2)$

$1.060 \mathrm{E}-022^{3} 1.060 \mathrm{E}-02{ }^{3} \mathrm{DCF} 3(3)$

$3.540 \mathrm{E}-03$ 3 $3.540 \mathrm{E}-03$ 3 DCF3( 4$)$

$2.673 \mathrm{E}-044^{3} 2.660 \mathrm{E}-04{ }^{3}$ DCF3( 5$)$

3 3

3 2.500E-03 3 2.500E-03 3 RTF $(1,1)$

$2.000 \mathrm{E}-055^{3} 2.000 \mathrm{E}-055^{3} \operatorname{RTF}(1,2)$

2. $000 \mathrm{E}-05$ 3 2. $2.00 \mathrm{E}-053 \operatorname{RTF}(1,3)$

3 4. $000 \mathrm{E}-02 \quad 3 \quad 4.000 \mathrm{E}-02 \quad 3 \operatorname{RTF}(2,1)$

3.

8. 000E-03 3 8.000E-03 3 RTF( 2,3)

3 3

1. $000 \mathrm{E}-02 \quad 3 \quad 1.000 \mathrm{E}-023 \operatorname{RTF}(3,1)$

3 5.

5. 5. 00०E-06 $35.000 \mathrm{E}-06$ 3 $\operatorname{RTF}(3,3)$

3 1. $000 \mathrm{E}-03 \quad 3 \quad 1.000 \mathrm{E}-033 \operatorname{RTF}(4,1)$

plant/soil concentration ratio, dimensionless beef/livestock-intake ratio, $(\mathrm{pCi} / \mathrm{kg}) /(\mathrm{pCi} / \mathrm{d})$

milk/livestock-intake ratio, $(\mathrm{pCi} / \mathrm{L}) /(\mathrm{pCi} / \mathrm{d})$

D-34 3

$\mathrm{D}-343 \mathrm{U}-235+\mathrm{D}$, plant/soil concentration ratio, dimensionless

$\mathrm{D}-34{ }^{3} \mathrm{U}-235+\mathrm{D}$, , beef/livestock-intake ratio, ( $\left.\mathrm{pCi} / \mathrm{kg}\right) /(\mathrm{pCi} / \mathrm{d})$

$\mathrm{D}-34$ $3 \mathrm{U}-235+\mathrm{D}$, milk/livestock-intake ratio, $(\mathrm{pCi} / \mathrm{L}) /(\mathrm{pCi} / \mathrm{d})$

1.000E-04 3 1.000E-04 3 RTF $(4,2)$

1.000E-06 3 1.000E-06 3 RTF( 4,3$)$

$2.500 \mathrm{E}-03 \quad 3 \quad 2.500 \mathrm{E}-03 \quad 3 \operatorname{RTF}(5,1)$

3. 400E- 04 3 $3.400 \mathrm{E}-04$ 3 $\operatorname{RTF}(5,2)$

3 6. $000 \mathrm{E}-044^{3}$ 6. $600 \mathrm{E}-04{ }^{3} \operatorname{RTF}(5,3)$

D-5 3 Bioaccumulation factors, fresh water, L/ kg:

$D-5 \quad 3$ AC-227+D, fish

D-5 3 AC-227+D， crustacea and mollusks

D $-5 \quad 3$

D-5 3 Cs $-137+D$, fish

D-5 3 Cs $-137+D$, crustacea and mollusks

D-5 3

D-5 3 Pa-231, fish

D-5 $3 \mathrm{~Pa}-231$, crustacea and mollusks

D-5 3 Pu-239, fish

D-5 3 Pu-239 , crustacea and mollusks

3

$31.500 \mathrm{E}+0131.500 \mathrm{~B}+013 \operatorname{BIOFAC}(1,1)$

$1.000 \mathrm{E}+03 \quad 31.000 \mathrm{E}+033^{3} \operatorname{BIOFAC}(1,2)$

$32.000 \mathrm{E}+03 \quad 3 \quad 2.000 \mathrm{E}+03 \quad 3 \quad \operatorname{BIOFAC}(2,1)$

$1.000 \mathrm{E}+\odot 2$ 3 $1.000 \mathrm{E}+\odot 2$ 3 $\operatorname{BIOFAC}(2,2)$

$31.000 \mathrm{E}+0131.000 \mathrm{E}+013 \operatorname{BIOFAC}(3,1)$

$1.100 \mathrm{E}+02 \quad 3 \quad 1.100 \mathrm{E}+02 \quad 3 \operatorname{BIOFAC}(3,2)$

$3.000 \mathrm{E}+0133.000 \mathrm{E}+013 \mathrm{BIOFAC}(4,1)$

1. $000 \mathrm{E}+0231.000 \mathrm{E}+02{ }^{3} \operatorname{BIOFAC}(4,2)$

$\begin{array}{lll}D-5 & 3 & \\ D-5 & 3 & U-235+D, f i s h\end{array}$

$\begin{array}{llll}D-5 & 3 & \mathrm{U}-235+D & \text {, fish } \\ \mathrm{D}-5 & 3 & \mathrm{U}-235+\mathrm{D} & \text {, crustacea and mollusks }\end{array}$

$\begin{array}{lllll}1.00 \odot E+01 & 3 & 1.000 E+01 & 3 & \operatorname{BIOFAC}(5,1)\end{array}$

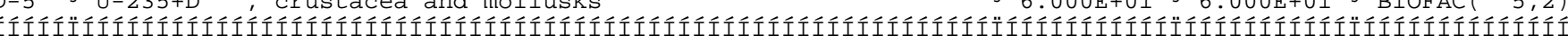

*Base Case means Default.Lib w/o Associate Nuclide contributions. 
Menu 3 Parameter 3 Input 3 Default 3 (If different from user input) 3 Name

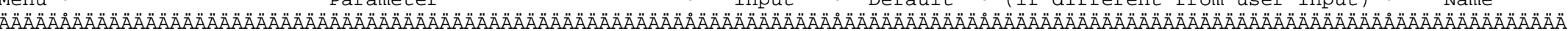

R011 3 Area of contaminated zone $\left(m^{* *} 2\right)$

R011 3 Thickness of contaminated zone $(\mathrm{m})$

R011 3 Length parallel to aquifer flow $(\mathrm{m})$

R011 3 Basic radiation dose limit (mrem/yr)

R011 3 Time since placement of material ( $y r$ )

R011 3 Times for calculations ( $y r$ )

R011 3 Times for calculations ( $\mathrm{yr}$ )

R011 3 Times for calculations ( $\mathrm{yr}$ )

R011 3 Times for calculations ( $y r$ )

R011 3 Times for calculations ( $\mathrm{yr}$ )

R011 3 Times for calculations ( $\mathrm{yr}$ )

R011 3 Times for calculations ( $y r$ )

R011 3 Times for calculations ( $y r$ )

R011 3 Times for calculations ( $y r$ )

R012 3 Initial principal radionuclide ( $\mathrm{pCi} / \mathrm{g}): \mathrm{Cs}-137$ $\mathrm{R} 0123$ Initial principal radionuclide (pCi/g): $\mathrm{Pu}-239$ $\mathrm{R} 0123$ Concentration in groundwater (pCi/L): RO12 Concentration in groundwater (pCi/L): Pu-239 R013 3 Cover depth (m)

R013 3 Density of cover material $\left(\mathrm{g} / \mathrm{cm}^{* *} 3\right)$

R013 3 Cover depth erosion rate ( $\mathrm{m} / \mathrm{yr}$ )

R013 3 Density of contaminated zone $\left(\mathrm{g} / \mathrm{cm}^{* *} 3\right)$

R013 3 Contaminated zone erosion rate $(\mathrm{m} / \mathrm{yr})$

R013 3 Contaminated zone total porosity

R013 3 Contaminated zone field capacity

R013 3 Contaminated zone hydraulic conductivity ( $\mathrm{m} / \mathrm{yr}$ ) R013 3 Contaminated zone b parameter

R013 3 Average annual wind speed $(\mathrm{m} / \mathrm{sec})$

R013 3 Humidity in air $\left(\mathrm{g} / \mathrm{m}^{* *} 3\right)$

R013 3 Evapotranspiration coefficient

R013 3 Precipitation ( $\mathrm{m} / \mathrm{yr}$ )

R013 3 Irrigation ( $\mathrm{m} / \mathrm{yr}$ )

R013 3 Irrigation mode

R013 3 Runoff coefficient

R013 3 Watershed area for nearby stream or pond $(m * * 2)$

R013 3 Accuracy for water/soil computations

$$
3
$$

R014 3 Density of saturated zone $\left(\mathrm{g} / \mathrm{cm}^{* *} 3\right)$

R014 3 Saturated zone total porosity

R014 3 Saturated zone effective porosity

R014 3 Saturated zone field capacity

R014 3 Saturated zone hydraulic conductivity (m/yr)

R014 3 Saturated zone hydraulic gradient

R014 3 Saturated zone b parameter

R014 3 water table drop rate $(\mathrm{m} / \mathrm{yr})$

R014 3 Well pump intake depth ( $\mathrm{m}$ below water table)

R014 3 Model: Nondispersion (ND) or Mass-Balance (MB)

R014 3 Well pumping rate $\left(m^{* *} 3 / \mathrm{yr}\right)$
$31.000 \mathrm{E}+02$ 3 $1.000 \mathrm{E}+04$

$1.500 \mathrm{E}-01$ 3 $2.000 \mathrm{E}+00$

3 not used $31.000 \mathrm{E}+022^{3}$

$\begin{array}{llll}3 & 2.500 \mathrm{E}+01 & 3 & 3.000 \mathrm{E}+01\end{array}$

$30.000 E+00 \quad 3 \quad 0.000 E+00$

1. $1.000 \mathrm{E}+00^{3} \quad 1.000 \mathrm{E}+00^{3}$

$3 \quad 3.000 \mathrm{E}+00 \quad 3 \quad 3.000 \mathrm{E}+00 \quad 3$

1. $000 \mathrm{E}+01$ 3 1. 1. $000 \mathrm{E}+01$

3. $000 \mathrm{E}+01$ 3 3. $3000 \mathrm{E}+01$

$5.000 \mathrm{E}+0131.000 \mathrm{E}+02$

7.500E+O1 3 3.000E+O2

$1.000 \mathrm{E}+02$ 3 1.000E+03

3. $1000 \mathrm{E}+02 \quad 3 \quad 0.000 \mathrm{E}+00$

$1.000 \mathrm{E}+03 \quad 3 \quad 0.000 \mathrm{E}+00$

2. $050 \mathrm{E}+013 \quad 0.000 \mathrm{E}+00$

$1.04 \odot \mathrm{E}+\odot \odot \quad 3 \quad 0.0 \odot \odot \mathrm{E}+\odot \odot 3$

not used $30.000 \mathrm{E}+00^{3}$

not used $30.000 \mathrm{E}+0 \odot^{3}$

3 ๑.

3 not used 3 1.500E +00

not used $31.000 \mathrm{E}-03$

$1.500 \mathrm{E}+00$ 3 $1.500 \mathrm{E}+00$

$1.500 E+00$ 3 $1.500 E+00$ 3

$1.000 \mathrm{E}-03$ 1.000E-03

4.

2.000E-01 3 2.000E-01

$1.000 \mathrm{E}+013$ 1.000E+01

$5.300 \mathrm{E}+00$ 3 $5.300 \mathrm{E}+00$

3. $300 \mathrm{E}+0 \odot \quad 3 \quad 2.000 \mathrm{E}+\odot \odot$

not used 3 8.000E+00

35 . $000 \mathrm{E}-013 \quad 5,000 \mathrm{E}-01$

$3.260 \mathrm{E}-0131.000 \mathrm{E}+0 \mathrm{C}^{3}$

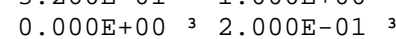

overhead 3 overhead

4.000E-01 3 2.000E-01

not used $31.000 \mathrm{E}+06$

not used 3 1.000E-03

3 not used 3 1.500E $+00^{3}$

not used 3 4.000E-01

not used 3 2.000E-01

not used $32.000 \mathrm{E}-01$

not used $31.000 \mathrm{E}+02$

not used 3 2.000E-02

not used 3 5.300E+00

not used 3 1. $000 \mathrm{E}-03$

3 not used $31.000 \mathrm{E}+01$

3 not used 3 ND

3 not used $32.500 \mathrm{E}+\odot 2^{3}$
- . $\quad 3$ AREA

3 THICKO

3 LCZPAQ

3 BRDL

3 TI

$3 \mathrm{~T}(2)$

$3 \mathrm{~T}(3)$

3 T( 4$)$

3 T $(5)$

$3 \mathrm{~T}(6)$

$\begin{array}{lll}3 & \mathrm{~T} & (8) \\ 3 & \mathrm{~T}(9) & 9\end{array}$

$\mathrm{T}(10)$

S1 ( 2)

S1( 4$)$

W1 ( 2)

3 W1 ( 4)

3 COVERO

DENSCV

VCV

3 DENSCZ

$3 \mathrm{VCZ}$

3 TPCZ

3 FCCZ

3 HCCZ

$3 \mathrm{BCZ}$

3 WIND

3 HUMID

3 EVAPTR

3 PRECIP

3 RI

3 IDITCH

3 RUNOFF

3 WAREA

3 EPS

3 DENSAQ

3 TPSZ

3 EPSZ

3 FCSZ

3 HCSZ

3 HGWT

3 BSZ

3 VWT

3 DWIBWT

3 MODEL

3 UW 
1RESRAD, Version $6.3 \quad$ T« Limit $=180$ days $\quad 05 / 11 / 2006$ 16:58 Page 4
Summary : CAU 383 0il Spill
File: CAU 383 0il Spill. RAD

Site-Specific Parameter Summary (continued)

$0 \quad 3$

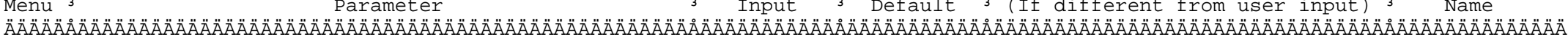

R015 3 number of unsaturated zone strata

R015 3 Unsat. zone 1, thickness (m)

R015 3 Unsat. zone 1 , soil density $\left(\mathrm{g} / \mathrm{cm}^{* *} 3\right)$

R015 3 Unsat. zone 1 , total porosity

R015 3 Unsat. zone 1, effective porosity

R015 3 Unsat. zone 1, field capacity

R015 3 Unsat. zone 1, soil-specific b parameter

R015 3 Unsat. zone 1, hydraulic conductivity (m/yr)

R016 3 Distribution coefficients for Cs -137

R016 3 Contaminated zone $\left(\mathrm{cm}^{* *} 3 / \mathrm{g}\right)$

R016 3 Unsaturated zone $1\left(\mathrm{~cm}^{* *} 3 / \mathrm{g}\right)$

R016 3 Saturated zone $\left(\mathrm{cm}^{* *} 3 / \mathrm{g}\right)$

R016 3 Leach rate (/yr)

R016 3 Solubility constant

R016 3 Distribution coefficients for Pu-239

R016 3 Contaminated zone $\left(\mathrm{cm}^{* *} 3 / \mathrm{g}\right)$

R016 3 Unsaturated zone $1\left(\mathrm{~cm}^{* *} 3 / \mathrm{g}\right)$

R016 3 Saturated zone $\left(\mathrm{cm}^{* *} 3 / \mathrm{g}\right)$

R016 3 Leach rate (/yr)

R016 3 Solubility constant

R016 3 Distribution coefficients for daughter AC-227

R016 3 Contaminated zone $\left(\mathrm{cm}^{* *} 3 / \mathrm{g}\right)$

R016 3 Unsaturated zone $1\left(\mathrm{~cm}^{* *} 3 / \mathrm{g}\right)$

R016 3 Saturated zone $\left(\mathrm{cm}^{* *} 3 / \mathrm{g}\right)$

R016 3 Leach rate (/yr)

Ro16 3 Solubility constant

R016 3 Distribution coefficients for daughter Pa-231

R016 3 Contaminated zone $\left(\mathrm{cm}^{* *} 3 / \mathrm{g}\right)$

R016 3 Unsaturated zone $1\left(\mathrm{~cm}^{* *} 3 / \mathrm{g}\right)$

R016 3 Saturated zone $\left(\mathrm{cm}^{* *} 3 / \mathrm{g}\right)$

R016 3 Leach rate (/yr)

R016 ${ }^{3}$ Solubility constant

R016 3 Distribution coefficients for daughter U-235

R016 3 Contaminated zone $\left(\mathrm{cm}^{* *} 3 / \mathrm{g}\right)$

R016 3 Unsaturated zone $1\left(\mathrm{~cm}^{* *} 3 / \mathrm{g}\right)$

R016 3 Saturated zone $\left(\mathrm{cm}^{* *} 3 / \mathrm{g}\right)$

R016 3 Leach rate (/yr)

R016 3 Solubility constant

R017 3 Inhalation rate $\left(m^{* *} 3 / y r\right)$

R017 3 Mass loading for inhalation $(\mathrm{g} / \mathrm{m} * * 3)$

R017 3 Exposure duration

R017 3 Shielding factor, inhalation

R017 3 Shielding factor, external gamma

R017 3 Fraction of time spent indoors

R017 3 Fraction of time spent outdoors (on site)

3 not used 31

not used 3 (

not used $3.000 \mathrm{E}+00$

not used $31.500 \mathrm{E}+00$

not used 3 4.000E-01

not used $32.000 \mathrm{E}-01$

3 not used 3 2.000E-01

3 not used $35.300 \mathrm{E}+0{ }^{3}$

not used 3 1.000E+O1

$4.600 \mathrm{E}+03 \quad 3 \quad 4.600 \mathrm{E}+03$

3 not used $34.600 \mathrm{E}+03$

3 not used 3 4.600E +03

$30.000 E+00 \quad 3 \quad 0.000 E+0 \odot 3$

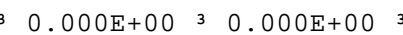

$2.000 \mathrm{E}+03 \quad 3 \quad 2.000 \mathrm{E}+03$

not used $32.000 \mathrm{E}+03$

not used $32.000 \mathrm{E}+03$

$0.000 \mathrm{E}+\odot \odot \quad 3 \quad 0.000 \mathrm{E}+\odot \odot$

$0.000 \mathrm{E}+0 \odot^{3} \quad 0.000 \mathrm{E}+00^{3}$

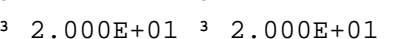

3 not used $32.000 \mathrm{E}+01$

not used $32.000 E+01$

$0.000 \mathrm{E}+00 \quad 3 \quad 0.000 \mathrm{E}+00$

$0.000 \mathrm{E}+003 \quad 0.000 \mathrm{E}+00$

$5.000 \mathrm{E}+01 \quad 3 \quad 5.000 \mathrm{E}+01$

not used $35.000 \mathrm{E}+01$

not used $35.000 \mathrm{E}+01$

$0.000 \mathrm{E}+0 \odot \quad 3 \quad 0.000 \mathrm{E}+0 \odot$

$\odot .000 \mathrm{E}+\odot \odot \quad 3 \quad \odot .00 \odot \mathrm{E}+\odot \odot$

$5.000 \mathrm{E}+01 \quad 3 \quad 5.000 \mathrm{E}+01$

3 not used 3 5. $000 \mathrm{E}+01$

not used 3 5. $000 E+01$

$0.000 \mathrm{E}+003 \quad 0.000 \mathrm{E}+003$

$0.000 E+003 \quad 0.000 E+00$

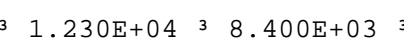

6.000E-04 3 1.000E-04

$32.500 \mathrm{E}+011^{3} 3.000 \mathrm{E}+011^{3}$

$1.000 \mathrm{E}+00 \quad 3 \quad 4.000 \mathrm{E}-01$

1. $000 \mathrm{E}+003 \quad 3.000 \mathrm{E}-013$

$0.000 \mathrm{E}+00$ 3 $5.000 \mathrm{E}-01$

$3.800 \mathrm{E}-\mathrm{O} 2$ 3 $2.500 \mathrm{E}-01$

Used by RESRAD
ferent from user input)

\begin{tabular}{|c|c|c|c|}
\hline-- & 3 & NS & \\
\hline-- & 3 & $H(1)$ & \\
\hline-- & 3 & DENSUZ( & \\
\hline-- & 3 & TPUZ(1) & \\
\hline-- & 3 & $\operatorname{EPUZ}(1)$ & \\
\hline-- & 3 & FCUZ(1) & \\
\hline-- & 3 & $\operatorname{BUZ}(1)$ & \\
\hline-- & 3 & $\operatorname{HCUZ}(1)$ & \\
\hline & 3 & & \\
\hline & 3 & & \\
\hline-- & 3 & DCNUCC ( & 2) \\
\hline-- & 3 & DCNUCU( & $2,1)$ \\
\hline$-\ldots$ & 3 & DCNUCSi & 2) \\
\hline $9.449 \mathrm{E}-05$ & 3 & ALEACH ( & 2) \\
\hline not used & 3 & SOLUBKi & 2) \\
\hline & 3 & & \\
\hline & 3 & & \\
\hline-- & 3 & DCNUCC ( & 4) \\
\hline - - & 3 & DCNUCU( & $4,1)$ \\
\hline-- & 3 & DCNUCSi & 4) \\
\hline $2.173 \mathrm{E}-04$ & 3 & ALEACH ( & 4) \\
\hline not used & 3 & SOLUBK( & 4) \\
\hline & 3 & & \\
\hline & 3 & & \\
\hline-- & 3 & DCNUCC ( & 1) \\
\hline$-\ldots$ & 3 & DCNUCU( & $1,1)$ \\
\hline-- & 3 & DCNUCSi & 1) \\
\hline $2.153 \mathrm{E}-02$ & 3 & ALEACH ( & 1) \\
\hline not used & 3 & SOLUBK( & 1) \\
\hline & 3 & & \\
\hline & 3 & & \\
\hline-- & 3 & DCNUCC ( & 3) \\
\hline-- & 3 & DCNUCU( & $3,1)$ \\
\hline - - - & 3 & DCNUCSI & 3) \\
\hline $8.660 \mathrm{E}-03$ & 3 & ALEACH ( & 3) \\
\hline not used & 3 & SOLUBK( & 3) \\
\hline & $\begin{array}{l}3 \\
3\end{array}$ & & \\
\hline - - - & 3 & DCNUCC ( & 5) \\
\hline-- & 3 & DCNUCU ( & $5,1)$ \\
\hline-- & 3 & DCNUCS & 5) \\
\hline $8.660 \mathrm{E}-03$ & 3 & ALEACH ( & 5) \\
\hline not used & 3 & SOLUBK( & 5) \\
\hline & 3 & & \\
\hline-- & ${ }^{3}$ & INHALR & \\
\hline-- & $\begin{array}{l}3 \\
3\end{array}$ & MLINH & \\
\hline-- & $\begin{array}{l}3 \\
3\end{array}$ & $\begin{array}{l}\text { ED } \\
\text { SHF3 }\end{array}$ & \\
\hline - . - & 3 & SHF1 & \\
\hline - - - & 3 & FIND & \\
\hline-- & 3 & FOTD & \\
\hline
\end{tabular}

Uncontrolled When Printed 
Site-Specific Parameter Summary (continued)

$0 \quad 3$

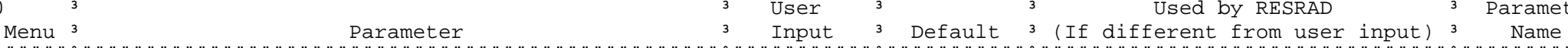

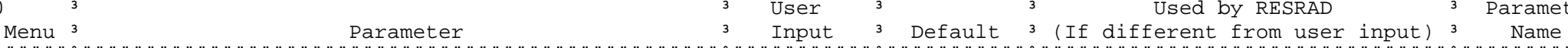

Used by RESRAD

3 Parameter

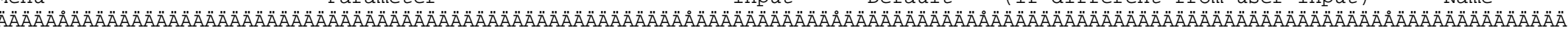
R017 3 Shape factor flag, external gamma

R017 3 Radii of shape factor array (used if FS = -1):

R017 3 Outer annular radius $(\mathrm{m})$, ring 1 :

$\mathrm{R} 017$ 3 3 Outer annular radius $(\mathrm{m})$, ring 2 :

R017 3 Outer annular radius $(\mathrm{m})$, ring 4 :

$\mathrm{R} 017$ O 3 Outer annular radius $(\mathrm{m})$, ring 5 :

$\begin{array}{lllll}\mathrm{R} 017 & 3 & \text { Outer annular radius }(\mathrm{m}), & \text { ring } & 5: \\ \mathrm{R} 017 & 3 & \text { Outer annular radius }(\mathrm{m}), & \text { ring } & 6:\end{array}$

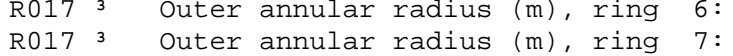

R017 3 Outer annular radius $(\mathrm{m})$, ring 8:

R017 3 Outer annular radius $(\mathrm{m})$, ring 9:

$\mathrm{R} 017$ 3 $\quad$ Outer annular radius $(\mathrm{m})$, ring 10:

R017 3 Outer annular radius (m), ring 11:

R017 3 Outer annular radius $(\mathrm{m})$, ring 12 :

R017 3 Fractions of annular areas within AREA:

R017 3 Ring 1

R017 3 Ring 2

R017 3 Ring 3

$\begin{array}{ll}1.000 \mathrm{E}+00^{3} & 1.000 \mathrm{E}+\odot \odot\end{array}$

$>\odot$ shows circular AREA.

3 FS

3

not used $35.000 \mathrm{E}+01$

not used 3 7.071E+01

not used ${ }^{3} 0.000 \mathrm{E}+00^{3}$

3 not used $30.000 \mathrm{E}+00^{3}$

3 not used 3 ๑.

3 not used $30.000 \mathrm{E}+\odot \odot^{3}$

3 not used $30.000 \mathrm{E}+0 \odot^{3}$

3 not used $30.000 \mathrm{E}+\Theta \Theta^{3}$

3 not used $30 . \odot \odot \odot E+\odot \odot$

3 not used $30.000 \mathrm{E}+\Theta \odot$

3 not used $30.000 \mathrm{E}+00^{3}$

3 not used $30.000 \mathrm{E}+00^{3}$

not used $31.000 \mathrm{E}+00^{3}$

not used $32.732 \mathrm{E}-01$

not used $30.000 \mathrm{E}+\odot \odot$

not used $30.000 \mathrm{E}+0 \odot$

3 not used $30.000 \mathrm{E}+\Theta \odot$

3 not used $30.000 \mathrm{E}+0 \Theta^{3}$

$\begin{array}{llll}R 017 & 3 & \text { Ring } & 5 \\ R 017 & 3 & \text { Ring } & 6\end{array}$

R017 3 Ring 7

not used $30.000+\odot 0^{3}$

not used 3 O.

$\begin{array}{llll}\mathrm{R} 017^{3} & \text { Ring } 8\end{array}$

R017 3 Ring 9

R017 3 Ring 10

not used $30.000 \mathrm{E}+00$

not used $30.000 \mathrm{E}+00$

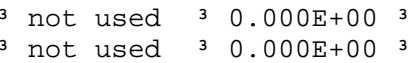

R017 3 Ring 12

not used

$\odot .000 \mathrm{E}+0 \odot$

R018 3 Fruits, vegetables and grain consumption ( $\mathrm{kg} / \mathrm{yr}$ )

R018 3 Leafy vegetable consumption ( $\mathrm{kg} / \mathrm{yr}$ )

R018 3 Milk consumption ( $\mathrm{L} / \mathrm{yr}$ )

R018 3 Meat and poultry consumption ( $\mathrm{kg} / \mathrm{yr}$ )

R018 3 Fish consumption ( $\mathrm{kg} / \mathrm{yr}$ )

R018 3 Other seafood consumption ( $\mathrm{kg} / \mathrm{yr}$ )

R018 3 Soil ingestion rate (g/yr)

R018 3 Drinking water intake (L/yr)

R018 3 Contamination fraction of drinking water

R018 3 Contamination fraction of household water

R018 3 Contamination fraction of livestock water

R018 3 Contamination fraction of irrigation water

RO18 3 Contamination fraction of aquatic food

R018 3 Contamination fraction of plant food

R018 3 Contamination fraction of meat

R018 3 Contamination fraction of milk 3

R019 3 Livestock fodder intake for meat ( $\mathrm{kg} /$ day)

R019 3 Livestock fodder intake for milk ( $\mathrm{kg} /$ day)

R019 3 Livestock water intake for meat (L/day)

R019 3 Livestock water intake for milk (L/day)

$1.600 \mathrm{E}+02$

3 not used 3 1.400E+01

3 not used $39.200 \mathrm{E}+01$

3 not used $36.300 \mathrm{E}+01$

not used $35.400 \mathrm{E}+0 \odot$

not used 3 9.000E-01

$1.752 \mathrm{E}+02 \quad 3 \quad 3.650 \mathrm{E}+01$

not used 3 5.100E +02

3 not used $31.000 \mathrm{E}+00$

not used $31.000 \mathrm{E}+00$

3 not used $31.000 E+00^{3}$

not used 3 1.000E+00 3

not used 3 5.000E-01

not used $3-1$

not used $3-1$

not used $3-1$

3

not used $36.800 \mathrm{E}+01$

3 not used $35.500 \mathrm{E}+01^{3}$

3 not used $35.000 \mathrm{E}+01$

3 not used $31.600 \mathrm{E}+02$

3 not used 3 5.000E-01

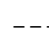

$-$

$-$

$-$

$-$

$-$

-

$-$

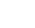

$-$

$-$

$-$

$-$

$-$

$-$

$-$

$-\cdot$

$--\cdot$

$-\cdot-$

- - -

$--$

$--$

$---$

$--$

$--$

$--$

$-\cdot$

$-\cdot$

$--$

$--$

$--$

- -

- - -

$-\cdot$

$-\cdot-$

$--$
3 RAD_SHAPE( 1$)$

3 RAD_SHAPE ( 2)

RAD_SHAPE ( 3$)$

3 RAD_SHAPE (5)

RAD_SHAPE (5)

3 RAD_SHAPE ( 7 )

3 RAD_SHAPE ( 8$)$

3 RAD_SHAPE ( 9)

3 RAD_SHAPE (10)

3 RAD_SHAPE (11)

RAD_SHAPE (12)

$\operatorname{FRACA}(1)$

FRACA( 2)

FRACA( 3 )

FRACA ( 4)

3 FRACA ( 5 )

3 FRACA ( 6)

FRACA ( 7)

3 FRACA ( 8)

3 FRACA ( 9)

FRACA (10)

FRACA (11)

$\operatorname{DIET}(1)$

$\operatorname{DIET}(2)$

3 DIET(3)

3 DIET (4)

3 DIET (6)

3 SOIL

3 DWI

3 FDW

3 FHHW

$3 \mathrm{FLW}$

FIRW

FR9

$\begin{array}{ll}3 & \text { FPLANT } \\ 3 & \text { FMEAT }\end{array}$

3 FMILK

3 LFI5

3 LFI6

3 LWI5

3 LWI6

3 LSI 
Site-Specific Parameter Summary (continued)

$0 \quad 3$

Menu 3 Parameter 3 Input 3 Default 3 (If different from user input) 3 Name

Used by RESRAD

3 Parameter

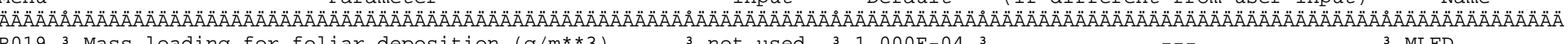

R019 3 Mass loading for foliar deposition $\left(\mathrm{g} / \mathrm{m}^{* *} 3\right) \quad 3$ not used $31.000 \mathrm{E}-044^{3} \quad{ }_{3}$

R019 3 Depth of soil mixing layer $(\mathrm{m})$

R019 3 Depth of roots $(m)$

R019 3 Drinking water fraction from ground water

R019 3 Household water fraction from ground water

3 1.500E-01 3 1.500E- 01

3 not used 3 9.000E- 01

3 not used $31.000 \mathrm{E}+\Theta 0^{3}$

3 not used $31.000 \mathrm{E}+00$

R019 3 Livestock water fraction from ground water

not used $31.000 \mathrm{E}+00^{3}$

R019 3 Irrigation fraction from ground water

R19B 3 Wet weight crop yield for Non-Leafy $(\mathrm{kg} / \mathrm{m} * * 2)$

3 not used $31.000 \mathrm{E}+00$

3 not used 3 1.000E+00 3

R19B Wet weight crop yield for Leafy $\left(\mathrm{kg} / \mathrm{m}^{* *} 2\right) \quad 3$ not used $31.500 \mathrm{E}+00^{3}$

R19B Wet weight crop yield for Fodder $\left(\mathrm{kg} / \mathrm{m}^{* *} 2\right) \quad{ }^{3}$ not used $31.100 \mathrm{E}+00$

R19B 3 Growing Season for Non-Leafy (years) 3 not used $31.700 \mathrm{E}-01$

R19B 3 Growing Season for Leafy (years) 3 not used $32.500 \mathrm{E}^{3} 01^{3}$

R19B 3 Growing Season for Fodder (years) 3 not used $38.000 \mathrm{E}-02$

R19B 3 Translocation Factor for Non-Leafy 3 not used $31.000 E-013$

R19B 3 Translocation Factor for Leafy 3 not used $31.000 \mathrm{E}+00^{3}$

R19B 3 Translocation Factor for Fodder 3 not used $31.000 \mathrm{E}+00^{3}$

R19B 3 Dry Foliar Interception Fraction for Non-Leafy 3 not used $32.500 \mathrm{E}-01^{3}$

R19B 3 Dry Foliar Interception Fraction for Leafy 3 not used $32.500 \mathrm{E}-01^{3}$

R19B 3 Dry Foliar Interception Fraction for Fodder 3 not used $32.500 \mathrm{E}-01$

R19B 3 wet Foliar Interception Fraction for Non-Leafy 3 not used $32.500 \mathrm{E}-01$

R19B 3 Wet Foliar Interception Fraction for Leafy 3 not used $32.500 \mathrm{E}-013$

R19B 3 wet Foliar Interception Fraction for Fodder 3 not used 3 2.500E-01

3. $2.500 \mathrm{E}-01$

R19B 3 Weathering Removal Constant for Vegetation

C14 3 C-12 concentration in water $\left(\mathrm{g} / \mathrm{cm}^{* *} 3\right)$

C14 3 C-12 concentration in contaminated soil $(\mathrm{g} / \mathrm{g})$

C14 3 Fraction of vegetation carbon from soil

C14 3 Fraction of vegetation carbon from air

C14 3 C-14 evasion layer thickness in soil (m)

C14 3 C-14 evasion flux rate from soil (1/sec)

$\begin{array}{llll}\text { C14 } & 3 & \text { C-14 evasion flux rate from soil }(1 / \mathrm{sec}) \\ \text { C14 } & 3 & \text { C-12 evasion flux rate from soil }(1 / \mathrm{sec})\end{array}$

C14 3 Fraction of grain in beef cattle feed

C14 3 Fraction of grain in milk cow feed

C14 3 DCF correction factor for gaseous forms of C14

3

not used $32.000 \mathrm{E}+01$

not used $32.000 \mathrm{E}-05$

3 not used $33.000 \mathrm{E}-022^{3}$

not used $32.000 \mathrm{E}-02$

not used 3 9.800E-01

not used 3 3.000E-01

not used 3 7.000E- 07

3 not used 3 1.000E-10 3

3 not used 3 8.000E- 013

3 not used 32 2.000E- 01

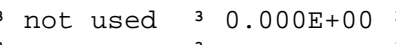

STOR 3 Storage times of contaminated foodstuffs (days): 3

STOR 3 Fruits, non-leafy vegetables, and grain

STOR 3 Leafy vegetables

3

$\begin{array}{ll}3 & \text { MLFD } \\ 3 & \text { DM }\end{array}$

3 DROOT

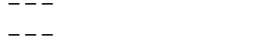

3 FGWHH

3 FGWLW

3 FGWIR

$3 \mathrm{YV}(1)$

.. 3 YV(3)

$3 \mathrm{TE}(1)$

3 TE (2)

-. $3 \operatorname{TE}(3)$

$\ldots \quad 3 \operatorname{TIV}(1)$

-.. $3 \operatorname{TIV}(2)$

$3 \operatorname{TIV}(3)$

$-\cdot-$

$\begin{array}{ll}--- & 3 \operatorname{RDRY}(2) \\ --- & 3 \operatorname{RDRY}(3)\end{array}$

$3 \operatorname{RWET}(1)$

3 RWET (2)

- . 3 RWET (3)

.. 3 WLAM

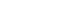

3 C12WTR

$-\cdot-$

$-\cdot-$

3 CAIR

3 EVSN

3 REVSN

3 AVFG5

-.- 3 CO2F

$1.400 \mathrm{E}+0131.400 \mathrm{E}+01$

$1.000 \mathrm{E}+00 \quad 3 \quad 1.000 \mathrm{E}+00$

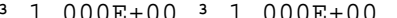

$32.000 \mathrm{E}+01332.000 \mathrm{E}+01$

$3 \quad 7.000 \mathrm{E}+003 \quad 7.000 \mathrm{E}+003$

$7.000 \mathrm{E}+00$ 3 $7.000 \mathrm{E}+00$

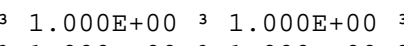

3 1. $1.000 \mathrm{E}+00 \quad 3 \quad 1.000 \mathrm{E}+00$

$4.500 \mathrm{E}+01 \quad 3 \quad 4.500 \mathrm{E}+013$

3

3 not used 3 1.500E- 01

$\begin{array}{lllll}\mathrm{R} 021 & 3 & \text { Bulk density of building foundation }\left(\mathrm{g} / \mathrm{cm}^{* *} 3\right) & 3 \text { not used } & 32.400 \mathrm{E}+00^{3} \\ \mathrm{R} 021 & 3 & \text { Total porosity of the cover material } & 3 \text { not used } & 34.000 \mathrm{E}-01\end{array}$

$\begin{array}{lllll}\mathrm{R} 021 & 3 & \text { Bulk density of building foundation }\left(\mathrm{g} / \mathrm{cm}^{* *} 3\right) & 3 \text { not used } & 32.400 \mathrm{E}+00^{3} \\ \mathrm{R} 021 & 3 & \text { Total porosity of the cover material } & 3 \text { not used } & 34.000 \mathrm{E}-01\end{array}$

R021 3 Total porosity of the cover material

3 not used $31.000 \mathrm{E}-01$

$-\cdot$

STOR_T(1)

3 STOR_T( 2$)$

3 STOR_T(3)

3 STOR_T( 4 )

3 STOR_T( 5)

3 STOR_T( 6)

3 STOR_T( (9)

- - 3 FLOOR1

3 DENSFL

3 TPCV

3 TPFL 
Site-Specific Parameter Summary (continued)

$0 \quad 3$

Menu 3 Parameter 3 Input 3 Default 3 (If different from user input) 3 Name

3 User

Menu 3 Parameter 3 Input 3 Default 3 (If different from user input) 3 Name

Used by RESRAD

3 Parameter

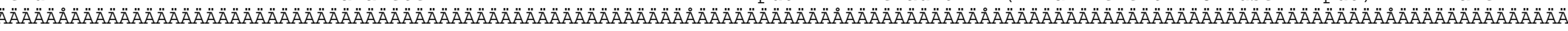

R021 3 Volumetric water content of the cover material 3 not used $35.000 \mathrm{E}-02^{3}$

R021 3 Volumetric water content of the foundation

R021 3 Diffusion coefficient for radon gas $(\mathrm{m} / \mathrm{sec})$ :

$\mathrm{R} 021^{3}$ in cover material

$\mathrm{R} 021^{3}$ in foundation material

$\mathrm{R} \odot 21^{3}$ in contaminated zone soil $\quad 3$ not used $32.000 \mathrm{E}-06$

R021 3 Radon vertical dimension of mixing $(\mathrm{m}) \quad 3$ not used 32 . $300 \mathrm{E}+00$

R०21 3 Average building air exchange rate $(1 / \mathrm{hr}) \quad 3$ not used $35.000 \mathrm{E}-01^{3}$

R021 3 Height of the building (room) (m)

R021 3 Building interior area factor

R०21 3 Building depth below ground surface ( $m$ )

not used $33.000 \mathrm{E}-\mathrm{O} 2$

not used $32.000 \mathrm{E}-066^{3}$

not used

not used $32.500 \mathrm{E}+00^{3}$

not used $30.000 \mathrm{E}+\odot \odot$

not used $3-1.000 \mathrm{E}+\odot \odot^{3}$

$\mathrm{R} 0213$ Emanating power of $\mathrm{Rn}-220$ gas

not used 3 2.500E- $011^{3}$

TITL 3 Number of graphical time points

TITL 3 Maximum number of integration points for dose

TITL 3 Maximum number of integration points for risk

3 not used $31.500 \mathrm{E}-01^{3}$

$--$

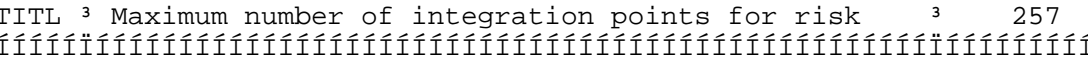

3 PH2OFL

3 DIFCV

3 DIFFL

3 DIFCZ

3 HIFCZ

3 REXG

3 HRM

3 FAI

3 DMFL

3 EMANA (1)

3 EMANA (2)

3 NPTS

3 LYMAX

3 KYMAX

Summary of Pathway Selections

Pathway 3 User Selection

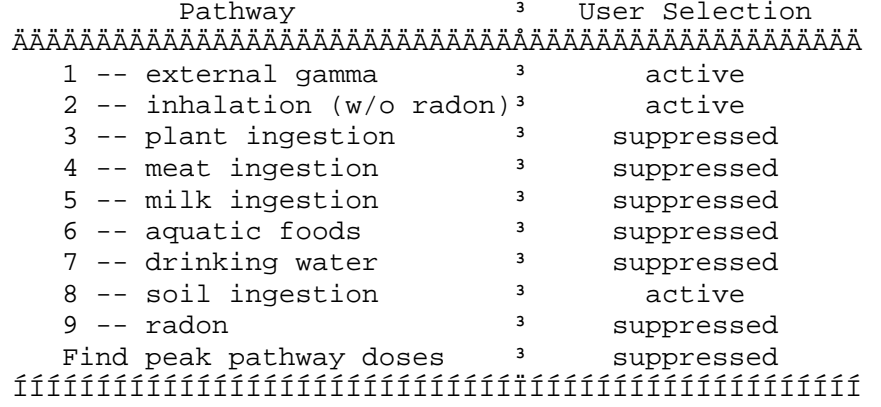

Uncontrolled When Printed 
1RESRAD, Version $6.3 \quad$ T« Limit $=180$ days Summary : CAU 383 Oil Spill

Contaminated Zone Dimensions

ÄÄÄÄÄÄÄÄÄÄÄÄÄÄÄÄÄÄÄÄÄÄÄÄÄÄÄ

Area: 100.00 square meters

Thickness: $\quad 0.15$ meters

$\odot$

$\odot .15$ meters
0.00 meters

05/11/2006 16:58 Page 8

File: CAU 383 oil spill. RAD

Initial Soil Concentrations, pCi/g

ÄÄÄÄÄÄÄÄÄÄÄÄÄÄÄÄÄÄÄÄÄÄÄÄÄÄÄÄÄÄÄÄÄÄ

CS $-137 \quad 2.050 \mathrm{E}+01$

Pu-239 $1.040 \mathrm{E}+00$

Total Dose TDOSE(t), mrem/yr

Basic Radiation Dose Limit $=2.500 \mathrm{E}+01 \mathrm{mrem} / \mathrm{yr}$

Total Mixture Sum $M(t)=$ Fraction of Basic Dose Limit Received at Time $(t)$

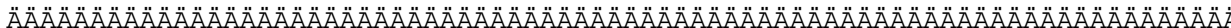

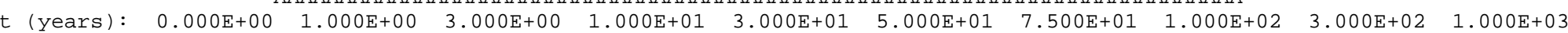

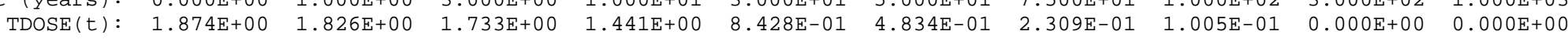

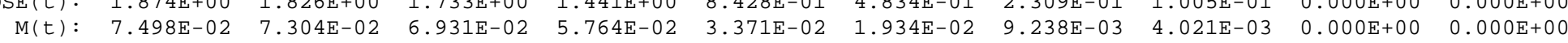

@Maximum TDOSE $(\mathrm{t}): 1.874 \mathrm{E}+0 \odot \mathrm{mrem} / \mathrm{yr}$ at $\mathrm{t}=0.000 \mathrm{E}+\odot \odot$ years 

1RESRAD, Version 6.3
T« Limit $=180$ days
05/11/2006 16:58 Page 9
File: CAU 383 oil spill. RAD

Total Dose Contributions TDOSE( $i, p, t)$ for Individual Radionuclides (i) and Pathways $(p)$ As $\mathrm{mrem} / \mathrm{yr}$ and Fraction of Total Dose At $\mathrm{t}=0.000 \mathrm{E}+0 \odot$ years
Water Independent Pathways (Inhalation excludes radon)

0 Ground
Radio-
ÄÄÄ̈̈̈̈̈̈̈̈̈̈̈̈A Inhalation Radon Plant Meat Muclide mrem/yr fract. Ӓ̈̈̈̈̈̈̈̈̈̈̈̈ÄÄÄÄÄÄÄ ÄÄÄÄÄÄÄÄÄÄÄÄÄÄÄ ÄÄÄÄÄÄÄÄÄÄÄÄÄÄÄÄÄ ӒӒÄÄÄÄÄÄÄÄÄÄÄÄÄÄ ÄÄÄÄÄÄ̈̈̈̈̈̈̈ ÄÄÄÄÄÄÄ Ä ÄÄÄÄÄÄÄÄÄ ÄÄÄÄÄÄ Cs-137 1.862E+00 0.9932 Pu-239 9.341E-06 0.0000 Íííííi íííííííi ííííi mrem/yr fract. maAAAAAAAAAAAA
mrem $/ y r$ fract. $\mathrm{mrem} / \mathrm{yr}$ fract mrem/yr fract A mrem/yr fract.

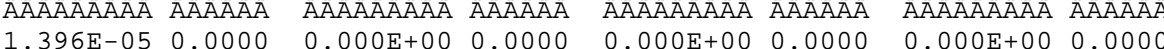

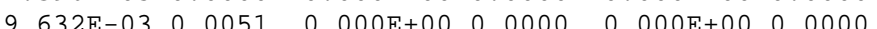
Total $1.862 \mathrm{E}+0 \odot$ 0.9932

IIIII IIIII

$0.000 \mathrm{E}+000.0000$ IT́TE+ 0.0000 $0.000+000.0000$ $0.000 \mathrm{E}+00$ 0. 0000 Аล̈ÄÄÄÄÄÄÄÄÄÄÄÄÄÄ Total $1.862 \mathrm{E}+0 \odot \quad 0.9932$

Total Dose Contributions $\operatorname{TDOSE}(i, p, t)$ for Individual Radionuclides (i) and Pathways $(p)$ As mrem/yr and Fraction of Total Dose At $t=0.000 \mathrm{E}+\odot \odot$ years Radon Water Dependent Pathways Water Fish

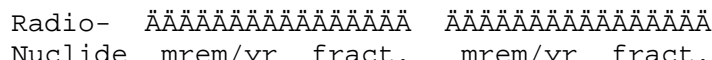
Nuclide mrem/yr fract. ÄÄÄÄÄÄÄÄÄÄÄÄÄÄÄA Plant Meat Milk All Pathways*

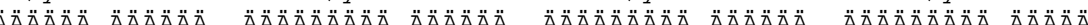

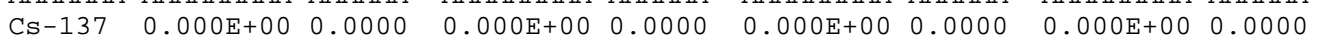

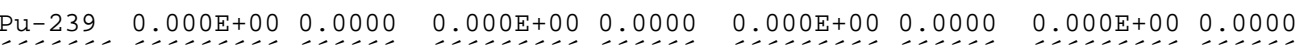

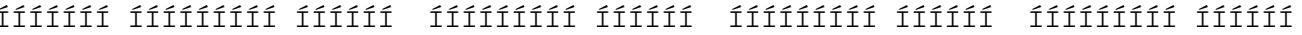

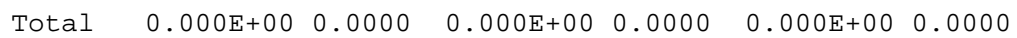
$\odot .000 \mathrm{E}+00 \quad 0.0000$ ÄÄÄÄÄÄÄÄÄÄÄÄÄÄÄÄÄ mrem/yr fract $0.000 \mathrm{E}+00$ ०. 0000 $0.000 \mathrm{E}+000.00000$ IIIIIIIII IIIIII AAAAAAAAAAAAAAA mrem/yr fract. 0. 000E 00 0.0000 AAAAAAAA AAÄAAA

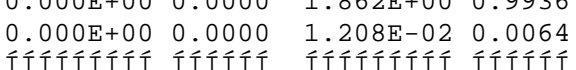
$\Theta *$ Sum of all water independent and dependent pathways. 

1RESRAD, Version 6.3
T« Limit $=180$ days
05/11/2006 16:58 Page 10
File: CAU 383 Oil spill. RAD

Total Dose Contributions TDOSE( $i, p, t)$ for Individual Radionuclides (i) and Pathways $(p)$ As $\mathrm{mrem} / \mathrm{yr}$ and Fraction of Total Dose At $\mathrm{t}=1.000 \mathrm{E}+0 \odot$ years
Water Independent Pathways (Inhalation excludes radon)

0

Radio- Ground Inhalation Radon

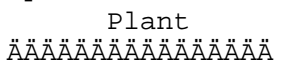
Meat

Ruclide mrem/yr fract. АÄÄÄÄÄÄÄÄÄÄÄÄÄÄ ÄÄÄÄÄÄÄÄÄÄÄÄÄÄÄ Ӓล̈ÄÄÄÄÄÄÄÄÄÄÄÄÄ ÄӒӒÄÄÄ̈̈̈̈̈ ÄÄÄÄÄÄÄ Cs-137 1.813E+00 0.9931 Pu-239 9.322E-06 0.0000 mrem/yr fract. maAAAAAAAAAAAA
rem fract. $\mathrm{mrem} / \mathrm{yr}$ fract mrem/yr fract АAАÄÄÄÄÄÄÄÄÄÄÄÄ mrem/yr fract.

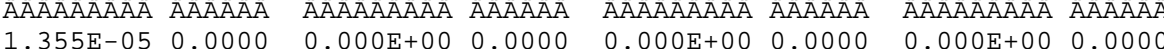
íííííi ííííííí ííííi

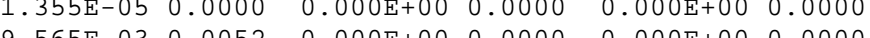
O. $0.00 E+000.0000$ $\odot .0 \odot \odot E+\odot \odot \odot .0 \odot \odot \odot$ Аล̈ÄÄÄÄÄÄÄÄÄÄÄÄÄÄ

Total Dose Contributions $\operatorname{TDOSE}(i, p, t)$ for Individual Radionuclides (i) and Pathways (p) As $\mathrm{mrem} / \mathrm{yr}$ and Fraction of Total Dose At $t=1.000 \mathrm{E}+\odot \odot$ years

$\odot$ Fish Radon Water Dependent Pathways

Water Radio- ÄÄÄÄÄÄÄÄÄÄÄÄÄÄÄÄ ÄÄÄÄÄÄÄÄÄÄÄÄÄÄÄÄ Nuclide mrem/yr fract. mrem/yr fract. ÄÄÄÄÄÄÄÄÄÄÄÄÄÄÄ Plant

Meat Milk All Pathways* AАAAAAAAAAAAAAAA ÄÄÄÄÄÄÄÄÄÄÄÄÄÄÄĂ . ÄÄÄÄÄÄ ÄÄÄÄÄÄÄÄÄ ÄÄÄÄÄÄ ÄÄÄÄÄÄÄÄÄ ÄÄÄAÄ AÄ ÄAAAAAA AAAAÄÄ ÄÄÄÄÄÄÄÄÄ ÄÄÄÄÄÄ

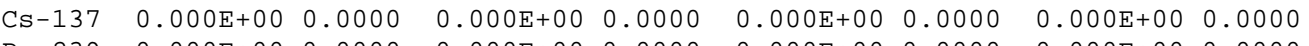

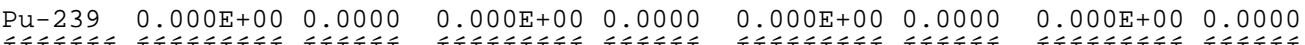

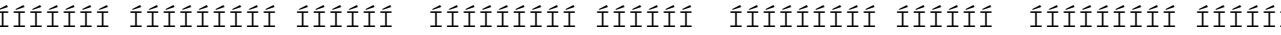

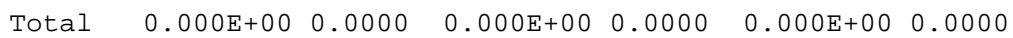
$0.000 \mathrm{E}+000.0000$ ÄÄÄÄÄÄÄÄÄÄÄÄÄÄÄÄÄ mrem/yr fract. ÄÄÄÄÄÄÄÄ ÄÄÄÄÄÄ $\odot .000 \mathrm{E}+00$ 0.0000 $0.000 \mathrm{E}+00 \quad 0.0000$ mrem/yr fract. mrem/yr fract. mrem/yr fract. $0.000 \mathrm{E}+00 \quad 0.0000 \quad 1.814 \mathrm{E}+00 \quad 0.9934$ $\odot .00 \odot \mathrm{E}+\odot \odot \quad \odot .00 \odot \odot \quad 1.200 \mathrm{E}-\odot 2 \quad 0.0066$ $\Theta *$ Sum of all water independent and dependent pathways. 

1RESRAD, Version 6.3
T« Limit $=180$ days
05/11/2006 16:58 Page 11
Summary : CAU 383 Oil Spill
File: CAU 383 oil spill. RAD

Total Dose Contributions TDOSE( $i, p, t)$ for Individual Radionuclides (i) and Pathways $(p)$ As $\mathrm{mrem} / \mathrm{yr}$ and Fraction of Total Dose At $\mathrm{t}=3.000 \mathrm{E}+0 \odot$ years
Water Independent Pathways (Inhalation excludes radon)

0

Ground Inhalation Radon Plant Meat

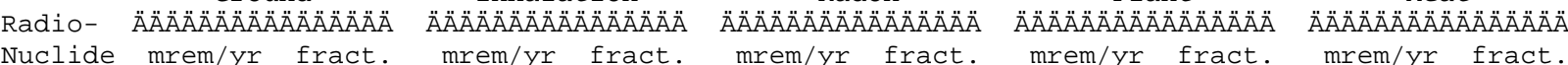
Nuclide $\mathrm{mrem} / \mathrm{yr}$ fract. mrem/yr fract mrem/yr fract mrem/yr fract $\operatorname{Mil}$ ÄÄÄÄÄÄÄ ÄÄÄÄÄÄÄÄÄ ÄÄÄÄÄA Cs-137 $1.720 \mathrm{E}+00 \quad 0.9928$ Pu-239 9.285E-06 9.0000 A ÄÄÄÄÄÄÄ ÄÄÄÄÄẢ ÄÄÄÄÄÄÄÄÄ ÄÄÄÄÄÄ Ä̈̈ÄÄÄÄÄÄÄ ÄÄÄÄÄÄ

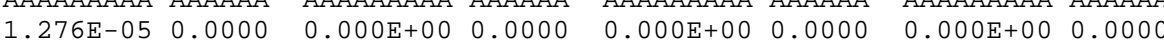
$\begin{array}{llllll}9.432 \mathrm{E}-03 & 0.0054 & 0.000 \mathrm{E}+00 & 0.0000 & 0.000 \mathrm{E}+0 \odot & 0.0000\end{array}$ $\begin{array}{lll}\text { Total } & 1.720 \mathrm{E}+00 & 0.9928\end{array}$

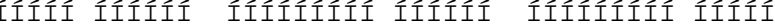
A.AAAAAAAA ÄÄÄÄÄ mrem/yr fract. ÄÄÄÄÄÄÄÄÄÄÄÄÄÄÄÄ

$0.444 \mathrm{E}-030.0055$ 0.000E+00 0.0000

tifititit tititi

$0.000 \mathrm{E}+00 \quad 0.0000$

$0.000 \mathrm{E}+00 \quad 0.0000$ mrem/yr fract $\odot$

Total Dose Contributions $\operatorname{TDOSE}(i, p, t)$ for Individual Radionuclides (i) and Pathways $(p)$ As $\mathrm{mrem} / \mathrm{yr}$ and Fraction of Total Dose At $t=3.000 \mathrm{E}+\odot \odot$ years

$\odot$ Water Dependent Pathways

\section{Water Fish Radon}

Milk

All Pathways*

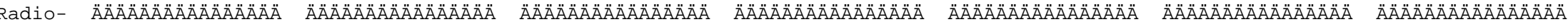
Nuclide mrem/yr fract mrem/yr fract mrem/yr fract mrem/yr fract mrem/yr fract mrem/yr fract. mrem/yr fract. ÄÄÄA ÄÄÄÄ̈ ÄÄÄ̈ ÄÄÄÄÄ ÄÄÄ̈ Ä $\begin{array}{llllllllllllllllllllllll}\text { CS-137 } & 0.000 \mathrm{E}+00 & 0.0000 & 0.000 \mathrm{E}+00 & 0.0000 & 0.000 \mathrm{E}+00 & 0.0000 & 0.000 \mathrm{E}+00 & 0.0000 & 0.000 \mathrm{E}+00 & 0.0000 & 0.000 \mathrm{E}+00 & 0.0000 & 1.721 \mathrm{E}+00 & 0.9932\end{array}$ Pu-239 0.000E+00 0.0000 0.000E+00 0.0000 0.000E+00 0.0000 0.000E+00 0.0000 Íf́tít

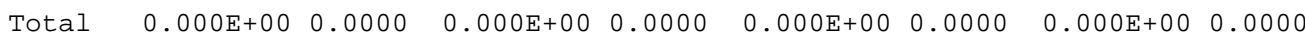
$0.000 \mathrm{E}+000.0000$ $\Theta *$ Sum of all water independent and dependent pathways. 


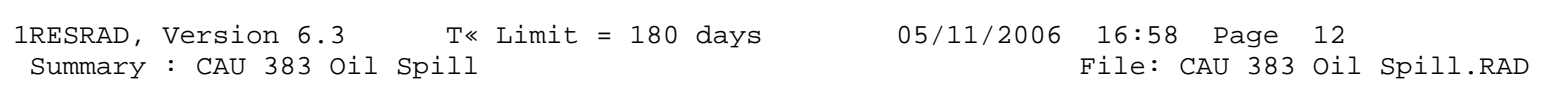

Total Dose Contributions $\operatorname{TDOSE}(i, p, t)$ for Individual Radionuclides (i) and Pathways (p) As $\mathrm{mrem} / \mathrm{yr}$ and Fraction of Total Dose At $\mathrm{t}=1.000 \mathrm{E}+01$ years
Water Independent Pathways (Inhalation excludes radon) Nuclide mrem $y$ r fract. ÄÄÄÄÄÄ Cs-137 1.429E+00 0.9918 $\mathrm{Pu}-239 \quad 9.148 \mathrm{E}-06 \quad 0.0000$ IIIííí íííííííi ííííi

Total 1.429E+00 0.9918 Inhalation Radon Plant Meat

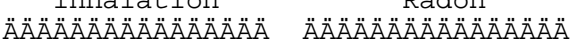
ÄÄÄÄÄÄÄÄÄÄÄÄÄÄÄÄÄ АAАAÄÄÄÄÄÄÄÄÄÄÄ

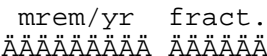
mrem/yr fract. AิAAAAÄÄÄÄÄÄÄÄÄ $\mathrm{mrem} / \mathrm{yr}$ fract

\section{ÄÄÄÄÄÄÄÄ ÄÄÄÄÄÄ} ÄÄÄÄÄÄÄÄÄ ÄÄÄÄÄA $1.033 \mathrm{E}-05 \quad 0.0000$ $\odot .000 \mathrm{E}+0 \odot \quad 0.000 \odot$ $\begin{array}{llllll}8.965 \mathrm{E}-03 & 0.0062 & 0.000 \mathrm{E}+00 & 0.0000 & 0.000 \mathrm{E}+00 & 0.0000\end{array}$

位 $\odot .000 \mathrm{E}+0 \odot$ ०. $000 \odot$

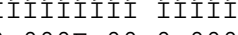
$0.000 \mathrm{O}+00$ 0. 0000 $\odot .000 \mathrm{E}+00 \quad 0.0000$ Ííííííí ííííi 0.0000000 $\odot .000 E+00 \quad 0.0000$ \\ $\operatorname{Mil}$} АААААААААӒ̈̈̈̈̈̈̈̆ mrem/yr fract. $0.0 \odot \odot E+0 \odot$ ๑. $\odot \odot \odot \odot$ $0.000 E+00 \quad 0.0000$ Íííííííí ííííi

$0.000 \mathrm{t} 00$ 0.0000
Soil ÄÄÄÄÄÄÄÄÄÄÄÄÄÄÄÄ mrem/yr fract ÄÄÄÄÄÄÄÄÄ ÄÄÄÄÄÄ 4.974E- $04 \quad 0.0003$ $2.274 \mathrm{E}-03 \quad 0.0016$ Íííííííí Íííííi

Total Dose Contributions TDOSE $(i, p, t)$ for Individual Radionuclides ( $i$ ) and Pathways ( $p$ ) As $\mathrm{mrem} / \mathrm{yr}$ and Fraction of Total Dose At $t=1.000 \mathrm{E}+01$ years

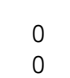
Fish Water Dependent Pathways

Water Radon Plant

Meat Milk All Pathways* ÄÄÄÄÄÄÄÄÄÄÄÄÄÄÄÄ ÄÄÄÄÄÄÄÄÄÄÄÄÄÄÄÄ ÄÄÄÄÄÄÄÄÄÄÄÄÄÄÄÄ mrem/yr fract. mrem/yr fract. mrem/yr fract. ÄÄÄÄÄÄÄÄÄ ÄÄÄÄÄÄ ÄÄÄÄÄÄÄÄÄ ÄÄÄÄÄÄ ÄÄÄÄÄÄÄÄÄ ÄÄÄÄÄÄ

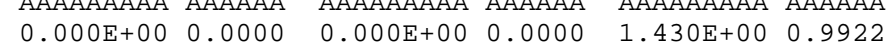
$\begin{array}{llllll}0.000 \mathrm{C}+00 & 0.0000 & 0.000 \mathrm{E}+00 & 0.000 \odot & 1.430 \mathrm{E}+0 \odot & 0.9922 \\ 0.000 \mathrm{E}+00 & 0.0000 & 0.000 \mathrm{E}+00 & 0.0000 & 1.125 \mathrm{E}-02 & 0.0078\end{array}$

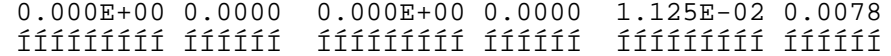

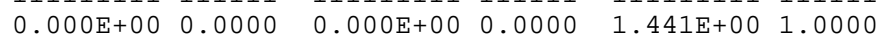

ÄÄÄÄÄÄÄÄÄ̈̈̈̈ÄÄÄÄÄÄ
mrem/yr fract

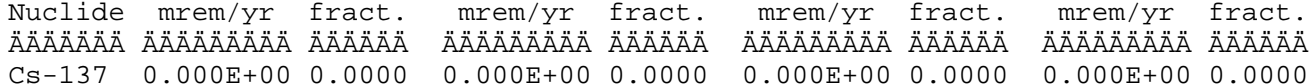

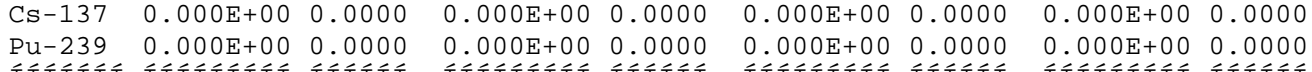

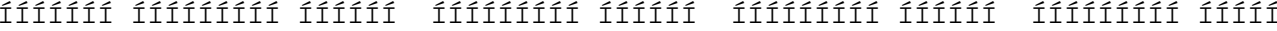

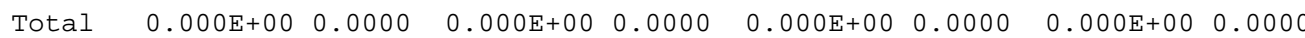
$\odot$ Sum of all water independent and dependent pathways. 

1RESRAD, Version 6.3
T« Limit $=180$ days
05/11/2006 16:58 Page 13
File: CAU 383 Oil spill. RAD

Total Dose Contributions TDOSE( $i, p, t)$ for Individual Radionuclides (i) and Pathways $(p)$ As $\mathrm{mrem} / \mathrm{yr}$ and Fraction of Total Dose At $\mathrm{t}=3.000 \mathrm{E}+01$ years
Water Independent Pathways (Inhalation excludes radon)

0 Ground
Radio-
ÄÄÄ̈̈̈̈̈̈̈̈̈̈̈̈A Inhalation Radon Plant Meat luclide mrem yr fract. ÄÄÄÄÄÄÄÄÄÄÄÄÄÄÄ ÄÄÄÄÄÄÄÄÄÄÄÄÄÄÄ ÄÄÄÄÄÄÄÄÄÄÄÄÄÄÄÄ ӒӒÄÄÄÄÄÄÄÄÄÄÄÄÄÄ ÄÄÄÄÄÄ̈̈̈̈̈̈̈ ÄÄÄÄÄÄÄ Cs-137 8.329E-01 0.9883 $\mathrm{Pu}-239$ 8.713E-06 0.0000 maAAAAAAAAAAAA
mrem $/ y r$ fract. $\mathrm{mrem} / \mathrm{yr}$ fract mrem/yr fract АลАล̈ÄÄÄÄÄÄÄÄÄÄ mrem/yr fract. ÄÄÄÄÄÄÄÄÄ ÄÄÄÄÄÄ Ä ÄÄÄÄÄÄÄÄÄ ÄÄÄÄÄÄ ÄÄÄÄÄÄÄÄÄ ÄÄÄÄÄÄ ÄÄÄÄÄÄÄÄÄ ÄÄÄÄÄÄA $\begin{array}{llllll}5.562 \mathrm{E}-06 & 0.0000 & 0.000 \mathrm{E}+00 & 0.0000 & 0.000 \mathrm{E}+00 & 0.0000\end{array}$ íííííi íííííííi ííííi $\begin{array}{llllll}7.642 \mathrm{E}-03 & 0.0091 & 0.000 \mathrm{E}+00 & 0.0000 & 0.000 \mathrm{E}+00 & 0.0000 \\ 0\end{array}$ $0.000 \mathrm{E}+00 \quad 0.0000$ IIIIIII IIIIIIII

7.64 $0.000 \mathrm{E}+00 \quad 0.0000$ $\odot .000 \mathrm{E}+0 \odot$ $0.000 \odot$ Аล̈ÄÄÄÄÄÄÄÄÄÄÄÄÄÄ Total 8.329E-01 0.9883

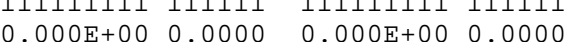

IIIIIIII IIIII

Total Dose Contributions $\operatorname{TDOSE}(i, p, t)$ for Individual Radionuclides (i) and Pathways $(p)$ As mrem/yr and Fraction of Total Dose At $t=3.000 \mathrm{E}+01$ years Radon Water Dependent Pathways

Radio- ÄÄÄÄÄÄÄÄÄÄÄÄÄÄÄÄ ÄÄÄÄÄÄÄÄÄÄÄÄÄÄÄÄ Nuclide mrem/yr fract. mrem/yr fract. mrem/yr fract. mrem/yr fract. mrem/yr fract.

ÄÄÄÄÄÄÄÄÄÄÄÄÄÄÄÄÄÄ Plant

Meat

Milk

All Pathways* ÄÄÄÄÄÄ ӒÄÄÄÄÄÄÄÄ ÄÄÄÄÄÄ ÄÄÄÄÄÄÄÄÄ ÄÄÄÄÄÄ ÄÄÄÄÄÄÄÄÄ ÄÄÄÄÄÄ ÄÄÄÄÄÄÄÄ ÄÄÄÄÄÄ

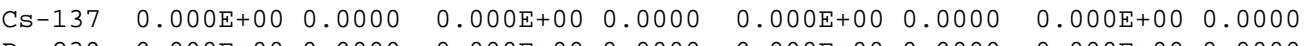

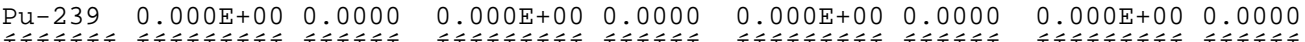

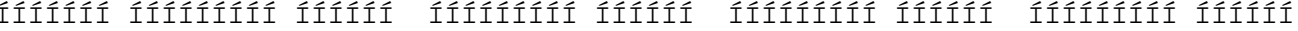

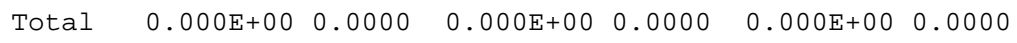
$\odot .000 \mathrm{E}+00 \quad 0.0000$ ÄÄÄÄÄÄÄÄÄÄÄÄÄÄÄÄ mrem/yr fract. ÄÄÄÄÄÄÄÄ ÄÄÄÄÄÄ $0.000 \mathrm{E}+00$ 0.0000 $0.000 \mathrm{E}+00 \quad 0.0000$ mrAAAAAAAAAA mrem/yr fract. mrem/yr fract.

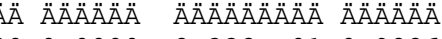
$\odot .0000108$ $\begin{array}{llll}0.000 \mathrm{E}+00 & 0.0000 & 9.589 \mathrm{E}-03 & 0.0114\end{array}$ $\Theta *$ Sum of all water independent and dependent pathways. 

1RESRAD, Version 6.3
T« Limit $=180$ days
05/11/2006 16:58 Page 14
File: CAU 383 Oil spill. RAD

Total Dose Contributions TDOSE( $i, p, t)$ for Individual Radionuclides (i) and Pathways ( $p$ ) As $\mathrm{mrem} / \mathrm{yr}$ and Fraction of Total Dose At $\mathrm{t}=5.000 \mathrm{E}+01$ years
Water Independent Pathways (Inhalation excludes radon)

0 Ground
Radio-
ÄÄÄ̈̈̈̈̈̈̈̈̈̈̈̈A Inhalation Radon Plant Meat luclide mrem yr fract. ÄÄÄÄÄÄÄÄÄÄÄÄÄÄÄ ÄÄÄÄÄÄÄÄÄÄÄÄÄÄÄ ÄÄÄÄÄÄÄÄÄÄÄÄÄÄÄÄÄ АAАÄÄÄÄÄÄÄÄÄÄÄA ӒӒӒӒ̈̈̈̈̈̈̈̈̈̈̈̈̈̈̈̈̈̈̈̈̈̈̈ ( mrem/yr fract. mrem/yr fract. mrem/yr fract. mrem/yr fract. AAAAAAA AÄÄÄÄÄÄ ÄÄÄÄA $\mathrm{Pu}-239$ 8.186E-06 0.0000 íííííi íííííííi ííííi ÄÄÄÄÄÄÄÄÄ ÄÄÄÄÄÄ $m r e m / y r$
Ä fact. Ä̈̈̈̈̈̈̈̈̈̈̈̈̈̈̈ ÄÄÄÄÄ̈

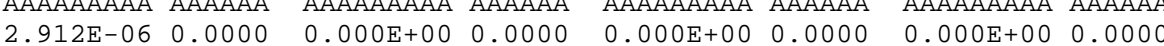
$\begin{array}{llllll}6.332 \mathrm{E}-03 & 0.0131 & 0.000 \mathrm{E}+00 & 0.0000 & 0.000 \mathrm{E}+00 & 0.0000\end{array}$ Total 4.753E-01 0.9833

作 ÄÄÄÄÄÄÄÄ ÄÄÄÄÄÄ $\begin{array}{ll}0.000 E+0 \odot & 0.000 \odot \\ 0.000 \mathrm{E}+0 \odot & 0.0000\end{array}$ $000 \mathrm{E}+000.0000$

Total Dose Contributions TDOSE( $i, p, t)$ for Individual Radionuclides (i) and Pathways ( $p$ ) As $\mathrm{mrem} / \mathrm{yr}$ and Fraction of Total Dose At $t=5.000 \mathrm{E}+01$ years Water Dependent Pathways Radon Plant

Meat Milk All Pathways* Radio- ÄÄÄÄÄÄÄÄÄÄÄÄÄÄÄÄ ÄÄÄÄÄÄÄÄÄÄÄÄÄÄÄÄ ÄÄÄÄÄÄÄÄÄÄÄÄÄÄÄÄ ÄÄÄÄÄÄÄÄÄÄÄÄÄÄÄÄ Nuclide mrem/yr fract. mrem/yr fract. mrem/yr fract. mrem/yr fract. ÄÄÄÄÄÄÄ ÄÄÄÄÄÄÄÄÄ ÄÄÄÄÄÄ ÄÄÄÄÄÄÄÄÄ ÄÄÄÄÄÄ ÄÄÄÄÄÄÄÄÄ ÄÄÄÄÄÄ ÄÄÄÄÄÄÄÄÄ ÄÄÄÄÄÄ

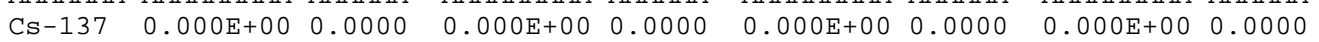

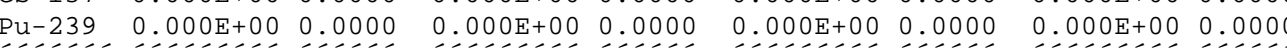

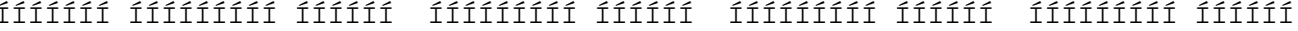

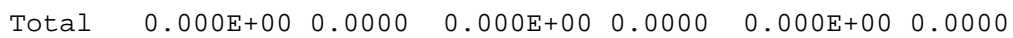
$\odot .0 \odot \odot \mathrm{E}+\odot \odot$ $\odot .0 \odot \odot 0$ ÄÄÄÄÄÄÄÄÄÄÄÄÄÄÄÄ mrem/yr fract 0. 000E+00 0.0000 $0.000 \mathrm{E}+000.0000$ mrem/yr fract. mrem/yr fract. mrem/yr fract. $\odot .00050 .9836$ $\begin{array}{llll}0.000 E+00 & 0.0000 & 7.946 \mathrm{E}-03 & 0.0164\end{array}$ * Sum of all water independent and dependent pathways. 

1RESRAD, Version 6.3
T« Limit $=180$ days
05/11/2006 16:58 Page 15
Summary : CAU 383 Oil Spill
File: CAU 383 oil spill. RAD

Total Dose Contributions TDOSE( $i, p, t)$ for Individual Radionuclides (i) and Pathways $(p)$ As $\mathrm{mrem} / \mathrm{yr}$ and Fraction of Total Dose At $\mathrm{t}=7.500 \mathrm{E}+01$ years
Water Independent Pathways (Inhalation excludes radon)

0

Ground Inhalation Radon Plant Meat

Milk

Soil

Radio- ÄÄÄÄÄÄÄÄÄÄÄÄÄÄÄÄ ÄÄÄÄÄÄÄÄÄÄÄÄÄÄÄÄ ÄÄÄÄÄÄÄÄÄÄÄÄÄÄÄÄ ÄÄÄÄÄÄÄÄÄÄÄÄÄÄÄÄ ÄÄÄÄÄÄÄÄÄÄÄÄÄÄÄÄ Nuclide mrem/yr fract. mrem/yr fract. mrem/yr fract. mrem/yr fract mrem/yr fract ÄÄ̈̈̈̈̈̈̈̈̈̈̈̈̈̈̈̈̈̈̈̈̈̈̈̈̈̈̈̈̈ Äล̈ล̈ÄÄÄÄÄÄÄÄÄÄÄÄÄ ÄÄÄÄÄÄÄ ÄÄÄÄÄÄÄÄÄ ÄÄÄÄÄÄ Cs-137 2.250E-01 0.9741 Pu-239 7.333E-06 0.0000 íííííi íííííííi ííííi

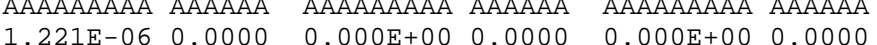
Total 2.250E-01 0.9742 $\begin{array}{llllll}4.712 \mathrm{E}-03 & 0.0204 & 0.000 \mathrm{E}+0 \odot & 0.000 \odot & 0.000 \mathrm{E}+0 \odot & 0.000 \odot\end{array}$

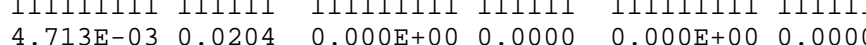

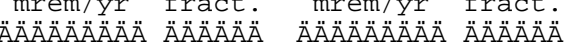
$0.000 \mathrm{E}+00$ $0.0000 \quad 5.881 \mathrm{E}-05 \quad 0.00003$ $0.000 \mathrm{E}+0 \odot \quad 0.0000 \quad 1.195 \mathrm{E}-03 \quad 0.0052$ IIIIIIII IIIIIí IIIIIIIIIí IIIIIII

Total Dose Contributions $\operatorname{TDOSE}(i, p, t)$ for Individual Radionuclides (i) and Pathways $(p)$ As $\mathrm{mrem} / \mathrm{yr}$ and Fraction of Total Dose At $t=7.500 \mathrm{E}+01$ years

$\odot$ Fish Water Dependent Pathways

Water Radon Plant

Meat Milk All Pathways*

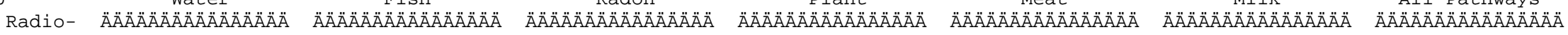
Nuclide mrem/yr fract. mrem/yr fract. mrem/yr fract. mrem/yr fract. mrem/yr fract. mrem/yr fract. mrem/yr fract. AAAAAAA AAAAAAAAA AAAAAA AAAAAAAAA AAAAAÄ AAAAAAAA AAAAAA AAAAAAAAA AAAAAA

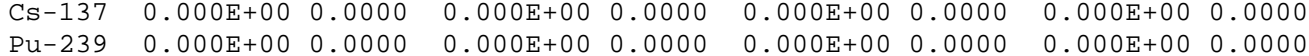

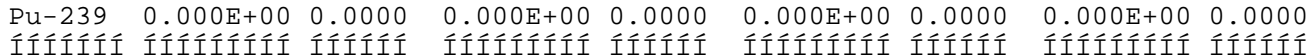

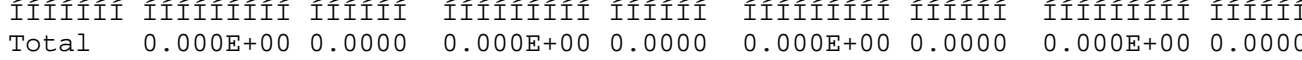

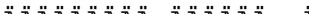
mrem/yr fract.: А.

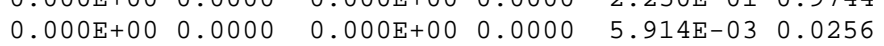

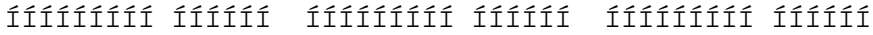
${ }^{*}$ Sum of all water independent and dependent pathways. 

1RESRAD, Version 6.3
T« Limit $=180$ days
05/11/2006 16:58 Page 16
File: CAU 383 Oil spill. RAD

Total Dose Contributions TDOSE( $i, p, t)$ for Individual Radionuclides (i) and Pathways ( $p$ ) As $\mathrm{mrem} / \mathrm{yr}$ and Fraction of Total Dose At $\mathrm{t}=1.000 \mathrm{E}+02$ years
Water Independent Pathways (Inhalation excludes radon) Inhalation Radon

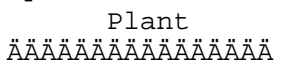
Meat Radio - АÄ̈̈̈̈̈̈̈̈̈ÄÄÄÄÄÄÄÄ АÄÄÄÄÄÄÄÄÄÄÄÄÄÄ ÄÄÄÄÄÄÄÄÄÄÄÄÄÄÄ Аล̈ÄÄÄÄÄÄÄÄÄÄÄÄÄÄ ӒӒӒӒӒ̈̈̈̈̈̈̈̈̈̈̈̈̈̈̈̈̈̈̈̈̈̈ ÄÄÄÄÄÄÄÄÄÄÄÄÄÄÄÄ vuclide mrem/yr fract. mrem/yr fract. mrem/yr fract. mrem/yr fract. mrem/yr fract.

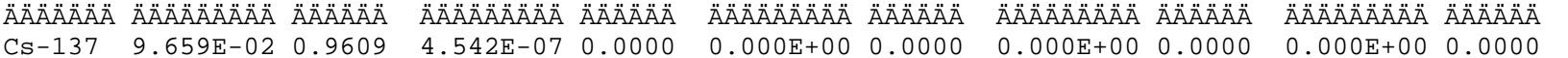
Pu-239 6.123E-06 0.0001 IIIIII IIIIIIII IIIIII $\begin{array}{llllll}3.112 \mathrm{E}-03 & 0.0310 & 0.000 \mathrm{E}+00 & 0.0000 & 0.000 \mathrm{E}+00 & 0.000 \odot\end{array}$

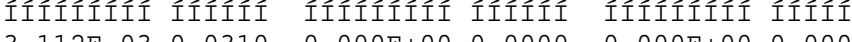

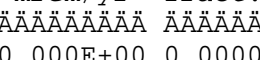
$0.000 \mathrm{E}+00 \quad 0.0000 \quad 0.000 \mathrm{E}+000.0000$ Ä̈̈̈̈̈̈̈̈̈̈̈̈̈̈ Ӓ̈̈̈̈̈̈̈̈̈̈ $2.188 \mathrm{E}-05 \quad 0.0002$ $7.891 E-040.0079$

Total Dose Contributions $\operatorname{TDOSE}(i, p, t)$ for Individual Radionuclides (i) and Pathways (p) As $\mathrm{mrem} / \mathrm{yr}$ and Fraction of Total Dose At $t=1.000 \mathrm{E}+\odot 2$ years

$\odot$ Fish Radon Water Dependent Pathways

water

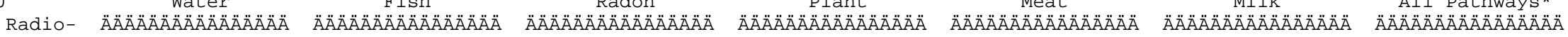
Plant Meat Milk All Pathways * Nuclide mrem/yr fract. mrem/yr fract. mrem/yr fract. mrem/yr fract. mrem/yr fract. $\mathrm{mrem} / \mathrm{yr}$ fract. mrem/yr fract.

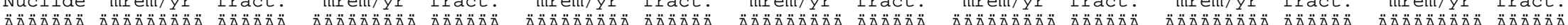

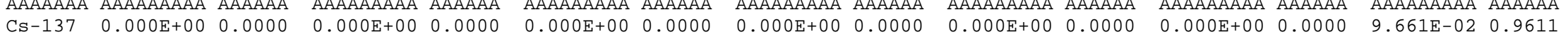

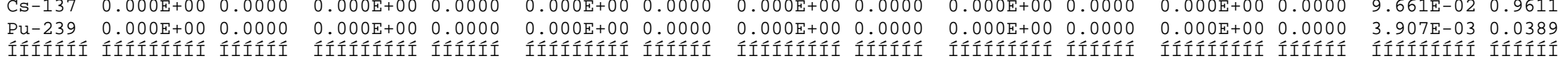

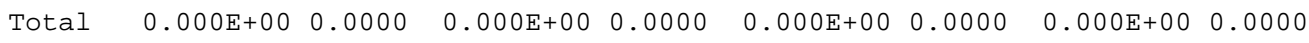
$\theta *$ Sum of all water independent and dependent pathways. 

1RESRAD, Version 6.3
T« Limit $=180$ days
05/11/2006 16:58 Page 17
File: CAU 383 Oil Spill. RAD

Total Dose Contributions TDOSE( $i, p, t)$ for Individual Radionuclides (i) and Pathways $(p)$ As $\mathrm{mrem} / \mathrm{yr}$ and Fraction of Total Dose At $\mathrm{t}=3.000 \mathrm{E}+02$ years
Water Independent Pathways (Inhalation excludes radon)

0 Ground
Radio-
ÄÄÄ̈̈̈̈̈̈̈̈̈̈̈̈A Inhalation Radon Plant Meat Muclide mrem/yr fract. Ӓ̈̈̈̈̈̈̈̈̈̈̈̈̈̈̈̈̈̈̈̈̈̈̈̈̈

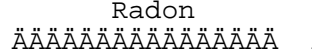

\begin{abstract}
ӒӒÄÄÄÄÄÄÄÄÄÄÄÄÄÄ
\end{abstract}
ÄÄÄÄÄÄÄÄÄÄÄÄÄÄÄÄ ÄÄÄÄÄÄÄÄ̈̈̈ ÄÄÄÄÄÄ ÄÄ̈̈̈̈̈̈̈̈̈̈̈̈ ÄÄÄÄÄ̈. CS-137 $0.000 \mathrm{E}+00 \quad 0.000 \odot$ Pu-239 $0.000 \mathrm{E}+00 \quad 0.0000$ mrem/yr fract. m
mrem/yr fract. mrem/yr fract.

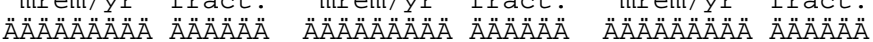

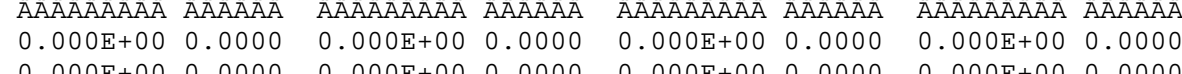
IIIIII IIIIIIII IIIII $\begin{array}{llll}0.000 E+00 & 0.0000 & 0.000 E+00 & 0.0000\end{array}$ Total $\odot .000 \mathrm{E}+0 \odot \quad 0.0000$

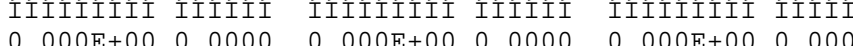
AАÄÄAAAA ÄÄÄÄÄ̈

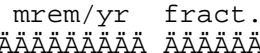

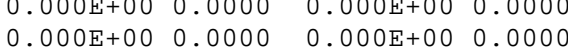
IIIIIIIII IIIIII $\odot$

Total Dose Contributions TDOSE( $i, p, t)$ for Individual Radionuclides (i) and Pathways ( $p$ ) As mrem/yr and Fraction of Total Dose At $t=3.000 \mathrm{E}+\odot 2$ years

$\odot$ Fish Water Dependent Pathways

Water Radon Plant

Meat Milk All Pathways*

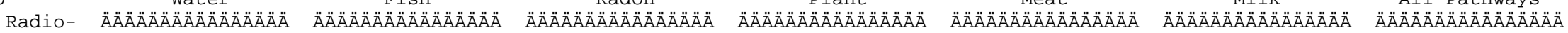
Nuclide mrem/yr fract mrem/yr fract mrem/yr fract mrem/yr fract mrem/yr fract m mrem/yr fract mrem/yr fract. AAAAAAA AAAAAAAAA AAAAAA AAAAAAAAA AAAAAA AAAAAAAA AAAAAA AAAAAAAAA AAAAAA

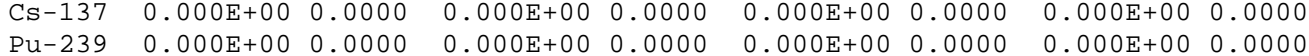

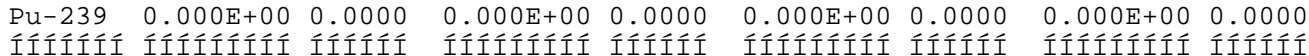

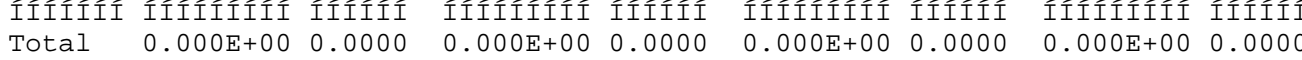
mrem/yr fract AAAAAAAAA AAAAAA mrem/yr fract. mrem/yr fract. (1)

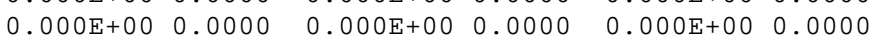

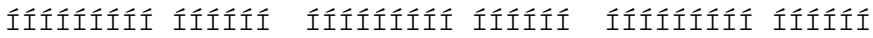
$0^{*}$ Sum of all water independent and dependent pathways. 

1RESRAD, Version 6.3
T« Limit $=180$ days
05/11/2006 16:58 Page 18
File: CAU 383 Oil spill. RAD

Total Dose Contributions TDOSE( $i, p, t)$ for Individual Radionuclides (i) and Pathways $(p)$ As $\mathrm{mrem} / \mathrm{yr}$ and Fraction of Total Dose At $\mathrm{t}=1.000 \mathrm{E}+03$ years
Water Independent Pathways (Inhalation excludes radon)

0 Ground
Radio-
ÄÄÄ̈̈̈̈̈̈̈̈̈̈̈̈A Inhalation Radon Plant Meat Muclide mrem/yr fract. ÄÄÄÄÄÄÄÄÄÄÄÄÄÄÄ ÄÄÄÄÄÄÄÄÄÄÄÄÄÄÄ ÄÄÄÄÄÄÄÄÄÄÄÄÄÄÄÄÄ ӒӒÄÄÄÄÄÄÄÄÄÄÄÄÄÄ ÄÄÄÄÄÄ̈̈̈̈̈̈̈ ÄÄÄÄÄÄÄ Cs-137 $0.000 \mathrm{E}+0 \odot \quad \odot .000 \odot$ Pu-239 $0.000 \mathrm{E}+00 \quad 0.000 \odot$ mrem/yr fract. maAAAAAAAAAAAA
rem fract. $\mathrm{mrem} / \mathrm{yr}$ fract mrem/yr fract АAАÄÄÄÄÄÄÄÄÄÄÄÄ mrem/yr fract.

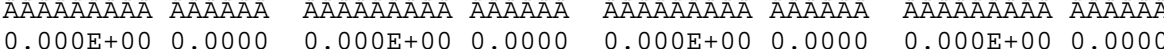
Íííííi ííííííí ííííí

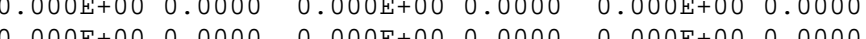
$0.000+00$ O.0000 $\odot .000 \mathrm{E}+0 \odot$ $0.000 \odot$ Аล̈ÄÄÄÄÄÄÄÄÄÄÄÄÄÄ $\begin{array}{lll}\text { Total } & 0.000 \mathrm{E}+\odot \odot & 0.00 \odot \odot\end{array}$

$0.000 \mathrm{E}+000.0000$

Iifitifition

$0.000 \mathrm{E}+000.0000$

Ííííííí ÍÍÍíí $\mathrm{mrem} / \mathrm{yr}$ fract. $\odot$

Total Dose Contributions $\operatorname{TDOSE}(i, p, t)$ for Individual Radionuclides (i) and Pathways (p) As $\mathrm{mrem} / \mathrm{yr}$ and Fraction of Total Dose At $t=1.000 \mathrm{E}+03$ years

$\odot$
$\odot$ Water Dependent Pathways

$$
\text { Water Fish r Radon }
$$

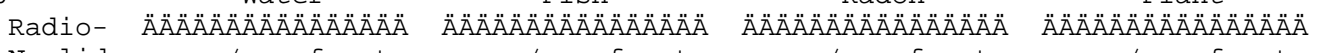
Nuclide mrem/yr fract. mrem/yr fract. mrem/yr fract. mrem/yr fract.

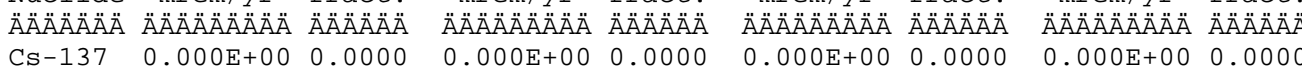
$\begin{array}{lllllllll}\mathrm{CS}-137 & 0.000 \mathrm{E}+00 & 0.0000 & 0.000 \mathrm{E}+00 & 0.0000 & 0.000 \mathrm{E}+00 & 0.0000 & 0.000 \mathrm{E}+00 & 0.0000 \\ \mathrm{Pu}-239 & 0.000 \mathrm{E}+00 & 0.0000 & 0.000 \mathrm{E}+00 & 0.0000 & 0.000 \mathrm{E}+00 & 0.0000 & 0.000 \mathrm{E}+00 & 0.0000\end{array}$

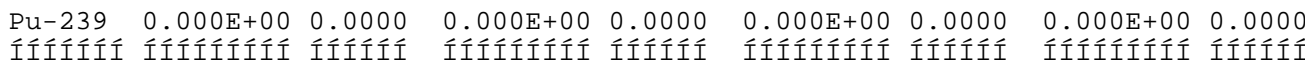

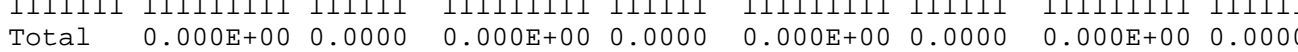
Milk All Pathways* ÄÄÄÄÄÄÄÄÄÄÄÄÄÄÄÄ ÄÄÄÄÄÄÄÄÄÄÄÄÄÄÄÄ ÄÄÄÄÄÄÄÄÄÄÄÄÄÄÄÄ mrem/yr fract. mrem/yr fract. mrem/yr fract. ÄÄÄÄÄÄÄÄÄ ÄÄÄÄÄÄ ÄÄÄÄÄÄÄÄÄ ÄÄÄÄÄÄ ÄÄÄÄÄÄÄÄÄ ÄÄÄÄÄÄ

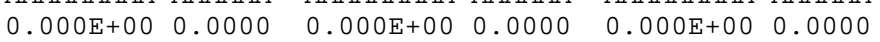
$\begin{array}{llllll}0.000 \mathrm{E}+00 & 0.0000 & 0.000 \mathrm{E}+00 & 0.0000 & 0.000 \mathrm{E}+00 & 0.0000\end{array}$

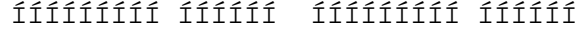
${ }^{*}$ Sum of all water independent and dependent pathways. 
Dose/Source Ratios Summed Over All Pathways

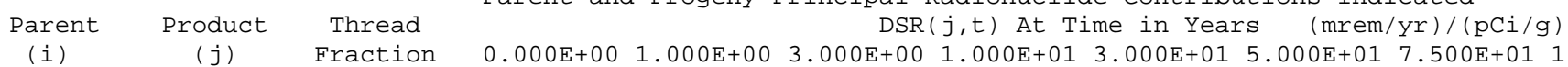

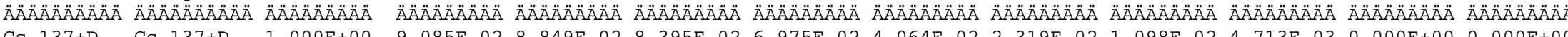
Cs-137+D CS-137+D $\quad 1.000 \mathrm{E}+00 \quad 9.085 \mathrm{E}-02$ 8.849E-02 8.395E-02 6.975E-02 4.064E-02 2.319E-02 $1.098 \mathrm{E}-02 \quad 4.713 \mathrm{E}-03 \quad 0.000 \mathrm{E}+00 \quad 0.000 \mathrm{E}+00$

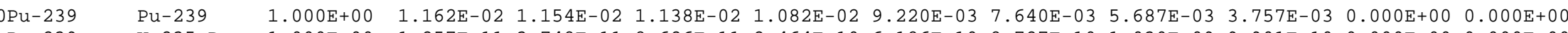

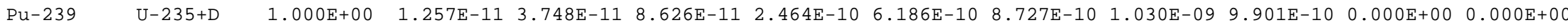

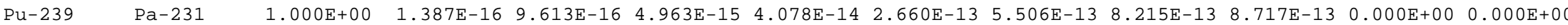

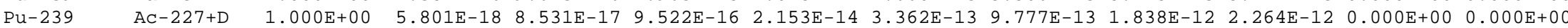
Pu-239 äDSR $(j) \quad 1.162 \mathrm{E}-02 \quad 1.154 \mathrm{E}-02 \quad 1.138 \mathrm{E}-02 \quad 1.082 \mathrm{E}-02 \quad 9.220 \mathrm{E}-03 \quad 7.640 \mathrm{E}-03 \quad 5.687 \mathrm{E}-03 \quad 3.757 \mathrm{E}-03 \quad 0.000 \mathrm{E}+00 \quad 0.000 \mathrm{E}+00$

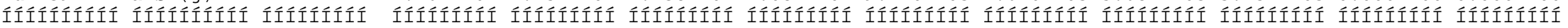
The DSR includes contributions from associated (half-life ó 180 days) daughters.

Single Radionuclide Soil Guidelines $G(i, t)$ in $\mathrm{pCi} / \mathrm{g}$ Basic Radiation Dose Limit $=2.500 \mathrm{E}+01 \mathrm{mrem} / \mathrm{yr}$

onuclide

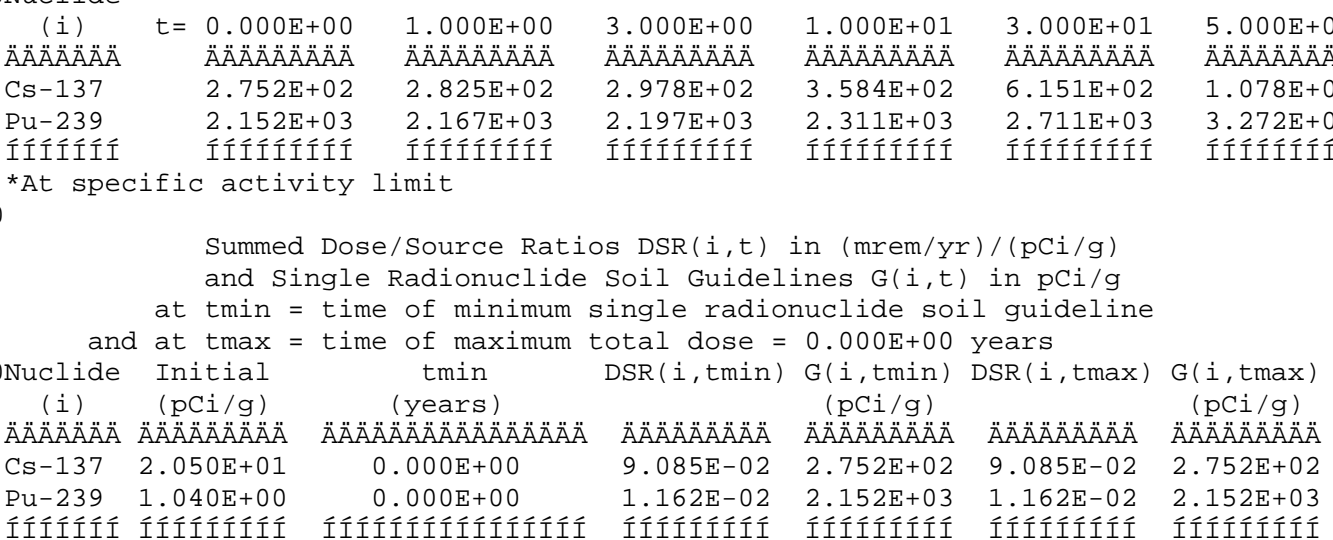


ONuclide Parent THF(i) (j) (i) Äล̈ÄÄÄÄÄ ÄÄÄÄÄÄÄ ÄÄÄÄÄÄÄÄÄ Cs-137 Cs-137 1.000E+OO ๑Pu-239 Pu-239 1. OU-235 PU-239 $1.000 \mathrm{E}+00$

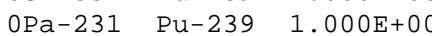
०AC-227 Pu-239 1. $1000 \mathrm{E}+00$ íííííi íííííi ííííííi IIIIII IIIIIII IIIIIIII
Individual Nuclide Dose Summed Over All Pathways

Parent Nuclide and Branch Fraction Indicated

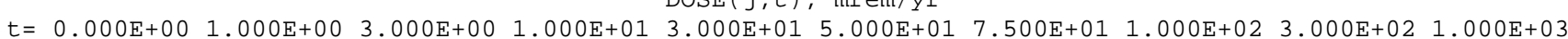
ÄÄÄÄÄÄÄÄ

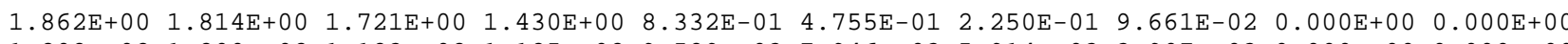

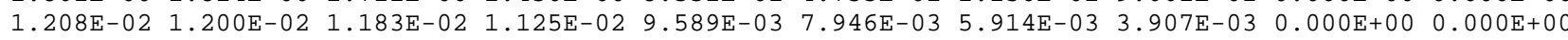

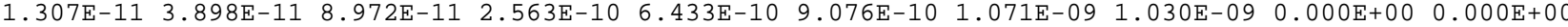
1.443E-16 9.997E-16 5.162E-15 4.241E-14 2.766E-13 5.726E-13 8.544E-13 9.065E-13 0.000E+00 $0.000 \mathrm{E}+00$

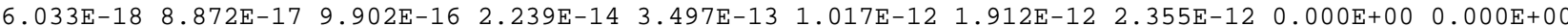

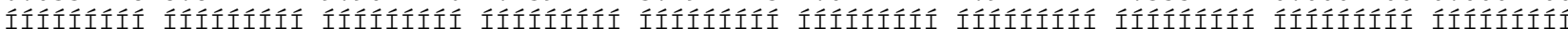
ion of the parent nuclide.

Individual Nuclide Soil Concentration

ONuclide Parent THF(i) (j) (i)

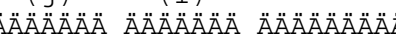
Cs-137 CS-137 1.000E+@०

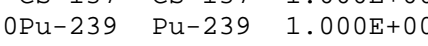

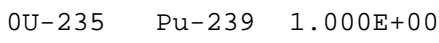
$\odot \mathrm{Pa}-231$ Pu-239 $1.000 \mathrm{E}+\odot \odot$ $\odot \mathrm{AC}-227 \mathrm{Pu}-239 \quad 1.000 \mathrm{E}+\odot \odot$ IIÍíííi íííííí íííííííi

THF(i) is the thread fraction ORESCALC. EXE execution time =
Parent Nuclide and Branch Fraction Indicated

$$
\mathrm{S}(j, \mathrm{t}), \mathrm{pCi} / \mathrm{g}
$$

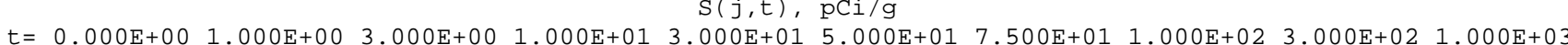

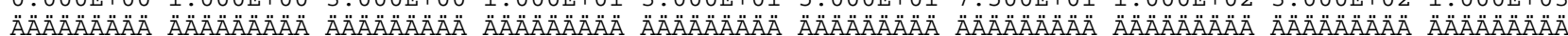

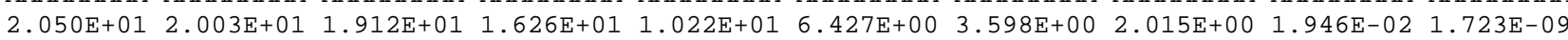

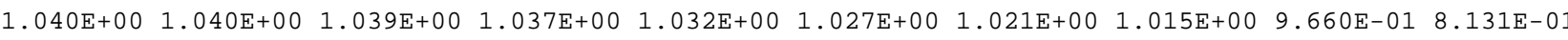

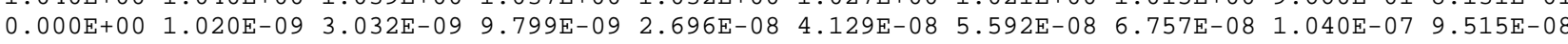
$0.000 \mathrm{E}+0 \odot$ 1. $1.077 \mathrm{E}-14$ 9.582E-14 $1.022 \mathrm{E}-12 \quad 8.194 \mathrm{E}-12 \quad 2.031 \mathrm{E}-11 \quad 3.972 \mathrm{E}-11$ 6.153E-11 $2.037 \mathrm{E}-10 \quad 2.382 \mathrm{E}-10$ $0.000 \mathrm{E}+0 \odot$ 1.130E-16 $2.945 \mathrm{E}-15$ 9.671E-14 $1.891 \mathrm{E}-12 \quad 6.516 \mathrm{E}-12 \quad 1.570 \mathrm{E}-11$ 2.737E-11 $1.161 \mathrm{E}-10$ 1.427E-10

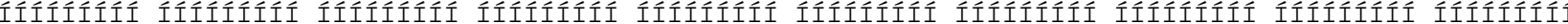
of the parent nuclide.

1.05 seconds 


\section{Exhibit 3}

\section{RESRAD Summary Report: \\ CAU 383 CAS 12-28-02}

RMA: 23 Pages 
Table of Contents

ÄÄÄÄÄÄÄÄÄÄÄÄÄÄÄÄ

Part I: Mixture Sums and Single Radionuclide Guidelines

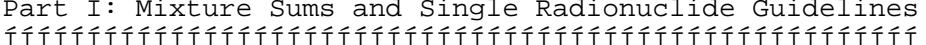

Dose Conversion Factor (and Related) Parameter Summary ... Site-Specific Parameter Summary ................... 4 Summary of Pathway Selections $\ldots \ldots \ldots \ldots \ldots \ldots \ldots \ldots \ldots \ldots$ Contaminated Zone and Total Dose Summary .............. 10 Total Dose Components

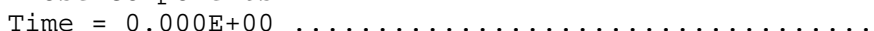

Time $=1.000 \mathrm{E}+00$.

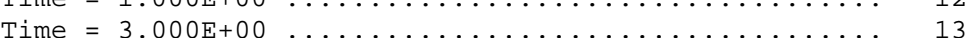

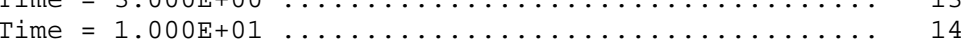

Time $=3.000 \mathrm{E}+01, \ldots \ldots \ldots \ldots \ldots \ldots \ldots \ldots \ldots \ldots \ldots \ldots \ldots, 15$

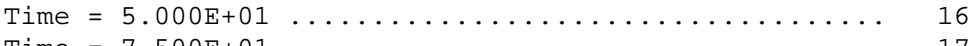

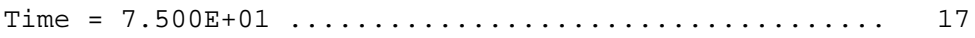

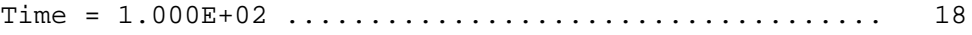

Time $=3.000 \mathrm{E}+02 \ldots \ldots \ldots \ldots \ldots \ldots \ldots \ldots \ldots \ldots \ldots \ldots, 19$

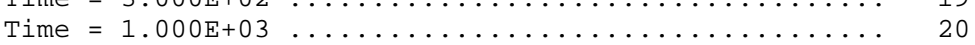

Dose/Source Ratios Summed over All Pathways .......... 21

Single Radionuclide Soil Guidelines $\ldots \ldots \ldots \ldots \ldots \ldots \ldots \ldots .21$

Dose Per Nuclide Summed Over All Pathways $\ldots \ldots \ldots \ldots \ldots \ldots \ldots$
Soil Concentration Per Nuclide $\ldots \ldots \ldots \ldots \ldots \ldots \ldots \ldots \ldots \ldots$ 
Dose Conversion Factor (and Related) Parameter Summary File: FGR 13 MORBIDITY

$\odot$

Parameter

3 Current ${ }^{3}$ Base ${ }_{3}$ Parameter
Menu ${ }^{3}$
Value
Case*
Name

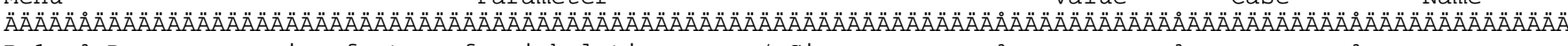

B-1 3 Dose conversion factors for inhalation, $\mathrm{mrem} / \mathrm{pCi}$ :

$B-1 \quad 3 \quad A C-227+D$

$B-1 \quad 3$ CS $-137+D$

$\mathrm{B}-1 \quad 3 \mathrm{~Pa}-231$

$\mathrm{B}-1 \quad 3 \mathrm{~Pb}-210+\mathrm{D}$

$\mathrm{B}-1$ 1 PU-238

$\mathrm{B}-1$ - $12 \mathrm{Pu}-238$

B-1 3 Ra-226+D

B-1 3 Th-230

$\mathrm{B}-1 \quad 3 \quad \mathrm{U}-234$

B-1 3 U-235+D

3

D-1 3 Dose conversion factors for ingestion, $\mathrm{mrem} / \mathrm{pCi}$ :

D-1 3 AC-227+D

$D-1 \quad 3 \quad$ Cs $-137+D$

$\mathrm{D}-1 \quad 3 \mathrm{~Pa}-231$

$\mathrm{D}-1 \quad 3 \mathrm{~Pb}-210+\mathrm{D}$

D-1 3 Pu-238

D-1 3 Pu-239

D-1 3 Ra-226+D

D-1 3 Th -230

D-1 3 U -234

D-1 $3 \quad$ U $-235+D$

D-34 3 Food transfer factors:

$\mathrm{D}-343 \mathrm{AC}-227+\mathrm{D}$, plant/soil concentration ratio, dimensionless

$\mathrm{D}-343 \mathrm{AC}-227+\mathrm{D}$, beef/livestock-intake ratio, $(\mathrm{pCi} / \mathrm{kg}) /(\mathrm{pCi} / \mathrm{d})$

$\mathrm{D}-343$ AC-227+D, milk/livestock-intake ratio, $(\mathrm{pCi} / \mathrm{L}) /(\mathrm{pCi} / \mathrm{d})$

D -343

D-34 3 Cs-137+D, plant/soil concentration ratio, dimensionless

$\mathrm{D}-343 \mathrm{Cs}-137+\mathrm{D}$, beef/livestock-intake ratio, $(\mathrm{pCi} / \mathrm{kg}) /(\mathrm{pCi} / \mathrm{d})$

$\mathrm{D}-34{ }^{3} \mathrm{Cs}-137+\mathrm{D}$, milk/livestock-intake ratio, (pCi/L)/(pCi/d)

$\mathrm{D}-343$

$\mathrm{D}-34 \quad \mathrm{~Pa}-231$

$\mathrm{D}-34 \quad 3 \mathrm{~Pa}-231$

$\mathrm{D}-34{ }^{3} \mathrm{~Pa}-231$

$\mathrm{D}-343$

$\mathrm{D}-344^{3} \mathrm{~Pb}-210+\mathrm{D}$

plant/soil concentration ratio, dimensionless

, beef/livestock-intake ratio, $(\mathrm{pCi} / \mathrm{kg}) /(\mathrm{pCi} / \mathrm{d})$

, milk/livestock-intake ratio, $(\mathrm{pCi} / \mathrm{L}) /(\mathrm{pCi} / \mathrm{d})$ $\mathrm{D}-343 \mathrm{~Pb}-210+\mathrm{D}$, beef/livestock-intake ratio, $(\mathrm{pCi} / \mathrm{kg}) /(\mathrm{pCi} / \mathrm{d})$

$\mathrm{D}-343 \mathrm{~Pb}-210+\mathrm{D}$, milk/livestock-intake ratio, $(\mathrm{pCi} / \mathrm{L}) /(\mathrm{pCi} / \mathrm{d})$

D-34 3

$\mathrm{D}-344^{3} \mathrm{Pu}-238$

$\mathrm{D}-34$ 3 $\mathrm{Pu}-238$

$\mathrm{D}-34{ }^{3} \mathrm{Pu}-238$

D-34 3

plant/soil concentration ratio, dimensionless

, beef/livestock-intake ratio, $(\mathrm{pCi} / \mathrm{kg}) /(\mathrm{pCi} / \mathrm{d})$

, milk/livestock-intake ratio, $(\mathrm{pCi} / \mathrm{L}) /(\mathrm{pCi} / \mathrm{d})$

$\mathrm{D}-34 \quad 3$ Pu-239

D-34 3 Pu-239

plant/soil concentration ratio, dimensionless , beef/livestock-intake ratio, $(\mathrm{pCi} / \mathrm{kg}) /(\mathrm{pCi} / \mathrm{d})$

, milk/livestock-intake ratio, $(\mathrm{pCi} / \mathrm{L}) /(\mathrm{pCi} / \mathrm{d})$

\begin{tabular}{|c|c|c|c|c|}
\hline \multirow{2}{*}{$6.724 \mathrm{E}+0 \odot$} & & \multicolumn{2}{|l|}{3} \\
\hline & & $6.700 \mathrm{E}+0 \odot$ & 3 DCF2( & 1) \\
\hline $3.190 \mathrm{E}-05$ & 3 & $3.190 \mathrm{E}-05$ & 3 DCF2( & 2) \\
\hline $1.280 \mathrm{E}+\odot \odot$ & 3 & $1.280 \mathrm{E}+0 \odot$ & 3 DCF2( & 3) \\
\hline $2.320 E-02$ & 3 & 1. $360 \mathrm{E}-02$ & 3 DCF2( & 4) \\
\hline $3.920 E-01$ & 3 & 3. $920 \mathrm{E}-01$ & 3 DCF2( & 5) \\
\hline $4.290 E-01$ & 3 & $4.290 \mathrm{E}-01$ & 3 DCF2( & 7) \\
\hline $8.594 \mathrm{E}-03$ & 3 & $8.580 \mathrm{E}-\odot 3$ & 3 DCF2( & 8) \\
\hline $3.260 \mathrm{E}-01$ & 3 & $3.26 \odot E-\odot 1$ & 3 DCF2( & 9) \\
\hline 1. $320 \mathrm{E}-01$ & 3 & $1.320 \mathrm{E}-01$ & 3 DCF2( & 10) \\
\hline $1.230 \mathrm{E}-01$ & $\begin{array}{l}3 \\
3\end{array}$ & $1.230 \mathrm{E}-01$ & $\begin{array}{l}3 \text { DCF2 } \\
3\end{array}$ & 11) \\
\hline & 3 & & 3 & \\
\hline 1. $480 E-\odot 2$ & 3 & $1.410 \mathrm{E}-02$ & 3 DCF3( & 1) \\
\hline $5.000 E-05$ & 3 & $5.000 \mathrm{E}-05$ & 3 DCF3( & 2) \\
\hline 1. $060 \mathrm{E}-\odot 2$ & 3 & 1. $\odot 6 \odot E-\odot 2$ & 3 DCF3( & 3) \\
\hline $7.276 \mathrm{E}-03$ & 3 & $5.37 \odot E-\odot 3$ & 3 DCF3( & 4) \\
\hline $3.200 E-03$ & 3 & $3.200 \mathrm{E}-\odot 3$ & 3 DCF3( & 5) \\
\hline $3.540 E-03$ & 3 & $3.540 E-03$ & 3 DCF3( & 7) \\
\hline 1. $321 \mathrm{E}-03$ & 3 & 1.320E- 03 & 3 DCF3( & 8) \\
\hline $5.480 E-04$ & 3 & $5.480 E-\odot 4$ & 3 DCF3( & 9) \\
\hline $2.830 E-04$ & 3 & $2.830 \mathrm{E}-\odot 4$ & 3 DCF3 & 10) \\
\hline $2.673 \mathrm{E}-04$ & 3 & $2.660 \mathrm{E}-04$ & 3 DCF3 & 11) \\
\hline & 3 & & 3 & \\
\hline & 3 & & 3 & \\
\hline $2.500 \mathrm{E}-03$ & 3 & $2.500 \mathrm{E}-\odot 3$ & 3 RTF ( & 1,1) \\
\hline 2. $.000 E-\odot 5$ & 3 & 2. $00 \odot E-05$ & 3 RTF( & $1,2)$ \\
\hline $2.000 E-05$ & 3 & 2. $000 \mathrm{E}-\odot 5$ & $\begin{array}{l}3 \\
3\end{array}$ & $1,3)$ \\
\hline $4.00 \odot E-\odot 2$ & 3 & 4. $\odot \odot \odot E-\odot 2$ & 3 RTF ( & $2,1)$ \\
\hline $3.000 E-\odot 2$ & 3 & $3.0 \odot \odot E-\odot 2$ & 3 RTF ( & $2,2)$ \\
\hline $8.000 E-03$ & $\begin{array}{l}3 \\
3\end{array}$ & 8. $000 \mathrm{E}-03$ & 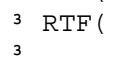 & $2,3)$ \\
\hline 1. $000 \mathrm{E}-\odot 2$ & 3 & 1. $\odot \odot \odot E-\odot 2$ & 3 RTF( & $3,1)$ \\
\hline $5.000 E-03$ & 3 & $5 . \odot \odot \odot E-\odot 3$ & 3 RTF ( & $3,2)$ \\
\hline $5.000 \mathrm{E}-06$ & 3 & $5.000 \mathrm{E}-06$ & $\begin{array}{l}3 \\
3\end{array}$ & $3,3)$ \\
\hline 1. $\odot \odot \odot E-\odot 2$ & 3 & 1. $\odot \odot \odot E-\odot 2$ & 3 RTF( & $4,1)$ \\
\hline 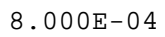 & 3 & 8. $0 \odot \odot E-\odot 4$ & 3 RTF( & $4,2)$ \\
\hline $3 . \odot \odot \odot E-\odot 4$ & 3 & $3 . \odot \odot \odot E-\odot 4$ & ${ }_{3}^{3} \mathrm{RTF}($ & $4,3)$ \\
\hline 1. $000 \mathrm{E}-\odot 3$ & 3 & 1. $000 \mathrm{E}-\odot 3$ & 3 RTF( & $5,1)$ \\
\hline 1. $000 \mathrm{E}-04$ & 3 & 1. $00 \odot E-\odot 4$ & 3 RTF( & $5,2)$ \\
\hline 1. $000 \mathrm{E}-06$ & & 1. $000 \mathrm{E}-06$ & 3 RTF( & $5,3)$ \\
\hline & & & 3 & \\
\hline 1. $000 \mathrm{E}-\odot 3$ & 3 & 1. $00 \odot E-\odot 3$ & 3 RTF( & $7,1)$ \\
\hline 1. $000 \mathrm{E}-\odot 4$ & & 1. $\odot \odot \odot E-\odot 4$ & 3 RTF ( & $7,2)$ \\
\hline 1. $000 \mathrm{E}-06$ & & 1. $\odot \odot \odot E-\odot 6$ & 3 RTF ( & $7,3)$ \\
\hline
\end{tabular}




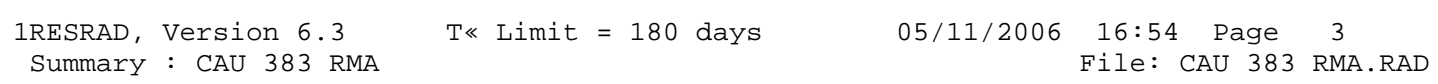

Dose Conversion Factor (and Related) Parameter Summary (continued) File: FGR 13 MORBIDITY

0

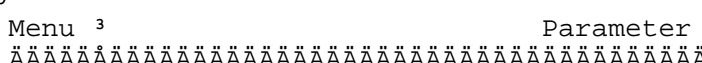

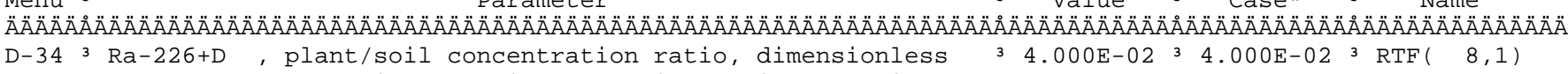

$\mathrm{D}-34 \mathrm{Ra}-226+\mathrm{D}$, beef/livestock-intake ratio, $(\mathrm{pCi} / \mathrm{kg}) /(\mathrm{pCi} / \mathrm{d})$

$\mathrm{D}-343 \mathrm{Ra}-226+\mathrm{D}$, milk/livestock-intake ratio, (pCi/L)/(pCi/d)

$\mathrm{D}-344^{3}$

D-34 3 Th-230, plant/soil concentration ratio, dimensionless

D- $-34 \quad 3$ Th -230

D- 34

D-34 3

D -34 - 3 U -234

$\mathrm{D}-34$ - 3 U-234

D-34 3 U-234

$\mathrm{D}-344^{3}$

D-34 3 U-235+D

, milk/livestock-intake ratio, $(\mathrm{pCi} / \mathrm{L}) /(\mathrm{pCi} / \mathrm{d})$

plant/soil concentration ratio, dimensionless

, beef/livestock-intake ratio, $(\mathrm{pCi} / \mathrm{kg}) /(\mathrm{pCi} / \mathrm{d})$

, milk/livestock-intake ratio, $(\mathrm{pCi} / \mathrm{L}) /(\mathrm{pCi} / \mathrm{d})$

$D-34 \quad 3 \quad U-235+D$

, plant/soil concentration ratio, dimensionless

D-34 3 U-235+D

blant/soil concentration ratio, dimensionless

, milk/livestock-intake ratio, $(\mathrm{pCi} / \mathrm{L}) /(\mathrm{pCi} / \mathrm{d})$

D-5 3 Bioaccumulation factors, fresh water, $\mathrm{L} / \mathrm{kg}$ :

$D-5 \quad 3$ AC-227+D, fish

D-5 3 AC-227+D, crustacea and mollusks

D $-5 \quad 3$

D-5 3 Cs $-137+D$, fish

D-5 3 Cs $-137+D$, crustacea and mollusks

$\mathrm{D}-5 \quad 3$

D-5 3 Pa-231, fish

D-5 3 Pa-231 , crustacea and mollusks

$D-5 \quad 3$

D-5 $3 \mathrm{~Pb}-210+\mathrm{D}$, fish

D-5 3 Pb-210+D, crustacea and mollusks

D $-5 \quad 3$

D-5 3 Pu-238, fish

D-5 3 Pu-238, crustacea and mollusks

$D-5 \quad 3$

D-5 3 Pu-239, fish

D-5 3 Pu-239 , crustacea and mollusks

D-5 3 Ra-226+D, fish

D-5 3 Ra-226+D, crustacea and mollusks

D- 53

D-5 3 Th-230 fish

D-5 3 Th-230 , crustacea and mollusks

D-5 3

D-5 3 U-234 , fish

D-5 3 U-234 , crustacea and mollusks

D-5 3 U $-235+D$, fish

$\begin{array}{lllllllllll}\mathrm{D}-5 & 3 & \mathrm{U}-235+\mathrm{D} & \text { crustacea and mollusks } & 3 & 6.000 \mathrm{E}+01 & 3 & 6.000 \mathrm{E}+01 & 3 & \mathrm{BIOFAC}(11,2)\end{array}$

1. $000 \mathrm{E}-03$ 3 $1.000 \mathrm{E}-03$ 3 $\operatorname{RTF}(8,3)$

$31.000 \mathrm{E}-03 \quad 3 \quad 1.000 \mathrm{E}-03 \quad 3 \operatorname{RTF}(9,1)$

1. $\odot \odot \odot E-\odot 4 \quad 3 \quad 1, \odot \odot \odot E-\odot 4 \quad 3 \operatorname{RTF}(9,2)$

5. $000 \mathrm{E}-06$ 3 5.000E-06 3 RTF ( 9,3)

$2.500 \mathrm{E}-03 \quad 3 \quad 2.500 \mathrm{E}-03 \quad 3 \operatorname{RTF}(10,1)$

3 3.400E-04 3 3.400E-04 $3 \operatorname{RTF}(10,2)$

$6.000 \mathrm{E}-044^{3} 6.000 \mathrm{E}-04{ }^{3} \operatorname{RTF}(10,3)$

3 2.500E-03 3 2.500E- $03 \quad 3 \operatorname{RTF}(11,1)$

$3.400 \mathrm{E}-04 \quad 3 \quad 3.400 \mathrm{E}-04 \quad 3 \operatorname{RTF}(11,2)$

3 $6.000 \mathrm{E}-04$ 3 $6.000 \mathrm{E}-043 \operatorname{RTF}(11,3)$

$1.500 \mathrm{E}+011^{3} 1.500 \mathrm{E}+011^{3} \operatorname{BIOFAC}(1,1)$

1. $000 \mathrm{E}+03$ 3 $1.000 \mathrm{E}+03$ 3 $\operatorname{BIOFAC}(1,2)$

$32.000 \mathrm{E}+03 \quad 3 \quad 2.000 \mathrm{E}+03 \quad 3 \operatorname{BIOFAC}(2,1)$

1. $000 \mathrm{E}+0231.000 \mathrm{E}+02{ }^{3} \operatorname{BIOFAC}(2,2)$

$1.000 \mathrm{E}+0131.000 \mathrm{E}+013 \operatorname{BIOFAC}(3,1)$

$1.100 \mathrm{E}+02$ 3 $1.100 \mathrm{E}+02$ 3 $\operatorname{BIOFAC}(3,2)$

$3 \quad 3.000 \mathrm{E}+02 \quad 3 \quad 3.000 \mathrm{E}+023 \operatorname{BIOFAC}(4,1)$

1. $000 \mathrm{E}+02 \quad 3 \quad 1.000 \mathrm{E}+023 \operatorname{BIOFAC}(4,2)$

$3.000 \mathrm{E}+01 \quad 3 \quad 3.000 \mathrm{E}+01 \quad 3 \quad \operatorname{BIOFAC}(5,1)$

1. $000 \mathrm{E}+02 \quad 3 \quad 1.000 \mathrm{E}+023 \operatorname{BIOFAC}(5,2)$

$3 \quad 3.000 \mathrm{E}+013 \quad 3.000 \mathrm{E}+011^{3} \operatorname{BIOFAC}(7,1)$

$1.000 \mathrm{E}+02$ 3 $1.000 \mathrm{E}+02 \quad 3 \quad \operatorname{BIOFAC}(7,2)$

$35.000 \mathrm{E}+01 \quad 3 \quad 5.000 \mathrm{E}+013 \operatorname{BIOFAC}(8,1)$

$32.500 \mathrm{E}+02 \quad 3 \quad 2.500 \mathrm{E}+02 \quad 3 \operatorname{BIOFAC}(8,2)$

1. $000 \mathrm{E}+02 \quad 3 \quad 1.000 \mathrm{E}+02 \quad 3 \quad \operatorname{BIOFAC}(9,1)$

5. $5.000 \mathrm{E}+02 \quad 3 \quad 5.000 \mathrm{E}+02$ 3 $\operatorname{BIOFAC}(9,2)$

$1.000 \mathrm{E}+01 \quad 3 \quad 1.000 \mathrm{E}+013 \operatorname{BIOFAC}(10,1)$

3 $6.000 \mathrm{E}+01$ 3 $6.000 \mathrm{E}+01$ 3 $\operatorname{BIOFAC}(10,2)$

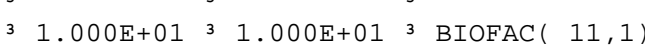

*Base Case means Default.Lib w/o Associate Nuclide contributions. 
Site-Specific Parameter Summary

0

3 User

Used by RESRAD

Used by RESRAD 3 Parameter

Menu 3 Parameter 3 Input 3 Default 3 (If different from user input) 3 Name

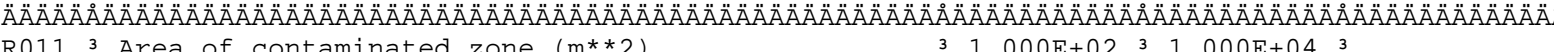

R011 3 Area of contaminated zone $\left(\mathrm{m}^{* *} 2\right)$

R011 3 Thickness of contaminated zone $(\mathrm{m})$

R011 3 Length parallel to aquifer flow $(\mathrm{m})$

R011 3 Basic radiation dose limit (mrem/yr)

R011 3 Time since placement of material (yr)

R011 3 Times for calculations ( $y r$ )

R011 3 Times for calculations ( $\mathrm{yr}$ )

R011 3 Times for calculations ( $\mathrm{yr}$ )

R011 3 Times for calculations ( $y r$ )

R011 3 Times for calculations ( $\mathrm{yr}$ )

R011 3 Times for calculations ( $\mathrm{yr}$ )

R011 3 Times for calculations ( $y r$ )

R011 3 Times for calculations ( $y r$ )

R011 3 Times for calculations ( $\mathrm{yr}$ )

3

R012 3 Initial principal radionuclide ( $\mathrm{pCi} / \mathrm{g}$ )

R012 3 Initial principal radionuclide (pCi/g) :

R012 3 Concentration in groundwater ( $\mathrm{pCi} / \mathrm{L})$ : $\mathrm{Cs}-137$

$\mathrm{R} 0123$ Concentration in groundwater ( $\mathrm{pCi} / \mathrm{L})$ : $\mathrm{Pu}-238$

R012 3 Concentration in groundwater (pCi/L):

R013 3 Cover depth (m)

R013 3 Density of cover material $\left(\mathrm{g} / \mathrm{cm}^{* *} 3\right)$

R013 3 Cover depth erosion rate (m/yr)

R013 3 Density of contaminated zone $\left(\mathrm{g} / \mathrm{cm}^{* *} 3\right)$

R013 3 Contaminated zone erosion rate $(\mathrm{m} / \mathrm{yr})$

R013 3 Contaminated zone total porosity

R013 3 Contaminated zone field capacity

R013 3 Contaminated zone hydraulic conductivity (m/yr)

R013 3 Contaminated zone b parameter

R013 3 Average annual wind speed ( $\mathrm{m} / \mathrm{sec}$ )

R013 3 Humidity in air $\left(\mathrm{g} / \mathrm{m}^{* *} 3\right)$

R013 3 Evapotranspiration coefficient

R013 3 Precipitation ( $\mathrm{m} / \mathrm{yr}$ )

R013 3 Irrigation ( $\mathrm{m} / \mathrm{yr}$ )

R013 3 Irrigation mode

R013 3 Runoff coefficient

R013 3 Watershed area for nearby stream or pond $\left(m^{* * 2}\right)$

R013 3 Accuracy for water/soil computations

R014 3 Density of saturated zone $\left(\mathrm{g} / \mathrm{cm}^{* *} 3\right)$

R014 3 Saturated zone total porosity

R014 3 Saturated zone effective porosity

R014 3 Saturated zone field capacity

R014 3 Saturated zone hydraulic conductivity ( $\mathrm{m} / \mathrm{yr}$ )

R014 3 Saturated zone hydraulic gradient

R014 3 Saturated zone b parameter

R014 3 water table drop rate (m/yr)

R014 3 Well pump intake depth ( $\mathrm{m}$ below water table)

R014 3 Model: Nondispersion (ND) or Mass-Balance (MB
$1.000 \mathrm{E}+02 \quad 3 \quad 1.000 \mathrm{E}+04$

$1.500 \mathrm{E}-0132.000 \mathrm{E}+00$

3 not used $31.000 \mathrm{E}+022^{3}$

$\begin{array}{llll}3 & 2.500 \mathrm{E}+01 & 3 & 3.000 \mathrm{E}+01\end{array}$

$30.000 \mathrm{E}+00 \quad 3 \quad 0.000 \mathrm{E}+0{ }^{3}$

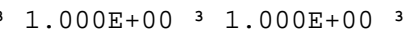

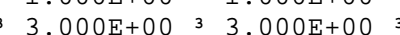

1. $000 \mathrm{E}+01$ 3 1. 1. $000 \mathrm{E}+01$

3. $000 \mathrm{E}+01$ 3 3. 3. $000 \mathrm{E}+01$

$5.000 \mathrm{E}+0131.000 \mathrm{E}+02$

$7.500 \mathrm{E}+01$ 3 $3.000 \mathrm{E}+02$

$1.000 \mathrm{E}+02 \quad 3 \quad 1.000 \mathrm{E}+03$

3. $1000 \mathrm{E}+02 \quad 3 \quad 0.000 \mathrm{E}+00$

$1.000 \mathrm{E}+03 \quad 3 \quad 0.000 \mathrm{E}+00$

$1.14 \odot \mathrm{E}+02 \quad 3 \quad \odot . \odot \odot \Theta \mathrm{E}+\Theta \odot$

$2.320 \mathrm{E}-01$ 3 $\odot$. $00 \odot \mathrm{E}+0 \odot$

$2.090 \mathrm{E}+\odot \odot$ 3 $\odot .000 \mathrm{E}+0 \odot$

not used $30.000 \mathrm{E}+00$

not used 3 ๑. $000 \mathrm{E}+00$

$3.0 \odot \odot \mathrm{E}+\odot \odot \quad 3 \quad \odot ., \odot \odot \mathrm{E}+\odot \odot$

not used $1.000+\odot \odot$

not used $1.500 \mathrm{E}+00$

not used $31.000 \mathrm{E}-03$

$1.500 \mathrm{E}+\odot \odot{ }^{3} 1.500 \mathrm{E}+\odot \odot$

1.00०E-03 3 1.00०E- 03

4.000E-01 3 4.000E-01

2. $000 \mathrm{E}-013 \quad 2.000 \mathrm{E}-01$

$1.000 \mathrm{E}+011^{3} 1.000 \mathrm{E}+01$

$5.300 \mathrm{E}+00 \quad 3 \quad 5.300 \mathrm{E}+00$

$3.400 \mathrm{E}+00 \quad 3 \quad 2.000 \mathrm{E}+0 \odot$

not used 3 8.000E+00

5. $000 \mathrm{E}-013 \quad 5.000 \mathrm{E}-01$

$3.260 \mathrm{E}-01$ 3 $1.000 \mathrm{E}+00$

$0.000 \mathrm{E}+0 \odot \quad 3 \quad 2.000 \mathrm{E}-01$

3 overhead 3 overhead 3

4.000E-01 3 2.000E-01

not used $31.000 \mathrm{E}+06$

not used 3 1.000E-03

not used 3 1.500E+00

not used 3 4.000E-01

not used 3 2.000E-01

not used 32 2.000E-01

not used 3 1.000E +02

3 not used 3 2. $000 \mathrm{E}-02$

3 not used $35.300 E+00$

3 not used $31.000 \mathrm{E}-03$

3 not used $31.000 \mathrm{E}+01$

3 not used 3 ND
-.- 3 AREA

-. 3 LCZPAQ

$-\cdot-$

$3 \mathrm{~T}(2)$

- - 3 T( 3$)$

3 T( 4$)$

$3 \mathrm{~T}(5)$

$3 \mathrm{~T}(6)$

$\begin{array}{lll}3 & \mathrm{~T}( & 8 \\ 3 & \mathrm{~T}(9)\end{array}$

S1( 2)

$-\cdot$

$\mathrm{S} 1(5)$

S1 $(7)$

3 W1 ( 2)

3 W1 ( 5)

W1 ( 7$)$

$\begin{array}{ll}3 & \text { COVERO } \\ 3 & \text { DENSCV }\end{array}$

3 VCV

3 DENSCZ

$3 \mathrm{VCZ}$

3 TPCZ

3 FCCZ

$3 \mathrm{HCCZ}$

$3 \mathrm{BCZ}$

3 WIND

3 HUMID

3 EVAPTR

3 PRECIP

3 RI

3 IDITCH

3 RUNOFF

WAREA

3 EPS

3 DENSAQ

3 TPSZ

3 EPSZ

3 FCSZ

3 HCSZ

3 HGWT

3 BSZ

3 VWT

3 VTT

3 MODEL 
Site-Specific Parameter Summary (continued)

$\odot \quad 3$

$$
3 \text { User }
$$

3 Default

Menu 3

Parameter

3 Input

З

Used by RESRAD

ÄÄÄÄÅÄÄÄÄÄÄÄÄÄÄÄÄÄÄÄÄÄÄÄÄÄ̈̈̈̈̈̈̈̈̈̈̈AA
R014 3 Well pumping rate $\left(m^{* *} 3 / y r\right)$ 3

R015 3 Number of unsaturated zone strata

R015 3 Unsat. zone 1, thickness (m)

R015 3 Unsat. zone 1 , soil density $\left(\mathrm{g} / \mathrm{cm}^{* *} 3\right)$

R015 3 Unsat. zone 1 , total porosity

R015 3 Unsat. zone 1, effective porosity

R015 3 Unsat. zone 1, field capacity

R015 3 Unsat. zone 1, soil-specific b parameter

R015 3 Unsat. zone 1, hydraulic conductivity (m/yr)

R016 3 Distribution coefficients for Cs-137

R016 3 Contaminated zone $\left(\mathrm{cm}^{* *} 3 / \mathrm{g}\right)$

R016 3 Unsaturated zone $1\left(\mathrm{~cm}^{* *} 3 / \mathrm{g}\right)$

$\mathrm{R} 016$ Saturated zone $\left(\mathrm{cm}^{* *} 3 / \mathrm{g}\right)$

R016 3 Leach rate (/yr)

R016 3 Solubility constant

R016 3 Distribution coefficients for $\mathrm{Pu}-238$

R016 3 Contaminated zone $\left(\mathrm{cm}^{* *} 3 / \mathrm{g}\right)$

R016 3 Unsaturated zone $1(\mathrm{~cm} * * 3 / \mathrm{g})$

R016 3 Saturated zone $\left(\mathrm{cm}^{* *} 3 / \mathrm{g}\right)$

R016 3 Leach rate (/yr)

R016 3 Solubility constant

R016 3 Distribution coefficients for Pu-239

R016 3 Contaminated zone $\left(\mathrm{cm}^{* *} 3 / \mathrm{g}\right)$

R016 3 Unsaturated zone $1\left(\mathrm{~cm}^{* *} 3 / \mathrm{g}\right)$

R016 3 Saturated zone $\left(\mathrm{cm}^{* *} 3 / \mathrm{g}\right)$

R016 3 Leach rate (/yr)

R016 3 Solubility constant

R016 3 Distribution coefficients for daughter Ac-227

RO16 3 Contaminated zone $\left(\mathrm{cm}^{* *} 3 / \mathrm{g}\right)$

R016 3 Unsaturated zone $1(\mathrm{~cm} * * 3 / \mathrm{g})$

R016 3 Saturated zone $\left(\mathrm{cm}^{* *} 3 / \mathrm{g}\right)$

R016 3 Leach rate (/yr)

R016 3 Solubility constant

R016 3 Distribution coefficients for daughter Pa-231

R016 3 Contaminated zone $\left(\mathrm{cm}^{* *} 3 / \mathrm{g}\right)$

R016 3 Unsaturated zone $1\left(\mathrm{~cm}^{* *} 3 / \mathrm{g}\right)$

R016 3 Saturated zone $\left(\mathrm{cm}^{* *} 3 / \mathrm{g}\right)$

R016 ${ }^{3}$ Leach rate (/yr)

R016 ${ }^{3} \quad$ Solubility constant

R016 3 Distribution coefficients for daughter Pb-210

R016 3 Contaminated zone $\left(\mathrm{cm}^{* *} 3 / \mathrm{g}\right)$

R016 3 Unsaturated zone $1\left(\mathrm{~cm}^{* *} 3 / \mathrm{g}\right)$

R016 3 Saturated zone $\left(\mathrm{cm}^{\star *} 3 / \mathrm{g}\right)$

R016 3 Leach rate (/yr)

R016 3 Solubility constant

$\begin{array}{lllll}3 & \text { not used } & 3 & 2.500 \mathrm{E}+02 & 3 \\ 3 & & 3 & 3 \\ 3 & \text { not used } & 3 & 1 & 3 \\ 3 & \text { not used } & 3 & 4.000 \mathrm{E}+\odot \odot & 3 \\ 3 & \text { not used } & 3 & 1.500 \mathrm{E}+0 \odot & 3 \\ 3 & \text { not used } & 3 & 4.000 \mathrm{E}-01 & 3 \\ 3 & \text { not used } & 3 & 2.000 \mathrm{E}-01 & 3 \\ 3 & \text { not used } & 3 & 2.000 \mathrm{E}-01 & 3 \\ 3 & \text { not used } & 3 & 5.300 \mathrm{E}+00 & 3 \\ 3 & \text { not used } & 3 & 1.000 \mathrm{E}+01 & 3 \\ 3 & & 3 & & 3 \\ 3 & & 3 & & 3\end{array}$

$34.600 \mathrm{E}+03$ 3 $4.600 \mathrm{E}+03$

3 not used 3 4.600E+03 3

3 not used 3 4.600E+O3

$0.000 \mathrm{E}+00 \quad 3 \quad 0.000 \mathrm{E}+00^{3}$

$\odot .00 \odot \mathrm{E}+\odot \odot \odot^{3} \odot . \odot \odot \odot \mathrm{E}+\odot \odot$

$2.000 \mathrm{E}+\odot 3 \quad 3 \quad 2.000 \mathrm{E}+03$

not used 3 2.000E +03

not used $32.000 \mathrm{E}+03$

๑. $000 \mathrm{E}+\odot \odot \quad 3 \quad 0.000 \mathrm{E}+\odot \odot^{3}$

$\odot .000 \mathrm{E}+0 \odot \quad 3 \quad \odot .000 \mathrm{E}+\odot \odot^{3}$

$2.000 \mathrm{E}+03 \quad 3 \quad 2.000 \mathrm{E}+03$

not used $32.000 \mathrm{E}+\mathrm{O}_{3}$

3 not used $32.000 \mathrm{E}+03$

$0.000 \mathrm{E}+003 \quad 0.000 \mathrm{E}+0 \odot$

$0.000 \mathrm{E}+\odot \odot \quad 3 \quad 0.000 \mathrm{E}+0 \mathrm{O}^{3}$

$2.000 \mathrm{E}+01 \quad 3 \quad 2.000 \mathrm{E}+01$

not used $32.000 \mathrm{E}+01$

not used $32.000 \mathrm{E}+01$

$0.000 \mathrm{E}+00 \quad 3 \quad 0.000 \mathrm{E}+00$

$0.000 \mathrm{E}+\odot \mathrm{O}^{3} \quad 0.000 \mathrm{E}+0 \odot{ }^{3}$

5. $\odot \odot \odot E+\odot 1 \quad 3 \quad 5.0 \odot \odot \mathrm{E}+\odot 1$

not used $35.000 \mathrm{E}+01$

not used 3 5.000E+01

०.०००E+०० $3 \quad 0.000 \mathrm{E}+0 \mathrm{O}^{3}$

$0.000 \mathrm{E}+00{ }^{3} \quad 0.000 \mathrm{E}+0 \odot$

3

3 1. $1.000 \mathrm{E}+02 \quad 3 \quad 1.000 \mathrm{E}+02$

3 not used $31.000 \mathrm{E}+02$

3 not used $31.000 \mathrm{E}+02$

$30.000 \mathrm{E}+0 \odot \quad 3 \quad 0.000 \mathrm{E}+0 \odot$

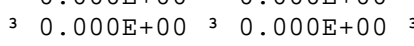
3 Parameter

ferent from user input)

Name

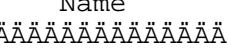

$-$

NS

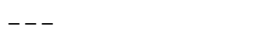

3 DENSUZ(1)

$3 \operatorname{TPUZ}(1)$

-. - $3 \operatorname{EPUZ}(1)$

-. 3 FCUZ (1)

$3 \operatorname{BUZ}(1)$

$3 \operatorname{HCUZ}(1)$

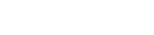

$--$

9. $449 \mathrm{E}-05$

not used

$\operatorname{DCNUCC}(2)$

$\operatorname{DCNUCU}(2,1)$

DCNUCS( 2)

ALEACH ( 2$)$

SOLUBK( 2)

DCNUCC ( 5 )

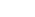

$-1$

.173E- -04

not used

$\operatorname{DCNUCU}(5,1)$

DCNUCS ( 5)

ALEACH $(5)$

SOLUBK ( 5)

$\operatorname{DCNUCC}(7)$

$-$

$2.173 \mathrm{E}-04$

not used

DCNUCS $(7)$

3 ALEACH $(7)$

SOLUBK( 7$)$

$-$

$2.153 \mathrm{E}-02$

not used

$\operatorname{DCNUCC}(1)$

$\operatorname{DCNUCU}(1,1)$

DCNUCS( 1)

3 ALEACH ( 1)

SOLUBK( 1$)$

DCNUCC ( 3)

$-$

$8.660 \mathrm{E}-03$

not used

DCNUCS ( 3)

ALEACH ( 3)

$3 \operatorname{SOLUBK}(3)$

$-\cdot$

$-$

$4.338 \mathrm{E}-\mathrm{03}$

$\operatorname{DCNUCC}(4)$

$3 \operatorname{DCNUCU}(4,1)$

3 DCNUCS( 4 )

$\begin{array}{ll}3 & \text { ALEACH ( } 4) \\ 3 & \text { SOLUBK }(4)\end{array}$ 
$0 \quad 3$

Site-Specific Parameter Summary (continued)

Menu 3

Menu 3 Parameter 3 Input 3 Default 3 (If different from user input) 3 Name

3 User

Menu 3 Parameter 3 Input 3 Default 3 (If different from user input) 3 Name

Used by RESRAD

3 Parameter

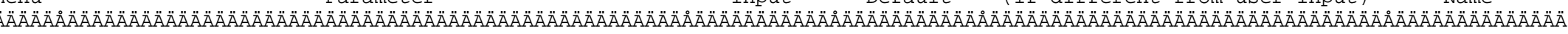

3

R016 3 Contaminated zone $\left(\mathrm{cm}^{* *} 3 / \mathrm{g}\right)$

RO16 3 Unsaturated zone $1\left(\mathrm{~cm}^{* *} 3 / \mathrm{g}\right)$

R016 3 Saturated zone $\left(\mathrm{cm}^{* *} 3 / \mathrm{g}\right)$

R016 3 Leach rate (/yr)

R016 3 Solubility constant

R016 3 Distribution coefficients for daughter Th-230

R016 3 Contaminated zone $\left(\mathrm{cm}^{* *} 3 / \mathrm{g}\right)$

R016 3 Unsaturated zone $1\left(\mathrm{~cm}^{* *} 3 / \mathrm{g}\right)$

R016 3 Saturated zone $\left(\mathrm{cm}^{* *} 3 / \mathrm{g}\right)$

R016 3 Leach rate (/yr)

R016 3 Solubility constant

R016 3 Distribution coefficients for daughter U-234

R016 3 Contaminated zone $\left(\mathrm{cm}^{* *} 3 / \mathrm{g}\right)$

R016 3 Unsaturated zone $1\left(\mathrm{~cm}^{* *} 3 / \mathrm{g}\right)$

R016 3 Saturated zone $\left(\mathrm{cm}^{* *} 3 / \mathrm{g}\right)$

R016 3 Leach rate (/yr)

R016 ${ }^{3}$ Solubility constant

R016 3 Distribution coefficients for daughter U-235

R016 3 Contaminated zone $\left(\mathrm{cm}^{* *} 3 / \mathrm{g}\right)$

R016 3 Unsaturated zone $1\left(\mathrm{~cm}^{* *} 3 / \mathrm{g}\right)$

R016 3 Saturated zone $\left(\mathrm{cm}^{* *} 3 / \mathrm{g}\right)$

R016 3 Leach rate (/yr)

R016 3 Solubility constant

R017 3 Inhalation rate $\left(m^{* *} 3 / y r\right)$

R017 3 Mass loading for inhalation $\left(\mathrm{g} / \mathrm{m}^{* *} 3\right)$

R017 3 Exposure duration

R017 3 Shielding factor, inhalation

R017 3 Shielding factor, external gamma

R017 3 Fraction of time spent indoors

R017 3 Fraction of time spent outdoors (on site)

R017 3 Shape factor flag, external gamma

R017 3 Radii of shape factor array (used if FS = -1)

$\mathrm{R} 017^{3}$ Outer annular radius $(\mathrm{m})$, ring 1 :

$\mathrm{R} 0173$ Outer annular radius $(\mathrm{m})$, ring 2 :

$\mathrm{R} 017^{3}$ Outer annular radius $(\mathrm{m})$, ring 3 :

$\mathrm{R} 017^{3}$ Outer annular radius $(\mathrm{m})$, ring 4 :

R017 3 Outer annular radius $(\mathrm{m})$, ring 5 :

R017 3 Outer annular radius ( $\mathrm{m})$, ring 6:

R017 3 Outer annular radius $(\mathrm{m})$, ring 7 :

R017 3 Outer annular radius $(\mathrm{m})$, ring 8:

R017 3 Outer annular radius $(\mathrm{m})$, ring 9:

R017 3 Outer annular radius $(\mathrm{m})$, ring 10:

$\mathrm{R} 0173$ Outer annular radius $(\mathrm{m})$, ring 11:

$\mathrm{R} 017^{3}$ Outer annular radius $(\mathrm{m})$, ring 12 :

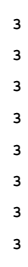

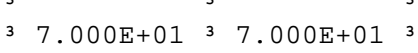

3 not used $37.000 \mathrm{E}+01^{3}$

not used 3 7.000E+01

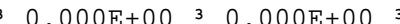

$0.000 \mathrm{E}+0 \odot^{3} \quad 0.000 \mathrm{E}+0 \odot^{3}$

$6.00 \odot \mathrm{E}+\odot 4 \quad 3 \quad 6.00 \odot \mathrm{E}+\odot 4$

not used $36.000 \mathrm{E}+04$

not used $36.000 \mathrm{E}+04$

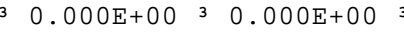

$0.000 E+00 \quad 3 \quad 0.000 E+00 \quad 3$ 3

$5.000 \mathrm{E}+01 \quad 3 \quad 5.000 \mathrm{E}+01$ $\begin{array}{lll}5 . \odot \odot \odot E+\odot 1 ~ & 5.0 \odot \odot E+\odot 1 \\ \text { not used } 3 & 5.00 \odot E+\odot 1\end{array}$ $\begin{array}{lll}\text { not used } & 5.000 \mathrm{E}+01 \\ \text { not used } 3 & 5.000 \mathrm{E}+01\end{array}$

$\begin{array}{lll}\text { not used } & 3 & 5.00 \odot E+\odot 1 \\ 0.000 E+\odot \odot ~ & 3 & 0.000 \mathrm{E}+\odot \odot\end{array}$

$\odot .0 \odot \odot E+\odot \odot \quad 3 \quad \odot . \odot \odot \odot E+\odot \odot$

3

$5.000 \mathrm{E}+0135.000 \mathrm{E}+01$ not used $5.000 \mathrm{E}+01$

not used $35.000 \mathrm{E}+01$

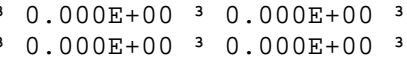

3 1.230E+04 3 8.400E+03 3

6.000E-04 3 1.000E-04

$2.500 \mathrm{E}+01 \quad 3 \quad 3.000 \mathrm{E}+013$

1. $000 \mathrm{E}+00$ 3 4.000E- 01

1. $1.00 \mathrm{E}+00$ 3 $7.000 \mathrm{E}-01$

$\odot .000 \mathrm{E}+00$ 3 5. $500 \mathrm{E}-01{ }^{3}$

$3.800 \mathrm{E}-02$ 3 $2.500 \mathrm{E}-01$

$1.000 \mathrm{E}+0031.000 \mathrm{E}+\odot \odot$

not used $35.000 \mathrm{E}+01$

3 not used $37.071 \mathrm{E}+013$

3 not used 3 ०. $000 \mathrm{E}+0 \odot^{3}$

not used 3 0.000E+00

not used $30.000 \mathrm{E}+00$

not used $30.000 \mathrm{E}+0 \odot$

not used $30.000 \mathrm{E}+0 \odot$

not used $30.000 \mathrm{E}+0 \odot$

3 not used $30.000 \mathrm{E}+00^{3}$

3 not used $3 \odot, \odot \odot \odot E+\odot \odot$

3 not used $30.000 E+00$

3 not used $30.00 \odot \mathrm{E}+\odot \odot^{3}$

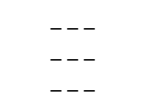

$6.193 E-03$

not used

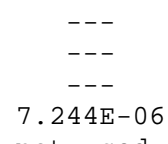

not used

---
---
---
8. $660 \mathrm{E}-03$
not used

not used

$$
\text { - - - }
$$

$8.660 \mathrm{E}-03$

not used

- - -

$-\cdot$

$-\cdot$

$-\cdot$

$--$

$>0$ shows circular AREA.

$--$

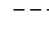

$-\cdot$

$--$

$---$

$---$

$--$

$-\cdot$

$--$
3 DCNUCC ( 8)

3 DCNUCU $(8,1)$

3 ALEACH ( 8)

SOLUBK( 8)

$\operatorname{DCNUCC}(9)$

$\operatorname{DCNUCU}(9,1)$

DCNUCS( 9)

SOLUBK $(9)$

$\operatorname{DCNUCC}(10)$

$\operatorname{DCNUCU}(10,1)$

$\operatorname{DCNUCS}(10)$

ALEACH (10)

$\operatorname{DCNUCC}(11)$

$3 \operatorname{DCNUCU}(11,1)$

DCNUCS(11)

ALEACH $(11)$

SOLUBK (11)

3 INHALR

MLINH

ED

3 SHF3

3 SHF1

3 FIND

3 FOTD

3

3 RAD_SHAPE( 1$)$

3 RAD_SHAPE ( 2)

3 RAD_SHAPE( 3 )

3 RAD_SHAPE( 4$)$

3 RAD_SHAPE( 5 )

3 RAD_SHAPE $(6)$

3 RAD_SHAPE ( 7 )

3 RAD_SHAPE ( 8$)$

3 RAD_SHAPE ( 9)

3 RAD_SHAPE $(10)$

3 RAD_SHAPE (11)

3 RAD_SHAPE (12) 
Site-Specific Parameter Summary (continued)

$0 \quad 3$$$
3 \text { User }
$$

Menu 3 Parameter 3 Input 3 Default 3 (If different from user input) 3 Name

Used by RESRAD

3 Parameter

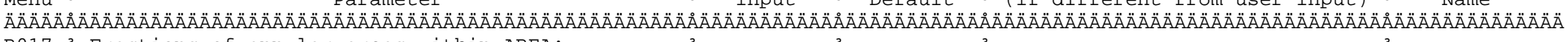
R017 3 Fractions of annular areas within AREA:

R017 3 Ring 1

$\begin{array}{llll}R 017 & 3 & \text { Ring } & 2\end{array}$

$\begin{array}{llll}\mathrm{R} 017 & 3 & \text { Ring } & 3\end{array}$

RO17 3 Ring 5

3 not used $3^{3} 1.000 \mathrm{E}+00^{3}$

3 not used $32.732 \mathrm{E}-01^{3}$

3 not used 3 ๑. $000 \mathrm{E}+0 \mathrm{O}^{3}$

3 not used $30.000 \mathrm{E}+0 \odot^{3}$

3 not used $30.000 \mathrm{E}+0 \odot^{3}$

R017 3 Ring 6

R017 3 Ring 7

$\mathrm{R} 0177^{3}$ Ring 8

$\mathrm{R} 0177^{3}$ Ring 9

$\begin{array}{llll}R 017 & 3 & \text { Ring } 10 \\ R 017 & 3 & \text { Ring } 11\end{array}$

R017 3 Ring 12

not used $30.000 \mathrm{E}^{3} 0 \mathrm{O}^{3}$

3 not used 3 ०. $000 \mathrm{E}+\odot \odot^{3}$

3 not used $30.000 \mathrm{E}+0{ }^{3}$

not used $30.000 \mathrm{E}+\odot \odot^{3}$

3 not used $30.00 \odot \mathrm{E}+\odot \odot$

3 not used $30.000 \mathrm{E}+0 \odot^{3}$

3

R018 ${ }^{3}$ Fruits, vegetables and grain consumption (kg/yr) ${ }^{3}$ not used ${ }^{3} 1.600 \mathrm{E}+02$

R018 3 Leafy vegetable consumption ( $\mathrm{kg} / \mathrm{yr}$ )

R018 3 Milk consumption ( $\mathrm{L} / \mathrm{yr}$ )

R018 3 Meat and poultry consumption ( $\mathrm{kg} / \mathrm{yr}$ )

R018 3 Fish consumption ( $\mathrm{kg} / \mathrm{yr}$ )

R018 3 Other seafood consumption ( $\mathrm{kg} / \mathrm{yr}$ )

R018 3 Soil ingestion rate (g/yr)

R018 3 Drinking water intake (L/yr)

R018 3 Contamination fraction of drinking water

R018 3 Contamination fraction of household water

R018 3 Contamination fraction of livestock water

R018 3 Contamination fraction of irrigation water

R018 3 Contamination fraction of aquatic food

Ro18 3 Contamination fraction of plant food

R018 3 Contamination fraction of meat

R018 3 Contamination fraction of milk 3

R019 3 Livestock fodder intake for meat ( $\mathrm{kg} /$ day)

R019 3 Livestock fodder intake for milk ( $\mathrm{kg} / \mathrm{day}$ )

R019 3 Livestock water intake for meat (L/day)

R019 3 Livestock water intake for milk (L/day)

R019 3 Livestock soil intake ( $\mathrm{kg} /$ day)

R019 3 Mass loading for foliar deposition $(\mathrm{g} / \mathrm{m} * * 3)$

R019 3 Depth of soil mixing layer $(\mathrm{m})$

R019 3 Depth of roots $(\mathrm{m})$

R019 3 Drinking water fraction from ground water

R019 3 Household water fraction from ground water R019 3 Livestock water fraction from ground water R019 3 Irrigation fraction from ground water

R19B 3 Wet weight crop yield for Non-Leafy $\left(\mathrm{kg} / \mathrm{m}^{* *} 2\right)$

R19B 3 Wet weight crop yield for Leafy $\left(\mathrm{kg} / \mathrm{m}^{* *} 2\right)$

R19B 3 Wet weight crop yield for Fodder

R19B 3 Growing Season for Non-Leafy (years)

R19B 3 Growing Season for Leafy (years)

R19B 3 Growing Season for Fodder (years)

R19B 3 Translocation Factor for Non-Leafy
3 not used $31.400 E+01$

3 not used 3 9.200E+01

3 not used $36.300 E+01$

3 not used $35.400 \mathrm{E}+\Theta \odot$

3 not used 3 9.000E-01

$3 \quad 1.752 \mathrm{E}+02 \quad 3 \quad 3.650 \mathrm{E}+01$

3 not used $35.100 \mathrm{E}+02^{3}$

3 not used $31.000 \mathrm{E}+00^{3}$

3 not used $31.000 \mathrm{E}+00^{3}$

not used $31.000 \mathrm{E}+00$

not used 3 5.000E-01

not used $3-1$

not used $3-1$

not used $3-1$

3 not used 3 6.800E+01

$-1$

not used $35.500 \mathrm{E}+01$

not used 3 5.000E+01

not used $31.600 \mathrm{E}+02$

not used 3 5.000E-01

3 1.500E-01 3 1.500E-01 3

not used 3 9.000E-01

not used 3 1.000E $+00^{3}$

not used 3 1. $1.000 \mathrm{E}+00$

not used $31.000 \mathrm{E}+0{ }^{3}$

not used $31.000 \mathrm{E}+\odot \odot^{3}$

not used 3 7.00९E-01

3 not used 3 1.500E +00

3 not used $31.100 \mathrm{E}+0 \circ$

3 not used $31.700 \mathrm{E}-01$

3 not used $32.500 \mathrm{E}-01$

3 not used 3 8.000E- -2

3 not used $31.000 \mathrm{E}-013$
3 not used $31.000 \mathrm{E}+0{ }^{3}$

not used $31.000 \mathrm{E}-04$
$--$

-

$-$

$-$

$-$

$-$

$-$

$-\ldots$

$-$

$--$

$-\cdots$

$--$

$--$

$(--$

$--$

$--$

$--$

$--$

$---$

- . -

- -

$--$

- - -

$--$

- - -

$--$

- -

$-\cdot$

- -

$--$

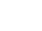

$-\cdot$

$-$.

$-$.

$---$

$--$
FRACA( 1)

3 FRACA ( 2)

3 FRACA ( 3$)$

3 FRACA( 5 )

FRACA( 6)

3 FRACA ( 7)

FRACA ( 8)

3 FRACA ( 9)

FRACA (10)

FRACA (12)

3 DIET(1)

3 DIET (2)

3 DIET (3)

DIET (4)

$\operatorname{DIET}(5)$
$\operatorname{DIET}(6)$

3 SOIL

3 DWI

3 FDW

3 FHHW

3 FLW

3 FIRW

3 FR9

3 FPLANT

3 FMEAT

3 FMILK

LFI5

3 LFI6

3 LWI5

3 LWI6

3 LSI

3 MLFD

3 DM

3 DROOT

3 FGWDW

3 FGWHH

3 FGWLW

3 FGWIR

$3 \mathrm{YV}(1)$

$3 \mathrm{YV}(2)$

3 YV(3)

3 TE (1)

3 TE(2)

$\begin{array}{ll}3 & \operatorname{TE}(3) \\ 3 & \operatorname{TIV}(1)\end{array}$ 
Site-Specific Parameter Summary (continued)

Menu 3

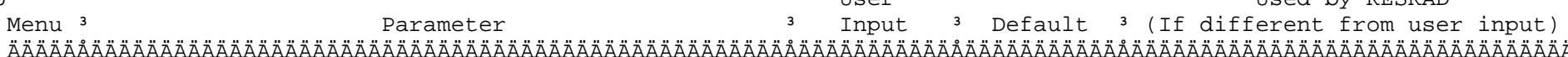

3 Default

R19B 3 Translocation factor for Leafy 3 not used $31.000 \mathrm{n}+00^{3}$

R19B 3 Translocation Factor for Fodder 3 not used $31.000 \mathrm{E}+00^{3}$

R19B 3 Dry Foliar Interception Fraction for Non-Leafy 3 not used $32.500 \mathrm{E}-01$

R19B 3 Dry Foliar Interception Fraction for Leafy 3 not used $32.500 \mathrm{E}-01$

R19B 3 Dry Foliar Interception Fraction for Fodder 3 not used $32.500 \mathrm{E}-013$

R19B 3 Wet Foliar Interception Fraction for Non-Leafy 3 not used $32.500 E-013$

R19B 3 Wet Foliar Interception Fraction for Leafy 3 not used $32.500 \mathrm{E}-013$

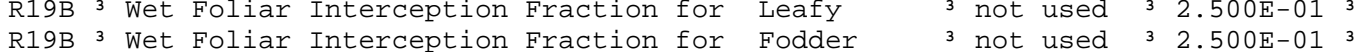

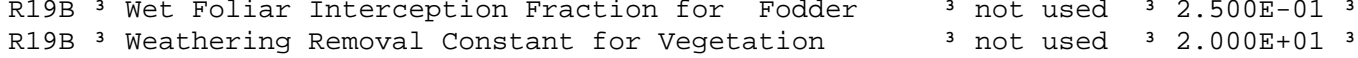
3

C14 3 C-12 concentration in water $\left(\mathrm{g} / \mathrm{cm}^{* *} 3\right)$

C14 3 C-12 concentration in contaminated soil $(\mathrm{g} / \mathrm{g})$

C14 3 Fraction of vegetation carbon from soil

C14 3 Fraction of vegetation carbon from air

C14 3 C-14 evasion layer thickness in soil (m)

C14 3 C-14 evasion flux rate from soil ( $1 / \mathrm{sec})$

C14 3 C-12 evasion flux rate from soil ( $1 / \mathrm{sec})$

C14 3 Fraction of grain in beef cattle feed

C14 3 Fraction of grain in milk cow feed

C14 3 DCF correction factor for gaseous forms of C14 3

STOR 3 Storage times of contaminated foodstuffs (days): STOR 3 Fruits, non-leafy vegetables, and grair

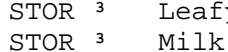

STOR 3 Milk

STOR ${ }^{3}$ Meat and poultry

STOR 3 Fish

STOR 3 Crustacea and mollusks

STOR ${ }^{3}$ Well water

STOR 3 Surface water

STOR 3 Livestock fodder

R021 3 Thickness of building foundation $(\mathrm{m})$

RO21 3 Bulk density of building foundation $\left(\mathrm{g} / \mathrm{cm}^{* *} 3\right)$

R021 3 Total porosity of the cover material

3

not used $32.000 \mathrm{E}-05$

3 not used $33.000 \mathrm{E}-02$

3 not used 3 2.000E-02

3 not used 3 9.800E-01

not used $33.000 \mathrm{E}-01$

3 not used 3.000 - 01

3 not used 3 7.000E-07

3 not used $31.000 \mathrm{E}-10$

not used 3 8.000E-01

not used 3 2.000E-01

$\odot .000 \mathrm{E}+0 \odot$

$$
3
$$

3 1. $400 \mathrm{E}+01 \quad 3 \quad 1.400 \mathrm{E}+01$

$31.000 \mathrm{E}+\odot \odot \quad 3 \quad 1.00 \ominus \mathrm{E}+\Theta \odot$

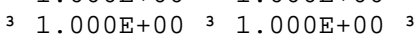

$2.000 \mathrm{E}+01$ 3 $2.000 \mathrm{E}+01$

$\begin{array}{lll}7 & 7.00 \odot \mathrm{E}+\odot \odot \quad 3 & 7.00 \odot \mathrm{E}+\odot \odot \quad 3\end{array}$

$7.000 \mathrm{E}+003 \quad 7.000 \mathrm{E}+00$

$31.000 \mathrm{E}+0 \odot$ 3 $1.000 \mathrm{E}+0 \odot^{3}$

1. 1.000E+00 3 1.000E+00 3

$4.500 \mathrm{E}+011^{3} \quad 4.500 \mathrm{E}+01$

not used $31.500 \mathrm{E}-013$

not used $32.400 \mathrm{E}+00^{3}$

not used 3 4.000E- $011^{3}$

not used 3 1.000E-01

R021 3 Volumetric water content of the cover material 3 not used 3 5.000E-02

R021 3 Volumetric water content of the foundation

R021 3 Diffusion coefficient for radon gas $(\mathrm{m} / \mathrm{sec})$ :

R०21 3 in cover material

$\mathrm{R} \odot 21^{3}$ in foundation material

RO21 3 in contaminated zone soil

R021 3 Radon vertical dimension of mixing $(\mathrm{m})$

R021 3 Average building air exchange rate $(1 / \mathrm{hr})$

R021 3 Height of the building (room) (m)

R021 3 Building interior area factor

not used 3 3.000E- 023

not used ${ }^{3}$

$3 \quad 2.000 \mathrm{E}-06$

not used $32.000 \mathrm{E}-06$

3 not used $32.000 \mathrm{E}-066^{3}$

not used $32.000 \mathrm{E}+00$

not used 3 5.000E-01

not used $32.500 \mathrm{E}+00$

not used $30.000 \mathrm{E}+00$

not used $3-1.000 \mathrm{E}+0 \odot$

3 not used $32.500 \mathrm{E}-01$

3 not used 3 1.500E-01 3

R021 3 Emanating power of $\mathrm{Rn}-222$ gas

RO21 3 Emanating power of Rn-220 gas

TITL 3

uamer of graphical time points

TITL 3 Maximum number of integration points for dose

$\begin{array}{cccc}\text { used } & 3 & 1.500 \mathrm{E}-01 & 3 \\ & 3 & & 3 \\ 32 & 3 & -.- & 3 \\ 17 & 3 & -.- & 3\end{array}$

Used by RESRAD

3 Parameter

$3 \quad$ Name

Name

3
.--1

3 RDRY (1)

$\begin{array}{ll}3 & \operatorname{RDRY}(2) \\ 3 & \operatorname{RDRY}(3)\end{array}$

$--$

3 RWET (2)

-. $3 \operatorname{RWET}(3)$

-. 3 WLAM

3 C12CZ

-.- 3 CSOIL

3 CAIR

$-$

$\begin{array}{ll}--- & 3 \text { EVSN } \\ --- & 3 \text { REVSN }\end{array}$

- - 3 AVFG4

3 AVFG5

$---$

3

STOR_T(1)

$-\cdot-$

$-\cdot-$

3 STOR_T(3)

3 STOR_T(4)

3 STOR_T(7)

-. 3 STOR_T(9)

$-$

$--$

$--$

$--$

FLOOR1

3 DENSFL

3 TPCV

3 TPFL

$3 \mathrm{PH} 2 \mathrm{OCV}$

3 PH2OFL

-. 3 DIFCV

-. 3 DIFFL

3 DIFCZ

3 EMANA(1)

$--$

$-\cdot-$

EMANA $(1)$

3 NPTS

3 LYMAX 


\section{$\odot{ }^{3}$}

Site-Specific Parameter Summary (continued)

Parameter

$$
3 \text { User }{ }_{3}{ }_{3} \text { Used by RESRAD }
$$

3 Parameter

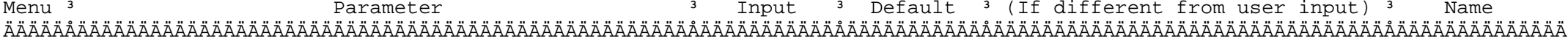
TITL 3 maximum number of integration points for risk 33257,3

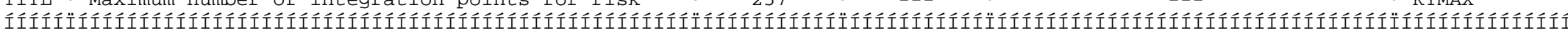

Summary of Pathway Selections

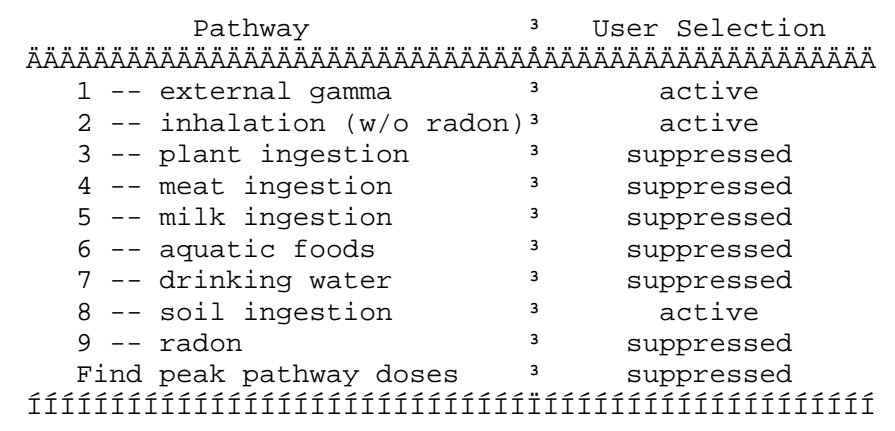


Contaminated Zone Dimensions

ÄÄÄÄÄÄÄÄÄÄÄÄÄÄÄÄÄÄÄÄÄÄÄÄÄÄÄÄÄ

Area: 100.00 square meters

Thickness: $\quad 0.15$ meters

$\odot$
Initial Soil Concentrations, $\mathrm{pCi} / \mathrm{g}$

ӒÄÄÄÄÄÄÄÄÄÄÄÄÄÄÄÄÄÄÄÄÄÄÄÄÄÄÄÄÄÄÄÄÄ

$$
\begin{array}{ll}
\mathrm{Cs}-137 & 1.140 \mathrm{E}+02 \\
\mathrm{Pu}-238 & 2.320 \mathrm{E}-01
\end{array}
$$

Pu-239

$$
\text { 2. } \odot 90 \mathrm{E}+\odot \odot
$$

Total Dose TDOSE(t), mrem/yr

Basic Radiation Dose Limit $=2.500 \mathrm{E}+01 \mathrm{mrem} / \mathrm{yr}$

Total Mixture Sum $\mathrm{M}(\mathrm{t})=$ Fraction of Basic Dose Limit Received at Time $(\mathrm{t})$

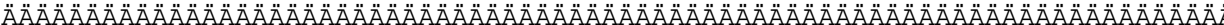

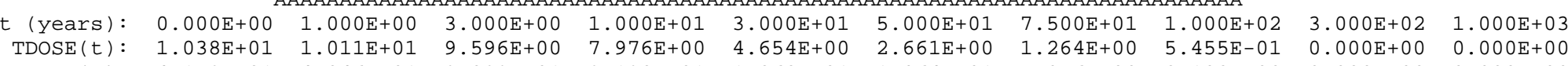
$M(t): \quad 4.153 \mathrm{E}-01 \quad 4.046 \mathrm{E}-01$
$\begin{array}{ll}5.455 \mathrm{E}-01 & 0.000 \mathrm{E}+0 \odot \\ 2.182 \mathrm{E}-02 & 0.000 \mathrm{E}+\odot \odot\end{array}$

๑Maximum TDOSE $(\mathrm{t}): 1.038 \mathrm{E}+01 \mathrm{mrem} / \mathrm{yr}$ at $\mathrm{t}=0.00 \odot \mathrm{E}+\odot \odot$ years 
Total Dose Contributions $\operatorname{TDOSE}(i, p, t)$ for Individual Radionuclides (i) and Pathways $(p)$ As mrem/yr and Fraction of Total Dose At $t=0.000 \mathrm{E}+0 \odot$ years
water Independent Pathways (Inhalation excludes radon) Radio- Ä̈̈Ä Nuclide mrem/yr fract. ÄÄÄÄÄÄÄ ÄÄÄÄÄÄÄÄÄ ÄÄÄÄÄÄ $\begin{array}{lll}\mathrm{CS}-137 & 1.035 \mathrm{E}+01 & 0.9971 \\ \mathrm{Pu}-238 & 1.202 \mathrm{E}-06 & 0.0000\end{array}$ Pu-239 1.877E-05 0.0000 íííííí íííííííí ííííít Total 1.035E+01 0.9971 Inhalation Radon Plant
ÄÄÄÄÄÄÄÄÄÄÄÄÄÄÄÄ Meat

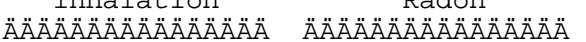
Ӓ̈̈̈ӒÄÄÄÄÄÄÄÄÄÄÄÄ mrem/yr fract Ä̈̈̈А mrem/yr fract. maAAAAAAAAAAAA
mrem $/$ yr fract. $\mathrm{mrem} / \mathrm{yr}$ fract ÄÄÄÄÄÄÄÄ ÄÄÄÄÄĂ

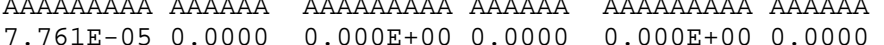

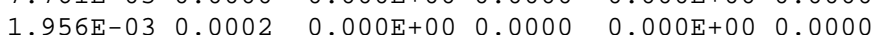

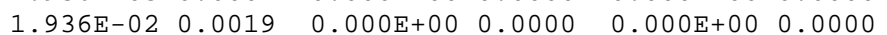
$0.000 \mathrm{E}+00 \quad 0.0000$ $0.000 E+00 \quad 0.0000$ $0 . \odot \odot \odot E+\odot \odot \odot$ IIÍíííííi ííííí íííííííí íííííi íííííííí Íííít

IIIIIII IIIII Total Dose Contributions TDOSE $(i, p, t)$ for Individual Radionuclides ( $i$ ) and Pathways $(p)$ As $\mathrm{mrem} / \mathrm{yr}$ and Fraction of Total Dose At $t=0.000 \mathrm{E}+\odot \odot$ years Fish Radon Water Dependent Pathways

Water Plant

Meat Milk

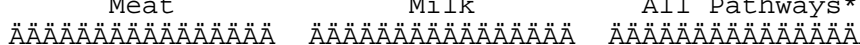
mrem/yr fract mrem/yr fract mrem/yr fract. ÄÄÄÄÄÄÄÄÄ ÄÄÄÄÄÄ ÄÄÄÄÄÄÄÄÄ ÄÄÄÄÄÄ ÄÄÄÄÄÄÄÄÄ ÄÄÄÄÄÄ Radio - ÄÄÄÄÄÄÄÄÄÄÄÄÄÄÄÄ ÄÄÄÄÄÄÄÄÄÄÄÄÄÄÄÄ ÄÄÄÄÄÄÄÄÄÄÄÄÄÄÄÄ ÄÄÄÄÄÄÄÄÄÄÄÄÄÄÄ Nuclide mrem/yr fract. mrem/yr fract. mrem/yr fract. mrem/yr fract. ÄÄÄÄÄÄÄ ÄÄÄÄÄÄÄÄÄ ÄÄÄÄÄÄ ÄÄÄÄÄÄÄÄÄ ÄÄÄÄÄÄ ÄÄÄÄÄÄÄÄÄ ÄÄÄÄÄÄ ÄÄÄÄÄÄÄÄÄ ÄÄÄÄÄ

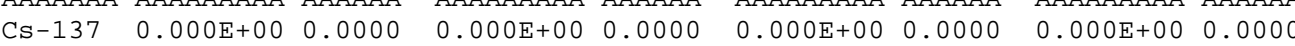

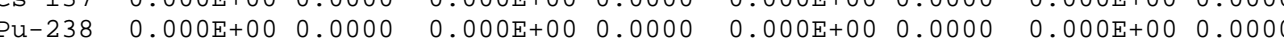

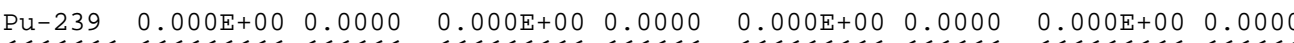
íííííi ííííííí ííííi ííííííí ííííi ííííííi ííííi $\begin{array}{lllllll}\text { Total } & 0.000 \mathrm{E}+00 & 0.0000 & 0.000 \mathrm{E}+00 & 0.0000 & 0.000 \mathrm{E}+00 & 0.0000\end{array}$ IÍíííííi ÍÍÍíí A $0.000 \mathrm{E}+000.0000$ $\odot .00 \odot \mathrm{E}+0 \odot \quad 0.000 \odot$ $\odot .000 \mathrm{E}+0 \odot$ ०. $0.00 \odot$ $1.036 \mathrm{E}+010.9974$ $2.447 \mathrm{E}-03 \quad 0.0002$ $\theta^{*}$ Sum of all water independent and dependent pathways. 
Total Dose Contributions $\operatorname{TDOSE}(i, p, t)$ for Individual Radionuclides (i) and Pathways $(p)$ As mrem/yr and Fraction of Total Dose At $t=1.000 \mathrm{E}+0 \odot$ years
water Independent Pathways (Inhalation excludes radon) Radio- Ä̈̈Ä Nuclide $\mathrm{mrem} / \mathrm{yr}$ fract. ÄÄÄÄÄÄÄ ÄÄÄÄÄÄÄÄÄ ÄÄÄÄÄA $\begin{array}{lll}\mathrm{CS}-137 & 1.008 \mathrm{E}+01 & 0.9970 \\ \mathrm{Pu}-238 & 1.192 \mathrm{E}-06 & 0.000 \odot\end{array}$ Pu-239 1.873E- 050.0000 íííííi ííííííí íííít Iilifilifili IIII $\odot$ Inhalation Radon Plant Meat

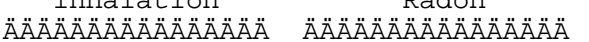

mrem/yr fract. AАAAAAAAÄÄÄÄÄÄ Ӓ̈̈̈ÄÄÄÄÄÄÄÄÄÄÄÄÄ ÄÄÄÄÄÄÄÄÄÄÄÄÄÄÄÄ $\mathrm{mrem} / \mathrm{yr}$ fract $\operatorname{Mil}$ ААลААลӒล̈ÄÄÄÄÄÄÄ mrem/yr fract.

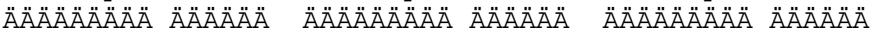
$\begin{array}{llll}7.533 \mathrm{E}-05 & 0.0000 & 0.000 \mathrm{E}+00 & 0.0000\end{array}$ $0.000 \mathrm{E}+000.0000$ $0.000 \mathrm{E}+00 \quad 0.0000 \quad 0.000 \mathrm{E}+0 \odot \quad 0.0000$ $\odot .000 \mathrm{E}+0 \odot \quad 0.000 \odot$ $0.000 \mathrm{E}+00 \quad 0.0000$ $\odot .00 \odot \mathrm{E}+\odot \odot$ ० $0.000 \odot$ $0.000 \mathrm{E}+000.0000$ Íííííííí ííííi

IIIííííi Ííííí íííííííi ííííi

$2.122 \mathrm{E}-020.00210 .00 \odot \mathrm{E}+000.0000 \quad 0.00 \odot \mathrm{E}+000.0000 \quad 0.00 \odot \mathrm{E}+000.0000$

Total Dose Contributions TDOSE( $i, p, t)$ for Individual Radionuclides (i) and Pathways $(p)$ As mrem/yr and Fraction of Total Dose At $t=1.000 \mathrm{E}+00$ years Fish Radon Water Dependent Pathways

Water Plant

Meat Milk

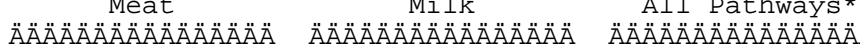
mrem/yr fract mrem/yr fract mrem/yr fract. ÄÄÄÄÄÄÄÄÄ ÄÄÄÄÄÄ ÄÄÄÄÄÄÄÄÄ ÄÄÄÄÄÄ ÄÄÄÄÄÄÄÄÄ ÄÄÄÄÄÄ Radio- ÄÄÄÄÄÄÄÄÄÄÄÄÄÄ ÄÄÄÄÄÄÄÄÄÄÄÄÄÄ ÄÄÄÄÄÄÄÄÄÄÄÄÄÄÄ ÄÄÄÄÄÄÄÄÄÄÄÄÄ Nuclide mrem/yr fract. mrem/yr fract. mrem/yr fract. mrem/yr fract. ÄÄÄÄÄÄÄ ÄÄÄÄÄÄÄÄÄ ÄÄÄÄÄÄ ÄÄÄÄÄÄÄÄÄ ÄÄÄÄÄÄ ÄÄÄÄÄÄÄÄÄ ÄÄÄÄÄÄ ÄÄÄÄÄÄÄÄÄ ÄÄÄÄÄ

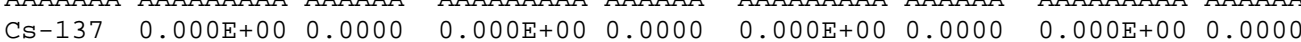

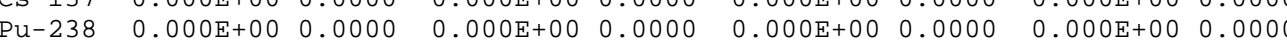

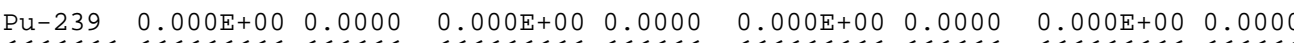
íííííi ííííííí ííííi ííííííí ííííi ííííííi ííííi $\begin{array}{lllllll}\text { Total } & 0.000 \mathrm{E}+00 & 0.0000 & 0.000 \mathrm{E}+00 & 0.0000 & 0.000 \mathrm{E}+00 & 0.0000\end{array}$ Íííííííi ííííí A $0.000 \mathrm{E}+000.0000$ $\odot .000 \mathrm{E}+0 \odot \odot .000 \odot$ $\odot .000 E+0 \odot$ ०. $000 \odot$ $1.009 \mathrm{E}+01 \quad 0.9974$ $2.411 \mathrm{E}-03 \quad 0.0002$ $2.412 E-02 \quad 0.0024$ $0^{*}$ Sum of all water independent and dependent pathways 
Total Dose Contributions $\operatorname{TDOSE}(i, p, t)$ for Individual Radionuclides (i) and Pathways $(p)$ As mrem/yr and Fraction of Total Dose At $t=3.000 \mathrm{E}+0 \odot$ years
water Independent Pathways (Inhalation excludes radon) Radio- Ä̈̈Ä

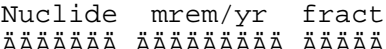
CS-137 9.567E+0๑ 0.9969 $\mathrm{Pu}-238 \quad 1.172 \mathrm{E}-06 \quad 0.0000$ $\mathrm{Pu}-239$ 1.866E-05 0.0000 íííííí íííííííí ííííí Total $9.567 \mathrm{E}+00$ 0.9969 Inhalation Radon

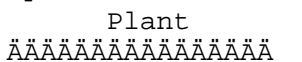
Meat

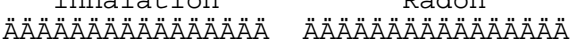
Ӓ̈̈̈ӒÄÄÄÄÄÄÄÄÄÄÄÄ mrem/yr fract mrem/yr fract. mrem/yr fract. ÄÄÄÄÄÄÄÄÄ ÄÄÄÄÄÄ mrem/yr fract

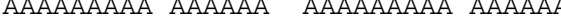
$\begin{array}{llllll}7.094 \mathrm{E}-05 & 0.000 \odot & 0.000 \mathrm{E}+0 \odot & 0.0000 & 0.00 \odot \mathrm{E}+\odot \odot & 0.000 \\ 1.870 \mathrm{E}-03 & 0.0002 & 0.000 \mathrm{E}+00 & 0.0000 & 0.000 \mathrm{E}+00 & 0.000 \odot\end{array}$

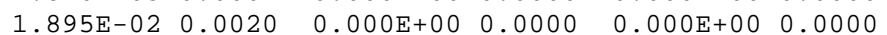
$0.000 \mathrm{E}+00 \quad 0.0000$ $\odot .000 \mathrm{E}+0 \odot \quad 0.0000$ $0.000 \mathrm{E}+00 \quad 0.0000$ Í

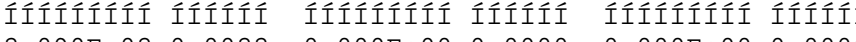

IIIÍíííi Ííííi

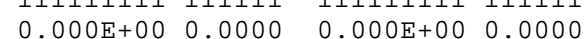

Milk

\section{mrem/yr fract.} Aremyr fract. $0.000 \mathrm{E}+00$ ๑. 0.000 $\odot .000 \mathrm{E}+000.0000$ $0.000 \mathrm{E}+00 \quad 0.0000$ ÍÍIííííí ÍÍÍíí

$\odot, 00 \odot \mathrm{E}+0 \odot$ ०. $000 \odot$
Soil ӒÄÄÄÄÄÄÄÄÄÄÄÄÄÄÄ mrem/yr fract ÄÄÄÄÄÄÄÄÄ ÄÄÄÄÄÄ $3.417 \mathrm{E}-03 \quad 0.0004$ 4.692E-04 0.0000 $4.807 \mathrm{E}-03 \quad 0.0005$ Íííííííí íííííi

Total Dose Contributions TDOSE $(i, p, t)$ for Individual Radionuclides ( $i$ ) and Pathways ( $p$ ) As mrem/yr and Fraction of Total Dose At $t=3.000 \mathrm{E}+00$ years Fish Radon Water Dependent Pathways

Water Plant

Meat Milk

All Pathways* ÄӒÄÄÄÄÄÄÄÄÄÄÄÄ ÄÄÄÄÄÄÄÄÄÄÄÄÄÄÄ ÄÄÄÄÄÄÄÄÄÄÄÄÄÄÄ $\mathrm{mrem} / \mathrm{yr}$ fract. $\mathrm{mrem} / \mathrm{yr}$ fract. $\mathrm{mrem} / \mathrm{yr}$ fract. Radio- AÄÄÄÄÄÄÄÄÄÄÄÄÄ ÄÄÄÄÄÄÄÄÄÄÄÄÄÄÄ ÄÄÄÄÄÄÄÄÄÄÄÄÄÄÄ ÄÄÄÄÄÄÄÄÄÄÄÄÄĂ Nuclide mrem/yr fract. mrem/yr fract. mrem/yr fract. mrem/yr fract.

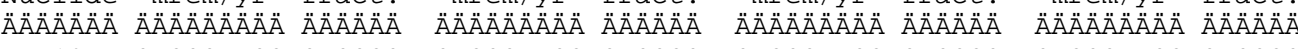

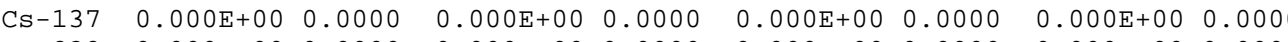

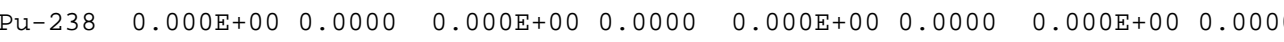

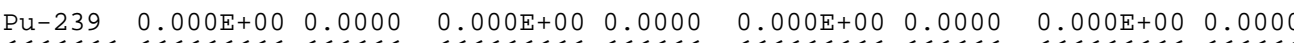
íííííi ííííííí ííííi ííííííí ííííi ííííííi ííííi $\begin{array}{lllllll}\text { Total } & 0.000 \mathrm{E}+00 & 0.0000 & 0.000 \mathrm{E}+00 & 0.0000 & 0.000 \mathrm{E}+00 & 0.0000\end{array}$ Ííííííí Íííít $0.000 \mathrm{E}+00$ 0.0000 $\odot .000 \mathrm{E}+000.0000$ ÄÄÄÄÄÄÄ ÄÄÄÄÄ

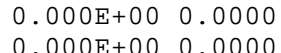

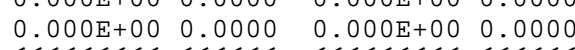
ÄÄÄÄÄÄÄÄÄ ÄÄÄÄÄÄ $9.570 E+0 \odot \quad 0.9973$ * Sum of all water independent and dependent pathways. 
Total Dose Contributions $\operatorname{TDOSE}(i, p, t)$ for Individual Radionuclides (i) and Pathways $(p)$ As mrem/yr and Fraction of Total Dose At $t=1.000 \mathrm{E}+01$ years
water Independent Pathways (Inhalation excludes radon) Radio- Ä̈̈̈̈̈A Ä̈̈̈̈̈̈̈̈̈̈̈̈ Cs-137 7.948E+00 0.9965 Pu-238 1.107E-06 0.0000 $\mathrm{Pu}-239$ 1.838E-05 0.0000 íííííí íííííííí ííííít Total 7.948E+0๑ 0.9966 Inhalation Radon Plant
ÄÄÄÄÄÄÄÄÄÄÄÄÄÄÄÄ Meat Soil

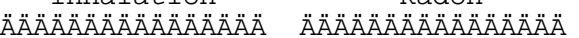
Ӓ̈̈ÄÄÄÄÄÄÄÄÄÄÄÄÄ ÄÄÄÄÄ̈̈̈̈̈̈̈̈̈ mrem/yr fract. mrem/yr fract. $\mathrm{mrem} / \mathrm{yr}$ fract ÄÄÄÄÄÄÄÄÄ ÄÄÄÄÄÄ

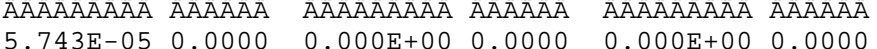

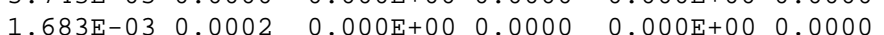
$\begin{array}{llllll}1.802 \mathrm{E}-02 & 0.0023 & 0.000 \mathrm{E}+00 & 0.0000 & 0.000 \mathrm{E}+00 & 0.0000\end{array}$ $\odot .000 \mathrm{E}+0 \odot \quad 0.000 \odot$ $0.000 \mathrm{E}+00 \quad 0.0000$

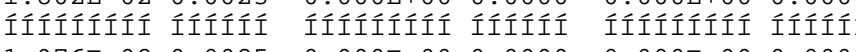

IIIÍííí ÍÍÍíi

$\odot . \odot \odot \odot E+\odot \odot \quad \odot . \odot \odot \odot \odot \quad \odot . \odot \odot \odot E+\odot \odot \odot . \odot \odot \odot \odot$ A Ä̈ÄÄÄ $\odot .000 \mathrm{E}+00$ 0.00०९ $0.000 \mathrm{E}+000.0000$ $0.000 \mathrm{E}+00 \quad 0.0000$

$\odot .000 \mathrm{E}+0 \odot$ ० 0.0000 IÍIIIIIIIIII ÍÍÍÍI
ÄÄÄÄÄÄÄÄÄÄÄÄÄÄÄÄ mrem/yr fract. ÄÄÄÄÄÄÄÄ ÄÄÄÄÄÄ $2.766 \mathrm{E}-03 \quad 0.0003$ $4.569 \mathrm{E}-03 \quad 0.0006$ Íííííííi ííííí

Total Dose Contributions TDOSE( $i, p, t)$ for Individual Radionuclides (i) and Pathways ( $p$ ) As mrem/yr and Fraction of Total Dose At $t=1.000 \mathrm{E}+01$ years Fish Radon ter Dependent Pathways Plant

Meat Milk

All Pathways* ÄÄÄÄÄÄÄÄÄÄÄÄÄÄÄÄ ÄÄÄÄÄÄÄÄÄÄÄÄÄÄÄÄ Ä ÄÄÄÄÄÄÄÄÄÄÄÄÄÄÄÄ

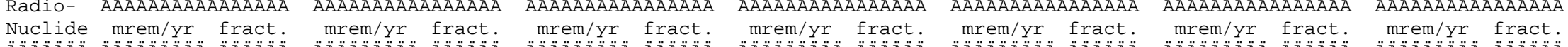
ÄÄÄÄÄÄÄ

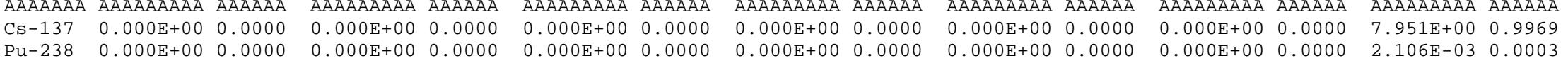

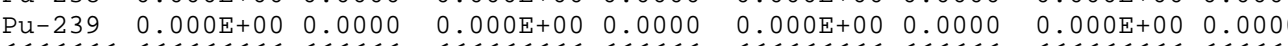

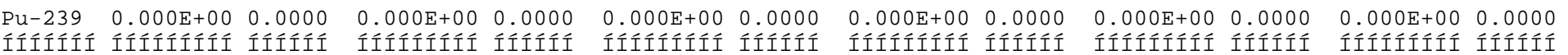
$\odot$ *Sum of all water independent and dependent pathways. 
Total Dose Contributions TDOSE(i,p,t) for Individual Radionuclides (i) and Pathways (p)

As mrem/yr and Fraction of Total Dose At $t=3.000 \mathrm{E}+01$ years Water Independent Pathways (Inhalation excludes radon) Inhalation Radon
ÄӒÄÄÄÄ̈̈̈̈̈̈̈̈̈̈̈̈̈ Plant
Slant Meat
$M i l k$ Radio - ÄÄÄÄÄÄÄÄÄÄÄÄÄÄÄA Ä̈̈̈̈̈̈̈̈̈̈̈̈ÄÄÄÄÄÄÄÄ Nuclide mrem/yr fract ÄÄÄÄÄÄÄ ÄÄÄÄÄÄÄÄÄ Ä ÄÄÄÄÄÄ $\begin{array}{lll}\text { CS-137 } & 4.632 \mathrm{E}+0 \odot & 0.9952 \\ \mathrm{Pu}-238 & 9.379 \mathrm{E}-07 & 0.0000\end{array}$ Pu-239 1.751E-05 0.0000 íííííi íííííííi ííííí IIIIIIII IIIIIIIII IIIIII $\odot$ mrem/yr fract. mrem/yr fract. m mrem/yr fract. mrem/yr fract.
ÄÄÄÄÄÄÄÄ ÄÄÄÄÄ̈

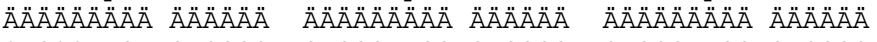

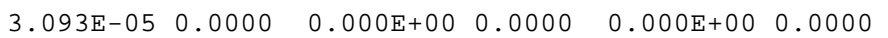
$\begin{array}{llllll}1.225 \mathrm{E}-03 & 0.0003 & 0.000 \mathrm{E}+00 & 0.0000 & 0.000 \mathrm{E}+00 & 0.0000\end{array}$

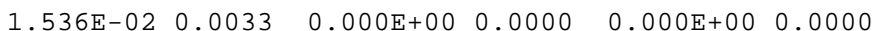
$0.000 \mathrm{E}+00 \quad 0.0000$ $0.000 \mathrm{E}+00 \quad 0.0000$ mrem/yr fract.
maAAAAA ÄÄÄÄÄÄÄÄ ÄÄÄÄÄÄ $\odot . \odot \odot \odot E+\odot \odot ~ \odot . ๑ \odot \odot \odot$ Íííííííí ííííí íííííííi íííííi íííííííí ííííí ííííííí ííííi ííííííí ííííi

Total Dose Contributions $\operatorname{TDOSE}(i, p, t)$ for Individual Radionuclides (i) and Pathways (p) As $\mathrm{mrem} / \mathrm{yr}$ and Fraction of Total Dose At $t=3.000 \mathrm{E}+01$ years Fish Radon Water Dependent Pathways Plant

Meat Milk

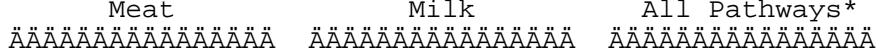

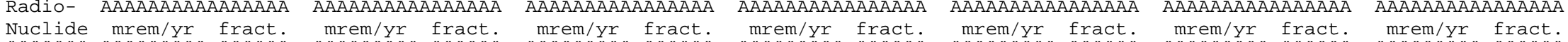

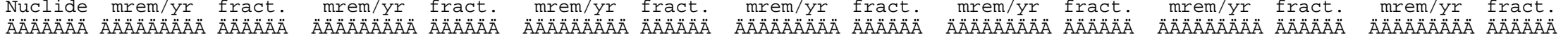

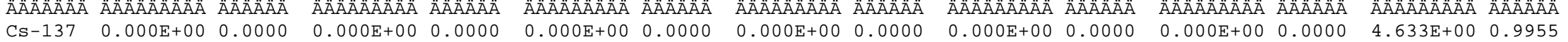

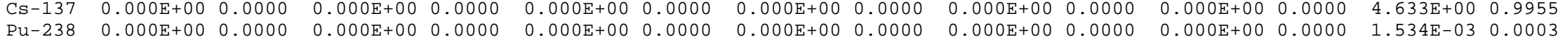

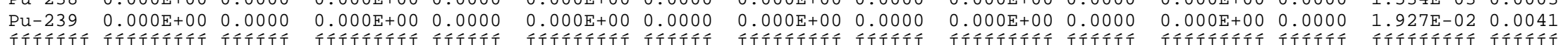

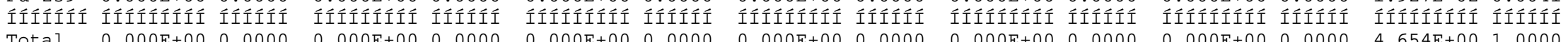
$\odot *$ Sum of all water independent and dependent pathways. 
Total Dose Contributions $\operatorname{TDOSE}(i, p, t)$ for Individual Radionuclides (i) and Pathways $(p)$ As $\mathrm{mrem} / \mathrm{yr}$ and Fraction of Total Dose At $\mathrm{t}=5.000 \mathrm{E}+01$ years
Water Independent Pathways (Inhalation excludes radon) Radio- Ä̈̈Ä

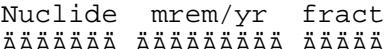
Cs-137 2.643E+0๑ $\odot .9933$ $\mathrm{Pu}-238 \quad 7.923 \mathrm{E}-07 \quad 0.0000$ $\mathrm{Pu}-239$ 1.645E-05 0.0000 íííííí íííííííí ííííí Total 2.643E+00 0.9933 Inhalation Radon

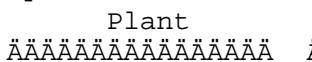
Meat

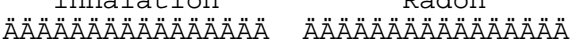
Ӓ̈ÄÄÄÄÄÄÄÄÄÄÄÄÄÄÄ

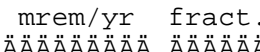
mä̈̈̈̈̈̈̈̈̈̈̈̈̈ fract.

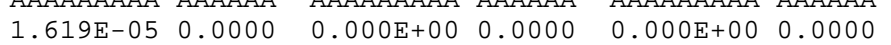

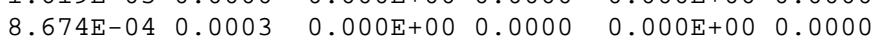
$\begin{array}{llllll}1.273 E-02 & 0.0048 & 0.000 E+00 & 0.000 \odot & 0.000 \mathrm{E}+0 \odot & 0.0000\end{array}$ Íííííííi ííííi íííííííi ííííí íííííííi íííít $0.000 \mathrm{E}+00$ 0. 0.0000 $\odot .000 \mathrm{E}+0 \odot \quad 0.0000$ $0.000 \mathrm{E}+00 \quad 0.0000$ Íííííííi ííííi

$0.000 \mathrm{E}+000.0000$ \\ Ӓ̈Ӓ̈̈̈̈̈̈̈}

\section{Arem/yr fract.} Arem/yr fract. $0.000 \mathrm{E}+00$ $\odot .0000$ $\odot .000 \mathrm{E}+000.0000$ $0.000 \mathrm{E}+00 \quad 0.0000$ Íííííííi ííííí

$\odot .000 \mathrm{E}+0 \odot$ ०. 0000
Soil ӒÄÄÄÄÄÄÄÄÄÄÄÄÄÄ $\mathrm{mrem} / \mathrm{yr}$ fract ÄÄÄÄÄÄÄÄÄ ÄÄÄÄÄÄ $7.801 \mathrm{E}-04 \quad 0.0003$ $2.176 \mathrm{E}-04 \quad 0.0001$ $3.227 \mathrm{E}-03 \quad 0.0012$ Íííííííi ííííí

Total Dose Contributions TDOSE $(i, p, t)$ for Individual Radionuclides ( $i$ ) and Pathways ( $p$ ) As mrem/yr and Fraction of Total Dose At $t=5.000 E+01$ years Water Dependent Pathways Fish Radon Plant

Meat Milk

All Pathways* ÄӒÄÄÄÄÄÄÄÄÄÄÄÄ ÄÄÄÄÄÄÄÄÄÄÄÄÄÄÄ ÄÄÄÄÄÄÄÄÄÄÄÄÄÄÄ $\mathrm{mrem} / \mathrm{yr}$ fract. $\mathrm{mrem} / \mathrm{yr}$ fract. $\mathrm{mrem} / \mathrm{yr}$ fract. adio- ÄÄÄÄÄÄÄÄÄÄÄÄÄÄ ÄÄÄÄÄÄÄÄÄÄÄÄÄÄÄ ÄÄÄÄÄÄÄÄÄÄÄÄÄÄÄ Ä ÄÄÄÄÄÄÄÄÄÄÄÄÄ Nuclide mrem/yr fract. mrem/yr fract. mrem/yr fract. mrem/yr fract.

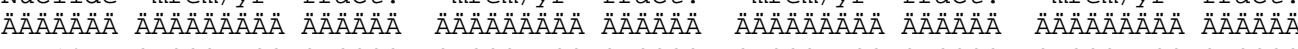

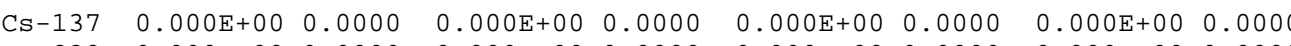

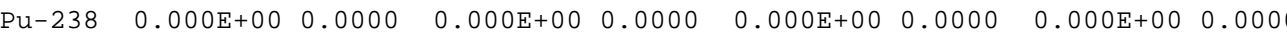

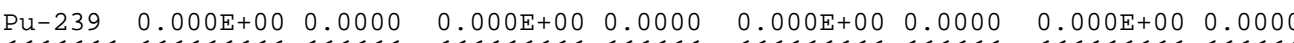
íííííi ííííííí ííííi ííííííí ííííi ííííííi ííííi $\begin{array}{lllllll}\text { Total } & 0.000 \mathrm{E}+00 & 0.0000 & 0.000 \mathrm{E}+00 & 0.0000 & 0.000 \mathrm{E}+00 & 0.0000\end{array}$ Ííííííí Íííít $0.000 \mathrm{E}+000.0000$ $\odot .000 \mathrm{E}+000.0000$ ÄÄÄÄÄÄÄÄÄ ÄÄÄÄÄÄ $0.000 \mathrm{E}+00 \quad 0.0000 \quad 0.000 \mathrm{E}+00 \quad 0.0000$ ÄÄÄÄÄÄÄÄÄ ÄÄÄÄÄÄ 2.644E+0๑ 0.9936

* Sum of all water independent and dependent pathways. 
Total Dose Contributions $\operatorname{TDOSE}(i, p, t)$ for Individual Radionuclides (i) and Pathways $(p)$ As mrem/yr and Fraction of Total Dose At $t=7.500 \mathrm{E}+01$ years
water Independent Pathways (Inhalation excludes radon) Radio- ÄÄÄÄÄÄÄÄÄÄÄÄÄÄÄ Nuclide mrem/yr fract. ÄÄÄÄÄÄÄ ÄÄÄÄÄÄÄÄÄ ÄÄÄÄÄA $\begin{array}{lll}\mathrm{CS}-137 & 1.251 \mathrm{E}+0 \odot & 0.9898 \\ \mathrm{Pu}-238 & 6.366 \mathrm{E}-07 & 0.000 \odot\end{array}$ Pu-239 1.474E-05 0.0000 ííííí ííííííí ííííi $\begin{array}{lll}\text { IIIIII } & \text { IIIIIII } \\ \text { Total } & 1.251 \mathrm{E}+00 & 0.9898\end{array}$ $\odot$ Inhalation Radon Plant Meat

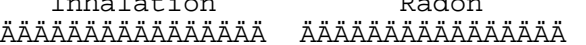

mrem/yr fract. AАAАAAÄÄÄÄÄÄÄÄ ÄÄÄÄÄÄÄÄÄÄÄÄÄÄÄÄ Ӓ̈̈ÄÄÄÄÄÄÄÄÄÄÄÄÄÄ $\mathrm{mrem} / \mathrm{yr}$ fract $\operatorname{Mil}$ ААลААลӒӒÄÄÄÄÄÄÄ mrem/yr fract. AAAAAAAA АAAAAA ÄÄÄÄÄÄÄÄÄ ÄÄÄÄÄÄ ÄÄÄÄÄÄÄÄÄ ÄÄÄÄÄA

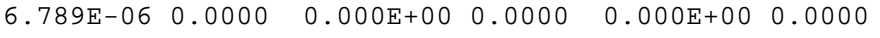
$\begin{array}{llllll}5.302 \mathrm{E}-\odot 4 & 0.0004 & 0.00 \odot \mathrm{E}+\odot \odot & 0.000 \odot & 0.00 \odot \mathrm{E}+0 \odot & 0.00 \odot \odot\end{array}$ $\begin{array}{llllll}9.469 \mathrm{E}-03 & 0.0075 & 0.000 \mathrm{E}+00 & 0.000 \odot & 0.000 \mathrm{E}+0 \odot & 0.0000\end{array}$ IIIIIIííi ííííí Íííííííi ííííí íííííííi íííít A. $\odot .000 \mathrm{E}+0 \odot \quad 0.000 \odot$ $0.000 \mathrm{E}+00 \quad 0.0000$

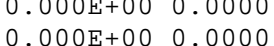
$0.000 \mathrm{E}+000.0000$ Íííííííi ííííi íííííííi ííííi

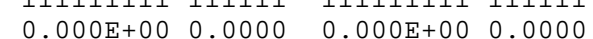

Total Dose Contributions $\operatorname{TDOSE}(i, p, t)$ for Individual Radionuclides (i) and Pathways $(p)$ As mrem/yr and Fraction of Total Dose At $t=7.500 E+01$ years Water Dependent Pathways Fish Radon Plant

Meat Milk All Pathways* ÄÄÄÄÄÄÄÄÄÄÄÄÄÄÄÄ ÄÄÄÄÄÄÄÄÄÄÄÄÄÄÄÄÄ ÄÄÄÄÄÄÄÄÄÄÄÄÄÄÄÄÄ mrem/yr fract. mrem/yr fract. mrem/yr fract. Radio- ÄÄÄÄÄÄÄÄÄÄÄÄÄÄ ÄÄÄÄÄÄÄÄÄÄÄÄÄÄÄ ÄÄÄÄÄÄÄÄÄÄÄÄÄÄ ÄÄÄÄÄÄÄÄÄÄÄÄÄ Nuclide mrem/yr fract. mrem/yr fract. mrem/yr fract. mrem/yr fract.

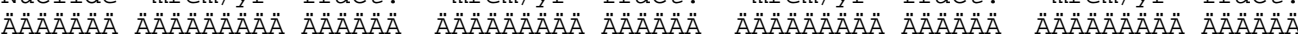

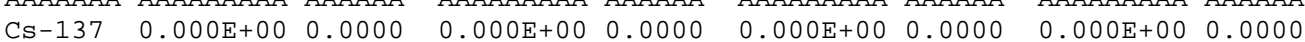

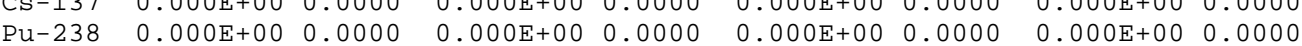

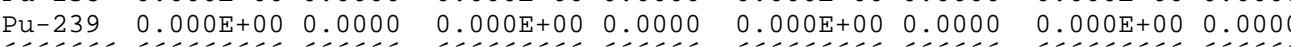

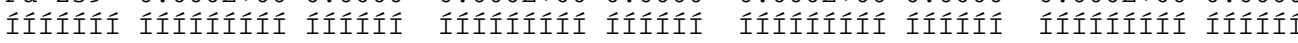
$\begin{array}{lllllll}\text { Total } & 0.000 \mathrm{E}+00 & 0.0000 & 0.000 \mathrm{E}+00 & 0.0000 & 0.000 \mathrm{E}+00 & 0.0000\end{array}$ $\odot .000 \mathrm{E}+00 \odot .0000$ $\odot .000 \mathrm{E}+00 \quad 0.0000$ $0.000 \mathrm{E}+000.0000$ $\odot .000 \mathrm{E}+00 \quad 0.0000$ ÄÄÄÄÄÄÄÄÄ ÄÄÄÄÄÄ $0.000 \mathrm{E}+00 \quad 0.0000$ $\odot .000 \mathrm{E}+00 \quad 0.0000$

* Sum of all water independent and dependent pathways. 
Total Dose Contributions $\operatorname{TDOSE}(i, p, t)$ for Individual Radionuclides (i) and Pathways $(p)$ As mrem/yr and Fraction of Total Dose At $t=1.000 \mathrm{E}+02$ years
water Independent Pathways (Inhalation excludes radon) Radio- Ä̈̈Ä

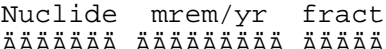
CS-137 5.371E-01 0.9847 Pu-238 5.019E- $07 \quad 0.000 \odot$ $\mathrm{Pu}-239$ 1.230E-05 0.0000 Íííííí íííííííi ííííi Total 5.371E-01 0.9847 Inhalation Radon Plant
ÄÄÄÄÄÄÄÄÄÄÄÄÄÄÄÄ Meat Soil

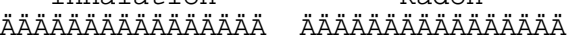
Ӓ̈̈ÄÄÄÄÄÄÄÄÄÄÄÄÄ ÄÄÄÄÄ̈̈̈̈̈̈̈̈̈

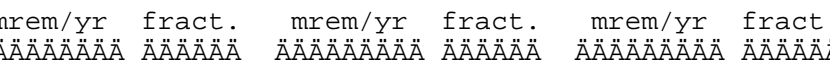
$\mathrm{mrem} / \mathrm{yr}$ fract mrem/yr fract. ÄÄÄÄÄÄÄÄÄ ÄÄÄÄÄA $\odot .000 \mathrm{E}+0 \odot \quad 0.0000$ $\odot .000 \mathrm{E}+0 \odot$ $\odot .000 \odot$ .526E - $06 \quad 0.0000$ $2.876 \mathrm{E}-04 \quad 0.0005 \quad 0.000 \mathrm{E}+00 \quad \odot .0000$ (1) $0.000 \mathrm{E}+00 \quad 0.0000$ $0.000 \mathrm{E}+00 \quad 0.0000$ $0.000 E+00 \quad 0.0000$ ÍÍÍt Íííííííi ííííi Íííííííí ÍÍííí

$0.000 \mathrm{E}+000.0000$

$\odot .000 \mathrm{E}+00$ ๑. 0.000 $0.000 \mathrm{E}+000.0000$ $0.000 \mathrm{E}+00 \quad 0.0000$ IIIIIIIIIIÍí ÍÍÍÍí

$6.543 \mathrm{E}-03 \quad 0.0120 \quad 0.000 \mathrm{E}+00 \quad 0.0000 \quad 0.000 \mathrm{E}+00 \quad 0.0000 \quad 0.000 \mathrm{E}+00 \quad 0.00000 .000 \mathrm{E}+000.0000$

Total Dose Contributions $\operatorname{TDOSE}(i, p, t)$ for Individual Radionuclides (i) and Pathways $(p)$ As $\mathrm{mrem} / \mathrm{yr}$ and Fraction of Total Dose At $t=1.000 \mathrm{E}+02$ years Fish Radon Water Dependent Pathways

Water Plant

Meat Milk ÄÄÄÄÄÄÄÄÄÄÄÄÄÄ ÄÄÄÄÄÄÄÄÄÄÄÄÄÄ Ä̈̈̈̈̈̈̈̈̈̈̈̈̈̈̈̈̈̈̈̈̈̈

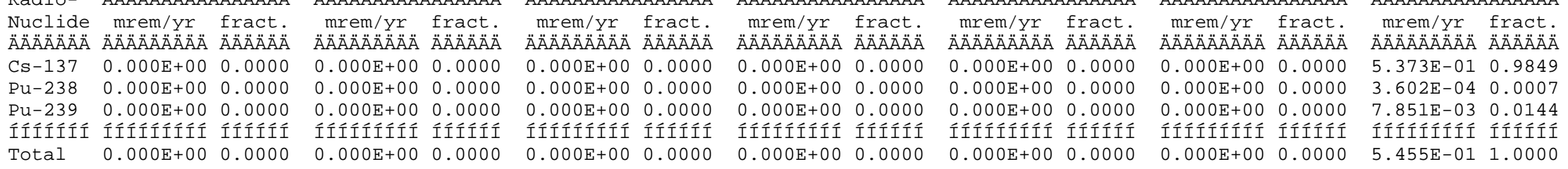

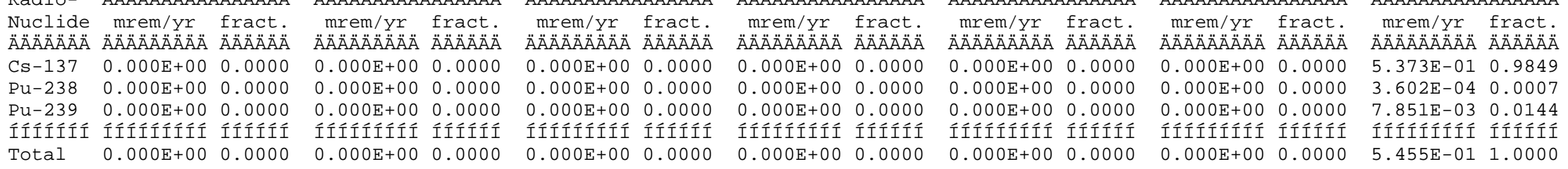

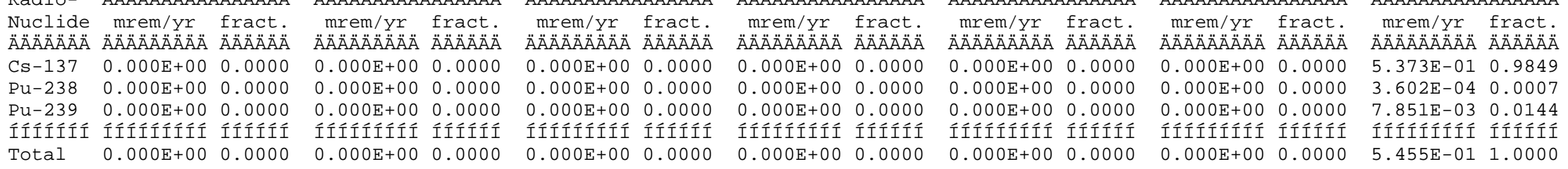

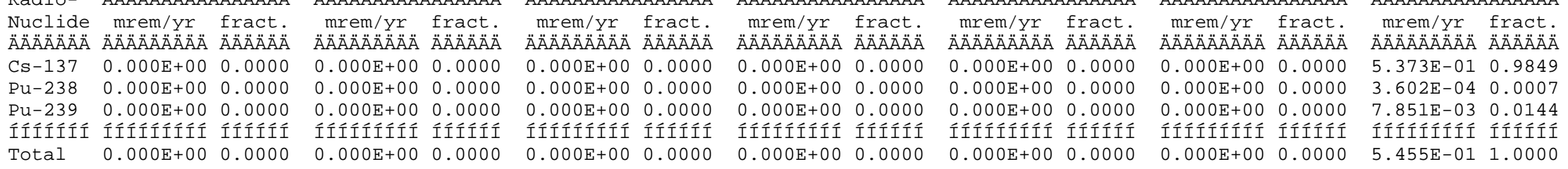

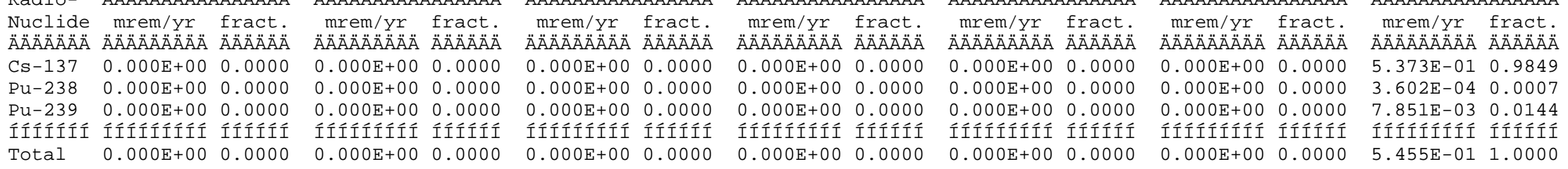

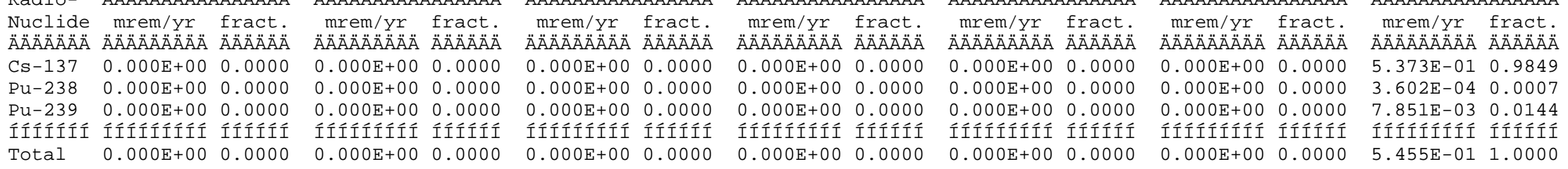

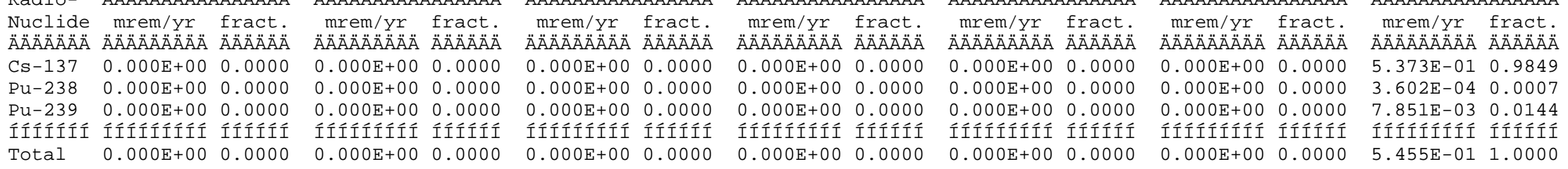
$0^{*}$ Sum of all water independent and dependent pathways. 
Total Dose Contributions $\operatorname{TDOSE}(i, p, t)$ for Individual Radionuclides (i) and Pathways $(p)$ As mrem/yr and Fraction of Total Dose At $t=3.000 \mathrm{E}+02$ years
water Independent Pathways (Inhalation excludes radon) Radio- Ä̈̈Ä Nuclide mrem/yr fract. Inhalation Radon Plant Meat

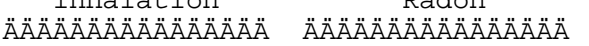
ÄÄÄÄÄÄÄÄÄÄÄÄÄÄÄÄÄ Ӓ̈̈̈ӒÄÄÄÄÄÄÄÄÄÄÄÄ mrem/yr fract mrem/yr fract. mrem/yr fract.

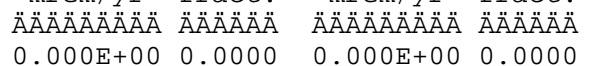
Cs-137 $0.0 \odot \odot E+\odot \odot \quad \odot .0 \odot \odot \odot$ $\mathrm{Pu}-238 \quad 0.000 \mathrm{E}+00 \quad 0.0000$ Pu-239 $0.000 \mathrm{E}+00 \quad 0.0000$ íííííí íííííííí ííííít Total $0.000 \mathrm{E}+0 \odot \quad 0.0000$ mrem/yr fract AÁAAAAAAA AAAAAÁ $\odot . \odot \odot \odot E+\odot \odot \odot . \odot \odot \odot \odot$ $\begin{array}{llllll}0.000 \mathrm{E}+00 & 0.0000 & 0.000 \mathrm{E}+00 & 0.0000 & 0.000 \mathrm{E}+0 \odot & 0.0000\end{array}$ $\begin{array}{llllll}0.000 E+00 & 0.000 \odot & 0.000 E+0 \odot & 0.000 \odot & 0.000 E+0 \odot & 0.000 \odot\end{array}$ $0.000 \mathrm{E}+0 \odot$ $\odot .0000$ $0.000 \mathrm{E}+000.0000$ $0.000 \mathrm{E}+00 \quad 0.0000$

Íííííííi ííííí íííííííi ííííi ííííííí ííííi

IIIIIIÍíi Ííííi $\odot$ Total Dose Contributions TDOSE $(i, p, t)$ for Individual Radionuclides $(i)$ and Pathways $(p)$
As mrem/yr and Fraction of Total Dose At $t=3.000 E+02$ years Fish Radon Water Dependent Pathways Plant

Meat Milk

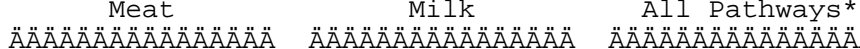
mrem/yr fract mrem/yr fract mrem/yr fract. mrem/yr Radio- ÄÄÄÄÄÄÄÄÄÄÄÄÄÄ ÄÄÄÄÄÄÄÄÄÄÄÄÄÄÄ ÄÄÄÄÄÄÄÄÄÄÄÄÄÄÄ ÄÄÄÄÄÄÄÄÄÄÄÄÄ Nuclide mrem/yr fract. mrem/yr fract. mrem/yr fract. mrem/yr fract. ÄÄÄÄÄÄÄ ÄÄÄÄÄÄÄÄÄ ÄÄÄÄÄÄ ÄÄÄÄÄÄÄÄÄ ÄÄÄÄÄÄ ÄÄÄÄÄÄÄÄÄ ÄÄÄÄÄÄ ÄÄÄÄÄÄÄÄÄ ÄÄÄÄÄ

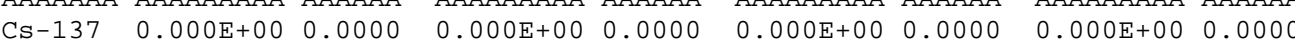

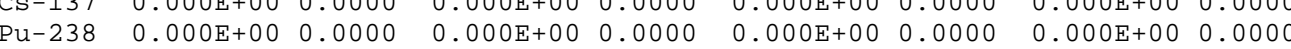

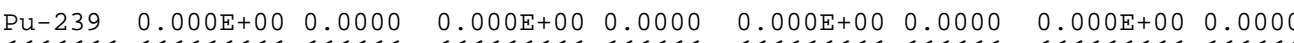
íííííi ííííííí ííííi ííííííí ííííi ííííííi ííííi $\begin{array}{lllllll}\text { Total } & 0.000 \mathrm{E}+00 & 0.0000 & 0.000 \mathrm{E}+00 & 0.0000 & 0.000 \mathrm{E}+00 & 0.0000\end{array}$ Íííííííí ÍÍÍíí $\Theta *$ Sum of all water independent and dependent pathways.
ÄÄÄÄÄÄÄÄÄÄÄÄÄÄÄÄÄ ÄÄÄÄÄÄÄÄÄÄÄÄÄÄÄÄÄ mrem/yr fract. mrem/yr fract. AАAAAAAAA ÄÄÄÄÄÄ ÄÄÄÄÄÄÄÄÄ ÄÄÄÄÄ̈ $0.000 E+00 \quad 0.0000 \quad 0.000 E+00 \quad 0.00000$ $0.000+00$ 0.0000 $0.000 E+00$ 0.0000 Íííííííi ÍÍííí 
Total Dose Contributions $\operatorname{TDOSE}(i, p, t)$ for Individual Radionuclides (i) and Pathways $(p)$ As mrem/yr and Fraction of Total Dose At $t=1.000 \mathrm{E}+03$ years
water Independent Pathways (Inhalation excludes radon) Radio- Ä̈̈Ä Nuclide mrem/yr fract. Inhalation Radon Plant Meat

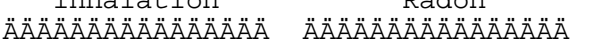
ÄÄÄÄÄÄÄÄÄÄÄÄÄÄÄÄÄ Ӓ̈ӒÄÄÄÄÄÄÄÄÄÄÄÄÄÄ mrem/yr fract

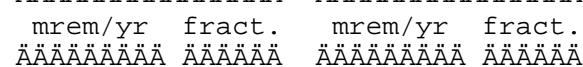

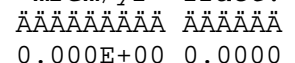
Cs-137 $0.0 \odot \odot E+\odot \odot \quad \odot . \odot \odot \odot \odot$ $\mathrm{Pu}-238 \quad 0.000 \mathrm{E}+00 \quad 0.0000$ Pu-239 $0.000 \mathrm{E}+00 \quad 0.0000$ íííííí íííííííí ííííít Total $0.000 \mathrm{E}+0 \odot \quad 0.0000$ mrem/yr fract AÁÄÄÁÄÄ ÄÄÁÄA $\begin{array}{llllll}0.000 \mathrm{E}+00 & 0.0000 & 0.000 \mathrm{E}+00 & 0.0000 & 0.000 \mathrm{E}+00 & 0.0000\end{array}$ $\begin{array}{llllll}0.000 \mathrm{E}+00 & 0.0000 & 0.000 \mathrm{E}+00 & 0.0000 & 0.000 \mathrm{E}+00 & 0.0000\end{array}$ $\begin{array}{lllllll}0.000 E+00 & 0.0000 & 0.000 E+00 & 0.0000 & 0.000 E+00 & 0.0000\end{array}$

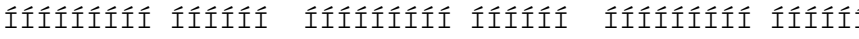
$0.000 \mathrm{E}+00$ 0. 0000 $\odot .000 \mathrm{E}+0 \odot \quad \odot .0000$ $0.000 \mathrm{E}+00 \quad 0.0000$ Íííííííi Ííííí $0.000 \mathrm{C}+00$ 0.0000 $0.000 \mathrm{E}+00$ 0.0000 M $0.000 \mathrm{E}+00$ - 0.0000 $\odot .00 \odot \mathrm{E}+\odot \odot \quad 0.000 \odot$ $0.000 E+00 \quad 0.0000$ íííííííí ííííí

$\odot, 00 \odot \mathrm{E}+0 \odot$ ०. $00 \odot \odot$
Soil ӒÄÄÄÄÄÄÄÄÄÄÄÄÄÄÄ $\mathrm{mrem} / \mathrm{yr}$ fract ÄÄÄÄÄÄÄÄÄ ÄÄÄÄÄÄ $0.000 \mathrm{E}+00 \quad 0.0000$ $0.000 E+00 \quad 0.0000$ $0.000 E+00 \quad 0.0000$

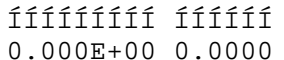

Total Dose Contributions TDOSE( $i, p, t)$ for Individual Radionuclides (i) and Pathways ( $p$ ) As $\mathrm{mrem} / \mathrm{yr}$ and Fraction of Total Dose At $t=1.000 \mathrm{E}+03$ years water Dependent Pathways Radon Plant

Meat Milk

All Pathways*

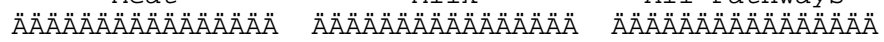
mrem/yr fract. mrem/yr fract. mrem/yr fract. Nuclide mrem/yr fract. mrem/yr fract. mrem/yr fract. mrem/yr fract.

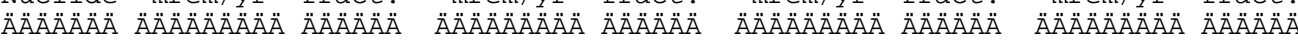

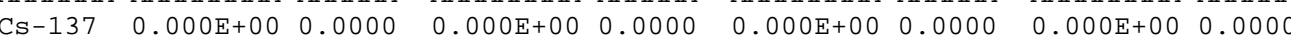

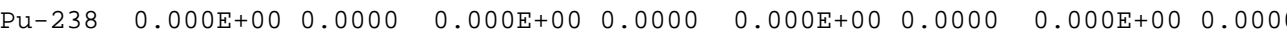

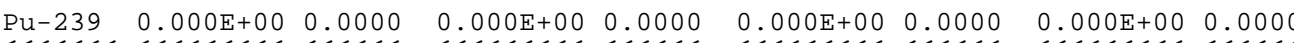
íííííi ííííííí ííííi ííííííí ííííi ííííííi ííííi $\begin{array}{lllllll}\text { Total } & 0.000 \mathrm{E}+00 & 0.0000 & 0.000 \mathrm{E}+00 & 0.0000 & 0.000 \mathrm{E}+00 & 0.0000\end{array}$ Íííííííi Ííííi AAAAAAAÄ $0.000 \mathrm{E}+00 \quad 0.0000$ $\odot .000 \mathrm{E}+000.0000$ A.ÄÄÄÄÄÄ ÄÄÄÄÄ

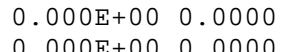
$\begin{array}{llll}0.000 E+0 \odot & 0.000 \odot & 0.000 \mathrm{E}+0 \odot & 0.000 \odot\end{array}$ ÄÄÄÄÄÄÄÄÄ ÄÄÄÄÄÄ ๑. $0 \odot \odot E+\odot \odot$ $0.0 \odot \odot \odot$

* Sum of all water independent and dependent pathways. 
Parent and Dose/Source Ratios Summed Over All Pathways

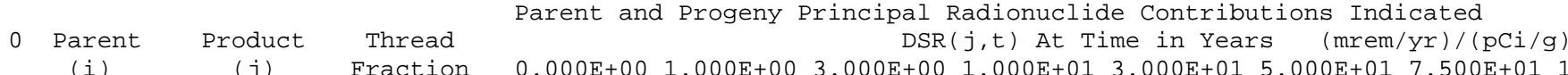

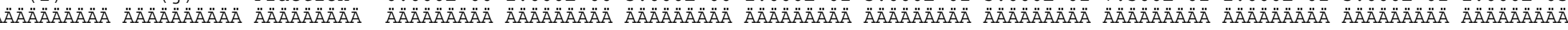

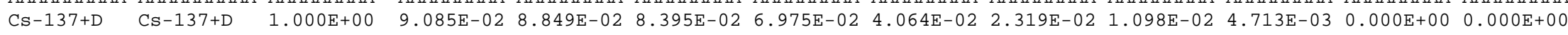

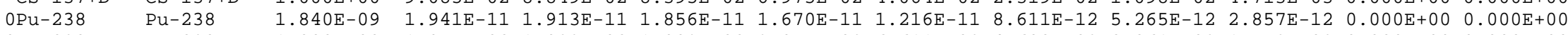

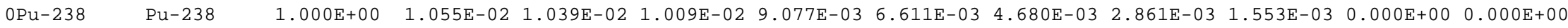

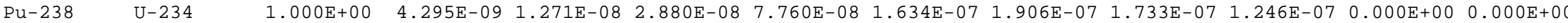
$\mathrm{Pu}-238 \quad \mathrm{Th}-230 \quad 1.000 \mathrm{E}+00 \quad 3.146 \mathrm{E}-14 \quad 2.179 \mathrm{E}-13 \quad 1.125 \mathrm{E}-12 \quad 9.219 \mathrm{E}-12 \quad 5.968 \mathrm{E}-11 \quad 1.223 \mathrm{E}-10 \quad 1.794 \mathrm{E}-10 \quad 1.858 \mathrm{E}-10 \quad 0.000 \mathrm{E}+000.000 \mathrm{E}+00$

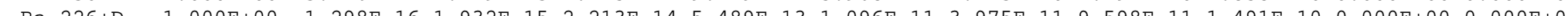
Pu-238

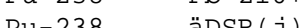
Pu Pu 239 Pu-239 U-235+ Pu-239 Pa-231 $1.000 \mathrm{E}+00$ PU-239 AC-227+D $1.000 \mathrm{E}+00$ äDSR $(j)$ $1.298 \mathrm{E}-16 \quad 1.932 \mathrm{E}-15 \quad 2.213 \mathrm{E}-14 \quad 5.489 \mathrm{E}-13 \quad 1.096 \mathrm{E}-11 \quad 3.975 \mathrm{E}-11 \quad 9.598 \mathrm{E}-111.491 \mathrm{E}-10 \quad 0.000 \mathrm{E}+00 \quad 0.000 \mathrm{E}+00$

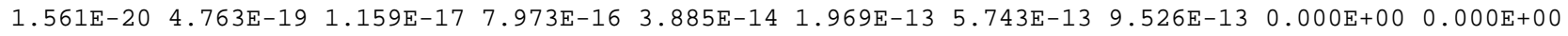

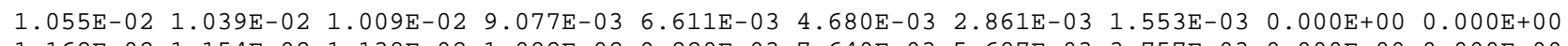
1.162E-02 1.154E-02 1.138E-02 1.082E-02 9.220E-03 7.640E-03 5.687E-03 3.757E-03 $0.000 \mathrm{E}+00$ 0.000E+00

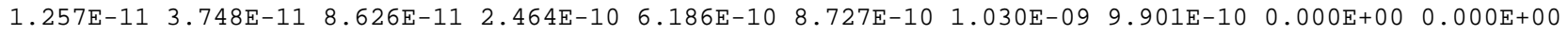
$\begin{array}{llllllllllll}1.387 \mathrm{E}-16 & 9.613 \mathrm{E}-16 & 4.963 \mathrm{E}-15 & 4.078 \mathrm{E}-14 & 2.660 \mathrm{E}-13 & 5.506 \mathrm{E}-13 & 8.215 \mathrm{E}-13 & 8.717 \mathrm{E}-13 & 0.000 \mathrm{E}+00 & 0.000 \mathrm{E}+00\end{array}$

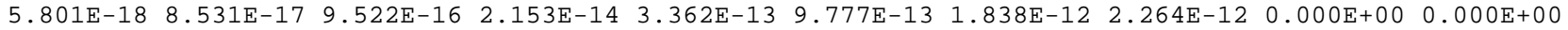

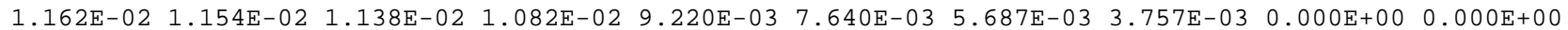

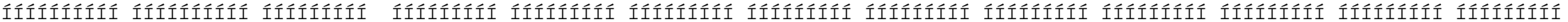
The DSR includes contributions from associated (half-life ó 180 days) daughters.

Single Radionuclide Soil Guidelines $G(i, t)$ in $\mathrm{pCi} / \mathrm{g}$ Basic Radiation Dose Limit $=2.500 \mathrm{E}+01 \mathrm{mrem} / \mathrm{yr}$

ONuclide

\begin{tabular}{|c|c|c|c|c|c|c|c|c|c|c|}
\hline 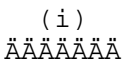 & 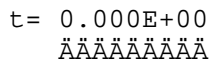 & $\begin{array}{l}1 . \odot \odot \odot E+\odot \odot \\
\ddot{A} \ddot{A} A \ddot{A} \ddot{A} A \ddot{A} A \ddot{A}\end{array}$ & 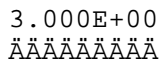 & 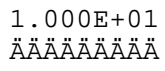 & $\begin{array}{l}3.000 E+\odot 1 \\
\ddot{A} A \ddot{A} A \ddot{A} A \ddot{A} A \ddot{A} \ddot{A}\end{array}$ & 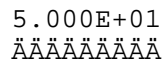 & $\begin{array}{l}7.500 E+01 \\
\ddot{A} \ddot{A} A \ddot{A} A \ddot{A} A ̈ A ̈ A ̈\end{array}$ & $\begin{array}{l}1 . \odot \odot \odot E+\odot 2 \\
\ddot{A} A \ddot{A} \ddot{A} A \ddot{A} A \ddot{A} \ddot{A}\end{array}$ & 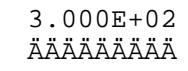 & $\begin{array}{l}1 . \odot \odot \odot E+\odot 3 \\
\ddot{A} \ddot{A} A \ddot{A} A \ddot{A} A \ddot{A} A \ddot{A}\end{array}$ \\
\hline-137 & $2.752 \mathrm{E}+02$ & $2.825 \mathrm{E}+02$ & $2.978 \mathrm{E}+02$ & $3.584 \mathrm{E}+02$ & $6.151 \mathrm{E}+02$ & $1.078 \mathrm{E}+03$ & $2.277 \mathrm{E}+03$ & $5.305 E+03$ & *8.704E+13 & $* 8.704 \mathrm{E}+13$ \\
\hline & $2.370 E+03$ & $2.405 \mathrm{E}+03$ & $2.478 \mathrm{E}+03$ & +03 & +03 & 5 & 3 & 94 & -13 & +13 \\
\hline & $2.152 \mathrm{E}+03$ & 2.16 & $2.197 \mathrm{E}+03$ & 2.31 & 2.71 & 3. & 4. & & ${ }^{*} 6$ & *6. \\
\hline & Ííííííííí & Íííííííí & Íííííííí & Íííííííí & Íííííííí & Íííííííí & Íííííííí & Íííííííít & Íííííííí & Íííííít \\
\hline
\end{tabular}
$\odot$ ${ }^{\star}$ At specific activity limit

Summed Dose/Source Ratios DSR(i,t) in (mrem/yr)/(pCi/g)

and Single Radionuclide Soil Guidelines $\mathrm{G}(i, \mathrm{t})$ in $\mathrm{pCi} / \mathrm{g}$

at $\operatorname{tmin}=$ time of minimum single radionuclide soil guideline and at $\mathrm{tmax}=\mathrm{time}$ of maximum total dose $=0.000 \mathrm{E}+00$ years

\begin{tabular}{|c|c|c|c|c|c|c|}
\hline$(i)$ & 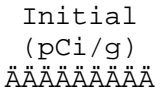 & $\begin{array}{l}(\text { years }) \\
\text { ÄÄÄÄÄÄÄÄÄÄÄÄÄÄÄ }\end{array}$ & 1) & 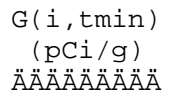 & $\operatorname{DSR}(i, \operatorname{tmax})$ & \\
\hline & & & & & & \\
\hline & & & & & & \\
\hline $1-23$ & & $+\odot \odot$ & $\begin{array}{l}1.162 \mathrm{E}-02 \\
-162\end{array}$ & $\begin{array}{ll}03 \\
03\end{array}$ & :162E-02 & \\
\hline
\end{tabular}




\section{ONuclide Parent THF(i)}

ÄÄÄÄÄÄÄ ÄÄÄÄÄÄÄ ÄÄÄÄÄÄÄÄA

Cs-137 Cs-137 1.000E+@O

๑Pu-238 Pu-238 1.840E- 99

$\mathrm{Pu}-238$ Pu-238 1.000E+००

$\mathrm{Pu}-238$ äDOSE $(j)$

OU-234 PU-238 1.000E+०0

OTh-230 Pu-238 1.000E+00

ORa-226 Pu-238 $1.000 \mathrm{E}+00$

$\odot \mathrm{Pb}-210 \quad \mathrm{Pu}-238 \quad 1.000 \mathrm{E}+\Theta 0$

๑Pu-239 Pu-239 $1.000 \mathrm{E}+\odot \odot$

OU-235 Pu-239 1.000E+०O

$\odot \mathrm{Pa}-231 \quad \mathrm{Pu}-239 \quad 1.000 \mathrm{E}+\odot 0$

OAC-227 Pu-239 1. $000 \mathrm{E}+00$

IIIITIIÍ ÍÍÍÍÍí ÍÍÍÍÍÍI

Individual Nuclide Dose Summed Over All Pathways

Parent Nuclide and Branch Fraction Indicated

$\operatorname{DOSE}(j, t), \quad \mathrm{mrem} / \mathrm{yr}$

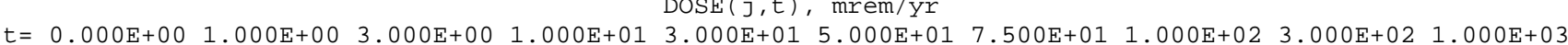

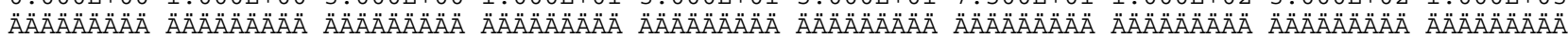

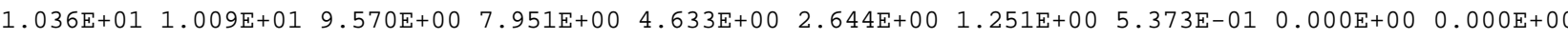
4.503E-12 4.437E-12 4.307E-12 3.875E-12 2.822E-12 1.998E-12 $1.221 \mathrm{E}-12 \quad 6.628 \mathrm{E}-13 \quad 0.000 \mathrm{E}+00 \quad 0.000 \mathrm{E}+00$ 2.447E-03 2.411E-03 2.341E-03 2.106E-03 1.534E-03 1.086E-03 6.638E-04 3.602E-04 $0.000 \mathrm{E}+00 \quad 0.000 \mathrm{E}+00$ $2.447 \mathrm{E}-03$ 2.411E-03 2.341E-03 2.106E-03 1.534E-03 1.086E-03 6.638E-04 3.602E-04 $0.000 \mathrm{E}+00 \quad 0.000 \mathrm{E}+00$

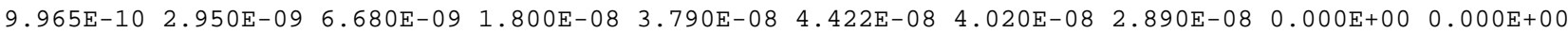

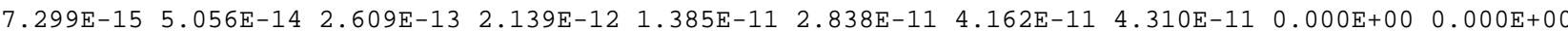

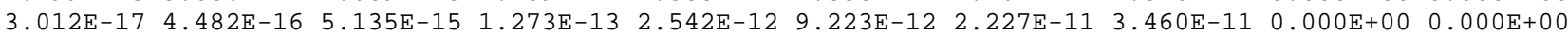

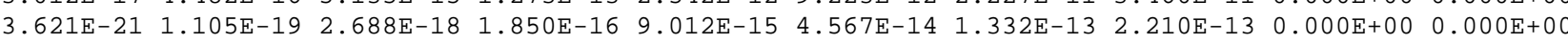

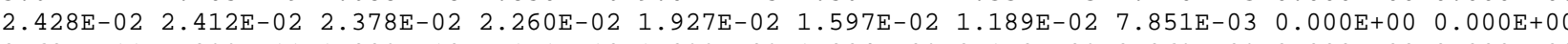

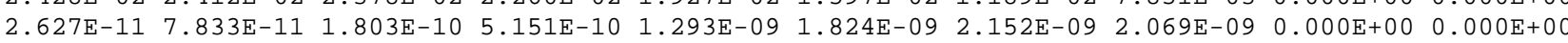
2.900E-16 2.009E-15 1.037E-14 8.522E-14 5.559E-13 1.151E-12 $1.717 \mathrm{E}-12$ 1.822E-12 $0.000 \mathrm{E}+000.0000 \mathrm{E}+00$ 1.212E-17 1.783E-16 1.990E-15 4.500E-14 7.027E-13 2.043E-12 3.842E-12 $4.733 \mathrm{E}-12 \quad 0.000 \mathrm{E}+00 \quad 0.000 \mathrm{E}+00$

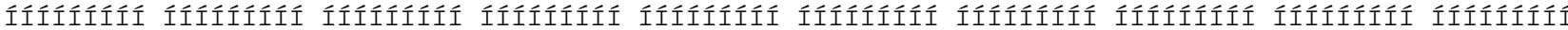

THF( $i)$ is the thread fraction of the parent nuclide. 
1RESRAD, Version 6.3 Summary : CAU 383 RMA

$\mathrm{T} \ll$ Limit $=180$ days

05/11/2006 16:54 Page 23

File: CAU 383 RMA. RAD

\section{onuclide Parent THF(i)}

$(j)$ (i)

ÄÄÄÄÄÄÄ ÄÄÄÄÄÄÄ ÄÄÄÄÄÄÄÄ̈̈

Cs-137 Cs-137 1.000E+00

OPU-238 Pu-238 1.840E- 09

$\mathrm{Pu}-238$ Pu-238 1.000E+००

$\mathrm{Pu}-238$ äS $(j)$ :

OU-234 Pu-238 $1.000 \mathrm{E}+00$

OTh-230 Pu-238 1.000E+00

ORa-226 Pu-238 $1.000 \mathrm{E}+00$

$\odot \mathrm{Pb}-210 \quad \mathrm{Pu}-238 \quad 1.000 \mathrm{E}+00$

๑Pu-239 Pu-239 $1.000 \mathrm{E}+\odot \odot$

๑U-235 PU-239 1.000E+०९

$\odot \mathrm{Pa}-231 \quad \mathrm{Pu}-239 \quad 1.000 \mathrm{E}+\odot 0$

$\odot A C-227 \quad \mathrm{Pu}-239 \quad 1.000 \mathrm{E}+00$

IIIIIííí Íííííí íííííííi

THF( $i)$ is the thread fraction

ORESCALC. EXE execution time =

Individual Nuclide Soil Concentration

Parent Nuclide and Branch Fraction Indicated

$$
\mathrm{S}(j, \mathrm{t}), \mathrm{pCi} / \mathrm{g}
$$

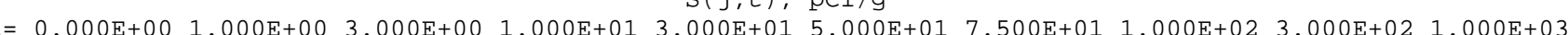
ÄÄÄÄÄÄÄÄA $\begin{array}{llllllllll}1.140 \mathrm{E}+02 & 1.114 \mathrm{E}+02 & 1.063 \mathrm{E}+02 & 9.040 \mathrm{E}+01 & 5.684 \mathrm{E}+01 & 3.574 \mathrm{E}+01 & 2.001 \mathrm{E}+01 & 1.120 \mathrm{E}+01 & 1.082 \mathrm{E}-01 & 9.584 \mathrm{E}-09\end{array}$ 4.269E-10 4.234E-10 4.166E-10 3.936E-10 3.346E-10 2.845E-10 2.322E-10 $1.896 \mathrm{E}-10 \quad 3.739 \mathrm{E}-11 \quad 1.273 \mathrm{E}-13$ 2.320E-01 2.301E-01 2.264E-01 2.139E-01 1.819E-01 1.546E-01 $1.262 \mathrm{E}-01$ 1.030E-01 2.032E-02 6.921E-05 2.320E-01 2.301E-01 2.264E-01 2.139E-01 1.819E-01 1.546E-01 1.262E-01 1.030E-01 2.032E-02 6.921E-05 $0.000 \mathrm{E}+00$ 6.522E-07 1.924E-06 6.048E-06 1.534E-05 2.162E-05 2.629E-05 2.843E-05 1.594E-05 1.512E-07 $0.000 \mathrm{E}+00$ 2.944E-12 $2.620 \mathrm{E}-11 \quad 2.800 \mathrm{E}-10 \quad 2.256 \mathrm{E}-09 \quad 5.622 \mathrm{E}-09$ 1.107E-08 $1.726 \mathrm{E}-08$ 6.011E-08 $\quad$ 8.298E-08

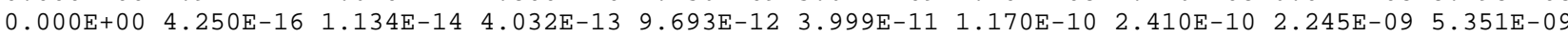
$\odot .00 \odot \mathrm{E}+0 \odot$ 3.283E-18 $2.598 \mathrm{E}-16 \quad 2.957 \mathrm{E}-14$ 1.911E-12 $1.187 \mathrm{E}-11$ 4.630E-11 $1.141 \mathrm{E}-10$ 1.690E-09 $4.681 \mathrm{E}-09$

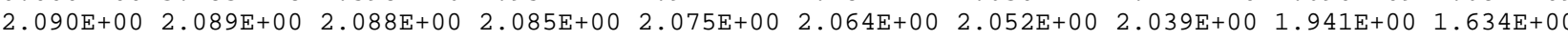

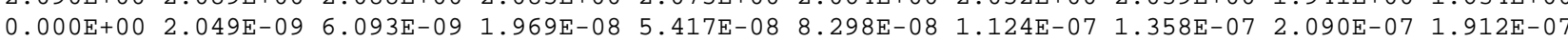
$0.000 \mathrm{E}+00$ 2.165E-14 1.926E-13 2.054E-12 1.647E-11 4.081E-11 7.981E-11 $1.237 \mathrm{E}-10$ 4.094E-10 4.787E-10 $0.000 \mathrm{E}+00 \quad 2.270 \mathrm{E}-16 \quad 5.918 \mathrm{E}-15$ 1.943E-13 $3.800 \mathrm{E}-12 \quad 1.310 \mathrm{E}-11$ 3.156E-11 $5.500 \mathrm{E}-11 \quad 2.333 \mathrm{E}-10 \quad 2.868 \mathrm{E}-10$

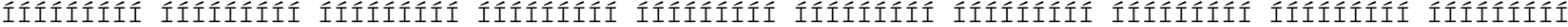
of the parent nuclide.

1.47 seconds 


\section{Appendix E}

Closure Summary

Uncontrolled When Printed 


\title{
E.1.0 Closure Summary
}

A land-use restriction will be applied as part of the closure in place alternative for CAU 383. These restrictions will be applied to control use and limit access to the site to prevent inadvertent exposure to the radionuclide contaminated soil identified in the muckpile (CAS 12-06-06) and the benzo(a)pyrene identified in the oil spill (CAS 12-25-02). The radionuclide contamination was identified at various depths in the muckpile at levels which exceeded the FALs. The benzo(a)pyrene was only found in one sample at the surface in the oil spill CAS at a level which exceeded the FAL. The completed land-use restriction form and associated documentation are included.

The following warning appears on the signs posted around CAU 383.

\author{
WARNING \\ Radiologically Contaminated Areas \\ FFACO Sites CAU 383, Area 12 E-Tunnel Sites \\ CAS 12-28-02, Radioactive Material Area \\ CAS 12-06-06, E-Tunnel Muckpile \\ CAS 12-25-02, Oil Spill
}

Access to this area is not permitted without U.S. Government permission.

Before working in this area,

Contact Real Estate Services at 295-2528

This site can be closed without further corrective action. 


\section{CAU Use Restriction Information}

CAU Number/Description: 383/Area 12 E-Tunnel Sites

Applicable CAS Numbers/Descriptions: 12-06-06/Muckpile, 12-25-02/Oil Spill, 1228-02/Radioactive Material

Contact (organization/project): DTRA Environmental Program Manager

Surveyed Area (UTM, Zone 11, NAD 27, meters): See Attached Figure NW Corner $4,115,986.2 \mathrm{~N} \quad 571.510 .5 \mathrm{E}$

W Center $4,115,938.0 \mathrm{~N} \quad 571,475.6 \mathrm{E}$

SW Corner $4,115,685.5 \mathrm{~N} \quad 571,517.4 \mathrm{E}$

SE Corner $4,115,656.1 \mathrm{~N} \quad 572,005.6 \mathrm{E}$

NE Corner $4,115,814.5 \mathrm{~N} \quad 572,067.8 \mathrm{E}$

Survey Date: 6/23/2004_Survey Method (GPS, etc): GPS

Site Monitoring Requirements: Certify that markers and postings are in place, intact. and readable.

Required Frequency (quarterly, annually?): Annually

If Monitoring Has Started, Indicate last Completion Date:

\section{Use Restrictions}

The future use of any land related to this Corrective Action Unit (CAU), as described by the above surveyed location, is restricted from any DOE or Air Force activity that may alter or modify the containment control as approved by the state and identified in the CAU Closure Report or other CAU documentation unless appropriate concurrence is obtained in advance.

Comments: See the Closure Report for additional information on the condition of the site(s). Results of the annual inspection will be provided in the annual Post Closure Inspection Monitoring Report.

Submitted By:

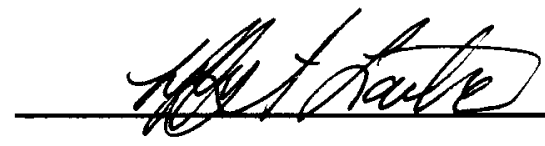

Submitted By:
Date:

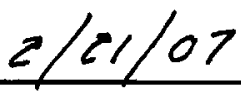

Date: 
cc with copy of survey map (paper and digital (.dgn) formats):

CAU Files ( 2 copies) 


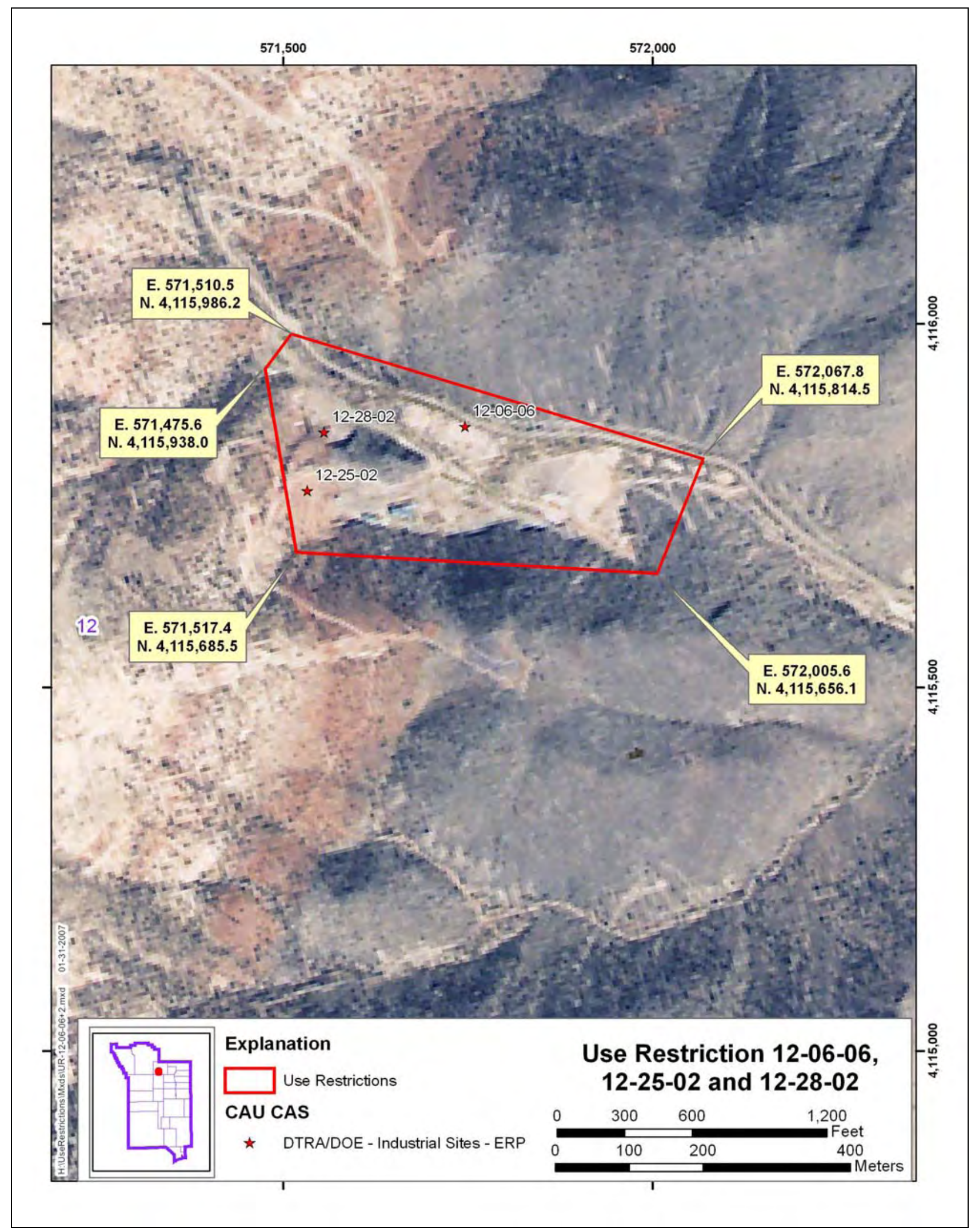

Figure E.1-1

CAU 383 Area 12 E-Tunnel Sites 


\section{LIBRARY DISTRIBUTION LIST}

U.S. Department of Energy

National Nuclear Security Administration

Nevada Site Office

Technical Library

P.O. Box 98518, M/S 505

Las Vegas, NV 89193-8518

U.S. Department of Energy

Office of Scientific and Technical Information

P.O. Box 62

Oak Ridge, TN 37831-0062

Southern Nevada Public Reading Facility

c/o Nuclear Testing Archive

P.O. Box 98521, M/S 400

Las Vegas, NV 89193-8521

Manager, Northern Nevada FFACO

Public Reading Facility

c/o Nevada State Library \& Archives

Carson City, NV 89701-4285
1 (Uncontrolled, electronic copy)

1 (Uncontrolled, electronic copy)

2 (Uncontrolled, electronic copies)

1 (Uncontrolled, electronic copy) 